\title{
Site Observational Worik Plan For the UMTRa PROJect SITE at Ambrosia LaKe, NeW MeXico
}

February 1995

\section{DISCLAIMER}

This report was prepared as an account of work sponsored by an agency of the United States Government. Neither the United States Government nor any agency thereof, nor any of their employees, makes any warranty, express or implied, or assumes any legal liability or responsibility for the accuracy, completeness, or usefulness of any information, apparatus, product, or process disclosed, or represents that its use would not infringe privately owned rights. Reference herein to any specific commercial product, process, or service by trade name, trademark, manufacturer, or otherwise does not necessarily constitute or imply its endorsement, recommendation, or favoring by the United States Government or any agency thereof. The views and opinions of authors expressed herein do not necessarily state or reflest those of the United States Government or any agency thereof.

\section{DISTRIBUTION OF THIS DOCUMENT IS UNLIMTTED WW}

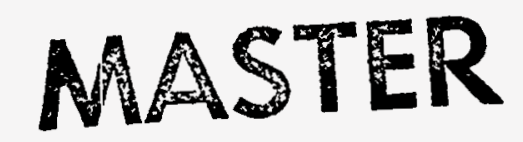




\section{INTENDED FOR PUBLIC RELEASE}

This report has been reproduced from the best available copy. Available in paper copy and microfiche.

Number of pages in this report: 552

DOE and DOE contractors can obtain copies of this report from:

Office of Scientific and Technical Information

P.O. Box 62

Oak Ridge, TN 37831

(615) 576-8401

This report is publicly available from:

National Technical Information Service

Department of Commerce

5285 Port Royal Road

Springfield, VA 22161

(703) $487-4650$ 


\section{DISCLAIMER}

Portions of this document may be illegible in electronic image products. Images are produced from the best available original document. 


\section{SITE OBSERVATIONAL WORK PLAN \\ FOR THE UMTRA PROJECT SITE \\ AT AMBROSIA LAKE, NEW MEXICO}

February 1995

Prepared for

U.S. Department of Energy

UMTRA Project Office

Albuquerque, New Mexico

Prepared by

Jacobs Engineering Group Inc.

Albuquerque, New Mexico

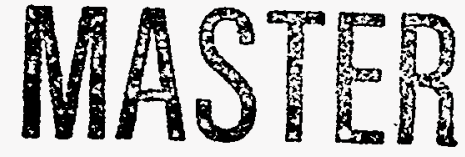




\section{EXECUTIVE SUMMARY}

Ground water compliance for the Uranium Mill Tailings Remedial Action (UMTRA) Project sites, including the Ambrosia Lake, New Mexico, site, is governed by the Uranium Mills Tailings Radiation Control Act (42 USC $\$ 7901$ et seq.) and the U.S. Environmental Protection Agency's Health and Environmental Protection Standards for Uranium and Thorium Mill Tailings (40 CFR Part 192; 60 FR 2854). The EPA standards describe specific conditions for which the U.S. Department of Energy (DOE) may apply for supplemental standards for contaminated ground water rather than meeting background levels or numerical standards.

To achieve compliance with Subpart A of the EPA standards the residual radioactive materials are currently being consolidated on the site by the DOE in a disposal cell, isolating them from direct human or ecological contact and further dispersion into the environment. Completion of the disposal cell is scheduled for early 1995. An environmental assessment (DOE, 1987a) and a Finding of No Significant Impact (FONSI) (DOE, 1987b) were completed in 1987. Concurrence with the UMTRA Surface Project Ambrosia Lake remedial action plan (RAP) (DOE, 1991) was granted by the U.S. Nuclear Regulatory Commission (NRC) and state of New Mexico in 1990 (NRC, 1990). The DOE deferred compliance with Subpart B of the EPA standards in the Surface Project RAP. This site observational work plan (SOWP) is the first document to address ground water compliance under Subpart B at the Ambrosia Lake site.

The Ambrosia Lake UMTRA Project site is within the Grants Mineral Belt and was one of numerous uranium mills supplied by many local mines. Ground water contamination at the site occurred as a result of uranium mill operations. During the period from 1958 to 1963 , approximately 3.0 million tons ( 2.7 million tonnes) of uranium-bearing ore were processed by crushing and grinding, and uranium was removed through a chemical process (DOE, 1991). Contamination of ground water resulted from discharge of waste water, infiltration of water through the tailings pile, hydraulic placement of mill tailings in nearby mines, and water pumped from mine shafts.

The uppermost aquifer at the Ambrosia Lake site is defined as the alluvium and Tres Hermanos-C Sandstones. Saturation of the aquifer was artificially created solely by discharge of mill process water and the extent of saturation is limited in both surface area and vertical extent.

The uppermost aquifer does not represent a ground water resource because of the limited extent of saturation and the inability to consistently sustain a yield of 150 gallons (gal) per day (570 liters [L] per day) to wells, based on a February 1989 aquifer test on DOE Well 675 (DOE, 1991). As demonstrated in this document, and previously in the RAP (DOE, 1991), ground water within the uppermost aquifer meets the qualifications for supplemental standards, Class III ("limited use"), based on limited vield. The major sources of recharge, waste water disposal and infiltration, and water from mine pumping, no longer exist. Therefore, the unit is expected to return to its premilling and mining condition of little-to-no saturation, further eliminating the unit as a potential future ground water resource. 
The potential for impacts to human health and the environment from contaminated ground water currently does not exist. No domestic or livestock wells accessing ground water from the uppermost aquifer have been identified within a 5 mile (mi) (8 kilometers [km]) radius from the site. Wells located further than $5 \mathrm{mi}(8 \mathrm{~km})$ from the site access ground water from deeper geologic units. Ground water does not discharge to the land surface and the nearest surface water is located approximately $1.5 \mathrm{mi}(2.4 \mathrm{~km})$ southwest of the site. Land use in the area is not expected to change in the future because of the poor quality of the range land, poor soils, and the limited surface or shallow ground water resources available in this semiarid region. Therefore, no current exposure pathways to humans, livestock, or wildlife exist, nor are any foreseen.

The proposed ground water compliance strategy under consideration for application at the Ambrosia Lake site is to perform no remediation, based on the application of supplemental standards because the ground water has "limited use" (40 CFR $\$ 192.21$ (g) (1994)) (60 FR 2854). "Limited use" means the ground water is not a current or potential source of drinking water because the quantity of water available is less than $150 \mathrm{gal}(570 \mathrm{~L})$ per day (40 CFR \$192.11(e)) (60 FR 2854). The NRC and state of New Mexico concurred with application of supplemental standards for the UMTRA Surface Project in 1990. The Surface Project supplemental standards compliance strategy was substantiated by many of the arguments presented in this SOWP for the Ground Water Project. The Surface Project compliance strategy can logically be applied to the Ground Water Project at the Ambrosia Lake site. 
TABLE OF CONTENTS

Section

Page

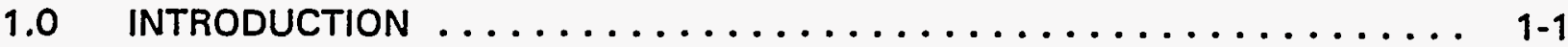

1.1 Purpose and content ......................... 1-1

1.2 Ground water compliance strategy ................... 1-1

1.3 Relationship to other programmatic documents ............ 1-1

1.4 Relationship to site-specific documents . . . . . . . . . . . . . 1-2

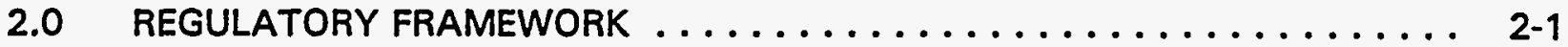

2.1 Uranium Mill Tailings Radiation Control Act . . . . . . . . . . . . 2-1

2.1.1 EPA ground water standards ................ 2-1

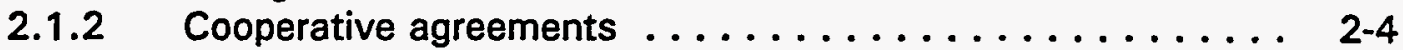

2.2 National Environmental Policy Act .................. 2-4

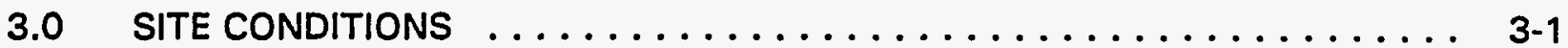

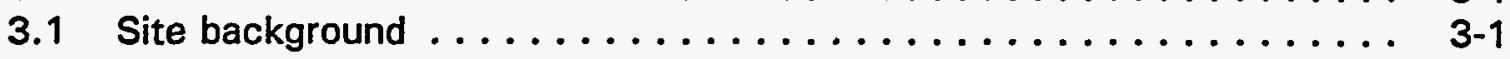

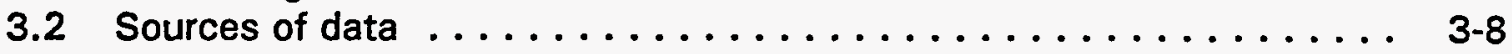

3.3 Conceptual site model . . . . . . . . . . . . . . . . $3-8$

3.3.1 Hydrogeologic setting $\ldots \ldots \ldots \ldots \ldots \ldots \ldots \ldots \ldots \ldots \ldots . \ldots \ldots$

3.3.2 Ground water quality ................. 3-19

3.3.3 Contaminant fate and transport .............. 3-26

3.3.4 Risk evaluation . . . . . . . . . . . . . . . . 3-28

3.4 Assessment of data needs . . . . . . . . . . . . . . . 3-30

3.5 Evaluation of interim remedial action $\ldots \ldots \ldots \ldots \ldots \ldots . \ldots . \ldots . \ldots$

4.0 GROUND WATER COMPLIANCE STRATEGY RECOMMENDATION . . . . . . 4-1

4.1 Ground water compliance strategy evaluation process . . . . . . . . . . 4-1

4.2 Site-specific ground water compliance strategy . . . . . . . . . . . . 4-3

4.3 Deviations, contingencies, and rules . . . . . . . . . . . . . . . . 4-4

4.4 Data collection and assessment ................... 4-5

$5.0 \quad$ LIST OF CONTRIBUTORS $\ldots \ldots \ldots \ldots \ldots \ldots \ldots \ldots \ldots \ldots \ldots \ldots \ldots$

6.0 REFERENCES $\ldots \ldots \ldots \ldots \ldots \ldots \ldots \ldots \ldots \ldots \ldots \ldots \ldots \ldots \ldots \ldots$

APPENDIX A LITHOLOGIC LOGS, WELL RECORDS, AND WELL COMPLETION LOGS, AMBROSIA LAKE, NEW MEXICO, UMTRA PROJECT' SITE

APPENDIX B HYDROGEOLOGIC INFORMATION, AMBROSIA LAKE, NEW MEXICO, UMTRA PROJECT SITE

APPENDIX C GROUND WATER QUALITY DATA BY LOCATION, AMBROSIA LAKE, NEW MEXICO, UMTRA PROJECT SITE 


\section{LIST OF FIGURES}

\section{Figure}

Page

3.1 Location of Ambrosia Lake UMTRA site . . . . . . . . . . . . . . $3-2$

3.2 Ambrosia Lake UMTRA site and surrounding area . . . . . . . . . . . 3-3

3.3 Preremedial action Ambrosia Lake UMTRA site . . . . . . . . . . . . . . . . 3-4

3.4 Generalized regional geologic construction for the Ambrosia Lake area, New Mexico . . . . . . . . . . . . . . . . . . . . . 3-11

3.5 Locations of geologic cross sections, Ambrosia Lake UMTRA site, New Mexico . . . . . . . . . . . . . . . . . . . . . . . 3-12

3.6 Site-specific hydrogeologic cross section (A-A'), Ambrosia Lake UMTRA site, New Mexico ... . . . . . . . . . . . . . . . . . . . . 3-13

3.7 Site-specific hydrogeologic cross section (B-B'), Ambrosia Lake UMTRA site, New Mexico . . . . . . . . . . . . . . . . . . . . . . . . 3-14

3.8 Sulfate isopleth map for ground water in the alluvium and the weathered Mancos Shale at the Ambrosia Lake site, New Mexico . . . . . . 3-21

3.9 Sulfate isopleth map for ground water in the Tres Hermanos $\mathrm{C}_{2}$ Sandstone at the Ambrosia Lake site, New Mexico . . . . . . . . . . . . . 3-25

3.10 Nitrate concentrations versus time in monitor well 678 located the Tres Hermanos-B Sandstone, Ambrosia Lake site, New Mexico . . . . . . . . 3-27

4.1 Ambrosia Lake compliance selection framework . . . . . . . . . . . 4-2 


\section{LIST OF TABLES}

Table

Page

2.1 Maximum concentration of constituents for ground water protection $\ldots \ldots$ 2-3

3.1 Water levels for all existing wells, July 13, 1994, Ambrosia Lake UMTRA site .............................. 3-16

3.2 Maximum observed concentrations of EPA MCL constituerits in monitor wells located in the alluvium/weathered Mancos Shale at the Ambrosia Lake site during the years from 1980 to $1994 \ldots \ldots$. . . . . . . . . . . 3-22

3.3 Maximum observed concentrations of EPA MCL constituerits in monitor wells located in the Tres Hermanos- $\mathrm{C}_{1}$ Sandstone at the Ambrosia Lake site during the years from 1980 to $1994 \ldots \ldots \ldots \ldots \ldots \ldots \ldots$. . . . . . . . . . . . .

3.4 Maximum observed concentrations of EPA MCL constituerits in monitor wells located in the Tres Hermanos- $\mathrm{C}_{2}$ Sandstone at the Ambrosia Lake site during the years from 1980 to $1994 \ldots \ldots \ldots \ldots \ldots \ldots$. . . . . . . . . . . . . 


\section{LIST OF ACRONYMS AND ABBREVIATIONS}

\section{Acronym Definition}

$A C L$

AEC

$\mathrm{cm} / \mathrm{s}$

$\mathrm{cm}$

DOE

EPA

FONSI

$\mathrm{ft}$

$\mathrm{ft} / \mathrm{day}$

gal

$\mathrm{km}$

L

m

MCL

$\mathrm{mg} / \mathrm{L}$

$\mathrm{mi}$

MSL

NEPA

NRC

PEIS

pCi/L

RAP

RRM

SOWP

SPEAR

TAGR

TER

UMTRA

UMTRCA alternate concentration limit

Atomic Energy Commission

centimeters per second

centimeter

U.S. Department of Energy

U.S. Environmental Protection Agency

Finding of No Significant Impact

foot

feet per day

gallon

kilometer

liter

meter

maximum concentration limit

milligrams per liter

mile

mean sea level

National Environmental Policy Act

U.S. Nuclear Regulatory Commission

Programmatic Environmental Impact Statement

picocuries per liter

remedial action plan

residual radioactive material

site observational work plan

Software Program for Environmental Analysis and Reporting

Technical Approach to Ground Water Restoration

Technical Evaluation Report

Uranium Mill Tailings Remedial Action

Uranium Mill Tailings Radiation Control Act 


\subsection{INTRODUCTION}

The "Site Observational Work Plan for Ground Water Investigation at Ambrosia Lake, New Mexico" is a response to requirements for ground water compliance established in the Uranium Mill Tailings Radiation Control Act (UMTRCA) (42 USC $\$ 7901$ et seq. (1978)). This site observational work plan (SOWP) is the first ground water document of the Ambrosia Lake Uranium Mill Tailings Remedial Action (UMTRA) Project to address sitespecific activities necessary to meet compliance with U.S. Environmental Protection Agency (EPA) ground water protection standards (60 FR 2854).

\subsection{PURPOSE AND CONTENT}

This SOWP, Revision a, recommends an appropriate, site-specific ground water compliance strategy for the Ambrosia Lake site.

Section 2.0 describes the requirements for meeting the ground water protection standards at UMTRA Project sites. Section 3.0 present:s a summary of the site's hydrogeological setting, describes the presence and distribution of contaminants in ground water resulting from the milling process, and describes and assesses the potential environmental and human health risks at the site. Section 4.0 presents and provides justifications for the recommended ground water compliance strategy at the Ambrosia Lake site.

\subsection{GROUND WATER COMPLIANCE STRATEGY}

The ground water compliance strategy recommended for the Ambrosia Lake site is to perform no remediation, based on the application of supplemental standards, because the ground water is classified as Class III ("limited use") ground water (40 CFR \$192.21(g) (1994)) (60 FR 2854). "Limited use" means ground water that is not a current or potential source of drinking water because the quantity of water available is less than 150 gallons (gal) (570 liters [L]) per day (40 CFR \$192.11(e)) (60 FR 2854).

\subsection{PROGRAMMATIC DOCUMENTS}

Programmatic documents that provide guidance for this SOWP include the U.S. Department of Energy (DOE) UMTRA Ground Water Program Plan (DOE, 1992a), Programmatic Environmental Impact Statement for the Uranium Mill Tailings Remedial Action Ground Water Project (PEIS) (DOE, 1994) and Technical Approach to Ground Water Restoration (TAGR) (DOE, 1993a). The UMTRA Ground Water Program Plan states the mission needs and objectives for the UMTRA Ground Water Compliance Program and provides an overall technical and management approach for conducting the program. The PEIS provides an objective programmatic decision-making framework for conducting the UMTRA Ground Water Project, assesses the potential programmatic impacts of conducting the project, provides a method for determining the site-specific ground water compliance strategies, and provides data and information that can 
be used to prepare site-specific National Environmental Policy Act (NEPA) (42 USC $\$ 4321$ et seq. (1970)) compliance documents more efficiently. A record of decision on the PEIS framework is anticipated in 1995. The preferred alternative from the PEIS for conducting the UMTRA Ground Water Project will be published as the record of decision in the Federal Register. The TAGR provides technical guidance for conducting the ground water program.

\subsection{RELATIONSHIP TO SITE-SPECIFIC DOCUMENTS}

The surface remedial action plan (RAP) provides site characterization information (DOE, 1991). This information is updated for the SOWP to formulate the site conceptual model. If a ground water compliance strategy requiring remedial action activities is selected for this site, a ground water RAP or surface RAP modification will be prepared.

Potential risks are considered in this SOWP to ensure that the proposed compliance strategy is protective of human health and the environment. The environmental impacts from the recommended ground water compliance strategy presented in this SOWP will be assessed in a separate, appropriate, site-specific document that will meet the requirements of the NEPA (42 USC $\$ 4321$ et seq. (1970)). The site-specific NEPA document will be prepared by the DOE and be based on the framework in the approved PEIS. 


\subsection{REGULATORY FRAMEWORK}

This SOWP proposes a ground water compliance strategy for the Ambrosia site to achieve compliance with the EPA ground water protection standards applicable to Title I UMTRA Project sites. This section identifies the relationship of the UMTRCA (42 USC $\$ 7901$ et seq.), the EPA ground water protection standards (40 CFR Part 192; 60 FR 2854), the cooperative agreements, and the NEPA to the UMTRA Ground Water Project.

\section{$2.1 \quad$ URANIUM MILL TAILINGS RADIATION CONTROL ACT}

The U.S. Congress passed the UMTRCA (42 USC $\$ 7901$ et seq.) in 1978 in response to public concerns about the potential health hazards from exposure to uranium mill tailings over long periods of time. The UMTRCA authorized the DOE to stabilize, dispose of, and control uranium mill tailings and other residual radioactive material (RRM) at uranium mill processing sites and associated vicinity properties.

The UMTRCA has three titles. The UMTRA Project has responsibility for administering only Title I of UMTRCA. Title I of the Act: designates 24 inactive processing sites that will undergo remediation; directs the EPA to promulgate standards; mandates remedial action in accordance with standards prescribed by the EPA; directs remedial action to be selected and performed with the concurrence of the U.S. Nuclear Regulatory Commission (NRC) and in consultation with the status and indian tribes; directs the NRC to license the disposal sites for long-term disposal; and directs the DOE to enter into cooperative agreements with the affected states and Indian tribes.

In 1988, Congress passed the Uranium Mill Tailings Rernedial Action Amendments Act (Amendments Act) (42 USC $\$ 7922$ et seq.) authorizing the DOE to extend without limitation the time needed to complete ground water restoration activities at the processing sites.

\subsubsection{EPA ground water standards}

The UMTRCA requires that the EPA promulgate standards for protecting public health, safety, and the environment from radiological and nonradiological contaminants associated with processing uranium and the resulting RRM. On January 5, 1983, the EPA promulgated final standards (40 CFR Part 192) for the disposal and cleanup of RRM. On September 3, 1985, the Tenth Circuit Court of Appeals set aside the ground water provisions of the regulations at 40 CFR \$192.20(a)(2) and (3) and remanded them to the EPA. The standards were revised and a final rule was published on January 11, 1995 (60 FR 2854).

The EPA ground water protection standards address two ground water contamination scenarios: the first addresses future ground water contamination that may occur from tailings piles after disposal (completion of surface remedial action); the second addresses the cleanup of residual contamination that 
occurred at the processing sites before disposal of the tailings piles (60 FR 2854). Protection of the ground water at the disposal sites is being addressed in the UMTRA Surface Project. The UMTRA Ground Water Project addresses the contamination that occurred at the processing sites and is regulated by Subparts $B$ and $C$ of the ground water protection standards. The maximum concentration limits of constituents for ground water protection are presented in Table 2.1 (60 FR 2854).

\section{Subpart B}

Subpart B ("Standards for Cleanup of Land and Buildings Contaminated with Residual Radioactive Materials from Inactive Uranium Processing Sites") requires that remedial action at processing sites be conducted to ensure that the amounts of RRM in ground water meet one of the three following standards presented in 60 FR 2854:

- Background levels: concentrations of constituents in nearby ground water unaffected by processing activities.

- Maximum concentration limits (MCL): the EPA's maximum concentration limits of certain constituents in ground water, as proposed for the UMTRA Project.

- Alternate concentration limits $(A C L)$ : an alternate concentration limit for a constituent that will not pose a substantial present or potential hazard to human health or the environment as long as the alternate concentration limit is not exceeded.

\section{Subpart C}

Under certain specific conditions, the DOE may apply supplemental standards, as described in Subpart C, in lieu of the standards of Subparts A or B 160 FR 2854) (background levels, MCLs, or ACLs). The supplemental standard applicable at the Ambrosia Lake site for the surface remedial action program, and discussed for further analysis in this SOWP, may be applied because the ground water is classified as having "limited use" (40 CFR \$192.21(g) (1994)) (60 FR 2854). Subpart B of the standards defines "limited use" as ground water that 1) is not a current or potential source of drinking water because the concentration of total dissolved solids is in excess of 10,000 milligrams per liter (mg/L); or, 2) because widespread, ambient contamination not due to activities involving RRM from a designated processing site exists, that cannot be cleaned up using treatment methods reasonably employed in public water-supply systems; or, 3) because the quantity of water available is less than $150 \mathrm{gal}$ (570 L) per day (40 CFR \$192.11(e)) (60 FR 2854).

When supplemental standards apply, implementing agencies shall apply any remedial actions for the restoration of ground water contaminated by RRM that are required to assure, at a minimum, protection of human health and the 
Table 2.1 Maximum concentration of constituents for ground water protection

\begin{tabular}{|c|c|}
\hline Constituent & $\begin{array}{c}\text { Maximum } \\
\text { concentration }\end{array}$ \\
\hline Arsenic & 0.05 \\
\hline Barium & 1 \\
\hline Cadmium & 0.01 \\
\hline Chromium & 0.05 \\
\hline Lead & 0.05 \\
\hline Mercury & 0.002 \\
\hline Selenium & 0.01 \\
\hline Silver & 0.05 \\
\hline Nitrate (as N) & 10 \\
\hline Molybdenum & 0.1 \\
\hline Combined radium-226 and radium -228 & $5 \mathrm{pCi} / \mathrm{L}$ \\
\hline Combined uranium-234 and uranium-238 ${ }^{\mathrm{b}}$ & $30 \mathrm{pCi} / \mathrm{L}$ \\
\hline Gross alpha-particle activity (excluding radon and uranium) & $15 \mathrm{pCi} / \mathrm{L}$ \\
\hline $\begin{array}{l}\text { Endrin }(1,2,3,4,10 \text {-hexachloro-6,7-epoxy-1,4,4a,5,6,7,8,8a- } \\
\text { octahydro-1,4-endo,endo-5,8-dimethanonaphthalene) }\end{array}$ & 0.0002 \\
\hline Lindane $(1,2,3,4,5,6$-hexachlorocyclohexane, gamma isomer $)$ & 0.004 \\
\hline Methoxychlor (1,1,1-trichloro-2,2'-bis(p-methoxyphenylethane)) & 0.1 \\
\hline $\begin{array}{l}\text { Toxaphene }\left(\mathrm{C}_{10} \mathrm{H}_{10} \mathrm{Cl}_{6} \text {, technical chlorinated camphene, }\right. \\
67-69 \text { percent chlorine })\end{array}$ & 0.005 \\
\hline 2,4-D (2,4-dichlorophenoxyacetic acid) & 0.1 \\
\hline 2,4,5-TP Silvex (2,4,5-trichlorophenoxypropionic acid) & 0.01 \\
\hline
\end{tabular}

Source: (60 FR 2854).

a Milligrams per liter, unless stated otherwise.

bWhere secular equilibrium obtains, this criterion will be satisfied by a concentration of $0.044 \mathrm{mg} / \mathrm{L}$. For conditions of other than secular equilibrium, a corresponding value may be derived and applied, based on the measured site-specific ration of the two isotopes of uranium.

pCi/L - picocuries per liter. 
environment. In addition, when limited use ground water applies, supplemental standards shall ensure that current and reasonably projected uses of the affected ground water are preserved (60 FR 2854).

Subpart B also provides for selecting natural flushing as a means to meet the proposed standards. Natural flushing means allowing natural ground water processes to reduce the contamination in ground water to the standards (background levels, MCLs, or ACLs) within 100 years. Institutional controls that protect human health (measures that restrict access to contamination and satisfy beneficial uses of ground water), and an adequate monitoring program must be established and maintained during the period of natural flushing. Ground water also must not be currently, or be projected to become, a source of drinking water during the period of natural flushing (40 CFR §192.12(c)(4)) (60 FR 2854).

Subpart C (Implementation) provides guidance for implementing methods and procedures to provide reasonable assurance that the provisions of Subpart $B$ are satisfied. Subpart $C$ requires the following: that the conditions of Subpart $B$ be met on a site-specific basis using information gathered from site characterization and monitoring; and that the compliance strategy plan or RAP contain the proposed compliance strategy, a demonstration of effectiveness, and a characterization and monitoring program, as appropriate to meet the conditions of Subpart B.

\subsubsection{Cooperative agreements}

The UMTRCA authorizes the DOE to enter into cooperative agreements with affected states (Section 103) and Indian tribes (Section 105) for the purpose of performing remedial action at each designated site. No Indian tribes are affected by the activities at the Ambrosia Lake site. The DOE and state of New Mexico are in the process of amending the Surface Project Cooperative Agreement to include the Ground Water Project (Cooperative Agreement Number DE-FC04-85AL20533, dated September 11, 1985, as amended) (DOE, 1985).

\subsection{NATIONAL ENVIRONMENTAL POLICY ACT}

Implementation of the UMTRCA represents a major federal action subject to the requirements of the NEPA (42 USC $\$ 4321$ et seq. (1970). The Council on Environmental Quality's regulations that implement the NEPA are codified in 40 CFR Parts 1500-1508 (1994) and require that each federal agency develop its own implementing procedures (40 CFR \$1507.3). The DOE NEPA regulations are contained in 10 CFR Part 1021 (1994), "National Environmental Policy Act; Implementing Procedures." DOE guidance is provided in Recommendations for the Preparation of Environmental Assessments and Environmental Impact Statements (DOE, 1993b). 
Pursuant to the NEPA, the DOE drafted a PEIS in 1994 to analyze the potential impacts of implementing the programmatic alternatives proposed for conducting the Ground Water Project. The preferred alternative for conducting the UMTRA Ground Water Project will be recommended by the DOE following a public participation effort, and the record of decision will be published in the Federal Register. All subsequent actions on the UMTRA Ground Water Project must comply with this record of decision, anticipated in 1995.

The Ambrosia Lake site-specific NEPA documentation will be prepared by the DOE and be based on the framework in the approved PEIS that has been published as the record of decision in the Federal Register. The site-specific NEPA document will analyze the potential impacts of the strategy of no remediation, based on the application of supplemental standards, as the recommended site-specific ground water compliance strategy. 


\subsection{SITE CONDITIONS}

The appropriate ground water compliance strategy can be recomrnended only after the evaluation of site location, surrounding area land use, site history, and hydrogeologic conditions. Evaluation of site location, surrounding area land use, and hydrogeologic conditions assists in the evaluation of potential pathways for risks to human health and the environment. The site history highlights processing operations that have caused contamination, and hydrogeologic conditions describe the mechanisms that control contaminant distribution.

\subsection{SITE BACKGROUND}

\section{Location}

The Ambrosia Lake site is located in McKinley County, New Mexico, about 25 miles (mi) (40 kilometers [km]) north of Grants, New/ Mexico, and $1 \mathrm{mi}$ $(1.6 \mathrm{~km}$ ) east of New Mexico Highway 509 (Figure 3.1). The town nearest to the site is San Mateo, about $10 \mathrm{mi}(16 \mathrm{~km})$ to the southeast (Figure 3.2).

The site is within the Ambrosia Lake Mining District, near the center of the Grants Mineral Belt. Decommissioned uranium mills, abandoned underground mines, mine shafts and vents, ore piles, tailings piles, and heap leach piles are in proximity to the site. The former mill, former main holding ponds, and tailings pile were in Section 28, and two former small holding ponds were in Section 33, Township 14 North, Range 9 West. A former heap leaching operation associated with the Ambrosia Lake mill was located in SSection 27 (Figure 3.3) (USGS, 1980). The approximate elevation of the site is; 7,000 feet $(\mathrm{ft})(2,100$ meters [m]) above sea level.

\section{Surrounding land use}

McKinley County is rural and sparsely populated. The town closest to the site is San Mateo, about $10 \mathrm{mi}(16 \mathrm{~km})$ to the southeast. The largest city in the area is Grants (in adjacent Cibola County), about $25 \mathrm{mi}(40 \mathrm{~km})$ south of the site, which had a population of 8626 in 1990 (DOC, 1990).

The sites of former uranium mines and mills, and a limited amount of grazing constitute current land use surrounding the site. The "7railer Park" shown on the USGS topographic map (Figure 3.3) (USGS, 1980) is within about $1 \mathrm{mi}$ $(1.6 \mathrm{~km})$ of the site, but was abandoned when the demiand for uranium declined during the late $1970 \mathrm{~s}$. More than one dozen former uranium mines are within a $5-\mathrm{mi}(8-\mathrm{km})$ radius of the site (Figure 3.2), and the closest residence is approximately $5 \mathrm{mi}(8 \mathrm{~km})$ south of the site. Not shown on the figure but present throughout the area are numerous abandoned mine spoil piles, ore piles, tailings piles, and heap leach piles resulting from former mining and milling 


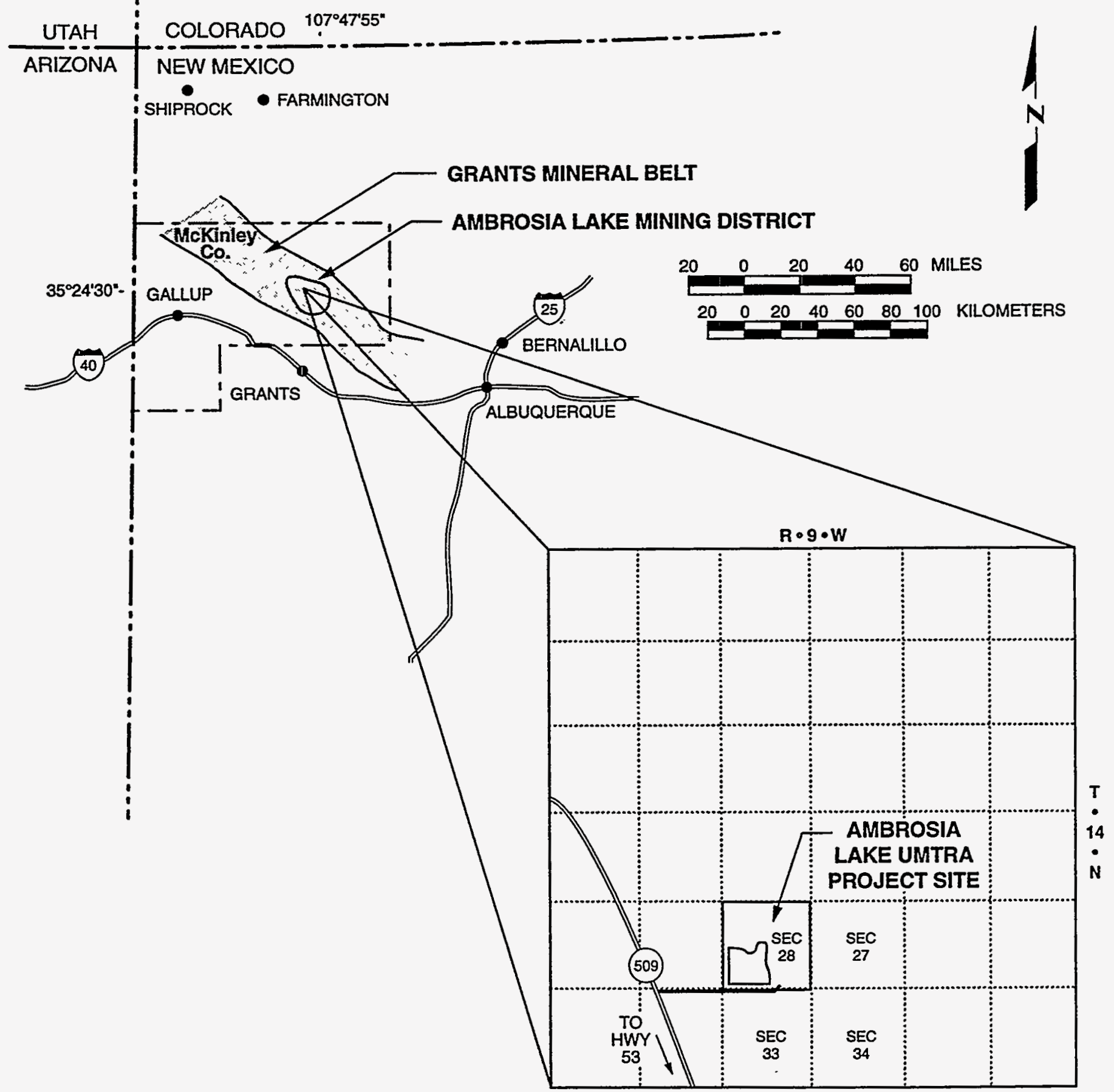

LEGEND

(40) INTERSTATE HIGHWAY

(509) STATE HIGHWAY

MODIFIED FROM DOE, 1991.

FIGURE 3.1

LOCATION OF AMBROSIA LAKE UMTRA SITE 


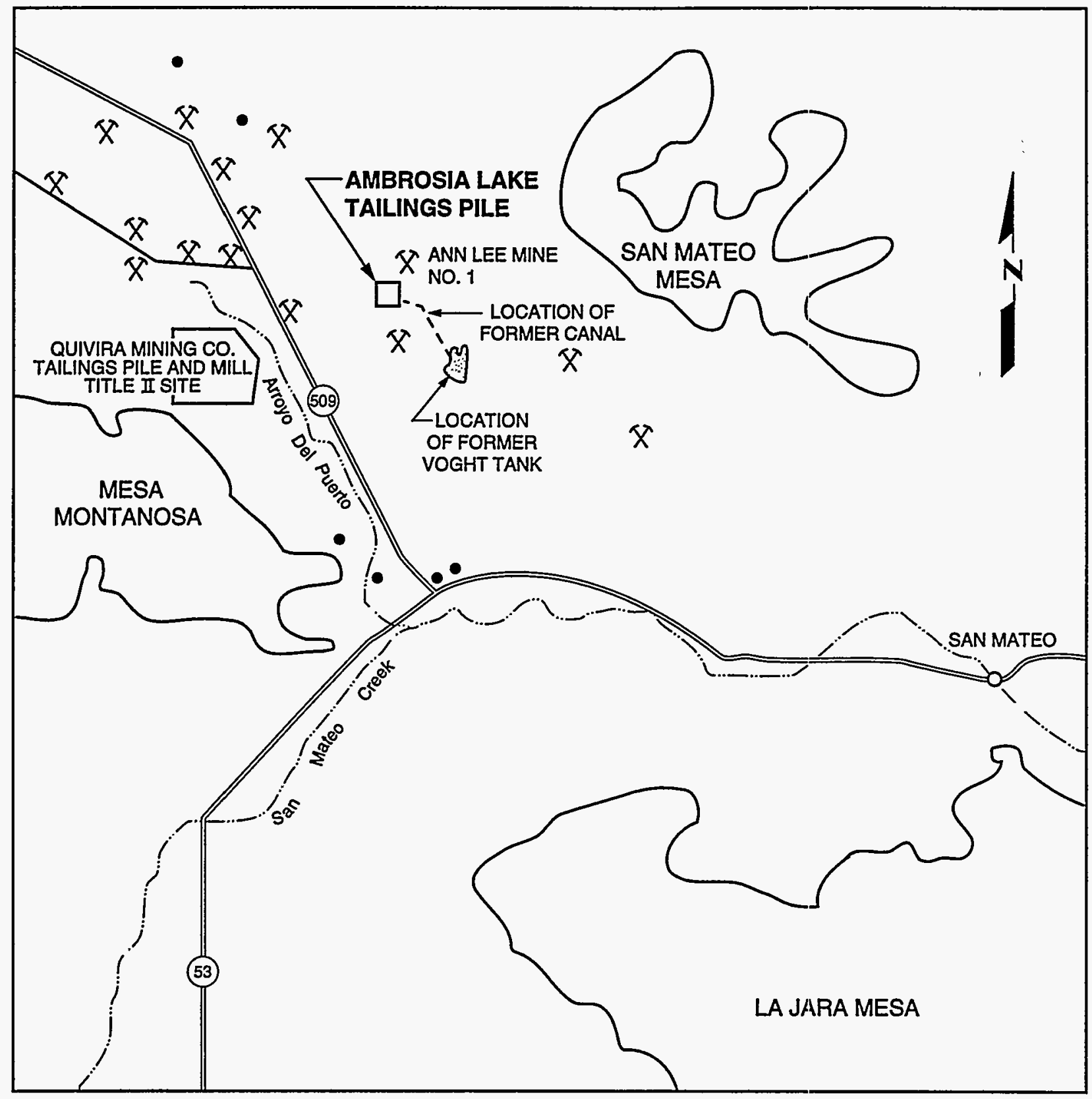

LEGEND

- DOMESTIC OR STOCK WELL

$X$ UNDERGROUND MINE

..... EPHEMERAL STREAM

$\begin{array}{lllll}1 & 0 & 1 & 2 & 3\end{array}$ MILES

$\begin{array}{llllllll}1 & 0 & 1 & 2 & 3 & 4 & 5\end{array}$ KILOMETERS

MODIFIED FROM DOE, 1991.

FIGURE 3.2

AMBROSIA LAKE UMTRA SITE AND SURROUNDING AREA 


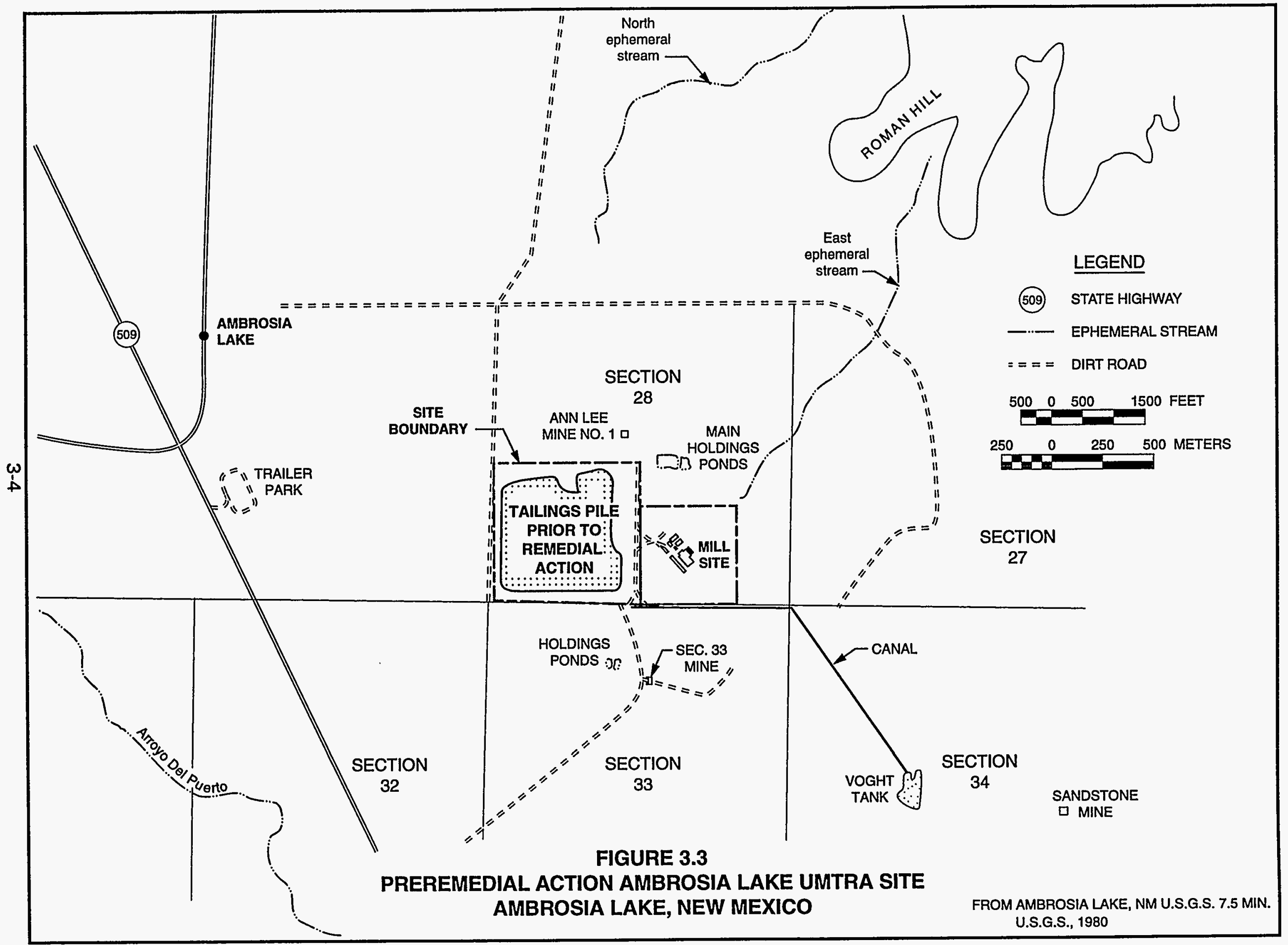


operations (Figure 3.2). Piles associated with the Ambrosia Lake UMTRA Project site have been cleaned up and placed in the disposal cell.

Many of the surrounding uranium mills are undergoing remediation. The former mill closest to the site is Quivira Mining Company, which is about $1 \mathrm{mi}(1.6 \mathrm{~km})$ to the southwest of the Ambrosia Lake site.

\section{Surrounding water use}

Ground water use in the Ambrosia Lake area was dominated by the uranium mining and milling industry, but has declined drastically as a result of the abandonment of mining and milling. The only surrounding ground water uses are for cleanup activities at nearby former mill sites, and for domestic and ranch supplies. The nearest surface water is located approximately $1.5 \mathrm{mi}(2.4 \mathrm{~km})$ southwest of the Ambrosia Lake site.

Water supplies associated with mill cleanup activities are from wells completed in the uranium ore zone (the Westwater Canyon Member of the Morrison Formation) and deeper hydrogeologic units. Domestic arid stock wells are also completed in the ore zone or deeper units and obtain water at depths from 300 to greater than $800 \mathrm{ft}(100$ to greater than $240 \mathrm{~m})$. No domestic or livestock wells are completed in any of the shallower zones (alluvium and Tres Hermanos Sandstones) within at least a 5-mi $(8-\mathrm{km})$ radius of the site, with the exception of monitor wells installed by the DOE (DOE, 1991). The nearest municipality operating a public water supply is the town of San Mateo, $10 \mathrm{mi}(16 \mathrm{~km})$ southeast of the Ambrosia Lake site. The water for San Mateo is derived from the Point Lookout Sandstone (Brod, 1979), which is stratigraphically higher than, and not connected with, any of the geologic units at the Ambrosia Lake site.

\section{Contaminant sources}

Contaminant sources described in this section result from two factors: 1) surrounding land uses and 2) the UMTRA Project site. These two factors are significant because of the location of the Ambrosia Lake UMTRA site. A large number of contaminant sources are within the vicinity.

The Ambrosia Lake UMTRA Project site has contributed relatively little contamination within an area that experienced intensive uranium mining and milling activities from the mid-1950s through the late 1970s. Chemical processes for uranium concentration within the area were similar to those employed at the UMTRA Project site; therefore, contaminants from the surrounding mills are similar. For example, the former Quivira Mining Company mill, adjacent to the UMTRA Project site, has produced more than ten times the tailings produced by the UMTRA site, representing a volume of tailings greater than all of the 24 UMTRA Project sites combined. Because similar processing techniques were employed, it is logical to assume that the Quivira Mining Company mill produced at least ten times the volume of waste water as the 
UMTRA Project mill produced. It is unknown how much additional ground water contamination has been produced from the other surrounding mills.

As stated earlier, the area surrounding the site has numerous abandoned mines and vent shafts, and associated spoil piles, ore piles, tailings piles, and heap leach piles. It is important to mention these conditions again, because of their net contribution to widespread ground water contamination. Many of the mines and vent shafts were legally backfilled with RRM, which contain contaminants that can leach into the water-bearing zones adjacent to the mines and vent shafts. Additionally, the mines and vent shafts provide interconnection between water-bearing zones, allowing the mixing of contaminated and noncontaminated ground water. If the piles are located over recharge areas for deeper aquifers, then the contaminants would not be limited to the uppermost aquifer.

The Ambrosia Lake UMTRA site was a former uranium mill that operated as a mechanical and chemical uranium-concentrating plant from 1958 to 1963 . It operated as an ion-exchange plant for uranium concentration from 1971 to 1983. The sources of water for the ion-exchange plant were three mines (the Ann Lee, Sandstone, and Section 27 mines), as well as return water from heap leach piles in Section 27.

Site contamination was caused primarily by the discharge of waste water and tailings from uranium milling during the 1958 to 1963 period. The ion-exchange and heap leach operations contributed little additional contamination when compared to the uranium mill process.

Approximately 3.0 million tons (2.7 million tonnes) of uranium-bearing ore were processed during the period of 1958 to 1963 by crushing and grinding, and then by removing the uranium through a chemical process (DOE, 1991). The operations resulted in the production of a relatively small percentage of uranium concentrate and a relatively large percentage of wastes, consisting of waste water and mill tailings. Contamination of ground water resulted from discharge of waste water, hydraulic placement of mill tailings, and infiltration of water discharged at the surface.

Mill tailings are the fine-grained materials (sand, silt, and clay) resulting from the uranium removal process. Because the uranium represented only a small portion of the ore, the volume of tailings is approximately equal to the amount of ore processed (i.e. approximately 3 million tons [2.7 million tonnes] of tailings were produced) (DOE, 1991). The tailings were mixed with water from the ore zone (the Westwater Canyon Member of the Morrison Formation) and with waste water from the chemical concentration process and this mixture was piped to the tailings pile.

Waste water was produced from the former mill at a rate of 1 to 5 tons 10.9 to 4.5 tonnes) for every ton of ore processed (Merritt, 1971). Therefore, from 3.0 to 15 million tons (2.7 to 13.6 million tonnes) of waste water were produced during the 5 years of ore milling. This is equivalent to between $7.2 \times 10^{8}$ to 
$3.6 \times 10^{9} \mathrm{gal}\left(2.7 \times 10^{9}\right.$ and $\left.1.4 \times 10^{10} \mathrm{~L}\right)$ or 2200 to 11,000 acre-feet of waste water. Waste water was disposed of with the tailings and also collected in two main holding ponds (formerly near the southeast corner of the tailings pile in Section 28); some was discharged to a canal which led to a holding pond called the Voght Tank, previously located in Section 34 (Figure 3.3). Some waste water was also discharged to two holding ponds near the southeast corner of the tailings pile in Section 33 (Figure 3.3).

Approximately 400,000 tons (360,000 tonnes) of the total 3 million tons of (2.7 million tonnes) tailings were piped into the Ann Lee Mine using water from the mines and possibly process waste water (DOE, 1991). This was a legal and common practice in the area to protect against subsiderice. The Ann Lee Mine was the only mine backfilled with tailings from the Ambrosia Lake site; however, this practice was undertaken at numerous other mines and contributes to widespread ground water contamination in all geologic units from the ore zone to the surface. Section 3.3, conceptual site modell, provides additional detail regarding site and surrounding area geology and water quality.

The carbonate process for uranium concentration was used at the Ambrosia Lake site (Merritt, 1971). Process materials contributing to site contamination were uranium ore, caustic soda, soda ash, sulfate salts, and ammonia. Major indicators of contamination resulting from the Ambrosia Lake site process materials and byproducts were uranium, nitrate, sulfate, and sodium.

The ion-exchange process contributed little if any additional contamination to the site. Water that was passed through the ion-exchange columns was returned to its source (the mines), and not discharged to the land surface. Water applied to the heap leach piles was collected with a drain system for recovery of uranium using the ion-exchange columns.

\section{Site status}

The Phillips Petroleum Company constructed, owned, and operated the former mill from 1957 until 1963, when United Nuclear Corporation purchased the operation. Uranium concentrate was sold to the Atomic; Energy Commission (AEC) throughout the mill's operational history. The state of New Mexico owns the site at present; ownership will be transferred to the DOE upon certification of completion of the surface remedial actions.

As part of the UMTRA project surface remediation activities, RRM is being consolidated on the site in a disposal cell that is scheduled for completion in early 1995. Contents of the cell include the tailings pile, former mill buildings, wind blown tailings, the heap leach pads (Section 27), the former main holding ponds (Section 28), and materials associated with the two former ponds near the southeast portion of the disposal cell (Section 33), the canal, and Voght Tank (Section 34) (Figure 3.3). An environmental assessment (DOE, 1987a) and a Finding of No Significant Impact (FONSI) (DOE, 1.987b) were completed in 
1987. The RAP for surface remediation activities was concurred with by the NRC and state of New Mexico in 1990 (NRC, 1990).

Tailings removal from the Ann Lee or other surrounding mines is technically unfeasible and their removal would pose a threat to personnel. The tailings within these underground mines are currently isolated from contact with human or environmental receptors. The tailings backfilled into the Ann Lee Mine, therefore, are anticipated to qualify for supplemental standards under the surface remediation project and will remain in place. A final application for supplemental surface remediation standards for the backfilled tailings in the Ann Lee Mine will be submitted by the DOE Remedial Action Contractor. Ambrosia Lake site tailings in the Ann Lee Mine have little additional adverse effect on area ground water quality because of the presence of tailings in numerous surrounding mines and the presence of uranium in the ore zone.

In their 1990 technical evaluation of the RAP, the NRC concurred with the finding that the uppermost aquifer at the site (the alluvium, weathered Mancos Shale, and Tres Hermanos-C Sandstones) is incapable of a sustained well yield of 150 gallons per day. Therefore ground water within the uppermost aquifer meets the criteria for Class III, supplemental standards, based on limited use (NRC, 1990). As described in Section 3.3, conceptual site model, the factors controlling well yield will not change over time, with the exception of the saturated thickness of the aquifer, which will decrease. Potential well yield will also decline as the saturated thickness decreases. Therefore, the "limited use" aquifer status should also apply to the ground water portion of this project.

\subsection{SOURCES OF DATA}

Information presented in this SOWP includes observations from site visits and review of data and documentation available at the UMTRA Project Document Control Center in Albuquerque, New Mexico.

Detailed descriptions of uranium concentration, mill byproducts, and process waste streams are provided in Merritt (1971). Site hydrogeologic conditions are described in detail in the RAP (DOE, 1991) and the NRC's final technical evaluation report (TER) (NRC, 1990). Additional site-specific information was obtained through a review of geologic and hydrologic reports cited in the RAP, an examination of monitor well completion reports, and ground water chemistry data contained in the DOE's Software Program for Environmental Analysis and Reporting (SPEAR) Program System (DOE, 1992b). The data contained in the SPEAR Program System are the results from periodic water quality monitoring at the site and encompass the years from 1980 to 1994.

\subsection{CONCEPTUAL SITE MODEL}

The following paragraphs present a summary of the site conceptual model. Additional details regarding the site conceptual model are in the subsections following this summary. 
Surrounding land use was predominantly uranium mining and milling. Mining companies still own the land surrounding the site, but conventional mining and milling are no longer performed because of economic conditions. However, Quivira Mining Company has resumed old stope leach well injections and is recovering uranium by ion exchange. Low-density grazing is the current predominant land use.

The tailings in the Ann Lee Mine are isolated from direct: contact to human and environmental receptors, and will remain in place. Ground water does not discharge at land surface; therefore a direct exposure pathway to contaminated ground water currently does not exist. No water supply wells are completed in the hydrogeologic zones with contamination attributable to the UMTRA Ambrosia Lake site, and water contaminated by the former uranium mill does not affect other ground water supplies in the area. Also, there are no nearby uses of surface water. Therefore, no current exposure pathways to humans, livestock, or wildlife exist.

As discussed previously, milling operations created a relatively large amount of waste water. Based on geochemical evaluation and the limited amount of natural recharge to the uppermost aquifer, saturation of the uppermost aquifer was created primarily by discharge of the mill waste water. The extent of saturation resulting from Ambrosia Lake site operations is therefore limited in both areal and vertical extent. The aquifer does not represent a ground water resource because of the inability to sustain a yield of $150 \mathrm{gal}(570 \mathrm{~L})$ per day to wells. Therefore, potential future exposure pathways to humans, livestock, or wildlife are also unlikely. Ground water within the uppermost aquifer meets the qualifications for supplemental standards, "limited use," based on limited yield (40 CFR $\$ 192.21(\mathrm{~g})$ ) (60 FR 2854). Because the potential for future exposure pathways to humans, livestock, or wildlife is unlikely, the proposed recommended ground water compliance strategy is no remediation based on the application for supplemental standards.

\subsubsection{Hydrogeologic setting}

\section{Physiographic setting}

The site lies within the Ambrosia Lake valley and west of San Mateo Mesa, a northwest trending ridge. The site is on a relatively flat area at an elevation of approximately $7000 \mathrm{ft}(2100 \mathrm{~m})$. San Mateo Mesa is at an elevation of about $8100 \mathrm{ft}(2500 \mathrm{~m})$.

The site is within the Arroyo del Puerto drainage basin, which slopes downward toward the southeast. The approximate elevation of the drainage channel nearest the site is about $6900 \mathrm{ft}(2100 \mathrm{~m})$. The streams in the area of the site and Ambrosia Lake are primarily ephemeral, containing water only after heavy rainfall. However, a portion of the Arroyo del Puerto approximately $1.5 \mathrm{mi}$ $(2.4 \mathrm{~km})$ southwest of the site (Figure 3.3$)$ appears to contain water for extended periods of time as a result of drainage from the Quivira Mining Company uranium mill site. 


\section{Geology}

In descending order, geologic units of interest at the site are as follows (Brod and Stone, 1981):

1. Alluvium: alluvial and eolian deposits of clay, silt, sand, and gravel;

2. Mancos Shale: dark gray, calcareous marine shale containing four silty sandstone interbeds, known as the Tres Hermanos- $C_{1},-C_{2},-B$, and $-A$ Sandstones;

3. Dakota Sandstone: fine- to medium-grained sandstone, probably representative of a marine shore zone; and

4. Morrison Formation: interbedded mudstones, siltstones, and sandstones that have been divided into three members, Brushy Basin Member (mudstones and sandstones that contain uranium ore in some areas), Westwater Canyon Member (sandstones and mudstones that contain the majority of the uranium ore mined in the Ambrosia Lake area and also the primary aquifer), and Recapture Member (interbedded shale, siltstone, and sandstone).

The geologic units beneath the site, with the exception of the alluvium, dip (on a regional basis) toward the northeast at approximately 2 degrees. The alluvium has been deposited nearly horizontally on top of the Mancos Shale. The direction of the dip, relative depths, and relative thicknesses of the units are shown on Figure 3.4, a regional, generalized geologic cross section. The Recapture Member of the Morrison Formation is below the ore zone and is considered an aquitard, retarding the exchange of water between units above it and below it (Brod and Stone, 1981). Site activities should not have impacted deeper units because mining operations did not extend deeper than the Westwater Canyon member (the ore zone) and because the Recapture Member retards the exchange of water between units above and below it. These deeper units are excluded from further description.

The locations of site-specific geologic cross sections constructed from wells installed by the DOE for the UMTRA project are shown on Figure 3.5. Cross sections are shown on Figures 3.6 and 3.7. The cross sections show sitespecific relationships between the shallow geologic units and the thickness of those units. Logs of the Ambrosia Lake UMTRA Project site wells shown on the figures are presented in Appendix $A$. There are a number of additional UMTRA Project site wells, but because of the large amount of information, logs for those wells are not included with this document. However, well construction details and water levels contained in the SPEAR data base (DOE, 1992b) for all of the wells are in Appendix $B$, Hydrogeologic Information. Additional information is available for review in the UMTRA Project Document Control Center, Albuquerque, New Mexico. Not contained in the SPEAR data base are water 


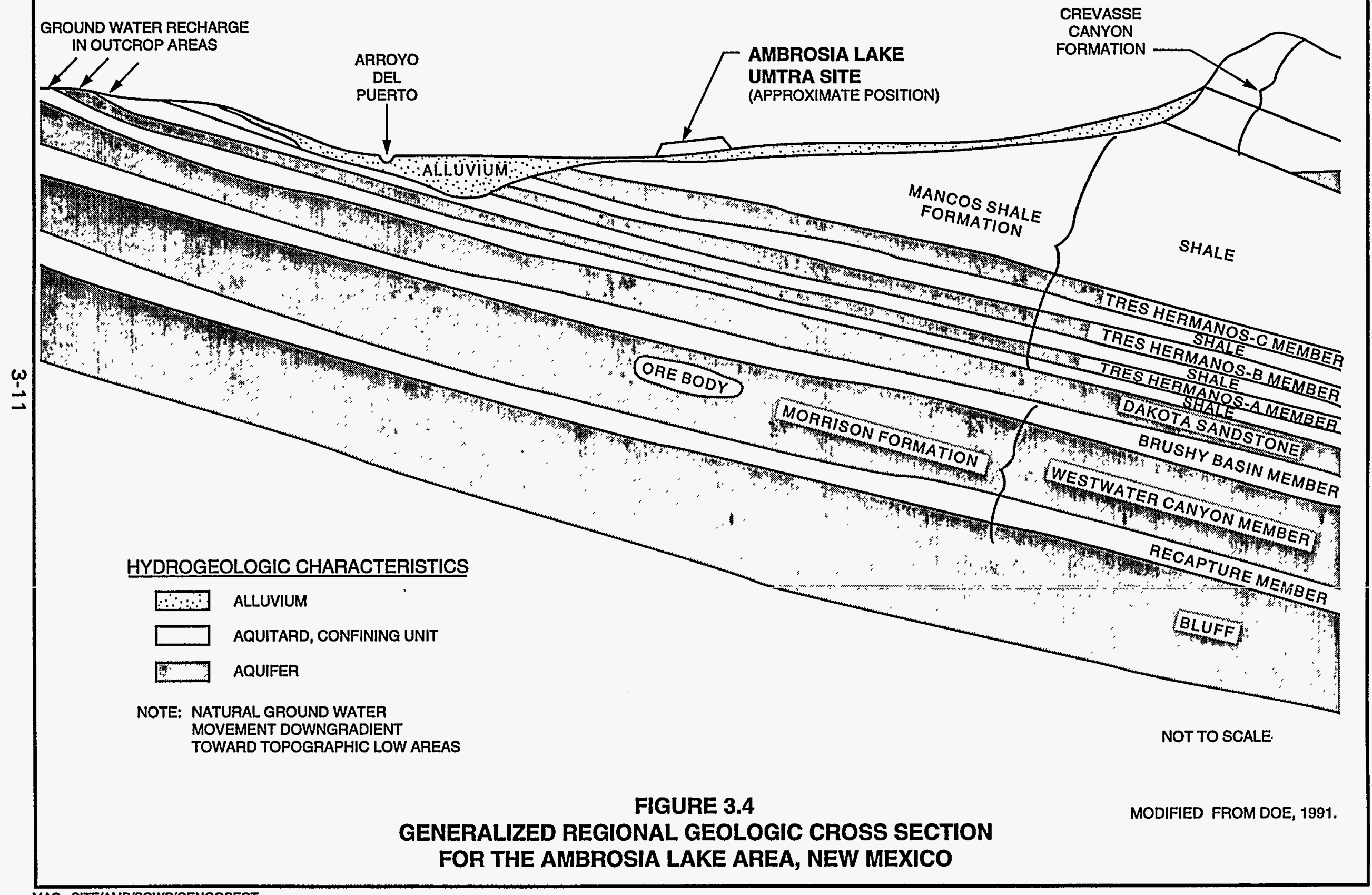




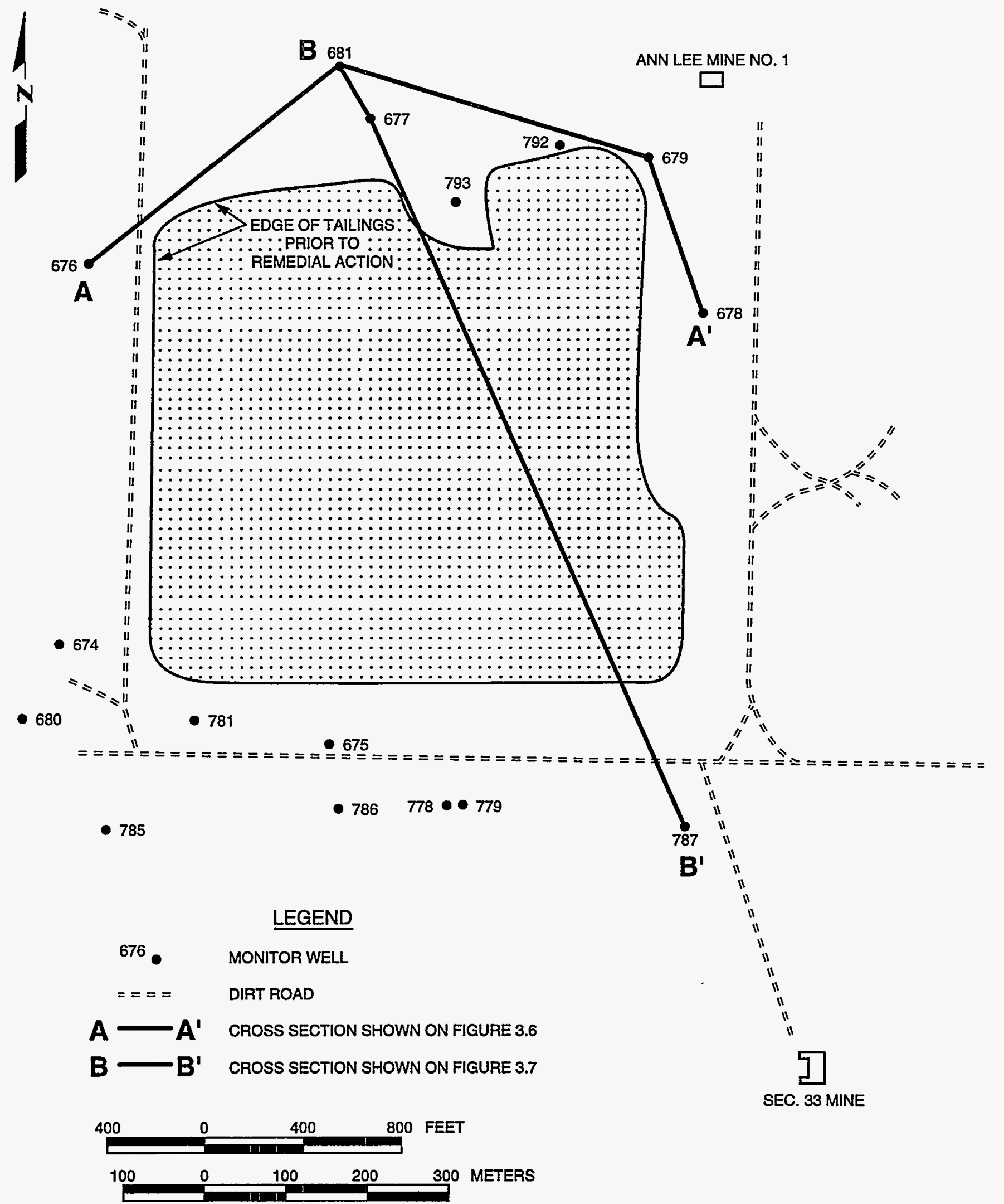

MODIFIED FROM DOE, 1991.

FIGURE 3.5

LOCATIONS OF GEOLOGIC CROSS SECTIONS

AMBROSIA LAKE UMTRA SITE, NEW MEXICO 
A

676

681

679

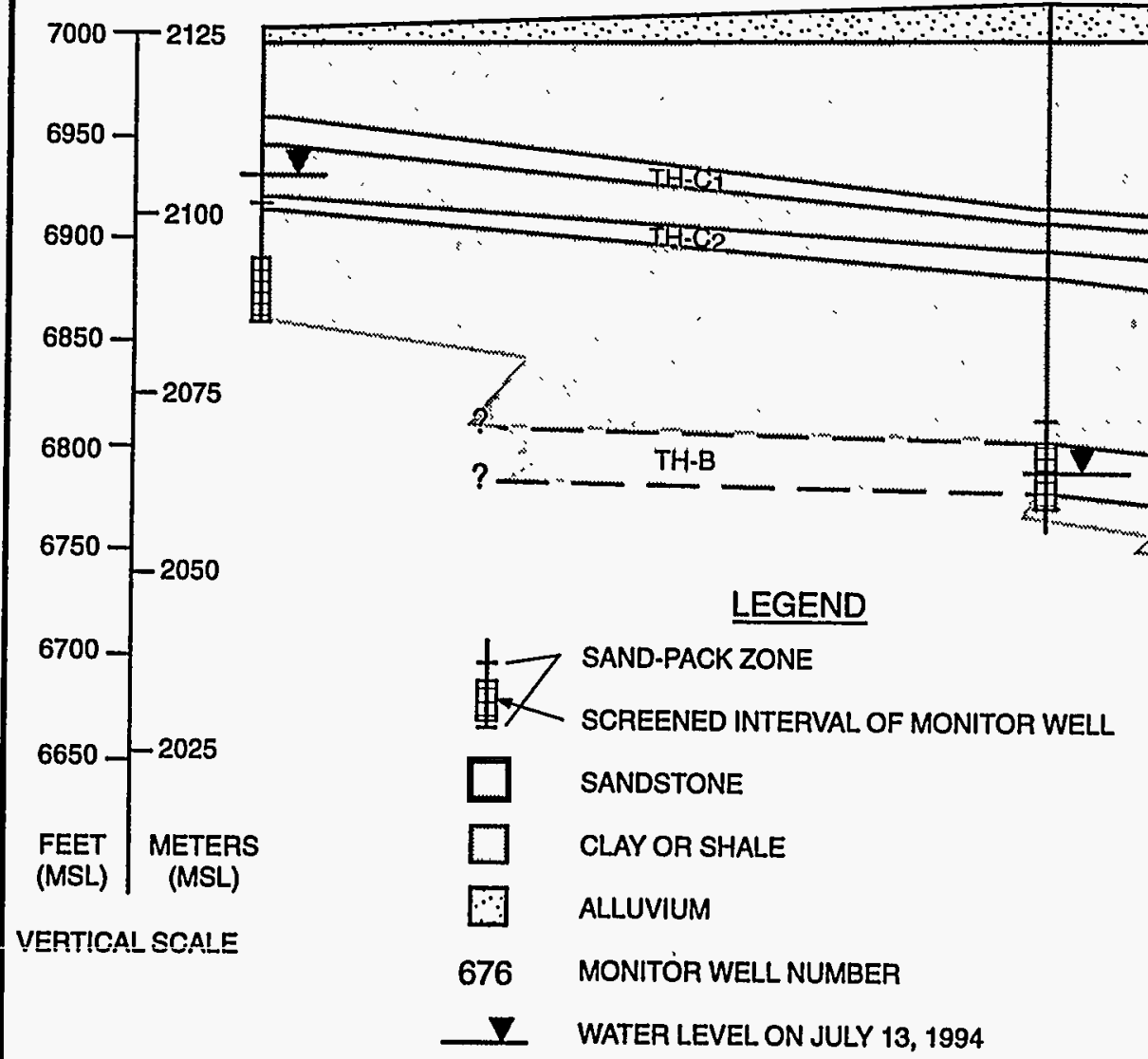

TRES HERMANOS SANDSTONE UNITS:

TH-C $\mathrm{C}_{1}$

$\mathrm{TH}-\mathrm{C}_{2}$

TH-B

TH-A

SITE-SPECIFIC HYDROGEOLOGIC CROSS SECTION (A-A')

$\begin{array}{llll}100 \quad 0 \quad 100 \quad 300 & \text { FEET }\end{array}$

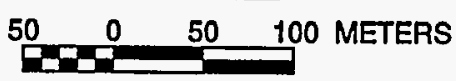

HORIZONTAL SCALE

AMBROSIA LAKE UMTRA SITE, NEW MEXICO

NOTE: WELL 679 WAS DRY 


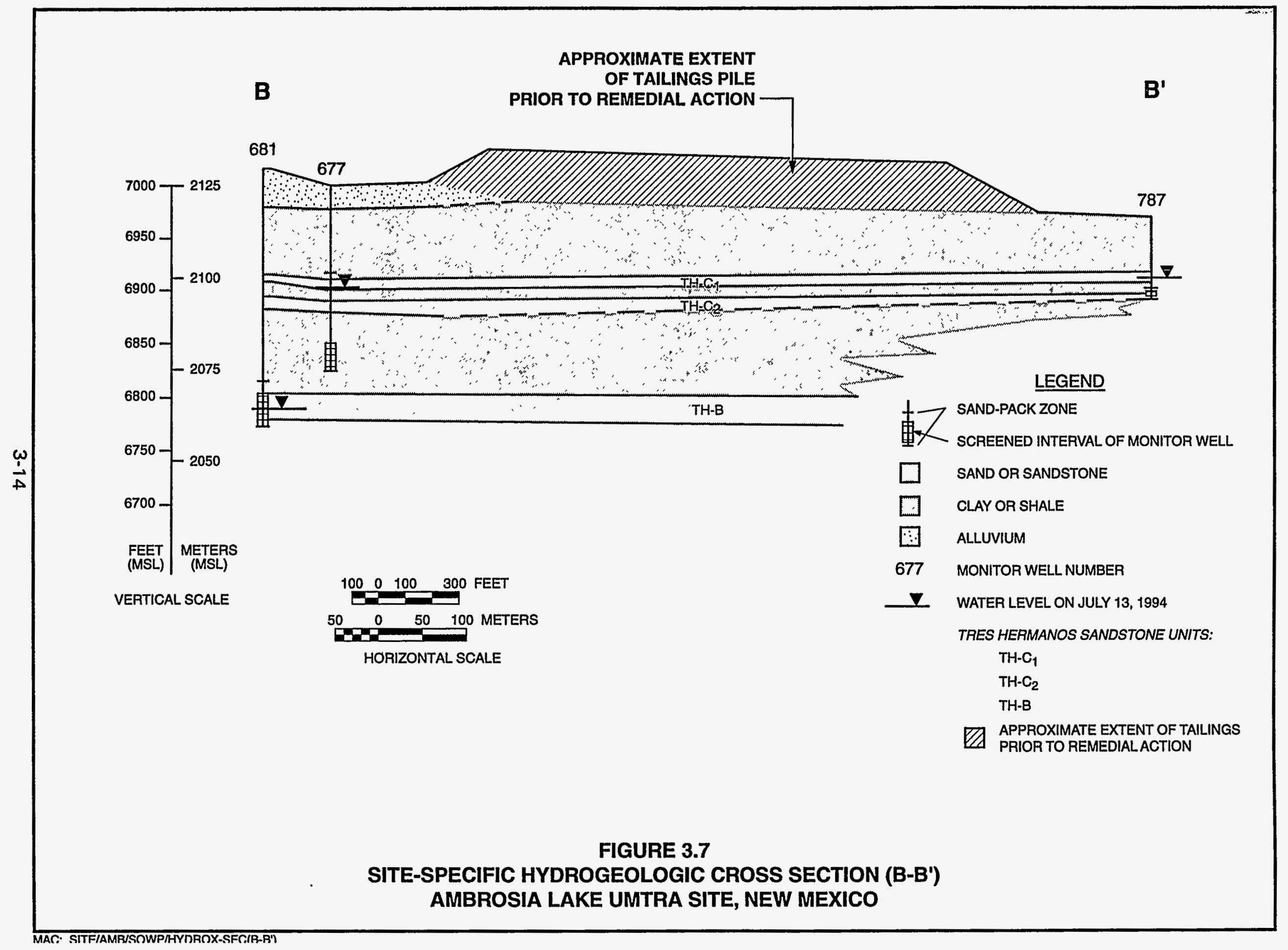


level data from the most recent sampling event, in July 1994. These data are shown in Table 3.1 and on the cross sections (Figures 3.6 and 3.7).

\section{Hydrology}

\section{Ground water}

Hydrogeologic units at the site, in descending order, and the hydraulic properties of each, are as follows:

1. Uppermost aquifer: By definition in the RAP (DOE, 1991), the uppermost aquifer consists of the Tres Hermanos- $C_{1}$ and $-C_{2}$ silty sandstone units, weathered Mancos Shale, and alluvium. The maximum thickness (and depth below grade) of the aquifer is approximately $175 \mathrm{ft}(53 \mathrm{~m})$. However, the maximum saturated thickness is only about $25 \mathrm{ft}(8 \mathrm{~m})$.

To determine the source of water in the uppermost aquifer, it is necessary to evaluate the relationship between recharge and discharge. The major sources of recharge, waste water disposal and water from mine pumping, no longer exist. The only current source of recharge to the uppermost aquifer is rainfall infiltration, which is limited by the semiarid climate.

A primary factor in determining recharge from rainfall is a comparison between rainfall and evapotranspiration. Annual rainfall is low, at 8.8 inches (22 centimeters [cm]) (OMC, 1981), and evapotranspiration is high, at a rate of 54 inches $(140 \mathrm{~cm})$ (NOAA, 1979). Other indicators of the semiarid conditions are the sparse vegetation and poor quality soils. Therefore, the potential for recharge from precipitation is low.

A further indicator of little-to-no recharge is the interaction between surface water and ground water. There have been no uses of ground water in the shallow aquifer, since there are no wells, other than DOE monitor wells, at the site. The Mancos Shale beneath the Tres Hermanos- $C$ Sandstone units acts as a lower confining unit, retarding the infiltration of water into lower units. Therefore, there are no mechanisms for significant reductions or withdrawals of ground water. If there was a significant amount of recharge from rainfall, water in the upper aquifer would build to a level at which the water level would be at ground surface, or it would clischarge to surface water bodies. Neither scenario is valid, based on records of water levels and because the surrounding surface water bodies are ephemeral, containing water only after significant rainfall. Therefore, the saturated thickness currently in the unit is expected to either remain about the same or decrease as water spreads laterally and a portion of it migrates upward into the vadose (unsaturated) zone.

An aquifer test conducted February 2-3, 1989 on well 675 and described in the RAP (DOE, 1991), shows that the uppermost aquifer is incapable of a sustained yield of $150 \mathrm{gal}(570 \mathrm{~L})$ per day to a well. Therefore, the unit is 
Table 3.1 Water levels for all existing wells, July 13, 1994, Ambrosia Lake UMTRA site

\begin{tabular}{|c|c|c|c|}
\hline Well No. & TOC elev ${ }^{a}$ & DTW & WL elev ${ }^{c}$ \\
\hline 674 & 6972.00 & 44.60 & 6927.40 \\
\hline 675 & 6966.00 & 22.35 & 6943.65 \\
\hline 676 & 6991.80 & 64.85 & 6926.95 \\
\hline 677 & 7001.60 & 89.37 & 6912.23 \\
\hline 678 & $\left.69751^{*}\right)$ & 220.80 & 6754.20 \\
\hline 679 & 7011.00 & $<300$ & $(* *)$ \\
\hline 680 & 6972.00 & $<300$ & $(* *)$ \\
\hline 681 & 7004.00 & 213.41 & 6790.59 \\
\hline 778 & 6962.70 & 27.93 & 6934.77 \\
\hline 779 & 6963.00 & 36.65 & 6926.35 \\
\hline 781 & 6968.40 & 39.64 & 6928.76 \\
\hline 785 & 6961.30 & 43.25 & 6918.05 \\
\hline 786 & 6963.40 & 21.86 & 6941.54 \\
\hline 787 & 6971.30 & 56.33 & 6914.97 \\
\hline 789 & 7003.90 & Dry & $(* *)$ \\
\hline 792 & 7001.10 & Dry & $(* *)$ \\
\hline 793 & 6998.60 & 27.55 & 6971.05 \\
\hline 797 & 6972.00 & Dry & $(* *)$ \\
\hline 798 & 6980.40 & Dry & $(* *)$ \\
\hline
\end{tabular}

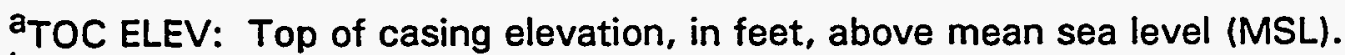

bDTW: Depth to water below top of casing, in feet.

CWL ELEV: Water level elevation, in feet, MSL.

* - Estimated for Well 678 because the well head was destroyed.

* * No water level elevation because well was dry or DTW exceeded length of water level indicator. 
considered a "limited use" aquifer, based on limited yield. The calculation set detailing the aquifer test is in Appendix B of this document. The NRC interpreted the test results as follows (NRC, 1990):

As defined in the standards, Class III ground water is not a current or potential source of drinking water for any of three reasons, including inability of the aquifer to provide a sustained yield exceeding $150 \mathrm{gal}$ per day. The DOE determined that the upper aquifer, composed of alluvium, weathered Mancos Shale, and the Tres Hermanos-C Sandstone members, is incapable of sustained yield of $150 \mathrm{gal}$ per day. The DOE's determination is based on the following:

(1) A pump test was performed on Well 675. It produced $0.35 \mathrm{gal}$ per minute (500 gal per day). The water level was drawn down $13 \mathrm{ft}$ in 12 hours, and the pumping could not be sustained.

(2) Well 675 has the greatest saturated thickness of any wells completed in the uppermost aquifer, and is screened to the base of the saturated zone. Therefore, it probably produces the maximum available water.

(3) The DOE calculated that long-term output of $150 \mathrm{gal}$ per day at Well 675 could not be sustained for more than 1 day. DOE further justifies application of supplemental standards at Ambrosia Lake because water in the uppermost aquifer is of limited extent, and is derived solely from infiltration of mining and milling water sources.

Therefore, there is reasonable assurance that $\mathrm{DCE}$ is eligible to apply for supplemental standards because the uppermost aquifer at Ambrosia Lake contains Class III ground water.

2. Mancos Shale: The Mancos Shale acts primarily as an aquitard, but by definition in the RAP (DOE, 1991), it contains a portion of the uppermost aquifer, the Tres Hermanos- $C_{1}$ and $-C_{2}$ Sandstones. Also contained within the Mancos Shale are the Tres Hermanos-B and -A Sandstone units which are silty sandstone units interbedded within the Mancos Shale. Aquifer tests were not performed on the Tres Hermanos-B and -A Sandstone units. However, based on a lithology similar to the Tres Hermanos-C Sandstone units, the water-bearing capacity of the Tres Hermarios-B and $-A$ Sandstone units is not much greater than the Mancos Shale.

Recharge to the Tres Hermanos-B and -A Sandstone units is from surface water at the locations where the units outcrop, or appear at the surface (Figure 3.4). The Tres Hermanos-B Sandstone unit outcrops about $1.5 \mathrm{mi}$ $(2.4 \mathrm{~km})$ southwest of the Ambrosia Lake site and is recharged by drainage 
from the nearby Quivira Mining Company uranium mill. Similarly, the Tres Hermanos-A Sandstone unit outcrops slightly further to the southwest and is likely recharged by water affected by the same mill (Bostick, 1985).

Recharge to these units has likely decreased since the report by Bostick (1985) was prepared, because the mill is no longer in operation.

Based on water-level data contained in the SPEAR data base (DOE, 1992b), there is an indication that the Tres-Hermanos-B Sandstone unit is only partially saturated. The information is shown graphically on Figures 3.6 and 3.7. Because the unit is relatively thin and only partially saturated, its use as a potential aquifer is limited.

The Mancos Shale units that separate the Tres Hermanos Sandstones and the units that are above the Dakota Sandstone are continuous and act as aquitards because of their thickness and low hydraulic conductivity. According to the RAP (DOE, 1991), "...a hydraulic conductivity of $4.3 \times 10^{-8}$ centimeter per second $(\mathrm{cm} / \mathrm{s})\left(1.2 \times 10^{-4}\right.$ feet per day) was measured in undisturbed Mancos Shale and is probably representative of the vertical hydraulic conductivity...." The low hydraulic conductivity of the Mancos Shale also makes it suitable as a source of low-permeability cover material for UMTRA Project disposal cells, and is used for several Project cells, including the Ambrosia Lake site.

3. Dakota Sandstone: The Dakota Sandstone is considered a secondary aquifer because of the relatively low yield (less than $10 \mathrm{gal}$ [ $40 \mathrm{~L}$ ] per minute) and poor water quality, when compared to the Westwater Canyon Member of the Morrison Formation (DOE, 1987a). Bostick (1985) reports that the Dakota Sandstone is present at land surface near the Quivira Mill and that surface discharge of water related to the Quivira Mill onto the outcrop of the Dakota Sandstone has caused contamination of the unit. The discharge of contaminated water at the outcrop and the discharge of water possibly from mill tailings placed in surrounding mines are the sources of contaminants found in the Dakota Sandstone beneath the Ambrosia Lake site.

4. Morrison Formation:

a. The Brushy Basin Member acts as an aquitard between the Dakota Sandstone and Westwater Canyon Member.

b. The Westwater Canyon Member is the principal aquifer in the Ambrosia Lake Mining District, but it is also the major source of uranium ore. Mine pumping began in the mid-1950s to facilitate ore removal from the Westwater Canyon Member. The ground water was discharged to surface drainages where it evaporated, and some infiltrated into the shallower zones. A portion of the water was used for mill process water. Because of the regional mine pumping, a large ground water depression has been created and ground water flow is probably toward the northeast (DOE, 1987a). 
c. The Recapture Member acts as an aquitard beneath the Westwater Canyon Member because of its thickness of $165 \mathrm{ft}(50 \mathrm{~m})$ and low permeability (DOE, 1987a).

\section{Surface water}

The Ambrosia Lake tailings site lies in the drainage basin of the Arroyo del Puerto, an ephemeral stream. Surface water flow occurs only after heavy rain; however, there are ponds approximately $1.5 \mathrm{mi}(2.4 \mathrm{~km})$ southwest of the site. Based on the presence of cattails and marsh grasses, it appears that the ponds contain water throughout the year. The ponds are located in the recharge area for the Tres Hermanos-B Sandstone unit and are fed by water from the Quivira Mining Company uranium mill operation.

Two unnamed drainage channels originate to the west (north ephemeral stream) and east (east ephemeral stream) of the site (Figure 3.3). There are no perennial streams close to the site.

\subsubsection{Ground water quality}

In order to define the extent of ground water contamination caused by the former uranium milling at the Ambrosia Lake site, water samples were collected from a DOE monitor well network from 1980 through 1994. Figure 3.5 shows a portion of the DOE monitor well network which is representative of current site conditions. Ground water samples collected from the monitor well network have been analyzed to determine the quality of ground water in the alluvium and the weathered Mancos Shale, the Tres Hermanos-C, $-B$ and $-A$ Sandstones, and the Westwater Canyon Member of the Morrison Formation. Ground water quality data by location for all Ambrosia Lake UMTRA Project site wells, including all sampling events, are presented in Appendix $C$ of this document.

\section{Background water quality}

Background ground water quality in the alluvium and the weathered Mancos Shale and Tres Hermanos-C Sandstone at the Ambrosia Lake site is considered to be the same as existing water quality because former mining and milling activities have created the saturated conditions (Bostick; 1985). It has been demonstrated, using geochemical modeling, that the ground water within the alluvium and the weathered Mancos Shale is derived entirely from tailings seepage and mill-makeup water (DOE, 1991). Mill-makeup water was generated from mine pumping discharge. Details of the modeling results are presented in Table D.8.25 of the RAP (DOE, 1991).

\section{Extent of contamination}

Because all water contained in the alluvium and the weathered Mancos Shale and Tres Hermanos-C Sandstone in the area of the milling site is primarily a mixture of tailings seepage and mine discharge, the extent of contamination is 
the same as the extent of ground water. Because of the large ground water depression created by mine pumping, all potential pathways for contamination from the Ambrosia Lake UMTRA site to enter units between the Tres Hermanos-C Sandstone and the Morrison Formation are points of discharge. Thus, there is no UMTRA site-related contamination in those units. Discharge of water from the Quivira Mining Company Mill to the outcrop of the Tres Hermanos-A Sandstone, Tres Hermanos-B Sandstone, and the Dakota Formation caused contamination of these units (Bostick, 1985). The Westwater Canyon Member is potentially contaminated by regional mining and milling processes because water from all overlying units drains into this unit through mine shafts and vent holes.

\section{Alluvium and the weathered Mancos Shale}

Tailings pore water at the site contained as much as $11,000 \mathrm{mg} / \mathrm{L}$ sulfate (DOE, 1991). The sulfate isopleth map (Figure 3.8) is used to define the extent of tailings-related contamination and, very likely, the extent of ground water in the alluvium and the weathered Mancos Shale at the site. Monitor well 620 is more than $1200 \mathrm{ft}(370 \mathrm{~m})$ southeast of the primary ground water mound. Sulfate concentrations and ground water in well 620 are assumed to be derived from the former holding ponds to the east (Figure 3.8). During the period of sampling (1980-1994), concentrations of arsenic, cadmium, chromium, molybdenum, nitrate, selenium, silver, uranium, and the activities of gross alpha and radium226 plus radium-228 exceeded the proposed EPA ground water standards in the alluvium and the weathered Mancos Shale (Table 3.2).

\section{Tres Hermanos-C Sandstone}

The Tres Hermanos- $\mathrm{C}_{1}$ Sandstone is separated from the underlying Tres Hermanos- $\mathrm{C}_{2}$ Sandstone by a 10-to-15 ft-(3-to-4.5 m-)thick shale unit. This shale unit retards downward migration of contaminants, resulting in slightly higher levels of contamination in the Tres Hermanos $-C_{1}$ Sandstone (Table 3.3), compared to values in the Tres Hermanos $-C_{2}$ Sandstone (Table 3.4). There are insufficient data to draw a sulfate isopleth map for the Tres Hermanos- $C_{1}$ Sandstone. The sulfate isopleth map (Figure 3.9) shows the approximate extent of tailings-related contamination in the Tres Hermanos- $\mathrm{C}_{2}$ Sandstone. This map indicates that contamination has moved farther downgradient to the northeast (as compared to Figure D.8.24 in DOE, 1991). In sulfate data from the January, 1991 sampling round (used for Figure 3.9), downgradient values are higher and upgradient values are lower, compared to those in the February, 1989 sampling round (used in Figure D.8.24 of DOE, 1991).

Maximum observed concentrations of cadmium, chromium, molybdenum, nitrate, selenium, silver, uranium, and the activities of gross alpha and radium-226 plus radium-228, exceed the EPA MCLs in ground water from wells completed in the Tres Hermanos- $C_{1}$ Sandstone (Table 3.3). Maximum observed concentrations of cadmium, chromium, molybdenum, selenium, silver, uranium and the activities of gross alpha and radium-226 plus radium-228 exceed the 


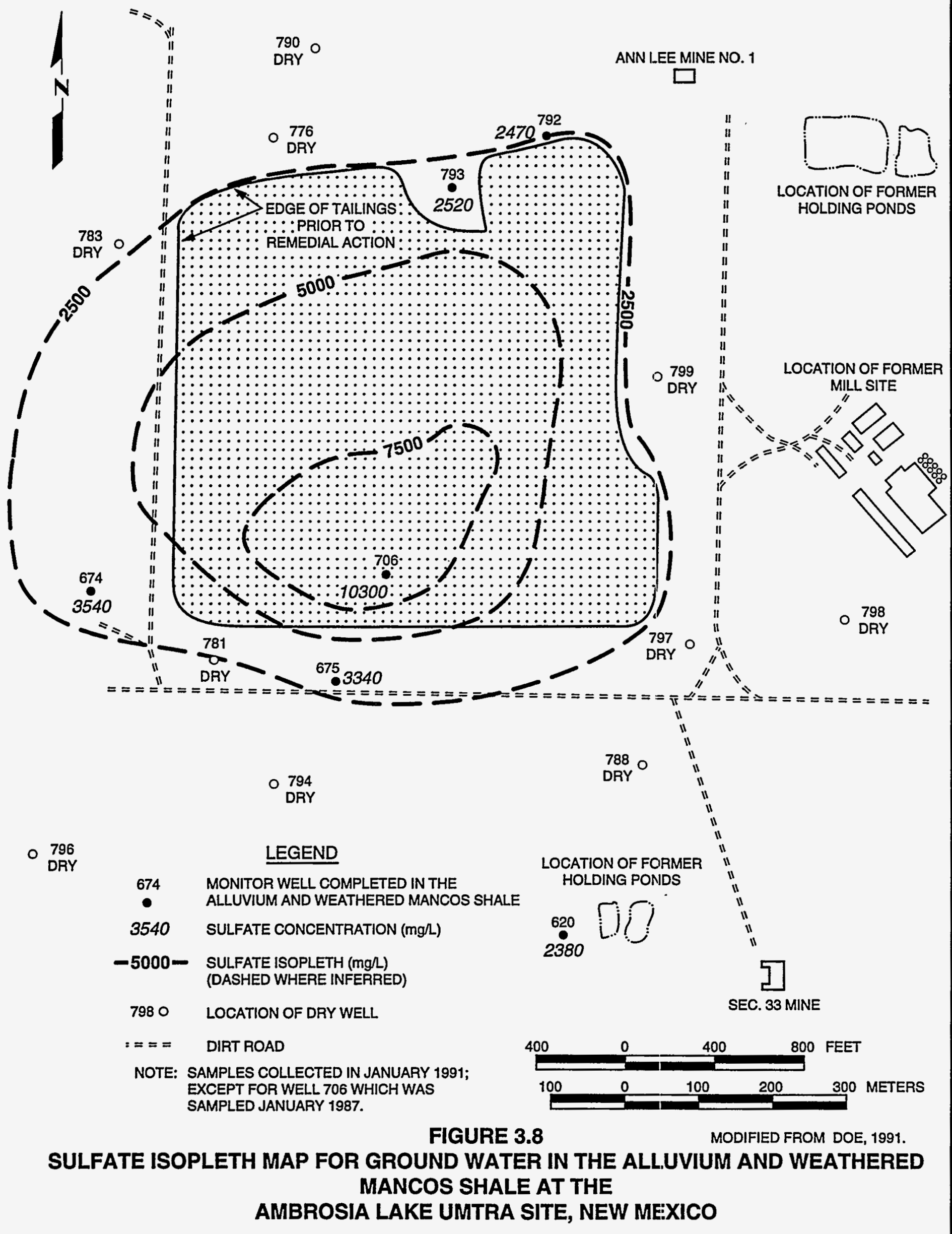


Table 3.2 Maximum observed concentrations of EPA MCL constituents in monitor wells located in the alluvium/weathered Mancos Shale at the Ambrosia Lake site during the years from 1980 to 1994

\begin{tabular}{|c|c|c|c|c|c|c|c|}
\hline \multirow[b]{2}{*}{ Constituent $^{\mathbf{a}}$} & \multirow{2}{*}{$\begin{array}{l}M^{M C L}{ }^{b} \\
(\mathrm{mg} / \mathrm{L})\end{array}$} & \multicolumn{6}{|c|}{ Monitor wells } \\
\hline & & 620 & 674 & 675 & 706 & 792 & 793 \\
\hline Arsenic & 0.05 & 0.01 & 0.02 & 0.02 & $0.33^{c}$ & 0.016 & 0.016 \\
\hline Barium & 1 & 0.1 & $<0.1$ & 0.01 & $<0.1$ & $<0.1$ & $<0.1$ \\
\hline Cadmium & 0.01 & $<0.001$ & 0.003 & 0.003 & $<0.001$ & 0.01 & $0.013^{c}$ \\
\hline Chromium & 0.05 & $0.16^{C}$ & $<0.01$ & $<0.01$ & $0.06^{c}$ & $0.28^{\mathrm{c}}$ & $0.28^{c}$ \\
\hline Lead & 0.05 & 0.02 & 0.03 & 0.02 & 0.02 & 0.02 & 0.02 \\
\hline Mercury & 0.002 & $<0.0002$ & $<0.0002$ & $<0.0002$ & $<0.002$ & 0.0007 & 0.0003 \\
\hline Molybdenum & 0.1 & $0.5^{c}$ & $9.81^{C}$ & $2.72^{C}$ & $225^{c}$ & $1.87^{\mathrm{C}}$ & $2.01^{c}$ \\
\hline Nitrate & $44^{d}$ & 12.1 & $69^{c}$ & $252^{c}$ & 25 & 1.8 & $830^{c}$ \\
\hline Selenium & 0.01 & $0.07^{c}$ & $3.1^{c}$ & $0.51^{c}$ & $0.088^{c}$ & $2.22^{c}$ & $2.1^{c}$ \\
\hline Silver & 0.05 & 0.04 & 0.01 & 0.02 & $0.15^{c}$ & $0.11^{c}$ & $0.11^{c}$ \\
\hline $\begin{array}{l}\text { Combined } \\
\text { Uranium-234 } \\
\text { and }-238\end{array}$ & $0.044^{e}$ & $8.22^{\mathrm{C}}$ & $10.7^{c}$ & $2.083^{c}$ & $11.1^{\mathrm{c}}$ & $3.31^{\mathrm{C}}$ & $0.393^{c}$ \\
\hline $\begin{array}{l}\text { Gross alpha } \\
\text { (excluding } \\
\text { radon and } \\
\text { uranium) } \\
\mathrm{pCi} / \mathrm{L}^{\mathrm{f}}\end{array}$ & 15 & $5300^{c}$ & $2200^{c}$ & $1700^{c}$ & $15,000^{c}$ & $2400^{c}$ & $320^{c}$ \\
\hline $\begin{array}{l}\text { Combined } \\
\text { Radium-226 } \\
\text { and }-228 \\
\text { pCi/L } f\end{array}$ & 5 & $9.13^{C}$ & 0.8 & 4.03 & $131.8^{C}$ & $6.85^{c}$ & 2.3 \\
\hline
\end{tabular}

${ }^{a}$ Constituents from Table 1 of the U.S. Environmental Protection Agency Draft Final Rule, Groundwater Standards for Remedial Actions at Inactive Uranium Processing Sites (60 FR 2854). All concentrations are in $\mathrm{mg} / \mathrm{L}$ unless stated otherwise.

BCL: maximum concentration limit, from Table 1 of the U.S. Environmental Protection Agency Draft Final Rule, Groundwater Standards for Remedial Actions at Inactive Uranium Processing Sites (60 FR 2854).

'Exceeds MCL.

The MCL for nitrate as (N) is $10 \mathrm{mg} / \mathrm{L}$.

The uranium concentration of $0.044 \mathrm{mg} / \mathrm{L}$ is equivalent to $30 \mathrm{pCi} / \mathrm{L}$, which is the $\mathrm{MCL}$.

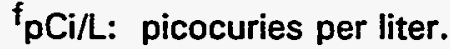


Table 3.3. Maximum observed concentrations of EPA MCL constituents in monitor wells located in the Tres Hermanos- $C_{1}$ Sandstone at the Ambrosia Lake site during the years from 1980 to 1994

\begin{tabular}{|c|c|c|c|}
\hline \multirow[b]{2}{*}{ Constituent $^{\mathbf{a}}$} & \multirow{2}{*}{$\begin{array}{c}\mathrm{MCL}^{\mathrm{b}} \\
(\mathrm{mg} / \mathrm{L})\end{array}$} & \multicolumn{2}{|c|}{ Monitor wells } \\
\hline & & 778 & 786 \\
\hline Arsenic & 0.05 & 0.022 & 0.02 \\
\hline Barium & 1 & $<0.1$ & 0.3 \\
\hline Cadmium & 0.01 & $0.014^{c}$ & $0.013^{c}$ \\
\hline Chromium & 0.05 & $0.22^{c}$ & $0.14^{c}$ \\
\hline Lead & 0.05 & 0.02 & 0.02 \\
\hline Mercury & 0.002 & 0.0005 & 0.0003 \\
\hline Molybdenum & 0.1 & $0.16^{c}$ & $0.34^{c}$ \\
\hline Nitrate & $44^{d}$ & $4: 30^{c}$ & $55^{c}$ \\
\hline Selenium & 0.01 & $0.28^{c}$ & $0.78^{c}$ \\
\hline Silver & 0.05 & $0.06^{c}$ & 0.02 \\
\hline Combined Uranium-234 and -238 & $0.044^{e}$ & $11.8^{c}$ & $2.88^{c}$ \\
\hline $\begin{array}{l}\text { Gross alpha (excluding radon and } \\
\text { uranium) } \mathrm{pCi} / \mathrm{L}^{f}\end{array}$ & 15 & $9400^{c}$ & $1300^{c}$ \\
\hline Combined Radium-226 and $-228 \mathrm{pCi} / \mathrm{L}^{f}$ & 5 & $7.93^{\mathrm{C}}$ & $9.92^{c}$ \\
\hline \multicolumn{4}{|c|}{ 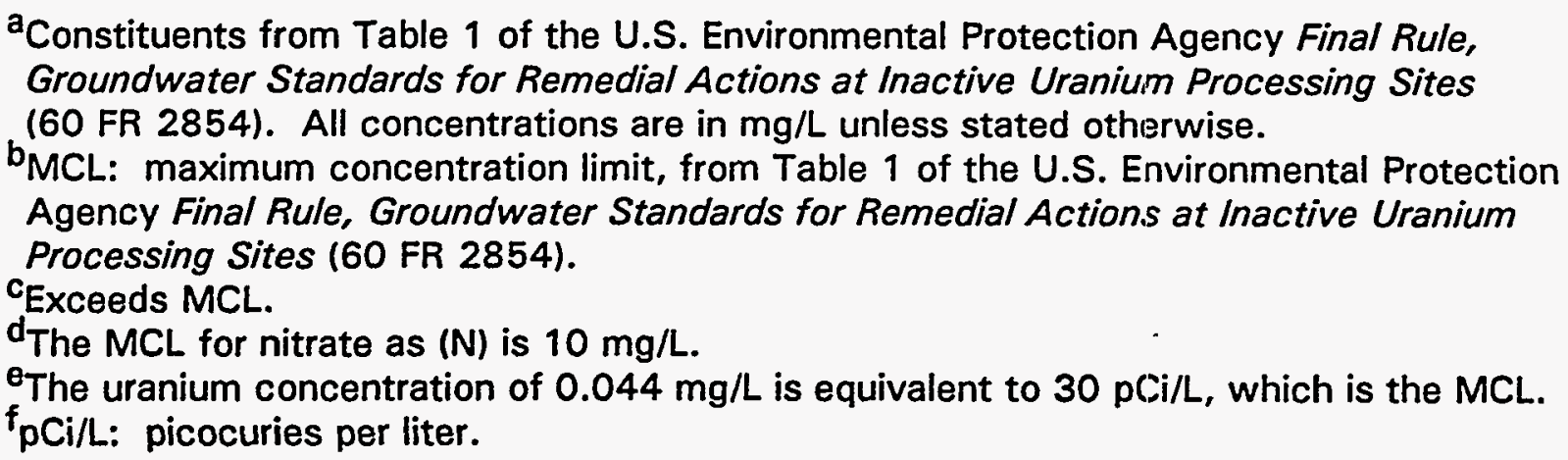 } \\
\hline
\end{tabular}


Table 3.4 Maximum observed concentrations of EPA MCL constituents in monitor wells located in the Tres Hermanos- $\mathrm{C}_{2}$ Sandstone at the Ambrosia Lake site during the years from 1980 to 1994

\begin{tabular}{|c|c|c|c|c|c|c|}
\hline \multirow[b]{2}{*}{ Constituent $^{\mathrm{a}}$} & \multirow{2}{*}{$\begin{array}{l}M C^{b} \\
(\mathrm{mg} / \mathrm{L})\end{array}$} & \multicolumn{5}{|c|}{ Monitor wells } \\
\hline & & 779 & 785 & 787 & 677 & 676 \\
\hline Arsenic & 0.05 & 0.025 & 0.012 & 0.019 & $<0.01$ & $<0.01$ \\
\hline Barium & 1 & 0.3 & 0.1 & 0.01 & 0.01 & 0.01 \\
\hline Cadmium & 0.01 & $0.016^{c}$ & $0.024^{c}$ & $0.012^{C}$ & 0.004 & 0.003 \\
\hline Chromium & 0.05 & $0.22^{c}$ & $0.24^{c}$ & $0.23^{c}$ & $<0.01$ & $<0.01$ \\
\hline Lead & 0.05 & 0.02 & 0.02 & 0.02 & 0.05 & 0.04 \\
\hline Mercury & 0.002 & 0.0006 & 0.0006 & 0.0004 & $<0.0002$ & $<0.0002$ \\
\hline Molybdenum & 0.1 & $0.14^{c}$ & $0.35^{c}$ & $0.25^{c}$ & 0.024 & $0.595^{c}$ \\
\hline Nitrate & $44^{d}$ & 2.29 & 20 & 29 & 6.9 & 25.6 \\
\hline Selenium & 0.01 & $0.092^{c}$ & $0.324^{c}$ & $0.054^{c}$ & $0.037^{c}$ & $0.091^{c}$ \\
\hline Silver & 0.05 & 0.05 & $0.08^{c}$ & $0.09^{c}$ & $<0.01$ & $<0.01$ \\
\hline $\begin{array}{l}\text { Combined } \\
\text { Uranium-234 and } \\
-238\end{array}$ & $0.044^{e}$ & 0.0238 & $3.30^{C}$ & 0.018 & 0.016 & $0.207^{c}$ \\
\hline $\begin{array}{l}\text { Gross alpha } \\
\text { (excluding radon and } \\
\text { uranium) pCi/L } f\end{array}$ & 15 & $74^{c}$ & $1900^{c}$ & $57.4^{c}$ & $29^{c}$ & $85.9^{C}$ \\
\hline $\begin{array}{l}\text { Combined } \\
\text { Radium-226 and } \\
-228 \mathrm{pCi} / \mathrm{L}^{f}\end{array}$ & 5 & $5.6^{\mathrm{C}}$ & $10.51^{c}$ & 4.2 & $6.45^{c}$ & $24.12^{c}$ \\
\hline
\end{tabular}

${ }^{a}$ Constituents from Table 1 of the U.S. Environmental Protection Agency Final Rule, Groundwater Standards for Remedial Actions at Inactive Uranium Processing Sites (60 FR 2854). All concentrations are in $\mathrm{mg} / \mathrm{L}$ unless stated otherwise.

bMCL: maximum concentration limit, from Table 1 of the U.S. Environmental Protection Agency Final Rule, Groundwater Standards for Remedial Actions at Inactive Uranium Processing Sites (60 FR 2854).

CExceeds MCL.

dTe MCL for nitrate as $(\mathrm{N})$ is $10 \mathrm{mg} / \mathrm{L}$.

eThe uranium concentration of $0.044 \mathrm{mg} / \mathrm{L}$ is equivalent to $30 \mathrm{pCi} / \mathrm{L}$, which is the $\mathrm{MCL}$. $f_{\mathrm{pCi} \text { L: }}$ picocuries per liter. 


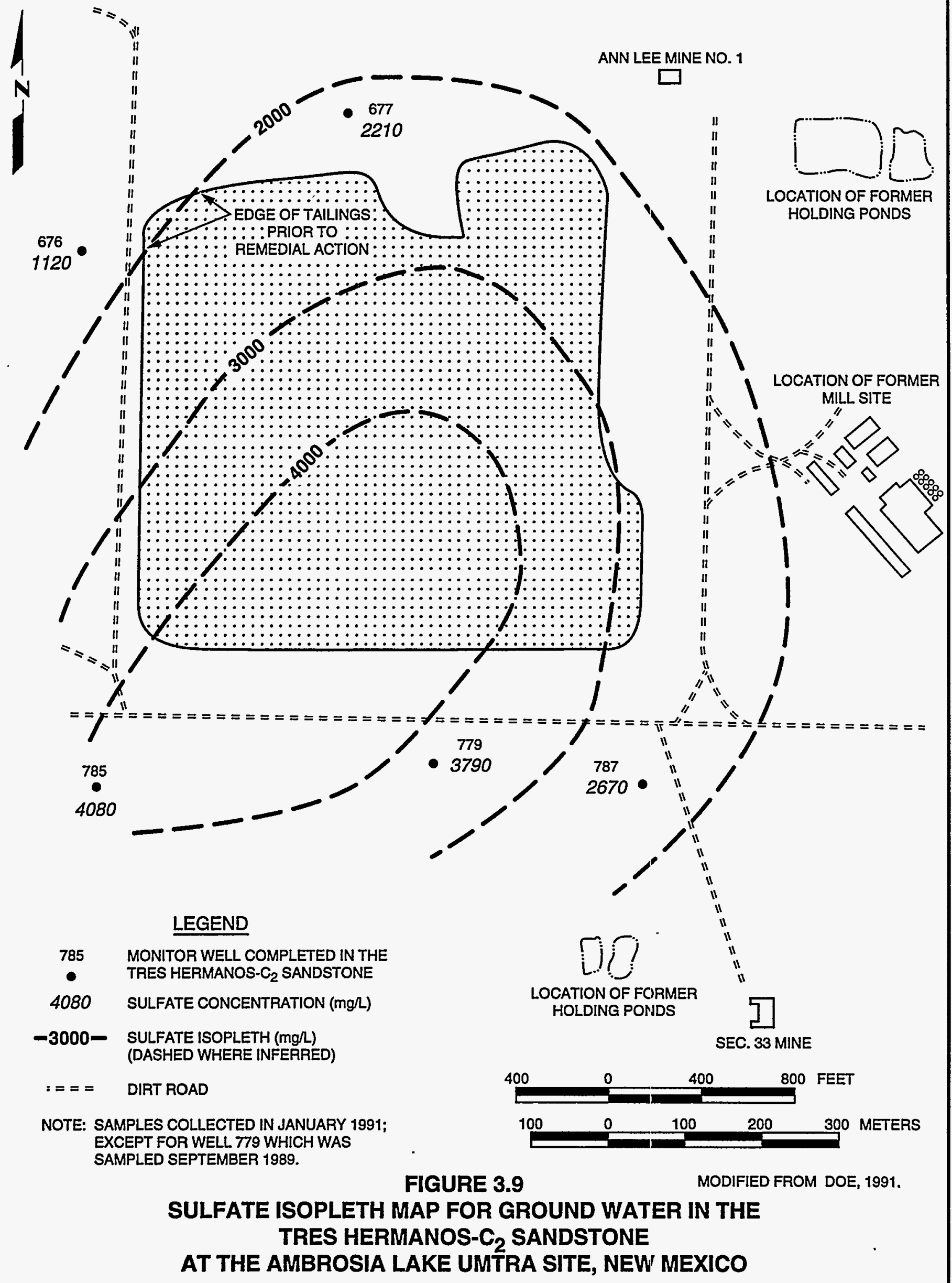


EPA MCLs in ground water from wells completed in the Tres Hermanos- $\mathrm{C}_{2}$ Sandstone (Table 3.4). Maximum concentrations of several parameters in the Tres Hermanos-C Sandstone have increased over those of previous years (compare Tables 3.3 and 3.4 to Tables D.8.13 and D.8.14, respectively in DOE, 1991), indicating that contamination is moving downgradient.

\section{Units below the Tres Hermanos-C Sandstone}

Units between the bottom of the Tres Hermanos-C Sandstone and the top of the Westwater Canyon Member include, in descending order, the Tres Hermanos-B and $-A$ Sandstones, and the Dakota Formation. Ground water from monitor wells completed in these units does not appear to be affected by siterelated contamination.

Nitrate levels are increasing in monitor well 678, completed in the Tres Hermanos-B Sandstone (3410 mg/L in the December, 1992 sampling round) (Figure 3.10). This increase is not related to uranium processing at the Ambrosia Lake site because: 1) it is not accompanied by increases in other relatively mobile site-related parameters (e.g. molybdenum, uranium, or sulfate), and 2) nitrate concentrations in monitor well 678 are much higher than average concentrations found in tailings pore fluids (approximately $1400 \mathrm{mg} / \mathrm{L}$ ). Further, the Tres Hermanos-B Sandstone is hydrologically isolated from alluvium and the weathered Mancos Shale at the site (see section 3.3.1). Relatively high nitrate levels are most likely related to the nearby Quivira Mining Company tailings pile.

\section{Westwater canyon member}

The Westwater Canyon Member is the primary source of uranium ore in the area and was the focus of intense mining-related activity. Thus, mining activities not related to UMTRA Project site tailings seepage introduced many other sources of contamination into the Westwater Canyon Member. The DOE made a comparison of concentrations of contaminants in the Tres Hermanos-C Sandstone and the Westwater Canyon Member (Table D.8.26 of DOE, 1991) and concluded that, in general, concentrations in the Tres Hermanos-C Sandstone are lower. This suggests that seepage from the Tres Hermanos-C Sandstone ground water will produce no increases in the concentrations of contaminants in the Westwater Canyon Member.

\subsubsection{Contaminant fate and transport}

Percolation of alkaline tailings leachate from the Ambrosia Lake site into ground water has not raised the $\mathrm{pH}$ of ground water in monitor wells at the site. The near-neutral pH (6.84-7.91) measured in the Tres Hermanos- C Sandstone during the November-December 1992 sampling round indicates that the baseneutralization potential of the alluvium and the weathered Mancos Shale and the Tres Hermanos-C Sandstone has not been exhausted. Although an alkaline $\mathrm{pH}$ front has migrated through subsoils beneath the former tailings site, the change in $\mathrm{pH}$ is relatively insignificant and does not affect the solubility of contaminants 


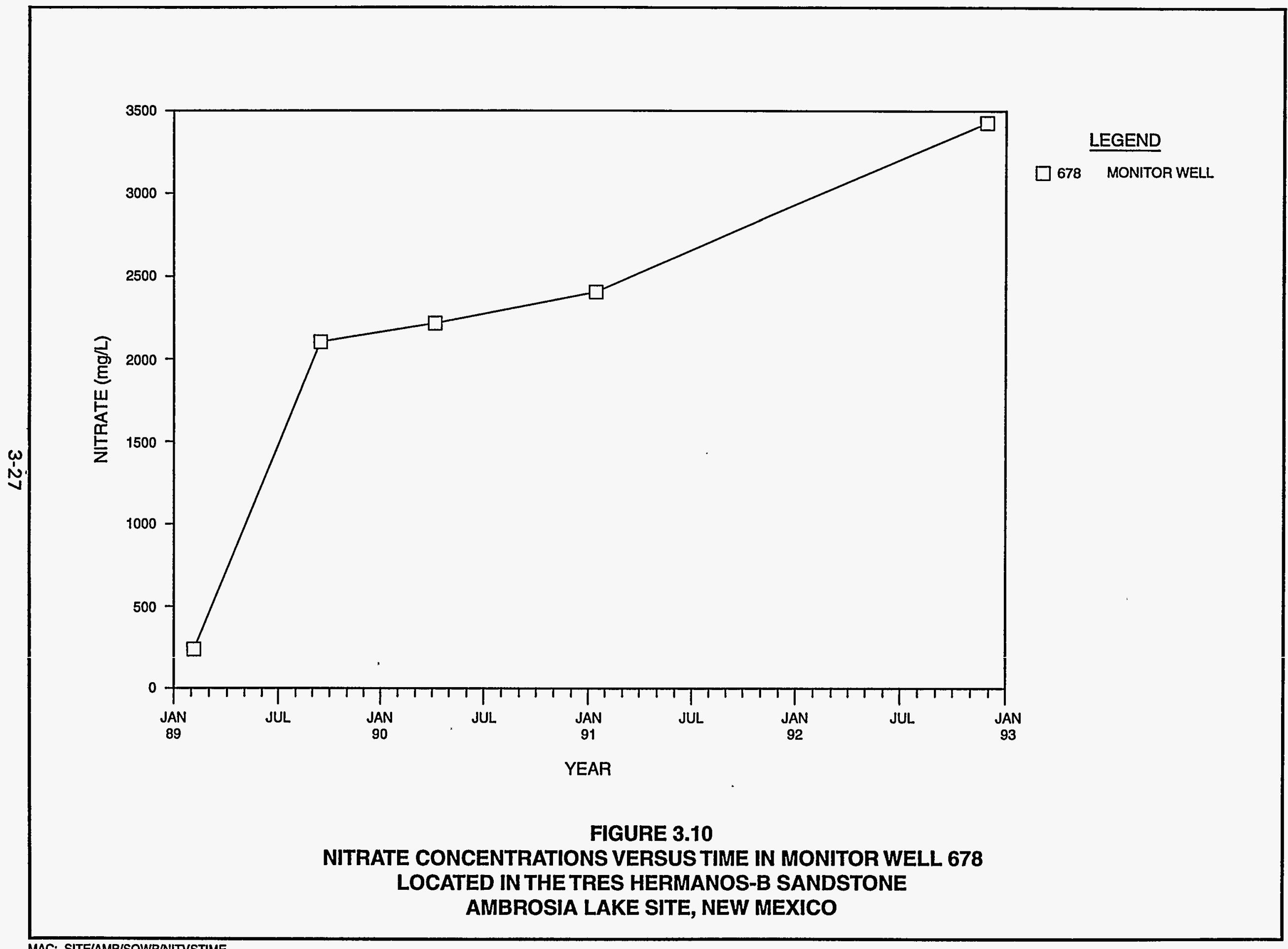


in tailings seepage (Table D.8.22 of DOE, 1991). The tailings fluid and ground water in the alluvium and the weathered Mancos Shale is oxidizing, but becomes somewhat less oxidizing as it flows from the alluvium and weathered Mancos Shale into the Tres Hermanos-C Sandstone (Table D.8.22 of DOE, 1991). The change, however, does not significantly affect the solubility of most contaminants. The most important EPA MCL-list contaminants in the ground water are nitrate, selenium, molybdenum, and uranium which occur as anions (DOE, 1991). Adsorption by the cation exchange process is not an important mechanism for removal of most of these contaminants at the Ambrosia Lake site. Dilution of tailings seepage by mill makeup water that drained into the alluvium and the weathered Mancos Shale reduced concentrations of these contaminants in the alluvium and the weathered Mancos Shale. Ultimately, contamination at the site will drain through mine shafts and drill holes into the Westwater Canyon Member of the Morrison Formation or be retained with residual moisture in the unsaturated alluvium and the weathered Mancos Shale or Tres Hermanos-C Sandstone.

\subsubsection{Risk evaluation}

Information on site-related risk to human health and the environment is integral to this SOWP to help guide the recommendation of a remediation strategy for the site. An initial risk assessment of ground water contamination at the Ambrosia Lake site was published in the RAP (DOE, 1991). This preliminary risk assessment discussed the potential health risks to humans from ingesting contaminated ground water in the uppermost aquifer beneath the former uranium mill processing site.

\section{Potential public health impacts}

The initial risk assessment presented in the 1991 RAP (DOE, 1991), qualitatively investigated potential risks to public health from exposure to ground water, assuming the most conservative pathway, exposure through drinking water, from the most contaminated wells at the processing site. The evaluation was based on ground water data and followed the hazard index approach proposed by the EPA (EPA, 1989). This initial identification of hazards, assessment of toxicity, assessment of potential exposure, and characterization of risk showed that levels of contaminants that would result in potential adverse health effects, if the ground water were ingested on a longterm basis, are present in shallow ground water beneath and adjacent to the site. Three indicator chemicals were selected for the evaluation: selenium, radium-226, and natural uranium. Hazard indices for selenium and uranium exceeded an acceptable index of one, and the excess lifetime cancer risks calculated exceeded the EPA acceptable limit of $1 \times 10^{-4}$ (DOE, 1991).

Currently, no domestic wells accessing the contaminated ground water in the uppermost aquifer exist within $5 \mathrm{mi}(8 \mathrm{~km})$ of the site, and surface expression of ground water does not occur. Therefore, with no current exposure pathway present there are no current human health or environmental risks associated 
with the contaminated ground water attributable to the Ambrosia Lake UMTRA site.

The potential for future use of the most contaminated ground water at the site is not probable. Several site conditions exist that greatly reduce the possibility of future exposure. The NRC and the state of New Mexico concurred with application of supplemental standards for "limited use" ground water at the Ambrosia Lake site for the UMTRA Surface Project (NRI, 1990). The ground water in the saturated zone where contamination occurs has been attributed primarily to previous mill-processing activities. An aquifer test on well 675 performed in February 1989 and described in the RAP (IDOE, 1991), shows that the uppermost aquifer is incapable of a sustained yield of $150 \mathrm{gal}(570 \mathrm{~L})$ per day and is considered a "limited use" aquifer based on limited vield. Therefore, the uppermost aquifer lacks both a long-term yield sufficient to provide a continuous drinking water source and threat of chronic exposure for a potential future resident.

Since the major sources of aquifer recharge (waste water disposal and water from mine pumping) no longer exist, the saturated thickness of the aquifer is expected to either remain about the same, or decrease as ground water spreads laterally and a portion of it migrates vertically upward into the vadose zone. Furthermore, ground water contamination attributable to the Ambrosia Lake site has not migrated from the area beneath the site, and no shallow domestic wells have been identified within a $5 \mathrm{mi}(8 \mathrm{~km})$ radius from the site. Land use in the area is not expected to change in the future because of the poor quality of the range land, poor soils, and the limited surface or shallow ground water resources available in this semiarid region. These conditions make it very unlikely that a future resident would install a shallow well immediately adjacent to the fenced disposal site. Therefore, future long-term exposure to ground water is unlikely.

\section{Environmental evaluation}

Current exposure and the potential for future exposure to contaminated ground water were evaluated for ecological receptors (livestock and terrestrial and aquatic biota). Because surface expression of contaminated ground water does not occur, and no other current pathway (such as a well used to water livestock) has been identified, there are no current expossure pathways for contaminated ground water to be used by terrestrial and aquatic biota and livestock. Therefore, current environmental risks do not exist.

The potential for future exposure of contaminated ground water to terrestrial or aquatic biota and livestock is unlikely because 1) the contaminated ground water is within a "limited use" aquifer, 2) aquifer yield will decrease as the saturated thickness decreases, and 3) surface expression of contaminated ground water will not occur, based on existing information. Therefore, the possibility for potential future use of ground water contaminated by the Ambrosia Lake UMTRA site is unlikely. 


\subsection{ASSESSMENT OF DATA NEEDS}

The hydrogeologic site description presented is supported by regional and sitespecific data. The information includes hydrogeologic reports prepared by state and federal agencies, site-specific and regional water quality data, site and surrounding area well completion reports, and well hydraulic testing data. Because the data base indicates little variability in the hydrogeologic conditions, no additional data needs have been identified.

\subsection{EVALUATION OF INTERIM REMEDIAL ACTION}

Interim remedial action is not required because no existing risks to human health or livestock result from site contamination, nor can any potential future risks be foreseen. 


\subsection{GROUND WATER COMPLIANCE STRATEGY RECOMMENDATION}

This section explains the application of site-specific data to the PEIS ground water compliance evaluation framework; recommends a ground water compliance strategy for the Ambrosia Lake processing site based on current conditions; analyzes possible deviations from the recommended ground water compliance strategy; and notes contingencies (conceivable reason for recommendation of a different compliance strategy) and rules (criteria) for application of contingencies.

\subsection{GROUND WATER COMPLIANCE STRATEGY EVALUATION PROCESS}

Ground water compliance recommendations at the former Ambrosia Lake processing site were made by using the compliance evaluation framework shown in Figure 4.1. This compliance evaluation framework was developed for analysis in the DOE UMTRA Ground Water PEIS (DOE, 1994). The recommended ground water compliance strategy for the Ambrosia Lake site will be selected by applying site-specific data to the approved PEIS compliance evaluation framework.

The compliance evaluation framework proposed in the PEIS provides for the identification of one or more of three ground water compliance strategies, based upon site-specific data. The three compliance strategies developed in the UMTRA Ground Water PEIS are defined as follows:

- No Remediation: Application of the strategy of no remediation would mean that compliance with the ground water protection standards would be met without altering the ground water or cleaning it up in any way. This strategy could be applied at sites that have no contamination above MCLs or background levels, or at sites that have contamination above MCLs or background levels but qualify for supplemental standards or ACLs.

- Natural flushing: Natural flushing allows for the natural ground water movement and geochemical processes to decrease the contaminant concentrations to levels within regulatory limits within a given time period. This could be applied at sites where ground water compliance would be achieved with the application of natural flushing within 100 years, where effective monitoring and institutional controls could be maintained, and where the ground water is not currently and is not projected to be a drinking water source.

- Active ground water remediation: Active ground water remediation would require the application of engineered ground water remediation methods such as gradient manipulation, ground water extraction and treatment, or in situ ground water treatment to achieve compliance with the ground water protection standards. 


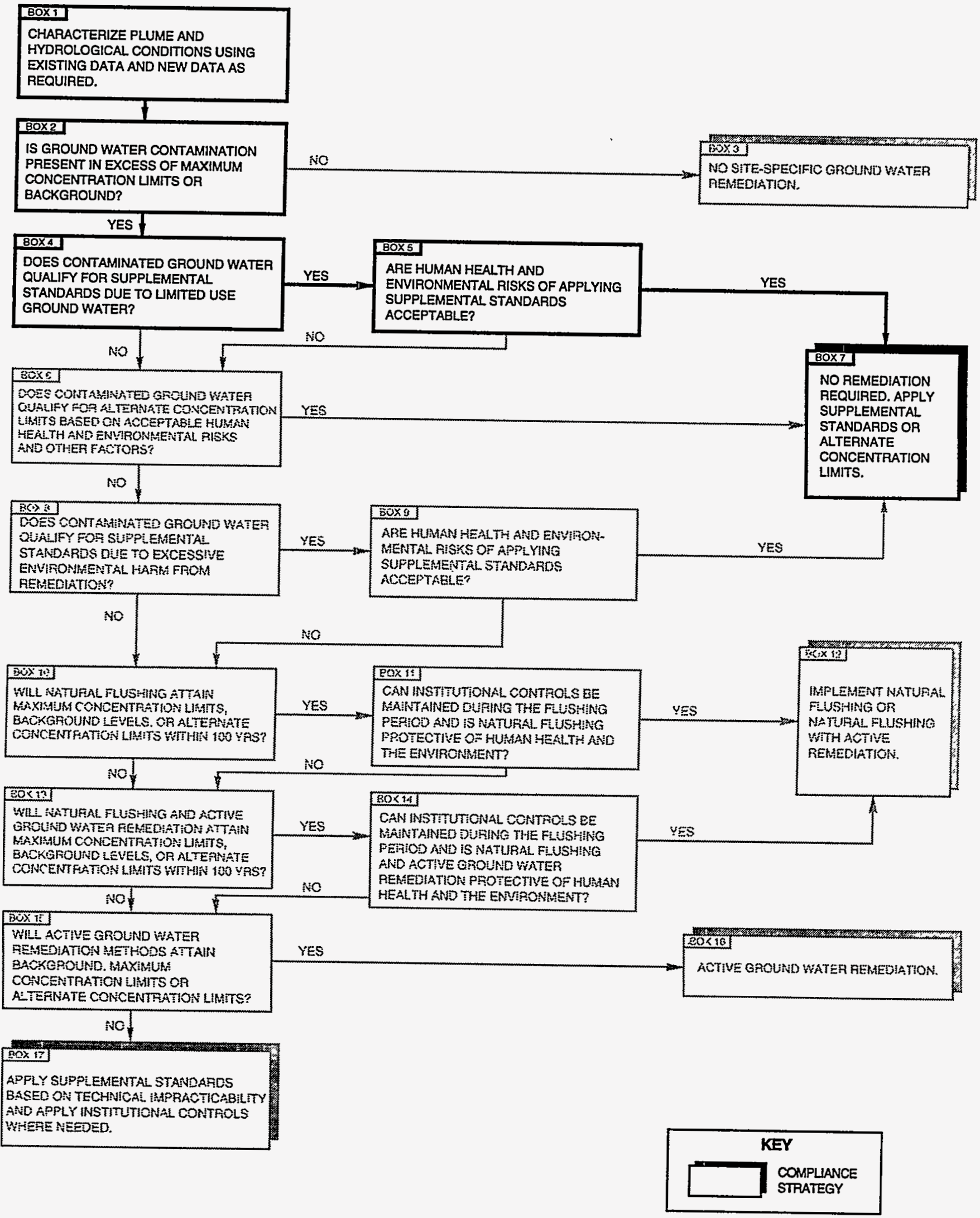

FIGURE 4.1

AMBROSIA LAKE COMPLIANCE SELECTION FRAMEWORK 
The ground water compliance strategy recommended for the Ambrosia Lake site is to perform no remediation, based on the application of supplemental standards to the affected ground water. The recommended compliance strategy is an appropriate compliance strategy to carry forward for analysis in an Ambrosia Lake site-specific NEPA document because the NRC and the state of New Mexico concurred with the RAP and application of supplemental standards for the UMTRA Surface Project (NRC, 1990).

\subsection{SITE-SPECIFIC GROUND WATER COMPLIANCE STRATEGY}

This section describes how the proposed PEIS decision-making framework was used to evaluate and recommend an appropriate ground water compliance strategy for the Ambrosia Lake site.

The ground water compliance strategy for the Ambrosia Lake site was identified through the application of site conditions to the proposed PEIS framework. The evaluation resulted in the identification of the strategy of performing no ground water remediation, based on the application of supplemental standards. The following is a description of the evaluation process, illustrated in Figure 4.1:

\section{First and second steps}

The first two steps (boxes 1 and 2 of Figure 4.1) confirm that ground water quality data obtained from the Ambrosia Lake site have resulted in ground water contamination exceeding background levels or MCLs. The uppermost aquifer was created primarily by discharge of mill process water and therefore the presence of water is primarily artificial. Because all water contained in the alluvium and the weathered Mancos Shale and Tres Hermanos-C Sandstone (uppermost aquifer) in the area of the milling site is primarily a mixture of tailings seepage and mine discharge, the extent of contamination is the same as the extent of background ground water.

\section{Third step}

The third step (box 4 of Figure 4.1) determines whether compliance with the proposed standards could be achieved by applying supplemental standards based on the presence of "limited use" ground water. "Limited use" means the ground water is not a current or potential source of drinking water because the quantity of water available is less than $150 \mathrm{gal}(570 \mathrm{~L})$ per day $(40 \mathrm{CFR}$ \$192.11(e)) (60 FR 2854). The uppermost aquifer at the Ambrosia Lake site, comprised of the alluvium, upper Mancos Shale, and Tres Hermanos-C Sandstones, is incapable of sustaining a well yield of greater than $150 \mathrm{gal}$ $(570 \mathrm{~L})$ per day based on an aquifer test (DOE, 1991). Therefore, the criterion is met for the Ambrosia Lake site for application of "limited use" ground water (supplemental standards), based on an insufficient yield of ground water. The major sources of recharge, waste water disposal and water from mine pumping, no longer exist. Therefore, the alluvium, upper Mancos Shale, and Tres Hermanos-C Sandstones are expected to return to premilling and mining 
conditions of little-to-no saturation, further eliminating the unit as a potential future ground water resource.

\section{Fourth step}

The fourth step (box 5 of Figure 4.1) determines whether applying supplemental standards results in acceptable human health and environmental risks. Levels of contaminants presenting potential adverse human health effects occur at the site, but would have effect only if the ground water were ingested on a longterm basis. Since there are no domestic wells accessing the contaminated ground water in the uppermost aquifer, and ground water does not discharge to the land surface, there are currently no human health risks associated with the contaminated ground water.

The potential for impacts to the ecological environment from contaminated ground water currently does not exist. Surface expression of ground water contaminated by the UMTRA Project site does not occur, and no other pathway (such as a well used to water livestock) has been identified. Without an exposure pathway for contaminated ground water to be used by terrestrial and aquatic biota and livestock, current environmental risks do not exist.

The potential for future use of the most contaminated ground water at the site is not probable. Ground water data show that the saturated zone at the site has a yield insufficient to provide a continuous drinking water source for a potential future resident. The saturated zone where contamination occurs is primarily attributed to previous processing activities. Since the major sources of aquifer recharge (waste water disposal and water from mine pumping) no longer exist, the saturated thickness of the existing ground water is expected to either remain about the same or decrease, as water spreads laterally and a portion of it migrates upward into the vadose zone. Therefore, future long-term exposure to ground water is unlikely.

\section{Fifth step}

The last step (box 7 of Figure 4.1 ) is the recommended site-specific compliance strategy. The strategy of performing no remediation, based on the application of supplemental standards to the site ground water is proposed as the recommended compliance strategy for the Ambrosia Lake site. The recommended compliance strategy is appropriate because the proposed PEIS process evaluation criteria and conclusions in the preceding steps are supported by existing site data.

\subsection{DEVIATIONS, CONTINGENCIES, AND RULES}

Elevation of site conditions through the proposed PEIS process supports the preliminary conclusion. The determination of the Ambrosia Lake site-specific hydrogeologic conditions is based on and supported by both regional and sitespecific data. Therefore, deviations that would impact the interpretation of the 
hydrogeologic conditions are not expected. Contingency plans and rules to implement those plans are necessary only if significant deviations are anticipated. Because deviations are not expected, contingency plans will not be required.

\subsection{DATA COLLECTION AND ASSESSMENT}

Additional data collection activities for the Ambrosia Lake site will not be required. Existing regional and site-specific data are aclequate to evaluate site hydrologic conditions and site risks. 


\subsection{LIST OF CONTRIBUTORS}

The following individuals contributed to the preparation of this report.

\begin{tabular}{ll}
\hline Name & Contribution \\
\hline M.D. Hansen & $\begin{array}{l}\text { Document coordinator, } \\
\text { National Environmental Policy Act coordinator }\end{array}$ \\
M.B. Leaf & Site manager, document sponsor, document review \\
A. Miller & Primary author, hydrogeology \\
M. Day & Risk evaluation \\
D. Erskine & Geochemistry \\
D. Bierley, R. Bowen, P. Briggs, & Document review \\
J. Gibb, A. Holm, L. Ulland & \\
J. Torline, R. Woodward & Editing \\
L. Sanchez, J. Martin & Text processing \\
B. Harvey & Graphic design \\
\hline
\end{tabular}




\subsection{REFERENCES}

Bostick, K., 1985. Ground-Water Discharge Plan Analysis For Kerr-McGee Nuclear Corporation, Ambrosia Lake Uranium Mill, Quivira Mining Company, February 1985, Ground Water Section, Ground Water Quality and Hazardous Waste Bureau, New Mexico Environmental Improvement Division, Santa Fe, New Mexico.

Brod, R.C., 1979. Hydrogeology and Water Resources of the Ambrosia Lake-San Mateo area, McKinley and Valencia Counties, New Mexico, June 1979, submitted in partial fulfillment of the requirements for the degree of Master of Science in Geology, New Mexico Institute of Mining and Technology, Socorro, New Mexico.

Brod, R.C. and W. J. Stone, 1981. Hydrogeology of Ambrosia Lake-San Mateo area, McKinley and Cibola Counties, New Mexico, Hydrogeologic Sheet 2, 1981, New Mexico Bureau of Mines \& Mineral Resources, a Division of New Mexico Institute of Mining \& Technology, Socorro, New Mexico.

DOC (U.S. Department of Commerce), 1990. Census of Population: General Population Characteristics, Economics and Statistics Administration, Bureau of the Census, Washington, D.C.

DOE (U.S. Department of Energy), 1994. "Programmatic Environmental Impact Statement for the Uranium Mill Tailings Remedial Action Ground Water Project," DOE/EIS-0198, Rev. 3, April 1994, prepared by the U.S. Department of Energy, UMTRA Project Office, Albuquerque Operations Office, Albuquerque, New Mexico.

DOE (U.S. Department of Energy), 1993a. Technical Approach to Groundwater Restoration, DOE/AL/62350-20F, Rev. 1, November 1993, prepared by the U.S. Department of Energy, UMTRA Project Office, Albuquerque Operations Office, Albuquerque, New Mexico.

DOE (U.S. Department of Energy), 1993b. Recommendations for the Preparation of Environmental Assessments and Environmental Impact Statements, May 1993, Office of NEPA Oversight, U.S. Department of Energy, Washington, D.C.

DOE (U.S. Department of Energy), 1992a. UMTRA Groundwater Frogram Plan, May 1992, prepared by the U.S. Department of Energy, UMTRA Project Office, Albuquerque Operations Office, Albuquerque, New Mexico.

DOE (U.S. Department of Energy), 1992b. Software Program for Environmental Analysis and Reporting (SPEAR) System, U.S. Department of Energy, UMTRA Project Office, Albuquerque Operations Office, Albuquerque, New Mexico. 
DOE (U.S. Department of Energy), 1991. Remedial Action Plan and Site Conceptual Design for Stabilization of the Inactive Uranium Mill Tailings Site at Ambrosia Lake, New Mexico, UMTRA-DOE/AL-050516.0000, November 1991, final, prepared by the U.S. Department of Energy, UMTRA Project Office, Albuquerque Operations Office, Albuquerque, New Mexico.

DOE (U.S. Department of Energy), 1987a. Environmental Assessment, Remedial Action at the Ambrosia Lake Uranium Mill Tailings Site, Ambrosia Lake, New Mexico, DOE/EA-0322, June 1987, prepared by the U.S. Department of Energy, UMTRA Project Office, Albuquerque Operations Office, Albuquerque, New Mexico.

DOE (U.S. Department of Energy), 1987b. Publication of Finding of No Significant Impact, Remedial Action at the Ambrosia Lake Uranium Mill Tailings Site, Ambrosia Lake, New Mexico, UMTRA Project Document Control Center file location number AMB EA, June 17, 1987, prepared by the U.S. Department of Energy, UMTRA Project Office, Albuquerque Operations Office, Albuquerque, New Mexico.

DOE (U.S. Department of Energy), 1985. Cooperative Agreement Between the United States Department of Energy and the State of New Mexico, DOE Cooperative Agreement Number DE-FC04-85AL20533, September 11, 1985, as amended, Uranium Mill Tailings Remedial Action Project, U.S. Department of Energy, Albuquerque Operations Office, Albuquerque, New Mexico.

EPA (U.S. Environmental Protection Agency), 1989. Risk Assessment Guidance for Superfund, Volume II, Environmental Evaluation Manual, March 1989, EPA/504/1-89/001, interim final, Office of Emergency and Remedial Response, U.S. Environmental Protection Agency, Washington, D.C.

Merritt, R.C., 1971. The Extractive Metallurgy of Uranium, 1971, Colorado School of Mines Research Institute, Golden, Colorado, prepared for the U.S. Atomic Energy Commission.

NOAA (National Oceanic and Atmospheric Administration), 1979. Climatic Atlas of the United States, prepared by Gale Research Company, Detroit, Michigan, for the National Oceanic and Atmospheric Administration, U.S. Department of Commerce, Washington, D.C.

NRC (U.S. Nuclear Regulatory Commission), 1990. Final Technical Evaluation Report for DOE's Proposed Remedial Action, Ambrosia Lake UMTRA Project Site, New Mexico, December 1990, prepared by the Uranium Recovery Field Office, Region IV, U.S. Nuclear Regulatory Commission, Denver, Colorado.

OMC (Quivira Mining Company), 1981. "Ambrosia Lake Mill, License Renewal Report," unpublished report, prepared by Quivira Mining Company, Oklahoma City, Oklahoma, for the New Mexico Environmental Improvement Division, Radiation Protection Bureau, Santa Fe, New Mexico. 
USGS (U.S. Geological Survey), 1980. U.S. Department of the Interior Geological Survey, Ambrosia Lake Quadrangle, New Mexico-McKinley County, 7.5 minute series (topographic), N3522.5 - W10745/7.5, photorevised 1980.

\section{CODE OF FEDERAL REGULATIONS}

10 CFR Part 1021, "National Environmental Policy Act; Implementation Procedures," U.S. Department of Energy, 1994.

40 CFR Part 192, "Health and Environmental Protection Standards for Uranium and Thorium Mill Tailings," U.S. Environmental Protection Agency, 1994.

40 CFR Part 1500, "Purpose, Policy, and Mandate" (Regulations for Implementing the Procedural Provisions of the National Environmental Policy Act), Council on Environmental Quality, 1994.

\section{FEDERAL REGISTER}

60 FR 2854, "Groundwater Standards for Remedial Actions at Inactive Uranium Processing Sites," Final Rule, January 11, 1995.

\section{UNITED STATES CODE}

42 USC §4321, et seq. National Environmental Policy Act, January 1, 1970.

42 USC \$7901, et seq. Uranium Mill Tailings Radiation Control Act, November 8, 1978.

42 USC $\$ 7922$, et seq. Uranium Mill Tailings Remedial Action Amendments Act, November 51988. 
APPENDIX A

LITHOLOGIC LOGS, WELL RECORDS, AND WELL CONJPLETION LOGS AMBROSIA LAKE, NEW MEXICO, UMTRA PROJECT SITE 
AMBROSIA LAKE UMTRA SITE SOWP

WELL LOGS FOR APPENDIX A

RECORD OF DOCUMENTS 
AMBROSIA LAKE UMTRA SITE SOWP

WELL LOGS FOR APPENDIX A

(as of 8/17/94)

\begin{tabular}{|c|c|c|c|}
\hline WELL NO & LITH LOG & WELL REC & WELL LOG \\
\hline 620 & 哱 & $6 \%$ & $6 \%$ \\
\hline 674 & $x$ & $x$ & $x$ \\
\hline 675 & $x$ & $x$ & $x$ \\
\hline 676 & $x$ & $x$ & $x$ \\
\hline 677 & $X$ & $x$ & $x$ \\
\hline 678 & $x$ & $x$ & $x$ \\
\hline 679 & $x$ & $x$ & $x$ \\
\hline 680 & $x$ & $x$ & $x$ \\
\hline 681 & $x$ & $x$ & $x$ \\
\hline 706 & 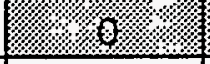 & 8 & $6.1 .1 \%$ \\
\hline 776 & $x$ & $x$ & 80. \\
\hline 777 & $x$ & $x$ & $x$ \\
\hline 778 & $x$ & $x$ & $x$ \\
\hline 779 & $x$ & $x$ & $x$ \\
\hline 781 & $x$ & $x$ & 18.8. \\
\hline 782 & $\underline{x}$ & $x$ & $\underline{x}$ \\
\hline 783 & $x$ & $x$ & $x$ \\
\hline 785 & 48 & $X$ & $x$ \\
\hline 786 & $x$ & $x$ & $x$ \\
\hline 787 & $x$ & $x$ & $x$ \\
\hline 790 & $x$ & $x$ & $x$ \\
\hline 792 & $\mathrm{X}$ & $x$ & $x$ \\
\hline 793 & $x$ & $x$ & $x$ \\
\hline 794 & $\mathrm{X}$ & $x$ & $x$ \\
\hline 796 & $\mathrm{X}$ & $x$ & $x$ \\
\hline 797 & $X$ & $x$ & $X$ \\
\hline 798 & $x$ & $x$ & $x$ \\
\hline 799 & $x$ & $x$ & $x$ \\
\hline
\end{tabular}

WELL RECORD = DIAGRAM

WELL LOG = 1 PG FORM

$X=$ RECORD OBTAINED

$0=$ RECORD MISSING 
WELL 674 LOGS 


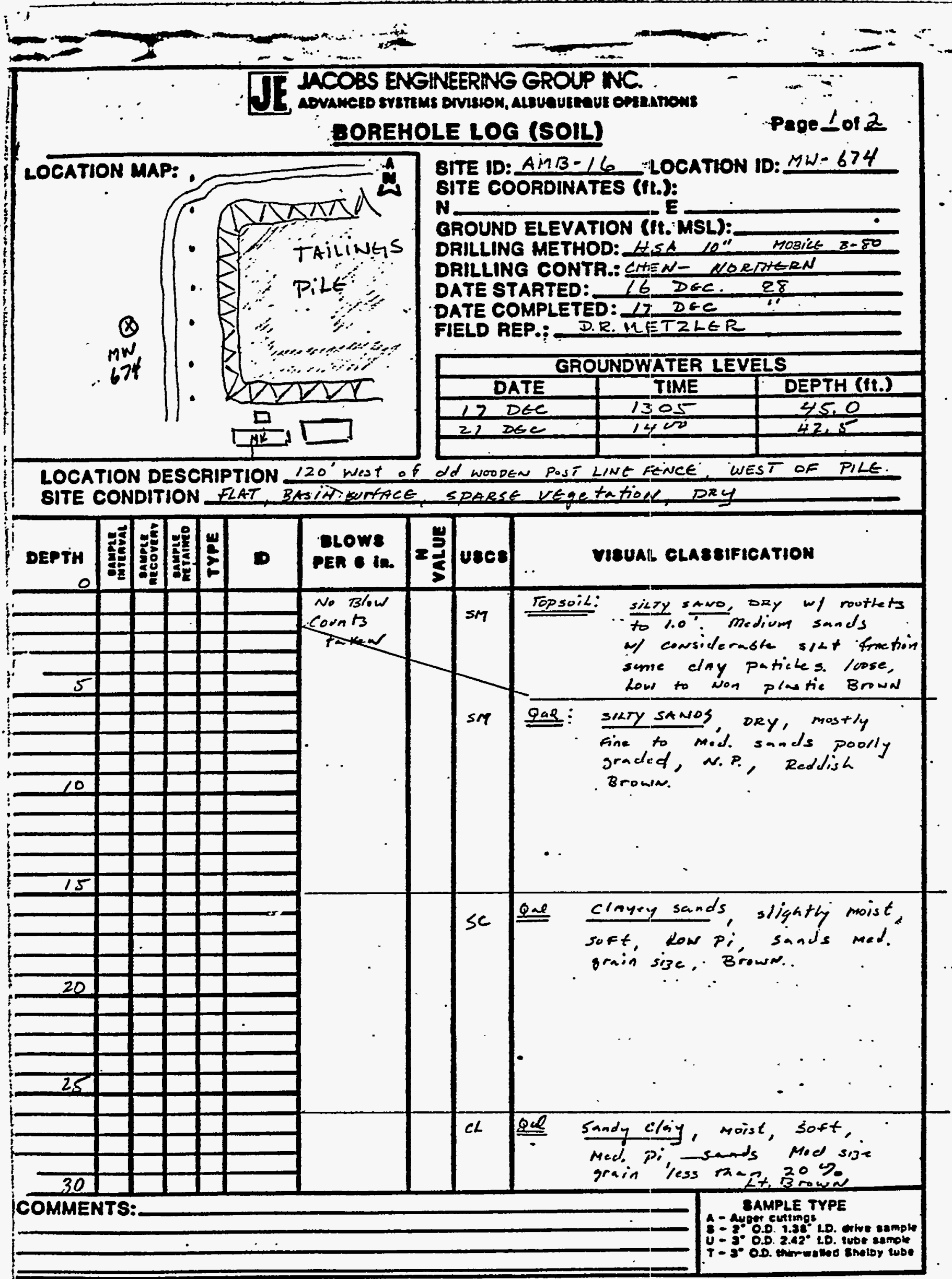




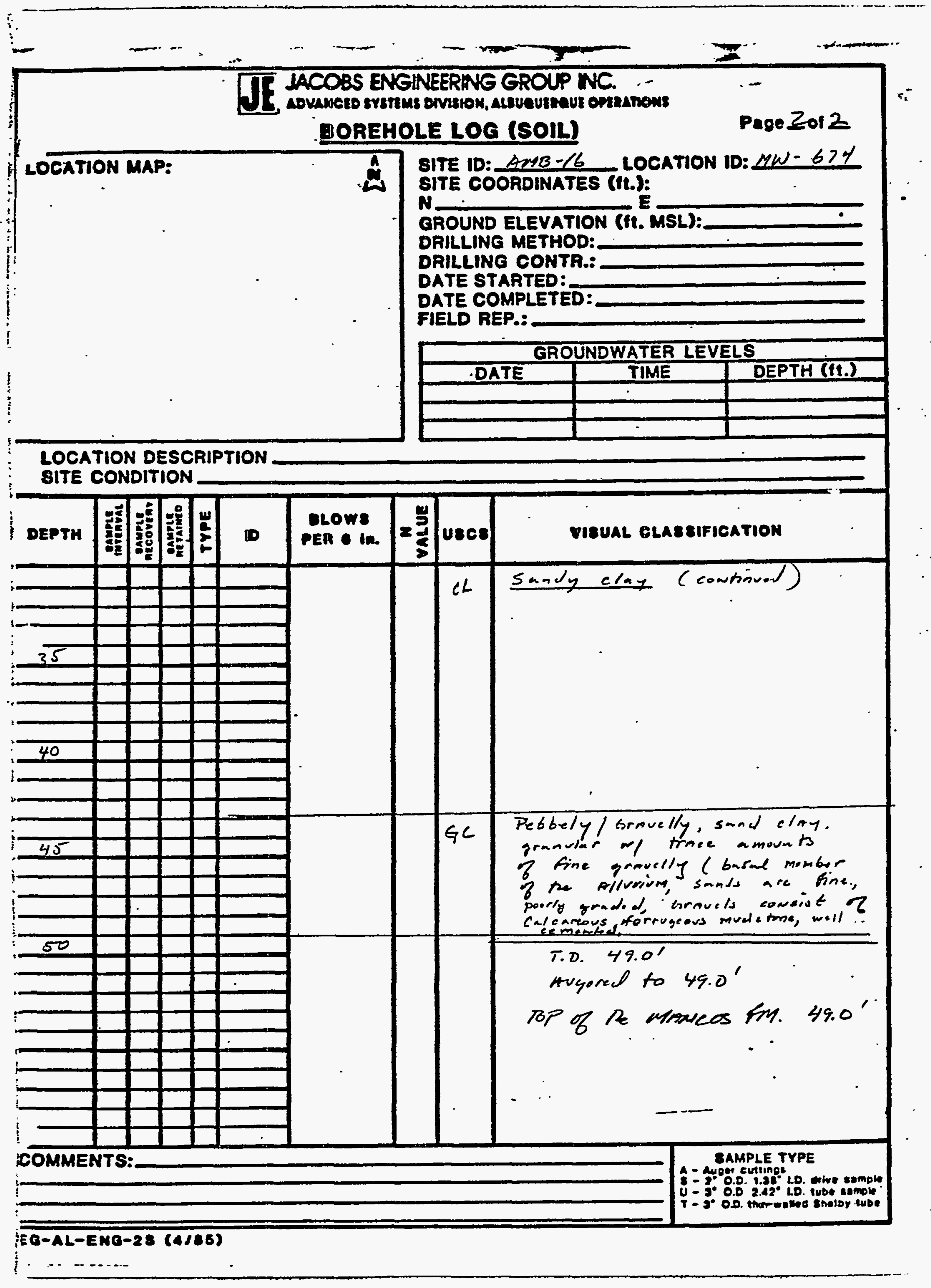




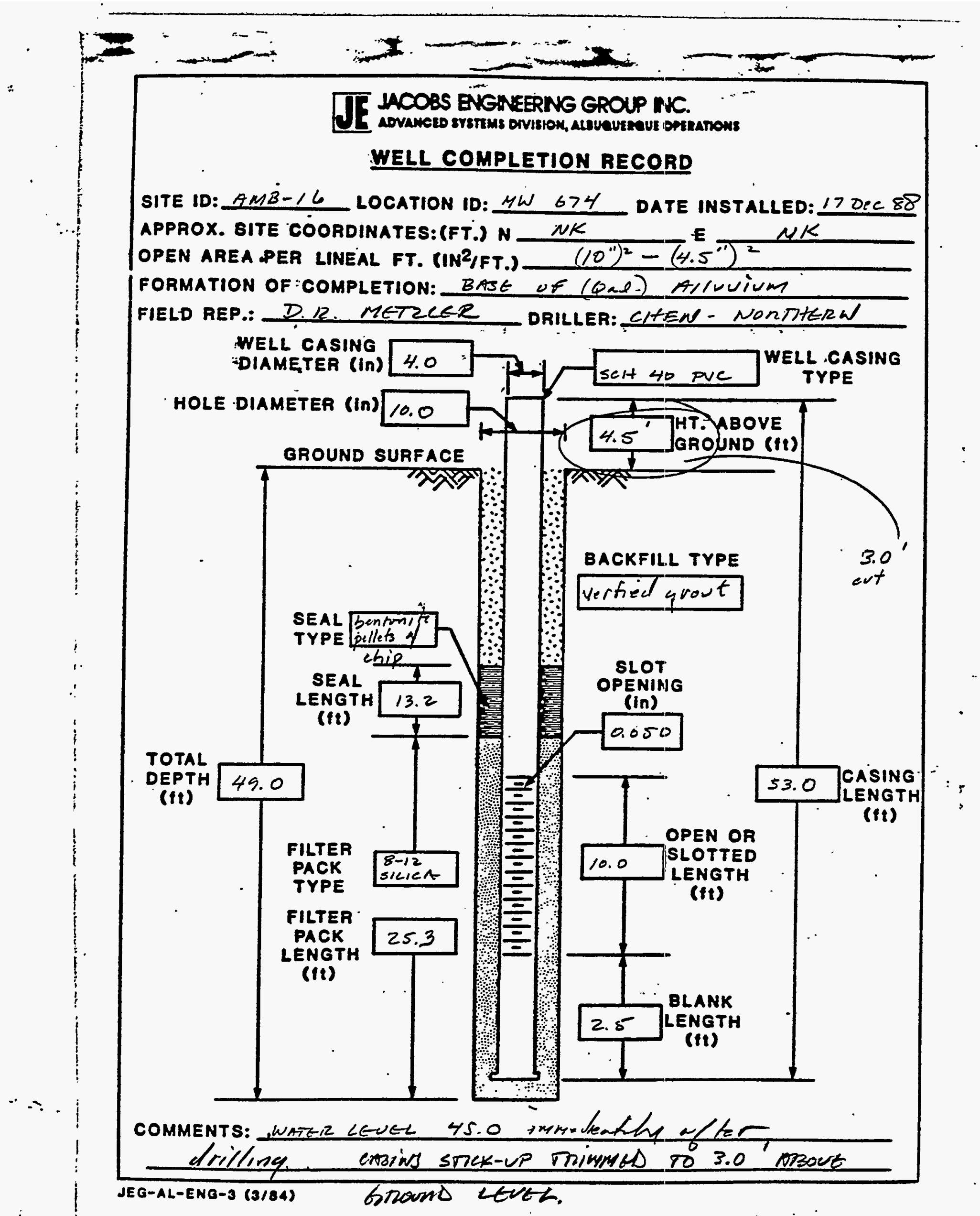




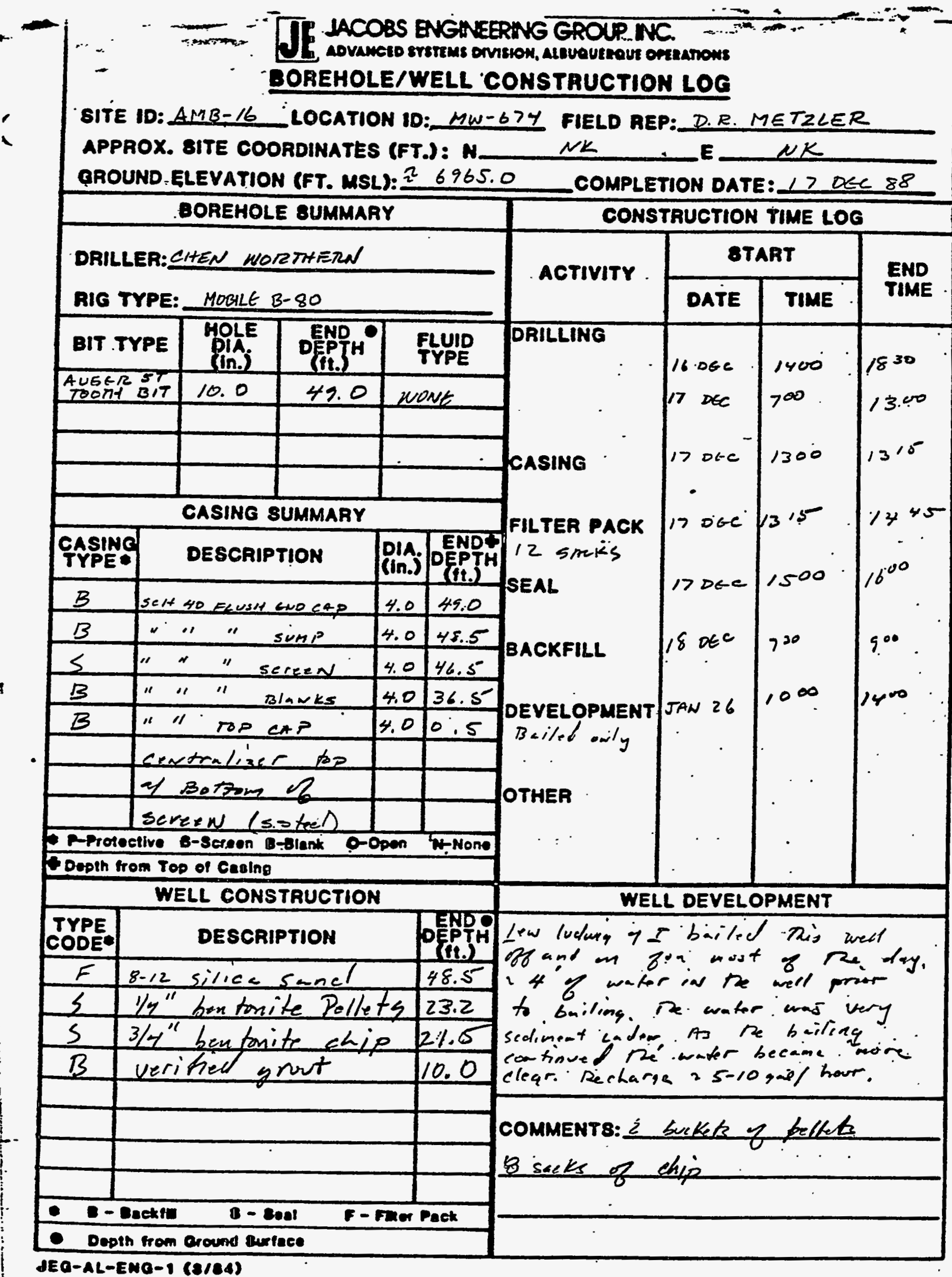


WELL 675 LOGS 


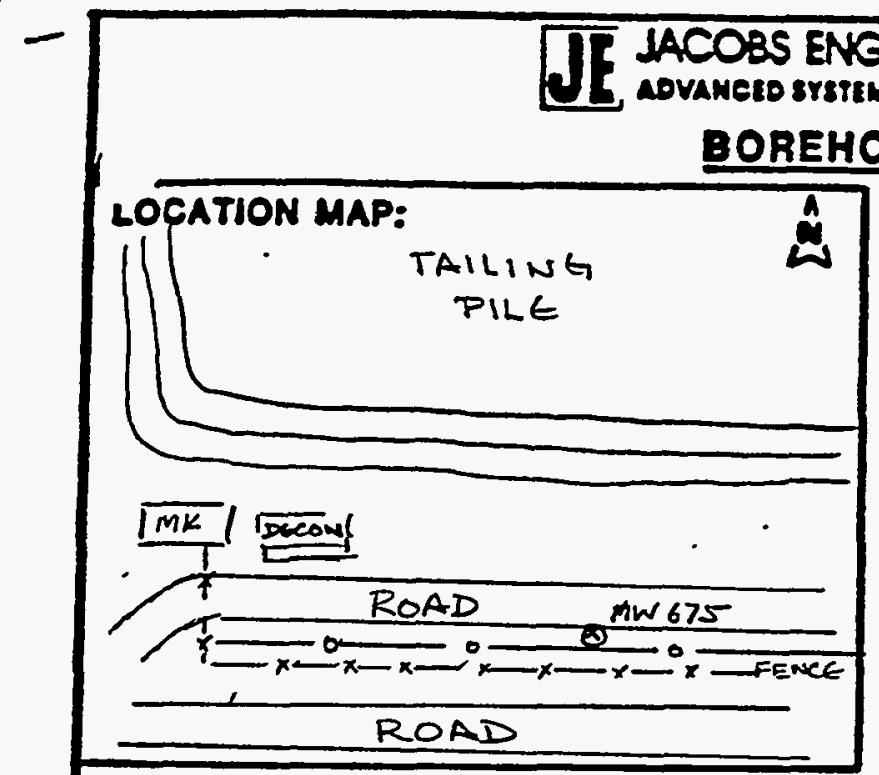

LOCATION DESCRIPTION BETWEEN THE E-W BTMINS ROSD $25^{\prime} \mathrm{N}$ R CONTROC EEMEE SITE CONDITION MIOWAY DOWN DHE PILE ON SOUTA SIDG, FLAT NEED COVERGS

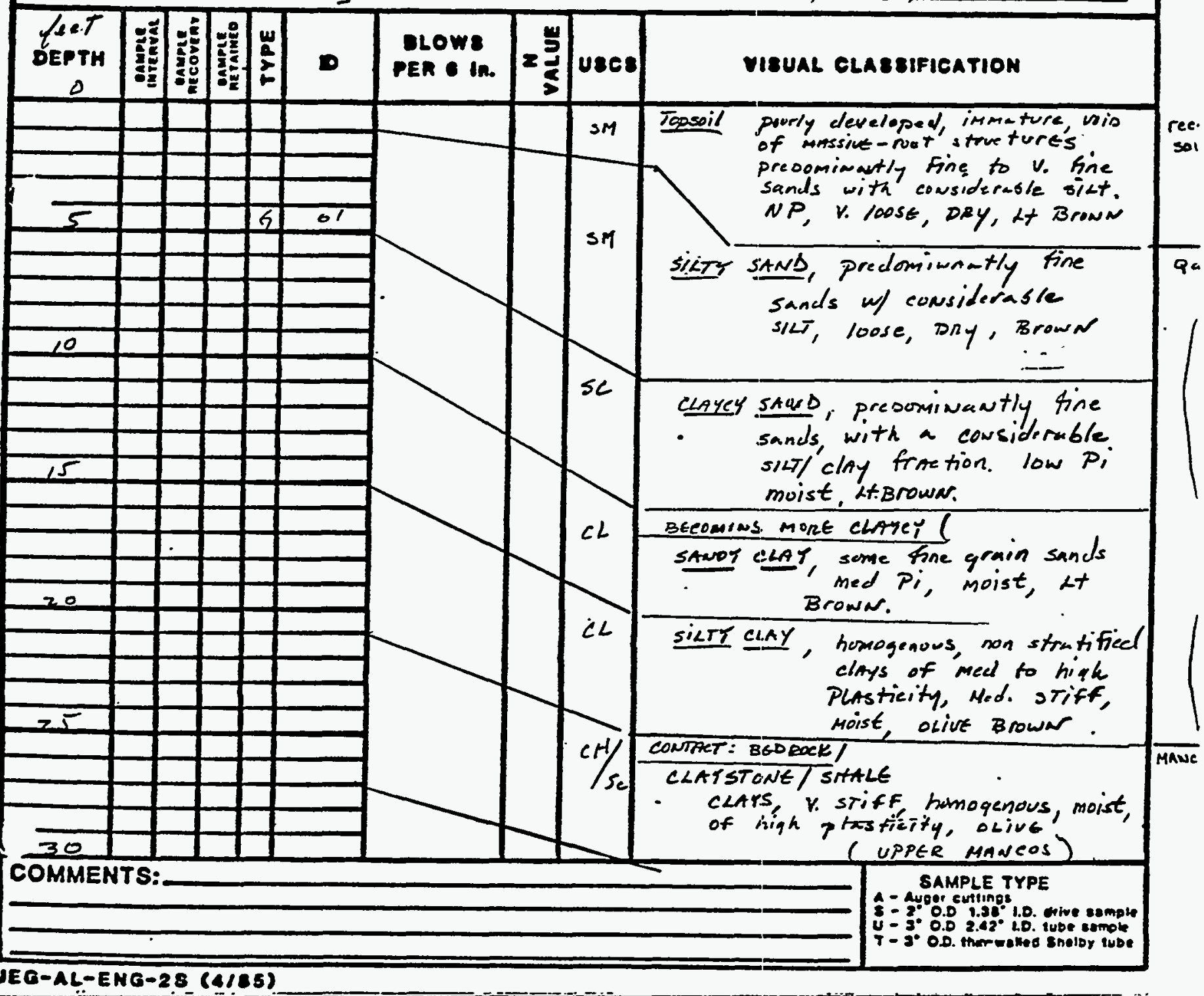




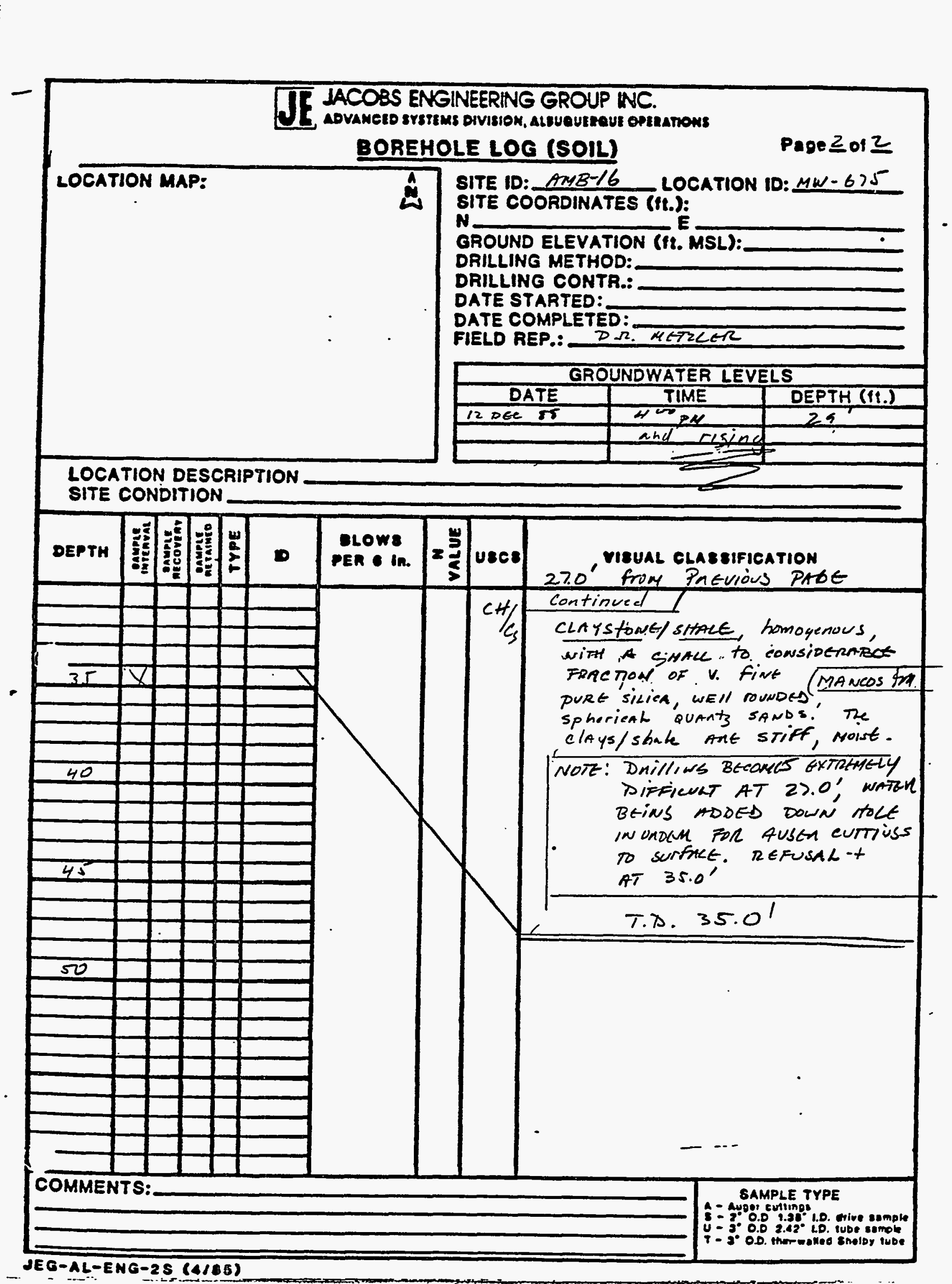




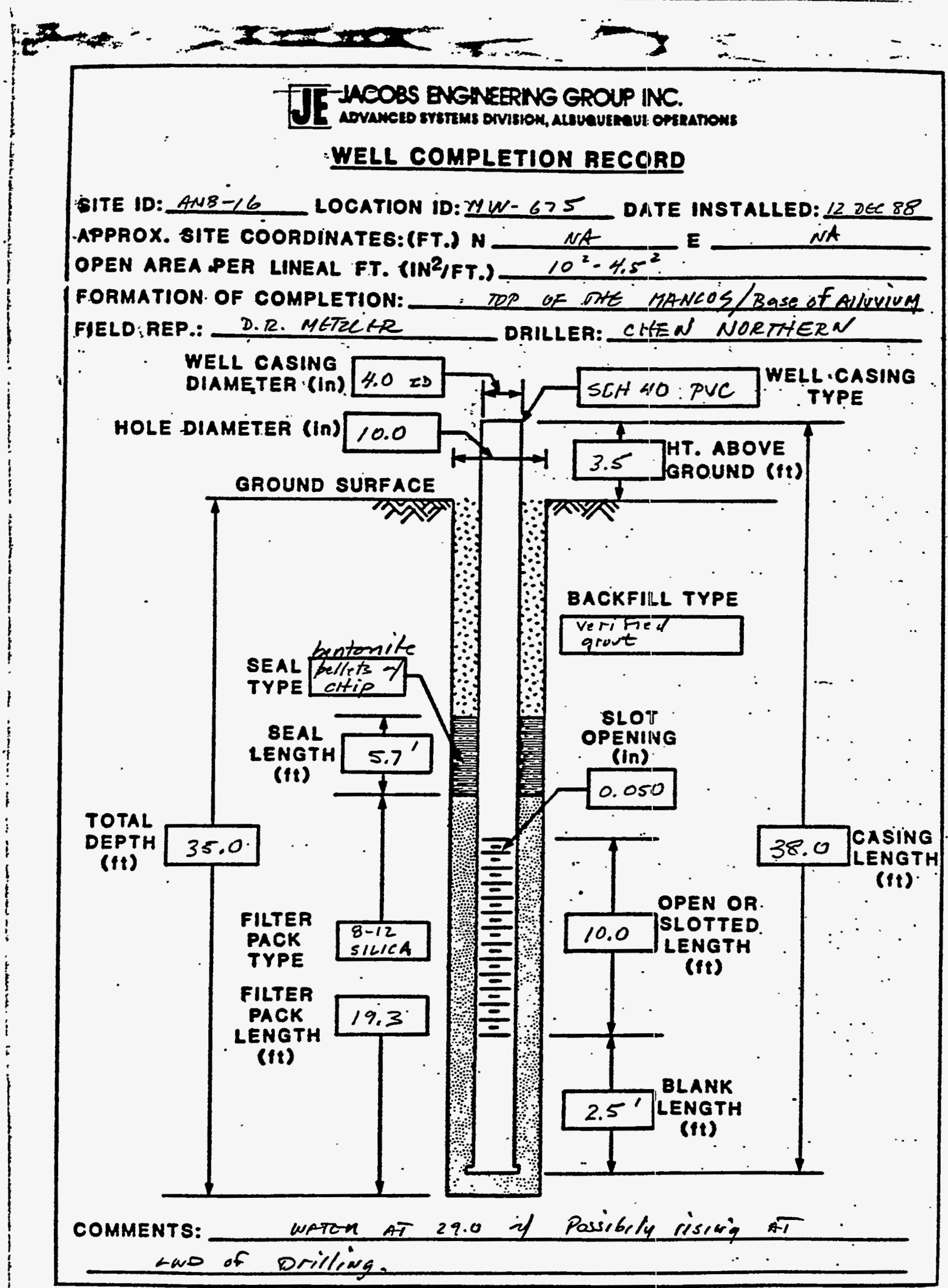

JEG-AL-ENG-3 (3/BA) 


\section{LACOBS ENGNEERIV GROUP NC.

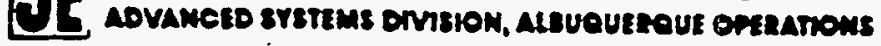 \\ BOREHOLE/WELL CONSTRUCTION LOG}

SITE ID: ANR-16 LOCATION 10:MW-675 FIELD AEP:DR. METULTR

APPROX. 8ITE COORDINATES (FT.): N_NA NA E W W

CROUND ELEVATION (FT. MSL): $26965.0^{\prime}$ COMPLETION DATE: 12 DEC 88

BOREHOLE BUMMARY

DRILLER: CHEN NURTAERN

RIG TYPE: MOZILE $80 B$

\begin{tabular}{|c|c|c|c|}
\hline BIT TYPE & $\begin{array}{l}\text { HOLE } \\
\text { PIA. } \\
\text { In. }\end{array}$ & $\begin{array}{c}\text { ENDP } \\
\text { Defit. }\end{array}$ & $\begin{array}{l}\text { FLUID } \\
\text { TYPE }\end{array}$ \\
\hline HSA & 10.0 & 35 & NONE:: \\
\hline & & & A AeN \\
\hline & & & Bucked \\
\hline & & & Ats \\
\hline
\end{tabular}

\begin{tabular}{|c|c|c|c|}
\hline \multicolumn{4}{|c|}{ CASING SUMMARY } \\
\hline $\begin{array}{l}\text { CASING } \\
\text { TYPE }\end{array}$ & DESCRIPTION & $\begin{array}{l}\text { pin. } \\
\text { (ing. }\end{array}$ & $\begin{array}{l}\text { ENDF } \\
\text { DEPTH } \\
\text { oft. }\end{array}$ \\
\hline$B$ & SCH 40 PVE SUMPP + CAP & 1.0 & 35.6 \\
\hline 5 & $"$ " $" S C R \in E N$ & 11 & 32.5 \\
\hline$B$ & " " BLANKS & $" 1$ & 22.5 \\
\hline$P$ & Protective steal croins & 858 & 6,0 \\
\hline & & & \\
\hline & & & \\
\hline & & & \\
\hline & & & \\
\hline 6 & 1e $8-8 \operatorname{cosen} 8-2$ & & \\
\hline
\end{tabular}

WELL CONSTRUCTION

\begin{tabular}{|c|c|c|}
\hline TYPE & DESCRIPTION & DEPPSH \\
\hline$E$ & B-12 sILICA SAnD & 35.0 \\
\hline$S_{1}$ & Bentowite Pellets & 15.7 \\
\hline$S_{2}$ & Bentowite chip J/4" & 13.5 \\
\hline$B$ & beatowite- brunt-cement & 10.0 \\
\hline
\end{tabular}

CONSTRUCTION TIME LOG

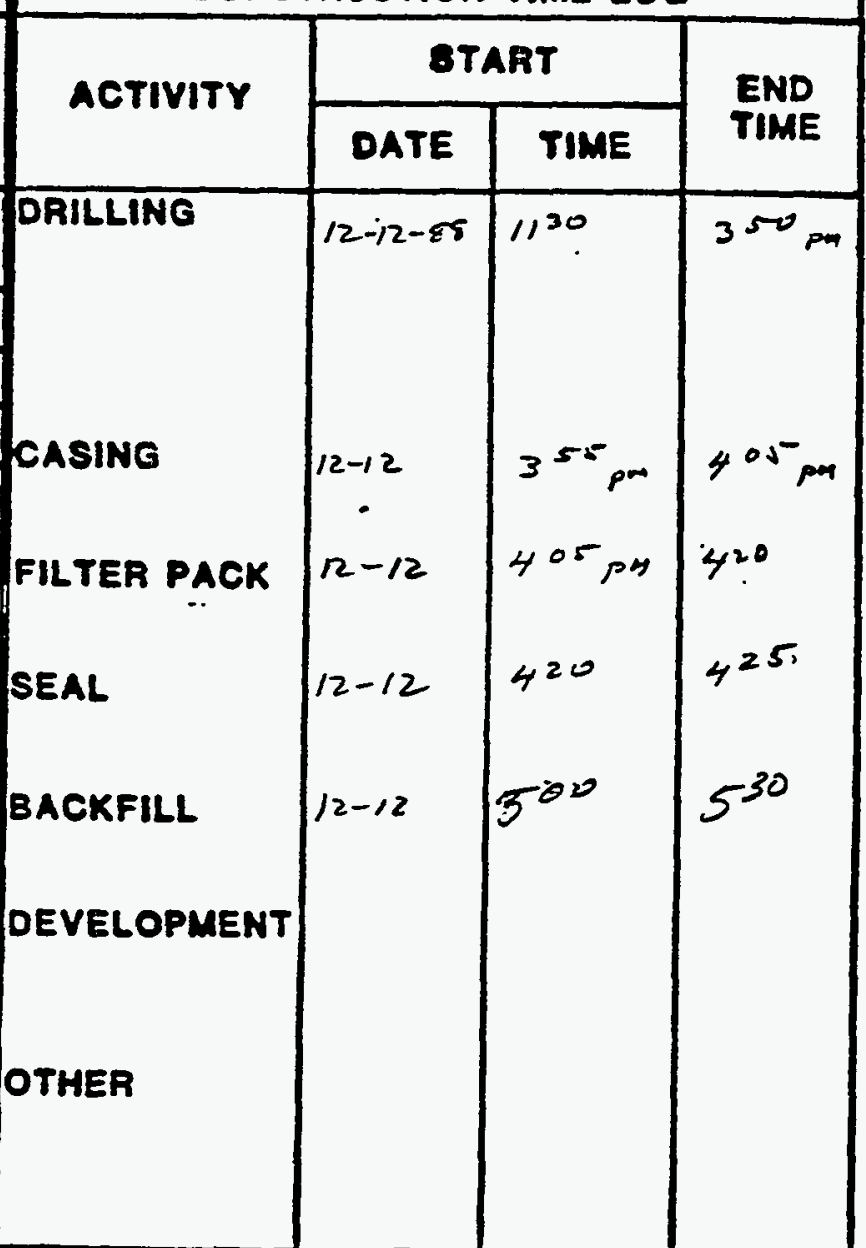

WELL DEVELOPMENT

InenTZER LEVEL 1.6 DEC 88 , is 22.5 from T. a C. 2 19,5 from brusuosureferea.

COMMENT8: 9 bags 2 8-12 silien

1 bucket of bentowith Rullets

3 bags 7 hole plug 3/4"

itydrated beutonite Pellets w/ 5 gad

- 8-Dackfe 8- Boal F-Fmar Paek

- Depth trom Ground eurface

JEO-AL-ENO-1 (2184) 
WELL 676 LOGS 


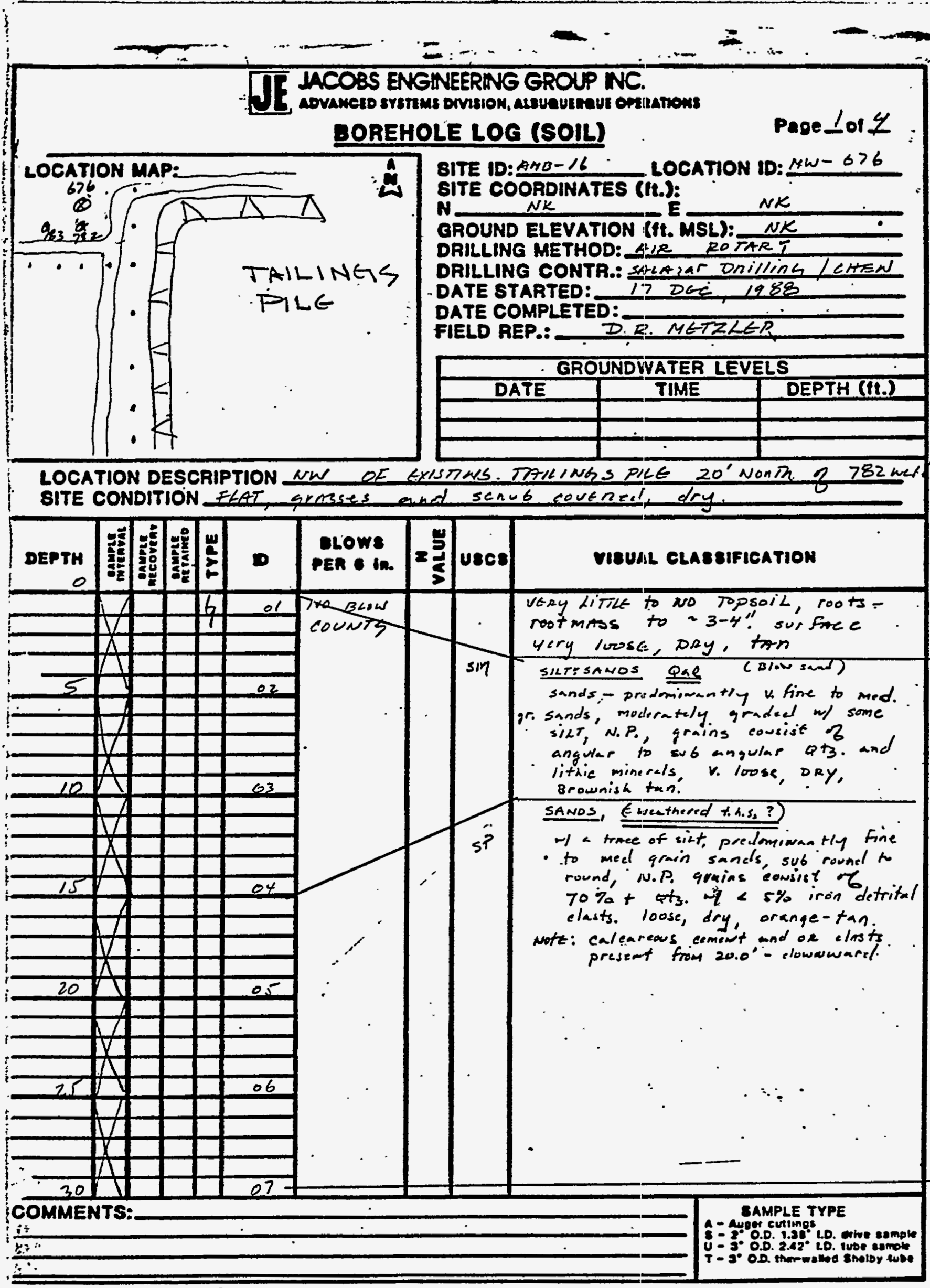

IEg-AL-ENO-28 (AIBE) 


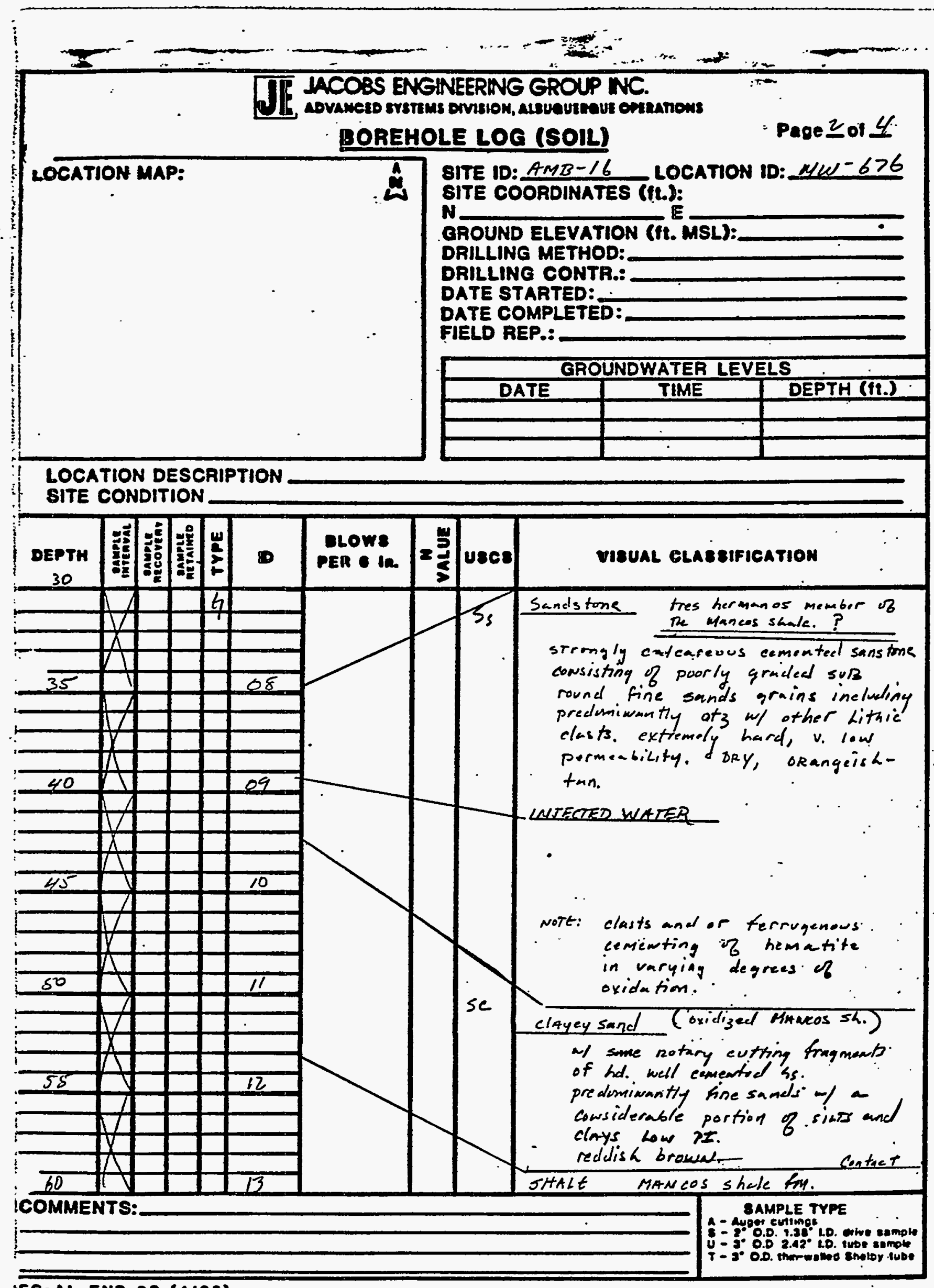




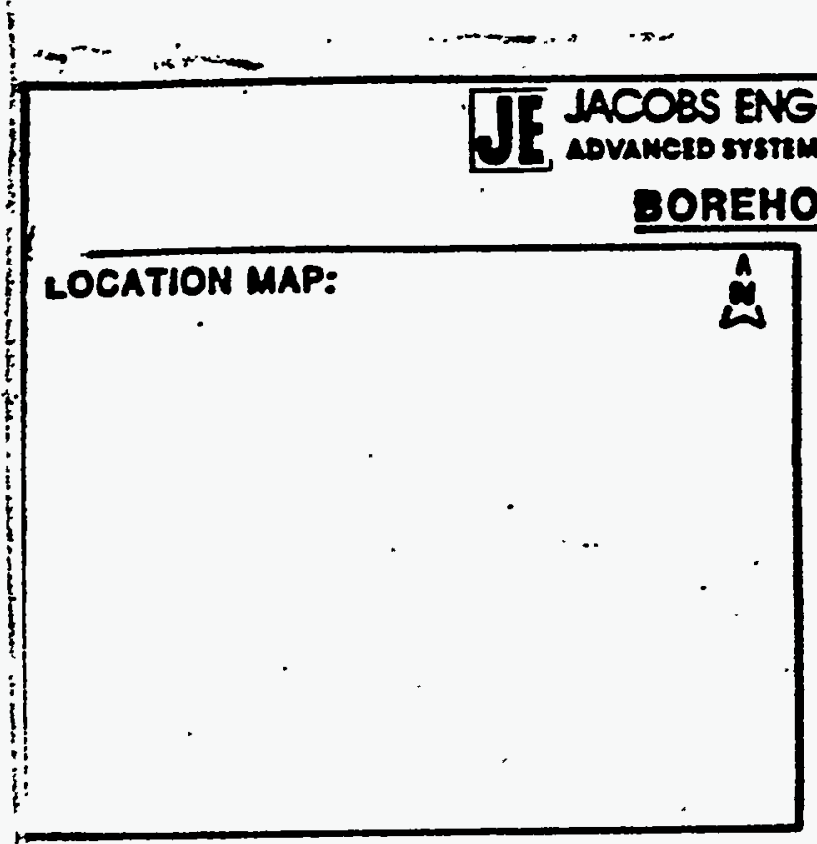

$\cos$

$+35$

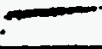

EERING GROUP NC.

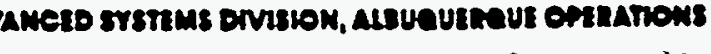

OREHOLE LOG (SOIL)

Page 3 or 4

BITE ID: $\frac{\text { AMB }-16}{\text { OAD LOCATION ID: } \mu W-676}$

SITE COORDINATES (It.):

N-

CROUND ELEVATION (It. MSL):

DRILLING METHOD:

DRILLING CONTR.:

DATE STARTED:

DATE COMPLETED:

FIELD REP.:

\begin{tabular}{|c|c|c|}
\hline \multicolumn{3}{|c|}{ GROUNDWATER LEVELS } \\
\hline DATE & TIME & DEPTH (fI.) \\
\hline & & $\cdots$ \\
\hline & & \\
\hline
\end{tabular}

LOCATION DESCRIPTION

SITE CONDITION

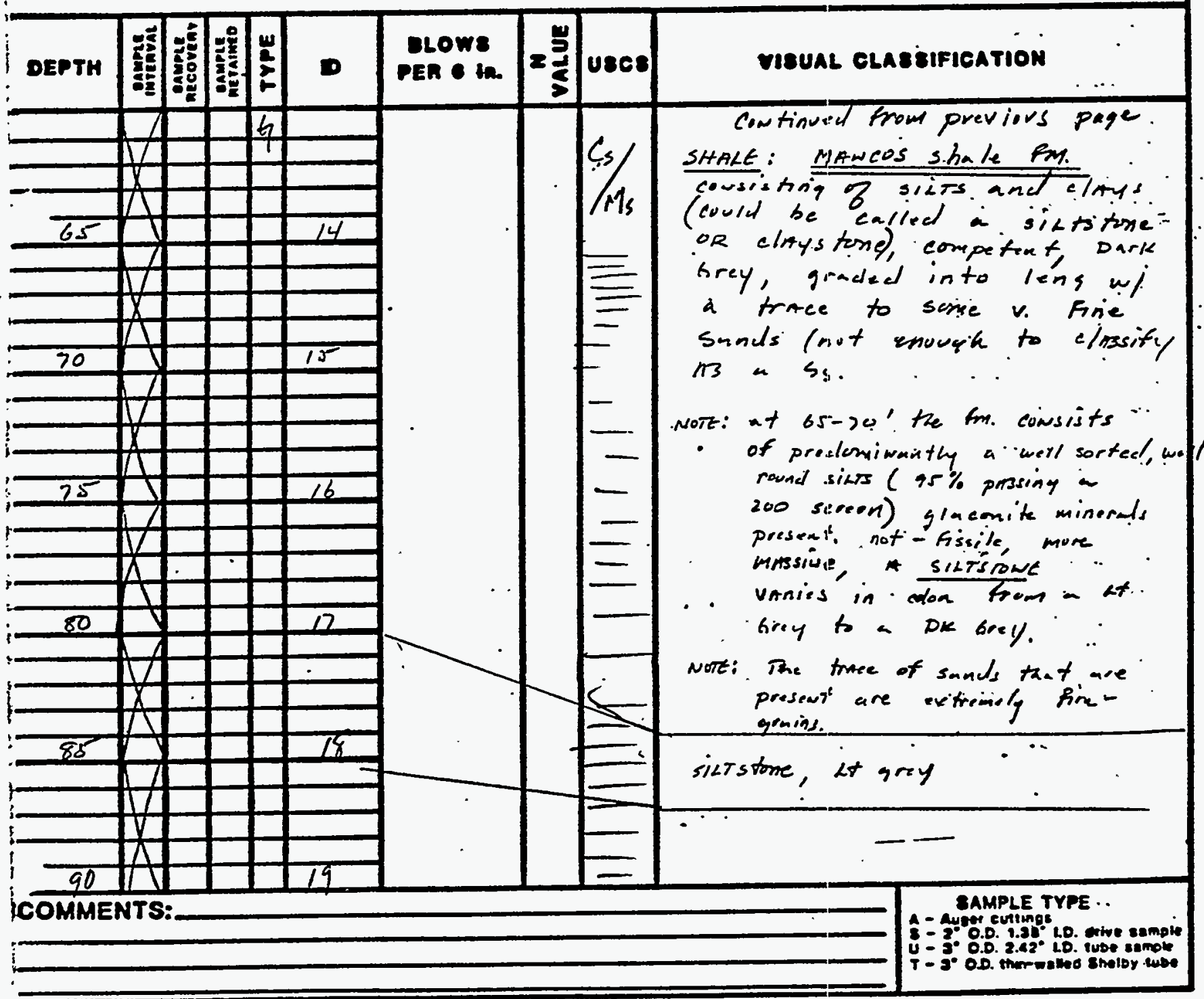

|EO-AL-ENO-2S (4185) 


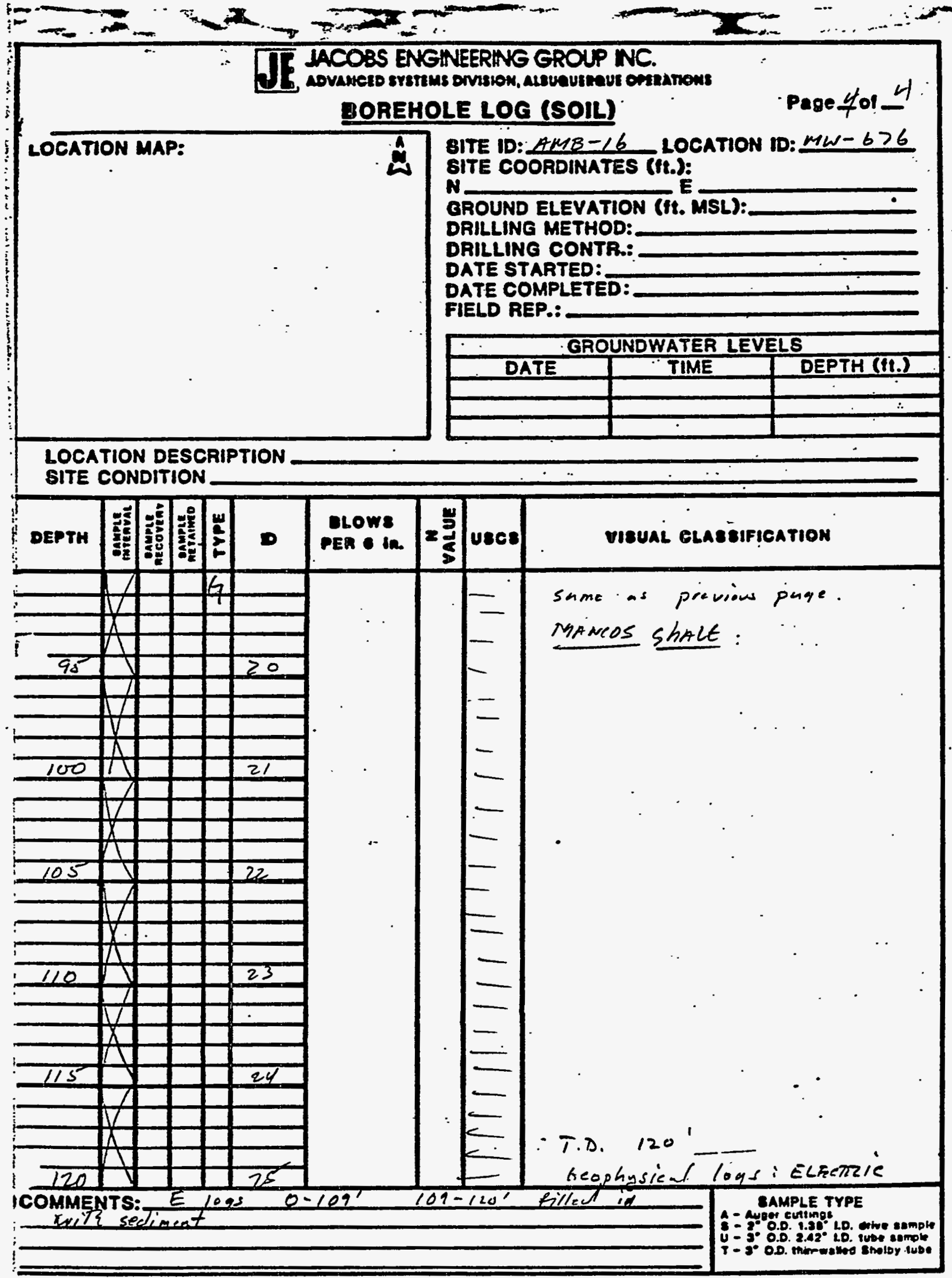

FO-AL-ENO-28 (4JES) 


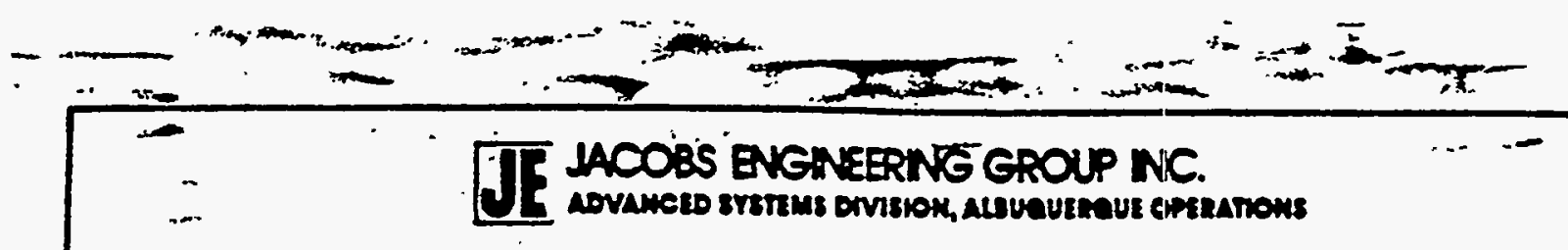

\section{WELL COMPLETION RECOIBD I}

SITE ID: $\frac{A M B-16}{16}$ LOCATION ID: MW 676 DATE INSTALLED: $12-22-88$ APPROX. SITE CÖORDINATES:(FT.) $N$ OPEN AREA PER LINEAL FT. (IN2/FT.) $\frac{8^{2}-4.5^{2}}{45^{2}}$

(FORMATION OF COMPLETION: _ Maucos Ekale $(C 2 ?$ ) Tres $C$

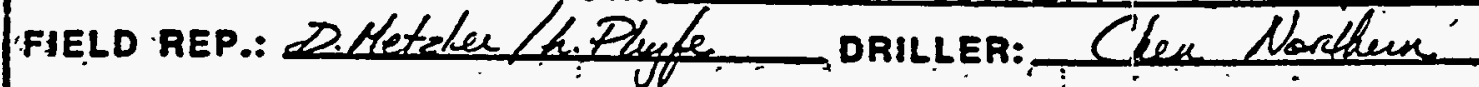

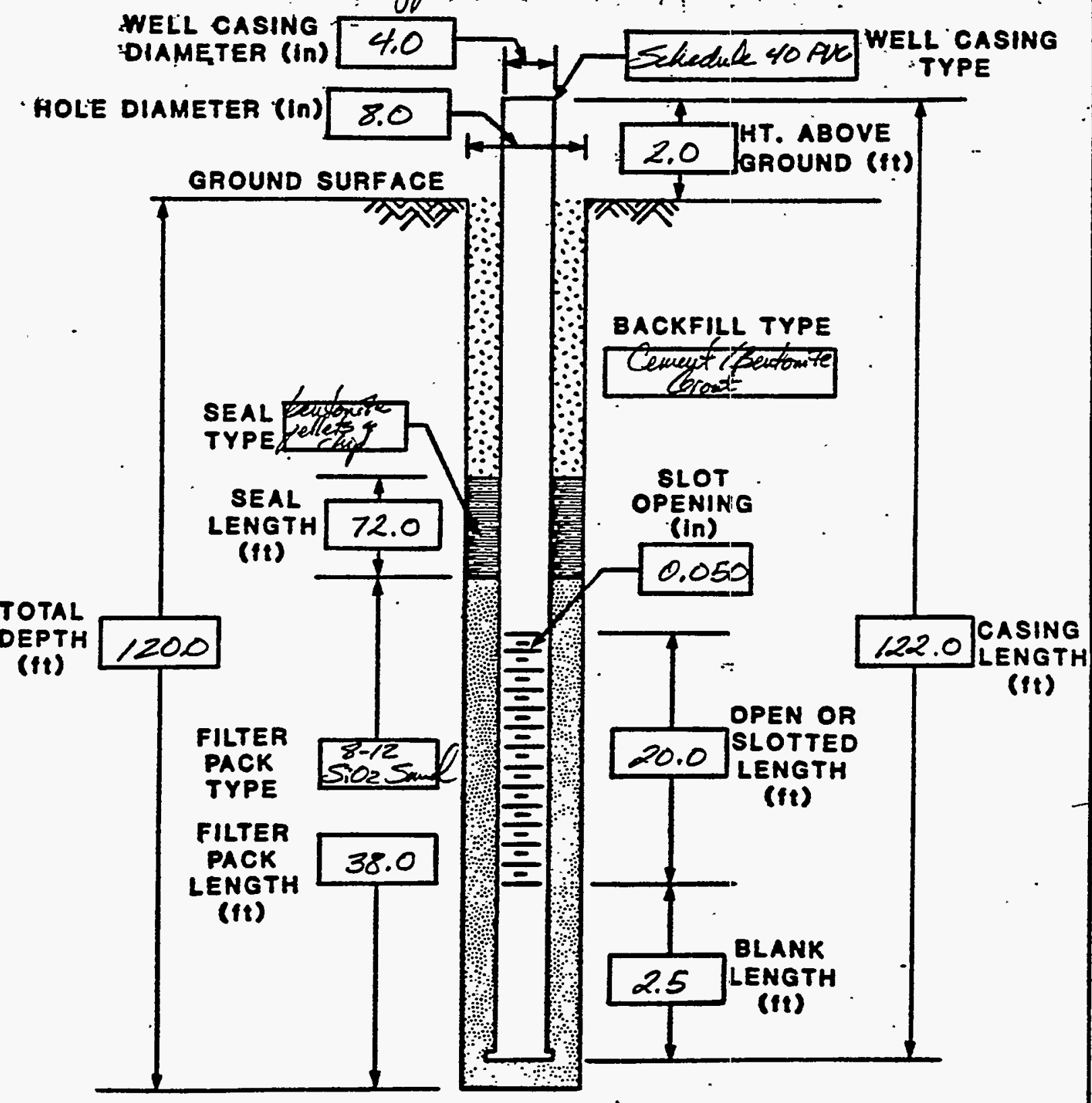

coMMENTS: left opew fon TAC desision

ri) empletel under lamy phyte's superivieoner

Gl 


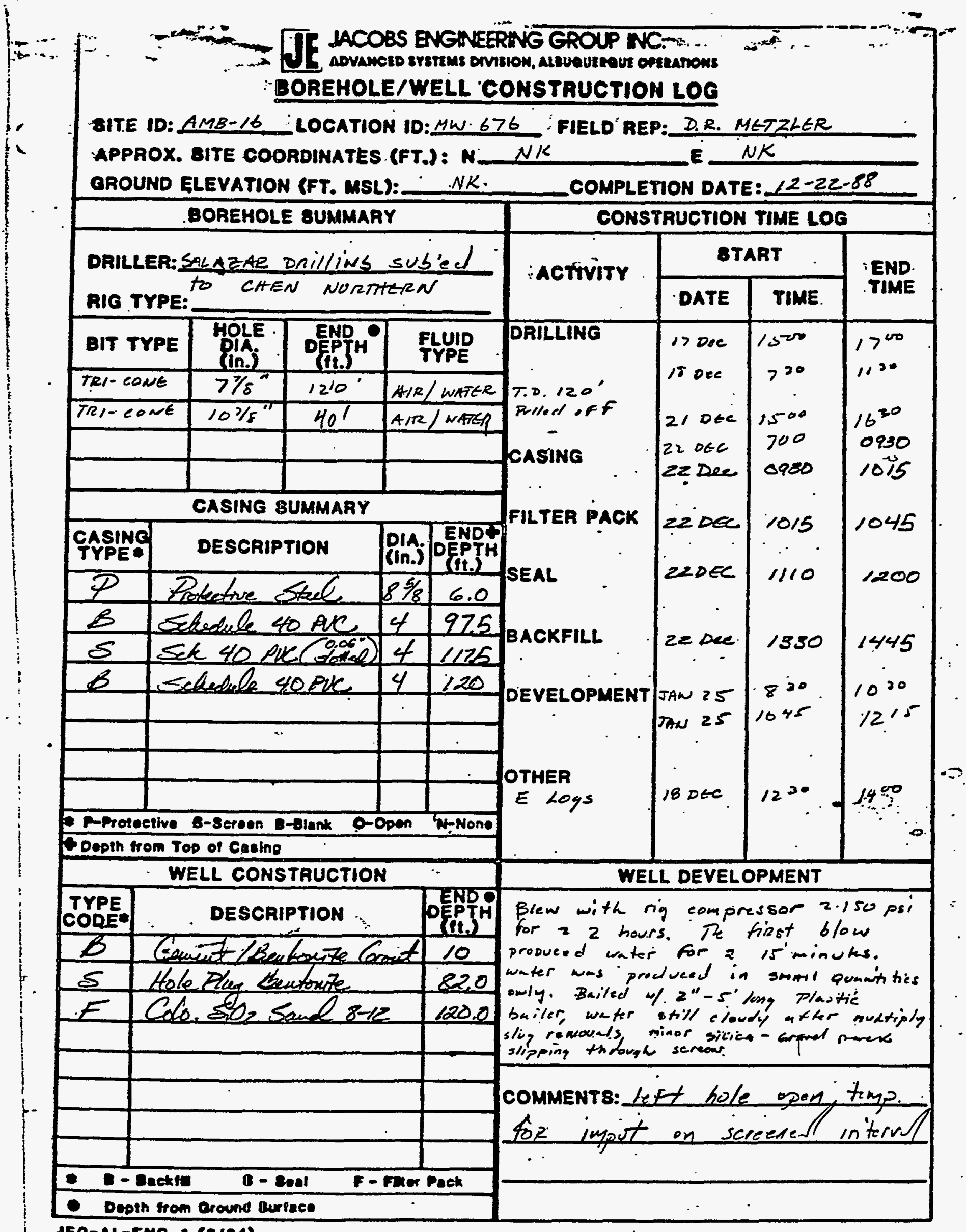

dEO-AL-ENO-1 (are4) 
WELL 677 LOGS 


\section{JE JACOBS ENGINEERING GROUP NC.}

BOREHOLE LOG (SOIL)

Page $\perp$ of 6

LOCATION MAP:

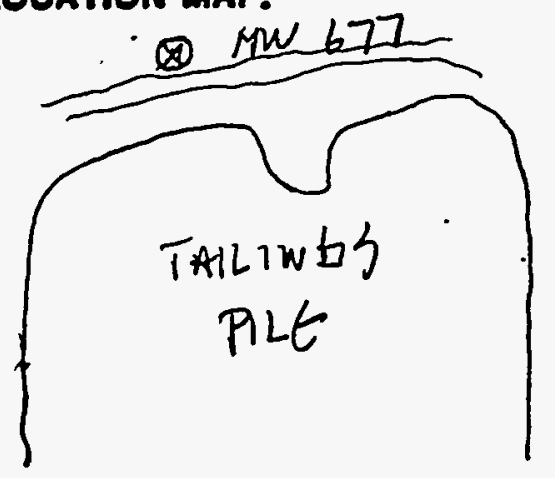

A

SITE ID: AMB-16

SITE COORDINATES (fit.)

$N$ NK

GROUND ELEVATION (It. MSL): NK -

DRILLING METHOD: ZZUAAX Y

DRILLING CONTR.: $\frac{1}{1+r n}$ ben 7 ern $/$ salez

DATE STARTED: $1 \frac{1}{}$ DEC $\overline{8}$

DATE COMPLETED: 22 DEC $8 \&$

FIELD REP.:

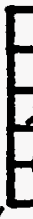

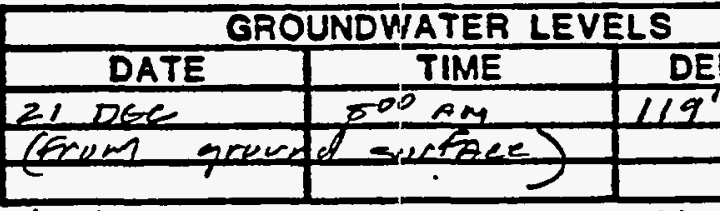

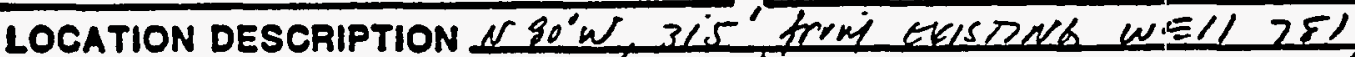

SITE CONDITION FLAL $100 \mathrm{se}$ sa

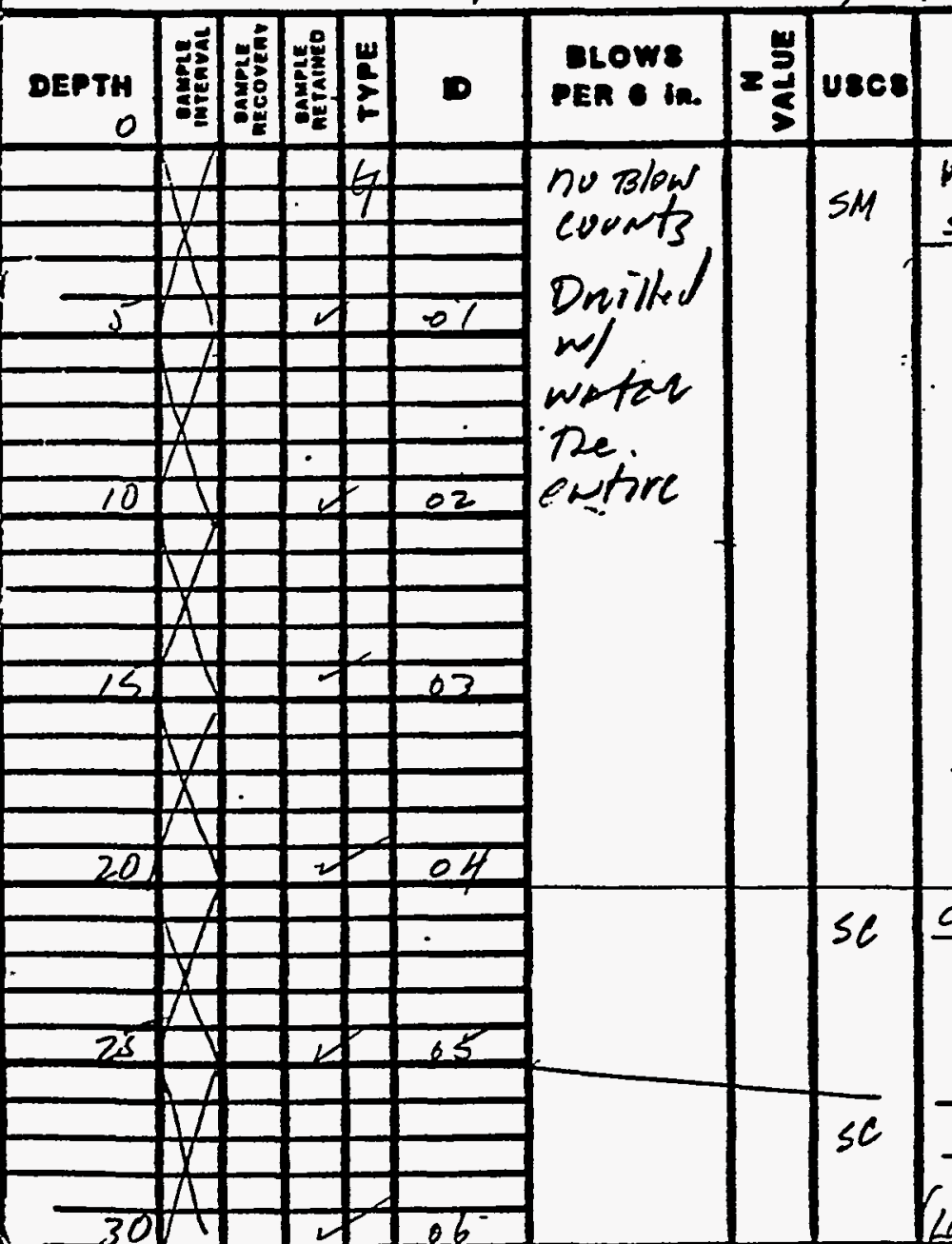

COMMENTS: $t=6 g a b$ sample Dnilling with water

clapey sand, predmivantly $r$.

Fine to fine sunds $w$

traze of elay.

orangisl -tan. (nP)

sandy dry. most chry with $\alpha$

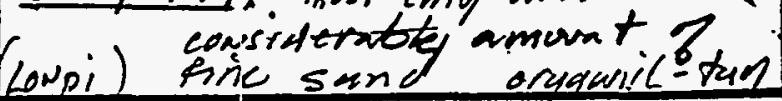

SAMPLE TYPE

$A$ - AYger cutsings

8: 2:0.0. 1.98 to. Hive sumple

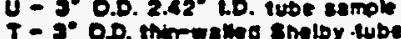


IACOBS ENGINEERING GROUP NC.

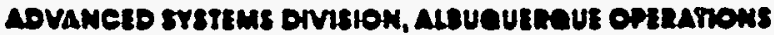

BOREHOLE LOG (SOIL)

Page 2 of 6

LOCATION MAP:

岀

8ITE ID: $A M B-16$

LOCATION ID: MN: 677

GITE COORDINATES (ft.):

GROUND ELEVATION (ft. MSL):

DRILLING METHOD:

DRILLING CONTR.:

DATE STARTED:

DATE COMPLETED:

FIELD REP.:

\begin{tabular}{|c|c|c|}
\hline \multicolumn{3}{|c|}{ GROUNDWATER LEVELS } \\
\hline DATE & TIME & DEPTH (fI.) \\
\hline & & \\
\hline & & \\
\hline & & \\
\hline
\end{tabular}

LOCATION DESCRIPTION

SITE CONDITION

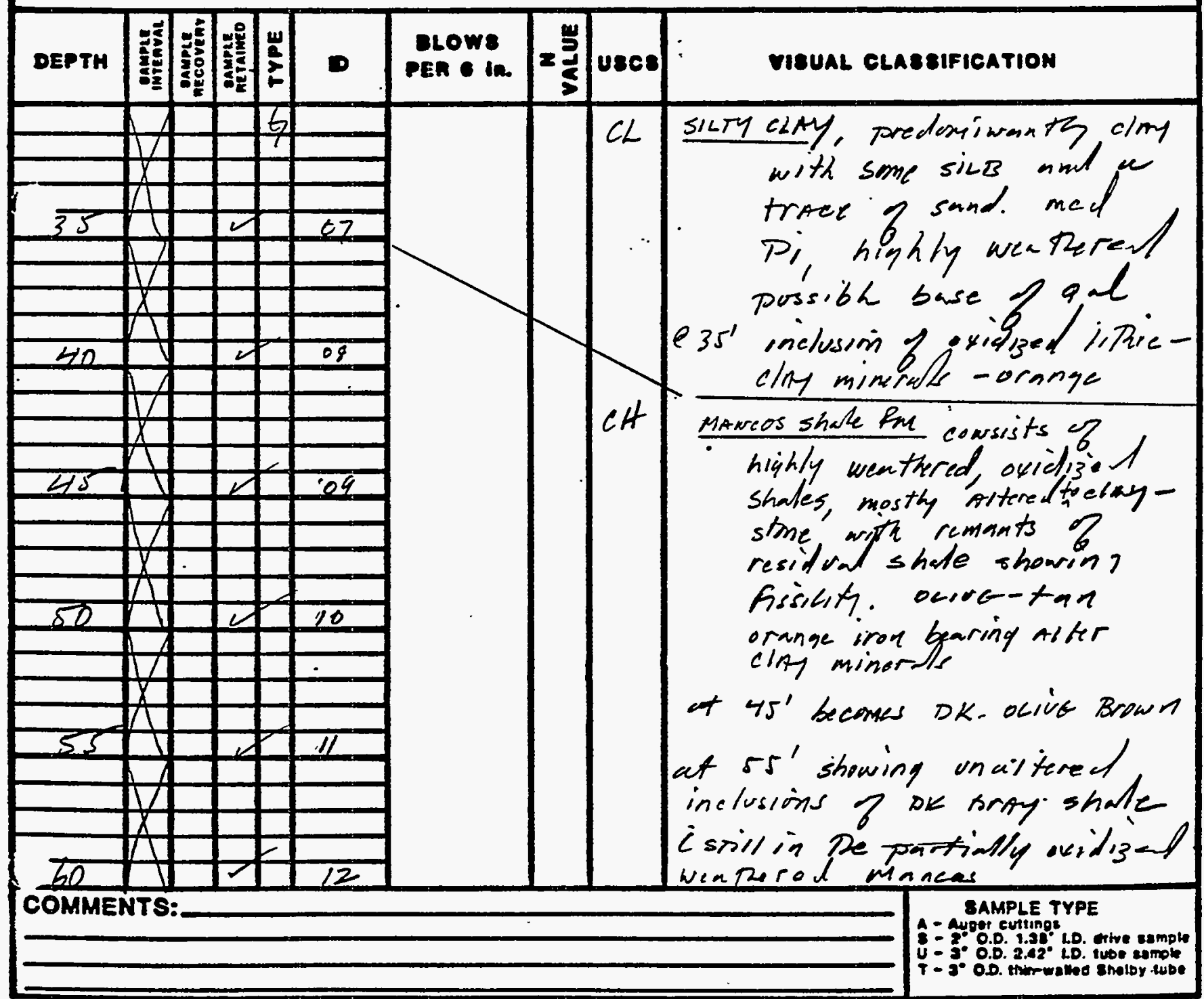




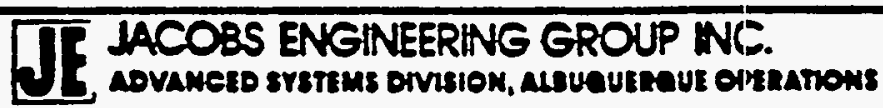

BOREHOLE LOG (SOIL)

LOCATION MAP:

8ITE ID: AMB - 16 LOCATION ID: $\angle W W 777$

SITE COORDINATES (ft.):

GROUND ELEVATION (ft. MSL):

DRILLING METHOD:

DRILLING CONTR.:

DATE STARTED:

DATE COMPLETED:

FIELD REP.:

\begin{tabular}{|c|c|c|}
\hline \multicolumn{3}{|c|}{ GROUNDWATER LEVELS } \\
\hline DATE & TIME & DEPTH (11.) \\
\hline & & \\
\hline & & \\
\hline & & \\
\hline
\end{tabular}

LOCATION DESCRIPTION

SITE CONDITION

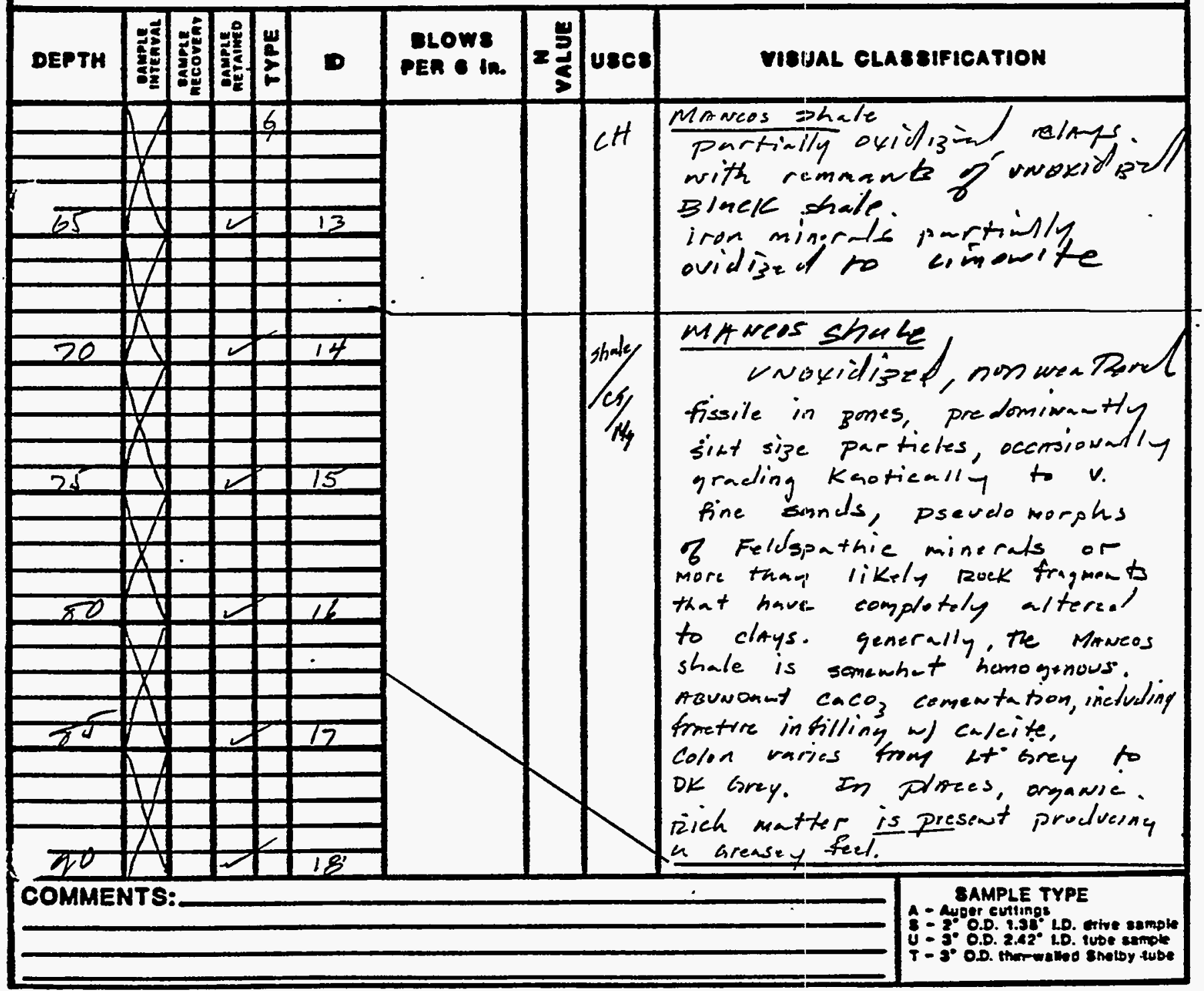




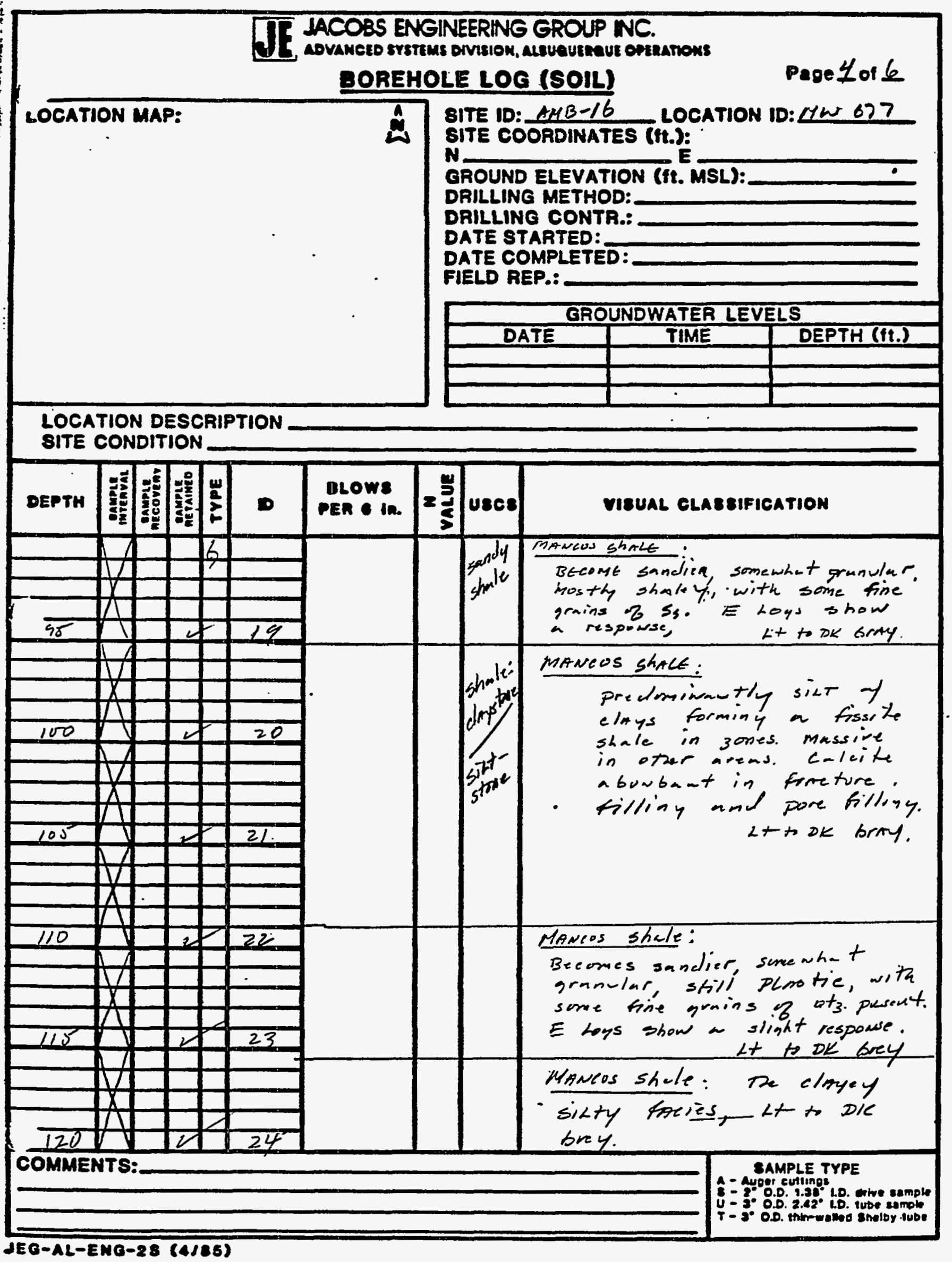




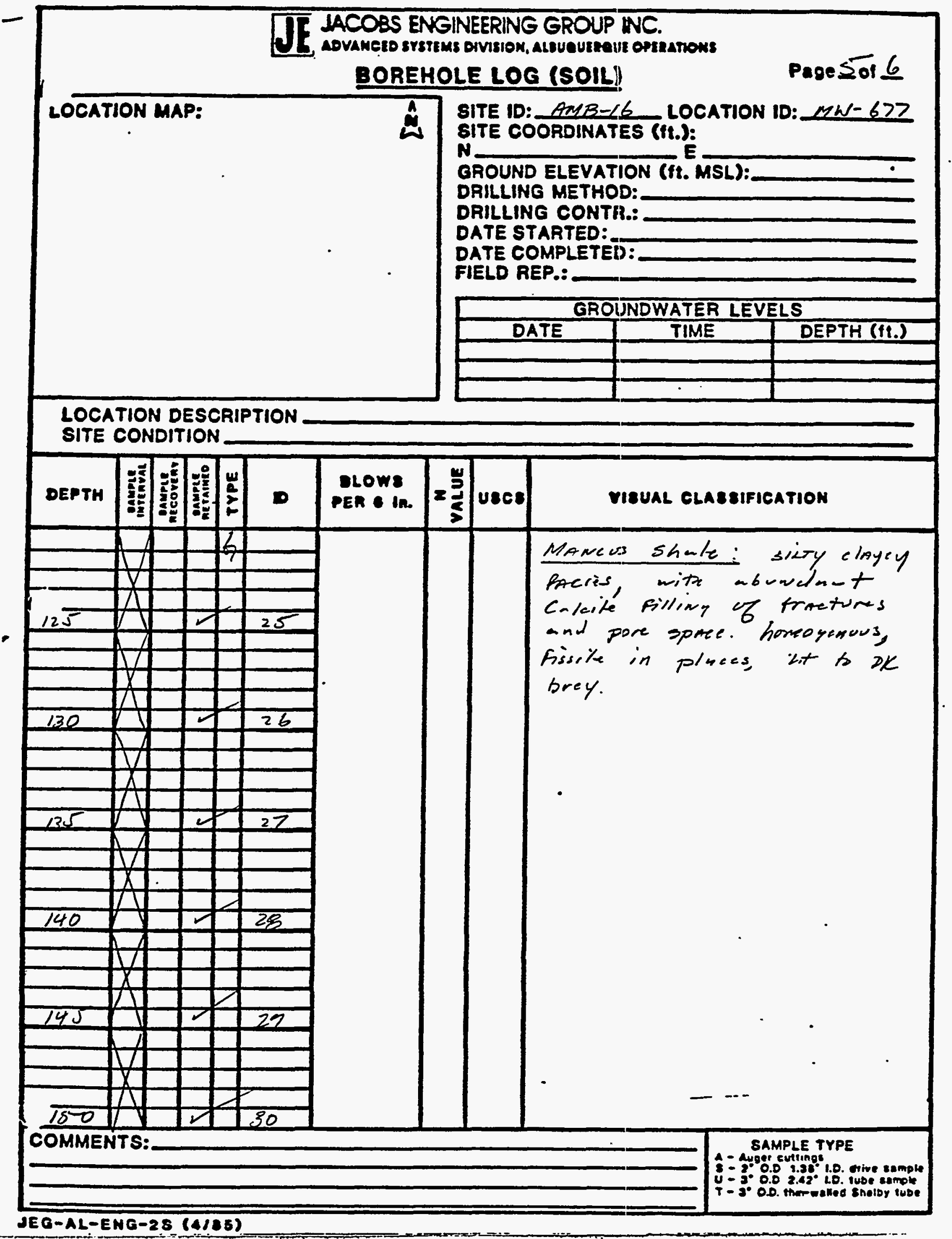




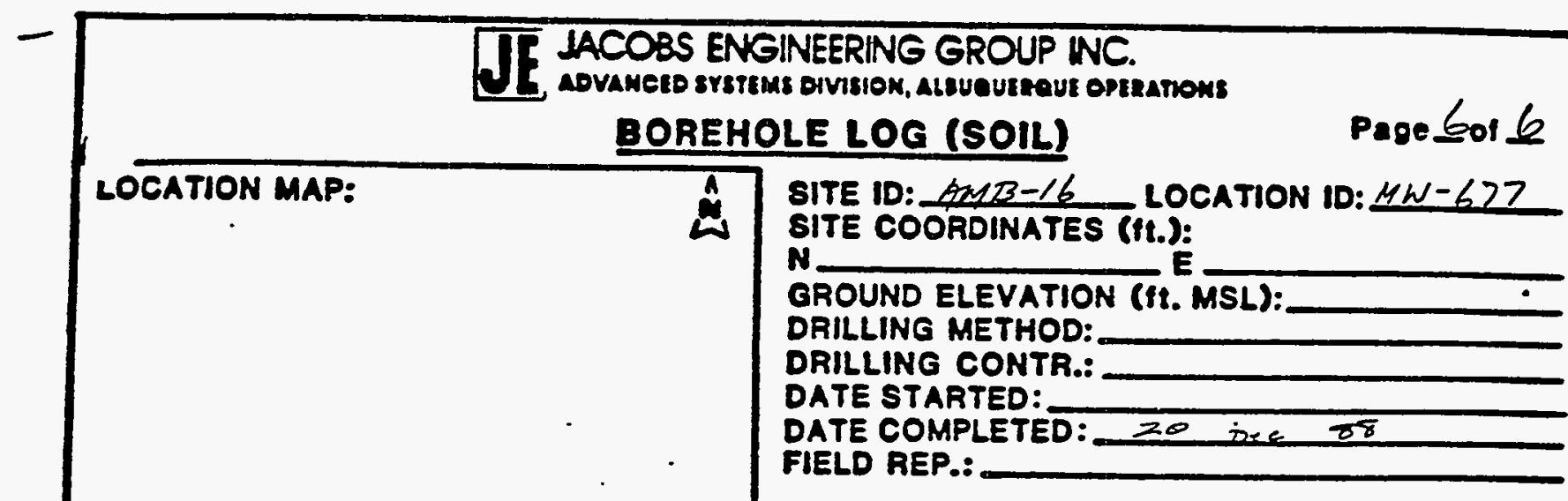

LOCATION DESCRIPTION

SITE CONDITION

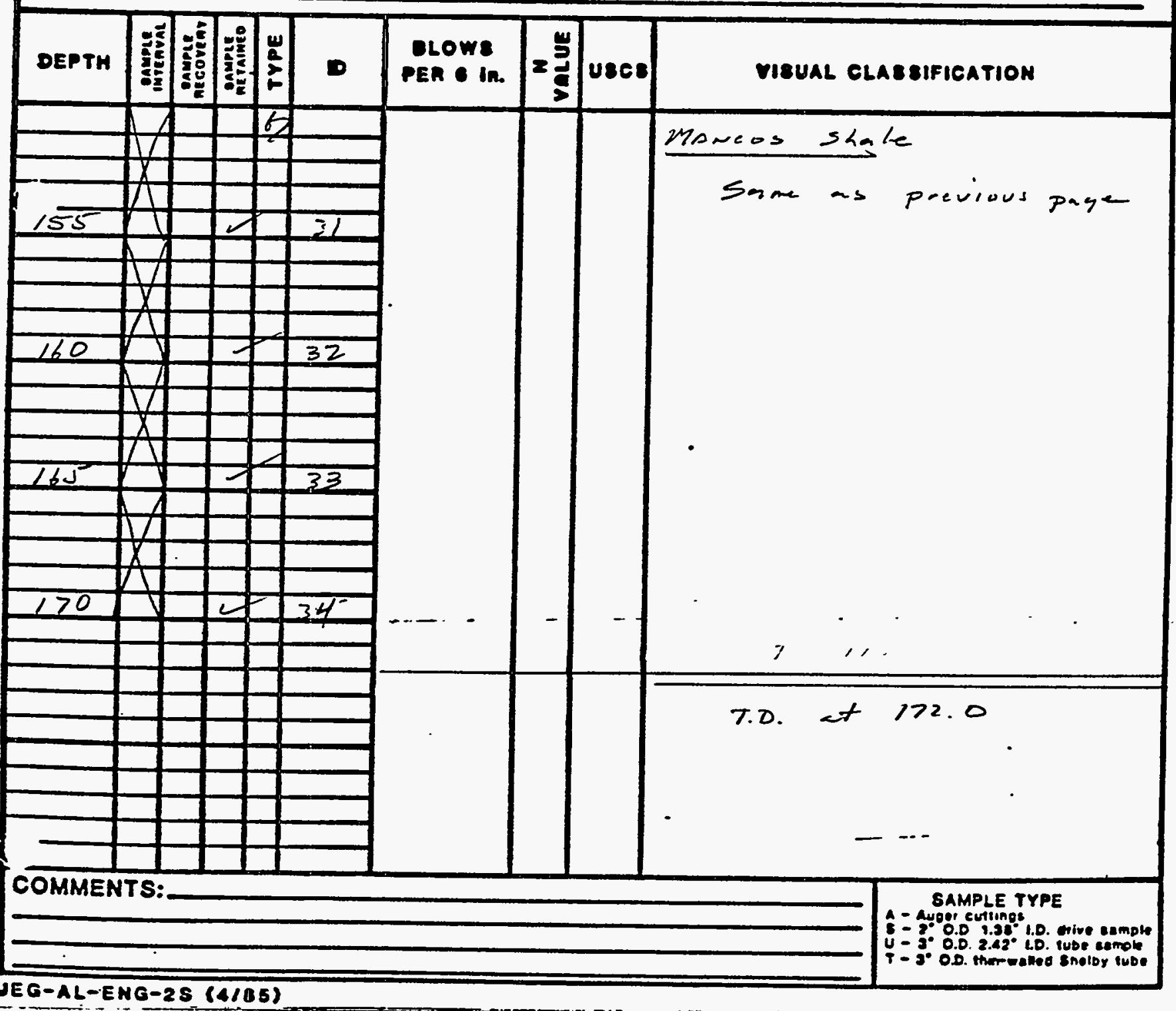




\section{TT IACOBS ENGNEERNG GROUP NK.

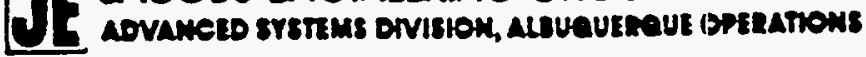

\section{WELL COMPLETION RECORD}

SITE ID: $A M B-16$ LOCATION ID: MW 677 DATE INSTALLED: DEC 20,88 APPROX. SITE COORDINATES:(FT.) N NK

OPEN AREA PER LINEAL FT. (IN2/FT.) $(8)^{2}-(4.5)^{2}$ EK

FORMATION OF COMPLETION: MANCOS SAALE; TTES $C$

FIELD REP.: D.R. METZLCR DRILLER: CHEN NORMHERW

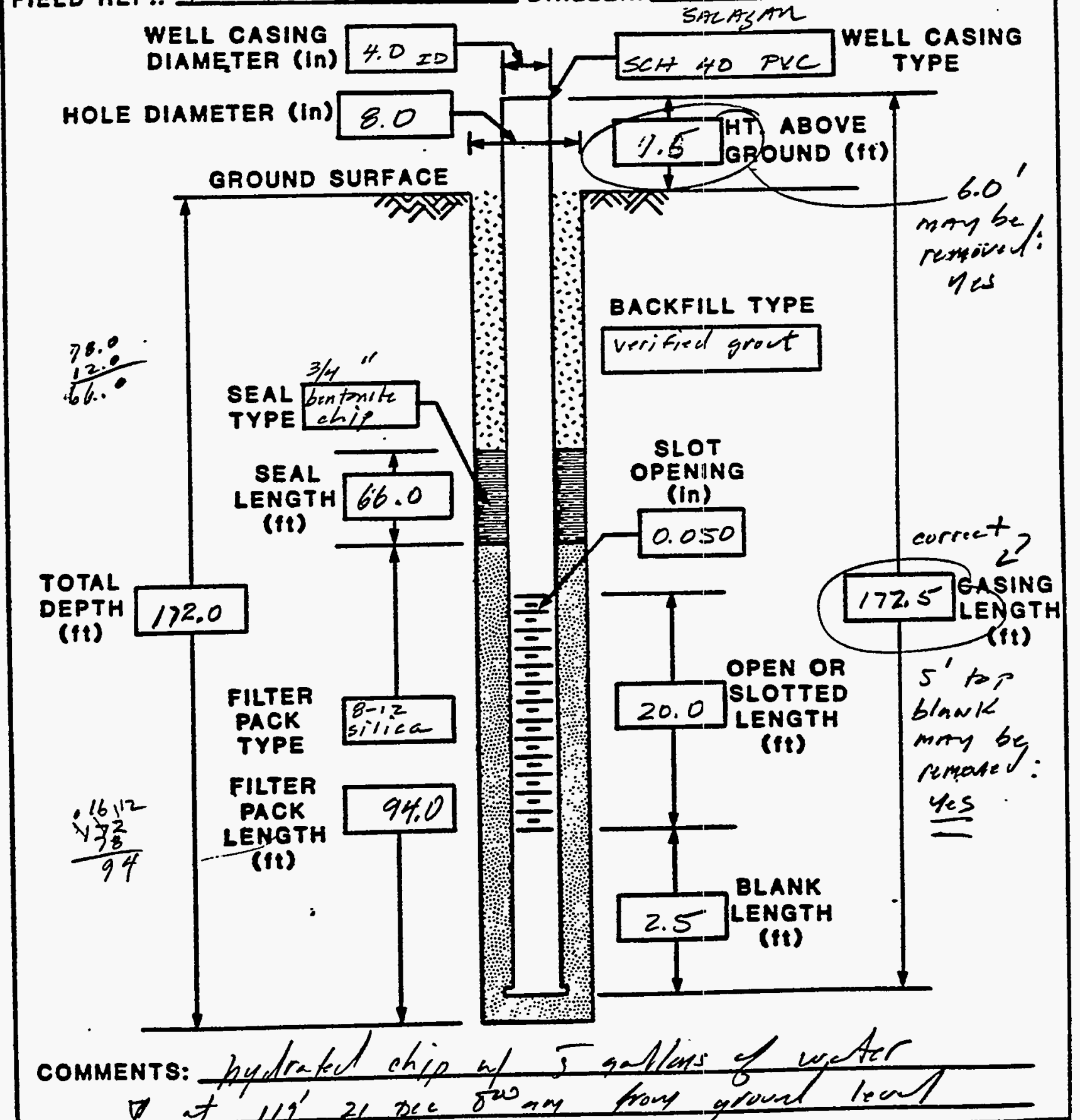




\section{.DE LACOBS ANGMEERNG GROUP NC. BOREHOLE/WELL CONSTRUCTION LOG}

GITE ID:AMB-16 LOCATION 10:MW-677 FIELO AEP:D.R. METZLER

APPROX. BITE COORDINATES (FT.): $N$ OROUND ELEVATION (FT. MSL):

\section{. DOREHOLE BUMMARY}

DRILLER: SALALAM DnilliNG SUBंA RIG TYPE:

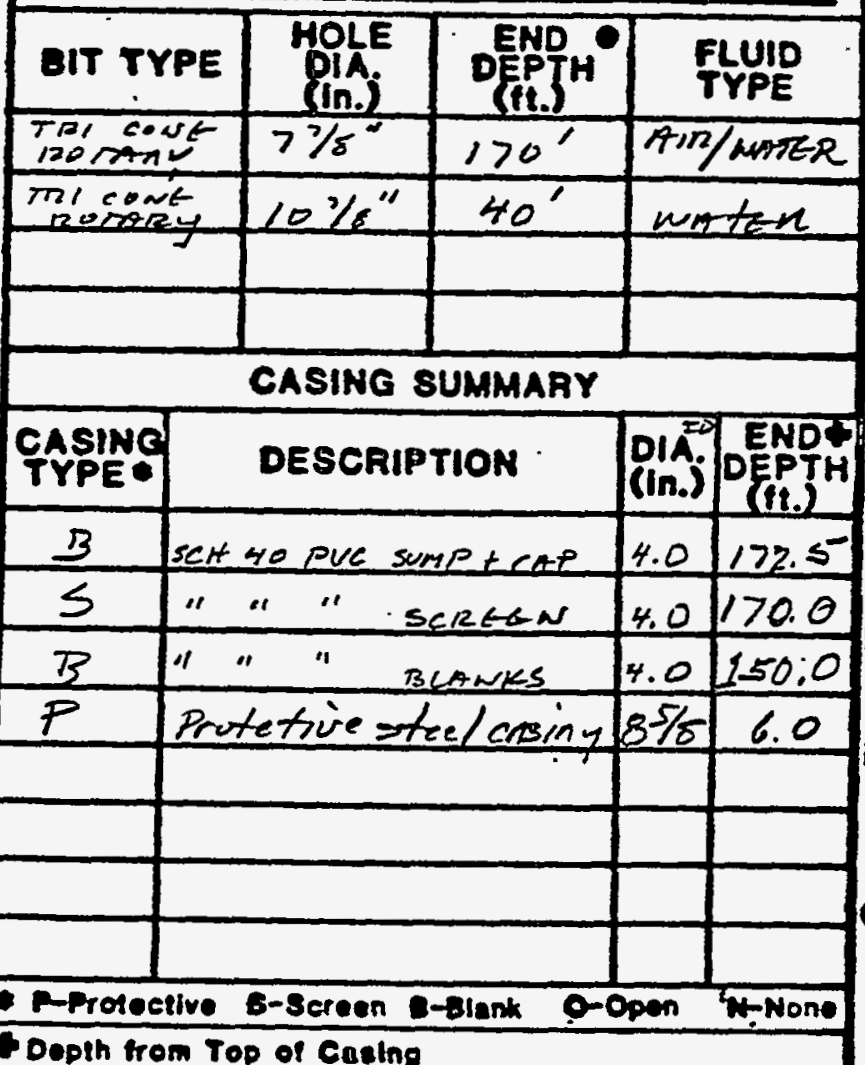

WELL CONSTRUCTION

\begin{tabular}{|c|c|c|}
\hline $\begin{array}{l}\text { TYPE } \\
\text { CODE }\end{array}$ & DESCAIPTION & $\begin{array}{l}\text { ENPD } \\
\text { DEPTH } \\
\text { (ie.) }\end{array}$ \\
\hline$E$ & $E-12$ siCiCA SAND & 172.0 \\
\hline \multirow[t]{2}{*}{$\leq$} & Enviroplun $3 / 4$ " bentanite & 78.0 \\
\hline & chip & \\
\hline$B$ & bentenite cenent youst & 12.0 \\
\hline & & \\
\hline & & \\
\hline & & \\
\hline & & \\
\hline e- & - Eoal & Peek \\
\hline
\end{tabular}

- Depin from around eurface

dEO-AL-ENO-1 (ereA)
E NK. NK COMPLETION DATE:

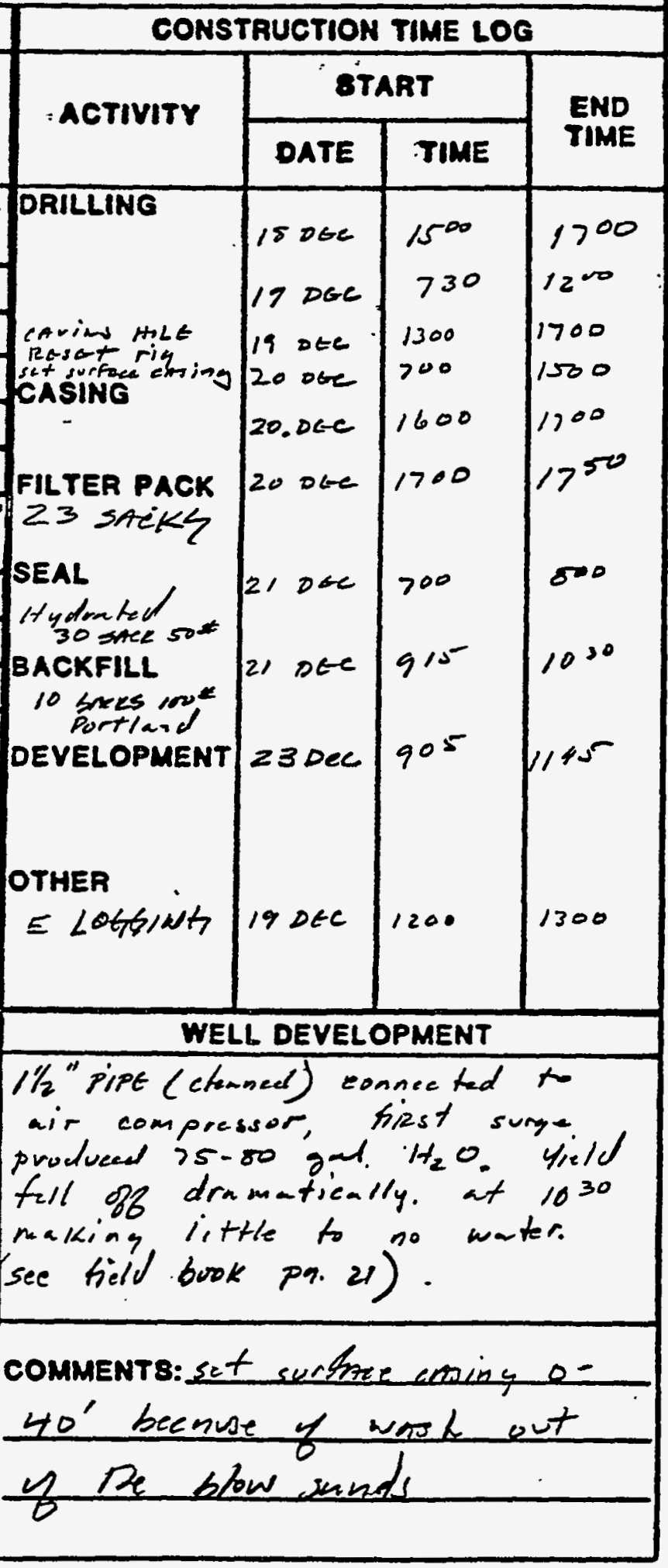


WELL 678 LOGS 


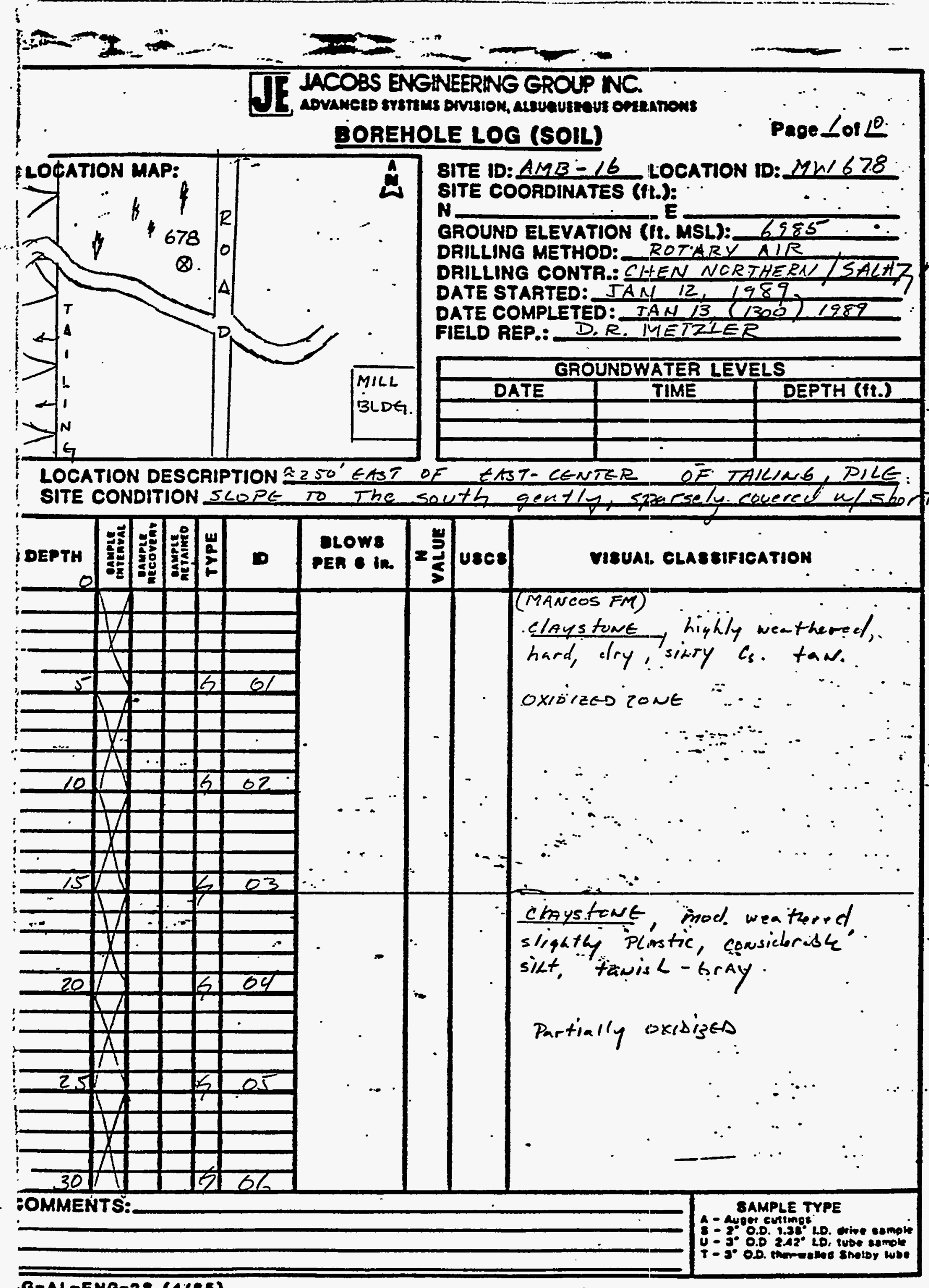




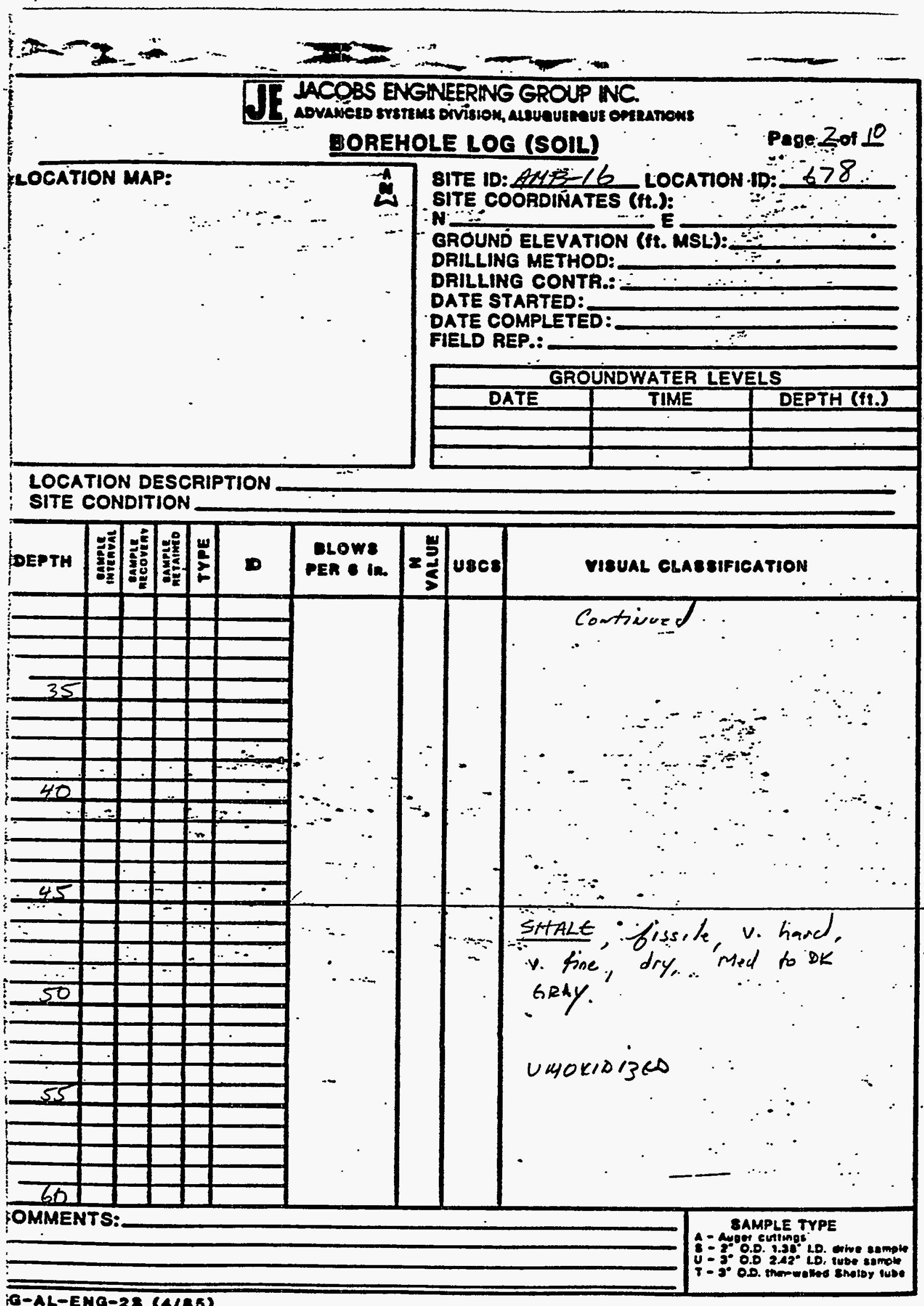




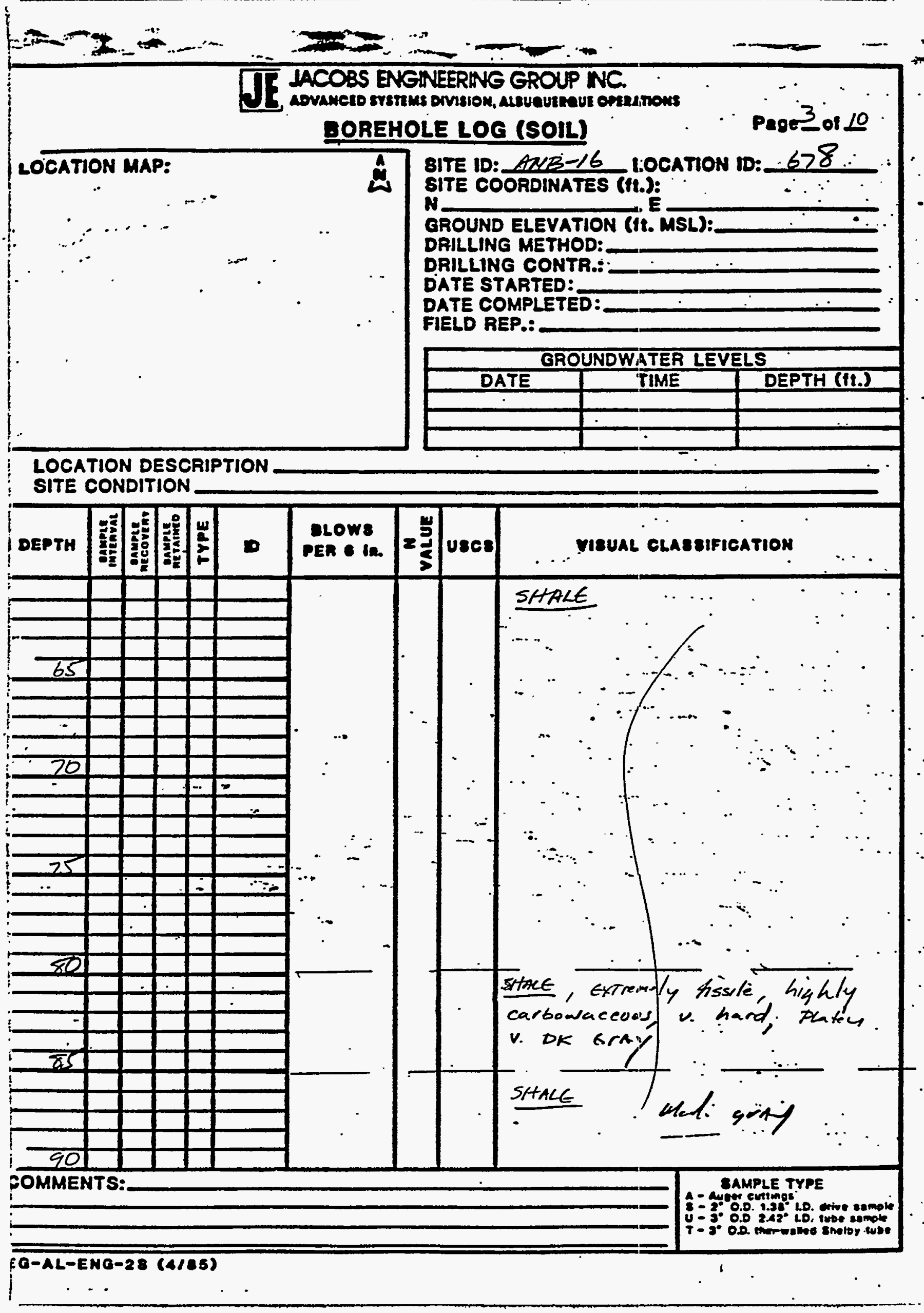




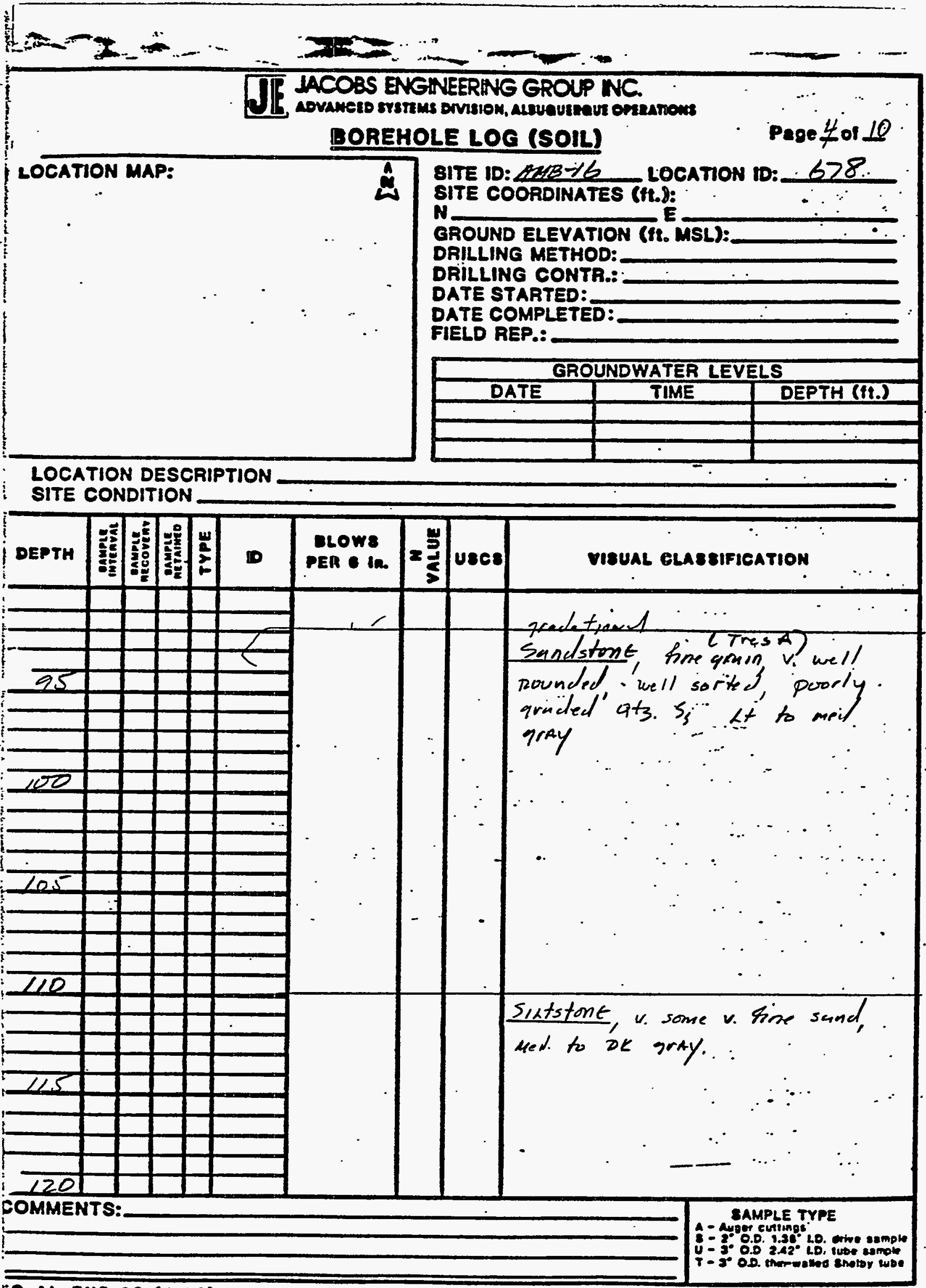

EO-AL-ENO-28 (4/EB) 


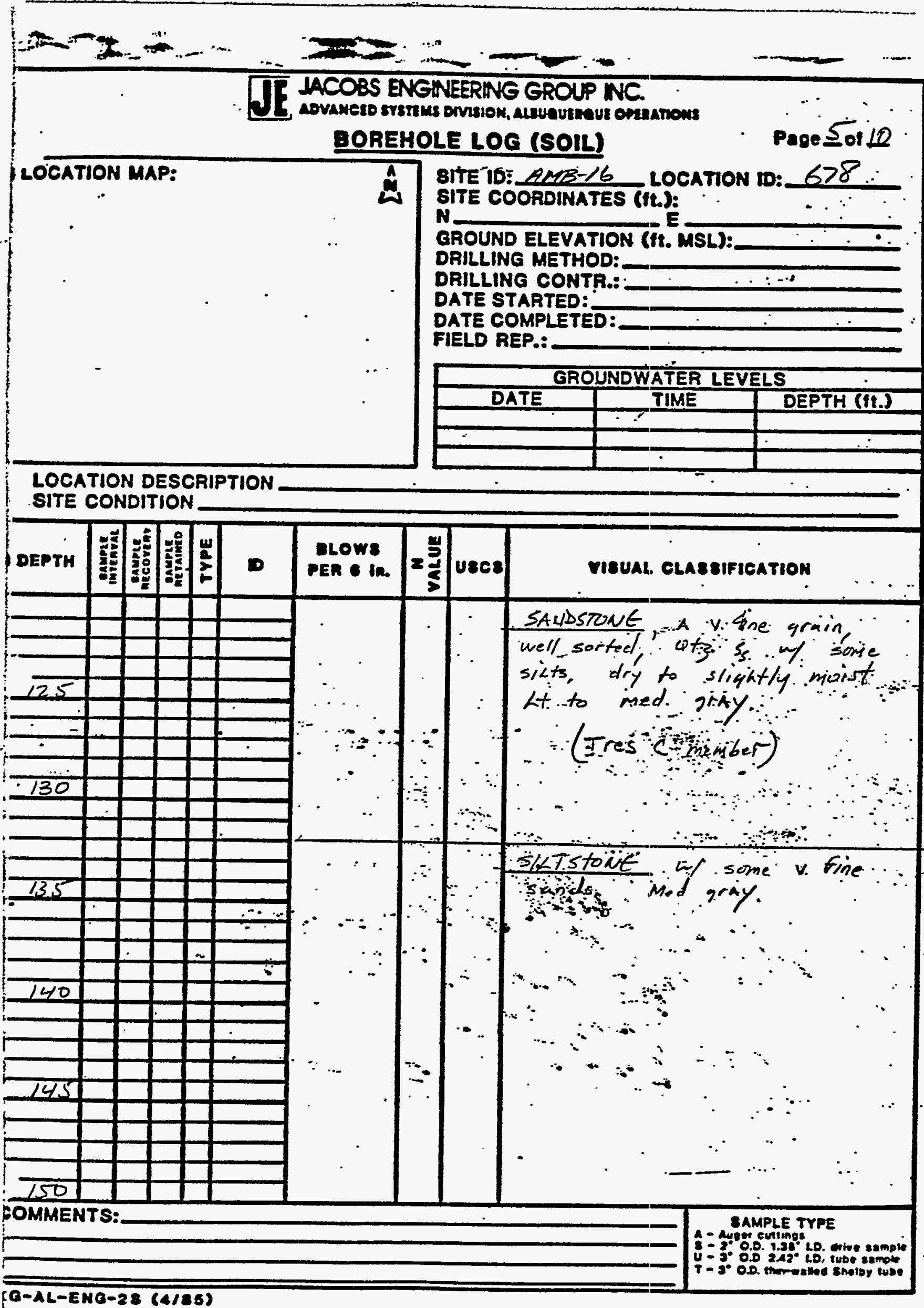




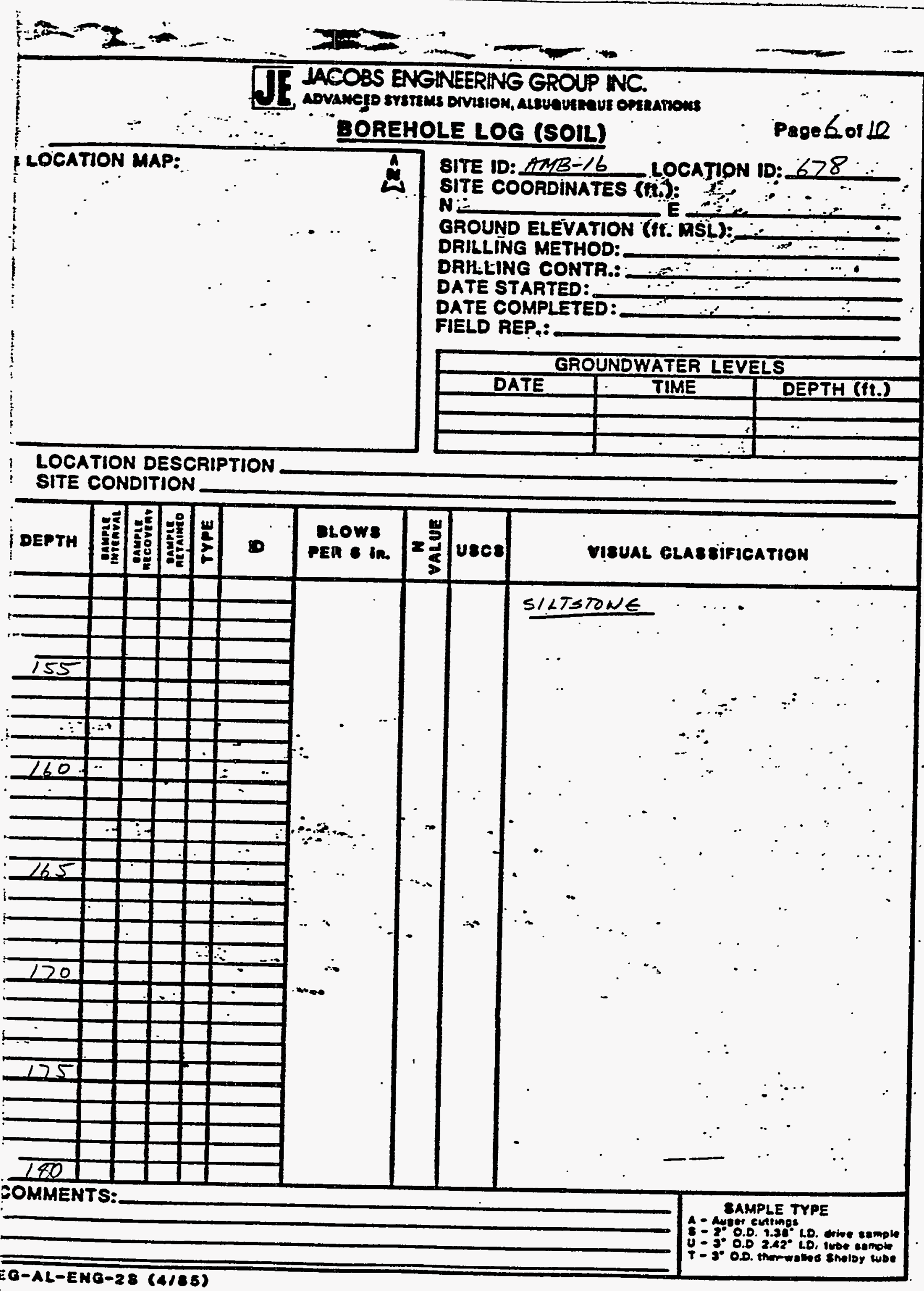


$=-2 x$

TI IACOBS ENGINEERING GROUP NC.

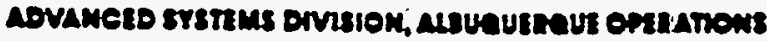

BOREHOLE LOG (SOIL)

PageZ of 10

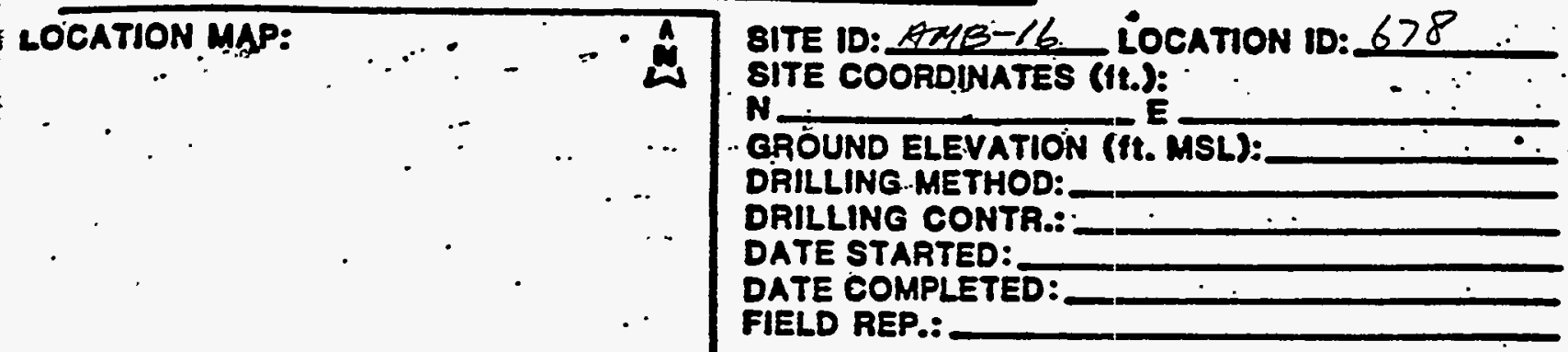

LOCATION DESCRIPTION

SITE CONDITION

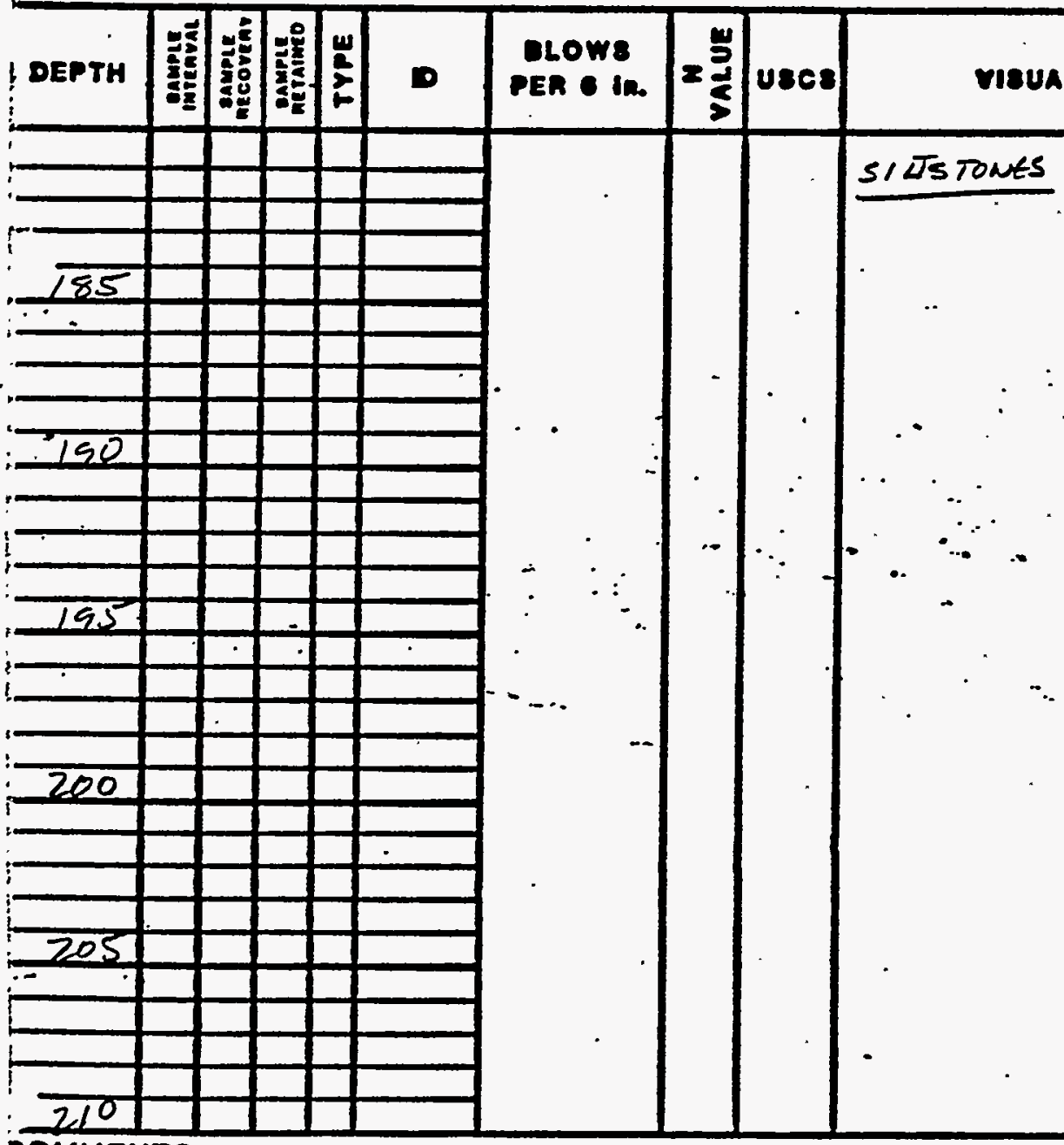

DOMMENTS:

\begin{tabular}{|c|c|c|}
\hline \multicolumn{3}{|c|}{ GROUNDWIATER LEVELS } \\
\hline DATE & TIME & DEPTH (ft.) \\
\hline & & \\
\hline & & \\
\hline
\end{tabular}




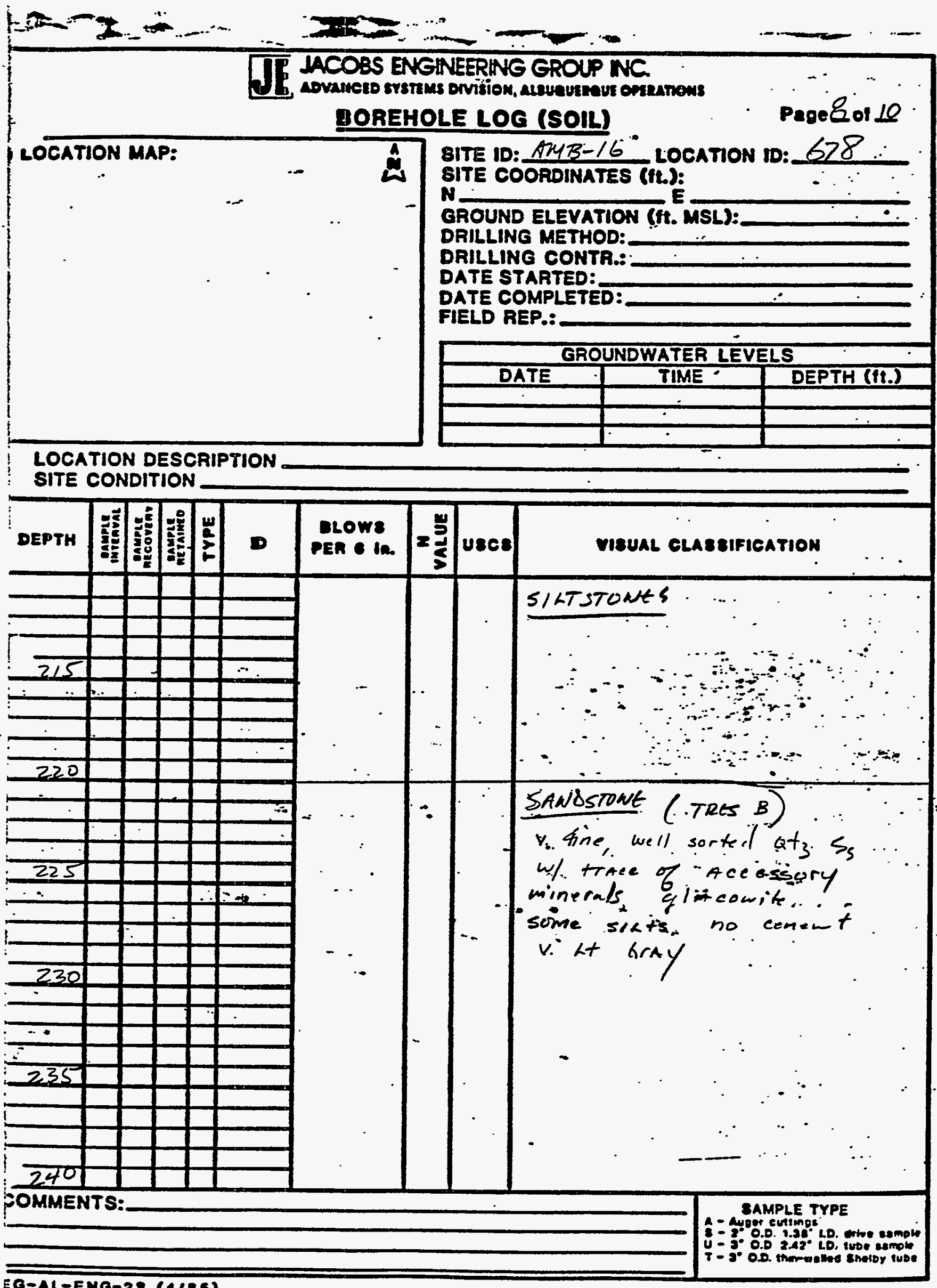




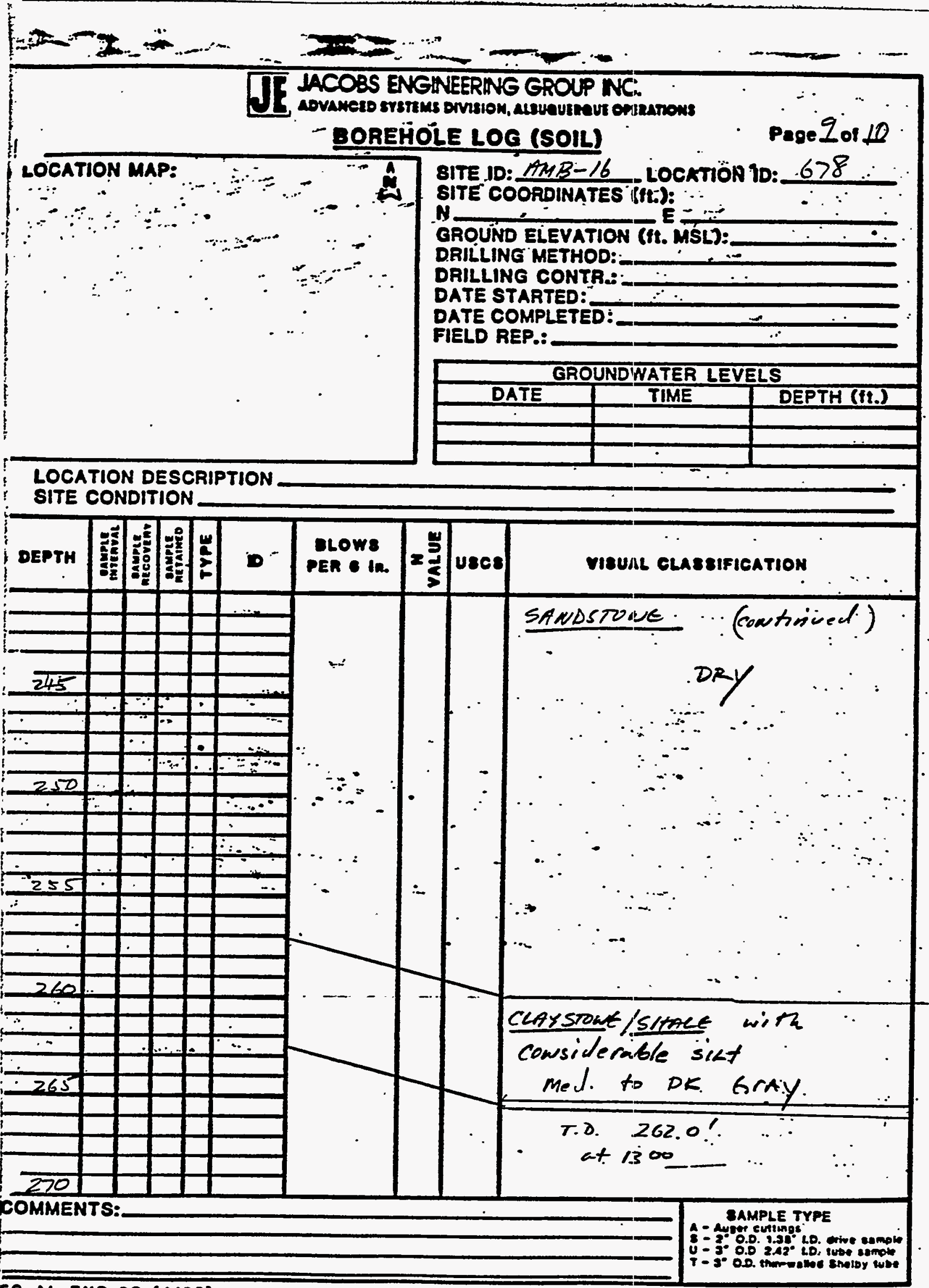




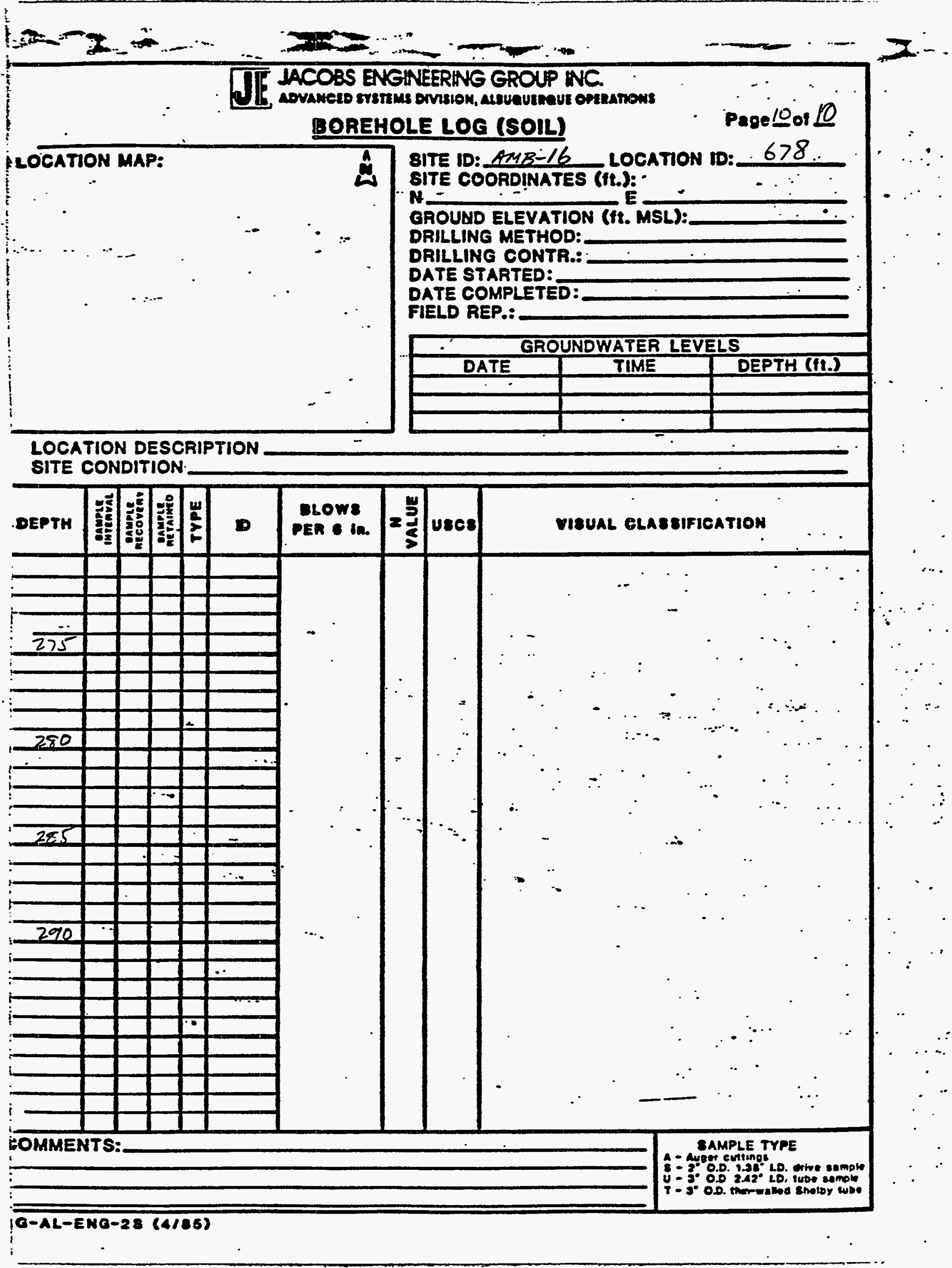




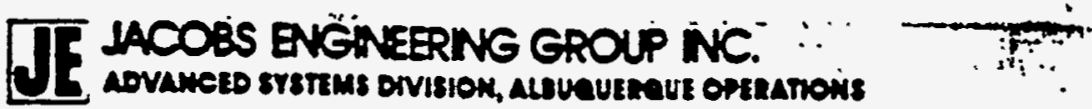

\section{WELL COMPLETION RECORD}

SITE ID: AMB-16 LOCATION ID: MWN-678 DATE INSTALLED: $-14-84$ APPROX. SITE COORDINATES:(FT.) N E

OPEN AREA PER LINEAL FT. (IN2/FT.)

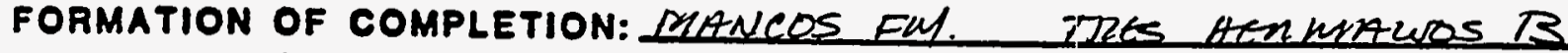

FIELD REP.: D.Z: METZLER

DRILLER:CHEN-NORTHERN

WELL CASING

DIAMETER (in)

$\therefore$.

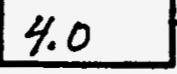

HOLE DIAMETER (In)
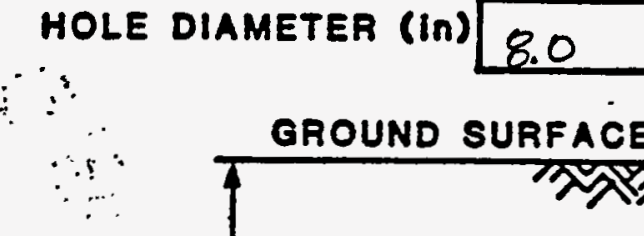

TOTAL

DEPTH

(ft)

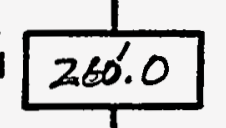

SEAL BEWTOMit

TYPELCHIP
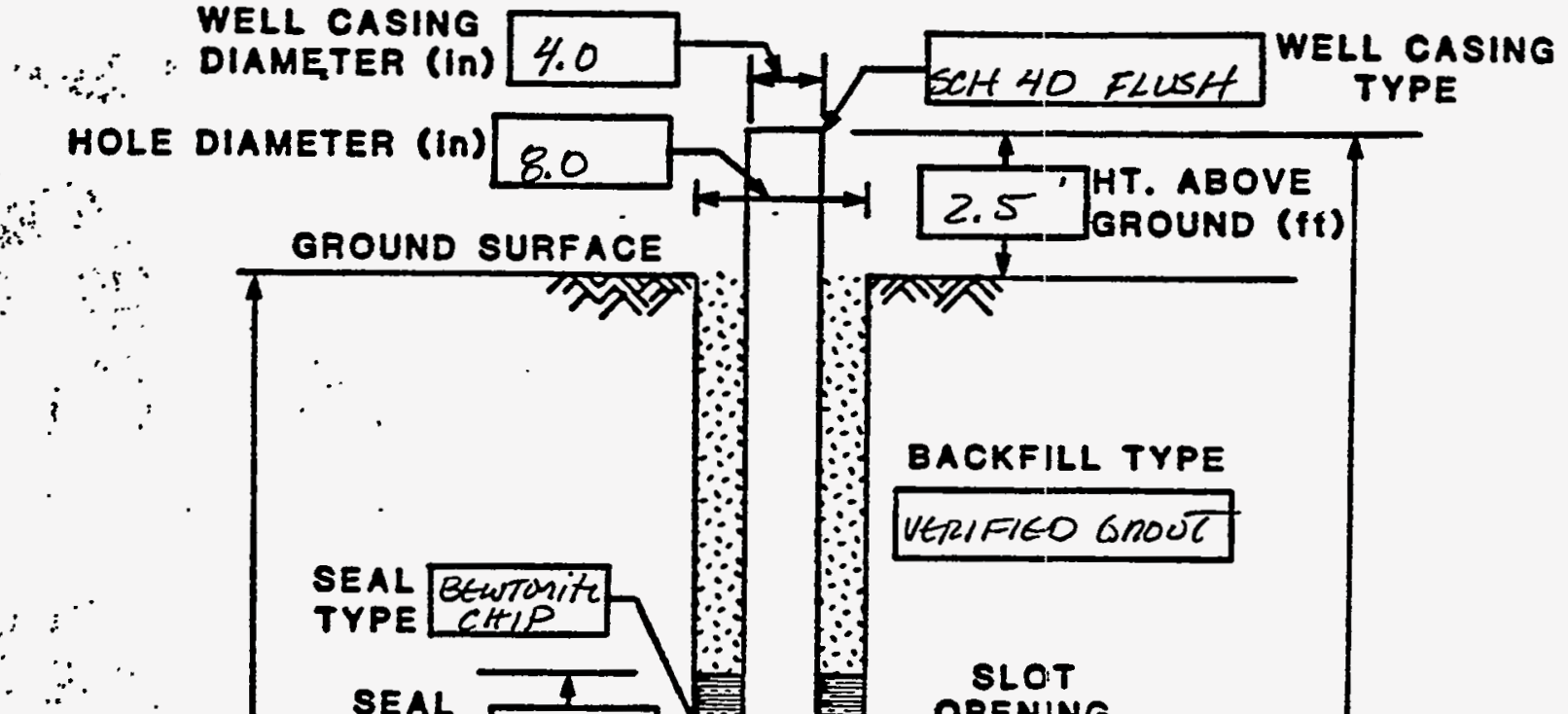

SEAL

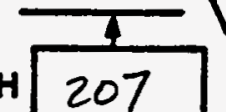

(ft)
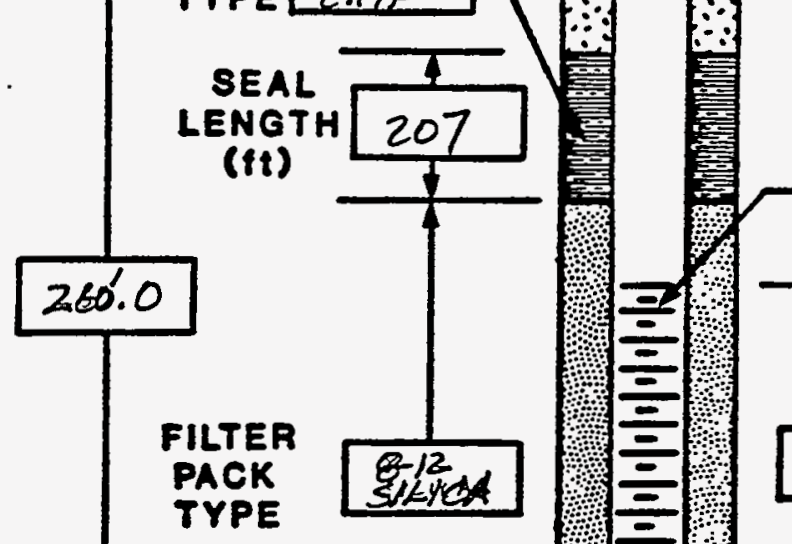

BACKFILL TYPE

VETIFIED GNOUT

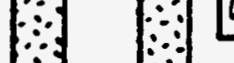

$\because \quad \therefore$

SLOT

OPENING

(in)
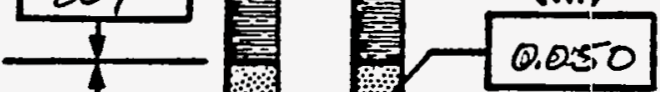

0.050

TYPE

FILTER

PACK

LENGTH

(it)
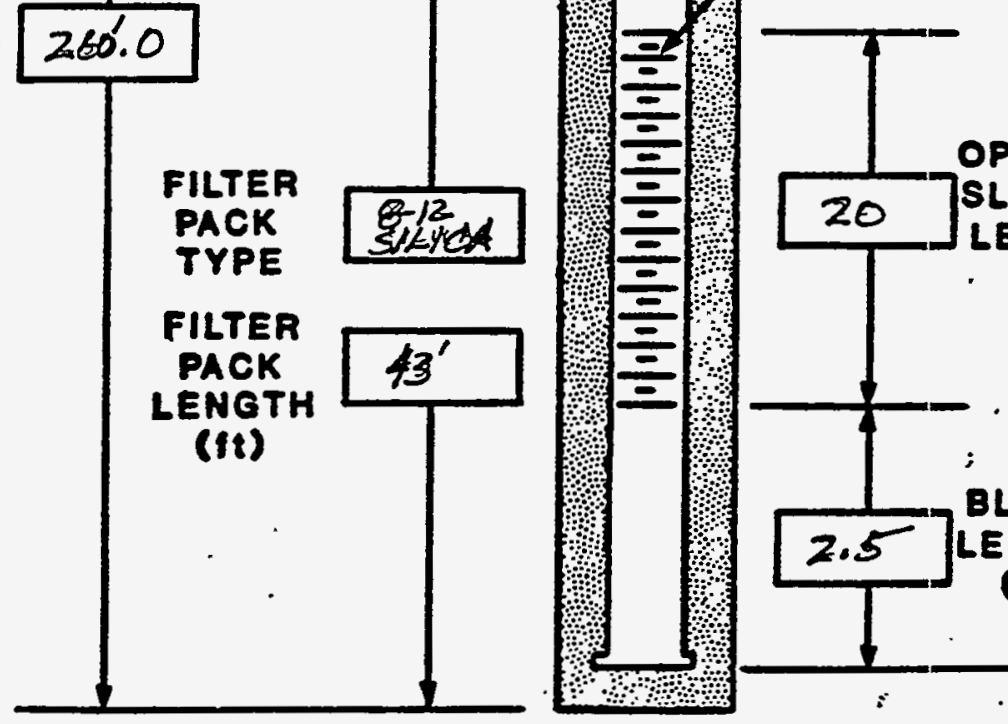

CASING LENGTH

(fi)

comments: Lew workil on well

$\because n$

(ti)

\subsection{LENGTH}

(fi) 


\section{IT - LACOBS ENGMEERHE GROUP NC.

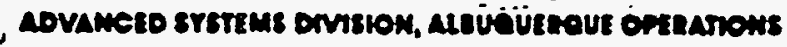 \\ BOREHOLE/WELL CONSTRUCTION LOG}

SITE. ID: $\angle N B-16$ LOCATION ID: 678 FIELD REP:D.R. METRLER APPROX. BITE COORDINATES (FT.): $N$ E OROUND ELEVATION (FT. MSL): 6985 COMPLETION DATE: $1-13-89$ BOREHOLE BUMMARY

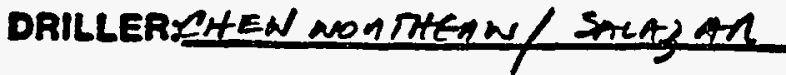
RIG TYPE:

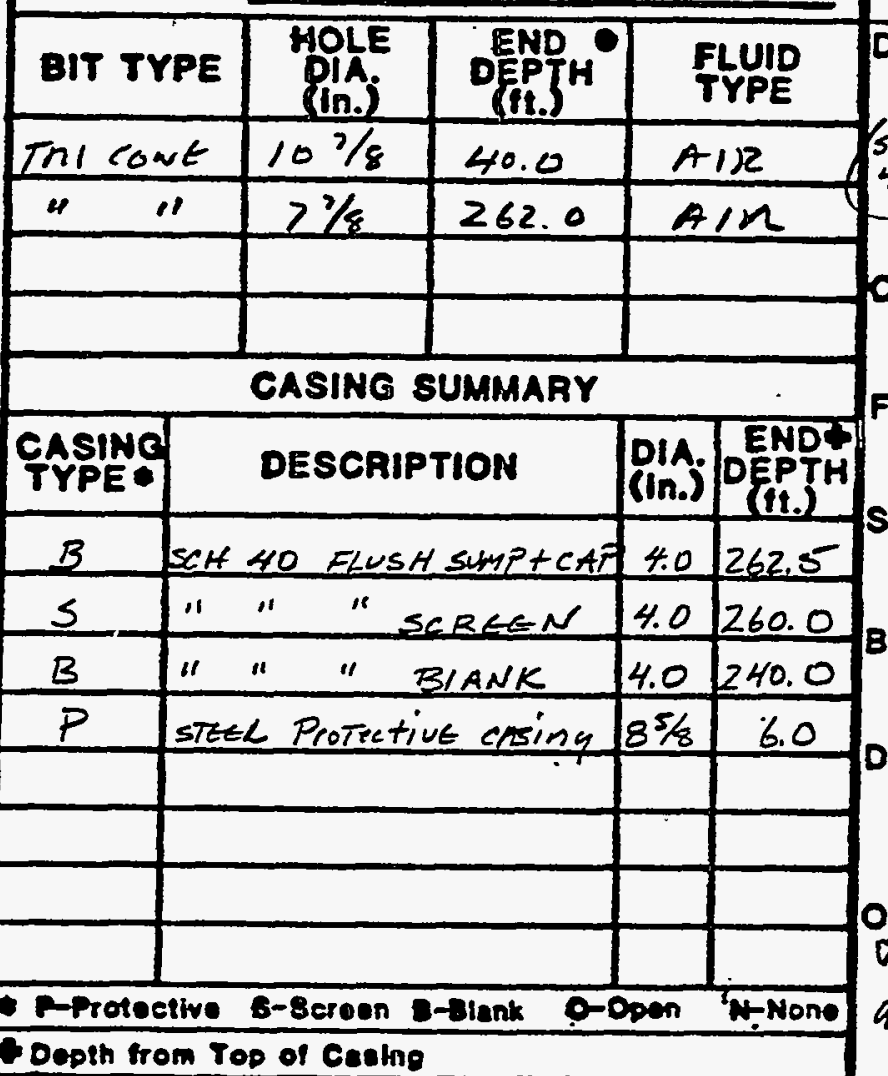
WELL CONSTRUCTION

\begin{tabular}{|c|c|c|}
\hline $\begin{array}{l}\text { TYPE } \\
\text { CODE }\end{array}$ & DESCRIPTION & DENPR \\
\hline $\bar{F}$ & $8-12$ SILIEA SANA & 260.0 \\
\hline 5 & $3 / 4$ "bentonite chip & 217.0 \\
\hline$B$ & bentorite-cement spout & 10.0 \\
\hline & & \\
\hline & & \\
\hline & & \\
\hline & & \\
\hline & & \\
\hline E- & $8-\cos 1$ & Peet \\
\hline \multicolumn{3}{|c|}{ - Dopth from Ground euriace } \\
\hline
\end{tabular}

JEQ-AL-EMQ-1 (s/QA)

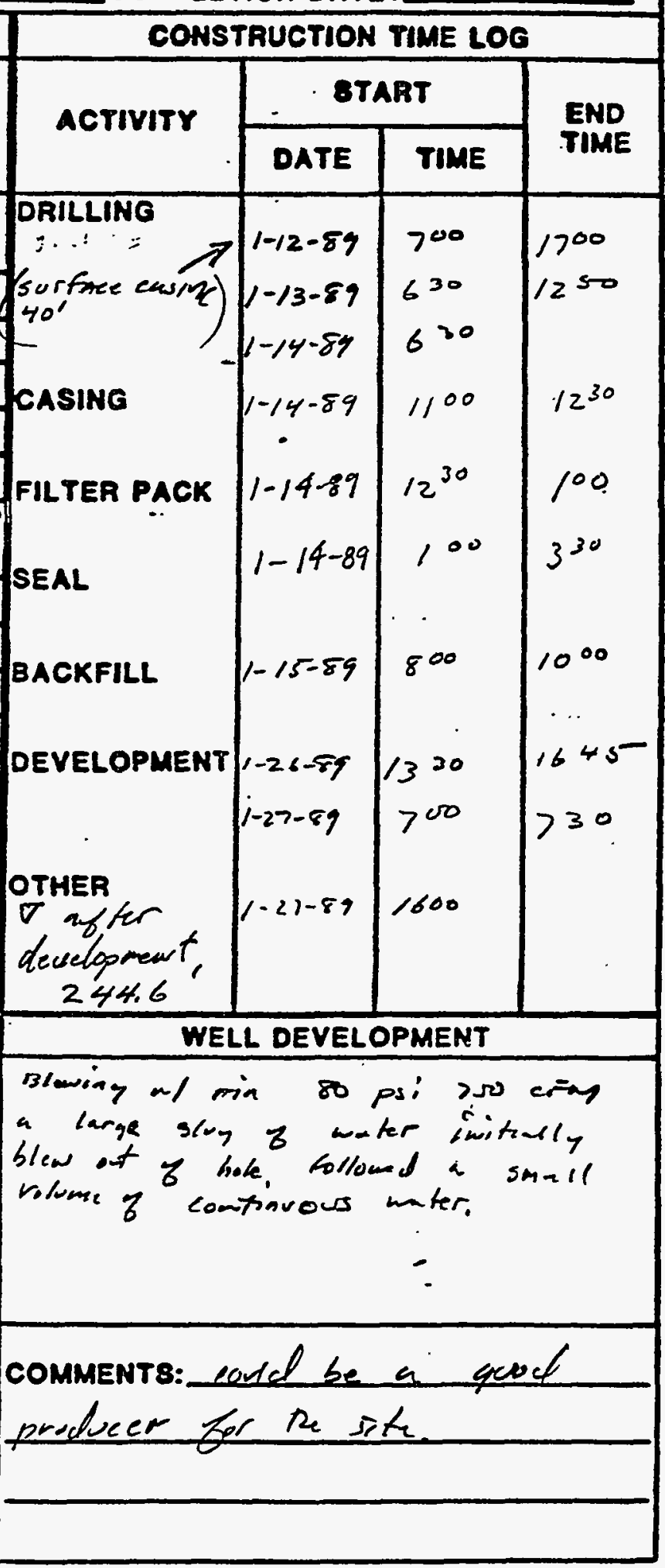


WELL 679 LOGS 


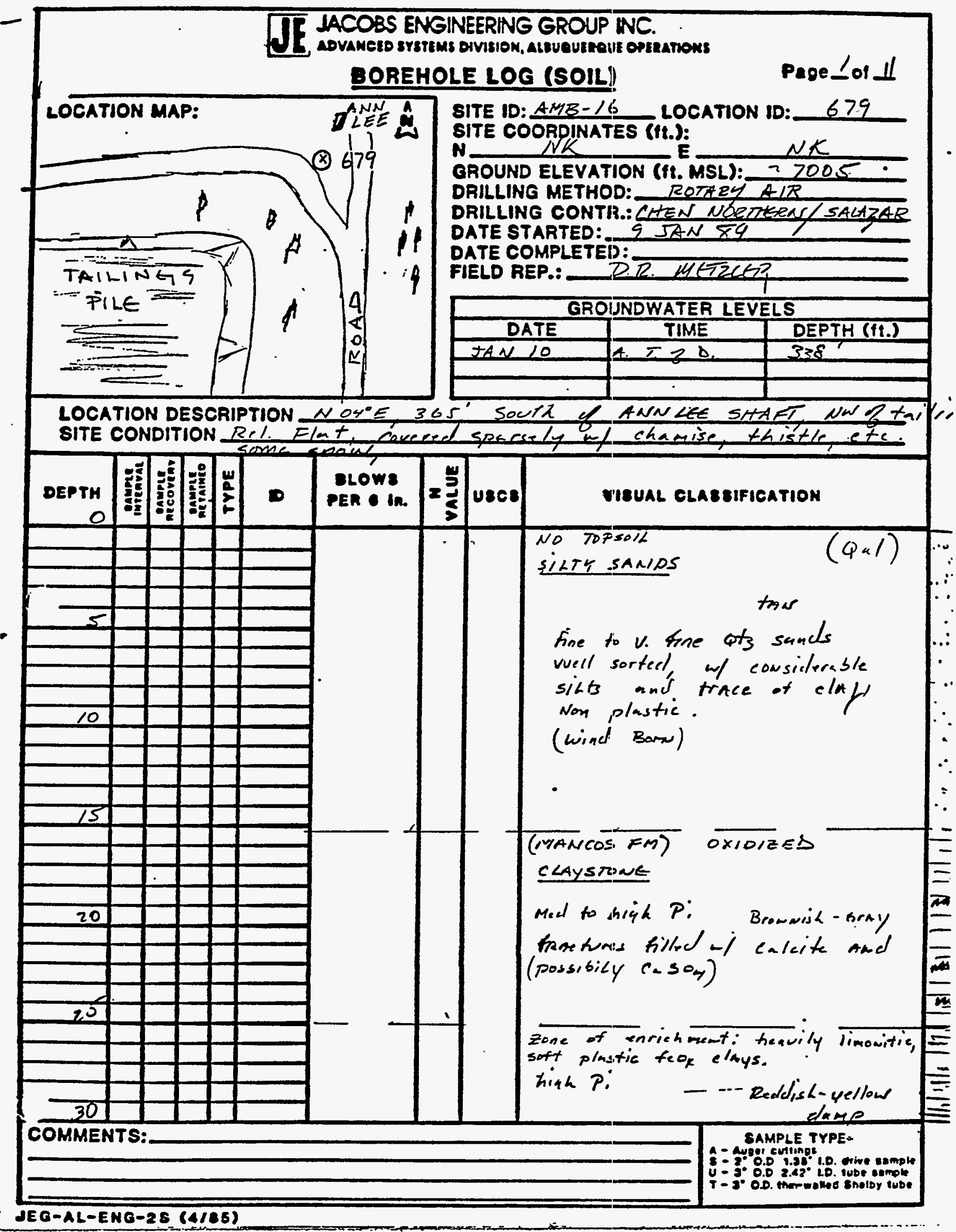




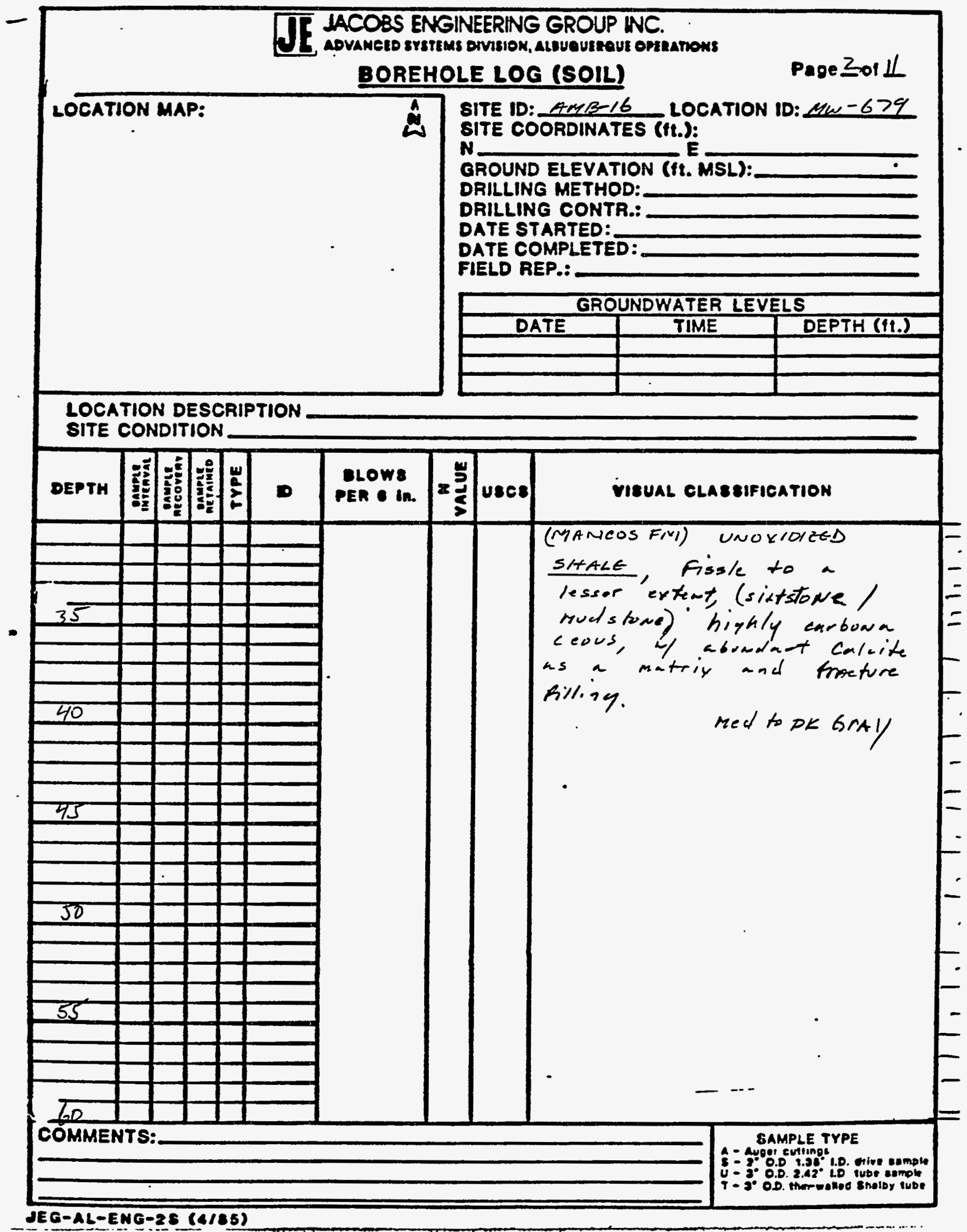




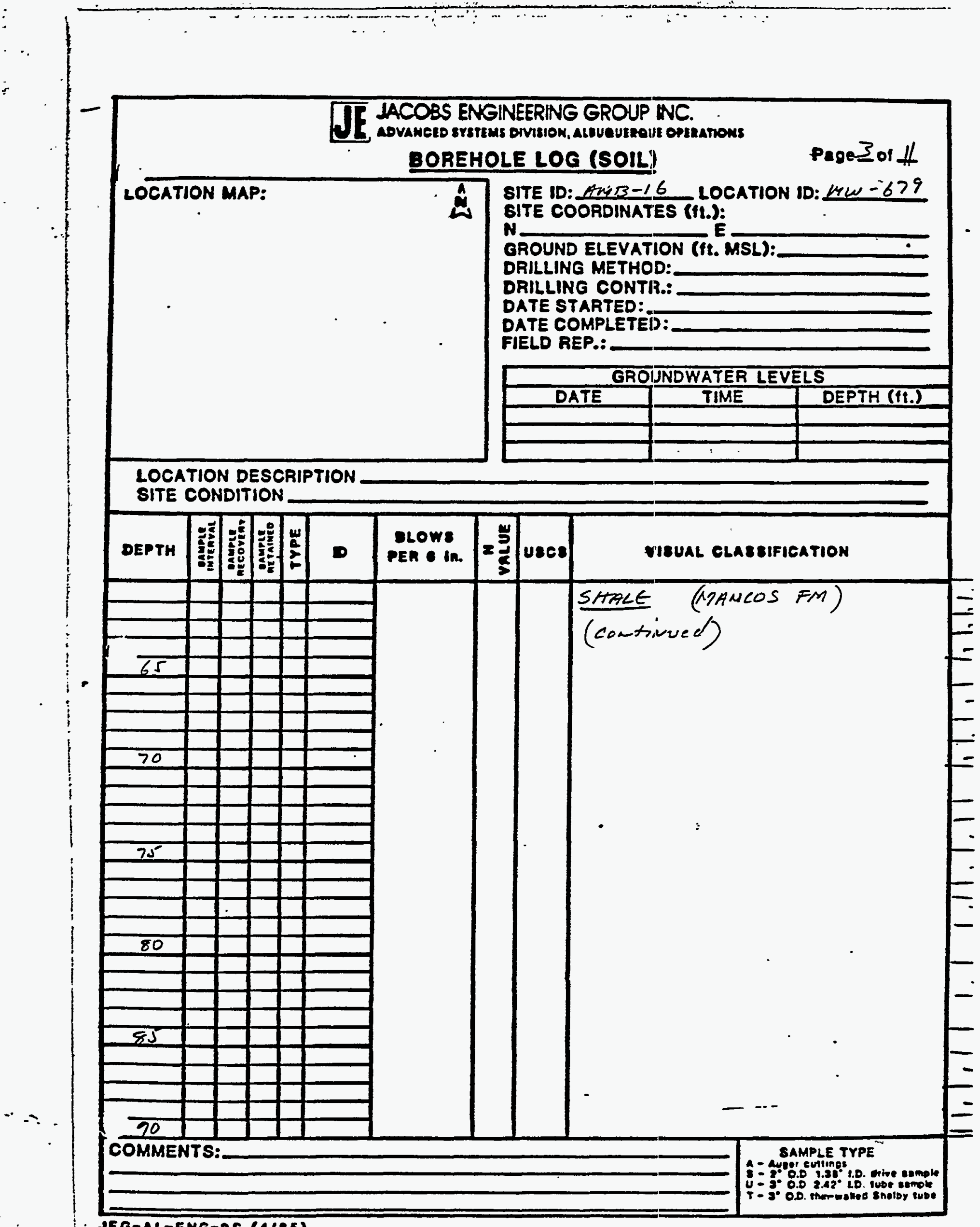




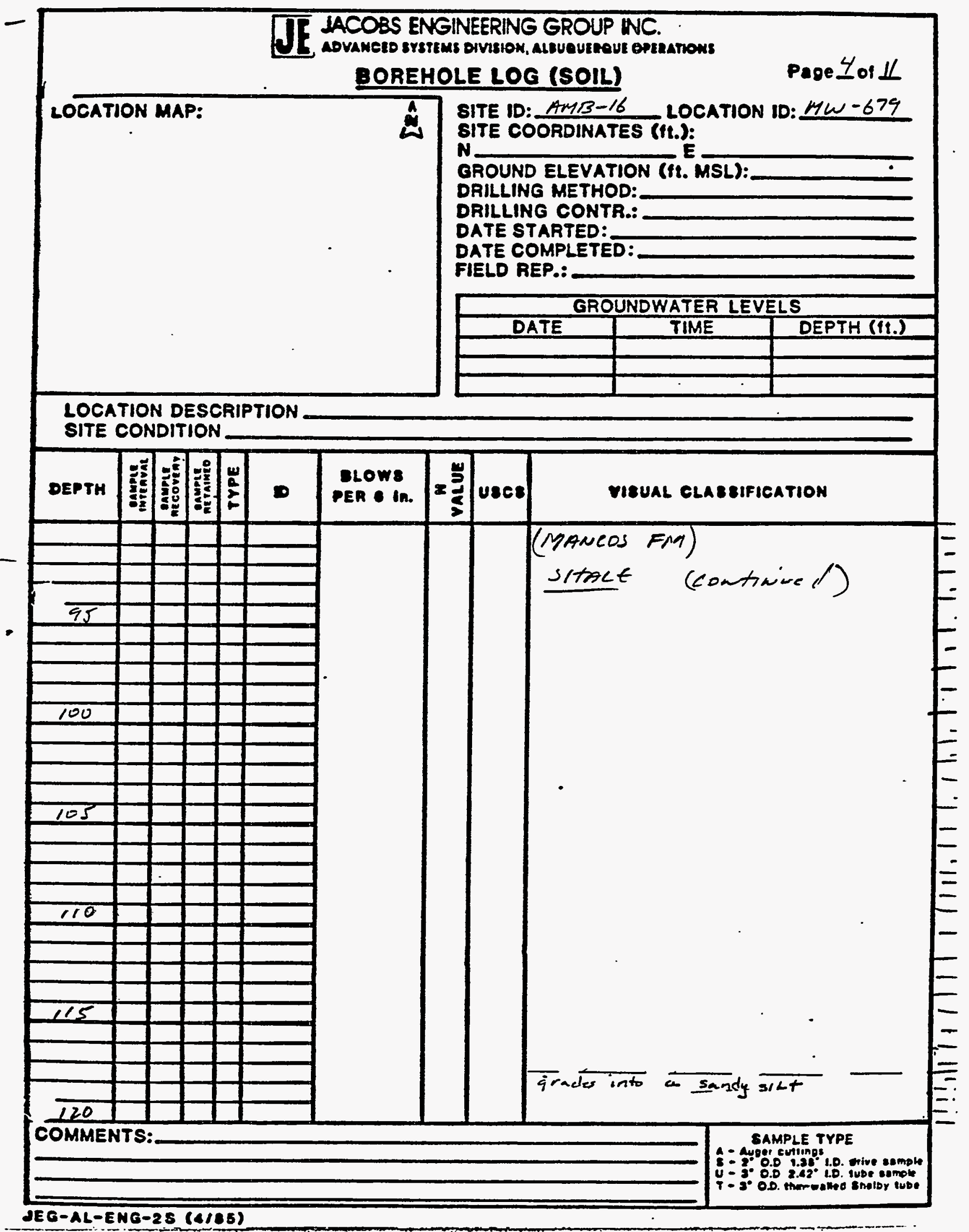




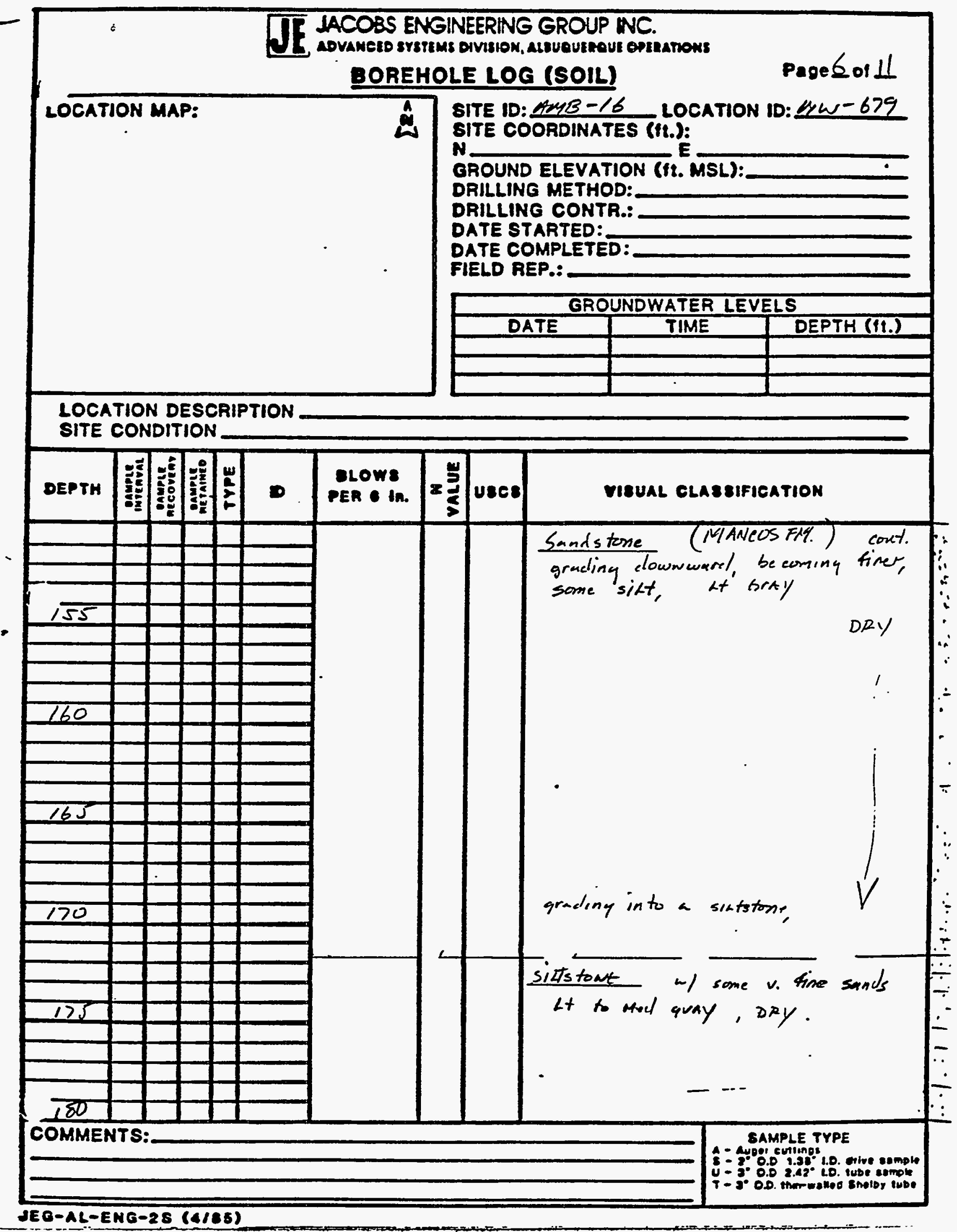




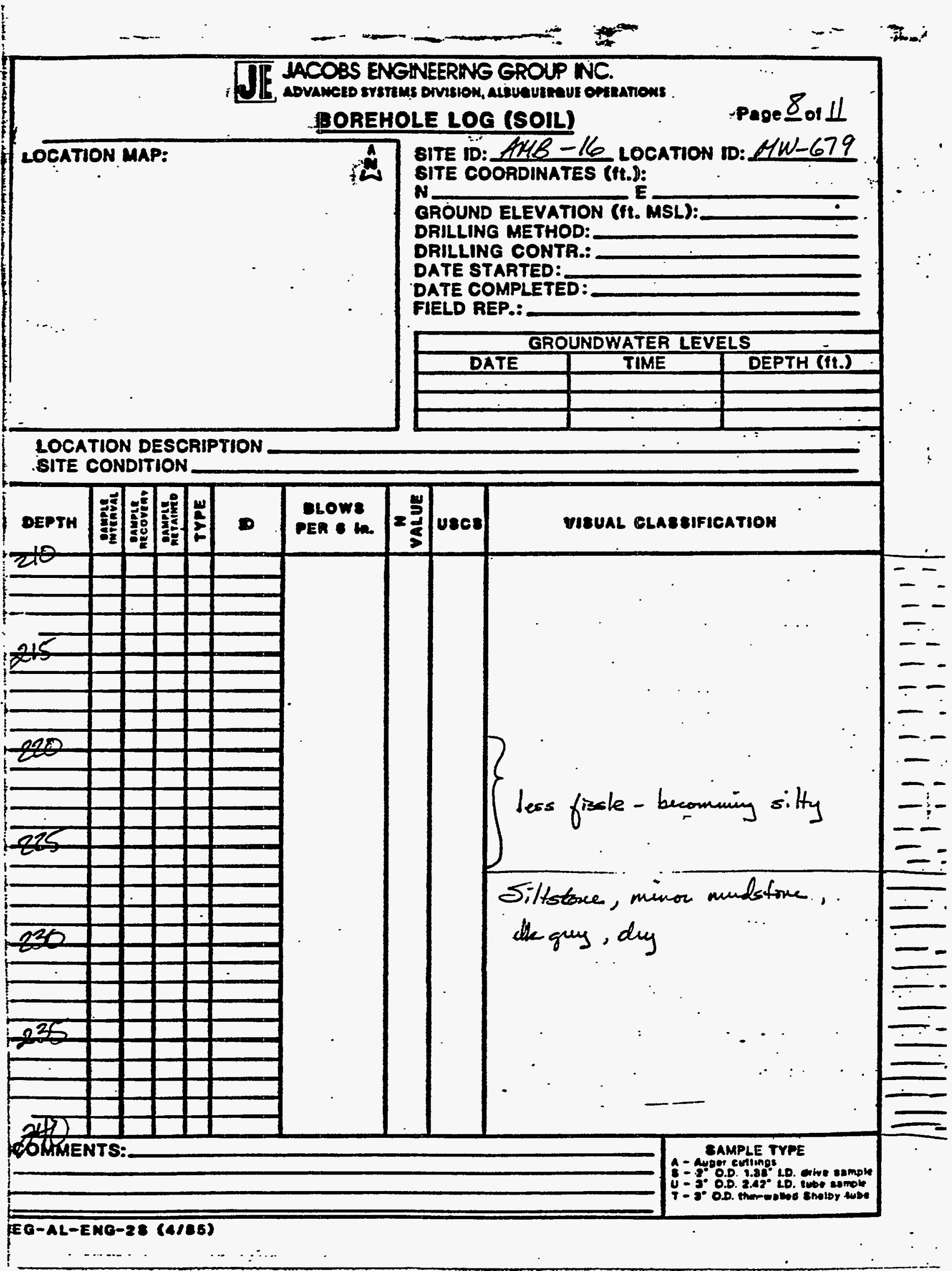




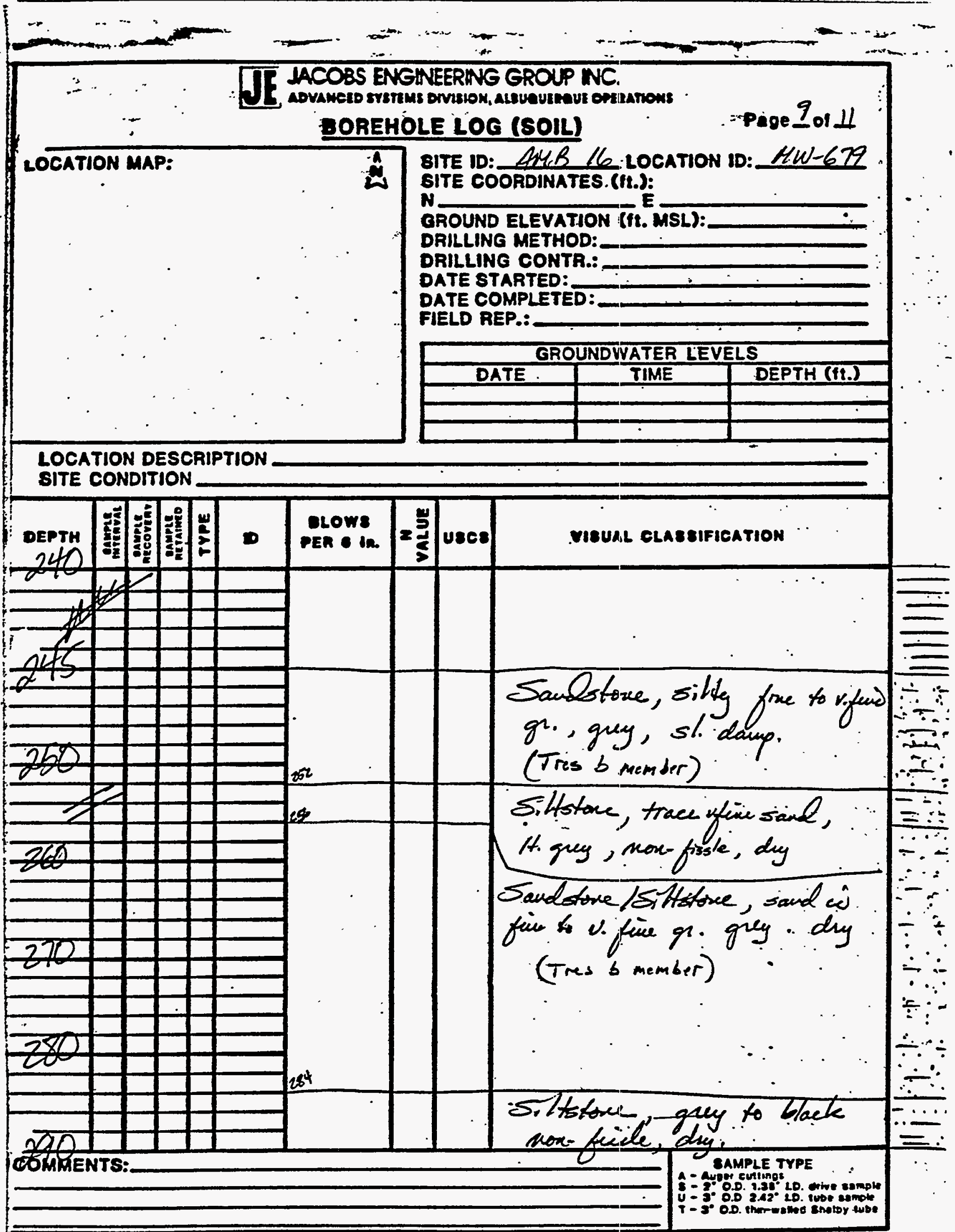

EO-AL-EMO-28 (A/CS) 


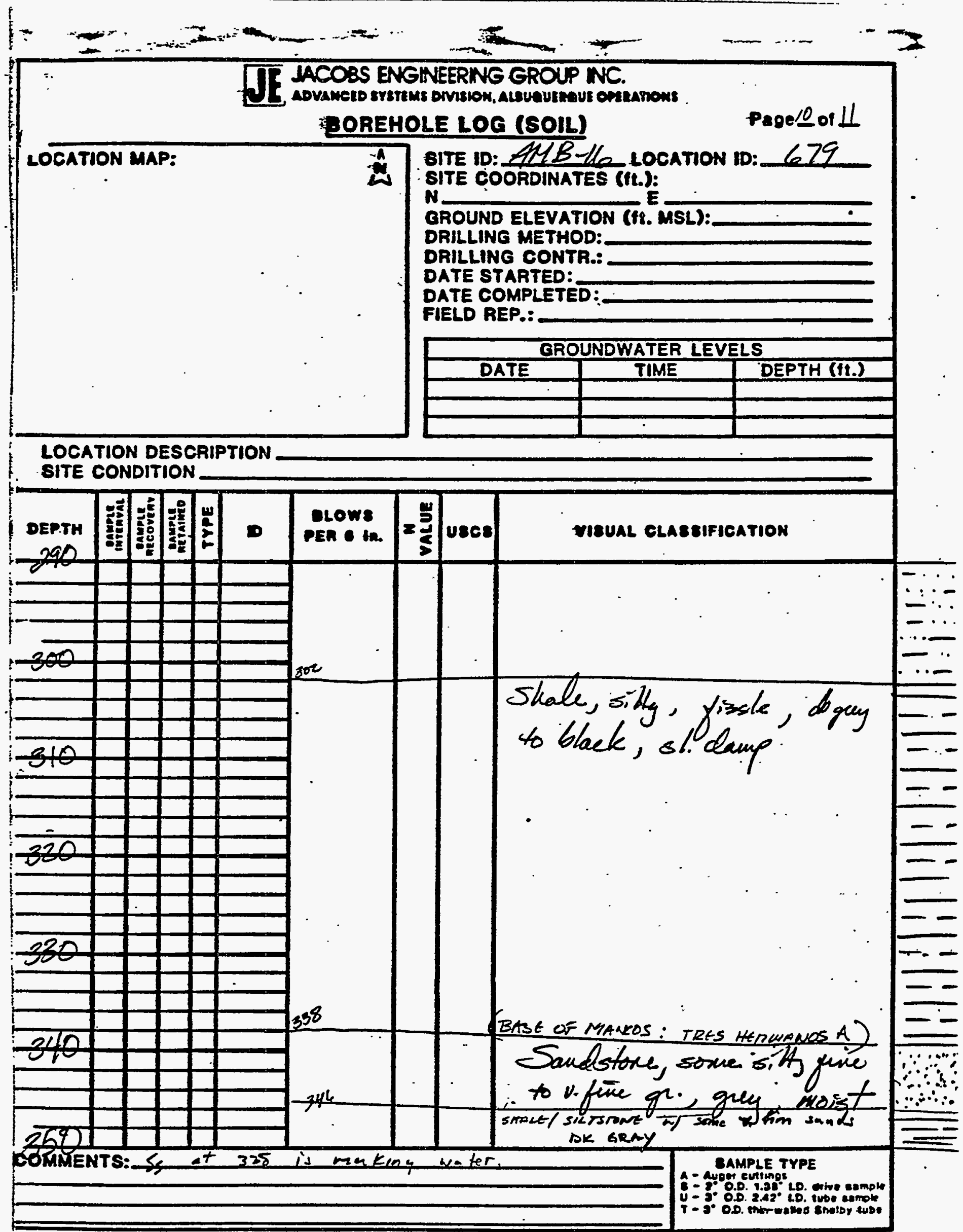

EO-AL-EMQ-28 (4)ES) 


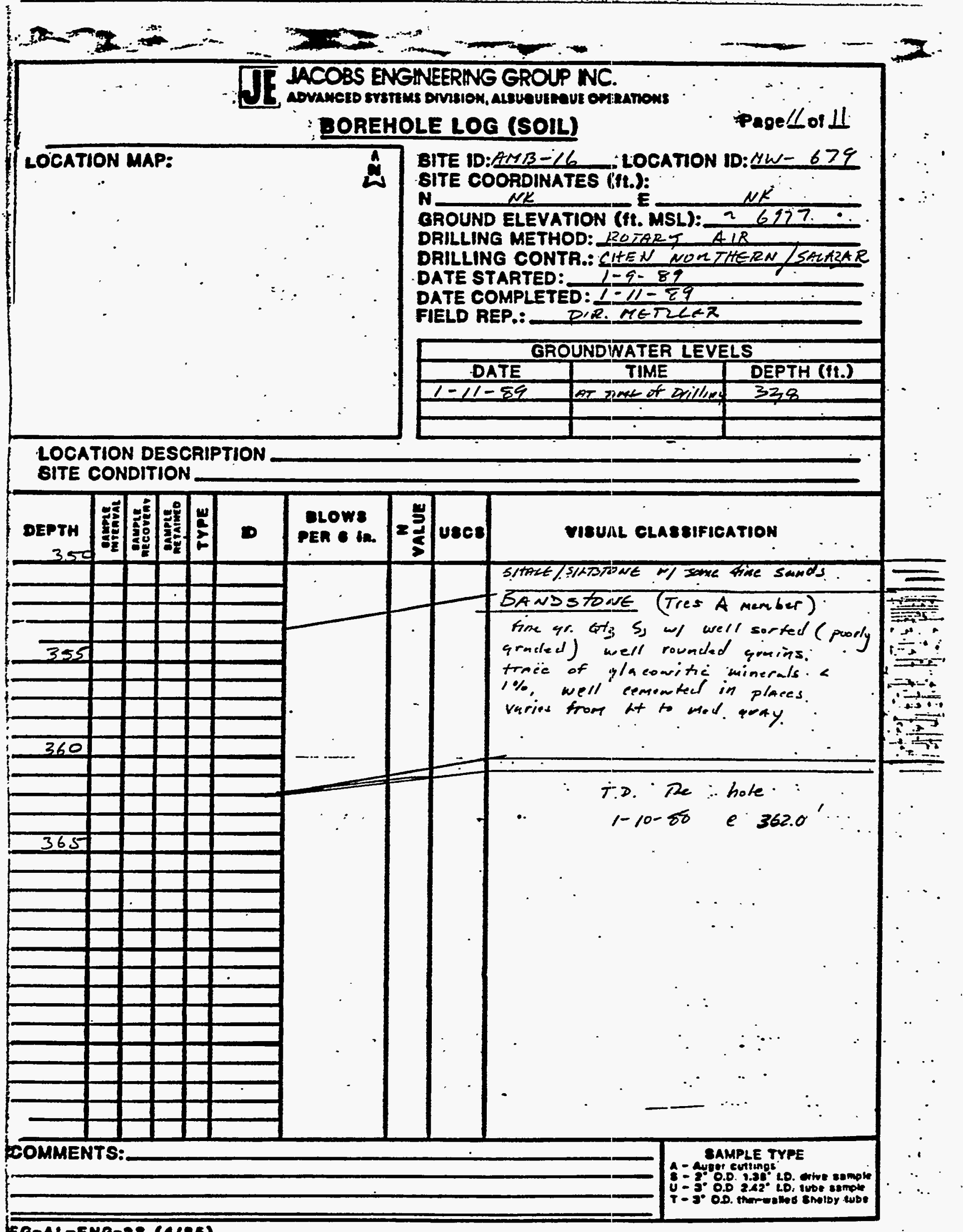


TT HOOBS ENGNEERNG GROUP NC.

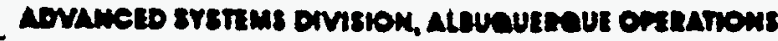

\section{WELL COMPLETION RECORD}

SITE ID: $A M B$ LOCATION ID: 679

DATE INSTALLED: JAN 11,89

APPROX. SITE COORDINATES:(FT.) N

OPEN : AREA PPER LINEAL FT. (IN2/FT.)

(FORMATION OF COMPLETION: Momeac i

$8^{2}-4.5^{2}$ E

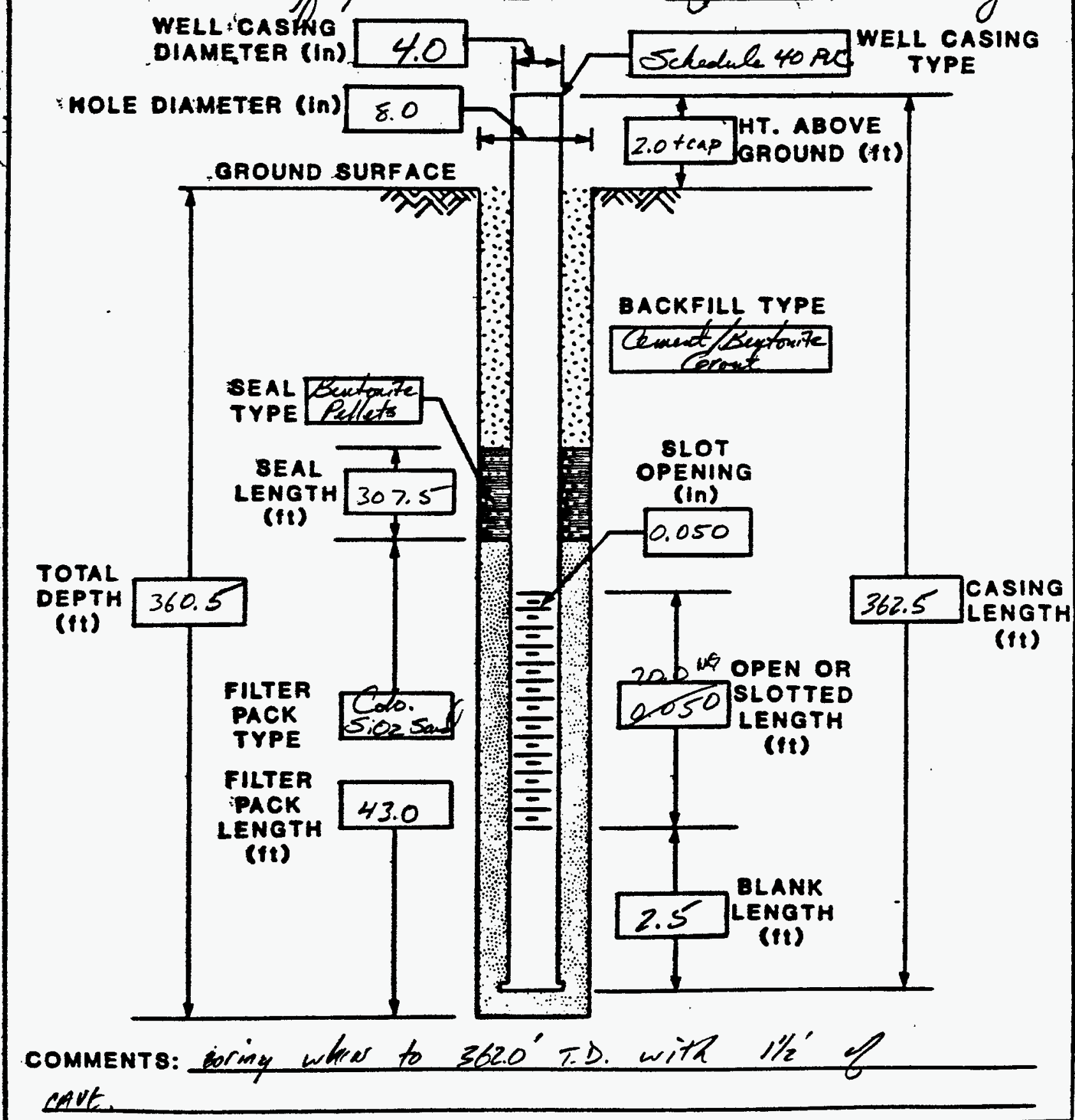

JEQ-AL-ENO-3 (S1CA) 


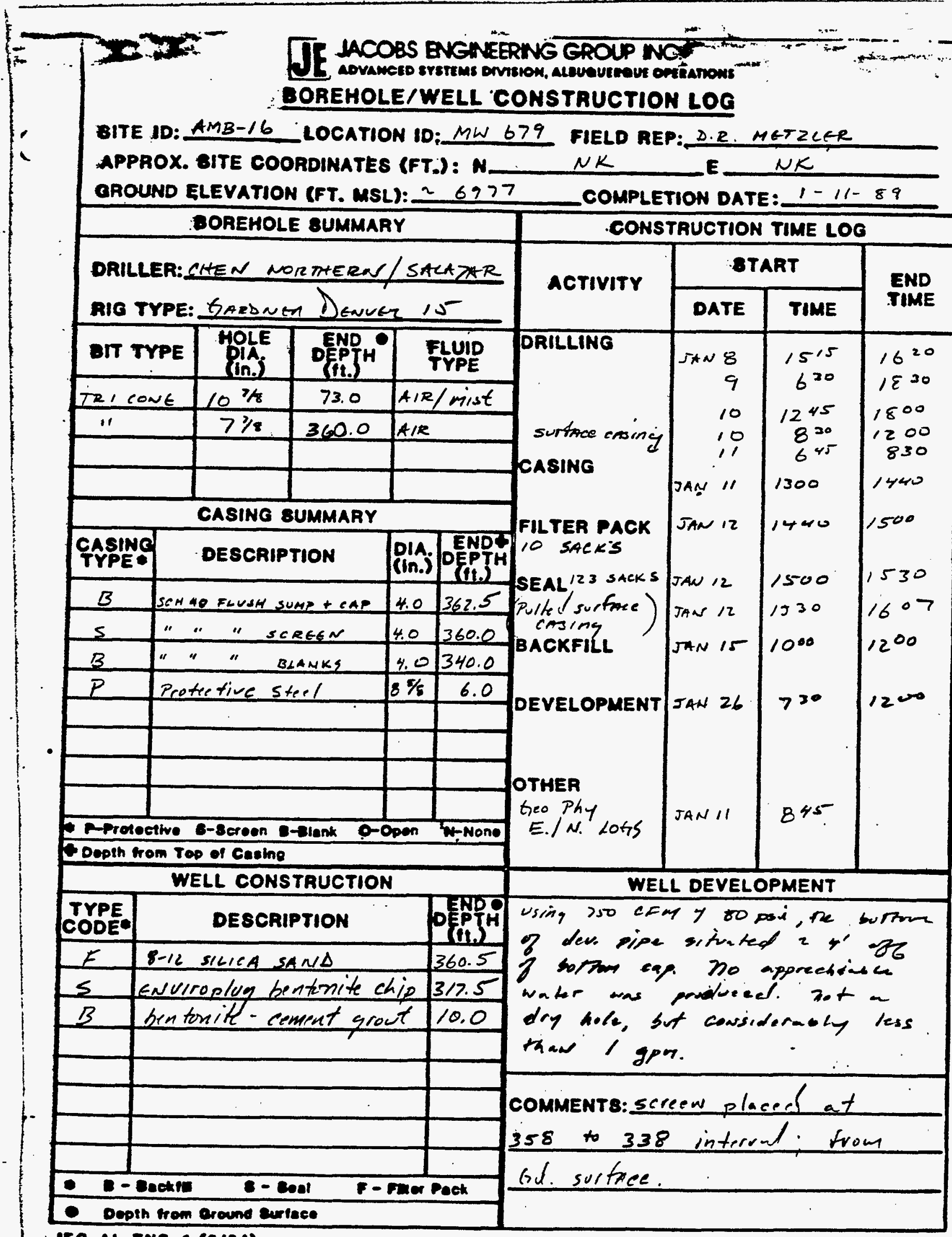

JEO-AL-ENa-1 (s/O4) 
WELL 680 LOGS 


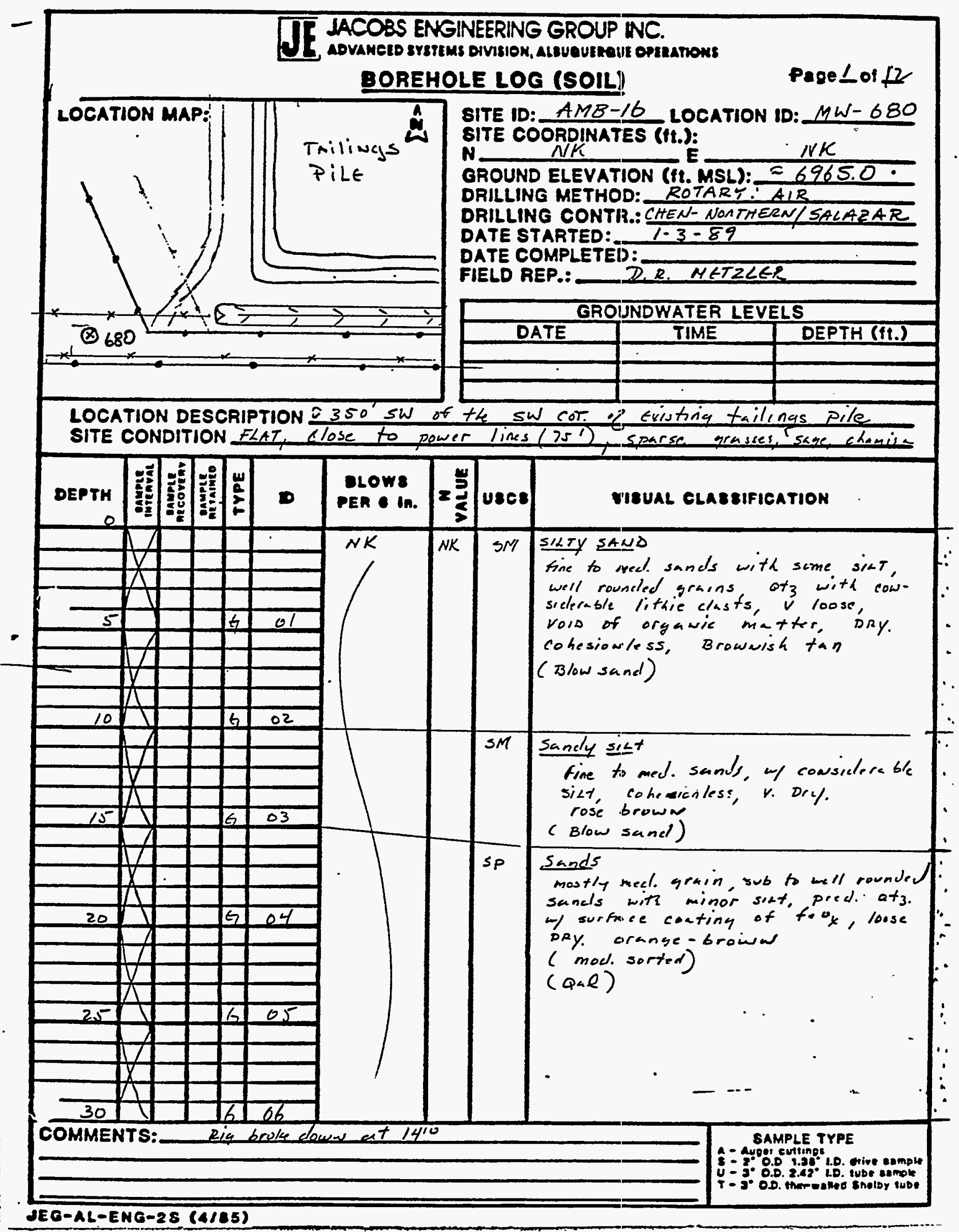




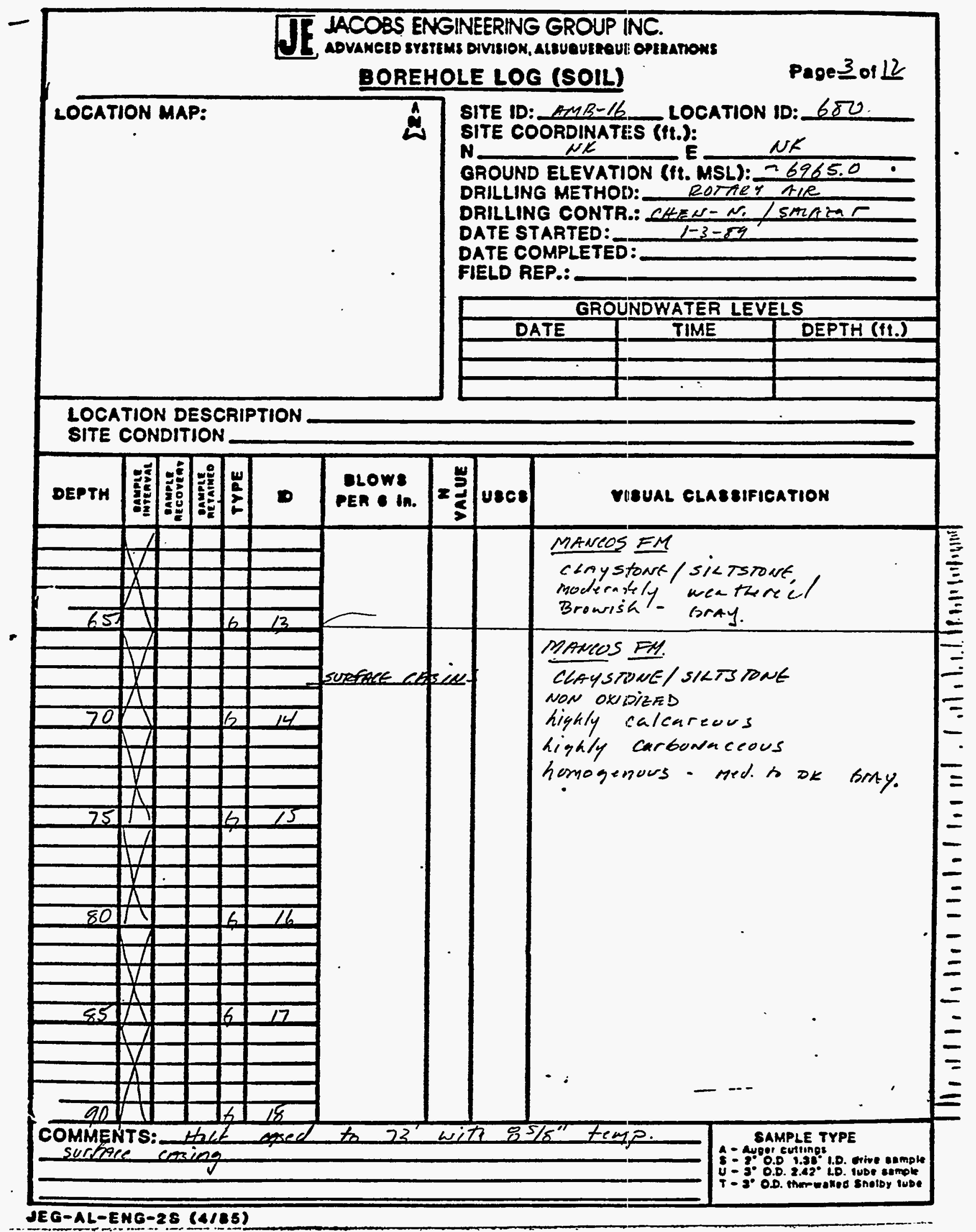




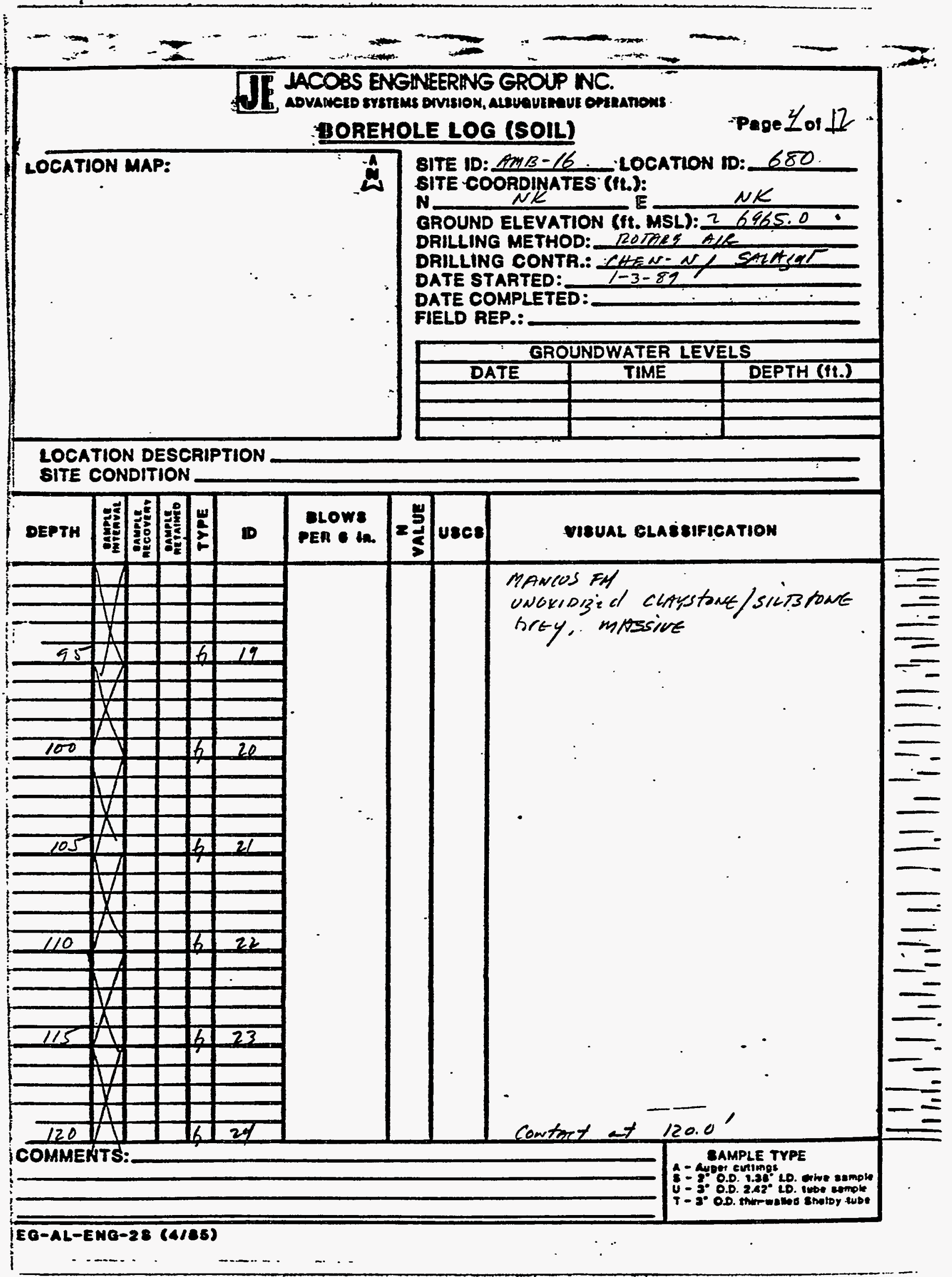




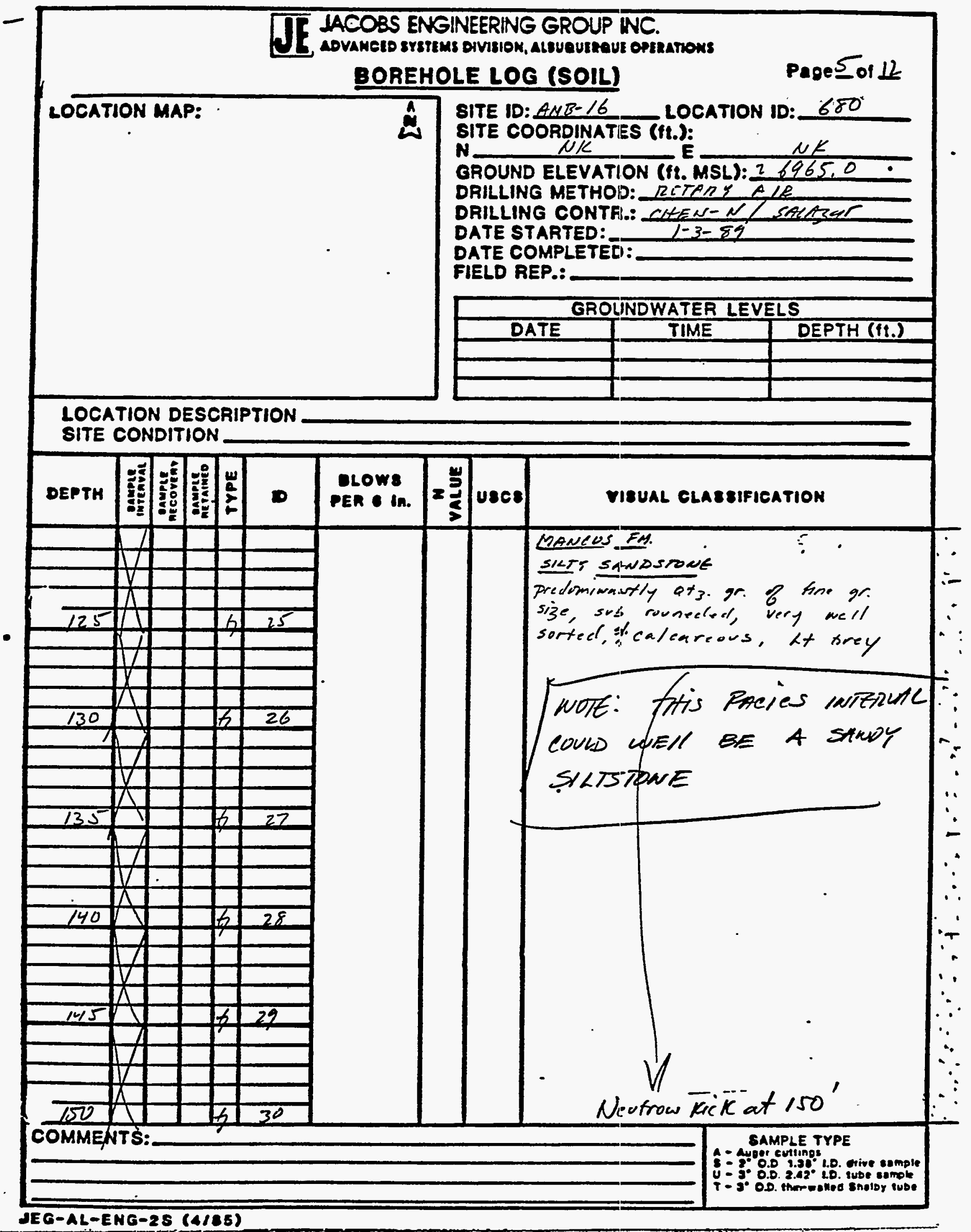




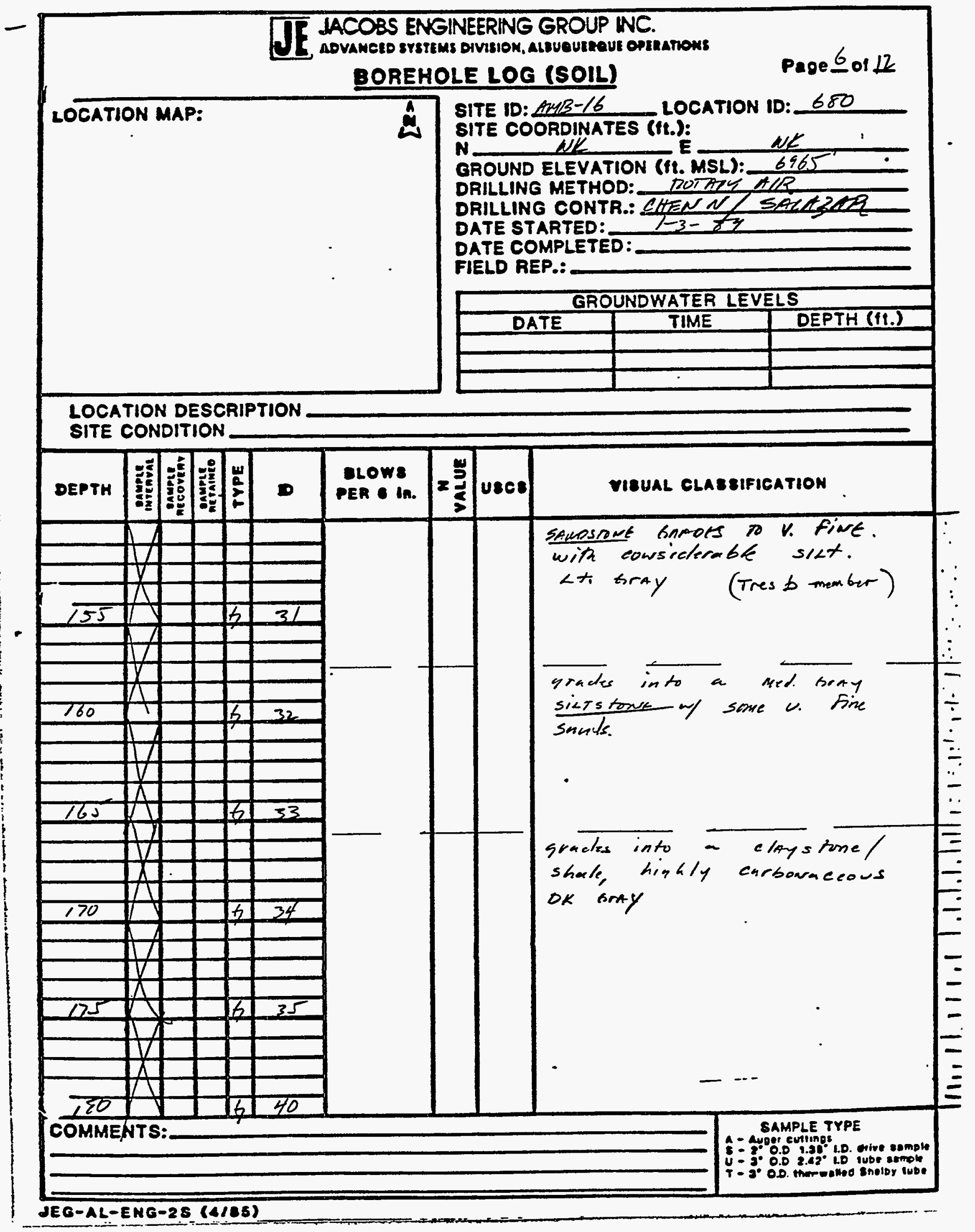




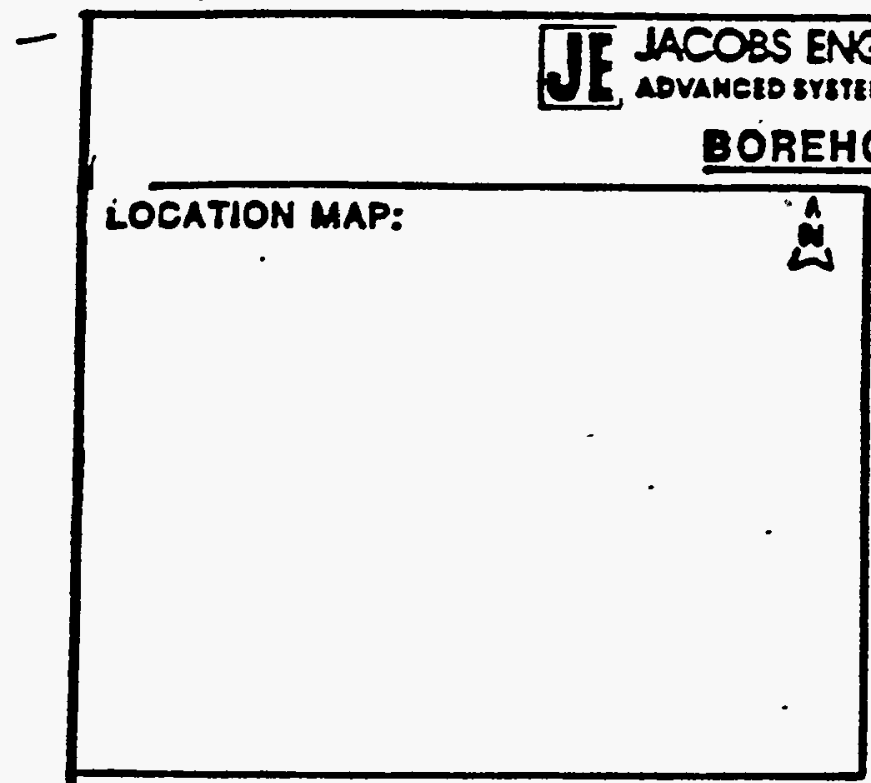

GINEERING GROUP INC.

MS DIVIBION, ALEUQUEREUI OPRATIONS

LOCATION DESCRIPTION

SITE CONDITION

$r$

\begin{tabular}{|c|c|c|c|c|c|c|}
\hline DEPTH & 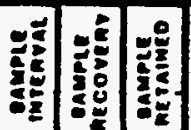 & $\frac{a}{a}$ & D & $\begin{array}{l}\text { OLOW8 } \\
\text { PER I In. }\end{array}$ & $=\frac{\omega}{g}$ & |usce \\
\hline
\end{tabular}

DATE STARTED:

DATE COMPLETED: $1-3-59$

FIELD REP.:

\begin{tabular}{|c|c|c|}
\hline \multicolumn{2}{|c|}{ GROUNDWATER LEVELS } \\
\hline DATE & TIME & DEPTH (ft.) \\
\hline & & \\
\hline & & \\
\hline & & \\
\hline
\end{tabular}

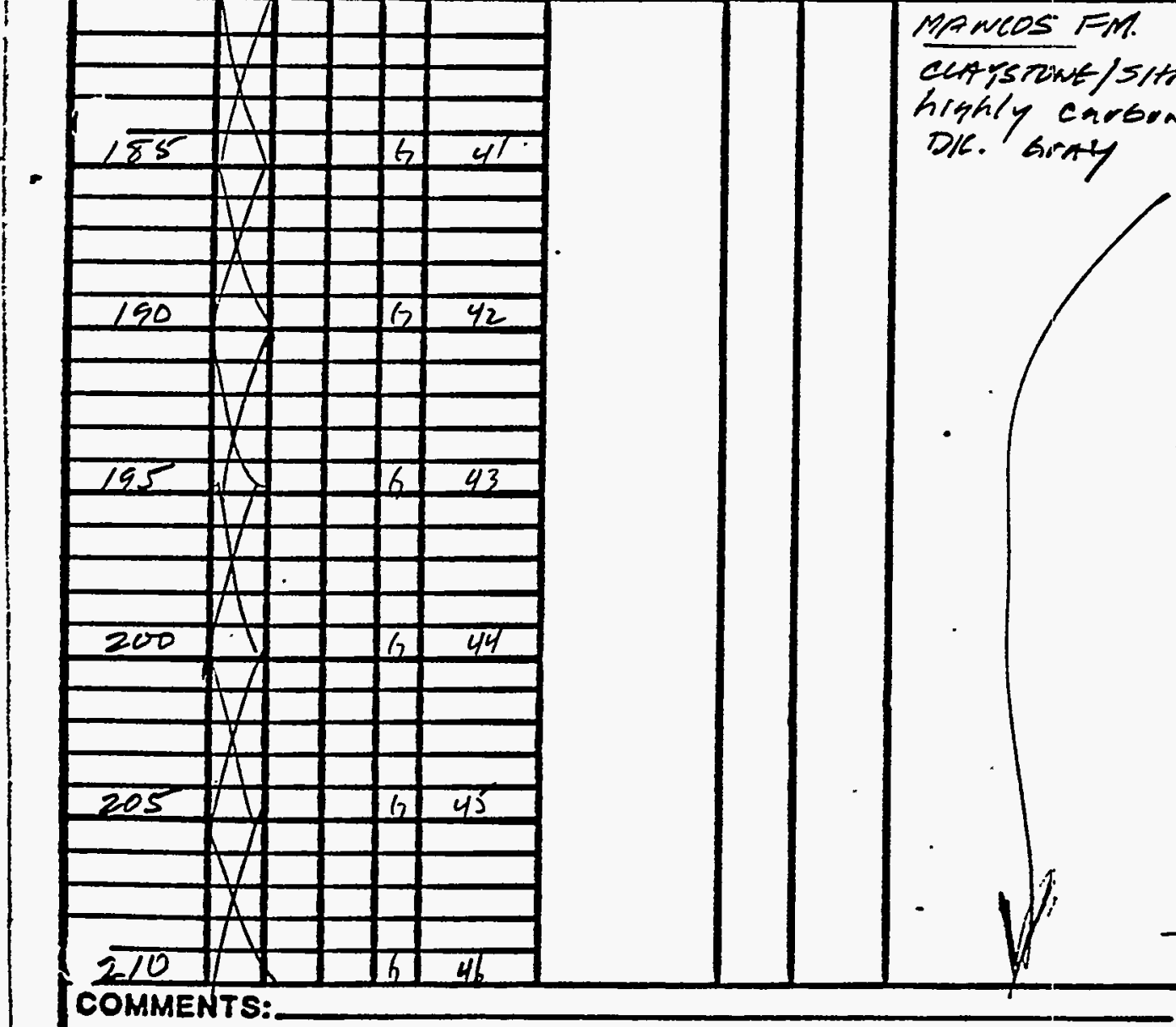

SAMPLE TYPE

A - Ayour curings

8 - 2.0.0 1.30 1.0. stive eample U - 30 0.0 2.42 id. Tube sumple

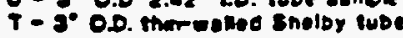

Page Zor 12

LOCATION ID: 680

NK E NK

OUND ELEVATION (ft. MSL): 6965 -

DRILLING METHOD: TOTRZY The

DRILLING CONTR.: CHEN 4 L LWLART

vivual glaseification

MANCOS FM.

Sitact

carbonereevos 


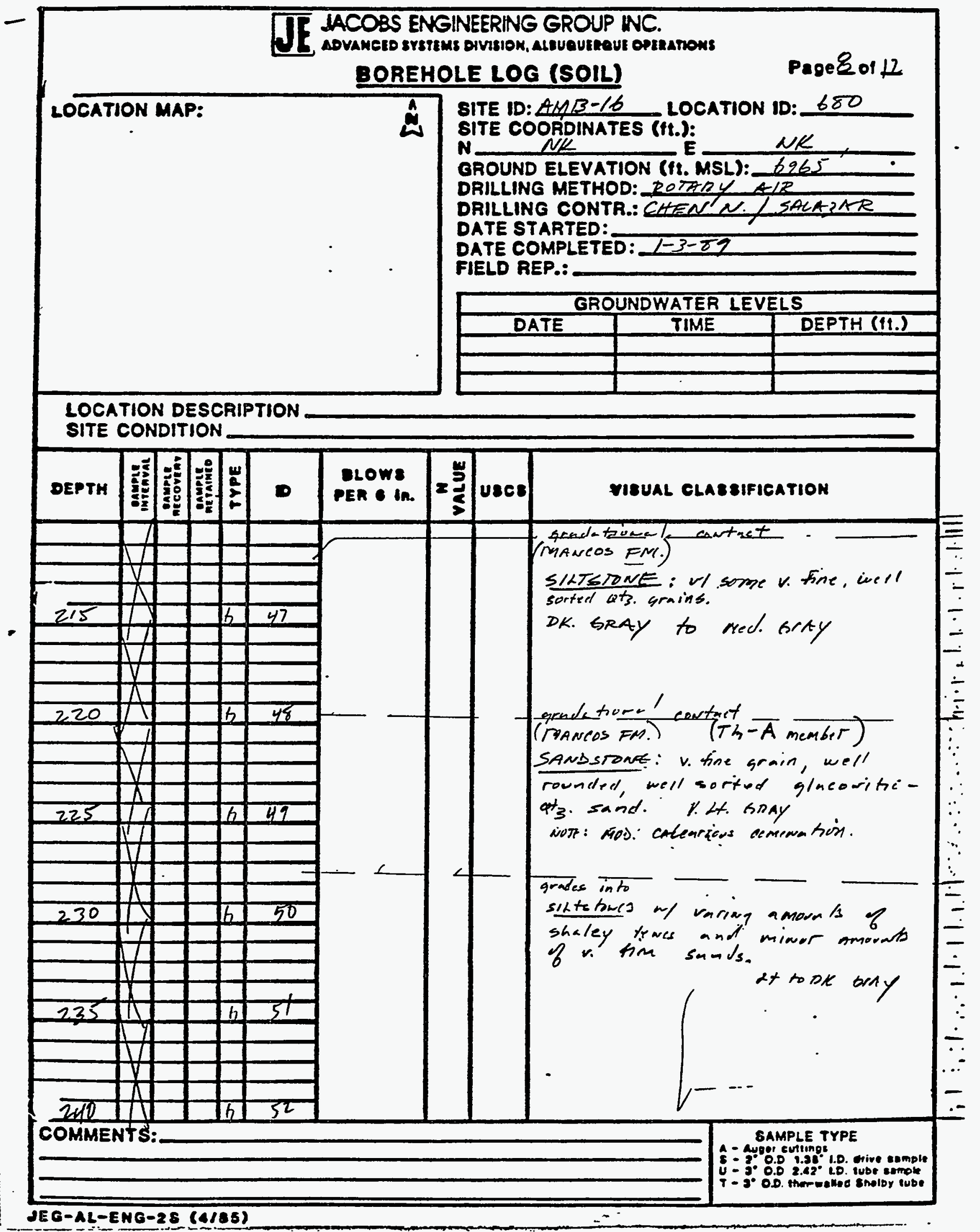




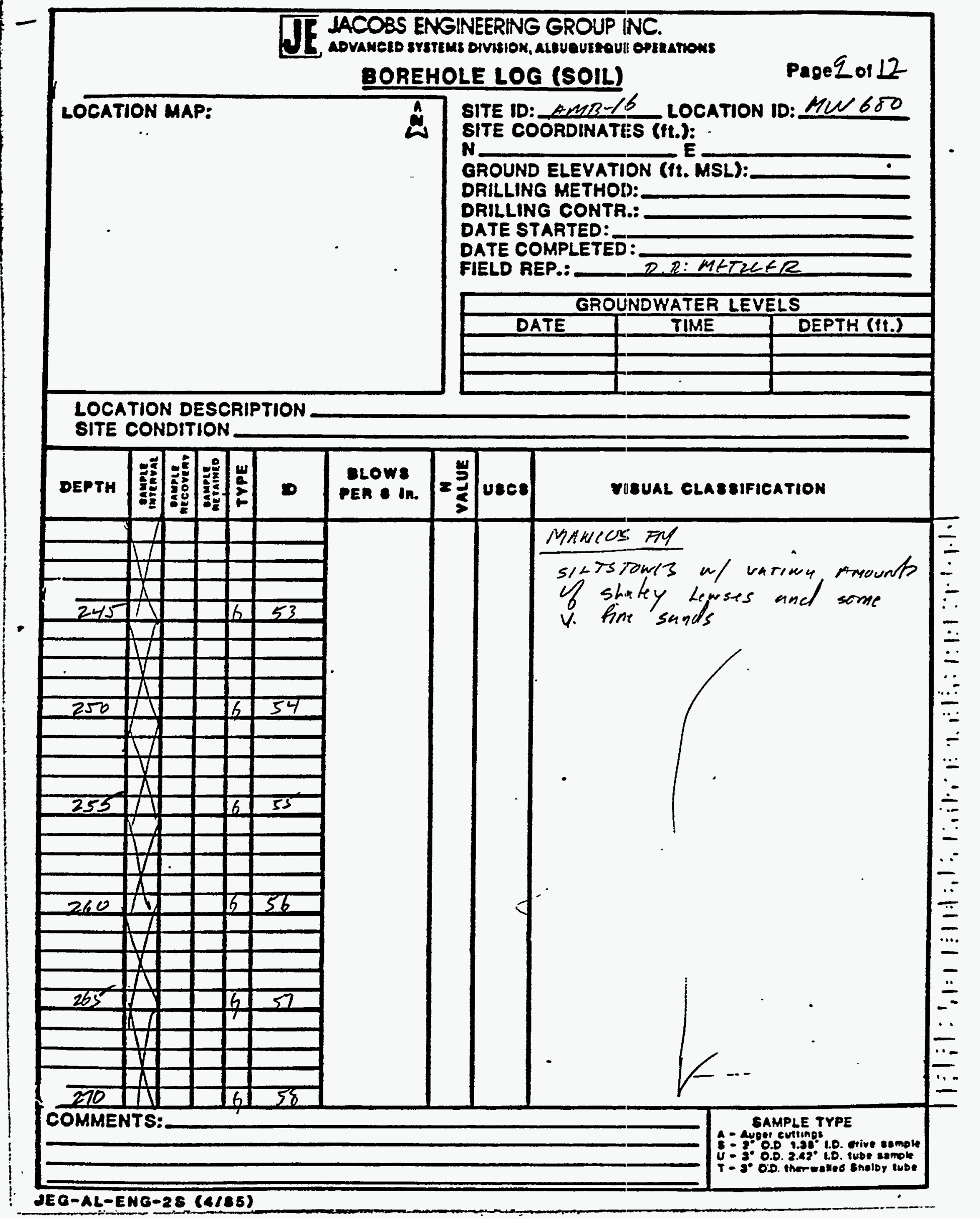




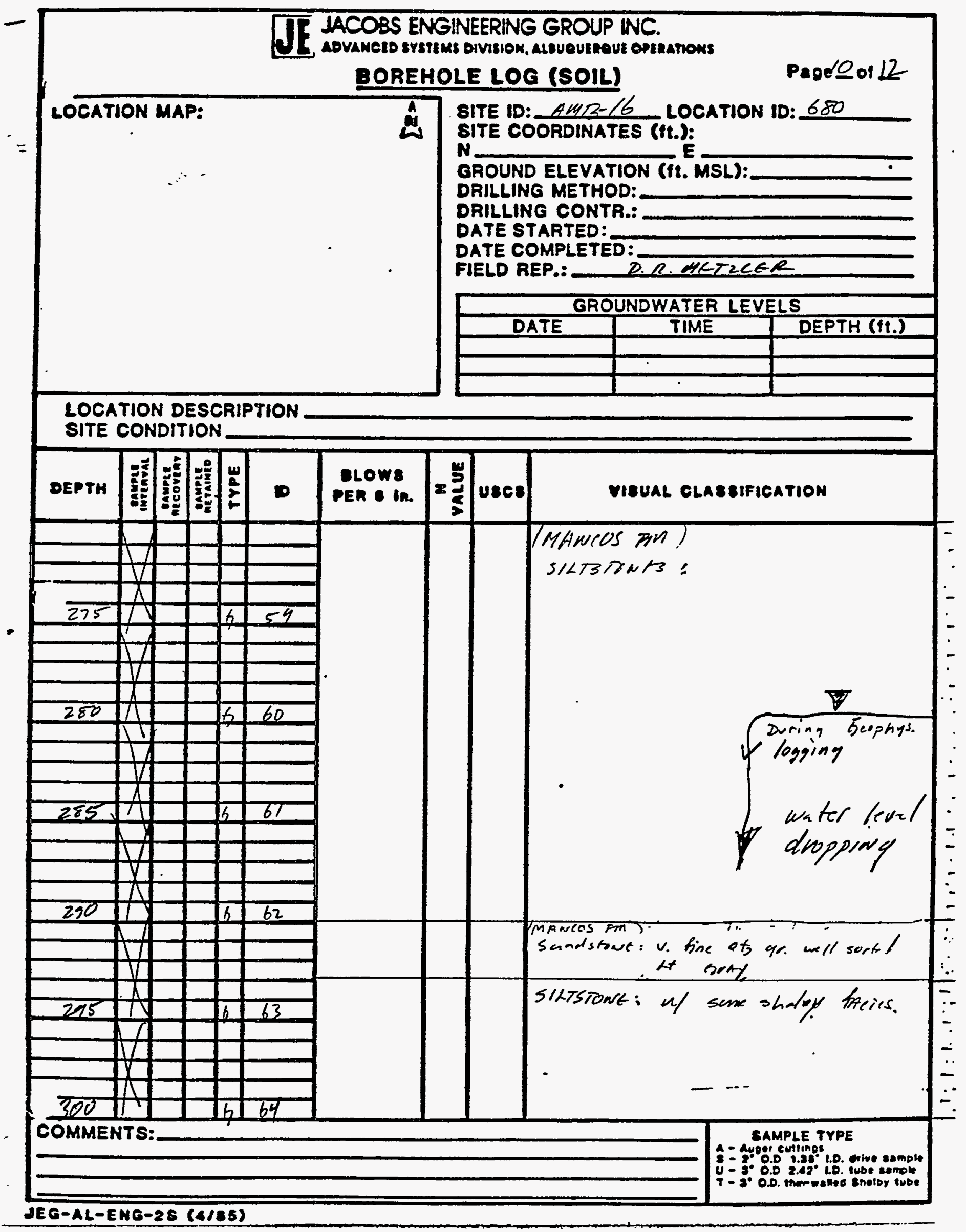




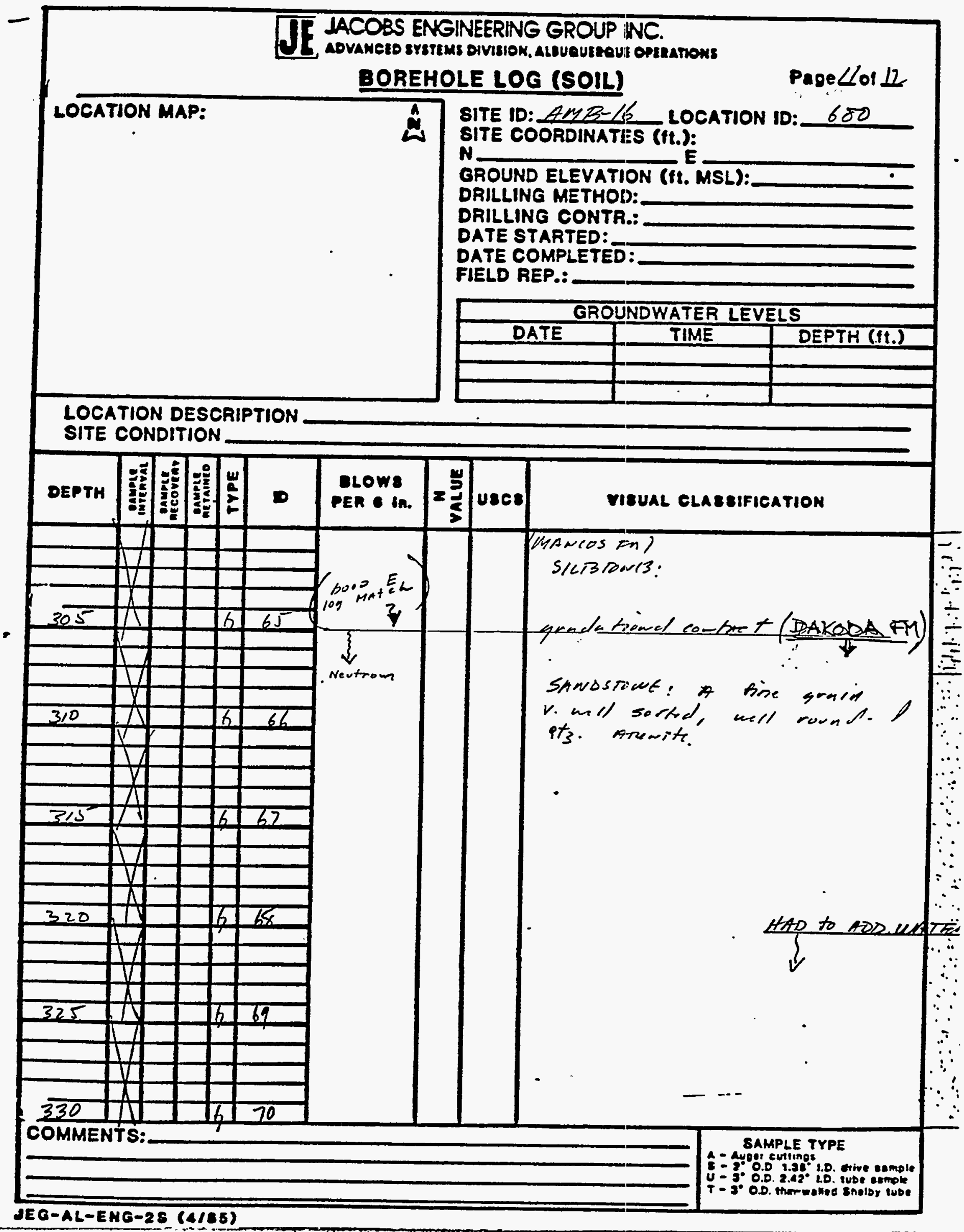




\section{BOREHOLE LOG (SOIL)}

LOCATION MAP:

SITE ID:ANB-16

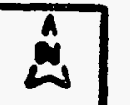

SITE COORDINATES (ft.):

$N$ GROUND ELEVATION (ft. MSL):

DRILLING METHOD:

DRILLING CONTR.:

DATE STARTED:

DATE COMPLETED: $\frac{1-6-\pi 9}{2-69}$

FIELD REP.: D. M. HET ZLE

\begin{tabular}{|c|c|c|}
\hline \multicolumn{3}{|c|}{ GROUNDWATER LEVELS } \\
\hline DATE & TIME & DEPTH (11.) \\
\hline & & \\
\hline & & \\
\hline
\end{tabular}

LOCATION DESCRIPTION

SITE CONDITION

\begin{tabular}{|c|c|c|c|c|c|c|c|c|c|}
\hline DEPTH & 熟 & 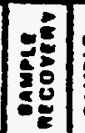 & 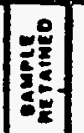 & $\frac{w}{\frac{w}{2}}$ & 0 & $\begin{array}{l}\text { DLOW } \\
\text { PER in. }\end{array}$ & $=\frac{\omega}{s}$ & uses & VIEUAL CLABEIFICATION \\
\hline
\end{tabular}

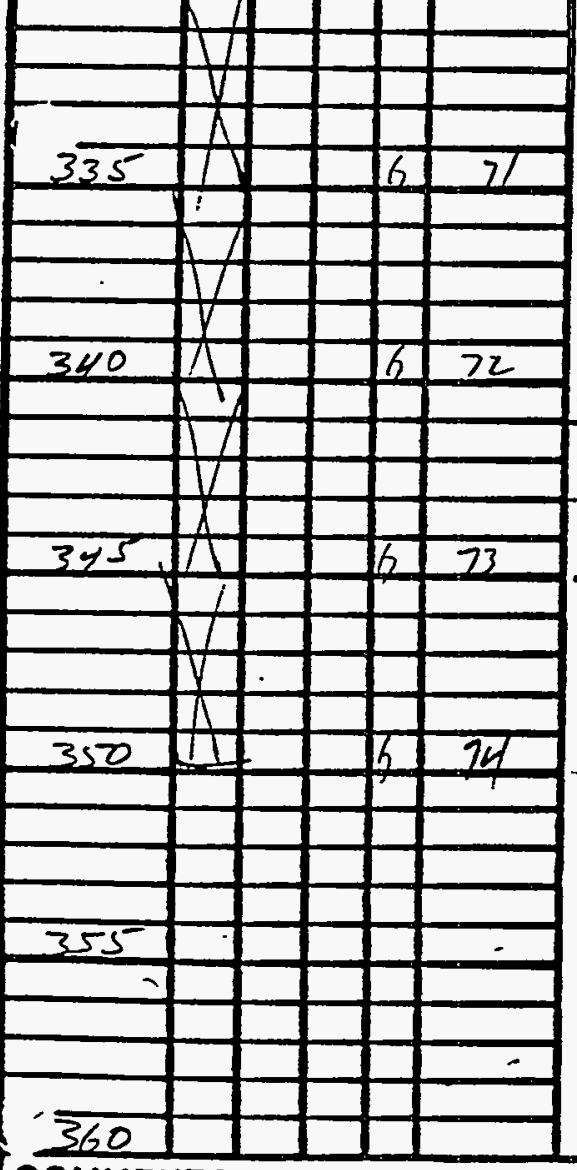

SANOSTONE

contiauset

FM. MAKINA WATER

T.D. hir

Loy

COMMENTS:

cowfret

ligarsite? hiqhly oryanic shale?

Shavosiowt:? Gine gro ot 3 arewith

strowgly cemeated / miros

af be Ligurte- oryanie shde.

$T . \Delta$

Sacor curtings

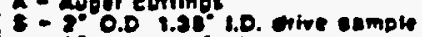

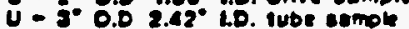

$T=3 \circ 0.0$. ther wellied snolby tube 


\section{TF LACOBS ENGNEERING GROUP MYC.

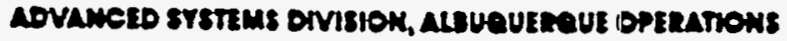

\section{WELL COMPLETION RECORD}

SITE ID: $A M B-16$ LOCATION ID: 688

DATE INSTALLED: $1 / 7 / 89$ APPROX. SITE COORDINATES:(FT.) $\mathrm{N} \frac{N K}{\left(\delta^{2}-H^{2}\right)^{\pi}} \mathrm{E}-\mathrm{NK}$ OPEN AREA PER LINEAL FT. (IN'/FT.)

FORMATION OF COMPLETION: Kd DAKODA SS $\zeta \ldots$, FIELD REP.: I MaCkuzie DRILLER: Cher Nothern Sclazar

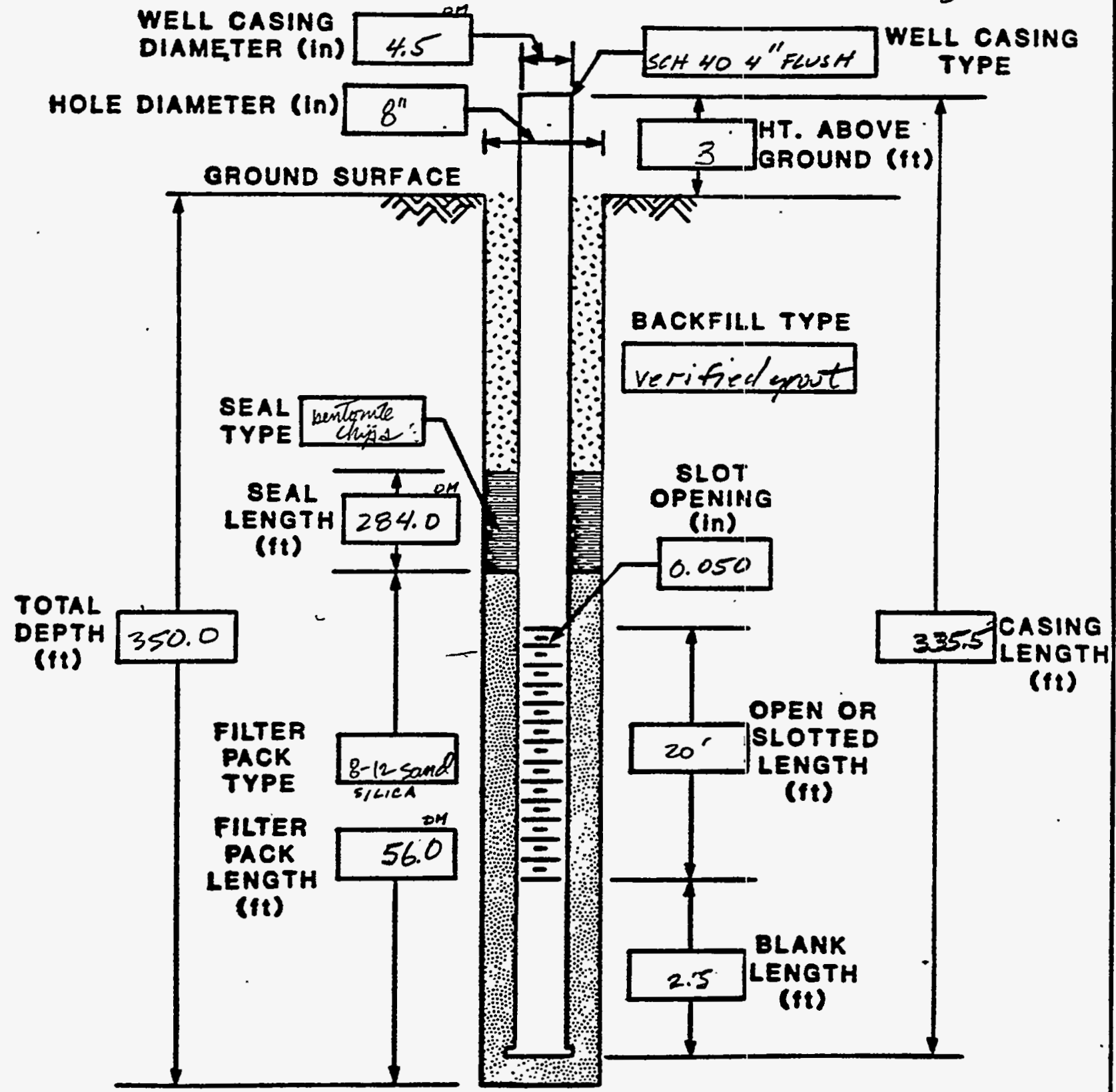

COMMENTS: wedl Awulos filled w/ siliex from $333.5^{\prime}$ to $350.0^{\prime}$ 


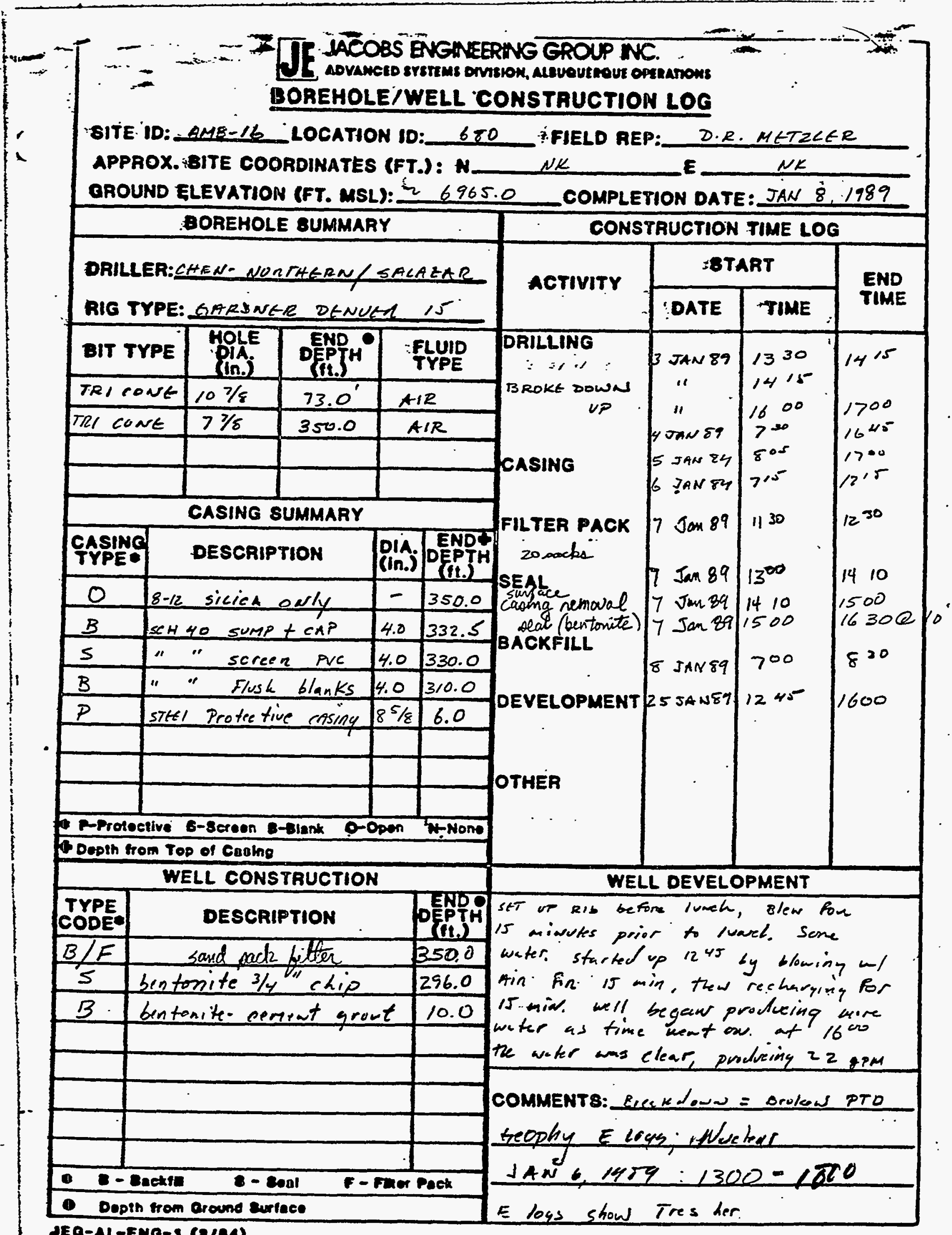


WELL 681 LOGS 


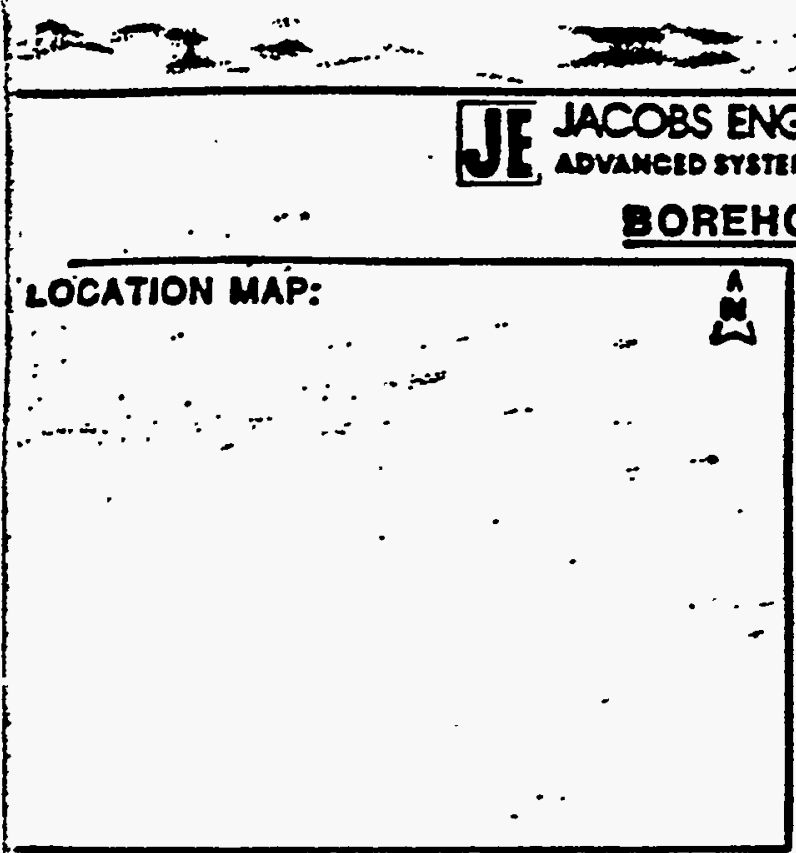

LOCATION DESCRIPTION N $45^{\circ} W$

GNEERING GROUP NC.

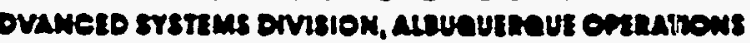

BOREHOLE LOG (SOIL)

Page Lor 8

A BITE ID: $A M B-16^{-}$LOCATION ID: 681 OFT.

SITE COORDINAFES (ft.):

N ROUND ELEVATION CIR

GROUND ELEVATION ( $f$ MSL):

DRILLING METHOD: ROTARY AIR

DRILLING CONTR.: CHEN NOMTTIMN/SALAZ AR

DATE STARTED: $\quad 1-23 .-88$

DATE COMPLETED:

FIELD REP.: DON MITZLER

\begin{tabular}{|c|c|c|}
\hline \multicolumn{3}{|c|}{ GROUNDWATER LEVELS } \\
\hline DATE & TIME & DEPTH (ft.) \\
\hline $1-23-88$ & 15.70 & 205 \\
\hline & & \\
\hline & & \\
\hline
\end{tabular}

SITE CONDITION

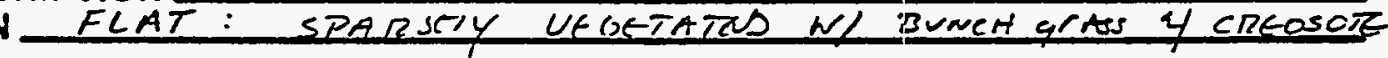

677

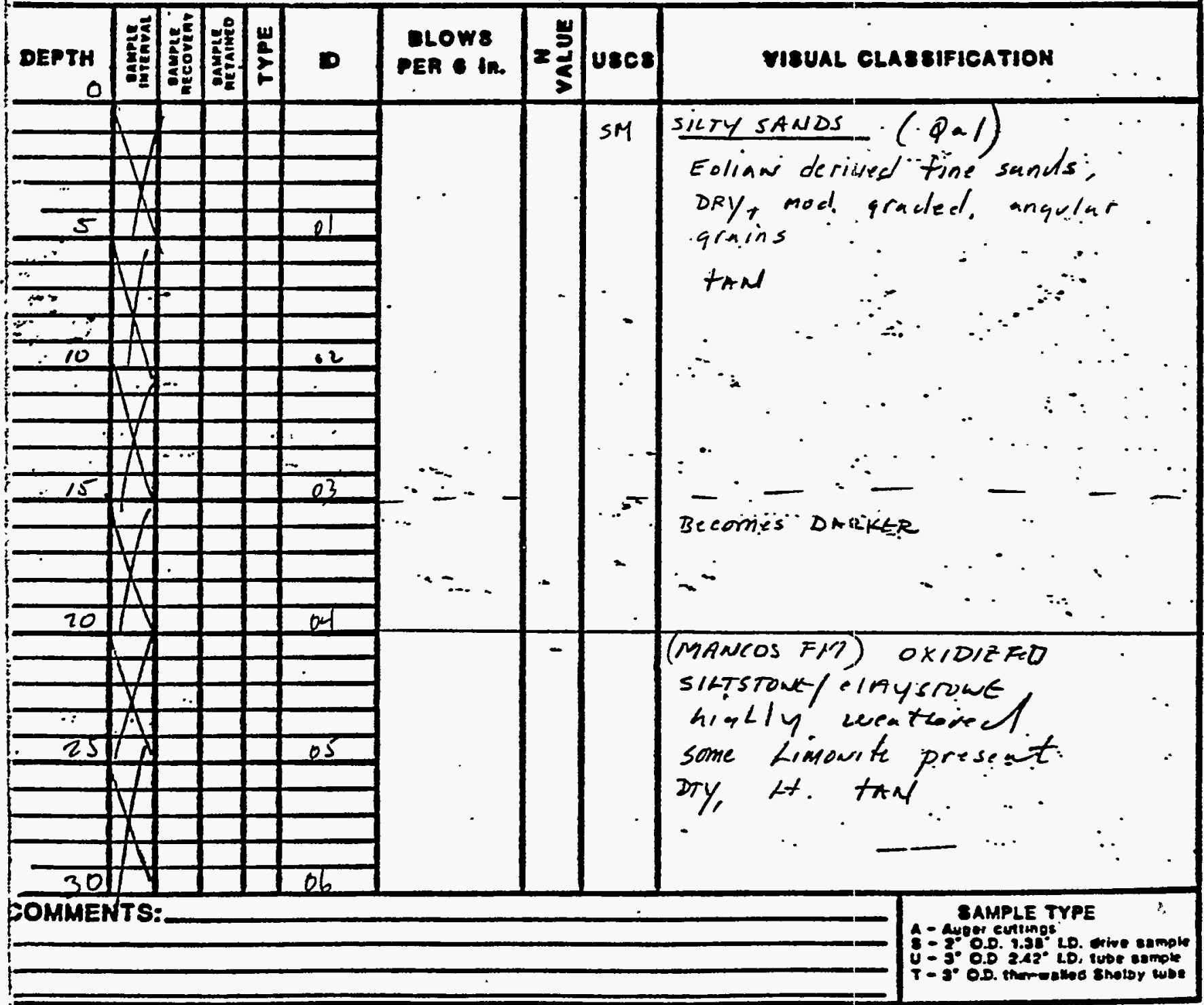


$x=-2$

ACOBS ENGINEERING GROUP NC.

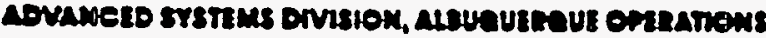

BOREHOLE LOG (SOIL)

Page zor 8

LOCATION MAP:

LOCATION DESCRIPTION

SITE CONDITION

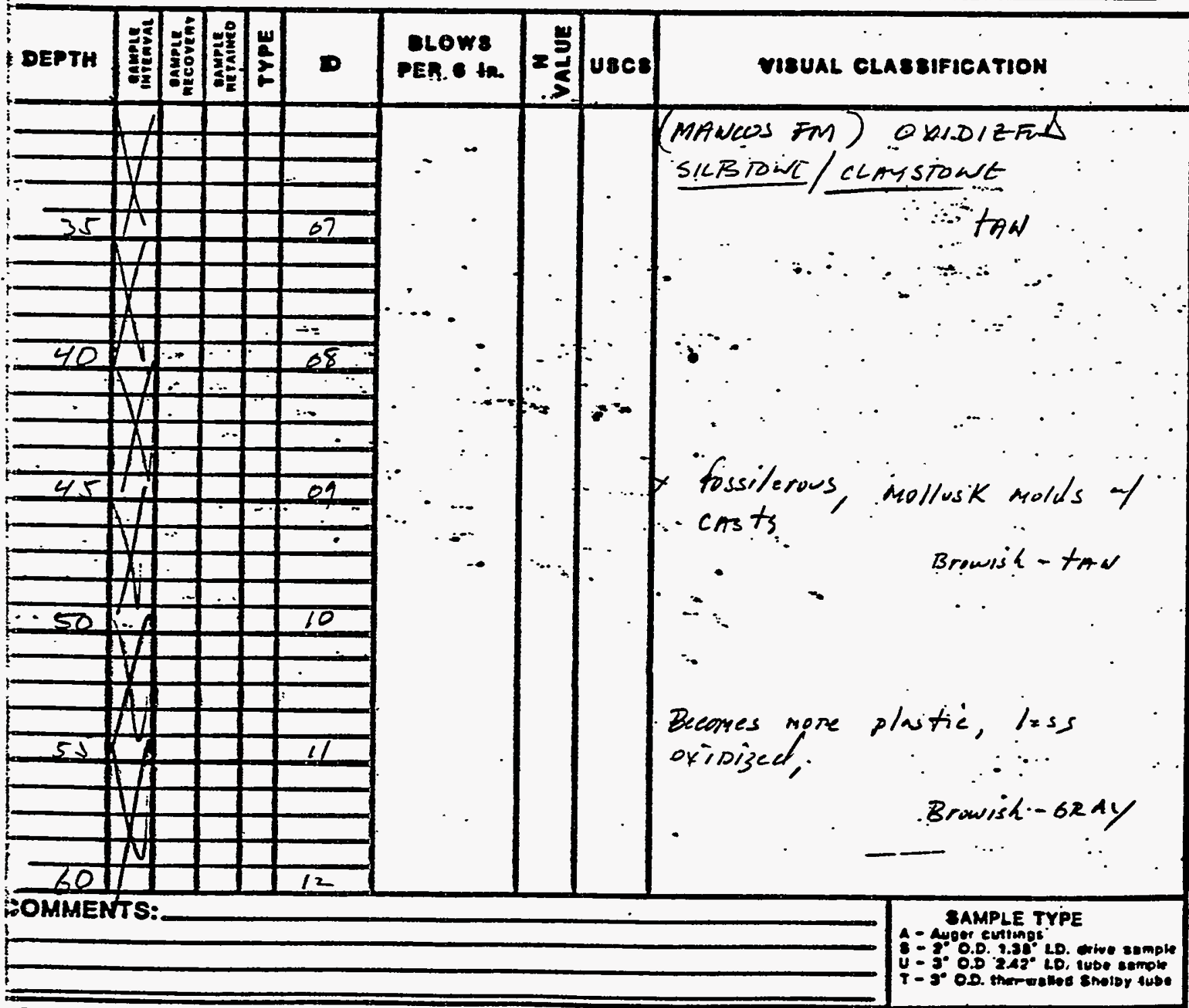

EQ-AL-ENO-2S (AISE)

$\therefore$

SITE 1D: MMB-76 LOCATTOON $10:-681$ ORI

SITE COORDINATES (ft.)

GROUND.ELEVATION (ft. MSL):

DRILLING METHOD:

DRILLING CONTR.:

DATE STARTED:

DATE COMPLETED:

FIELD REP.:

\begin{tabular}{|c|c|c|}
\hline \multicolumn{3}{|c|}{ GROUNDWATER LEVELS } \\
\hline DATE & TIME & DEPTH (f1.) \\
\hline & & \\
\hline & & \\
\hline & & \\
\hline
\end{tabular}




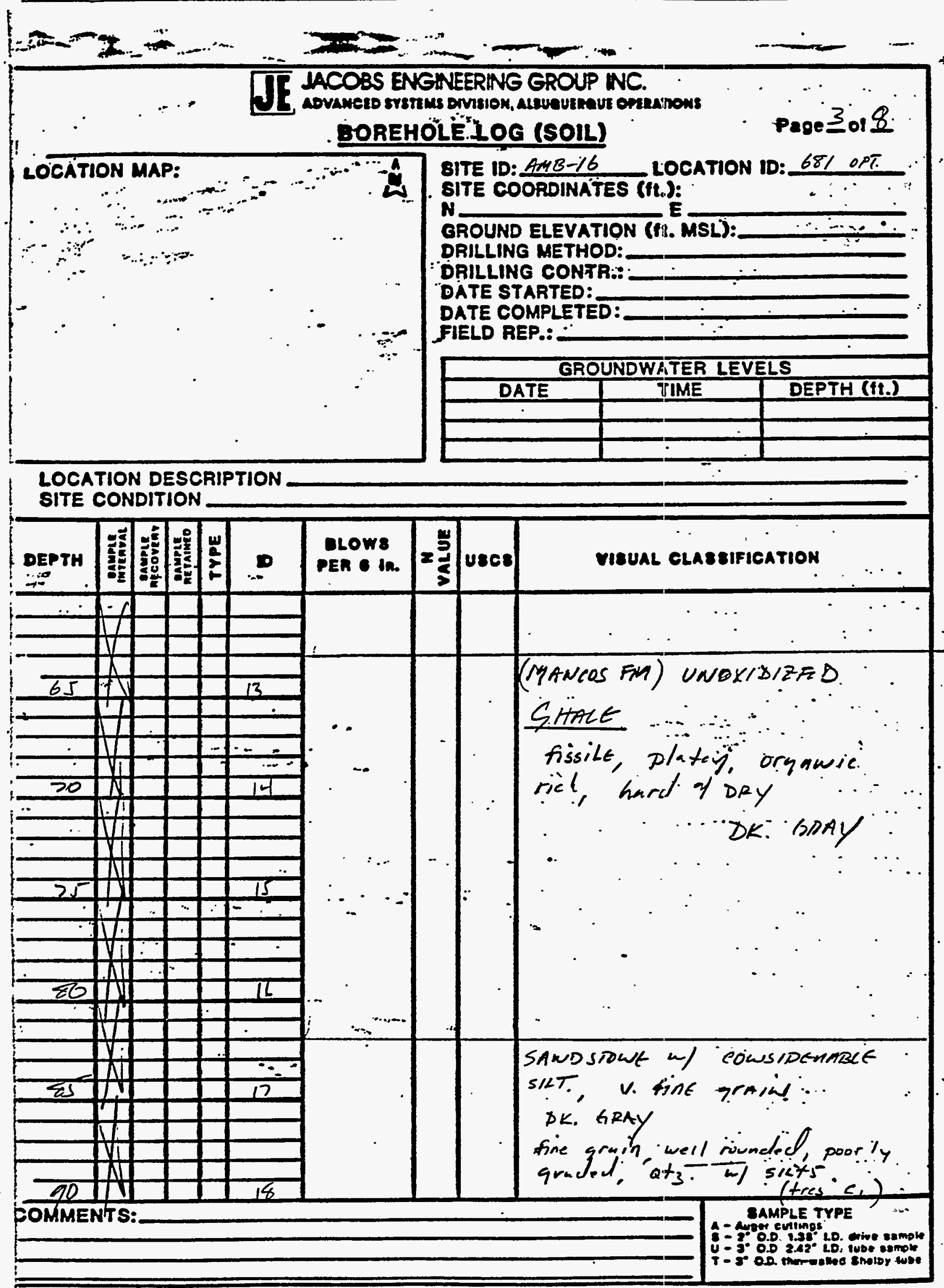


$3-2-\ldots$

IACOBS ENGNEERING GROUP NC.

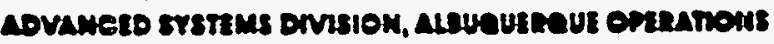

BOREHOLE LOG (SOIL)

Paoe $\not$ for 8

LOCATION MAP:

1...

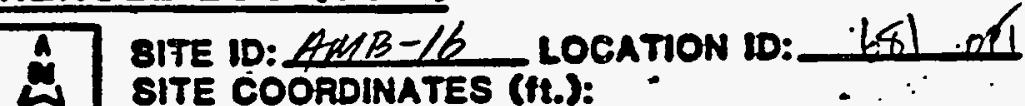

SITE COORDINATES (ft.):

GROUND ELEVATION (ft. MSL):

DRILLING METHOD:

DRILLING CONTR.:

DATE STARTED:

DATE COMPLETED:

FIELD REP.:

\begin{tabular}{|c|c|c|}
\hline \multicolumn{3}{|c|}{ GROUNDWATER LEVELS } \\
\hline DATE TIME & DEPTH (II.) \\
\hline & & \\
\hline & & \\
\hline & & \\
\hline
\end{tabular}

LOCATION DESCRIPTION

SITE CONDITION

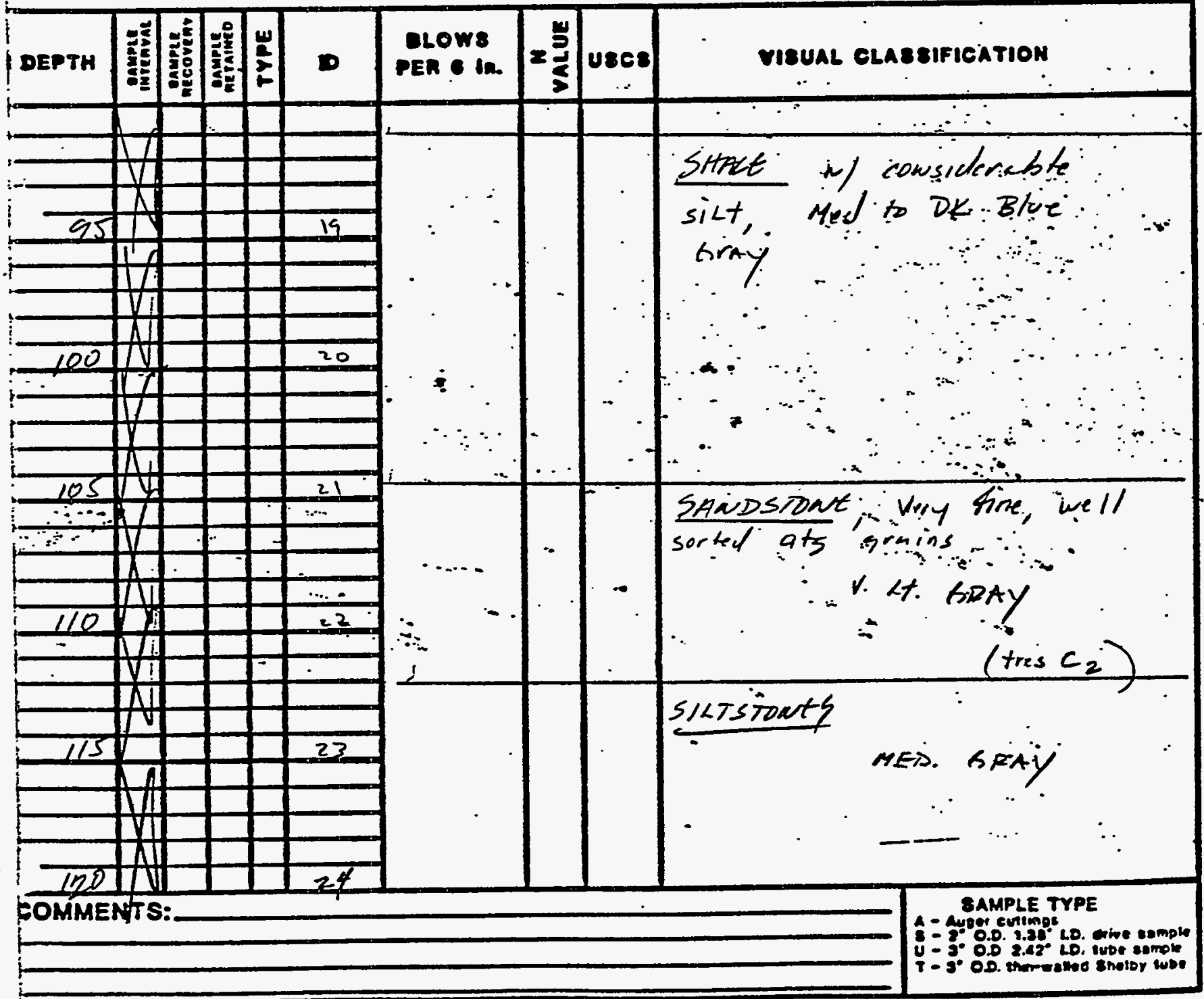




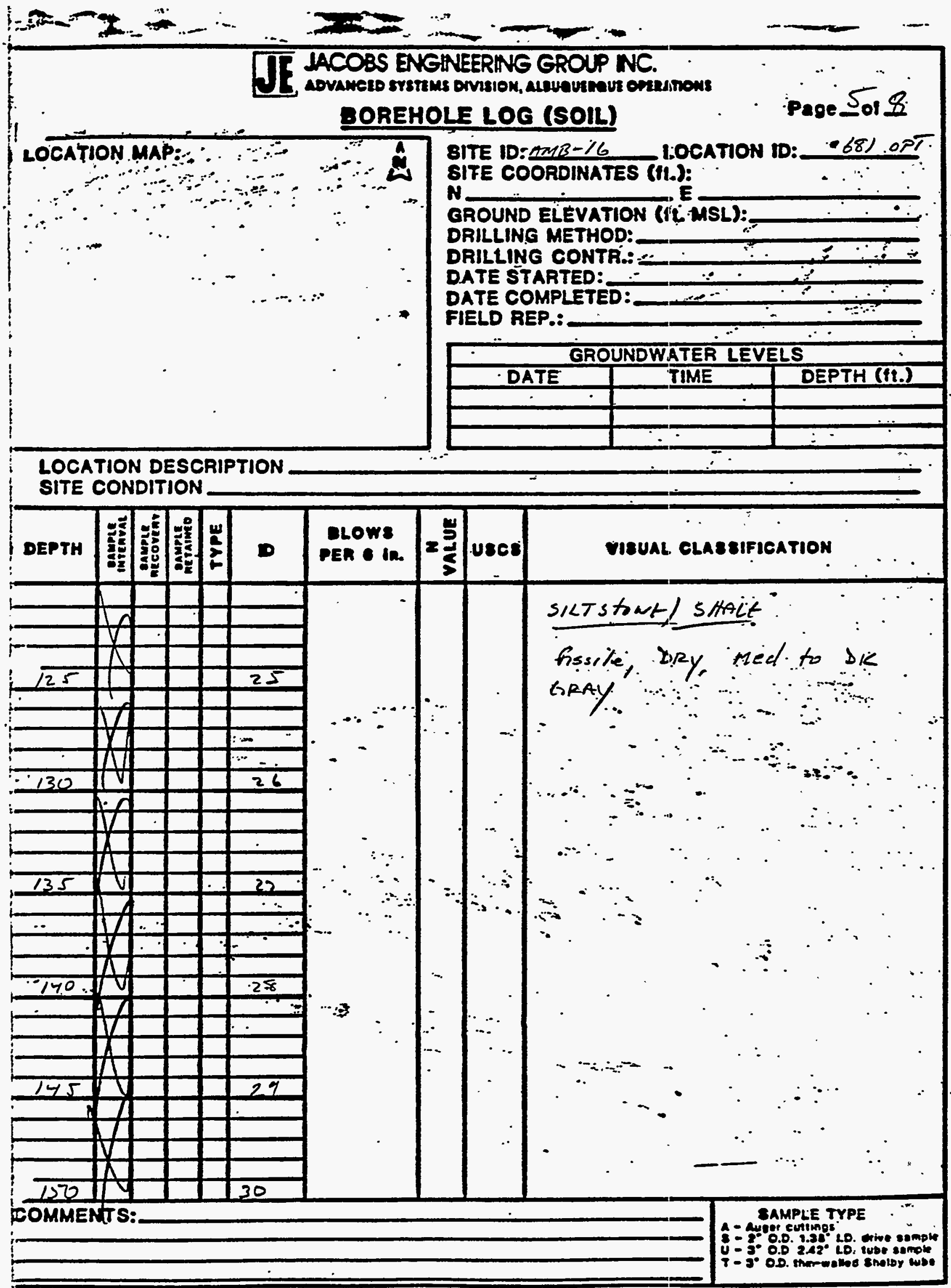

EO-AL-EMO-28 (4/8B) 


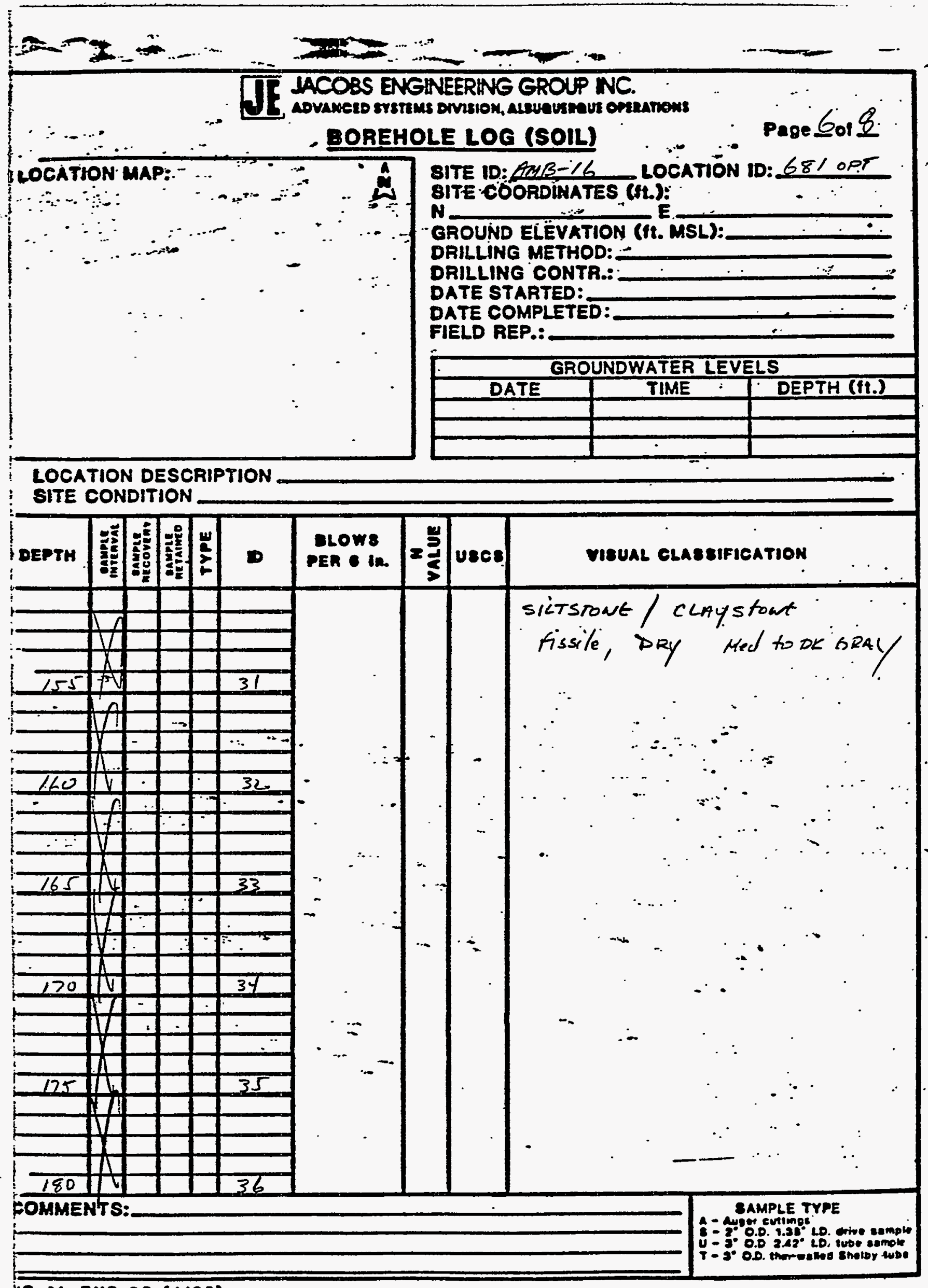




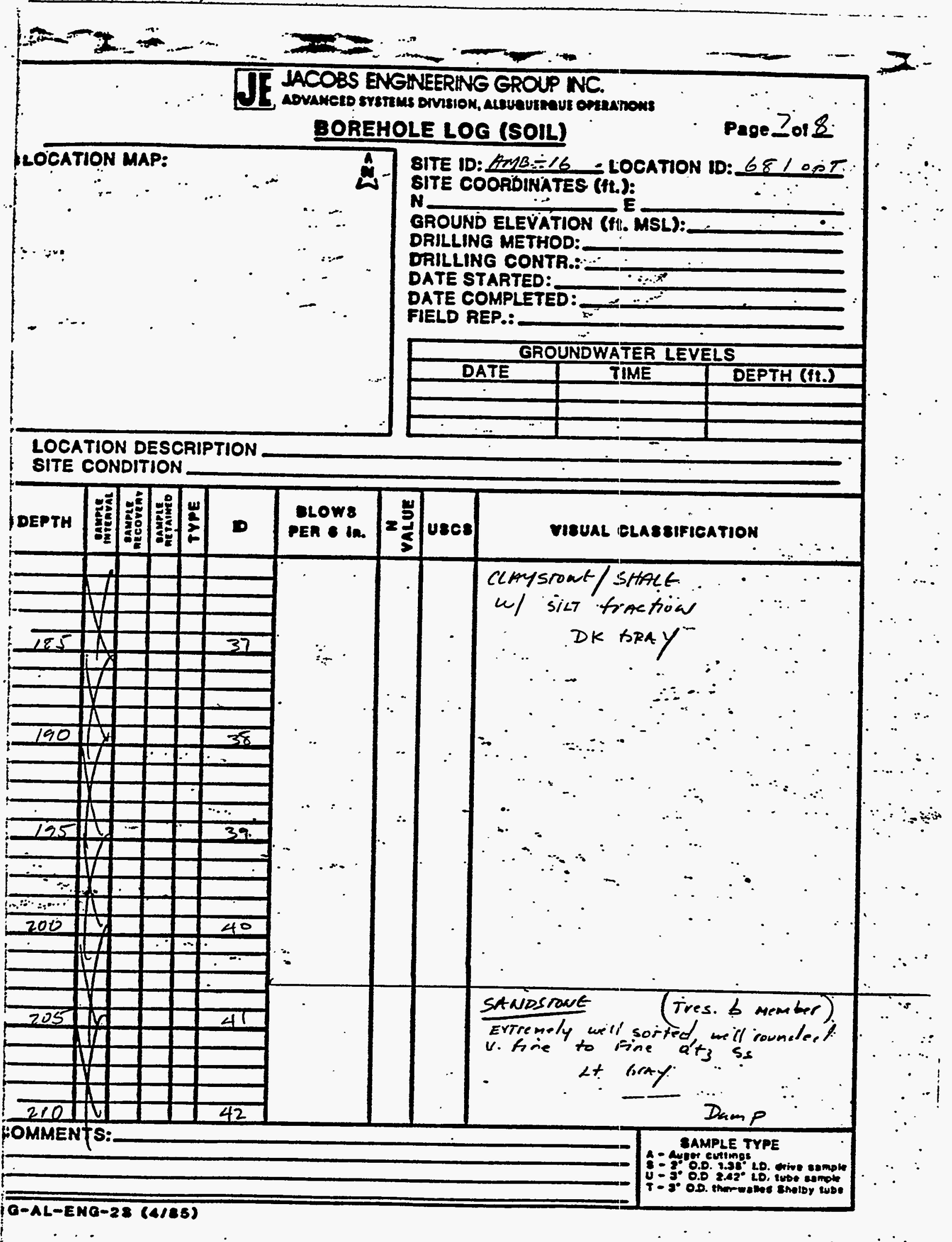




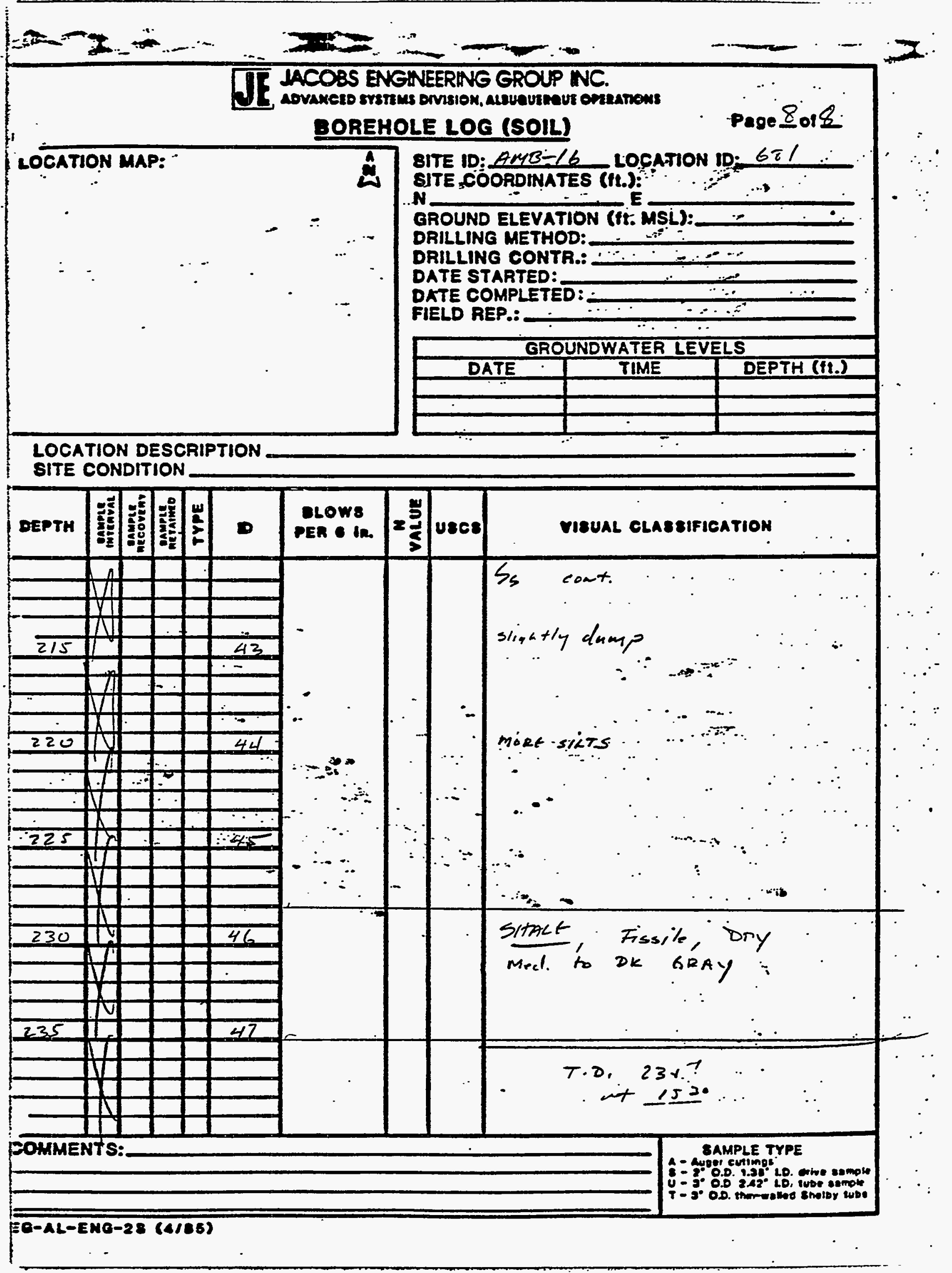




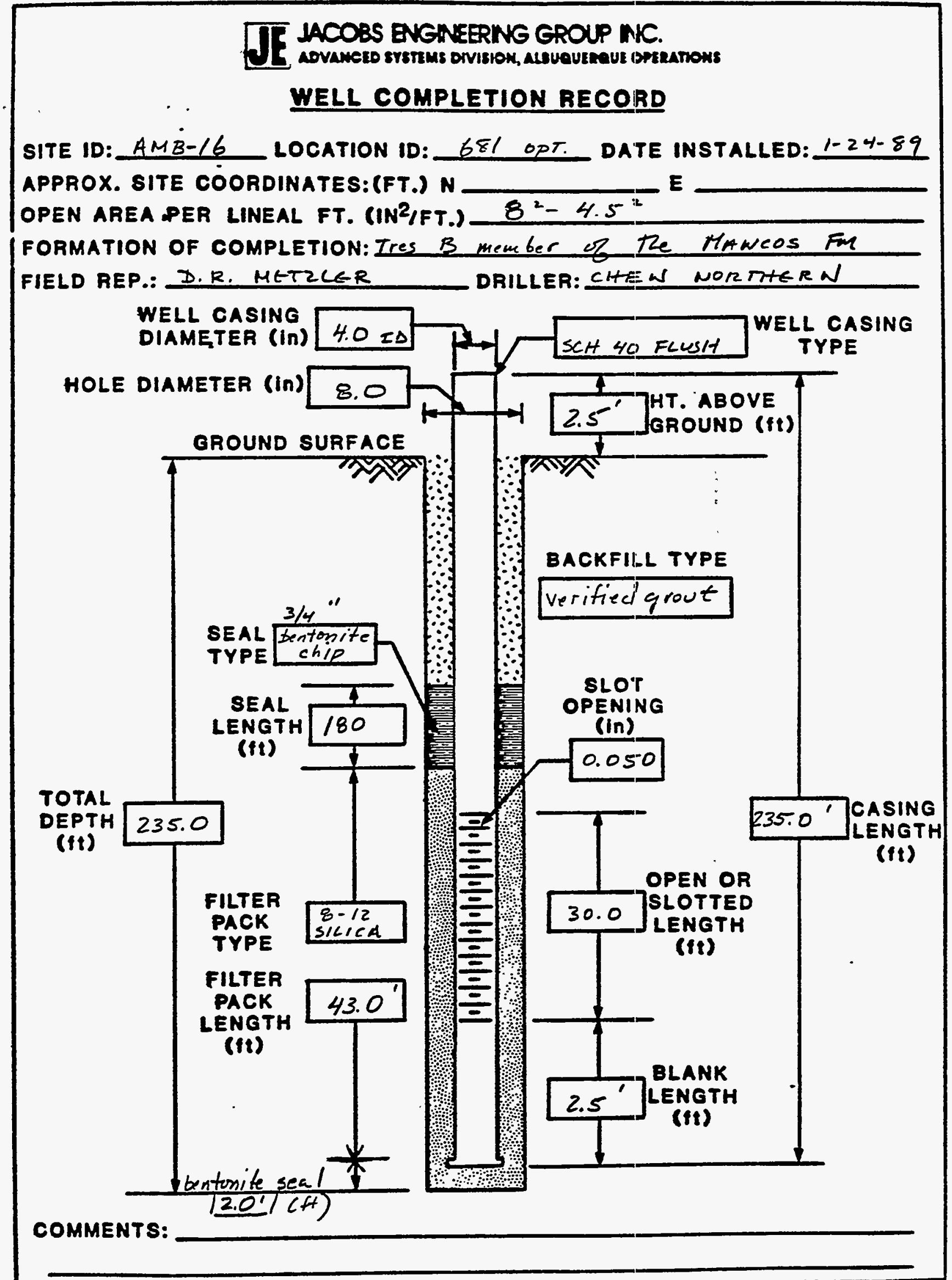




\section{TE BOREHOLE/WELL CONSTRUCTION LOG}

OITE ID: AMB-16 LOCATION 10: 681 APPROXX. giTE COORDIHATES (FT.): $N$ GROUND ELEVATION (FT̈. MSL): $2-7000$ GOREHOLE 8UMMARY

DRILLER:CHENI INOR THERN/SALAZAR RIG TYPE:

\begin{tabular}{|c|c|c|c|}
\hline BIT TYPE & $\begin{array}{l}\text { HOLE } \\
\text { OIA. } \\
\text { In.) }\end{array}$ & DEND & $\begin{array}{l}\text { FLUID } \\
\text { TYPE }\end{array}$ \\
\hline Tír-cont & $9^{1 / 8}$ & 40.0 & $A I R$ \\
\hline$\because$ & $71 / 8$ & 235.0 & $A I R$ \\
\hline & & & \\
\hline & & & \\
\hline
\end{tabular}

\begin{tabular}{|l|l|}
\hline \multicolumn{2}{|c|}{ CASIN } \\
\hline CASING & DE \\
\hline TYPE: &
\end{tabular}

B \begin{tabular}{|l|l|l|} 
& $\begin{array}{c}\text { DIA. } \\
(\text { In.) }\end{array}$ & $\begin{array}{c}\text { ENPPTH } \\
\text { DEPTH }\end{array}$ \\
\hline SCH 40 FLUSH SUMP T CAP & 4.0 & 232.5 \\
\hline
\end{tabular} \begin{tabular}{l|ll|l|l|} 
S. " $"$ SCREEN & 4.0 & 230.0 \\
\hline
\end{tabular} B..." "BlanK CAS in 4.0200 .0

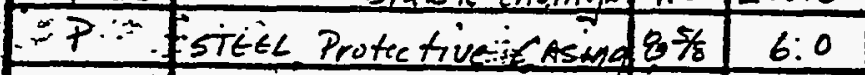
$\therefore \therefore \therefore$

- P-Protective 5-Sereen O-Blank O-Opon H-Nono Oopth from Top ol Caeling-

\begin{tabular}{|c|c|c|}
\hline & WELL GONSTRUCTION & \\
\hline $\begin{array}{l}\text { TYPE } \\
\text { CODE }\end{array}$ & DESCRIPTION & \\
\hline 5 & $3 / 4$ bentonite chip & $235.0^{\circ}$ \\
\hline$F$ & 8-12 SIIIICA SANS ${ }^{2} \cdot \cdots$ & $233.0^{\circ}$ \\
\hline$\leq$ & $3 / 4$ bentonite chip & 190.0 \\
\hline$\underline{B}$ & bentonite - cement grout & 10.0 \\
\hline & & \\
\hline & & \\
\hline & & \\
\hline & & \\
\hline e. & $8-8001$ & Peet \\
\hline-0 & in from cround burfece & \\
\hline
\end{tabular}

FIELD REP: D.R. METZLER $E$ COMÁLETION DATE: $1-24 \cdot 89$ - CONSTRUCTHON TIME LOG

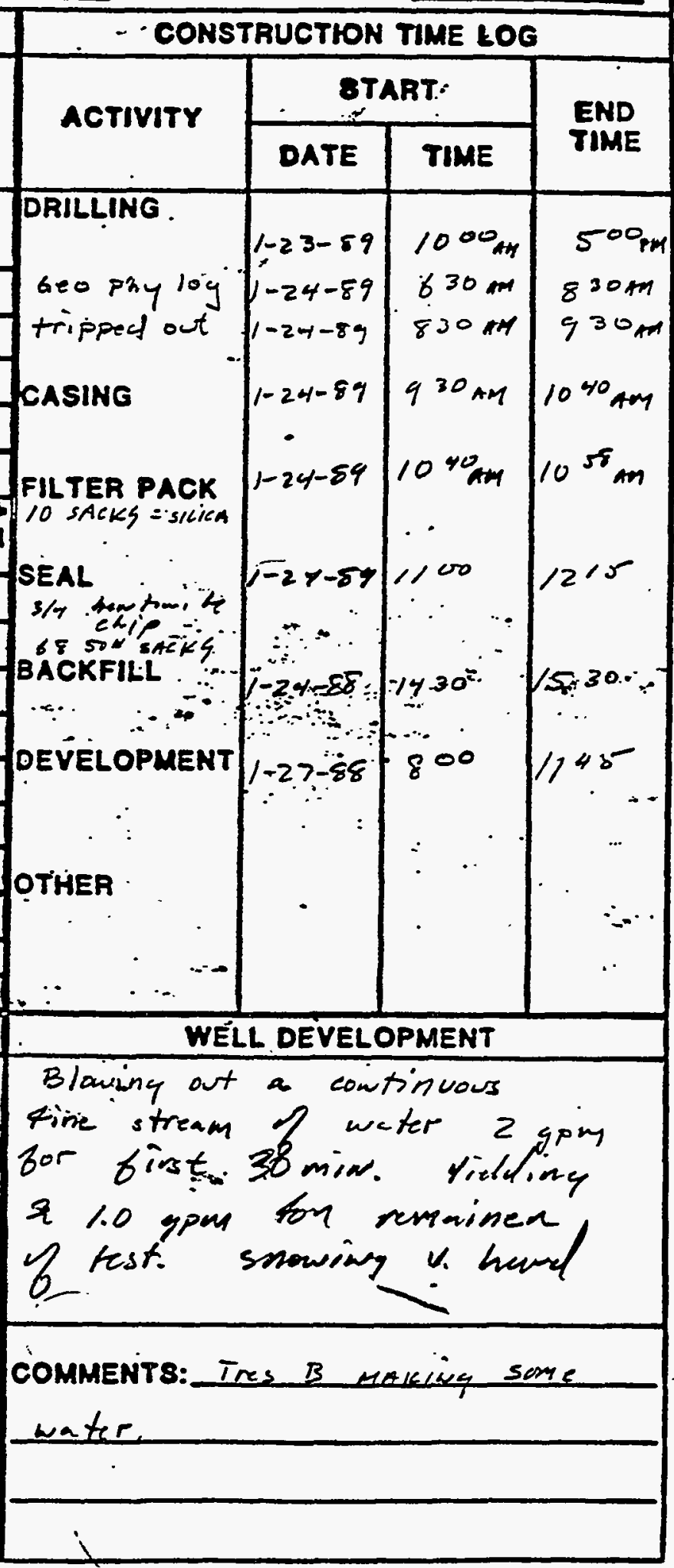


WELL 776 LOGS 


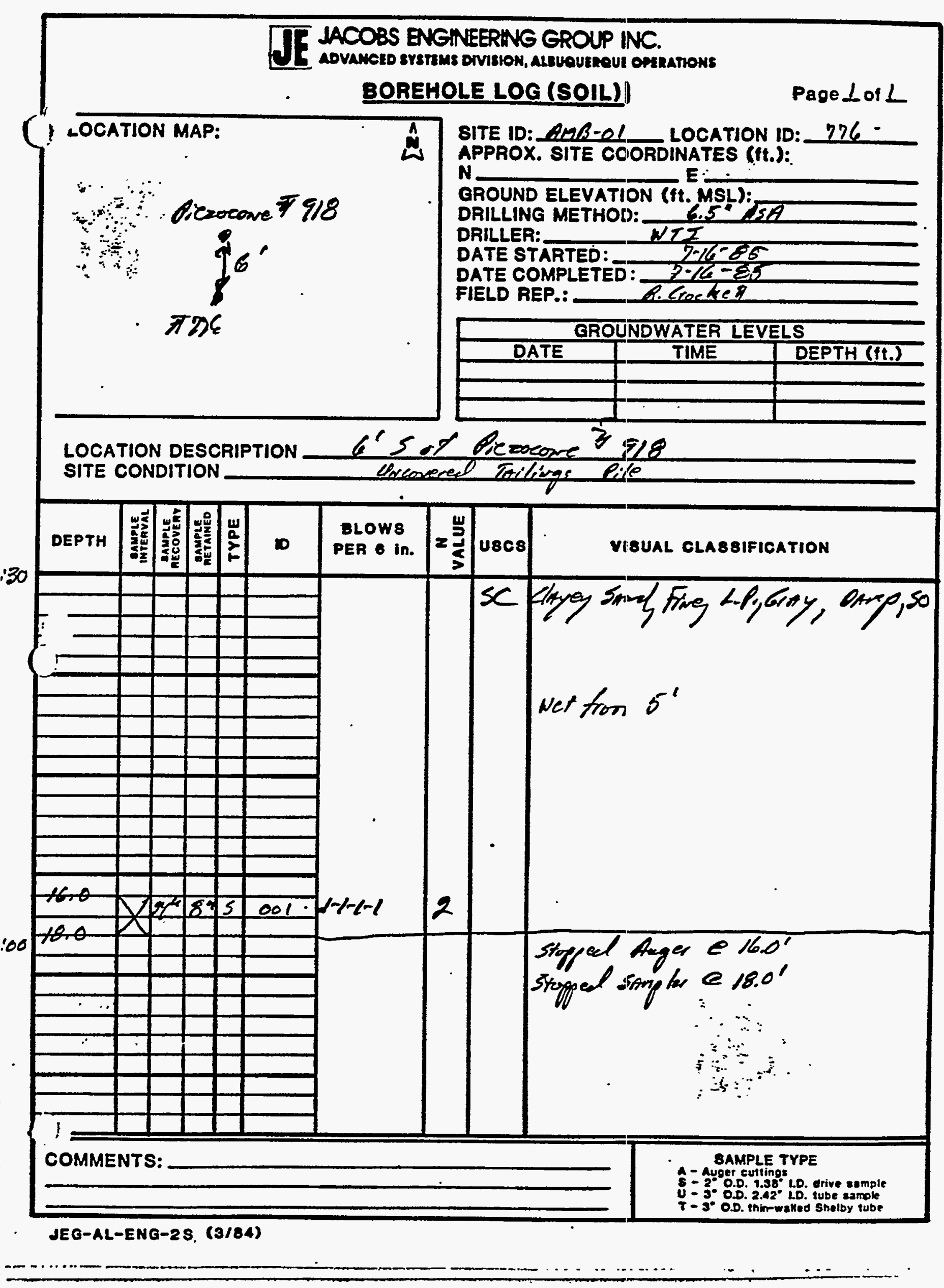




\section{TT MAOOBS BNGAEERTG CROUP NC.

\section{WELL COMPLETION RECORD}

SITE ID: AMBOL LOCATION ID: 276

DATE INSTALLED: \&/OS/OS APPROX. SITE COORDINATES:(FT.) N NK E NK OPEN AREA PER LINEAL FT. $\left(I N^{2} / F T.\right) \quad 150.2$ for $4.0^{N}$. FORMATION OF COMPLETION: FIELD -REP.: Rager talland DRILLER: A(bert)

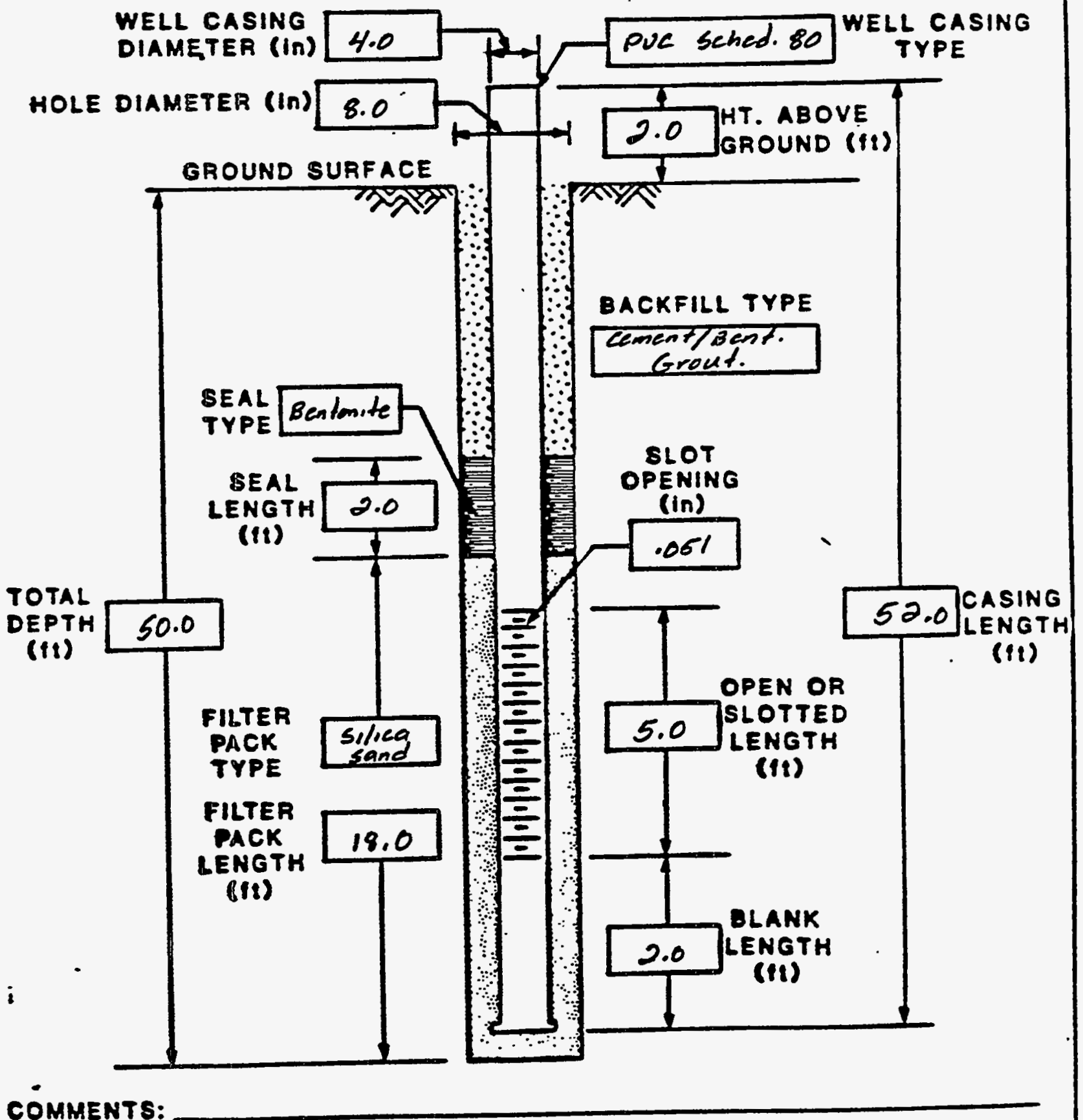

COMMENTS: 
WELL 777 LOGS 


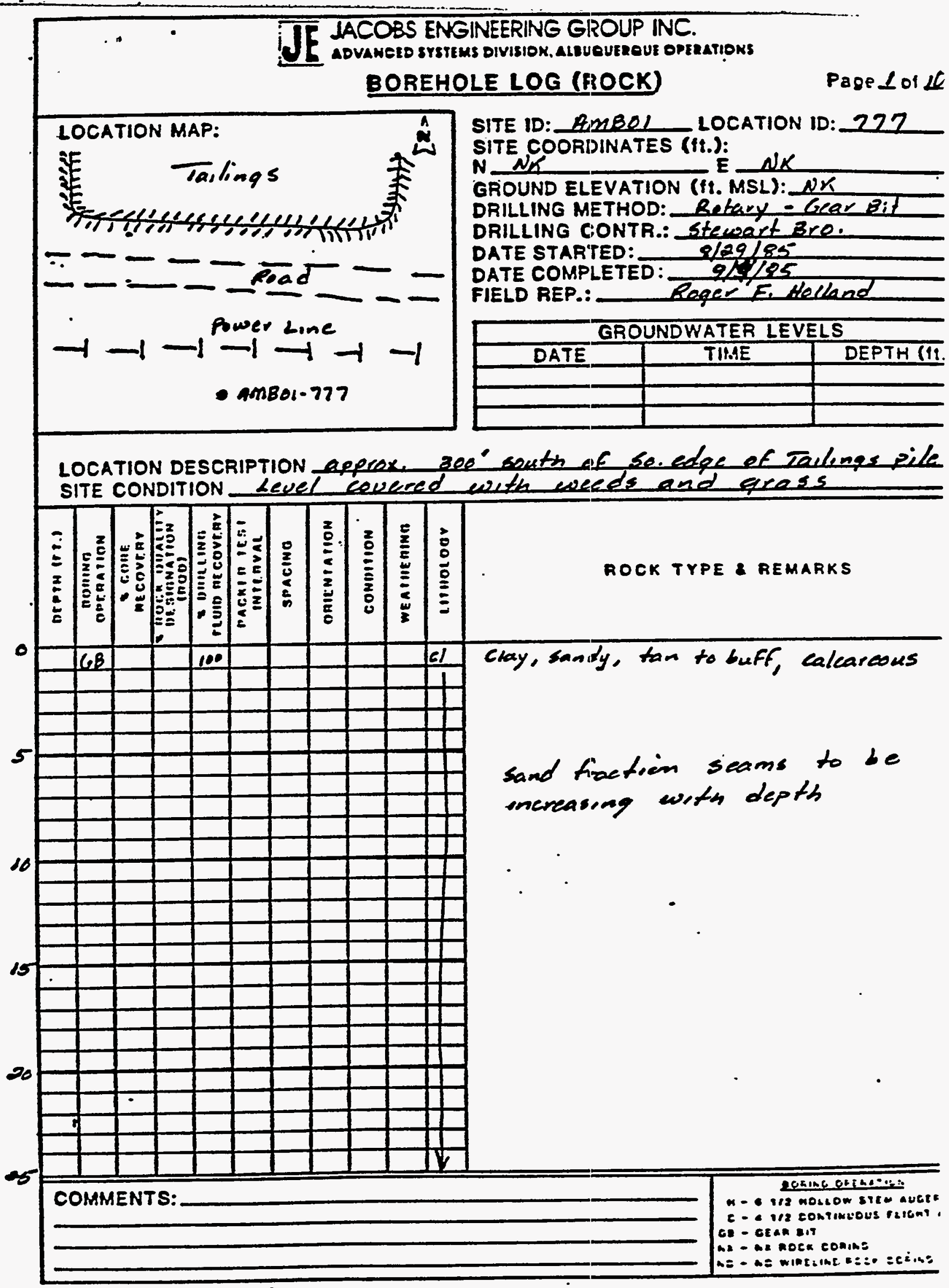

JEG-AL-ENG-2A (4/85) 


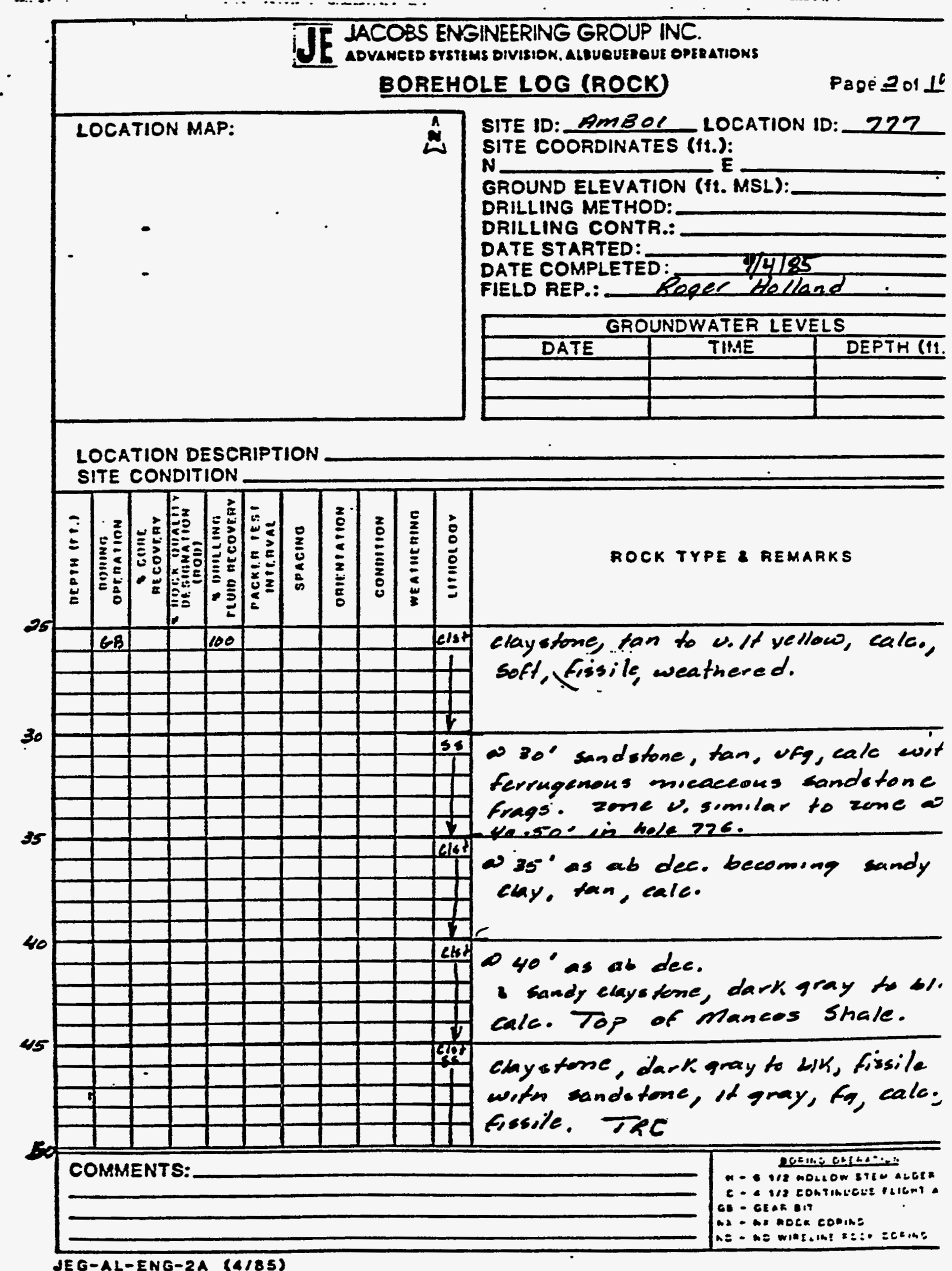




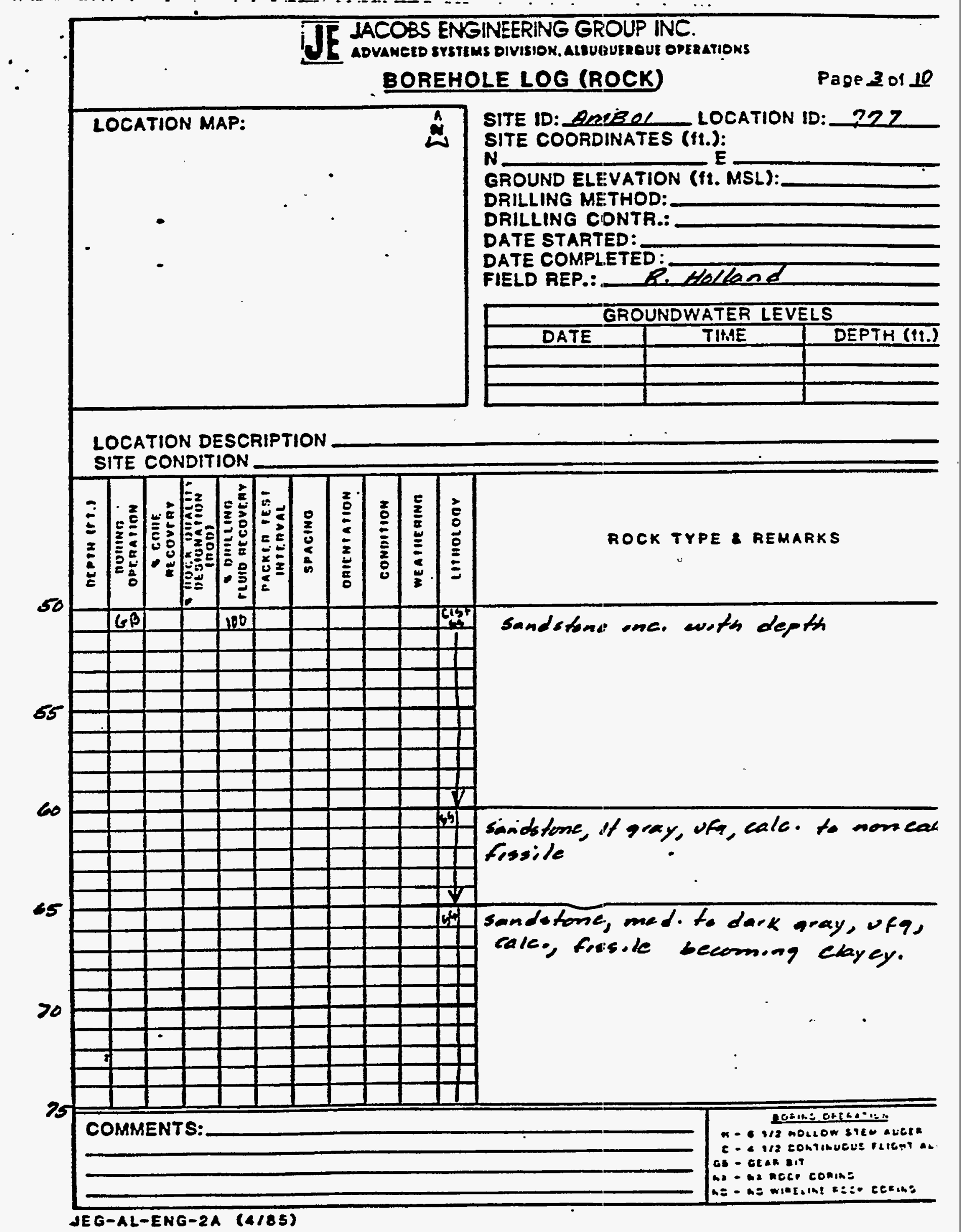




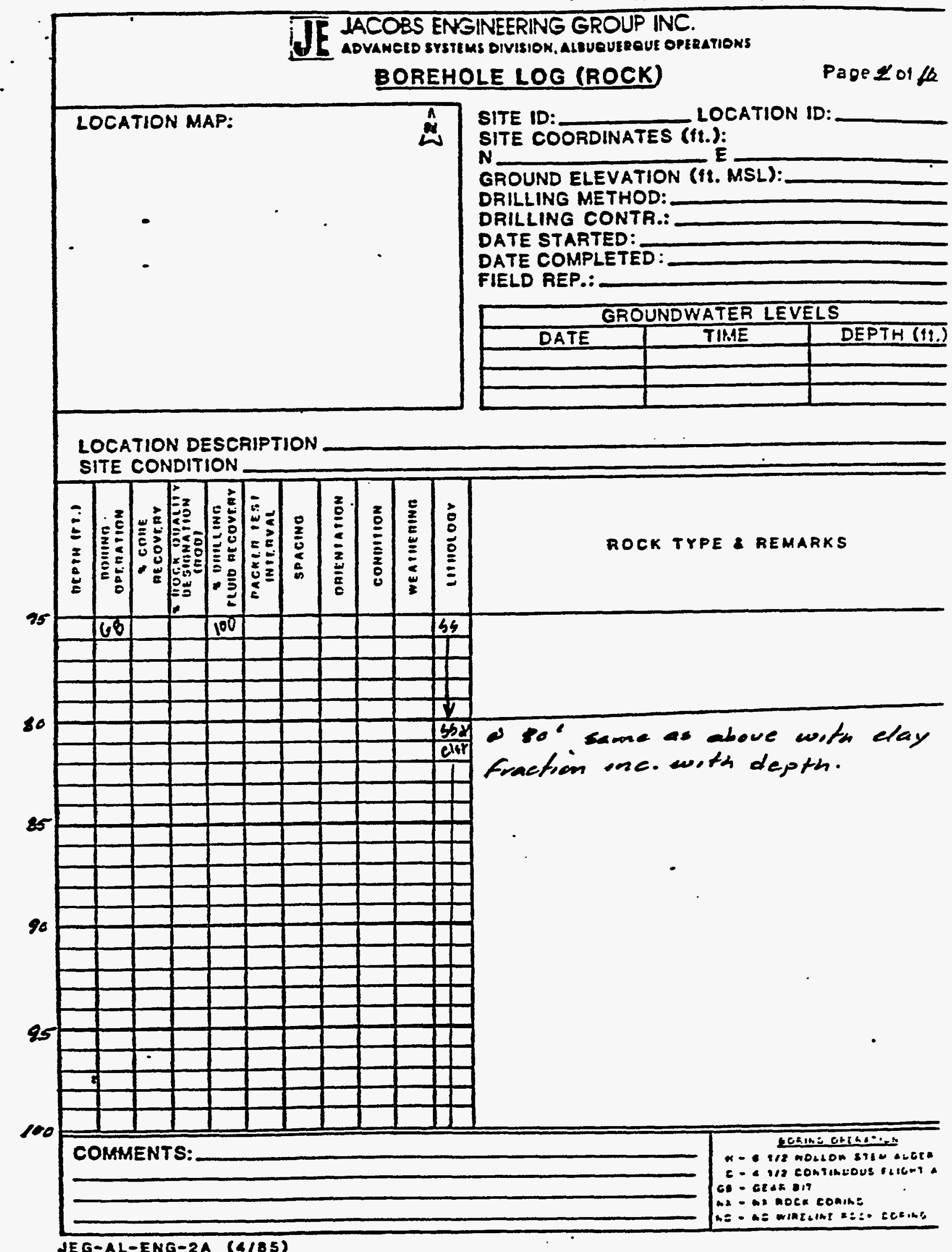




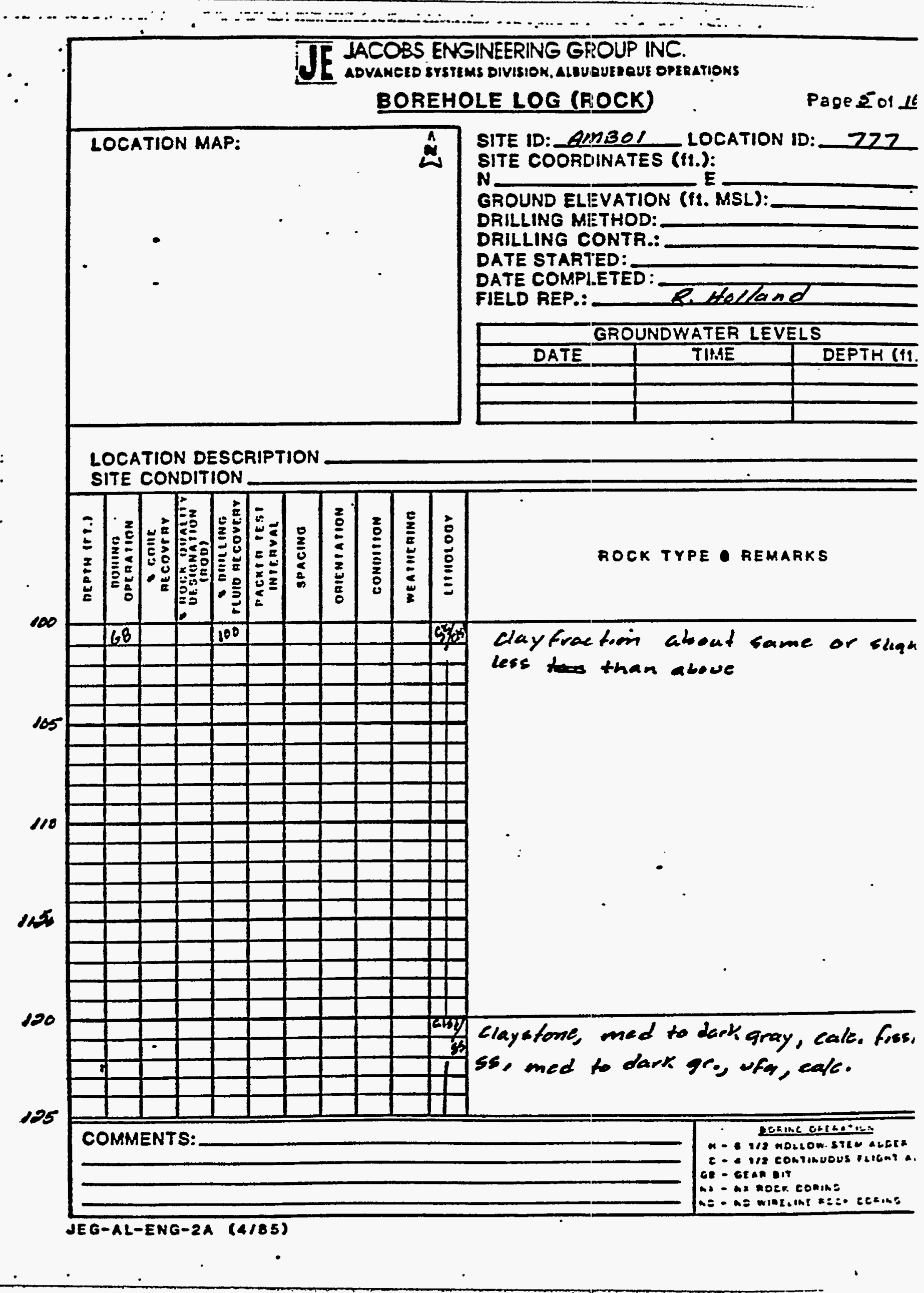




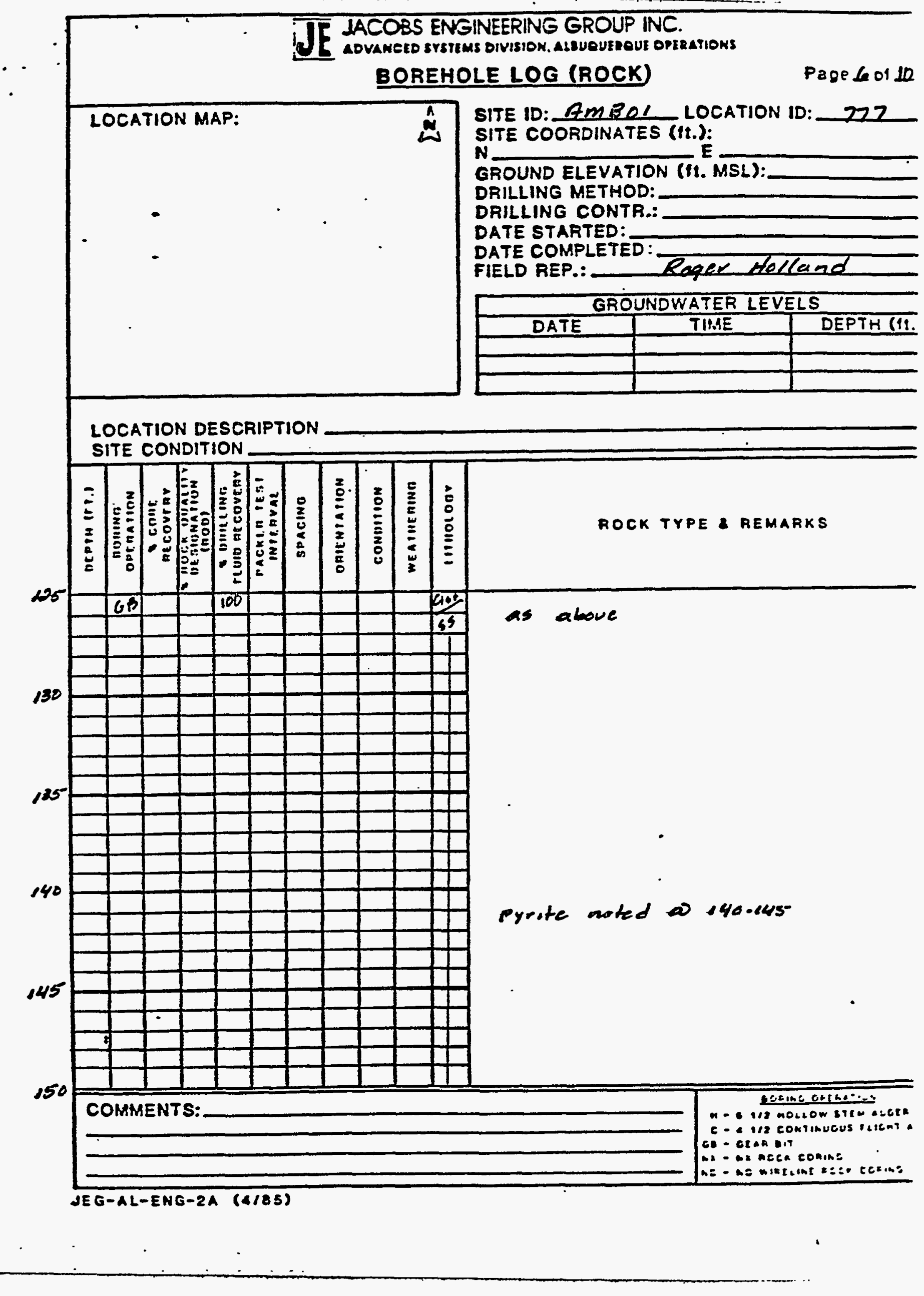




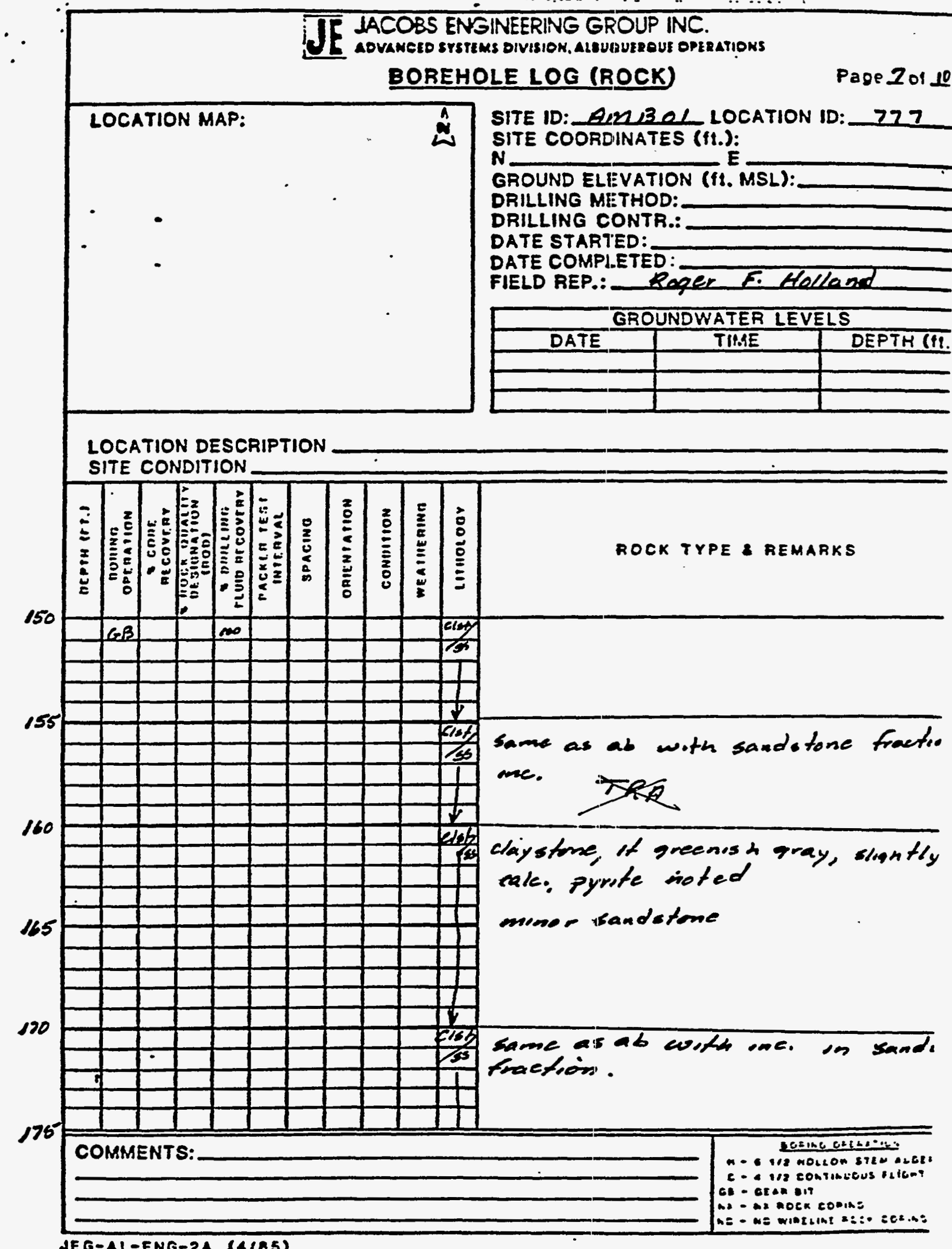

JEG-AL-ENG-2A (4/85) 
IF IACOBS ENSINEERING GROUP INC.

LOCATION MAP:

$\stackrel{a}{\omega}$

SITE ID: AMBOL LOCATION ID: 725

SITE COORDINATES (1t.):

\section{N}

GROUND ELEVATION (ft. MSL):

DRILLING METHOD:

DRILLING CONTR.:

DATE STARTED:

DATE COMPLETED:

FIELD REP.:

\begin{tabular}{|c|c|c}
\hline \multicolumn{3}{|c|}{ GROUNDWATER LEVELS } \\
\hline DATE & TIM.AE & DEPTH (11.: \\
\hline & & \\
\hline & & \\
\hline & & \\
\hline
\end{tabular}

LOCATION DESCRIPTION

SITE CONDITION

175

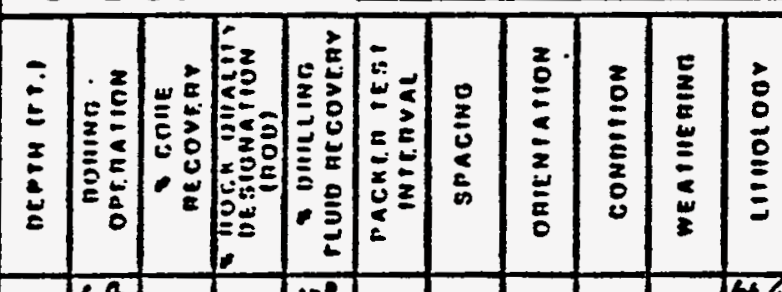

ROCK TYPE \& REMARKS

180

185 68

$10^{\circ}$

as ab

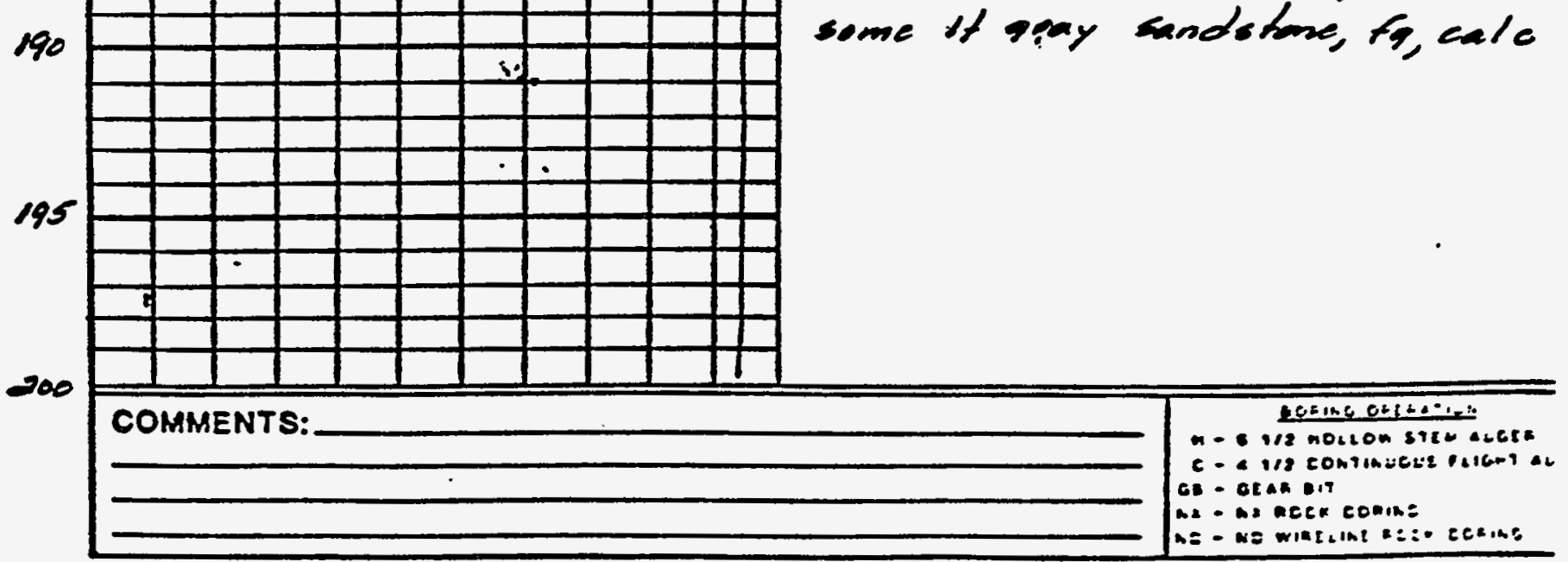

qreenish qray elaystone as ab dec. sandy claystaice. med pray to blx some of spay sindotone, fo, cale 


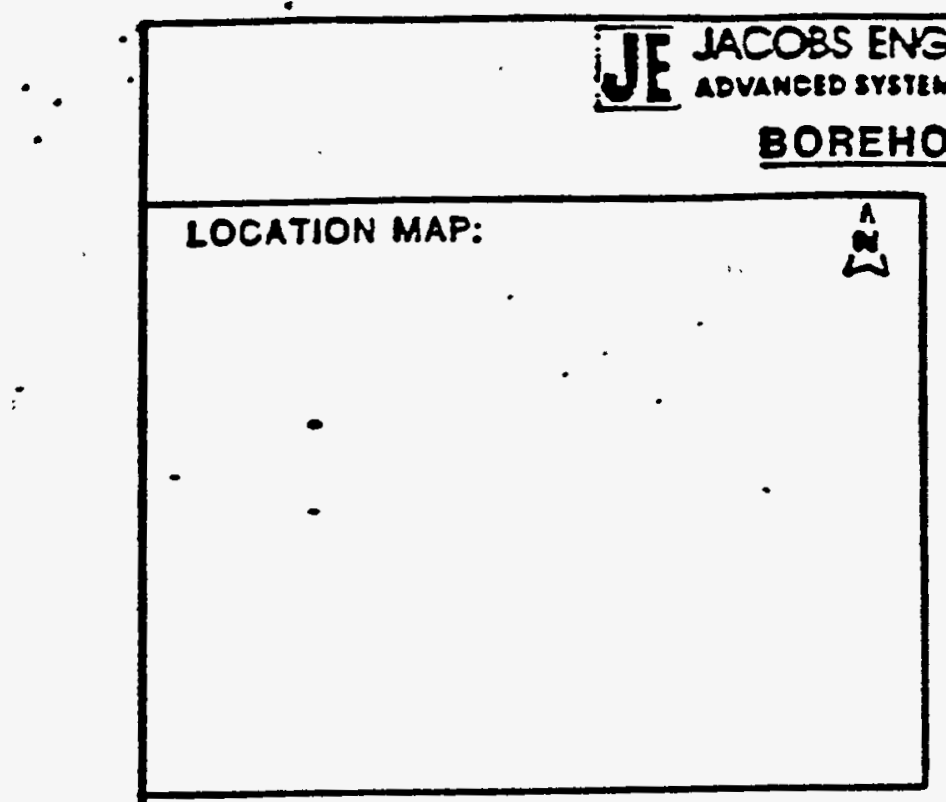

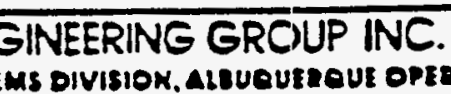

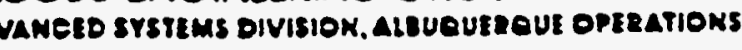

BOREHOLE LOG (ROCK)

Page 9 or 16

SITE ID: AMIZ OL LOCATION ID: 272

SITE COORDINATES (it.):

GROUND ELEVATION (II. MSL):

DRILLING METHOD:

DRILLING CONTR.:

DATE STARTED:

DATE COMPLETED:

FIELD REP.:

\section{i.}

\begin{tabular}{|c|c|c|}
\hline \multicolumn{3}{|c}{ GROUNDWATER LEVELS } \\
\hline DATE & TIA.AE & DEPTH (11.) \\
\hline & & \\
\hline & & \\
\hline & & \\
\hline
\end{tabular}

LOCATION DESCRIPTION

SITE CONDITION

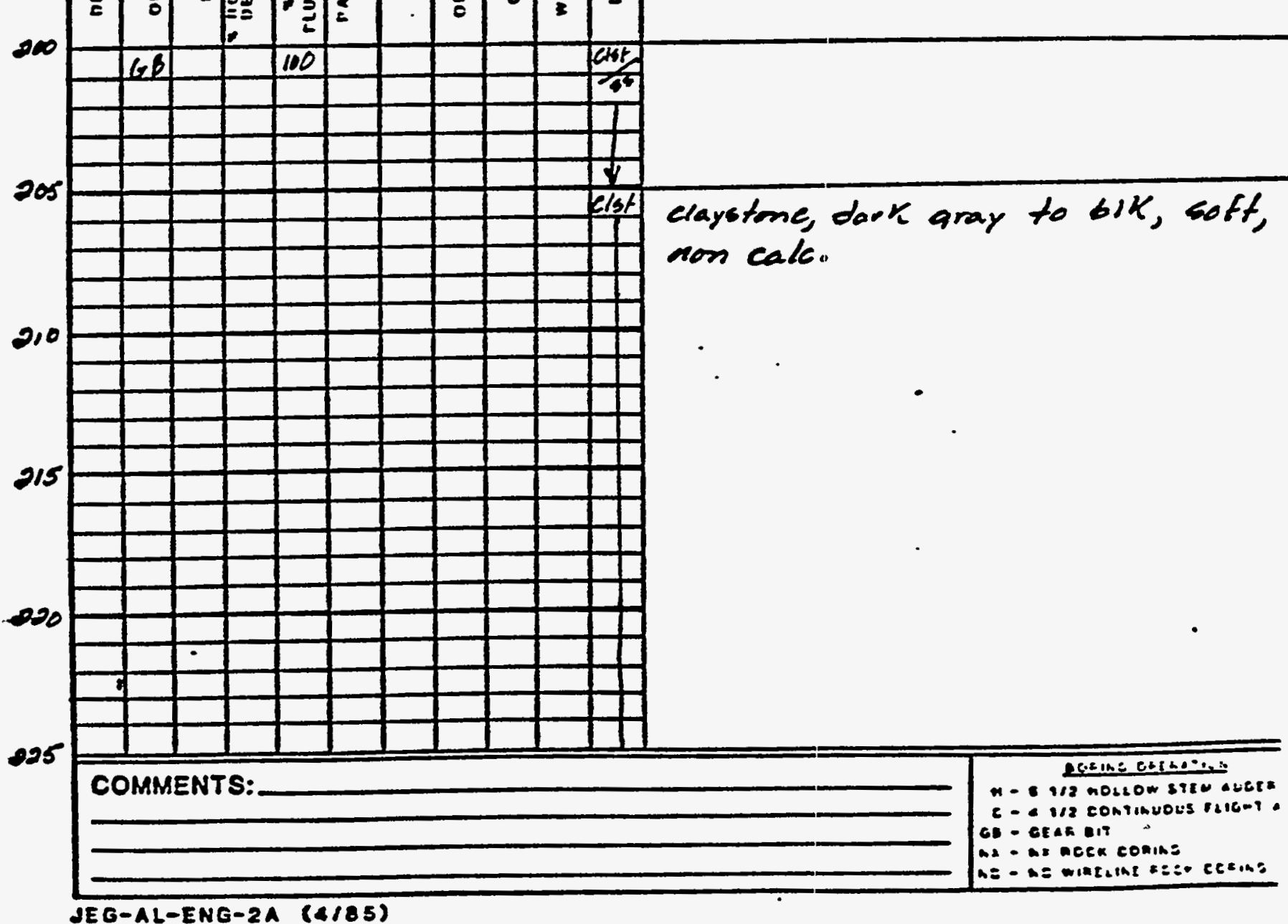




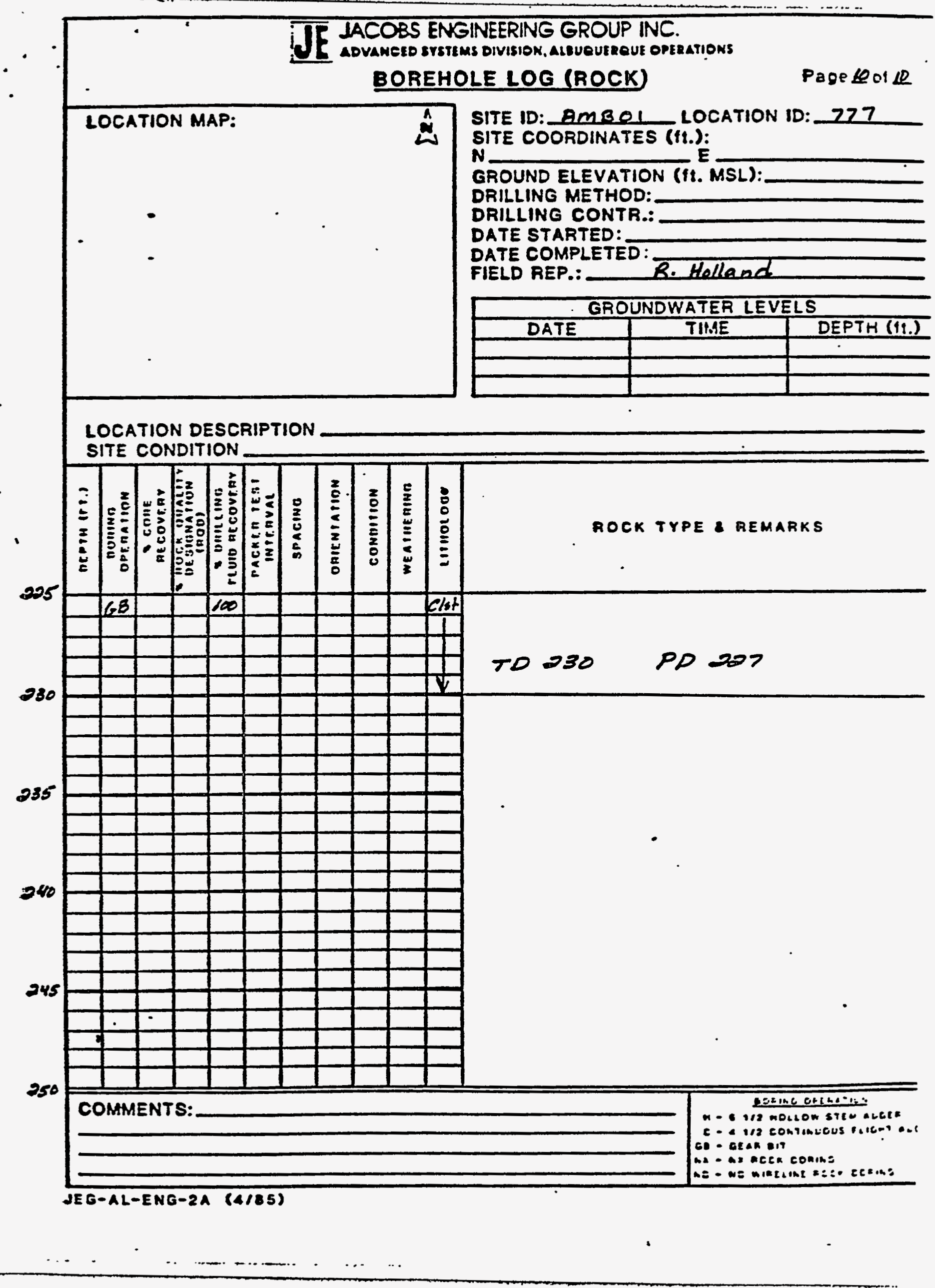




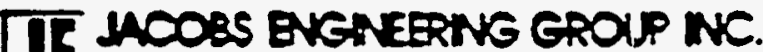

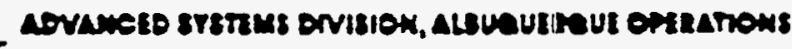

\section{WELL COMPLETION RECORD}

SITE ID: AMBOL LOCATION ID: 727 APPROX. SITE COORDINATES:(FT.) $N$ NK DATE INSTALLED: $9 / 4 / 85$

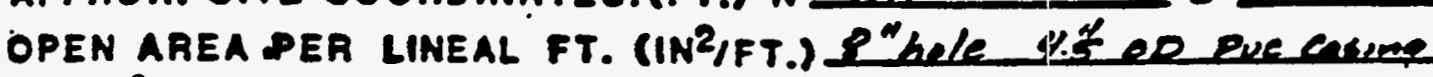
FORMATION OF COMPLETION: Tres termanes: $2 B$ FIELD-REP.: Reger folland DRILLER: stewart Bre.

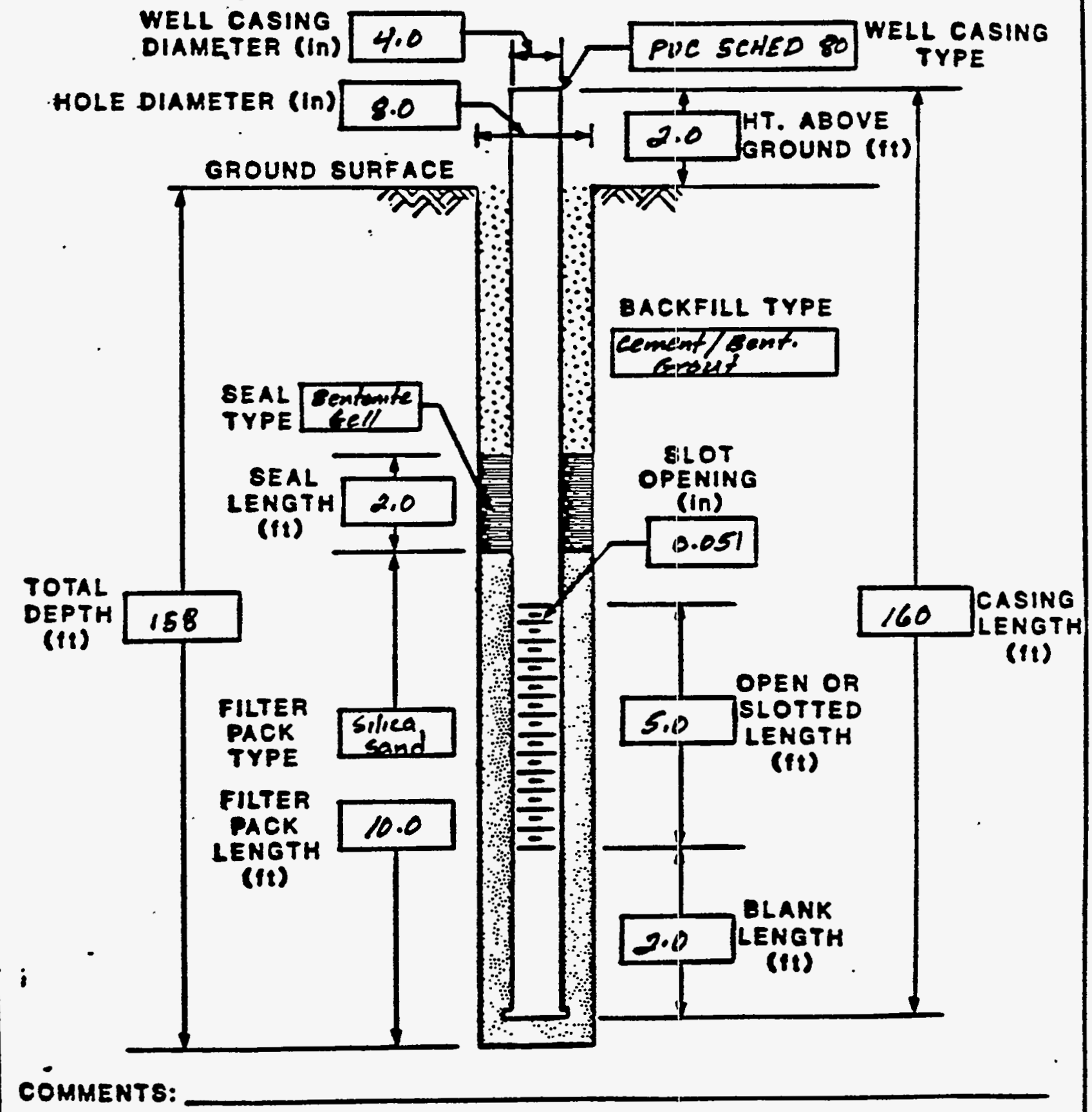




\section{TE MCOBS ENGNEERTG GROUP INC.

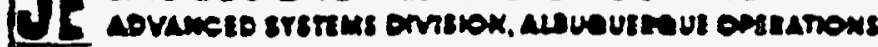 DOREHOLE/WELL CONSTRUCTION LOG}
SITE ID: AMBOI
LOCATION ID: 272 FIELD REP:
APPROX. BITE COORDINATES (FT.): N NK
CE AX

GROUND ELEVATIONN (FT. MSL): NK

OOREHOLE SUMMARY

DRILLER:_Stewart Bros.

RIG TYPE: Cordner Denver 15 W

\begin{tabular}{|c|c|c|c|}
\hline BIT TYPE & $\begin{array}{l}\text { HOLE } \\
\text { DiA. } \\
\text { (in.) }\end{array}$ & $\begin{array}{c}\text { END } \\
\text { De } \\
(\text { E }\end{array}$ & $\begin{array}{l}\text { FLUID } \\
\text { TYPE }\end{array}$ \\
\hline Amphos & 8.0 & $158^{\circ}$ & Textogite \\
\hline & & & \\
\hline & & & \\
\hline - & & & \\
\hline
\end{tabular}

\begin{tabular}{|c|c|c|c|}
\hline $\begin{array}{l}\text { CASINO } \\
\text { TYPE: }\end{array}$ & DESCRIPTION & $\begin{array}{l}\text { pia } \\
(\text { in. }\end{array}$ & $\begin{array}{l}\text { END' } \\
\text { DEPTH } \\
(f i .)\end{array}$ \\
\hline$B$ & pue sehed 80 & 4.0 & 153 \\
\hline 5 & Poe .051" slotied & 4.0 & 158 \\
\hline$B$ & Pue siched 80 & 4.0 & 160 \\
\hline & & & \\
\hline & & & \\
\hline & & & \\
\hline & & & \\
\hline & & & \\
\hline p-piotec & tive G-Beroon O-Blank & opon & A-Mono \\
\hline
\end{tabular}

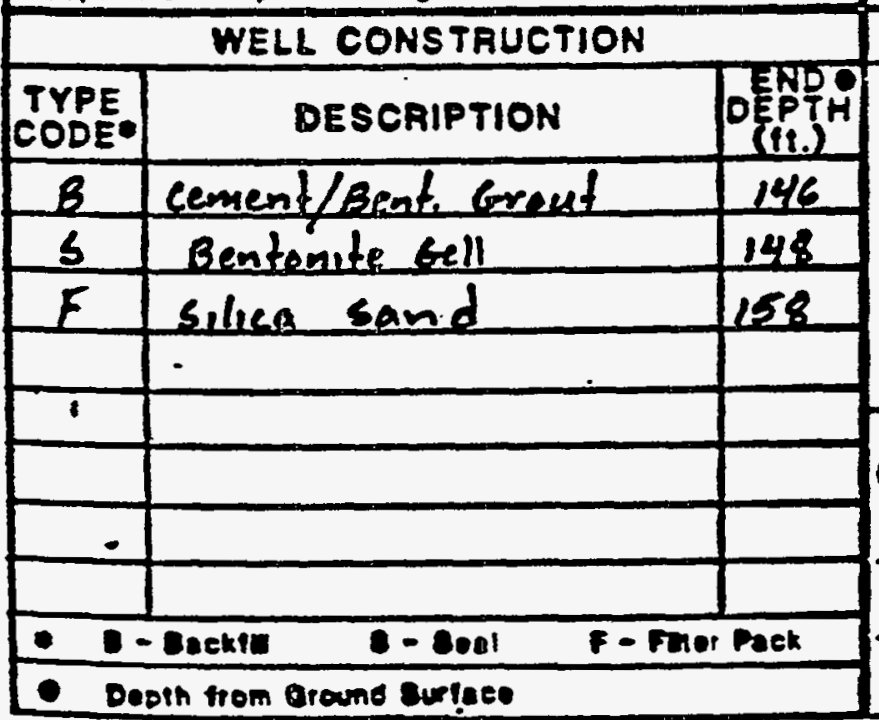

JEO-AL-ENO-1 (28104)
COMPLETION DATE: $9 / 5 / 85$

\begin{tabular}{|c|c|c|c|}
\hline \multicolumn{4}{|c|}{ CONSTRUCTION TIME LOG } \\
\hline \multirow{2}{*}{ ACTIVITY } & \multicolumn{2}{|c|}{ START } & \multirow{2}{*}{$\begin{array}{l}\text { END } \\
\text { TIME }\end{array}$} \\
\hline & DATE & TIME & \\
\hline DRILLINGF & $9 / 4 / 85$ & $12: 20 \mathrm{pm}$ & s:appm \\
\hline CASING & $9 / 4 / 85$ & s:25 pm & $4: 10 \mathrm{sm}$ \\
\hline FILTER PACK & $9 / 4 / 85$ & $4: 59 \mathrm{gm}$ & $85: 45 \%$ \\
\hline SEAL & $9 / 4 / 85$ & $6: 00 \mathrm{pm}$ & $6: 05 \mathrm{sm}$ \\
\hline BACKFILL & $17 / 3 / 85$ & PRIIAm & $7: 20 \mathrm{Am}$ \\
\hline DEVELOPMEN & $\begin{array}{l}9 / 6 / 85^{-} \\
9 / 7 / 85^{-}\end{array}$ & 3:12 $\mathrm{pm}$ & $\begin{array}{l}6: 30 \mathrm{~cm} \\
8: 46 \mathrm{Am}\end{array}$ \\
\hline OTHER & & & \\
\hline \multicolumn{4}{|c|}{ WELL DEVELOPMENT } \\
\hline $\begin{array}{l}\text { Hole wown } \\
\text { Pn until } \\
\text { g.9 } \\
\text { Hole slown } \\
\text { No water } \\
\text { Bail isen }\end{array}$ & $\begin{array}{l}\text { Flusned } \\
\text { clean. } \\
\text { dry. le } \\
\text { gettie }\end{array}$ & $\begin{array}{l}\text { repecate } \\
\text { water oua } \\
\text { f set, ble } \\
\text { claal re }\end{array}$ & $\begin{array}{l}\text { dly } \\
\text { dity: } \\
\text { ow - } \\
\text { moved }\end{array}$ \\
\hline $\begin{array}{l}\text { COMMENTS: - } \\
\text { te } 250 \mathrm{en}\end{array}$ & Lesere & cempuedx & drelled \\
\hline dere cement & bened th & $\frac{\text { en }}{\text { onde }}$ & $\frac{\text { eame }}{\text { to } 150}$ \\
\hline
\end{tabular}


WELL 778 LOGS 


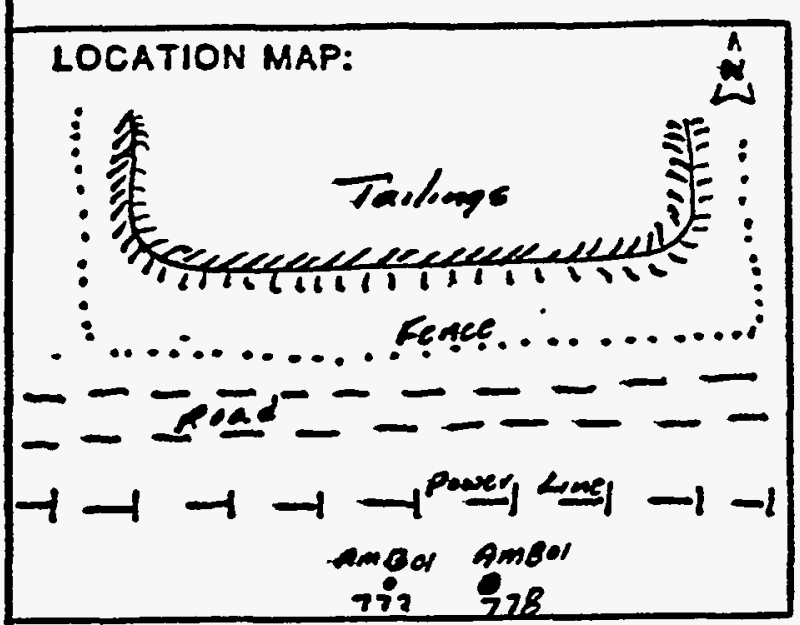

SITE ID:AMBOL LOCATION ID: 778 SITE COORDINATES (ft.): N NK E NK GROUND ELEVATION (11. MSL): NK DRILLING METHOD: Retary - Geat Bit DRILLING CONTR.: Stewait Bre,

DATE STARTED: $8 / 20 / 85$

DATE COMPLETED:__ \&/30/85

FIELD REP.: Reger thelland

\begin{tabular}{|c|c|c|}
\hline \multicolumn{3}{|c|}{ GROUNDWATER LEVELS } \\
\hline DATE & TIMA & DEPTH (11.) \\
\hline & & \\
\hline & & \\
\hline & & \\
\hline
\end{tabular}

LOCATION DESCRIPTION Apprex $300^{\circ}$ senet he serside ef Tailings pile SITE CONDITION Lewel leamy rael weth weed and peass levex

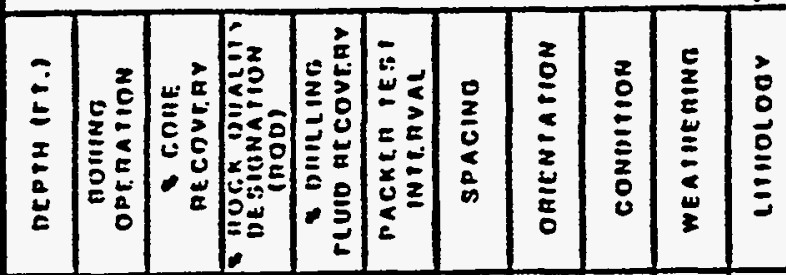

ROCK TYPE REMARKS

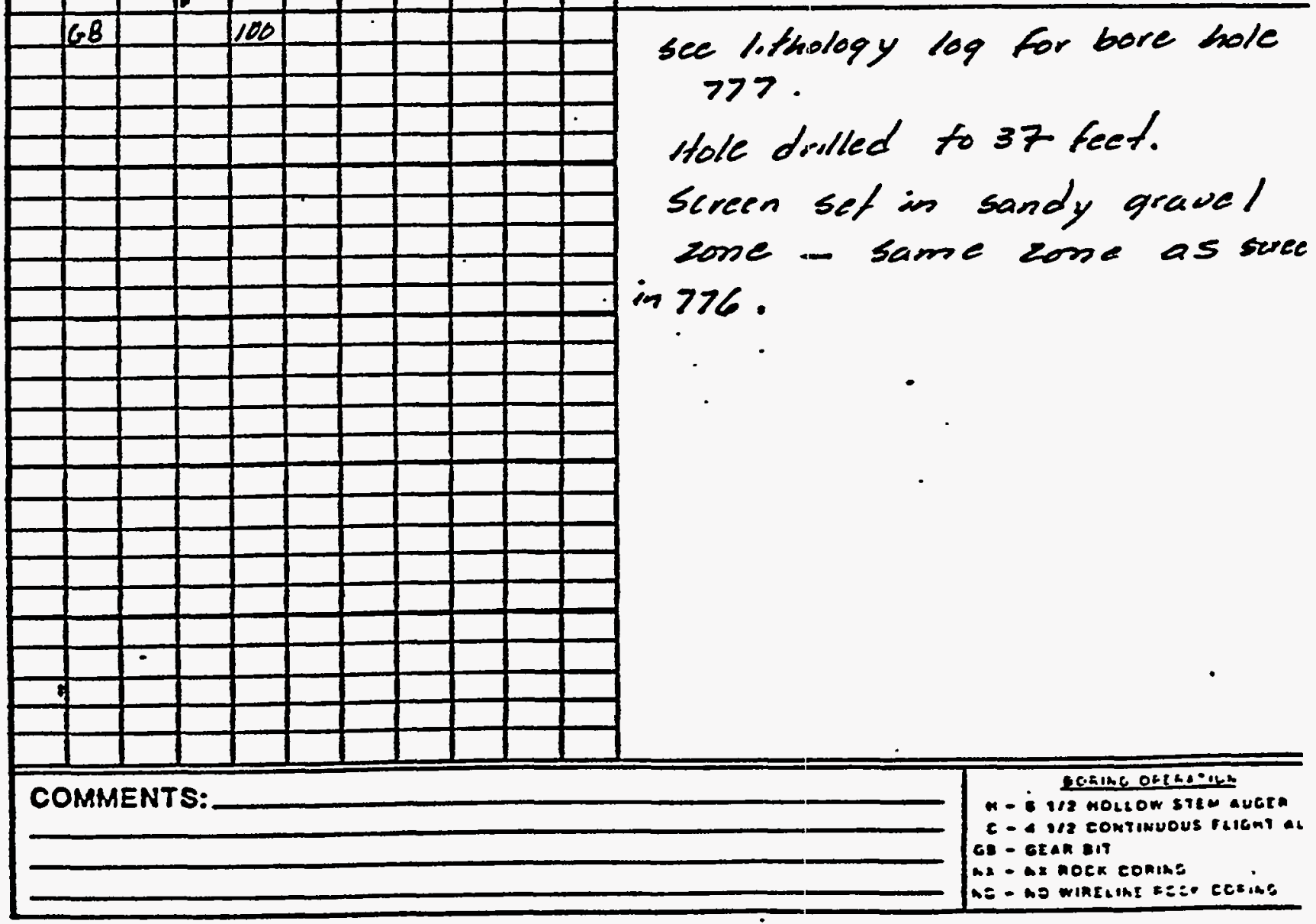

JEG-AL-ENG-2A (4/85) 


\section{TT MCOBS EVENERNG EROUP NC.

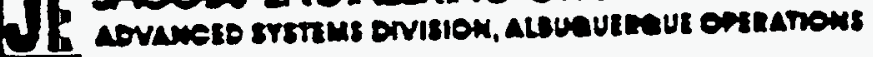

\section{WELL COMPLETION RECORD}

SITE ID: AMBOL LOCATION ID: 728

DATE INSTALLED: $8 / 30 / 85$ APPROX. SITE COORDINATES:(FT.) N NK

OPEN AREA PER LINEAL FT. (IN2/FT.) s.0" bore hole 4.0 "OD casing FORMATION OF COMPLETION:

FIELD-REP.: Reger Helland IRCI

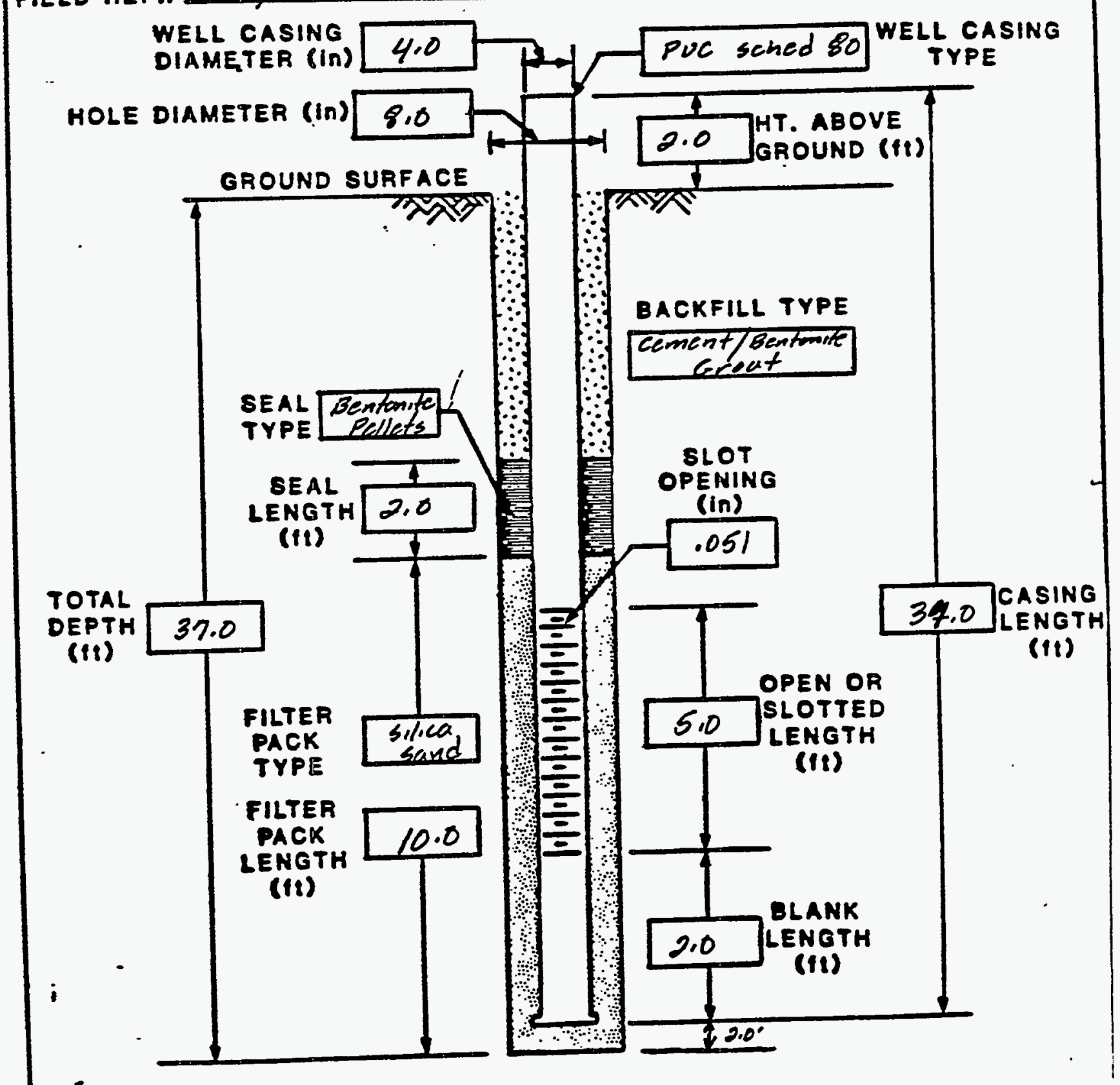

COMMENTS: 


\section{TIF HCOSS ENCN EERTE GROUP INC.

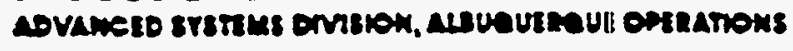 \\ BOREHOLE/WELL CONSTRUCTION LOG}

SITE ID: PMBOLLLOCATION ID: 278 FIELD REP: feqer Holland APPROX. BITE COORDINATES (FT.): N NK E AKK

GROUND ELEVATION (FT. MSL): NK COMPLETION DATE: \$/30/85

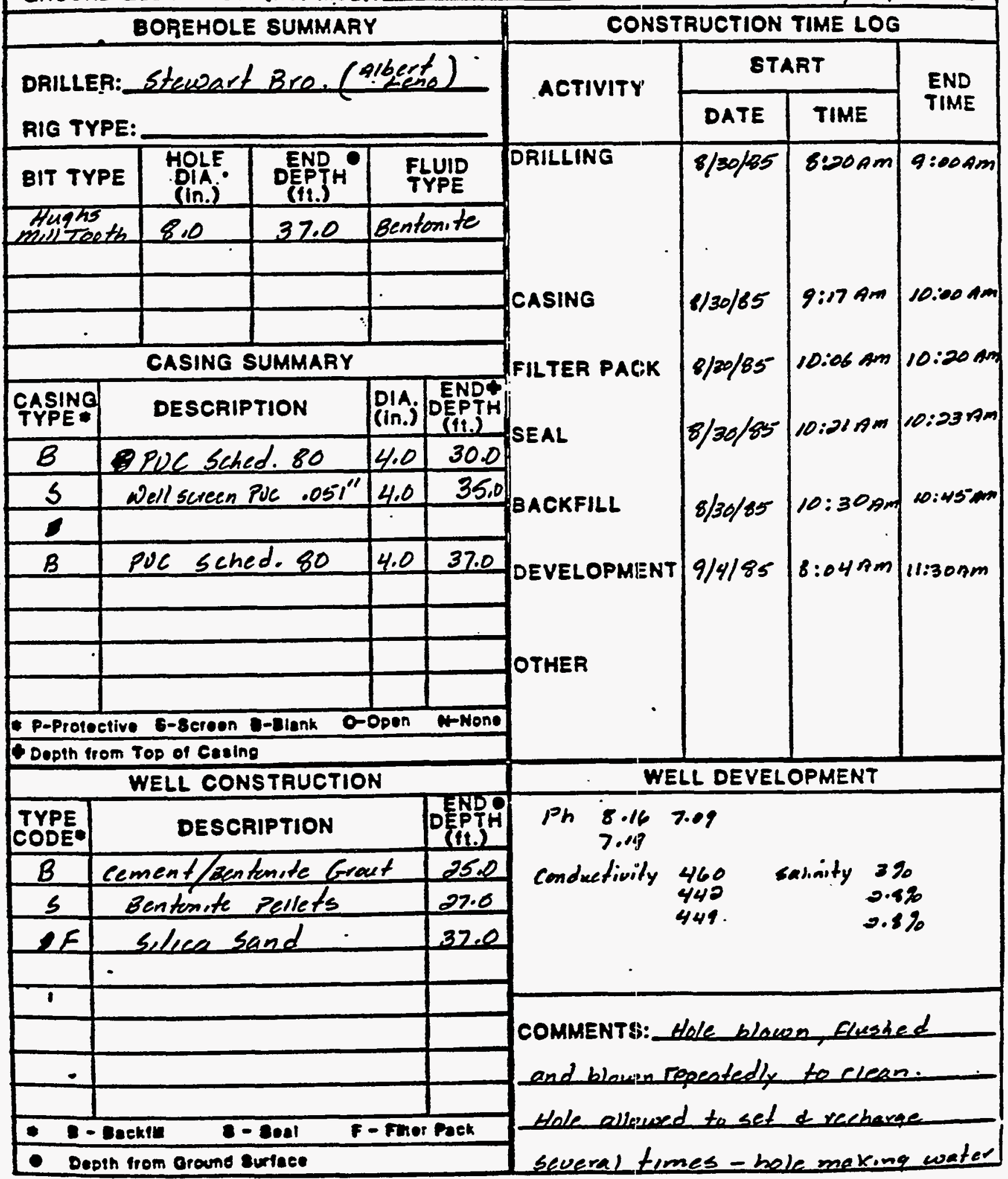

JEG-AL-ENO-I (8104) 
WELL 779 LOGS 


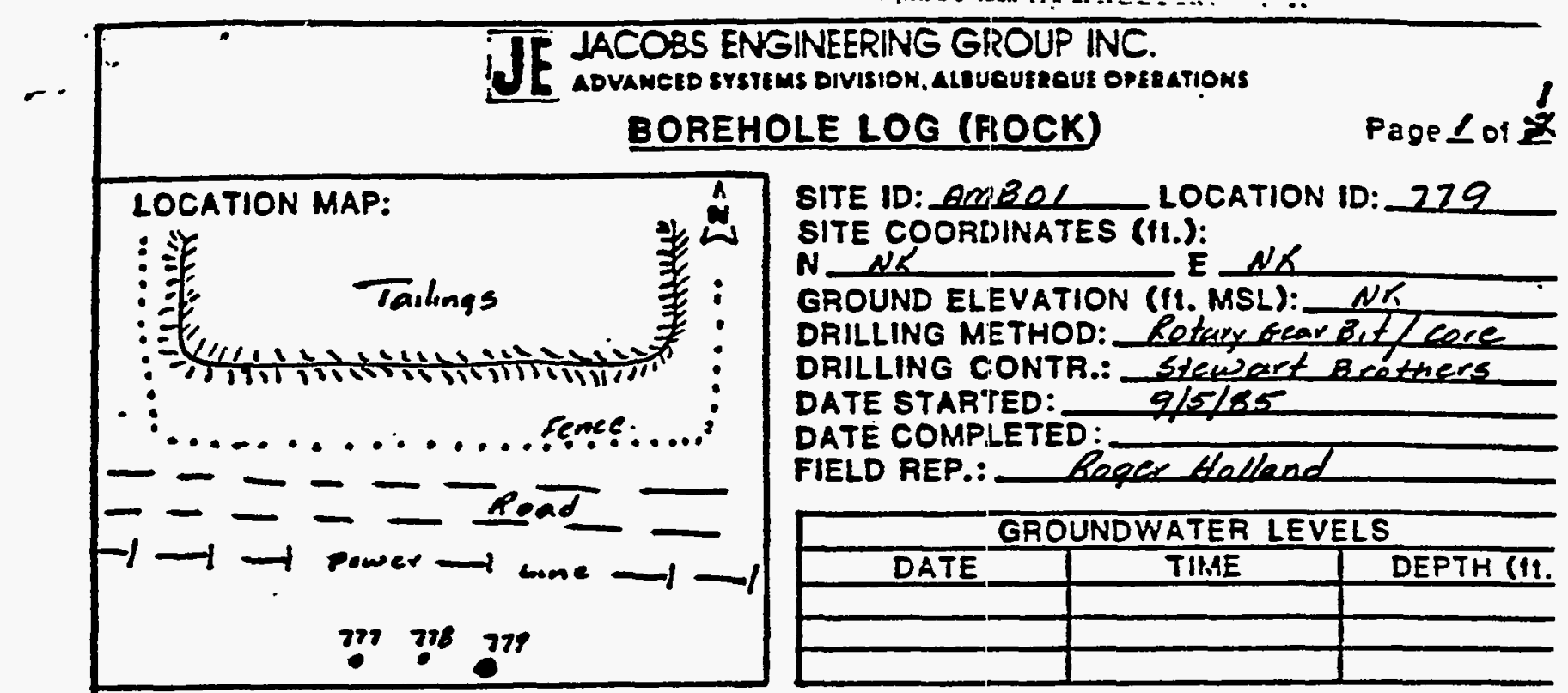

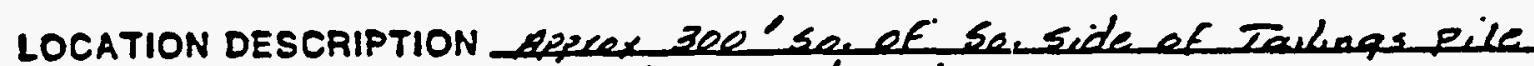
SITE CONDITION bewel weth weed and griass eswer

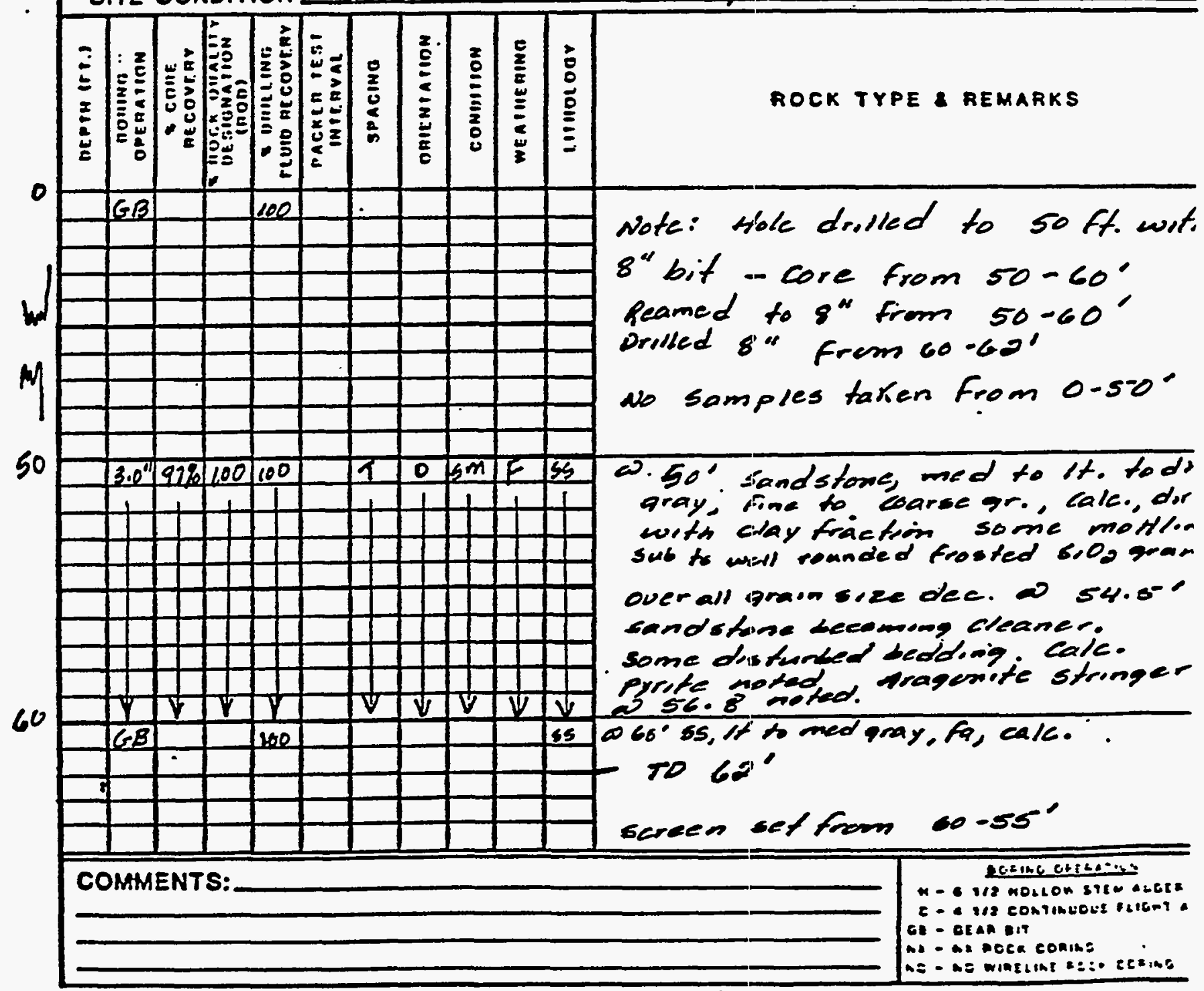

JEG-AL-ENG-2A (4/85) 


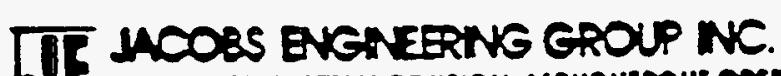

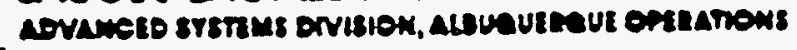

\section{WELL COMPLETION RECORD}

SITE ID: AmBOI
DATE INSTALLED: 9/5/85 E $1 K$

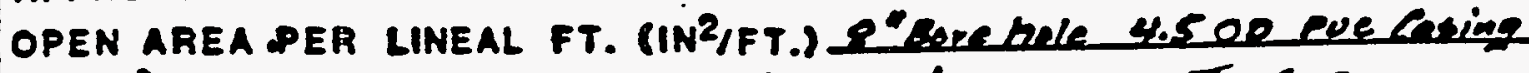
FORMATION OF COMPLETION: Tres termands $\mathrm{C} z$

FIELD -REP.: DRILLER: Steubet Bre.

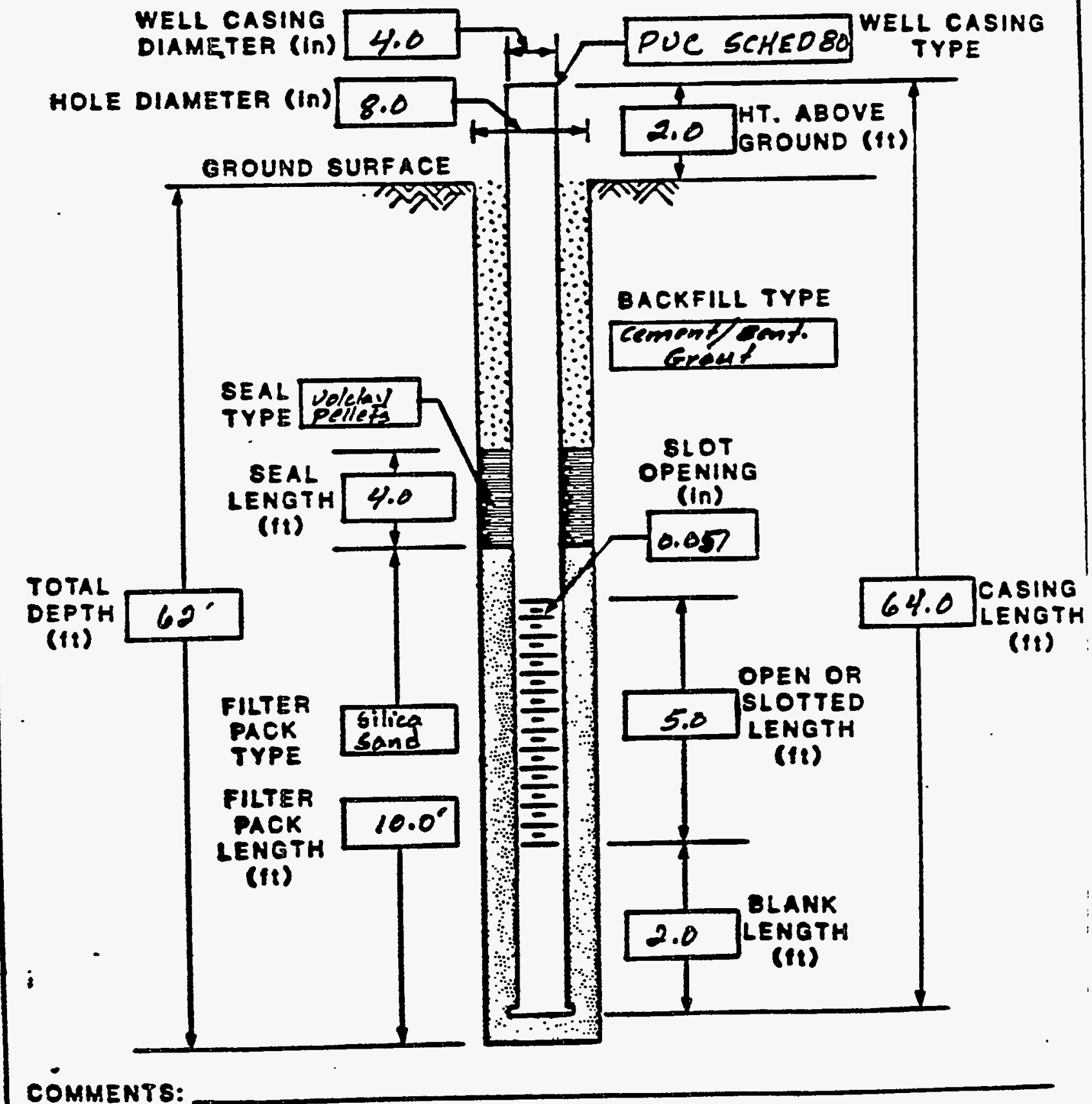




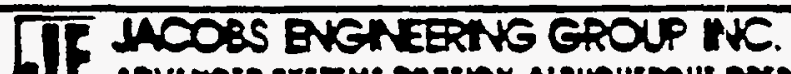

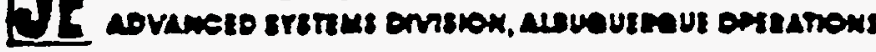 BOREHOLE/WELL CONSTRUCTION LOG}

SITE ID:AMBA LOCATION ID: 279 FIELD REP: Peger flelland APPROX. SITE COORDINATES (FT.): N NK GROUND ELEVATION (F́T. MSL): NK ENK

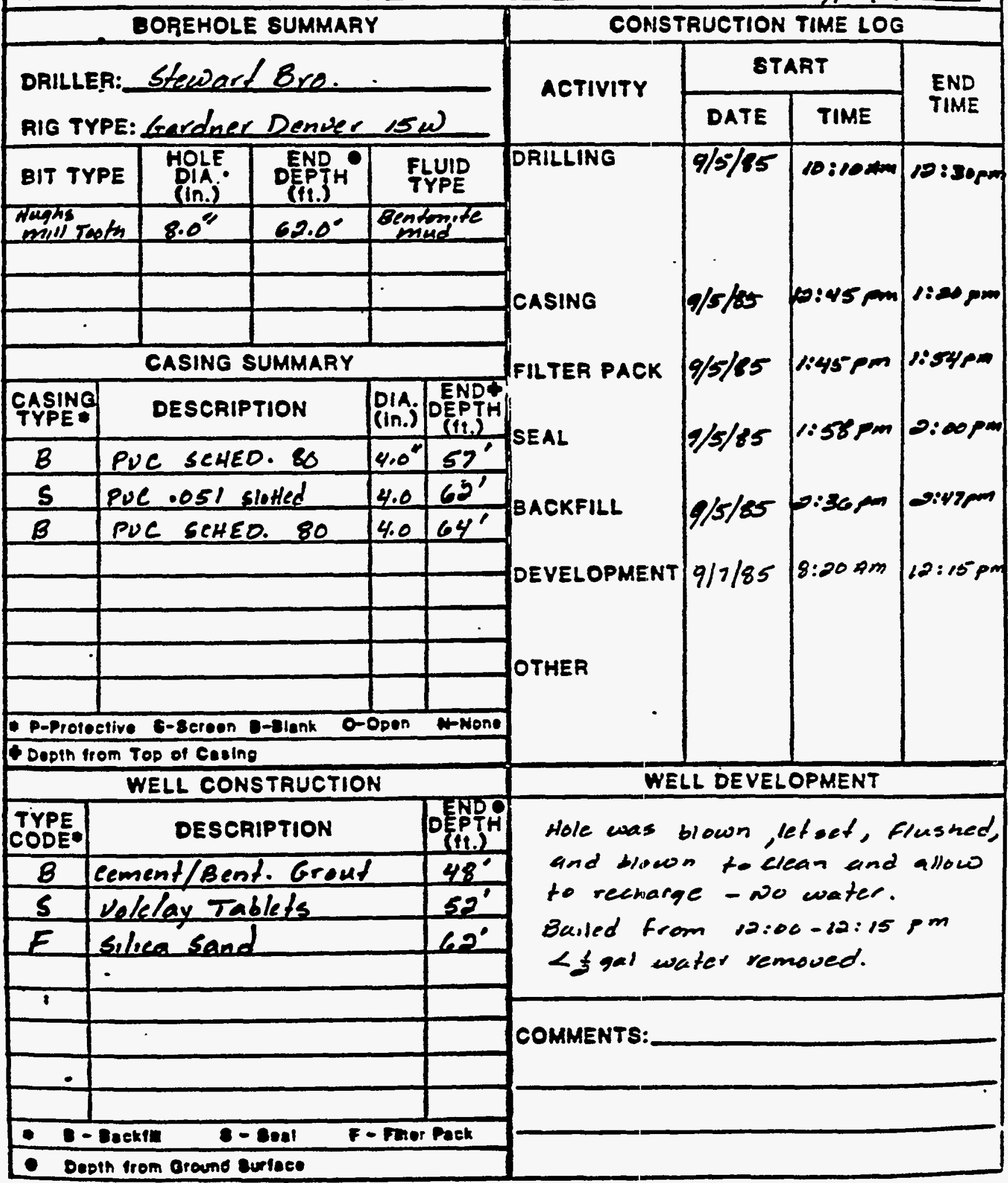


WELL 781 LOGS 


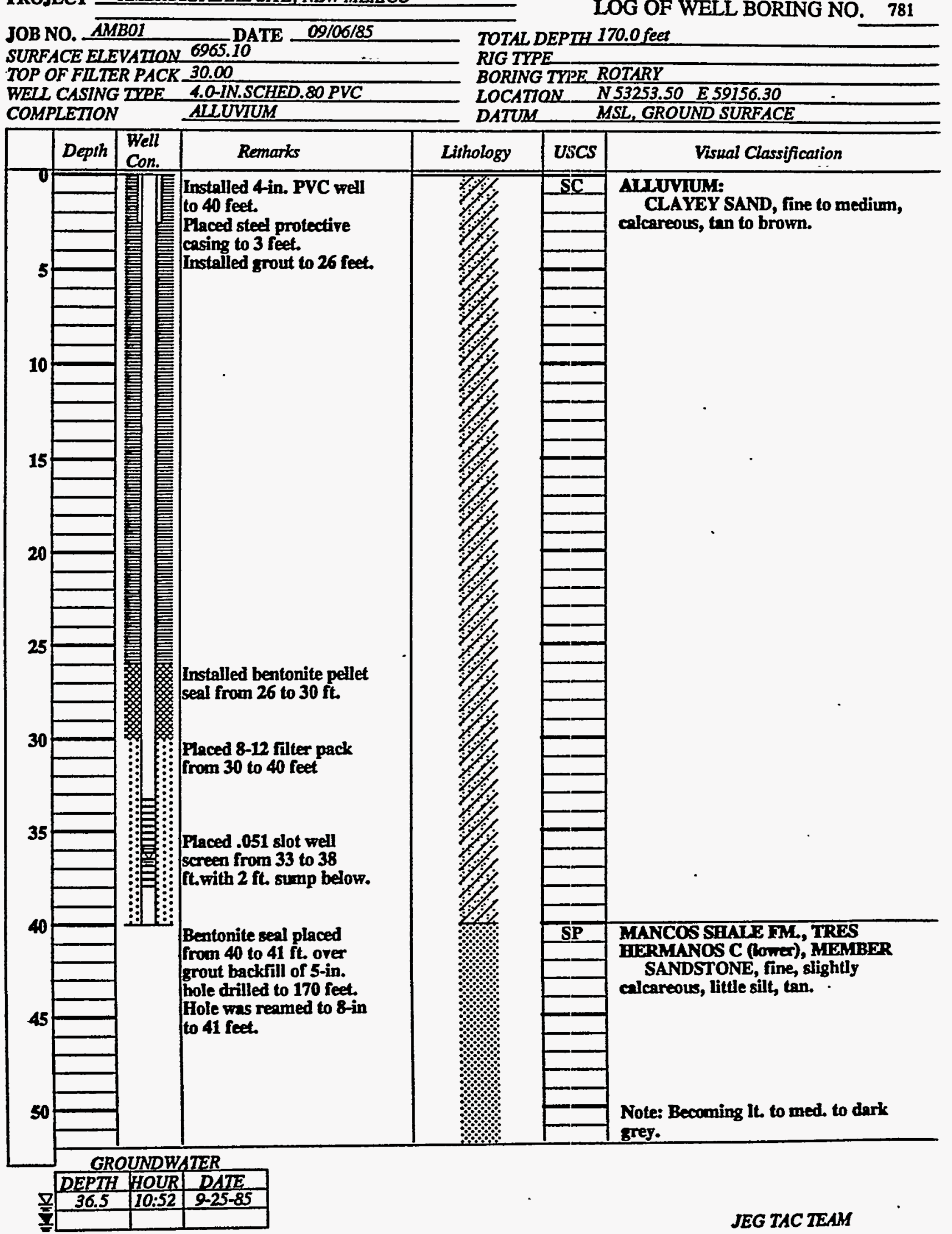




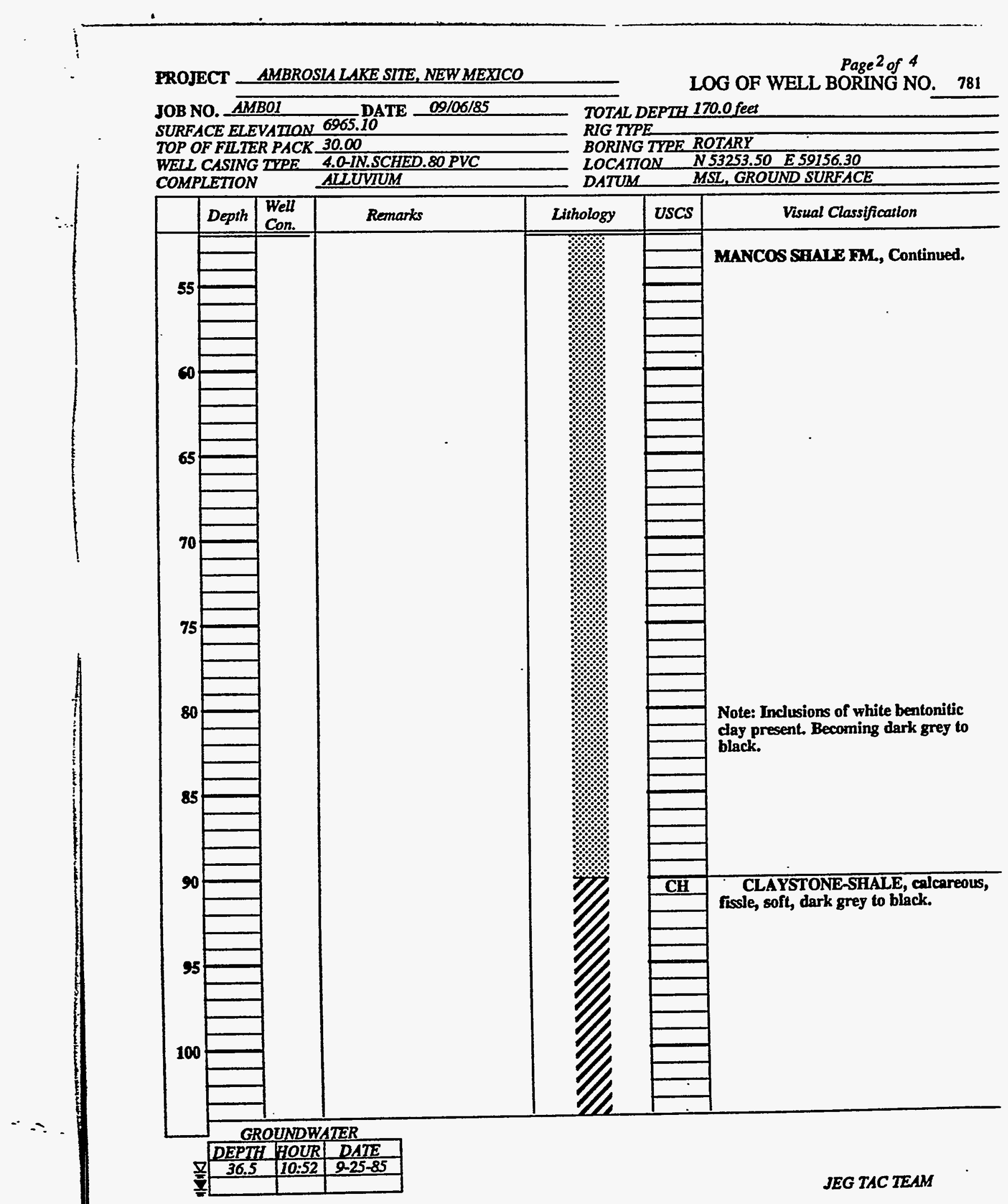

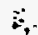




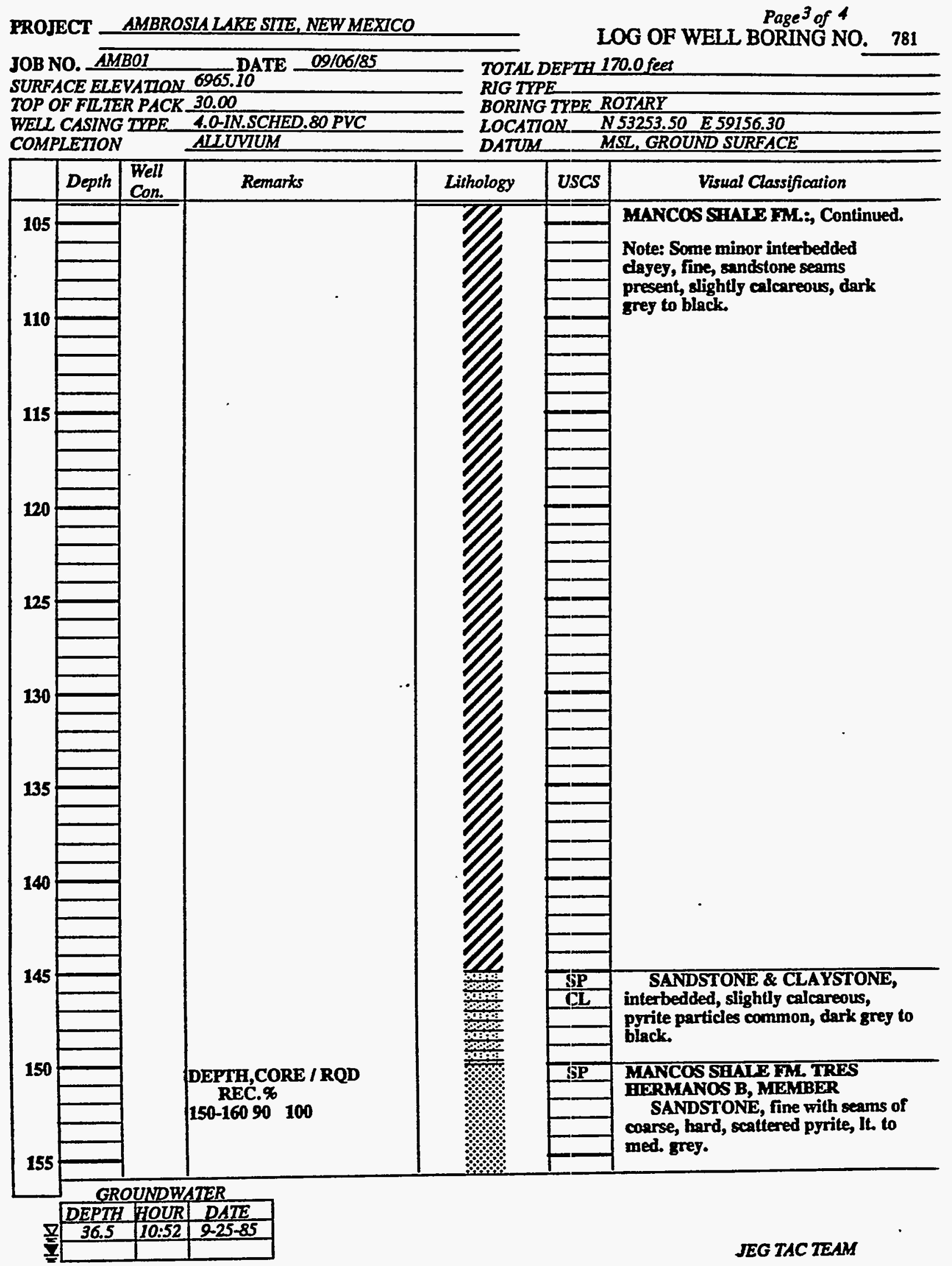


PROJECT AMBROSLA LAKE SITE, NEW MEXICO

JOB NO. AMBOI DATE 09/06/85

SURFACE ELEVATION 6965.10

TOP OF FIITER PACK 30.00

WELL CASING TIPE 4.0-IN.SCHED.80 PVC

COMPLETION ALLUVIUM

LOG OF WELL BORING NO.

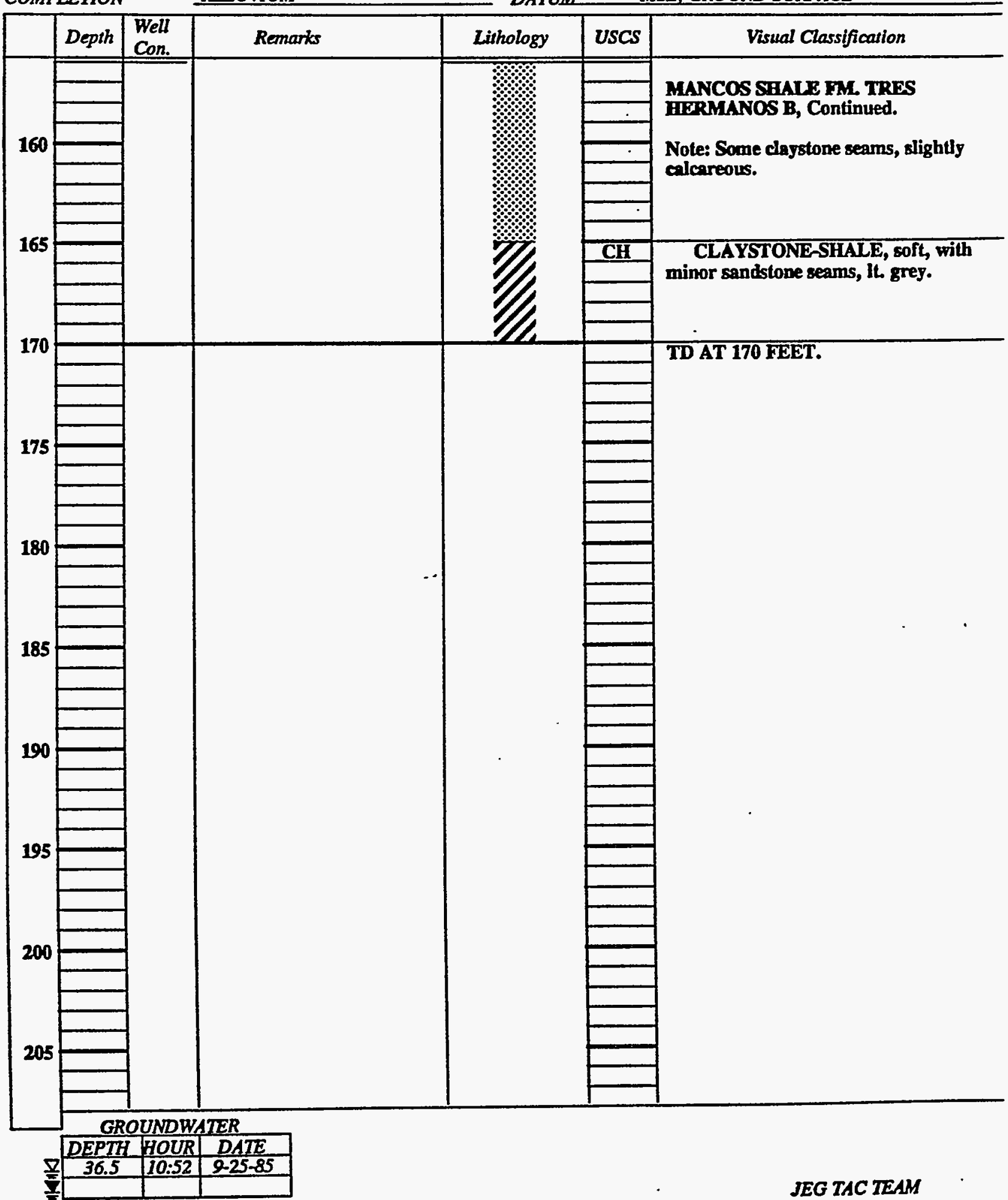




\section{TT MOOBS BNGNERTE EROUP INC.

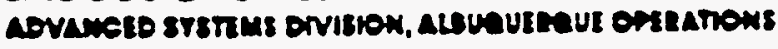

\section{WELL COMPLETION RECORD}

SITE ID: AMBOL LOCATION ID: 781

DATE INSTALLED: $q / 7 / 85$ APPROX. SITE COORDINATES:(FT.) $N$ NK $E N K$

OPEN AREA PER LINEAL FT. $\left(I^{2} / F T.\right) 8^{\prime \prime}$ Bore hate with $4.5^{\prime \prime}$ oD cesing FORMATION OF COMPLETION:

FIELD -REP.: Allwieum

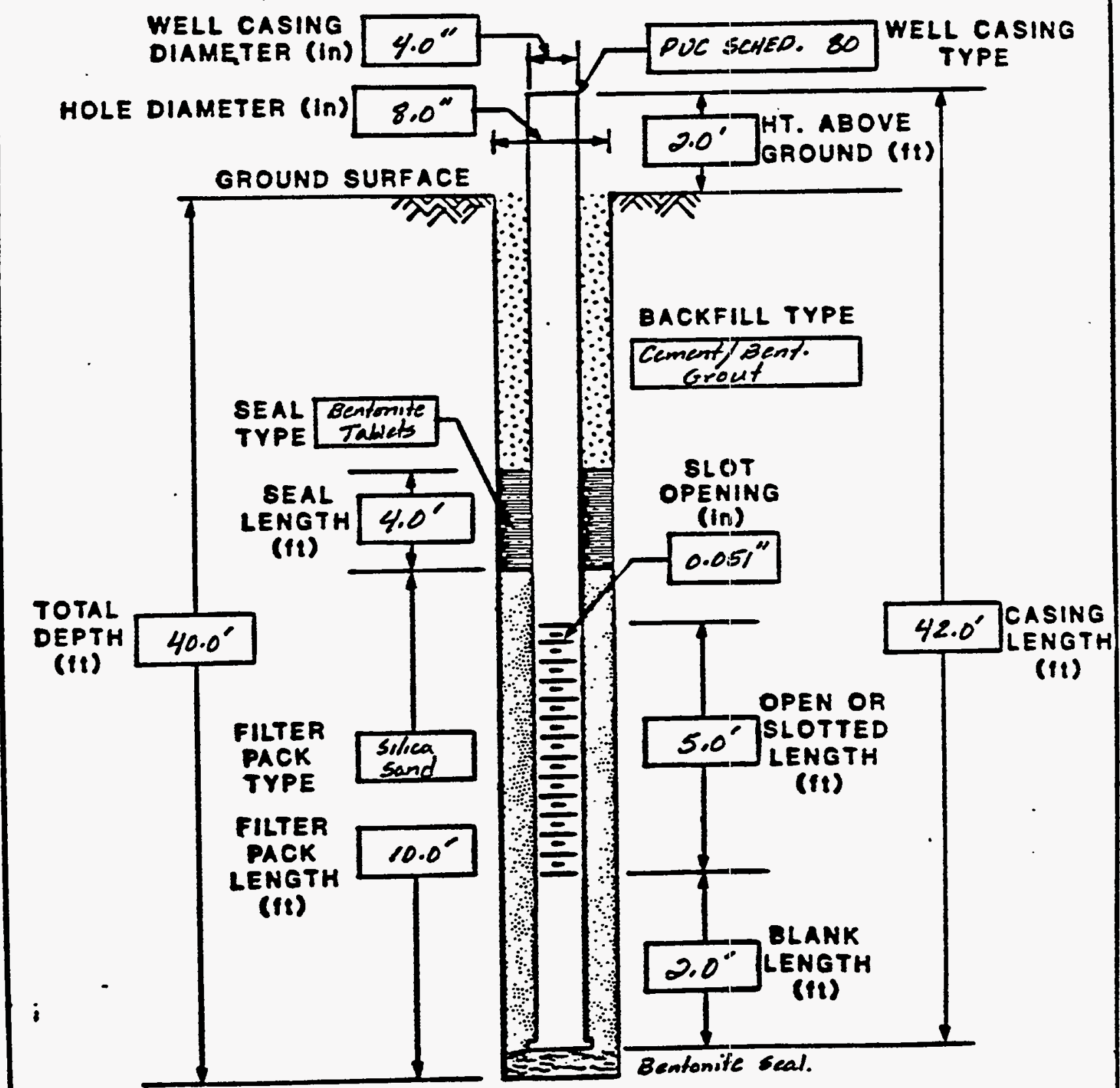

COMMENTs: A small amt. of sentemite Tablets was pet in hole

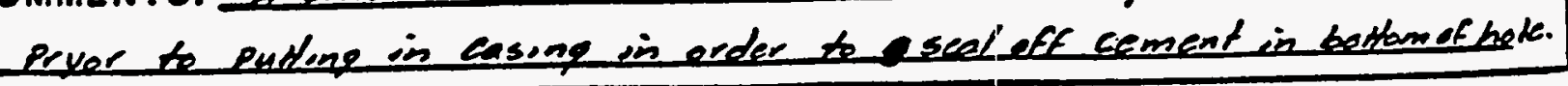


WELL 782 LOGS 


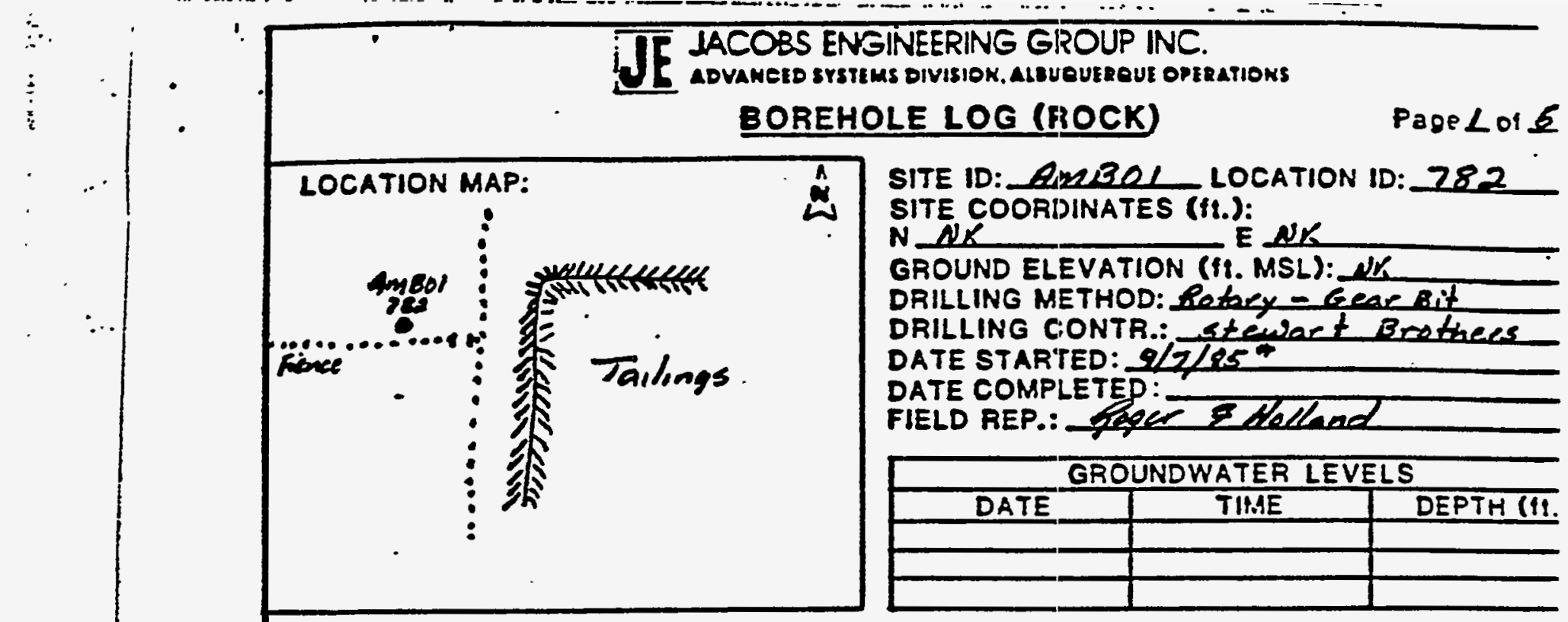

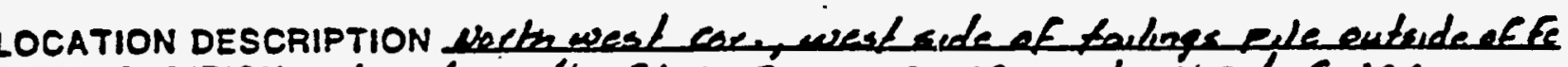
SITE CONDITION Level with slue Gemene press and wieed cever

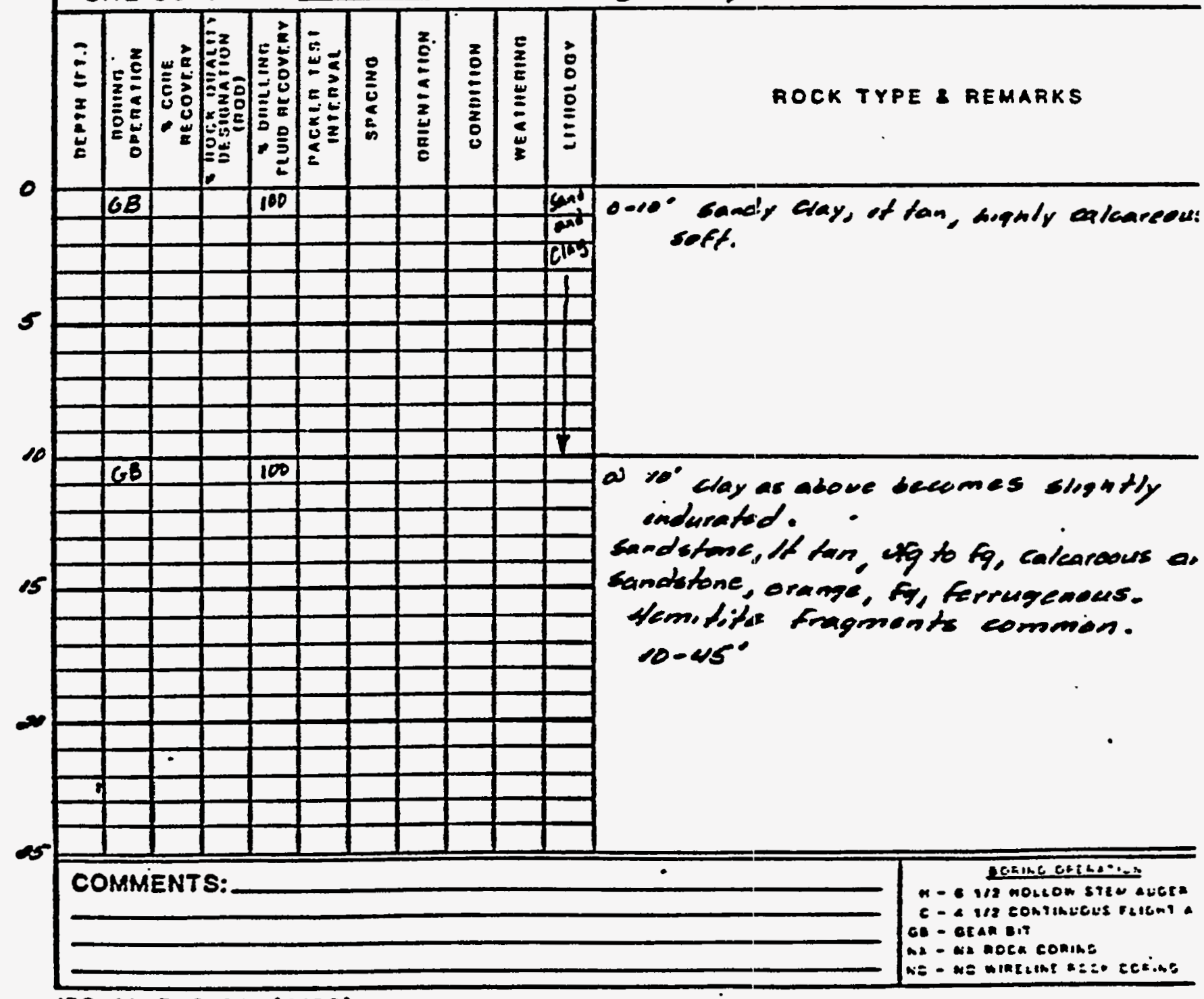

JEG-AL-ENG-2A (4185)

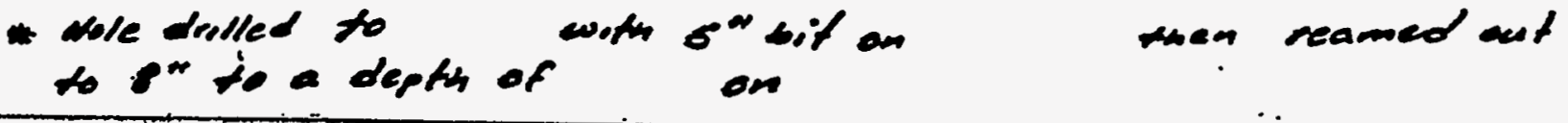




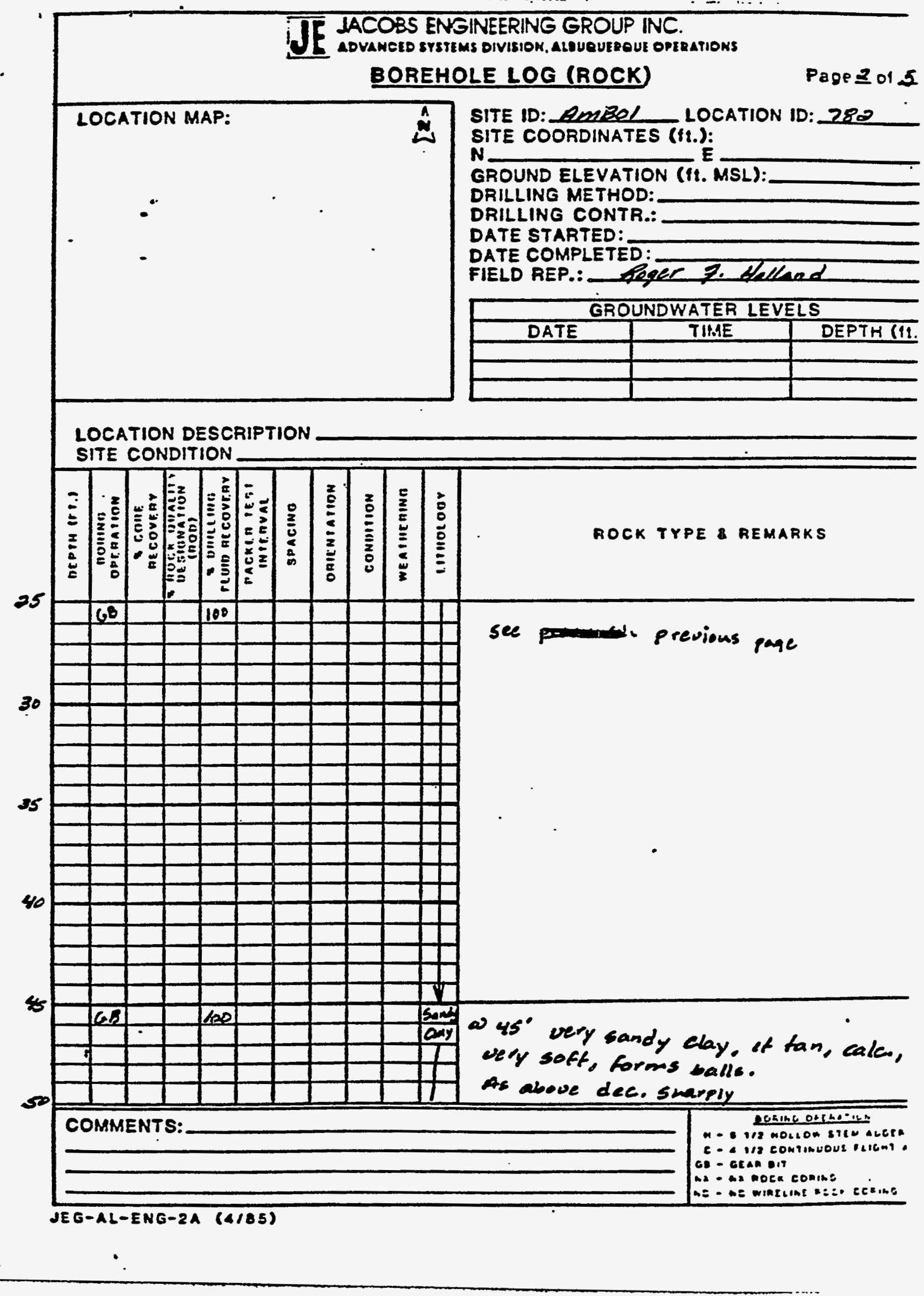




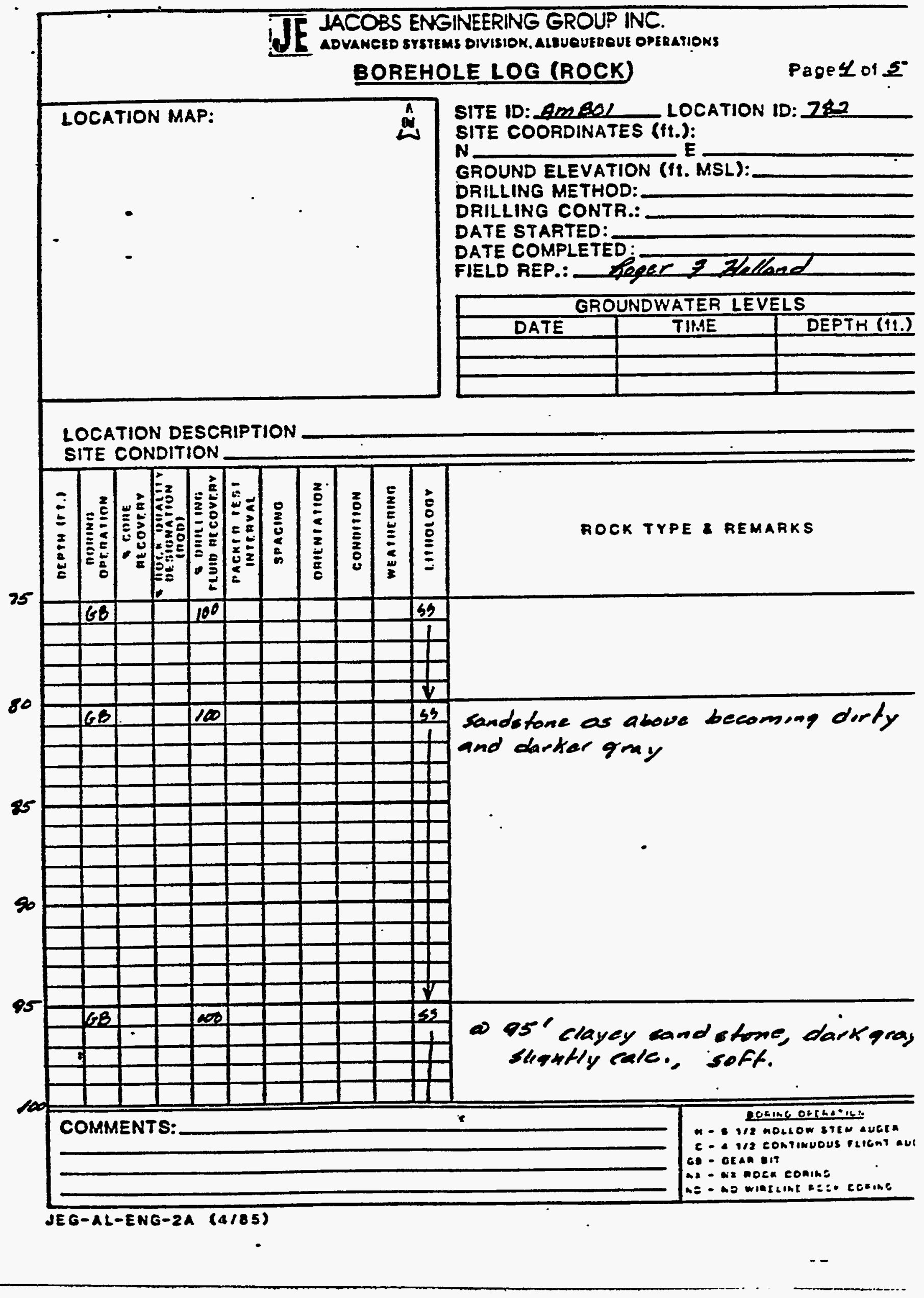




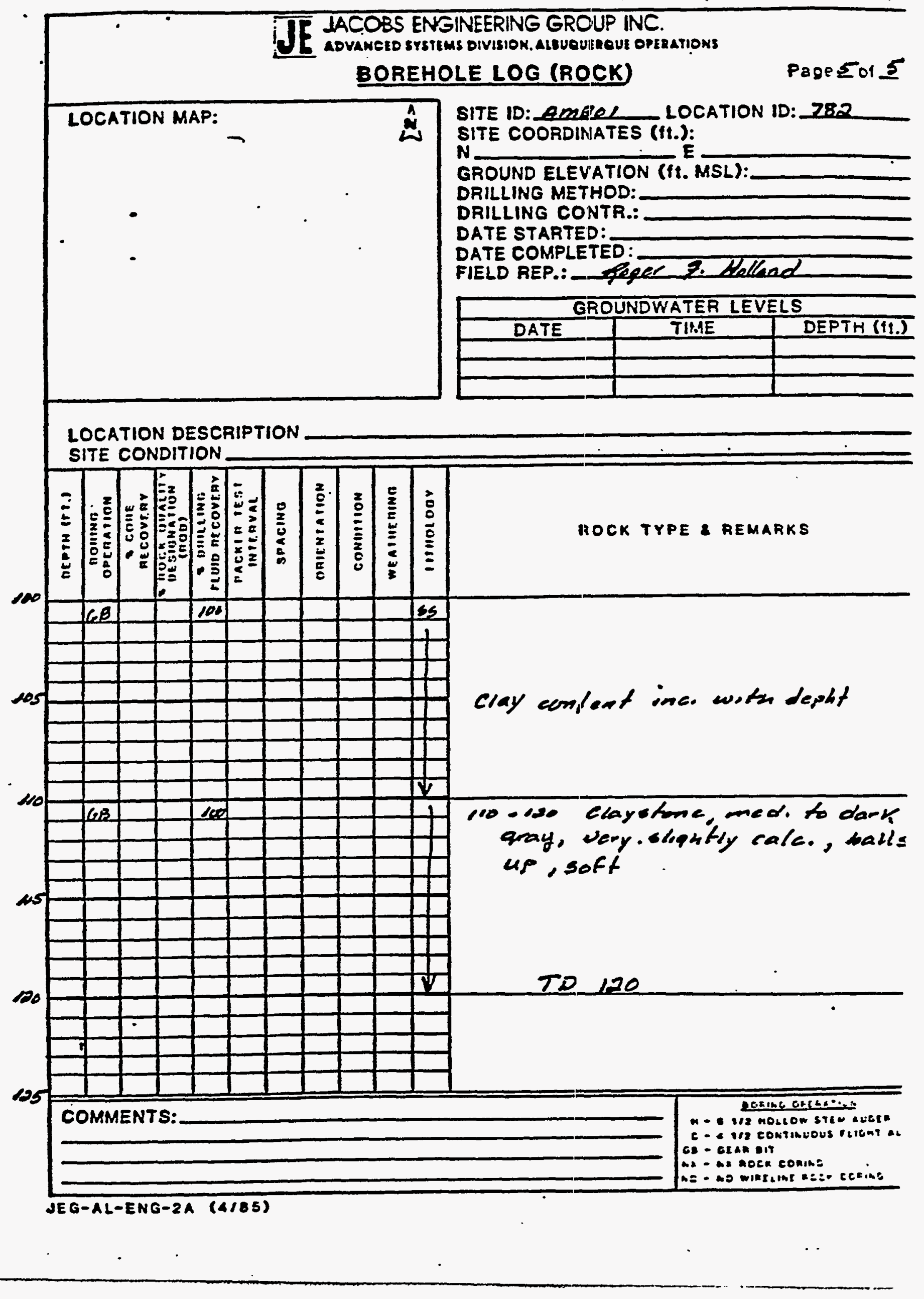




\section{TT MCOSS ENGNERTIS GROUP NC. aOVMneto srstrus orvisiok, ALbuautneut orteanows}

\section{MELL COMPLETION RECORD}

SITE ID:AMBOL LOCATION ID: 282 APPROX. SITE COORDINATES:(FT.) N NA

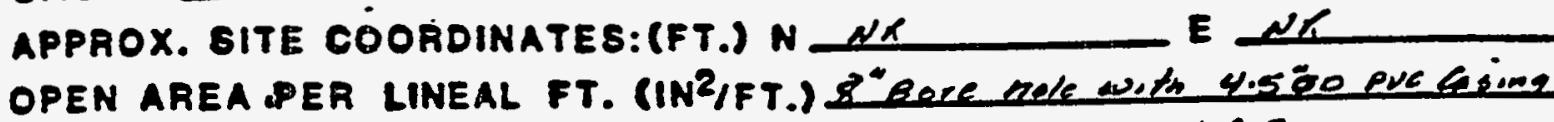
FORMATION OF COMPLETION: Tres Heconnes $\alpha \mathrm{CZ}$

FIELD-REP.: Reger Halland DRILLER: Steweset Brethers

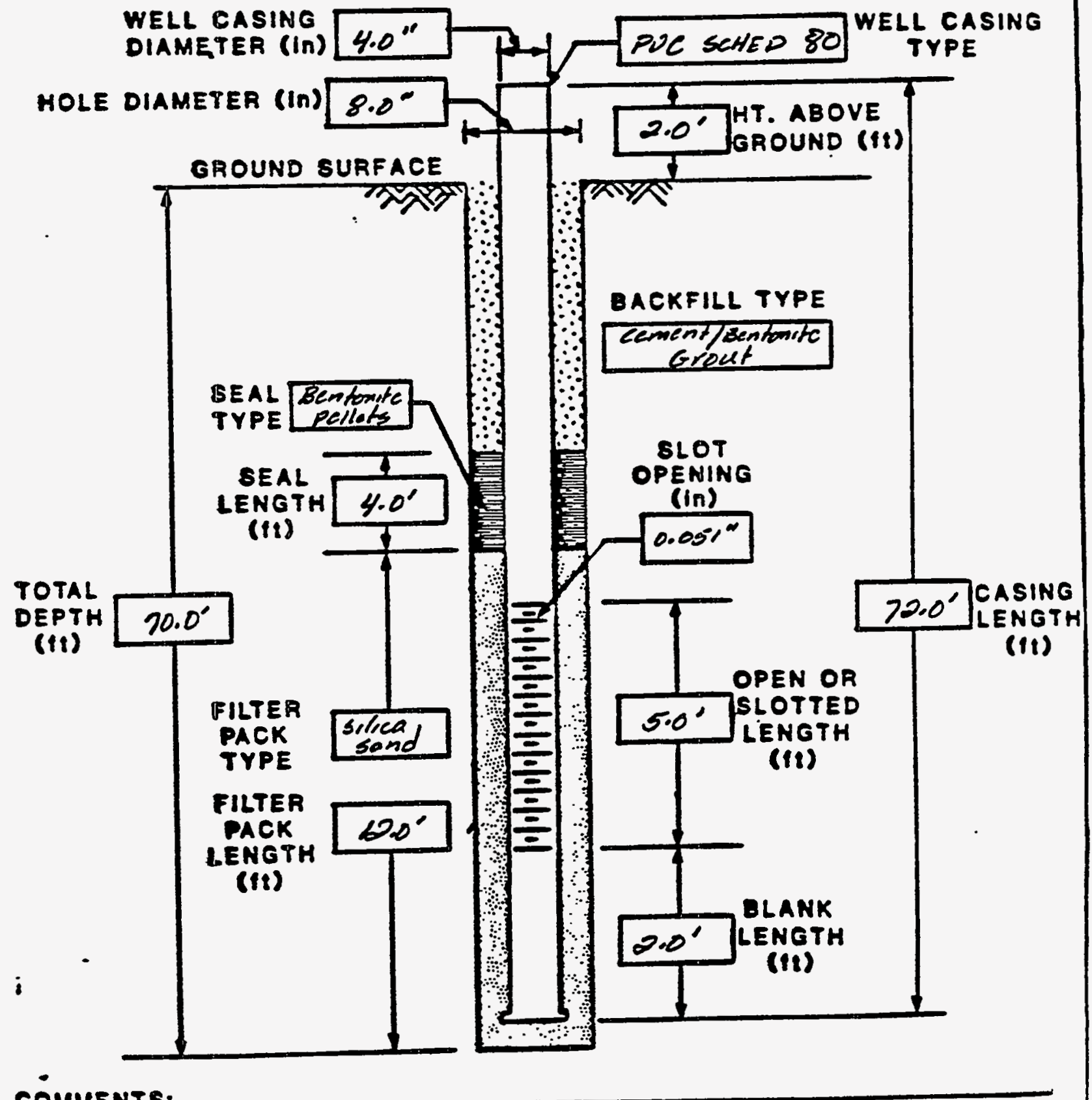

COMMENTS:
DATE INSTALLED: $\mathcal{I} / 9 / 85$ 


\section{TT INCOBS BNENEERIV CROUP INC. aDVAMCID srstrus omisiox, alsuautraut ertations BOREHOLE/WELL CONSTRUCTION LOG}

SITE ID: AMBOI LOCATION 1D:782 FIELD REP: Peqeer Helland APPROX. BITE COORDINATES (FT.): $N$ NK GROUND ELEVATION (FT. MSL): NK E EL

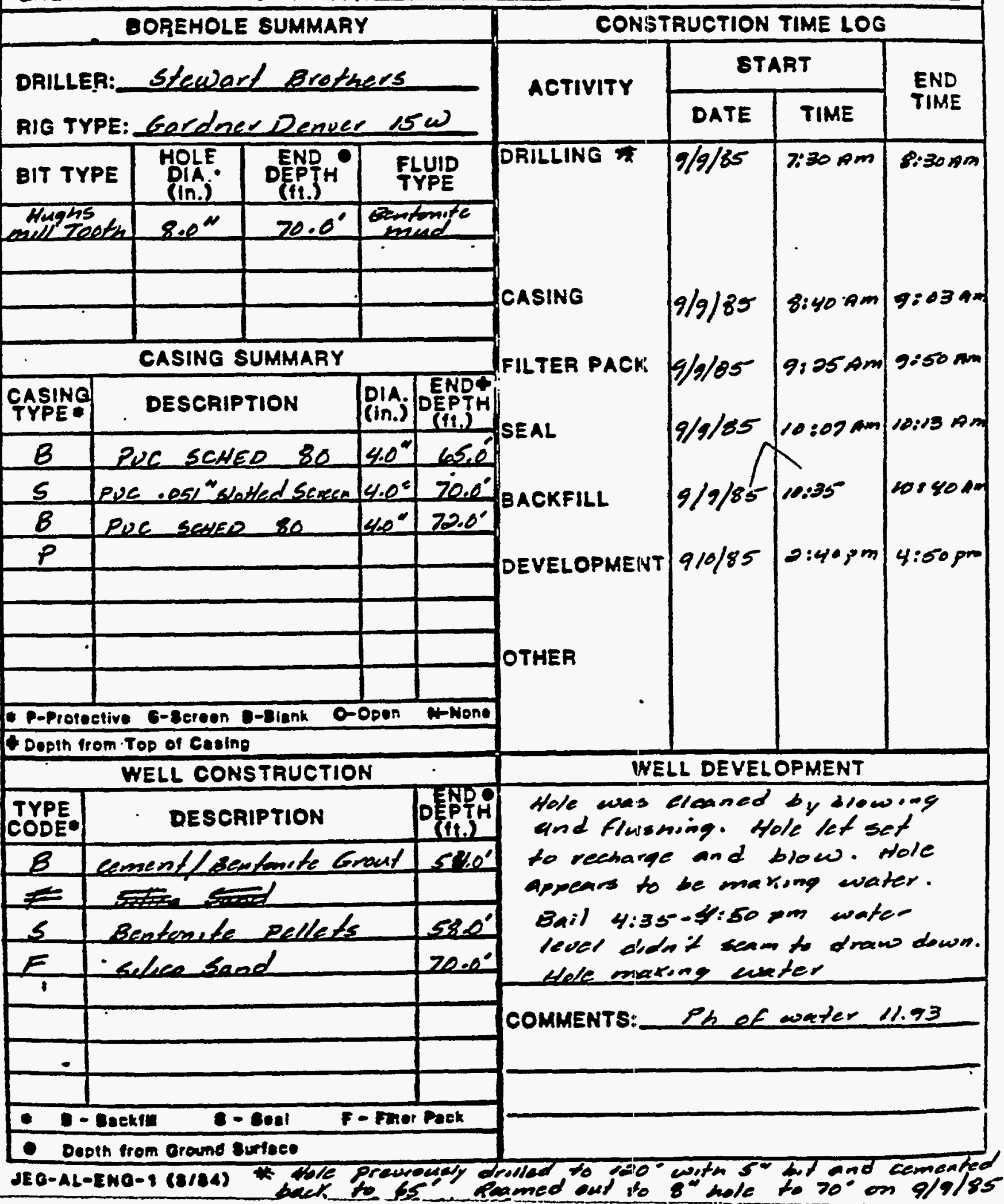


WELL 783 LOGS 


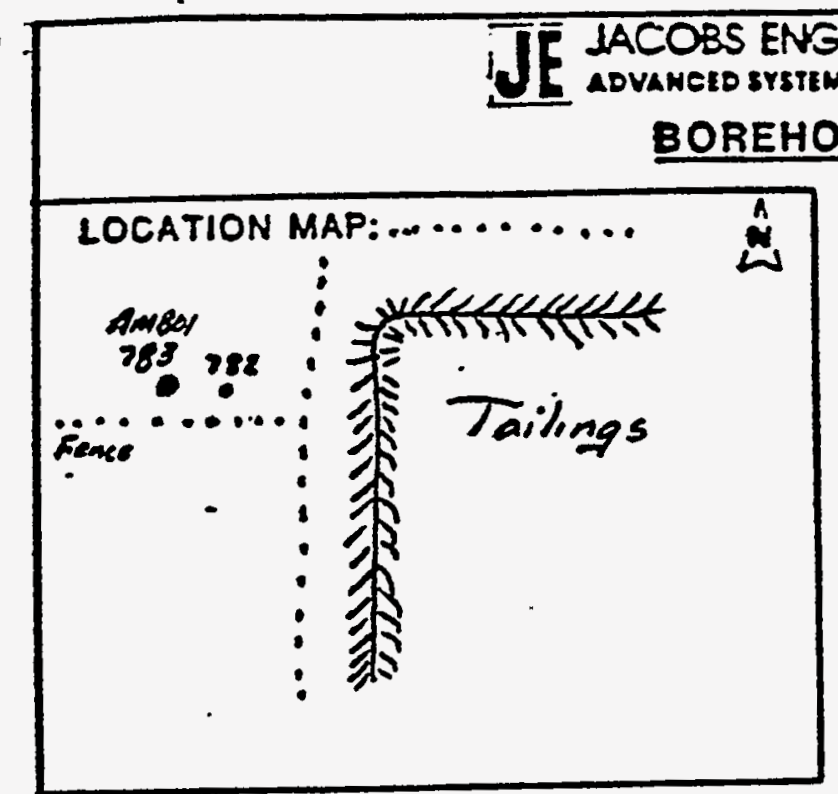

LOCATION DESCRIPTION west ef the Newest Con of Taukage pile SITE CONDITION tewel with arask and weed cover.

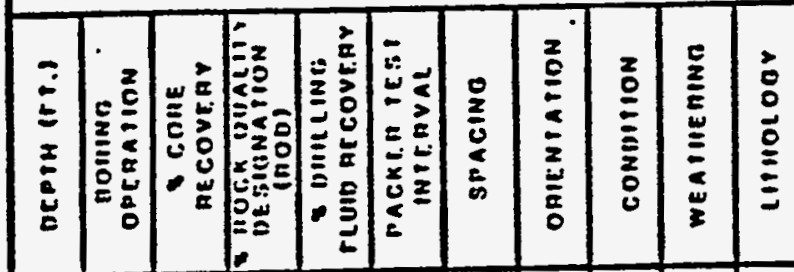

SITE COORDINATES ( $f t$. ):

N AL

GROUND ELEVATION (1t. MSL): NK

DRILLING METHOD: Petary - Gear Bit

DRILLING CONTR.: Gtwwart Brothers

DATE STARTED: Q/S/85-

DATE COMPLETED: 2/8/85

FIELD REP.: legee E. flelland

\begin{tabular}{|c|c|c}
\hline \multicolumn{3}{|c}{ GROUNDWATER LEVELS } \\
\hline DATE & TIMA & DEPTH (11.) \\
\hline & & \\
\hline & & \\
\hline & & \\
\hline
\end{tabular}

$G B$

\begin{tabular}{|llllllllll}
\hline & & & & & & & & & \\
\hline & & & & & & & & & \\
\hline & & & & & & & & & \\
\hline
\end{tabular}

ROCK TYPE R REMAKS

ב

Bore sole drilled to 5o' 6 "dia see log for AmBor-782 for detail dithology and electric 1

Formation - Qal 


\section{TT MCOsS BNENERING EROUP NC.

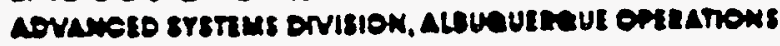

\section{WELL COMPLETION RECORD}

SITE ID:AMBO LOCATION ID: DATE INSTALLED: $2 / 8 / 85$ APPROX. SITE COORDINATES:(FT.) N WK E AK OPEN AREA PER LINEAL FT. (IN2/FT.) $Q^{\prime \prime b o r e ~ h a l e ~ w i t h ~} 4.5$ "0D casing FORMATION OF COMPLETION: Alluvium FIELD-REP.: Roger F. Helland DRILLER: Stewart Brethers

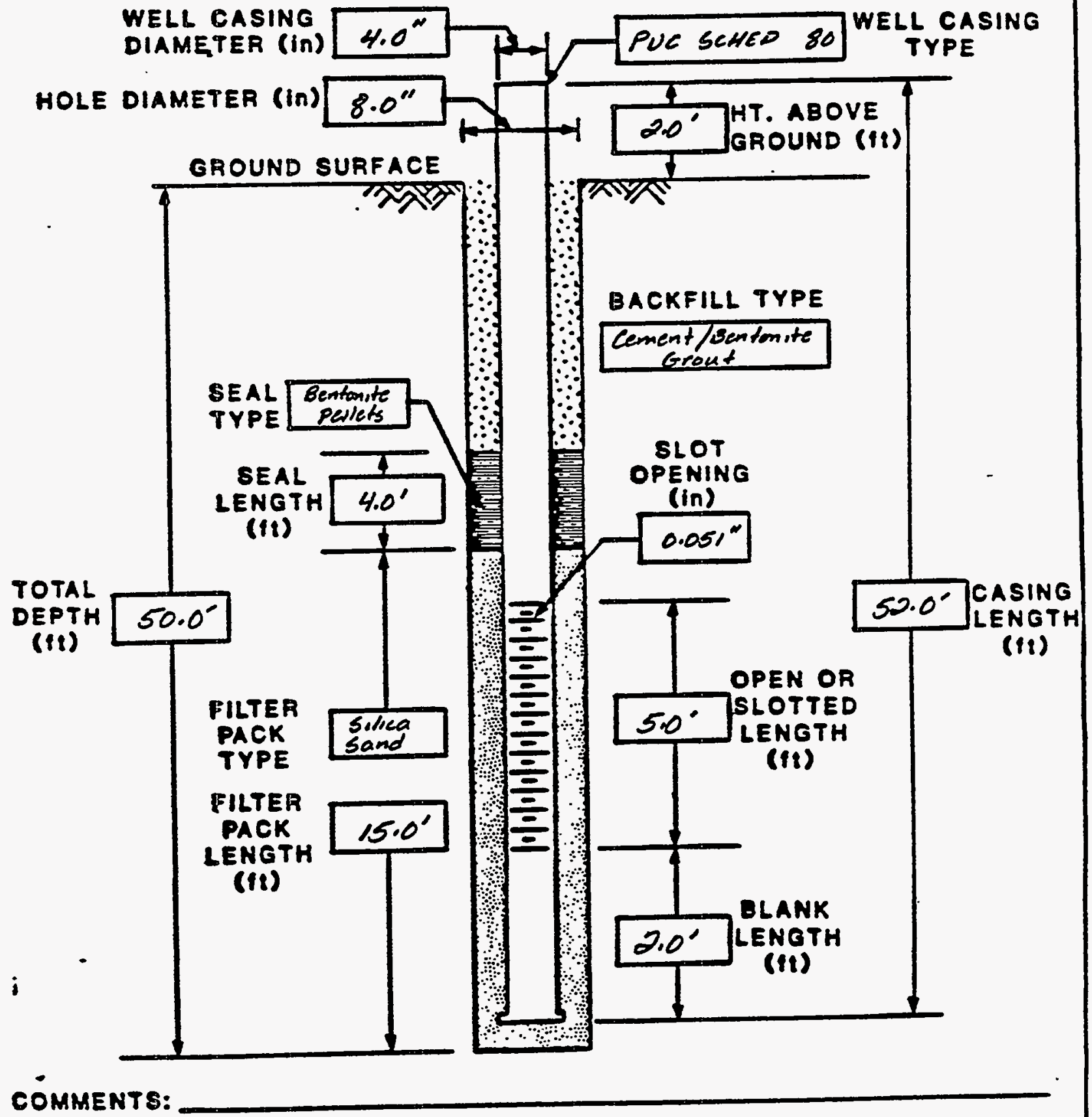




\section{If HCOBS BNENEERTE GROUP NK.

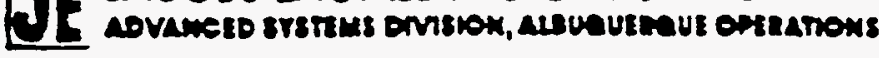 BOREHOLE/WELL CONSTRUCTION LOG}

SITE ID: AMBOI LOCATION ID: 783 APPROX. BITE COORDINATES (FT.): N NK GROUND ELEVATION (FT. MSL): NK
BOREHOLE SUMMARY

DRILLER: Stewart Brothers

RIG TYPE: Gardner Denver 15W

\begin{tabular}{|c|c|c|c|}
\hline BIT TYPE & $\begin{array}{l}\text { HOLEE } \\
\text { DIA . } \\
\text { (In.) }\end{array}$ & $\begin{array}{l}\text { END } \\
\text { DEPTH } \\
(f i .)^{\circ}\end{array}$ & $\begin{array}{l}\text { FLUID } \\
\text { TYPE }\end{array}$ \\
\hline Hughs & $8.0^{4}$ & $50^{\prime}$ & $\begin{array}{l}\text { Semponite } \\
\text { mude }\end{array}$ \\
\hline & & & \\
\hline & & & \\
\hline & & & \\
\hline
\end{tabular}

\begin{tabular}{|c|c|c|c|}
\hline $\begin{array}{l}\text { CASING } \\
\text { TYPE }\end{array}$ & DESCRIPTION & \begin{tabular}{|l|}
$p i A$ \\
$(i n)$.
\end{tabular} & $\begin{array}{l}\text { END' } \\
\text { EEPTH } \\
\text { (it,) }\end{array}$ \\
\hline$B$ & PUC SCHEO 80 & $4.02 \mathrm{E}$ & 5.0 \\
\hline$s$ & PUC .0s\%" slomed & $4.0^{-1}$ & .0 \\
\hline$B$ & PUC SCHEP 80 & $4.0^{\circ}$ & 52.0 \\
\hline$P$ & steel casing & $8.0^{\circ}$ & $5.0^{\circ}$ \\
\hline & & & \\
\hline & & & \\
\hline & & & \\
\hline & & & \\
\hline P-Pioloc & ive S-8eroen E-2ienk & & A-Nor \\
\hline
\end{tabular}

Dopin from Top of Cesing

\begin{tabular}{|c|c|c|}
\hline \multicolumn{3}{|c|}{ WELL CONSTRUCTION } \\
\hline $\begin{array}{l}\text { TYPE } \\
\text { CODE }\end{array}$ & DESCRIPTION & DEPTH \\
\hline$B$ & cement/Bentonete & $28.0^{\prime}$ \\
\hline 5 & sentemite Pellets & 32.0 \\
\hline$F$ & Selica bend & $50.0^{\circ}$ \\
\hline & - & \\
\hline 7 & & \\
\hline & & \\
\hline - & & \\
\hline & & \\
\hline 0 & $-3-3021$ & Poek \\
\hline - Do & in from Ground surface & \\
\hline
\end{tabular}

FIELD REP: Poger Fi Holland E NK

COMFLETION DATE: $9 / 8 / 85$ CONSTRUCTION TIME LOG

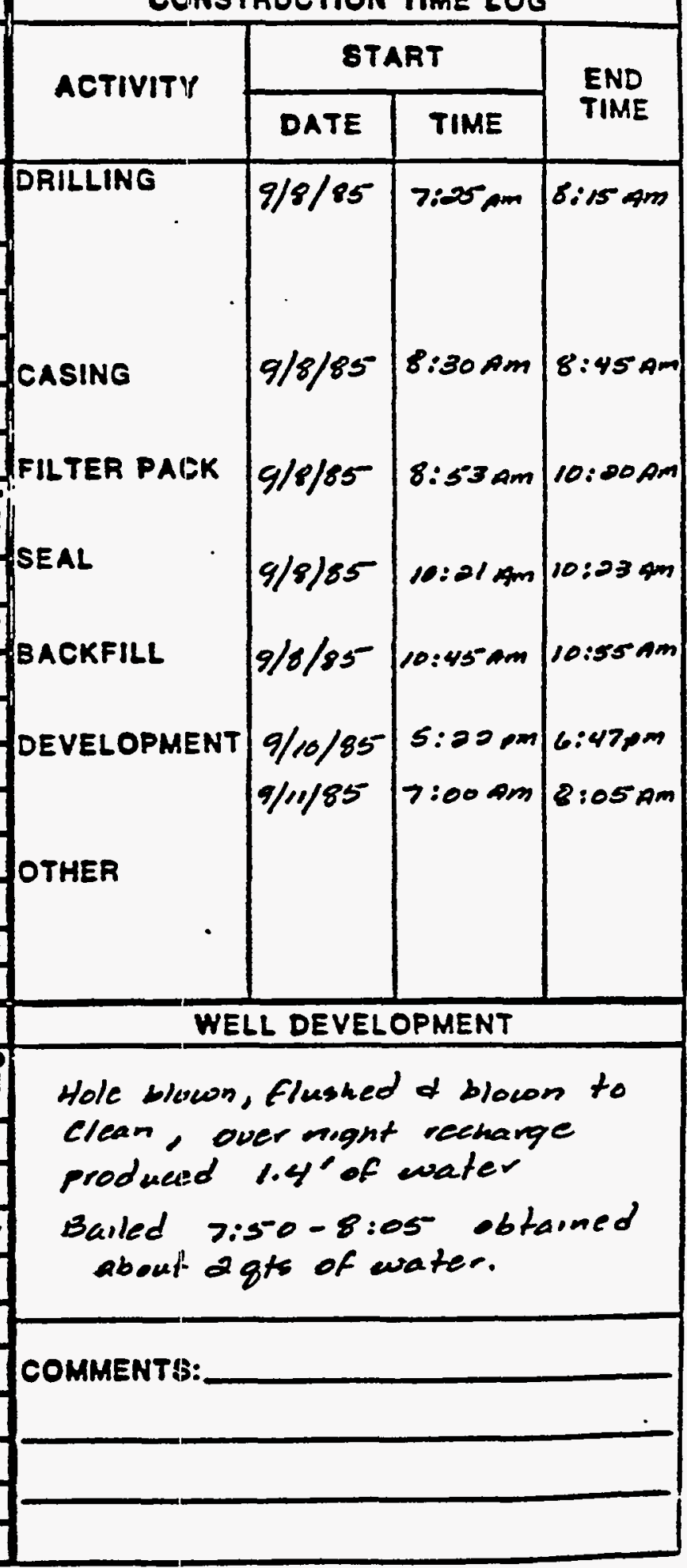


WELL 785 LOGS 


\section{TT MCOSS ENGNERTE GROUP NC.

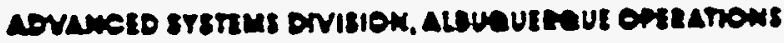

\section{WELL COMPLETION RECORD}

SITE ID: AMBOL LOCATION ID: 285 DATE INSTALLED: $9 / 11 / 85$ APPROX. SITE COORDINATES:(FT.) $N$ NK E NK

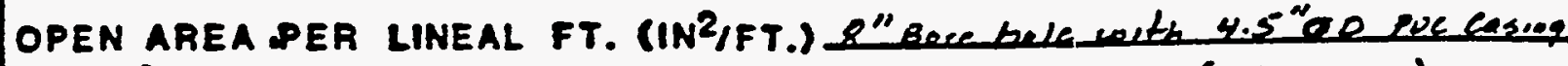
FORMATION OF COMPLETION: Tres Hecoanes 8 (mances) FIELD-REP.: Roger Helland DRILLER: Ltewart Brethers

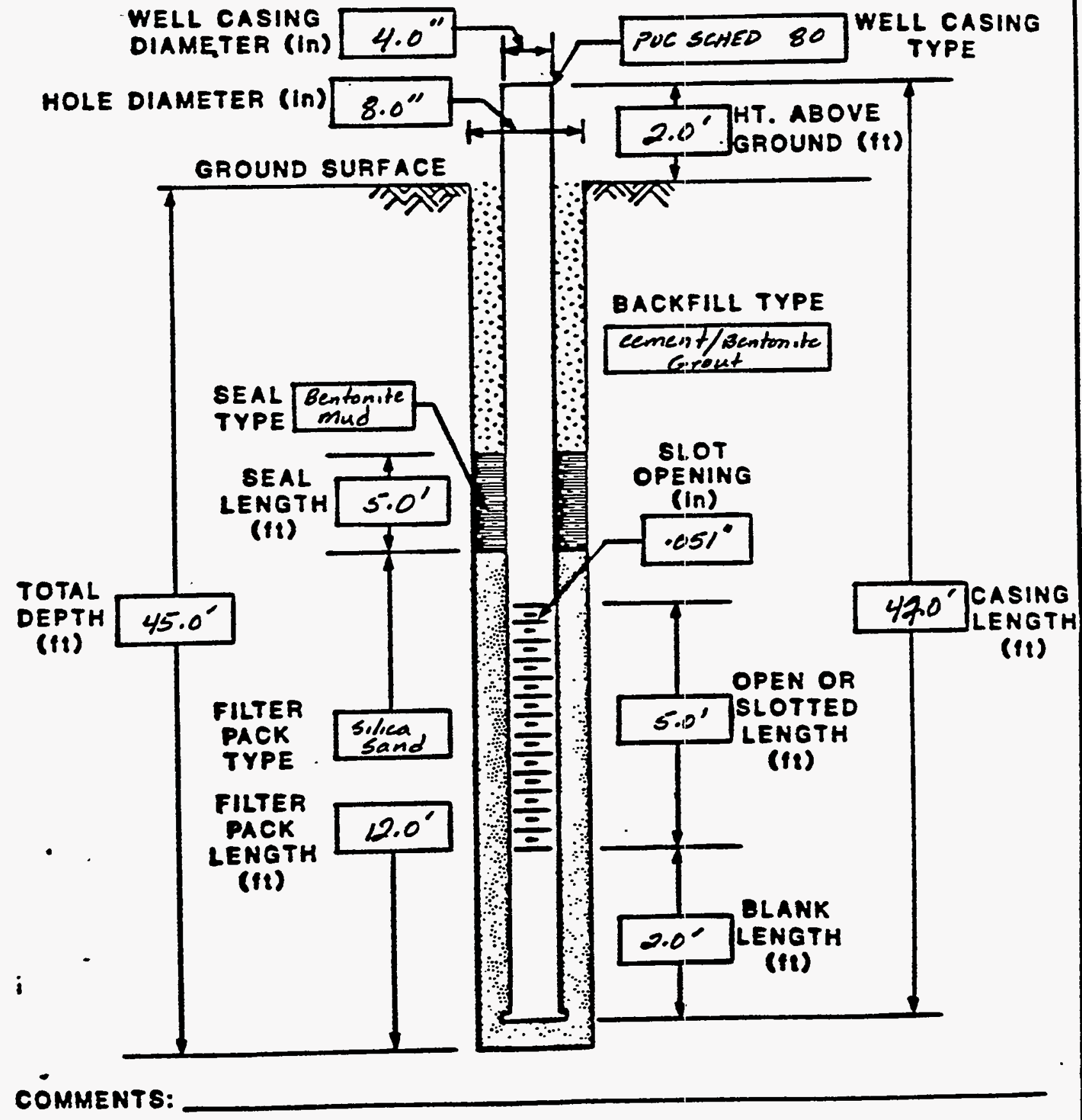




\section{IT IACOBS BNGN EERTE GROUP NK.

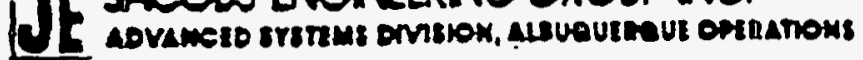 \\ BOREHOLE/WELL CONSTRUCTION LOG}

SITE ID: AMBOI LOCATION ID: 785 FIELD REP: Roger Holland

APPROX. BITE COORDINATES (FT.): N NK

GROUND ELEVATION (FT. MSL): NK BOREHOLE SUMMARY

DRILLER: Stewart Brothers

RIG TYPE: Gardner Denver is w

\begin{tabular}{|c|c|c|c|}
\hline BIT TYPE & $\begin{array}{l}\text { HOLE. } \\
\text { OIA } \\
\text { (In.j }\end{array}$ & 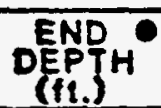 & $\begin{array}{l}\text { FLUID } \\
\text { TYPE }\end{array}$ \\
\hline 4ugks & $8.0^{\prime \prime}$ & $45^{\prime}$ & $\begin{array}{l}\text { Oantonite } \\
\text { mude }\end{array}$ \\
\hline & & & \\
\hline & & & \\
\hline & & & \\
\hline
\end{tabular}

\begin{tabular}{|c|c|c|c|}
\hline $\begin{array}{l}\text { CASING } \\
\text { TYPEE }\end{array}$ & DESCAIPTION & 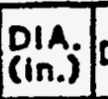 & (it. \\
\hline $\bar{B}$ & PUC SCHEO 80 & 4.0 .0 & 70 \\
\hline 5 & Lue $.051^{\prime \prime}$ slotted & $4.0^{*}$ & \\
\hline B & PUC GCHEO SO & $4.0^{\prime \prime}$ & \\
\hline $\boldsymbol{P}$ & 8" Se steel casing & $8.0^{\circ}$ & \\
\hline & & & \\
\hline & & & \\
\hline & & & \\
\hline & & & \\
\hline
\end{tabular}

- P-Protective 8-Bcreon e-blank O-Open N-None

Copin trom Top of Cecing

\begin{tabular}{|c|c|c|}
\hline \multicolumn{3}{|c|}{ WELL CONSTRUCTION } \\
\hline $\begin{array}{l}\text { TYPE } \\
\text { CODE- }\end{array}$ & DESCRIPTION & DEpe $(f,)^{H}$ \\
\hline$B$ & Cement/Bentente Grout & 28 \\
\hline 5 & Heavy Bentonite mad & 320 \\
\hline $\bar{F}$ & - Silica band & 4 \\
\hline i & & \\
\hline & & \\
\hline & & \\
\hline & & \\
\hline -- & $0-8021$ & Pack \\
\hline & 10 eurfoes & \\
\hline
\end{tabular}
COMPLETION DATE: $9 / 4 / 85-$ CONSTRUCTION TIME LOG

CASING

FILTER PACK

SEAL

BACKFILL

DEVELOPMENT

OTHER probe

\begin{tabular}{|c|c|c|}
\hline \multicolumn{2}{|c|}{ START } & \multirow{2}{*}{ END } \\
\hline TIME \\
\hline $9 / 11 / 85$ & $9: 34 A \mathrm{Am}$ & $11: 00 \mathrm{Am}$ \\
\hline
\end{tabular}

$9 / 11 / 45-11: 300 \mathrm{~m} \mid 10: 00$ anom

$9 / 11 / 85 \quad 12: 04 \mathrm{pm} \quad 10: 24 \mathrm{pm}$

$9 / 4 / 85 \quad 10: 26 \mathrm{pm} / 0: 36 \mathrm{pm}$

$9 / \mathrm{u} / 85$ 12:38pm 1:00 pm

9/12/85 $3: 80 \mathrm{pm} 6: 17 \mathrm{pm}$

9/11/85 11:0040m 11:30000

\section{WELL DEVELOPMENT}

siew and flushed hole to clean.

Blew hole 10 men to dry.

Let set $15 \mathrm{~m}$ in - blow-very

little water. Igject to cican

4 develop further. Blew dry.

Bail 6:00 om - 6:17 pon < 2 gt Hab. waterlevel on 9/13/85 49.7' 
WELL 786 LOGS 
IT IACOBS ENGINEERING GROUP INC.

AOVAMEED SVSTEMS DIVISIOK. ALQUEUIERQUE DPERATIONS

BOREHOLE LOG (ROCK)

Page 1 of 2

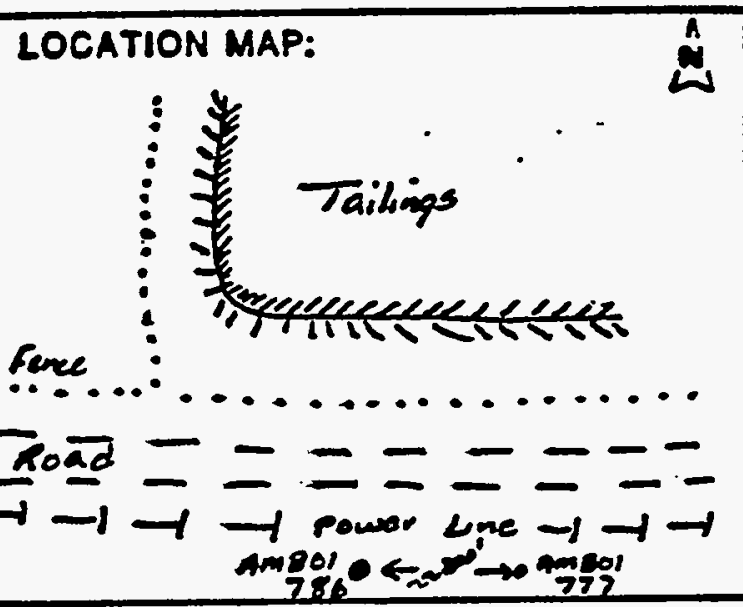

SITE ID: AMBOI

LOCATION 1D: 786

SITE COORDINATES (ft.):

$N$ NK

GROUND ELEVATION (ft. MSL): NW.

DRILLING METHOD: Patory - Gear Bit

DRILLING CONTR.: Stentart Brothers

DATE STARTED: $\frac{a / m / 85}{2}$

DATE COMPLETED:

FIELD REP.: - Boger talland

\begin{tabular}{|c|c|c|}
\hline \multicolumn{3}{|c}{ GROUNDWATER LEVELS } \\
\hline DATE & TIMA & DEPTH (f1.) \\
\hline & & \\
\hline & & \\
\hline
\end{tabular}

LOCATION DESCRIPTION

SITE CONDITION

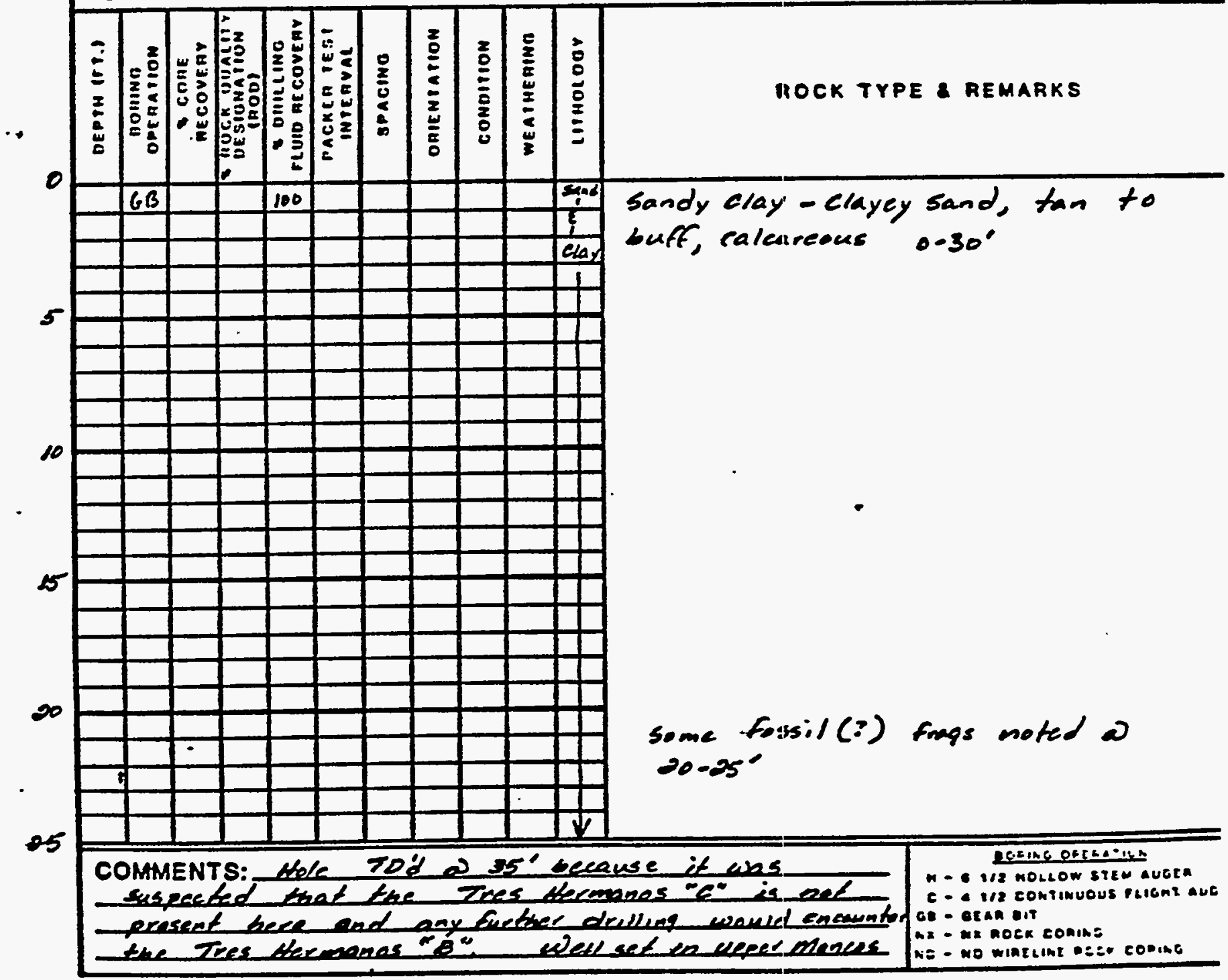

JEG-AL-ENG-2A (4/8S) 


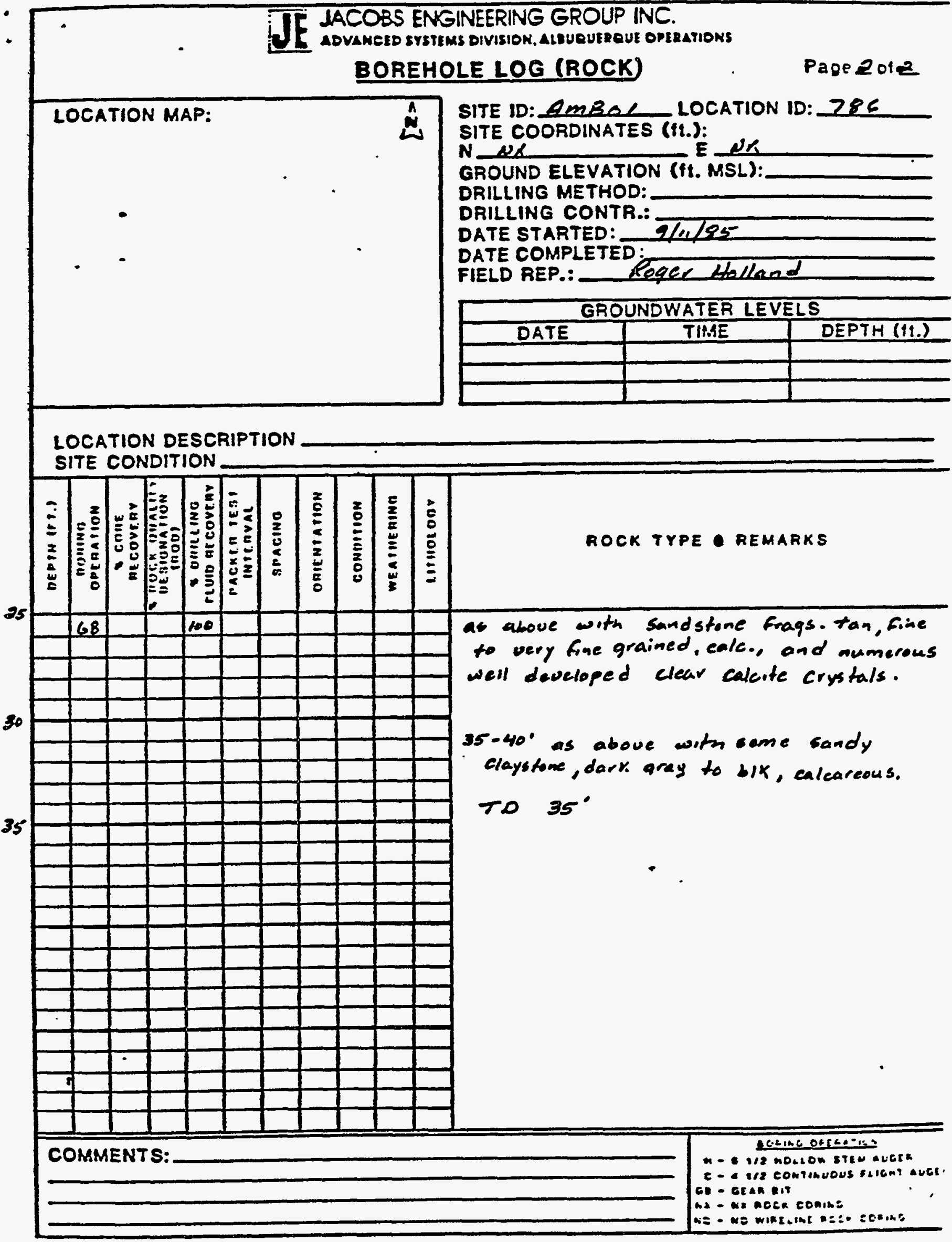

JEG-AL-ENG-2A (4IB5) 


\section{TT MCOSS BNGANERIVG GROUP NK.

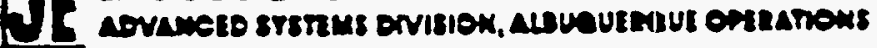

\section{WELL COMPLETION RECORD}

SITE ID: AMBOI LOCATION ID: 786 DATE INSTALLED: $q / 1 / 85$ APPROX. SITE COORDINATES:(FT.) N NK E NR

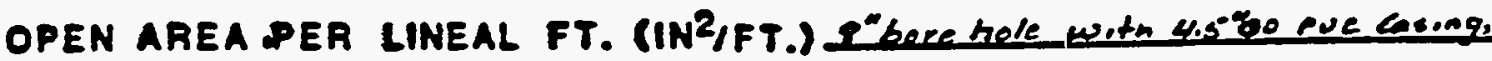
FORMATTION OF COMPLETION: UPeer meaces/alwiel TrCI? FIELD-REP.: Reger fllland DRILLER: stewart Brothers

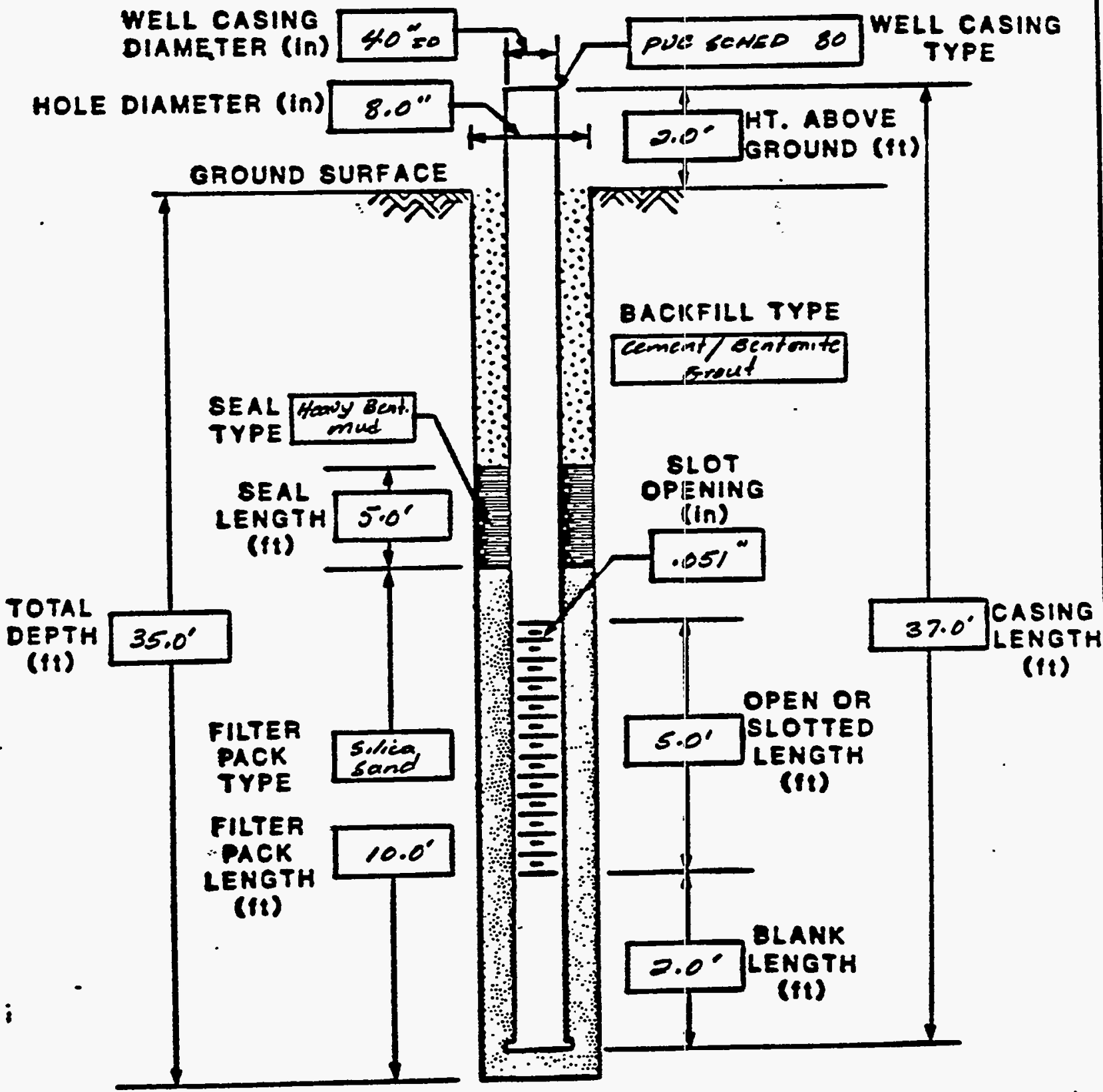

COMMENTS:

The seal leneth wes encreased feom $2.0^{\circ}$ to $5.0^{\prime}$

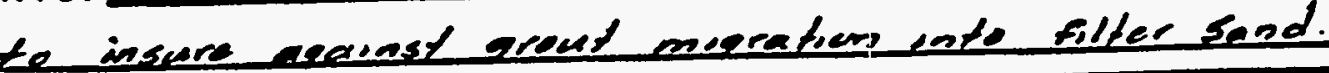




\section{DE MOOBS BNEN EERTVG EROUP NC. BOREHOLE/WELL CONSTRUCTION LOG}

SITE ID: AMBOL LOCATION ID: 286 FIELD REP: Peger Halland APPROX. 8ITE COORDINATES (FT.): $N$ AKK GROUND ELEVATION (FT. MSL): NK BOREHOLE SUMMARY

DRILLER: Stemenet Brothers

RIG TYPE: Gardner Denver is W (Botary)

\begin{tabular}{|c|c|c|c|}
\hline BIT TYPE & $\begin{array}{l}\text { HOLE. } \\
\text { DiA }\end{array}$ & $\begin{array}{c}\text { END } \\
\text { DEPTH }\end{array}$ & $\begin{array}{l}\text { FLUID } \\
\text { TYPE }\end{array}$ \\
\hline 4aphos & $8.0^{\circ}$ & $35.0^{\prime}$ & Bentugho \\
\hline & & & \\
\hline & & & \\
\hline & & & \\
\hline
\end{tabular}

\begin{tabular}{|c|c|c|c|}
\hline $\begin{array}{l}\text { CASING } \\
\text { TYPE : }\end{array}$ & DESCRIPTION & $\left|\begin{array}{c}p i A_{0} \\
(\text { in. })\end{array}\right|$ & $\begin{array}{l}\text { END } \\
\text { DEPTH } \\
(11 .)\end{array}$ \\
\hline$B$ & PUE SEHED. 80 & $4 \cdot 0^{\prime \prime}$ & $30.0^{\circ}$ \\
\hline 5 & pue .051" slotted & $4.0^{\circ}$ & $35.0^{\circ}$ \\
\hline$B$ & PuC scheo. 80 & $4.0^{\circ}$ & $37.0^{\circ}$ \\
\hline$p$ & 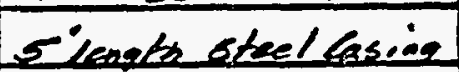 & $8.0^{41}$ & 3.08 \\
\hline & & & \\
\hline & & & \\
\hline & & & \\
\hline & & & \\
\hline
\end{tabular}

- P-Proloctive S-8eroen O-Elank O-Opon A-Mono A Depin from Top of Cesing

\begin{tabular}{|c|c|c|}
\hline \multicolumn{3}{|c|}{ WELL CONSTRUCTION } \\
\hline $\begin{array}{l}\text { TYPE } \\
\text { CODE }\end{array}$ & DESCRIPTION & D(t..) \\
\hline$B$ & Cement/Bentonite Grout & $20.0^{\circ}$ \\
\hline 5 & Heavy Bentonite mud & $25.0^{\prime}$ \\
\hline$E$ & solece Sand & $35.0^{\circ}$ \\
\hline & - & \\
\hline$T$ & & \\
\hline & & \\
\hline-1 & & \\
\hline 10 & Eekin & \\
\hline$-D$ & oth from grount surtaet & \\
\hline
\end{tabular}

E NK COMPLETION DATE: $2 / 11 / 55$ CONSTRUCTION TIME LOG

\begin{tabular}{|c|c|c|c|}
\hline \multicolumn{4}{|c|}{ CONSTRUCTION TIME LOG } \\
\hline \multirow{2}{*}{ ACTIVITY } & \multicolumn{2}{|c|}{ START } & \multirow{2}{*}{$\begin{array}{l}\text { END } \\
\text { TIMEE }\end{array}$} \\
\hline & DATE & TIME & \\
\hline RILLING & $9 / 11 / 85$ & $2: 18 \mathrm{gm}$ & $2055 \mathrm{pm}$ \\
\hline CASING & $9 / 10 / 85$ & $3: 50 \mathrm{pm}$ & $4: 04 \mathrm{pm}$ \\
\hline FILTER PACK & $9 / 11 / 85$ & 4:10 pom & $4: 28 \mathrm{pm}$ \\
\hline SEAL & $9 / 10 / 85$ & $4: 29 \mathrm{pm}$ & $5: 05 \mathrm{pm}$ \\
\hline BACKFILL & $9 / 10 / 8 s^{-}$ & $4: 57 \mathrm{rm}$ & 6.808\%a \\
\hline DEVELOPMENI & $9 / 13 / 65$ & 2:05 $\mathrm{Am}$ & $11: 08 \mathrm{Am}$ \\
\hline $\begin{array}{l}\text { OTHER } \\
\text { Probing. }\end{array}$ & ofo/ss & $3: 1$ spm & $3=4800$ \\
\hline \multicolumn{4}{|c|}{ WELL DEVELOPMENT } \\
\hline $\begin{array}{l}\text { Flush \& b } \\
8: 33 \text { am } \\
\text { Blow : se: } \\
\text { set: } 8: 0 \\
\text { Blow: } 8: 5 \\
\text { flubh } 5 \text { s } \\
\text { Blow dry } \\
\text { let set }\end{array}$ & $\begin{array}{l}\text { ow hole } \\
-8: 38 \\
-8: 50 \\
0-9: 00 \\
\text { une } \\
9: 20-\end{array}$ & $\begin{array}{l}\text { to clean } \\
\text { in } \\
\text { m No me } \\
\because 30 \times m\end{array}$ & until \\
\hline $\begin{array}{r}\text { COMMENTS: } \\
\text { ELUS } \\
\end{array}$ & $\frac{\text { Blow } 9}{4 y \text { lotie }}$ & $\begin{array}{l}30-9: 35 \\
\text { water p } \\
0 \text { till }\end{array}$ & $\frac{\text { - pm }}{\text { rodued }}$ \\
\hline Basl & $0: 50-11$ & ios an & \\
\hline $\begin{array}{l}\text { atole } \\
\text { anese }\end{array}$ & $=4$ & ede & \\
\hline
\end{tabular}
JEO-AL-ENO-1 (STS4) 
WELL 787 LOGS 
IF IACOBS ENGINEERING GROUP INC.

ADVANELD STSTIMS DIVISION. ALQUQULREUE OPERATIONS

BOREHOLE LOG (ROCK)

Page 1 of 4

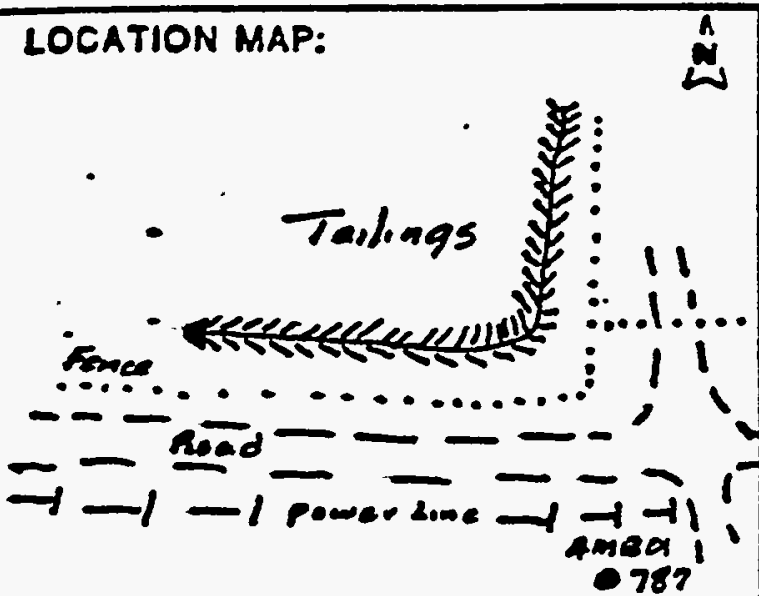

SITE ID: AMEOI LOCATION ID: 787

SITE COORDINATES (ft.):

$N$ NX

GROUND ELEVATION (ft. MSL): NK

DRILLING METHOD: Retary - Gear B.t

DRILLING CONTR.: Stewart Rothers

DATE STARTEL: $2 / 12 / 85$

DATE COMPLEUED:

FIELD REP.: Aleger thelland

\begin{tabular}{|c|c|c}
\hline \multicolumn{2}{|c|}{ GF OUNDWATER LEVELS } \\
\hline DATE & TIMAE & DEPTH (II.) \\
\hline & & \\
\hline & & \\
\hline & & \\
\hline
\end{tabular}

LOCATION DESCRIPTION Due suth of the Sa East Cor at Truelings pile SITE CONDITION Leves kandy with weed cover

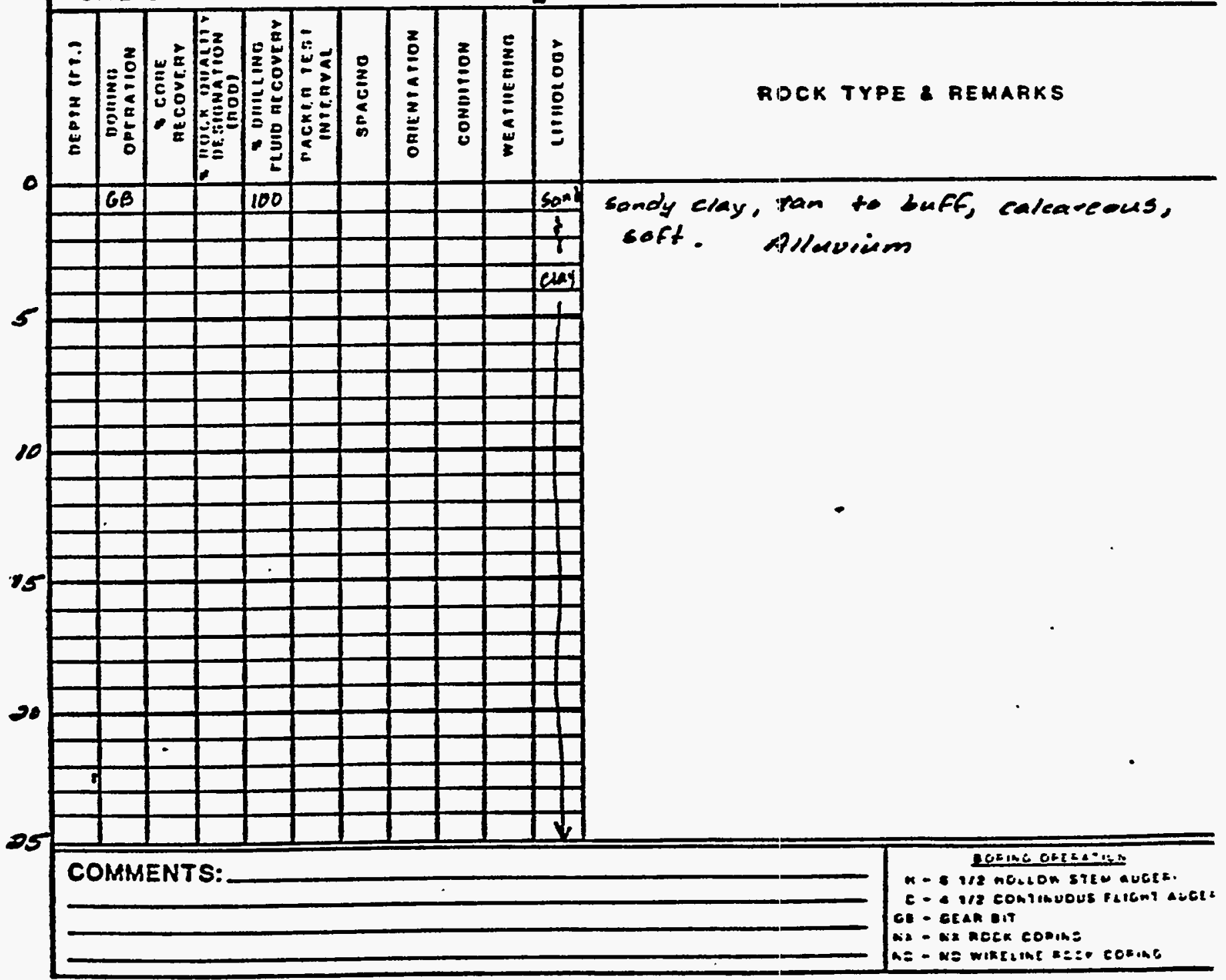

JEG-AL-ENG-2A (4/85) 
IACOBS ENGINEERING GROUP INC.

L $\triangle$ DVANEED SYSTEMS DIVISIOK. ALLUEUERQUE OPIRATIONS

BOREHOLE LOG (ROCK)

Page 2 or 4

LOCATION MAP:

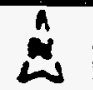

SITE ID: AmBQI

LOCATION ID: 782

SITE COORDINATES (ft.):

N E

GROUND ELEVATION (fi. MSL):

DRILLING METHOD:

DRILLING CONTR.:

DATE STARTED:

DATE COMPLETED

FIELD REP.:

Pegen

\begin{tabular}{|c|c|c|}
\hline \multicolumn{3}{|c|}{ GROUNDWATER LEVELS } \\
\hline DATE & TIME & DEPTH (II.) \\
\hline & & \\
\hline & & \\
\hline & & \\
\hline
\end{tabular}

LOCATION DESCRIPTION

SITE CONDITION

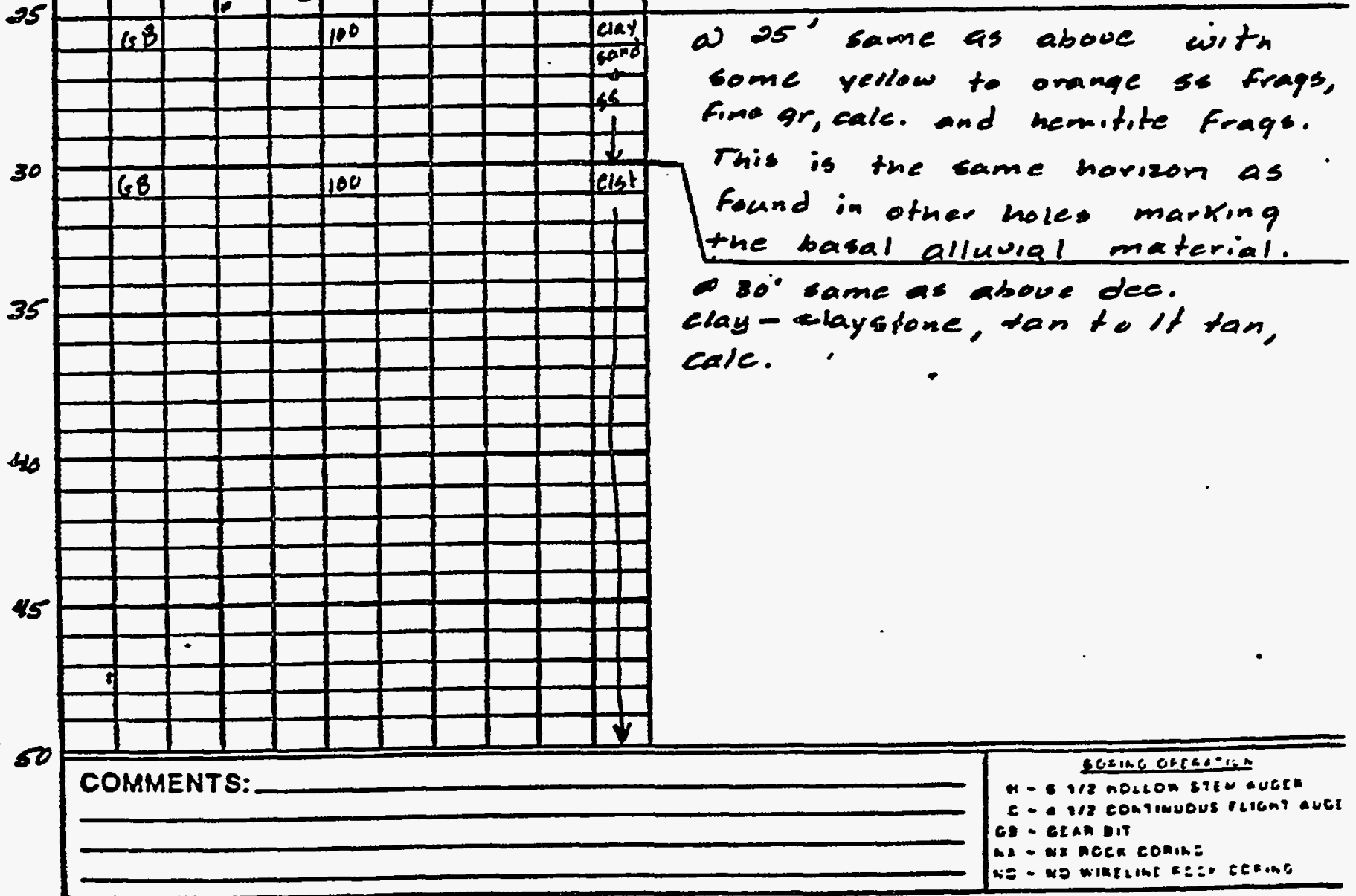

ROCK TYPE \& REMARKS

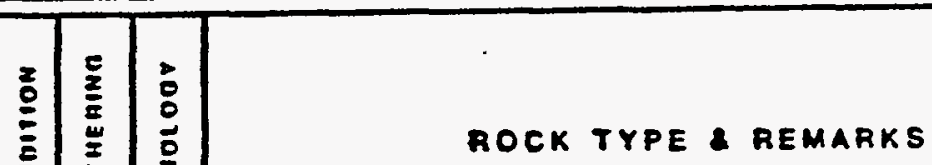

a $25^{\prime}$ same as above with come yedlow to orange so frags, fine gr, cale. and hemitite frags. This is the same horizon as in ofher holes marking tasal alluviol material - 30. came as aboue dec. elay - Elaysfone, tan to if tan cale. 


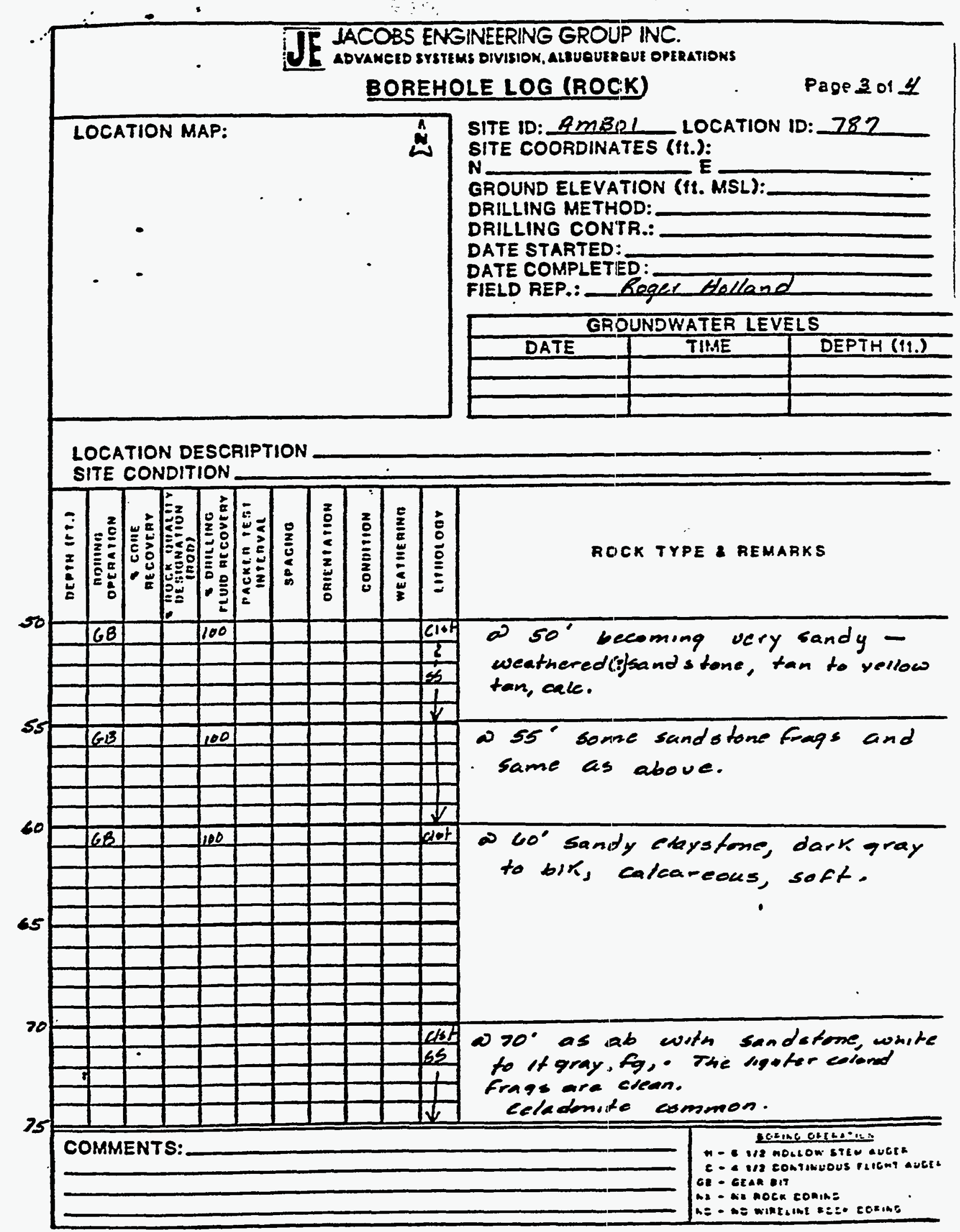

JEG-AL-ENG-2A (4/85) 


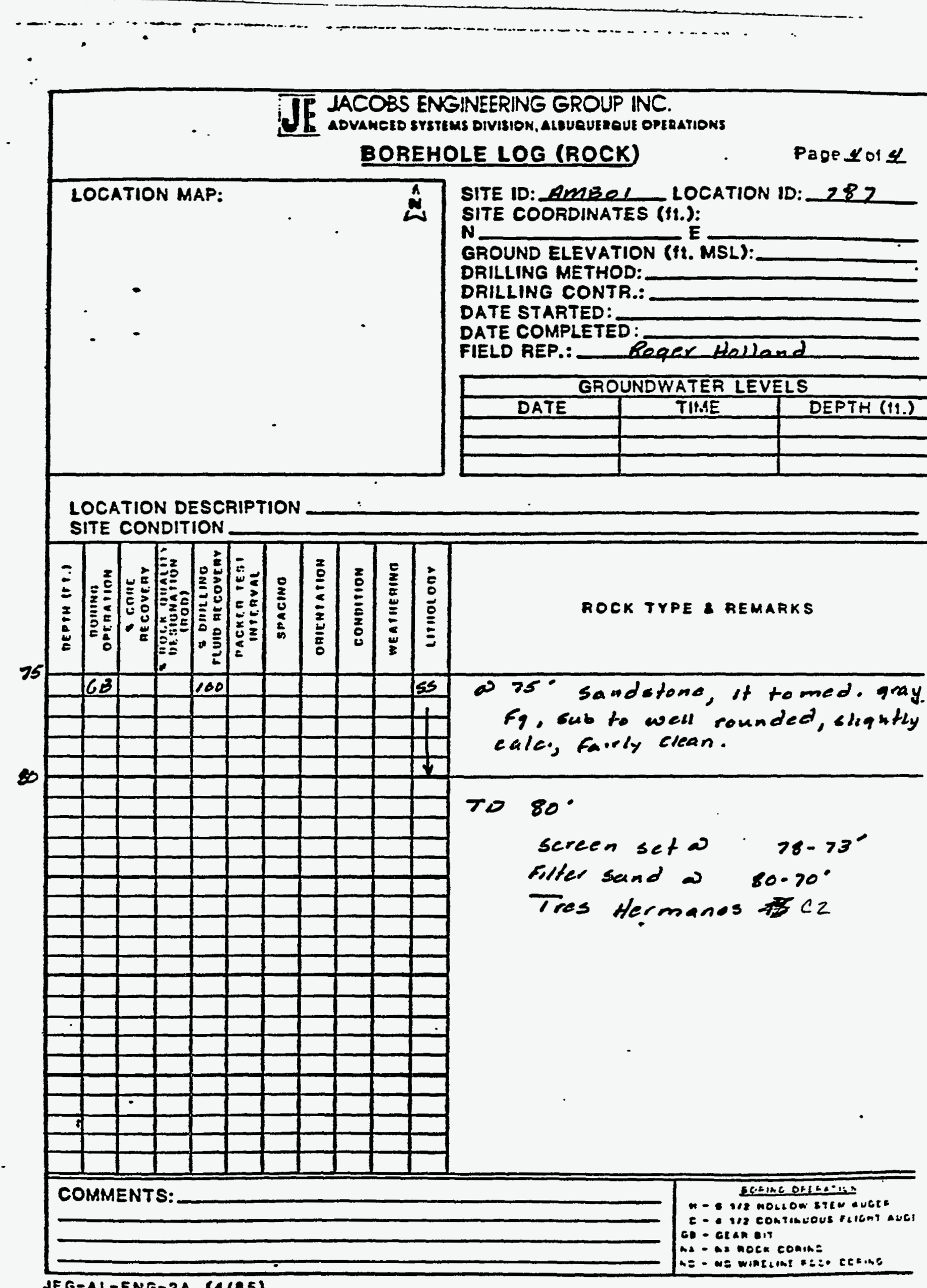

JE G-AL-ENG-2A_(4185) 


\section{$\sqrt{1}$ MCOzS BNGA}

\section{WELL COMPLETION RECORD}

SITE ID: AMBOI LOCATION ID: 787 APPROX. SITE COORDINATES:(FT.) $N$ PK

DATE INSTALLED: $\% / 12 / 85$

FORMATION OF COMPLETION: Tres dermanes $B$

FIELD-REP.: Beger Helland DRILLER: Stewart Brethers

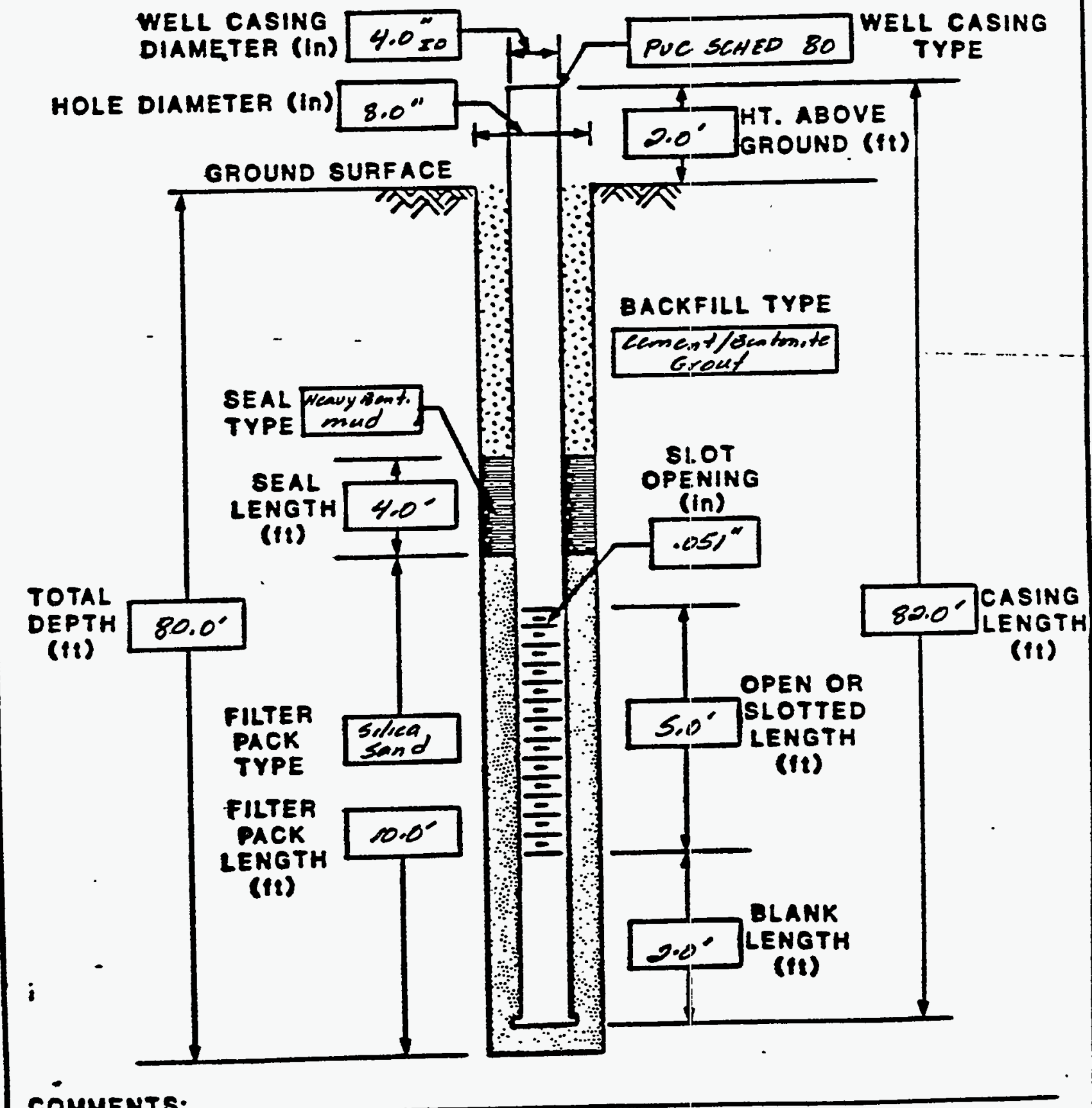

COMMENTS: 


\section{TT MCOSS BNENEERTE GROUP WC.

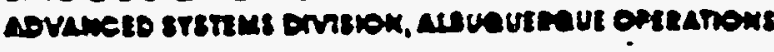 BOREHOLE/WELL CONSTRUCTION LOG}

SITE ID:AmBol LOCATION ID: 787 FIELD REP: Aleger blelland APPROX. BITE COORDINATES (FT.): N NK E NK GROUND ELEVATION (FT. MSL): NK COMPLETION DATE: $1 / 12 / 35$

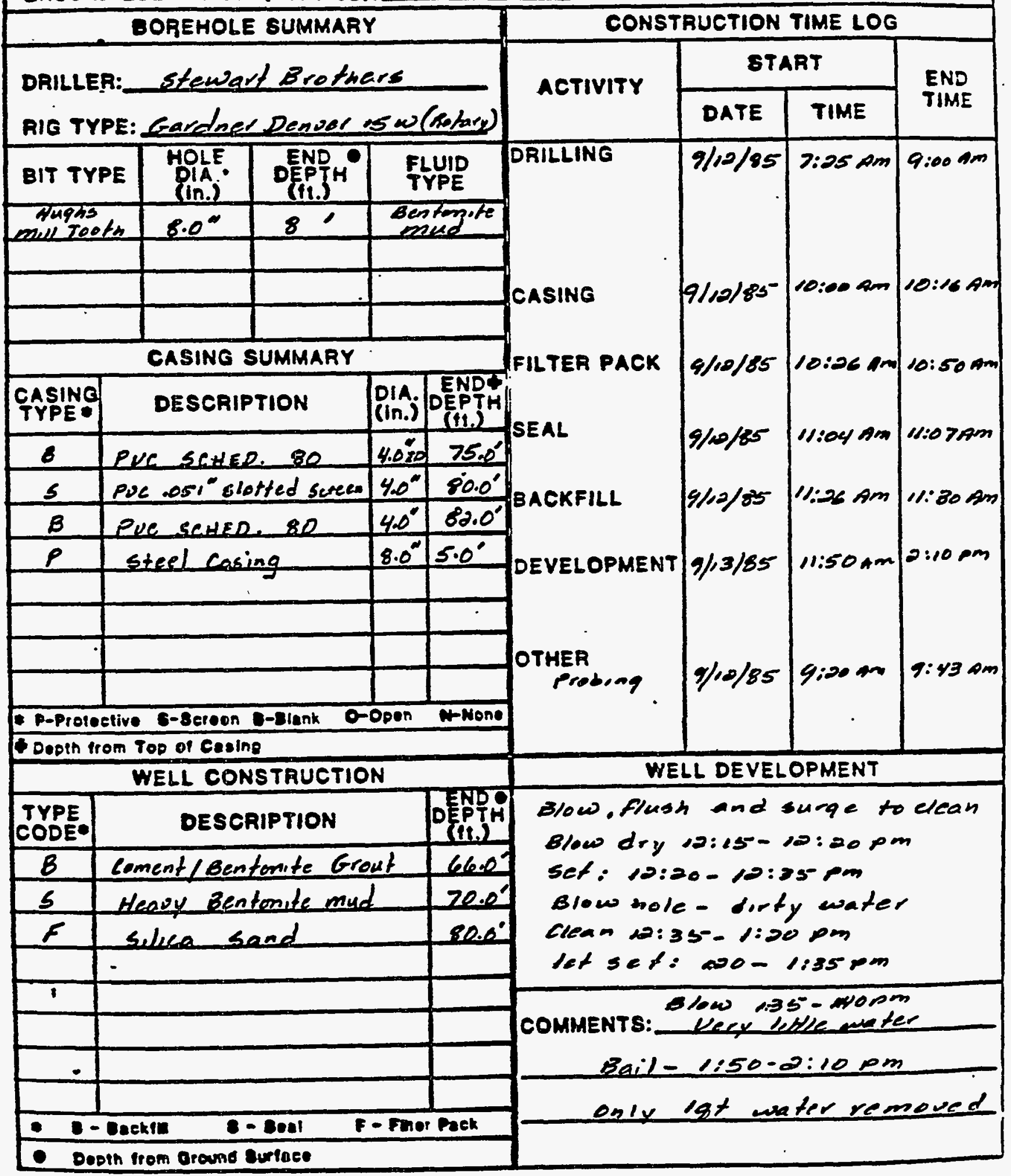


WELL 790 LOGS

, 
PROJECT AMBROSLA LAKE SITE, NEW MEXICO

Pagel of 1

JOB NO. AMBOI DATE 09/22/85
SURFACE ELEVATION 7002.90

LOG OF WELL BORING NO.

790

TOP OF FLIER PACK 21.00

WELL CASING TIPE 4.0-IN.SCHED.80 PVC

COMPLETION

TRES HERMANOS C

TOTAL DEY'TH 30.0 feet

RIG TIPE

BORNG TFE R 5613590 E5059020

\begin{tabular}{|c|c|c|c|c|c|}
\hline Depth & $\begin{array}{l}\text { Well } \\
\text { Con. }\end{array}$ & Remarks & Lithology & USCS & Visual Classification \\
\hline
\end{tabular}

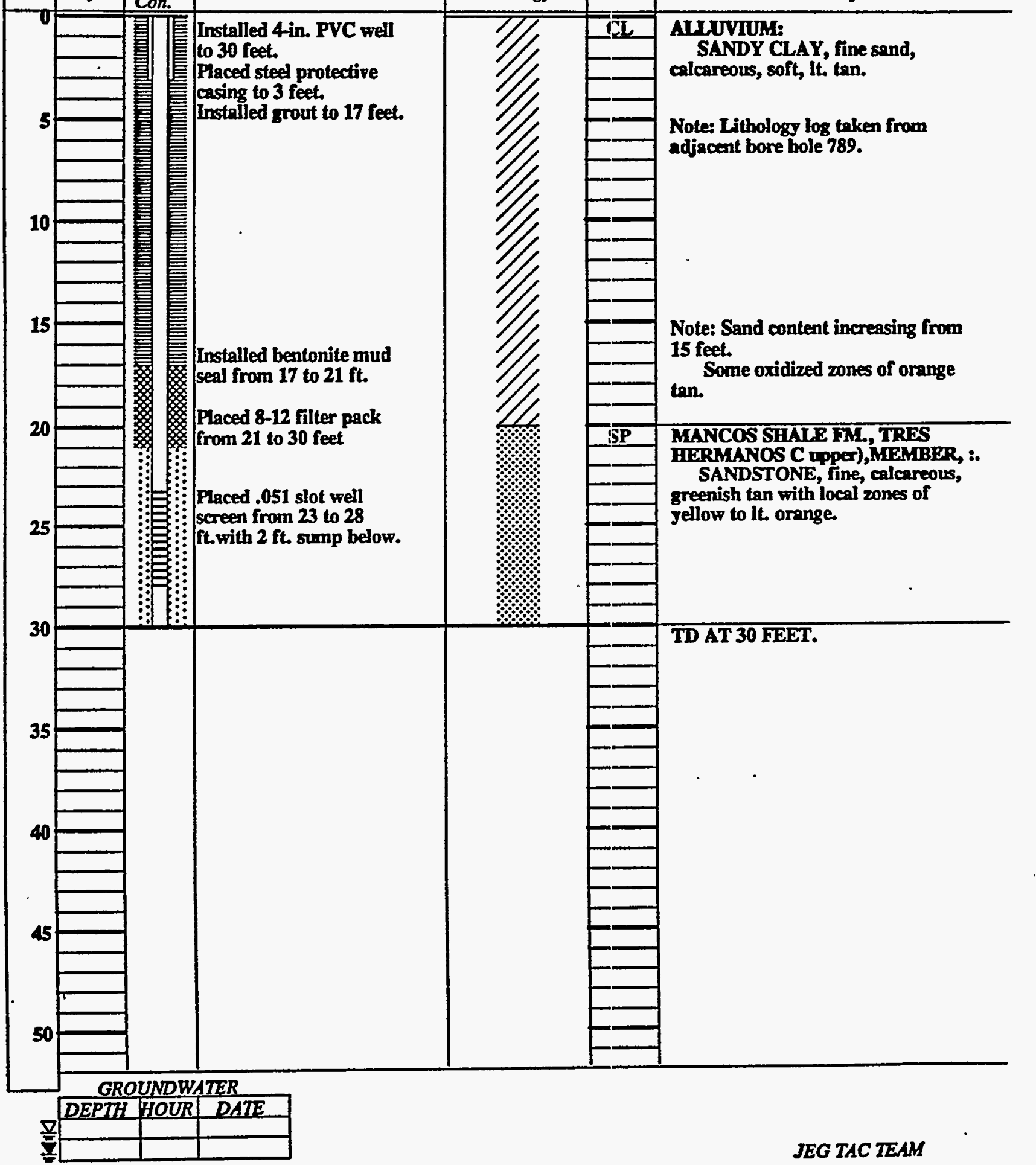




\section{TT MCOSS ENGNERTHG CROUP NC.

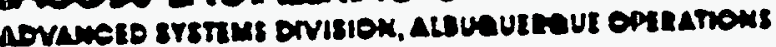

\section{WELL COMPLETION RECORD}

SITE ID: AmBOL LOCATION ID: DATE INSTALLED: $9 / 23 / 85$ APPROX. SITE COORDINATES:(FT.) $N$ NK

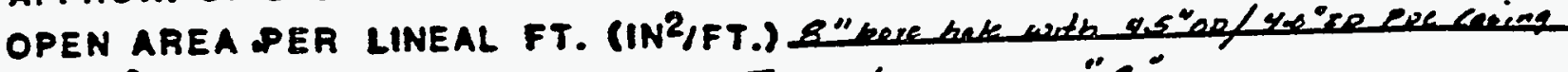
FORMATION OF COMPLETION:

FIELD -REP.: Boger dellond Tees deremanes " $\mathrm{C}$ " DRILLER:

stewart Brethers

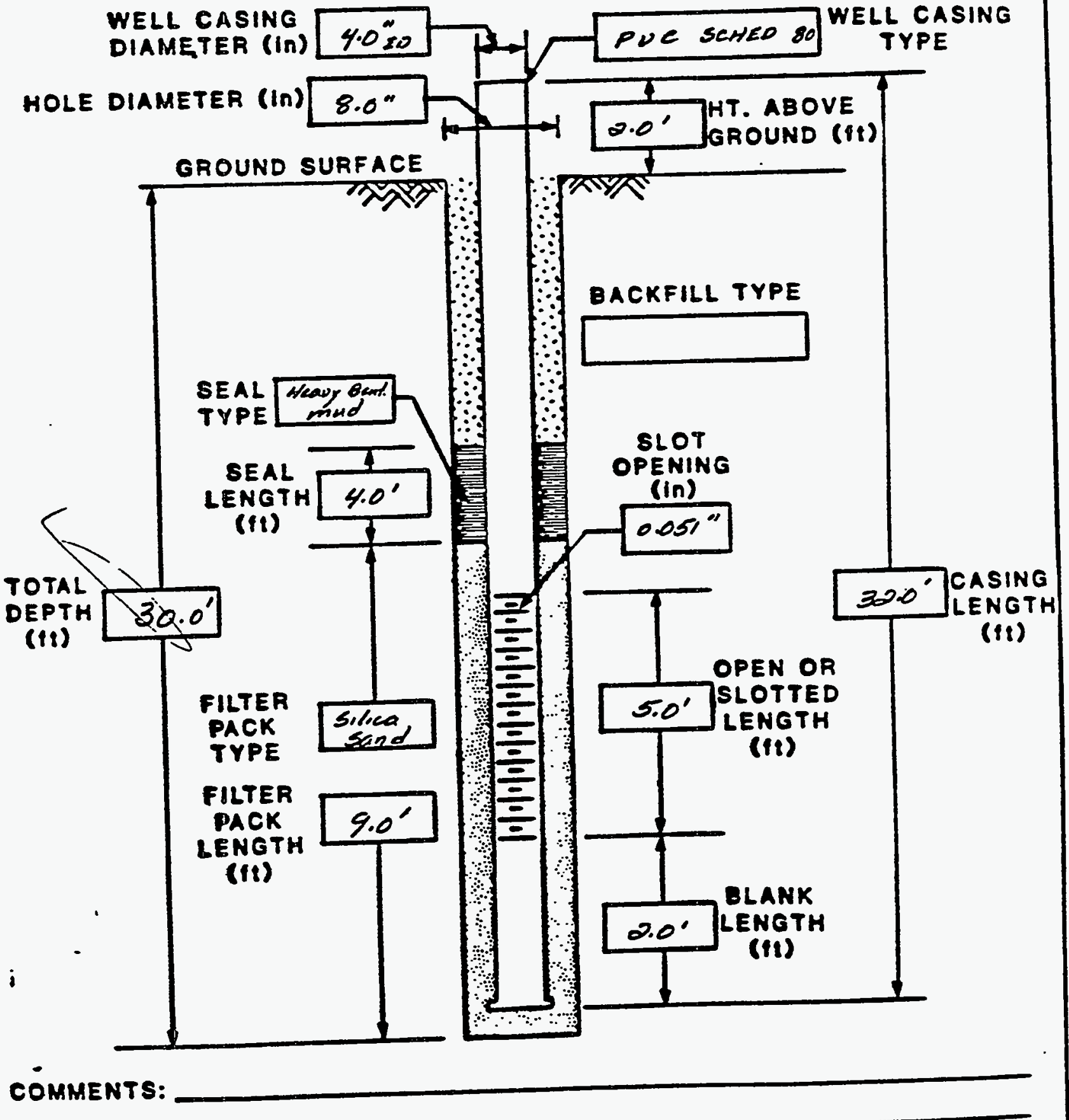




\section{TT ACOBS ENCNEERIVG GROUP MIC.

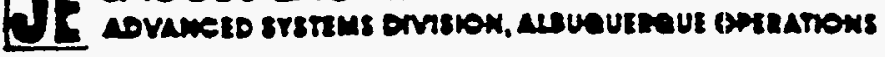 \\ BOREHOLE/WELL CONSTRUCTION LOG}

SITE ID:AMBOL LOCATION ID: 790 FIELD RIEP: Reger Holland

APPROX. BITE COORDINATES (FT.): N_NK

GROUND ELEVATION (FT. MSL): NK

COMPLETION DATE: $9 / 23 / 85$.

BOREHOLE SUMMARY

DRILLER: Stewart Brothers

RIG TYPE: Gardner Denver 15w (Rotary)

\begin{tabular}{|c|c|c|c|}
\hline BIT TYPE & $\begin{array}{c}\text { HOLE. } \\
\text { DIR. } \\
\text { (In.) }\end{array}$ & $\begin{array}{c}\text { END } \\
\text { DEPH } \\
\text { (P1.) }\end{array}$ & $\begin{array}{c}\text { FLUID } \\
\text { TYPE }\end{array}$ \\
\hline mughs & $8.0^{\prime \prime}$ & $30.0^{\prime}$ & Bentorite \\
\hline & & & \\
\hline & & & \\
\hline & & & \\
\hline & & & \\
\hline
\end{tabular}

CASING SUMMARY

\begin{tabular}{|c|c|c|c|}
\hline \begin{tabular}{|l|} 
CASINQ \\
TYPE
\end{tabular} & DESCRIPTION & 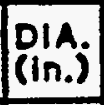 & $\begin{array}{c}\text { ENDW } \\
\text { DEPTH } \\
(\mathrm{fH} .)\end{array}$ \\
\hline$B$ & PUC SCHED 80 & $4.0^{\prime \prime}$ & 25 \\
\hline$s$ & PuC .05i" slotted & $4.0^{\circ}$ & $30^{\prime}$ \\
\hline$B$ & PUC SCH ED 80 & & $32^{\prime}$ \\
\hline$p$ & steel casing & $8.0^{\prime \prime}$ & $50^{\prime}$ \\
\hline & & & \\
\hline & & & \\
\hline & & & \\
\hline & & & \\
\hline
\end{tabular}

- P-Protective 6-8creen B-Slank O-Opon N-Mone

Gopth trom Top of cesing

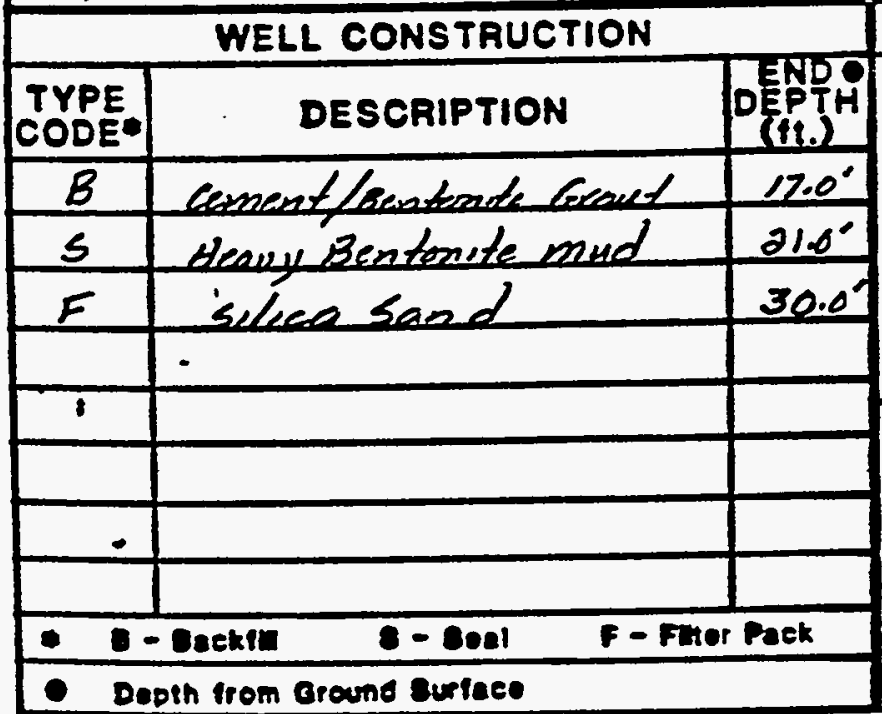

CONSTRUCTION TIME LOG

ACTIVITY

DRILLING

CASING

FILTER PACH:

SEAL

BACKFILL

DEVELOPMENT

OTHER Probiong

START

\begin{tabular}{|l|l|l|}
\hline DATE & END \\
\hline TIME & \\
\hline
\end{tabular}

\begin{tabular}{|l|l|l|}
\hline $9 / 20 / 86$ & $5: 30 \mathrm{pm}$ & $60: 55 \mathrm{pm}$ \\
\hline
\end{tabular}

\section{(9)}

$9 / 24 / 85$

$0: 20 \mathrm{pm}$ $9 / 25 / 85$

$7: 45 \mathrm{am}$

$6: 45 \mathrm{pm}$

ro: 20 an

9/23/85- 7:42000

$8: 13 \mathrm{Am}$

sble developed using portable aircompressor

we was contianously blown, Flusted, surged and bown dry for apprex. 7 hes. Hole wown dry and let set several fimes. Hole making somall amst. of cuater.

COMMENTS: 
WELL 792 LOGS 
PROJECT AMBROSL LAKE SITE, NEW MEXICO

Page 1 of 1

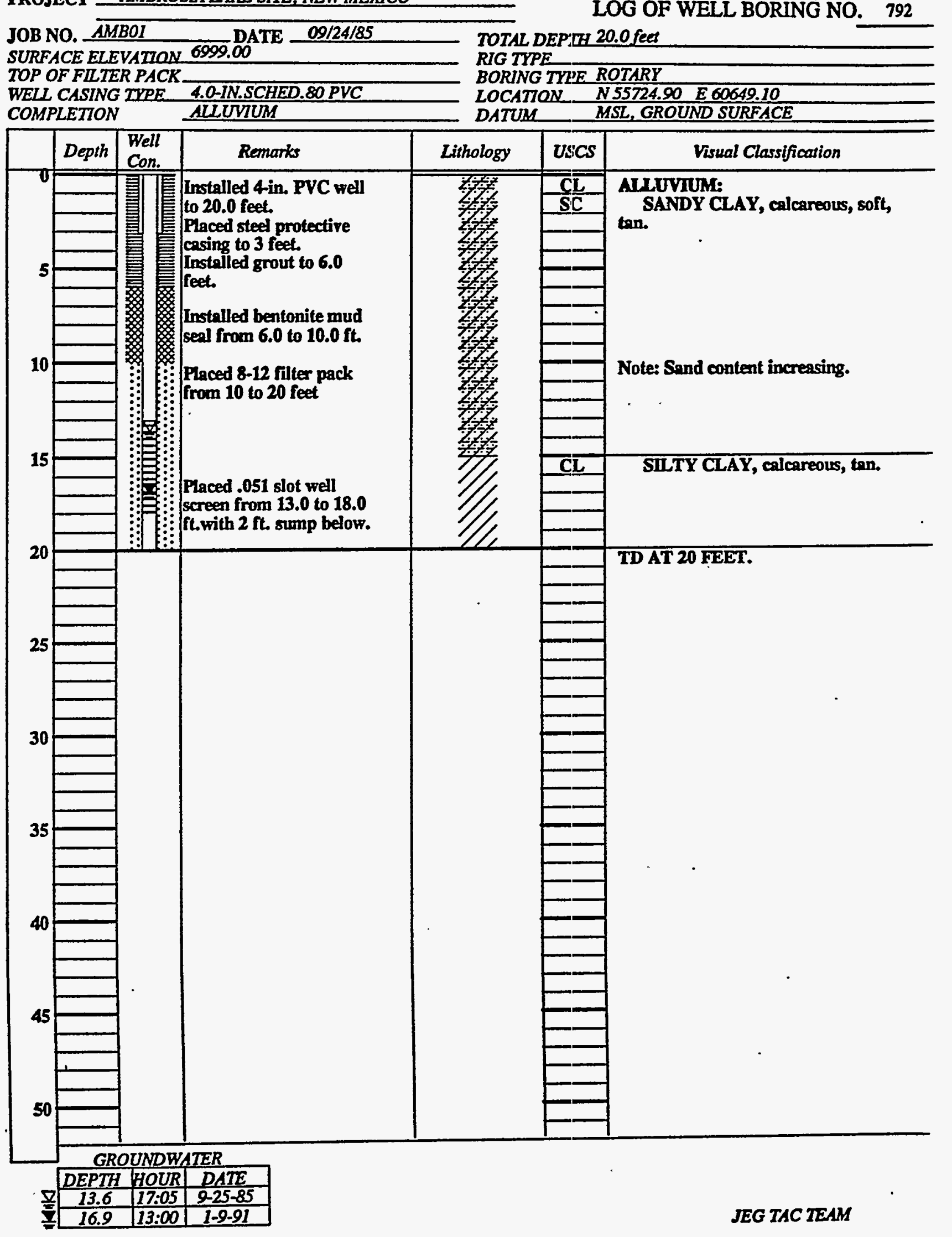




\section{TT MCOBS ENGNEERTE CROUP NK.

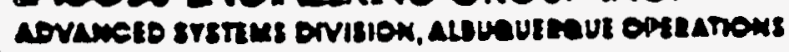

\section{WELL COMPLETION RECORD}

SITE ID:AMBOI LOCATION ID: 792 DATE INSTALLED: $9 / 24 / 85$ APPROX. SITE COORDINATES:(FT.) N NK E NK

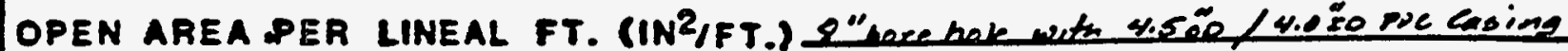
FORMATION OF COMPLETION: FIELD -REP.: Reger Hollond Alluerimen

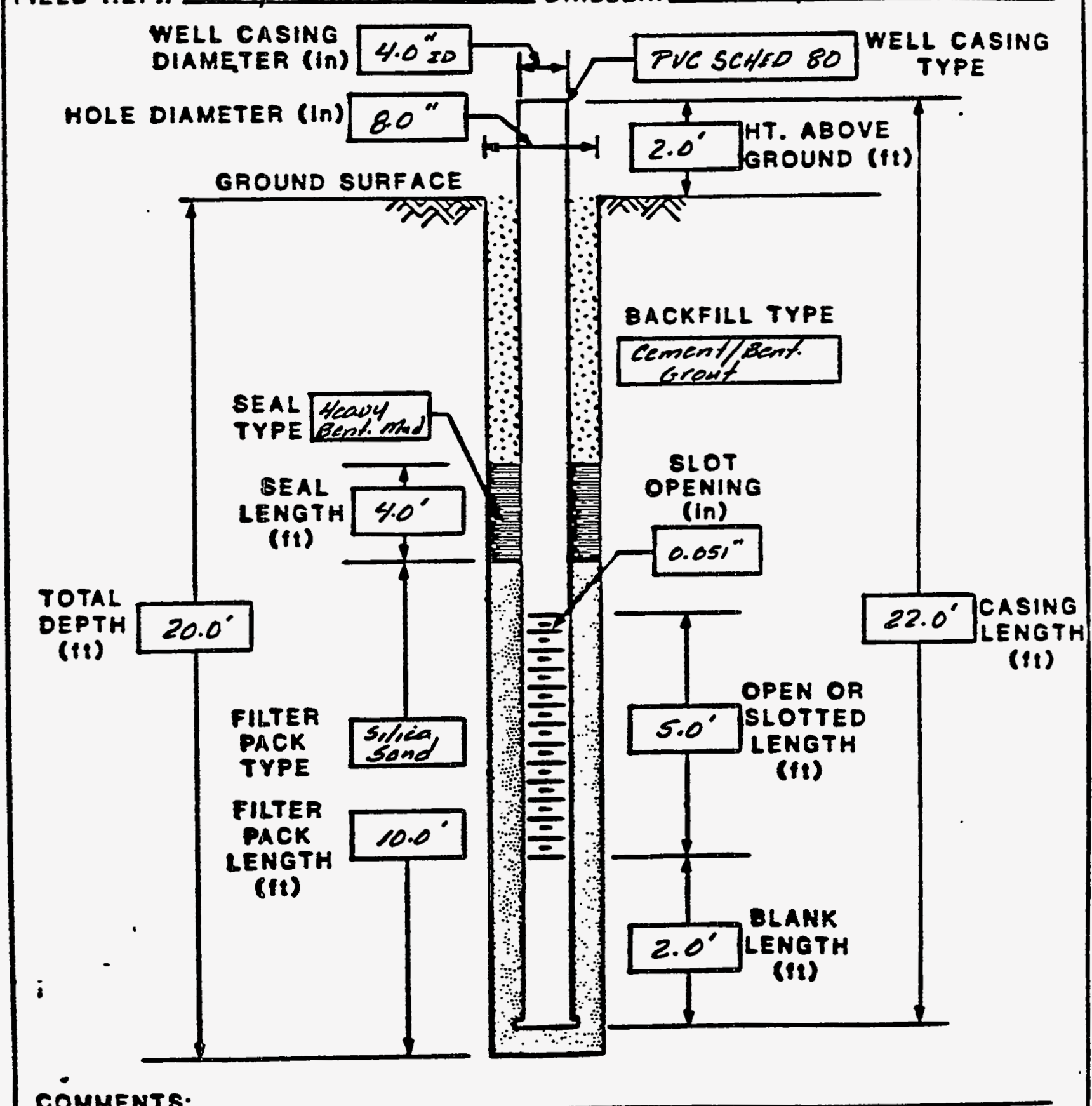

COMMENTS: 


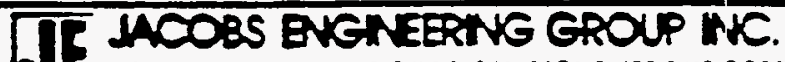

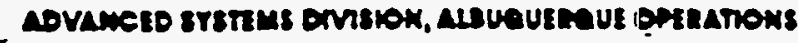 BOREHOLE/WELL CONSTRUCTION LOG}

SITE ID: AmBOL LOCATION ID: 792 FIELD REP: Reger Holland APPROX. BITE COORDINATES (FT.): N_NX N_E NK GROUND ELEVATION (FT. MSL): NK COMPLETION DATE: $9 / 24 / 85$

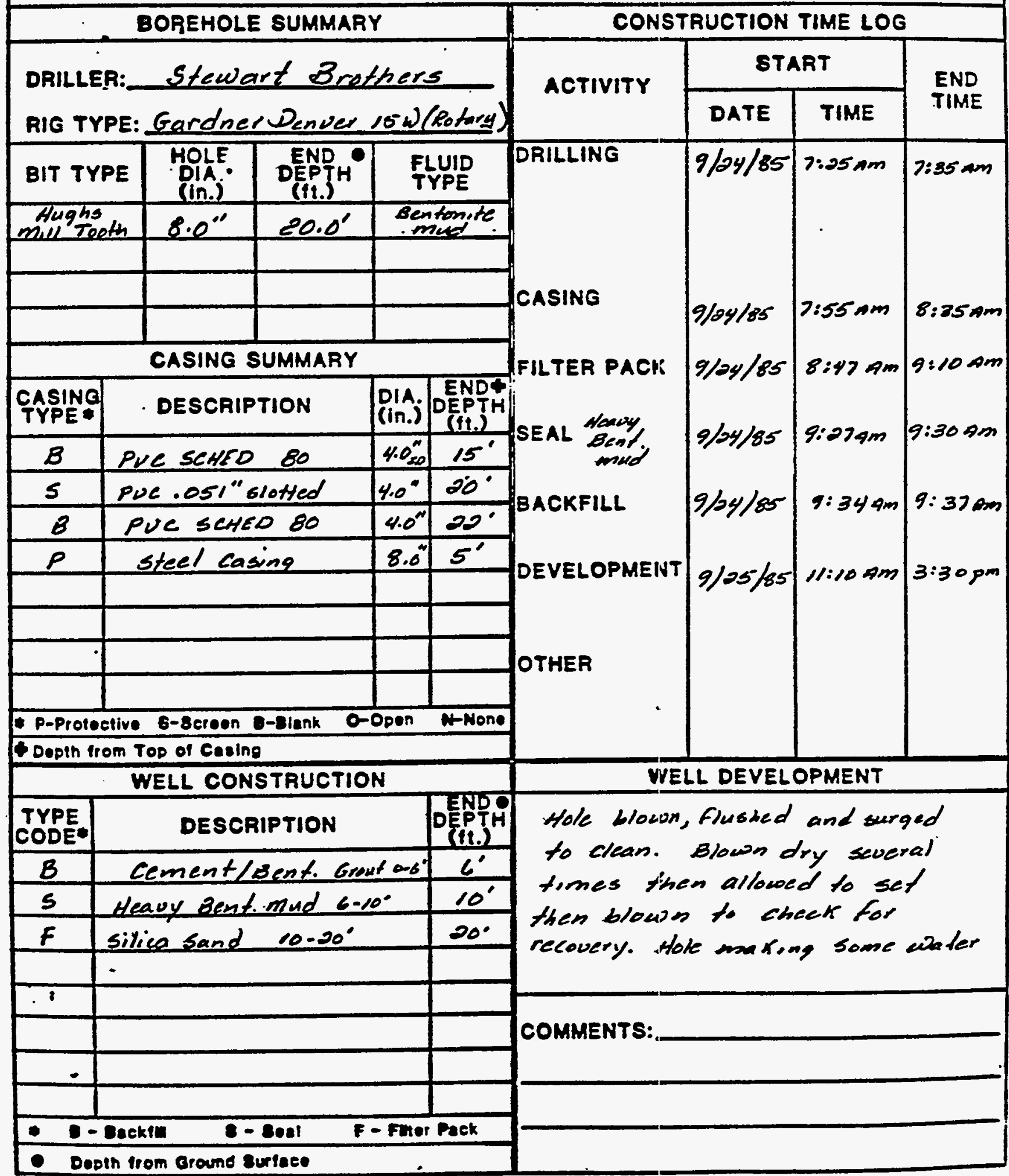


WELL 793 LOGS 


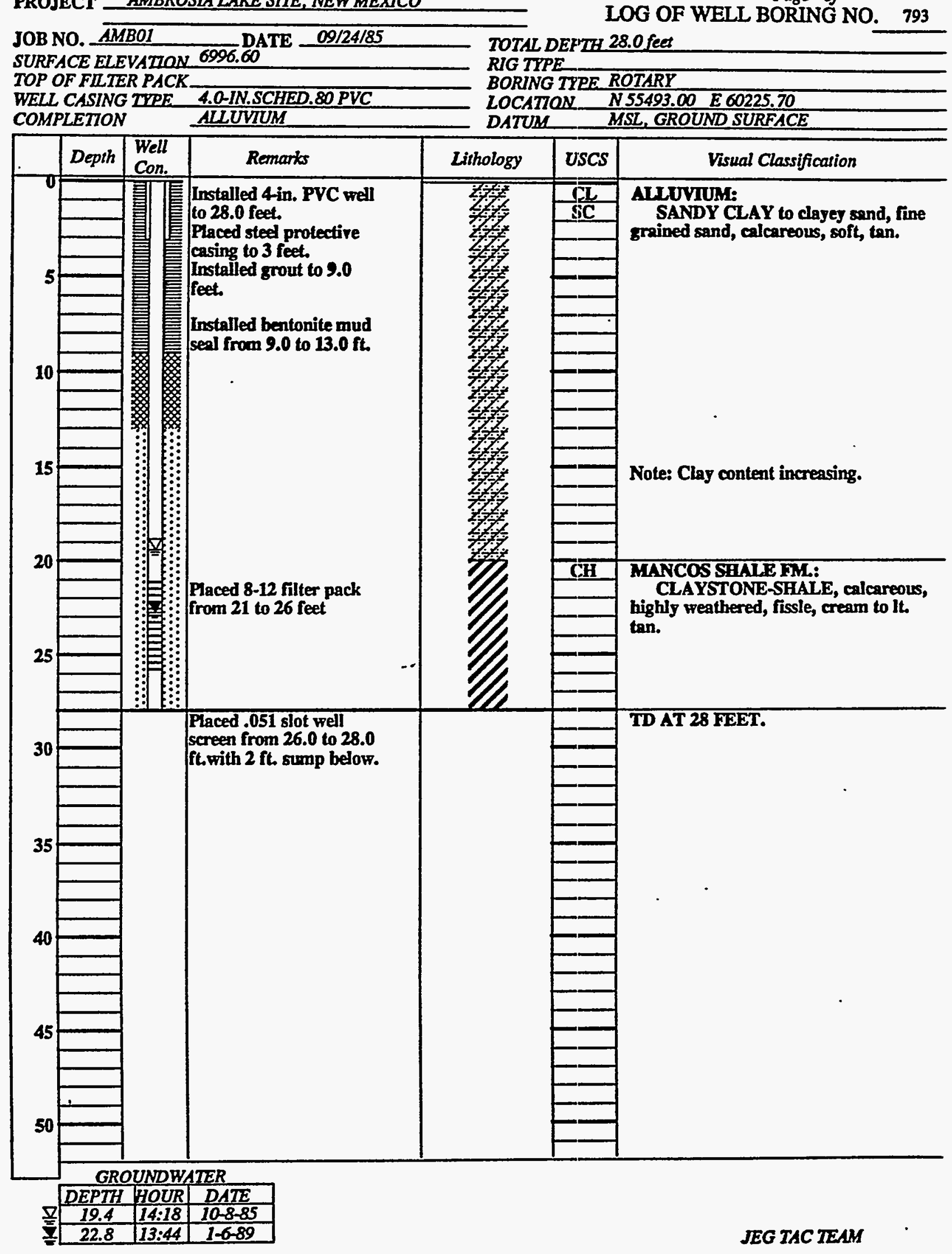




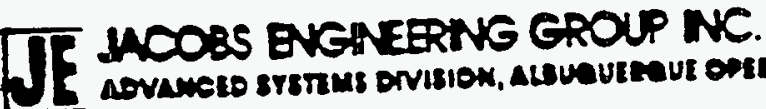

\section{WELL COMPLETION RECORD}

SITE 10: AMBOI LOCATION ID: 793

DATE INSTALLED: $9 / 24 / 85$ APPROX. SITE COORDINATES:(FT.) $N$ NK E NK

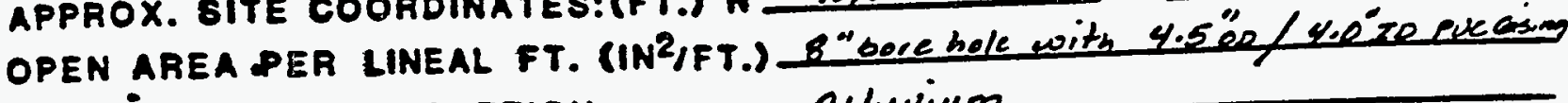
IFORMATION OF COMPLETION:

FIELD -REP.: Reger thalland Alleweum

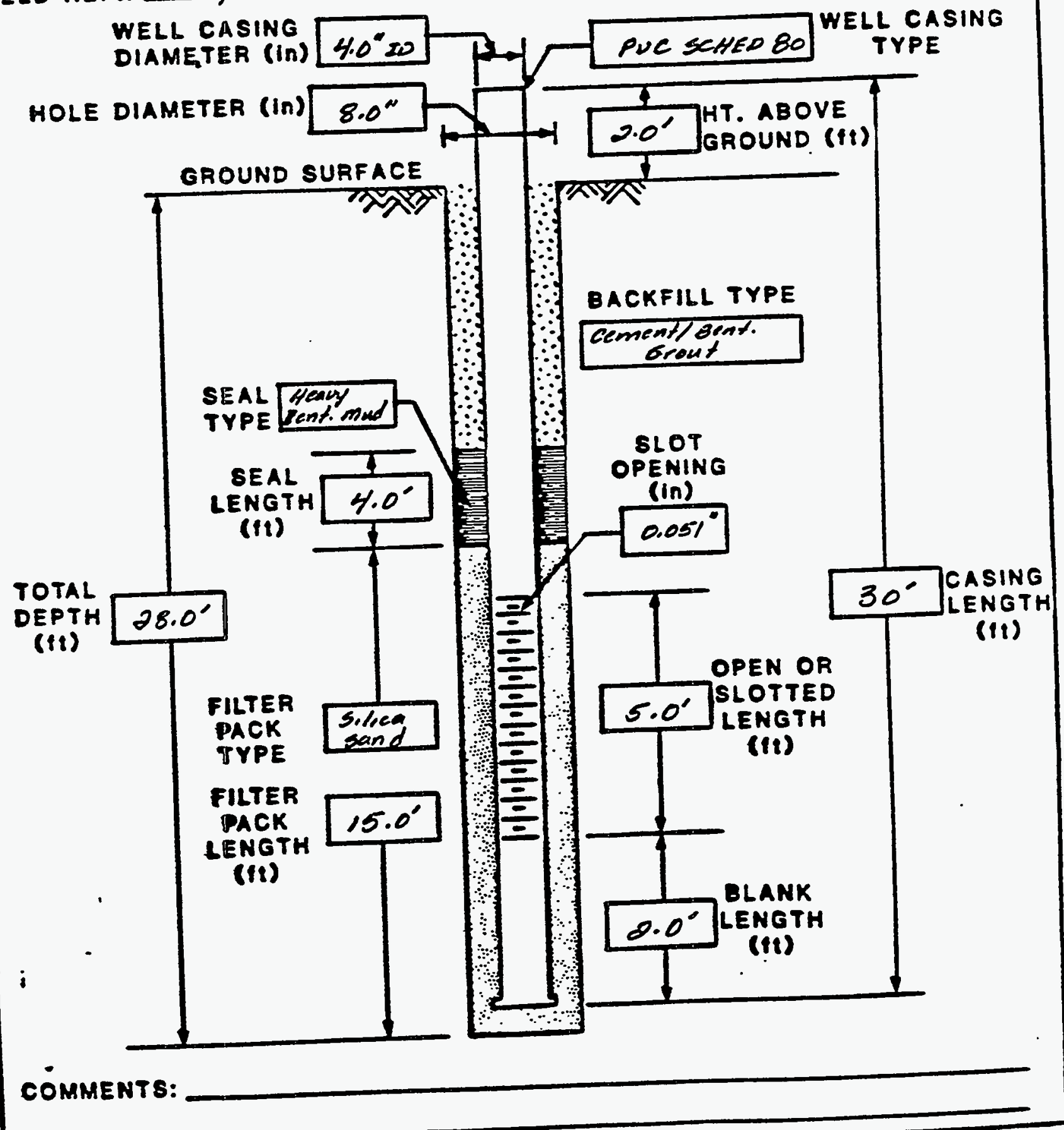




\section{TT ACOBS BNGN EERTV GROUP WN.

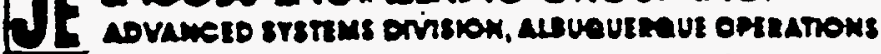 \\ BOREHOLE/WELL CONSTRUCTION LOG}

SITE ID: AMBOL LOCATION ID: 793 APPROX. BITE COORDINATES (FT.): $N$ NK GROUND ELEVATION (FT. MSL): NK BOREHOLE SUMMARY

DRILLER:_stewart Brothers

RIG TYPE: Gardner Denuel $15 \omega$ (Rotary)

\begin{tabular}{|c|c|c|c|}
\hline BIT TYPE & $\begin{array}{l}\text { HOLE } \\
\text { DIA. } \\
\text { (In.) }\end{array}$ & 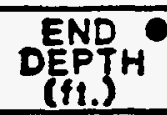 & $\begin{array}{l}\text { FLUID } \\
\text { TYPE }\end{array}$ \\
\hline mull footh & $8.0^{\prime \prime}$ & $28.0^{\prime}$ & $\begin{array}{l}\text { Bentomite } \\
\text { mud }\end{array}$ \\
\hline & & & \\
\hline & & & \\
\hline & & & \\
\hline
\end{tabular}

\begin{tabular}{|c|c|c|c|}
\hline $\begin{array}{l}\text { CASINO } \\
\text { TYPE }\end{array}$ & DESCRIPTION & \begin{tabular}{|} 
Din. \\
(in.)
\end{tabular} & $\begin{array}{l}\text { END中 } \\
\text { EPTH } \\
(14 .)^{2}\end{array}$ \\
\hline$B$ & PUC SCHED 80 & $4.0 \%$ & $23^{\circ}$ \\
\hline 5 & PUC 0.05 " slotted & $4.0^{11}$ & 28 \\
\hline B & PUC SCHED 80 & 4.07 & $30^{\prime}$ \\
\hline$p$ & steel casing & $8.0^{11}$ & $5.0^{\circ}$ \\
\hline & & & \\
\hline & & & \\
\hline & & & \\
\hline & & & \\
\hline & \multicolumn{3}{|l|}{ ve 6-8croen e-8iznk } \\
\hline
\end{tabular}

WELL CONSTRUCTION

\begin{tabular}{|c|c|c|}
\hline \begin{tabular}{l|} 
TYPE \\
CODEE
\end{tabular} & DESCRIPTION & $\begin{array}{l}\text { EEPP } \\
\text { Defi.) } \\
\text { (it.) }\end{array}$ \\
\hline$B$ & Gement/Bent broul a'to surfo & 2 \\
\hline .5 & Heavy Bent. mud $19-13^{\prime}$ & $13^{\prime}$ \\
\hline$E$ & silecesand 13-28' & $28^{\circ}$ \\
\hline & - & \\
\hline T & & \\
\hline & & \\
\hline-1 & & \\
\hline & & \\
\hline-10 & Decktil $\quad-$ - soal & \\
\hline
\end{tabular}

FIELD REP: Roger tolland E NK COMPLETION DATE: $9 / 24 / 85$ CONSTRUCTION TIME LOG

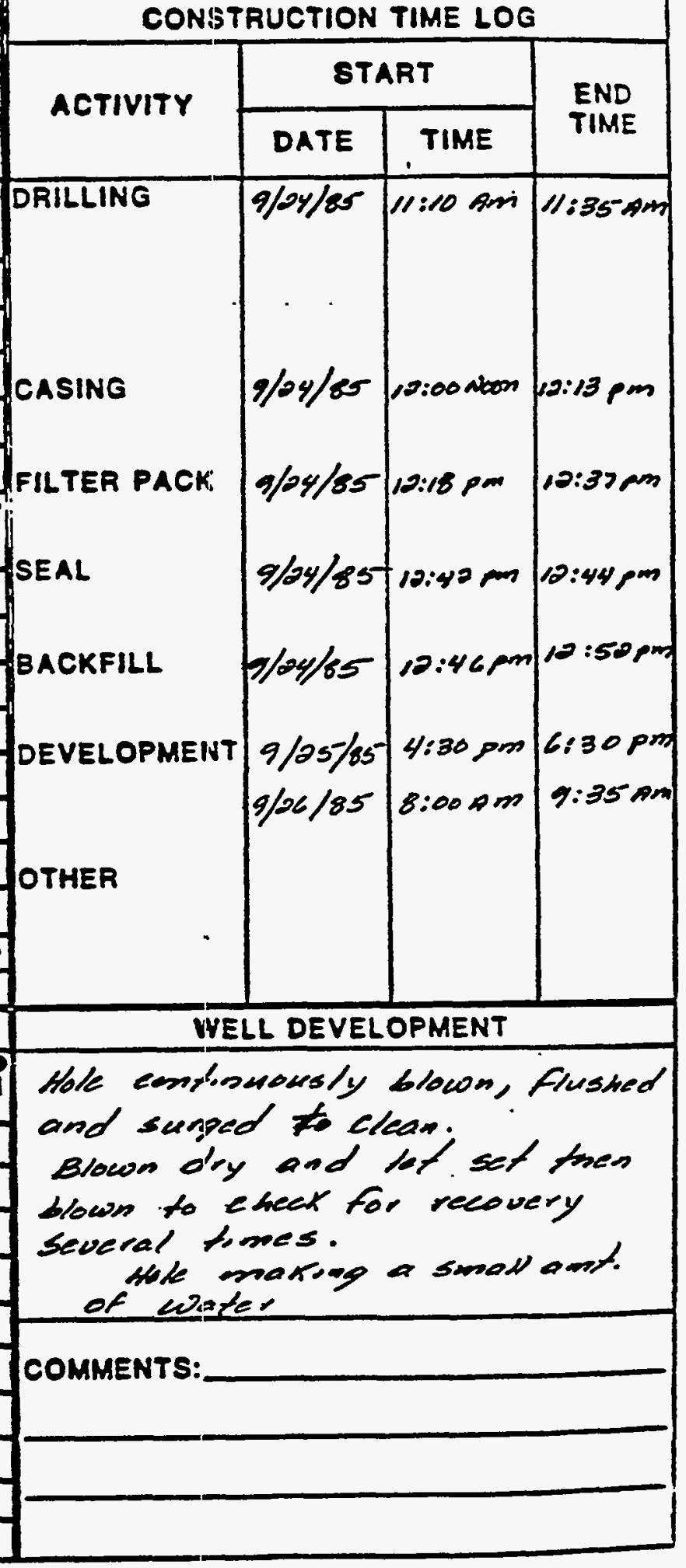


WELL 794 LOGS 


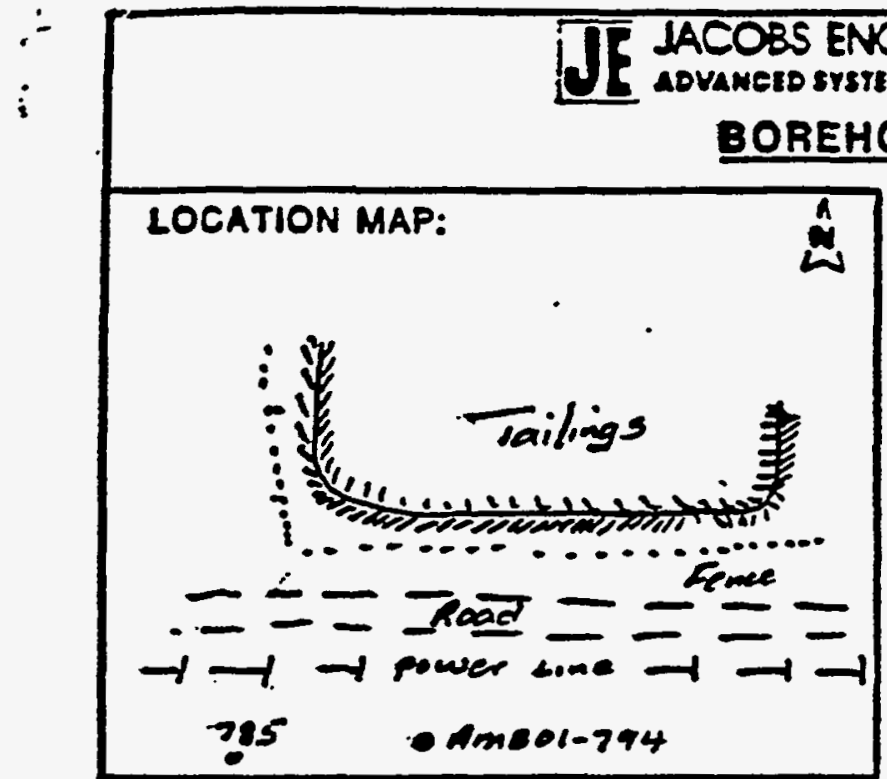

LOCATION MAP:

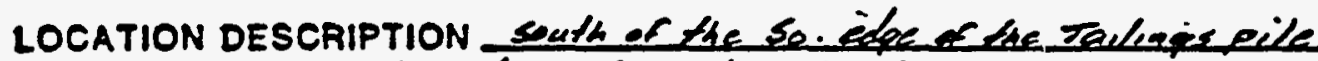

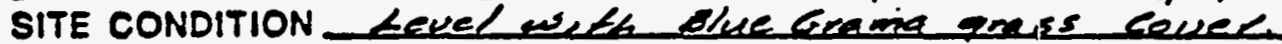

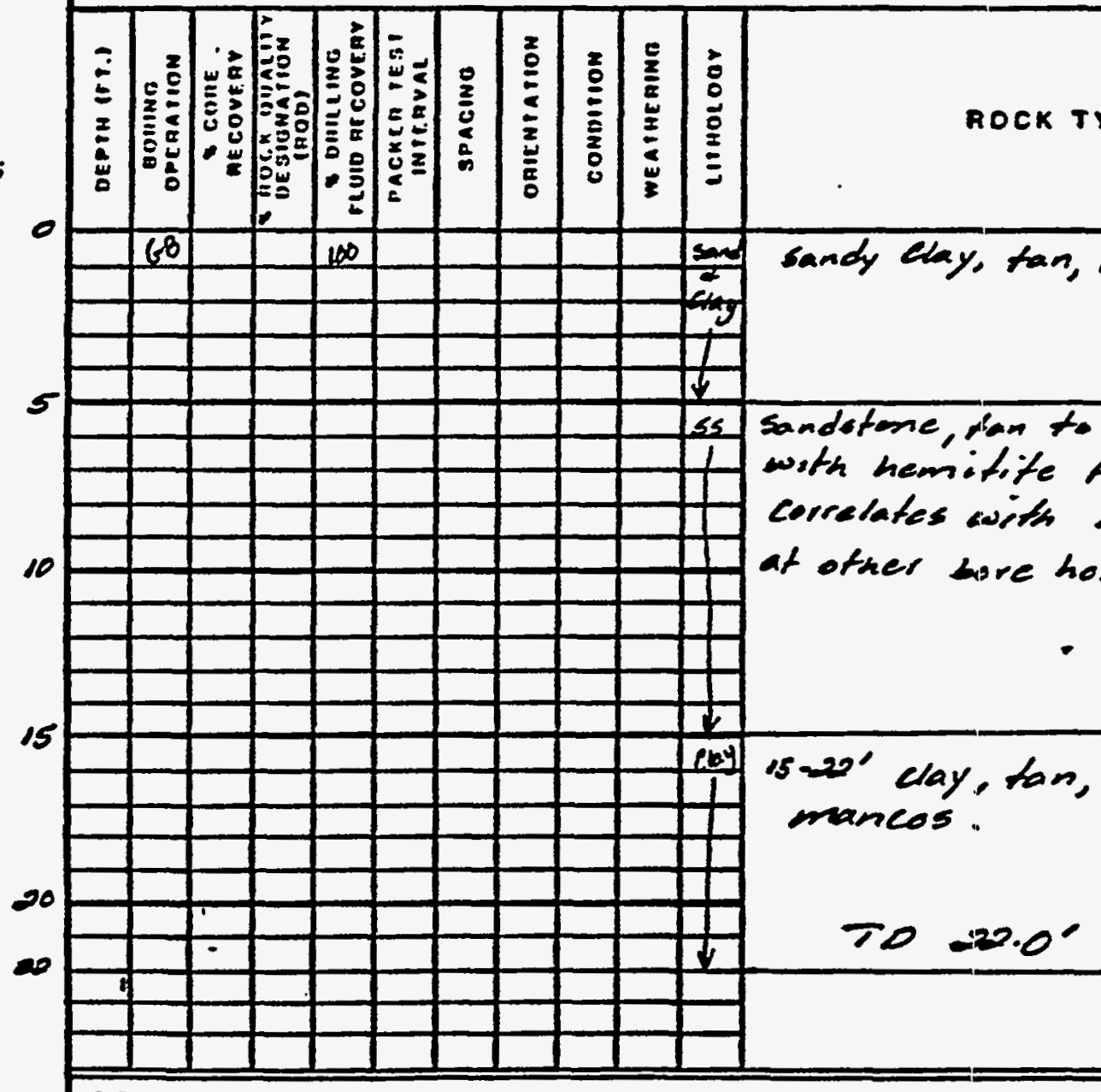

COMMENTS:

JEG-AL-ENG-2A (4185).

sandy Chy, tan, F, calc.

at ofher hore holes mancos.
SITE ID: AMEEOL LOCATION ID: 794 SITE COORDINATES (14.):

N AK

GROUND ELEVATION (ft. MSL): NK

DRILLING METHOD: Patary - Gear Bit

DRILLING CONTR.: Stewart Brethers

DATE STARTED: $2 / 24 / 85$

DATE COMPLETED: g/24/15

FIELD REP.: _ Repex thllad

\begin{tabular}{|c|c|c}
\hline \multicolumn{3}{|c|}{ GROUNDWATER LEVELS } \\
\hline DATE & TIMA & DEPTH (11.) \\
\hline & & \\
\hline & & \\
\hline & & \\
\hline
\end{tabular}

Sandefone, than to vellow to red orange

with hemitite frags - pravely.

correlates with basal alluvium

$1522^{\prime}$ clay, tan, soft, calc. weatherec

na - $x$ a noek coning 


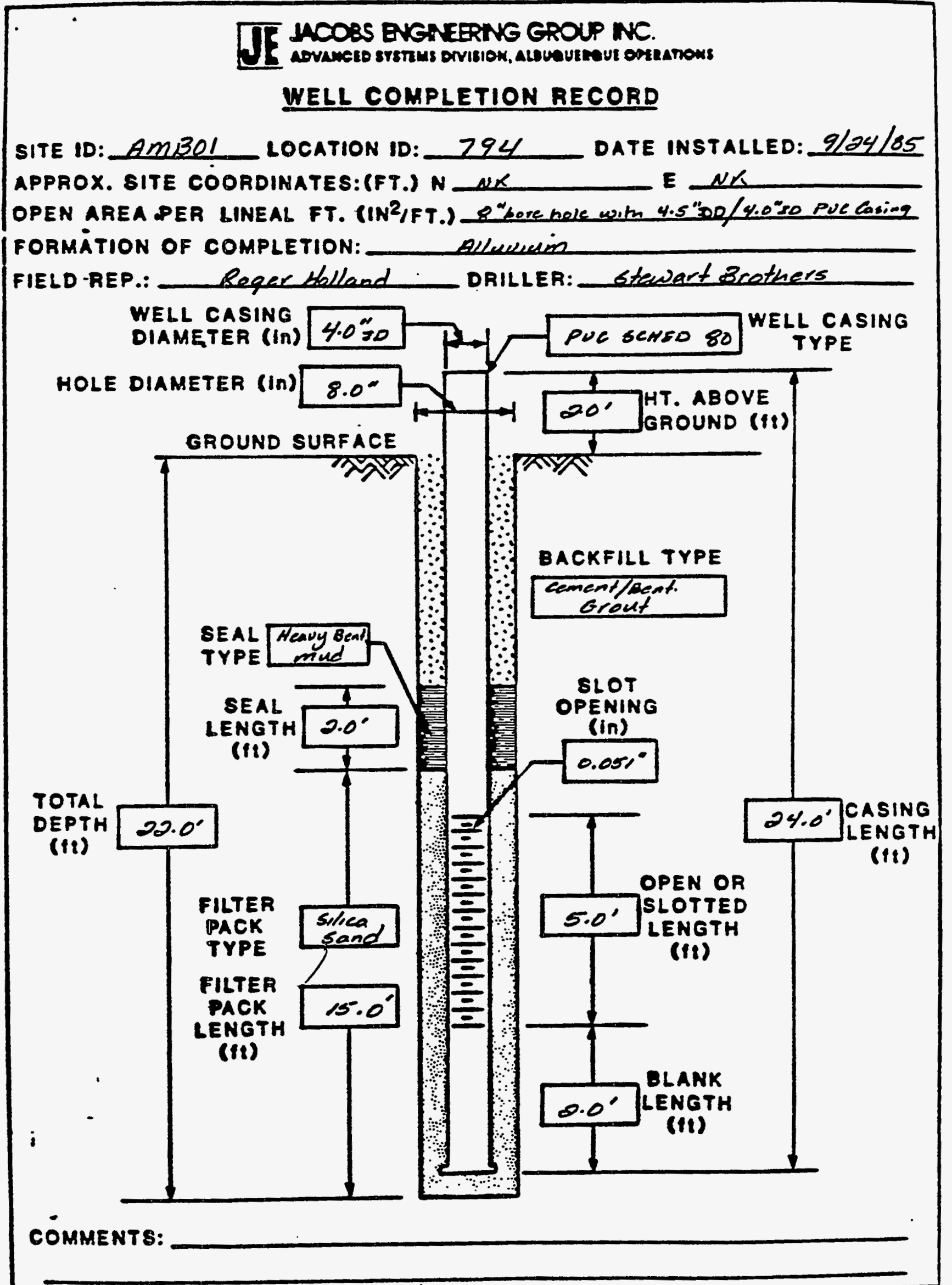

JEG-AL-ENO-B (SICA) 


\section{TE MCOSS BNGNEERTV GROUP WNE.

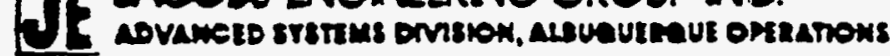 \\ BOREHOLE/WELL CONSTRUCTION LOG}

SITE ID: AmBel LOCATION ID: 294
APPROX. 8ITE COORDINATES (FT.): N_NK FIELD REP: Roger Holland

COMPLETION DATE: $2 / 04 / 55$

GROUND ELEVATION (FT. MSL): NK CONSTRUCTION TIME LOG

DRILLER:_stewart Brothars

RIG TYPE: Gardner Denver 15w (Ratary)

\begin{tabular}{|c|c|c|c|}
\hline BIT TYPE & $\begin{array}{l}\text { HOLE. } \\
\text { DIA } \\
\text { (in.) }\end{array}$ & 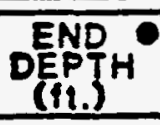 & $\begin{array}{l}\text { FLUID } \\
\text { TYPE } \\
\end{array}$ \\
\hline $\begin{array}{l}\text { Hughs } \\
\text { mill }\end{array}$ & 8.01 & $22.0^{\prime}$ & Benterife \\
\hline & & & \\
\hline & & & \\
\hline & & & \\
\hline
\end{tabular}

CASING

CASING SUMMARY

\begin{tabular}{|c|c|c|}
\hline $\begin{array}{l}\text { CASING } \\
\text { TYPE }\end{array}$ & DESCRIPTION & $\left|p_{i n}{ }_{j}\right| c \mid$ \\
\hline
\end{tabular}

FILTER PACK.

\begin{tabular}{|c|c|c|c|}
\hline & & & \\
\hline$\underline{B}$ & PUC BCHEO & 8.020 & 1 \\
\hline 5 & Puc $0.051^{\circ}$ 66otted & $4.0^{-\infty}$ & $22^{\circ}$ \\
\hline$B$ & PUE SCHED. 80 & $4.0^{\circ}$ & $24^{\circ}$ \\
\hline$p$ & steid Casing & $8 \infty$ & 5 \\
\hline & & & \\
\hline & & & \\
\hline & & & \\
\hline & & & \\
\hline -Pros & 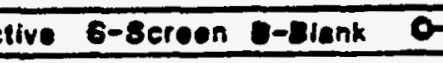 & OpQn & A-Nono \\
\hline
\end{tabular}

SEAL Heavy Buid

BACKFILL

DEVELOPMENT

\section{START}

DRILLING

\begin{tabular}{|c|c|c|c|}
\hline \multirow{2}{*}{ ACTIVITY } & \multicolumn{2}{|c|}{ START } & \multirow{2}{*}{$\begin{array}{l}\text { END } \\
\text { TIME }\end{array}$} \\
\hline & DATE & TIME & \\
\hline RILLING & $9 / 24 / 65$ & $3: 50 \mathrm{pm}$ & $4.00 \mathrm{pm}$ \\
\hline CASING & 9/2y/85 & $4: 20 \mathrm{pm}$ & 4:30 pm \\
\hline =ILTER PACK. & $9 / 24 / 85$ & $4: 37 \mathrm{pm}$ & $4: 55 \mathrm{pm}$ \\
\hline $\begin{array}{c}\text { SEAL Heavy } \\
\text { Bent } \\
\text { mud }\end{array}$ & $9 / 04 / 85$ & $4: 58 \mathrm{pm}$ & 4:59 pm \\
\hline BACKFILL & $9 / 24 / 85$ & 6:01 pm & $5: 11 \mathrm{pm}$ \\
\hline DEVELOPMENT & $9 / 06 / 85$ & $11: 200 \mathrm{~m}$ & 3:00 pm \\
\hline
\end{tabular}

END

TIME

\section{OTHER}

WELL CONSTRUCTION

\begin{tabular}{|c|c|c|}
\hline $\begin{array}{l}\text { TYPE } \\
\text { CODE }\end{array}$ & DESCRIPTION & $\begin{array}{l}\text { ENP } \\
\text { DEP } \\
\text { (it.) }\end{array}$ \\
\hline 8 & Cement/Bentenste Geruf O-5-' & $\sigma^{\prime}$ \\
\hline 5 & Heavin Bentemite $5-7^{\prime}$ & $7^{\prime}$ \\
\hline$E$ & sdien sand $7-20^{\circ}$ & $22^{\prime}$ \\
\hline & - & \\
\hline$T$ & & \\
\hline & & \\
\hline - & & \\
\hline & & \\
\hline$\frac{1}{0}$ & - Saekifl & \\
\hline
\end{tabular}

WELL DEVELOPMENT

Hote blown, fluobed and surged to ckan. Hole bicwn dry, allowed to set then blown dry to eneck recovery several tomes. Hole morisong same nater.

\section{COMMENTS:}


WELL 796 LOGS 
PROJECT AMBROSLA LAKE SITE, NEW MEXICO

Page 1 of 1

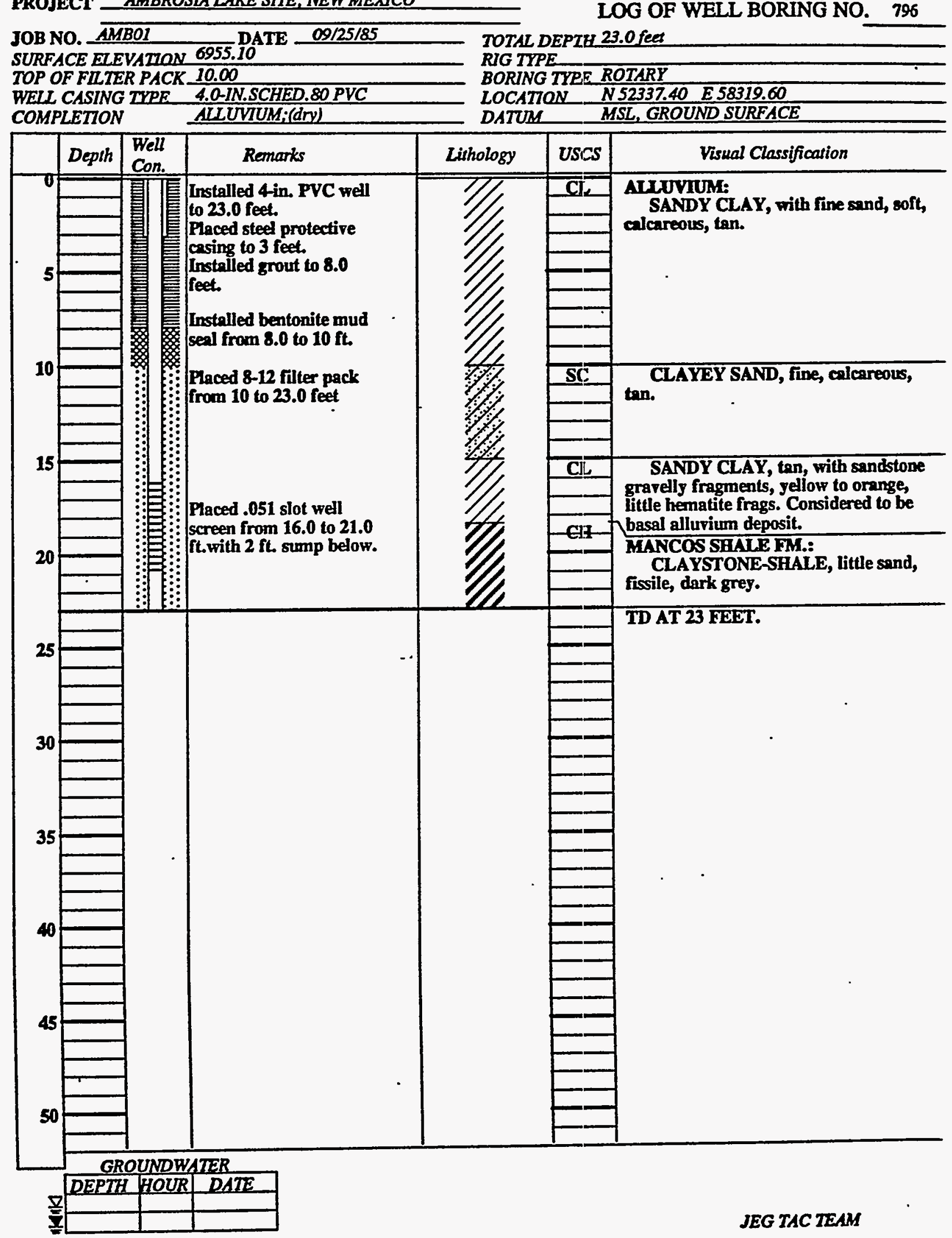




\section{TT MOOBS ENGNEERTG EROUP NC.

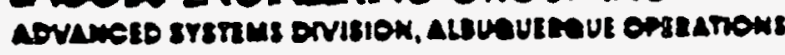

\section{WELL COMPLETION RECORD}

SITE 10: AMBOL LOCATION 1D: 796 DATE INSTALLED: $2 / 25 / 85$ APPROX. SITE COORDINATES:(FT.) $N$

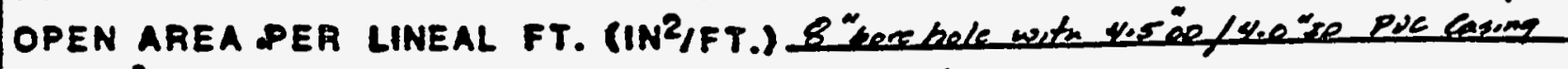
FORMATION OF COMPLETION: FIELD-REP.: Geper Helland Qlluevim

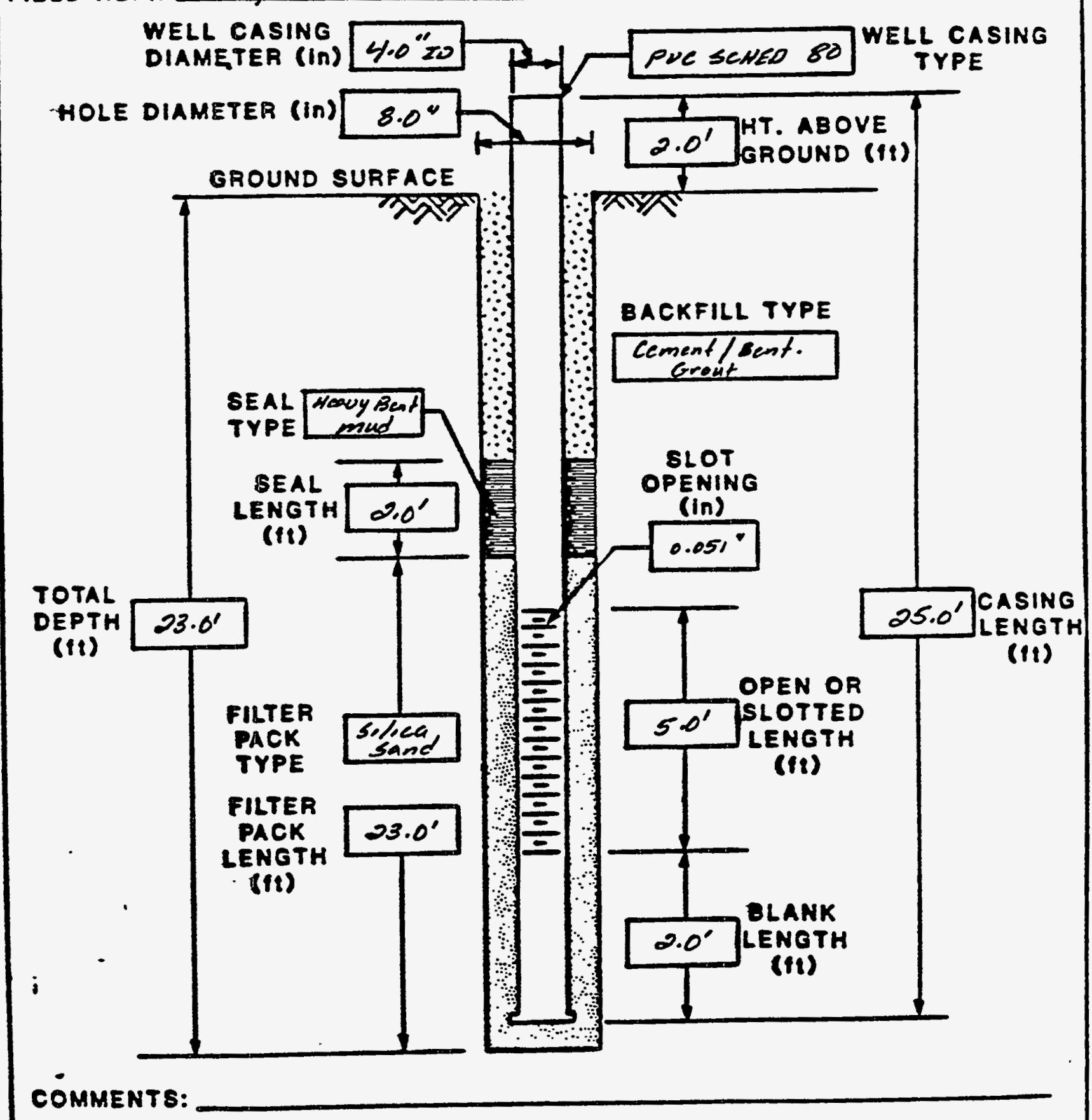




\section{TT MCOSS ENGN EERTIG GROUP WK.

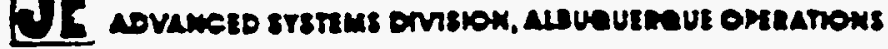 \\ BOREHOLE/WELL CONSTRUCTION LOG}

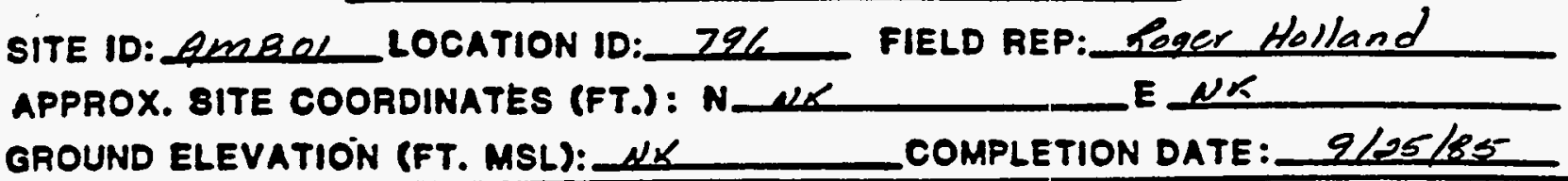

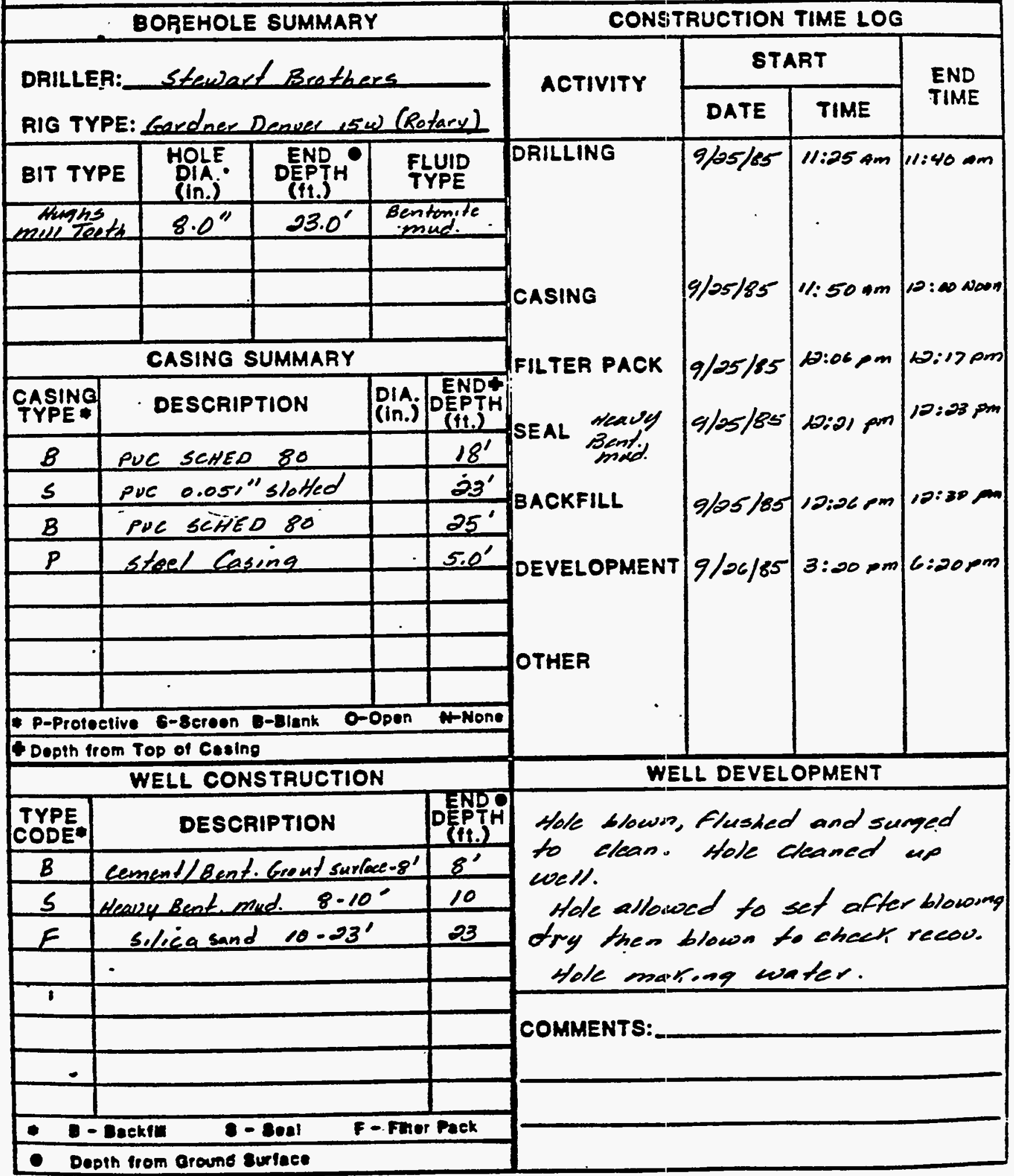


WELL 797 LOGS 


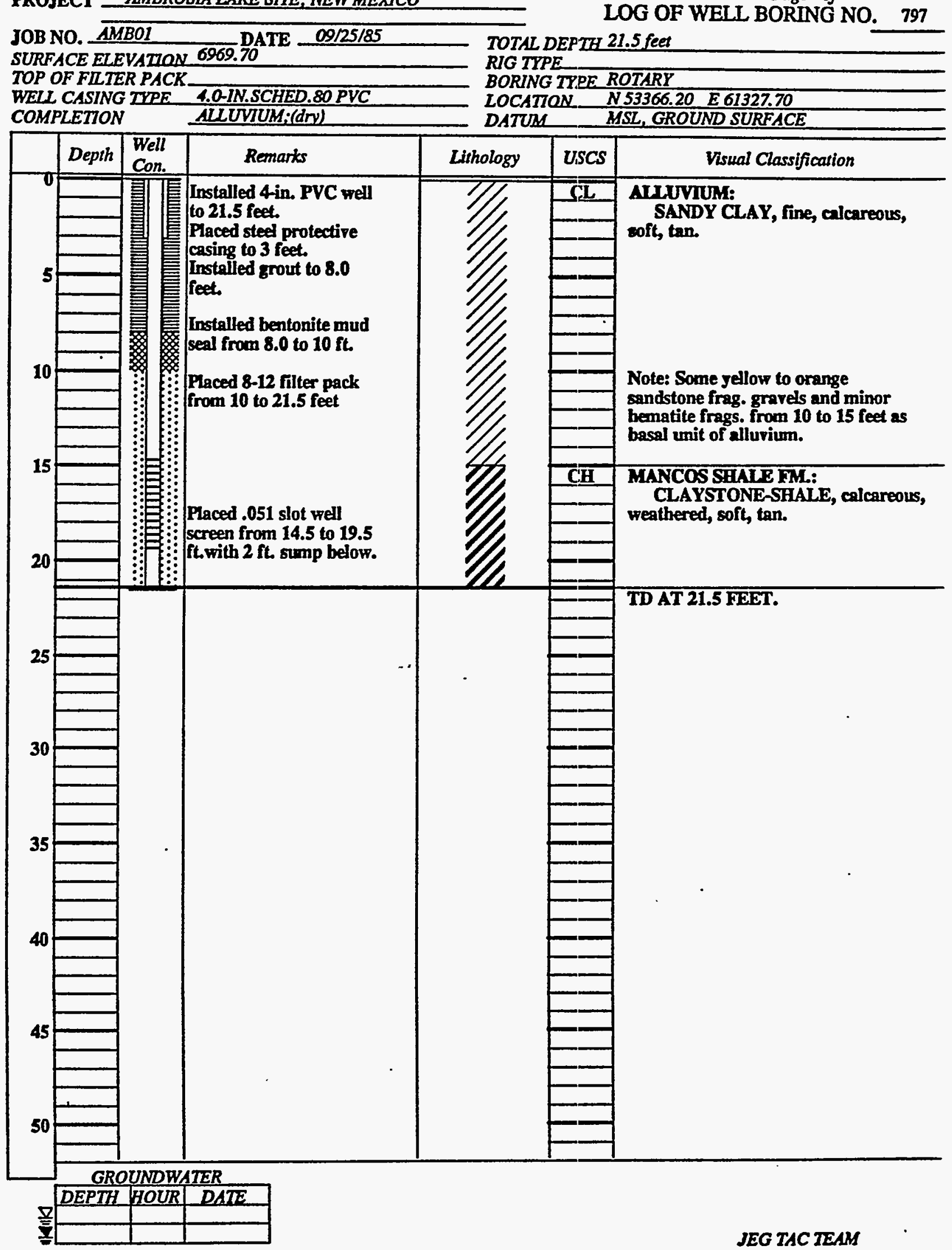




\section{DE MOOBS ENGNEERTG GROUP NC.}

\section{WELL COMPLETION RECORD}

SITE ID: $A M B O$ LOCATION ID:

DATE INSTALLED: 7/25/85 APPROX. SITE COORDINATES:(FT.) $N$ WK E NK

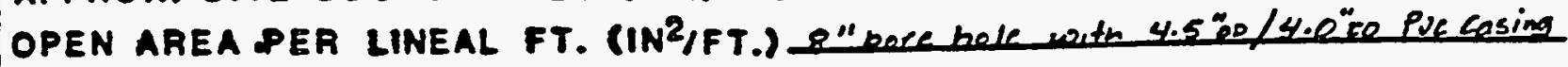
FORMATION OF COMPLETION:

FIELD-REP.: Roger Holland Allecriven

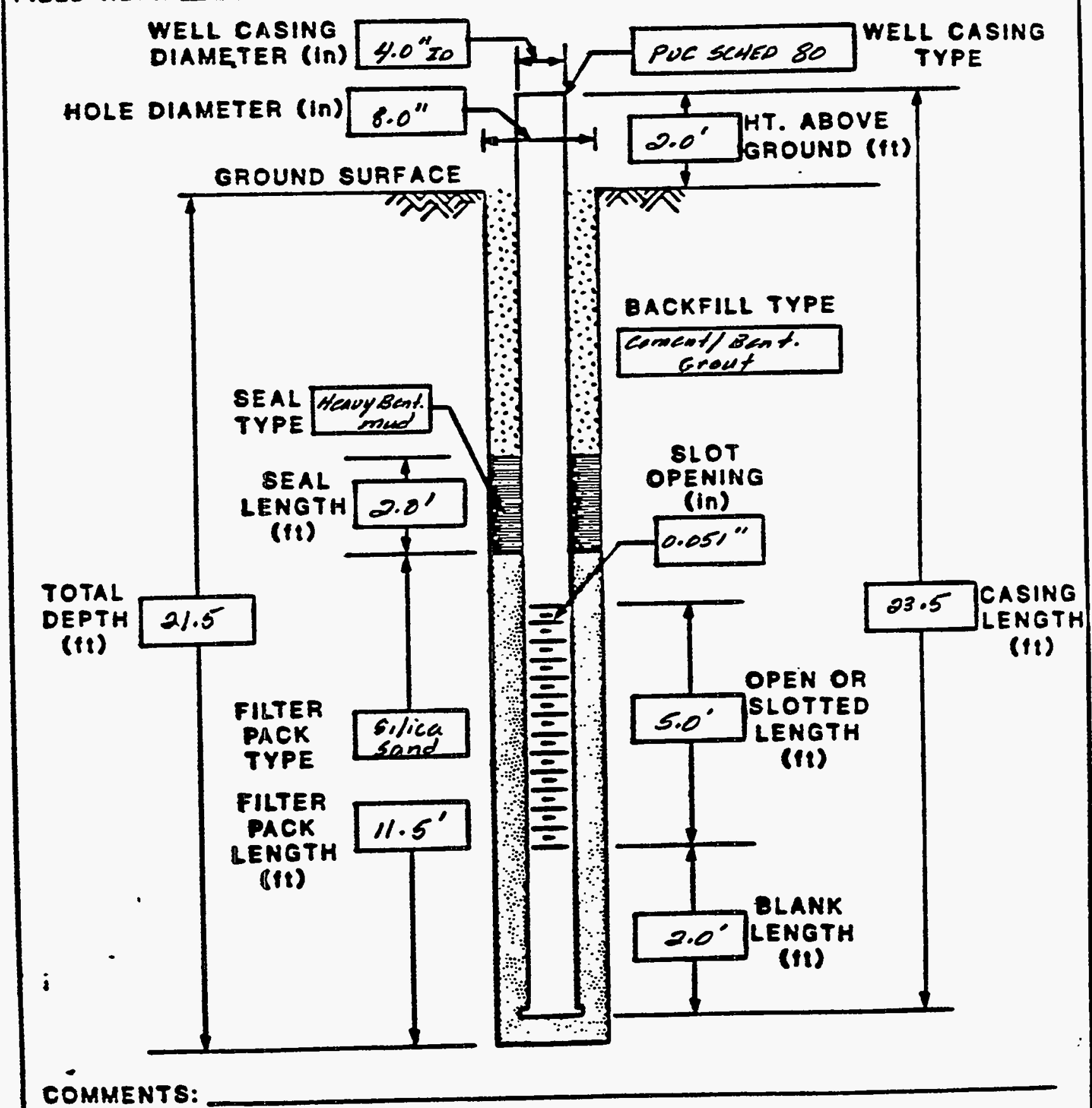

COMMENTS: 
$16 \cdot 19 \cdot 2 \cdot 2 \cdot 3$

\section{TT MCOSS GNENEERTG EROUP WT.

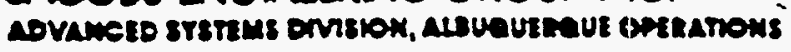 BOREHOLE/WELL CONSTRUCTION LOG}

SITE ID: AMBBOL LOCATION ID: 792 FIELD REP: Reger Holland. APPROX. BITE COORDINATES (FT.): N_NS GROUND ELEVATION (FT. MSL): NK COMPLETION DATE: $2 / 25 / 85$

BOREHOLE SUMMARY

DRILLER:_ Stewart Brothers

RIG TYPE: Gardner Denver isw (Rotary)

\begin{tabular}{|c|c|c|c|}
\hline BIT TYPE & $\begin{array}{c}\text { HOLE. } \\
\text { DIA } \\
\text { (In.j }\end{array}$ & $\begin{array}{c}\text { DEND } \\
\text { (I1.) }\end{array}$ & $\begin{array}{c}\text { FLUID } \\
\text { TYPE }\end{array}$ \\
\hline $\begin{array}{c}\text { Hughs } \\
\text { mell Teeth }\end{array}$ & $8.0^{*}$ & & Bentonite \\
\hline & & & \\
\hline & & & \\
\hline & & & \\
\hline
\end{tabular}

\begin{tabular}{|l|l|l|l|}
\hline \multicolumn{3}{|c|}{ CASING SUMMARY } \\
\hline $\begin{array}{l}\text { CASINQ } \\
\text { TYPE }\end{array}$ & DESCRIPTION & $\begin{array}{l}\text { DIA.j } \\
\text { (IN.) }\end{array}$ & $\begin{array}{c}\text { END EPTH } \\
\text { (I1.) }\end{array}$ \\
\hline
\end{tabular}

\begin{tabular}{|c|l|l|l|}
\hline$B$ & PUC SCHED 80 & $4.0^{\prime \prime}$ & $16.5^{\prime}$ \\
\hline$S$ & PUC 0.05, slotted & $4.0^{\circ}$ & $21.5^{\prime}$ \\
\hline$B$ & PUC SCHEO 80 & $4.0^{\circ}$ & $23.5^{\circ}$ \\
\hline$P$ & Steel Casing & $8.0^{\circ \prime}$ & 5.0 \\
\hline & & & \\
\hline & & & \\
\hline & & & \\
\hline & & & \\
\hline
\end{tabular}

* P-Protective 6-8creen 8-Slank O-Opon N-Mono Dopin from Tod of Casing

\begin{tabular}{|c|c|c|}
\hline \multicolumn{3}{|c|}{ WELL CONSTRUCTION } \\
\hline $\begin{array}{l}\text { TYPE } \\
\text { CODE }\end{array}$ & \multicolumn{2}{|l|}{ DESCRIPTION } \\
\hline$B$ & Cement/Bent. Grout $0-b^{\prime}$ & $8^{\prime}$ \\
\hline$\underline{s}$ & Heary sent and s-10- & $10.0^{5}$ \\
\hline$F$ & Selcea sand $10-21.5$ & $2 \%$ \\
\hline & - & \\
\hline 8 & & \\
\hline & & \\
\hline$=$ & & \\
\hline a- & $8-8021$ & Paek \\
\hline 0 & enen & \\
\hline
\end{tabular}

CASING CONSTRUCTION TIME LOG

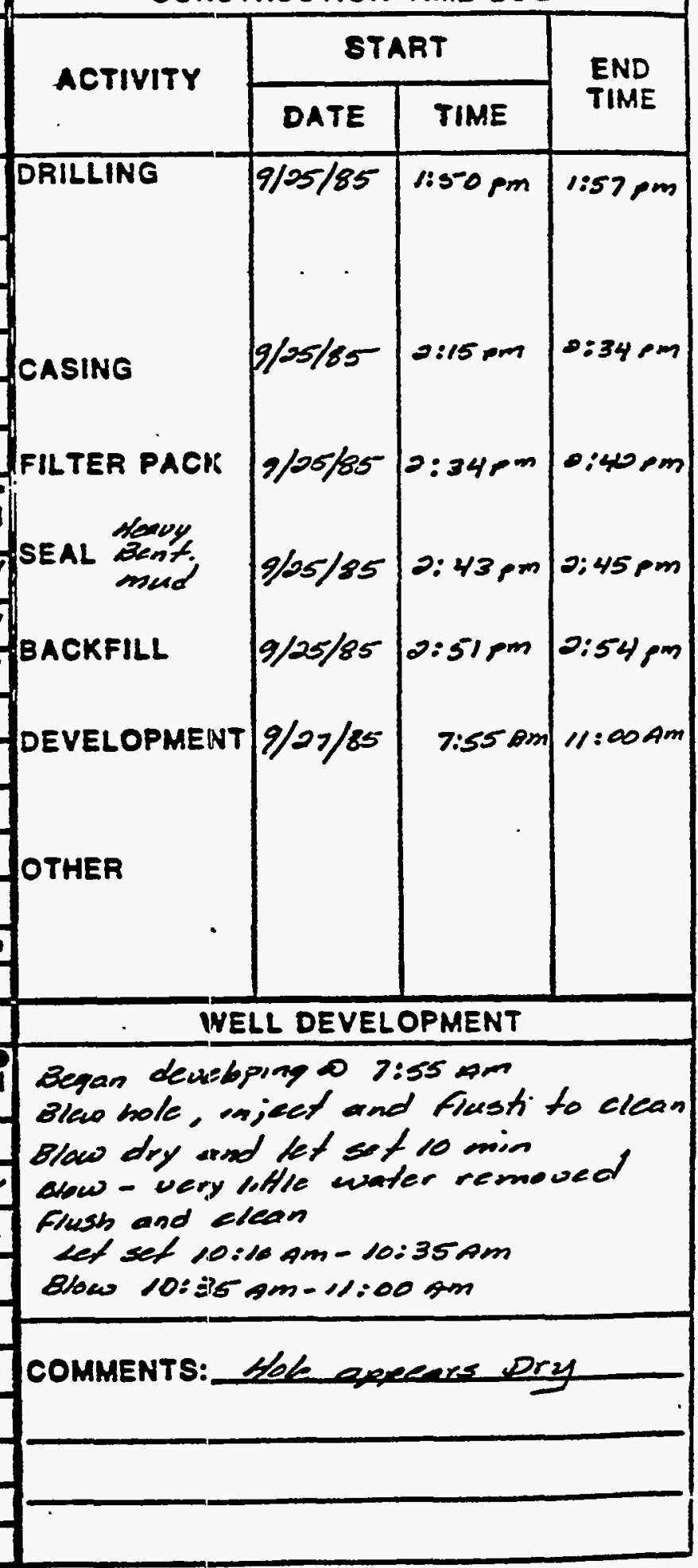


WELL 798 LOGS 
PROJECT AMBROSLA LAKE SITE, NEW MEXICO

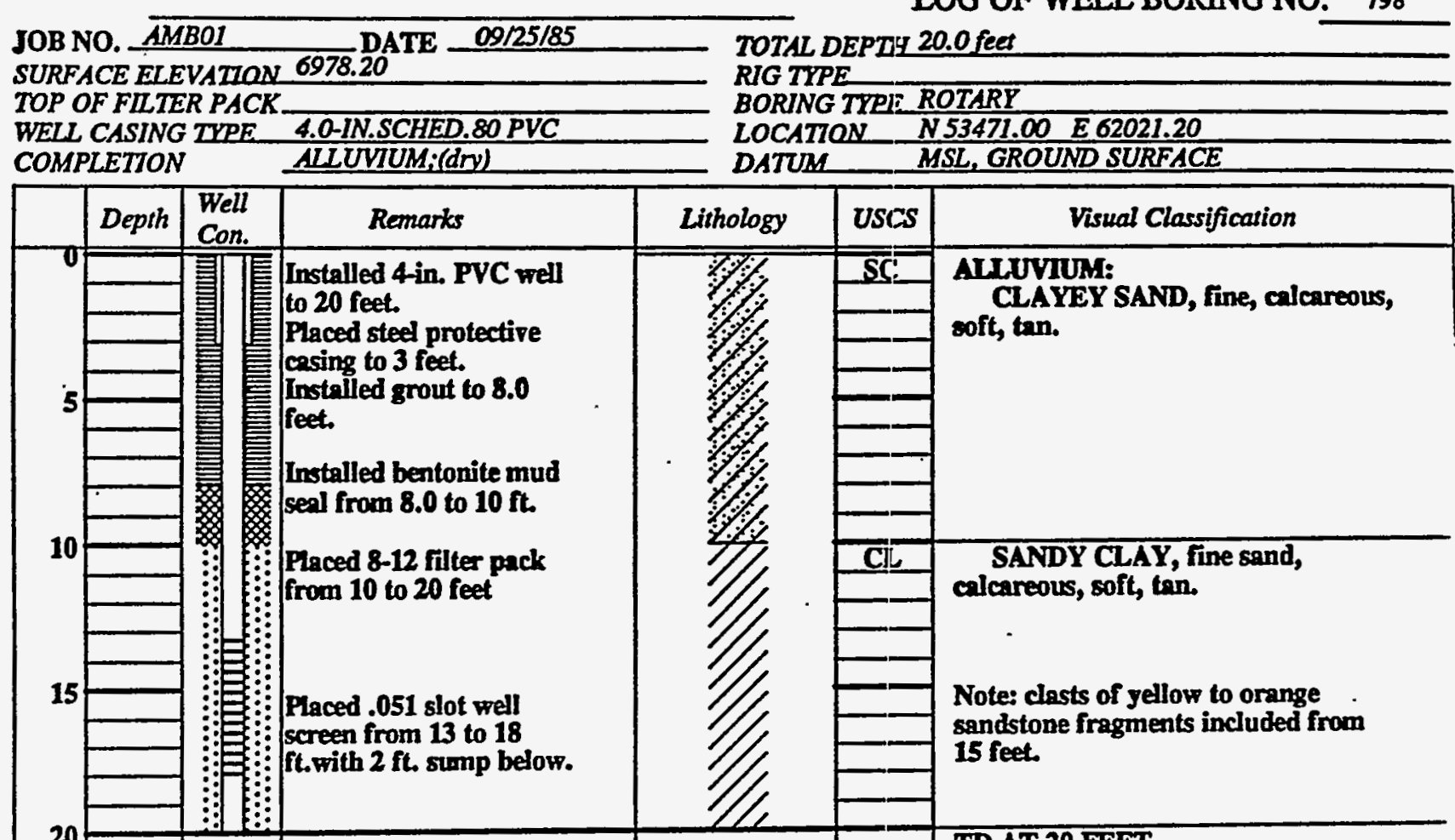

LOG OF WELL BORING NO. 798

JOB NO. AMBO1 DATE 09/25/85

SURFACE ELEVATZON 6978.20

WELL CASING TIPE.

4.0-IN.SCHED.80 PVC

BORING TYPIE ROTARY

COMPLETION

20

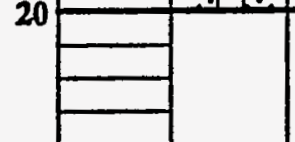

25

25

30

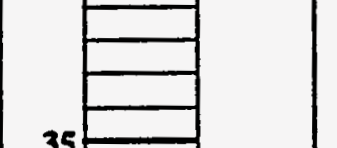

35
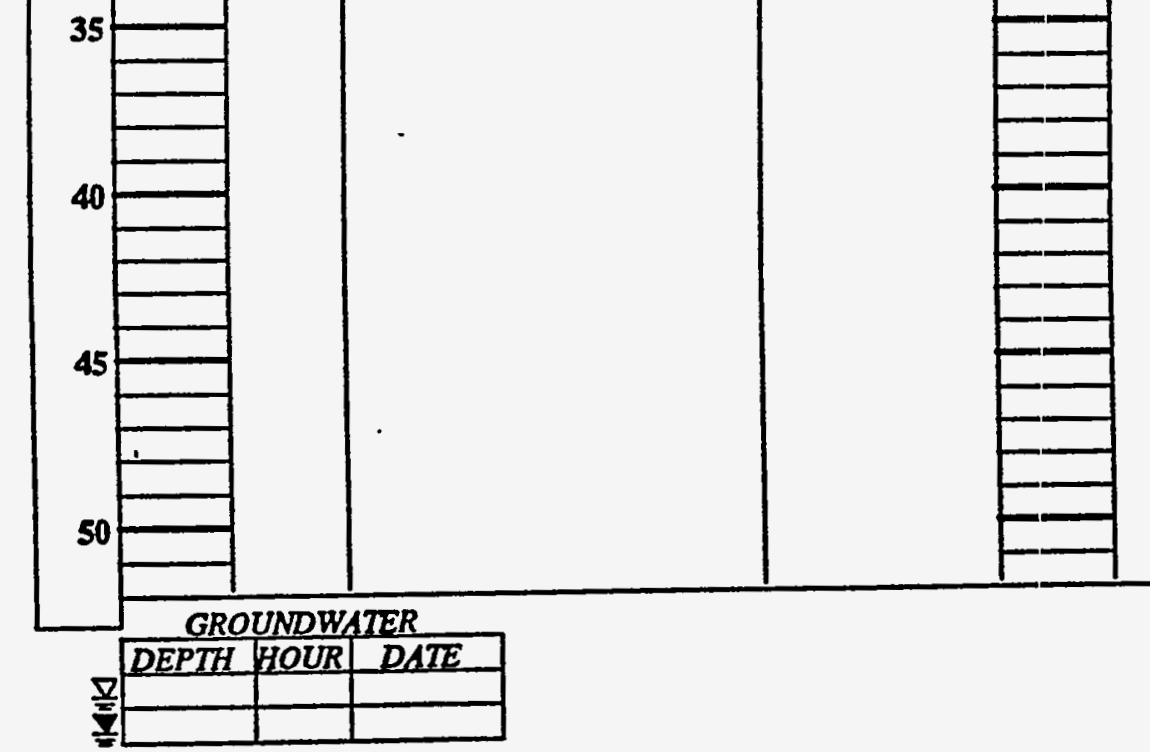


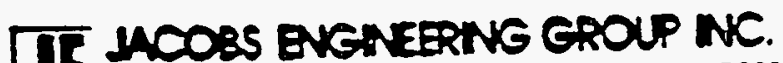

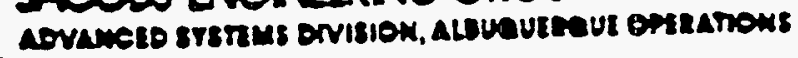

\section{WELL COMPLETION RECORD}

SITE ID: AmBOL LOCATION ID: 798

DATE INSTALLED: $2 / 25 / 85$ APPROX. SITE COORDINATES:(FT.) N NK E NK

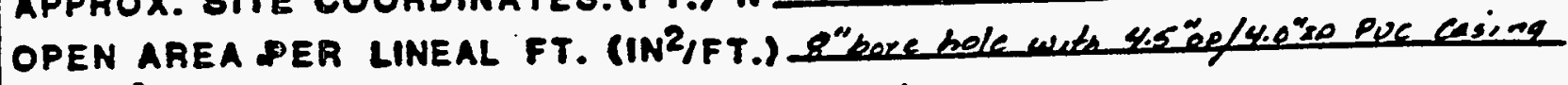
IFORMATION OF COMPLETION:

FIELD -REP.: Leper thllond Allciviums

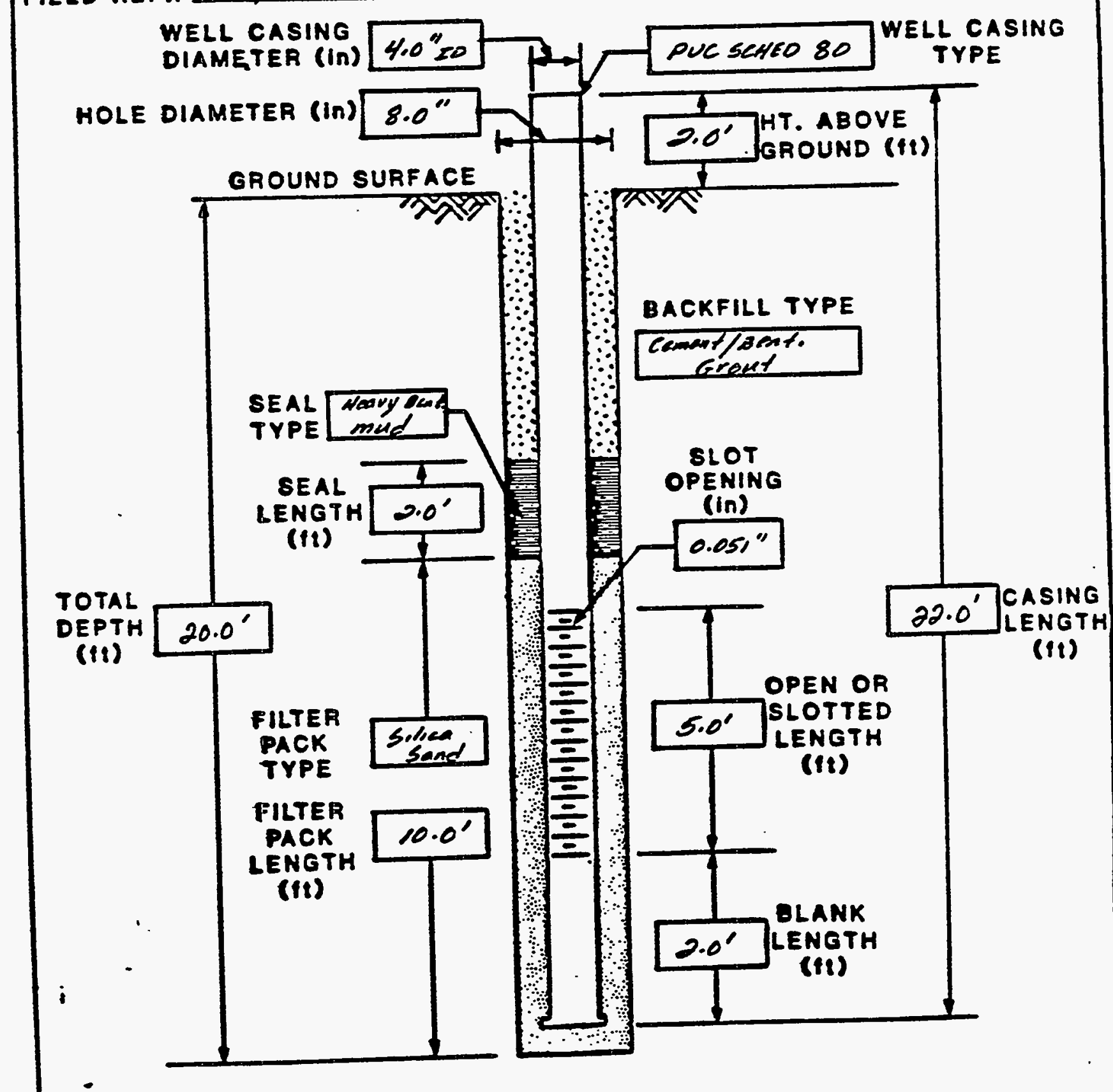

COMAENTE: 
$16.19 \cdot 2 \cdot 2 \cdot 3$

\section{TT IACOSS ENGNEERIVG GROUP NV.

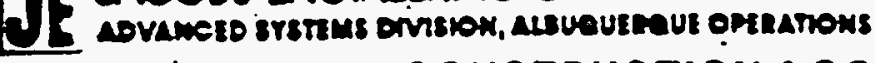 \\ BOREHOLE/WELL CONSTRUCTION LOG}

SITE ID: AmBel LOCATION ID: 298 FIELD REP: Roger F. Helland APPROX. BITE COORDINATES (FT.): N_NK N NK

GROUND ELEVATION (FT. MSL): NK BOREHOLE SUMMARY

DRILLER:Stewart Brothers.

RIG TYPE: Gordner Denver 15w (Rotary)

\begin{tabular}{|c|c|c|c|}
\hline BIT TYPE & $\begin{array}{l}\text { HOLE } \\
\text { DIA: } \\
\text { (In.; }\end{array}$ & $\underset{\text { DEPTH }}{\text { DENP }}$ & $\begin{array}{l}\text { FLUID } \\
\text { TYPE }\end{array}$ \\
\hline Hashis intents & $8.0^{\prime \prime}$ & & $\begin{array}{l}\text { Santanote } \\
\text { mude }\end{array}$ \\
\hline & & & \\
\hline & & & \\
\hline & & & \\
\hline
\end{tabular}

\begin{tabular}{|l|l|l|l|}
\hline CASING & DESCRIPTION & DIA.j & $\begin{array}{c}\text { END } \\
\text { TYPE }\end{array}$ \\
\hline (H.
\end{tabular}

\begin{tabular}{|c|c|c|c|}
\hline & & m.1. & \\
\hline$B$ & RUC SCHED 80 & $4.0^{\prime \prime}$ & $15^{\prime}$ \\
\hline 5 & puc $0.051^{\circ}$ slotted & $4.0^{\circ}$ & $20^{\prime}$ \\
\hline$B$ & PUC SCHED 80 & $4.0^{\prime \prime}$ & $22^{\prime}$ \\
\hline$p$ & steel casing & $8.0^{4}$ & $5.0^{\prime}$ \\
\hline & & & \\
\hline & & & \\
\hline & & & \\
\hline & & & \\
\hline
\end{tabular}
- Dopth from TOP of Casing

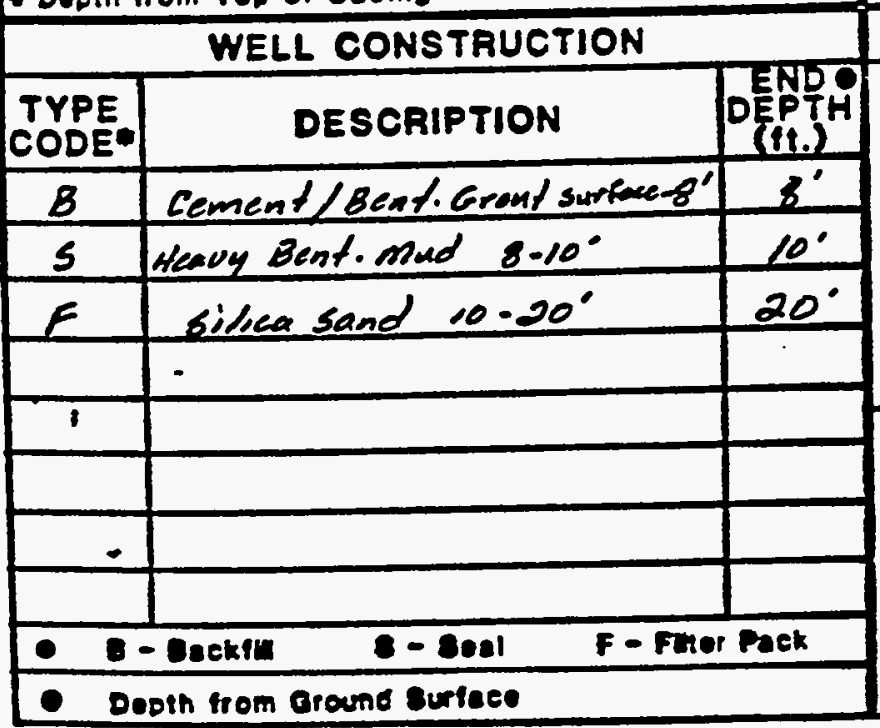

JEG-AL-ENO-1 (S/OA)
COMPLETION DATE: 2/25/85

CONSTRUCTION TIME LOG

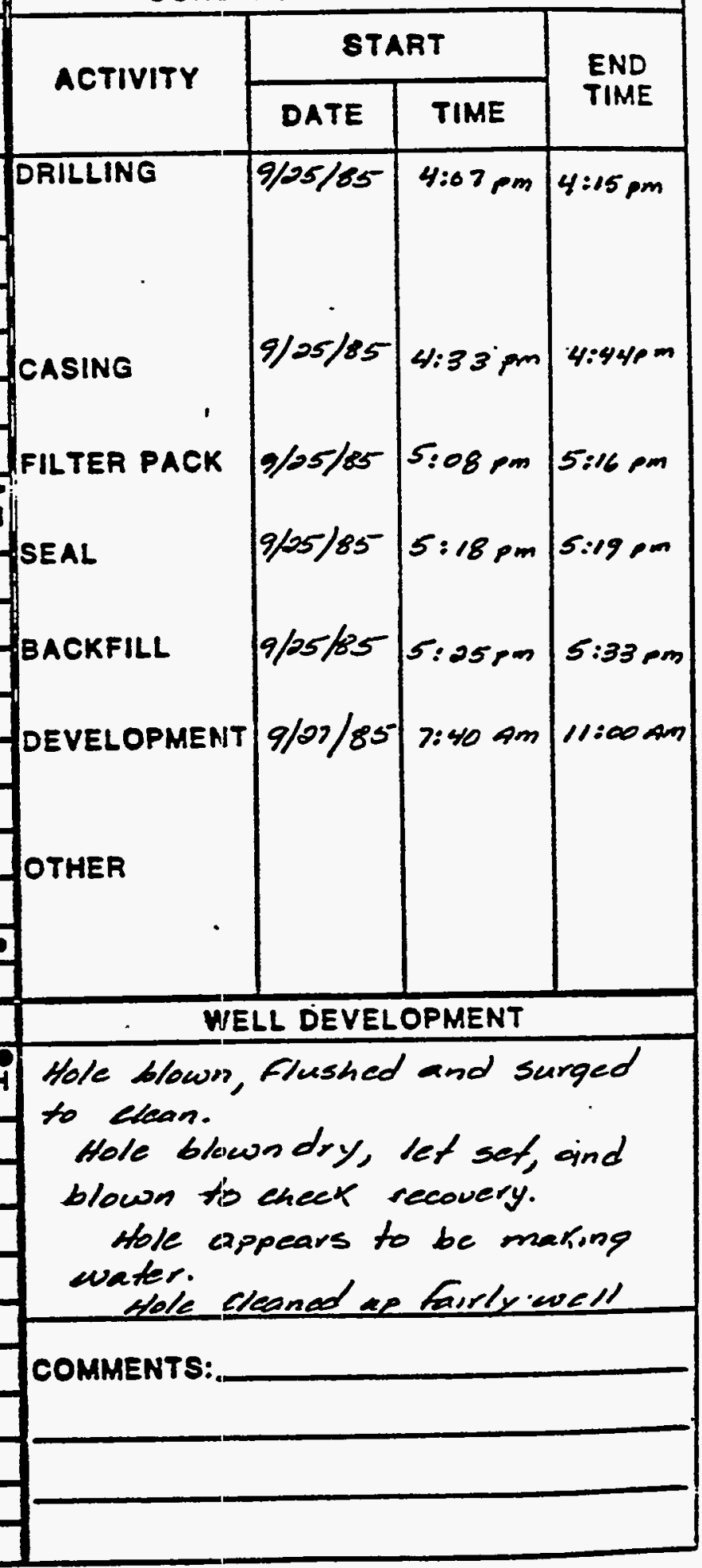


WELL 799 LOGS 


\section{MOOBS BNENERRV CROUP NK.}

\section{WELL COMPLETION RECORD}

SITE ID: AMBOL LOCATION ID:

DATE INSTALLED: $9 / 26 / 85$ APPROX. SITE COORDINATES:(FT.) N NK E AN

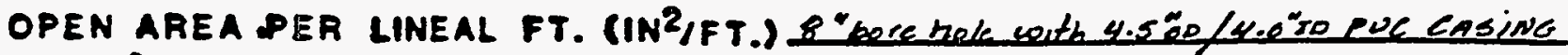
FORMATION OF COMPLETION: Alluvinion FIELD REP.: DRILLER:

steubort Brothers

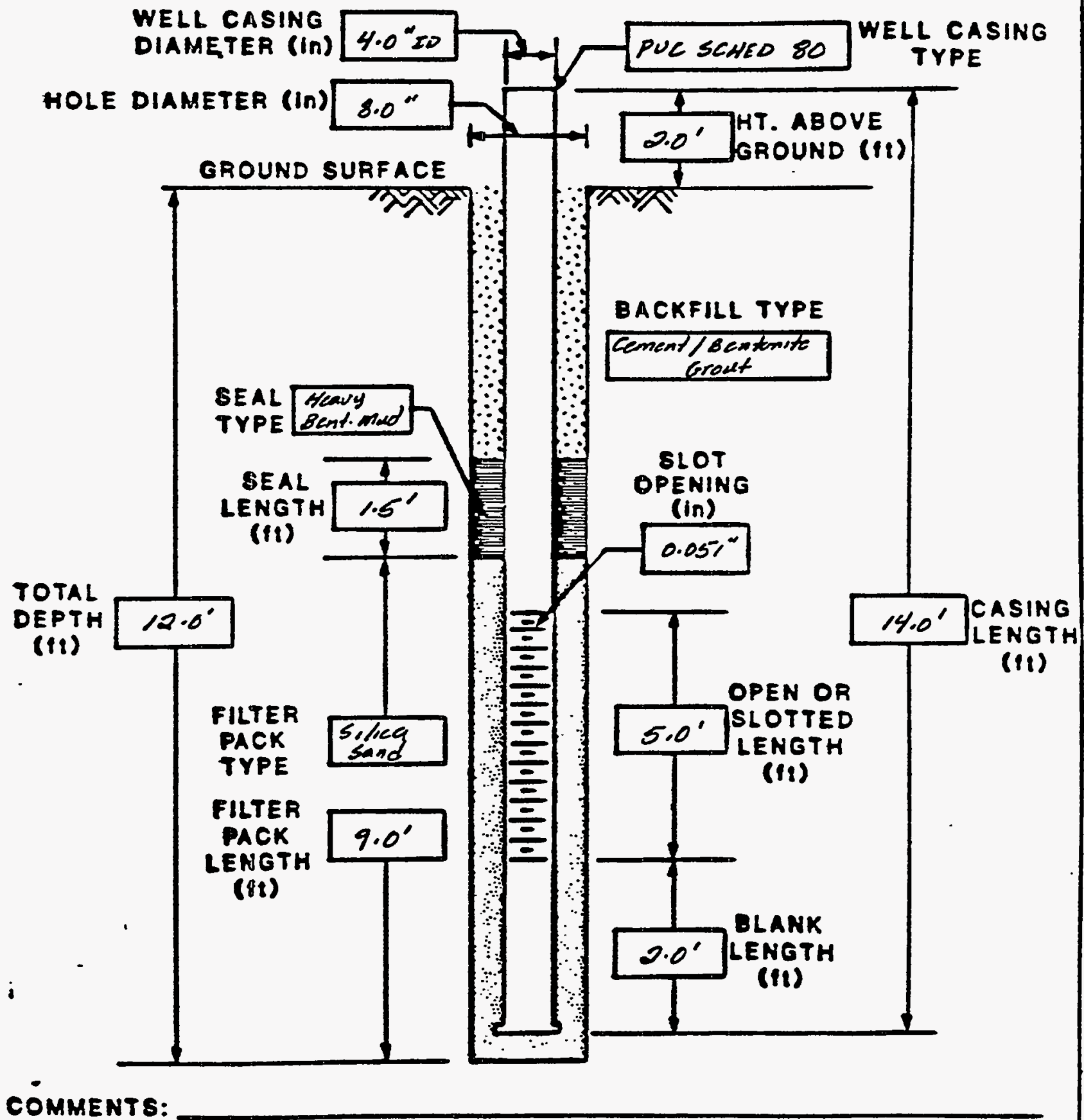

COMMENTS: 


\section{TT JACOSS QNGNERTE GROUP INE.

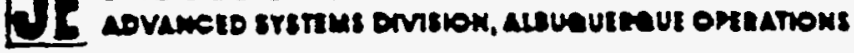 BOREHOLE/WELL CONSTRUCTION LOG}

SITE 1D: AmBol LOCATION ID: 799 FIELD REP:- Reger Hellend GROUND ELEVATIOON (FT. MSL): NK COMPLETION DATE: 2/06/85

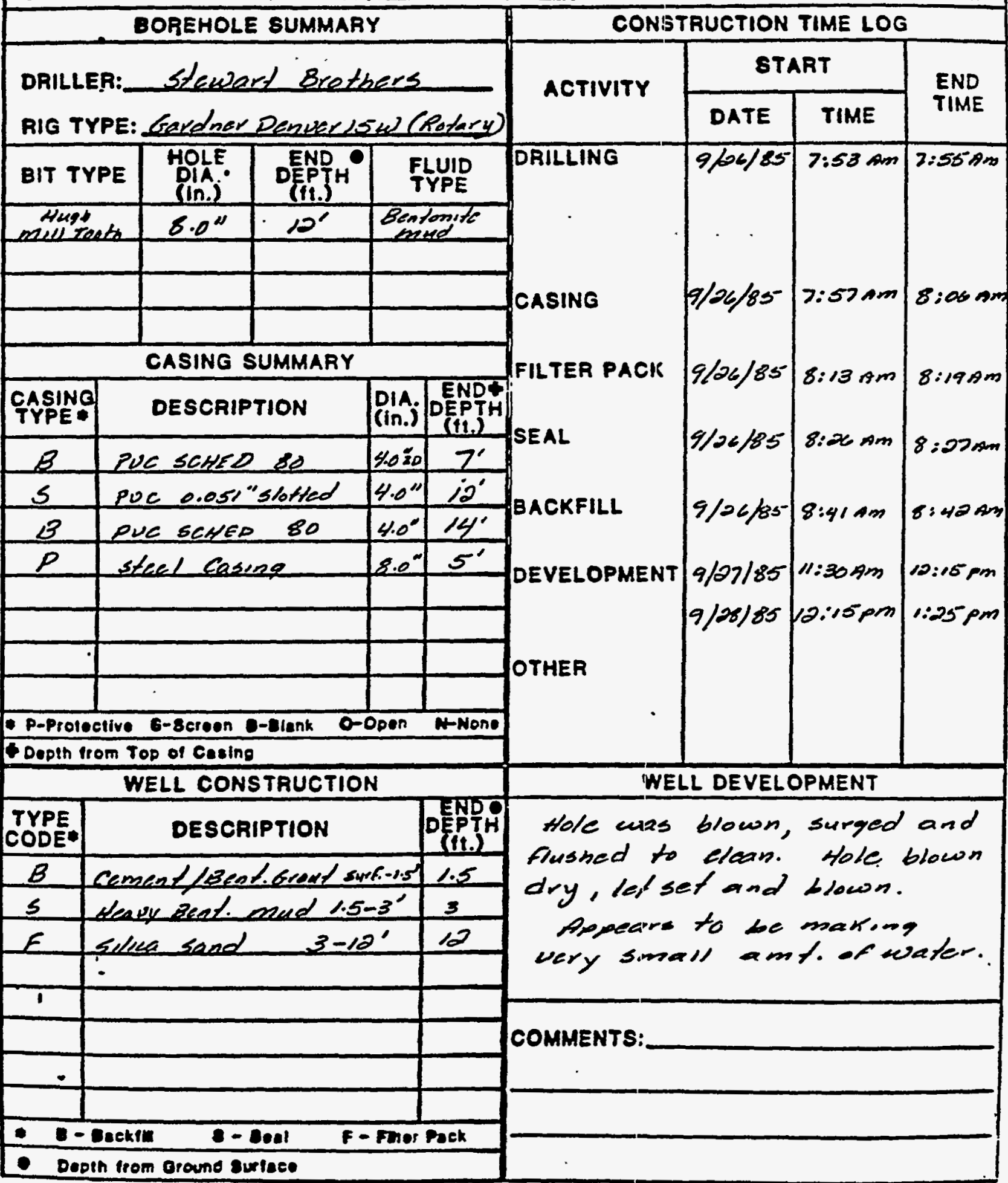


APPENDIX B

HYDROGEOLOGIC INFORMATION

AMBROSIA LAKE, NEW MEXICO, UMTRA PROJECT SITE 


\section{TT IACOBS ENGNEERING GROUP NC. -

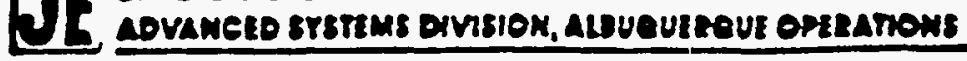

\section{calculation COVER gHEEI}

CALC NO.AMB-03-88-15-01- OI DISCIPLINE HYOROLOGY NO. OF SHEETS PROJECT:

UMTRA

\section{BITE:}

$$
\text { AMBROSIA LAKE, NEW MEXICO }
$$

\section{FEATURE:}

CALCULATION OF TRANSMISSIVITY \&' STORATIUITY WITHIN ALLUVIUM/WEATHERED MANCOS SHALE

USING JACOB STRAIGHT-LINE METHOD.

\section{SOURCES OF DATA:}

AquifER PUMPING TEST DATA COLLELTED

BY TAC - FEB. $2 \xi 3,1989$

SOURCES OF FORMULAE REFERENCES:

GROUNDWATER AND WELLS, 1986, JOHNSON, PP. $219-$ 257.

PRELIMIMARY CALC. $\square$ FinAL CALC. $\square$ supersiedes CaLC. NO.

\begin{tabular}{|l|l|l|l|l|l|l|l}
\hline & & & & & & & \\
\hline & & & & & & & \\
\hline & & & & & & & \\
\hline & & & & & & & \\
\hline $\begin{array}{c}\text { AEV. } \\
\text { MO. }\end{array}$ & GEVIUION & & & & & & \\
\hline
\end{tabular}


BY

CHKD.

JOB NO

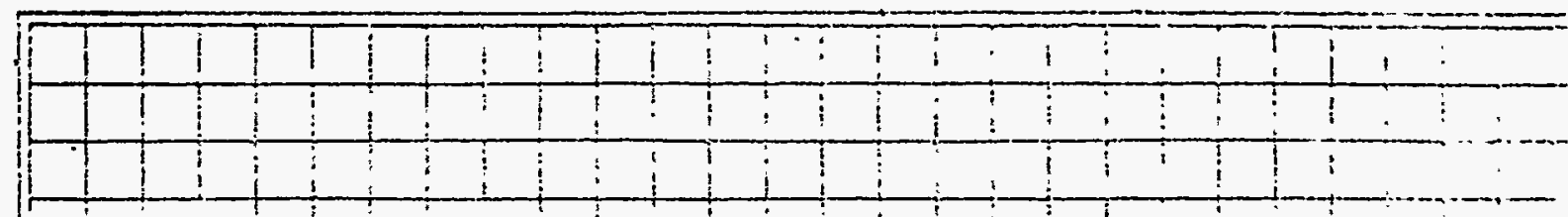

A. PURPOSE OF CALCULATION

To calculate the : transmissivity and storativity in the ali Juvium/weatherted -Mancos - Shale :-at-the -4mbrosia Lake (AMB-01)-UMTRA Project Site.

B.- METHOD AND PROCEQURES An aquifer pumping test was performed on Well 675 with wells OBS-1 and OBS-2 used as observation wells. The pumping test occurred in two phases:-1f-drawdown - and :2f-irecovery - The -drawdown-phase -cons isted of a 12-hour ttest where Hell 675 was pumped at a constant rate of 0.35 $\mathrm{gpm}$ before the water level fell below the intake of the pump ending the -drawdown -phase of the test:- - The -recovery phase began immediately after. :-Water level measurements were recorded electronically and manua77y throughout both phases of the test from the pumping and observation -wells. - - The -discharge -was routed to a nearby drainage ditch. Where it could not recharge the alluvial aquifer. Discharge rate was monitored manually throughout the duration of the drawdown -phase. -

The Jacob straight-7ine technique (Johnson, 1986) was used to analyse the -drawdown and recovery data to obtain values for transmissivity and storativity. :... Time-drawdown graphs : (Figures 1 through 3). Were generated from the drawdown data collected from pumping and observation -wells. - Residual-drawdown and time-recovery curves were generated from the recovery data collected from observation well OBS-1. : The graphs were analysed according to methods described in - "Groundwater and Hel1s," - (Johnson, -1986).

C.-ASSUMPTIONS'

i.-1) . The aquifer is homogenous and isotropic.

2) The formation has uniform thickness:

3) The formation receives no recharge from any source.

4) The pumped weil] penetrates and receives water from the fult thickness of the water-bearing formation.:

5) The-aquifer is infinite in areal extent. 6) The water removed from torage is discharged instantaneousty with ${ }^{-1}$ D. MATERIAL PROPERTIES

Attachment A contains the well $\log$ and well construction summary for We11 675: Doservation points OBS-1 and OBS-2 are located within 30 feet of -We 17 -675-and penetrated similiar - lithologic-conditions. 
DATE

BY

CHKD.

JOB NO.

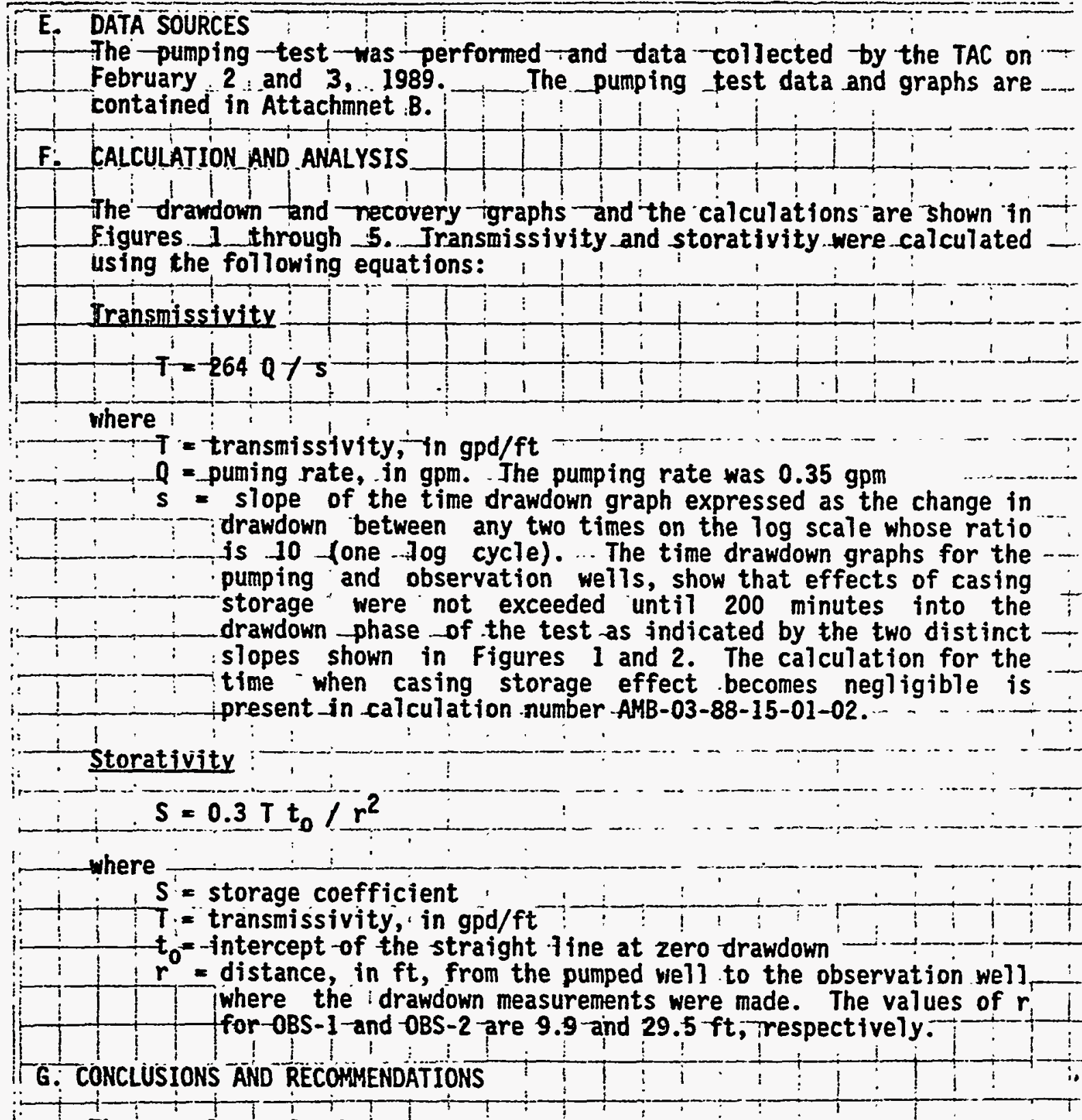

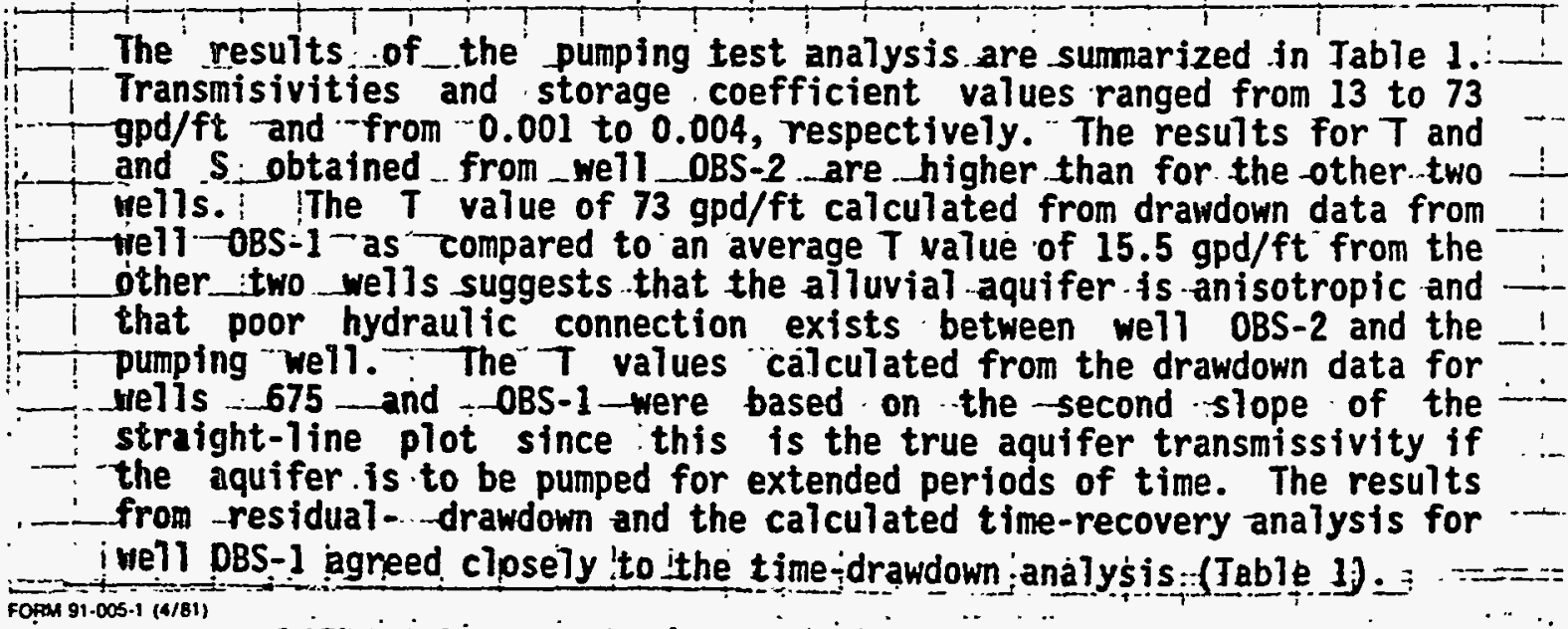


CKK.

JOBNO

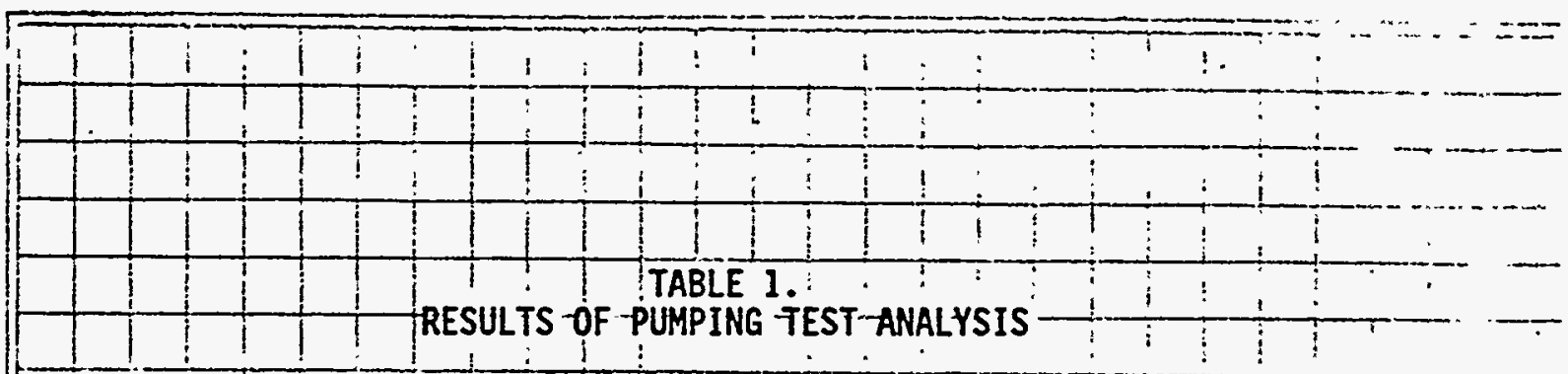

Time-drawdown analysis

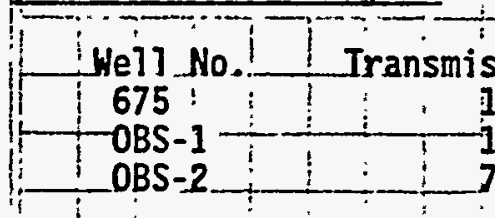

Time-recovery anajysis

:

He 17 No.

Transmissivity (gpd/ft) OBS-1

$+$

Residual-drawdown analysis

\section{..... Hell No.}

OBS-1
Transmissivity (gpd/ft) 22
Storage Coefficient 0.001

!

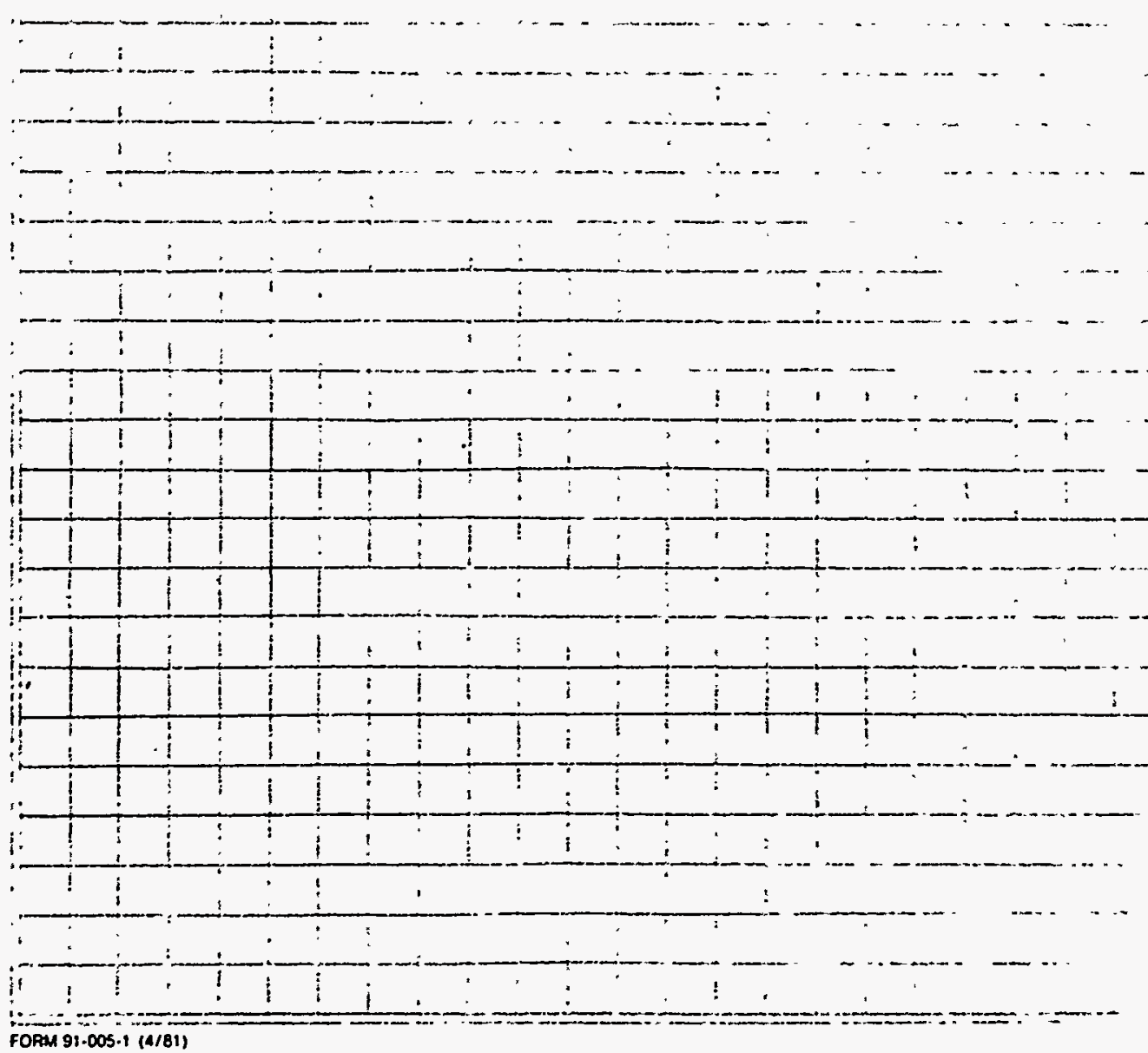

Storage Coefficient Not applicable 


\section{ATTACHMENT A \\ WELL LOG AND CONSTRUCTION SUMMARY}

WELL 675 


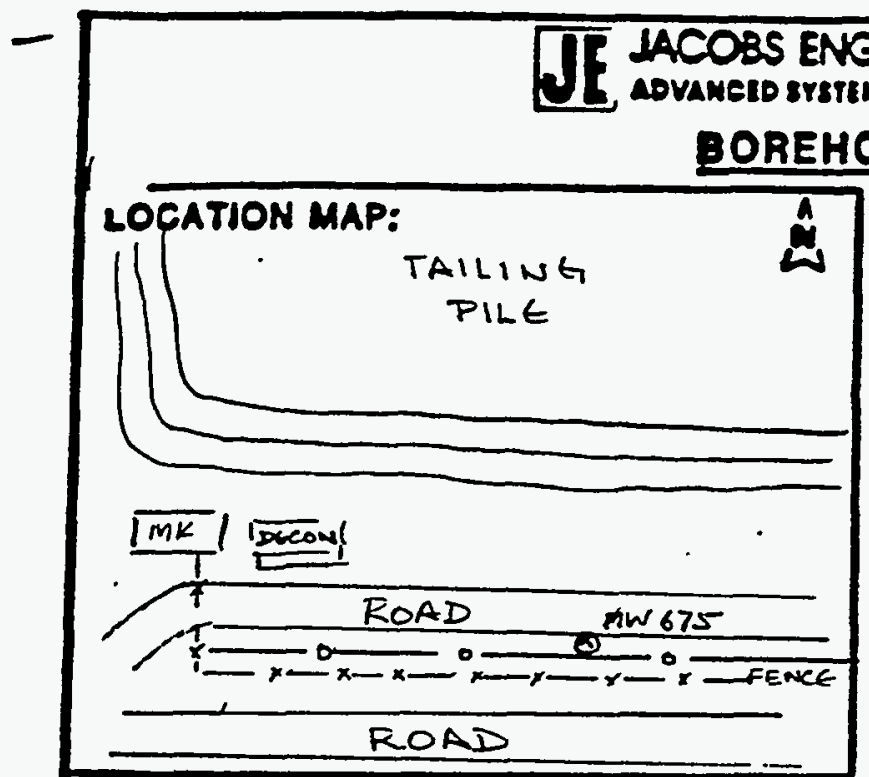

LOCATION DESCRIPTION BETWEN THE E-W DEAMINS ROAD $25^{\prime} N$ O CONTHOC EENE SITE CONDITION HIO WAY DOWN DEE PILE ON SOUTA SIbE, FLAT NEES COVERES

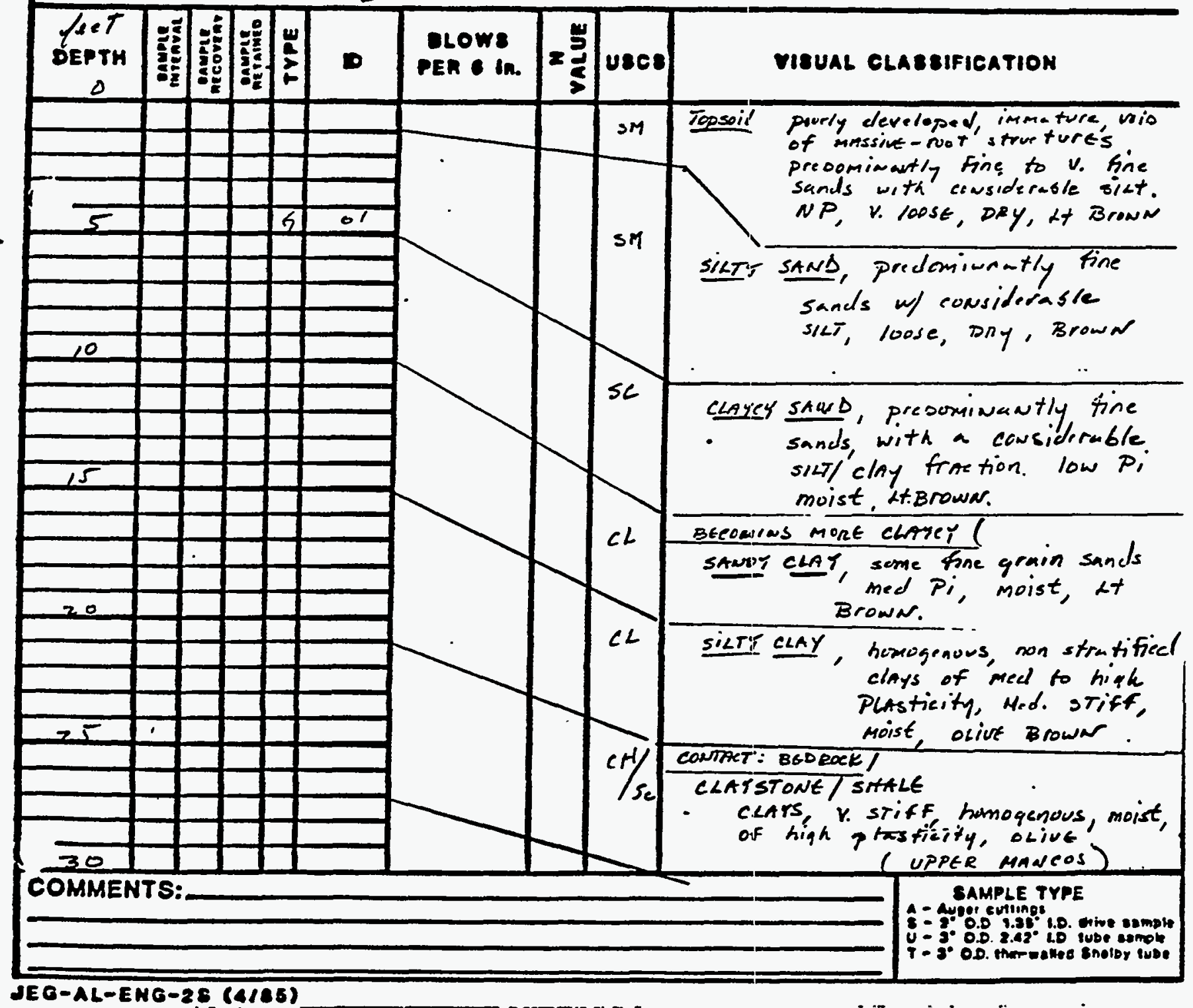




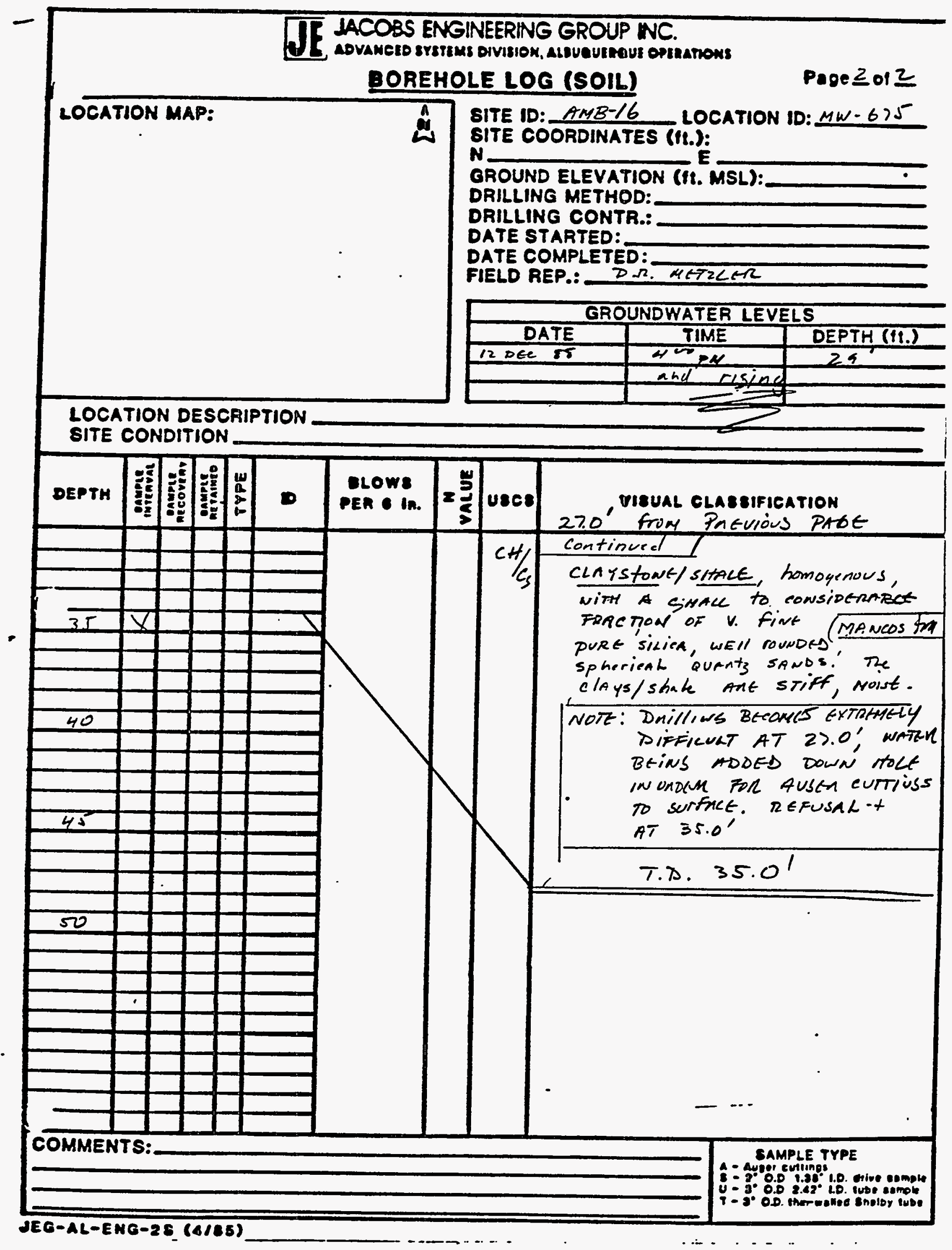




\section{FT-MeOsS ENeNEERIN GROLP NC.

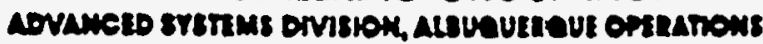

\section{WELL COMPLETION RECORD}

SITE 10: $A M B-16$ LOCATION ID: YWW- 675 Date installed: 12 dee 88 APPROX. SITE COORDINATES:(FT.) $N$ OPEN AREA PER LINEAL FT. (IN2/FT.)
$\frac{N A}{10^{2}-40}$ E $E$ NA /FORMATION OF COMPLETION: FIELD REP.: D.R. METZLAR IDP OE ONE MANCOS/Base of AllwivM DRILLER: CItEN NORTHERN

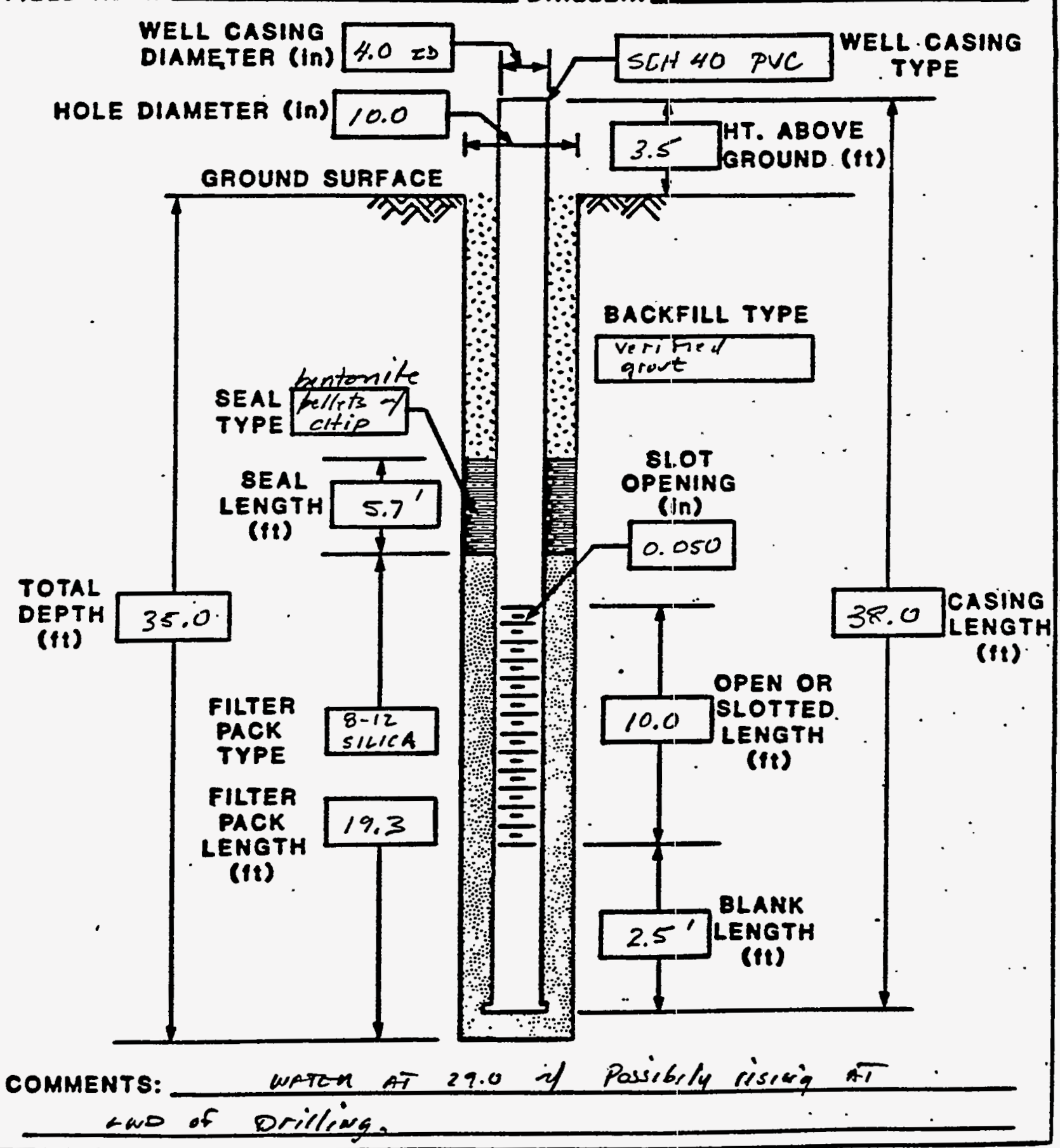




\section{IIE LACOBS ENGNEERHG GROUP NK.

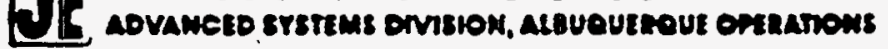 \\ BOREHOLE/WELL CONSTRUCTION LOG}

SITE ID: AMB-16 LOCATION 10:MW-675 FIELD REP:DR. METZLCR

APPROX. 8ITE COORDINATES (FT.): N NA NA

OROUND ELEVATION (FT. MSL): $26965.0^{\prime}$ COMPLETION DATE: 12 DEC 88

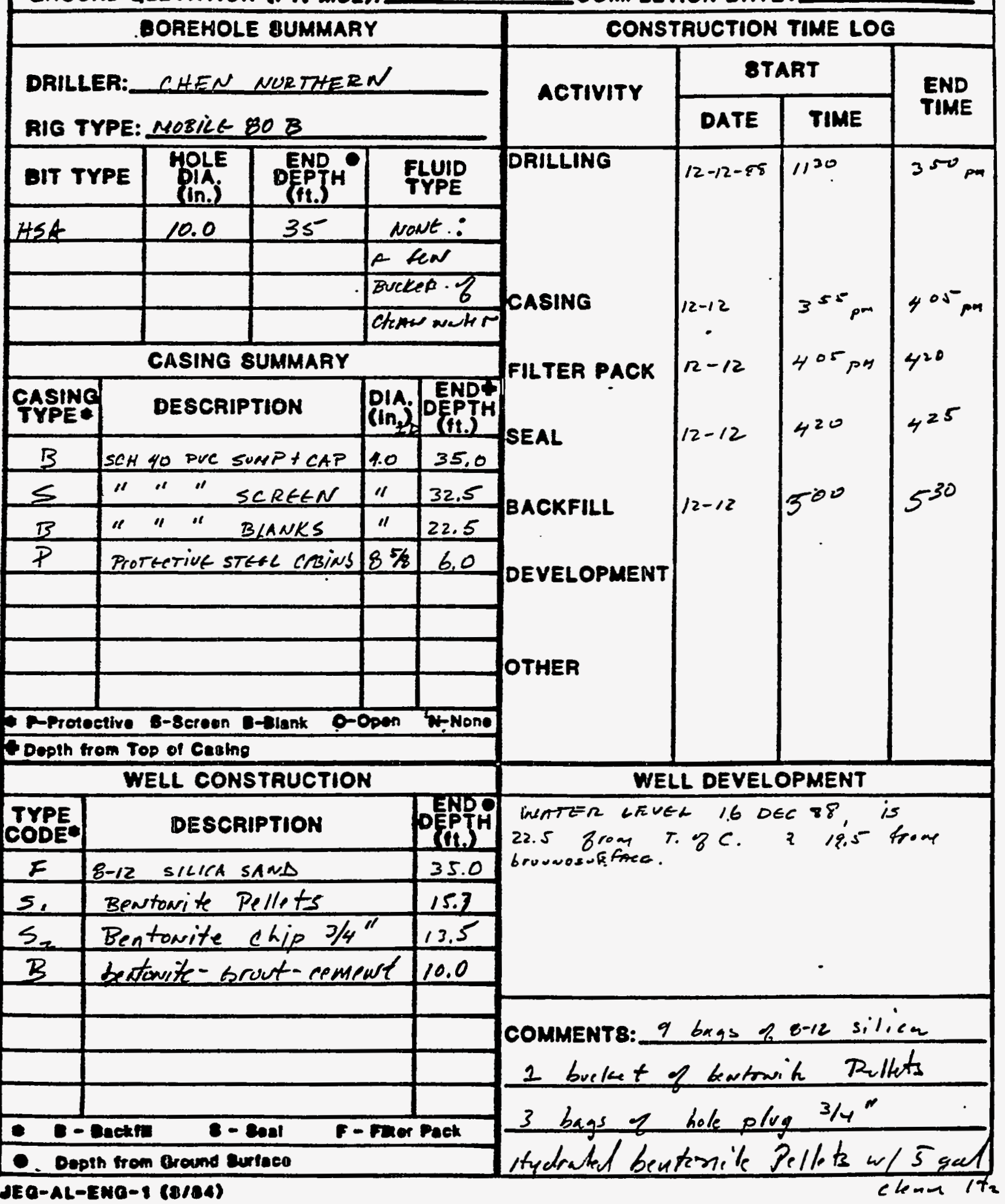




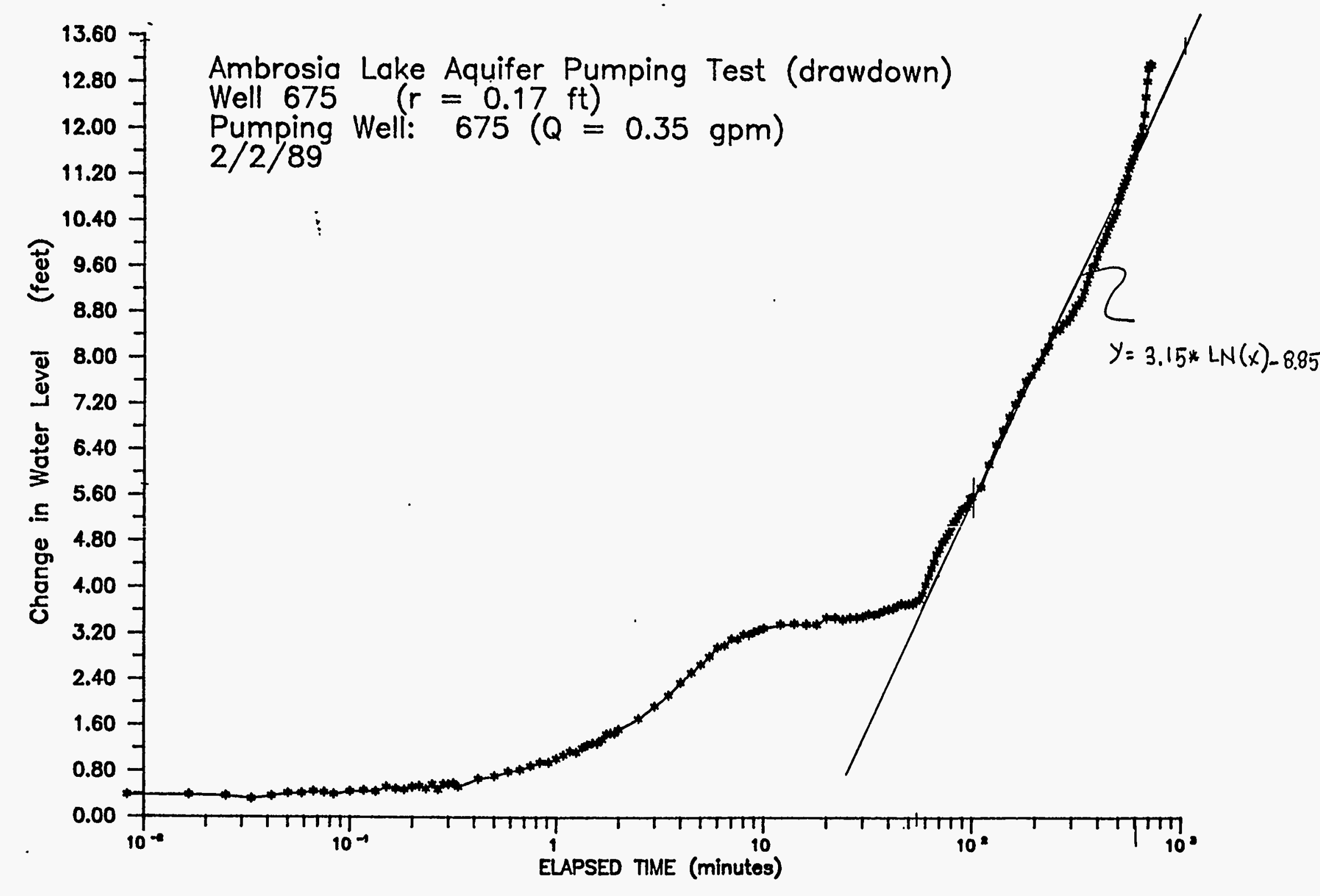


ATTACHMENT B

PUMP TEST DATA AND FIGURES 


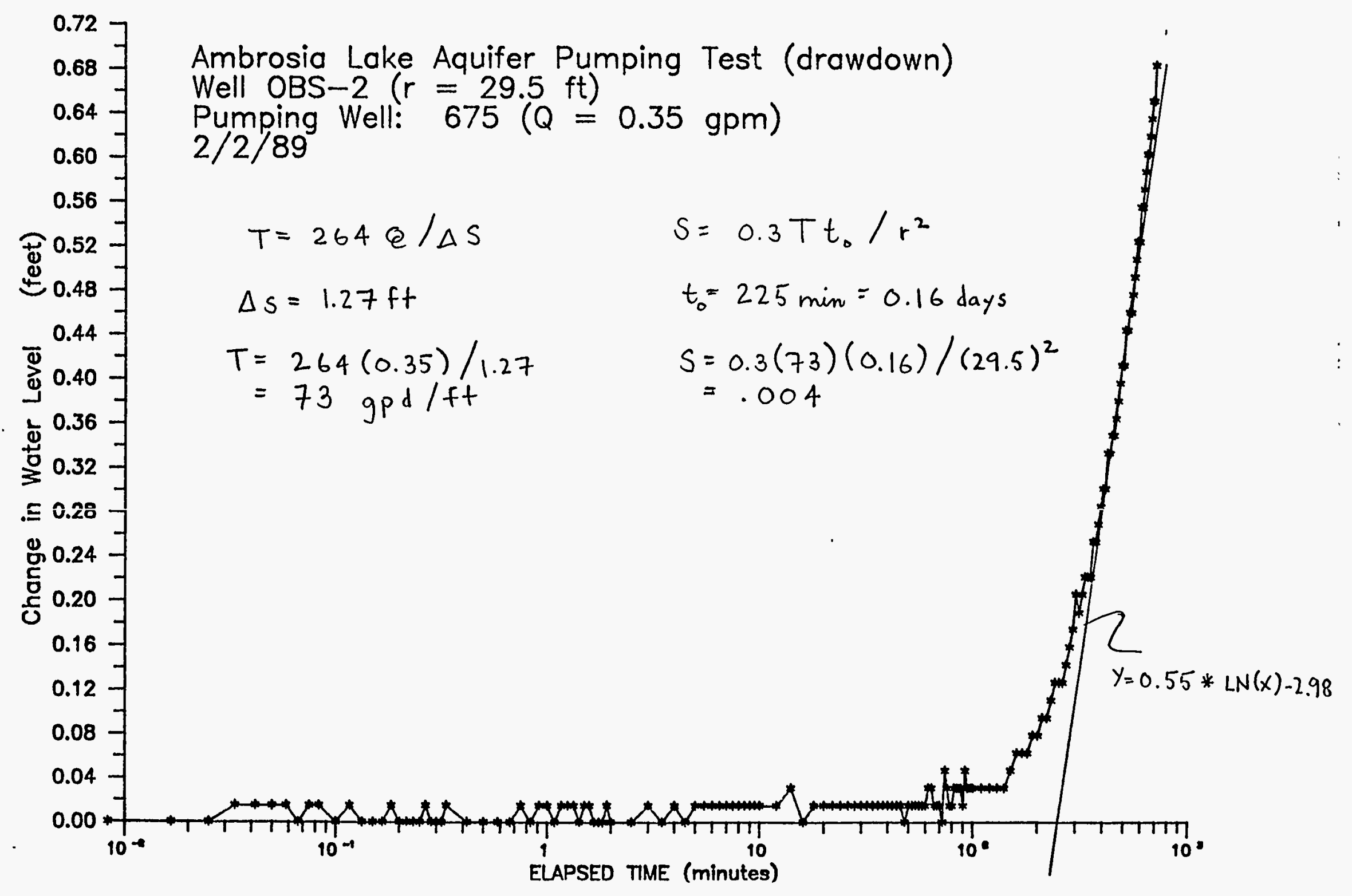




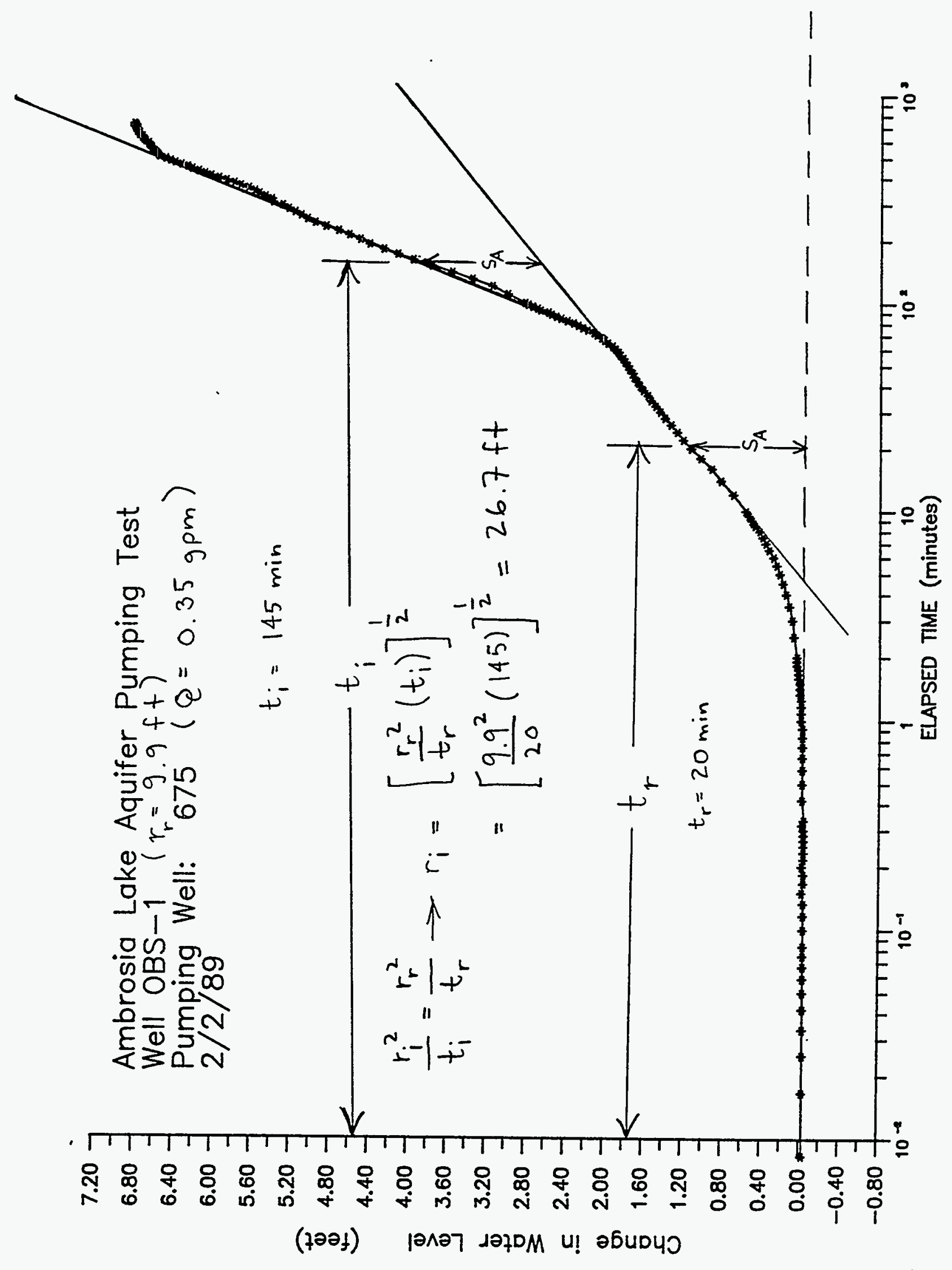




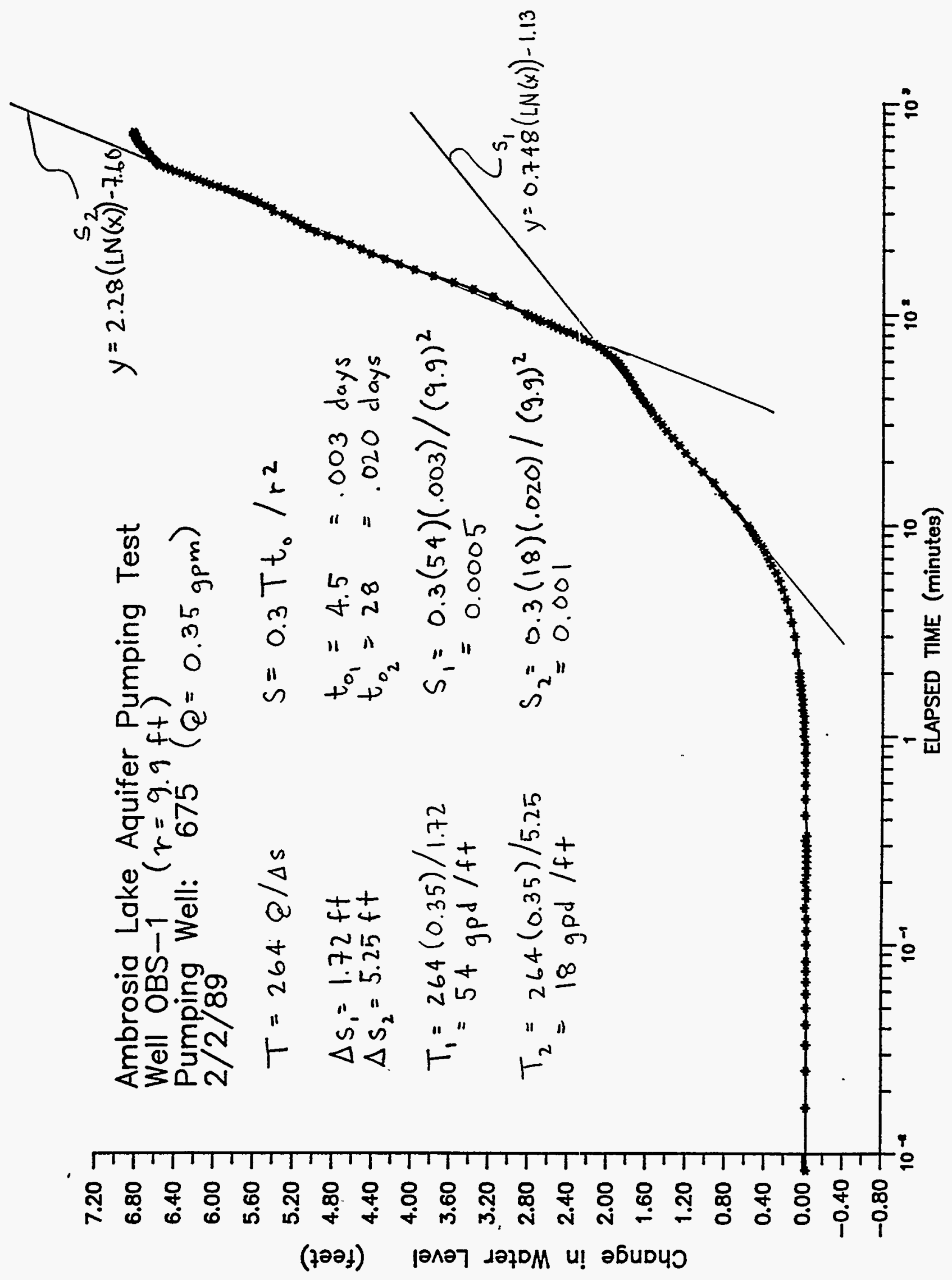


S

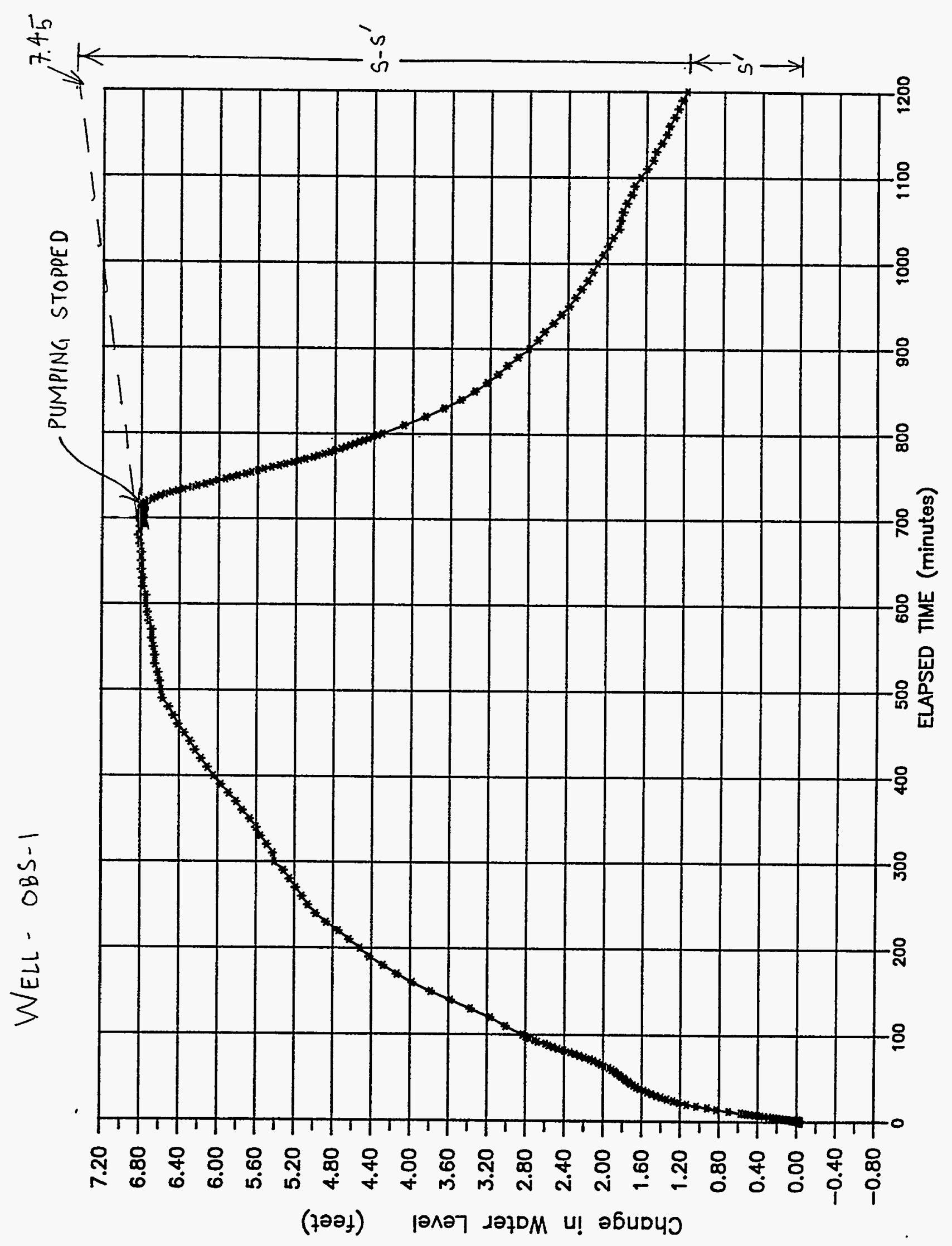




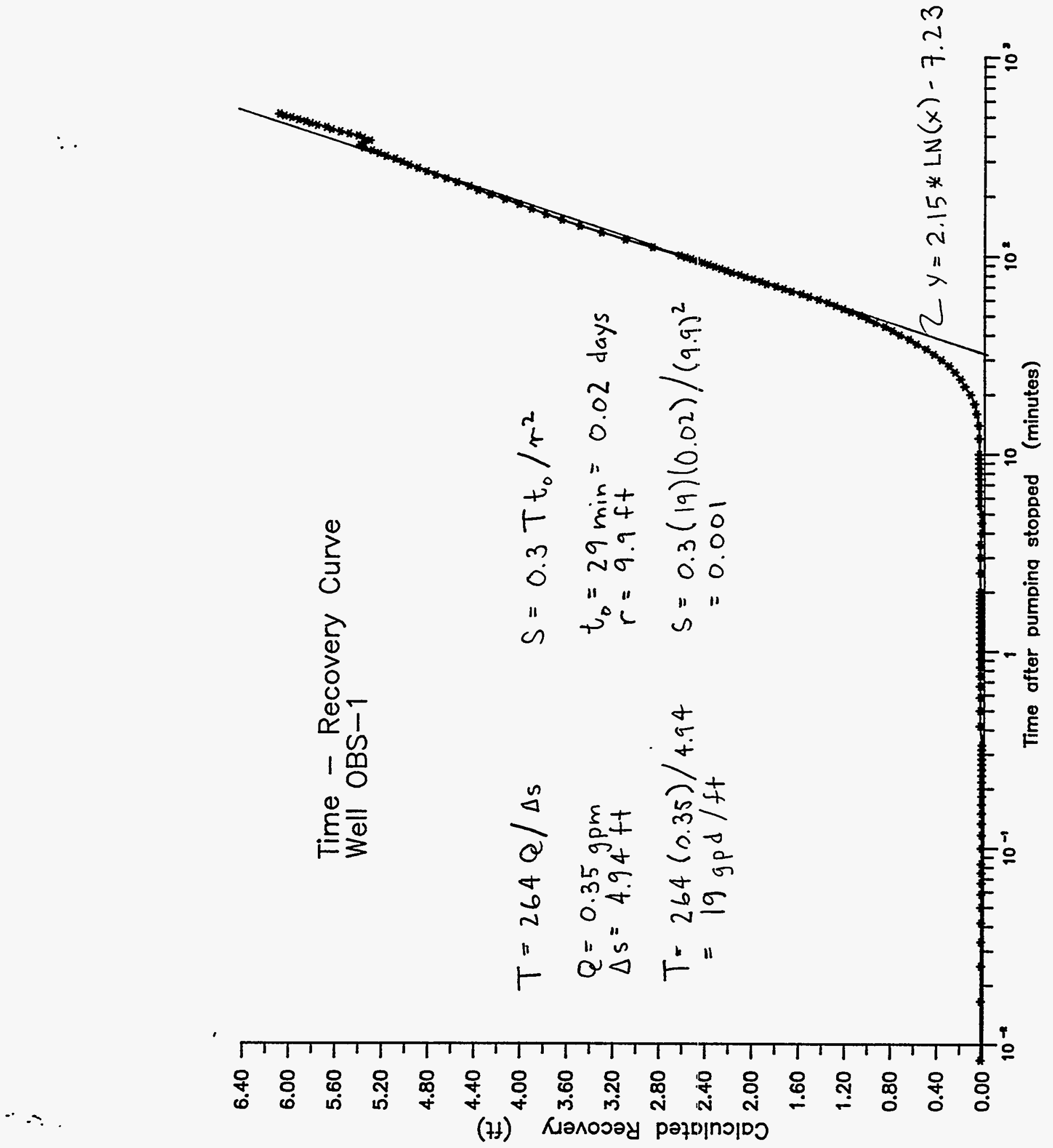




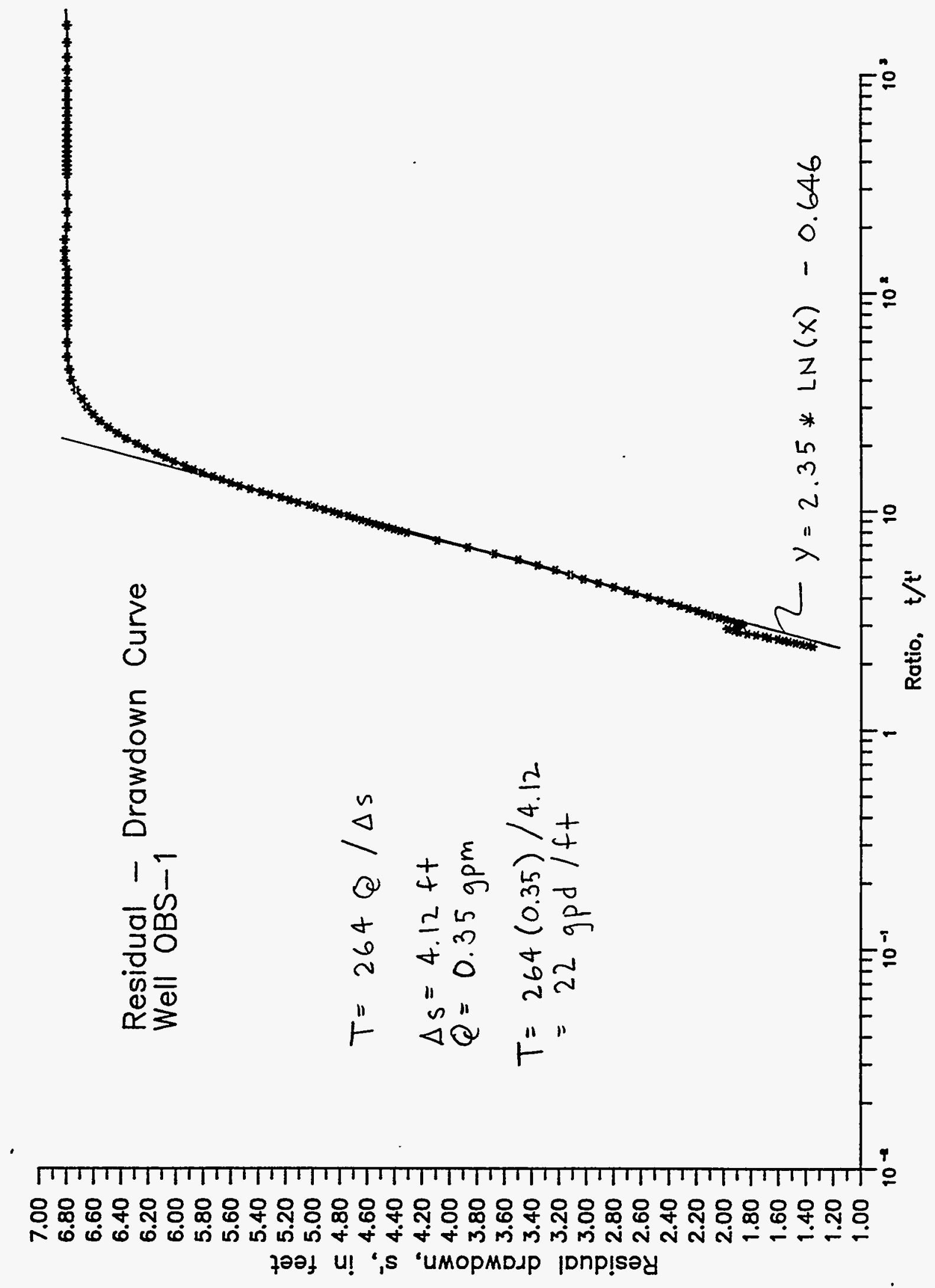




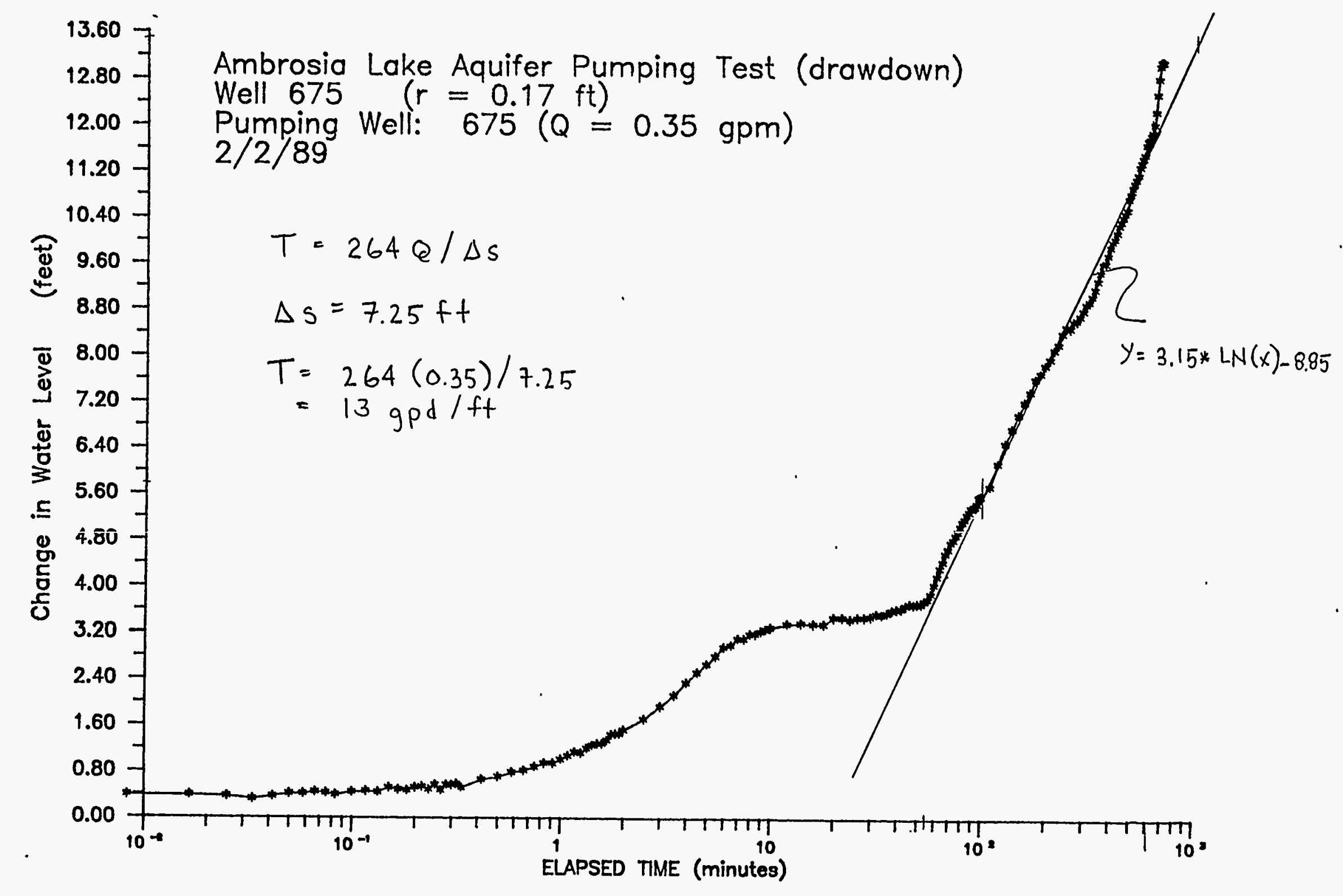




\begin{abstract}
APPENDIX C
GROUND WATER QUALITY DATA BY LOCATION

AMBROSIA LAKE, NEW MEXICO, UMTRA PROJECT SITE
\end{abstract}


GROUNDWATER QUALITY DATA BY LOCATION

SITE: AMBO1 AMBROSIA LAKE

LOCATIOH: 0568

HORTH COORDIHATE: 51550.0 FT

EAST COORDIHATE: $\quad 50990.0$ FT

$06 / 10 / 80$ TO $12 / 04 / 92$

REPORT DATE: 08/04/94

FORMATION OF COMPLETION: TRES HERMANOS - B SAHDSTOHE (TB)

HYDRAULIC FLOW RELATIONSHIP: UHKHOWH ( $H$ )

\begin{tabular}{|c|c|c|c|c|c|c|c|}
\hline PARAMETER NAME & LOG DATE & $\underset{10}{\text { SAMPLE }}$ & $\begin{array}{l}\text { UHITS OF } \\
\text { MEASURE }\end{array}$ & PVI & $\begin{array}{l}\text { PARAMETER } \\
\text { VALUE FLAGS }\end{array}$ & $\begin{array}{l}\text { DETECTION } \\
\text { LIHIT }\end{array}$ & $\begin{array}{l}\text { PARAMETER } \\
\text { UNCERTAINTY }\end{array}$ \\
\hline ALKALIKITY & $08 / 16 / 83$ & 0001 & MG/L CACO3 & & 90.20 & - &.- \\
\hline ARSENIC & $\begin{array}{l}10 / 21 / 80 \\
08 / 96 / 83\end{array}$ & $\begin{array}{l}0001 \\
0001\end{array}$ & MG/L & & $\begin{array}{l}0.012 \\
0.18\end{array}$ & $\dot{-}$ & $\dot{-}$ \\
\hline BARIUM & $\begin{array}{l}10 / 21 / 80 \\
08 / 16 / 83\end{array}$ & $\begin{array}{l}0001 \\
.0001\end{array}$ & MG/L & & $\begin{array}{l}0.01 \\
0.014\end{array}$ & $\dot{-}$ & $\dot{-}$ \\
\hline BICARBOHATE - HCO3 & $\begin{array}{l}10 / 21 / 80 \\
08 / 16 / 83\end{array}$ & $\begin{array}{l}0001 \\
0001\end{array}$ & $\mathrm{MG} / \mathrm{L}$ & & $\begin{array}{l}152.00 \\
220.00\end{array}$ & - & $\therefore$ \\
\hline BORON & $\begin{array}{l}10 / 21 / 80 \\
08 / 16 / 83\end{array}$ & $\begin{array}{l}0001 \\
0001\end{array}$ & $M \dot{G} / L$ & & $\begin{array}{l}0.44 \\
0.45\end{array}$ & $\dot{-}$ & $\dot{-}$ \\
\hline CALCIUM & $\begin{array}{l}10 / 21 / 80 \\
08 / 16 / 83\end{array}$ & $\begin{array}{l}0001 \\
0001\end{array}$ & $M G / L$ & & $\begin{array}{l}200.00 \\
210.00\end{array}$ & - & - \\
\hline CARBOHATE & $08 / 16 / 83$ & 0001 & MG/L & $<$ & 10.00 & 10. & - \\
\hline CHLORIDE & $\begin{array}{l}10 / 21 / 80 \\
08 / 16 / 83\end{array}$ & $\begin{array}{l}0001 \\
0001\end{array}$ & MG/L & $<$ & $\begin{array}{l}10.00 \\
18.00\end{array}$ & 10. & $\dot{-}$ \\
\hline CHROMIUM & $\begin{array}{l}10 / 21 / 80 \\
08 / 16 / 83\end{array}$ & $\begin{array}{l}0001 \\
0001\end{array}$ & MG/L & $<$ & $\begin{array}{l}0.001 \\
0.036\end{array}$ & 0.001 & $\dot{-}$ \\
\hline COBALT & $\begin{array}{l}10 / 21 / 80 \\
08 / 16 / 83\end{array}$ & $\begin{array}{l}0001 \\
0001\end{array}$ & $M G / L$ & $<$ & $\begin{array}{l}0.006 \\
0.029\end{array}$ & 0.006 & - \\
\hline LEAD & $\begin{array}{l}10 / 21 / 80 \\
08 / 16 / 83\end{array}$ & $\begin{array}{l}0001 \\
0001\end{array}$ & $M G / L$ & $<$ & $\begin{array}{l}0.001 \\
0.19\end{array}$ & 0.001 & $\because$ \\
\hline MAGKESIUM & $\begin{array}{l}10 / 21 / 80 \\
08 / 16 / 83\end{array}$ & $\begin{array}{l}0001 \\
0001\end{array}$ & MG/L & & $\begin{array}{l}92.00 \\
83.00\end{array}$ & - & : \\
\hline MAKGAHESE & $\begin{array}{l}10 / 21 / 80 \\
08 / 16 / 83\end{array}$ & $\begin{array}{l}0001 \\
0001\end{array}$ & $M G / L$ & & $\begin{array}{l}0.021 \\
0.11\end{array}$ & - & 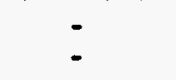 \\
\hline MERCURY & $10 / 21 / 80$ & 0001 & MG/L & $<$ & 0.001 & 0.001 & - \\
\hline HOLYBDEEUU & $\begin{array}{l}10 / 21 / 80 \\
08 / 16 / 83\end{array}$ & $\begin{array}{l}0001 \\
0001\end{array}$ & $M G / L$ & & $\begin{array}{l}0.035 \\
0.019\end{array}$ & - & - \\
\hline
\end{tabular}


GROUNDWATER QUALITY DATA BY LOCATION

SITE: AMBOI AMBROSIA LAKE

LOCATION: 0568

NORTH COORDIHATE: $\quad 51550.0$ FT

EAST COORDINATE: $\quad 50990.0$ FT

06/10/80 TO $12 / 04 / 92$

REPORT DATE: $08 / 04 / 94$

FORMATION OF COMPLETION: TRES HERMANOS - B SANDSTONE (TB)

HYDRAULIC FLON RELATIONSHIP: UHKNOWN (N)

\begin{tabular}{|c|c|c|c|c|c|c|c|}
\hline PARAMETER NAME & LOG DATE & $\underset{\text { ID }}{\text { SAMPLE }}$ & $\begin{array}{l}\text { UNITS OF } \\
\text { MEASURE }\end{array}$ & PVI & $\begin{array}{l}\text { PARAMETER } \\
\text { VALUE FLAGS }\end{array}$ & $\begin{array}{l}\text { DETECTIOH } \\
\text { LIMIT }\end{array}$ & $\begin{array}{l}\text { PARAMETER } \\
\text { UHCERTAIHTY }\end{array}$ \\
\hline HICKEL & $\begin{array}{l}10 / 21 / 80 \\
08 / 16 / 83\end{array}$ & $\begin{array}{l}0001 \\
0001\end{array}$ & $M G / L$ & & $\begin{array}{l}0.038 \\
0.077\end{array}$ & - & - \\
\hline HITRATE & $\begin{array}{l}10 / 21 / 80 \\
08 / 16 / 83\end{array}$ & $\begin{array}{l}0001 \\
0001\end{array}$ & MG/L & $<$ & $\begin{array}{l}1.30 \\
1.00\end{array}$ & i. & - \\
\hline PH & $\begin{array}{l}10 / 21 / 80 \\
08 / 16 / 83\end{array}$ & $\begin{array}{l}0001 \\
0001\end{array}$ & SU & & $\begin{array}{l}7.80 \\
7.30\end{array}$ & - & - \\
\hline POTASSIUA & $\begin{array}{l}10 / 21 / 80 \\
08 / 16 / 83\end{array}$ & $\begin{array}{l}0001 \\
0001\end{array}$ & MG/L & & $\begin{array}{l}6.40 \\
9.00\end{array}$ & - & - \\
\hline RADIUN-226 & $\begin{array}{l}10 / 21 / 80 \\
08 / 16 / 83\end{array}$ & $\begin{array}{l}0001 \\
0001\end{array}$ & PCI/L & & $\begin{array}{l}2.45 \\
1.10\end{array}$ & - & - \\
\hline RADIUN-226 + RADIUN-228 & $08 / 16 / 83$ & 0001 & $\mathrm{PCI} / \mathrm{L}$ & & 2.20 & - & - \\
\hline RADIUM-228 & $08 / 16 / 83$ & 0001 & $\mathrm{PCI} / \mathrm{L}$ & & 1.10 & - & - \\
\hline SELENIUM & $\begin{array}{l}10 / 21 / 80 \\
08 / 16 / 83\end{array}$ & $\begin{array}{l}0009 \\
0001\end{array}$ & MG/L & & $\begin{array}{l}0.009 \\
0.21\end{array}$ & : & - \\
\hline SILVER & $\begin{array}{l}10 / 21 / 80 \\
08 / 16 / 83\end{array}$ & $\begin{array}{l}0001 \\
0001\end{array}$ & $M G / L$ & $<$ & $\begin{array}{l}0.002 \\
0.065\end{array}$ & 0.002 & - \\
\hline SOOIUA & $\begin{array}{l}10 / 21 / 80 \\
08 / 16 / 83\end{array}$ & $\begin{array}{l}0001 \\
0001\end{array}$ & MG/L & & $\begin{array}{l}100.00 \\
110.00\end{array}$ & - & - \\
\hline SPECIFIC CONDUCTANCE & $10 / 21 / 80$ & 0001 & UМНО/CM & & 1860.00 & - & - \\
\hline SULFATE & $\begin{array}{l}10 / 21 / 80 \\
08 / 16 / 83\end{array}$ & $\begin{array}{l}0001 \\
0001\end{array}$ & $M G / L$ & & $\begin{array}{l}990.00 \\
960.00\end{array}$ & - & - \\
\hline TOTAL DISSOLVED SOLIDS & $\begin{array}{l}10 / 21 / 80 \\
08 / 16 / 83\end{array}$ & $\begin{array}{l}0001 \\
0001\end{array}$ & MG/L & & $\begin{array}{l}1590.00 \\
1480.00\end{array}$ & - & $\dot{-}$ \\
\hline TOTAL SUSPENDED SOLIDS & $10 / 21 / 80$ & 0001 & MG/L & & 124.00 & - & - \\
\hline URAHIUN & $\begin{array}{l}10 / 21 / 80 \\
08 / 16 / 83\end{array}$ & $\begin{array}{l}0001 \\
0001\end{array}$ & MG/L. & & $\begin{array}{l}0.027 \\
0.016\end{array}$ & $\dot{-}$ & - \\
\hline VANADIUA & $\begin{array}{l}10 / 21 / 80 \\
08 / 16 / 83\end{array}$ & $\begin{array}{l}0001 \\
0001\end{array}$ & HG/L & $<$ & $\begin{array}{l}0.003 \\
0.046\end{array}$ & 0.003 & - \\
\hline ZIMC & $\begin{array}{l}10 / 21 / 80 \\
08 / 16 / 83\end{array}$ & $\begin{array}{l}0001 \\
0001\end{array}$ & MG/L & & $\begin{array}{l}0.17 \\
0.045\end{array}$ & - & - \\
\hline
\end{tabular}

PARAMETER VALUE INDICATOR (PVI):

< - Less than detection limit

SAMPLE ID COOES:

OOO1 - FILTERED SAMPLE (.45 MICRONS) 
GRONWDATER OUALITY DATA BY LOCATION

SITE: NMBO1 AMBROSIA LAKE

LOCATIOH: 0569

MORTH COORDIHATE: $\quad 49990.0$ FT

EAST COORDIHATE: $\quad 51010.0$ FT

$06 / 10 / 80$ TO $12 / 04 / 92$

REPORT OATE: $08 / 04 / 94$

FORMATIOH OF COMPLETIOH: TRES HERHANOS-A SAMDSTOHE (TR)

HYDRAULIC FLON RELATIOHSHIP: DONN GRADIENT (D)

\begin{tabular}{|c|c|c|c|c|c|c|c|}
\hline PARAMETER NAME & LOG DATE & $\underset{10}{\text { SAMPLE }}$ & $\begin{array}{l}\text { UHITS OF } \\
\text { MEASURE }\end{array}$ & PVI & $\begin{array}{l}\text { PARAMETER } \\
\text { VALUE FLAGS }\end{array}$ & $\begin{array}{l}\text { DETECTION } \\
\text { LIMIT }\end{array}$ & $\begin{array}{l}\text { PARAMETER } \\
\text { UNCERTAINTY }\end{array}$ \\
\hline ALKALIHITY & $\begin{array}{l}11 / 14 / 83 \\
10 / 15 / 85\end{array}$ & $\begin{array}{l}0009 \\
0001\end{array}$ & MG/L CACO3 & & $\begin{array}{l}49.20 \\
43 .\end{array}$ & - & - \\
\hline ALCHIRUM & $\begin{array}{l}11 / 04 / 80 \\
11 / 14 / 83\end{array}$ & $\begin{array}{l}0009 \\
0001\end{array}$ & MG/L & $<$ & $\begin{array}{l}0.72 \\
0.008\end{array}$ & 0.008 & - \\
\hline BARIUY & $\begin{array}{l}11 / 04 / 80 \\
11 / 14 / 83\end{array}$ & $\begin{array}{l}0001 \\
0001\end{array}$ & MG/L & $<$ & $\begin{array}{l}0.009 \\
0.013\end{array}$ & 0.013 & - \\
\hline BICARBONATE - HCOZ & $\begin{array}{l}11 / 04 / 80 \\
11 / 14 / 83\end{array}$ & $\begin{array}{l}0001 \\
0001\end{array}$ & $M G / L$ & & $\begin{array}{l}232.00 \\
120.00\end{array}$ & - & - \\
\hline CALCIUM & $\begin{array}{l}11 / 04 / 80 \\
11 / 14 / 83 \\
10 / 15 / 85\end{array}$ & $\begin{array}{l}0001 \\
0001 \\
0001\end{array}$ & $M G / L$ & & $\begin{array}{l}98.00 \\
65.00 \\
161 .\end{array}$ & - & : \\
\hline CARBOHATE & $11 / 14 / 83$ & 0001 & $M G / L$ & $<$ & 10.00 & 10. & - \\
\hline CHLORIDE & $\begin{array}{l}11 / 04 / 80 \\
11 / 14 / 83 \\
10 / 15 / 85\end{array}$ & $\begin{array}{l}0001 \\
0001 \\
0001\end{array}$ & HG/L & & $\begin{array}{l}24.00 \\
25.00 \\
35 .\end{array}$ & :- & : \\
\hline CHROMIUM & $\begin{array}{l}11 / 04 / 80 \\
11 / 14 / 83\end{array}$ & $\begin{array}{l}0001 \\
0001\end{array}$ & MG/L & $<$ & $\begin{array}{l}0.001 \\
0.026\end{array}$ & $\begin{array}{l}0.001 \\
0.026\end{array}$ & $\dot{-}$ \\
\hline FLUORIDE & $\begin{array}{l}11 / 04 / 80 \\
11 / 14 / 83\end{array}$ & $\begin{array}{l}0001 \\
0001\end{array}$ & MG/L & $<$ & $\begin{array}{l}0.20 \\
1.00\end{array}$ & $\begin{array}{l}0.2 \\
1 .\end{array}$ & - \\
\hline IROM & $\begin{array}{l}11 / 04 / 80 \\
11 / 14 / 83\end{array}$ & $\begin{array}{l}0001 \\
0001\end{array}$ & $M G / L$ & & $\begin{array}{l}0.30 \\
0.14\end{array}$ & - & $\because$ \\
\hline LEAD & $\begin{array}{l}11 / 04 / 80 \\
11 / 14 / 83\end{array}$ & $\begin{array}{l}0001 \\
0001\end{array}$ & $M G / L$ & $<$ & $\begin{array}{l}0.001 \\
0.03\end{array}$ & 0.001 & - \\
\hline MAGHESIUM & $\begin{array}{l}11 / 04 / 80 \\
11 / 14 / 83 \\
10 / 15 / 85\end{array}$ & $\begin{array}{l}0001 \\
0001 \\
0001\end{array}$ & HG/L & & $\begin{array}{l}92.00 \\
84.00 \\
167 .\end{array}$ & 0.001 & : \\
\hline MAHGAHESE & $\begin{array}{l}11 / 04 / 80 \\
11 / 14 / 83\end{array}$ & $\begin{array}{l}0001 \\
0001\end{array}$ & $M O / L$ & & $\begin{array}{l}0.44 \\
0.41\end{array}$ & - & : \\
\hline
\end{tabular}

PARAMETER VALUE INDICATOR (PVI): < - LESS THAN DETECTION LIHIT

SAMPLE ID COOES:

0009 - FILTERED SAMPLE (.45 MICRONS) 
GROUNDHATER QUALITY DATA BY LOCATIOH

SITE: AMBO1 AMBROSIA LAKE

LOCATION: 0569

NORTH COORDIHATE: 49990.0 FT

EAST COORDINATE: $\quad 51010.0$ FT

06/10/80 TO $12 / 04 / 92$

REPORT DATE: $08 / 04 / 94$

FORMATION OF COMPLETION: TRES HERMANOS-A SANDSTONE (TR) HYDRAULIC FLOW RELATIONSHIP: DOWH GRADIENT (D)

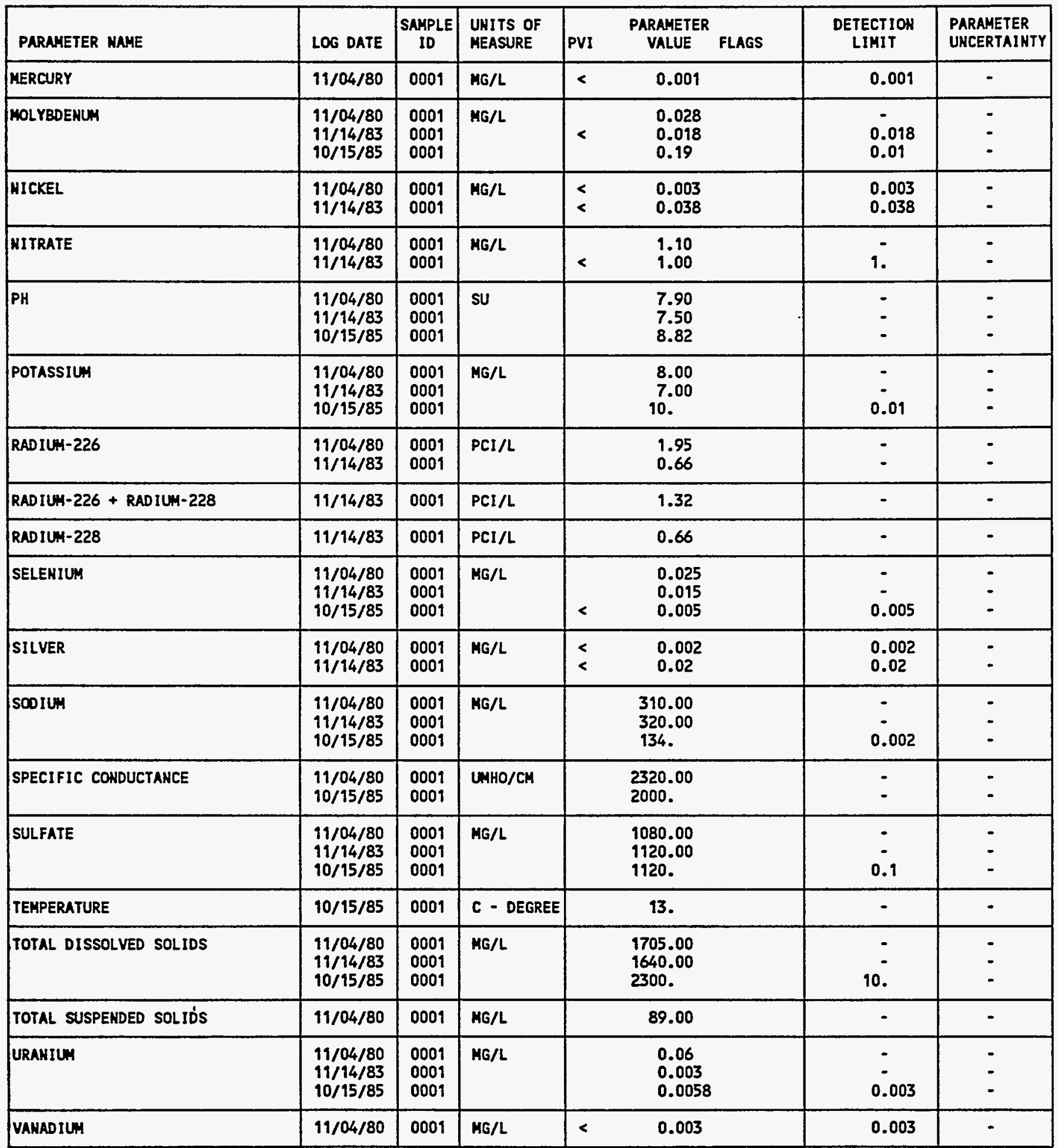

PARAMETER VALUE INDICATOR (PVI): < - LESS THAN DETECTIOH LIMIT

SAMPLE ID CODES:

0001 - FILTERED SAMPLE (.45 MICRONS) 
GROUNDWATER QUALITY DATA BY LOCATIOH

SITE: AMBO1 AMBROSIA LAKE

LOCATION: 0569

MORTH COORDINATE: 49990.0 FT

EAST COORDIHATE: $\quad 51010.0$ FT

06/10/80 TO 12/04/92

REPORT DATE: 08/04/94

FORHATIOH OF COMPLETIOH: TRES HERMAHOS-A SAMDSTONE (TR)

HYDRAULIC FLOW RELATIONSHIP: DOWN GRADIENT (D)

\begin{tabular}{|c|c|c|c|c|c|c|c|}
\hline PARAMETER NAME & LOG DATE & $\underset{\text { ID }}{\text { SAMPLE }}$ & $\begin{array}{l}\text { UNITS OF } \\
\text { MEASURE }\end{array}$ & PVI & $\begin{array}{l}\text { PARAMETER } \\
\text { VALUE FLAGS }\end{array}$ & $\begin{array}{l}\text { DETECTION } \\
\text { LIMIT }\end{array}$ & $\begin{array}{l}\text { PARAMETER } \\
\text { UNCERTAINTY }\end{array}$ \\
\hline VAMADIUA & $11 / 14 / 83$ & 0001 & MG/L & & 0.007 & - & - \\
\hline ZIHC & $\begin{array}{l}11 / 04 / 80 \\
11 / 14 / 83\end{array}$ & $\begin{array}{l}0001 \\
0009\end{array}$ & MG/L & & $\begin{array}{l}0.079 \\
0.022\end{array}$ & - & - \\
\hline
\end{tabular}

PARAMETER VALUE IMDICATOR (PVI): < - LESS THAM DETECTION LIMIT SAMPLE ID COOES:

0001 - FILTERED SAMPLE (.45 MICRONS) 
GROUNDWATER OUALITY DATA BY LOCATIOH

SITE: AMBOI AMBROSIA LAKE

LOCATION: 0570

NORTH COORDIHATE: 51730.0 FT

EAST COORDINATE: $\quad 47650.0$ FT

06/10/80 TO $12 / 04 / 92$

REPORT DATE: 08/04/94

FORMATION OF COMPLETION: TRES HERMANOS - B SAMDSTONE (TB) HYDRAULIC FLOW RELATIONSHIP: UNKHOWN (N)

\begin{tabular}{|c|c|c|c|c|c|c|c|}
\hline PARAMETER NAME & LOG DATE & $\underset{\text { ID }}{\text { SAMPLE }}$ & $\begin{array}{l}\text { UNITS OF } \\
\text { MEASURE }\end{array}$ & PVI & $\begin{array}{l}\text { PARAMETER } \\
\text { VALUE FLAGS }\end{array}$ & $\begin{array}{l}\text { DETECTIOH } \\
\text { LIMIT }\end{array}$ & $\begin{array}{l}\text { PARAMETER } \\
\text { UHCERTAIKTY }\end{array}$ \\
\hline ALKALINITY & $05 / 24 / 83$ & 0001 & MG/L CACO3 & & 86.10 & - & - \\
\hline ALUAINUH & $\begin{array}{l}90 / 02 / 80 \\
05 / 24 / 83\end{array}$ & $\begin{array}{l}0001 \\
0001\end{array}$ & MG/L & & $\begin{array}{l}0.088 \\
0.22\end{array}$ & $\dot{-}$ & $\ddot{-}$ \\
\hline ARSENIC & $\begin{array}{l}10 / 02 / 80 \\
05 / 24 / 83\end{array}$ & $\begin{array}{l}0001 \\
0001\end{array}$ & MG/L & & $\begin{array}{l}0.004 \\
0.21\end{array}$ & : & - \\
\hline BARIUA & $\begin{array}{l}10 / 02 / 80 \\
05 / 24 / 83\end{array}$ & $\begin{array}{l}0001 \\
0001\end{array}$ & MG/L & $<$ & $\begin{array}{l}0.018 \\
0.02\end{array}$ & 0.02 & $\overline{-}$ \\
\hline BICARBOHATE - HCO3 & $\begin{array}{l}10 / 02 / 80 \\
05 / 24 / 83\end{array}$ & $\begin{array}{l}0001 \\
0001\end{array}$ & MG/L & & $\begin{array}{l}397.00 \\
210.00\end{array}$ & - & $\dot{-}$ \\
\hline BORON & $\begin{array}{l}10 / 02 / 80 \\
05 / 24 / 83\end{array}$ & $\begin{array}{l}0001 \\
0001\end{array}$ & HG/L & & $\begin{array}{l}0.38 \\
0.25\end{array}$ & - & $\dot{-}$ \\
\hline CADMIUM & $\begin{array}{l}10 / 02 / 80 \\
05 / 24 / 83\end{array}$ & $\begin{array}{l}0001 \\
0001\end{array}$ & HG/L & $<$ & $\begin{array}{l}0.008 \\
0.017\end{array}$ & 0.008 & 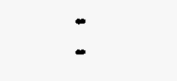 \\
\hline CALCIUM & $\begin{array}{l}10 / 02 / 80 \\
05 / 24 / 83\end{array}$ & $\begin{array}{l}0001 \\
0001\end{array}$ & MG/L & & $\begin{array}{l}420.00 \\
430.00\end{array}$ & - & - \\
\hline CARBOHATE & $05 / 24 / 83$ & 0001 & MG/L & $<$ & 10.00 & 10. & - \\
\hline CHLORIDE & $\begin{array}{l}10 / 02 / 80 \\
05 / 24 / 83\end{array}$ & $\begin{array}{l}0001 \\
0001\end{array}$ & $M G / L$ & & $\begin{array}{l}2398.00 \\
1840.00\end{array}$ & - & $\dot{-}$ \\
\hline CHROMIUM & $\begin{array}{l}10 / 02 / 80 \\
05 / 24 / 83\end{array}$ & $\begin{array}{l}0001 \\
0001\end{array}$ & MG/L & & $\begin{array}{l}0.061 \\
0.01\end{array}$ & - & 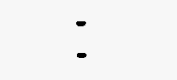 \\
\hline COBALT & $\begin{array}{l}10 / 02 / 80 \\
05 / 24 / 83\end{array}$ & $\begin{array}{l}0001 \\
0001\end{array}$ & $M G / L$ & $<$ & $\begin{array}{l}0.006 \\
0.04\end{array}$ & $\begin{array}{l}0.006 \\
0.04\end{array}$ & $\dot{-}$ \\
\hline COPPER & $\begin{array}{l}10 / 02 / 80 \\
05 / 24 / 83\end{array}$ & $\begin{array}{l}0001 \\
0001\end{array}$ & MG/L. & $<$ & $\begin{array}{l}0.001 \\
0.03\end{array}$ & 0.03 & $\ddot{-}$ \\
\hline CYAKIDE & $05 / 24 / 83$ & 0001 & MG/L & $<$ & 1.00 & 1. & - \\
\hline FLUORIDE & $\begin{array}{l}10 / 02 / 80 \\
05 / 24 / 83\end{array}$ & $\begin{array}{l}0001 \\
0001\end{array}$ & MG/L & $<$ & $\begin{array}{l}0.10 \\
1.00\end{array}$ & 1. & - \\
\hline IRON & $\begin{array}{l}10 / 02 / 80 \\
05 / 24 / 83\end{array}$ & $\begin{array}{l}0001 \\
0001\end{array}$ & MG/L & & $\begin{array}{r}0.11 \\
28.00\end{array}$ & - & - \\
\hline LEAD & $\begin{array}{l}10 / 02 / 80 \\
05 / 24 / 83\end{array}$ & $\begin{array}{l}0001 \\
0001\end{array}$ & MG/L & & $\begin{array}{l}0.002 \\
0.27\end{array}$ & 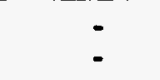 & - \\
\hline MAGNESIUM & $\begin{array}{l}10 / 02 / 80 \\
05 / 24 / 83\end{array}$ & $\begin{array}{l}0001 \\
0001\end{array}$ & MG/L & & $\begin{array}{l}1400.00 \\
1900.00\end{array}$ & - & - \\
\hline MANGAHESE & $\begin{array}{l}10 / 02 / 80 \\
05 / 24 / 83\end{array}$ & $\begin{array}{l}0001 \\
0001\end{array}$ & MG/L & & $\begin{array}{l}2.00 \\
1.70\end{array}$ & - & - \\
\hline MERCURY & $10 / 02 / 80$ & 0001 & $M G / L$ & $<$ & 0.001 & 0.009 & - \\
\hline MOLYBDEKUM & $\begin{array}{l}10 / 02 / 80 \\
05 / 24 / 83\end{array}$ & $\begin{array}{l}0001 \\
0001\end{array}$ & MG/L & & $\begin{array}{l}0.002 \\
0.031\end{array}$ & - & - \\
\hline
\end{tabular}

PARAMETER VALUE INDICATOR (PVI): < - LESS THAN DETECTION LIMIT 
GROUNDWATER QUALITY DATA BY LOCATION

SITE: AMBO1 AMBROSIA LAKE

LOCATIOH: 0570

HORTH COORDIHATE: $\quad 51730.0$ FT

EAST COORDIKATE: $\quad 47650.0$ FT

$06 / 10 / 80$ TO $12 / 04 / 92$

REPORT DATE: 08/04/94

FORMATION OF COMPLETION: TRES HERMANOS - B SAHDSTONE (TB)

HYDRAULIC FLOW RELATIONSHIP: UNKHOWH (H)

\begin{tabular}{|c|c|c|c|c|c|c|c|}
\hline PARAMETER NAME & LOG DATE & $\underset{\text { ID }}{\text { SAMPLE }}$ & $\begin{array}{l}\text { UNITS OF } \\
\text { MEASURE }\end{array}$ & $\begin{array}{lc} & \text { PARAMETER } \\
\text { PVI } & \text { VALUE }\end{array}$ & FLAGS & $\begin{array}{l}\text { DETECTION } \\
\text { LIMIT }\end{array}$ & $\begin{array}{l}\text { PARAMETER } \\
\text { UNCERTAIKTY }\end{array}$ \\
\hline HICKEL & $\begin{array}{l}10 / 02 / 80 \\
05 / 24 / 83\end{array}$ & $\begin{array}{l}0001 \\
0001\end{array}$ & MG/L & $\begin{array}{l}0.01 \\
0.075\end{array}$ & & - & $\therefore$ \\
\hline HITRATE & $\begin{array}{l}10 / 02 / 80 \\
05 / 24 / 83\end{array}$ & $\begin{array}{l}0009 \\
0001\end{array}$ & MG/L & $\begin{array}{r}24.00 \\
6.00\end{array}$ & & - & - \\
\hline PH & $\begin{array}{l}10 / 02 / 80 \\
05 / 24 / 83\end{array}$ & $\begin{array}{l}0001 \\
0001\end{array}$ & SU & $\begin{array}{l}7.00 \\
6.20\end{array}$ & & - & $\dot{-}$ \\
\hline potassium & $\begin{array}{l}10 / 02 / 80 \\
05 / 24 / 83\end{array}$ & $\begin{array}{l}0001 \\
0001\end{array}$ & $M G / L$ & $\begin{array}{l}15.00 \\
11.00\end{array}$ & & - & - \\
\hline RADIUH-226 & $\begin{array}{l}10 / 02 / 80 \\
05 / 24 / 83\end{array}$ & $\begin{array}{l}0001 \\
0001\end{array}$ & PCI/L & $\begin{array}{l}1.41 \\
1.77\end{array}$ & & $\cdot$ & $\because$ \\
\hline RADIUH-226 + RADIUH-228 & $05 / 24 / 83$ & 0001 & $\mathrm{PCI} / \mathrm{L}$ & 3.54 & & - & - \\
\hline RADIUH-228 & $05 / 24 / 83$ & 0001 & $\mathrm{PCI} / \mathrm{L}$ & 1.77 & & - & - \\
\hline SELENIUAM & $\begin{array}{l}10 / 02 / 80 \\
05 / 24 / 83\end{array}$ & $\begin{array}{l}0001 \\
0001\end{array}$ & MG/L & $\begin{array}{l}0.68 \\
0.24\end{array}$ & & - & - \\
\hline SILVER & $\begin{array}{l}10 / 02 / 80 \\
05 / 24 / 83\end{array}$ & $\begin{array}{l}0001 \\
0001\end{array}$ & MG/L & $\begin{array}{l}0.002 \\
0.035\end{array}$ & & 0.002 & : \\
\hline scoIU⿴囗十 & $\begin{array}{l}10 / 02 / 80 \\
05 / 24 / 83\end{array}$ & $\begin{array}{l}0001 \\
0001\end{array}$ & MG/L & $\begin{array}{l}800.00 \\
730.00\end{array}$ & & $\dot{-}$ & $\dot{-}$ \\
\hline SPECIFIC CONDUCTANCE & $10 / 02 / 80$ & 0001 & UAKO/CM & 12400.00 & & - & - \\
\hline SULFATE & $\begin{array}{l}10 / 02 / 80 \\
05 / 24 / 83\end{array}$ & $\begin{array}{l}0001 \\
0001\end{array}$ & MG/L & $\begin{array}{l}4780.00 \\
7630.00\end{array}$ & & - & - \\
\hline TOTAL DISSOLVED SOLIDS & $\begin{array}{l}10 / 02 / 80 \\
05 / 24 / 83\end{array}$ & $\begin{array}{l}0001 \\
0001\end{array}$ & MG/L & $\begin{array}{r}10710.00 \\
12: 70\end{array}$ & & - & $\because$ \\
\hline TOTAL ORGAKIC CARBOH & $05 / 24 / 83$ & 0001 & MG/L & 18.00 & & - & - \\
\hline TOTAL SUSPEKDED SOLIOS & $10 / 02 / 80$ & 0009 & MG/L & 36.00 & & - & - \\
\hline URAHILM & $\begin{array}{l}10 / 02 / 80 \\
05 / 24 / 83\end{array}$ & $\begin{array}{l}0001 \\
0001\end{array}$ & MG/L & $\begin{array}{l}0.04 \\
0.08\end{array}$ & & - & - \\
\hline VANADIUM & $\begin{array}{l}10 / 02 / 80 \\
05 / 24 / 83\end{array}$ & $\begin{array}{l}0001 \\
0001\end{array}$ & MG/L & $\begin{array}{l}0.028 \\
0.04\end{array}$ & & - & - \\
\hline ZINC & $\begin{array}{l}10 / 02 / 80 \\
05 / 24 / 83\end{array}$ & $\begin{array}{l}0001 \\
0001\end{array}$ & MG/L & $\begin{array}{l}0.23 \\
0.70\end{array}$ & & - & : \\
\hline
\end{tabular}

PARAMETER VALUE IMDICATOR (PVI): < - LESS THAN DETECTIOH LIMIT

SAKPLE ID COOES:

0001 - FILTIERED SAMPLE (.45 MICROWS) 
FORMATION OF COMPLETION: TRES HERMANOS-A SAMDSTONE (TR) HYDRAULIC FLON RELATIOHSHIP: UNKNOWN (N)

\begin{tabular}{|c|c|c|c|c|c|c|c|}
\hline PARAMETER MAME & LOG DATE & $\underset{10}{\text { SAMPLE }}$ & $\begin{array}{l}\text { UNITS OF } \\
\text { MEASURE }\end{array}$ & PV & $\begin{array}{l}\text { PARAMETER } \\
\text { VALUE FLAGS }\end{array}$ & $\begin{array}{l}\text { DETECTION } \\
\text { LIMIT }\end{array}$ & $\begin{array}{l}\text { PARAMETER } \\
\text { UHCERTAIHTY }\end{array}$ \\
\hline ALKALINITY & $08 / 15 / 83$ & 0001 & MG/L CACO3 & & 28.70 & - & - \\
\hline ALUHINUM & $\begin{array}{l}11 / 05 / 80 \\
08 / 15 / 83\end{array}$ & $\begin{array}{l}0001 \\
0001\end{array}$ & MG/L & $<$ & $\begin{array}{l}0.05 \\
0.04\end{array}$ & $\begin{array}{l}0.05 \\
0.04\end{array}$ & 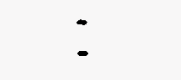 \\
\hline ARSENIC & $\begin{array}{l}11 / 05 / 80 \\
08 / 15 / 83\end{array}$ & $\begin{array}{l}0001 \\
0001\end{array}$ & MG/L & $<$ & $\begin{array}{l}0.009 \\
0.10\end{array}$ & 0.1 & $\dot{-}$ \\
\hline BARIUH & $\begin{array}{l}11 / 05 / 80 \\
08 / 15 / 83\end{array}$ & $\begin{array}{l}0001 \\
0001\end{array}$ & MG/L & & $\begin{array}{l}0.063 \\
0.021\end{array}$ & - & - \\
\hline BICARBONATE - HCO3 & $\begin{array}{l}11 / 05 / 80 \\
08 / 15 / 83\end{array}$ & $\begin{array}{l}0001 \\
0001\end{array}$ & MG/L & & $\begin{array}{l}71.00 \\
70.00\end{array}$ & - & - \\
\hline BORON & $\begin{array}{l}11 / 05 / 80 \\
08 / 15 / 83\end{array}$ & $\begin{array}{l}0001 \\
0001\end{array}$ & MG/L & & $\begin{array}{l}0.27 \\
0.38\end{array}$ & $\dot{-}$ & - \\
\hline CADMIUM & $\begin{array}{l}11 / 05 / 80 \\
08 / 15 / 83\end{array}$ & $\begin{array}{l}0001 \\
0001\end{array}$ & MG/L & $<$ & $\begin{array}{l}0.008 \\
0.007\end{array}$ & $\begin{array}{l}0.008 \\
0.007\end{array}$ & 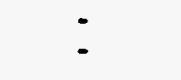 \\
\hline CALCIUM & $\begin{array}{l}11 / 05 / 80 \\
08 / 15 / 83\end{array}$ & $\begin{array}{l}0001 \\
0001\end{array}$ & MG/L & & $\begin{array}{r}100.00 \\
22.00\end{array}$ & 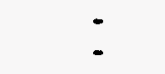 & $\dot{-}$ \\
\hline CARBONATE & $08 / 15 / 83$ & 0001 & $M G / L$ & $<$ & 10.00 & 10. & - \\
\hline CHLORIDE & $\begin{array}{l}11 / 05 / 80 \\
08 / 15 / 83\end{array}$ & $\begin{array}{l}0001 \\
0001\end{array}$ & $M G / L$ & & $\begin{array}{r}30.00 \\
2.40\end{array}$ & - & - \\
\hline CHROMIUH & $\begin{array}{l}11 / 05 / 80 \\
08 / 15 / 83\end{array}$ & $\begin{array}{l}0001 \\
0001\end{array}$ & MG/L & $<$ & $\begin{array}{l}0.005 \\
0.03\end{array}$ & $\overline{0.03}$ & $\dot{-}$ \\
\hline COBALT & $\begin{array}{l}11 / 05 / 80 \\
08 / 15 / 83\end{array}$ & $\begin{array}{l}0001 \\
0001\end{array}$ & $M G / L$ & $<$ & $\begin{array}{l}0.006 \\
0.01\end{array}$ & $\begin{array}{l}0.006 \\
0.01\end{array}$ & - \\
\hline COPPER & $\begin{array}{l}11 / 05 / 80 \\
08 / 15 / 83\end{array}$ & $\begin{array}{l}0001 \\
0001\end{array}$ & HG/L & $<$ & $\begin{array}{l}0.021 \\
0.02\end{array}$ & 0.02 & - \\
\hline CYANIDE & $08 / 15 / 83$ & 0001 & MG/L & $<$ & 0.10 & 0.1 & - \\
\hline FLUORIDE & $\begin{array}{l}11 / 05 / 80 \\
08 / 15 / 83\end{array}$ & $\begin{array}{l}0001 \\
0001\end{array}$ & HG/L & $<$ & $\begin{array}{l}0.20 \\
0.30\end{array}$ & 0.2 & - \\
\hline IRON & $\begin{array}{l}11 / 05 / 80 \\
08 / 15 / 83\end{array}$ & $\begin{array}{l}0001 \\
0001\end{array}$ & HG/L & & $\begin{array}{l}0.19 \\
0.028\end{array}$ & - & - \\
\hline LEAD & $\begin{array}{l}11 / 05 / 80 \\
08 / 15 / 83\end{array}$ & $\begin{array}{l}0001 \\
0001\end{array}$ & MG/L & $<$ & $\begin{array}{l}0.001 \\
0.07\end{array}$ & $\begin{array}{l}0.001 \\
0.07\end{array}$ & - \\
\hline MAGMESIUN & $\begin{array}{l}11 / 05 / 80 \\
08 / 15 / 83\end{array}$ & $\begin{array}{l}0001 \\
0001\end{array}$ & MG/L & & $\begin{array}{r}37.00 \\
3.70\end{array}$ & - & - \\
\hline MANGANESE & $\begin{array}{l}11 / 05 / 80 \\
08 / 15 / 83\end{array}$ & $\begin{array}{l}0001 \\
0001\end{array}$ & MG/L & & $\begin{array}{l}0.071 \\
0.017\end{array}$ & - & - \\
\hline MERCURY & $11 / 05 / 80$ & 0001 & MG/L & $<$ & 0.001 & 0.001 & - \\
\hline MOLYBDENUM & $\begin{array}{l}11 / 05 / 80 \\
08 / 15 / 83\end{array}$ & $\begin{array}{l}0001 \\
0001\end{array}$ & MG/L & $<$ & $\begin{array}{l}0.027 \\
0.02\end{array}$ & 0.02 & - \\
\hline
\end{tabular}


GROUHDHATER OUALITY DATA BY LOCATIOH

SITE: AMBO1 AMBROSIA LAKE

LOCATION: 0571

HORTH COORDINATE: 57990.0 FT

EAST COOROINATE: $\quad 47500.0$ FT

06/10/80 TO $12 / 04 / 92$

REPORT DATE: 08/04/94

FORMATION OF COMPLETIOH: TRES HERMANOS-A SAMDSTOWE (TR)

HYORAULIC FLOW RELATIONSHIP: UNKHOWH (H)

\begin{tabular}{|c|c|c|c|c|c|c|c|}
\hline PARAMETER MAME & LOS DATE & $\begin{array}{c}\text { SAMPLE } \\
10\end{array}$ & $\begin{array}{l}\text { UNITS OF } \\
\text { MEASURE }\end{array}$ & PVI & $\begin{array}{l}\text { PARAMETER } \\
\text { VALUE FLAGS }\end{array}$ & $\begin{array}{l}\text { DETECTION } \\
\text { LIHIT }\end{array}$ & $\begin{array}{l}\text { PARAMETER } \\
\text { UMCERTAINTY }\end{array}$ \\
\hline HICKEL & $\begin{array}{l}11 / 05 / 80 \\
08 / 15 / 83\end{array}$ & $\begin{array}{l}0001 \\
0001\end{array}$ & MG/L & $<$ & $\begin{array}{l}0.003 \\
0.04\end{array}$ & $\begin{array}{l}0.003 \\
0.04\end{array}$ & 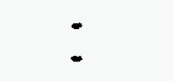 \\
\hline MITRATE & $\begin{array}{l}11 / 05 / 80 \\
08 / 15 / 83\end{array}$ & $\begin{array}{l}0001 \\
0001\end{array}$ & MG/L & $<$ & $\begin{array}{l}1.80 \\
1.00\end{array}$ & 1. & - \\
\hline $\mathbf{P H}$ & $\begin{array}{l}11 / 05 / 80 \\
08 / 15 / 83\end{array}$ & $\begin{array}{l}0001 \\
0001\end{array}$ & SU & & $\begin{array}{l}7.80 \\
8.20\end{array}$ & - & $\dot{-}$ \\
\hline POTASSIUN & $\begin{array}{l}11 / 05 / 80 \\
08 / 15 / 83\end{array}$ & $\begin{array}{l}0001 \\
0001\end{array}$ & MG/L & & $\begin{array}{r}20.00 \\
8.90\end{array}$ & - & $\dot{-}$ \\
\hline RADIUA-226 & $\begin{array}{l}11 / 05 / 80 \\
08 / 15 / 83\end{array}$ & $\begin{array}{l}0001 \\
0001\end{array}$ & $\mathrm{PCI} / \mathrm{L}$ & & $\begin{array}{l}1.901 \\
2.52\end{array}$ & $\dot{-}$ & $\dot{-}$ \\
\hline RADIUA-226 + RADIUH -228 & $08 / 15 / 83$ & 0001 & $\mathrm{PCI} / \mathrm{L}$ & & 5.04 & - & - \\
\hline RADIUH-228 & $08 / 15 / 83$ & 0001 & PCI/L & & 2.52 & - & - \\
\hline SELENIUM & $\begin{array}{l}11 / 05 / 80 \\
08 / 15 / 83\end{array}$ & $\begin{array}{l}0001 \\
0001\end{array}$ & MG/L & $<$ & $\begin{array}{l}0.011 \\
0.10\end{array}$ & 0.1 & $\dot{-}$ \\
\hline SILVER & $\begin{array}{l}11 / 05 / 80 \\
08 / 15 / 83\end{array}$ & $\begin{array}{l}0001 \\
0001\end{array}$ & $M G / L$ & $<$ & $\begin{array}{l}0.002 \\
0.02\end{array}$ & $\begin{array}{l}0.002 \\
0.02\end{array}$ & - \\
\hline soorum & $\begin{array}{l}11 / 05 / 80 \\
08 / 15 / 83\end{array}$ & $\begin{array}{l}0001 \\
0001\end{array}$ & $M G / L$ & & $\begin{array}{r}170.00 \\
3.30\end{array}$ & $\dot{-}$ & $\dot{-}$ \\
\hline SPECIFIC COMDUCTAHCE & $11 / 05 / 80$ & 0001 & ЧнНО/СК & & 1530.00 & - & - \\
\hline SULFATE & $\begin{array}{l}11 / 05 / 80 \\
08 / 15 / 83\end{array}$ & $\begin{array}{l}0001 \\
0001\end{array}$ & MG/L & & $\begin{array}{r}760.00 \\
28.00\end{array}$ & - & 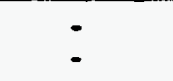 \\
\hline TOTAL DISSOLVED SOLIDS & $\begin{array}{l}11 / 05 / 80 \\
08 / 15 / 83\end{array}$ & $\begin{array}{l}0001 \\
0001\end{array}$ & $M G / L$ & & $\begin{array}{r}1130.00 \\
120.00\end{array}$ & - & $\dot{-}$ \\
\hline TOTAL CRGAMIC CARBON & $08 / 15 / 83$ & 0001 & $M G / L$ & & 21.00 & - & - \\
\hline TOTAL SUSPENDED SOLIDS & $11 / 05 / 80$ & 0001 & $\mathrm{MG} / \mathrm{L}$ & & 48.00 & - & - \\
\hline URAKIUM & $\begin{array}{l}11 / 05 / 80 \\
08 / 15 / 83\end{array}$ & $\begin{array}{l}0001 \\
0001\end{array}$ & HG/L & & $\begin{array}{l}0.10 \\
0.005\end{array}$ & 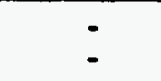 & $\because$ \\
\hline VANADIUM & $\begin{array}{l}11 / 05 / 80 \\
08 / 15 / 83\end{array}$ & $\begin{array}{l}0001 \\
0001\end{array}$ & MG/L & $<$ & $\begin{array}{l}0.003 \\
0.005\end{array}$ & $\begin{array}{l}0.003 \\
0.005\end{array}$ & - \\
\hline ZINC & $\begin{array}{l}11 / 05 / 80 \\
08 / 15 / 83\end{array}$ & $\begin{array}{l}0001 \\
0001\end{array}$ & MG/L & $<$ & $\begin{array}{l}0.14 \\
0.008\end{array}$ & 0.008 & - \\
\hline
\end{tabular}

PARAMETER VALUE INDICATOR (PVI): < - LESS THAN DETECTION LIMIT

SAMPLE ID CODES:

OOO1 - FILTERED SAMPLE (.45 MICROHS) 
GROUNDWATER QUALITY DATA BY LOCATION

SITE: AMBO1 AMBROSIA LAKE

LOCATION: 0572

NORTH COORDINATE: 52200.0 FT

EAST COORDINATE: $\quad 51100.0$ FT

06/10/80 TO $12 / 04 / 92$

REPORT DATE: 08/04/94

FORMATION OF COMPLETION: TRES HERMAHOS-A SAMDSTOHE (TR)

HYDRAULIC FLOW RELATIONSHIP: UNKNOWN (N)

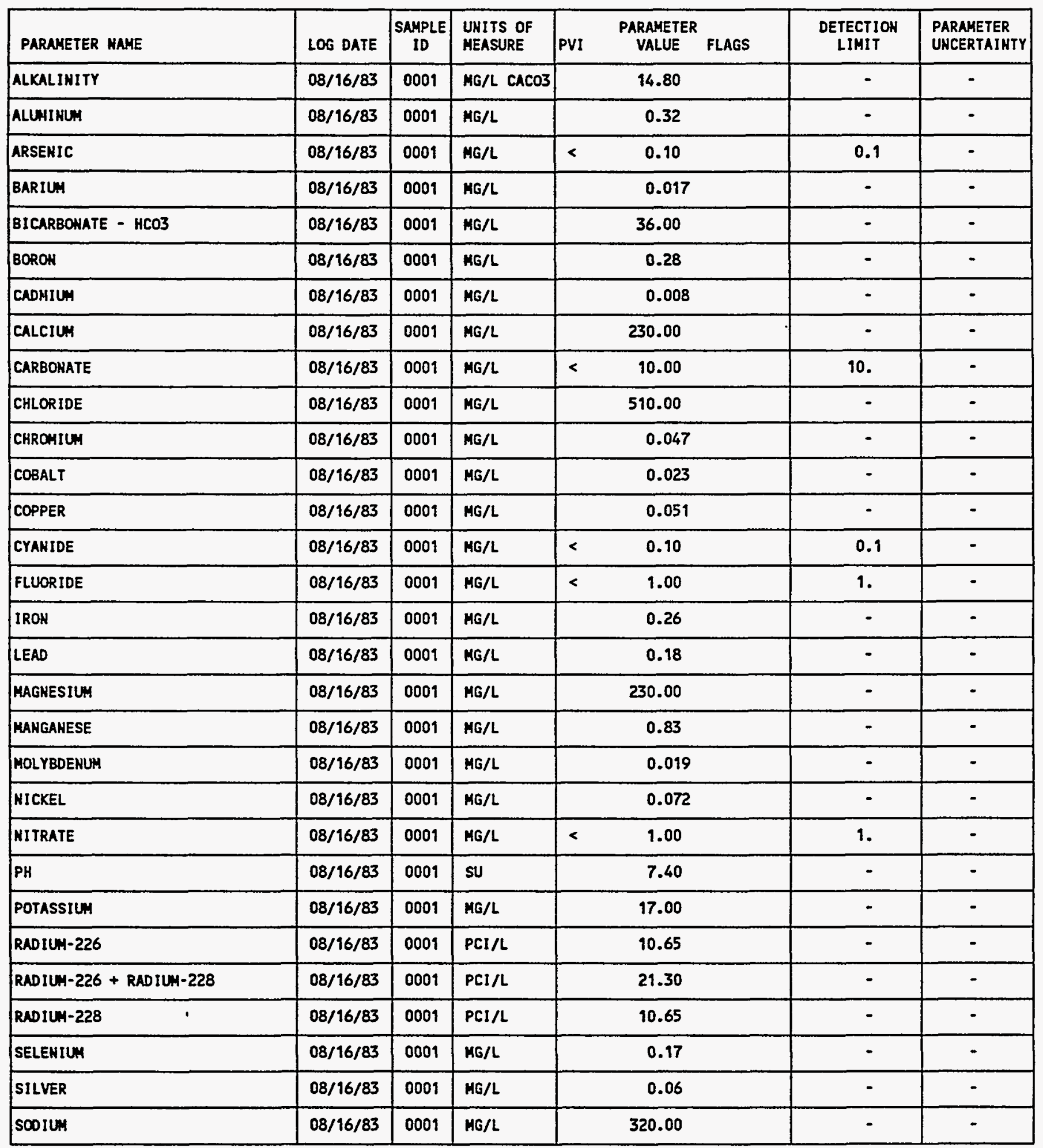

PARAMETER VALUE INDICATOR (PVI):

< - LESS THAN DETECTION LIMIT

SAMPLE ID CODES:

0001 - FILTERED SAMPLE (.45 MICROHS) 
GROUNDWATER QUALITY DATA BY LOCATION

SITE: AMBOI AMBROSIA LAKE

LOCATION: 0572

NORTH COORDINATE: $\quad 52200.0$ FT

EAST COORDIHATE: $\quad 51100.0$ FT

06/10/80 TO $12 / 04 / 92$

REPORT DATE: 08/04/94

FORMATION OF COMPLETIOH: TRES HERMANOS-A SAHDSTOHE (TR)

HYDRAULIC FLOW RELATIONSHIP: UNKHOWN (H)

\begin{tabular}{|c|c|c|c|c|c|c|c|}
\hline PARAMETER MAME & LOG DATE & $\begin{array}{c}\text { SAMPLE } \\
\text { ID }\end{array}$ & $\begin{array}{l}\text { UNITS OF } \\
\text { MEASURE }\end{array}$ & PVI & $\begin{array}{l}\text { PARAMETER } \\
\text { VALUE FLAGS }\end{array}$ & $\begin{array}{l}\text { DETECTION } \\
\text { LIMIT }\end{array}$ & $\begin{array}{l}\text { PARAMETER } \\
\text { UNCERTAINTY }\end{array}$ \\
\hline SULFATE & $08 / 16 / 83$ & 0001 & $M G / L$ & & 1650.00 & - & - \\
\hline TOTAL DISSOLVED SOLIOS & $08 / 16 / 83$ & 0001 & $M G / L$ & & 2940.00 & - & - \\
\hline TOTAL ORGANIC CARBOH & $08 / 16 / 83$ & 0001 & $M G / L$ & & 12.00 & - & - \\
\hline URAHIU⿴囗十 & $08 / 16 / 83$ & 0001 & MG/L & & 0.016 & - & - \\
\hline VANADIUA & $08 / 16 / 83$ & 0001 & MG/L & & 0.059 & - & - \\
\hline ZIHC & $08 / 16 / 83$ & 0001 & $M G / L$ & & 0.10 & - & - \\
\hline
\end{tabular}

PARAMETER VALUE IMDICATOR (PVI):

$<$ - LESS THAN DETECTION LIMIT

SAMPLE ID COOES:

0001 - FILTERED SAMPLE (.45 MICRONS) 
GROUNDWATER QUALITY DATA BY LOCATION

SITE: AHBO1 AMBROSIA LAKE

LOCATION: 0583

NORTH COORDINATE: $\quad 45780.0$ FT

EAST COORDINATE: $\quad 57270.0$ FT

06/10/80 TO 12/04/92

REPORT DATE: $08 / 04 / 94$

FORMATION OF COMPLETION: DAKOTA SANDSTOHE (KD)

HYDRAULIC FLOW RELATIONSHIP: UNKHOWN (N)

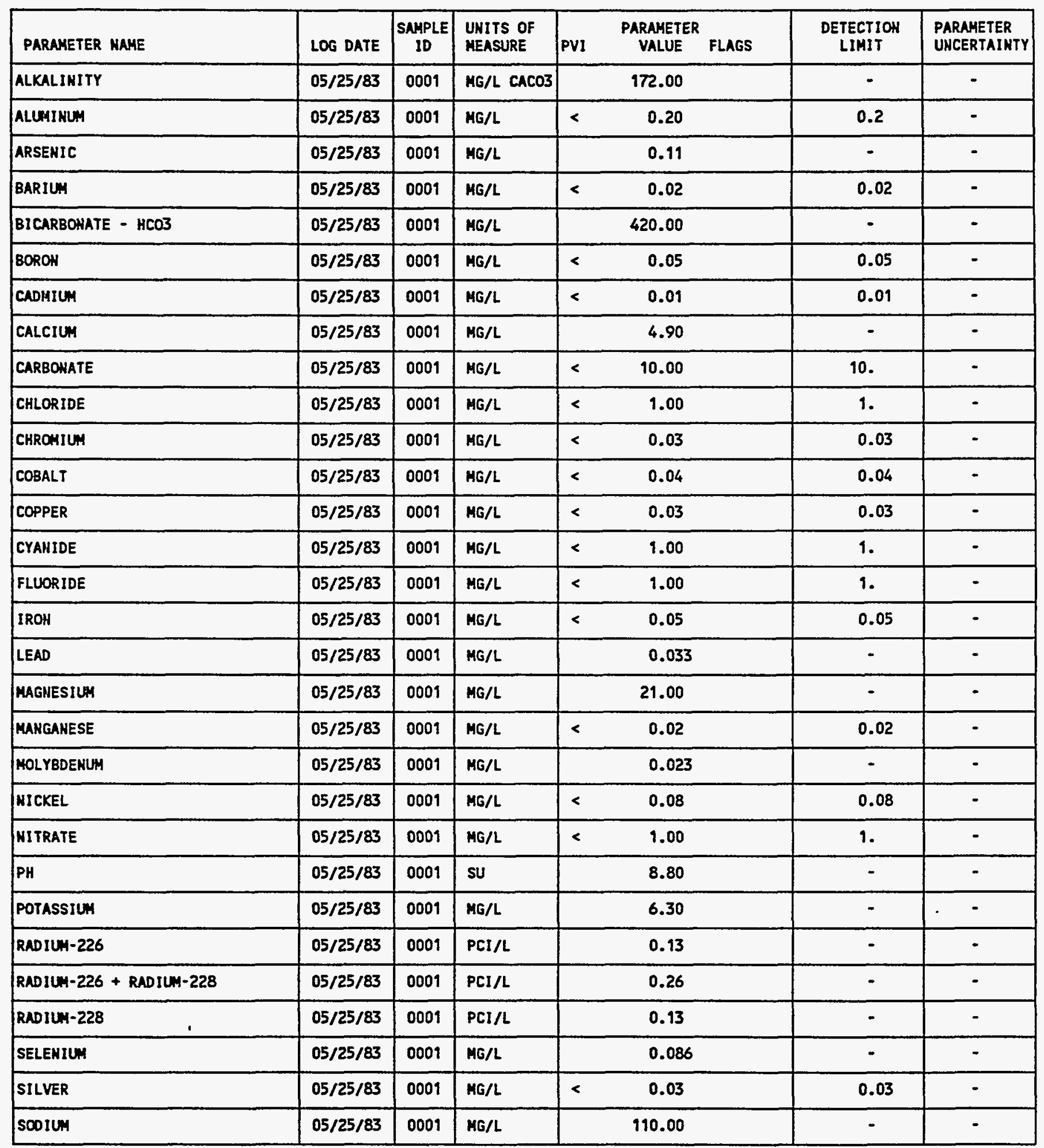

PARAMETER VALUE INDICATOR (PVI): < - LESS THAN DETECTION LIMIT

SAMPLE ID COOES:

0001 - FILTERED SAMPLE (.45 MICRONS) 
GROUMDHATER OUALITY DATA BY LOCATION

SITE: AMBO1 AMBROSIA LAKE

LOCATION: 0583

NORTH COORDINATE: $\quad 45780.0$ FT

EAST COORDINATE: $\quad 57270.0$ FT

06/10/80 TO $12 / 04 / 92$

REPORT DATE: $08 / 04 / 94$

FORMATION OF COMPLETION: DAKOTA SANOSTONE (KD)

HYDRAULIC FLON RELATIONSHIP: UNKHOWN (H)

\begin{tabular}{|c|c|c|c|c|c|c|c|}
\hline PARANETER NANE & LOG DATE & $\underset{\text { ID }}{\text { SAHPLE }}$ & $\begin{array}{l}\text { UNITS OF } \\
\text { MEASURE }\end{array}$ & PVI & $\begin{array}{l}\text { PARAMETER } \\
\text { VALUE FLAGS }\end{array}$ & $\begin{array}{l}\text { DETECTIOH } \\
\text { LIMIT }\end{array}$ & $\begin{array}{l}\text { PARAMETER } \\
\text { UXCERTAINTY }\end{array}$ \\
\hline SULFATE & $05 / 25 / 83$ & 0001 & MG/L & & 36.00 & - & - \\
\hline TOTAL ORGANIC CARBOH & $05 / 25 / 83$ & 0001 & $M G / L$ & $<$ & 5.00 & 5. & - \\
\hline VANADIUM & $05 / 25 / 83$ & 0001 & $M G / L$ & $<$ & 0.04 & 0.04 & - \\
\hline ZINC & $05 / 25 / 83$ & 0001 & $M G / L$ & & 0.014 & - & - \\
\hline
\end{tabular}

PARAMETER VALUE INDICATOR (PVI): < - LESS THAN DETECTION LIMIT

SAMPLE ID CODES:

0001 - FILTERED SAMPLE (.45 MICRONS) 
GROUHDHATER QUALITY DATA BY LOCATION

SITE: AMBOI AMBROSIA LAKE

LOCATIOH: 0584

NORTH COORDINATE: $\quad 45290.0$ FT

EAST COORDINATE: $\quad 56310.0$ FT

06/10/80 TO $12 / 04 / 92$

REPORT DATE: 08/04/94

FORMATION OF COMPLETION: DAKOTA SAMDSTONE (KD)

HYDRAULIC FLON RELATIONSHIP: UNKNOWN (N)

\begin{tabular}{|c|c|c|c|c|c|c|c|}
\hline PARAMETER MAME & LOG DATE & $\mid \begin{array}{c}\text { SAMPLE } \\
10\end{array}$ & $\begin{array}{l}\text { UNITS OF } \\
\text { MEASURE }\end{array}$ & PVI & $\begin{array}{l}\text { PARAMETER } \\
\text { VALUE FLAGS }\end{array}$ & $\begin{array}{l}\text { DETECTIOH } \\
\text { LIMIT }\end{array}$ & $\begin{array}{l}\text { PARAMETER } \\
\text { UHCERTAIHTY }\end{array}$ \\
\hline ALUAINUM & $05 / 26 / 83$ & 0001 & HG/L & $<$ & 0.20 & 0.2 & - \\
\hline ARSENIC & $05 / 26 / 83$ & 0001 & $\mathrm{HG} / \mathrm{L}$ & $<$ & 0.10 & 0.1 & - \\
\hline BARIUM & $05 / 26 / 83$ & 0001 & HG/L & & 0.05 & - & - \\
\hline BICARBOHATE - HCO3 & $05 / 26 / 83$ & 0001 & MG/L & & 110.00 & - & - \\
\hline BOROM & $05 / 26 / 83$ & 0001 & MG/L & $<$ & 0.05 & 0.05 & - \\
\hline CADMIUM & $05 / 26 / 83$ & 0001 & MG/L & $<$ & 0.01 & 0.01 & - \\
\hline CALCIUN & $05 / 26 / 83$ & 0001 & MG/L & & 110.00 & - & - \\
\hline CARBOHATE & $05 / 26 / 83$ & 0001 & MG/L & $<$ & 10.00 & 10. & - \\
\hline CHLORIDE & $05 / 26 / 83$ & 0001 & $M G / L$ & & 27.00 & - & - \\
\hline CHROMIUM & $05 / 26 / 83$ & 0001 & $M G / L$ & $<$ & 0.03 & 0.03 & - \\
\hline COBALT & $05 / 26 / 83$ & 0001 & $M G / L$ & $<$ & 0.04 & 0.04 & - \\
\hline COPPER & $05 / 26 / 83$ & 0001 & $M G / L$ & $<$ & 0.03 & 0.03 & - \\
\hline CYANIDE & $05 / 26 / 83$ & 0001 & $M G / L$ & $<$ & 1.00 & 1. & - \\
\hline IROH & $05 / 26 / 83$ & 0001 & $M G / L$ & $<$ & 0.05 & 0.05 & - \\
\hline LEAD & $05 / 26 / 83$ & 0001 & $M G / L$ & & 0.047 & - & - \\
\hline MAGHESIUM & $05 / 26 / 83$ & 0001 & $M G / L$ & & 56.00 & - & - \\
\hline MANGANESE & $05 / 26 / 83$ & 0001 & $M G / L$ & & 0.60 & - & - \\
\hline HOLYBDENUM & $05 / 26 / 83$ & 0001 & $M G / L$ & $<$ & 0.02 & 0.02 & - \\
\hline HICKEL & $05 / 26 / 83$ & 0001 & HG/L & $<$ & 0.08 & 0.08 & - \\
\hline HITRATE & $05 / 26 / 83$ & 0001 & $M G / L$ & & 1.00 & - & - \\
\hline POTASSIUN & $05 / 26 / 83$ & 0001 & $M G / L$ & & 10.00 & - & - \\
\hline RADIUH-226 & $05 / 26 / 83$ & 0001 & PCI/L & $<$ & 1.30 & 1.3 & - \\
\hline RADIUH-226 + RADIUH-228 & $05 / 26 / 83$ & 0001 & $\mathrm{PCI} / \mathrm{L}$ & & 1.30 & - & - \\
\hline RADIUN-228 & $05 / 26 / 83$ & 0001 & PCI/L & $<$ & 1.30 & 1.3 & - \\
\hline SELENIUM & $05 / 26 / 83$ & 0001 & MG/L & & 0.048 & - & - \\
\hline SILVER & $05 / 26 / 83$ & 0001 & $M G / L$ & $<$ & 0.03 & 0.03 & - \\
\hline SOOIUH & $05 / 26 / 83$ & 0001 & ME/L & & 56.00 & - & - \\
\hline SULFATE & $05 / 26 / 83$ & 0001 & MG/L & & 420.00 & - & - \\
\hline URANIU:H & $05 / 26 / 83$ & 0001 & $M G / L$ & & 0.004 & - & - \\
\hline VANADIUN & $05 / 26 / 83$ & 0001 & MG/L & $<$ & 0.04 & 0.04 & - \\
\hline
\end{tabular}

PARAMETER VALUE INDICATOR (PVI): < - LESS THAN DETECTION LIMIT 
GROUNDHATER QUALITY DATA BY LOCATION

SITE: AMBOI AMBROSIA LAKE

LOCATIOH: 0584

NORTH COORDIHATE: $\quad 45290.0$ FT

EAST COORDINATE: $\quad 56310.0$ FT

O6/10/80 TO $12 / 04 / 92$

REPORT DATE: $08 / 04 / 94$

FORHATION OF CONPLETION: DAKOTA SAKDSTONE (KD)

HYDRAULIC FLON RELATIOHSHIP: UHKHOWH (H)

\begin{tabular}{|l|c|c|l|c|c|c|c|}
\hline PARAMETER NAHE & LOG DATE & $\begin{array}{l}\text { SAMPLE } \\
\text { ID }\end{array}$ & $\begin{array}{l}\text { UHITS OF } \\
\text { MEASURE }\end{array}$ & PVI & $\begin{array}{l}\text { PARAMETER } \\
\text { VALUE }\end{array}$ FLAGS & $\begin{array}{c}\text { DETECTION } \\
\text { LIHIT }\end{array}$ & $\begin{array}{l}\text { PARAMETER } \\
\text { UHCERTAINTY }\end{array}$ \\
\hline ZIHC & $05 / 26 / 83$ & 0001 & MG/L & 0.044 & - & - \\
\hline
\end{tabular}

PARAMETER VALUE IMDICATOR (PVI): < - LESS THAN DETECTION LIMIT

SAMPLE ID CODES:

0001 - FILTERED SAMPLE (.45 MICRONS) 
GROUNDWATER QUALITY DATA BY LOCATIOH

SITE: AMBO1 AMBROSIA LAKE

LOCATION: 0585

NORTH COORDINATE: 56520.0 FT

EAST COORDINATE: $\quad \mathbf{4 7 0 9 0 . 0}$ FT

06/10/80 TO $12 / 04 / 92$

REPORT DATE: $08 / 04 / 94$

FORMATION OF COMPLETIOH: DAKOTA SAMDSTOHE (KD)

HYDRAULIC FLOA RELATIONSHIP: UHKNOWH (H)

\begin{tabular}{|c|c|c|c|c|c|c|c|}
\hline PARAMETER MAME & LOG DATE & $\begin{array}{c}\text { SAMPLE } \\
10\end{array}$ & $\begin{array}{l}\text { UNITS OF } \\
\text { MEASURE }\end{array}$ & PVI & $\begin{array}{l}\text { PARAMETER } \\
\text { VALUE FLAGS }\end{array}$ & $\begin{array}{l}\text { DETECTION } \\
\text { LIMIT }\end{array}$ & $\begin{array}{l}\text { PARAMETER } \\
\text { UHCERTAINTY }\end{array}$ \\
\hline ALKALINITY & $11 / 14 / 83$ & 0001 & MG/L CACO3 & & 8.20 & - & - \\
\hline ALUHINUM & $\begin{array}{l}11 / 05 / 80 \\
11 / 14 / 83\end{array}$ & $\begin{array}{l}0001 \\
0001\end{array}$ & $M G / L$ & $<$ & $\begin{array}{l}0.05 \\
0.091\end{array}$ & 0.05 & - \\
\hline ARSENIC & $\begin{array}{l}11 / 05 / 80 \\
11 / 14 / 83\end{array}$ & $\begin{array}{l}0001 \\
0001\end{array}$ & $M G / L$ & $<$ & $\begin{array}{l}0.001 \\
0.057\end{array}$ & 0.001 & - \\
\hline BARIUA & $\begin{array}{l}11 / 05 / 80 \\
11 / 14 / 83\end{array}$ & $\begin{array}{l}0001 \\
0001\end{array}$ & $M G / L$ & & $\begin{array}{l}0.03 \\
0.046\end{array}$ & - & - \\
\hline BICARBOHATE - HCO3 & $\begin{array}{l}11 / 05 / 80 \\
19 / 14 / 83\end{array}$ & $\begin{array}{l}0001 \\
0001\end{array}$ & MG/L & & $\begin{array}{l}24.00 \\
20.00\end{array}$ & - & - \\
\hline BORON & $\begin{array}{l}11 / 05 / 80 \\
11 / 14 / 83\end{array}$ & $\begin{array}{l}0001 \\
0001\end{array}$ & MG/L & & $\begin{array}{l}7.20 \\
4.70\end{array}$ & - & - \\
\hline CADMIUH & $\begin{array}{l}11 / 05 / 80 \\
11 / 14 / 83\end{array}$ & $\begin{array}{l}0001 \\
0001\end{array}$ & MG/L & $<$ & $\begin{array}{l}0.008 \\
0.007\end{array}$ & $\begin{array}{l}0.008 \\
0.007\end{array}$ & - \\
\hline CALCIUM & $\begin{array}{l}11 / 05 / 80 \\
11 / 14 / 83\end{array}$ & $\begin{array}{l}0001 \\
0001\end{array}$ & HG/L & & $\begin{array}{r}29.00 \\
110.00\end{array}$ & - & - \\
\hline CARBONATE & $11 / 14 / 83$ & 0001 & MG/L & $<$ & 10.00 & 10. & - \\
\hline CHLORIDE & $\begin{array}{l}11 / 05 / 80 \\
11 / 14 / 83\end{array}$ & $\begin{array}{l}0001 \\
0001\end{array}$ & $M G / L$ & $<$ & $\begin{array}{l}10.00 \\
90.00\end{array}$ & 10. & - \\
\hline CHRONIUH & $\begin{array}{l}11 / 05 / 80 \\
11 / 14 / 83\end{array}$ & $\begin{array}{l}0001 \\
0001\end{array}$ & $M G / L$ & $\begin{array}{l}< \\
<\end{array}$ & $\begin{array}{l}0.001 \\
0.026\end{array}$ & $\begin{array}{l}0.001 \\
0.026\end{array}$ & - \\
\hline COBALT & $\begin{array}{l}11 / 05 / 80 \\
11 / 14 / 83\end{array}$ & $\begin{array}{l}0001 \\
0001\end{array}$ & MG/L & $<$ & $\begin{array}{l}0.006 \\
0.011\end{array}$ & $\begin{array}{l}0.006 \\
0.011\end{array}$ & - \\
\hline COPPER & $\begin{array}{l}11 / 05 / 80 \\
11 / 14 / 83\end{array}$ & $\begin{array}{l}0001 \\
0001\end{array}$ & MG/L & $<$ & $\begin{array}{l}0.19 \\
0.017\end{array}$ & 0.017 & - \\
\hline CYANIDE & $11 / 14 / 83$ & 0001 & $M G / L$ & $<$ & 1.00 & 1. & - \\
\hline FLUORIDE & $\begin{array}{l}11 / 05 / 80 \\
11 / 14 / 83\end{array}$ & $\begin{array}{l}0001 \\
0001\end{array}$ & $M G / L$ & $\begin{array}{l}< \\
<\end{array}$ & $\begin{array}{l}0.20 \\
1.00\end{array}$ & $\begin{array}{l}0.2 \\
1 .\end{array}$ & - \\
\hline IRON & $\begin{array}{l}11 / 05 / 80 \\
11 / 14 / 83\end{array}$ & $\begin{array}{l}0001 \\
0001\end{array}$ & MG/L & $<$ & $\begin{array}{l}0.053 \\
0.024\end{array}$ & 0.024 & - \\
\hline LEAD & $\begin{array}{l}11 / 05 / 80 \\
11 / 14 / 83\end{array}$ & $\begin{array}{l}0001 \\
0001\end{array}$ & MG/L & $<$ & $\begin{array}{l}0.001 \\
0.023\end{array}$ & 0.001 & - \\
\hline MAGNESIUM & $\begin{array}{l}11 / 05 / 80 \\
11 / 14 / 83\end{array}$ & $\begin{array}{l}0001 \\
0001\end{array}$ & HG/L & & $\begin{array}{l}0.27 \\
9.90\end{array}$ & - & - \\
\hline MAMGAHESE & $\begin{array}{l}11 / 05 / 80 \\
11 / 14 / 83\end{array}$ & $\begin{array}{l}0001 \\
0001\end{array}$ & $\mathrm{MG} / \mathrm{L}$ & $<$ & $\begin{array}{l}0.004 \\
0.003\end{array}$ & 0.003 & - \\
\hline MERCURY & $11 / 05 / 80$ & 0001 & MG/L & $<$ & 0.001 & 0.001 & - \\
\hline MOLYBDENUM & $\begin{array}{l}11 / 05 / 80 \\
11 / 14 / 83\end{array}$ & $\begin{array}{l}0001 \\
0001\end{array}$ & $M G / L$ & & $\begin{array}{l}0.066 \\
0.021\end{array}$ & - & - \\
\hline
\end{tabular}

PARNMETER VALUE IMDICATOR (PVI): < - LESS THAN DETECTION LIHIT 
GROUNDWATER QUALITY DATA BY LOCATION

SITE: AMBOI - AMBROSIA LAKE

LOCATION: 0585

NORTH COORDINATE: 56520.0 FT.

EAST COORDINATE: $\quad 47090.0$ FT

06/10/80 TO $12 / 04 / 92$

REPORT DATE: 08/04/94

FORHATION OF COMPLETION: DAKOTA SAMDSTONE (KD)

HYDRAULIC FLOW RELATIONSHIP: UHKNONN (H)

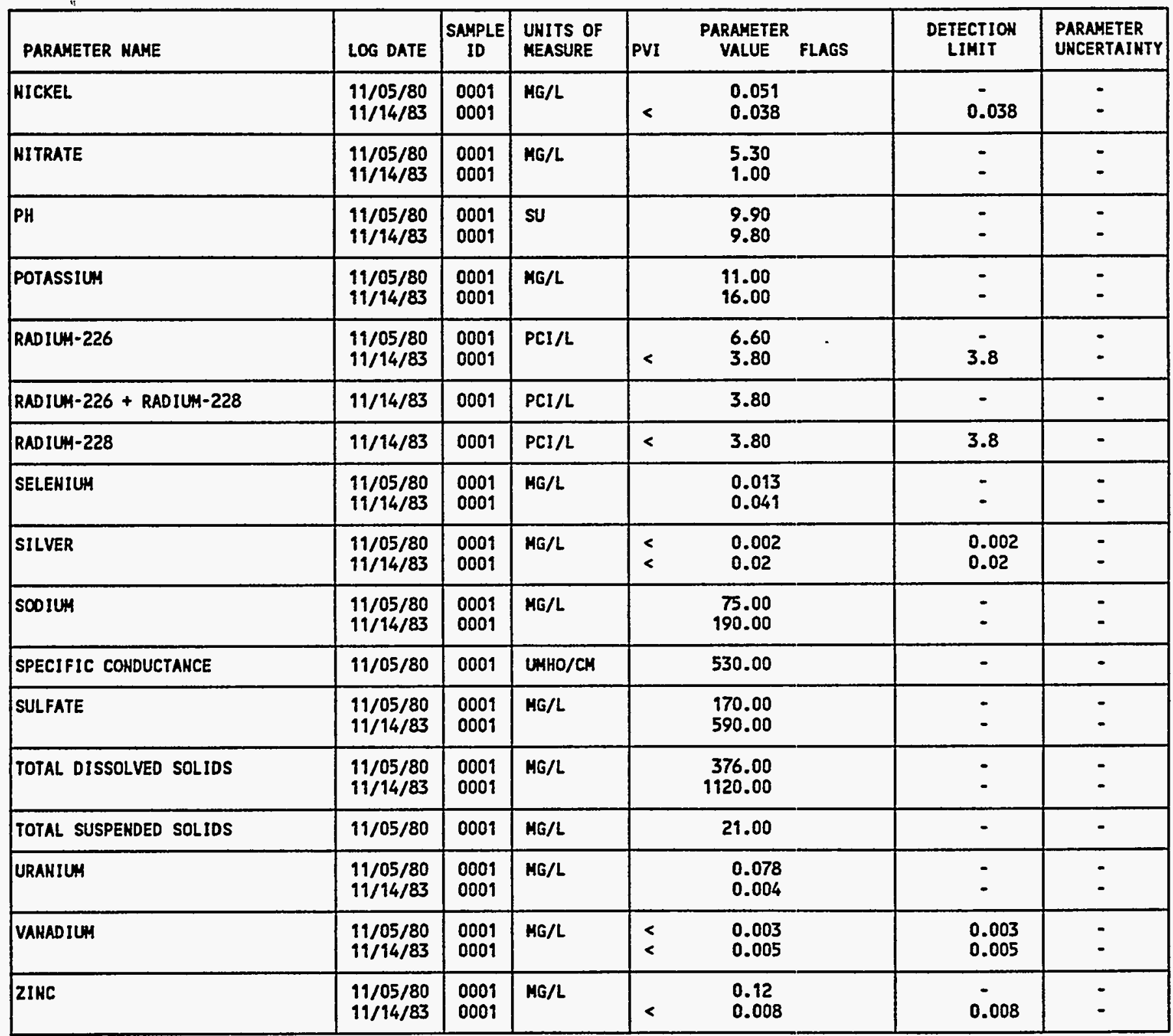

PARAMETER VALUE INDICATOR (PVI): < - LESS THAK DETECTION LIMIT

SAKPLE ID IOODES:

0001 - FILUERED SAMPLE (.45 MICRONS) 
GROUNDWATER OUALITY OATA BY LOCATION

SITE: AMBOI AMBROSIA LAKE

LOCATION: 0586

HORTH COORDINATE: 53590.0 FT

EAST COORDIHATE: $\quad 48410.0$ FT

06/10/80 TO $12 / 04 / 92$

REPORT DATE: 08/04/94

FORMATION OF COMPLETION: DAKOTA SANDSTONE (KD)

HYORAULIC FLOW RELATIONSHIP: UNKHOWH (N)

\begin{tabular}{|c|c|c|c|c|c|c|c|}
\hline PARAMETER NAME & LOG DATE & $\mid \begin{array}{c}\text { SNMPLE } \\
\text { ID }\end{array}$ & $\begin{array}{l}\text { UHITS OF } \\
\text { MEASURE }\end{array}$ & PVI & $\begin{array}{l}\text { PARAMETER } \\
\text { VALUE FLAGS }\end{array}$ & $\begin{array}{l}\text { DETECTION } \\
\text { LIMIT }\end{array}$ & $\begin{array}{l}\text { PARAMETER } \\
\text { UHCERTAINTY }\end{array}$ \\
\hline ALUAINUM & $\begin{array}{l}10 / 20 / 80 \\
05 / 26 / 83\end{array}$ & $\begin{array}{l}0001 \\
0001\end{array}$ & $M G / L$ & $<$ & $\begin{array}{l}0.05 \\
0.43\end{array}$ & 0.05 & - \\
\hline ARSENIC & $\begin{array}{l}10 / 20 / 80 \\
05 / 26 / 83\end{array}$ & $\begin{array}{l}0001 \\
0001\end{array}$ & MG/L & & $\begin{array}{l}0.033 \\
0.18\end{array}$ & - & 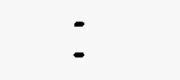 \\
\hline BICARBONATE - HCO3 & $\begin{array}{l}90 / 20 / 80 \\
05 / 26 / 83\end{array}$ & $\begin{array}{l}0001 \\
0001\end{array}$ & MG/L & $<$ & $\begin{array}{r}279.00 \\
10.00\end{array}$ & 10. & - \\
\hline BORON & $\begin{array}{l}10 / 20 / 80 \\
05 / 26 / 83\end{array}$ & $\begin{array}{l}0001 \\
0001\end{array}$ & MG/L & & $\begin{array}{l}0.58 \\
0.48\end{array}$ & $\dot{-}$ & - \\
\hline CARBONATE & $05 / 26 / 83$ & 0001 & MG/L & $<$ & 10.00 & 10. & - \\
\hline CHLORIDE & $\begin{array}{l}10 / 20 / 80 \\
05 / 26 / 83\end{array}$ & $\begin{array}{l}0001 \\
0001\end{array}$ & MG/L & & $\begin{array}{r}375.00 \\
1070.00\end{array}$ & - & - \\
\hline CHROHIUH & $\begin{array}{l}10 / 20 / 80 \\
05 / 26 / 83\end{array}$ & $\begin{array}{l}0001 \\
0001\end{array}$ & $M G / L$ & & $\begin{array}{l}0.006 \\
0.096\end{array}$ & - & - \\
\hline COBALT & $\begin{array}{l}10 / 20 / 80 \\
05 / 26 / 83\end{array}$ & $\begin{array}{l}0009 \\
0001\end{array}$ & $M G / L$ & $<$ & $\begin{array}{l}0.006 \\
0.066\end{array}$ & 0.006 & - \\
\hline COPPER & $\begin{array}{l}10 / 20 / 80 \\
05 / 26 / 83\end{array}$ & $\begin{array}{l}0001 \\
0001\end{array}$ & $M G / L$ & & $\begin{array}{l}0.011 \\
0.073\end{array}$ & - & - \\
\hline LEAD & $\begin{array}{l}10 / 20 / 80 \\
05 / 26 / 83\end{array}$ & $\begin{array}{l}0001 \\
0001\end{array}$ & MG/L & & $\begin{array}{l}0.003 \\
0.46\end{array}$ & $\dot{-}$ & - \\
\hline MAGHESIUN & $\begin{array}{l}10 / 20 / 80 \\
05 / 26 / 83\end{array}$ & $\begin{array}{l}0001 \\
0001\end{array}$ & $M G / L$ & & $\begin{array}{l}230.00 \\
330.00\end{array}$ & - & - \\
\hline MANGANESE & $\begin{array}{l}10 / 20 / 80 \\
05 / 26 / 83\end{array}$ & $\begin{array}{l}0001 \\
0001\end{array}$ & $M G / L$ & & $\begin{array}{l}0.74 \\
2.90\end{array}$ & $\dot{-}$ & : \\
\hline MERCURY & $10 / 20 / 80$ & 0001 & $M G / L$ & $<$ & 0.001 & 0.001 & - \\
\hline MOLYBDENUM & $\begin{array}{l}10 / 20 / 80 \\
05 / 26 / 83\end{array}$ & $\begin{array}{l}0001 \\
0001\end{array}$ & $M G / L$ & & $\begin{array}{l}0.08 \\
0.04\end{array}$ & $\dot{-}$ & - \\
\hline MICKEL & $10 / 20 / 80$ & 0001 & $M G / L$ & & 0.05 & - & - \\
\hline
\end{tabular}

PARAHETER VALUE IMDICATOR (PVI): < - LESS THAN DETECTION LIMIT

SAMPLE ID CODES:

0001 - FILTERED SAMPLE (.45 MICRONS) 
GROUNDHATER OUALITY DATA BY LOCATIOH

SITE: AMBO1 AMBROSIA LAKE

LOCATIOH: 0586

HORTH COORDIHATE: 53590.0 FंT

EAST COORDINATE: $\quad 48410.0$ FT

06/10/80 TO $12 / 04 / 92$

REPORT DATE: 08/04/94

FORMATION OF COMPLETION: DAKOTA SAMDSTONE (KD)

HYDRAULIC FLOW RELATIONSHIP: UNKHOWH (N)

\begin{tabular}{|c|c|c|c|c|c|c|c|}
\hline PARAMETER MAME & LOG DATE & ${ }_{\text {ID }}^{\text {SAMPLE }}$ & $\begin{array}{l}\text { UNITS OF } \\
\text { MEASURE }\end{array}$ & PVI & $\begin{array}{l}\text { PARAMETEIR } \\
\text { VALUE FLAGS }\end{array}$ & $\begin{array}{l}\text { DETECTIOH } \\
\text { LIMIT }\end{array}$ & $\begin{array}{l}\text { PARAMETER } \\
\text { UNCERTAINTY }\end{array}$ \\
\hline HICKEL & $05 / 26 / 83$ & 0001 & MG/L & & 0.18 & - & - \\
\hline HITRATE & $\begin{array}{l}10 / 20 / 80 \\
05 / 26 / 83\end{array}$ & $\begin{array}{l}0001 \\
0001\end{array}$ & MG/L & & $\begin{array}{l}2.80 \\
2.00\end{array}$ & - & - \\
\hline PH & $\begin{array}{l}10 / 20 / 80 \\
05 / 26 / 83\end{array}$ & $\begin{array}{l}0009 \\
0001\end{array}$ & su & & $\begin{array}{l}7.70 \\
5.00\end{array}$ & - & - \\
\hline POTASSIUM & $\begin{array}{l}10 / 20 / 80 \\
05 / 26 / 83\end{array}$ & $\begin{array}{l}0001 \\
0001\end{array}$ & MG/L & & $\begin{array}{l}19.00 \\
26.00\end{array}$ & - & $\because$ \\
\hline RADILA-226 & $\begin{array}{l}10 / 20 / 80 \\
05 / 26 / 83\end{array}$ & $\begin{array}{l}0001 \\
0001\end{array}$ & $\mathrm{PCI} / \mathrm{L}$ & & $\begin{array}{l}3.07 \\
2.86\end{array}$ & - & 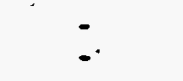 \\
\hline RADIUM-226 + RADIUM-228 & $05 / 26 / 83$ & 0001 & PCI /L & & 5.72 & - & - \\
\hline RADIUM-228 & $05 / 26 / 83$ & 0001 & $\mathrm{PCI} / \mathrm{L}$ & & 2.86 & - & - \\
\hline SELEHIUM & $\begin{array}{l}10 / 20 / 80 \\
05 / 26 / 83\end{array}$ & $\begin{array}{l}0001 \\
0001\end{array}$ & MG/L & & $\begin{array}{l}0.025 \\
0.26\end{array}$ & - & $\therefore$ \\
\hline SILVER & $\begin{array}{l}10 / 20 / 80 \\
05 / 26 / 83\end{array}$ & $\begin{array}{l}0001 \\
0001\end{array}$ & MG/L & $<$ & $\begin{array}{l}0.002 \\
0.10\end{array}$ & 0.002 & - \\
\hline SoOIUM & $\begin{array}{l}10 / 20 / 80 \\
05 / 26 / 83\end{array}$ & $\begin{array}{l}0001 \\
0001\end{array}$ & MG/L & & $\begin{array}{l}210.00 \\
500.00\end{array}$ & - & - \\
\hline SPECIFIC CONDUCTANCE & $10 / 20 / 80$ & 0001 & ЧМHO/CM & & 4020.00 & - & - \\
\hline SULFATE & $\begin{array}{l}10 / 20 / 80 \\
05 / 26 / 83\end{array}$ & $\begin{array}{l}0001 \\
0001\end{array}$ & $M G / L$ & & $\begin{array}{l}1615.00 \\
2770.00\end{array}$ & - & - \\
\hline TOTAL DISSOLVED SOLIDS & $\begin{array}{l}10 / 20 / 80 \\
05 / 26 / 83\end{array}$ & $\begin{array}{l}0001 \\
0001\end{array}$ & MG/L & & $\begin{array}{l}3280.00 \\
4880.00\end{array}$ & - & $\dot{-}$ \\
\hline TOTAL SUSPENDED SOLIOS & $10 / 20 / 80$ & 0001 & $M G / L$ & & 124.00 & - & - \\
\hline URANIUM & $\begin{array}{l}10 / 20 / 80 \\
05 / 26 / 83\end{array}$ & $\begin{array}{l}0001 \\
0001\end{array}$ & MG/L & & $\begin{array}{l}0.06 \\
0.004\end{array}$ & - & - \\
\hline VANADIUM & $\begin{array}{l}10 / 20 / 80 \\
05 / 26 / 83\end{array}$ & $\begin{array}{l}0001 \\
0001\end{array}$ & MG/L & $<$ & $\begin{array}{l}0.003 \\
0.091\end{array}$ & 0.003 & - \\
\hline ZIнс & $\begin{array}{l}10 / 20 / 80 \\
05 / 26 / 83\end{array}$ & $\begin{array}{l}0001 \\
0001\end{array}$ & $M G / L$ & & $\begin{array}{l}0.21 \\
0.23\end{array}$ & - & - \\
\hline
\end{tabular}

PARAMETER VALUE IMDICATOR (PVI): < - LESS THAH DETECTION LIMIT

SAMPLE ID COOES:

DOO1 - FILTERED SAMPLE (.45 MICRONS) 
GROUNDWATER OUALITY DATA BY LOCATION

SITE: AMBOI AMBROSIA LAKE

LOCATIOH: 0587

MORTH COORDIHATE: $\quad 54400.0$ FT

EAST COORDINATE: $\quad 51480.0$ FT

06/10/80 TO 12/04/92

REPORT DATE: 08/04/94

FORHATION OF COAPLETION: DAKOTA SAMDSTONE (KD)

HYDRAULIC FLOW RELATIOHSHIP: UHKHOWN (H)

\begin{tabular}{|c|c|c|c|c|c|c|c|}
\hline PARAMETER MAME & LOG DATE & $\underset{\text { ID }}{\text { SAMPLE }}$ & $\begin{array}{l}\text { UNITS OF } \\
\text { MEASURE }\end{array}$ & PVI & $\begin{array}{l}\text { PARAMETER } \\
\text { VALUE FLAGS }\end{array}$ & $\begin{array}{l}\text { DETECTION } \\
\text { LIMIT }\end{array}$ & $\begin{array}{l}\text { PARAMETER } \\
\text { UNCERTAIHTY }\end{array}$ \\
\hline ALKALIMITY & $08 / 15 / 83$ & 0001 & $\mathrm{MG} / \mathrm{L}$ CACO3 & & 258.00 & - & - \\
\hline ARSENIC & $08 / 15 / 83$ & 0001 & MG/L & & 0.45 & - & - \\
\hline BICARBOHATE - HCO3 & $08 / 15 / 83$ & 0001 & MG/L & & 630.00 & - & - \\
\hline BORON & $08 / 15 / 83$ & 0001 & MG/L & & 0.36 & - & - \\
\hline CADHIUN & $08 / 15 / 83$ & 0001 & $M G / L$ & & 0.02 & - & - \\
\hline CALCIUH & $08 / 15 / 83$ & 0001 & MG/L & & 640.00 & - & - \\
\hline CHROAIUM & $08 / 15 / 83$ & 0001 & $M G / L$ & & 0.096 & - & - \\
\hline COBALT & $08 / 15 / 83$ & 0001 & $M G / L$ & & 0.072 & - & - \\
\hline COPPER & $08 / 15 / 83$ & 0001 & MG/L & & 0.12 & - & - \\
\hline CYALIDE & $08 / 15 / 83$ & 0001 & $M G / L$ & $<$ & 1.00 & 1. & - \\
\hline FLUORIDE & $08 / 15 / 83$ & 0001 & MG/L & & 1.10 & - & - \\
\hline IRON & $08 / 15 / 83$ & 0001 & MG/L & & 0.82 & - & - \\
\hline LEAD & $08 / 15 / 83$ & 0001 & MG/L & & 0.39 & - & - \\
\hline PH & $08 / 15 / 83$ & 0001 & su & & 6.70 & - & - \\
\hline POTASSIUM & $08 / 15 / 83$ & 0001 & MG/L & & 15.00 & - & - \\
\hline RADIUH-226 & $08 / 15 / 83$ & 0001 & PCI/L & $<$ & 3.30 & 3.3 & - \\
\hline RADIUH-226 + RADIUH-228 & $08 / 15 / 83$ & 0001 & PCI/L & & 3.30 & - & - \\
\hline RADIUH-228 & $08 / 15 / 83$ & 0001 & PCI/L & $<$ & 3.30 & 3.3 & - \\
\hline SELENIUNH & $08 / 15 / 83$ & 0001 & MG/L & & 0.49 & - & - \\
\hline SILVER & $08 / 15 / 83$ & 0001 & MG/L & & 0.14 & - & - \\
\hline SoOIUn & $08 / 15 / 83$ & 0001 & $M G / L$ & & 330.00 & - & - \\
\hline
\end{tabular}

PARAMETER VALUE INDICATOR (PVI):

< - LESS than DETECTION LIMIT 
GROUNDWATER QUALITY DATA BY LOCATION

SITE: AMBO1 AMBROSIA LAKE

LOCATION: 0587

HORTH COORDINATE: 54400.0 FT

EAST COORDINATE: $\quad 51480.0$ FT

06/10/80 TO $12 / 04 / 92$

REPORT DATE: $08 / 04 / 94$

FORMATION OF COMPLETIOH: DAKOTA SAMDSTONE (KD)

HYORAULIC FLON RELATIOHSHIP: UNKWOWN (N)

\begin{tabular}{|c|c|c|c|c|c|c|c|}
\hline PARAMETER MAME & LOG DATE & $\begin{array}{c}\text { SAMPLE } \\
\text { ID }\end{array}$ & $\begin{array}{l}\text { UHITS OF } \\
\text { HEASURE }\end{array}$ & PVI & $\begin{array}{l}\text { PARAMETER' } \\
\text { VALUE FLAGS }\end{array}$ & $\begin{array}{l}\text { DETECTIOH } \\
\text { LIMIT }\end{array}$ & $\begin{array}{l}\text { PARAMETER } \\
\text { UNCERTAINTY }\end{array}$ \\
\hline SULFATE & $08 / 15 / 83$ & 0001 & MG/L & & 2590.00 & - & - \\
\hline TOTAL DISSOLVED SOLIDS & $08 / 15 / 83$ & 0001 & $M G / L$ & & 5380.00 & - & - \\
\hline URANIUY & $08 / 15 / 83$ & 0001 & MG/L & & 0.038 & - & - \\
\hline VAHADIUN & $08 / 15 / 83$ & 0001 & HG/L & & 0.098 & - & - \\
\hline ZINC & $08 / 15 / 83$ & 0001 & $M G / L$ & & 0.14 & - & - \\
\hline
\end{tabular}

PARAMETER VALUE IMDICATOR (PVI): < - LESS THAN DETECTION LIMIT

SAMPLE ID COOES:

0001 - FILTERED SAMPLE (.45 MICRONS) 
GROUNDWATER OUALITY DATA BY LOCATIOH

SITE: AMBOT AMBROSIA LAKE

LOCATION: 0588

NORTH COORDINATE: $\quad 51630.0$ FT

EAST COORDINATE: $\quad 50720.0$ FT

DS/10/80 TO $12 / 04 / 92$

REPORT DATE: $08 / 04 / 94$

FORHATION OF COMPLETIOH: DAKOTA SAMDSTONE (KO)

HYDRAULIC FLON RELATIONSHIP: UNKHOWN (N)

\begin{tabular}{|c|c|c|c|c|c|c|c|}
\hline PARAMETER MAME & LOG DATE & $\begin{array}{c}\text { SAMPLE } \\
\text { ID }\end{array}$ & $\begin{array}{l}\text { UNITS OF } \\
\text { MEASURE }\end{array}$ & PVI & $\begin{array}{l}\text { PARAMETER } \\
\text { VALUE FLAGS }\end{array}$ & $\begin{array}{l}\text { DETECTIOH } \\
\text { LIMIT }\end{array}$ & $\begin{array}{l}\text { PARAMETER } \\
\text { UNCERTAINTY }\end{array}$ \\
\hline ALKALINITY & $08 / 18 / 83$ & 0001 & MG/L CACO3 & & 49.20 & - & - \\
\hline ALCHINLY & $\begin{array}{l}10 / 21 / 80 \\
08 / 18 / 83\end{array}$ & $\begin{array}{l}0001 \\
0001\end{array}$ & MG/L & & $\begin{array}{l}0.03 \\
0.14\end{array}$ & - & - \\
\hline ARSENIC & $\begin{array}{l}10 / 21 / 80 \\
08 / 98 / 83\end{array}$ & $\begin{array}{l}0001 \\
0001\end{array}$ & MG/L & & $\begin{array}{l}0.035 \\
0.12\end{array}$ & - & - \\
\hline BARIUA & $\begin{array}{l}10 / 21 / 80 \\
08 / 18 / 83\end{array}$ & $\begin{array}{l}0001 \\
0001\end{array}$ & MG/L & & $\begin{array}{l}0.016 \\
0.017\end{array}$ & - & - \\
\hline BICARBONATE - HCO3 & $\begin{array}{l}10 / 21 / 80 \\
08 / 18 / 83\end{array}$ & $\begin{array}{l}0001 \\
0001\end{array}$ & $M G / L$ & & $\begin{array}{l}200.00 \\
120.00\end{array}$ & - & - \\
\hline BORON & $\begin{array}{l}10 / 21 / 80 \\
08 / 98 / 83\end{array}$ & $\begin{array}{l}0001 \\
0001\end{array}$ & MG/L & & $\begin{array}{l}0.078 \\
0.23\end{array}$ & - & - \\
\hline CADHIUM & $\begin{array}{l}10 / 21 / 80 \\
08 / 18 / 83\end{array}$ & $\begin{array}{l}0001 \\
0001\end{array}$ & MG/L & $<$ & $\begin{array}{l}0.008 \\
0.008\end{array}$ & 0.008 & - \\
\hline CALCIUM & $\begin{array}{l}10 / 21 / 80 \\
08 / 98 / 83\end{array}$ & $\begin{array}{l}0001 \\
0001\end{array}$ & $M G / L$ & & $\begin{array}{l}160.00 \\
180.00\end{array}$ & - & - \\
\hline CARBONATE & $08 / 98 / 83$ & 0001 & $M G / L$ & $<$ & 10.00 & 10. & - \\
\hline CHLORIDE & $\begin{array}{l}10 / 21 / 80 \\
08 / 18 / 83\end{array}$ & $\begin{array}{l}0001 \\
0001\end{array}$ & $M G / L$ & & $\begin{array}{l}80.00 \\
95.00\end{array}$ & - & - \\
\hline CHROMIUM & $\begin{array}{l}10 / 21 / 80 \\
08 / 18 / 83\end{array}$ & $\begin{array}{l}0001 \\
0001\end{array}$ & MG/L & $\begin{array}{l}< \\
<\end{array}$ & $\begin{array}{l}0.001 \\
0.026\end{array}$ & $\begin{array}{l}0.001 \\
0.026\end{array}$ & - \\
\hline COBALT & $\begin{array}{l}10 / 21 / 80 \\
08 / 18 / 83\end{array}$ & $\begin{array}{l}0001 \\
0001\end{array}$ & MG/L & $<$ & $\begin{array}{l}0.006 \\
0.015\end{array}$ & 0.006 & - \\
\hline COPPER & $\begin{array}{l}10 / 21 / 80 \\
08 / 18 / 83\end{array}$ & $\begin{array}{l}0001 \\
0001\end{array}$ & MG/L & $<$ & $\begin{array}{l}0.001 \\
0.036\end{array}$ & 0.001 & - \\
\hline CYAHIDE & $08 / 18 / 83$ & 0001 & MG/L & $<$ & 0.10 & 0.1 & - \\
\hline FLUORIDE & $\begin{array}{l}10 / 21 / 80 \\
08 / 18 / 83\end{array}$ & $\begin{array}{l}0001 \\
0001\end{array}$ & $M G / L$ & $<$ & $\begin{array}{l}0.38 \\
1.00\end{array}$ & 1. & - \\
\hline IROH & $\begin{array}{l}10 / 21 / 80 \\
08 / 18 / 83\end{array}$ & $\begin{array}{l}0001 \\
0001\end{array}$ & $M G / L$ & & $\begin{array}{l}0.15 \\
0.025\end{array}$ & - & - \\
\hline LEAD & $\begin{array}{l}10 / 21 / 80 \\
08 / 18 / 83\end{array}$ & $\begin{array}{l}0001 \\
0001\end{array}$ & MG/L & $<$ & $\begin{array}{l}0.001 \\
0.14\end{array}$ & 0.001 & - \\
\hline MagNESIUH & $\begin{array}{l}10 / 21 / 80 \\
08 / 18 / 83\end{array}$ & $\begin{array}{l}0001 \\
0001\end{array}$ & MG/L & & $\begin{array}{l}42.00 \\
44.00\end{array}$ & - & - \\
\hline MANGANESE & $\begin{array}{l}10 / 21 / 80 \\
08 / 18 / 83\end{array}$ & $\begin{array}{l}0001 \\
0001\end{array}$ & $M G / L$ & & $\begin{array}{l}0.082 \\
0.025\end{array}$ & $\dot{-}$ & - \\
\hline MERCURY & $10 / 21 / 80$ & 0001 & MG/L & $<$ & 0.001 & 0.001 & - \\
\hline MOLYBDENUM & $\begin{array}{l}10 / 21 / 80 \\
08 / 18 / 83\end{array}$ & $\begin{array}{l}0001 \\
0001\end{array}$ & MG/L & & $\begin{array}{l}0.09 \\
0.041\end{array}$ & - & - \\
\hline
\end{tabular}

PARAMETER VALUE INDICATOR (PVI): < - LESS THAN DETECTIOH LIMIT

SAMPLE ID COOES:

0001 - FILTERED SAMPLE (.45 MICRONS) 
GROUNDUATER QUALITY DATA BY LOCATION

SITE: AMBOI AMBROSIA LAKE

LOCATION: 0588

NORTH COORDIHATE: 51630.0 FT

EAST COORDINATE: 50720.0 FT

06/10/80 TO 12/04/92

REPORT DATE: 08/04/94

FORHATION OF COMPLETION: DAKOTA SANDSTOHE (KD)

HYORAULIC FLON RELATIONSHIP: UHKNOWN (N)

\begin{tabular}{|c|c|c|c|c|c|c|c|}
\hline PARAMETER NAME & LOG DATE & $\begin{array}{c}\text { SNMPLE } \\
\text { ID }\end{array}$ & $\begin{array}{l}\text { UNITS OF } \\
\text { MEASURE }\end{array}$ & PVI & $\begin{array}{l}\text { PARAMETER } \\
\text { VALUE FLAGS }\end{array}$ & $\begin{array}{l}\text { DETECTIOH } \\
\text { LIMIT }\end{array}$ & $\begin{array}{l}\text { PARAMETER } \\
\text { UHCERTAINTY }\end{array}$ \\
\hline HICKEL & $\begin{array}{l}10 / 21 / 80 \\
08 / 18 / 83\end{array}$ & $\begin{array}{l}0001 \\
0001\end{array}$ & $M G / L$ & $<$ & $\begin{array}{l}0.003 \\
0.049\end{array}$ & 0.003 & $\dot{-}$ \\
\hline HITRATE & $\begin{array}{l}10 / 21 / 80 \\
08 / 18 / 83\end{array}$ & $\begin{array}{l}0009 \\
0001\end{array}$ & HG/L & $<$ & $\begin{array}{l}1.30 \\
1.00\end{array}$ & 1. & - \\
\hline PH & $\begin{array}{l}10 / 21 / 80 \\
08 / 18 / 83\end{array}$ & $\begin{array}{l}0001 \\
0001\end{array}$ & su & & $\begin{array}{l}7.80 \\
7.40\end{array}$ & - & - \\
\hline POTASSIUH & $\begin{array}{l}10 / 21 / 80 \\
08 / 18 / 83\end{array}$ & $\begin{array}{l}0001 \\
0001\end{array}$ & MG/L & & $\begin{array}{l}12.00 \\
12.00\end{array}$ & - & - \\
\hline RADIUH-226 & $\begin{array}{l}10 / 21 / 80 \\
08 / 98 / 83\end{array}$ & $\begin{array}{l}0001 \\
0001\end{array}$ & $\mathrm{PCI} / \mathrm{L}$ & & $\begin{array}{r}15.48 \\
2.33\end{array}$ & - & - \\
\hline RADIUH-226 + RADIUM-228 & $08 / 18 / 83$ & 0001 & $\mathrm{PCI} / \mathrm{L}$ & & 4.66 & - & - \\
\hline RADIUM-228 & $08 / 18 / 83$ & 0001 & PCI/L & & 2.33 & - & - \\
\hline SELENIUM & $\begin{array}{l}10 / 21 / 80 \\
08 / 18 / 83\end{array}$ & $\begin{array}{l}0001 \\
0001\end{array}$ & MG/L & & $\begin{array}{l}0.11 \\
0.12\end{array}$ & - & $\dot{-}$ \\
\hline SILVER & $\begin{array}{l}10 / 21 / 80 \\
08 / 18 / 83\end{array}$ & $\begin{array}{l}0001 \\
0001\end{array}$ & $M G / L$ & $<$ & $\begin{array}{l}0.002 \\
0.049\end{array}$ & 0.002 & $\dot{-}$ \\
\hline soolum & $\begin{array}{l}10 / 21 / 80 \\
08 / 18 / 83\end{array}$ & $\begin{array}{l}0001 \\
0001\end{array}$ & $M G / L$ & & $\begin{array}{l}300.00 \\
280.00\end{array}$ & - & $\dot{-}$ \\
\hline SPECIFIC CONDUCTANCE & $10 / 21 / 80$ & 0001 & UМНО/CH & & 2340.00 & - & - \\
\hline SULFATE & $\begin{array}{l}10 / 21 / 80 \\
08 / 18 / 83\end{array}$ & $\begin{array}{l}0001 \\
0001\end{array}$ & MG/L & & $\begin{array}{l}970.00 \\
900.00\end{array}$ & - & - \\
\hline TOTAL DISSOLVED SOLIDS & $\begin{array}{l}10 / 21 / 80 \\
08 / 18 / 83\end{array}$ & $\begin{array}{l}0001 \\
0001\end{array}$ & $M G / L$ & & $\begin{array}{l}1800.00 \\
1560.00\end{array}$ & - & - \\
\hline TOTAL SUSPENDED SOLIDS & $10 / 21 / 80$ & 0001 & $M G / L$ & & 9830.00 & - & - \\
\hline URANIUH & $\begin{array}{l}10 / 21 / 80 \\
08 / 18 / 83\end{array}$ & $\begin{array}{l}0001 \\
0001\end{array}$ & MG/L & & $\begin{array}{l}0.11 \\
0.022\end{array}$ & - & - \\
\hline VANADIUN & $\begin{array}{l}10 / 21 / 80 \\
08 / 18 / 83\end{array}$ & $\begin{array}{l}0001 \\
0001\end{array}$ & MG/L & & $\begin{array}{l}0.006 \\
0.032\end{array}$ & - & - \\
\hline ZINC & $\begin{array}{l}10 / 21 / 80 \\
08 / 18 / 83\end{array}$ & $\begin{array}{l}0001 \\
0001\end{array}$ & MG/L & & $\begin{array}{l}0.026 \\
0.03\end{array}$ & - & - \\
\hline
\end{tabular}

PARAMETER VALUE IHDICATOR (PVI): < - LESS THAN DETECTIOH LIHIT

SAMPLE ID COOES

0001 - FILTERED SAMPLE (.45 MICROHS) 
GROUNDWATER QUALITY DATA BY LOCATION

SITE: AMBO1 AMBROSIA LAKE

LOCATION: 0589

NORTH COORDIHATE: 50500.0 FT

EAST COORDIHATE: $\quad 54790.0$ FT

06/10/80 TO $12 / 04 / 92$

REPORT DATE: $08 / 04 / 94$

FORMATION OF COMPLETION: DAKOTA SANDSTONE (KD)

HYDRAULIC FLON RELATIONSHIP: UHKNOWN (H)

\begin{tabular}{|c|c|c|c|c|c|c|c|}
\hline PARAMETER MAME & LOG DATE & $\underset{\text { ID }}{\text { SAMPLE }}$ & $\begin{array}{l}\text { UNITS OF } \\
\text { MEASURE }\end{array}$ & PVI & $\begin{array}{l}\text { PARAMETER } \\
\text { VALUE FLAGS }\end{array}$ & $\begin{array}{l}\text { DETECTIOH } \\
\text { LIMIT }\end{array}$ & $\begin{array}{l}\text { PARAMETER } \\
\text { UNCERTAINTY }\end{array}$ \\
\hline ALUMINUM & $\begin{array}{l}10 / 20 / 80 \\
05 / 25 / 83\end{array}$ & $\begin{array}{l}0001 \\
0001\end{array}$ & MG/L & & $\begin{array}{l}0.33 \\
0.51\end{array}$ & - & $\dot{-}$ \\
\hline ARSENIC & $\begin{array}{l}10 / 20 / 80 \\
05 / 25 / 83\end{array}$ & $\begin{array}{l}0001 \\
0001\end{array}$ & MG/L & $<$ & $\begin{array}{l}0.015 \\
0.20\end{array}$ & 0.2 & - \\
\hline BARIUM & $\begin{array}{l}10 / 20 / 80 \\
05 / 25 / 83\end{array}$ & $\begin{array}{l}0001 \\
0001\end{array}$ & $M G / L$ & & $\begin{array}{l}0.092 \\
0.06\end{array}$ & - & - \\
\hline BICARBOHATE - HCO3 & $05 / 25 / 83$ & 0001 & $M G / L$ & $<$ & 10.00 & 10. & - \\
\hline BORON & $\begin{array}{l}10 / 20 / 80 \\
05 / 25 / 83\end{array}$ & $\begin{array}{l}0001 \\
0001\end{array}$ & $M G / L$ & & $\begin{array}{l}0.97 \\
0.54\end{array}$ & - & - \\
\hline CADMIUM & $\begin{array}{l}10 / 20 / 80 \\
05 / 25 / 83\end{array}$ & $\begin{array}{l}0001 \\
0001\end{array}$ & MG/L & $<$ & $\begin{array}{l}0.008 \\
0.50\end{array}$ & $\begin{array}{l}0.008 \\
0.5\end{array}$ & - \\
\hline CALCIUH & $\begin{array}{l}10 / 20 / 80 \\
05 / 25 / 83\end{array}$ & $\begin{array}{l}0001 \\
0001\end{array}$ & $M G / L$ & & $\begin{array}{l}120.00 \\
110.00\end{array}$ & - & - \\
\hline CARBOHATE & $05 / 25 / 83$ & 0001 & $M G / L$ & $<$ & 10.00 & 10. & - \\
\hline CHLORIDE & $\begin{array}{l}10 / 20 / 80 \\
05 / 25 / 83\end{array}$ & $\begin{array}{l}0001 \\
0001\end{array}$ & MG/L & & $\begin{array}{l}146.00 \\
210.00\end{array}$ & : & - \\
\hline CHROMIUH & $\begin{array}{l}10 / 20 / 80 \\
05 / 25 / 83\end{array}$ & $\begin{array}{l}0001 \\
0001\end{array}$ & $M G / L$ & $<$ & $\begin{array}{l}0.001 \\
0.50\end{array}$ & $\begin{array}{l}0.001 \\
0.5\end{array}$ & - \\
\hline COBALT & $\begin{array}{l}10 / 20 / 80 \\
05 / 25 / 83\end{array}$ & $\begin{array}{l}0001 \\
0001\end{array}$ & $M G / L$ & $<$ & $\begin{array}{l}0.006 \\
0.50\end{array}$ & $\begin{array}{l}0.006 \\
0.5\end{array}$ & - \\
\hline COPPER & $\begin{array}{l}10 / 20 / 80 \\
05 / 25 / 83\end{array}$ & $\begin{array}{l}0001 \\
0001\end{array}$ & HG/L & $<$ & $\begin{array}{l}0.005 \\
0.50\end{array}$ & 0.5 & $\dot{-}$ \\
\hline CYANIDE & $05 / 25 / 83$ & 0001 & MG/L & $<$ & 1.00 & 1. & - \\
\hline FLUORIDE & $\begin{array}{l}10 / 20 / 80 \\
05 / 25 / 83\end{array}$ & $\begin{array}{l}0001 \\
0001\end{array}$ & MG/L & & $\begin{array}{l}0.27 \\
1.00\end{array}$ & - & - \\
\hline IRON & $\begin{array}{l}10 / 20 / 80 \\
05 / 25 / 83\end{array}$ & $\begin{array}{l}0001 \\
0001\end{array}$ & $M G / L$ & $<$ & $\begin{array}{l}0.093 \\
0.25\end{array}$ & 0.25 & - \\
\hline LEAD & $\begin{array}{l}10 / 20 / 80 \\
05 / 25 / 83\end{array}$ & $\begin{array}{l}0001 \\
0001\end{array}$ & MG/L & $<$ & $\begin{array}{l}0.001 \\
0.027\end{array}$ & 0.001 & - \\
\hline HAGMESIUN & $\begin{array}{l}10 / 20 / 80 \\
05 / 25 / 83\end{array}$ & $\begin{array}{l}0001 \\
0001\end{array}$ & $M G / L$ & $<$ & $\begin{array}{l}0.11 \\
0.50\end{array}$ & 0.5 & - \\
\hline MANGANESE & $\begin{array}{l}10 / 20 / 80 \\
05 / 25 / 83\end{array}$ & $\begin{array}{l}0001 \\
0001\end{array}$ & $\mathrm{HG} / \mathrm{L}$ & $<$ & $\begin{array}{l}0.002 \\
0.05\end{array}$ & $\begin{array}{l}0.002 \\
0.05\end{array}$ & - \\
\hline MERCURY & $10 / 20 / 80$ & 0001 & MG/L & $<$ & 0.001 & 0.001 & - \\
\hline MOLYBDENLA & $\begin{array}{l}10 / 20 / 80 \\
05 / 25 / 83\end{array}$ & $\begin{array}{l}0001 \\
0001\end{array}$ & HG/L & $<$ & $\begin{array}{l}0.27 \\
0.011\end{array}$ & 0.011 & - \\
\hline MICKEL & $\begin{array}{l}10 / 20 / 80 \\
05 / 25 / 83\end{array}$ & $\begin{array}{l}0001 \\
0001\end{array}$ & HG/L & $<$ & $\begin{array}{l}0.041 \\
0.01\end{array}$ & 0.01 & - \\
\hline
\end{tabular}

PARAMETER VALUE INDICATOR (PVI):

< - LESS THAN DETECTION LIMIT

SAMPLE ID COOES:

0001 - FILTERED SAMPLE (.45 MICRONS) 
GROUHDHATER QUALITY DATA BY LOCATIOH

SITE: AMBOI AMBROSIA LAKE

LOCATIOH: 0589

HORTH COORDINATE: 50500.0 FT

EAST COORDIHATE: $\quad 54790.0 \mathrm{FT}$

06/10/80 TO $12 / 04 / 92$

REPORT DATE: 08/04/94

FORHATION OF COMPLETION: DAKOTA SANDSTONE (KD)

HYDRAULIC FLON RELATIONSHIP: UNKHOWN (H)

\begin{tabular}{|c|c|c|c|c|c|c|c|}
\hline PARAMETER NAME & LOG DATE & $\underset{\text { ID }}{\text { SMMPLE }}$ & $\begin{array}{l}\text { UNITS OF } \\
\text { MEASURE }\end{array}$ & PVI & $\begin{array}{l}\text { PARAMETER } \\
\text { VALUE FLAGS }\end{array}$ & $\begin{array}{l}\text { DETECTION } \\
\text { LIMIT }\end{array}$ & $\begin{array}{l}\text { PARÄMETER } \\
\text { UHCERTAINTY }\end{array}$ \\
\hline HITRATE & $\begin{array}{l}10 / 20 / 80 \\
05 / 25 / 83\end{array}$ & $\begin{array}{l}0001 \\
0001\end{array}$ & MG/L & $<$ & $\begin{array}{l}4.60 \\
1.00\end{array}$ & i. & $\dot{-}$ \\
\hline PH & $\begin{array}{l}10 / 20 / 80 \\
05 / 25 / 83\end{array}$ & $\begin{array}{l}0001 \\
0001\end{array}$ & SU & & $\begin{array}{l}11.50 \\
11.40\end{array}$ & : & - \\
\hline POTASSIUH & $\begin{array}{l}10 / 20 / 80 \\
05 / 25 / 83\end{array}$ & $\begin{array}{l}0009 \\
0001\end{array}$ & MG/L & & $\begin{array}{r}13.00 \\
9.40\end{array}$ & - & - \\
\hline RADIUN-226 & $\begin{array}{l}10 / 20 / 80 \\
05 / 25 / 83\end{array}$ & $\begin{array}{l}0001 \\
0001\end{array}$ & PCI/L & $<$ & $\begin{array}{l}1.097 \\
2.00\end{array}$ & 2. & $\overline{-}$ \\
\hline RADIUH-226 + RADIUN-228 & $05 / 25 / 83$ & 0001 & PCI/L & & 2.00 & - & $\bullet$ \\
\hline RADIUM-228 & $05 / 25 / 83$ & 0001 & PCI/L & $<$ & 2.00 & 2. & - \\
\hline SELEHIUM & $\begin{array}{l}10 / 20 / 80 \\
05 / 25 / 83\end{array}$ & $\begin{array}{l}0001 \\
0001\end{array}$ & $M G / L$ & & $\begin{array}{l}0.16 \\
0.053\end{array}$ & - & - \\
\hline SILVER & $\begin{array}{l}10 / 20 / 80 \\
05 / 25 / 83\end{array}$ & $\begin{array}{l}0001 \\
0001\end{array}$ & $M G / L$ & $<$ & $\begin{array}{l}0.002 \\
0.50\end{array}$ & $\begin{array}{l}0.002 \\
0.5\end{array}$ & $\dot{-}$ \\
\hline SOOIUH & $\begin{array}{l}10 / 20 / 80 \\
05 / 25 / 83\end{array}$ & $\begin{array}{l}0001 \\
0001\end{array}$ & $M G / L$ & & $\begin{array}{l}190.00 \\
170.00\end{array}$ & - & - \\
\hline SPECIFIC CONDUCTANCE & $10 / 20 / 80$ & 0001 & UHHO/CH & & 1730.00 & - & - \\
\hline SULFATE & $\begin{array}{l}10 / 20 / 80 \\
05 / 25 / 83\end{array}$ & $\begin{array}{l}0001 \\
0001\end{array}$ & MG/L & & $\begin{array}{l}400.00 \\
220.00\end{array}$ & : & $\dot{-}$ \\
\hline TOTAL DISSOLVED SOLIDS & $\begin{array}{l}10 / 20 / 80 \\
05 / 25 / 83\end{array}$ & $\begin{array}{l}0001 \\
0001\end{array}$ & $M G / L$ & & $\begin{array}{l}969.00 \\
820.00\end{array}$ & - & $\dot{-}$ \\
\hline TOTAL SUSPEHDED SOLIDS & $10 / 20 / 80$ & 0001 & MG/L & & 3.00 & - & - \\
\hline URAKIUM & $\begin{array}{l}10 / 20 / 80 \\
05 / 25 / 83\end{array}$ & $\begin{array}{l}0001 \\
0001\end{array}$ & MG/L & & $\begin{array}{l}0.05 \\
0.006\end{array}$ & $\dot{-}$ & $\dot{-}$ \\
\hline VANADIUN & $\begin{array}{l}10 / 20 / 80 \\
05 / 25 / 83\end{array}$ & $\begin{array}{l}0001 \\
0001\end{array}$ & MG/L & $<$ & $\begin{array}{l}0.003 \\
0.04\end{array}$ & $\begin{array}{l}0.003 \\
0.04\end{array}$ & - \\
\hline ZINC & $\begin{array}{l}10 / 20 / 80 \\
05 / 25 / 83\end{array}$ & $\begin{array}{l}0001 \\
0001\end{array}$ & $M G / L$ & & $\begin{array}{l}0.059 \\
0.013\end{array}$ & - & 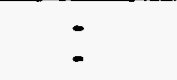 \\
\hline
\end{tabular}

PARAMETER VALUE INDICATOR (PVI): < - LeSS ThaN DETECTION LIHIT

SAMPLE ID COOES:

0001 - FILTERED SAMPLE (.45 MICRONS) 
GROUHDWATER OUALITY DATA BY LOCATION

SITE: AMBOI AMBROSIA LAKE

LOCATION: 0590

MORTH COORDINATE: $\quad 51910.0$ FT

EAST COORDINATE: $\quad 54880.0$ FT

06/10/80 TO $12 / 04 / 92$

REPORT DATE: 08/04/94

FORMATION OF COHPLETION: DAKOTA SANDSTONE (KD)

HYORAULIC FLON RELATIONSHIP: UNKWONH (H)

\begin{tabular}{|c|c|c|c|c|c|c|c|}
\hline PARAMETER MAME & LOG DATE & $\underset{\text { ID }}{\text { SAMPLE }}$ & $\begin{array}{l}\text { UNITS OF } \\
\text { MEASURE }\end{array}$ & PVI & $\begin{array}{l}\text { PARAMETER } \\
\text { VALUE FLAGS }\end{array}$ & $\begin{array}{l}\text { DETECTIOH } \\
\text { LIMIT }\end{array}$ & $\begin{array}{l}\text { PARAMETER } \\
\text { UMCERTAINTY }\end{array}$ \\
\hline ALKALINITY & $11 / 14 / 83$ & 0001 & $\mathrm{HG} / \mathrm{L} \mathrm{CACO}_{\mathrm{C}}$ & & 8.20 & - & - \\
\hline ALCMINUM & $\begin{array}{l}06 / 23 / 80 \\
11 / 14 / 83\end{array}$ & $\begin{array}{l}0001 \\
0001\end{array}$ & HG/L & & $\begin{array}{l}1.60 \\
0.15\end{array}$ & - & - \\
\hline ARSENIC & $\begin{array}{l}06 / 23 / 80 \\
11 / 14 / 83\end{array}$ & $\begin{array}{l}0001 \\
0001\end{array}$ & MG/L & & $\begin{array}{l}0.13 \\
0.064\end{array}$ & - & - \\
\hline BARIUM & $\begin{array}{l}06 / 23 / 80 \\
11 / 14 / 83\end{array}$ & $\begin{array}{l}0001 \\
0001\end{array}$ & $M G / L$ & $<$ & $\begin{array}{l}0.001 \\
0.014\end{array}$ & 0.001 & - \\
\hline BICARBOMATE - HCO3 & $\begin{array}{l}06 / 23 / 80 \\
11 / 14 / 83\end{array}$ & $\begin{array}{l}0001 \\
0001\end{array}$ & MG/L & & $\begin{array}{r}293.00 \\
20.00\end{array}$ & $\dot{-}$ & $\dot{-}$ \\
\hline BORON & $\begin{array}{l}06 / 23 / 80 \\
11 / 14 / 83\end{array}$ & $\begin{array}{l}0001 \\
0001\end{array}$ & $M G / L$ & & $\begin{array}{l}1.00 \\
0.44\end{array}$ & - & - \\
\hline CADHIUM & $\begin{array}{l}06 / 23 / 80 \\
11 / 14 / 83\end{array}$ & $\begin{array}{l}0001 \\
0001\end{array}$ & MG/L & $<$ & $\begin{array}{l}0.008 \\
0.007\end{array}$ & $\begin{array}{l}0.008 \\
0.007\end{array}$ & - \\
\hline CALCIUM & $\begin{array}{l}06 / 23 / 80 \\
11 / 14 / 83\end{array}$ & $\begin{array}{l}0001 \\
0001\end{array}$ & $M G / L$ & & $\begin{array}{l}670.00 \\
240.00\end{array}$ & - & - \\
\hline CARBONATE & $11 / 14 / 83$ & 0001 & $M G / L$ & $<$ & 10.00 & 10. & - \\
\hline CHLORIDE & $\begin{array}{l}06 / 23 / 80 \\
11 / 14 / 83\end{array}$ & $\begin{array}{l}0001 \\
0001\end{array}$ & MG/L & & $\begin{array}{l}2120.00 \\
1310.00\end{array}$ & - & - \\
\hline CHROMIUM & $\begin{array}{l}06 / 23 / 80 \\
11 / 14 / 83\end{array}$ & $\begin{array}{l}0001 \\
0001\end{array}$ & $H G / L$ & & $\begin{array}{l}0.037 \\
0.038\end{array}$ & - & - \\
\hline COBALT & $\begin{array}{l}06 / 23 / 80 \\
11 / 14 / 83\end{array}$ & $\begin{array}{l}0001 \\
0001\end{array}$ & $M G / L$ & $<$ & $\begin{array}{l}0.006 \\
0.014\end{array}$ & 0.006 & - \\
\hline COPPER & $\begin{array}{l}06 / 23 / 80 \\
11 / 14 / 83\end{array}$ & $\begin{array}{l}0001 \\
0001\end{array}$ & $M G / L$ & & $\begin{array}{l}0.01 \\
0.019\end{array}$ & - & - \\
\hline CYANIDE & $19 / 14 / 83$ & 0001 & $M G / L$ & $<$ & 1.00 & 1. & - \\
\hline FLUORIDE & $\begin{array}{l}06 / 23 / 80 \\
11 / 14 / 83\end{array}$ & $\begin{array}{l}0001 \\
0001\end{array}$ & MG/L & $<$ & $\begin{array}{l}0.05 \\
1.00\end{array}$ & $i$ & - \\
\hline IRON & $\begin{array}{l}06 / 23 / 80 \\
11 / 14 / 83\end{array}$ & $\begin{array}{l}0001 \\
0001\end{array}$ & MG/L & $<$ & $\begin{array}{l}0.001 \\
0.17\end{array}$ & 0.001 & - \\
\hline LEAD & $\begin{array}{l}06 / 23 / 80 \\
11 / 14 / 83\end{array}$ & $\begin{array}{l}0001 \\
0001\end{array}$ & MG/L & & $\begin{array}{l}0.008 \\
0.033\end{array}$ & - & - \\
\hline MAGNESIUA & $\begin{array}{l}06 / 23 / 80 \\
11 / 14 / 83\end{array}$ & $\begin{array}{l}0001 \\
0001\end{array}$ & $M G / L$ & & $\begin{array}{l}400.00 \\
510.00\end{array}$ & - & - \\
\hline MANGANESE & $\begin{array}{l}06 / 23 / 80 \\
11 / 14 / 83\end{array}$ & $\begin{array}{l}0001 \\
0001\end{array}$ & MG/L & & $\begin{array}{l}0.51 \\
0.70\end{array}$ & $:$ & - \\
\hline MERCURY & $06 / 23 / 80$ & 0001 & HG/L & $<$ & 0.001 & 0.001 & - \\
\hline MOLYBDENUN & $\begin{array}{l}06 / 23 / 80 \\
11 / 14 / 83\end{array}$ & $\begin{array}{l}0001 \\
0001\end{array}$ & MG/L & $<$ & $\begin{array}{l}0.002 \\
0.018\end{array}$ & 0.018 & - \\
\hline
\end{tabular}

PARAMETER VALUE INDICATOR (PVI): < - LESS THAN DETECTION LIMIT

SAMPLE ID COOES:

0001 - FILTERED SAMPLE (.45 MICRONS) 
GROUHDHATER QUALITY DATA BY LOCATIOH

SITE: AMBOI AMBROSIA LAKE

LOCATION: 0590

NORTH COORDINATE: $\quad 51910.0$ FT

EAST COORDIHATE: $\quad 54880.0$ FT

06/10/80 TO 12/04/92

REPORT DATE: 08/04/94

FORHATIOH OF COMPLETIOH: DAKOTA SANDSTONE (KD)

HYDRAULIC FLOH RELATIONSHIP: UNKHOWN (N)

\begin{tabular}{|c|c|c|c|c|c|c|c|}
\hline PARAMETER MAME & LOG DATE & $\begin{array}{c}\text { SAMPLE } \\
\text { ID }\end{array}$ & $\begin{array}{l}\text { UNITS OF } \\
\text { MEASURE }\end{array}$ & PVI & $\begin{array}{l}\text { PARAMETER } \\
\text { VALUE FLAGS }\end{array}$ & $\begin{array}{l}\text { DETECTION } \\
\text { LIHIT }\end{array}$ & $\begin{array}{l}\text { PARAMETER } \\
\text { UKCERTAINTY }\end{array}$ \\
\hline HICKEL & $\begin{array}{l}06 / 23 / 80 \\
11 / 14 / 83\end{array}$ & $\begin{array}{l}0001 \\
0001\end{array}$ & MG/L & & $\begin{array}{l}0.014 \\
0.038\end{array}$ & - & - \\
\hline HITRATE & $\begin{array}{l}06 / 23 / 80 \\
11 / 14 / 83\end{array}$ & $\begin{array}{l}0001 \\
0001\end{array}$ & MG/L & & $\begin{array}{l}50.00 \\
10.00\end{array}$ & $\dot{-}$ & - \\
\hline PH & $\begin{array}{l}06 / 23 / 80 \\
11 / 14 / 83\end{array}$ & $\begin{array}{l}0001 \\
0001\end{array}$ & SU & & $\begin{array}{l}7.30 \\
6.00\end{array}$ & - & - \\
\hline POTASSIUN & $\begin{array}{l}06 / 23 / 80 \\
11 / 14 / 83\end{array}$ & $\begin{array}{l}0001 \\
0001\end{array}$ & MG/L & & $\begin{array}{l}7.80 \\
7.70\end{array}$ & - & - \\
\hline RADIUN-226 & $\begin{array}{l}06 / 23 / 80 \\
11 / 14 / 83\end{array}$ & $\begin{array}{l}0001 \\
0001\end{array}$ & $\mathrm{PCI} / \mathrm{L}$ & $<$ & $\begin{array}{l}0.50 \\
3.60\end{array}$ & 3.6 & - \\
\hline RADIUH-226 + RADIUH-228 & $11 / 14 / 83$ & 0001 & $\mathrm{PCl} / \mathrm{L}$ & & 3.60 & - & - \\
\hline RADIUH-228 & $11 / 14 / 83$ & 0001 & $\mathrm{PCI} / \mathrm{L}$ & $<$ & 3.60 & 3.6 & - \\
\hline SELENIUH & $\begin{array}{l}06 / 23 / 80 \\
11 / 14 / 83\end{array}$ & $\begin{array}{l}0001 \\
0001\end{array}$ & $M G / L$ & & $\begin{array}{l}0.32 \\
0.11\end{array}$ & - & - \\
\hline SILVER & $\begin{array}{l}06 / 23 / 80 \\
11 / 14 / 83\end{array}$ & $\begin{array}{l}0001 \\
0001\end{array}$ & MG/L & & $\begin{array}{l}0.009 \\
0.028\end{array}$ & - & - \\
\hline soolum & $\begin{array}{l}06 / 23 / 80 \\
11 / 14 / 83\end{array}$ & $\begin{array}{l}0001 \\
0001\end{array}$ & $M G / L$ & & $\begin{array}{l}1100.00 \\
1000.00\end{array}$ & - & - \\
\hline SPECIFIC CONDUCTANCE & $06 / 23 / 80$ & 0001 & ЧМно/CH & & 8770.00 & - & - \\
\hline SULFATE & $\begin{array}{l}06 / 23 / 80 \\
11 / 14 / 83\end{array}$ & $\begin{array}{l}0001 \\
0001\end{array}$ & $M G / L$ & & $\begin{array}{l}2810.00 \\
2630.00\end{array}$ & - & - \\
\hline TOTAL DISSOLVED SOLIDS & $\begin{array}{l}06 / 23 / 80 \\
11 / 14 / 83\end{array}$ & $\begin{array}{l}0001 \\
0001\end{array}$ & MG/L & & $\begin{array}{l}7510.00 \\
6270.00\end{array}$ & - & - \\
\hline TOTAL SUSPEHDED SOLIDS & $06 / 23 / 80$ & 0001 & MG/L & & 33.00 & - & - \\
\hline URAKIUM & $\begin{array}{l}06 / 23 / 80 \\
11 / 14 / 83\end{array}$ & $\begin{array}{l}0001 \\
0001\end{array}$ & $M G / L$ & & $\begin{array}{l}0.31 \\
0.015\end{array}$ & - & - \\
\hline VAKADIUM & $\begin{array}{l}06 / 23 / 80 \\
11 / 14 / 83\end{array}$ & $\begin{array}{l}0001 \\
0001\end{array}$ & MG/L & $<$ & $\begin{array}{l}0.003 \\
0.021\end{array}$ & 0.003 & : \\
\hline ZINC & $\begin{array}{l}06 / 23 / 80 \\
11 / 14 / 83\end{array}$ & $\begin{array}{l}0001 \\
0001\end{array}$ & MG/L & & $\begin{array}{l}0.096 \\
0.14\end{array}$ & - & - \\
\hline
\end{tabular}

PARAMETER VALUE IHDICATOR (PVI): < - LESS THAH DETECTIOH LIMIT

SIMPLE 10 COOES

0001 - FILTERED SAMPLE (.45 MICRONS) 
GROUNDWATER QUALITY DATA BY LOCATION

SITE: AMBO1 AMBROSIA LAKE

LOCATION: 0591

NORTH COORDINATE: 50580.0 FT

EAST COORDIHATE: $\quad 55640.0$ FT

06/10/80 TO $12 / 04 / 92$

REPORT DATE: $08 / 04 / 94$

FORMATION OF COMPLETIOH: DAKOTA SAHDSTOHE (KD)

HYDRAULIC FLON RELATIONSHIP: UNKHOWH (N)

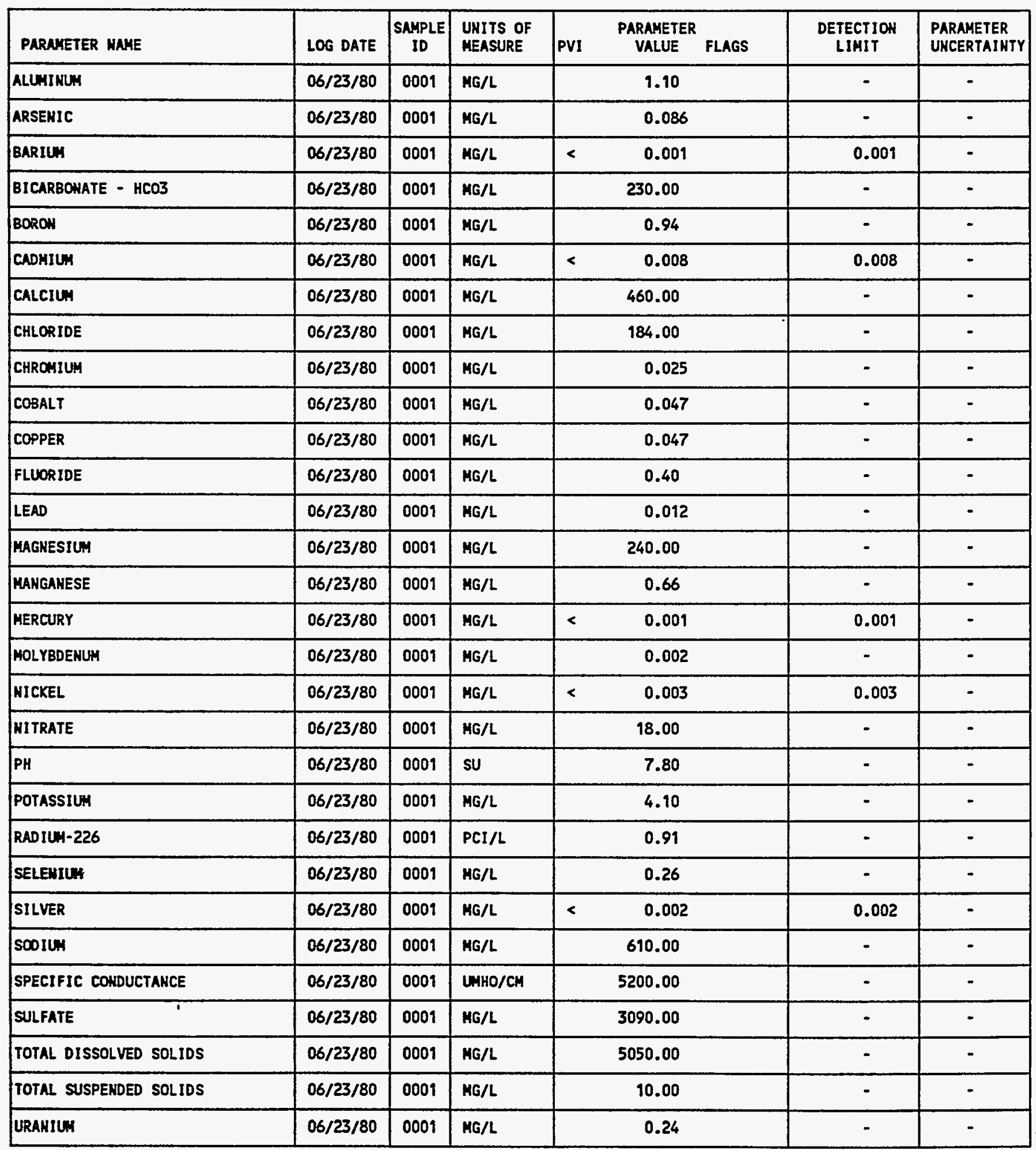

PARAHETER VALUE INDICATOR (PVI): < - LESS THAH DETECTION LIMIT 
GROUNDWATER OUALITY DATA BY LOCATION

SITE: AMBO1 AMBROSIA LAKE

LOCATION: 0591

NORTH COORDIHATE: $\quad 50580.0$ FT

EAST COORDINATE: $\quad 55640.0$ FT

06/10/80 TO $12 / 04 / 92$

REPORT DATE: 08/04/94

FORMATION OF COMPLETION: DAKOTA SAMDSTOHE (KD)

HYORAULIC FLOW RELATIONSHIP: UNKHOWN (H)

\begin{tabular}{|c|c|c|c|c|c|c|c|}
\hline $\begin{array}{c}\text { ' } \\
\text { PARAMETER MAME }\end{array}$ & LOG DATE & $\begin{array}{c}\text { SAMPLE } \\
\text { ID }\end{array}$ & $\begin{array}{l}\text { UNITS OF } \\
\text { MEASURE }\end{array}$ & PVI & $\begin{array}{l}\text { PARAMETER } \\
\text { VALUE FLAGS }\end{array}$ & $\begin{array}{l}\text { DETECTION } \\
\text { LINIT }\end{array}$ & $\begin{array}{l}\text { PARAMETER } \\
\text { UNCERTAINTY }\end{array}$ \\
\hline VANADIUM & $06 / 23 / 80$ & 0001 & $M G / L$ & $<$ & 0.003 & 0.003 & - \\
\hline ZINC & $06 / 23 / 80$ & 0001 & MG/L & & 0.063 & - & - \\
\hline
\end{tabular}

PARAMETER VALUE IMDICATOR (PVI):

< - LESS THAN DETECTION LIMIT

SAMPLE ID COOES:

0001 - FILTERED SAMPLE (.45 MICRONS) 
GROUNDWATER QUALITY OATA BY LOCATION

SITE: AMBO1 AMBROSIA LAKE

LOCATION: 0592

NORTH COORDIMATE: $\quad 48150.0$ FT

EAST COORDINATE: $\quad 56680.0$ FT

06/10/80 TO 12/04/92

REPORT DATE: 08/04/94

FORMATION OF COMPLETION: DAKOTA SANDSTONE (KD)

HYDRAULIC FLOW RELATIONSHIP: UNKNOWH (N)

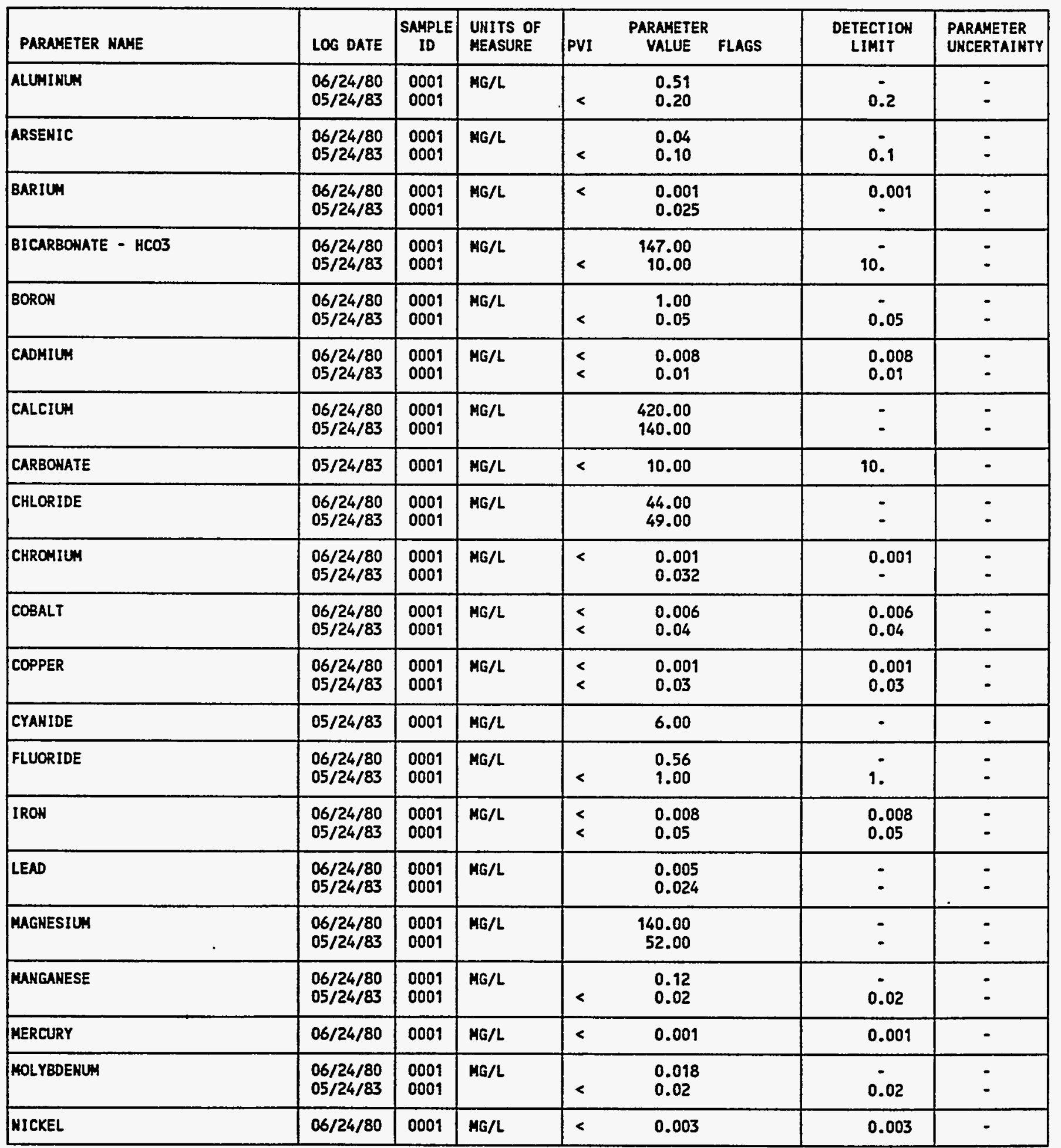

PARAMETER VALUE INDICATOR (PVI): < - LESS THAN DETECTIOH LIMIT

SAMPLE ID COOES:

0001 - FILTERED SAMPLE (.45 MICROHS) 
GROUHDHATER QUALITY DATA BY LOCATION

SITE: AMBOI AMBROSIA LAKE

LOCATIOH: 0592

NORTH COORDINATE: 48150.0 FT

EAST COORDINATE: $\quad 56680.0$ FT

06/10/80 TO 12/04/92

REPORT DATE: 08/04/94

FORMATIOH OF COMPLETION: DAKOTA SAHDSTONE (KD)

HYORAULIC FLOW RELATIONSHIP: UHKNOWH (H)

\begin{tabular}{|c|c|c|c|c|c|c|c|}
\hline $\begin{array}{c}\vdots \\
\text { PARAMETER MAME } \\
\end{array}$ & LOG DATE & $\underset{\text { SAMPLE }}{\text { ID }}$ & $\begin{array}{l}\text { UHITS OF } \\
\text { MEASURE }\end{array}$ & PVI & $\begin{array}{l}\text { PARAMETER } \\
\text { VALUE FLAGS }\end{array}$ & $\begin{array}{l}\text { DETECTION } \\
\text { LIMIT }\end{array}$ & $\begin{array}{l}\text { PARAMETER } \\
\text { UNCERTAINTY }\end{array}$ \\
\hline HICKEL & $05 / 24 / 83$ & 0001 & $M G / L$ & $<$ & 0.08 & 0.08 & - \\
\hline NITRATE & $\begin{array}{l}06 / 24 / 80 \\
05 / 24 / 83\end{array}$ & $\begin{array}{l}0001 \\
0001\end{array}$ & MG/L & & $\begin{array}{r}42.00 \\
1.00\end{array}$ & i. & - \\
\hline PH & $\begin{array}{l}06 / 24 / 80 \\
05 / 24 / 83\end{array}$ & $\begin{array}{l}0001 \\
0001\end{array}$ & su & & $\begin{array}{l}7.90 \\
9.00\end{array}$ & - & - \\
\hline POTASSIUH & $\begin{array}{l}06 / 24 / 80 \\
05 / 24 / 83\end{array}$ & $\begin{array}{l}0001 \\
0001\end{array}$ & MG/L & & $\begin{array}{l}4.70 \\
9.20\end{array}$ & - & - \\
\hline RADIUH-226 & $\begin{array}{l}06 / 24 / 80 \\
05 / 24 / 83\end{array}$ & $\begin{array}{l}0001 \\
0001\end{array}$ & $\mathrm{PCl} / \mathrm{L}$ & & $\begin{array}{l}1.42 \\
3.16\end{array}$ & - & $\therefore$ \\
\hline RADIUN-226 + RADIUH-228 & $05 / 24 / 83$ & 0001 & $\mathrm{PCI} / \mathrm{L}$ & & 6.32 & - & - \\
\hline RADIUH-228 & $05 / 24 / 83$ & 0001 & PCI/L & & 3.16 & - & - \\
\hline SELENIUM & $\begin{array}{l}06 / 24 / 80 \\
05 / 24 / 83\end{array}$ & $\begin{array}{l}0001 \\
0001\end{array}$ & MG/L & & $\begin{array}{l}0.26 \\
0.052\end{array}$ & $\div$ & - \\
\hline SILVER & $\begin{array}{l}06 / 24 / 80 \\
05 / 24 / 83\end{array}$ & $\begin{array}{l}0001 \\
0001\end{array}$ & MG/L & $<$ & $\begin{array}{l}0.002 \\
0.03\end{array}$ & $\begin{array}{l}0.002 \\
0.03\end{array}$ & - \\
\hline SOOIUN & $\begin{array}{l}06 / 24 / 80 \\
05 / 24 / 83\end{array}$ & $\begin{array}{l}0001 \\
0001\end{array}$ & MG/L & & $\begin{array}{l}290.00 \\
130.00\end{array}$ & - & - \\
\hline SPECIFIC COHDUCTAMCE & $06 / 24 / 80$ & 0001 & UMHO/CH & & 3570.00 & - & - \\
\hline SULFATE & $\begin{array}{l}06 / 24 / 80 \\
05 / 24 / 83\end{array}$ & $\begin{array}{l}0001 \\
0001\end{array}$ & HG/L & & $\begin{array}{r}2040.00 \\
760.00\end{array}$ & : & - \\
\hline TOTAL DISSOLVED SOLIDS & $\begin{array}{l}06 / 24 / 80 \\
05 / 24 / 83\end{array}$ & $\begin{array}{l}0001 \\
0001\end{array}$ & MG/L & & $\begin{array}{l}3440.00 \\
1220.00\end{array}$ & - & - \\
\hline TOTAL SUSPEKDED SOLIDS & $06 / 24 / 80$ & 0001 & HG/L & & 70.00 & - & - \\
\hline URAKIUA & $\begin{array}{l}06 / 24 / 80 \\
05 / 24 / 83\end{array}$ & $\begin{array}{l}0001 \\
0001\end{array}$ & MG/L & & $\begin{array}{l}0.085 \\
0.008\end{array}$ & - & - \\
\hline VAHADIUH & $06 / 24 / 80$ & 0001 & MG/L & $<$ & 0.003 & 0.003 & - \\
\hline ZIMC & $\begin{array}{l}06 / 24 / 80 \\
05 / 24 / 83\end{array}$ & $\begin{array}{l}0001 \\
0001\end{array}$ & MG/L & & $\begin{array}{l}0.059 \\
0.04\end{array}$ & - & $=$ \\
\hline
\end{tabular}

PARAMETER VALUE INDICATOR (PVI): < - LESS THAM DETECTIOH LIMIT

SAMPLE ID CODES:

0001 - FILTERED SAMPLE (.45 MICRONS) 
GROUHDWATER QUALITY DATA BY LOCATION

SITE: AMBO1 AMBROSIA LAKE

LOCATION: 0593

MORTH COORDINATE: 52240.0 FT

EAST COORDIHATE: $\quad \mathbf{4 5 5 9 0 . 0}$ FT

06/10/80 TO $12 / 04 / 92$

REPORT DATE: 08/04/94

FORHATION OF COMPLETION: DAKOTA SANDSTONE (KD)

HYORAULIC FLON RELATIONSHIP: UNKNOWN (N)

\begin{tabular}{|c|c|c|c|c|c|c|c|}
\hline PARAMETER NAME & LOG DATE & $\begin{array}{c}\text { SAMPLE } \\
\text { ID }\end{array}$ & $\begin{array}{l}\text { UNITS OF } \\
\text { MEASURE }\end{array}$ & PVI & $\begin{array}{l}\text { PARAMETER } \\
\text { VALUE FLAGS }\end{array}$ & $\begin{array}{l}\text { DETECTIOH } \\
\text { LIMIT }\end{array}$ & $\begin{array}{l}\text { PARAMETER } \\
\text { UHCERTAINTY }\end{array}$ \\
\hline ALCHINUM & $08 / 16 / 83$ & 0001 & $M G / L$ & & 0.65 & - & - \\
\hline ARSEHIC & $08 / 16 / 83$ & 0001 & MG/L & & 0.26 & - & - \\
\hline BARIUA & $08 / 16 / 83$ & 0001 & MG/L & & 0.028 & - & - \\
\hline BORON & $08 / 16 / 83$ & 0001 & MG/L & & 1.30 & - & - \\
\hline CADHIUH & $08 / 16 / 83$ & 0001 & MG/L & & 0.042 & - & - \\
\hline CALCIUN & $08 / 16 / 83$ & 0001 & HG/L & & 510.00 & - & - \\
\hline CARBONATE & $08 / 16 / 83$ & 0001 & $H G / L$ & $<$ & 10.00 & 10. & - \\
\hline COBALT & $08 / 16 / 83$ & 0001 & MG/L & & 0.074 & - & - \\
\hline COPPER & $08 / 96 / 83$ & 0001 & MG/L & & 0.12 & - & - \\
\hline CYANIDE & $08 / 16 / 83$ & 0001 & $M G / L$ & $<$ & 0.10 & 0.1 & - \\
\hline FLLORIDE & $08 / 16 / 83$ & 0001 & MG/L & & 9.90 & - & - \\
\hline IRON & $08 / 16 / 83$ & 0001 & $M G / L$ & & 500.00 & - & - \\
\hline LEAD & $08 / 16 / 83$ & 0001 & HG/L & & 0.46 & - & - \\
\hline MAGNESIUM & $08 / 16 / 83$ & 0001 & $M G / L$ & & 700.00 & - & - \\
\hline POTASSIUM & $08 / 16 / 83$ & 0001 & MG/L & & 28.00 & - & - \\
\hline RADIUH-226 & $08 / 16 / 83$ & 0001 & $\mathrm{PCI} / \mathrm{L}$ & $<$ & 2.30 & 2.3 & - \\
\hline RADIUH -226 + RADIUH -228 & $08 / 16 / 83$ & 0001 & $\mathrm{PCI} / \mathrm{L}$ & & 2.30 & - & - \\
\hline RADILA-228 & $08 / 16 / 83$ & 0001 & $\mathrm{PCI} / \mathrm{L}$ & $<$ & 2.30 & 2.3 & - \\
\hline SELEHIUH & $08 / 16 / 83$ & 0001 & MG/L & & 0.43 & - & - \\
\hline SILVER & $08 / 16 / 83$ & 0001 & $M G / L$ & & 0.14 & - & - \\
\hline SOOIUM & $08 / 16 / 83$ & 0001 & HG/L & & 1200.00 & - & - \\
\hline SULFATE & $08 / 16 / 83$ & 0001 & $M G / L$ & & 3770.00 & - & - \\
\hline
\end{tabular}

PARAMETER VALUE INDICATOR (PVI):

< - LESS THAN DETECTION LIMIT

SAMPLE ID COOES:

D001 - FILTERED SAMPLE (.45 MICROHS) 
GROUHDWATER OUALITY DATA BY LOCATIOH

SITE: AMBOI AMBROSIA LAKE

LOCATIOH: 0593

MORTH COORDINATE: 52240.0 FT

EAST COORDINATE: $\quad 45590.0$ FT

06/10/80 TO 12/04/92

REPORT DATE: 08/04/94

FORMATION OF COMPLETIOH: DAKOTA SAMDSTONE (KD)

HYDRAULIC FLON RELATIONSHIP: UHKNOWH (H)

\begin{tabular}{|c|c|c|c|c|c|c|c|}
\hline PARAMETER MAME & LOG DATE & $\begin{array}{c}\text { SAMPLE } \\
\text { ID }\end{array}$ & $\begin{array}{l}\text { UHITS OF } \\
\text { MEASURE }\end{array}$ & PVI & $\begin{array}{l}\text { PARAMETER } \\
\text { VALUE FLAGS }\end{array}$ & $\begin{array}{l}\text { DETECTION } \\
\text { LIMIT }\end{array}$ & $\begin{array}{l}\text { PARÁKETER } \\
\text { UNCERTAINTY }\end{array}$ \\
\hline TOTAL DISSOLVED SOLIDS & $08 / 16 / 83$ & 0001 & MG/L & & 9320.00 & - & - \\
\hline URAKIUM & $08 / 16 / 83$ & 0001 & MG/L & & 0.003 & - & - \\
\hline VAKADIUH & $08 / 96 / 83$ & 0001 & $M G / L$ & & 0.12 & - & - \\
\hline ZIHC & $08 / 16 / 83$ & 0001 & $M G / L$ & & 0.25 & - & - \\
\hline
\end{tabular}

PARAMETER VALUE INDICATOR (PVI): < - LESS THAN DETECTION LIMIT

SUMPLE ID COOES:

0001 - FILTERED SAMPLE (.45 HICRONS) 
GROUNDWATER OUALITY DATA BY LOCATION

SITE: AMBOI AMBROSIA LAKE

LOCATION: 0594

NORTH COORDINATE: 31650.0 FT

EAST COORDINATE: 72440.0 FT

$06 / 10 / 80$ TO $12 / 04 / 92$

REPORT DATE: 08/04/94

FORHATION OF COMPLETION: ALLUVIUN (AL)

HYDRAULIC FLON RELATIONSHIP: UNKNOWN (N)

\begin{tabular}{|c|c|c|c|c|c|c|c|}
\hline PARAMETER MAME & LOG DATE & $\begin{array}{c}\text { SAMPLE } \\
10\end{array}$ & $\begin{array}{l}\text { UNITS OF } \\
\text { MEASURE }\end{array}$ & PVI & $\begin{array}{l}\text { PARAMETER } \\
\text { VALUE FLAGS }\end{array}$ & $\begin{array}{l}\text { DETECTIOH } \\
\text { LIMIT }\end{array}$ & $\begin{array}{l}\text { PARAMETER } \\
\text { UMCERTAIHTY }\end{array}$ \\
\hline ALKALIHITY & $\begin{array}{l}06 / 10 / 80 \\
07 / 01 / 81\end{array}$ & $\begin{array}{l}0001 \\
0001\end{array}$ & HG/L CACO3 & & $\begin{array}{r}101.00 \\
99.20\end{array}$ & - & - \\
\hline ARSENIC & $\begin{array}{l}06 / 10 / 80 \\
07 / 01 / 81\end{array}$ & $\begin{array}{l}0001 \\
0002\end{array}$ & MG/L & & $\begin{array}{l}0.005 \\
0.005\end{array}$ & : & - \\
\hline BARIUH & $\begin{array}{l}06 / 10 / 80 \\
07 / 01 / 81\end{array}$ & $\begin{array}{l}0001 \\
0002\end{array}$ & $M G / L$ & & $\begin{array}{l}0.10 \\
0.10\end{array}$ & - & - \\
\hline BICARBOHATE - HCO3 & $\begin{array}{l}06 / 10 / 80 \\
07 / 01 / 81\end{array}$ & $\begin{array}{l}0001 \\
0002\end{array}$ & $M G / L$ & & $\begin{array}{l}246.00 \\
242.00\end{array}$ & - & - \\
\hline CADMIUM & $\begin{array}{l}06 / 10 / 80 \\
07 / 01 / 81\end{array}$ & $\begin{array}{l}0001 \\
0002\end{array}$ & $M G / L$ & & $\begin{array}{l}0.001 \\
0.001\end{array}$ & - & - \\
\hline CALCIUM & $\begin{array}{l}06 / 10 / 80 \\
07 / 01 / 81\end{array}$ & $\begin{array}{l}0001 \\
0002\end{array}$ & $\mathrm{MG} / \mathrm{L}$ & & $\begin{array}{l}57.20 \\
60.20\end{array}$ & - & - \\
\hline CHLORIDE & $\begin{array}{l}06 / 10 / 80 \\
07 / 01 / 81\end{array}$ & $\begin{array}{l}0001 \\
0002\end{array}$ & MG/L & & $\begin{array}{l}15.00 \\
18.00\end{array}$ & - & - \\
\hline LEAD & $\begin{array}{l}06 / 10 / 80 \\
07 / 01 / 81\end{array}$ & $\begin{array}{l}0001 \\
0002\end{array}$ & MG/L & & $\begin{array}{l}0.005 \\
0.005\end{array}$ & - & - \\
\hline MAGNESIUM & $\begin{array}{l}06 / 10 / 80 \\
07 / 01 / 81\end{array}$ & $\begin{array}{l}0001 \\
0002\end{array}$ & $M G / L$ & & $\begin{array}{r}10.70 \\
7.60\end{array}$ & - & $\dot{-}$ \\
\hline MOLYBDEKUH & $\begin{array}{l}06 / 10 / 80 \\
07 / 01 / 81\end{array}$ & $\begin{array}{l}0001 \\
0002\end{array}$ & $M G / L$ & & $\begin{array}{l}0.159 \\
0.155\end{array}$ & - & - \\
\hline PH & $\begin{array}{l}06 / 10 / 80 \\
07 / 01 / 81\end{array}$ & $\begin{array}{l}0001 \\
0002\end{array}$ & SU & & $\begin{array}{l}7.45 \\
7.40\end{array}$ & $\dot{-}$ & - \\
\hline POTASSIUA & $\begin{array}{l}06 / 10 / 80 \\
07 / 01 / 81\end{array}$ & $\begin{array}{l}0001 \\
0002\end{array}$ & MG/L & & $\begin{array}{l}3.50 \\
2.70\end{array}$ & - & $\dot{-}$ \\
\hline SELENIUA & $\begin{array}{l}06 / 10 / 80 \\
07 / 01 / 81\end{array}$ & $\begin{array}{l}0001 \\
0002\end{array}$ & $M G / L$ & & $\begin{array}{l}0.022 \\
0.028\end{array}$ & - & - \\
\hline scolum & $\begin{array}{l}06 / 10 / 80 \\
07 / 01 / 81\end{array}$ & $\begin{array}{l}0001 \\
0002\end{array}$ & $\mathrm{MG} / \mathrm{L}$ & & $\begin{array}{l}142.60 \\
144.90\end{array}$ & - & - \\
\hline SPECIFIC CONDUCTANCE & $06 / 10 / 80$ & 0001 & UHHO/CM & & 968.00 & - & - \\
\hline SULFATE & $\begin{array}{l}06 / 10 / 80 \\
07 / 01 / 81\end{array}$ & $\begin{array}{l}0001 \\
0002\end{array}$ & MG/L. & & $\begin{array}{l}259.00 \\
265.00\end{array}$ & - & - \\
\hline TOTAL DISSOLVED SOLIDS & $\begin{array}{l}06 / 10 / 80 \\
07 / 01 / 81\end{array}$ & $\begin{array}{l}0001 \\
0002\end{array}$ & MG/L & & $\begin{array}{l}656.00 \\
665.00\end{array}$ & - & - \\
\hline URANIUA & $\begin{array}{l}06 / 10 / 80 \\
07 / 01 / 81\end{array}$ & $\begin{array}{l}0001 \\
0002\end{array}$ & $M G / L$ & & $\begin{array}{l}0.178 \\
0.22\end{array}$ & - & - \\
\hline VAHADIUN & $\begin{array}{l}06 / 10 / 80 \\
07 / 01 / 81\end{array}$ & $\begin{array}{l}0001 \\
0002\end{array}$ & MG/L & & $\begin{array}{l}0.01 \\
0.10\end{array}$ & - & - \\
\hline $21 \mathrm{KC}$ & $06 / 10 / 80$ & 0001 & HG/L & & 0.25 & - & - \\
\hline
\end{tabular}

PARAMETER VALUE INDICATOR (PVI): < - LESS THAN DETECTION LIHIT

SAMPLE ID CODES:

OOO1 - FILTERED SAMPLE (.45 MICROHS)

0002 - FILTERED REPLICATE SAMPLE (.45 MICRONS) 
GROUNDHATER QUALITY DATA BY LOCATION

SITE: AMBOI AHBROSIA LAKE

LOCATION: 0595

NORTH COORDINATE: 30640.0 FT

EAST COORDINATE: $\quad 67540.0$ FT

06/10/80 TO $12 / 04 / 92$

REPORT DATE: 08/04/94

FORMATION OF COMPLETION: ALLUVIUM (AL)

HYDRAULIC FLON RELATIOHSHIP: UHKHOUK (N)

\begin{tabular}{|c|c|c|c|c|c|c|c|}
\hline PARAMETER HAHE & LOG DATE & $\begin{array}{c}\text { SAMPLE } \\
\text { ID }\end{array}$ & $\begin{array}{l}\text { UHITS OF } \\
\text { MEASURE }\end{array}$ & PVI & $\begin{array}{l}\text { PARAMETER } \\
\text { VALUE FLAGS }\end{array}$ & $\begin{array}{l}\text { DETECTIOH } \\
\text { LIMIT }\end{array}$ & $\begin{array}{l}\text { PARANETER } \\
\text { UNCERTAIHTY }\end{array}$ \\
\hline ALKALINITY & $07 / 01 / 81$ & 0001 & MG/L CACO3 & & 108.00 & - & - \\
\hline ARSENIC & $07 / 01 / 81$ & 0001 & $M G / L$ & & 0.005 & - & - \\
\hline BARIUH & $07 / 01 / 81$ & 0001 & MG/L & & 0.10 & - & - \\
\hline BICARBONATE - HCO3 & $07 / 01 / 81$ & 0001 & MG/L & & 264.00 & - & - \\
\hline CADMILA & $07 / 01 / 81$ & 0001 & MG/L & & 0.001 & - & - \\
\hline CALCIUH & $07 / 01 / 81$ & 0001 & MG/L & & 58.60 & - & - \\
\hline CHLORIDE & $07 / 01 / 81$ & 0001 & $M G / L$ & & 19.00 & - & - \\
\hline LEAD & $07 / 01 / 81$ & 0001 & MG/L & & 0.005 & - & - \\
\hline MAGHESIUM & $07 / 01 / 81$ & 0001 & $M G / L$ & & 4.60 & - & - \\
\hline MOLYBDEKUM & $07 / 01 / 81$ & 0001 & $M G / L$ & & 0.155 & - & - \\
\hline PH & $07 / 01 / 81$ & 0001 & SU & & 7.50 & - & - \\
\hline POTASSIUH & $07 / 01 / 81$ & 0001 & $M G / L$ & & 2.30 & - & - \\
\hline SELENIUH & $07 / 01 / 81$ & 0001 & $M G / L$ & & 0.023 & - & - \\
\hline SODIUN & $07 / 01 / 81$ & 0001 & MG/L & & 158.70 & - & - \\
\hline SPECIFIC CONDUCTANCE & $07 / 01 / 81$ & 0001 & UMHO/CH & & 879.00 & - & - \\
\hline SULFATE & $07 / 01 / 81$ & 0001 & MG/L & & 259.00 & - & - \\
\hline TEMPERATURE & $07 / 01 / 81$ & 0001 & C - DEGREE & & 12.20 & - & - \\
\hline TOTAL DISSOLVED SOLIOS & $07 / 01 / 81$ & 0009 & $M G / L$ & & 668.00 & - & - \\
\hline URANIUM-234 & $07 / 01 / 81$ & 0009 & $\mathrm{PCI} / \mathrm{L}$ & & 0.18 & - & - \\
\hline VANADIUH & $07 / 01 / 81$ & 0001 & MG/L & & 0.01 & - & - \\
\hline ZIHC & $07 / 01 / 81$ & 0001 & HG/L & & 0.10 & - & - \\
\hline
\end{tabular}

PARAMETER VALUE IHDICATOR (PVI): < - LESS THAH DETECTIOH LIMIT

SAMPLE ID COOES

0001 - FILTERED SAMPLE (.45 MICRONS) 
GROUNDWATER QUALITY DATA BY LOCATION

SITE: AMBOI AMBROSIA LAKE

LOCATION: 0596

MORTH COORDINATE: 20790.0 FT

EAST COORDINATE: $\quad 64530.0$ FT

06/10/80 TO $12 / 04 / 92$

REPORT DATE: 08/04/94

FORHATION OF COMPLETION: ALLUVIUM (AL)

HYORAULIC FLON RELATIONSHIP: UNKNOWN (N)

\begin{tabular}{|c|c|c|c|c|c|c|}
\hline PARAMETER MAME & LOG DATE & $\begin{array}{c}\text { SAMPLE } \\
\text { ID }\end{array}$ & $\begin{array}{l}\text { UHITS OF } \\
\text { MEASURE }\end{array}$ & $\begin{array}{l}\text { PARAMETER } \\
\text { PVI VALUE FLAGS }\end{array}$ & $\begin{array}{l}\text { DETECTION } \\
\text { LIMIT }\end{array}$ & $\begin{array}{l}\text { PARAMETER } \\
\text { UMCERTAINTY }\end{array}$ \\
\hline ALKALIMITY & $01 / 26 / 82$ & 0001 & MG/L CACO3 & 155.00 & - & - \\
\hline ALUHIKUY & $01 / 26 / 82$ & 0001 & MG/L & 0.25 & - & - \\
\hline ARSENIC & $01 / 26 / 82$ & 0001 & MG/L & 0.005 & - & - \\
\hline BARIUH & $01 / 26 / 82$ & 0001 & MG/L & 0.10 & - & - \\
\hline BICARBONATE - HCO3 & $01 / 26 / 82$ & 0001 & $M G / L$ & 377.00 & - & - \\
\hline CADKIUN & $01 / 26 / 82$ & 0001 & MG/L & 0.002 & - & - \\
\hline CALCIUM & $01 / 26 / 82$ & 0001 & $M G / L$ & 73.60 & - & - \\
\hline CHLORIDE & $01 / 26 / 82$ & 0001 & $M G / L$ & 20.00 & - & - \\
\hline FLUORIDE & $01 / 26 / 82$ & 0001 & $M G / L$ & 1.15 & - & - \\
\hline IRON & $01 / 26 / 82$ & 0001 & $M G / L$ & 0.10 & - & - \\
\hline LEAD & $01 / 26 / 82$ & 0001 & $M G / L$ & 0.005 & - & - \\
\hline MAGKESIUN & $01 / 26 / 82$ & 0001 & $M G / L$ & 19.50 & - & - \\
\hline MOLYBDENUM & $01 / 26 / 82$ & 0001 & $M G / L$ & 0.01 & - & - \\
\hline PH & $01 / 26 / 82$ & 0001 & SU & 7.40 & - & - \\
\hline POTASSIUN & $01 / 26 / 82$ & 0001 & $M G / L$ & 0.00 & - & - \\
\hline SELEKIU⿴囗十 & $01 / 26 / 82$ & 0001 & MG/L & 0.005 & - & - \\
\hline SILICON & $01 / 26 / 82$ & 0001 & $M G / L$ & 13.00 & - & - \\
\hline SOOIUM & $01 / 26 / 82$ & 0001 & $M G / L$ & 101.20 & - & - \\
\hline SPECIFIC CONDUCTANCE & $01 / 26 / 82$ & 0001 & UMHO/CM & 638.00 & - & - \\
\hline SULFATE & $01 / 26 / 82$ & 0001 & MG/L & 84.00 & - & - \\
\hline TEMPERATURE & $01 / 26 / 82$ & 0001 & C - DEGREE & 12.50 & - & - \\
\hline TOTAL DISSOLVED SOLIDS & $01 / 26 / 82$ & 0001 & $M G / L$ & 505.00 & - & - \\
\hline URANIUM-234 & $01 / 26 / 82$ & 0001 & $\mathrm{PCI} / \mathrm{L}$ & 0.005 & - & - \\
\hline VAHADIUA & $01 / 26 / 82$ & 0001 & $M G / L$ & 0.01 & - & - \\
\hline ZINC & $01 / 26 / 82$ & 0001 & $M G / L$ & 0.10 & - & - \\
\hline
\end{tabular}

PARAMETER VALUE INDICATOR (PVI): < - LESS THAN DETECTION LIMIT

SAMPLE ID CODES:

0001 - FILTERED SAMPLE (.45 MICRONS) 
GROUNDHATER QUAALITY DATA BY LOCATION

SITE: AMBOI AMBROSIA LAKE

LOCATION: 0597

NORTH COORDINATE: 19350.0 FT

EAST COORDINATE: $\quad 65380.0$ FT

06/10/80 TO $12 / 04 / 92$

REPORT DATE: 08/04/94

FORMATION OF COMPLETION: ALLUVIUM (AL)

HYDRAULIC FLON RELATIOWSHIP: UNKNONH (K)

\begin{tabular}{|c|c|c|c|c|c|c|}
\hline PARAMETER NAME & LOG DATE & $\begin{array}{c}\text { SAMPLE } \\
\text { ID }\end{array}$ & $\begin{array}{l}\text { UNITS OF } \\
\text { MEASURE }\end{array}$ & $\begin{array}{l}\text { PARAMETER } \\
\text { PVI VALUE }\end{array}$ & $\begin{array}{l}\text { DETECTIOH } \\
\text { LIMIT }\end{array}$ & $\begin{array}{l}\text { PARAMETER } \\
\text { UHCERTAINTY }\end{array}$ \\
\hline ALKALIKITY & $01 / 26 / 82$ & 0001 & MG/L CACO3 & 86.50 & - & - \\
\hline ALUMIKUM & $01 / 26 / 82$ & 0001 & $M G / L$ & 0.25 & - & - \\
\hline ARSENIC & $01 / 26 / 82$ & 0001 & MG/L & 0.007 & - & - \\
\hline BARIUA & $01 / 26 / 82$ & 0001 & $\mathrm{MG} / \mathrm{L}$ & 0.15 & - & - \\
\hline BICARBONATE - HCO3 & $01 / 26 / 82$ & 0001 & MG/L & 211.00 & - & - \\
\hline CADMIUH & $01 / 26 / 82$ & 0001 & MG/L & 0.001 & - & - \\
\hline CALCIUM. & $01 / 26 / 82$ & 0001 & MG/L & 21.20 & - & $-\cdot$ \\
\hline CHLORIDE & $01 / 26 / 82$ & 0001 & $M G / L$ & 8.00 & - & - \\
\hline FLUORIDE & $01 / 26 / 82$ & 0001 & $M G / L$ & 1.90 & - & - \\
\hline IRON & $01 / 26 / 82$ & 0001 & MG/L & 0.10 & - & - \\
\hline LEAD & $01 / 26 / 82$ & 0001 & MG/L & 0.005 & - & - \\
\hline MAGNESIUM & $01 / 26 / 82$ & 0001 & MG/L & 4.00 & - & - \\
\hline MOLYBDENUH & $01 / 26 / 82$ & 0001 & $M G / L$ & 0.012 & - & - \\
\hline PH & $01 / 26 / 82$ & 0001 & SU & 7.80 & - & - \\
\hline POTASSIUH & $01 / 26 / 82$ & 0001 & MG/L & 2.30 & - & - \\
\hline SELEHIUM & $01 / 26 / 82$ & 0001 & MG/L & 0.005 & - & - \\
\hline SILICOH & $01 / 26 / 82$ & 0001 & MG/L & 22.90 & - & - \\
\hline Soolum & $01 / 26 / 82$ & 0001 & MG/L & 126.50 & - & - \\
\hline SPECIFIC COHDUCTANCE & $01 / 26 / 82$ & 0001 & UHHO/CH & 628.00 & - & - \\
\hline SULFATE & $01 / 26 / 82$ & 0001 & MG/L & 31.00 & - & - \\
\hline TEMPERATURE & $01 / 26 / 82$ & 0001 & C - DEGREE & 12.20 & - & - \\
\hline TOTAL DISSOLVED SOLIDS & $01 / 26 / 82$ & 0001 & MG/L & 422.00 & - & - \\
\hline URAHIUH-234 & $01 / 26 / 82$ & 0001 & $\mathrm{PCI} / \mathrm{L}$ & 0.005 & - & - \\
\hline VAHADIUY & $01 / 26 / 82$ & 0001 & $\mathrm{KG} / \mathrm{L}$ & 0.01 & - & $\because$ \\
\hline ZINC & $01 / 26 / 82$ & 0001 & $M G / L$ & 0.10 & - & - \\
\hline
\end{tabular}

PARAMETER VALUE IMDICATOR (PVI): < - LESS THAH DETECTIOH LIMIT

SAMPLE ID COOES:

0001 - FILTERED SAMPLE (.45 MICROMS) 
GROUHDWATER QUALITY DATA BY LOCATION

SITE: AMBO1 AMBROSIA LAKE

LOCATION: 0609

NORTH COORDINATE: 55335.0 FT

EAST COORDINATE: 60670.0 FT

06/10/80 TO $12 / 04 / 92$

REPORT DATE: 08/04/94

FORMATION OF COMPLETION: ALLUVIUA (AL)

HYDRAULIC FLOH RELATIONSHIP: OH-SITE (O)

\begin{tabular}{|c|c|c|c|c|c|c|c|}
\hline PARAMETER MANE & LOG DATE & $\underset{\text { ID }}{\text { SAMPLE }}$ & $\begin{array}{l}\text { UHITS OF } \\
\text { MEASURE }\end{array}$ & PVI & $\begin{array}{l}\text { PARAMETER } \\
\text { VALUE FLAGS }\end{array}$ & $\begin{array}{l}\text { DETECTION } \\
\text { LIMIT }\end{array}$ & $\begin{array}{l}\text { PARAMETER } \\
\text { UHCERTAIHTY }\end{array}$ \\
\hline ALKALINITY & $\begin{array}{l}09 / 30 / 85 \\
09 / 30 / 85 \\
09 / 30 / 85 \\
09 / 30 / 85 \\
09 / 30 / 85 \\
05 / 13 / 86 \\
05 / 13 / 86 \\
05 / 13 / 86 \\
05 / 13 / 86 \\
05 / 13 / 86\end{array}$ & $\begin{array}{l}0001 \\
0002 \\
0003 \\
0004 \\
0005 \\
0001 \\
0002 \\
0003 \\
0004 \\
0005\end{array}$ & MG/L CACO3 & & $\begin{array}{l}462 . \\
462 . \\
462 . \\
462 . \\
462 . \\
463 . \\
463 . \\
463 . \\
463 . \\
463 .\end{array}$ & $\begin{array}{l}\text { - } \\
\dot{-} \\
\dot{-} \\
\dot{-} \\
- \\
-\end{array}$ & $\begin{array}{l}- \\
- \\
\dot{-} \\
\dot{-} \\
- \\
-\end{array}$ \\
\hline ALUMINUM & $\begin{array}{l}09 / 30 / 85 \\
09 / 30 / 85 \\
09 / 30 / 85 \\
09 / 30 / 85\end{array}$ & $\begin{array}{l}0002 \\
0003 \\
0004 \\
0005\end{array}$ & MG/L & & $\begin{array}{l}0.3 \\
0.3 \\
0.2 \\
0.3\end{array}$ & $\begin{array}{l}0.1 \\
0.1 \\
0.1 \\
0.1\end{array}$ & $\begin{array}{l}- \\
- \\
-\end{array}$ \\
\hline AMAONIUM & $\begin{array}{l}09 / 30 / 85 \\
09 / 30 / 85 \\
09 / 30 / 85 \\
09 / 30 / 85\end{array}$ & $\begin{array}{l}0002 \\
0003 \\
0004 \\
0005\end{array}$ & $M G / L$ & $\begin{array}{l}< \\
< \\
< \\
<\end{array}$ & $\begin{array}{l}0.1 \\
0.1 \\
0.1 \\
0.1\end{array}$ & $\begin{array}{l}0.1 \\
0.1 \\
0.1 \\
0.1\end{array}$ & $\begin{array}{l}- \\
- \\
-\end{array}$ \\
\hline AKT IMONY & $\begin{array}{l}09 / 30 / 85 \\
09 / 30 / 85 \\
09 / 30 / 85 \\
09 / 30 / 85\end{array}$ & $\begin{array}{l}0002 \\
0003 \\
0004 \\
0005\end{array}$ & MG/L & $\begin{array}{l}< \\
< \\
< \\
<\end{array}$ & $\begin{array}{l}0.003 \\
0.003 \\
0.003 \\
0.003\end{array}$ & $\begin{array}{l}0.003 \\
0.003 \\
0.003 \\
0.003\end{array}$ & $\begin{array}{l}- \\
- \\
-\end{array}$ \\
\hline ARSENIC & $\begin{array}{l}09 / 30 / 85 \\
09 / 30 / 85 \\
09 / 30 / 85 \\
09 / 30 / 85 \\
09 / 30 / 85 \\
05 / 13 / 86 \\
05 / 13 / 86 \\
05 / 13 / 86 \\
05 / 13 / 86 \\
05 / 13 / 86\end{array}$ & $\begin{array}{l}0001 \\
0002 \\
0003 \\
0004 \\
0005 \\
0001 \\
0002 \\
0003 \\
0004 \\
0005\end{array}$ & $\mathrm{MG} / \mathrm{L}$ & $\begin{array}{l}< \\
< \\
< \\
< \\
< \\
< \\
< \\
< \\
<\end{array}$ & $\begin{array}{l}0.01 \\
0.01 \\
0.01 \\
0.01 \\
0.01 \\
0.01 \\
0.01 \\
0.01 \\
0.01 \\
0.01\end{array}$ & $\begin{array}{l}0.01 \\
0.01 \\
0.01 \\
0.01 \\
0.01 \\
0.01 \\
0.01 \\
0.01 \\
0.01 \\
0.01\end{array}$ & $\begin{array}{l}- \\
- \\
- \\
- \\
- \\
- \\
-\end{array}$ \\
\hline BARIUM & $\begin{array}{l}09 / 30 / 85 \\
09 / 30 / 85 \\
09 / 30 / 85 \\
09 / 30 / 85\end{array}$ & $\begin{array}{l}0002 \\
0003 \\
0004 \\
0005\end{array}$ & $M G / L$ & & $\begin{array}{l}0.1 \\
0.1 \\
0.1 \\
0.1\end{array}$ & $\begin{array}{l}0.1 \\
0.1 \\
0.1 \\
0.1\end{array}$ & - \\
\hline BORON & $\begin{array}{l}09 / 30 / 85 \\
09 / 30 / 85 \\
09 / 30 / 85 \\
09 / 30 / 85 \\
05 / 13 / 86 \\
05 / 13 / 86 \\
05 / 13 / 86 \\
05 / 13 / 86 \\
05 / 13 / 86\end{array}$ & $\begin{array}{l}0002 \\
0003 \\
0004 \\
0005 \\
0001 \\
0002 \\
0003 \\
0004 \\
0005\end{array}$ & $\mathrm{HG} / \mathrm{L}$ & & $\begin{array}{l}0.4 \\
0.5 \\
0.6 \\
0.5 \\
1 . \\
1 . \\
1 . \\
1 . \\
1 .\end{array}$ & $\begin{array}{l}0.1 \\
0.1 \\
0.1 \\
0.1 \\
0.1 \\
0.1 \\
0.1 \\
0.1 \\
0.1\end{array}$ & $\begin{array}{l}- \\
- \\
- \\
- \\
- \\
- \\
-\end{array}$ \\
\hline CADMIUH & $\begin{array}{l}09 / 30 / 85 \\
09 / 30 / 85 \\
09 / 30 / 85 \\
09 / 30 / 85\end{array}$ & $\begin{array}{l}0002 \\
0003 \\
0004 \\
0005\end{array}$ & $M G / L$ & $\begin{array}{l}< \\
< \\
< \\
<\end{array}$ & $\begin{array}{l}0.001 \\
0.001 \\
0.001 \\
0.001\end{array}$ & $\begin{array}{l}0.001 \\
0.001 \\
0.001 \\
0.001\end{array}$ & $\begin{array}{l}- \\
- \\
-\end{array}$ \\
\hline
\end{tabular}

PARAMETER VALUE IRDICATOR (PVI): < - LESS THAN DETECTION LIMIT

SAMPLE ID COOES:

0001 - FILTERED SAMPLE (.45 MICRONS)

D002 - FILTERED REPLICATE SAMPLE (.45 MICRONS)

0003 - FILTERED REPLICATE SAMPLE (.45 MICRONS)

0004 - FILTERED REPLICATE SAMPLE (.45 MICRONS) 
GROUHDWATER OUALITY DATA BY LOCATION

SITE: AMBOI AMBROSIA LAKE

LOCATION: 0609

HORTH COORDIHATE: 55335.0 FT

EAST COORDIHATE: $\quad 60670.0$ FT

06/10/80 To $12 / 04 / 92$

REPORT DATE: 08/04/94

FORMATIOH OF COMPLETION: ALLUVILH (AL)

HYDRAULIC FLON RELATIONSHIP: ON-SITE (O)

\begin{tabular}{|c|c|c|c|c|c|c|c|}
\hline PARAMETER NAME & LOG DATE & $\underset{\text { SOMPLE }}{\text { SAMPL }}$ & $\begin{array}{l}\text { UNITS OF } \\
\text { MEASURE }\end{array}$ & PVI & $\begin{array}{l}\text { PARAMETER } \\
\text { VALUE FLAGS }\end{array}$ & $\begin{array}{l}\text { DETECTION } \\
\text { LIMIT }\end{array}$ & $\begin{array}{l}\text { PARAMETER } \\
\text { UNCERTAINTY }\end{array}$ \\
\hline CADHIUH & $\begin{array}{l}05 / 13 / 86 \\
05 / 13 / 86 \\
05 / 13 / 86 \\
05 / 13 / 86 \\
05 / 13 / 86\end{array}$ & $\begin{array}{l}0001 \\
0002 \\
0003 \\
0004 \\
0005\end{array}$ & MG/L & $\begin{array}{l}< \\
< \\
< \\
<\end{array}$ & $\begin{array}{l}0.001 \\
0.001 \\
0.001 \\
0.001 \\
0.001\end{array}$ & $\begin{array}{l}0.001 \\
0.001 \\
0.001 \\
0.001 \\
0.001\end{array}$ & $\begin{array}{l}- \\
- \\
-\end{array}$ \\
\hline CALCIUA & $\begin{array}{l}09 / 30 / 85 \\
09 / 30 / 85 \\
09 / 30 / 85 \\
09 / 30 / 85 \\
09 / 30 / 85 \\
05 / 13 / 86 \\
05 / 13 / 86 \\
05 / 13 / 86 \\
05 / 13 / 86 \\
05 / 13 / 86\end{array}$ & $\begin{array}{l}0001 \\
0002 \\
0003 \\
0004 \\
0005 \\
0001 \\
0002 \\
0003 \\
0004 \\
0005\end{array}$ & MG/L & & $\begin{array}{l}562 . \\
610 . \\
536 . \\
540 . \\
492 . \\
566 . \\
566 . \\
566 . \\
566 . \\
566 .\end{array}$ & $\begin{array}{l}0.01 \\
0.01 \\
0.01 \\
0.01 \\
0.01 \\
0.01 \\
0.01 \\
0.01 \\
0.01 \\
0.01\end{array}$ & $\begin{array}{l}- \\
- \\
- \\
- \\
- \\
-\end{array}$ \\
\hline CHLORIDE & $\begin{array}{l}09 / 30 / 85 \\
09 / 30 / 85 \\
09 / 30 / 85 \\
09 / 30 / 85 \\
09 / 30 / 85 \\
05 / 13 / 86 \\
05 / 13 / 86 \\
05 / 13 / 86 \\
05 / 13 / 86 \\
05 / 13 / 86\end{array}$ & $\begin{array}{l}0001 \\
0002 \\
0003 \\
0004 \\
0005 \\
0001 \\
0002 \\
0003 \\
0004 \\
0005\end{array}$ & $M G / L$ & & $\begin{array}{l}148 . \\
150 . \\
150 . \\
150 . \\
150 . \\
160 . \\
160 . \\
160 . \\
160 . \\
160 .\end{array}$ & $\begin{array}{l}1 . \\
1 . \\
1 . \\
1 . \\
1 . \\
1 . \\
1 . \\
1 . \\
1 .\end{array}$ & $\begin{array}{l}- \\
- \\
- \\
- \\
- \\
- \\
-\end{array}$ \\
\hline CHROMIUM & $\begin{array}{l}09 / 30 / 85 \\
09 / 30 / 85 \\
09 / 30 / 85 \\
09 / 30 / 85 \\
05 / 13 / 86 \\
05 / 13 / 86 \\
05 / 13 / 86 \\
05 / 13 / 86 \\
05 / 13 / 86\end{array}$ & $\begin{array}{l}0002 \\
0003 \\
0004 \\
0005 \\
0001 \\
0002 \\
0003 \\
0004 \\
0005\end{array}$ & MG/L & & $\begin{array}{l}0.04 \\
0.03 \\
0.05 \\
0.04 \\
0.2 \\
0.02 \\
0.02 \\
0.02 \\
0.02\end{array}$ & $\begin{array}{l}0.01 \\
0.01 \\
0.01 \\
0.01 \\
0.01 \\
0.01 \\
0.01 \\
0.01 \\
0.01\end{array}$ & $\begin{array}{l}- \\
- \\
- \\
- \\
- \\
-\end{array}$ \\
\hline COBALT & $\begin{array}{l}09 / 30 / 85 \\
09 / 30 / 85 \\
09 / 30 / 85 \\
09 / 30 / 85 \\
05 / 13 / 86 \\
05 / 13 / 86 \\
05 / 13 / 86 \\
05 / 13 / 86 \\
05 / 13 / 86\end{array}$ & $\begin{array}{l}0002 \\
0003 \\
0004 \\
0005 \\
0001 \\
0002 \\
0003 \\
0004 \\
0005\end{array}$ & $M G / L$ & & $\begin{array}{l}0.07 \\
0.06 \\
0.07 \\
0.09 \\
0.09 \\
0.09 \\
0.09 \\
0.09 \\
0.09\end{array}$ & $\begin{array}{l}0.05 \\
0.05 \\
0.05 \\
0.05 \\
0.05 \\
0.05 \\
0.05 \\
0.05 \\
0.05\end{array}$ & $\begin{array}{l}- \\
- \\
- \\
- \\
- \\
- \\
-\end{array}$ \\
\hline COPPER & $\begin{array}{l}09 / 30 / 85 \\
09 / 30 / 85 \\
09 / 30 / 85 \\
09 / 30 / 85\end{array}$ & $\begin{array}{l}0002 \\
0003 \\
0004 \\
0005\end{array}$ & MG/L & & $\begin{array}{l}0.03 \\
0.05 \\
0.04 \\
0.04\end{array}$ & $\begin{array}{l}0.02 \\
0.02 \\
0.02 \\
0.02\end{array}$ & : \\
\hline CYAKIDE & $\begin{array}{l}09 / 30 / 85 \\
09 / 30 / 85 \\
09 / 30 / 85\end{array}$ & $\begin{array}{l}0002 \\
0003 \\
0004\end{array}$ & $M G / L$ & $<$ & $\begin{array}{l}0.01 \\
0.01 \\
0.01\end{array}$ & $\begin{array}{l}0.01 \\
0.01 \\
0.01\end{array}$ & - \\
\hline
\end{tabular}

PARAMETER VALUE INDICATOR (PVI): < - LESS THAH DETECTION LIMIT SAMPLE ID CODES:

0001 - FILTERED SAMPLE (.45 MICRONS)

0002 - FILTERED REPLICATE SAMPLE (.45 MICROHS)

0003 - FILTERED REPLICATE SAMPLE (.45 MICRONS)

0004 - FILTERED REPLICATE SAMPLE (.45 MICROHS) 
GROUHDHATER QUALITY DATA BY LOCATION

SITE: AMBO1 AMBROSIA LAKE

LOCATION: 0609

NORTH COORDIMATE: 55335.0 FT

EAST COORDINATE: 60570.0 FT

06/10/80 TO 12/04/92

REPORT DATE: 08/04/94

FORMATION OF COMPLETION: ALLUVIUM (AL)

HYORAULIC FLON RELATIONSHIP: ON-SITE (O)

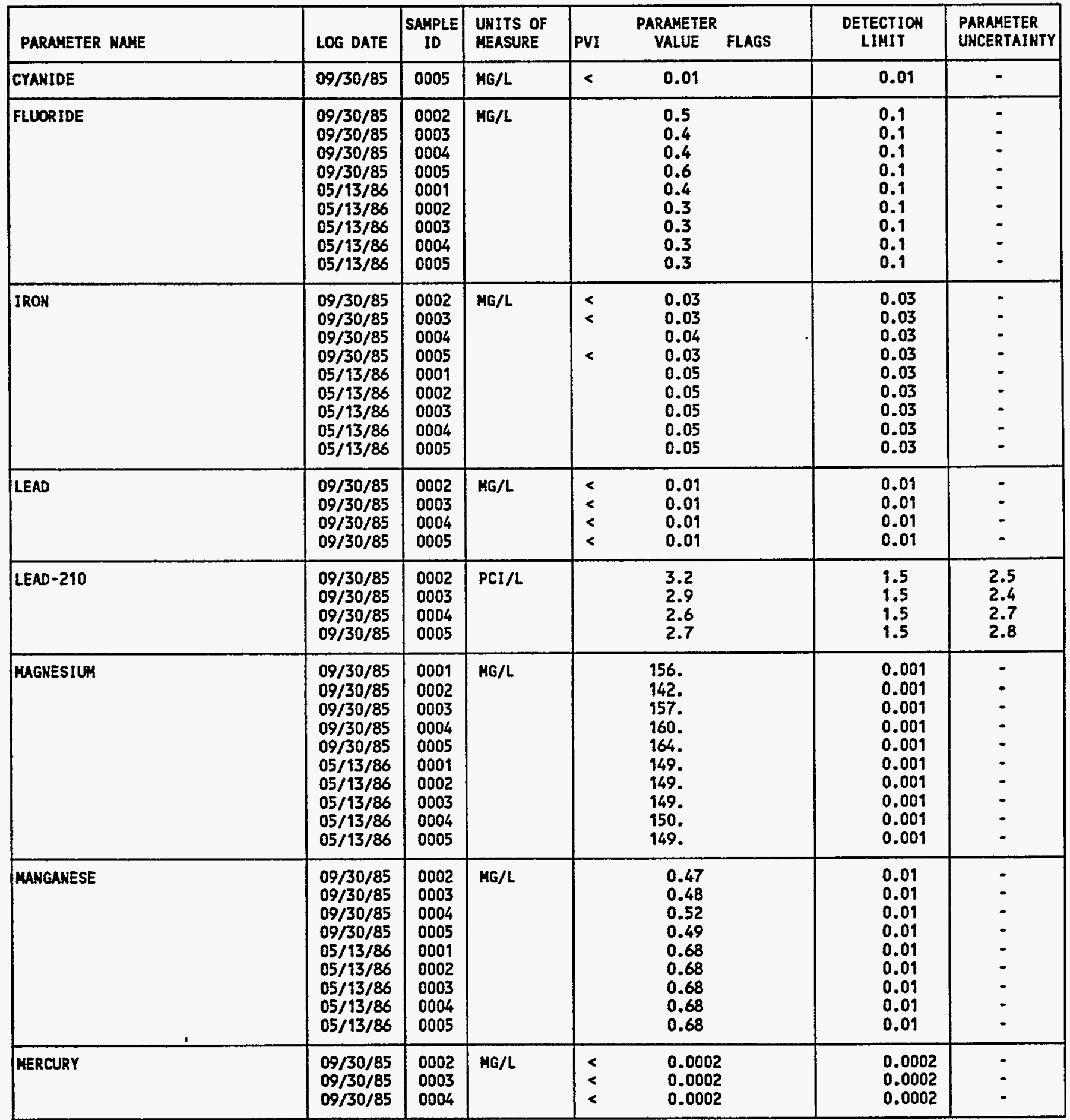

PARAMETER VALUE INDICATOR (PVI): < - LESS THAN DETECTION LIMIT

SAMPLE ID COOES:

0001 - FILTERED SAMPLE (.45 MICRONS)

0002 - FILTERED REPLICATE SAMPLE (.45 MICRONS)

0003 - FILTERED REPLICATE SAMPLE (.45 MICROHS)

0004 - FILTERED REPLICATE SAMPLE (.45 MICROHS) 
GROUNDHATER OUALITY DATA BY LOCATION

SITE: AMBO1 AMBROSIA LAKE

LOCATIOH: 0609

MORTH COORDIHATE: 55335.0 FT

EAST COORDIHATE: $\quad 60670.0$ FT

06/10/80 TO 12/04/92

REPORT DATE: 08/04/94

FORMATIOH OF COMPLETION: ALLUVILH (AL)

HYDRAULIC FLOH RELATIONSHIP: OH-SITE (O)

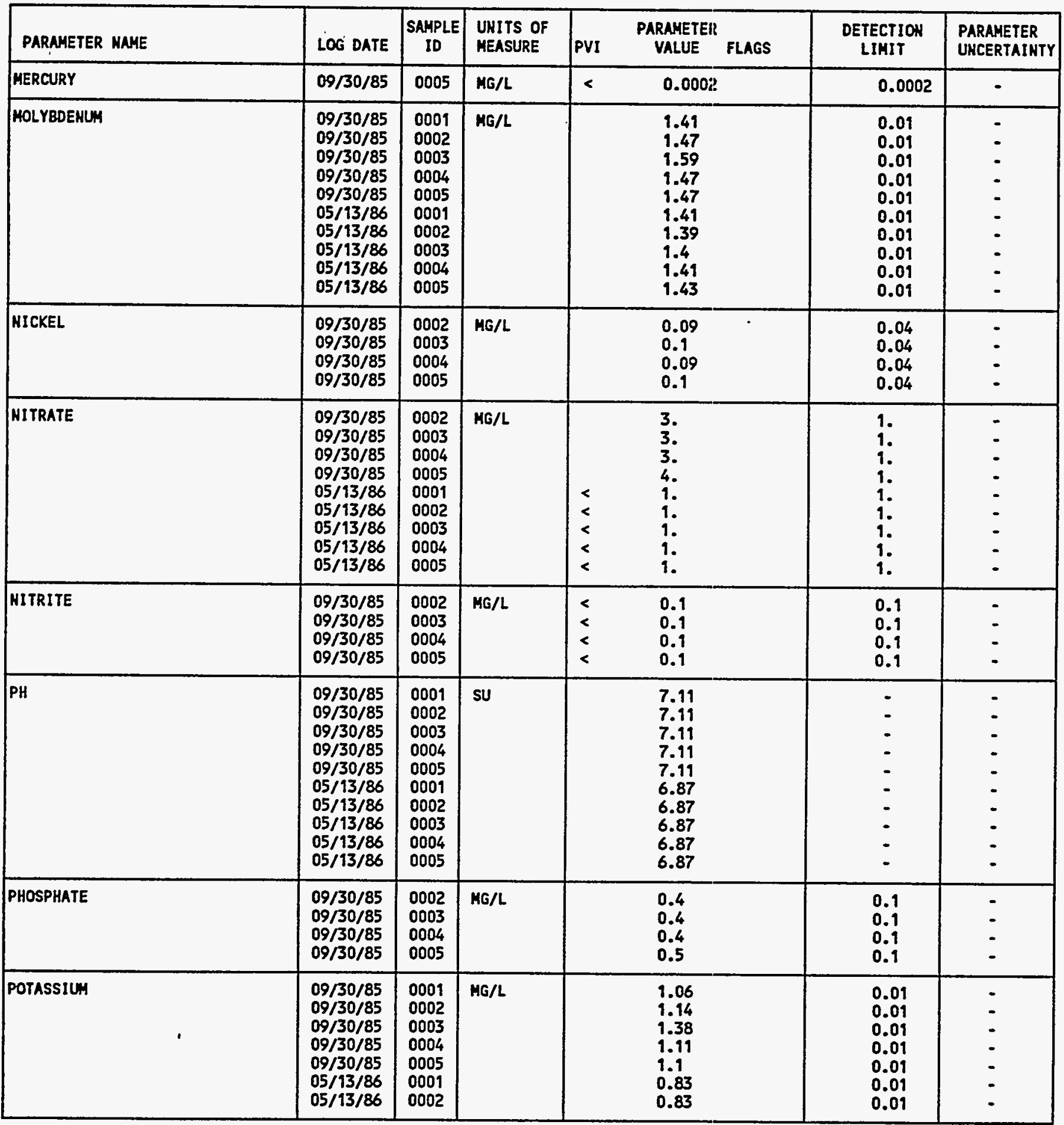

PARAMETER VALUE IMDICATOR (PVI): < - LESS THAN DETECTIOH LIMIT SAMPLE ID LOOES:

0001 - FILTERED SAMPLE (.45 MICROHS)

0002 - FILTERED REPLICATE SAMPLE (.45 MICRONS)

0003 - FILTERED REPLICATE SAMPLE (.45 MICROHS)

0004 - FILTERED REPLICATE SAMPLE (.45 MICRONS) 
GROUNDWATER OUALITY DATA BY LOCATIOH

SITE: AMBOI AMBROSIA LAKE

LOCATION: 0609

MORTK COORDINATE: 55335.0 FT

EAST COORDINATE: 60670.0 FT

06/10/80 TO $12 / 04 / 92$

REPORT DATE: 08/04/94

FORMATION OF COMPLETION: ALLUVIUM (AL)

HYDRAULIC FLOW RELATIOHSHIP: OW-SITE (O)

\begin{tabular}{|c|c|c|c|c|c|c|c|}
\hline PARAMETER NAME & LOG DATE & $\begin{array}{c}\text { SAMPLE } \\
\text { ID }\end{array}$ & $\begin{array}{l}\text { UNITS OF } \\
\text { MEASURE }\end{array}$ & PVI & $\begin{array}{l}\text { PARAMETER } \\
\text { VALUE FLAGS }\end{array}$ & $\begin{array}{l}\text { DETECTIOH } \\
\text { LIMIT }\end{array}$ & $\begin{array}{l}\text { PARAMETER } \\
\text { UNCERTAIHTY }\end{array}$ \\
\hline POTASSIUA & $\begin{array}{l}05 / 13 / 86 \\
05 / 13 / 86 \\
05 / 13 / 86\end{array}$ & $\begin{array}{l}0003 \\
0004 \\
0005\end{array}$ & $M G / L$ & & $\begin{array}{l}0.83 \\
0.83 \\
0.83\end{array}$ & $\begin{array}{l}0.01 \\
0.01 \\
0.01\end{array}$ & : \\
\hline RADIUM-226 & $\begin{array}{l}09 / 30 / 85 \\
09 / 30 / 85 \\
09 / 30 / 85 \\
09 / 30 / 85\end{array}$ & $\begin{array}{l}0002 \\
0003 \\
0004 \\
0005\end{array}$ & PCI/L & & $\begin{array}{r}410 . \\
11 . \\
8.3 \\
7.9\end{array}$ & $\begin{array}{l}1 . \\
1 . \\
1 .\end{array}$ & $\begin{array}{l}20 . \\
3 . \\
2.9 \\
3.2\end{array}$ \\
\hline RADIUA-226 + RADIUH-228 & $\begin{array}{l}09 / 30 / 85 \\
09 / 30 / 85 \\
09 / 30 / 85 \\
09 / 30 / 85\end{array}$ & $\begin{array}{l}0002 \\
0003 \\
0004 \\
0005\end{array}$ & $\mathrm{PCI} / \mathrm{L}$ & & $\begin{array}{r}410.00 \\
11.20 \\
8.60 \\
8.10\end{array}$ & $\begin{array}{l}- \\
- \\
-\end{array}$ & : \\
\hline RADIUA-228 & $\begin{array}{l}09 / 30 / 85 \\
09 / 30 / 85 \\
09 / 30 / 85 \\
09 / 30 / 85\end{array}$ & $\begin{array}{l}0002 \\
0003 \\
0004 \\
0005\end{array}$ & $\mathrm{PCI} / \mathrm{L}$ & & $\begin{array}{l}0 . \\
0.2 \\
0.3 \\
0.2\end{array}$ & $\begin{array}{l}1 . \\
1 . \\
1 .\end{array}$ & $\begin{array}{l}4 . \\
4.5 \\
5 . \\
3 .\end{array}$ \\
\hline SILICA - SIO2 & $\begin{array}{l}09 / 30 / 85 \\
09 / 30 / 85 \\
09 / 30 / 85 \\
09 / 30 / 85\end{array}$ & $\begin{array}{l}0002 \\
0003 \\
0004 \\
0005\end{array}$ & MG/L & & $\begin{array}{l}11 . \\
11 . \\
10 . \\
12 .\end{array}$ & $\begin{array}{l}2 . \\
2 . \\
2 . \\
2 .\end{array}$ & : \\
\hline SPECIFIC CONDUCTAKCE, & $\begin{array}{l}09 / 30 / 85 \\
09 / 30 / 85 \\
09 / 30 / 85 \\
09 / 30 / 85 \\
09 / 30 / 85\end{array}$ & $\begin{array}{l}0001 \\
0002 \\
0003 \\
0004 \\
0005\end{array}$ & UHKO/CM & & $\begin{array}{l}3200 . \\
3200 . \\
3200 \\
3200 . \\
3200 .\end{array}$ & $\begin{array}{l}- \\
- \\
- \\
-\end{array}$ & $\begin{array}{l}- \\
- \\
-\end{array}$ \\
\hline
\end{tabular}

PARAMETER VALUE IHDICATOR (PVI): < - LESS THAN DETECTIOH LIMIT SAMPLE ID CODES:

0001 - FILTERED SAMPLE (.45 HICROWS)

0002 - FILTERED REPLICATE SAMPLE (.45 MICRONS)

0003 - FILTERED REPLICATE SAMPLE (.45 MICRONS)

0004 - FILTERED REPLICATE SAMPLE (.45 MICRONS) 
GROUNDHATER OUALITY DATA BY LOCATIOH

SITE: AMBOI AHBROSIA LAKE

LOCATION: 0609

NORTH COORDINATE: 55335.0 FT

EAST COORDINATE: 60670.0 FT

$06 / 10 / 80$ TO $12 / 04 / 92$

REPORT DATE: 08/04/94

FORMATION OF COMPLETION: ALLUVIUH (AL)

HYDRAULIC FLON RELATIOHSHIP: OH-SITE (O)

\begin{tabular}{|c|c|c|c|c|c|c|c|}
\hline PARAMETER MAME & LOG DATE & $\begin{array}{c}\text { SAMPLE } \\
\text { ID }\end{array}$ & $\begin{array}{l}\text { UNITS OF } \\
\text { MEASURE }\end{array}$ & PVI & $\begin{array}{l}\text { ParaMETER } \\
\text { VALUE FLAGS }\end{array}$ & $\begin{array}{l}\text { OETECTION } \\
\text { LIMIT }\end{array}$ & $\begin{array}{l}\text { PARAMETER } \\
\text { UNCERTAINTY }\end{array}$ \\
\hline SPECIFIC CONDUCTANCE & $\begin{array}{l}05 / 13 / 86 \\
05 / 13 / 86 \\
05 / 13 / 86 \\
05 / 13 / 86 \\
05 / 13 / 86\end{array}$ & $\begin{array}{l}0001 \\
0002 \\
0003 \\
0004 \\
0005\end{array}$ & ШАHO/CH & & $\begin{array}{l}3450 . \\
3450 . \\
3450 . \\
3450 \\
3450 .\end{array}$ & $\begin{array}{l}- \\
- \\
- \\
-\end{array}$ & $\begin{array}{l}- \\
- \\
- \\
-\end{array}$ \\
\hline STRONTIUA & $\begin{array}{l}09 / 30 / 85 \\
09 / 30 / 85 \\
09 / 30 / 85 \\
09 / 30 / 85 \\
05 / 13 / 86 \\
05 / 13 / 86 \\
05 / 13 / 86 \\
05 / 13 / 86 \\
05 / 13 / 86\end{array}$ & $\begin{array}{l}0002 \\
0003 \\
0004 \\
0005 \\
0001 \\
0002 \\
0003 \\
0004 \\
0005\end{array}$ & MG/L & & $\begin{array}{l}9.8 \\
10 . \\
12 . \\
12 . \\
0.8 \\
0.8 \\
0.8 \\
0.8 \\
0.8\end{array}$ & $\begin{array}{l}0.1 \\
0.1 \\
0.1 \\
0.1 \\
0.1 \\
0.1 \\
0.1 \\
0.1 \\
0.1\end{array}$ & $\begin{array}{l}- \\
- \\
- \\
- \\
-\end{array}$ \\
\hline SULFATE & $\begin{array}{l}09 / 30 / 85 \\
09 / 30 / 85 \\
09 / 30 / 85 \\
09 / 30 / 85 \\
09 / 30 / 85 \\
05 / 13 / 86 \\
05 / 13 / 86 \\
05 / 13 / 86 \\
05 / 13 / 86 \\
05 / 13 / 86\end{array}$ & $\begin{array}{l}0001 \\
0002 \\
0003 \\
0004 \\
0005 \\
0001 \\
0002 \\
0003 \\
0004 \\
0005\end{array}$ & $M G / L$ & & $\begin{array}{l}2420 . \\
2400 . \\
2400 . \\
2400 . \\
2390 . \\
2540 . \\
2530 . \\
2520 . \\
2540 . \\
2530 .\end{array}$ & $\begin{array}{l}0.1 \\
0.1 \\
0.1 \\
0.1 \\
0.1 \\
0.1 \\
0.1 \\
0.1 \\
0.1 \\
0.1\end{array}$ & $\begin{array}{l}- \\
- \\
- \\
- \\
- \\
-\end{array}$ \\
\hline SULFIDE & $\begin{array}{l}09 / 30 / 85 \\
09 / 30 / 85 \\
09 / 30 / 85 \\
09 / 30 / 85\end{array}$ & $\begin{array}{l}0002 \\
0003 \\
0004 \\
0005\end{array}$ & $M G / L$ & $\begin{array}{l}< \\
< \\
< \\
<\end{array}$ & $\begin{array}{l}0.1 \\
0.1 \\
0.1 \\
0.1\end{array}$ & $\begin{array}{l}0.1 \\
0.1 \\
0.1 \\
0.1\end{array}$ & $\begin{array}{l}- \\
- \\
-\end{array}$ \\
\hline TEAPERATURE & $\begin{array}{l}09 / 30 / 85 \\
09 / 30 / 85 \\
09 / 30 / 85 \\
09 / 30 / 85 \\
09 / 30 / 85 \\
05 / 13 / 86 \\
05 / 13 / 86 \\
05 / 13 / 86 \\
05 / 13 / 86 \\
05 / 13 / 86\end{array}$ & $\begin{array}{l}0001 \\
0002 \\
0003 \\
0004 \\
0005 \\
0001 \\
0002 \\
0003 \\
0004 \\
0005\end{array}$ & C - DEGREE & & $\begin{array}{l}12 . \\
12 . \\
12 . \\
12 . \\
12 . \\
16 . \\
16 . \\
16 . \\
16 . \\
16 .\end{array}$ & $\begin{array}{l}- \\
- \\
- \\
- \\
- \\
-\end{array}$ & $\begin{array}{l}- \\
: \\
- \\
: \\
: \\
-\end{array}$ \\
\hline THORIUA-230 & $\begin{array}{l}09 / 30 / 85 \\
09 / 30 / 85 \\
09 / 30 / 85 \\
09 / 30 / 85\end{array}$ & $\begin{array}{l}0002 \\
0003 \\
0004 \\
0005\end{array}$ & $\mathrm{PCI} / \mathrm{L}$ & & $\begin{array}{l}1.4 \\
0.9 \\
0.9 \\
0.5\end{array}$ & $\begin{array}{l}1 . \\
1 . \\
1 .\end{array}$ & $\begin{array}{l}1.1 \\
0.9 \\
0.9 \\
0: 8\end{array}$ \\
\hline TIH & $\begin{array}{l}09 / 30 / 85 \\
09 / 30 / 85 \\
09 / 30 / 85 \\
09 / 30 / 85\end{array}$ & $\begin{array}{l}0002 \\
0003 \\
0004 \\
0005\end{array}$ & $M G / L$ & $\begin{array}{l}< \\
< \\
< \\
<\end{array}$ & $\begin{array}{l}0.005 \\
0.005 \\
0.005 \\
0.005\end{array}$ & $\begin{array}{l}0.005 \\
0.005 \\
0.005 \\
0.005\end{array}$ & $\begin{array}{l}- \\
- \\
-\end{array}$ \\
\hline TOTAL DISSOLVED SOLIDS & $\begin{array}{l}09 / 30 / 85 \\
09 / 30 / 85 \\
09 / 30 / 85\end{array}$ & $\begin{array}{l}0001 \\
0002 \\
0003\end{array}$ & MG/L & & $\begin{array}{l}4310 . \\
4370 . \\
4330 .\end{array}$ & $\begin{array}{l}10 . \\
10 . \\
10 .\end{array}$ & - \\
\hline
\end{tabular}

PARAMETER VALUE INOICATOR (PVI): < - LESS THAN DETECTION LIMIT

SAMPLE ID COOES:

0001 - FILTERED SAMPLE (.45 MICRONS)

0002 - FILTERED REPLICATE SAMPLE (.45 MICROHS)

0003 - FILTERED REPLICATE SAMPLE (.45 MICROHS)

0004 - FILTERED REPLICATE SAMPLE (.45 MICRONS) 
GROUHDWATER OUALITY DATA BY LOCATION

SITE: AMBOI AMBROSIA LAKE

LOCATION: 0609

NORTH COORDINATE: $\quad 55335.0$ FT

EAST COORDINATE: 60670.0 FT

06/10/80 TO $12 / 04 / 92$

REPORT DATE: $08 / 04 / 94$

FORHATION OF COMPLETIOH: ALLUVIUM (AL)

HYDRAULIC FLON RELATIONSHIP: ON-SITE (O)

\begin{tabular}{|c|c|c|c|c|c|c|c|}
\hline PARAMETER MAME & LOG DATE & $\underset{\text { SDAMPLE }}{\operatorname{SD}}$ & $\begin{array}{l}\text { UNITS OF } \\
\text { MEASURE }\end{array}$ & PVI & $\begin{array}{l}\text { PARAMETER } \\
\text { VALUE FLAGS }\end{array}$ & $\begin{array}{l}\text { DETECTIOH } \\
\text { LIMIT }\end{array}$ & $\begin{array}{l}\text { PARAMETER } \\
\text { UNCERTAIHTY }\end{array}$ \\
\hline TOTAL DISSOLVED SOLIOS & $\begin{array}{l}09 / 30 / 85 \\
09 / 30 / 85 \\
05 / 13 / 86 \\
05 / 13 / 86 \\
05 / 13 / 86 \\
05 / 13 / 86 \\
05 / 13 / 86\end{array}$ & $\begin{array}{l}0004 \\
0005 \\
0001 \\
0002 \\
0003 \\
0004 \\
0005\end{array}$ & MG/L & & $\begin{array}{l}4350 . \\
4360 . \\
4360 \\
4390 \\
4540 \\
4370 \\
4390\end{array}$ & $\begin{array}{l}10 . \\
10 . \\
10 . \\
10 . \\
10 . \\
10 . \\
10 .\end{array}$ & $\begin{array}{l}- \\
- \\
- \\
-\end{array}$ \\
\hline URAKIUM & $\begin{array}{l}09 / 30 / 85 \\
09 / 30 / 85 \\
09 / 30 / 85 \\
09 / 30 / 85 \\
09 / 30 / 85 \\
05 / 13 / 86 \\
05 / 13 / 86 \\
05 / 13 / 86 \\
05 / 13 / 86 \\
05 / 13 / 86\end{array}$ & $\begin{array}{l}0001 \\
0002 \\
0003 \\
0004 \\
0005 \\
0001 \\
0002 \\
0003 \\
0004 \\
0005\end{array}$ & MG/L & & $\begin{array}{l}0.0807 \\
0.102 \\
0.0991 \\
0.0848 \\
0.0885 \\
0.0537 \\
0.0534 \\
0.0517 \\
0.05 \\
0.0506\end{array}$ & $\begin{array}{l}0.003 \\
0.003 \\
0.003 \\
0.003 \\
0.003 \\
0.003 \\
0.003 \\
0.003 \\
0.003 \\
0.003\end{array}$ & $\begin{array}{l}: \\
: \\
: \\
: \\
- \\
-\end{array}$ \\
\hline ZINC & $\begin{array}{l}09 / 30 / 85 \\
09 / 30 / 85 \\
09 / 30 / 85 \\
09 / 30 / 85\end{array}$ & $\begin{array}{l}0002 \\
0003 \\
0004 \\
0005\end{array}$ & $M G / L$ & & $\begin{array}{l}0.069 \\
0.063 \\
0.075 \\
0.066\end{array}$ & $\begin{array}{l}0.005 \\
0.005 \\
0.005 \\
0.005\end{array}$ & $\begin{array}{l}- \\
- \\
-\end{array}$ \\
\hline
\end{tabular}

PARAMETER VALUE IMDICATOR (PVI): < - LESS THAN DETECTION LIMIT

SAMPLE ID CODES:

0001 - FILTERED SAMPLE (.45 MICROHS)

0002 - FILTERED REPLICATE SAMPLE (.45 MICRONS)

0003 - FILTERED REPLICATE SAMPLE (.45 MICRONS)

0004 - FILTERED REPLICATE SAMPLE (.45 MICRONS) 
GROUNDHATER QUALITY DATA BY LOCATION

SITE: AHBOI AMBROSIA LAKE

LOCATION: 0619

NORTH COORDIHATE: 50910.0 FT

EAST COORDINATE: 64040.0 FT

06/10/80 TO $12 / 04 / 92$

REPORT DATE: 08/04/94

FORHATION OF COHPLETIOH: ALLUVIUM (AL)

HYORAULIC FLON RELATIONSHIP: CROSS GRADIENT (C)

\begin{tabular}{|c|c|c|c|c|c|c|c|}
\hline PARAMETER NAME & LOG DATE & $\begin{array}{c}\text { SAMPLE } \\
\text { ID }\end{array}$ & $\begin{array}{l}\text { UNITS OF } \\
\text { MEASURE }\end{array}$ & PVI & $\begin{array}{l}\text { PARAMETEIR } \\
\text { VALUE FLAGS }\end{array}$ & $\begin{array}{l}\text { DETECTION } \\
\text { LIMIT }\end{array}$ & $\begin{array}{l}\text { PARAMETER } \\
\text { UNCERTAINTY }\end{array}$ \\
\hline ALKALINITY & $10 / 02 / 85$ & 0001 & $\mathrm{MG} / \mathrm{L} \mathrm{CACO} 3$ & & 597. & - & - \\
\hline ARSENIC & $10 / 02 / 85$ & 0001 & $M G / L$ & $<$ & 0.01 & 0.01 & - \\
\hline CALCIUM & $10 / 02 / 85$ & 0001 & MG/L & & 380. & 0.01 & - \\
\hline CHLORIDE & $10 / 02 / 85$ & 0001 & HG/L & & 165. & 1. & - \\
\hline MAGHESIUH & $10 / 02 / 85$ & 0001 & MG/L & & 110. & 0.001 & - \\
\hline HOLYBDERUM & $10 / 02 / 85$ & 0001 & $M G / L$ & & 0.22 & 0.01 & - \\
\hline PH & $10 / 02 / 85$ & 0001 & SU & & 7.01 & - & - \\
\hline POTASSIUH & $10 / 02 / 85$ & 0001 & $\mathrm{HG} / \mathrm{L}$ & & 5.38 & 0.01 & - \\
\hline SELENIUY & $10 / 02 / 85$ & 0001 & $M G / L$ & & 0.033 & 0.005 & - \\
\hline SCOIUM & $10 / 02 / 85$ & 0001 & MG/L & & 555. & 0.002 & - \\
\hline SPECIFIC CONDUCTANCE & $10 / 02 / 85$ & 0001 & UMHO/CM & & 2600. & - & - \\
\hline SULFATE & $10 / 02 / 85$ & 0001 & $M G / L$ & & 1770. & 0.1 & - \\
\hline TEHPERATURE & $10 / 02 / 85$ & 0001 & C - DEGREE & & 12. & - & - \\
\hline TOTAL DISSOLVED SOLIDS & $10 / 02 / 85$ & 0001 & $M G / L$ & & 3200. & 10. & - \\
\hline URANIUM & $10 / 02 / 85$ & 0001 & $M G / L$ & & 0.95 & 0.003 & - \\
\hline
\end{tabular}

PARAMETER VALUE IMDICATOR (PVI): < - LESS THAN DETECTION LIMIT

SAMPLE ID COOES

OOO1 - FILTERED SAMPLE (.45 MICRONS) 
GROUNDWATER QUALITY DATA BY LOCATION

SITE: AMBOI AMBROSIA LAKE

LOCATIOH: 0620

NORTH COOROINATE: 52040.0 FT

EAST COORDINATE: $\quad 60740.0$ FT

06/10/80 To $12 / 04 / 92$

REPORT DATE: $08 / 04 / 94$

FORHATION OF COHPLETION: ALLUVIUM (AL)

HYDRAULIC FLOW RELATIONSHIP: CROSS GRADIENT (C)

\begin{tabular}{|c|c|c|c|c|c|c|c|c|}
\hline PARAMETER MAME & LOG DATE & $\underset{\text { ID }}{\text { SAMPLE }}$ & $\begin{array}{l}\text { UNITS OF } \\
\text { MEASURE }\end{array}$ & PVI & $\begin{array}{l}\text { PARAMETER } \\
\text { VALUE }\end{array}$ & FLAGS & $\begin{array}{l}\text { DETECTION } \\
\text { LIHIT }\end{array}$ & $\begin{array}{l}\text { PARAMETER } \\
\text { UHCERTAINTY }\end{array}$ \\
\hline ALKALINITY & $\begin{array}{l}10 / 02 / 85 \\
01 / 09 / 87 \\
07 / 15 / 88 \\
01 / 12 / 89 \\
09 / 14 / 89 \\
03 / 31 / 90 \\
01 / 09 / 91\end{array}$ & $\begin{array}{l}0001 \\
0001 \\
0001 \\
0001 \\
0001 \\
0001 \\
0001\end{array}$ & $\mathrm{HG} / \mathrm{L} \mathrm{CACO}$ & & $\begin{array}{l}294 . \\
294 . \\
299 . \\
278 . \\
274 . \\
299 . \\
277 .\end{array}$ & & $\begin{array}{l}- \\
- \\
\dot{-} \\
-\end{array}$ & $\begin{array}{l}: \\
: \\
: \\
-\end{array}$ \\
\hline ALUMINUN & $\begin{array}{l}10 / 02 / 85 \\
07 / 15 / 88 \\
01 / 12 / 89 \\
09 / 14 / 89 \\
03 / 31 / 90 \\
01 / 09 / 91\end{array}$ & $\begin{array}{l}0001 \\
0001 \\
0001 \\
0001 \\
0001 \\
0001\end{array}$ & MG/L & $\begin{array}{l}< \\
< \\
<\end{array}$ & $\begin{array}{l}0.4 \\
0.3 \\
0.05 \\
0.1 \\
0.1 \\
0.05\end{array}$ & J & $\begin{array}{l}0.1 \\
0.1 \\
0.1 \\
0.1 \\
0.1 \\
0.05\end{array}$ & $\begin{array}{l}: \\
: \\
-\end{array}$ \\
\hline AMHOHIUM & $\begin{array}{l}10 / 02 / 85 \\
07 / 15 / 88 \\
01 / 12 / 89 \\
09 / 14 / 89 \\
03 / 31 / 90 \\
01 / 09 / 91\end{array}$ & $\begin{array}{l}0001 \\
0001 \\
0001 \\
0001 \\
0001 \\
0001\end{array}$ & MG/L & $\begin{array}{l}< \\
< \\
<\end{array}$ & $\begin{array}{l}0.1 \\
0.1 \\
0.10 \\
0.1 \\
0.1 \\
0.08\end{array}$ & & $\begin{array}{l}0.1 \\
0.1 \\
0.1 \\
0.1 \\
0.1 \\
0.01\end{array}$ & $\begin{array}{l}- \\
: \\
-\end{array}$ \\
\hline ANTIMONY & $\begin{array}{l}10 / 02 / 85 \\
07 / 15 / 88 \\
01 / 12 / 89 \\
09 / 14 / 89 \\
03 / 31 / 90 \\
01 / 09 / 91\end{array}$ & $\begin{array}{l}0001 \\
0001 \\
0001 \\
0001 \\
0001 \\
0001\end{array}$ & $H G / L$ & $\begin{array}{l}< \\
< \\
<\end{array}$ & $\begin{array}{l}0.003 \\
0.013 \\
0.003 \\
0.003 \\
0.021 \\
0.003\end{array}$ & & $\begin{array}{l}0.003 \\
0.003 \\
0.003 \\
0.003 \\
0.003 \\
0.003\end{array}$ & $\begin{array}{l}- \\
: \\
-\end{array}$ \\
\hline ARSEHIC & $\begin{array}{l}10 / 02 / 85 \\
01 / 09 / 87 \\
07 / 15 / 88 \\
01 / 12 / 89 \\
09 / 14 / 89 \\
03 / 31 / 90 \\
01 / 09 / 91\end{array}$ & $\begin{array}{l}0001 \\
0001 \\
0001 \\
0001 \\
0001 \\
0001 \\
0001\end{array}$ & $M G / L$ & $\begin{array}{l}< \\
< \\
< \\
<\end{array}$ & $\begin{array}{l}0.01 \\
0.003 \\
0.01 \\
0.001 \\
0.01 \\
0.01 \\
0.03\end{array}$ & $\begin{array}{l}J \\
J \\
I\end{array}$ & $\begin{array}{l}0.01 \\
0.01 \\
0.01 \\
0.01 \\
0.01 \\
0.01 \\
0.03\end{array}$ & $\begin{array}{l}- \\
- \\
- \\
- \\
-\end{array}$ \\
\hline BARIUM & $\begin{array}{l}10 / 02 / 85 \\
07 / 15 / 88 \\
01 / 12 / 89 \\
09 / 14 / 89 \\
03 / 31 / 90 \\
01 / 09 / 91\end{array}$ & $\begin{array}{l}0001 \\
0001 \\
0001 \\
0001 \\
0001 \\
0001\end{array}$ & MG/L & $\begin{array}{l}< \\
< \\
< \\
<\end{array}$ & $\begin{array}{l}0.1 \\
0.1 \\
0.01 \\
0.1 \\
0.1 \\
0.01\end{array}$ & $J$ & $\begin{array}{l}0.1 \\
0.1 \\
0.1 \\
0.1 \\
0.1 \\
0.01\end{array}$ & $\begin{array}{l}- \\
- \\
- \\
-\end{array}$ \\
\hline BERYLLIUY & $\begin{array}{l}01 / 12 / 89 \\
09 / 14 / 89 \\
03 / 31 / 90 \\
01 / 09 / 91\end{array}$ & $\begin{array}{l}0001 \\
0001 \\
0001 \\
0001\end{array}$ & $M G / L$ & $\begin{array}{l}< \\
< \\
< \\
<\end{array}$ & $\begin{array}{l}0.005 \\
0.01 \\
0.01 \\
0.005\end{array}$ & & $\begin{array}{l}0.005 \\
0.09 \\
0.01 \\
0.005\end{array}$ & $\begin{array}{l}- \\
-\end{array}$ \\
\hline BORON & $\begin{array}{l}10 / 02 / 85 \\
01 / 09 / 87 \\
07 / 15 / 88 \\
01 / 12 / 89 \\
09 / 14 / 89 \\
03 / 31 / 90 \\
01 / 09 / 91\end{array}$ & $\begin{array}{l}0001 \\
0001 \\
0001 \\
0001 \\
0001 \\
0001 \\
0001\end{array}$ & $M G / L$ & & $\begin{array}{l}0.4 \\
0.35 \\
0.3 \\
0.37 \\
0.4 \\
0.3 \\
0.35\end{array}$ & & $\begin{array}{l}0.1 \\
0.1 \\
0.1 \\
0.1 \\
0.1 \\
0.1 \\
0.05\end{array}$ & $\begin{array}{l}- \\
- \\
- \\
- \\
-\end{array}$ \\
\hline
\end{tabular}

PARAMETER YALUE IMDICATOR (PVI): < - LESS THAN DETECTIOM LIMIT

SAHPLE ID COOES:

0001 - FILTERED SAMPLE (.45 MICRONS)

OTHER PARAMETER VALUE FLAGS:

1 - INCREASED DETECTION LIMIT DUE TO REQUIRED DILUTIOH

- estimated value 
GROUNDWATER QUALITY DATA BY LOCATION

SITE: AMBOI AMBROSIA LAKE

LOCATIOH: 0620

MORTH COORDINATE: $\quad 52040.0$ FT

EAST COORDIHATE: $\quad 60740.0$ FT

$06 / 10 / 80$ TO $12 / 04 / 92$

REPORT DATE: 08/04/94

FORMATIOH OF COAPLETIOH: ALLUVIUM (AL)

HYDRAULIC FLOW RELATIONSHIP: CROSS GRADIEHT (C)

\begin{tabular}{|c|c|c|c|c|c|c|c|c|}
\hline PARAMETER NAME & LOG DATE & $\begin{array}{c}\text { SAMPLE } \\
\text { ID }\end{array}$ & $\begin{array}{l}\text { UMITS OF } \\
\text { MEASURE }\end{array}$ & PVI & $\begin{array}{l}\text { PARAMETER } \\
\text { VALUE }\end{array}$ & FLAGS & $\begin{array}{l}\text { DETECTIOH } \\
\text { LIMIT }\end{array}$ & $\begin{array}{l}\text { PARAMETER } \\
\text { UNCERTAINTY }\end{array}$ \\
\hline BROAIDE & $\begin{array}{l}07 / 15 / 88 \\
01 / 12 / 89 \\
09 / 14 / 89 \\
03 / 31 / 90 \\
01 / 09 / 91\end{array}$ & $\begin{array}{l}0001 \\
0001 \\
0001 \\
0001 \\
0001\end{array}$ & MG/L & & $\begin{array}{l}0.1 \\
0.42 \\
0.12 \\
0.2 \\
0.4\end{array}$ & & $\begin{array}{l}0.1 \\
0.01 \\
0.1 \\
0.1 \\
0.1\end{array}$ & $\begin{array}{l}- \\
- \\
-\end{array}$ \\
\hline CAOMIUA & $\begin{array}{l}10 / 02 / 85 \\
07 / 15 / 88 \\
01 / 12 / 89 \\
09 / 14 / 89 \\
03 / 31 / 90 \\
01 / 09 / 91\end{array}$ & $\begin{array}{l}0001 \\
0001 \\
0001 \\
0001 \\
0001 \\
0001\end{array}$ & MG/L & $\begin{array}{l}< \\
< \\
< \\
< \\
<\end{array}$ & $\begin{array}{l}0.001 \\
0.001 \\
0.0001 \\
0.001 \\
0.001 \\
0.001\end{array}$ & $\mathbf{J}$ & $\begin{array}{l}0.001 \\
0.001 \\
0.001 \\
0.001 \\
0.001 \\
0.001\end{array}$ & $\begin{array}{l}- \\
- \\
- \\
-\end{array}$ \\
\hline CALCIUN & $\begin{array}{l}10 / 02 / 85 \\
01 / 09 / 87 \\
07 / 15 / 88 \\
01 / 12 / 89 \\
09 / 14 / 89 \\
03 / 31 / 90 \\
01 / 09 / 91\end{array}$ & $\begin{array}{l}0001 \\
0001 \\
0001 \\
0001 \\
0001 \\
0001 \\
0001\end{array}$ & MG/L & & $\begin{array}{l}533 . \\
475 . \\
482 . \\
503 . \\
479 . \\
481 . \\
535 .\end{array}$ & & $\begin{array}{l}0.01 \\
0.01 \\
0.01 \\
0.01 \\
0.01 \\
0.01 \\
0.01\end{array}$ & $\begin{array}{l}- \\
- \\
- \\
-\end{array}$ \\
\hline CHLORIDE & $\begin{array}{l}10 / 02 / 85 \\
01 / 09 / 87 \\
07 / 15 / 88 \\
01 / 12 / 89 \\
09 / 14 / 89 \\
03 / 31 / 90 \\
01 / 09 / 91\end{array}$ & $\begin{array}{l}0001 \\
0001 \\
0001 \\
0001 \\
0001 \\
0001 \\
0001\end{array}$ & MG/L & & $\begin{array}{l}36 . \\
43 . \\
48 . \\
43 . \\
54 . \\
58 . \\
65 .\end{array}$ & & $\begin{array}{l}1 . \\
1 . \\
1 . \\
1 . \\
1 . \\
1.5\end{array}$ & $\begin{array}{l}- \\
\dot{-} \\
\dot{-} \\
\dot{-}\end{array}$ \\
\hline CHROMIUH & $\begin{array}{l}10 / 02 / 85 \\
01 / 09 / 87 \\
07 / 15 / 88 \\
01 / 12 / 89 \\
09 / 14 / 89 \\
03 / 31 / 90 \\
01 / 09 / 91\end{array}$ & $\begin{array}{l}0001 \\
0001 \\
0001 \\
0001 \\
0001 \\
0001 \\
0001\end{array}$ & MG/L & $\begin{array}{l}< \\
< \\
< \\
<\end{array}$ & $\begin{array}{l}0.04 \\
0.02 \\
0.16 \\
0.01 \\
0.01 \\
0.01 \\
0.01\end{array}$ & & $\begin{array}{l}0.01 \\
0.01 \\
0.01 \\
0.01 \\
0.01 \\
0.01 \\
0.01\end{array}$ & $\begin{array}{l}- \\
- \\
- \\
- \\
-\end{array}$ \\
\hline COBALT & $\begin{array}{l}10 / 02 / 85 \\
01 / 09 / 87 \\
07 / 15 / 88 \\
01 / 12 / 89 \\
09 / 14 / 89 \\
03 / 31 / 90 \\
01 / 09 / 91\end{array}$ & $\begin{array}{l}0001 \\
0001 \\
0001 \\
0001 \\
0001 \\
0001 \\
0001\end{array}$ & $M G / L$ & $\begin{array}{l}< \\
< \\
< \\
< \\
<\end{array}$ & $\begin{array}{l}0.06 \\
0.05 \\
0.05 \\
0.01 \\
0.05 \\
0.05 \\
0.03\end{array}$ & $J$ & $\begin{array}{l}0.05 \\
0.05 \\
0.05 \\
0.05 \\
0.05 \\
0.05 \\
0.03\end{array}$ & $\begin{array}{l}- \\
- \\
- \\
-\end{array}$ \\
\hline COPPER & $\begin{array}{l}10 / 02 / 85 \\
01 / 09 / 87 \\
07 / 15 / 88 \\
01 / 12 / 89 \\
09 / 14 / 89 \\
03 / 31 / 90 \\
01 / 09 / 91\end{array}$ & $\begin{array}{l}0001 \\
0001 \\
0001 \\
0001 \\
0001 \\
0001 \\
0001\end{array}$ & $\mathrm{MG} / \mathrm{L}$ & $\begin{array}{l}< \\
< \\
< \\
<\end{array}$ & $\begin{array}{l}0.03 \\
0.01 \\
0.03 \\
0.01 \\
0.02 \\
0.02 \\
0.01\end{array}$ & $\begin{array}{l}\mathbf{J} \\
\mathbf{J}\end{array}$ & $\begin{array}{l}0.02 \\
0.02 \\
0.02 \\
0.02 \\
0.02 \\
0.02 \\
0.01\end{array}$ & $\begin{array}{l}- \\
- \\
- \\
-\end{array}$ \\
\hline CYANIDE & $\begin{array}{l}10 / 02 / 85 \\
01 / 12 / 89 \\
09 / 14 / 89 \\
03 / 31 / 90\end{array}$ & $\begin{array}{l}0001 \\
0001 \\
0001 \\
0001\end{array}$ & $M G / L$ & $\begin{array}{l}< \\
< \\
< \\
<\end{array}$ & $\begin{array}{l}0.01 \\
0.002 \\
0.01 \\
0.01\end{array}$ & $J$ & $\begin{array}{l}0.01 \\
0.01 \\
0.01 \\
0.01\end{array}$ & $\begin{array}{l}- \\
- \\
-\end{array}$ \\
\hline
\end{tabular}

PARAMETER VALUE IMDICATOR (PVI): < - LESS thaH DETECTION LIMIT SAMPLE ID CCOES:

0001 - FILTERED SAMPLE (.45 MICRONS)

OTHER PARAMETER VALUE FLAGS:

J. ESTIMATED VALUE 
GROUNDHATER OUALITY DATA BY LOCATION

SITE: AMBOI AMBROSIA LAKE

LOCATION: 0620

NORTH COORDINATE: $\quad 52040.0$ FT

EAST COORDINATE: $\quad 60740.0$ FT

$06 / 10 / 80$ TO $12 / 04 / 92$

REPORT DATE: 08/04/94

FORMATIOH OF CONPLETION: ALLUVIUN (AL)

HYDRAULIC FLOW RELATIONSHIP: CROSS GRADIEHT (C)

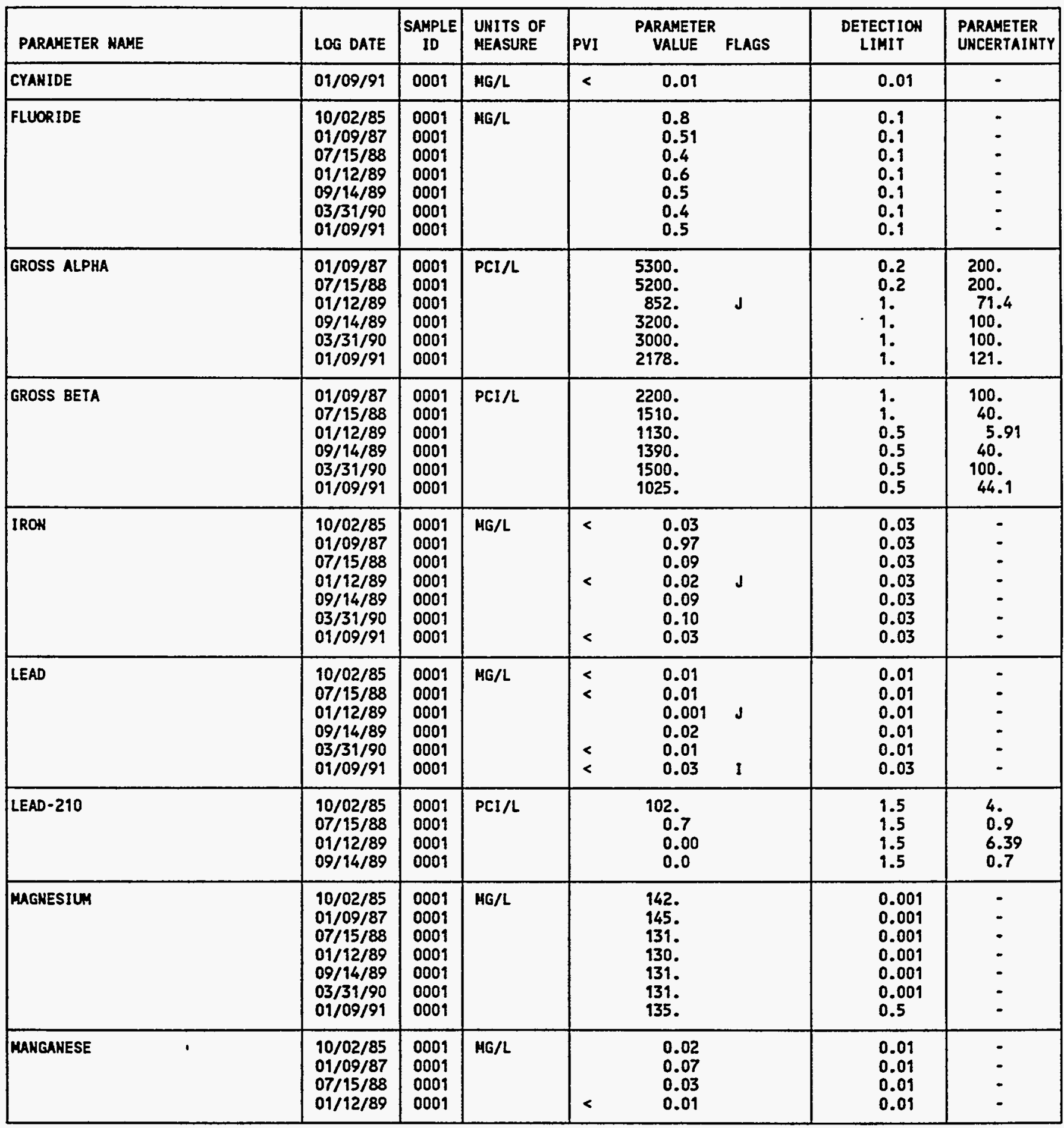

PARAMETER VALUE INDICATOR (PVI): < - LESS THAK DETECTION LIMIT

SAMPLE ID COOES:

0001 - FILTERED SAMPLE (.45 MICRONS)

OTHER PARAMETER VALUE FLAGS:

I - INCREASED DETECTION LIMIT DUE TO REQUIRED DILUTION

$J$ - ESTIMATED VALUE 
GROUNDHATER QUALITY DATA BY LOCATION

SITE: AMBO1 AMBROSIA LAKE

LOCATION: 0620

NORTH COORDINATE: 52040.0 FT

EAST COORDINATE: 60740.0 FT

06/10/80 TO 12/04/92

REPORT DATE: 08/04/94

FORMATION OF COMPLETIOH: ALLUVIUH (AL)

HYDRAULIC FLON RELATIONSHIP: CROSS GRADIENT (C)

\begin{tabular}{|c|c|c|c|c|c|c|c|c|}
\hline $\begin{array}{c}! \\
\text { PARAMETER HAME }\end{array}$ & LOG DATE & $\begin{array}{c}\text { SAMPLE } \\
10\end{array}$ & $\begin{array}{l}\text { UHITS OF } \\
\text { MEASURE }\end{array}$ & PVI & $\begin{array}{l}\text { PARAMETER } \\
\text { VALUE }\end{array}$ & FLAGS & $\begin{array}{l}\text { DETECTION } \\
\text { LIMIT }\end{array}$ & $\begin{array}{l}\text { PARAMETER } \\
\text { UNCERTAINTY }\end{array}$ \\
\hline MANGAMESE & $\begin{array}{l}09 / 14 / 89 \\
03 / 31 / 90 \\
01 / 09 / 91\end{array}$ & $\begin{array}{l}0001 \\
0001 \\
0001\end{array}$ & MG/L & & $\begin{array}{l}0.03 \\
0.02 \\
0.02\end{array}$ & & $\begin{array}{l}0.01 \\
0.01 \\
0.01\end{array}$ & $\ddot{-}$ \\
\hline MERCURY & $\begin{array}{l}10 / 02 / 85 \\
07 / 15 / 88 \\
01 / 12 / 89 \\
09 / 14 / 89 \\
03 / 31 / 90 \\
01 / 09 / 91\end{array}$ & $\begin{array}{l}0001 \\
0001 \\
0001 \\
0001 \\
0001 \\
0001\end{array}$ & MG/L & $\begin{array}{l}< \\
< \\
< \\
< \\
<\end{array}$ & $\begin{array}{l}0.0002 \\
0.0002 \\
0.0001 \\
0.0002 \\
0.0002 \\
0.0002\end{array}$ & 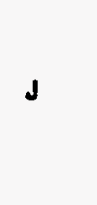 & $\begin{array}{l}0.0002 \\
0.0002 \\
0.0002 \\
0.0002 \\
0.0002 \\
0.0002\end{array}$ & $\begin{array}{l}- \\
- \\
-\end{array}$ \\
\hline HOLYBDENUM & $\begin{array}{l}10 / 02 / 85 \\
01 / 09 / 87 \\
07 / 15 / 88 \\
01 / 12 / 89 \\
09 / 14 / 89 \\
03 / 31 / 90 \\
01 / 09 / 91\end{array}$ & $\begin{array}{l}0001 \\
0001 \\
0001 \\
0001 \\
0001 \\
0001 \\
0001\end{array}$ & MG/L & $<$ & $\begin{array}{l}0.5 \\
0.1 \\
0.12 \\
0.076 \\
0.11 \\
0.11 \\
0.07\end{array}$ & & $\begin{array}{l}0.01 \\
0.1 \\
0.01 \\
0.01 \\
0.01 \\
0.01 \\
0.01\end{array}$ & $\begin{array}{l}- \\
- \\
- \\
- \\
-\end{array}$ \\
\hline NET GROSS ALPHA * & $\begin{array}{l}01 / 09 / 87 \\
07 / 15 / 88 \\
01 / 12 / 89 \\
09 / 14 / 89 \\
03 / 31 / 90 \\
01 / 09 / 91\end{array}$ & $\begin{array}{l}0001 \\
0001 \\
0001 \\
0001 \\
0001 \\
0001\end{array}$ & $\mathrm{PCI} / \mathrm{L}$ & & $\begin{array}{r}-338.92 \\
809.60 \\
-3038.99 \\
346.24 \\
269.72 \\
-847.26\end{array}$ & & $\begin{array}{l}- \\
- \\
- \\
-\end{array}$ & $\begin{array}{l}: \\
: \\
-\end{array}$ \\
\hline NICKEL & $\begin{array}{l}10 / 02 / 85 \\
07 / 15 / 88 \\
01 / 12 / 89 \\
09 / 14 / 89 \\
03 / 31 / 90 \\
01 / 09 / 91\end{array}$ & $\begin{array}{l}0001 \\
0001 \\
0001 \\
0001 \\
0001 \\
0001\end{array}$ & MG/L & $\begin{array}{l}< \\
< \\
< \\
<\end{array}$ & $\begin{array}{l}0.06 \\
0.04 \\
0.02 \\
0.04 \\
0.04 \\
0.04\end{array}$ & $\mathbf{J}$ & $\begin{array}{l}0.04 \\
0.04 \\
0.04 \\
0.04 \\
0.04 \\
0.04\end{array}$ & $\begin{array}{l}- \\
- \\
- \\
-\end{array}$ \\
\hline NITRATE & $\begin{array}{l}10 / 02 / 85 \\
01 / 09 / 87 \\
07 / 15 / 88 \\
01 / 12 / 89 \\
09 / 14 / 89 \\
03 / 31 / 90 \\
01 / 09 / 91\end{array}$ & $\begin{array}{l}0001 \\
0001 \\
0001 \\
0001 \\
0001 \\
0001 \\
0001\end{array}$ & HG/L & & $\begin{array}{l}3 . \\
7.5 \\
12 . \\
12.1 \\
3.8 \\
5.7 \\
8.0\end{array}$ & $J$ & $\begin{array}{l}1 . \\
1 . \\
1 . \\
1 . \\
1 . \\
1.1\end{array}$ & $\begin{array}{l}- \\
- \\
- \\
- \\
-\end{array}$ \\
\hline NITRITE & $10 / 02 / 85$ & 0001 & HG/L & $<$ & 0.1 & & 0.1 & - \\
\hline HITRITE AMD NITRATE & $\begin{array}{l}03 / 31 / 90 \\
01 / 09 / 91\end{array}$ & $\begin{array}{l}0001 \\
0001\end{array}$ & MG/L & & $\begin{array}{l}1.3 \\
1.79\end{array}$ & & $\begin{array}{l}1.05 \\
0.05\end{array}$ & - \\
\hline PH & $\begin{array}{l}10 / 02 / 85 \\
01 / 09 / 87 \\
07 / 15 / 88 \\
01 / 12 / 89 \\
09 / 14 / 89 \\
03 / 31 / 90 \\
01 / 09 / 91\end{array}$ & $\begin{array}{l}0001 \\
0001 \\
0001 \\
0001 \\
0001 \\
0001 \\
0001\end{array}$ & su & & $\begin{array}{l}7.22 \\
7.59 \\
7.08 \\
7.2 \\
7.30 \\
7.15 \\
7.39\end{array}$ & & $\begin{array}{l}- \\
- \\
- \\
- \\
-\end{array}$ & $\begin{array}{l}- \\
- \\
- \\
-\end{array}$ \\
\hline PHOSPHATE & $10 / 02 / 85$ & 0001 & MG/L & & 0.6 & & 0.1 & - \\
\hline
\end{tabular}

- het gROSS ALPHA (GROSS ALPHA - URANIUH) HITH 1 Hg URAHIUH = 686 PCI

PARAMETER VALUE IMDICATOR (PVI): < - LESS THAH DETECTION LIMIT

SAMPLE ID COOES:

0001 - FILTERED SAMPLE (.45 MICRONS)

OTHER PARAMETER VALUE fLAGS:

J - ESTIMATED VALUE 
GROUNDWATER OUALITY DATA BY LOCATIOH

SITE: AMBO1 AMBROSIA LAKE

LOCATION: 0620

MORTH COORDINATE: $\quad 52040.0$ FT

EAST COORDINATE: $\quad 60740.0$ FT

06/10/80 TO 12/04/92

REPORT DATE: 08/04/94

FORMATION OF COMPLETION: ALLUVIUN (AL)

HYDRAULIC FLON RELATIONSHIP: CROSS GRADIENT (C)

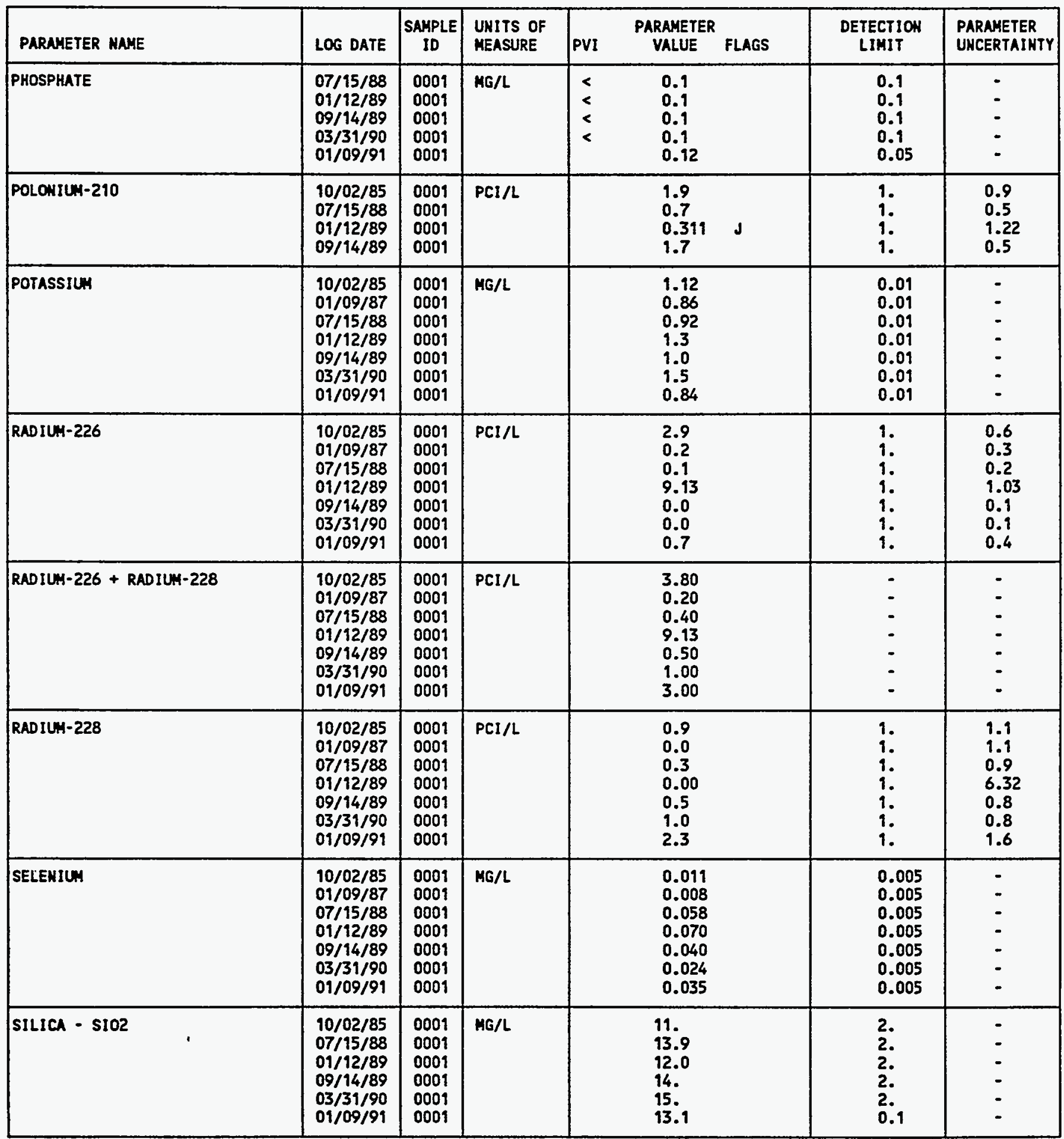

PARAMETER VALUE INDICATOR (PVI): < - LESS THAH DETECTIOH LIMIT

SAMPLE ID CODES:

0001 - FILTERED SAMPLE (.45 MICRONS)

OTHER PARAMETER VALUE FLAGS:

J ESTIMATED VALUE 
GROUNDHATER QUALITY DATA BY LOCATION

SITE: AMBO1 AMBROSIA LAKE

LOCATIOH: 0620

MORTH COORDINATE: 52040.0 FT

EAST COORDIHATE: 60740.0 FT

$06 / 10 / 80$ TO $12 / 04 / 92$

REPORT DATE: 08/04/94

FORMATIOH OF COAPLETIOH: ALLUVIUH (AL)

HYDRAULIC FLOW RELATIONSHIP: CROSS GRADIENT (C)

\begin{tabular}{|c|c|c|c|c|c|c|c|c|}
\hline PARAMETER MAME & LOG DATE & $\underset{\text { ID }}{\text { SAMPLE }}$ & $\begin{array}{l}\text { UNITS OF } \\
\text { MEASURE }\end{array}$ & PVI & $\begin{array}{l}\text { PARAMETER } \\
\text { VALUE }\end{array}$ & FLAGS & $\begin{array}{l}\text { DETECTION } \\
\text { LIMIT }\end{array}$ & $\begin{array}{l}\text { PARAMETER } \\
\text { UNCERTAINTY }\end{array}$ \\
\hline SILVER & $\begin{array}{l}10 / 02 / 85 \\
01 / 09 / 87 \\
07 / 15 / 88 \\
01 / 12 / 89 \\
09 / 14 / 89 \\
03 / 31 / 90 \\
01 / 09 / 91\end{array}$ & $\begin{array}{l}0001 \\
0001 \\
0001 \\
0001 \\
0001 \\
0001 \\
0001\end{array}$ & $M G / L$ & $\begin{array}{l}< \\
< \\
< \\
< \\
<\end{array}$ & $\begin{array}{l}0.01 \\
0.01 \\
0.04 \\
0.01 \\
0.01 \\
0.01 \\
0.01\end{array}$ & & $\begin{array}{l}0.01 \\
0.01 \\
0.01 \\
0.01 \\
0.01 \\
0.01 \\
0.01\end{array}$ & $\begin{array}{l}- \\
- \\
- \\
- \\
- \\
-\end{array}$ \\
\hline soo IUm & $\begin{array}{l}10 / 02 / 85 \\
01 / 09 / 87 \\
07 / 15 / 88 \\
01 / 12 / 89 \\
09 / 14 / 89 \\
03 / 31 / 90 \\
01 / 09 / 91\end{array}$ & $\begin{array}{l}0001 \\
0001 \\
0001 \\
0001 \\
0001 \\
0001 \\
0001\end{array}$ & MG/L & & $\begin{array}{l}434 . \\
439 . \\
366 . \\
440 . \\
415 . \\
421 . \\
435\end{array}$ & & $\begin{array}{l}0.002 \\
0.002 \\
0.002 \\
0.002 \\
0.002 \\
0.002 \\
5 .\end{array}$ & $\begin{array}{l}- \\
- \\
- \\
- \\
-\end{array}$ \\
\hline SPECIFIC CONDUCTANCE & $\begin{array}{l}10 / 02 / 85 \\
01 / 09 / 87 \\
07 / 15 / 88 \\
01 / 12 / 89 \\
09 / 14 / 89 \\
03 / 31 / 90 \\
01 / 09 / 91\end{array}$ & $\begin{array}{l}0001 \\
0001 \\
0001 \\
0001 \\
0001 \\
0001 \\
0001\end{array}$ & UWHO/CM & & $\begin{array}{l}3100 . \\
2850 . \\
2750 . \\
2600 . \\
1300 . \\
2700 . \\
2800 .\end{array}$ & & $\begin{array}{l}- \\
- \\
- \\
- \\
-\end{array}$ & $\begin{array}{l}: \\
: \\
: \\
:\end{array}$ \\
\hline STROHTIUM & $\begin{array}{l}10 / 02 / 85 \\
07 / 15 / 88 \\
01 / 12 / 89 \\
09 / 14 / 89 \\
03 / 31 / 90 \\
01 / 09 / 91\end{array}$ & $\begin{array}{l}0001 \\
0001 \\
0001 \\
0001 \\
0001 \\
0001\end{array}$ & MG/L & & $\begin{array}{l}8.4 \\
6.2 \\
7.40 \\
6.9 \\
6.86 \\
7.15\end{array}$ & & $\begin{array}{l}0.1 \\
0.1 \\
0.1 \\
0.1 \\
0.1 \\
0.01\end{array}$ & $\begin{array}{l}- \\
- \\
- \\
-\end{array}$ \\
\hline SULFATE & $\begin{array}{l}10 / 02 / 85 \\
01 / 09 / 87 \\
07 / 15 / 88 \\
01 / 12 / 89 \\
09 / 14 / 89 \\
03 / 31 / 90 \\
01 / 09 / 91\end{array}$ & $\begin{array}{l}0001 \\
0001 \\
0001 \\
0001 \\
0001 \\
0001 \\
0001\end{array}$ & $M G / L$ & & $\begin{array}{l}2440 \\
2310 \\
2100 \\
2354 \\
2370 \\
2380 \\
2380\end{array}$ & & $\begin{array}{c}0.1 \\
0.1 \\
0.1 \\
0.1 \\
0.1 \\
0.1 \\
10 .\end{array}$ & $\begin{array}{l}- \\
- \\
- \\
- \\
-\end{array}$ \\
\hline SULFIDE & $\begin{array}{l}10 / 02 / 85 \\
07 / 15 / 88 \\
01 / 12 / 89 \\
09 / 14 / 89 \\
03 / 31 / 90 \\
01 / 09 / 91\end{array}$ & $\begin{array}{l}0001 \\
0001 \\
0001 \\
0001 \\
0001 \\
0001\end{array}$ & MG/L & $\begin{array}{l}< \\
< \\
< \\
< \\
<\end{array}$ & $\begin{array}{l}0.1 \\
0.1 \\
0.01 \\
0.1 \\
0.1 \\
0.1\end{array}$ & $\begin{array}{l}\mathbf{J} \\
\mathbf{J}\end{array}$ & $\begin{array}{l}0.1 \\
0.1 \\
0.1 \\
0.1 \\
0.1 \\
0.1\end{array}$ & $\begin{array}{l}- \\
- \\
- \\
-\end{array}$ \\
\hline TEMPERATURE & $\begin{array}{l}10 / 02 / 85 \\
01 / 09 / 87 \\
07 / 15 / 88 \\
01 / 12 / 89 \\
09 / 14 / 89 \\
03 / 31 / 90 \\
01 / 09 / 91\end{array}$ & $\begin{array}{l}0001 \\
0001 \\
0001 \\
0001 \\
0001 \\
0001 \\
0001\end{array}$ & C - DEGREE & & $\begin{array}{l}12 . \\
12 . \\
13 . \\
11.0 \\
13.0 \\
12 . \\
12.5\end{array}$ & & $\begin{array}{l}- \\
- \\
- \\
- \\
- \\
-\end{array}$ & $\begin{array}{l}\dot{-} \\
\dot{-} \\
\dot{-} \\
\dot{-}\end{array}$ \\
\hline THALLILA & $\begin{array}{l}01 / 12 / 89 \\
09 / 14 / 89\end{array}$ & $\begin{array}{l}0001 \\
0001\end{array}$ & MG/L & $<$ & $\begin{array}{l}0.005 \\
0.01\end{array}$ & 1 & $\begin{array}{l}0.005 \\
0.01\end{array}$ & $\dot{-}$ \\
\hline
\end{tabular}

PARAMETER VALUE INDICATOR (PVI): < - LESS THAH DETECTION LIHIT

SAMPLE ID COOES:

0001 - FILTERED SAMPLE (.45 MICROHS)

OTHER PARAMETER VALUE FLAGS:

1 - IHCREASED DETECTION LIHIT DUE TO REQUIRED DILUTIOH

\$- estimated VALUE 
GROUHDWATER QUALITY DATA BY LOCATIOH

SITE: AMBO1 AMBROSIA LAKE

LOCATIOH: 0620

NORTH COORDINATE: 52040.0 FT

EAST COORDINATE: $\quad 60740.0$ FT

06/10/80 TO $12 / 04 / 92$

REPORT DATE: 08/04/94

FORMATION OF COMPLETION: ALLUVIUH (AL)

HYDRAULIC FLOW RELATIONSHIP: CROSS GRADIENT (C)

\begin{tabular}{|c|c|c|c|c|c|c|c|c|}
\hline PARAMETER MAME & LOG DATE & $\begin{array}{c}\text { SAMPLE } \\
10\end{array}$ & $\begin{array}{l}\text { UNITS OF } \\
\text { MEASURE }\end{array}$ & PVI & $\begin{array}{l}\text { PARAMETER } \\
\text { VALUE }\end{array}$ & FLAGS & $\begin{array}{l}\text { DETECTION } \\
\text { LIMIT }\end{array}$ & $\begin{array}{l}\text { PARAMETER } \\
\text { UNCERTAINTY }\end{array}$ \\
\hline THALLIUM & $\begin{array}{l}03 / 31 / 90 \\
01 / 09 / 91\end{array}$ & $\begin{array}{l}0001 \\
0001\end{array}$ & MG/L & $<$ & $\begin{array}{l}0.01 \\
0.03\end{array}$ & $I$ & $\begin{array}{l}0.01 \\
0.03\end{array}$ & - \\
\hline THORIUM-230 & $\begin{array}{l}10 / 02 / 85 \\
07 / 15 / 88 \\
01 / 12 / 89 \\
09 / 14 / 89\end{array}$ & $\begin{array}{l}0001 \\
0001 \\
0001 \\
0001\end{array}$ & $\mathrm{PCI} / \mathrm{L}$ & & $\begin{array}{l}4 . \\
0.2 \\
5.14 \\
0.1\end{array}$ & & $\begin{array}{l}1 . \\
1 . \\
1 .\end{array}$ & $\begin{array}{l}1.2 \\
0.4 \\
0.660 \\
0.4\end{array}$ \\
\hline TIN & $\begin{array}{l}10 / 02 / 85 \\
07 / 15 / 88 \\
01 / 12 / 89 \\
09 / 14 / 89 \\
03 / 31 / 90 \\
01 / 09 / 91\end{array}$ & $\begin{array}{l}0001 \\
0001 \\
0001 \\
0001 \\
0001 \\
0001\end{array}$ & MG/L & $\begin{array}{l}< \\
< \\
< \\
< \\
<\end{array}$ & $\begin{array}{l}0.005 \\
0.010 \\
0.001 \\
0.1 \\
0.005 \\
0.03\end{array}$ & $\begin{array}{l}\mathrm{J} \\
\mathrm{I}\end{array}$ & $\begin{array}{l}0.005 \\
0.005 \\
0.005 \\
0.1 \\
0.005 \\
0.03\end{array}$ & $\begin{array}{l}- \\
: \\
- \\
-\end{array}$ \\
\hline TOTAL DISSOLVED SOLIDS & $\begin{array}{l}10 / 02 / 85 \\
01 / 09 / 87 \\
07 / 15 / 88 \\
01 / 12 / 89 \\
09 / 14 / 89 \\
03 / 31 / 90 \\
01 / 09 / 91\end{array}$ & $\begin{array}{l}0001 \\
0001 \\
0001 \\
0001 \\
0001 \\
0001 \\
0001\end{array}$ & MG/L & & $\begin{array}{l}4060 . \\
4000 . \\
3940 . \\
3782 . \\
3680 . \\
3550 . \\
3860 .\end{array}$ & & $\begin{array}{l}10 . \\
10 . \\
10 . \\
10 . \\
10 . \\
10 . \\
10 .\end{array}$ & : \\
\hline TOTAL KJELDAHL NITROGEN & $01 / 09 / 91$ & 0001 & $M G / L$ & & 2. & & 1. & - \\
\hline TOTAL ORGANIC CARBON & $\begin{array}{l}10 / 02 / 85 \\
07 / 15 / 88 \\
01 / 12 / 89 \\
09 / 14 / 89 \\
03 / 31 / 90 \\
01 / 09 / 91\end{array}$ & $\begin{array}{l}0001 \\
0001 \\
0001 \\
0001 \\
0001 \\
0001\end{array}$ & MG/L & & $\begin{array}{l}63 . \\
78.6 \\
15 . \\
79.1 \\
5.2 \\
4 .\end{array}$ & J & $\begin{array}{l}1 . \\
1 . \\
1 . \\
1 .\end{array}$ & : \\
\hline URAMIUM & $\begin{array}{l}10 / 02 / 85 \\
01 / 09 / 87 \\
07 / 15 / 88 \\
01 / 12 / 89 \\
09 / 14 / 89 \\
03 / 31 / 90 \\
01 / 09 / 91\end{array}$ & $\begin{array}{l}0001 \\
0001 \\
0001 \\
0001 \\
0001 \\
0001 \\
0001\end{array}$ & MG/L & & $\begin{array}{l}5.34 \\
8.22 \\
6.40 \\
5.672 \\
4.16 \\
3.98 \\
4.41\end{array}$ & & $\begin{array}{l}0.003 \\
0.003 \\
0.003 \\
0.003 \\
0.003 \\
0.003 \\
0.001\end{array}$ & $\begin{array}{l}. \\
. \\
. \\
. \\
.\end{array}$ \\
\hline VAKADIUA & $\begin{array}{l}10 / 02 / 85 \\
01 / 09 / 87 \\
07 / 15 / 88 \\
01 / 12 / 89 \\
09 / 14 / 89 \\
03 / 31 / 90 \\
01 / 09 / 91\end{array}$ & $\begin{array}{l}0001 \\
0001 \\
0001 \\
0001 \\
0001 \\
0001 \\
0001\end{array}$ & MG/L & $\begin{array}{l}< \\
< \\
< \\
< \\
<\end{array}$ & $\begin{array}{l}0.01 \\
0.01 \\
0.07 \\
0.01 \\
0.01 \\
0.01 \\
0.01\end{array}$ & & $\begin{array}{l}0.01 \\
0.01 \\
0.01 \\
0.01 \\
0.01 \\
0.01 \\
0.01\end{array}$ & $\begin{array}{l}. \\
. \\
. \\
. \\
- \\
-\end{array}$ \\
\hline ZINC & $\begin{array}{l}10 / 02 / 85 \\
07 / 15 / 88 \\
01 / 12 / 89 \\
09 / 14 / 89 \\
03 / 31 / 90 \\
01 / 09 / 91\end{array}$ & $\begin{array}{l}0001 \\
0001 \\
0001 \\
0001 \\
0001 \\
0001\end{array}$ & MG/L & $\begin{array}{l}< \\
< \\
<\end{array}$ & $\begin{array}{l}0.017 \\
0.019 \\
0.01 \\
0.005 \\
0.005 \\
0.005\end{array}$ & & $\begin{array}{l}0.005 \\
0.005 \\
0.01 \\
0.005 \\
0.005 \\
0.005\end{array}$ & $\begin{array}{l}- \\
- \\
- \\
-\end{array}$ \\
\hline
\end{tabular}

PARAMETER VALUE IMDICATOR (PVI): < - LESS THAN DETECTION LIMIT SAMPLE ID CODES:

0001 - FILTERED SAMPLE (.45 HICRONS)

OTHER PARAMETER VALUE FLAGS:

I - IHCREASED DETECTION LIMIT DUE TO REOUIRED DILUTIOH

J - ESTIMATED VALUE 
GROUHDHATER OUALITY DATA BY LOCATION

SITE: AMBOI AMBROSIA LAKE

LOCATION: 0650

NORTH COORDIHATE: $\quad 49990.0$ FT

EAST COORDINATE: $\quad 51010.0$ FT

06/10/80 TO 12/04/92

REPORT DATE: 08/04/94

FORMATION OF COMPLETIOH: ALLUVIUH (AL)

HYORAULIC FLOW RELATIONSHIP: UNKHOWK (N)

\begin{tabular}{|c|c|c|c|c|c|c|c|c|}
\hline PARAMETER HAME & LOG DATE & $\begin{array}{c}\text { SAMPLE } \\
\text { ID }\end{array}$ & $\begin{array}{l}\text { UNITS OF } \\
\text { MEASURE }\end{array}$ & PVI & $\begin{array}{l}\text { PARAMETER } \\
\text { VALUE }\end{array}$ & FLAGS & $\begin{array}{l}\text { DETECTIOH } \\
\text { LIMIT }\end{array}$ & $\begin{array}{l}\text { PARAMETER } \\
\text { UNCERTAINTY }\end{array}$ \\
\hline ALKALIHITY & $\begin{array}{l}05 / 22 / 86 \\
05 / 22 / 86 \\
05 / 22 / 86 \\
05 / 22 / 86 \\
05 / 22 / 86 \\
01 / 09 / 87\end{array}$ & $\begin{array}{l}0001 \\
0002 \\
0003 \\
0004 \\
0005 \\
0001\end{array}$ & MG/L CACO3 & & $\begin{array}{l}260 . \\
260 . \\
260 . \\
260 . \\
260 . \\
283 .\end{array}$ & & $\begin{array}{l}- \\
\dot{-} \\
\dot{-} \\
-\end{array}$ & :- \\
\hline ARSENIC & $\begin{array}{l}05 / 22 / 86 \\
05 / 22 / 86 \\
05 / 22 / 86 \\
05 / 22 / 86 \\
05 / 22 / 86 \\
01 / 09 / 87\end{array}$ & $\begin{array}{l}0001 \\
0002 \\
0003 \\
0004 \\
0005 \\
0001\end{array}$ & MG/L & $\begin{array}{l}< \\
< \\
< \\
< \\
<\end{array}$ & $\begin{array}{l}0.01 \\
0.01 \\
0.01 \\
0.01 \\
0.01 \\
0.003\end{array}$ & J & $\begin{array}{l}0.01 \\
0.01 \\
0.01 \\
0.01 \\
0.09 \\
0.01\end{array}$ & $\begin{array}{l}- \\
- \\
- \\
-\end{array}$ \\
\hline BOROH & $\begin{array}{l}05 / 22 / 86 \\
05 / 22 / 86 \\
05 / 22 / 86 \\
05 / 22 / 86 \\
05 / 22 / 86 \\
01 / 09 / 87\end{array}$ & $\begin{array}{l}0009 \\
0002 \\
0003 \\
0004 \\
0005 \\
0001\end{array}$ & MG/L & & $\begin{array}{l}0.5 \\
0.6 \\
0.5 \\
0.5 \\
0.5 \\
0.21\end{array}$ & & $\begin{array}{l}0.1 \\
0.1 \\
0.1 \\
0.1 \\
0.1 \\
0.1\end{array}$ & $\begin{array}{l}- \\
\dot{-} \\
\dot{-} \\
-\end{array}$ \\
\hline CADMIUM & $\begin{array}{l}05 / 22 / 86 \\
05 / 22 / 86 \\
05 / 22 / 86 \\
05 / 22 / 86 \\
05 / 22 / 86\end{array}$ & $\begin{array}{l}0001 \\
0002 \\
0003 \\
0004 \\
0005\end{array}$ & HG/L & $\begin{array}{l}< \\
< \\
< \\
< \\
<\end{array}$ & $\begin{array}{l}0.001 \\
0.001 \\
0.001 \\
0.001 \\
0.001\end{array}$ & & $\begin{array}{l}0.001 \\
0.009 \\
0.001 \\
0.001 \\
0.001\end{array}$ & $\begin{array}{l}\ddot{-} \\
\dot{-} \\
\dot{-}\end{array}$ \\
\hline CALCIUA & $\begin{array}{l}05 / 22 / 86 \\
05 / 22 / 86 \\
05 / 22 / 86 \\
05 / 22 / 86 \\
05 / 22 / 86 \\
01 / 09 / 87\end{array}$ & $\begin{array}{l}0001 \\
0002 \\
0003 \\
0004 \\
0005 \\
0001\end{array}$ & MG/L & & $\begin{array}{l}565 . \\
565 . \\
565 . \\
565 . \\
565 . \\
551 .\end{array}$ & & $\begin{array}{l}0.01 \\
0.01 \\
0.01 \\
0.01 \\
0.01 \\
0.01\end{array}$ & $\begin{array}{l}- \\
- \\
- \\
-\end{array}$ \\
\hline CHLORIDE & $\begin{array}{l}05 / 22 / 86 \\
05 / 22 / 86 \\
05 / 22 / 86 \\
05 / 22 / 86 \\
05 / 22 / 86 \\
01 / 09 / 87\end{array}$ & $\begin{array}{l}0001 \\
0002 \\
0003 \\
0004 \\
0005 \\
0001\end{array}$ & $M G / L$ & & $\begin{array}{l}240 . \\
240 . \\
240 . \\
240 . \\
240 . \\
200 .\end{array}$ & & $\begin{array}{l}1 . \\
1 . \\
1 . \\
1 . \\
1 .\end{array}$ & $\begin{array}{l}: \\
: \\
:\end{array}$ \\
\hline CHROMIUM & $\begin{array}{l}05 / 22 / 86 \\
05 / 22 / 86 \\
05 / 22 / 86 \\
05 / 22 / 86 \\
05 / 22 / 86 \\
01 / 09 / 87\end{array}$ & $\begin{array}{l}0001 \\
0002 \\
0003 \\
0004 \\
0005 \\
0001\end{array}$ & HG/L & & $\begin{array}{l}0.04 \\
0.04 \\
0.04 \\
0.04 \\
0.04 \\
0.02\end{array}$ & & $\begin{array}{l}0.01 \\
0.01 \\
0.01 \\
0.01 \\
0.01 \\
0.01\end{array}$ & $\begin{array}{l}- \\
- \\
- \\
-\end{array}$ \\
\hline COBALT & $\begin{array}{l}05 / 22 / 86 \\
05 / 22 / 86 \\
05 / 22 / 86 \\
05 / 22 / 86 \\
05 / 22 / 86 \\
01 / 09 / 87\end{array}$ & $\begin{array}{l}0001 \\
0002 \\
0003 \\
0004 \\
0005 \\
0001\end{array}$ & MG/L & & $\begin{array}{l}0.08 \\
0.08 \\
0.08 \\
0.08 \\
0.08 \\
0.05\end{array}$ & & $\begin{array}{l}0.05 \\
0.05 \\
0.05 \\
0.05 \\
0.05 \\
0.05\end{array}$ & $\begin{array}{l}- \\
- \\
- \\
-\end{array}$ \\
\hline COPPER & $01 / 09 / 87$ & 0001 & MG/L & $<$ & 0.01 & J & 0.02 & - \\
\hline
\end{tabular}

PARAMETER VALUE IMOICATOR (PVI): < - LESS THAN DETECTIOH LIMIT

SAMPLE ID CODES:

0001 - FILTIERED SAMPLE (.45 MICRONS)

OTHER PARAMETER VALUE FLAGS:

0002 - FILTIERED REPLICATE SAMPLE (.45 MICROHS)

0003 - FILTIERED REPLICATE SAMPLE (.45 MICROHS)

J. ESTIMATED VALUE

0004 - FILTIERED REPLICATE SAHPLE (.45 MICRONS) 
GROUNDWATER QUALITY DATA BY LOCATION

SITE: AMBO1 AMBROSIA LAKE

LOCATIOH: 0650

NORTH COORDINATE: $\quad 49990.0$ FT

EAST COORDINATE: $\quad 51010.0$ FT

06/10/80 TO 12/04/92

REPORT DATE: 08/04/94

FORMATION OF COMPLETION: ALLUVIUM (AL)

HYDRAULIC FLON RELATIONSHIP: UNKNOWN (N)

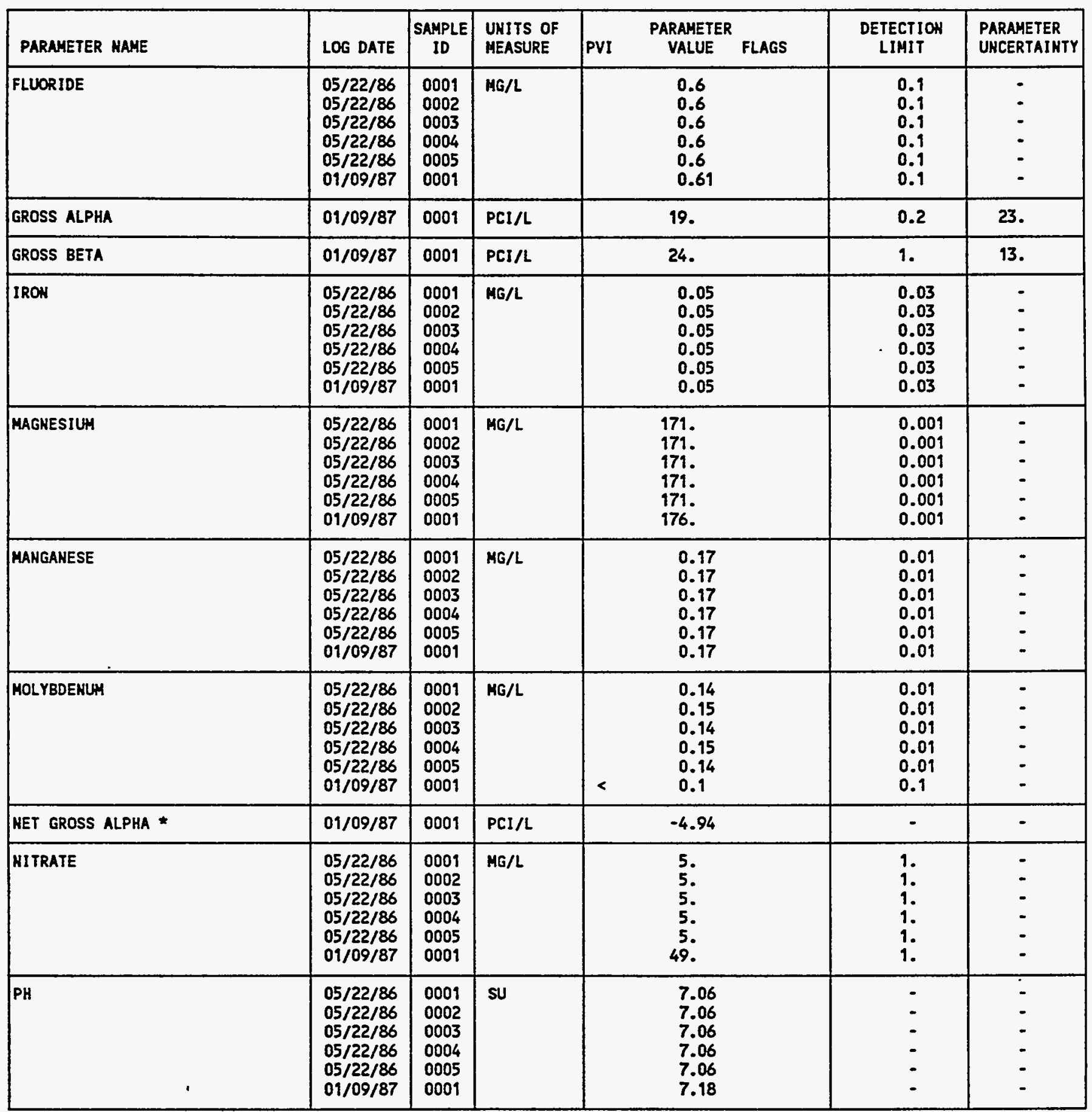

- MET GROSS ALPHA (GROSS ALPHA - URANIUH) WITH 1 HG URANIUH = 686 PCI

PARAMETER VALUE INDICATOR (PVI): < - LESS THAN DETECTION LIMIT

SAMPLE ID COOES:

0001 - FILTERED SAMPLE (.45 MICRONS)

0002 - FILTERED REPLICATE SAMPLE (.45 MICROHS)

0003 - FILTERED REPLICATE SAMPLE (.45 MICROHS)

0004 - FILTERED REPLICATE SAMPLE (.45 MICRONS) 
GROUNDWATER OUALITY DATA BY LOCATION

SITE: AMBO1 AMBROSIA LAKE

LOCATIOH: 0650

NORTH COORDINATE: 49990.0 FT

EAST COORDINATE: $\quad 51010.0$ FT

06/10/80 TO $12 / 04 / 92$

REPORT DATE: 08/04/94

FORMATIOH OF COAPLETION: ALLUVIUM (AL)

HYDRAULIC FLON RELATIOHSHIP: UNKWOWH (K)

\begin{tabular}{|c|c|c|c|c|c|c|c|}
\hline PARAMETER haHE & LOG DATE & $\underset{\text { SAMPLE }}{\text { ID }}$ & $\begin{array}{l}\text { UNITS OF } \\
\text { MEASURE }\end{array}$ & $\begin{array}{lc} & \text { PARAMETER } \\
\text { PVI } & \text { VALUE }\end{array}$ & FLAGS & $\begin{array}{l}\text { DETECTION } \\
\text { LIMIT }\end{array}$ & $\begin{array}{l}\text { PARAMETER } \\
\text { UNCERTAINTY }\end{array}$ \\
\hline Potassiun & $\begin{array}{l}05 / 22 / 86 \\
05 / 22 / 86 \\
05 / 22 / 86 \\
05 / 22 / 86 \\
05 / 22 / 86 \\
01 / 09 / 87\end{array}$ & $\begin{array}{l}0001 \\
0002 \\
0003 \\
0004 \\
0005 \\
0001\end{array}$ & $M G / L$ & $\begin{array}{l}7.92 \\
7.92 \\
7.92 \\
7.92 \\
7.92 \\
7.26\end{array}$ & & $\begin{array}{l}0.01 \\
0.01 \\
0.01 \\
0.01 \\
0.01 \\
0.01\end{array}$ & $\begin{array}{l}- \\
- \\
- \\
-\end{array}$ \\
\hline RADILH-226 & $01 / 09 / 87$ & 0001 & $\mathrm{PCI} / \mathrm{L}$ & 0.5 & & 1. & 0.4 \\
\hline RADIUH-226 + RADILN-228 & $01 / 09 / 87$ & 0001 & PCI/L & 0.70 & & - & - \\
\hline RADIUH-228 & $01 / 09 / 87$ & 0001 & $\mathrm{PCI} / \mathrm{L}$ & 0.2 & & 1. & 1.3 \\
\hline SELENIUM & $\begin{array}{l}05 / 22 / 86 \\
05 / 22 / 86 \\
05 / 22 / 86 \\
05 / 22 / 86 \\
05 / 22 / 86 \\
01 / 09 / 87\end{array}$ & $\begin{array}{l}0001 \\
0002 \\
0003 \\
0004 \\
0005 \\
0001\end{array}$ & MG/L & $\begin{array}{l}0.005 \\
0.005 \\
0.005 \\
0.005 \\
0.005 \\
0.007\end{array}$ & & $\begin{array}{l}0.005 \\
0.005 \\
0.005 \\
0.005 \\
0.005 \\
0.005\end{array}$ & $\begin{array}{l}- \\
- \\
- \\
-\end{array}$ \\
\hline SILVER & $01 / 09 / 87$ & 0001 & MG/L & 0.01 & & 0.01 & - \\
\hline Soolum & $\begin{array}{l}05 / 22 / 86 \\
05 / 22 / 86 \\
05 / 22 / 86 \\
05 / 22 / 86 \\
05 / 22 / 86 \\
01 / 09 / 87\end{array}$ & $\begin{array}{l}0001 \\
0002 \\
0003 \\
0004 \\
0005 \\
0001\end{array}$ & $M G / L$ & $\begin{array}{l}367 . \\
367 . \\
367 . \\
367 . \\
367 . \\
411 .\end{array}$ & & $\begin{array}{l}0.002 \\
0.002 \\
0.002 \\
0.002 \\
0.002 \\
0.002\end{array}$ & $\begin{array}{l}- \\
- \\
-\end{array}$ \\
\hline SPECIFIC COHDUCTANCE & $\begin{array}{l}05 / 22 / 86 \\
05 / 22 / 86 \\
05 / 22 / 86 \\
05 / 22 / 86 \\
05 / 22 / 86 \\
01 / 09 / 87\end{array}$ & $\begin{array}{l}0001 \\
0002 \\
0003 \\
0004 \\
0005 \\
0001\end{array}$ & UAHO/CM & $\begin{array}{l}3200 . \\
3200 . \\
3200 . \\
3200 . \\
3200 . \\
3100 .\end{array}$ & & $\begin{array}{c}- \\
- \\
- \\
0.02 \\
-\end{array}$ & $\begin{array}{l}- \\
- \\
- \\
-\end{array}$ \\
\hline STRONTIUM & $\begin{array}{l}05 / 22 / 86 \\
05 / 22 / 86 \\
05 / 22 / 86 \\
05 / 22 / 86 \\
05 / 22 / 86\end{array}$ & $\begin{array}{l}0001 \\
0002 \\
0003 \\
0004 \\
0005\end{array}$ & $M G / L$ & $\begin{array}{l}0.7 \\
0.7 \\
0.7 \\
0.7 \\
0.7\end{array}$ & & $\begin{array}{l}0.1 \\
0.1 \\
0.1 \\
0.1 \\
0.1\end{array}$ & $\begin{array}{l}- \\
- \\
-\end{array}$ \\
\hline SULFATE & $\begin{array}{l}05 / 22 / 86 \\
05 / 22 / 86 \\
05 / 22 / 86 \\
05 / 22 / 86 \\
05 / 22 / 86 \\
01 / 09 / 87\end{array}$ & $\begin{array}{l}0001 \\
0002 \\
0003 \\
0004 \\
0005 \\
0001\end{array}$ & MG/L & $\begin{array}{l}2360 . \\
2360 . \\
2360 \\
2360 \\
2360 \\
2250\end{array}$ & & $\begin{array}{l}0.1 \\
0.1 \\
0.1 \\
0.1 \\
0.1 \\
0.1\end{array}$ & $\begin{array}{l}- \\
- \\
- \\
-\end{array}$ \\
\hline TEMPERATURE & $\begin{array}{l}05 / 22 / 86 \\
05 / 22 / 86 \\
05 / 22 / 86 \\
05 / 22 / 86 \\
05 / 22 / 86 \\
01 / 09 / 87\end{array}$ & $\begin{array}{l}0001 \\
0002 \\
0003 \\
0004 \\
0005 \\
0001\end{array}$ & C - DEGREE & $\begin{array}{l}13 . \\
13 . \\
13 . \\
13 . \\
13 . \\
12.5\end{array}$ & & $\begin{array}{l}- \\
: \\
- \\
-\end{array}$ & $\begin{array}{l}- \\
- \\
- \\
-\end{array}$ \\
\hline TOTAL DISSOLVED SOLIDS & $05 / 22 / 86$ & 0001 & MG/L & 4330. & & 10. & - \\
\hline
\end{tabular}

PARAMETER VALUE INDICATOR (PVI): < - LESS THAN DETECTION LIMIT

SAMPLE ID COOES:

0001 - FILTERED SAMPLE (.45 MICRONS)

0002 - FILTERED REPLICATE SAMPLE (.45 HICRONS)

0003 - FILTERED REPLICATE SAMPLE (.45 MICRONS)

0004 - FILTERED REPLICATE SAMPLE (.45 MICRONS) 
GROUNDHATER QUALITY DATA BY LOCATIOH

SITE: AMBOI AMBROSIA LAKE

LOCATION: 0650

NORTH COORDINATE: 49990.0 FT

EAST COORDINATE: $\quad 51010.0$ FT

O6/10/80 TO $12 / 04 / 92$

REPORT DATE: 08/04/94

FORMATION OF COMPLETION: ALLUVIUH (AL)

HYDRAULIC FLON RELATIOHSHIP: UNKNOWN (H)

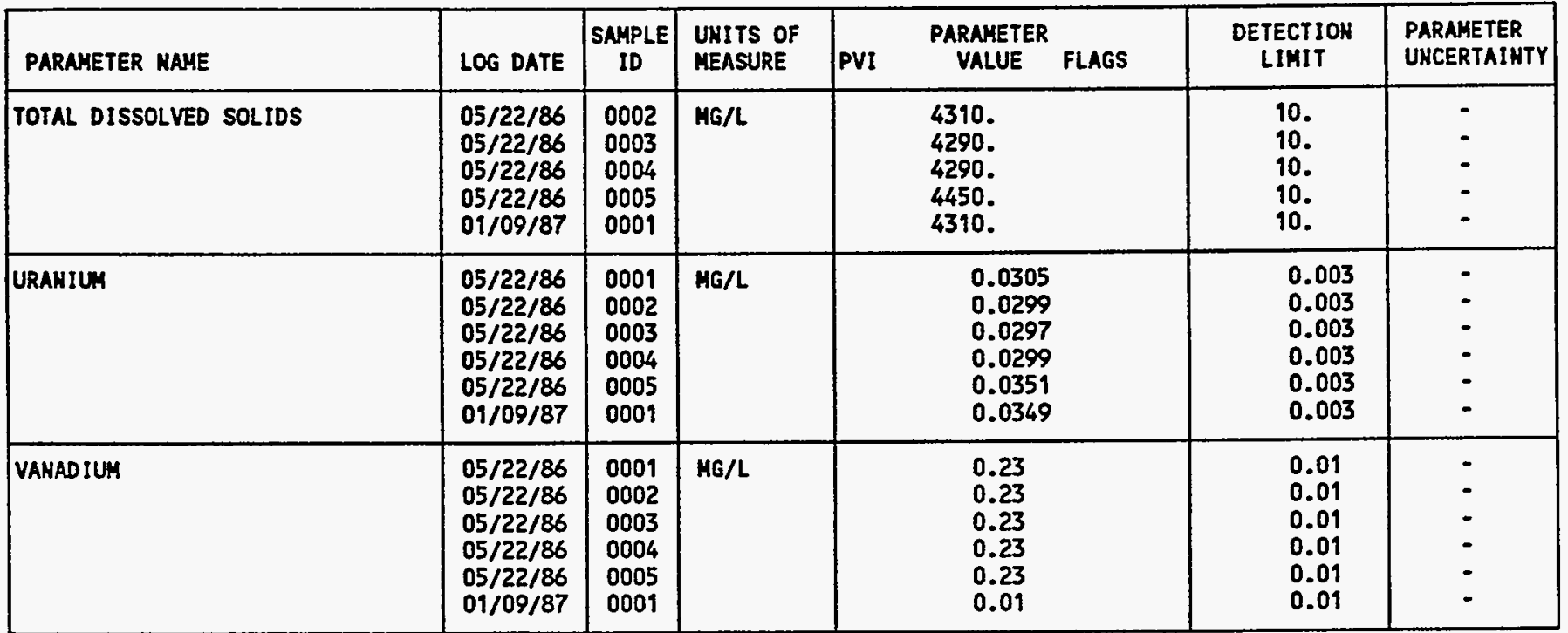

PARAMETER VALUE INDICATOR (PVI): < - LESS THAN DETECTION LIMIT

SAMPLE ID COOES:

0001 - FILTERED SAMPLE (.45 MICROHS)

0002 - FILTERED REPLICATE SAMPLE (.45 MICRONS)

0003 - FILTERED REPLICATE SAMPLE (.45 MICRONS)

0004 - FILTERED REPLICATE SAMPLE (.45 MICRONS) 
GROUNDHATER QUALITY DATA BY LOCATION

SITE: AMBO1 AMBROSIA LAKE

LOCATIOH: 0674 RESERVED ESTORHS, MH, 11/19/88

HORTH COORDIHATE: UKKNOWH

EAST COORDINATE: UNKNOWN

06/10/80 TO 12/04/92

REPORT DATE: 08/04/94

FORMATION OF COMPLETIOH: ALLUVIUM (AL)

HYDRAULIC FLOW RELATIONSHIP: DONH GRADIENT (D)

\begin{tabular}{|c|c|c|c|c|c|c|c|c|}
\hline PARAMETER MAME & LOG DATE & $\begin{array}{c}\text { SAMPLE } \\
\text { ID }\end{array}$ & $\begin{array}{l}\text { UNITS OF } \\
\text { MEASURE }\end{array}$ & PVI & $\begin{array}{l}\text { PARAMETER: } \\
\text { VALUE }\end{array}$ & FLAGS & $\begin{array}{l}\text { DETECTION } \\
\text { LIMIT }\end{array}$ & $\begin{array}{l}\text { PARAMETER } \\
\text { UNCERTAINTY }\end{array}$ \\
\hline ALKALINITY & $\begin{array}{l}02 / 01 / 89 \\
09 / 19 / 89 \\
04 / 11 / 90 \\
01 / 24 / 91 \\
12 / 03 / 92\end{array}$ & $\begin{array}{l}0001 \\
0001 \\
0001 \\
0001 \\
0001\end{array}$ & MG/L CACO3 & & $\begin{array}{l}466 . \\
463 . \\
538 . \\
356 . \\
447\end{array}$ & & $\begin{array}{l}- \\
- \\
-\end{array}$ & $\begin{array}{l}- \\
- \\
- \\
- \\
-\end{array}$ \\
\hline ALUAINUH & $\begin{array}{l}02 / 01 / 89 \\
09 / 19 / 89 \\
04 / 11 / 90 \\
01 / 24 / 91\end{array}$ & $\begin{array}{l}0001 \\
0001 \\
0001 \\
0001\end{array}$ & MG/L & $\begin{array}{l}< \\
<\end{array}$ & $\begin{array}{l}0.16 \\
0.1 \\
0.1 \\
0.05\end{array}$ & & $\begin{array}{l}0.1 \\
0.1 \\
0.1 \\
0.05\end{array}$ & $\dot{-}$ \\
\hline AHMOHIUNA & $\begin{array}{l}02 / 01 / 89 \\
09 / 19 / 89 \\
04 / 11 / 90 \\
12 / 03 / 92\end{array}$ & $\begin{array}{l}0001 \\
0001 \\
0001 \\
0001\end{array}$ & MG/L & $<$ & $\begin{array}{l}0.28 \\
0.1 \\
0.1 \\
0.2\end{array}$ & & $\begin{array}{l}0.1 \\
0.1 \\
0.1 \\
0.1\end{array}$ & - \\
\hline ANTIMONY & $\begin{array}{l}02 / 01 / 89 \\
09 / 19 / 89 \\
04 / 11 / 90 \\
01 / 24 / 91 \\
12 / 03 / 92\end{array}$ & $\begin{array}{l}0001 \\
0001 \\
0001 \\
0001 \\
0001\end{array}$ & MG/L & $<$ & $\begin{array}{l}0.003 \\
0.020 \\
0.040 \\
0.003 \\
0.003\end{array}$ & & $\begin{array}{l}0.003 \\
0.003 \\
0.003 \\
0.003 \\
0.003\end{array}$ & $\begin{array}{l}- \\
- \\
- \\
-\end{array}$ \\
\hline ARSEHIC & $\begin{array}{l}02 / 01 / 89 \\
09 / 19 / 89 \\
04 / 11 / 90 \\
01 / 24 / 91\end{array}$ & $\begin{array}{l}0001 \\
0001 \\
0001 \\
0001\end{array}$ & MG/L & $\begin{array}{l}< \\
<\end{array}$ & $\begin{array}{l}0.001 \\
0.02 \\
0.01 \\
0.03\end{array}$ & $\mathbf{J}$ & $\begin{array}{l}0.01 \\
0.01 \\
0.01 \\
0.03\end{array}$ & $\begin{array}{l}- \\
- \\
-\end{array}$ \\
\hline BARIUM & $\begin{array}{l}02 / 01 / 89 \\
09 / 19 / 89 \\
04 / 11 / 90 \\
01 / 24 / 91\end{array}$ & $\begin{array}{l}0001 \\
0001 \\
0001 \\
0001\end{array}$ & $M G / L$ & $\begin{array}{l}< \\
< \\
<\end{array}$ & $\begin{array}{l}0.01 \\
0.1 \\
0.1 \\
0.01\end{array}$ & 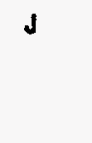 & $\begin{array}{l}0.1 \\
0.1 \\
0.1 \\
0.01\end{array}$ & - \\
\hline BERYLLIUM & $\begin{array}{l}02 / 01 / 89 \\
09 / 19 / 89 \\
04 / 11 / 90 \\
01 / 24 / 91\end{array}$ & $\begin{array}{l}0001 \\
0001 \\
0001 \\
0001\end{array}$ & $M G / L$ & $\begin{array}{l}< \\
< \\
< \\
<\end{array}$ & $\begin{array}{l}0.005 \\
0.01 \\
0.01 \\
0.005\end{array}$ & & $\begin{array}{l}0.005 \\
0.01 \\
0.01 \\
0.005\end{array}$ & $\begin{array}{l}- \\
- \\
-\end{array}$ \\
\hline BORON & $\begin{array}{l}02 / 01 / 89 \\
09 / 19 / 89 \\
04 / 11 / 90 \\
01 / 24 / 91\end{array}$ & $\begin{array}{l}0001 \\
0001 \\
0001 \\
0001\end{array}$ & MG/L & & $\begin{array}{l}0.50 \\
0.5 \\
0.5 \\
0.51\end{array}$ & & $\begin{array}{l}0.1 \\
0.1 \\
0.1 \\
0.05\end{array}$ & $\begin{array}{l}- \\
- \\
-\end{array}$ \\
\hline BROMIDE & $\begin{array}{l}02 / 01 / 89 \\
09 / 19 / 89 \\
04 / 11 / 90 \\
01 / 24 / 91\end{array}$ & $\begin{array}{l}0001 \\
0001 \\
0001 \\
0001\end{array}$ & MG/L & $<$ & $\begin{array}{l}1.28 \\
0.1 \\
0.3 \\
1.1\end{array}$ & $J$ & $\begin{array}{l}0.01 \\
0.1 \\
0.1 \\
0.1\end{array}$ & $\begin{array}{l}- \\
-\end{array}$ \\
\hline CADHIUH & $\begin{array}{l}02 / 01 / 89 \\
09 / 19 / 89 \\
04 / 11 / 90 \\
01 / 24 / 91 \\
12 / 03 / 92\end{array}$ & $\begin{array}{l}0001 \\
0009 \\
0001 \\
0001 \\
0001\end{array}$ & MG/L & $\begin{array}{l}< \\
<\end{array}$ & $\begin{array}{l}0.0005 \\
0.003 \\
0.001 \\
0.001 \\
0.001\end{array}$ & $\mathbf{J}$ & $\begin{array}{l}0.001 \\
0.001 \\
0.001 \\
0.001 \\
0.001\end{array}$ & $\begin{array}{l}- \\
- \\
-\end{array}$ \\
\hline CALCIUn & $\begin{array}{l}02 / 01 / 89 \\
09 / 19 / 89 \\
04 / 11 / 90\end{array}$ & $\begin{array}{l}0001 \\
0001 \\
0001\end{array}$ & MG/L & & $\begin{array}{l}444 . \\
472 . \\
411 .\end{array}$ & & $\begin{array}{l}0.01 \\
0.01 \\
0.01\end{array}$ & - \\
\hline
\end{tabular}

PARAMETER VALUE IHDICATOR (PVI): < - LESS THAN DETECTION LIMIT SAMPLE ID COOES:

0001 - FILTERED SAMPLE (.45 MICROHS)

OTHER PARAMETER VALUE FLAGS:

1 - IMCREASED DETECTIOH LIMIT DUE TO REQUIRED DILUTION

J - ESTIMATED VALUE 
GROUNDWATER QUALITY DATA BY LOCATION

SITE: AMBO1 AMBROSIA LAKE

LOCATION: 0674 RESERVED ESTORMS, MH, 11/14/88

HORTH COORD IMATE: UNKHOWH

EAST COORDINATE: UNKHOWN

06/10/80 TO $12 / 04 / 92$

REPORT DATE: 08/04/94

FORMATION OF COMPLETIOH: ALLUVIUM (AL)

HYDRAULIC FLON RELATIOHSHIP: DOWN GRADIENT (D)

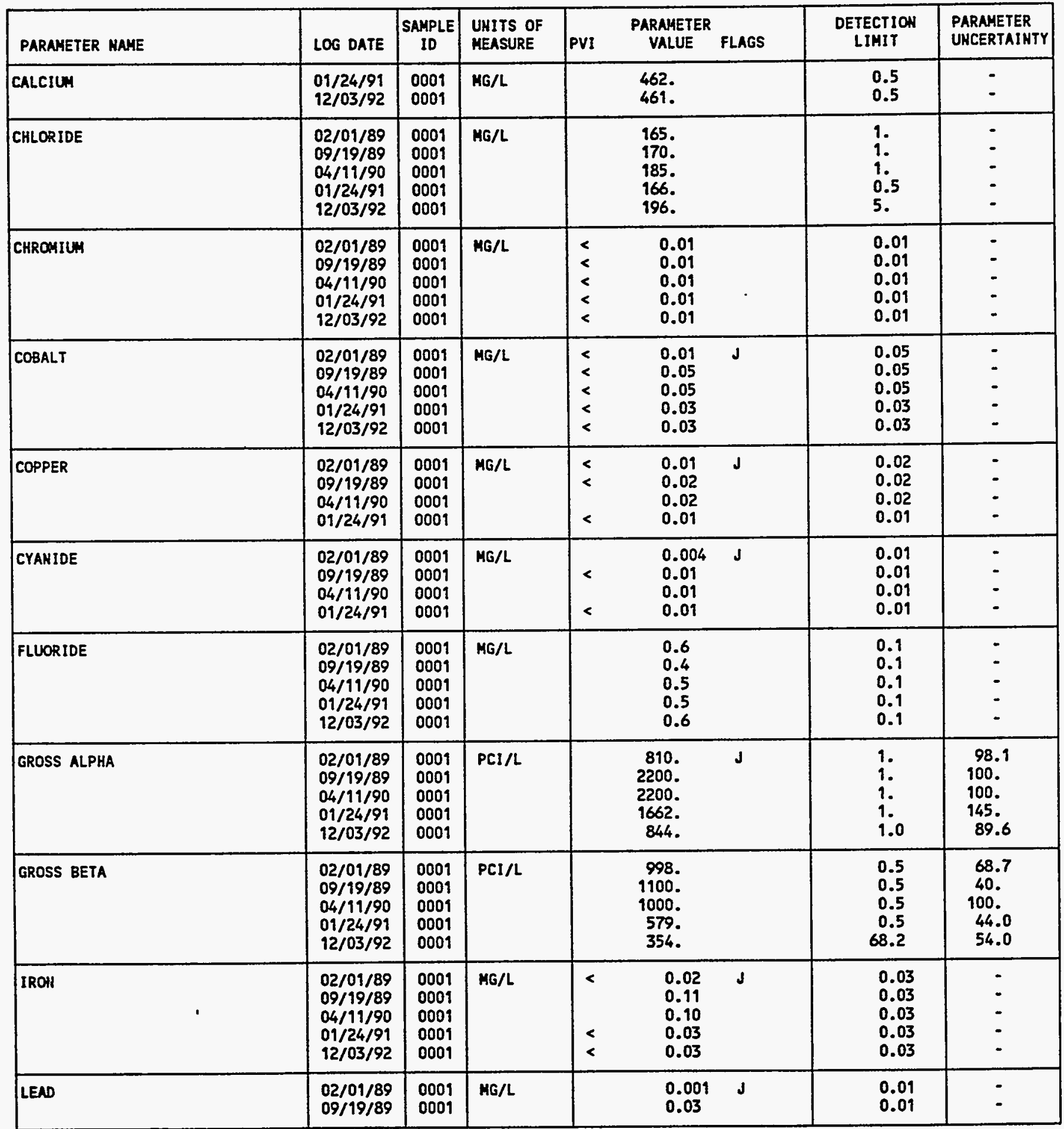

PARAMETER VALUE IMDICATOR (PVI): < - LESS THAN DETECTION LIMIT

SAMPLE ID CODES:

0001 - FILTERED SAMPLE (.45 MICRONS)

OTHER PARAMETER VALUE FLAGS:

$J$ - ESTIMATED VALUE 
GROUNDWATER QUALITY DATA BY LOCATIOH

SITE: AMBO1 AMBROSIA LAKE

LOCATIOH: 0674 RESERVED ESTORMS, MH, 11/14/88

HORTH COORDIHATE: UHKHOWH

EAST COORDIHATE: UHKNONM

06/10/80 TO $12 / 04 / 92$

REPORT DATE: 08/04/94

FORHATION OF CONPLETION: ALLUVIUM (AL)

HYDRAULIC FLON RELATIONSHIP: DON GRADIENT (D)

\begin{tabular}{|c|c|c|c|c|c|c|c|c|}
\hline PARAMETER NAME & LOG DATE & $\mid \begin{array}{c}\text { SAMPLE } \\
\text { ID }\end{array}$ & $\begin{array}{l}\text { UNITS OF } \\
\text { MEASURE }\end{array}$ & PVI & $\begin{array}{l}\text { PARAMETER } \\
\text { VALUE }\end{array}$ & FLAGS & $\begin{array}{l}\text { DETECTION } \\
\text { LIMIT }\end{array}$ & $\begin{array}{l}\text { PARAMETER } \\
\text { UNCERTAINTY }\end{array}$ \\
\hline LEAD & $\begin{array}{l}04 / 11 / 90 \\
01 / 24 / 91\end{array}$ & $\begin{array}{l}0001 \\
0001\end{array}$ & MG/L & $<$ & $\begin{array}{l}0.01 \\
0.03\end{array}$ & I & $\begin{array}{l}0.01 \\
0.03\end{array}$ & - \\
\hline LEAD-210 & $\begin{array}{l}02 / 01 / 89 \\
09 / 19 / 89\end{array}$ & $\begin{array}{l}0001 \\
0001\end{array}$ & $\mathrm{PCl} / \mathrm{L}$ & & $\begin{array}{l}0.00 \\
0.2\end{array}$ & & $\begin{array}{l}1.5 \\
1.5\end{array}$ & $\begin{array}{l}7.92 \\
0.6\end{array}$ \\
\hline MAGHESIUH & $\begin{array}{l}02 / 01 / 89 \\
09 / 19 / 89 \\
04 / 11 / 90 \\
01 / 24 / 91 \\
12 / 03 / 92\end{array}$ & $\begin{array}{l}0001 \\
0001 \\
0001 \\
0001 \\
0001\end{array}$ & MG/L & & $\begin{array}{l}230 . \\
243 . \\
229 . \\
246 \\
222 .\end{array}$ & & $\begin{array}{l}0.001 \\
0.001 \\
0.001 \\
0.5 \\
0.5\end{array}$ & $\begin{array}{l}- \\
- \\
- \\
-\end{array}$ \\
\hline MANGANESE & $\begin{array}{l}02 / 01 / 89 \\
09 / 19 / 89 \\
04 / 11 / 90 \\
01 / 24 / 91\end{array}$ & $\begin{array}{l}0001 \\
0001 \\
0001 \\
0001\end{array}$ & MG/L & & $\begin{array}{l}0.38 \\
0.06 \\
0.02 \\
0.03\end{array}$ & & $\begin{array}{l}0.01 \\
0.01 \\
0.01 \\
0.01\end{array}$ & 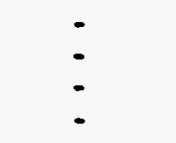 \\
\hline MERCURY & $\begin{array}{l}02 / 01 / 89 \\
09 / 19 / 89 \\
04 / 11 / 90 \\
01 / 24 / 91\end{array}$ & $\begin{array}{l}0001 \\
0001 \\
0001 \\
0001\end{array}$ & MG/L & $\begin{array}{l}< \\
< \\
<\end{array}$ & $\begin{array}{l}0.0001 \\
0.0002 \\
0.0002 \\
0.0002\end{array}$ & J & $\begin{array}{l}0.0002 \\
0.0002 \\
0.0002 \\
0.0002\end{array}$ & : \\
\hline MOLYBDENUM & $\begin{array}{l}02 / 01 / 89 \\
09 / 19 / 89 \\
04 / 11 / 90 \\
01 / 24 / 91 \\
12 / 03 / 92\end{array}$ & $\begin{array}{l}0001 \\
0001 \\
0001 \\
0001 \\
0001\end{array}$ & $M G / L$ & & $\begin{array}{l}9.81 \\
9.19 \\
8.62 \\
5.25 \\
5.75\end{array}$ & & $\begin{array}{l}0.01 \\
0.01 \\
0.01 \\
0.01 \\
0.05\end{array}$ & $\begin{array}{l}- \\
- \\
-\end{array}$ \\
\hline HET GROSS ALPHA * & $\begin{array}{l}02 / 01 / 89 \\
09 / 19 / 89 \\
04 / 11 / 90 \\
01 / 24 / 91 \\
12 / 03 / 92\end{array}$ & $\begin{array}{l}0001 \\
0001 \\
0001 \\
0001 \\
0001\end{array}$ & $\mathrm{PCI} / \mathrm{L}$ & & $\begin{array}{r}-1798.17 \\
-5140.20 \\
320.36 \\
-663.54 \\
-781.82\end{array}$ & & $\begin{array}{l}- \\
- \\
- \\
-\end{array}$ & :- \\
\hline NICKEL & $\begin{array}{l}02 / 01 / 89 \\
09 / 19 / 89 \\
04 / 11 / 90 \\
01 / 24 / 91 \\
12 / 03 / 92\end{array}$ & $\begin{array}{l}0001 \\
0001 \\
0001 \\
0001 \\
0001\end{array}$ & MG/L & $\begin{array}{l}< \\
< \\
<\end{array}$ & $\begin{array}{l}0.02 \\
0.04 \\
0.04 \\
0.04 \\
0.10\end{array}$ & $\mathbf{J}$ & $\begin{array}{l}0.04 \\
0.04 \\
0.04 \\
0.04 \\
0.04\end{array}$ & $\begin{array}{l}- \\
- \\
- \\
-\end{array}$ \\
\hline NITRATE & $\begin{array}{l}02 / 01 / 89 \\
09 / 19 / 89 \\
04 / 11 / 90 \\
12 / 03 / 92\end{array}$ & $\begin{array}{l}0001 \\
0001 \\
0001 \\
0001\end{array}$ & MG/L & & $\begin{array}{r}36.5 \\
9.7 \\
12.4 \\
69 .\end{array}$ & & $\begin{array}{l}1 . \\
1 . \\
10 .\end{array}$ & $\begin{array}{l}- \\
- \\
-\end{array}$ \\
\hline HITRITE AHD MITRATE & $04 / 11 / 90$ & 0001 & MG/L & & 2.8 & & 1. & - \\
\hline$P H$ & $\begin{array}{l}02 / 01 / 89 \\
09 / 19 / 89 \\
04 / 11 / 90 \\
01 / 24 / 91 \\
12 / 03 / 92\end{array}$ & $\begin{array}{l}0001 \\
0001 \\
0001 \\
0001 \\
0001\end{array}$ & su & & $\begin{array}{l}7.25 \\
7.27 \\
6.99 \\
7.61 \\
7.16\end{array}$ & & $\begin{array}{l}- \\
\dot{-} \\
\dot{-}\end{array}$ & $\begin{array}{l}- \\
- \\
-\end{array}$ \\
\hline PHOSPHATE & $09 / 19 / 89$ & 0001 & MG/L & $<$ & 0.1 & & 0.1 & - \\
\hline
\end{tabular}

* het gross alPHA (GROSS ALPHA - URANIUM) WITH I MG URAHIUM = 686 PCI

PARAMETER VALUE INDICATOR (PVI): < - LESS THAN DETECTION LIMIT

SAMPLE ID CODOS:

OOO1 - FILTERED SAMPLE (.45 MICROHS)

OTHER PARAMETER VALUE FLAGS:

1 - IMCREASED DETECTION LIMIT DUE TO REOUIRED DILUTION

J - estimated VALUE 
GROUNDWATER QUALITY DATA BY LOCATIOH

SITE: AMBOI AMBROSIA LAKE

LOCATION: 0674 RESERVED ESTORMS, MH, 11/14/88

NORTH COOROINATE: UNKHOWN

EAST COORDIHATE: UHKHONN

06/10/80 TO 12/04/92

REPORT DATE: 08/04/94

FORMATION OF COMPLETION: ALLUVIUM (AL)

HYDRAULIC FLON RELATIONSHIP: DONN GRADIENT (D)

\begin{tabular}{|c|c|c|c|c|c|c|c|}
\hline PARNMETER NAME & LOG DATE & $\underset{10}{\text { SAMPLE }}$ & $\begin{array}{l}\text { UNITS OF } \\
\text { MEASURE }\end{array}$ & PVI & $\begin{array}{l}\text { PARAMETER } \\
\text { VALUE FLAGS }\end{array}$ & $\begin{array}{l}\text { DETECTION } \\
\text { LIHIT }\end{array}$ & $\begin{array}{l}\text { PARAMETER } \\
\text { UMCERTAINTY }\end{array}$ \\
\hline PHOSPHATE & $\begin{array}{l}04 / 11 / 90 \\
01 / 24 / 91 \\
12 / 03 / 92\end{array}$ & $\begin{array}{l}0001 \\
0001 \\
0001\end{array}$ & $M G / L$ & $<$ & $\begin{array}{l}0.1 \\
0.05 \\
3.2\end{array}$ & $\begin{array}{l}0.1 \\
0.05 \\
0.2\end{array}$ & - \\
\hline POLONIUAH-210 & $\begin{array}{l}02 / 01 / 89 \\
09 / 19 / 89\end{array}$ & $\begin{array}{l}0001 \\
0001\end{array}$ & $\mathrm{PCI} / \mathrm{L}$ & & $\begin{array}{l}0.621 \mathrm{~J} \\
1.5\end{array}$ & 1. & $\begin{array}{l}1.29 \\
0.5\end{array}$ \\
\hline RADIUM-226 & $\begin{array}{l}02 / 01 / 89 \\
09 / 19 / 89 \\
04 / 11 / 90 \\
12 / 03 / 92\end{array}$ & $\begin{array}{l}0001 \\
0001 \\
0001 \\
0001\end{array}$ & $\mathrm{PCI} / \mathrm{L}$ & & $\begin{array}{l}0.744 \\
0.0 \\
0.1 \\
0.4\end{array}$ & $\begin{array}{l}1 . \\
1 . \\
1.0 \\
1.0\end{array}$ & $\begin{array}{l}0.404 \\
0.1 \\
0.1 \\
0.7\end{array}$ \\
\hline RADIUH-228 & $\begin{array}{l}02 / 01 / 89 \\
09 / 19 / 89 \\
04 / 11 / 90 \\
12 / 03 / 92\end{array}$ & $\begin{array}{l}0001 \\
0001 \\
0001 \\
0001\end{array}$ & PCI/L & & $\begin{array}{l}0.00 \\
0.8 \\
0.2 \\
0.3\end{array}$ & $\begin{array}{l}1 . \\
1 . \\
1.0\end{array}$ & $\begin{array}{l}6.57 \\
2.0 \\
1.1 \\
1.4\end{array}$ \\
\hline SELENIUM & $\begin{array}{l}02 / 01 / 89 \\
09 / 19 / 89 \\
04 / 11 / 90 \\
01 / 24 / 91 \\
12 / 03 / 92\end{array}$ & $\begin{array}{l}0001 \\
0001 \\
0001 \\
0001 \\
0001\end{array}$ & MG/L & & $\begin{array}{l}1.390 \\
1.05 \\
0.043 \\
0.800 \\
3.1\end{array}$ & $\begin{array}{l}0.005 \\
0.005 \\
0.005 \\
0.005 \\
0.1\end{array}$ & $\begin{array}{l}- \\
- \\
- \\
-\end{array}$ \\
\hline scolum & $\begin{array}{l}02 / 01 / 89 \\
09 / 19 / 89 \\
04 / 11 / 90 \\
01 / 24 / 91 \\
12 / 03 / 92\end{array}$ & $\begin{array}{l}0001 \\
0001 \\
0001 \\
0001 \\
0001\end{array}$ & MG/L & & $\begin{array}{c}945 . \\
913 . \\
952 . \\
1060 . \\
757 .\end{array}$ & $\begin{array}{l}0.002 \\
0.002 \\
0.002 \\
5 . \\
5 .\end{array}$ & $\begin{array}{l}: \\
- \\
- \\
-\end{array}$ \\
\hline SPECIFIC CONDUCTANCE & $\begin{array}{l}02 / 01 / 89 \\
09 / 19 / 89 \\
04 / 11 / 90 \\
01 / 24 / 91 \\
12 / 03 / 92\end{array}$ & $\begin{array}{l}0001 \\
0009 \\
0001 \\
0001 \\
0001\end{array}$ & UHHO/CH & & $\begin{array}{l}3200 . \\
2000 . \\
3450 \\
3950 \\
4100\end{array}$ & $\begin{array}{l}- \\
\dot{-} \\
\dot{-}\end{array}$ & $\begin{array}{l}- \\
- \\
- \\
-\end{array}$ \\
\hline
\end{tabular}

PARAMETER VALUE INDICATOR (PVI): < - LESS THAN DETECTION LIMIT

SAMPLE ID CODES:

0001 - FILTERED SAMPLE (.45 MICRONS)

OTHER PARAMETER VALUE FLAGS:

h - HOLD TIME EXPIRED, VALUE SUSPECT

J - estimated VAlUE 
GROUNDWATER QUALITY DATA BY LOCATION

SITE: AMBOT AMBROSIA LAKE

LOCATION: 0674 RESERVED ESTORMS, MW, 11/14/88

HORTH COORDINATE: UNKHOWN

EAST COORDINATE: UNKNOWN

O6/10/80 TO $12 / 04 / 92$

REPORT DATE: 08/04/94

FORMATION OF COMPLETION: ALLUVIUM (AL)

HYDRAULIC FLOW RELATIONSHIP: DOWN GRADIENT (D)

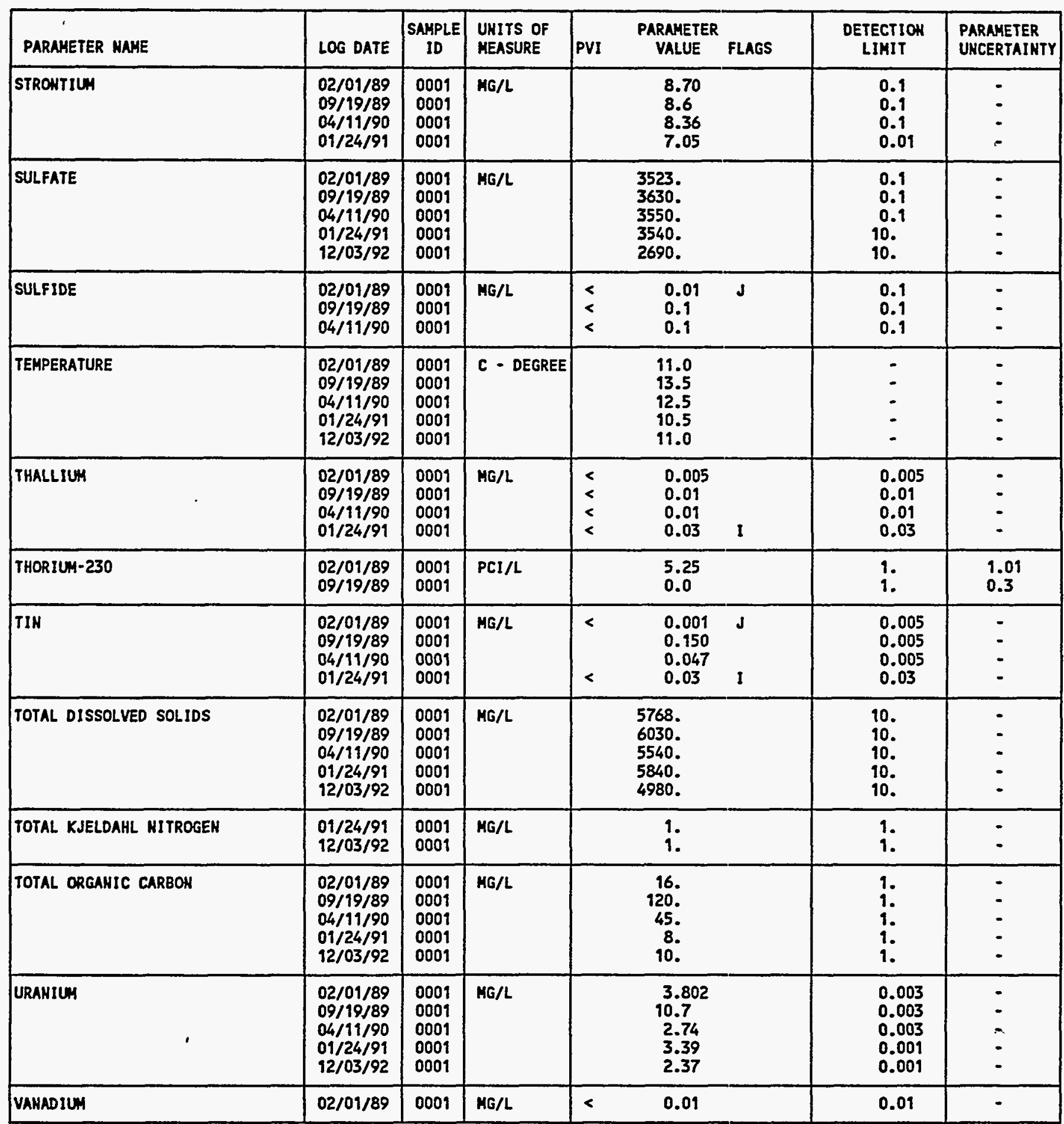

PARAMETER VALUE IMDICATOR (PVI): < - LESS THAM DETECTION LIMIT

SAMPLE ID CODES:

0001 - FILTIERED SAMPLE (.45 MICRONS)

OTHER PARAMETER VALUE FLAGS:

I - IHCREASED DETECTION LIMIT DUE TO REQUIRED DILUTION

J - estimated value 
GROUNDHATER QUALITY DATA BY LOCATION

SITE: AMBOI AMBROSIA LAKE

LOCATION: 0674 RESERVED ESTORMS, MH, 11/14/88

NORTH COORDIHATE: UNKHONN

EAST COORDIHATE: UNKNOWN

06/10/80 TO $12 / 04 / 92$

REPORT DATE: $08 / 04 / 94$

FORMATIOH OF COHPLETION: ALLUVIUN (AL)

HYDRAULIC FLOW RELATIONSHIP: DOWN GRADIENT (D)

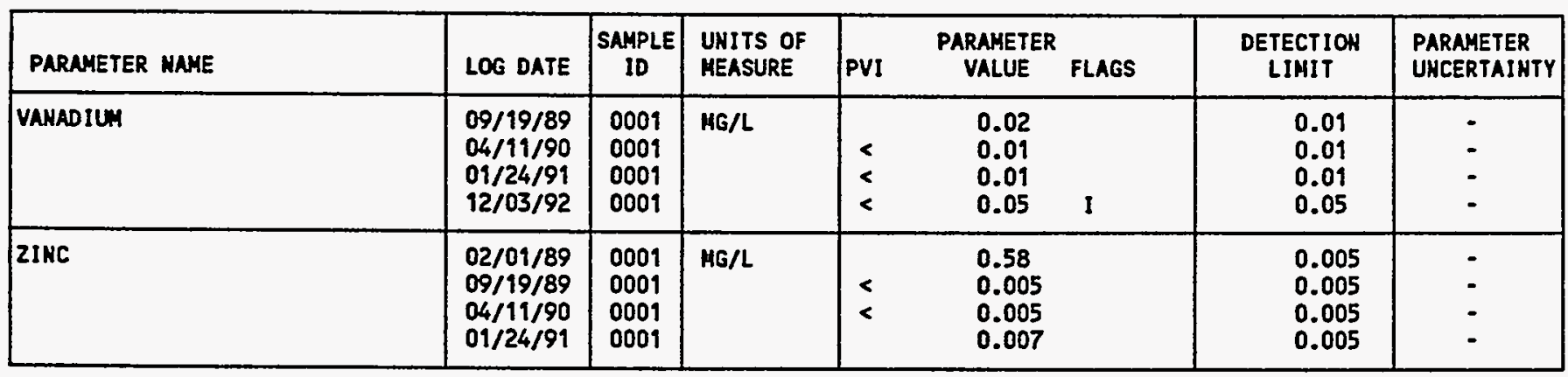

PARAMETER VALUE IMDICATOR (PVI): < - LESS THAN DETECTION LIMIT

SAMPLE ID COOES:

0001 - FILTERED SAMPLE (.45 MICRONS)

OTHER PARAMETER VALUE FLAGS:

I - INCREASED DETECTION LIMIT DUE TO REQUIRED DILUTION 
SITE: AMBO1 AMBROSIA LAKE

LOCATIOH: 0675 RESERVED ESTORMS, MH, 11/14/88

NORTH COORDINATE: UNKHOWN

EAST COORDINATE: UNKHOWH

06/10/80 TO $12 / 04 / 92$

REPORT DATE: 08/04/94

FORHATION OF COHPLETION: MAHCOS SHALE (KM)

HYDRAULIC FLOH RELATIOHSHIP: DOWH GRADIENT (D)

\begin{tabular}{|c|c|c|c|c|c|c|c|c|}
\hline PARAMETER HAHE & LOG DATE & 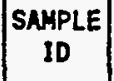 & $\begin{array}{l}\text { UHITS OF } \\
\text { MEASURE }\end{array}$ & PVI & $\begin{array}{l}\text { PARAMETER } \\
\text { VALUE }\end{array}$ & FLAGS & $\begin{array}{l}\text { DETECTIOH } \\
\text { LIMIT }\end{array}$ & $\begin{array}{l}\text { PARAMETER } \\
\text { UNCERTAINTY }\end{array}$ \\
\hline ALKALINITY & $\begin{array}{l}02 / 02 / 89 \\
09 / 14 / 89 \\
04 / 11 / 90 \\
01 / 16 / 91\end{array}$ & $\begin{array}{l}0001 \\
0001 \\
0001 \\
0001\end{array}$ & MG/L CACO3 & & $\begin{array}{l}551 . \\
428 . \\
484 . \\
278 .\end{array}$ & & $\begin{array}{l}- \\
\dot{-} \\
\dot{-}\end{array}$ & - \\
\hline ALUAINUH & $\begin{array}{l}02 / 02 / 89 \\
09 / 14 / 89 \\
04 / 11 / 90 \\
01 / 16 / 91\end{array}$ & $\begin{array}{l}0001 \\
0001 \\
0001 \\
0001\end{array}$ & HG/L & $\begin{array}{l}< \\
< \\
<\end{array}$ & $\begin{array}{l}0.05 \\
0.1 \\
0.1 \\
0.05\end{array}$ & $J$ & $\begin{array}{l}0.1 \\
0.1 \\
0.1 \\
0.05\end{array}$ & : \\
\hline AMMONILY & $\begin{array}{l}02 / 02 / 89 \\
09 / 14 / 89 \\
04 / 11 / 90 \\
01 / 16 / 91\end{array}$ & $\begin{array}{l}0001 \\
0001 \\
0001 \\
0001\end{array}$ & MG/L & $<$ & $\begin{array}{l}0.15 \\
0.1 \\
0.1 \\
0.28\end{array}$ & & $\begin{array}{l}0.1 \\
0.1 \\
0.1 \\
0.01\end{array}$ & $\begin{array}{l}- \\
- \\
-\end{array}$ \\
\hline ANT IMONY & $\begin{array}{l}02 / 02 / 89 \\
09 / 14 / 89 \\
04 / 11 / 90 \\
01 / 16 / 91\end{array}$ & $\begin{array}{l}0001 \\
0001 \\
0001 \\
0001\end{array}$ & MG/L & $<$ & $\begin{array}{l}0.003 \\
0.021 \\
0.053 \\
0.003\end{array}$ & & $\begin{array}{l}0.003 \\
0.003 \\
0.003 \\
0.003\end{array}$ & $\begin{array}{l}- \\
-\end{array}$ \\
\hline ARSENIC & $\begin{array}{l}02 / 02 / 89 \\
09 / 14 / 89 \\
04 / 11 / 90 \\
01 / 16 / 91\end{array}$ & $\begin{array}{l}0001 \\
0001 \\
0001 \\
0001\end{array}$ & MG/L & $\begin{array}{l}< \\
< \\
<\end{array}$ & $\begin{array}{l}0.001 \\
0.02 \\
0.01 \\
0.03\end{array}$ & $\begin{array}{l}J \\
I\end{array}$ & $\begin{array}{l}0.01 \\
0.01 \\
0.01 \\
0.03\end{array}$ & : \\
\hline BARIUM & $\begin{array}{l}02 / 02 / 89 \\
09 / 14 / 89 \\
04 / 11 / 90 \\
01 / 16 / 91\end{array}$ & $\begin{array}{l}0001 \\
0001 \\
0001 \\
0001\end{array}$ & MG/L & $<$ & $\begin{array}{l}0.02 \\
0.1 \\
0.1 \\
0.01\end{array}$ & J & $\begin{array}{l}0.1 \\
0.1 \\
0.1 \\
0.01\end{array}$ & : \\
\hline BERYLLIUHA & $\begin{array}{l}02 / 02 / 89 \\
09 / 14 / 89 \\
04 / 11 / 90 \\
01 / 16 / 91\end{array}$ & $\begin{array}{l}0001 \\
0001 \\
0001 \\
0001\end{array}$ & MG/L & $\begin{array}{l}< \\
< \\
<\end{array}$ & $\begin{array}{l}0.005 \\
0.01 \\
0.01 \\
0.005\end{array}$ & & $\begin{array}{l}0.005 \\
0.01 \\
0.01 \\
0.005\end{array}$ & $\ddot{-}$ \\
\hline BOROH & $\begin{array}{l}02 / 02 / 89 \\
09 / 14 / 89 \\
04 / 11 / 90 \\
01 / 16 / 91\end{array}$ & $\begin{array}{l}0001 \\
0001 \\
0001 \\
0001\end{array}$ & MG/L & & $\begin{array}{l}0.91 \\
0.9 \\
0.8 \\
0.73\end{array}$ & & $\begin{array}{l}0.1 \\
0.1 \\
0.1 \\
0.05\end{array}$ & $\begin{array}{l}- \\
- \\
-\end{array}$ \\
\hline BROMIDE & $\begin{array}{l}02 / 02 / 89 \\
09 / 14 / 89 \\
04 / 11 / 90 \\
01 / 96 / 91\end{array}$ & $\begin{array}{l}0001 \\
0001 \\
0001 \\
0001\end{array}$ & MG/L & & $\begin{array}{l}2.86 \\
2.2 \\
2.0 \\
7.1\end{array}$ & J & $\begin{array}{l}0.01 \\
0.1 \\
0.1 \\
0.1\end{array}$ & $\begin{array}{l}- \\
- \\
-\end{array}$ \\
\hline CADMIUH & $\begin{array}{l}02 / 02 / 89 \\
09 / 14 / 89 \\
04 / 11 / 90 \\
01 / 16 / 91\end{array}$ & $\begin{array}{l}0001 \\
0001 \\
0001 \\
0001\end{array}$ & MG/L & $<$ & $\begin{array}{l}0.0003 \\
0.003 \\
0.001 \\
0.001\end{array}$ & J & $\begin{array}{l}0.001 \\
0.001 \\
0.001 \\
0.001\end{array}$ & $\begin{array}{l}: \\
- \\
-\end{array}$ \\
\hline CALCIUA & $\begin{array}{l}02 / 02 / 89 \\
09 / 14 / 89 \\
04 / 11 / 90 \\
01 / 16 / 91\end{array}$ & $\begin{array}{l}0001 \\
0001 \\
0001 \\
0001\end{array}$ & MG/L & & $\begin{array}{l}464 . \\
505 . \\
431 . \\
496\end{array}$ & & $\begin{array}{l}0.01 \\
0.01 \\
0.01 \\
0.01\end{array}$ & $\begin{array}{l}- \\
. \\
-\end{array}$ \\
\hline CHLORIOE & $02 / 02 / 89$ & 0001 & MG/L & & 309. & & 1. & - \\
\hline
\end{tabular}

PARAMETER VALUE INDICATOR (PVI): < - LeSS than DETECTION LIMIT

SAMPLE ID COOES:

0009 - FILTIERED SAMPLE (.45 MICROHS)

OTHER PARAMETER VALUE FLAGS:

1 - IHCREASED DETECTION LIHIT DUE TO REQUIRED DILUTION

d - estimated VALUe 
GROUNDHATER OUALITY DATA BY LOCATION

SITE: AMBO1 AMBROSIA LAKE

LOCATION: 0675 RESERVED ESTORMS, MH, 11/14/88

NORTH COORDINATE: UHKNOWH

EAST COORDINATE: UKKNOWH

06/10/80 TO $12 / 04 / 92$

REPORT DATE: 08/04/94

FORMATION OF COMPLETION: MANCOS SHALE (XN)

HYORAULIC FLOW RELATIONSHIP: DONN GRADIENT (D)

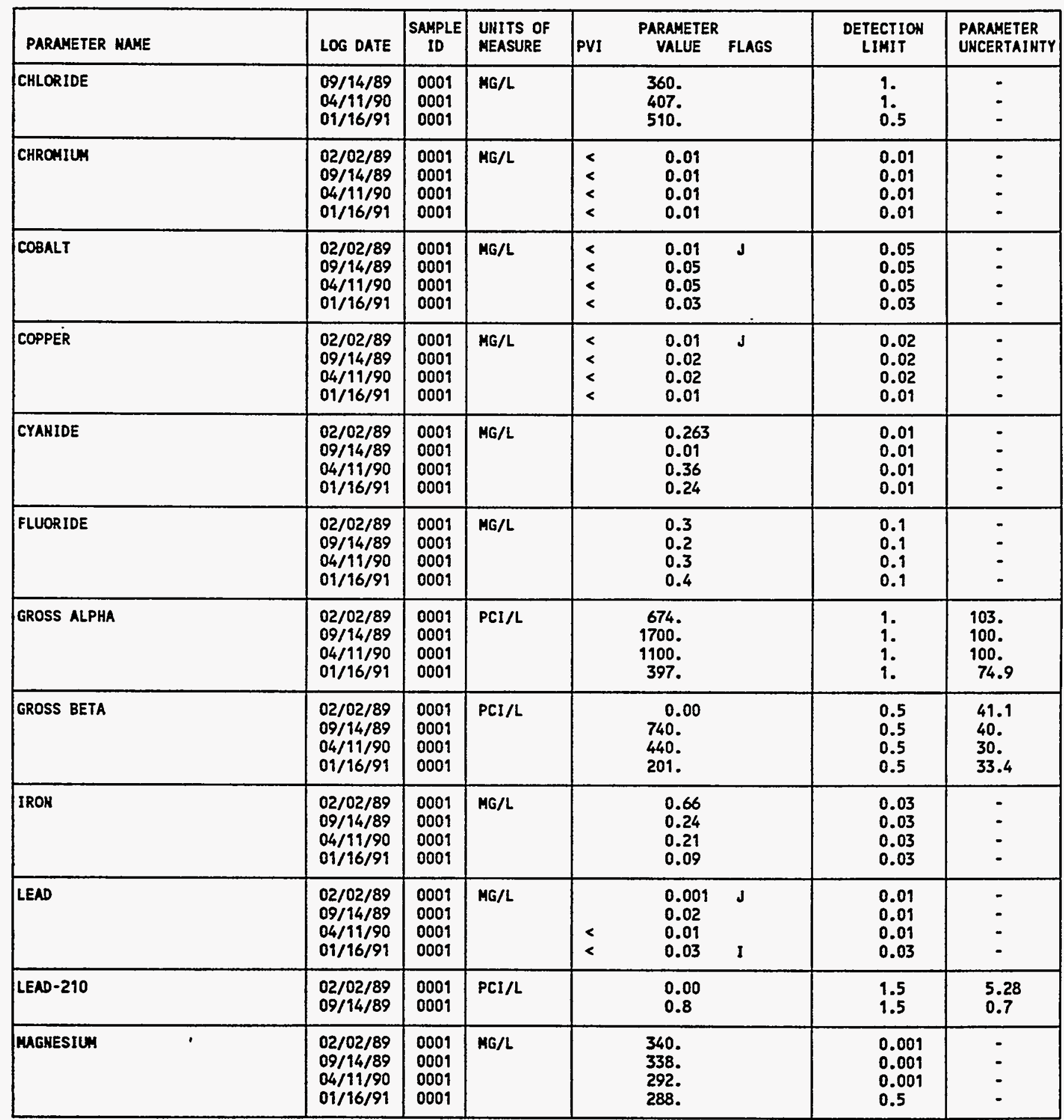

PARAMETER VALUE IMDICATOR (PVI): < - LESS THAN DETECTION LIMIT

SAMPLE ID COOES:

DOO1 - FILTERED SAMPLE (.45 MICRONS)

OTHER PARAMETER VALUE FLAGS:

I - INCREASED DETECTION LIMIT DUE TO REQUIRED DILUTION

d- estimated VALUe 
GROUHDHATER QUALITY DATA BY LOCATION

SITE: AMBOI AMBROSIA LAKE

LOCATION: 0675 RESERVED ESTORMS, MH, 11/14/88

NORTH COORDIHATE: UHKHOWN

EAST COORDIHATE: UHKNOWN

06/10/80 TO $12 / 04 / 92$

REPORT DATE: $08 / 04 / 94$

FORMATIOH OF COMPLETION: MANCOS SHALE (KM)

HYDRAULIC FLON RELATIONSHIP: DOWH GRADIENT (D)

\begin{tabular}{|c|c|c|c|c|c|c|c|c|}
\hline PARAMETER MAHE & LOG DATE & $\begin{array}{l}\text { SAMPLE } \\
\text { ID }\end{array}$ & $\begin{array}{l}\text { UNITS OF } \\
\text { MEASURE }\end{array}$ & PVI & $\begin{array}{l}\text { PARAMETER } \\
\text { VALUE }\end{array}$ & FLAGS & $\begin{array}{l}\text { DETECTION } \\
\text { LIMIT }\end{array}$ & $\begin{array}{l}\text { PARAMETER } \\
\text { UNCERTAIHTY }\end{array}$ \\
\hline MAHGAHESE & $\begin{array}{l}02 / 02 / 89 \\
09 / 14 / 89 \\
04 / 11 / 90 \\
01 / 16 / 91\end{array}$ & $\begin{array}{l}0001 \\
0001 \\
0001 \\
0001\end{array}$ & MG/L & $<$ & $\begin{array}{l}0.10 \\
0.04 \\
0.04 \\
0.01\end{array}$ & & $\begin{array}{l}0.01 \\
0.01 \\
0.01 \\
0.01\end{array}$ & $\begin{array}{l}- \\
-\end{array}$ \\
\hline MERCURY & $\begin{array}{l}02 / 02 / 89 \\
09 / 14 / 89 \\
04 / 11 / 90 \\
01 / 16 / 91\end{array}$ & $\begin{array}{l}0001 \\
0001 \\
0001 \\
0001\end{array}$ & $M G / L$ & $\begin{array}{l}< \\
< \\
<\end{array}$ & $\begin{array}{l}0.0001 \\
0.0002 \\
0.0002 \\
0.0002\end{array}$ & J & $\begin{array}{l}0.0002 \\
0.0002 \\
0.0002 \\
0.0002\end{array}$ & $\begin{array}{l}- \\
-\end{array}$ \\
\hline MOLYBDEHUM & $\begin{array}{l}02 / 02 / 89 \\
09 / 14 / 89 \\
04 / 11 / 90 \\
01 / 16 / 91\end{array}$ & $\begin{array}{l}0001 \\
0001 \\
0001 \\
0001\end{array}$ & $M G / L$ & & $\begin{array}{l}2.72 \\
1.77 \\
1.11 \\
0.28\end{array}$ & & $\begin{array}{l}0.01 \\
0.01 \\
0.01 \\
0.01\end{array}$ & $\begin{array}{l}- \\
- \\
-\end{array}$ \\
\hline HET GROSS ALPHA * & $\begin{array}{l}02 / 02 / 89 \\
09 / 14 / 89 \\
04 / 11 / 90 \\
01 / 16 / 91\end{array}$ & $\begin{array}{l}0001 \\
0001 \\
0001 \\
0001\end{array}$ & $\mathrm{PCI} / \mathrm{L}$ & & $\begin{array}{r}-754.94 \\
993.42 \\
478.48 \\
-302.72\end{array}$ & & $\begin{array}{l}- \\
-\end{array}$ & $\begin{array}{l}- \\
- \\
-\end{array}$ \\
\hline NICKEL & $\begin{array}{l}02 / 02 / 89 \\
09 / 14 / 89 \\
04 / 11 / 90 \\
01 / 16 / 91\end{array}$ & $\begin{array}{l}0001 \\
0001 \\
0001 \\
0001\end{array}$ & $M G / L$ & $\begin{array}{l}< \\
< \\
<\end{array}$ & $\begin{array}{l}0.02 \\
0.04 \\
0.04 \\
0.04\end{array}$ & J & $\begin{array}{l}0.04 \\
0.04 \\
0.04 \\
0.04\end{array}$ & $\dot{-}$ \\
\hline HITRATE & $\begin{array}{l}02 / 02 / 89 \\
09 / 14 / 89 \\
04 / 11 / 90 \\
01 / 16 / 91\end{array}$ & $\begin{array}{l}0001 \\
0001 \\
0001 \\
0001\end{array}$ & $M G / L$ & & $\begin{array}{l}138.6 \\
160 . \\
173 . \\
252 .\end{array}$ & & $\begin{array}{l}1 . \\
1 . \\
1 . \\
0.1\end{array}$ & - \\
\hline NITRITE AMD NITRATE & $\begin{array}{l}04 / 11 / 90 \\
01 / 16 / 91\end{array}$ & $\begin{array}{l}0001 \\
0001\end{array}$ & $M G / L$ & & $\begin{array}{l}39.0 \\
54.0\end{array}$ & & $\begin{array}{l}1.05 \\
0.05\end{array}$ & - \\
\hline $\mathrm{PH}$ & $\begin{array}{l}02 / 02 / 89 \\
09 / 14 / 89 \\
04 / 11 / 90 \\
01 / 16 / 91\end{array}$ & $\begin{array}{l}0001 \\
0001 \\
0001 \\
0001\end{array}$ & SU & & $\begin{array}{l}7.03 \\
6.95 \\
6.81 \\
7.20\end{array}$ & & $\dot{-}$ & $\dot{-}$ \\
\hline PHOSPHATE & $\begin{array}{l}02 / 02 / 89 \\
09 / 14 / 89 \\
04 / 11 / 90 \\
01 / 16 / 91\end{array}$ & $\begin{array}{l}0001 \\
0001 \\
0001 \\
0001\end{array}$ & $M G / L$ & $<$ & $\begin{array}{l}0.1 \\
0.1 \\
0.3 \\
0.10\end{array}$ & & $\begin{array}{l}0.1 \\
0.1 \\
0.1 \\
0.05\end{array}$ & $\begin{array}{l}- \\
-\end{array}$ \\
\hline POLONIUA-210 & $\begin{array}{l}02 / 02 / 89 \\
09 / 14 / 89\end{array}$ & $\begin{array}{l}0001 \\
0001\end{array}$ & $\mathrm{PCI} / \mathrm{L}$ & & $\begin{array}{l}0.00 \\
1.3\end{array}$ & & 1. & $\begin{array}{l}1.01 \\
0.5\end{array}$ \\
\hline POTASSIUA & $\begin{array}{l}02 / 02 / 89 \\
09 / 14 / 89 \\
04 / 11 / 90 \\
01 / 16 / 91\end{array}$ & $\begin{array}{l}0001 \\
0001 \\
0001 \\
0001\end{array}$ & HG/L & & $\begin{array}{r}15.6 \\
11.6 \\
1.8 \\
7.6\end{array}$ & & $\begin{array}{l}0.01 \\
0.01 \\
0.01 \\
0.01\end{array}$ & $\because$ \\
\hline RADIUA-226 & $\begin{array}{l}02 / 02 / 89 \\
09 / 14 / 89 \\
04 / 11 / 90 \\
01 / 16 / 91\end{array}$ & $\begin{array}{l}0001 \\
0001 \\
0001 \\
0001\end{array}$ & $\mathrm{PCI} / \mathrm{L}$ & & $\begin{array}{l}2.05 \\
0.4 \\
0.2 \\
1.6\end{array}$ & & $\begin{array}{l}1 . \\
1 . \\
1 .\end{array}$ & $\begin{array}{l}0.271 \\
0.2 \\
0.2 \\
0.6\end{array}$ \\
\hline
\end{tabular}

* Net gROSS ALPHA (GROSS ALPHA - URAHIUH) WITH 1 MG URANILH = 686 PCI

PARAMETER VALUE IMDICATOR (PVI): < - LESS THAH DETECTION LIMIT 
GROUNDWATER QUALITY DATA BY LOCATION

SITE: AMBO1 AMBROSIA LAKE

LOCATIOH: 0675 RESERVED ESTORMS, MH, 11/14/88

HORTH COORDIHATE: UHKHOWH

EAST COORDINATE: UNKNOWH

$06 / 10 / 80$ TO $12 / 04 / 92$

REPORT DATE: 08/04/94

FORMATION OF COMPLETION: MANCOS SHALE (KM)

HYDRAULIC FLOH RELATIOHSKIP: DOWN GRADIENT (D)

\begin{tabular}{|c|c|c|c|c|c|c|c|}
\hline PARAMETER MAME & LOG DATE & $\begin{array}{c}\text { SAMPLE } \\
\text { ID }\end{array}$ & $\begin{array}{l}\text { UHITS OF } \\
\text { MEASURE }\end{array}$ & PVI & $\begin{array}{l}\text { PARAMETER } \\
\text { VALUE FLAGS }\end{array}$ & $\begin{array}{l}\text { DETECTION } \\
\text { LIMIT }\end{array}$ & $\begin{array}{l}\text { PARAMETER } \\
\text { UNCERTAIMTY }\end{array}$ \\
\hline RADIUH-226 + RADIUA-228 & $\begin{array}{l}02 / 02 / 89 \\
09 / 14 / 89 \\
04 / 11 / 90 \\
01 / 16 / 91\end{array}$ & $\begin{array}{l}0001 \\
0001 \\
0001 \\
0001\end{array}$ & PCI/L & & $\begin{array}{l}4.03 \\
1.50 \\
1.50 \\
2.60\end{array}$ & $\begin{array}{l}- \\
- \\
-\end{array}$ & $\begin{array}{l}- \\
- \\
-\end{array}$ \\
\hline RADIUA-228 & $\begin{array}{l}02 / 02 / 89 \\
09 / 14 / 89 \\
04 / 11 / 90 \\
01 / 16 / 91\end{array}$ & $\begin{array}{l}0001 \\
0001 \\
0001 \\
0001\end{array}$ & PCI/L & & $\begin{array}{l}1.98 \\
1.1 \\
1.3 \\
1.0\end{array}$ & $\begin{array}{l}1 . \\
1 . \\
1 . \\
1 .\end{array}$ & $\begin{array}{l}8.10 \\
0.8 \\
0.9 \\
1.6\end{array}$ \\
\hline SELENIUM & $\begin{array}{l}02 / 02 / 89 \\
09 / 14 / 89 \\
04 / 11 / 90 \\
01 / 16 / 91\end{array}$ & $\begin{array}{l}0001 \\
0001 \\
0001 \\
0001\end{array}$ & MG/L & & $\begin{array}{l}0.510 \\
0.421 \\
0.030 \\
0.500\end{array}$ & $\begin{array}{l}0.005 \\
0.005 \\
0.005 \\
.0 .005\end{array}$ & $\begin{array}{l}- \\
- \\
=\end{array}$ \\
\hline SILICA - SIOZ & $\begin{array}{l}02 / 02 / 89 \\
09 / 14 / 89 \\
04 / 11 / 90 \\
01 / 16 / 91\end{array}$ & $\begin{array}{l}0001 \\
0001 \\
0001 \\
0001\end{array}$ & $M G / L$ & & $\begin{array}{l}10.0 \\
11 . \\
10 . \\
9.8\end{array}$ & $\begin{array}{l}2 . \\
2 . \\
2 . \\
0.1\end{array}$ & $\begin{array}{l}- \\
- \\
-\end{array}$ \\
\hline SILVER & $\begin{array}{l}02 / 02 / 89 \\
09 / 14 / 89 \\
04 / 11 / 90 \\
01 / 16 / 91\end{array}$ & $\begin{array}{l}0001 \\
0001 \\
0001 \\
0001\end{array}$ & MG/L & $\begin{array}{l}< \\
< \\
<\end{array}$ & $\begin{array}{l}0.01 \\
0.02 \\
0.01 \\
0.01\end{array}$ & $\begin{array}{l}0.01 \\
0.01 \\
0.01 \\
0.01\end{array}$ & $\begin{array}{l}- \\
- \\
-\end{array}$ \\
\hline soorum & $\begin{array}{l}02 / 02 / 89 \\
09 / 14 / 89 \\
04 / 11 / 90 \\
01 / 16 / 91\end{array}$ & $\begin{array}{l}0001 \\
0001 \\
0001 \\
0001\end{array}$ & MG/L & & $\begin{array}{l}1200 . \\
1090 . \\
1090 \\
1050 .\end{array}$ & $\begin{array}{l}0.002 \\
0.002 \\
0.002 \\
5 .\end{array}$ & $\begin{array}{l}- \\
- \\
-\end{array}$ \\
\hline SPECIFIC CONDUCTANCE & $\begin{array}{l}02 / 02 / 89 \\
09 / 14 / 89 \\
04 / 11 / 90 \\
01 / 16 / 91\end{array}$ & $\begin{array}{l}0001 \\
0001 \\
0001 \\
0001\end{array}$ & ЕнHО/CM & & $\begin{array}{l}3600 . \\
2700 \\
4500 \\
4600\end{array}$ & $\begin{array}{l}- \\
- \\
-\end{array}$ & - \\
\hline STRONTIUM & $\begin{array}{l}02 / 02 / 89 \\
09 / 14 / 89 \\
04 / 11 / 90 \\
01 / 16 / 91\end{array}$ & $\begin{array}{l}0001 \\
0001 \\
0001 \\
0001\end{array}$ & $M G / L$ & & $\begin{array}{l}12.2 \\
12.0 \\
11.5 \\
10.9\end{array}$ & $\begin{array}{l}0.1 \\
0.1 \\
0.1 \\
0.01\end{array}$ & - \\
\hline SULFATE & $\begin{array}{l}02 / 02 / 89 \\
09 / 14 / 89 \\
04 / 11 / 90 \\
01 / 16 / 91\end{array}$ & $\begin{array}{l}0001 \\
0001 \\
0001 \\
0001\end{array}$ & MG/L & & $\begin{array}{l}4141 . \\
4110 . \\
3770 . \\
3340 .\end{array}$ & $\begin{array}{c}0.1 \\
0.1 \\
0.1 \\
10 .\end{array}$ & $\begin{array}{l}- \\
- \\
-\end{array}$ \\
\hline SULFIDE & $\begin{array}{l}02 / 02 / 89 \\
09 / 14 / 89 \\
04 / 11 / 90 \\
01 / 16 / 91\end{array}$ & $\begin{array}{l}0001 \\
0001 \\
0001 \\
0001\end{array}$ & $M G / L$ & $\begin{array}{l}< \\
< \\
< \\
<\end{array}$ & $\begin{array}{l}0.01 \\
0.1 \\
0.1 \\
0.1\end{array}$ & $\begin{array}{l}0.1 \\
0.1 \\
0.1 \\
0.1\end{array}$ & - \\
\hline TEHPERATURE & $\begin{array}{l}02 / 02 / 89 \\
09 / 14 / 89 \\
04 / 11 / 90 \\
01 / 16 / 91\end{array}$ & $\begin{array}{l}0001 \\
0001 \\
0001 \\
0001\end{array}$ & C - DEGREE & & $\begin{array}{l}12.0 \\
12.0 \\
12 . \\
11.5\end{array}$ & $\begin{array}{l}- \\
- \\
-\end{array}$ & - \\
\hline THALLIUI & $\begin{array}{l}02 / 02 / 89 \\
09 / 14 / 89\end{array}$ & $\begin{array}{l}0001 \\
0001\end{array}$ & MG/L & $<$ & $\begin{array}{l}0.005 \\
0.01\end{array}$ & $\begin{array}{l}0.005 \\
0.01\end{array}$ & - \\
\hline
\end{tabular}

PARAHETER VALUE IMDICATOR (PVI): < - LESS THAN DETECTION LIMIT

SAMPLE ID CODES:

0001 - FILTERED SAMPLE (.45 HICRONS)

OTHER PARAHETER VALUE FLAGS:

J - estimated value 
GROUNDWATER QUALITY DATA BY LOCATION

SITE: AMBOI AMBROSIA LAKE

LOCATION: 0675 RESERVED ESTORMS, MH, 11/14/88

MORTH COORDIHATE: UHKHOWH

EAST COORDIHATE: UNKHONH

O6/10/80 TO 12/04/92

REPORT DATE: 08/04/94

FORHATION OF COMPLETION: MAHCOS SHALE (KH)

HYDRAULIC FLOW RELATIONSHIP: DOWN GRADIENT (D)

\begin{tabular}{|c|c|c|c|c|c|c|c|}
\hline PARAHETER haHe & LOG DATE & $\mid \begin{array}{c}\text { SAMPLE } \\
\text { ID }\end{array}$ & $\begin{array}{l}\text { UNITS OF } \\
\text { MEASURE }\end{array}$ & PVI & $\begin{array}{l}\text { PARAMETER } \\
\text { VALUE FLAGS }\end{array}$ & $\begin{array}{l}\text { DETECTIOH } \\
\text { LIMIT }\end{array}$ & $\begin{array}{l}\text { PARAMETER } \\
\text { UHCERTAINTY }\end{array}$ \\
\hline THALLIUH & $\begin{array}{l}04 / 11 / 90 \\
01 / 16 / 91\end{array}$ & $\begin{array}{l}0001 \\
0001\end{array}$ & HG/L & $<$ & $\begin{array}{l}0.01 \\
0.03\end{array}$ & $\begin{array}{l}0.01 \\
0.03\end{array}$ & $\dot{-}$ \\
\hline THORIUH-230 & $\begin{array}{l}02 / 02 / 89 \\
09 / 14 / 89\end{array}$ & $\begin{array}{l}0001 \\
0009\end{array}$ & $\mathrm{PCI} / \mathrm{L}$ & & $\begin{array}{l}1.02 \\
0.1\end{array}$ & $\begin{array}{l}1 . \\
1 .\end{array}$ & $\begin{array}{l}0.423 \\
0.4\end{array}$ \\
\hline TOTAL DISSOLVED SOLIDS & $\begin{array}{l}02 / 02 / 89 \\
09 / 14 / 89 \\
04 / 11 / 90 \\
01 / 16 / 91\end{array}$ & $\begin{array}{l}0001 \\
0001 \\
0001 \\
0001\end{array}$ & MG/L & & $\begin{array}{l}7208 . \\
6650 . \\
6420 . \\
6350 .\end{array}$ & $\begin{array}{l}10 . \\
10 . \\
10 . \\
10 .\end{array}$ & $\begin{array}{l}- \\
-\end{array}$ \\
\hline URANIUH & $\begin{array}{l}02 / 02 / 89 \\
09 / 14 / 89 \\
04 / 11 / 90 \\
01 / 16 / 91\end{array}$ & $\begin{array}{l}0001 \\
0001 \\
0001 \\
0001\end{array}$ & MG/L & & $\begin{array}{l}2.083 \\
1.03 \\
0.906 \\
1.02\end{array}$ & $\begin{array}{l}0.003 \\
0.003 \\
0.003 \\
0.001\end{array}$ & $\begin{array}{l}- \\
- \\
-\end{array}$ \\
\hline VANADIUH & $\begin{array}{l}02 / 02 / 89 \\
09 / 14 / 89 \\
04 / 11 / 90 \\
01 / 16 / 91\end{array}$ & $\begin{array}{l}0001 \\
0001 \\
0001 \\
0001\end{array}$ & MG/L & $\begin{array}{l}< \\
< \\
< \\
<\end{array}$ & $\begin{array}{l}0.01 \\
0.01 \\
0.01 \\
0.01\end{array}$ & $\begin{array}{l}0.01 \\
0.01 \\
0.01 \\
0.01\end{array}$ & $\begin{array}{l}- \\
- \\
-\end{array}$ \\
\hline
\end{tabular}

PARAMETER VALUE INDICATOR (PVI): < - LESS THAN DETECTION LIHIT

SAMPLE ID LOOES

O001 - FILTERED SAMPLE (.45 MICRONS)

OTHER PARAMETER VALUE fLAGS:

1 - IHCREASED DETECTIOH LIMIT DUE TO REQUIRED DILUTIOH

$J$ - ESTIHATED VALUE 
GROUNDWATER QUALITY DATA BY LOCATION

SITE: AMBOI AMBROSIA LAKE

LOCATION: 0676 RESERVED ESTORMS, HH, 11/14/88

NORTH COORDINATE: UNKNONN

EAST COORDIHATE: UNKMOWH

06/10/80 TO $12 / 04 / 92$

REPORT DATE: 08/04/94

FORHATION OF COMPLETIOH: TRES HERMANOS - C2 SANDSTONE (TT) HYDRAULIC FLON RELATIONSHIP: CROSS GRADIENT (C)

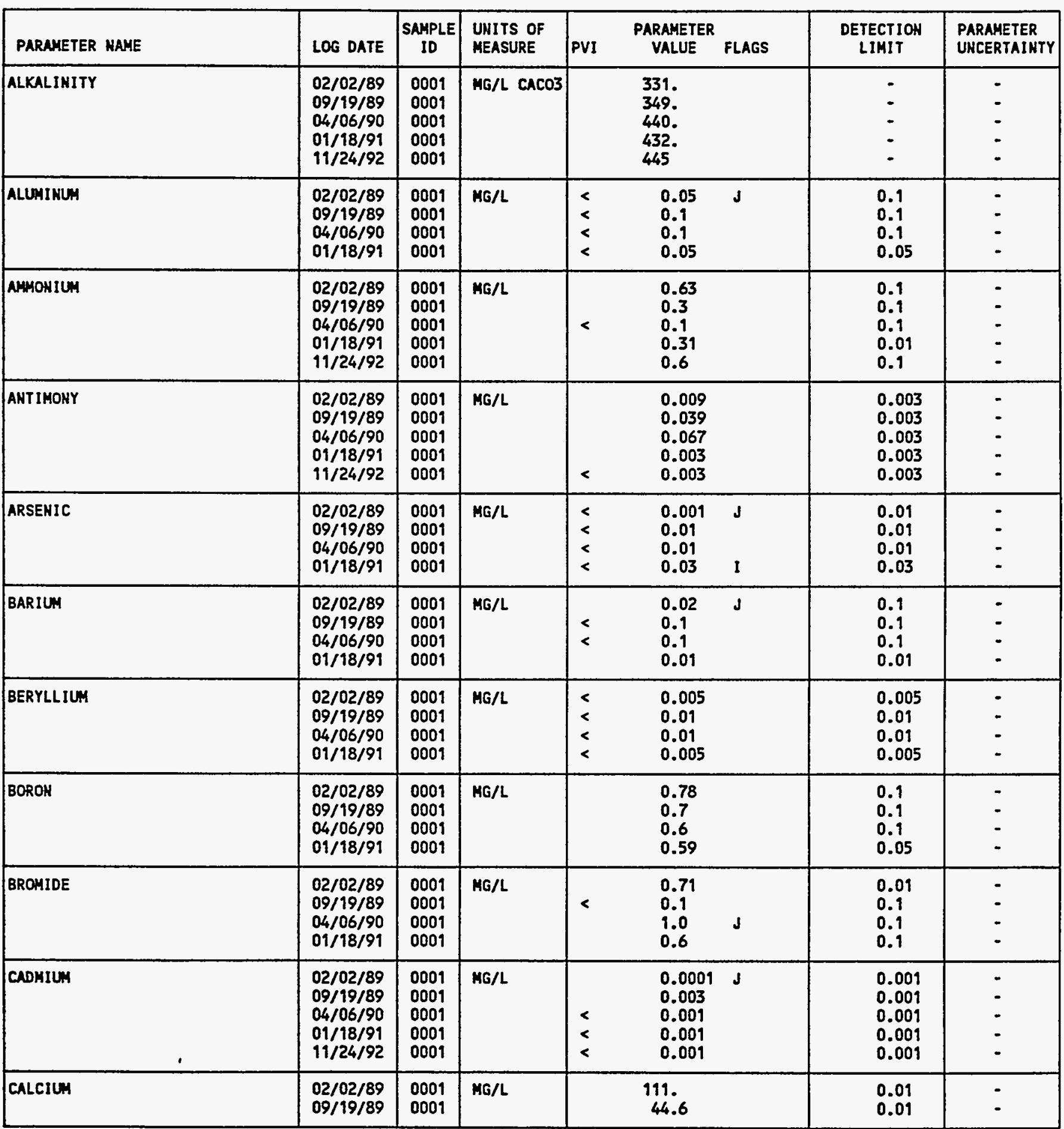

PARAMETER VALUE INDICATOR (PVI): < - LESS THAN DETECTION LIHIT

SAHPLE ID CODES

0001 - FILTERED SAMPLE (.45 MICRONS)

OTHER PARAMETER VALUE FLAGS:

1 - INCREASED DETECTIOH LIMIT DUE TO REQUIRED DILUTION

J. estimated VAlue 
GROUNDWATER QUALITY DATA BY LOCATIOH

SITE: AMBO1 AMBROSIA LAKE

LOCATIOH: 0676 RESERVED ESTORMS, MH, 11/14/88

HORTH COORDIMATE: UHKNOWH

EAST COORDIHATE: UNKHOWN

06/10/80 TO $12 / 04 / 92$

REPORT DATE: 08/04/94

FORMATION OF COMPLETIOH: TRES HERMANOS - C2 SANDSTONE (TT) HYORAULIC FLOW RELATIONSHIP: CROSS GRADIENT (C)

\begin{tabular}{|c|c|c|c|c|c|c|c|c|}
\hline $\begin{array}{c}\vdots \\
\text { PARAMETER MAME }\end{array}$ & LOG DATE & $\underset{10}{\text { SAMPLE }}$ & $\begin{array}{l}\text { UNITS OF } \\
\text { MEASURE }\end{array}$ & PVI & $\begin{array}{l}\text { PARAMETER } \\
\text { VALUE }\end{array}$ & FLAGS & $\begin{array}{l}\text { DETECTION } \\
\text { LIMIT }\end{array}$ & $\begin{array}{l}\text { PARAMETER } \\
\text { UNCERTAINTY }\end{array}$ \\
\hline CALCIUA & $\begin{array}{l}04 / 06 / 90 \\
01 / 18 / 91 \\
11 / 24 / 92\end{array}$ & $\begin{array}{l}0001 \\
0001 \\
0001\end{array}$ & MG/L & & $\begin{array}{l}34.4 \\
25.3 \\
26.0\end{array}$ & & $\begin{array}{l}0.01 \\
0.5 \\
0.5\end{array}$ & - \\
\hline CHLORIDE & $\begin{array}{l}02 / 02 / 89 \\
09 / 19 / 89 \\
04 / 06 / 90 \\
01 / 18 / 99 \\
11 / 24 / 92\end{array}$ & $\begin{array}{l}0001 \\
0001 \\
0001 \\
0001 \\
0001\end{array}$ & $M G / L$ & & $\begin{array}{l}210 . \\
150 . \\
31 . \\
32 . \\
28 .\end{array}$ & & $\begin{array}{l}1 . \\
1 . \\
1 . \\
0.5 \\
1 .\end{array}$ & $\begin{array}{l}\dot{-} \\
\dot{-} \\
\dot{-}\end{array}$ \\
\hline CHROAIUM & $\begin{array}{l}02 / 02 / 89 \\
09 / 19 / 89 \\
04 / 06 / 90 \\
01 / 18 / 91 \\
11 / 24 / 92\end{array}$ & $\begin{array}{l}0001 \\
0001 \\
0001 \\
0001 \\
0001\end{array}$ & MG/L & $\begin{array}{l}< \\
< \\
< \\
< \\
<\end{array}$ & $\begin{array}{l}0.01 \\
0.01 \\
0.01 \\
0.01 \\
0.01\end{array}$ & & $\begin{array}{l}0.01 \\
0.01 \\
0.01 \\
0.01 \\
0.01\end{array}$ & $\begin{array}{l}- \\
- \\
- \\
-\end{array}$ \\
\hline COBALT & $\begin{array}{l}02 / 02 / 89 \\
09 / 19 / 89 \\
04 / 06 / 90 \\
01 / 18 / 91 \\
11 / 24 / 92\end{array}$ & $\begin{array}{l}0001 \\
0001 \\
0001 \\
0001 \\
0001\end{array}$ & $M G / L$ & $\begin{array}{l}< \\
< \\
< \\
< \\
<\end{array}$ & $\begin{array}{l}0.01 \\
0.05 \\
0.05 \\
0.03 \\
0.03\end{array}$ & $J$ & $\begin{array}{l}0.05 \\
0.05 \\
0.05 \\
0.03 \\
0.03\end{array}$ & $\begin{array}{l}- \\
- \\
-\end{array}$ \\
\hline COPPER & $\begin{array}{l}02 / 02 / 89 \\
09 / 19 / 89 \\
04 / 06 / 90 \\
01 / 18 / 91\end{array}$ & $\begin{array}{l}0001 \\
0001 \\
0001 \\
0001\end{array}$ & HG/L & < & $\begin{array}{l}0.01 \\
0.02 \\
0.02 \\
0.01\end{array}$ & $J$ & $\begin{array}{l}0.02 \\
0.02 \\
0.02 \\
0.01\end{array}$ & $\begin{array}{l}- \\
-\end{array}$ \\
\hline CYANIDE & $\begin{array}{l}02 / 02 / 89 \\
09 / 19 / 89 \\
04 / 06 / 90 \\
01 / 18 / 91\end{array}$ & $\begin{array}{l}0001 \\
0001 \\
0001 \\
0001\end{array}$ & $M G / L$ & $\begin{array}{l}< \\
< \\
<\end{array}$ & $\begin{array}{l}0.002 \\
0.01 \\
0.01 \\
0.01\end{array}$ & J & $\begin{array}{l}0.01 \\
0.01 \\
0.01 \\
0.01\end{array}$ & $\dot{-}$ \\
\hline FLUORIDE & $\begin{array}{l}02 / 02 / 89 \\
09 / 19 / 89 \\
04 / 06 / 90 \\
01 / 18 / 91 \\
11 / 24 / 92\end{array}$ & $\begin{array}{l}0001 \\
0001 \\
0001 \\
0001 \\
0001\end{array}$ & MG/L & & $\begin{array}{l}1.1 \\
1.4 \\
0.7 \\
0.9 \\
0.9\end{array}$ & & $\begin{array}{l}0.1 \\
0.1 \\
0.1 \\
0.1 \\
0.1\end{array}$ & $\begin{array}{l}- \\
- \\
- \\
-\end{array}$ \\
\hline GROSS ALPHA & $\begin{array}{l}02 / 02 / 89 \\
04 / 06 / 90 \\
01 / 18 / 91 \\
11 / 24 / 92\end{array}$ & $\begin{array}{l}0001 \\
0001 \\
0001 \\
0001\end{array}$ & $\mathrm{PCI} / \mathrm{L}$ & & $\begin{array}{l}85.9 \\
24 . \\
48.1 \\
3.9\end{array}$ & H & $\begin{array}{l}1 . \\
1 . \\
1.0\end{array}$ & $\begin{array}{l}27.8 \\
11 . \\
23.1 \\
15.9\end{array}$ \\
\hline GROSS BETA & $\begin{array}{l}02 / 02 / 89 \\
04 / 06 / 90 \\
01 / 18 / 91 \\
11 / 24 / 92\end{array}$ & $\begin{array}{l}0001 \\
0001 \\
0001 \\
0001\end{array}$ & $\mathrm{PCI} / \mathrm{L}$ & & $\begin{array}{l}79.9 \\
15 . \\
20.7 \\
0.0\end{array}$ & & $\begin{array}{r}0.5 \\
0.5 \\
0.5 \\
29.5\end{array}$ & $\begin{array}{c}26.8 \\
8 . \\
9.8 \\
16: 3\end{array}$ \\
\hline IRON & $\begin{array}{l}02 / 02 / 89 \\
09 / 19 / 89 \\
04 / 06 / 90 \\
01 / 18 / 91 \\
11 / 24 / 92\end{array}$ & $\begin{array}{l}0001 \\
0001 \\
0001 \\
0001 \\
0001\end{array}$ & $M G / L$ & $\begin{array}{l}< \\
< \\
<\end{array}$ & $\begin{array}{l}0.02 \\
0.03 \\
0.03 \\
0.03 \\
0.12\end{array}$ & $J$ & $\begin{array}{l}0.03 \\
0.03 \\
0.03 \\
0.03 \\
0.03\end{array}$ & $\begin{array}{l}- \\
\dot{-} \\
-\end{array}$ \\
\hline LEAD & $\begin{array}{l}02 / 02 / 89 \\
09 / 19 / 89\end{array}$ & $\begin{array}{l}0001 \\
0001\end{array}$ & MG/L & $<$ & $\begin{array}{l}0.001 \\
0.04\end{array}$ & $J$ & $\begin{array}{l}0.01 \\
0.01\end{array}$ & $\dot{-}$ \\
\hline
\end{tabular}

PARAMETER VAlUe IHDICATOR (PVI): < - LESS than DETECTION LIMIT

OTHER PARAMETER VALUE FLAGS:

H - HOLD TIME EXPIRED, VALUE SUSPECT

$\checkmark$ - ESTIMATED VALUE 
GROUHDWATER QUALITY DATA BY LOCATION

SITE: AMBO1 AMBROSIA LAKE

LOCATION: 0676 RESERVED ESTORHS, MH, 11/14/88

NORTH COOROINATE: UHKNOWN

EAST COORDINATE: UNKNOWN

$06 / 10 / 80$ TO $12 / 04 / 92$

REPORT DATE: 08/04/94

FORMATION OF COMPLETIOH: TRES HERHANOS - C2 SAHDSTOHE (TT) HYDRAULIC FLOW RELATIOWSHIP: CROSS GRADIENT (C)

\begin{tabular}{|c|c|c|c|c|c|c|c|c|}
\hline PARAMETER NAKE & LOG DATE & $\begin{array}{l}\text { SAMPLE } \\
\text { ID }\end{array}$ & $\begin{array}{l}\text { UHITS OF } \\
\text { MEASURE }\end{array}$ & PVI & $\begin{array}{l}\text { PARAMETER } \\
\text { VALUE }\end{array}$ & FLAGS & $\begin{array}{l}\text { DETECTIOH } \\
\text { LIMIT }\end{array}$ & $\begin{array}{l}\text { PARAMETER } \\
\text { UNCERTAINTY }\end{array}$ \\
\hline LEAD & $\begin{array}{l}04 / 06 / 90 \\
01 / 18 / 91\end{array}$ & $\begin{array}{l}0001 \\
0001\end{array}$ & MG/L & $<$ & $\begin{array}{l}0.01 \\
0.03\end{array}$ & I & $\begin{array}{l}0.01 \\
0.03\end{array}$ & - \\
\hline LEAD -210 & $02 / 02 / 89$ & 0001 & $\mathrm{PCI} / \mathrm{L}$ & & 0.00 & & 1.5 & 6.21 \\
\hline MAGHESILH & $\begin{array}{l}02 / 02 / 89 \\
09 / 19 / 89 \\
04 / 06 / 90 \\
01 / 18 / 91 \\
11 / 24 / 92\end{array}$ & $\begin{array}{l}0001 \\
0001 \\
0001 \\
0001 \\
0001\end{array}$ & MG/L & & $\begin{array}{r}33 . \\
15.2 \\
14.3 \\
10.0 \\
9.5\end{array}$ & & $\begin{array}{l}0.001 \\
0.001 \\
0.001 \\
0.1 \\
0.1\end{array}$ & $\begin{array}{l}- \\
- \\
-\end{array}$ \\
\hline MAKGANESE & $\begin{array}{l}02 / 02 / 89 \\
09 / 19 / 89 \\
04 / 06 / 90 \\
01 / 18 / 91\end{array}$ & $\begin{array}{l}0001 \\
0001 \\
0001 \\
0001\end{array}$ & MG/L & & $\begin{array}{l}0.13 \\
0.43 \\
0.02 \\
0.03\end{array}$ & . & $\begin{array}{l}0.01 \\
0.01 \\
0.01 \\
0.01\end{array}$ & : \\
\hline MERCURY & $\begin{array}{l}02 / 02 / 89 \\
09 / 19 / 89 \\
04 / 06 / 90 \\
01 / 18 / 91\end{array}$ & $\begin{array}{l}0001 \\
0001 \\
0001 \\
0001\end{array}$ & $M G / L$ & $\begin{array}{l}< \\
< \\
<\end{array}$ & $\begin{array}{l}0.0001 \\
0.0002 \\
0.0002 \\
0.0002\end{array}$ & J & $\begin{array}{l}0.0002 \\
0.0002 \\
0.0002 \\
0.0002\end{array}$ & $\begin{array}{l}- \\
- \\
-\end{array}$ \\
\hline HOLYBDENUH & $\begin{array}{l}02 / 02 / 89 \\
09 / 19 / 89 \\
04 / 06 / 90 \\
01 / 18 / 91 \\
11 / 24 / 92\end{array}$ & $\begin{array}{l}0001 \\
0001 \\
0001 \\
0001 \\
0001\end{array}$ & $M G / L$ & & $\begin{array}{l}0.595 \\
0.24 \\
0.02 \\
0.02 \\
0.01\end{array}$ & & $\begin{array}{l}0.01 \\
0.01 \\
0.01 \\
0.01 \\
0.01\end{array}$ & $\begin{array}{l}- \\
- \\
- \\
-\end{array}$ \\
\hline NET GROSS ALPHA * & $\begin{array}{l}02 / 02 / 89 \\
04 / 06 / 90 \\
01 / 18 / 91 \\
11 / 24 / 92\end{array}$ & $\begin{array}{l}0001 \\
0001 \\
0001 \\
0001\end{array}$ & $\mathrm{PCI} / \mathrm{L}$ & & $\begin{array}{r}-56.10 \\
18.92 \\
47.76 \\
1.84\end{array}$ & & $\begin{array}{l}- \\
- \\
-\end{array}$ & $\begin{array}{l}- \\
- \\
-\end{array}$ \\
\hline HICKEL & $\begin{array}{l}02 / 02 / 89 \\
09 / 19 / 89 \\
04 / 06 / 90 \\
01 / 18 / 91 \\
11 / 24 / 92\end{array}$ & $\begin{array}{l}0001 \\
0001 \\
0001 \\
0001 \\
0001\end{array}$ & HG/L & $\begin{array}{l}< \\
< \\
< \\
<\end{array}$ & $\begin{array}{l}0.02 \\
0.04 \\
0.04 \\
0.04 \\
0.04\end{array}$ & J & $\begin{array}{l}0.04 \\
0.04 \\
0.04 \\
0.04 \\
0.04\end{array}$ & $\begin{array}{l}- \\
- \\
- \\
-\end{array}$ \\
\hline NITRATE & $\begin{array}{l}02 / 02 / 89 \\
09 / 19 / 89 \\
04 / 06 / 90 \\
01 / 18 / 91 \\
11 / 24 / 92\end{array}$ & $\begin{array}{l}0001 \\
0001 \\
0001 \\
0001 \\
0001\end{array}$ & MG/L & & $\begin{array}{r}4.5 \\
3.1 \\
25.6 \\
18.6 \\
1.8\end{array}$ & & $\begin{array}{l}1 . \\
1 . \\
1 . \\
0.1 \\
1.0\end{array}$ & $\begin{array}{l}- \\
- \\
- \\
-\end{array}$ \\
\hline MITRITE AKD HITRATE & $\begin{array}{l}04 / 06 / 90 \\
01 / 18 / 91\end{array}$ & $\begin{array}{l}0001 \\
0001\end{array}$ & $M G / L$ & & $\begin{array}{l}5.8 \\
4.74\end{array}$ & & $\begin{array}{l}9 . \\
0.05\end{array}$ & - \\
\hline PH & $\begin{array}{l}02 / 02 / 89 \\
09 / 19 / 89 \\
04 / 06 / 90 \\
01 / 18 / 91 \\
11 / 24 / 92\end{array}$ & $\begin{array}{l}0001 \\
0001 \\
0001 \\
0001 \\
0001\end{array}$ & SU & & $\begin{array}{l}8.20 \\
8.02 \\
7.64 \\
8.09 \\
7.91\end{array}$ & & : & : \\
\hline PHOSPHATE & $02 / 02 / 89$ & 0001 & $M G / L$ & $<$ & 0.1 & & 0.1 & - \\
\hline
\end{tabular}

- Met gross alPHa (gRoss ALPHA - URANIUM) HITH 1 Mg URANIUH = 686 PCI

PARAMETER VALUE INDICATOR (PVI): < - LESS THAN DETECTION LIMIT SAMPLE ID COOES:

0001 - FILTERED SAMPLE (.45 MICRONS)

OTHER PARAKETER VALUE FLAGS:

I - INCREASED DETECTION LIMIT DUE TO REQUIRED DILUTION

$J$ - estiHATED VALUE 
GROUNDWATER OUALITY DATA BY LOCATIOH

SITE: AMBOI AMBROSIA LAKE

LOCATIOH: 0676 RESERVED ESTORHS, MH, 11/14/88

NORTH COORDINATE: UNKHOWH

EAST COOROIMATE: UHKHOWH

$06 / 10 / 80$ To $12 / 04 / 92$

REPORT DATE: 08/04/94

FORMATION OF COMPLETION: TRES HERMAMOS - C2 SAMDSTONE (TT) HYDRAULIC FLOW RELATIONSHIP: CROSS GRADIENT (C)

\begin{tabular}{|c|c|c|c|c|c|c|c|c|}
\hline PARAMETER NAME & LOG DATE & $\begin{array}{c}\text { SAMPLE } \\
\text { ID }\end{array}$ & $\begin{array}{l}\text { UHITS OF } \\
\text { MEASURE }\end{array}$ & PVI & $\begin{array}{c}\text { PARAMETER } \\
\text { VALUE }\end{array}$ & FLAGS & $\begin{array}{l}\text { DETECTION } \\
\text { LIHIT }\end{array}$ & $\begin{array}{l}\text { PARAMETER } \\
\text { UHCERTAINTY }\end{array}$ \\
\hline PHOSPHATE & $\begin{array}{l}09 / 19 / 89 \\
04 / 06 / 90 \\
01 / 18 / 91 \\
11 / 24 / 92\end{array}$ & $\begin{array}{l}0001 \\
0001 \\
0001 \\
0001\end{array}$ & MG/L & $<$ & $\begin{array}{l}0.1 \\
0.3 \\
0.12 \\
0.6\end{array}$ & & $\begin{array}{l}0.1 \\
0.1 \\
0.05 \\
0.1\end{array}$ & : \\
\hline POLONIUA-210 & $02 / 02 / 89$ & 0001 & $\mathrm{PCI} / \mathrm{L}$ & & 0.777 & & 1. & 1.33 \\
\hline POTASSIUM & $\begin{array}{l}02 / 02 / 89 \\
09 / 19 / 89 \\
04 / 06 / 90 \\
01 / 18 / 91 \\
11 / 24 / 92\end{array}$ & $\begin{array}{l}0001 \\
0001 \\
0001 \\
0001 \\
0001\end{array}$ & MG/L & & $\begin{array}{c}10.4 \\
5.4 \\
4.8 \\
3.3 \\
3.82\end{array}$ & & $\begin{array}{l}0.01 \\
0.01 \\
0.01 \\
0.01 \\
0.01\end{array}$ & $\begin{array}{l}- \\
- \\
-\end{array}$ \\
\hline RADIUH-226 & $\begin{array}{l}02 / 02 / 89 \\
04 / 06 / 90 \\
01 / 18 / 91 \\
11 / 24 / 92\end{array}$ & $\begin{array}{l}0001 \\
0001 \\
0001 \\
0001\end{array}$ & PCI/L & & $\begin{array}{l}1.72 \\
0.4 \\
1.0 \\
0.9\end{array}$ & & $\begin{array}{l}1 . \\
1 . \\
1 . \\
0.3\end{array}$ & $\begin{array}{l}0.291 \\
0.3 \\
0.5 \\
0.4\end{array}$ \\
\hline RADIUH-226 + RADIUH-228 & $\begin{array}{l}02 / 02 / 89 \\
04 / 06 / 90 \\
01 / 18 / 91 \\
11 / 24 / 92\end{array}$ & $\begin{array}{l}0001 \\
0001 \\
0001 \\
0001\end{array}$ & $\mathrm{PCI} / \mathrm{L}$ & & $\begin{array}{r}24.12 \\
1.60 \\
4.00 \\
1.30\end{array}$ & & $\begin{array}{l}- \\
\dot{-} \\
-\end{array}$ & $\begin{array}{l}- \\
-\end{array}$ \\
\hline RADIUN-228 & $\begin{array}{l}02 / 02 / 89 \\
04 / 06 / 90 \\
01 / 18 / 91 \\
11 / 24 / 92\end{array}$ & $\begin{array}{l}0001 \\
0001 \\
0001 \\
0001\end{array}$ & $\mathrm{PCI} / \mathrm{L}$ & & $\begin{array}{r}22.4 \\
1.2 \\
3.0 \\
0.4\end{array}$ & H & $\begin{array}{l}1 . \\
1 . \\
1.0\end{array}$ & $\begin{array}{l}8.63 \\
0.9 \\
1.6 \\
1.5\end{array}$ \\
\hline SELENIUH & $\begin{array}{l}02 / 02 / 89 \\
09 / 19 / 89 \\
04 / 06 / 90 \\
01 / 18 / 91 \\
11 / 24 / 92\end{array}$ & $\begin{array}{l}0001 \\
0001 \\
0001 \\
0001 \\
0001\end{array}$ & MG/L & $<$ & $\begin{array}{l}0.091 \\
0.021 \\
0.005 \\
0.03 \\
0.05\end{array}$ & I & $\begin{array}{l}0.005 \\
0.005 \\
0.005 \\
0.03 \\
0.05\end{array}$ & $\begin{array}{l}- \\
- \\
- \\
-\end{array}$ \\
\hline SILICA - SIOZ & $\begin{array}{l}02 / 02 / 89 \\
09 / 19 / 89 \\
04 / 06 / 90 \\
01 / 18 / 91\end{array}$ & $\begin{array}{l}0001 \\
0001 \\
0001 \\
0001\end{array}$ & $M G / L$ & & $\begin{array}{l}13.3 \\
11 . \\
8 . \\
7.1\end{array}$ & & $\begin{array}{l}2 . \\
2 . \\
2 . \\
0.1\end{array}$ & : \\
\hline SILVER & $\begin{array}{l}02 / 02 / 89 \\
09 / 19 / 89 \\
04 / 06 / 90 \\
01 / 18 / 91 \\
11 / 24 / 92\end{array}$ & $\begin{array}{l}0001 \\
0001 \\
0001 \\
0001 \\
0001\end{array}$ & $M G / L$ & $\begin{array}{l}< \\
< \\
< \\
<\end{array}$ & $\begin{array}{l}0.01 \\
0.01 \\
0.01 \\
0.01 \\
0.01\end{array}$ & & $\begin{array}{l}0.01 \\
0.01 \\
0.01 \\
0.01 \\
0.01\end{array}$ & $\begin{array}{l}- \\
= \\
-\end{array}$ \\
\hline SCOIUH & $\begin{array}{l}02 / 02 / 89 \\
09 / 19 / 89 \\
04 / 06 / 90 \\
01 / 18 / 91 \\
11 / 24 / 92\end{array}$ & $\begin{array}{l}0001 \\
0001 \\
0001 \\
0001 \\
0001\end{array}$ & MG/L & & $\begin{array}{l}750 . \\
804 . \\
674 . \\
754 . \\
665 .\end{array}$ & J & $\begin{array}{l}0.002 \\
0.002 \\
0.002 \\
5 . \\
5 .\end{array}$ & $\begin{array}{l}- \\
- \\
- \\
-\end{array}$ \\
\hline SPECIFIC CONDUCTANCE & $\begin{array}{l}02 / 02 / 89 \\
09 / 19 / 89 \\
04 / 06 / 90 \\
01 / 18 / 91\end{array}$ & $\begin{array}{l}0001 \\
0001 \\
0001 \\
0001\end{array}$ & МHO/CM & & $\begin{array}{l}2000 . \\
1450 . \\
2000 \\
2150 .\end{array}$ & & $\dot{-}$ & $\begin{array}{l}- \\
- \\
-\end{array}$ \\
\hline
\end{tabular}

PARAMETER VALUE INDICATOR (PVI): < - LESS THAN DETECTION LIMIT

SAMPLE ID COOES:

DOO1 - FILTERED SAMPLE (.45 MICRONS)

OTHER PARAMETER VALUE FLAGS:

H - HOLD TIME EXPIRED, VALUE SUSPECT

I - IMCREASED DETECTION LIHIT DUE TO REOUIRED DILUTION

j - Estimated VALUE 
GROUNDWATER QUALITY DATA BY LOCATION

SITE: AMBO1 AMBROSIA LAKE

LOCATION: 0676 RESERVED ESTORMS, MW, 11/14/88

NORTH COOROIHATE: UNKNOWH

EAST COORDINATE: UNKNOWN

06/10/80 TO $12 / 04 / 92$

REPORT DATE: $08 / 04 / 94$

FORMATION OF COMPLETIOH: TRES HERHANOS - C2 SAMDSTONE (TT) HYDRAULIC FLOY RELATIONSHIP: CROSS GRADIENT (C)

\begin{tabular}{|c|c|c|c|c|c|c|c|c|}
\hline PARAMETER MAME & LOG DATE & $\underset{\text { SOMPLE }}{\text { SA }}$ & $\begin{array}{l}\text { UNITS OF } \\
\text { MEASURE }\end{array}$ & PVI & $\begin{array}{l}\text { PARAMETER } \\
\text { VALUE }\end{array}$ & FLAGS & $\begin{array}{l}\text { DETECTIOH } \\
\text { LIMIT }\end{array}$ & $\begin{array}{l}\text { PARAMETER } \\
\text { UNCERTAINTY }\end{array}$ \\
\hline SPECIFIC CONDUCTANCE & $11 / 24 / 92$ & 0001 & UHHO/CH & \multicolumn{3}{|c|}{2200} & - & - \\
\hline STRONTIUM & $\begin{array}{l}02 / 02 / 89 \\
09 / 19 / 89 \\
04 / 06 / 90 \\
01 / 18 / 91\end{array}$ & $\begin{array}{l}0001 \\
0001 \\
0001 \\
0001\end{array}$ & MG/L & \multicolumn{3}{|c|}{$\begin{array}{l}3.30 \\
1.7 \\
1.69 \\
1.47\end{array}$} & $\begin{array}{l}0.1 \\
0.9 \\
0.1 \\
0.01\end{array}$ & : \\
\hline SULFATE & $\begin{array}{l}02 / 02 / 89 \\
09 / 19 / 89 \\
04 / 06 / 90 \\
01 / 18 / 91 \\
11 / 24 / 92\end{array}$ & $\begin{array}{l}0001 \\
0001 \\
0001 \\
0001 \\
0001\end{array}$ & MG/L & \multicolumn{3}{|c|}{$\begin{array}{l}1494 . \\
1460 \\
1130 \\
1120 \\
920\end{array}$} & $\begin{array}{c}0.1 \\
0.1 \\
0.1 \\
10 . \\
10 .\end{array}$ & $\begin{array}{l}- \\
- \\
- \\
-\end{array}$ \\
\hline SULFIDE & $\begin{array}{l}02 / 02 / 89 \\
04 / 06 / 90 \\
01 / 18 / 91\end{array}$ & $\begin{array}{l}0001 \\
0001 \\
0001\end{array}$ & HG/L & $\begin{array}{l}< \\
< \\
<\end{array}$ & $\begin{array}{l}0.01 \\
0.1 \\
0.1\end{array}$ & $J$ & $\begin{array}{l}0.1 \\
0.1 \\
0.1\end{array}$ & - \\
\hline TEMPERATURE & $\begin{array}{l}02 / 02 / 89 \\
09 / 19 / 89 \\
04 / 06 / 90 \\
01 / 18 / 91 \\
11 / 24 / 92\end{array}$ & $\begin{array}{l}0001 \\
0001 \\
0001 \\
0001 \\
0001\end{array}$ & C - DEGREE & \multicolumn{3}{|c|}{$\begin{array}{l}11.0 \\
15.0 \\
13 . \\
11 . \\
11.5\end{array}$} & $\begin{array}{l}- \\
- \\
-\end{array}$ & $\begin{array}{l}- \\
- \\
- \\
-\end{array}$ \\
\hline THALLIUN & $\begin{array}{l}02 / 02 / 89 \\
09 / 19 / 89 \\
04 / 06 / 90 \\
01 / 18 / 91\end{array}$ & $\begin{array}{l}0001 \\
0001 \\
0001 \\
0001\end{array}$ & $M G / L$ & $\begin{array}{l}< \\
<\end{array}$ & $\begin{array}{l}0.005 \\
0.01 \\
0.01 \\
0.03\end{array}$ & $\begin{array}{l}1 \\
1\end{array}$ & $\begin{array}{l}0.005 \\
0.01 \\
0.01 \\
0.03\end{array}$ & $\begin{array}{l}- \\
\dot{-}\end{array}$ \\
\hline THORIUH-230 & $02 / 02 / 89$ & 0001 & PCI/L & \multicolumn{3}{|c|}{0.159} & 1. & 0.367 \\
\hline TIN & $\begin{array}{l}02 / 02 / 89 \\
09 / 19 / 89 \\
04 / 06 / 90 \\
01 / 18 / 91\end{array}$ & $\begin{array}{l}0001 \\
0001 \\
0001 \\
0001\end{array}$ & MG/L & $\begin{array}{l}< \\
<\end{array}$ & $\begin{array}{l}0.001 \\
0.141 \\
0.052 \\
0.03\end{array}$ & $\begin{array}{l}\text { J } \\
\text { I }\end{array}$ & $\begin{array}{l}0.005 \\
0.005 \\
0.005 \\
0.03\end{array}$ & $\begin{array}{l}- \\
- \\
-\end{array}$ \\
\hline TOTAL DISSOLVED SOLIDS & $\begin{array}{l}02 / 02 / 89 \\
09 / 19 / 89 \\
04 / 06 / 90 \\
01 / 18 / 91 \\
11 / 24 / 92\end{array}$ & $\begin{array}{l}0001 \\
0001 \\
0001 \\
0001 \\
0001\end{array}$ & MG/L & \multicolumn{3}{|c|}{$\begin{array}{l}2668 . \\
2530 . \\
2010 . \\
2180 . \\
2150 .\end{array}$} & $\begin{array}{l}10 . \\
10 . \\
10 . \\
10 \\
10\end{array}$ & $\begin{array}{l}- \\
- \\
- \\
-\end{array}$ \\
\hline TOTAL KJELDAHL NITROGEN & $\begin{array}{l}01 / 18 / 91 \\
11 / 24 / 92\end{array}$ & $\begin{array}{l}0001 \\
0001\end{array}$ & HG/L & \multicolumn{3}{|c|}{ i. } & 1. & - \\
\hline TOTAL ORGANIC CARBOH & $\begin{array}{l}02 / 02 / 89 \\
09 / 19 / 89 \\
04 / 06 / 90 \\
01 / 18 / 91 \\
11 / 24 / 92\end{array}$ & $\begin{array}{l}0001 \\
0001 \\
0001 \\
0001 \\
0001\end{array}$ & $M G / L$ & \multicolumn{3}{|c|}{$\begin{array}{r}20 . \\
12 . \\
101 . \\
8 . \\
2 .\end{array}$} & $\begin{array}{l}1 . \\
1 . \\
1 . \\
1 . \\
1 .\end{array}$ & $\begin{array}{l}: \\
: \\
-\end{array}$ \\
\hline URANIU:M & $\begin{array}{l}02 / 02 / 89 \\
09 / 19 / 89 \\
04 / 06 / 90 \\
01 / 18 / 91 \\
11 / 24 / 92\end{array}$ & $\begin{array}{l}0001 \\
0001 \\
0001 \\
0001 \\
0001\end{array}$ & $M G / L$ & \multicolumn{3}{|c|}{$\begin{array}{l}0.207 \\
0.016 \\
0.0074 \\
0.001 \\
0.003 \quad H\end{array}$} & $\begin{array}{l}0.003 \\
0.003 \\
0.003 \\
0.001 \\
0.001\end{array}$ & $\begin{array}{l}- \\
- \\
-\end{array}$ \\
\hline
\end{tabular}

PARAMETER VALUE INDICATOR (PVI): < - LESS THAH DETECTIOH LIMIT

SAMPLE ID COOES:

0001 - FILTERED SAMPLE (.45 MICRONS)

OTHER PARAMETER VALUE FLAGS:

H - HOLD TIME EXPIRED, VALUE SUSPECT

1 - INCREASED DETECTION LIMIT DUE TO REQUIRED DILUTION

J - estimated value 
GROUNDHATER QUALITY DATA BY LOCATION

SITE: AMBO1 AMBROSIA LAKE

LOCATION: 0676 RESERVED ESTORMS, MH, 11/1\%/88

NORTH COORDIHATE: UNKWOWN

EAST COORDINATE: UKKHOWN

06/10/80 TO $12 / 04 / 92$

REPORT DATE: 08/04/94

FORMATION OF COMPLETION: TRES HERMANOS - C2 SAMDSTONE (TT)

HYDRAULIC FLON RELATIONSHIP: CROSS GRADIEHT (C)

\begin{tabular}{|c|c|c|c|c|c|c|c|}
\hline PARAMETER NAME & LOG DATE & $\underset{\text { SAMPLE }}{\text { SAMP }}$ & $\begin{array}{l}\text { UNITS OF } \\
\text { MEASURE }\end{array}$ & PVI & $\begin{array}{l}\text { PARAMETER } \\
\text { VALUE FLAGS }\end{array}$ & $\begin{array}{l}\text { DETECTIOH } \\
\text { LIMIT }\end{array}$ & $\begin{array}{l}\text { PARÁMETER } \\
\text { UNCERTAINTY }\end{array}$ \\
\hline VAHADIUH & $\begin{array}{l}02 / 02 / 89 \\
09 / 19 / 89 \\
04 / 06 / 90 \\
01 / 18 / 91 \\
11 / 24 / 92\end{array}$ & $\begin{array}{l}0001 \\
0001 \\
0001 \\
0001 \\
0001\end{array}$ & MG/L & $\begin{array}{l}< \\
< \\
< \\
< \\
<\end{array}$ & $\begin{array}{l}0.01 \\
0.01 \\
0.01 \\
0.01 \\
0.01\end{array}$ & $\begin{array}{l}0.01 \\
0.01 \\
0.01 \\
0.01 \\
0.01\end{array}$ & $\begin{array}{l}- \\
- \\
- \\
-\end{array}$ \\
\hline ZINC & $\begin{array}{l}02 / 02 / 89 \\
09 / 19 / 89 \\
04 / 06 / 90 \\
01 / 18 / 91\end{array}$ & $\begin{array}{l}0001 \\
0001 \\
0001 \\
0001\end{array}$ & MG/L & $<$ & $\begin{array}{l}0.01 \\
0.048 \\
0.005 \\
0.030\end{array}$ & $\begin{array}{l}0.01 \\
0.005 \\
0.005 \\
0.005\end{array}$ & $\begin{array}{l}- \\
- \\
-\end{array}$ \\
\hline
\end{tabular}

PARAMETER VALUE INDICATOR (PVI): < - LESS THAK DETECTION LIMIT

SAMPLE ID COOES:

0001 - FILTERED SAMPLE (.45 MICRONS) 
GROUNDWATER OUALITY DATA BY LOCATION

SITE: AMBO1 AMBROSIA LAKE

LOCATION: 0677 RESERVED ESTORHS, MH, 11/14/88

NORTH COORDINATE: UNKNOWN

EAST COORDIHATE: UNKHOWH

06/10/80 TO $12 / 04 / 92$

REPORT DATE: 08/04/94

FORMATION OF COMPLETION: TRES HERHANOS - C2 SANDSTONE (TT) HYDRAULIC FLON RELATIONSHIP: CROSS GRADIENT (C)

\begin{tabular}{|c|c|c|c|c|c|c|c|c|}
\hline PARAMETER MAME & LOS DATE & $\begin{array}{c}\text { SAMPLE } \\
\text { ID }\end{array}$ & $\begin{array}{l}\text { UNITS OF } \\
\text { MEASURE }\end{array}$ & PVI & $\begin{array}{l}\text { PARAMETER } \\
\text { VALUE }\end{array}$ & FLAGS & $\begin{array}{l}\text { DETECTIOH } \\
\text { LIMIT }\end{array}$ & $\begin{array}{l}\text { PARAMETER } \\
\text { UHCERTAINTY }\end{array}$ \\
\hline ALKALIMITY & $\begin{array}{l}02 / 06 / 89 \\
09 / 19 / 89 \\
04 / 11 / 90 \\
01 / 18 / 91\end{array}$ & $\begin{array}{l}0001 \\
0001 \\
0001 \\
0001\end{array}$ & $\mathrm{MG} / \mathrm{L} \mathrm{CACO} 3$ & & $\begin{array}{l}296 . \\
428 . \\
394 . \\
398 .\end{array}$ & & $\begin{array}{l}- \\
-\end{array}$ & : \\
\hline ALUAINUA & $\begin{array}{l}02 / 06 / 89 \\
09 / 19 / 89 \\
04 / 11 / 90 \\
01 / 18 / 91\end{array}$ & $\begin{array}{l}0001 \\
0001 \\
0001 \\
0001\end{array}$ & $M G / L$ & $\begin{array}{l}< \\
< \\
<\end{array}$ & $\begin{array}{l}0.05 \\
0.1 \\
0.1 \\
0.05\end{array}$ & $\mathbf{J}$ & $\begin{array}{l}0.1 \\
0.1 \\
0.1 \\
0.05\end{array}$ & - \\
\hline AMHONIUN & $\begin{array}{l}02 / 06 / 89 \\
09 / 19 / 89 \\
04 / 11 / 90 \\
01 / 18 / 91\end{array}$ & $\begin{array}{l}0001 \\
0001 \\
0001 \\
0001\end{array}$ & HG/L & $<$ & $\begin{array}{l}0.58 \\
0.8 \\
0.1 \\
0.49\end{array}$ & & $\begin{array}{l}0.1 \\
0.1 \\
0.1 \\
0.01\end{array}$ & $\begin{array}{l}- \\
- \\
-\end{array}$ \\
\hline АNT IMONY & $\begin{array}{l}02 / 06 / 89 \\
09 / 19 / 89 \\
04 / 11 / 90 \\
01 / 18 / 91\end{array}$ & $\begin{array}{l}0001 \\
0001 \\
0001 \\
0001\end{array}$ & MG/L & $<$ & $\begin{array}{l}0.004 \\
0.053 \\
0.116 \\
0.003\end{array}$ & & $\begin{array}{l}0.003 \\
0.003 \\
0.003 \\
0.003\end{array}$ & $\begin{array}{l}- \\
- \\
-\end{array}$ \\
\hline ARSENIC & $\begin{array}{l}02 / 06 / 89 \\
09 / 19 / 89 \\
04 / 11 / 90 \\
01 / 18 / 91\end{array}$ & $\begin{array}{l}0001 \\
0001 \\
0001 \\
0001\end{array}$ & MG/L & $\begin{array}{l}< \\
< \\
< \\
<\end{array}$ & $\begin{array}{l}0.001 \\
0.01 \\
0.01 \\
0.05\end{array}$ & $\begin{array}{l}\text { J } \\
I\end{array}$ & $\begin{array}{l}0.01 \\
0.01 \\
0.01 \\
0.05\end{array}$ & $\begin{array}{l}- \\
- \\
-\end{array}$ \\
\hline BARIUM & $\begin{array}{l}02 / 06 / 89 \\
09 / 19 / 89 \\
04 / 11 / 90 \\
01 / 18 / 91\end{array}$ & $\begin{array}{l}0001 \\
0001 \\
0001 \\
0001\end{array}$ & $M G / L$ & $<$ & $\begin{array}{l}0.02 \\
0.1 \\
0.1 \\
0.01\end{array}$ & $\mathbf{J}$ & $\begin{array}{l}0.1 \\
0.1 \\
0.1 \\
0.01\end{array}$ & $\begin{array}{l}- \\
-\end{array}$ \\
\hline BERYLLIUH & $\begin{array}{l}02 / 06 / 89 \\
09 / 19 / 89 \\
04 / 11 / 90 \\
01 / 18 / 91\end{array}$ & $\begin{array}{l}0001 \\
0001 \\
0001 \\
0001\end{array}$ & MG/L & $\begin{array}{l}< \\
< \\
<\end{array}$ & $\begin{array}{l}0.005 \\
0.01 \\
0.01 \\
0.005\end{array}$ & & $\begin{array}{l}0.005 \\
0.01 \\
0.01 \\
0.005\end{array}$ & : \\
\hline BORON & $\begin{array}{l}02 / 06 / 89 \\
09 / 19 / 89 \\
04 / 11 / 90 \\
01 / 18 / 91\end{array}$ & $\begin{array}{l}0001 \\
0001 \\
0001 \\
0001\end{array}$ & HG/L & & $\begin{array}{l}0.64 \\
0.6 \\
0.5 \\
0.49\end{array}$ & & $\begin{array}{l}0.1 \\
0.1 \\
0.1 \\
0.05\end{array}$ & - \\
\hline BROMIDE & $\begin{array}{l}02 / 06 / 89 \\
09 / 19 / 89 \\
04 / 11 / 90 \\
01 / 18 / 91\end{array}$ & $\begin{array}{l}0001 \\
0001 \\
0001 \\
0001\end{array}$ & MG/L & & $\begin{array}{l}0.42 \\
0.2 \\
0.3 \\
0.3\end{array}$ & J & $\begin{array}{l}0.01 \\
0.1 \\
0.1 \\
0.1\end{array}$ & $\begin{array}{l}- \\
- \\
-\end{array}$ \\
\hline CADMIUA & $\begin{array}{l}02 / 06 / 89 \\
09 / 19 / 89 \\
04 / 11 / 90 \\
01 / 18 / 91\end{array}$ & $\begin{array}{l}0001 \\
0001 \\
0001 \\
0001\end{array}$ & $M G / L$ & $<$ & $\begin{array}{l}0.0009 \\
0.004 \\
0.001 \\
0.001\end{array}$ & J & $\begin{array}{l}0.001 \\
0.001 \\
0.001 \\
0.001\end{array}$ & $\begin{array}{l}- \\
-\end{array}$ \\
\hline Calciun & $\begin{array}{l}02 / 06 / 89 \\
09 / 19 / 89 \\
04 / 11 / 90 \\
01 / 18 / 91\end{array}$ & $\begin{array}{l}0001 \\
0001 \\
0001 \\
0001\end{array}$ & MG/L & & $\begin{array}{l}75 . \\
31.9 \\
28.2 \\
30.9\end{array}$ & & $\begin{array}{l}0.01 \\
0.01 \\
0.01 \\
0.5\end{array}$ & $\begin{array}{l}- \\
- \\
-\end{array}$ \\
\hline CHLORIDE & $02 / 06 / 89$ & 0001 & $M G / L$ & & 73. & & 1. & - \\
\hline
\end{tabular}

PARAMETER VALUE IMDICATOR (PVI): < - LESS THAN DETECTION LIMIT

OTHER PARAMETER VALUE FLAGS:

1 - IMCREASED DETECTION LIMIT DUE TO REQUIRED DILUTIOH

J- ESTIMATED VALUE 
GROUNDWATER QUALITY DATA BY LOCATIOH

SITE: AMBO1 AMBROSIA LAKE

LOCATIOH: 0677 RESERVED ESTORMS, HH, 11/14/88

HORTH COORDINATE: UNKNOWN

EAST COORDIKATE: UNKHOWN

06/10/80 TO 12/04/92

REPORT DATE: 08/04/94

FORHATION OF COMPLETION: TRES HERHANOS - C2 SAHDSTOHE (TT) HYDRAULIC FLOW RELATIONSHIP: CROSS GRADIENT (C)

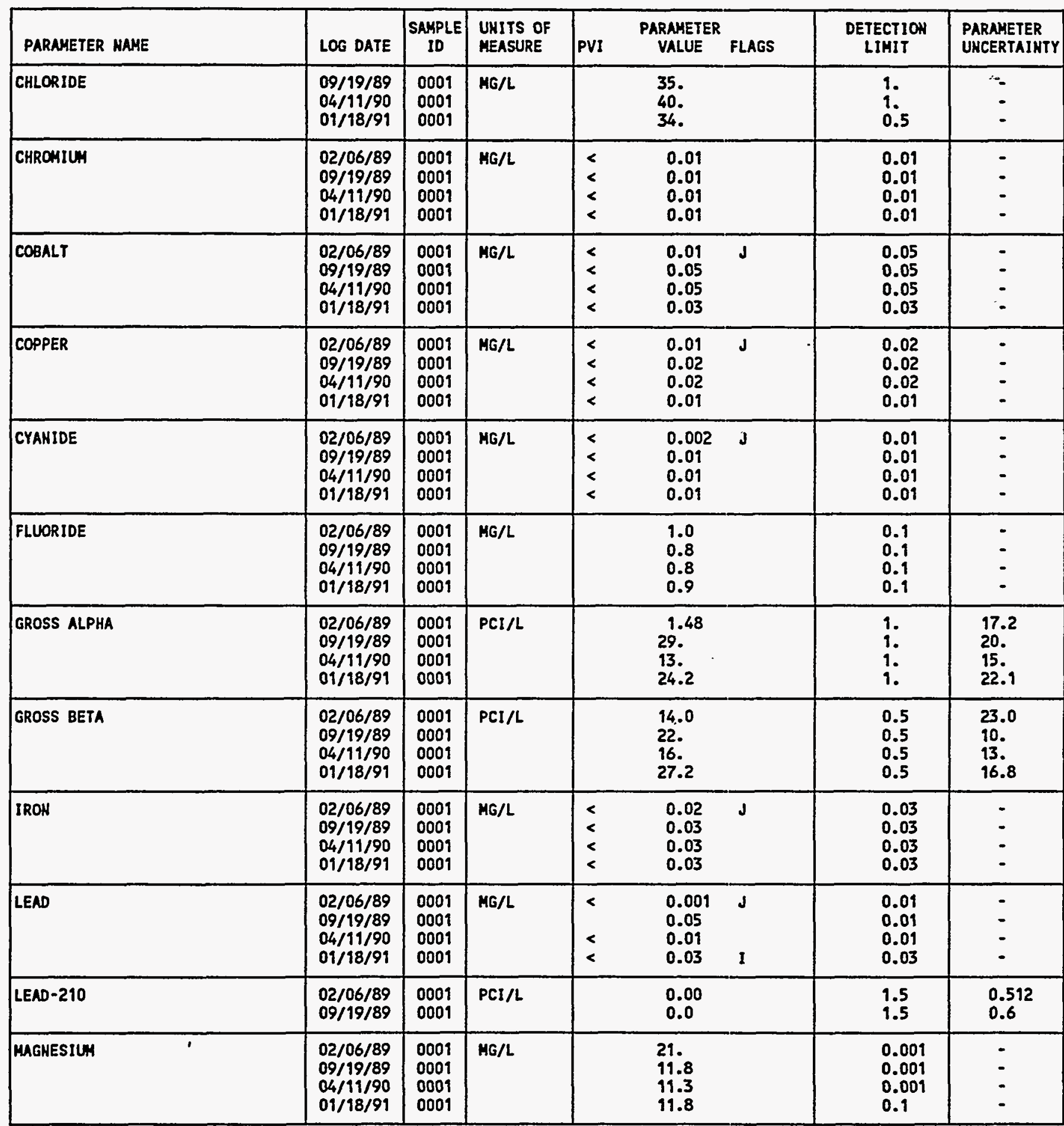

PARAMETER VALUE IMDICATOR (PVI): < - LESS THAN DETECTION LIMIT

OTHER PARAMETER VALUE FLAGS:

I - IHCREASED DETECTION LIMIT DUE TO REOUIRED DILUTIOH

J - ESTIMATED VALUE 


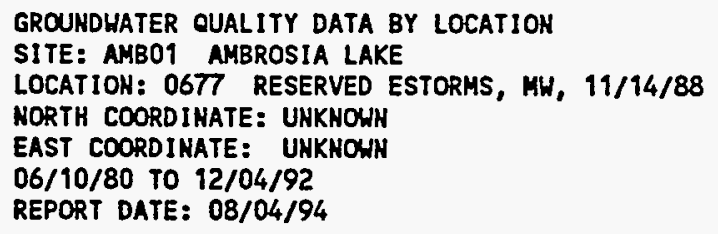

FORMATION OF COMPLETION: TRES HERMAMOS - C2 SANDSTONE (TT) HYDRAULIC FLON RELATIONSHIP: CROSS GRADIENT (C)

\begin{tabular}{|c|c|c|c|c|c|c|c|}
\hline PARAMETER MAME & LOG DATE & $\begin{array}{l}\text { SAMPLE } \\
\text { ID }\end{array}$ & $\begin{array}{l}\text { UNITS OF } \\
\text { MEASURE }\end{array}$ & PVI & $\begin{array}{l}\text { PARAMETER } \\
\text { VALUE FLAGS }\end{array}$ & $\begin{array}{l}\text { DETECTION } \\
\text { LIMIT }\end{array}$ & $\begin{array}{l}\text { PARAMETER } \\
\text { UMCERTAINTY }\end{array}$ \\
\hline MAKGANESE & $\begin{array}{l}02 / 06 / 89 \\
09 / 19 / 89 \\
04 / 11 / 90 \\
01 / 18 / 91\end{array}$ & $\begin{array}{l}0001 \\
0001 \\
0001 \\
0001\end{array}$ & MG/L & & $\begin{array}{l}0.03 \\
0.11 \\
0.04 \\
0.04\end{array}$ & $\begin{array}{l}0.01 \\
0.01 \\
0.01 \\
0.01\end{array}$ & $\begin{array}{l}- \\
\text { - } \\
-\end{array}$ \\
\hline MERCURY & $\begin{array}{l}02 / 06 / 89 \\
09 / 19 / 89 \\
04 / 11 / 90 \\
01 / 18 / 91\end{array}$ & $\begin{array}{l}0001 \\
0001 \\
0001 \\
0001\end{array}$ & MG/L & $\begin{array}{l}< \\
< \\
<\end{array}$ & $\begin{array}{l}0.0001 \mathrm{~J} \\
0.0002 \\
0.0002 \\
0.0002\end{array}$ & $\begin{array}{l}0.0002 \\
0.0002 \\
0.0002 \\
0.0002\end{array}$ & $\begin{array}{l}- \\
\text { - } \\
.\end{array}$ \\
\hline MOLYBDENUY & $\begin{array}{l}02 / 06 / 89 \\
09 / 19 / 89 \\
04 / 11 / 90 \\
01 / 18 / 91\end{array}$ & $\begin{array}{l}0001 \\
0001 \\
0001 \\
0001\end{array}$ & MG/L & $<$ & $\begin{array}{l}0.024 \\
0.02 \\
0.01 \\
0.01\end{array}$ & $\begin{array}{l}0.01 \\
0.01 \\
0.01 \\
0.01\end{array}$ & $\begin{array}{l}. \\
\dot{-} \\
.\end{array}$ \\
\hline MET GROSS ALPHA * & $\begin{array}{l}02 / 06 / 89 \\
09 / 19 / 89 \\
04 / 11 / 90 \\
01 / 18 / 91\end{array}$ & $\begin{array}{l}0001 \\
0001 \\
0001 \\
0001\end{array}$ & $\mathrm{PCI} / \mathrm{L}$ & & $\begin{array}{l}-9.50 \\
26.53 \\
11.01 \\
23.86\end{array}$ & $\begin{array}{l}- \\
- \\
-\end{array}$ & $\begin{array}{l}. \\
. \\
.\end{array}$ \\
\hline HICKEL & $\begin{array}{l}02 / 06 / 89 \\
09 / 19 / 89 \\
04 / 19 / 90 \\
01 / 18 / 91\end{array}$ & $\begin{array}{l}0001 \\
0001 \\
0001 \\
0001\end{array}$ & $M G / L$ & $\begin{array}{l}< \\
< \\
< \\
<\end{array}$ & $\begin{array}{l}0.02 \\
0.04 \\
0.04 \\
0.04\end{array}$ & $\begin{array}{l}0.04 \\
0.04 \\
0.04 \\
0.04\end{array}$ & $\begin{array}{l}. \\
. \\
.\end{array}$ \\
\hline MITRATE & $\begin{array}{l}02 / 06 / 89 \\
09 / 19 / 89 \\
04 / 11 / 90 \\
01 / 18 / 91\end{array}$ & $\begin{array}{l}0001 \\
0001 \\
0001 \\
0001\end{array}$ & $M G / L$ & $<$ & $\begin{array}{l}4.3 \\
1 . \\
6.9 \\
5.8\end{array}$ & $\begin{array}{l}1 . \\
1 . \\
1 . \\
0.1\end{array}$ & - \\
\hline KITRITE AND NITRATE & $\begin{array}{l}04 / 11 / 90 \\
01 / 18 / 91\end{array}$ & $\begin{array}{l}0001 \\
0001\end{array}$ & $M G / L$ & & $\begin{array}{l}1.6 \\
1.23\end{array}$ & $\begin{array}{l}1 . \\
0.05\end{array}$ & 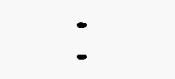 \\
\hline PH & $\begin{array}{l}02 / 06 / 89 \\
09 / 19 / 89 \\
04 / 11 / 90 \\
01 / 18 / 91\end{array}$ & $\begin{array}{l}0001 \\
0001 \\
0001 \\
0001\end{array}$ & SU & & $\begin{array}{l}7.85 \\
8.07 \\
7.87 \\
7.83\end{array}$ & : & $\begin{array}{l}. \\
. \\
.\end{array}$ \\
\hline PHOSPHATE & $\begin{array}{l}02 / 06 / 89 \\
09 / 19 / 89 \\
04 / 11 / 90 \\
01 / 18 / 91\end{array}$ & $\begin{array}{l}0001 \\
0001 \\
0001 \\
0001\end{array}$ & $M G / L$ & < & $\begin{array}{l}0.1 \\
0.1 \\
0.1 \\
0.10\end{array}$ & $\begin{array}{l}0.1 \\
0.1 \\
0.1 \\
0.05\end{array}$ & $\begin{array}{l}- \\
- \\
-\end{array}$ \\
\hline POLONIUN-210 & $\begin{array}{l}02 / 06 / 89 \\
09 / 19 / 89\end{array}$ & $\begin{array}{l}0001 \\
0001\end{array}$ & $\mathrm{PCI} / \mathrm{L}$ & & $\begin{array}{l}0.155 \\
0.0\end{array}$ & 9. & $\begin{array}{l}1.18 \\
0.3\end{array}$ \\
\hline POTASSIUA & $\begin{array}{l}02 / 06 / 89 \\
09 / 19 / 89 \\
04 / 11 / 90 \\
01 / 18 / 91\end{array}$ & $\begin{array}{l}0001 \\
0001 \\
0001 \\
0001\end{array}$ & HG/L & & $\begin{array}{l}9.4 \\
5.4 \\
5.4 \\
3.8\end{array}$ & $\begin{array}{l}0.01 \\
0.01 \\
0.01 \\
0.01\end{array}$ & $\begin{array}{l}- \\
- \\
-\end{array}$ \\
\hline RADIUA-226 & $\begin{array}{l}02 / 06 / 89 \\
09 / 19 / 89 \\
04 / 11 / 90 \\
01 / 18 / 91\end{array}$ & $\begin{array}{l}0001 \\
0001 \\
0001 \\
0001\end{array}$ & PCI/L & & $\begin{array}{l}1.75 \\
5.4 \\
3.0 \\
1.4\end{array}$ & $\begin{array}{l}1 . \\
1 . \\
1 . \\
1 .\end{array}$ & $\begin{array}{l}0.279 \\
1.4 \\
0.6 \\
0.6\end{array}$ \\
\hline
\end{tabular}

- MET GROSS ALPHA (GROSS ALPHA - URANIUM) WITH 1 MG URANIUM = 686 PCI

PARAMETER VALUE INDICATOR (PVI): < - LESS THAH DETECTION LIMIT
SAMPLE ID COOES:

0001 - FILTERED SAMPLE (.45 MICRONS)

OTHER PARAMETER VALUE fLAGS:

J. ESTIMATED VALUE 
GROUNDWATER QUALITY DATA BY LOCATION

SITE: AMBO1 AMBROSIA LAKE

LOCATIOH: 0677 RESERVED ESTORHS, MH, 11/14/88

HORTH COORDINATE: UHKHOWH

EAST COORDINATE: UNKNOWN

06/10/80 TO $12 / 04 / 92$

REPORT DATE: 08/04/94

FORMATIOH OF COHPLETION: TRES HERMANOS - C2 SAMDSTONE (TT) HYDRAULIC FLOW RELATIONSHIP: CROSS GRADIENT (C)

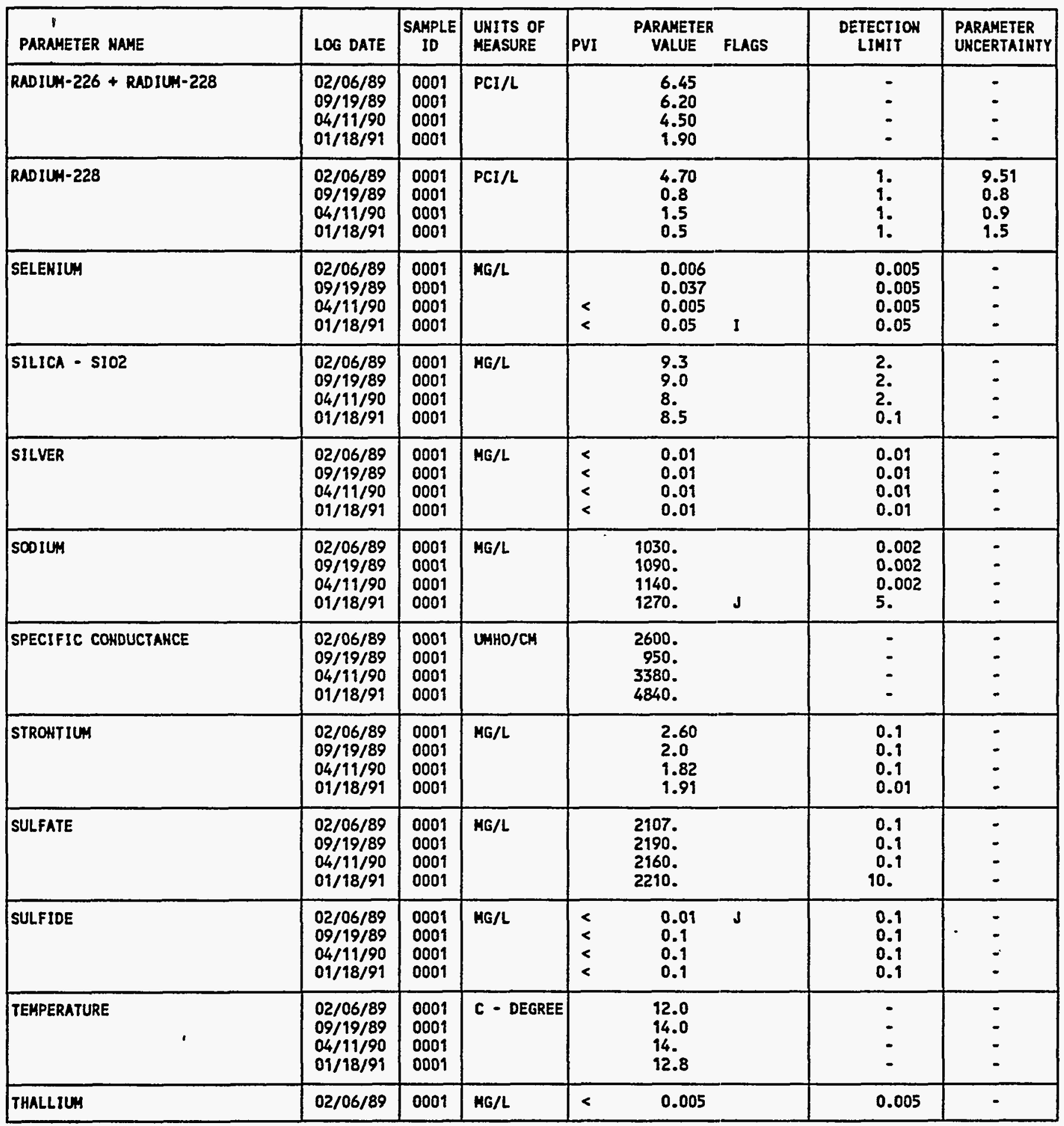

PARAMETER VALUE IMDICATOR (PVI): < - LESS THAN DETECTION LIMIT

OTHER PARAMETER VALUE FLAGS:

I - INCREASED OETECTION LIMIT DUE TO REQUIRED DILUTION

$J$ - estimated value 
GROUNDHATER QUALITY DATA BY LOCATIOH

SITE: AMBOI AMBROSIA LAKE

LOCATION: 0677 RESERVED ESTORHS, MW, 11/14/88

HORTH COORDIHATE: UHKMOWN

EAST COORDIMATE: UNKNOWN

06/10/80 TO $12 / 04 / 92$

REPORT DATE: 08/04/94

FORMATION OF COHPLETION: TRES HERHANOS - C2 SAHDSTONE (TT) HYDRAULIC FLOW RELATIOHSHIP: CROSS GRADIENT (C)

\begin{tabular}{|c|c|c|c|c|c|c|c|}
\hline PARAMETER MAME & LOS DATE & $\begin{array}{l}\text { SAMPLE } \\
\text { ID }\end{array}$ & $\begin{array}{l}\text { UHITS OF } \\
\text { MEASURE }\end{array}$ & PVI & $\begin{array}{l}\text { PARAMETER } \\
\text { VALUE FLAGS }\end{array}$ & $\begin{array}{l}\text { DETECTION } \\
\text { LIMIT }\end{array}$ & $\begin{array}{l}\text { PARAMETER } \\
\text { UHCERTAINTY }\end{array}$ \\
\hline THALLIUH & $\begin{array}{l}09 / 19 / 89 \\
04 / 11 / 90 \\
01 / 18 / 91\end{array}$ & $\begin{array}{l}0009 \\
0001 \\
0001\end{array}$ & $M G / L$ & $<$ & $\begin{array}{l}0.02 \\
0.01 \\
0.03\end{array}$ & $\begin{array}{l}0.09 \\
0.01 \\
0.03\end{array}$ & - \\
\hline THORIUH-230 & $\begin{array}{l}02 / 06 / 89 \\
09 / 19 / 89\end{array}$ & $\begin{array}{l}0001 \\
0001\end{array}$ & PCI/L & & $\begin{array}{l}0.285 \\
0.1\end{array}$ & 1. & $\begin{array}{l}0.463 \\
0.4\end{array}$ \\
\hline TIN & $\begin{array}{l}02 / 06 / 89 \\
09 / 19 / 89 \\
04 / 11 / 90 \\
01 / 18 / 91\end{array}$ & $\begin{array}{l}0001 \\
0001 \\
0001 \\
0001\end{array}$ & MG/L & $<$ & $\begin{array}{l}0.006 \\
0.173 \\
0.090 \\
0.03\end{array}$ & $\begin{array}{l}0.005 \\
0.005 \\
0.005 \\
0.03\end{array}$ & - \\
\hline TOTAL DISSOLVEO SOLIDS & $\begin{array}{l}02 / 06 / 89 \\
09 / 19 / 89 \\
04 / 11 / 90 \\
01 / 18 / 91\end{array}$ & $\begin{array}{l}0001 \\
0001 \\
0001 \\
0001\end{array}$ & $M G / L$ & & $\begin{array}{l}3372 . \\
3470 \\
3500 \\
3600\end{array}$ & $\begin{array}{l}10 . \\
10 . \\
10 \\
10\end{array}$ & $\begin{array}{l}- \\
-\end{array}$ \\
\hline TOTAL KJELDAHL NITROGEN & $01 / 18 / 91$ & 0001 & $M G / L$ & & 1. & 1. & - \\
\hline TOTAL ORGANIC CARBOH & $\begin{array}{l}02 / 06 / 89 \\
09 / 19 / 89 \\
04 / 11 / 90 \\
01 / 18 / 91\end{array}$ & $\begin{array}{l}0001 \\
0001 \\
0001 \\
0001\end{array}$ & $M G / L$ & & $\begin{array}{l}11 . \\
98 . \\
57 . \\
2 .\end{array}$ & $\begin{array}{l}1 . \\
1 . \\
1 .\end{array}$ & - \\
\hline URANIUN & $\begin{array}{l}02 / 06 / 89 \\
09 / 19 / 89 \\
04 / 11 / 90 \\
01 / 18 / 91\end{array}$ & $\begin{array}{l}0001 \\
0001 \\
0009 \\
0001\end{array}$ & $M G / L$ & $<$ & $\begin{array}{l}0.016 \\
0.0036 \\
0.0029 \mathrm{~J} \\
0.001\end{array}$ & $\begin{array}{l}0.003 \\
0.003 \\
0.003 \\
0.001\end{array}$ & $\begin{array}{l}- \\
-\end{array}$ \\
\hline VANADIUA & $\begin{array}{l}02 / 06 / 89 \\
09 / 19 / 89 \\
04 / 11 / 90 \\
01 / 18 / 91\end{array}$ & $\begin{array}{l}0009 \\
0001 \\
0001 \\
0001\end{array}$ & $M G / L$ & $\begin{array}{l}< \\
< \\
< \\
<\end{array}$ & $\begin{array}{l}0.01 \\
0.01 \\
0.01 \\
0.01\end{array}$ & $\begin{array}{l}0.01 \\
0.01 \\
0.01 \\
0.01\end{array}$ & $\begin{array}{l}- \\
- \\
-\end{array}$ \\
\hline ZIHC & $\begin{array}{l}02 / 06 / 89 \\
09 / 19 / 89 \\
04 / 19 / 90 \\
01 / 18 / 91\end{array}$ & $\begin{array}{l}0001 \\
0001 \\
0001 \\
0001\end{array}$ & $M G / L$ & & $\begin{array}{l}0.02 \\
0.015 \\
0.035 \\
0.008\end{array}$ & $\begin{array}{l}0.005 \\
0.005 \\
0.005 \\
0.005\end{array}$ & $\begin{array}{l}- \\
- \\
-\end{array}$ \\
\hline
\end{tabular}

PARAMETER VALUE INDICATOR (PVI): < - LESS THAN DETECTION LIMIT

SAMPLE ID CODES:

0001 - FILTERED SAMPLE (.45 MICRONS)

OTHER PARAMETER VALUE FLAGS:

I - INCREASED DETECTION LIMIT DUE TO REQUIRED DILUTION

J - estimated value 
GROUNDHATER QUALITY DATA BY LOCATION

SITE: AMBO 1 AMBROSIA LAKE

LOCATION: 0678 RESERVED ESTORMS, MW, 11/14/88

NORTH COORDIHATE: UNKNOWN

EAST COORDINATE: UNKNOWN

06/10/80 TO 12/04/92

REPORT DATE: 08/04/94

FORMATION OF COMPLETION: TRES HERHANOS - B SANDSTONE (TB) HYDRAULIC FLOW RELATIONSHIP: CROSS GRADIENT (C)

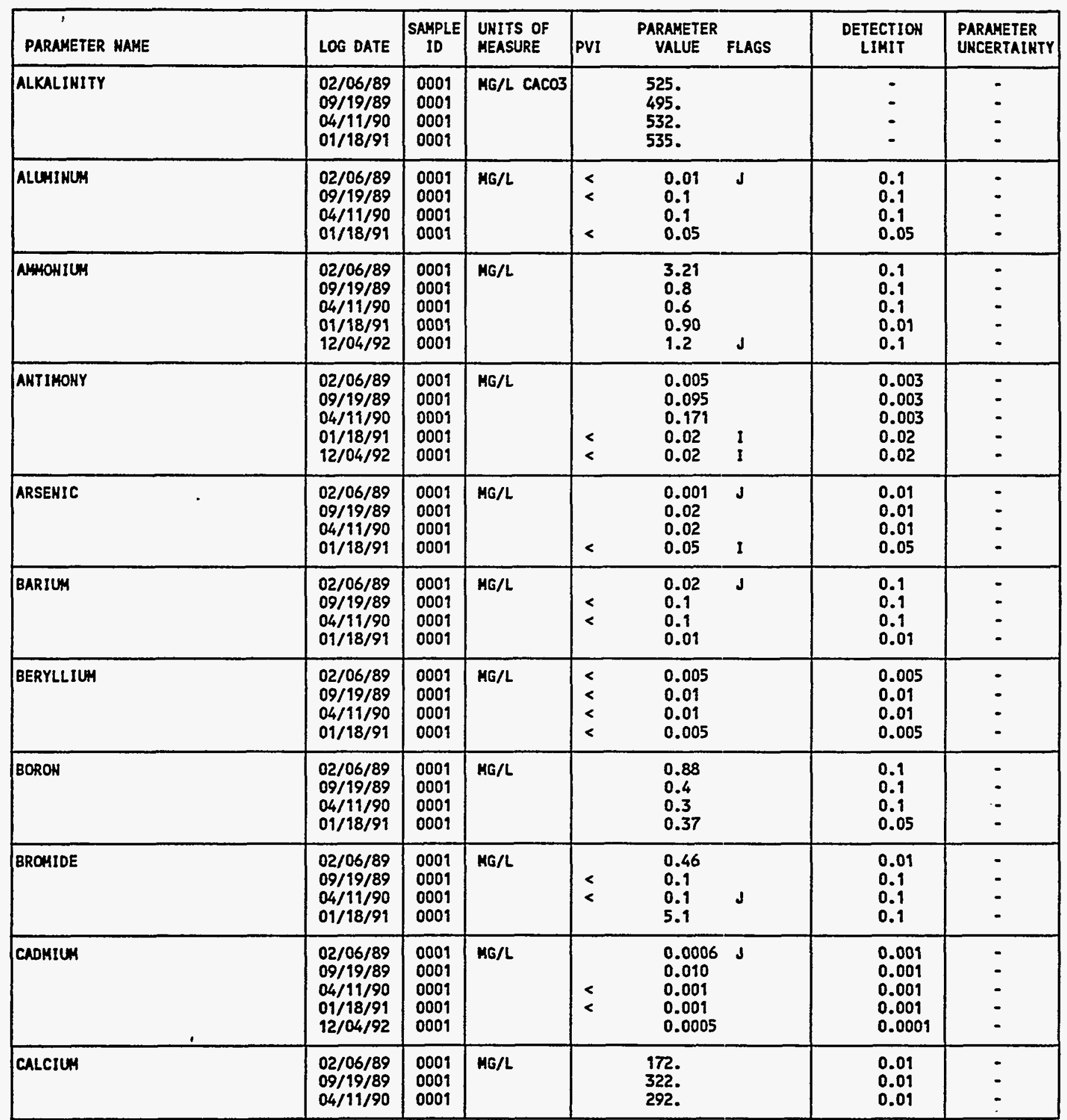

PARAMETER VALUE IMDICATOR (PVI): < - LESS THAM DETECTION LIMIT

SAMPLE ID COOES:

0001 - FILTERED SAMPLE (.45 MICRONS)

OTHER PARAMETER VALUE FLAGS:

1 - INCREASED DETECTION LIMIT DUE TO REOUIRED DILUTIOH

J - estimated VAlUe 
GROUNDWATER QUALITY DATA BY LOCATIOH

SITE: AMB01 AMBROSIA LAKE

LOCATION: 0678 RESERVED ESTORHS, HH, 11/14/88

NORTH COORDINATE: UNKNONN

EAST COORDINATE: UMKHOWH

06/10/80 TO 12/04/92

REPORT DATE: 08/04/94

FORHATION OF COHPLETION: TRES HERHANOS - B SANDSTONE (TB) HYDRAULIC FLOW RELATIONSHIP: CROSS GRADIENT (C)

\begin{tabular}{|c|c|c|c|c|c|c|c|c|}
\hline PARAMETER MAME & LOG DATE & $\underset{\text { ID }}{\text { SAMPLE }}$ & $\begin{array}{l}\text { UNITS OF } \\
\text { MEASURE }\end{array}$ & PVI & $\begin{array}{l}\text { PARAMETER } \\
\text { VALUE }\end{array}$ & FLAGS & $\begin{array}{l}\text { DETECTIOH } \\
\text { LIMIT }\end{array}$ & $\begin{array}{l}\text { PARAMETER } \\
\text { UHCERTAIHTY }\end{array}$ \\
\hline CALCIUH & $\begin{array}{l}01 / 18 / 91 \\
12 / 04 / 92\end{array}$ & $\begin{array}{l}0001 \\
0001\end{array}$ & HG/L & & $\begin{array}{l}322 . \\
392 .\end{array}$ & & $\begin{array}{l}0.5 \\
0.5\end{array}$ & - \\
\hline CHLORIDE & $\begin{array}{l}02 / 06 / 89 \\
09 / 19 / 89 \\
04 / 11 / 90 \\
01 / 18 / 91 \\
12 / 04 / 92\end{array}$ & $\begin{array}{l}0001 \\
0001 \\
0001 \\
0001 \\
0001\end{array}$ & MG/L & & $\begin{array}{l}248 . \\
280 \\
309 \\
286 \\
300\end{array}$ & & $\begin{array}{l}1 . \\
1 . \\
1 . \\
0.5 \\
10 .\end{array}$ & $\begin{array}{l}- \\
- \\
- \\
-\end{array}$ \\
\hline CHRONIUN & $\begin{array}{l}02 / 06 / 89 \\
09 / 19 / 89 \\
04 / 11 / 90 \\
01 / 18 / 91 \\
12 / 04 / 92\end{array}$ & $\begin{array}{l}0001 \\
0001 \\
0001 \\
0001 \\
0001\end{array}$ & $M G / L$ & $\begin{array}{l}< \\
< \\
< \\
<\end{array}$ & $\begin{array}{l}0.01 \\
0.01 \\
0.01 \\
0.01 \\
0.01\end{array}$ & & $\begin{array}{l}0.01 \\
0.01 \\
0.01 \\
0.01 \\
0.01\end{array}$ & $\begin{array}{l}- \\
- \\
- \\
-\end{array}$ \\
\hline COBALT & $\begin{array}{l}02 / 06 / 89 \\
09 / 19 / 89 \\
04 / 11 / 90 \\
01 / 18 / 91 \\
12 / 04 / 92\end{array}$ & $\begin{array}{l}0001 \\
0001 \\
0001 \\
0001 \\
0001\end{array}$ & $M G / L$ & $\begin{array}{l}< \\
< \\
<\end{array}$ & $\begin{array}{l}0.01 \\
0.05 \\
0.01 \\
0.03 \\
0.03\end{array}$ & $\begin{array}{l}\mathbf{J} \\
\mathbf{J}\end{array}$ & $\begin{array}{l}0.05 \\
0.05 \\
0.05 \\
0.03 \\
0.03\end{array}$ & $\begin{array}{l}- \\
\dot{-} \\
-\end{array}$ \\
\hline COPPER & $\begin{array}{l}02 / 06 / 89 \\
09 / 19 / 89 \\
04 / 11 / 90 \\
01 / 18 / 91\end{array}$ & $\begin{array}{l}0001 \\
0001 \\
0001 \\
0001\end{array}$ & $M G / L$ & $<$ & $\begin{array}{l}0.01 \\
0.02 \\
0.02 \\
0.02\end{array}$ & $J$ & $\begin{array}{l}0.02 \\
0.02 \\
0.02 \\
0.01\end{array}$ & $\begin{array}{l}- \\
- \\
-\end{array}$ \\
\hline CYANIDE & $\begin{array}{l}02 / 06 / 89 \\
09 / 19 / 89 \\
04 / 11 / 90 \\
01 / 98 / 91\end{array}$ & $\begin{array}{l}0001 \\
0001 \\
0001 \\
0001\end{array}$ & MG/L & $\begin{array}{l}< \\
< \\
<\end{array}$ & $\begin{array}{l}0.002 \\
0.01 \\
0.01 \\
0.01\end{array}$ & J & $\begin{array}{l}0.01 \\
0.01 \\
0.01 \\
0.01\end{array}$ & - \\
\hline FLUORIDE & $\begin{array}{l}02 / 06 / 89 \\
09 / 19 / 89 \\
04 / 91 / 90 \\
01 / 18 / 91 \\
12 / 04 / 92\end{array}$ & $\begin{array}{l}0001 \\
0001 \\
0001 \\
0001 \\
0001\end{array}$ & HG/L & $<$ & $\begin{array}{l}0.02 \\
0.3 \\
0.3 \\
0.2 \\
0.2\end{array}$ & J & $\begin{array}{l}0.1 \\
0.1 \\
0.1 \\
0.1 \\
0.1\end{array}$ & $\begin{array}{l}- \\
- \\
- \\
-\end{array}$ \\
\hline GROSS ALPHA & $\begin{array}{l}02 / 06 / 89 \\
09 / 19 / 89 \\
04 / 11 / 90 \\
01 / 18 / 91 \\
12 / 04 / 92\end{array}$ & $\begin{array}{l}0001 \\
0001 \\
0001 \\
0001 \\
0001\end{array}$ & $\mathrm{PCl} / \mathrm{L}$ & & $\begin{array}{c}48.2 \\
92 . \\
130 . \\
82.9 \\
45.2\end{array}$ & & $\begin{array}{l}1 . \\
1 . \\
1 . \\
1.0\end{array}$ & $\begin{array}{c}31.0 \\
67 . \\
80 . \\
121 . \\
95.7\end{array}$ \\
\hline GROSS BETA & $\begin{array}{l}02 / 06 / 89 \\
09 / 19 / 89 \\
04 / 11 / 90 \\
01 / 18 / 91 \\
12 / 04 / 92\end{array}$ & $\begin{array}{l}0001 \\
0001 \\
0001 \\
0001 \\
0001\end{array}$ & $\mathrm{PCI} / \mathrm{L}$ & & $\begin{array}{c}18.7 \\
77 . \\
100 . \\
120 . \\
0.0\end{array}$ & & $\begin{array}{r}0.5 \\
0.5 \\
0.5 \\
0.5 \\
204 .\end{array}$ & $\begin{array}{l}32.0 \\
33 . \\
60 . \\
67.4 \\
113 .\end{array}$ \\
\hline IRON & $\begin{array}{l}02 / 06 / 89 \\
09 / 19 / 89 \\
04 / 11 / 90 \\
01 / 18 / 91 \\
12 / 04 / 92\end{array}$ & $\begin{array}{l}0001 \\
0001 \\
0001 \\
0001 \\
0001\end{array}$ & MG/L & $\begin{array}{l}< \\
< \\
<\end{array}$ & $\begin{array}{l}0.02 \\
0.10 \\
0.09 \\
0.03 \\
0.03\end{array}$ & $J$ & $\begin{array}{l}0.03 \\
0.03 \\
0.03 \\
0.03 \\
0.03\end{array}$ & $\begin{array}{l}- \\
- \\
-\end{array}$ \\
\hline LEAD & $\begin{array}{l}02 / 06 / 89 \\
09 / 19 / 89\end{array}$ & $\begin{array}{l}0001 \\
0001\end{array}$ & MG/L & $<$ & $\begin{array}{l}0.001 \\
0.04\end{array}$ & $\mathbf{J}$ & $\begin{array}{l}0.01 \\
0.01\end{array}$ & 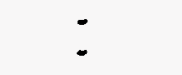 \\
\hline
\end{tabular}

PARAMETER VALUE IMDICATOR (PVI): < - LESS THAN DETECTIOH LIHIT 
GROUHDHATER QUALITY DATA BY LOCATIOH

SITE: AMBO1 AMBROSIA LAKE

LOCATION: 0678 RESERVED ESTORMS, MH, 11/14/,88

HORTH COORDINATE: UNKNOWH

EAST COORDINATE: UNKHOWN

06/10/80 TO $12 / 04 / 92$

REPORT DATE: $08 / 04 / 94$

FORMATION OF COMPLETION: TRES HERMAMOS - B SAMDSTONE (TB)

HYDRAULIC FLOW RELATIONSHIP: CROSS GRADIENT (C)

\begin{tabular}{|c|c|c|c|c|c|c|c|}
\hline PARAHETER NAHE & LOG DATE & $\begin{array}{c}\text { SAMPLE } \\
\text { ID }\end{array}$ & $\begin{array}{l}\text { UHITS OF } \\
\text { MEASURE }\end{array}$ & PVI & $\begin{array}{l}\text { PARAMETER } \\
\text { VALUE FLAGS }\end{array}$ & $\begin{array}{l}\text { DETECTIOH } \\
\text { LIMIT }\end{array}$ & $\begin{array}{l}\text { PARAMETER } \\
\text { UNCERTAINTY }\end{array}$ \\
\hline LEAD & $\begin{array}{l}04 / 11 / 90 \\
01 / 18 / 91\end{array}$ & $\begin{array}{l}0001 \\
0001\end{array}$ & $\mathrm{MG} / \mathrm{L}$ & $<$ & $\begin{array}{l}0.01 \\
0.03\end{array}$ & $\begin{array}{l}0.01 \\
0.03\end{array}$ & - \\
\hline LEAD-210 & $\begin{array}{l}02 / 06 / 89 \\
09 / 19 / 89\end{array}$ & $\begin{array}{l}0001 \\
0001\end{array}$ & PCI/L & & $\begin{array}{l}0.00 \\
0.4\end{array}$ & $\begin{array}{l}1.5 \\
9.5\end{array}$ & $\begin{array}{r}19.0 \\
0.6\end{array}$ \\
\hline MAMGAMESE & $\begin{array}{l}02 / 06 / 89 \\
09 / 19 / 89 \\
04 / 11 / 90 \\
01 / 18 / 91\end{array}$ & $\begin{array}{l}0001 \\
0001 \\
0001 \\
0001\end{array}$ & $M G / L$ & & $\begin{array}{l}0.12 \\
0.12 \\
0.10 \\
0.08\end{array}$ & $\begin{array}{l}0.01 \\
0.01 \\
0.01 \\
0.01\end{array}$ & : \\
\hline MOLYBDENUM & $\begin{array}{l}02 / 06 / 89 \\
09 / 19 / 89 \\
04 / 11 / 90 \\
01 / 18 / 91 \\
12 / 04 / 92\end{array}$ & $\begin{array}{l}0001 \\
0001 \\
0001 \\
0001 \\
0001\end{array}$ & MG/L & & $\begin{array}{l}0.016 \\
0.05 \\
0.03 \\
0.02 \\
0.05\end{array}$ & $\begin{array}{l}0.01 \\
0.01 \\
0.01 \\
0.01 \\
0.01\end{array}$ & - \\
\hline HET GROSS ALPHA * & $\begin{array}{l}02 / 06 / 89 \\
09 / 19 / 89 \\
04 / 11 / 90 \\
01 / 18 / 91 \\
12 / 04 / 92\end{array}$ & $\begin{array}{l}0001 \\
0001 \\
0001 \\
0001 \\
0001\end{array}$ & $\mathrm{PCI} / \mathrm{L}$ & & $\begin{array}{r}28.31 \\
66.62 \\
127.46 \\
57.52 \\
6.10\end{array}$ & $\begin{array}{l}- \\
- \\
- \\
-\end{array}$ & $\begin{array}{l}- \\
- \\
-\end{array}$ \\
\hline HITRITE AND NITRATE & $\begin{array}{l}04 / 11 / 90 \\
01 / 18 / 91\end{array}$ & $\begin{array}{l}0001 \\
0001\end{array}$ & $M G / L$ & & $\begin{array}{l}499 . \\
54.8\end{array}$ & 1.05 & $\therefore$ \\
\hline PH & $\begin{array}{l}02 / 06 / 89 \\
09 / 19 / 89 \\
04 / 11 / 90 \\
01 / 18 / 91 \\
12 / 04 / 92\end{array}$ & $\begin{array}{l}0001 \\
0001 \\
0001 \\
0001 \\
0001\end{array}$ & su & & $\begin{array}{l}7.71 \\
7.23 \\
7.23 \\
7.57 \\
7.23\end{array}$ & $\begin{array}{l}- \\
- \\
-\end{array}$ & $\begin{array}{l}- \\
- \\
-\end{array}$ \\
\hline
\end{tabular}

- het gRosS alPHA (GROSS ALPHA - URANIUM) UITH 1 Mg URANIUH = 686 PCI

PARAMETER VALUE IMDICATOR (PVI): < - LESS THAH DETECTION LIMIT SAMPLE ID LOOES:

0001 - FILTERED SAMPLE (.45 MICROHS)

OTHER PARAMETER VALUE FLAGS:

1 - IMCREASED DETECTIOH LIMIT DUE TO REQUIRED DILUTION

$J$ - ESTIMATED VALUE 
GROUNDHATER QUALITY DATA BY LOCATIOH

SITE: AMBO1 AMBROSIA LAKE

LOCATIOH: 0678 RESERVED ESTORHS, MH, 11/14/88

MORTH COORDINATE: UNKNOWN

EAST COORDIHATE: UHKNOUN

06/10/80 TO 12/04/92

REPORT DATE: 08/04/94

FORMATION OF COMPLETION: TRES HERMANOS - B SAMDSTONE (TB)

HYDRAULIC FLOU RELATIONSHIP: CROSS GRADIENT (C)

\begin{tabular}{|c|c|c|c|c|c|c|c|}
\hline PARAKETER MAME & LOG DATE & $\begin{array}{c}\text { SAMPLE } \\
\text { ID }\end{array}$ & $\begin{array}{l}\text { UNITS OF } \\
\text { MEASURE }\end{array}$ & PVI & $\begin{array}{l}\text { PARAMETER } \\
\text { VALUE FLAGS }\end{array}$ & $\begin{array}{l}\text { DETECTIOH } \\
\text { LIAIT }\end{array}$ & $\begin{array}{l}\text { PARAMETER } \\
\text { UHCERTAIHTY }\end{array}$ \\
\hline PHOSPHATE & $\begin{array}{l}02 / 06 / 89 \\
09 / 19 / 89 \\
04 / 11 / 90 \\
01 / 18 / 91 \\
12 / 04 / 92\end{array}$ & $\begin{array}{l}0001 \\
0001 \\
0001 \\
0001 \\
0001\end{array}$ & $M G / L$ & $\begin{array}{l}< \\
< \\
< \\
<\end{array}$ & $\begin{array}{l}0.1 \\
0.1 \\
0.1 \\
0.08 \\
0.1\end{array}$ & $\begin{array}{l}0.1 \\
0.1 \\
0.1 \\
0.05 \\
0.1\end{array}$ & $\begin{array}{l}- \\
- \\
-\end{array}$ \\
\hline POLONIUA-210 & $\begin{array}{l}02 / 06 / 89 \\
09 / 19 / 89\end{array}$ & $\begin{array}{l}0001 \\
0001\end{array}$ & PCI/L & & $\begin{array}{l}2.17 \\
0.2\end{array}$ & $\begin{array}{l}1 . \\
1 .\end{array}$ & $\begin{array}{l}1.61 \\
0.4\end{array}$ \\
\hline POTASSIUA & $\begin{array}{l}02 / 06 / 89 \\
09 / 19 / 89 \\
04 / 11 / 90 \\
01 / 18 / 91 \\
12 / 04 / 92\end{array}$ & $\begin{array}{l}0001 \\
0001 \\
0001 \\
0001 \\
0001\end{array}$ & MG/L & & $\begin{array}{l}23.2 \\
29.3 \\
32.8 \\
21 . \\
24 .\end{array}$ & $\begin{array}{l}0.01 \\
0.01 \\
0.01 \\
5 . \\
5 .\end{array}$ & $\begin{array}{l}- \\
- \\
-\end{array}$ \\
\hline RADIUM-226 & $\begin{array}{l}02 / 06 / 89 \\
09 / 19 / 89 \\
04 / 11 / 90 \\
01 / 18 / 91 \\
12 / 04 / 92\end{array}$ & $\begin{array}{l}0001 \\
0001 \\
0001 \\
0001 \\
0001\end{array}$ & $\mathrm{PCI} / \mathrm{L}$ & & $\begin{array}{l}1.71 \\
1.1 \\
0.5 \\
3.0 \\
2.4\end{array}$ & $\begin{array}{l}1 . \\
1 . \\
1 . \\
1 . \\
0.3\end{array}$ & $\begin{array}{l}0.257 \\
0.4 \\
0.3 \\
0.8 \\
0.6\end{array}$ \\
\hline RADIUH-226 + RADIUHA-228 & $\begin{array}{l}02 / 06 / 89 \\
09 / 19 / 89 \\
04 / 11 / 90 \\
01 / 18 / 91 \\
12 / 04 / 92\end{array}$ & $\begin{array}{l}0001 \\
0001 \\
0001 \\
0001 \\
0001\end{array}$ & PCI/L & & $\begin{array}{l}4.63 \\
4.80 \\
6.10 \\
3.90 \\
9.80\end{array}$ & $\begin{array}{l}- \\
- \\
-\end{array}$ & $\begin{array}{l}- \\
- \\
- \\
-\end{array}$ \\
\hline RADIUM-228 & $\begin{array}{l}02 / 06 / 89 \\
09 / 19 / 89 \\
04 / 11 / 90 \\
01 / 18 / 91 \\
12 / 04 / 92\end{array}$ & $\begin{array}{l}0001 \\
0001 \\
0001 \\
0001 \\
0001\end{array}$ & $\mathrm{PCI} / \mathrm{L}$ & & $\begin{array}{l}2.92 \\
3.7 \\
5.6 \\
0.9 \\
7.4\end{array}$ & $\begin{array}{l}1 . \\
1 . \\
1 . \\
1.0 \\
1.0\end{array}$ & $\begin{array}{l}8.08 \\
1.0 \\
1.1 \\
1.6 \\
2.0\end{array}$ \\
\hline SELENIUA & $\begin{array}{l}02 / 06 / 89 \\
09 / 19 / 89 \\
04 / 11 / 90 \\
01 / 18 / 91 \\
12 / 04 / 92\end{array}$ & $\begin{array}{l}0001 \\
0001 \\
0001 \\
0001 \\
0001\end{array}$ & $M G / L$ & & $\begin{array}{l}0.011 \\
0.212 \\
0.100 \\
0.250 \\
0.7\end{array}$ & $\begin{array}{l}0.005 \\
0.005 \\
0.005 \\
0.005 \\
0.1\end{array}$ & $\begin{array}{l}- \\
- \\
-\end{array}$ \\
\hline SILICA - SIOZ & $\begin{array}{l}02 / 06 / 89 \\
09 / 19 / 89 \\
04 / 11 / 90 \\
01 / 18 / 91\end{array}$ & $\begin{array}{l}0001 \\
0001 \\
0001 \\
0001\end{array}$ & MG/L & & $\begin{array}{l}9.4 \\
6 . \\
5 . \\
4.5\end{array}$ & $\begin{array}{l}2 . \\
2 . \\
2 . \\
0.1\end{array}$ & $\begin{array}{l}- \\
-\end{array}$ \\
\hline SILVER & $\begin{array}{l}02 / 06 / 89 \\
09 / 19 / 89 \\
04 / 11 / 90 \\
01 / 18 / 91 \\
12 / 04 / 92\end{array}$ & $\begin{array}{l}0001 \\
0001 \\
0001 \\
0001 \\
0001\end{array}$ & MG/L & $\begin{array}{l}< \\
< \\
< \\
<\end{array}$ & $\begin{array}{l}0.01 \\
0.01 \\
0.01 \\
0.01 \\
0.01\end{array}$ & $\begin{array}{l}0.01 \\
0.01 \\
0.01 \\
0.01 \\
0.01\end{array}$ & $\begin{array}{l}- \\
- \\
-\end{array}$ \\
\hline SODIU: & $\begin{array}{l}02 / 06 / 89 \\
09 / 19 / 89 \\
04 / 11 / 90 \\
01 / 18 / 91 \\
12 / 04 / 92\end{array}$ & $\begin{array}{l}0001 \\
0001 \\
0001 \\
0001 \\
0001\end{array}$ & $M G / L$ & & $\begin{array}{l}1320 . \\
2850 . \\
2980 . \\
3290 . \\
3450 .\end{array}$ & $\begin{array}{l}0.002 \\
0.002 \\
0.002 \\
5 . \\
20 .\end{array}$ & $\begin{array}{l}- \\
- \\
-\end{array}$ \\
\hline SPECIFIC CONDUCTANCE & $02 / 06 / 89$ & 0001 & UHН/CH & & 3700. & - & - \\
\hline
\end{tabular}

PARAMETER VALUE INDICATOR (PVI): < - LESS THAN DETECTION LIMIT

SAMPLE ID COOES:

0001 - FILTERED SAMPLE (.45 MICRONS)

OTHER PARAMETER VALUE FLAGS:

H - HOLD TIME EXPIRED, VALUE SUSPECT

1. estimated value 
GROUNDHATER QUALITY DATA BY LOCATION

SITE: AMBOI AMBROSIA LAKE

LOCATIOH: 0678 RESERVED ESTORHS, HH, 11/14/818

HORTH COORDIHATE: UNKHOUN

EAST COORDIHATE: UNKHOHN

$06 / 10 / 80$ TO $12 / 04 / 92$

REPORT DATE: $08 / 04 / 94$

FORHATION OF COMPLETIOH: TRES HERHANOS - B SAMDSTOHE (TB) HYDRAULIC FLON RELATIONSHIP: CROSS GRADIENT (C)

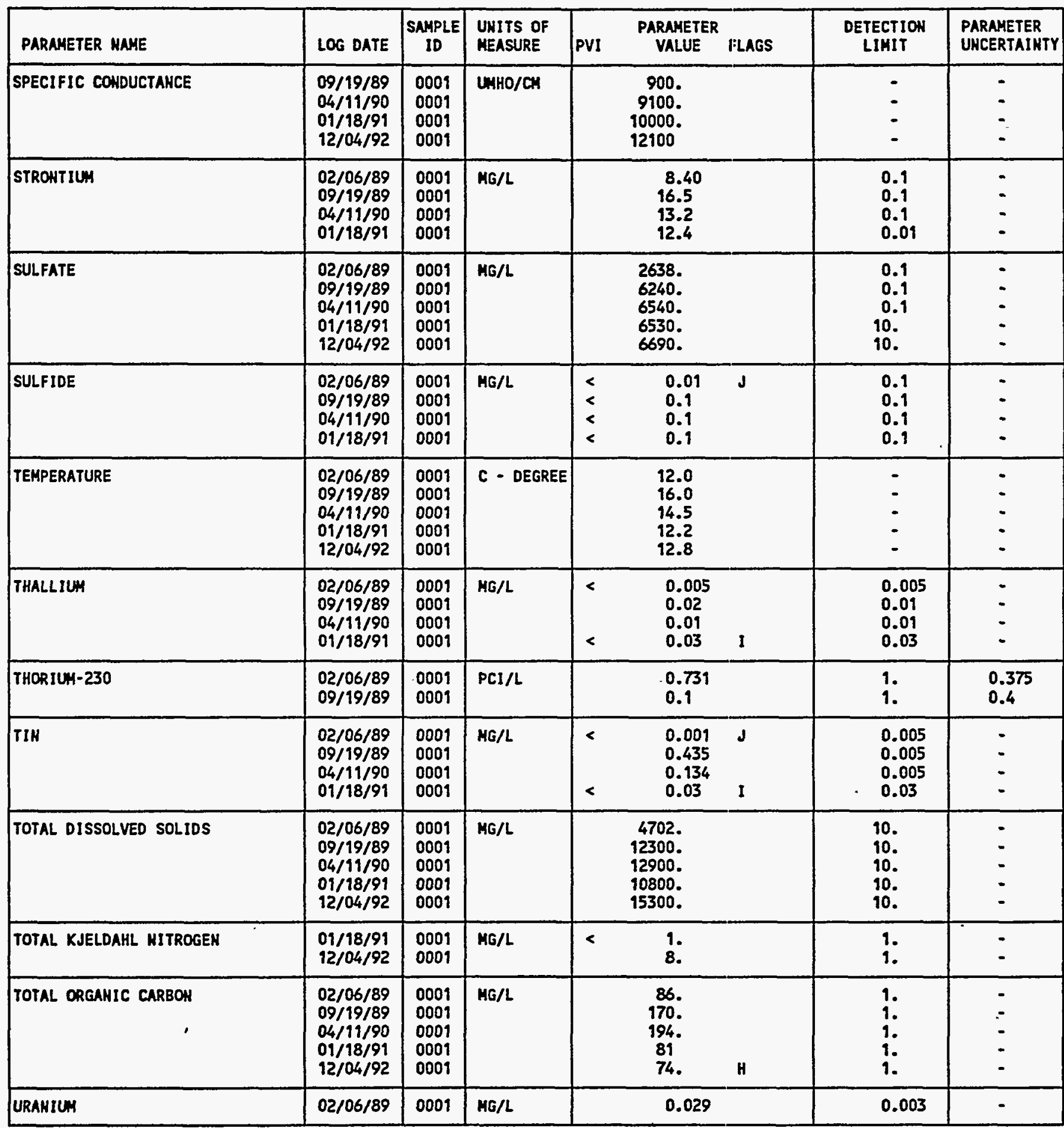

PARAMETER VALUE IMDICATOR (PVI): < - LESS THAN DETECTION LIMIT

OTHER PARAMETER VALUE FLAGS:

H - HOLD TIHE EXPIRED, VALUE SUSPECT

1 - IMCREASED DETECTION LIMIT DUE TO REQUIRED DILUTIOH

J - eStIMATEO VALUE 
GROUHDWATER QUALITY DATA BY LOCATION

SITE: AMBOI AMBROSIA LAKE

LOCATIOH: 0678 RESERVED ESTORMS, MH, 11/14/88

MORTH COORDIHATE: UMKNOWN

EAST COORDIMATE: UNKMONM

06/10/80 TO $12 / 04 / 92$

REPORT DATE: $08 / 04 / 94$

FORMATION OF CONPLETION: TRES HERMANOS - B SANDSTONE (TB) HYDRAULIC FLON RELATIONSHIP: CROSS GRADIEHT (C)

\begin{tabular}{|c|c|c|c|c|c|c|c|}
\hline PARAMETER NAME & LOG DATE & $\underset{\text { ID }}{\text { SAMPLE }}$ & $\begin{array}{l}\text { UNITS OF } \\
\text { MEASURE }\end{array}$ & PVI & $\begin{array}{l}\text { PARAMETER } \\
\text { VALUE FLAGS }\end{array}$ & $\begin{array}{l}\text { DETECTION } \\
\text { LIMIT }\end{array}$ & $\begin{array}{l}\text { PARAMETER } \\
\text { UNCERTAIHTY }\end{array}$ \\
\hline URANIUM & $\begin{array}{l}09 / 19 / 89 \\
04 / 11 / 90 \\
01 / 18 / 91 \\
12 / 04 / 92\end{array}$ & $\begin{array}{l}0001 \\
0001 \\
0001 \\
0001\end{array}$ & $M G / L$ & & $\begin{array}{l}0.037 \\
0.0037 \\
0.037 \\
0.057\end{array}$ & $\begin{array}{l}0.003 \\
0.003 \\
0.001 \\
0.001\end{array}$ & $:$ \\
\hline VANADIUH & $\begin{array}{l}02 / 06 / 89 \\
09 / 19 / 89 \\
04 / 11 / 90 \\
01 / 18 / 91 \\
12 / 04 / 92\end{array}$ & $\begin{array}{l}0001 \\
0001 \\
0001 \\
0001 \\
0001\end{array}$ & $M G / L$ & $\begin{array}{l}< \\
< \\
< \\
< \\
<\end{array}$ & $\begin{array}{l}0.01 \\
0.01 \\
0.01 \\
0.01 \\
0.01\end{array}$ & $\begin{array}{l}0.01 \\
0.01 \\
0.01 \\
0.01 \\
0.01\end{array}$ & : \\
\hline ZINC & $\begin{array}{l}02 / 06 / 89 \\
09 / 19 / 89 \\
04 / 11 / 90 \\
01 / 18 / 91\end{array}$ & $\begin{array}{l}0001 \\
0001 \\
0001 \\
0001\end{array}$ & $M G / L$ & $<$ & $\begin{array}{l}0.02 \\
0.006 \\
3.79 \\
0.005\end{array}$ & $\begin{array}{r}0.005 \\
0.005 \\
0.005 \\
0.005\end{array}$ & : \\
\hline
\end{tabular}

PARAMETER VALUE INDICATOR (PVI): < - LESS THAN DETECTION LIMIT SAMPLE ID COOES:

DOO1 - FILTERED SAMPLE (.45 MICROHS) 
GROUNDHATER QUALITY DATA BY LOCATIOH

SITE: AMBO1 AMBROSIA LAKE

LOCATION: 0679 RESERVED ESTORMS, MH, 11/14/88

NORTH COORDINATE: UNKNOWH

EAST COOROINATE: UKKHOWH

06/10/80 TO 12/04/92

REPORT DATE: 08/04/94

FORHATIOH OF COMPLETION: TRES HERMANOS-A SAHDSTONE (TR)

HYDRAULIC FLOW RELATIONSHIP: DOWN GRADIENT (D)

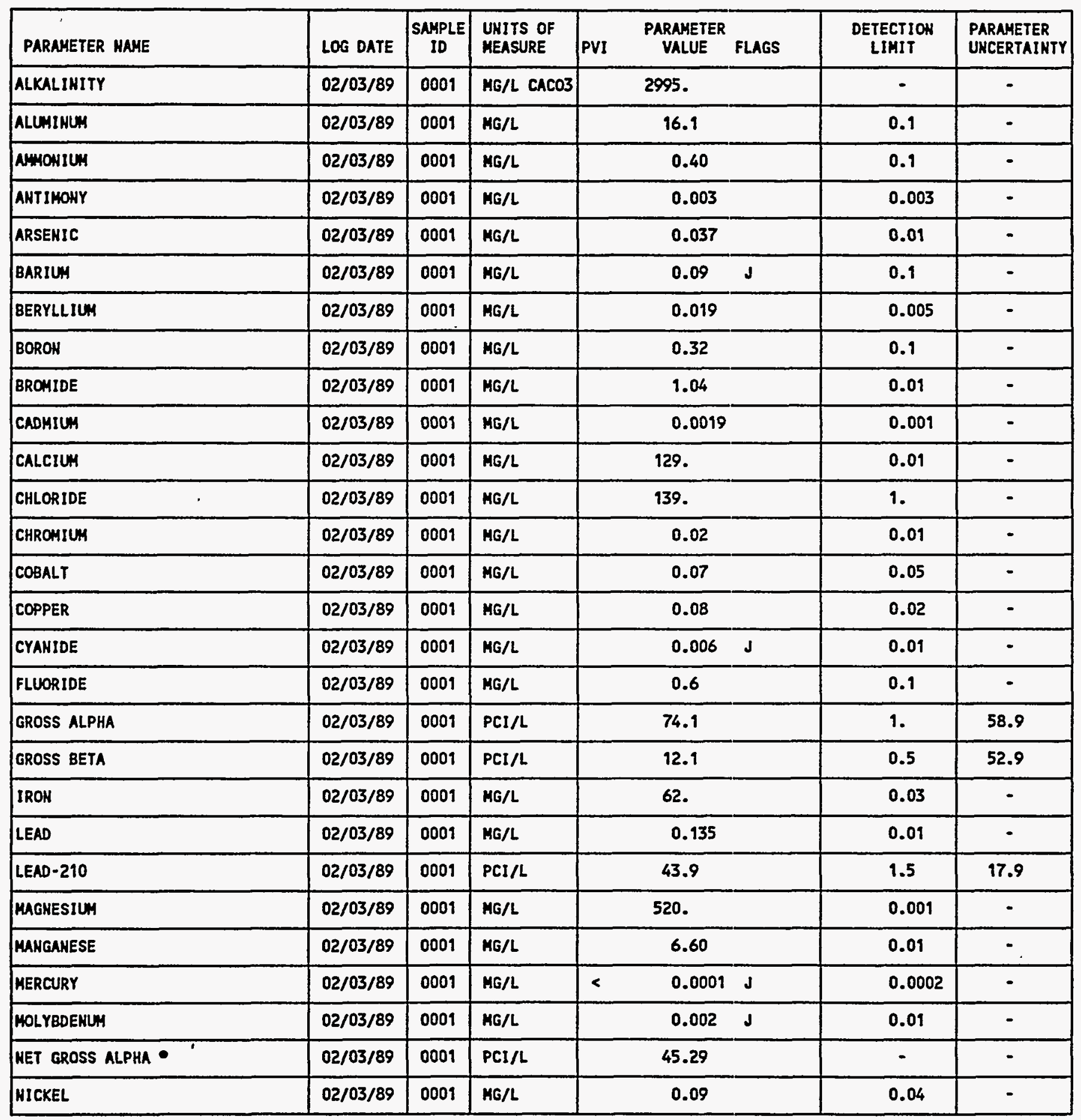

* het gROSS ALPHA (GROSS ALPHA - URAHIUM) MITH I MG URAHIUM = 686 PCI

PARAMETER VALUE IMDICATOR (PVI): < - LESS THAH DETECTION LIMIT SAMPLE ID COOES:

ODO1 - FILTERED SAMPLE (.45 MICRONS)

OTHER PARAMETER VALUE FLAGS:

$J$ - estimated VALUE 


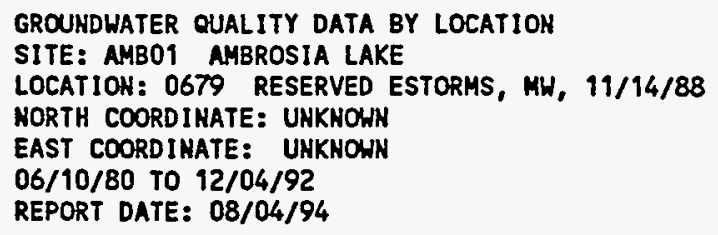

FORMATION OF COMPLETION: TRES HERMANOS-A SANDSTONE (TR) HYDRAULIC FLON RELATIONSHIP: DOWN GRADIENT (D)

\begin{tabular}{|c|c|c|c|c|c|c|c|}
\hline PARAMETER MAME & LOG DATE & $\underset{\text { IO }}{\text { SAMPLE }}$ & $\begin{array}{l}\text { UNITS OF } \\
\text { MEASURE }\end{array}$ & PVI & $\begin{array}{l}\text { PARAMETER } \\
\text { VALUE FLAGS }\end{array}$ & $\begin{array}{l}\text { DETECTION } \\
\text { LIMIT }\end{array}$ & $\begin{array}{l}\text { PARAMETER } \\
\text { UYCERTAIHTY }\end{array}$ \\
\hline NITRATE & $02 / 03 / 89$ & 0001 & MG/L & & 730. & 1. & - \\
\hline PH & $02 / 03 / 89$ & 0001 & su & & 8.08 & - & - \\
\hline PHOSPHATE & 02/03/89 & 0001 & $M G / L$ & $<$ & 0.1 & 0.1 & - \\
\hline POLONIUN-210 & $02 / 03 / 89$ & 0001 & $\mathrm{PCI} / \mathrm{L}$ & & 0.932 & 1. & 1.36 \\
\hline POTASSIUY & $02 / 03 / 89$ & 0001 & HG/L & & 19.5 & 0.01 & - \\
\hline RADIUH-226 & $02 / 03 / 89$ & 0001 & $\mathrm{PCI} / \mathrm{L}$ & & 5.09 & 1. & 1.30 \\
\hline RADIUH-226 + RADIUH-228 & $02 / 03 / 89$ & 0001 & $\mathrm{PCI} / \mathrm{L}$ & & 13.80 & $\cdot$ & $\cdot$ \\
\hline RADIUM-228 & $02 / 03 / 89$ & 0001 & $\mathrm{PCI} / \mathrm{L}$ & & 8.71 & 1. & 9.09 \\
\hline SELEHIUM & $02 / 03 / 89$ & 0001 & $M G / L$ & & 0.004 & 0.005 & - \\
\hline SILICA - SIOZ & $02 / 03 / 89$ & 0001 & $M G / L$ & & 5.0 & 2. & - \\
\hline SILVER & $02 / 03 / 89$ & 0001 & MG/L & $<$ & 0.01 & 0.01 & - \\
\hline SOOIUM & $02 / 03 / 89$ & 0001 & $M G / L$ & & 2400 & 0.002 & - \\
\hline SPECIFIC CONDUCTANCE & $02 / 03 / 89$ & 0001 & UMHO/CM & & 4000 & - & - \\
\hline STRONTIUM & $02 / 03 / 89$ & 0001 & MG/L & & 18.6 & 0.1 & - \\
\hline SULFATE & 02/03/89 & 0001 & MG/L & & 3935. & 0.1 & - \\
\hline SULFIDE & 02/03/89 & 0001 & MG/L & & 0.04 & 0.1 & - \\
\hline TEMPERATURE & $02 / 03 / 89$ & 0001 & C - DEGREE & & 10.5 & - & - \\
\hline THALLIUM & $02 / 03 / 89$ & 0001 & $M G / L$ & $<$ & 0.005 & 0.005 & - \\
\hline THORIUH-230 & $02 / 03 / 89$ & 0001 & $\mathrm{PCI} / \mathrm{L}$ & & 23.8 & 1. & 2.80 \\
\hline TIM & $02 / 03 / 89$ & 0001 & $M G / L$ & $<$ & $0.001 \mathrm{~J}$ & 0.005 & - \\
\hline TOTAL DISSOLVED SOLIDS & 02/03/89 & 0001 & MG/L & & 6778. & 10. & - \\
\hline TOTAL ORGANIC CARBOH & $02 / 03 / 89$ & 0001 & MG/L. & & 430. & 1. & $\cdot$ \\
\hline URAHIUH & $02 / 03 / 89$ & 0001 & MG/L & & 0.042 & 0.003 & - \\
\hline VANADIUA & $02 / 03 / 89$ & 0001 & HG/L & & 0.03 & 0.01 & - \\
\hline ZIMC & $02 / 03 / 89$ & 0001 & $M G / L$ & & 0.60 & 0.005 & - \\
\hline
\end{tabular}

PARAMETER VALUE INDICATOR (PVI): < - LESS THAK DETECTION LIMIT SAMPLE IO COOES:

0001 - FILTERED SAMPLE (.45 MICROHS)

OTHER PARAMETER VALUE FLAGS:

J - ESTIMATED VALUE 
GROUKDWATER QUALITY OATA BY LOCATIOH

SITE: AMBO1 AMBROSIA LAKE

LOCATION: 0680 RESERVED ESTORMS, MH, 11/1i//88

HORTH COORDIHATE: UNKHOWH

EAST COORDIMATE: UNKHOWN

06/10/80 TO 12/04/92

REPORT DATE: $08 / 04 / 94$

FORHATION OF COMPLETION: DAKOTA SANDSTONE (KD)

HYDRAULIC FLON RELATLOHSHIP: UPGRADIENT (U)

\begin{tabular}{|c|c|c|c|c|c|c|c|c|}
\hline PARAMETER NAME & LOG DATE & SAMPLE & $\begin{array}{l}\text { UNITS OF } \\
\text { MEASURE }\end{array}$ & PVI & $\begin{array}{l}\text { PARAMETER: } \\
\text { VALUE }\end{array}$ & FLAGS & $\begin{array}{l}\text { DETECTION } \\
\text { LIMIT }\end{array}$ & $\begin{array}{l}\text { PARAMETER } \\
\text { UHCERTAIHTY }\end{array}$ \\
\hline ALKALIKITY & $\begin{array}{l}02 / 03 / 89 \\
09 / 19 / 89 \\
04 / 11 / 90 \\
01 / 15 / 91 \\
12 / 03 / 92\end{array}$ & $\begin{array}{l}0001 \\
0001 \\
0001 \\
0001 \\
0001\end{array}$ & MG/L CACO3 & & $\begin{array}{l}322 . \\
359 . \\
354 . \\
344 . \\
329\end{array}$ & & $\begin{array}{l}- \\
- \\
-\end{array}$ & $\begin{array}{l}- \\
- \\
- \\
-\end{array}$ \\
\hline ALUMIHUM & $\begin{array}{l}02 / 03 / 89 \\
09 / 19 / 89 \\
04 / 11 / 90 \\
01 / 15 / 91\end{array}$ & $\begin{array}{l}0001 \\
0001 \\
0001 \\
0001\end{array}$ & MG/L & $\begin{array}{l}< \\
<\end{array}$ & $\begin{array}{l}0.15 \\
0.1 \\
0.1 \\
0.05\end{array}$ & & $\begin{array}{l}0.1 \\
0.1 \\
0.1 \\
0.05\end{array}$ & . \\
\hline Am,ONIU: & $\begin{array}{l}02 / 03 / 89 \\
09 / 19 / 89 \\
04 / 11 / 90 \\
01 / 15 / 91 \\
12 / 03 / 92\end{array}$ & $\begin{array}{l}0001 \\
0001 \\
0001 \\
0001 \\
0001\end{array}$ & MG/L & $<$ & $\begin{array}{l}0.13 \\
0.1 \\
0.1 \\
0.27 \\
0.2\end{array}$ & $J$ & $\begin{array}{l}0.1 \\
0.1 \\
0.1 \\
0.01 \\
0.1\end{array}$ & $\begin{array}{l}- \\
- \\
- \\
-\end{array}$ \\
\hline ANTIMONY & $\begin{array}{l}02 / 03 / 89 \\
09 / 19 / 89 \\
04 / 11 / 90 \\
01 / 15 / 91 \\
12 / 03 / 92\end{array}$ & $\begin{array}{l}0001 \\
0001 \\
0001 \\
0001 \\
0001\end{array}$ & MG/L & $<$ & $\begin{array}{l}0.003 \\
0.007 \\
0.021 \\
0.003 \\
0.003\end{array}$ & & $\begin{array}{l}0.003 \\
0.003 \\
0.003 \\
0.003 \\
0.003\end{array}$ & $\begin{array}{l}- \\
- \\
- \\
-\end{array}$ \\
\hline ARSEKIC & $\begin{array}{l}02 / 03 / 89 \\
09 / 19 / 89 \\
04 / 11 / 90 \\
01 / 15 / 91\end{array}$ & $\begin{array}{l}0001 \\
0001 \\
0001 \\
0001\end{array}$ & $M G / L$ & $<$ & $\begin{array}{l}0.005 \\
0.02 \\
0.01 \\
0.03\end{array}$ & $\begin{array}{l}\mathbf{J} \\
\mathbf{I}\end{array}$ & $\begin{array}{l}0.01 \\
0.01 \\
0.01 \\
0.03\end{array}$ & $\begin{array}{l}- \\
- \\
-\end{array}$ \\
\hline BARIUH & $\begin{array}{l}02 / 03 / 89 \\
09 / 19 / 89 \\
04 / 11 / 90 \\
01 / 15 / 91\end{array}$ & $\begin{array}{l}0001 \\
0001 \\
0001 \\
0001\end{array}$ & MG/L & $<$ & $\begin{array}{l}0.02 \\
0.1 \\
0.1 \\
0.01\end{array}$ & J & $\begin{array}{l}0.1 \\
0.1 \\
0.1 \\
0.01\end{array}$ & $\begin{array}{l}- \\
- \\
-\end{array}$ \\
\hline BERYLLIUUM & $\begin{array}{l}02 / 03 / 89 \\
09 / 19 / 89 \\
04 / 11 / 90 \\
01 / 15 / 91\end{array}$ & $\begin{array}{l}0001 \\
0001 \\
0001 \\
0001\end{array}$ & MG/L & $\begin{array}{l}< \\
< \\
< \\
<\end{array}$ & $\begin{array}{l}0.005 \\
0.01 \\
0.01 \\
0.005\end{array}$ & & $\begin{array}{l}0.005 \\
0.01 \\
0.01 \\
0.005\end{array}$ & $\begin{array}{l}- \\
-\end{array}$ \\
\hline BORON & $\begin{array}{l}02 / 03 / 89 \\
09 / 19 / 89 \\
04 / 11 / 90 \\
01 / 15 / 91\end{array}$ & $\begin{array}{l}0001 \\
0001 \\
0001 \\
0001\end{array}$ & MG/L & & $\begin{array}{l}0.77 \\
0.2 \\
0.1 \\
0.18\end{array}$ & & $\begin{array}{l}0.1 \\
0.1 \\
0.1 \\
0.05\end{array}$ & $\begin{array}{l}- \\
-\end{array}$ \\
\hline BROMIDE & $\begin{array}{l}02 / 03 / 89 \\
09 / 19 / 89 \\
04 / 11 / 90 \\
01 / 15 / 91\end{array}$ & $\begin{array}{l}0001 \\
0001 \\
0001 \\
0001\end{array}$ & MG/L & & $\begin{array}{l}0.67 \\
0.2 \\
0.9 \\
0.6\end{array}$ & J & $\begin{array}{l}0.01 \\
0.1 \\
0.1 \\
0.1\end{array}$ & : \\
\hline CADMIUY & $\begin{array}{l}02 / 03 / 89 \\
09 / 19 / 89 \\
04 / 11 / 90 \\
01 / 15 / 91 \\
12 / 03 / 92\end{array}$ & $\begin{array}{l}0001 \\
0001 \\
0001 \\
0001 \\
0001\end{array}$ & HG/L & $\begin{array}{l}< \\
<\end{array}$ & $\begin{array}{l}0.0006 \\
0.002 \\
0.001 \\
0.001 \\
0.001\end{array}$ & $\mathbf{J}$ & $\begin{array}{l}0.001 \\
0.001 \\
0.001 \\
0.001 \\
0.001\end{array}$ & $\begin{array}{l}- \\
- \\
-\end{array}$ \\
\hline CALCIUH & $\begin{array}{l}02 / 03 / 89 \\
09 / 19 / 89\end{array}$ & $\begin{array}{l}0001 \\
0001\end{array}$ & MG/L & & $\begin{array}{l}317 . \\
587 .\end{array}$ & & $\begin{array}{l}0.01 \\
0.01\end{array}$ & - \\
\hline
\end{tabular}

PARAMETER VALUE IHDICATOR (PVI): < - LESS THAN DETECTION LIMIT SAMPLE ID I:OOES:

D001 - FILIERED SAMPLE (.45 MICRONS)

OTHER PARAMETER VALUE FLAGS:

I - INCREASED DETECTION LIMIT DUE TO REQUIRED DILUTIOH

J - ESTIMATED VALUE 
GROUKDWATER OUALITY OATA BY LOCATION

SITE: AMBO1 AMBROSIA LAKE

LOCATIOH: 0680 RESERVED ESTORMS, MH, 11/14/88

MORTH COORDINATE: UNKHOWH

EAST COORDINATE: UNKHOWN

$06 / 10 / 80$ TO $12 / 04 / 92$

REPORT OATE: $08 / 04 / 94$

FORHATION OF CONPLETION: DAKOTA SANDSTOHE (KD)

HYORAULIC FLON RELATIONSHIP: UPGRADIENT (U)

\begin{tabular}{|c|c|c|c|c|c|c|c|}
\hline PARAMETER NAME & LOG DATE & $\underset{\text { SD }}{\text { SAMPLE }}$ & $\begin{array}{l}\text { UNITS OF } \\
\text { MEASURE }\end{array}$ & PVI & $\begin{array}{l}\text { PARAMETER } \\
\text { VALUE FLAGS }\end{array}$ & $\begin{array}{l}\text { DETECTION } \\
\text { LIMIT }\end{array}$ & $\begin{array}{l}\text { PARAMETER } \\
\text { UHCERTAINTY }\end{array}$ \\
\hline CALCIUH & $\begin{array}{l}04 / 11 / 90 \\
01 / 15 / 91 \\
12 / 03 / 92\end{array}$ & $\begin{array}{l}0001 \\
0001 \\
0001\end{array}$ & $M G / L$ & & $\begin{array}{l}529 . \\
595 . \\
600 .\end{array}$ & $\begin{array}{l}0.01 \\
0.01 \\
2 .\end{array}$ & $\dot{-}$ \\
\hline CHLORIDE & $\begin{array}{l}02 / 03 / 89 \\
09 / 19 / 89 \\
04 / 11 / 90 \\
01 / 15 / 91 \\
12 / 03 / 92\end{array}$ & $\begin{array}{l}0001 \\
0001 \\
0001 \\
0001 \\
0001\end{array}$ & MG/L & & $\begin{array}{c}178 . \\
61 . \\
73 . \\
69 . \\
80 .\end{array}$ & $\begin{array}{l}1 . \\
1 . \\
1 . \\
0.5 \\
5 .\end{array}$ & $\begin{array}{l}- \\
- \\
-\end{array}$ \\
\hline CHROAIUA & $\begin{array}{l}02 / 03 / 89 \\
09 / 19 / 89 \\
04 / 11 / 90 \\
01 / 15 / 91 \\
12 / 03 / 92\end{array}$ & $\begin{array}{l}0001 \\
0001 \\
0001 \\
0001 \\
0001\end{array}$ & MG/L & $\begin{array}{l}< \\
< \\
< \\
<\end{array}$ & $\begin{array}{l}0.01 \\
0.01 \\
0.01 \\
0.01 \\
0.01\end{array}$ & $\begin{array}{l}0.01 \\
0.01 \\
0.01 \\
0.01 \\
0.01\end{array}$ & $\begin{array}{l}: \\
:\end{array}$ \\
\hline COBALT & $\begin{array}{l}02 / 03 / 89 \\
09 / 19 / 89 \\
04 / 11 / 90 \\
01 / 15 / 91 \\
12 / 03 / 92\end{array}$ & $\begin{array}{l}0001 \\
0001 \\
0001 \\
0001 \\
0001\end{array}$ & MG/L & $\begin{array}{l}< \\
< \\
<\end{array}$ & $\begin{array}{l}0.01 \\
0.05 \\
0.05 \\
0.03 \\
0.03\end{array}$ & $\begin{array}{l}0.05 \\
0.05 \\
0.05 \\
0.03 \\
0.03\end{array}$ & $\begin{array}{l}- \\
- \\
.\end{array}$ \\
\hline COPPER & $\begin{array}{l}02 / 03 / 89 \\
09 / 19 / 89 \\
04 / 11 / 90 \\
01 / 15 / 91\end{array}$ & $\begin{array}{l}0001 \\
0001 \\
0001 \\
0001\end{array}$ & HG/L & $\begin{array}{l}< \\
<\end{array}$ & $\begin{array}{l}0.02 \\
0.02 \\
0.02 \\
0.01\end{array}$ & $\begin{array}{l}0.02 \\
0.02 \\
0.02 \\
0.01\end{array}$ & $\begin{array}{l}- \\
-\end{array}$ \\
\hline CYANIDE & $\begin{array}{l}02 / 03 / 89 \\
09 / 19 / 89 \\
04 / 11 / 90 \\
01 / 15 / 91\end{array}$ & $\begin{array}{l}0001 \\
0001 \\
0001 \\
0001\end{array}$ & MG/L & $\begin{array}{l}< \\
< \\
<\end{array}$ & $\begin{array}{l}0.002 \mathrm{~J} \\
0.01 \\
0.01 \\
0.01\end{array}$ & $\begin{array}{l}0.01 \\
0.01 \\
0.01 \\
0.01\end{array}$ & $\begin{array}{l}- \\
-\end{array}$ \\
\hline FLUORIDE & $\begin{array}{l}02 / 03 / 89 \\
09 / 19 / 89 \\
04 / 19 / 90 \\
01 / 15 / 91 \\
12 / 03 / 92\end{array}$ & $\begin{array}{l}0001 \\
0001 \\
0001 \\
0001 \\
0001\end{array}$ & MG/L & & $\begin{array}{l}0.6 \\
0.2 \\
0.2 \\
0.1 \\
0.3\end{array}$ & $\begin{array}{l}0.1 \\
0.1 \\
0.1 \\
0.1 \\
0.1\end{array}$ & $\begin{array}{l}- \\
- \\
-\end{array}$ \\
\hline GROSS ALPHA & $\begin{array}{l}02 / 03 / 89 \\
09 / 19 / 89 \\
04 / 11 / 90 \\
01 / 15 / 91 \\
12 / 03 / 92\end{array}$ & $\begin{array}{l}0001 \\
0001 \\
0001 \\
0001 \\
0001\end{array}$ & $\mathrm{PCI} / \mathrm{L}$ & & $\begin{array}{l}15.6 \\
12 . \\
29 . \\
0.0 \\
25.0\end{array}$ & $\begin{array}{l}1 . \\
1 . \\
1 . \\
1.0\end{array}$ & $\begin{array}{l}20.0 \\
20 . \\
18 . \\
28.5 \\
30.5\end{array}$ \\
\hline GROSS BETA & $\begin{array}{l}02 / 03 / 89 \\
09 / 19 / 89 \\
04 / 11 / 90 \\
01 / 15 / 91 \\
12 / 03 / 92\end{array}$ & $\begin{array}{l}0001 \\
0001 \\
0001 \\
0001 \\
0001\end{array}$ & PCI/L & & $\begin{array}{l}33.2 \\
33 . \\
30 . \\
22.8 \\
0.0\end{array}$ & $\begin{array}{r}0.5 \\
0.5 \\
0.5 \\
0.5 \\
50.3\end{array}$ & $\begin{array}{l}24.2 \\
11 . \\
14 . \\
18.4 \\
28.4\end{array}$ \\
\hline IRON & $\begin{array}{l}02 / 03 / 89 \\
09 / 19 / 89 \\
04 / 11 / 90 \\
01 / 15 / 91 \\
12 / 03 / 92\end{array}$ & $\begin{array}{l}0001 \\
0001 \\
0001 \\
0001 \\
0001\end{array}$ & MG/L & & $\begin{array}{l}1.61 \\
2.15 \\
2.23 \\
2.31 \\
1.82\end{array}$ & $\begin{array}{l}0.03 \\
0.03 \\
0.03 \\
0.03 \\
0.03\end{array}$ & $\begin{array}{l}- \\
- \\
- \\
-\end{array}$ \\
\hline LEAD & $02 / 03 / 89$ & 0001 & MG/L & & 0.010 & 0.01 & - \\
\hline
\end{tabular}

PARAMETER VALUE IKDICATOR (PVI): < - LESS THAN DETECTION LIMIT

OTHER PARAMETER VALUE FLAGS:

$d$ - ESTIMATED VALUE 
GROUHDWATER QUALITY DATA BY LOCATIOH

SITE: AMBO1 AMBROSIA LAKE

LOCATIOH: O68O RESERVED ESTORMS, MH, 11/14/88

MORTH COORDINATE: UHKHOWH

EAST COORDIHATE: UNKNONH

06/10/80 TO 12/04/92

REPORT OATE: 08/04/94

FORHATION OF COMPLETION: DAKOTA SANDSTONE (KD)

HYDRAULIC FLOU RELATIONSHIP: UPGRADIENT (U)

\begin{tabular}{|c|c|c|c|c|c|c|c|c|}
\hline PARAMETER NAME & LOG DATE & $\underset{10}{\text { SAMPLE }}$ & $\begin{array}{l}\text { UHITS OF } \\
\text { MEASURE }\end{array}$ & PVI & $\begin{array}{l}\text { PARAMETER } \\
\text { VALUE }\end{array}$ & FLAGS & $\begin{array}{l}\text { DETECTION } \\
\text { LIMIT }\end{array}$ & $\begin{array}{l}\text { PARAMETER } \\
\text { UNCERTAINTY }\end{array}$ \\
\hline LEAD & $\begin{array}{l}09 / 19 / 89 \\
04 / 11 / 90 \\
01 / 15 / 91\end{array}$ & $\begin{array}{l}0001 \\
0001 \\
0001\end{array}$ & MG/L & $<$ & $\begin{array}{l}0.01 \\
0.01 \\
0.03\end{array}$ & 1 & $\begin{array}{l}0.01 \\
0.01 \\
0.03\end{array}$ & $\dot{-}$ \\
\hline LEAD-210 & $\begin{array}{l}02 / 03 / 89 \\
09 / 19 / 89\end{array}$ & $\begin{array}{l}0001 \\
0001\end{array}$ & PCI/L & & $\begin{array}{l}0.00 \\
0.0\end{array}$ & & $\begin{array}{l}9.5 \\
1.5\end{array}$ & $\begin{array}{l}6.25 \\
0.8\end{array}$ \\
\hline MAGKESIUIM & $\begin{array}{l}02 / 03 / 89 \\
09 / 19 / 89 \\
04 / 11 / 90 \\
01 / 15 / 91 \\
12 / 03 / 92\end{array}$ & $\begin{array}{l}0001 \\
0001 \\
0001 \\
0001 \\
0001\end{array}$ & MG/L & & $\begin{array}{l}84 . \\
212 . \\
210 \\
222 \\
213 .\end{array}$ & & $\begin{array}{l}0.001 \\
0.001 \\
0.001 \\
0.5 \\
0.5\end{array}$ & $\begin{array}{l}- \\
- \\
- \\
-\end{array}$ \\
\hline MANGAMESE & $\begin{array}{l}02 / 03 / 89 \\
09 / 19 / 89 \\
04 / 11 / 90 \\
01 / 15 / 91\end{array}$ & $\begin{array}{l}0001 \\
0001 \\
0001 \\
0001\end{array}$ & MG/L & & $\begin{array}{l}0.51 \\
1.35 \\
1.35 \\
1.22\end{array}$ & & $\begin{array}{l}0.01 \\
0.01 \\
0.01 \\
0.01\end{array}$ & $\begin{array}{l}- \\
-\end{array}$ \\
\hline MERCURY & $\begin{array}{l}02 / 03 / 89 \\
09 / 19 / 89 \\
04 / 19 / 90 \\
01 / 15 / 91\end{array}$ & $\begin{array}{l}0001 \\
0009 \\
0009 \\
0001\end{array}$ & MG/L & $\begin{array}{l}< \\
< \\
<\end{array}$ & $\begin{array}{l}0.0001 \\
0.0002 \\
0.0002 \\
0.0002\end{array}$ & J & $\begin{array}{l}0.0002 \\
0.0002 \\
0.0002 \\
0.0002\end{array}$ & $\begin{array}{l}- \\
- \\
-\end{array}$ \\
\hline MOLYBDENUM & $\begin{array}{l}02 / 03 / 89 \\
09 / 19 / 89 \\
04 / 11 / 90 \\
01 / 15 / 91 \\
12 / 03 / 92\end{array}$ & $\begin{array}{l}0001 \\
0001 \\
0001 \\
0001 \\
0001\end{array}$ & MG/L & & $\begin{array}{l}0.058 \\
0.12 \\
0.10 \\
0.09 \\
0.08\end{array}$ & & $\begin{array}{l}0.01 \\
0.01 \\
0.01 \\
0.01 \\
0.01\end{array}$ & $\begin{array}{l}- \\
- \\
-\end{array}$ \\
\hline HET GROSS ALPHA * & $\begin{array}{l}02 / 03 / 89 \\
09 / 19 / 89 \\
04 / 11 / 90 \\
01 / 15 / 91 \\
12 / 03 / 92\end{array}$ & $\begin{array}{l}0001 \\
0001 \\
0001 \\
0001 \\
0001\end{array}$ & PCI/L & & $\begin{array}{l}-0.18 \\
10.63 \\
27.90 \\
-0.34 \\
24.31\end{array}$ & & $\begin{array}{l}- \\
- \\
- \\
-\end{array}$ & $\begin{array}{l}- \\
- \\
-\end{array}$ \\
\hline HICKEL & $\begin{array}{l}02 / 03 / 89 \\
09 / 99 / 89 \\
04 / 11 / 90 \\
01 / 15 / 91 \\
12 / 03 / 92\end{array}$ & $\begin{array}{l}0001 \\
0001 \\
0001 \\
0001 \\
0001\end{array}$ & MG/L & $\begin{array}{l}< \\
< \\
<\end{array}$ & $\begin{array}{l}0.02 \\
0.04 \\
0.04 \\
0.04 \\
0.10\end{array}$ & 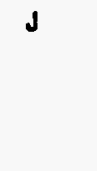 & $\begin{array}{l}0.04 \\
0.04 \\
0.04 \\
0.04 \\
0.04\end{array}$ & $\begin{array}{l}- \\
- \\
- \\
-\end{array}$ \\
\hline NITRATE & $\begin{array}{l}02 / 03 / 89 \\
09 / 19 / 89 \\
04 / 11 / 90 \\
01 / 15 / 91 \\
12 / 03 / 92\end{array}$ & $\begin{array}{l}0001 \\
0001 \\
0001 \\
0001 \\
0001\end{array}$ & $M G / L$ & $\begin{array}{l}< \\
<\end{array}$ & $\begin{array}{l}6.78 \\
1 . \\
0.8 \\
4.4 \\
1.0\end{array}$ & $\mathbf{J}$ & $\begin{array}{l}1 . \\
1 . \\
1 . \\
0.1 \\
1.0\end{array}$ & $\begin{array}{l}- \\
- \\
- \\
-\end{array}$ \\
\hline HITRITE AKD MITRATE & $\begin{array}{l}04 / 11 / 90 \\
01 / 15 / 91\end{array}$ & $\begin{array}{l}0001 \\
0001\end{array}$ & MG/L & & $\begin{array}{l}0.2 \\
0.94\end{array}$ & J & $\begin{array}{l}1.05 \\
0.05\end{array}$ & $\because$ \\
\hline PH & $\begin{array}{l}02 / 03 / 89 \\
09 / 19 / 89 \\
04 / 11 / 90 \\
01 / 15 / 91\end{array}$ & $\begin{array}{l}0001 \\
0001 \\
0001 \\
0001\end{array}$ & SU & & $\begin{array}{l}7.12 \\
6.59 \\
6.53 \\
6.9\end{array}$ & & : & : \\
\hline
\end{tabular}

- Met gross aLPHA (GROSS ALPHA - URAhIUM) HITH 1 MG URAMIUH = 686 PCI

PARAMETER VALUE IMDICATOR (PVI): < - LESS THAN DETECTIOH LIMIT

OTHER PARAMETER VALUE FLAGS:

1 - IHCREASED DETECTION LIMIT DUE TO REQUIRED DILUTION

J - ESTIMATED VALUE 
GROUNDWATER QUALITY DATA BY LOCATION

SITE: AMBOI AMBROSIA LAKE

LOCATION: 0680 RESERVED ESTORMS, MH, 11/14/88

NORTK COORDIHATE: UHKNOWN

EAST COORDINATE: UNKHOWH

06/10/80 TO $12 / 04 / 92$

REPORT DATE: $08 / 04 / 94$

FORMATION OF COMPLETIOH: DAKOTA SANDSTOHE (KD)

HYDRAULIC FLOH RELATIONSHIP: UPGRADIERT (U)

\begin{tabular}{|c|c|c|c|c|c|c|c|}
\hline PARAMETER NAHE & LOG DATE & $\underset{10}{\text { SAMPLE }}$ & $\begin{array}{l}\text { UNITS OF } \\
\text { MEASURE }\end{array}$ & PVI & $\begin{array}{l}\text { PARAMETER } \\
\text { VALUE FLAGS }\end{array}$ & $\begin{array}{l}\text { DETECTIOH } \\
\text { LIHIT }\end{array}$ & $\begin{array}{l}\text { PARAMETER } \\
\text { UNCERTAIRTY }\end{array}$ \\
\hline PH & $12 / 03 / 92$ & 0001 & SU & & 6.74 & - & - \\
\hline PHOSPHATE & $\begin{array}{l}02 / 03 / 89 \\
09 / 19 / 89 \\
04 / 11 / 90 \\
01 / 15 / 91 \\
12 / 03 / 92\end{array}$ & $\begin{array}{l}0001 \\
0001 \\
0001 \\
0001 \\
0001\end{array}$ & $M G / L$ & $\begin{array}{l}< \\
<\end{array}$ & $\begin{array}{l}0.1 \\
0.1 \\
0.1 \\
0.23 \\
0.3\end{array}$ & $\begin{array}{l}0.1 \\
0.1 \\
0.1 \\
0.05 \\
0.1\end{array}$ & $\begin{array}{l}- \\
- \\
-\end{array}$ \\
\hline POLOHIUM-210 & $\begin{array}{l}02 / 03 / 89 \\
09 / 19 / 89\end{array}$ & $\begin{array}{l}0001 \\
0001\end{array}$ & $\mathrm{PCI} / \mathrm{L}$ & & $\begin{array}{l}0.466 \\
0.3\end{array}$ & 1. & $\begin{array}{l}1.26 \\
0.4\end{array}$ \\
\hline POTASSIU: & $\begin{array}{l}02 / 03 / 89 \\
09 / 19 / 89 \\
04 / 11 / 90 \\
01 / 15 / 91 \\
12 / 03 / 92\end{array}$ & $\begin{array}{l}0001 \\
0001 \\
0001 \\
0001 \\
0001\end{array}$ & HG/L & & $\begin{array}{c}22.6 \\
9.8 \\
10.9 \\
8.3 \\
8.75\end{array}$ & $\begin{array}{l}0.01 \\
0.01 \\
0.01 \\
0.01 \\
0.01\end{array}$ & $\begin{array}{l}- \\
- \\
-\end{array}$ \\
\hline RADIUM-226 & $\begin{array}{l}02 / 03 / 89 \\
09 / 19 / 89 \\
04 / 11 / 90 \\
01 / 15 / 91 \\
12 / 03 / 92\end{array}$ & $\begin{array}{l}0001 \\
0001 \\
0001 \\
0001 \\
0001\end{array}$ & $\mathrm{PCI} / \mathrm{L}$ & & $\begin{array}{l}2.60 \\
1.5 \\
0.9 \\
3.5 \\
2.2\end{array}$ & $\begin{array}{l}1 . \\
1 . \\
1 . \\
0.3\end{array}$ & $\begin{array}{l}0.351 \\
0.4 \\
0.3 \\
0.9 \\
0.6\end{array}$ \\
\hline RADIUH-226 + RADIUM-228 & $\begin{array}{l}02 / 03 / 89 \\
09 / 19 / 89 \\
04 / 11 / 90 \\
01 / 15 / 91 \\
12 / 03 / 92\end{array}$ & $\begin{array}{l}0001 \\
0001 \\
0001 \\
0001 \\
0001\end{array}$ & $\mathrm{PCI} / \mathrm{L}$ & & $\begin{array}{l}3.82 \\
4.10 \\
4.50 \\
6.40 \\
5.30\end{array}$ & $\begin{array}{l}- \\
- \\
-\end{array}$ & $\begin{array}{l}- \\
- \\
-\end{array}$ \\
\hline RADIUH-228 & $\begin{array}{l}02 / 03 / 89 \\
09 / 19 / 89 \\
04 / 19 / 90 \\
01 / 15 / 91 \\
12 / 03 / 92\end{array}$ & $\begin{array}{l}0001 \\
0001 \\
0001 \\
0001 \\
0001\end{array}$ & $\mathrm{PCI} / \mathrm{L}$ & & $\begin{array}{l}1.22 \\
2.6 \\
3.6 \\
2.9 \\
3.1\end{array}$ & $\begin{array}{l}1 . \\
1 . \\
1.0\end{array}$ & $\begin{array}{l}8.64 \\
1.2 \\
1.0 \\
1.8 \\
1.7\end{array}$ \\
\hline SELENIUM & $\begin{array}{l}02 / 03 / 89 \\
09 / 19 / 89 \\
04 / 11 / 90 \\
01 / 15 / 91 \\
12 / 03 / 92\end{array}$ & $\begin{array}{l}0001 \\
0001 \\
0001 \\
0001 \\
0001\end{array}$ & $M G / L$ & $\begin{array}{l}< \\
< \\
<\end{array}$ & $\begin{array}{ll}0.001 & J \\
0.018 & \\
0.005 & \\
0.05 & 1 \\
0.05 & 1\end{array}$ & $\begin{array}{l}0.005 \\
0.005 \\
0.005 \\
0.05 \\
0.05\end{array}$ & $\begin{array}{l}- \\
- \\
-\end{array}$ \\
\hline SILICA - SIOZ & $\begin{array}{l}02 / 03 / 89 \\
09 / 19 / 89 \\
04 / 11 / 90 \\
01 / 15 / 91\end{array}$ & $\begin{array}{l}0001 \\
0001 \\
0001 \\
0001\end{array}$ & $M G / L$ & & $\begin{array}{l}14.2 \\
21 . \\
20 . \\
20.1\end{array}$ & $\begin{array}{l}2 . \\
2 . \\
2 . \\
0.1\end{array}$ & $\begin{array}{l}- \\
-\end{array}$ \\
\hline SILVER & $\begin{array}{l}02 / 03 / 89 \\
09 / 19 / 89 \\
04 / 11 / 90 \\
01 / 15 / 91 \\
12 / 03 / 92\end{array}$ & $\begin{array}{l}0001 \\
0001 \\
0001 \\
0001 \\
0001\end{array}$ & MG/L & $\begin{array}{l}< \\
< \\
<\end{array}$ & $\begin{array}{l}0.01 \\
0.01 \\
0.01 \\
0.01 \\
0.01\end{array}$ & $\begin{array}{l}0.01 \\
0.01 \\
0.01 \\
0.01 \\
0.01\end{array}$ & $\begin{array}{l}- \\
- \\
-\end{array}$ \\
\hline soolun & $\begin{array}{l}02 / 03 / 89 \\
09 / 19 / 89 \\
04 / 11 / 90\end{array}$ & $\begin{array}{l}0001 \\
0001 \\
0001\end{array}$ & MG/L & & $\begin{array}{l}515 . \\
309 . \\
374 .\end{array}$ & $\begin{array}{l}0.002 \\
0.002 \\
0.002\end{array}$ & : \\
\hline
\end{tabular}

PARAMETER VALUE IMDICATOR (PVI): < - LESS THAN DETECTION LIMIT

SAMPLE ID COOES:

0001 - FILTERED SAMPLE (.45 MICRONS)

OTHER PARAMETER VALUE FLAGS:

H - HOLD TIHE EXPIRED, VALUE SUSPECT

I - INCREASED DETECTION LIMIT DUE TO REOUIRED DILUTION

d - estimated value 
GROUNDWATER QUALITY DATA BY LOCATION

SITE: AMBO1 AMBROSIA LAKE

LOCATION: 0680 RESERVED ESTORHS, HH, 11/14/88

HORTH COORDIHATE: UHKNOWH

EAST COORDIHATE: UHKHOWH

06/10/80 TO $12 / 04 / 92$

REPORT DATE: 08/04/94

FORMATION OF COMPLETION: DAKOTA SAMDSTONE (KD)

HYDRAULIC FLON RELATIONSHIP: UPGRADIENT (U)

\begin{tabular}{|c|c|c|c|c|c|c|c|}
\hline PARAMETER MAME & LOG DATE & $\underset{\text { SD }}{\text { SAMPLE }}$ & $\begin{array}{l}\text { UNITS OF } \\
\text { MEASURE }\end{array}$ & PVI & $\begin{array}{l}\text { PARAMETER } \\
\text { VALUE FLAGS }\end{array}$ & $\begin{array}{l}\text { DETECTIOH } \\
\text { LIMIT }\end{array}$ & $\begin{array}{l}\text { PARAMETER } \\
\text { UNCERTAINTY }\end{array}$ \\
\hline soolun & $\begin{array}{l}01 / 15 / 91 \\
12 / 03 / 92\end{array}$ & $\begin{array}{l}0001 \\
0001\end{array}$ & MG/L & & $\begin{array}{l}332 . \\
308 .\end{array}$ & $\begin{array}{l}5 . \\
5 .\end{array}$ & - \\
\hline SPECIFIC CONDUCTANCE & $\begin{array}{l}02 / 03 / 89 \\
09 / 19 / 89 \\
04 / 11 / 90 \\
01 / 15 / 99 \\
12 / 03 / 92\end{array}$ & $\begin{array}{l}0001 \\
0001 \\
0001 \\
0001 \\
0001\end{array}$ & UNHO/CH & & $\begin{array}{l}2000 . \\
700 . \\
2850 \\
3050 \\
3200\end{array}$ & $\begin{array}{l}- \\
- \\
- \\
-\end{array}$ & $\begin{array}{l}- \\
- \\
- \\
-\end{array}$ \\
\hline STRONTIUMA & $\begin{array}{l}02 / 03 / 89 \\
09 / 19 / 89 \\
04 / 91 / 90 \\
01 / 15 / 91\end{array}$ & $\begin{array}{l}0001 \\
0001 \\
0001 \\
0001\end{array}$ & MG/L & & $\begin{array}{l}5.60 \\
5.7 \\
5.58 \\
5.50\end{array}$ & $\begin{array}{l}0.1 \\
0.1 \\
0.1 \\
0.01\end{array}$ & $\begin{array}{l}- \\
-\end{array}$ \\
\hline SULFATE & $\begin{array}{l}02 / 03 / 89 \\
09 / 19 / 89 \\
04 / 11 / 90 \\
01 / 15 / 91 \\
12 / 03 / 92\end{array}$ & $\begin{array}{l}0001 \\
0001 \\
0001 \\
0001 \\
0001\end{array}$ & MG/L & & $\begin{array}{l}1862 . \\
2600 . \\
2630 . \\
2430 . \\
2390 .\end{array}$ & $\begin{array}{c}0.1 \\
0.1 \\
0.1 \\
10 . \\
10 .\end{array}$ & $\begin{array}{l}- \\
- \\
- \\
-\end{array}$ \\
\hline SULFIDE & $\begin{array}{l}02 / 03 / 89 \\
09 / 19 / 89 \\
04 / 11 / 90 \\
01 / 15 / 91\end{array}$ & $\begin{array}{l}0001 \\
0001 \\
0001 \\
0001\end{array}$ & MG/L & $\begin{array}{l}< \\
< \\
< \\
<\end{array}$ & $\begin{array}{l}0.01 \\
0.1 \\
0.1 \\
0.1\end{array}$ & $\begin{array}{l}0.1 \\
0.1 \\
0.1 \\
0.1\end{array}$ & $\begin{array}{l}- \\
-\end{array}$ \\
\hline TEMPERATURE & $\begin{array}{l}02 / 03 / 89 \\
09 / 19 / 89 \\
04 / 11 / 90 \\
01 / 15 / 91 \\
12 / 03 / 92\end{array}$ & $\begin{array}{l}0001 \\
0001 \\
0001 \\
0001 \\
0001\end{array}$ & C - DEGREE & & $\begin{array}{l}11.0 \\
16.0 \\
14.5 \\
13 . \\
13.4\end{array}$ & $\begin{array}{l}- \\
- \\
- \\
-\end{array}$ & $\begin{array}{l}- \\
- \\
- \\
-\end{array}$ \\
\hline THALLIUM & $\begin{array}{l}02 / 03 / 89 \\
09 / 19 / 89 \\
04 / 11 / 90 \\
01 / 15 / 91\end{array}$ & $\begin{array}{l}0001 \\
0001 \\
0001 \\
0001\end{array}$ & $M G / L$ & $\begin{array}{l}< \\
< \\
< \\
<\end{array}$ & $\begin{array}{l}0.005 \\
0.01 \\
0.01 \\
0.03\end{array}$ & $\begin{array}{l}0.005 \\
0.01 \\
0.01 \\
0.03\end{array}$ & $\begin{array}{l}- \\
\dot{-} \\
.\end{array}$ \\
\hline THORIUH-230 & $\begin{array}{l}02 / 03 / 89 \\
09 / 19 / 89\end{array}$ & $\begin{array}{l}0001 \\
0001\end{array}$ & $\mathrm{PCI} / \mathrm{L}$ & & $\begin{array}{l}1.96 \\
0.0\end{array}$ & 1. & $\begin{array}{l}0.716 \\
0.2\end{array}$ \\
\hline TIH & $\begin{array}{l}02 / 03 / 89 \\
09 / 19 / 89 \\
04 / 11 / 90 \\
01 / 15 / 91\end{array}$ & $\begin{array}{l}0001 \\
0001 \\
0001 \\
0001\end{array}$ & $M G / L$ & $\begin{array}{l}< \\
< \\
< \\
<\end{array}$ & $\begin{array}{ll}0.009 & \mathrm{~J} \\
0.1 & \\
0.005 & \\
0.03 & 1\end{array}$ & $\begin{array}{l}0.005 \\
0.1 \\
0.005 \\
0.03\end{array}$ & $\begin{array}{l}- \\
- \\
-\end{array}$ \\
\hline TOTAL DISSOLVED SOLIDS & $\begin{array}{l}02 / 03 / 89 \\
09 / 19 / 89 \\
04 / 11 / 90 \\
01 / 15 / 91 \\
12 / 03 / 92\end{array}$ & $\begin{array}{l}0001 \\
0001 \\
0001 \\
0001 \\
0001\end{array}$ & MG/L & & $\begin{array}{l}3234 . \\
4050 . \\
4060 . \\
5570 \\
4140 .\end{array}$ & $\begin{array}{l}10 . \\
10 . \\
10 . \\
10 . \\
10 .\end{array}$ & $\begin{array}{l}- \\
\dot{-} \\
\dot{-}\end{array}$ \\
\hline TOTAL KJELDAHL HITROGEH & $\begin{array}{l}01 / 15 / 91 \\
12 / 03 / 92\end{array}$ & $\begin{array}{l}0001 \\
0001\end{array}$ & MG/L & & 1. & 1. & - \\
\hline TOTAL ORGANIC CARBOH & $\begin{array}{l}02 / 03 / 89 \\
09 / 19 / 89 \\
04 / 11 / 90\end{array}$ & $\begin{array}{l}0001 \\
0001 \\
0001\end{array}$ & $M G / L$ & & $\begin{array}{l}27 . \\
99.0 \\
49 .\end{array}$ & i. & $\begin{array}{l}- \\
\therefore\end{array}$ \\
\hline
\end{tabular}

PARAMETER VALUE IHDICATOR (PVI): < - LESS Than DETECTION LIMIT SAMPLE ID COOES:

0001 - FILIERED SAMPLE (.45 MICRONS)

OTHER PARAMETER VALUE FLAGS:

I - INCREASED DETECTION LIHIT DUE TO REQUIRED DILUTION

J - estimated value 
GROUNDWATER QUALITY DATA BY LOCATIOH

SITE: AMBO1 AMBROSIA LAKE

LOCATIOH: O680 RESERVED ESTORMS, MH, 11/14/88

HORTH COORDINATE: UNKNOWN

EAST COORDINATE: UNKNOWN

D6/10/80 TO $12 / 04 / 92$

REPORT DATE: 08/04/94

FORMATION OF COMPLETION: DAKOTA SAMDSTONE (KD)

HYDRAULIC FLON RELATIOHSHIP: UPGRADIENT (U)

\begin{tabular}{|c|c|c|c|c|c|c|c|}
\hline PARAMETER NAME & LOG DATE & $\underset{\text { SAMPLE }}{\text { ID }}$ & $\begin{array}{l}\text { UHITS OF } \\
\text { MEASURE }\end{array}$ & PVI & $\begin{array}{l}\text { PARAMETER } \\
\text { VALUE FLAGS }\end{array}$ & $\begin{array}{l}\text { DETECTIOH } \\
\text { LIHIT }\end{array}$ & $\begin{array}{l}\text { PARAMETER } \\
\text { UMCERTAINTY }\end{array}$ \\
\hline TOTAL ORGANIC CARBON & $\begin{array}{l}01 / 15 / 91 \\
12 / 03 / 92\end{array}$ & $\begin{array}{l}0001 \\
0001\end{array}$ & $\mathrm{MG} / \mathrm{L}$ & & $\begin{array}{l}4 . \\
8 .\end{array}$ & 1. & $\dot{-}$ \\
\hline URANIUA & $\begin{array}{l}02 / 03 / 89 \\
09 / 19 / 89 \\
04 / 11 / 90 \\
01 / 15 / 91 \\
12 / 03 / 92\end{array}$ & $\begin{array}{l}0001 \\
0001 \\
0001 \\
0001 \\
0001\end{array}$ & MG/L & $<$ & $\begin{array}{l}0.023 \\
0.0020 \mathrm{~J} \\
0.0016 \mathrm{~J} \\
0.001 \\
0.001\end{array}$ & $\begin{array}{l}0.003 \\
0.003 \\
0.003 \\
0.001 \\
0.001\end{array}$ & : \\
\hline VANADIUA & $\begin{array}{l}02 / 03 / 89 \\
09 / 19 / 89 \\
04 / 11 / 90 \\
01 / 15 / 91 \\
12 / 03 / 92\end{array}$ & $\begin{array}{l}0001 \\
0001 \\
0001 \\
0001 \\
0001\end{array}$ & HG/L & $\begin{array}{l}< \\
< \\
< \\
< \\
<\end{array}$ & $\begin{array}{l}0.01 \\
0.01 \\
0.01 \\
0.01 \\
0.01\end{array}$ & $\begin{array}{l}0.01 \\
0.01 \\
0.01 \\
0.01 \\
0.01\end{array}$ & $\begin{array}{l}- \\
- \\
- \\
-\end{array}$ \\
\hline ZIHC & $\begin{array}{l}02 / 03 / 89 \\
09 / 19 / 89 \\
04 / 11 / 90 \\
01 / 15 / 91\end{array}$ & $\begin{array}{l}0001 \\
0001 \\
0001 \\
0001\end{array}$ & $M G / L$ & $\begin{array}{l}< \\
<\end{array}$ & $\begin{array}{l}0.06 \\
0.005 \\
0.005 \\
0.005\end{array}$ & $\begin{array}{l}0.005 \\
0.005 \\
0.005 \\
0.005\end{array}$ & $\begin{array}{l}- \\
- \\
-\end{array}$ \\
\hline
\end{tabular}

PARAMETER VALUE IHDICATOR (PVI):

< - LESS THAN DETECTION LIMIT

SAMPLE ID COOES:

0001 - FILTERED SAMPLE (.45 MICRONS)

OTHER PARAMETER VALUE FLAGS:

H - HOLD TIME EXPIRED, VALUE SUSPECT

J - estimated VALUE 
GROUNDWATER QUALITY DATA BY LOCATION

SITE: AMBOI AMBROSIA LAKE

LOCATIOH: 0681 RESERVED ESTORMS, MH, 11/14/88

HORTH COORDINATE: UNKHOWH

EAST COORDINATE: UNKHOWN

06/10/80 TO $12 / 04 / 92$

REPORT DATE: 08/04/94

FORHATION OF COMPLETION: TRES HERMANOS - B SAMDSTONE (TB) HYORAULIC FLON RELATIOHSHIP: CROSS GRADIENT (C)

\begin{tabular}{|c|c|c|c|c|c|c|c|c|}
\hline PARAMETER MAME & LOG DATE & $\begin{array}{c}\text { SNAPLE } \\
\text { ID }\end{array}$ & $\begin{array}{l}\text { UHITS OF } \\
\text { MEASURE }\end{array}$ & PVI & $\begin{array}{l}\text { PARAMETER } \\
\text { VALUE }\end{array}$ & FLAGS & $\begin{array}{l}\text { DETECTION } \\
\text { LIMIT }\end{array}$ & $\begin{array}{l}\text { PARAMETER } \\
\text { UNCERTAINTY }\end{array}$ \\
\hline ALKALIHITY & $\begin{array}{l}02 / 02 / 89 \\
09 / 19 / 89 \\
04 / 06 / 90 \\
01 / 09 / 91 \\
12 / 04 / 92\end{array}$ & $\begin{array}{l}0001 \\
0001 \\
0001 \\
0001 \\
0001\end{array}$ & MG/L CACO3 & & $\begin{array}{l}363 . \\
329 . \\
307 . \\
309 . \\
283\end{array}$ & & $\begin{array}{l}- \\
- \\
- \\
-\end{array}$ & $\begin{array}{l}- \\
- \\
. \\
.\end{array}$ \\
\hline ALUMINUA & $\begin{array}{l}02 / 02 / 89 \\
09 / 19 / 89 \\
04 / 06 / 90 \\
01 / 09 / 91\end{array}$ & $\begin{array}{l}0001 \\
0001 \\
0001 \\
0001\end{array}$ & MG/L & $\begin{array}{l}< \\
< \\
<\end{array}$ & $\begin{array}{l}0.05 \\
0.1 \\
0.1 \\
0.05\end{array}$ & $J$ & $\begin{array}{l}0.1 \\
0.1 \\
0.1 \\
0.05\end{array}$ & - \\
\hline AN4ONIUY & $\begin{array}{l}02 / 02 / 89 \\
09 / 19 / 89 \\
04 / 06 / 90 \\
01 / 09 / 91 \\
12 / 04 / 92\end{array}$ & $\begin{array}{l}0001 \\
0001 \\
0001 \\
0001 \\
0001\end{array}$ & $M G / L$ & $<$ & $\begin{array}{l}0.50 \\
0.1 \\
0.1 \\
0.40 \\
0.3\end{array}$ & J & $\begin{array}{l}0.1 \\
0.1 \\
0.1 \\
0.01 \\
0.1\end{array}$ & $\begin{array}{l}- \\
- \\
- \\
-\end{array}$ \\
\hline ANTIMONY & $\begin{array}{l}02 / 02 / 89 \\
09 / 19 / 89 \\
04 / 06 / 90 \\
01 / 09 / 91 \\
12 / 04 / 92\end{array}$ & $\begin{array}{l}0001 \\
0001 \\
0001 \\
0001 \\
0001\end{array}$ & $M G / L$ & $<$ & $\begin{array}{l}0.019 \\
0.029 \\
0.077 \\
0.005 \\
0.003\end{array}$ & & $\begin{array}{l}0.003 \\
0.003 \\
0.003 \\
0.003 \\
0.003\end{array}$ & $\begin{array}{l}- \\
- \\
- \\
-\end{array}$ \\
\hline ARSENIC & $\begin{array}{l}02 / 02 / 89 \\
09 / 19 / 89 \\
04 / 06 / 90 \\
01 / 09 / 91\end{array}$ & $\begin{array}{l}0001 \\
0001 \\
0001 \\
0001\end{array}$ & $M G / L$ & $\begin{array}{l}< \\
<\end{array}$ & $\begin{array}{l}0.003 \\
0.01 \\
0.01 \\
0.03\end{array}$ & $\begin{array}{l}\text { J } \\
\text { I }\end{array}$ & $\begin{array}{l}0.01 \\
0.01 \\
0.01 \\
0.03\end{array}$ & $\begin{array}{l}- \\
-\end{array}$ \\
\hline BARIUM & $\begin{array}{l}02 / 02 / 89 \\
09 / 19 / 89 \\
04 / 06 / 90 \\
01 / 09 / 91\end{array}$ & $\begin{array}{l}0001 \\
0001 \\
0001 \\
0001\end{array}$ & $M G / L$ & $\begin{array}{l}< \\
<\end{array}$ & $\begin{array}{l}0.02 \\
0.1 \\
0.1 \\
0.01\end{array}$ & J & $\begin{array}{l}0.1 \\
0.1 \\
0.1 \\
0.01\end{array}$ & $\begin{array}{l}- \\
\dot{-} \\
\dot{-}\end{array}$ \\
\hline BERYLLIUM & $\begin{array}{l}02 / 02 / 89 \\
09 / 19 / 89 \\
04 / 06 / 90 \\
01 / 09 / 91\end{array}$ & $\begin{array}{l}0001 \\
0001 \\
0001 \\
0001\end{array}$ & MG/L & $\begin{array}{l}< \\
< \\
<\end{array}$ & $\begin{array}{l}0.005 \\
0.01 \\
0.01 \\
0.005\end{array}$ & & $\begin{array}{l}0.005 \\
0.01 \\
0.01 \\
0.005\end{array}$ & $\dot{-}$ \\
\hline BORON & $\begin{array}{l}02 / 02 / 89 \\
09 / 19 / 89 \\
04 / 06 / 90 \\
01 / 09 / 91\end{array}$ & $\begin{array}{l}0001 \\
0001 \\
0001 \\
0001\end{array}$ & MG/L & & $\begin{array}{l}0.47 \\
0.3 \\
0.2 \\
0.25\end{array}$ & & $\begin{array}{l}0.1 \\
0.1 \\
0.1 \\
0.05\end{array}$ & $\begin{array}{l}- \\
- \\
-\end{array}$ \\
\hline BROAIDE & $\begin{array}{l}02 / 02 / 89 \\
09 / 19 / 89 \\
04 / 06 / 90 \\
01 / 09 / 91\end{array}$ & $\begin{array}{l}0001 \\
0001 \\
0001 \\
0001\end{array}$ & MG/L & $<$ & $\begin{array}{l}0.33 \\
0.1 \\
0.4 \\
0.2\end{array}$ & $J$ & $\begin{array}{l}0.01 \\
0.1 \\
0.1 \\
0.1\end{array}$ & $\begin{array}{l}- \\
- \\
-\end{array}$ \\
\hline CADHIU: & $\begin{array}{l}02 / 02 / 89 \\
09 / 19 / 89 \\
04 / 06 / 90 \\
01 / 09 / 91 \\
12 / 04 / 92\end{array}$ & $\begin{array}{l}0001 \\
0001 \\
0001 \\
0001 \\
0001\end{array}$ & MG/L & $\begin{array}{l}< \\
< \\
<\end{array}$ & $\begin{array}{l}0.0001 \\
0.002 \\
0.001 \\
0.001 \\
0.001\end{array}$ & J & $\begin{array}{l}0.001 \\
0.001 \\
0.001 \\
0.001 \\
0.001\end{array}$ & $\begin{array}{l}- \\
\therefore \\
-\end{array}$ \\
\hline Calcium & $\begin{array}{l}02 / 02 / 89 \\
09 / 19 / 89\end{array}$ & $\begin{array}{l}0001 \\
0001\end{array}$ & $M G / L$ & & $\begin{array}{r}26 . \\
7193 .\end{array}$ & & $\begin{array}{l}0.01 \\
0.01\end{array}$ & 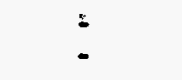 \\
\hline
\end{tabular}

PARAMETER VALUE IHDICATOR (PVI): < - LESS THAN DETECTION LIMIT

OTHER PARAMETER VALUE FLAGS:

1 - IHCREASED DETECTIOH LIMIT DUE TO REQUIRED DILUTION

d - estimated VALUE 
GROUNDWATER QUALITY DATA BY LOCATIOH

SITE: AMBO1 AMBROSIA LAKE

LOCATIOH: 0681 RESERVED ESTORMS, HW, 11/14/88

NORTH COORDINATE: UHKHOWH

EAST COORDIHATE: UHKHOWH

$06 / 10 / 80$ TO $12 / 04 / 92$

REPORT DATE: 08/04/94

FORMATION OF COMPLETIOH: TRES HERHANOS - B SANDSTONE (TB)

HYDRAULIC FLON RELATIONSHIP: CROSS GRADIENT (C)

\begin{tabular}{|c|c|c|c|c|c|c|c|c|}
\hline PARAMETER NAME & LOG DATE & $\begin{array}{c}\text { SAMPLE } \\
10\end{array}$ & $\begin{array}{l}\text { UHITS OF } \\
\text { MEASURE }\end{array}$ & PVI & $\begin{array}{l}\text { PARAMETER } \\
\text { VALUE }\end{array}$ & FLAGS & $\begin{array}{l}\text { DETECTIOH } \\
\text { LIHIT }\end{array}$ & $\begin{array}{l}\text { PARAMETER } \\
\text { UNCERTAIHTY }\end{array}$ \\
\hline CALCIUN & $\begin{array}{l}04 / 06 / 90 \\
01 / 09 / 91 \\
12 / 04 / 92\end{array}$ & $\begin{array}{l}0001 \\
0001 \\
0001\end{array}$ & MG/L & & $\begin{array}{l}6.22 \\
6.1 \\
6.2\end{array}$ & & $\begin{array}{l}0.09 \\
0.1 \\
0.1\end{array}$ & - \\
\hline CHLORIDE & $\begin{array}{l}02 / 02 / 89 \\
09 / 19 / 89 \\
04 / 06 / 90 \\
01 / 09 / 91 \\
12 / 04 / 92\end{array}$ & $\begin{array}{l}0001 \\
0001 \\
0001 \\
0001 \\
0001\end{array}$ & MG/L & & $\begin{array}{l}72 . \\
15 . \\
19 . \\
13.6 \\
12.9\end{array}$ & & $\begin{array}{l}1 . \\
1 . \\
1 . \\
0.5 \\
0.5\end{array}$ & $\begin{array}{l}- \\
- \\
- \\
-\end{array}$ \\
\hline CHROMIUH & $\begin{array}{l}02 / 02 / 89 \\
09 / 19 / 89 \\
04 / 06 / 90 \\
01 / 09 / 91 \\
12 / 04 / 92\end{array}$ & $\begin{array}{l}0001 \\
0001 \\
0001 \\
0001 \\
0001\end{array}$ & MG/L & $\begin{array}{l}< \\
< \\
< \\
< \\
<\end{array}$ & $\begin{array}{l}0.01 \\
0.01 \\
0.01 \\
0.01 \\
0.01\end{array}$ & & $\begin{array}{l}0.01 \\
0.01 \\
0.01 \\
0.01 \\
0.01\end{array}$ & $\begin{array}{l}- \\
\text { - } \\
\text { - } \\
\text { - }\end{array}$ \\
\hline COBALT & $\begin{array}{l}02 / 02 / 89 \\
09 / 19 / 89 \\
04 / 06 / 90 \\
01 / 09 / 91 \\
12 / 04 / 92\end{array}$ & $\begin{array}{l}0001 \\
0001 \\
0001 \\
0001 \\
0001\end{array}$ & MG/L & $\begin{array}{l}< \\
< \\
< \\
< \\
<\end{array}$ & $\begin{array}{l}0.01 \\
0.05 \\
0.05 \\
0.03 \\
0.03\end{array}$ & J & $\begin{array}{l}0.05 \\
0.05 \\
0.05 \\
0.03 \\
0.03\end{array}$ & $\begin{array}{l}- \\
- \\
\dot{-}\end{array}$ \\
\hline COPPER & $\begin{array}{l}02 / 02 / 89 \\
09 / 19 / 89 \\
04 / 06 / 90 \\
01 / 09 / 91\end{array}$ & $\begin{array}{l}0001 \\
0001 \\
0001 \\
0001\end{array}$ & MG/L & $\begin{array}{l}< \\
<\end{array}$ & $\begin{array}{l}0.01 \\
0.02 \\
0.02 \\
0.01\end{array}$ & 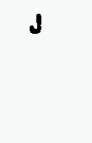 & $\begin{array}{l}0.02 \\
0.02 \\
0.02 \\
0.01\end{array}$ & $\dot{-}$ \\
\hline CYANIDE & $\begin{array}{l}02 / 02 / 89 \\
09 / 19 / 89 \\
04 / 06 / 90 \\
01 / 09 / 91\end{array}$ & $\begin{array}{l}0001 \\
0001 \\
0001 \\
0001\end{array}$ & $M G / L$ & $\begin{array}{l}< \\
< \\
< \\
<\end{array}$ & $\begin{array}{l}0.002 \\
0.01 \\
0.01 \\
0.01\end{array}$ & $\mathbf{J}$ & $\begin{array}{l}0.01 \\
0.01 \\
0.01 \\
0.01\end{array}$ & $\dot{-}$ \\
\hline FLUORIDE & $\begin{array}{l}02 / 02 / 89 \\
09 / 19 / 89 \\
04 / 06 / 90 \\
01 / 09 / 91 \\
12 / 04 / 92\end{array}$ & $\begin{array}{l}0001 \\
0001 \\
0001 \\
0001 \\
0001\end{array}$ & MG/L & & $\begin{array}{l}0.9 \\
0.8 \\
0.8 \\
0.8 \\
0.8\end{array}$ & & $\begin{array}{l}0.1 \\
0.1 \\
0.1 \\
0.1 \\
0.1\end{array}$ & $\begin{array}{l}- \\
\dot{-} \\
\dot{-}\end{array}$ \\
\hline GROSS ALPHA & $\begin{array}{l}02 / 02 / 89 \\
09 / 19 / 89 \\
04 / 06 / 90 \\
01 / 09 / 91 \\
12 / 04 / 92\end{array}$ & $\begin{array}{l}0001 \\
0001 \\
0001 \\
0001 \\
0001\end{array}$ & $\mathrm{PCl} / \mathrm{L}$ & & $\begin{array}{c}14.6 \\
9.4 \\
29 . \\
41.6 \\
0.0\end{array}$ & & $\begin{array}{l}1 . \\
1 . \\
1 . \\
1.0\end{array}$ & $\begin{array}{c}16.2 \\
9.7 \\
11 . \\
18.2 \\
10.9\end{array}$ \\
\hline GROSS BETA & $\begin{array}{l}02 / 02 / 89 \\
09 / 19 / 89 \\
04 / 06 / 90 \\
01 / 09 / 91 \\
12 / 04 / 92\end{array}$ & $\begin{array}{l}0001 \\
0001 \\
0001 \\
0001 \\
0001\end{array}$ & $\mathrm{PCl} / \mathrm{L}$ & & $\begin{array}{c}4.37 \\
11 . \\
9.7 \\
32.0 \\
9.5\end{array}$ & & $\begin{array}{r}0.5 \\
0.5 \\
0.5 \\
0.5 \\
23.1\end{array}$ & $\begin{array}{c}21.4 \\
5 . \\
6.5 \\
8.8 \\
14.2\end{array}$ \\
\hline IRON & $\begin{array}{l}02 / 02 / 89 \\
09 / 19 / 89 \\
04 / 06 / 90 \\
01 / 09 / 91 \\
12 / 04 / 92\end{array}$ & $\begin{array}{l}0001 \\
0001 \\
0001 \\
0001 \\
0001\end{array}$ & $M G / L$ & $\begin{array}{l}< \\
< \\
< \\
<\end{array}$ & $\begin{array}{l}0.02 \\
0.03 \\
0.04 \\
0.03 \\
0.03\end{array}$ & J & $\begin{array}{l}0.03 \\
0.03 \\
0.03 \\
0.03 \\
0.03\end{array}$ & $\begin{array}{l}- \\
\dot{-} \\
\dot{-}\end{array}$ \\
\hline LEAD & $02 / 02 / 89$ & 0001 & MG/L & $<$ & 0.001 & J & 0.01 & - \\
\hline
\end{tabular}

PARAMETER VALUE IMDICATOR (PVI): < - LESS THAN DETECTION LIMIT

SAMPLE ID CODES:

0001 - FILTERED SAMPLE (.45 MICRONS)

OTHER PARAHETER VALUE FLAGS:

J - estIMUTED VALUE 


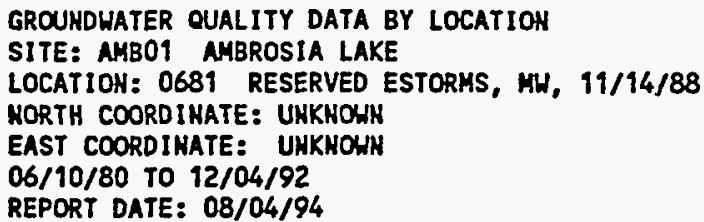

FORHATIOH OF COMPLETIOH: TRES HERMAHOS - B SAHDSTOHE (TB) HYDRAULIC FLOW RELATIOHSHIP: CROSS GRADIENT (C)

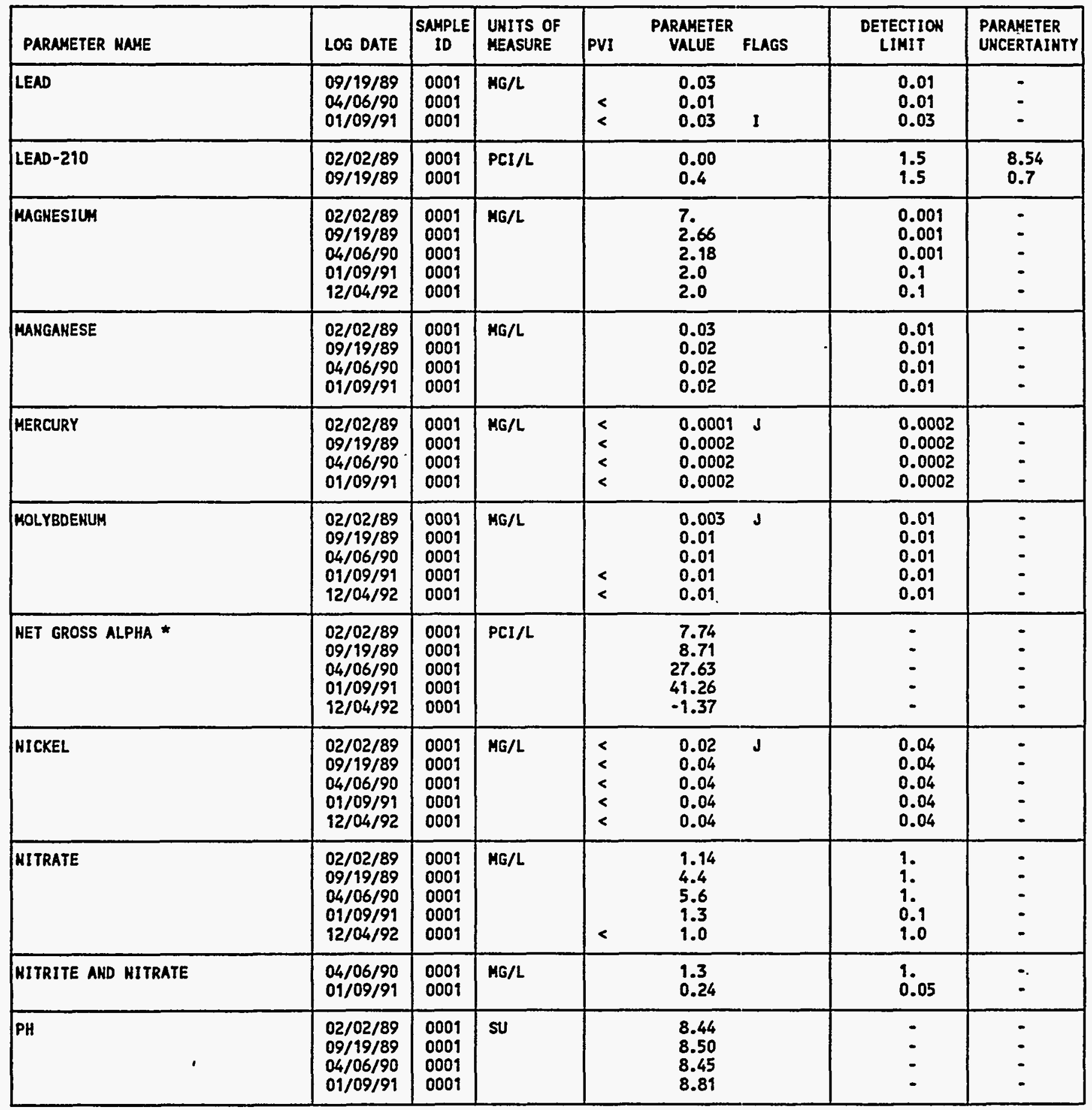

* MET GROSS ALPHA (GROSS ALPHA - URAKIUH) HITH 1 HG URAHIUH = 686 PCI

PARAMETER VALUE IHDICATOR (PVI): < - LeSS thaN DETECTION LIMIT

OTHER PARAMETER VALUE FLAGS:

I - INCREASED DETECTION LIMIT DUE TO REQUIRED DILUTION

$\jmath$ - estimated VALUE 
GROUHDHATER QUALITY DATA BY LOCATION

SITE: AMBOI AMBROSIA LAKE

LOCATION: 0681 RESERVED ESTORMS, MH, $11 / 14 / 88$

NORTH COORDIHATE: UKKHOWN

EAST COORDINATE: UNKHOWH

06/10/80 TO $12 / 04 / 92$

REPORT DATE: 08/04/94

FORHATIOH OF COMPLETIOH: TRES HERMANOS - B SAKDSTOHE (TB) HYDRAULIC FLOW RELATIONSHIP: CROSS GRADIENT (C)

\begin{tabular}{|c|c|c|c|c|c|c|c|}
\hline PARAMETER MAME & LOG DATE & SAMPLE & $\begin{array}{l}\text { UHITS OF } \\
\text { MEASURE }\end{array}$ & PVI & $\begin{array}{l}\text { PARAMETER } \\
\text { VALUE FLAGS }\end{array}$ & $\begin{array}{l}\text { DETECTION } \\
\text { LIMIT }\end{array}$ & $\begin{array}{l}\text { PARAMETER } \\
\text { UNCERTAINTY }\end{array}$ \\
\hline PH & $12 / 04 / 92$ & 0001 & SU & & 8.71 & - & - \\
\hline PHOSPHATE & $\begin{array}{l}02 / 02 / 89 \\
09 / 19 / 89 \\
04 / 06 / 90 \\
01 / 09 / 91 \\
12 / 04 / 92\end{array}$ & $\begin{array}{l}0001 \\
0001 \\
0001 \\
0001 \\
0001\end{array}$ & $M G / L$ & $\begin{array}{l}< \\
<\end{array}$ & $\begin{array}{l}0.1 \\
0.1 \\
0.1 \\
0.13 \\
0.3\end{array}$ & $\begin{array}{l}0.1 \\
0.1 \\
0.1 \\
0.05 \\
0.1\end{array}$ & $\begin{array}{l}- \\
- \\
- \\
-\end{array}$ \\
\hline POLONIUH-210 & $\begin{array}{l}02 / 02 / 89 \\
09 / 19 / 89\end{array}$ & $\begin{array}{l}0001 \\
0001\end{array}$ & PCI/L & & $\begin{array}{l}1.55 \\
0.0\end{array}$ & 9. & $\begin{array}{l}1.49 \\
0.3\end{array}$ \\
\hline POTASSIUM & $\begin{array}{l}02 / 02 / 89 \\
09 / 19 / 89 \\
04 / 06 / 90 \\
01 / 09 / 91 \\
12 / 04 / 92\end{array}$ & $\begin{array}{l}0001 \\
0001 \\
0001 \\
0001 \\
0001\end{array}$ & MG/L & & $\begin{array}{l}5.6 \\
2.3 \\
2.6 \\
1.83 \\
2.15\end{array}$ & $\begin{array}{l}0.01 \\
0.01 \\
0.01 \\
0.01 \\
0.01\end{array}$ & $\begin{array}{l}- \\
- \\
-\end{array}$ \\
\hline RADIUM-226 & $\begin{array}{l}02 / 02 / 89 \\
09 / 19 / 89 \\
04 / 06 / 90 \\
01 / 09 / 91 \\
12 / 04 / 92\end{array}$ & $\begin{array}{l}0001 \\
0001 \\
0001 \\
0001 \\
0001\end{array}$ & PCI/L & & $\begin{array}{l}0.648 \\
0.4 \\
0.7 \\
0.3 \\
0.5\end{array}$ & $\begin{array}{l}1 . \\
1 . \\
1 . \\
1 . \\
0.3\end{array}$ & $\begin{array}{l}0.225 \\
0.3 \\
0.3 \\
0.4 \\
0.3\end{array}$ \\
\hline RADIUN-226 + RADIUM-228 & $\begin{array}{l}02 / 02 / 89 \\
09 / 19 / 89 \\
04 / 06 / 90 \\
01 / 09 / 91 \\
12 / 04 / 92\end{array}$ & $\begin{array}{l}0001 \\
0001 \\
0001 \\
0001 \\
0001\end{array}$ & PCI/L & & $\begin{array}{l}0.65 \\
1.00 \\
1.10 \\
0.30 \\
1.40\end{array}$ & $\begin{array}{l}- \\
- \\
-\end{array}$ & $\begin{array}{l}- \\
- \\
- \\
-\end{array}$ \\
\hline RADIUM-228 & $\begin{array}{l}02 / 02 / 89 \\
09 / 19 / 89 \\
04 / 06 / 90 \\
01 / 09 / 91 \\
12 / 04 / 92\end{array}$ & $\begin{array}{l}0001 \\
0001 \\
0001 \\
0001 \\
0001\end{array}$ & $\mathrm{PCI} / \mathrm{L}$ & & $\begin{array}{l}0.00 \\
0.6 \\
0.4 \\
0.0 \\
0.9\end{array}$ & $\begin{array}{l}1 . \\
1 . \\
1 . \\
1.0 \\
1.0\end{array}$ & $\begin{array}{l}6.19 \\
1.1 \\
0.7 \\
1.6 \\
0.7\end{array}$ \\
\hline SELENIUM & $\begin{array}{l}02 / 02 / 89 \\
09 / 19 / 89 \\
04 / 06 / 90 \\
01 / 09 / 91 \\
12 / 04 / 92\end{array}$ & $\begin{array}{l}0001 \\
0001 \\
0001 \\
0001 \\
0001\end{array}$ & $M G / L$ & $\begin{array}{l}< \\
<\end{array}$ & $\begin{array}{ll}0.001 & \mathrm{~J} \\
0.015 & \\
0.005 & \\
0.005 & \\
0.05 & \mathrm{I}\end{array}$ & $\begin{array}{l}0.005 \\
0.005 \\
0.005 \\
0.005 \\
0.05\end{array}$ & $\begin{array}{l}- \\
- \\
- \\
-\end{array}$ \\
\hline SILICA - SIOZ & $\begin{array}{l}02 / 02 / 89 \\
09 / 19 / 89 \\
04 / 06 / 90 \\
01 / 09 / 91\end{array}$ & $\begin{array}{l}0001 \\
0001 \\
0009 \\
0001\end{array}$ & MG/L & & $\begin{array}{l}8.4 \\
9.0 \\
8 . \\
8.2\end{array}$ & $\begin{array}{l}2 . \\
2 . \\
2 . \\
0.1\end{array}$ & : \\
\hline SILVER & $\begin{array}{l}02 / 02 / 89 \\
09 / 19 / 89 \\
04 / 06 / 90 \\
01 / 09 / 91 \\
12 / 04 / 92\end{array}$ & $\begin{array}{l}0001 \\
0001 \\
0001 \\
0001 \\
0001\end{array}$ & $M G / L$ & $\begin{array}{l}< \\
< \\
< \\
< \\
<\end{array}$ & $\begin{array}{l}0.01 \\
0.01 \\
0.01 \\
0.01 \\
0.01\end{array}$ & $\begin{array}{l}0.01 \\
0.01 \\
0.01 \\
0.01 \\
0.01\end{array}$ & : \\
\hline SOOIUN & $\begin{array}{l}02 / 02 / 89 \\
09 / 19 / 89 \\
04 / 06 / 90\end{array}$ & $\begin{array}{l}0009 \\
0009 \\
0001\end{array}$ & $M G / L$ & & $\begin{array}{l}720 . \\
576 . \\
593 .\end{array}$ & $\begin{array}{l}0.002 \\
0.002 \\
0.002\end{array}$ & : \\
\hline
\end{tabular}

PARAMETER VALUE INDICATOR (PVI): < - LESS THAN DETECTION LIMIT

SAMPLE ID COOES:

0001 - FILTERED SAMPLE (.45 HICROHS)

OTHER PARAMETER VALUE FLAGS:

H - HOLD TIME EXPIRED, VALUE SUSPECT

1 - IHCREASED DETECTION LIHIT DUE TO REQUIRED DILUTION

d - estimated value 
GROUNDWATER QUALITY DATA BY LOCATION

SITE: AMBO1 AMBROSIA LAKE

LOCATIOH: 0681 RESERVED ESTORMS, HH, 11/14./88

MORTH COORDINATE: UNKNOWM

EAST COORDINATE: UNKWONH

06/10/80 TO $12 / 04 / 92$

REPORT DATE: $08 / 04 / 94$

FORHATIOH OF COMPLETION: TRES HERMANOS - B SAMDSTONE (TB)

HYDRAULIC FLON RELATIOHSHIP: CROSS GRADIENT (C)

\begin{tabular}{|c|c|c|c|c|c|c|c|c|}
\hline PARAMETER MAME & LOG DATE & $\begin{array}{c}\text { SAMPLE } \\
\text { ID }\end{array}$ & $\begin{array}{l}\text { UNITS OF } \\
\text { MEASURE }\end{array}$ & PVI & $\begin{array}{l}\text { PARAMETER: } \\
\text { VALUE }\end{array}$ & FLAGS & $\begin{array}{l}\text { DETECTIOH } \\
\text { LIMIT }\end{array}$ & $\begin{array}{l}\text { PARAMETER } \\
\text { UNCERTAINTY }\end{array}$ \\
\hline SoOIU: & $\begin{array}{l}01 / 09 / 91 \\
12 / 04 / 92\end{array}$ & $\begin{array}{l}0001 \\
0001\end{array}$ & $M G / L$ & & $\begin{array}{l}601 . \\
589 .\end{array}$ & $\mathbf{j}$ & $\begin{array}{l}5 . \\
5 .\end{array}$ & - \\
\hline SPECIFIC CONDUCTANCE & $\begin{array}{l}02 / 02 / 89 \\
09 / 19 / 89 \\
04 / 06 / 90 \\
01 / 09 / 91 \\
12 / 04 / 92\end{array}$ & $\begin{array}{l}0001 \\
0001 \\
0001 \\
0001 \\
0001\end{array}$ & UHO/CH & & $\begin{array}{l}1900 . \\
800 . \\
1790 . \\
1880 . \\
2100\end{array}$ & & $\begin{array}{l}- \\
- \\
-\end{array}$ & $\begin{array}{l}- \\
- \\
- \\
-\end{array}$ \\
\hline STRONTIUA & $\begin{array}{l}02 / 02 / 89 \\
09 / 19 / 89 \\
04 / 06 / 90 \\
01 / 09 / 91\end{array}$ & $\begin{array}{l}0001 \\
0001 \\
0001 \\
0001\end{array}$ & MG/L & & $\begin{array}{l}1.00 \\
0.4 \\
0.39 \\
0.41\end{array}$ & & $\begin{array}{l}0.1 \\
0.1 \\
0.1 \\
0.01\end{array}$ & $\begin{array}{l}- \\
- \\
-\end{array}$ \\
\hline SULFATE & $\begin{array}{l}02 / 02 / 89 \\
09 / 19 / 89 \\
04 / 06 / 90 \\
01 / 09 / 91 \\
12 / 04 / 92\end{array}$ & $\begin{array}{l}0001 \\
0001 \\
0001 \\
0001 \\
0001\end{array}$ & MG/L & & $\begin{array}{c}1163 . \\
967 . \\
1040 . \\
1030 . \\
911 .\end{array}$ & & $\begin{array}{l}0.1 \\
0.1 \\
0.1 \\
10 . \\
10 .\end{array}$ & $\begin{array}{l}- \\
- \\
- \\
-\end{array}$ \\
\hline SULFIDE & $\begin{array}{l}02 / 02 / 89 \\
09 / 19 / 89 \\
04 / 06 / 90 \\
01 / 09 / 91\end{array}$ & $\begin{array}{l}0001 \\
0001 \\
0001 \\
0001\end{array}$ & MG/L & $\begin{array}{l}< \\
< \\
< \\
<\end{array}$ & $\begin{array}{l}0.01 \\
0.1 \\
0.1 \\
0.1\end{array}$ & $J$ & $\begin{array}{l}0.1 \\
0.1 \\
0.1 \\
0.1\end{array}$ & : \\
\hline TEMPERATURE & $\begin{array}{l}02 / 02 / 89 \\
09 / 19 / 89 \\
04 / 06 / 90 \\
01 / 09 / 91 \\
12 / 04 / 92\end{array}$ & $\begin{array}{l}0001 \\
0001 \\
0001 \\
0001 \\
0001\end{array}$ & C - DEGREE & & $\begin{array}{l}13.0 \\
14.0 \\
14.0 \\
13.8 \\
13.5\end{array}$ & & $\begin{array}{l}- \\
- \\
- \\
-\end{array}$ & $\begin{array}{l}- \\
- \\
-\end{array}$ \\
\hline THALLIUH & $\begin{array}{l}02 / 02 / 89 \\
09 / 19 / 89 \\
04 / 06 / 90 \\
01 / 09 / 91\end{array}$ & $\begin{array}{l}0001 \\
0001 \\
0001 \\
0001\end{array}$ & $M G / L$ & $\begin{array}{l}< \\
< \\
<\end{array}$ & $\begin{array}{l}0.005 \\
0.01 \\
0.01 \\
0.03\end{array}$ & 1 & $\begin{array}{l}0.005 \\
0.01 \\
0.01 \\
0.03\end{array}$ & $\begin{array}{l}- \\
-\end{array}$ \\
\hline THORIUM-230 & $\begin{array}{l}02 / 02 / 89 \\
09 / 19 / 89\end{array}$ & $\begin{array}{l}0001 \\
0001\end{array}$ & $\mathrm{PCI} / \mathrm{L}$ & \multicolumn{3}{|c|}{$\begin{array}{l}0.00854 \\
0.0\end{array}$} & 1. & $\begin{array}{l}0.245 \\
0.3\end{array}$ \\
\hline TIH & $\begin{array}{l}02 / 02 / 89 \\
09 / 19 / 89 \\
04 / 06 / 90 \\
01 / 09 / 91\end{array}$ & $\begin{array}{l}0001 \\
0001 \\
0001 \\
0001\end{array}$ & $M G / L$ & $\begin{array}{l}< \\
<\end{array}$ & $\begin{array}{l}0.001 \\
0.104 \\
0.056 \\
0.03\end{array}$ & $\begin{array}{l}J \\
I\end{array}$ & $\begin{array}{l}0.005 \\
0.005 \\
0.005 \\
0.03\end{array}$ & : \\
\hline TOTAL DISSOLVED SOLIDS & $\begin{array}{l}02 / 02 / 89 \\
09 / 19 / 89 \\
04 / 06 / 90 \\
01 / 09 / 91 \\
12 / 04 / 92\end{array}$ & $\begin{array}{l}0001 \\
0001 \\
0001 \\
0001 \\
0001\end{array}$ & MG/L & & $\begin{array}{l}2064 . \\
1620 \\
1710 . \\
1790 . \\
1770 .\end{array}$ & & $\begin{array}{l}10 . \\
10 . \\
10 . \\
10 . \\
10 .\end{array}$ & $\begin{array}{l}- \\
- \\
- \\
-\end{array}$ \\
\hline TOTAL KJELDAHL HITROGEN & $\begin{array}{l}01 / 09 / 91 \\
12 / 04 / 92\end{array}$ & $\begin{array}{l}0001 \\
0001\end{array}$ & $M G / L$ & & i. & & $i$. & - \\
\hline TOTAL ORGAHIC CARBOH & $\begin{array}{l}02 / 02 / 89 \\
09 / 19 / 89 \\
04 / 06 / 90\end{array}$ & $\begin{array}{l}0001 \\
0001 \\
0001\end{array}$ & MG/L & & $\begin{array}{l}44 . \\
67 . \\
45 .\end{array}$ & & $\begin{array}{l}1 . \\
1 .\end{array}$ & $\because$ \\
\hline
\end{tabular}

PARAMETER VALUE IMDICATOR (PVI): < - LESS THAN DETECTIOH LIMIT

SAMPLE ID COOES:

0001 - FILTERED SAMPLE (.45 MICRONS)

OTHER PARAMETER VALUE FLAGS:

I - IHCREASED DETECTION LIMIT DUE TO REQUIRED DILUTION

J- estiMated VAlue 
GROUNDWATER QUALITY DATA BY LOCATION

SITE: AHBOI AMBROSIA LAKE

LOCATIOH: 0681 RESERVED ESTORHS, MH, 11/14/88

NORTH COORDIHATE: UNKHOWH

EAST COORDINATE: UNKNOWN

$06 / 10 / 80$ TO $12 / 04 / 92$

REPORT DATE: 08/04/94

FORMATION OF COMPLETION: TRES HERMAHOS - B SANDSTONE (TB) HYDRAULIC FLON RELATIOHSHIP: CROSS GRADIENT (C)

\begin{tabular}{|c|c|c|c|c|c|c|c|}
\hline PARANETER NAME & LOG DATE & $\underset{\text { SD }}{\text { SAMPLE }}$ & $\begin{array}{l}\text { UHITS OF } \\
\text { NEASURE }\end{array}$ & PVI & $\begin{array}{l}\text { PARAMETER } \\
\text { VALUE FLAGS }\end{array}$ & $\begin{array}{l}\text { DETECTIOH } \\
\text { LIMIT }\end{array}$ & $\begin{array}{l}\text { PARAHETER } \\
\text { UNCERTAINTY }\end{array}$ \\
\hline TOTAL ORGANIC CARBON & $\begin{array}{l}01 / 09 / 91 \\
12 / 04 / 92\end{array}$ & $\begin{array}{l}0001 \\
0001\end{array}$ & MG/L & & $\begin{array}{l}5 . \\
2 .\end{array}$ & 1. & - \\
\hline URANIUY & $\begin{array}{l}02 / 02 / 89 \\
09 / 19 / 89 \\
04 / 06 / 90 \\
01 / 09 / 91 \\
12 / 04 / 92\end{array}$ & $\begin{array}{l}0001 \\
0001 \\
0001 \\
0001 \\
0001\end{array}$ & MG/L & $<$ & $\begin{array}{l}0.010 \\
0.0010 \mathrm{~J} \\
0.0020 \mathrm{~J} \\
0.001 \\
0.002\end{array}$ & $\begin{array}{l}0.003 \\
0.003 \\
0.003 \\
0.001 \\
0.001\end{array}$ & $\begin{array}{l}- \\
- \\
- \\
-\end{array}$ \\
\hline VAMADIUM & $\begin{array}{l}02 / 02 / 89 \\
09 / 19 / 89 \\
04 / 06 / 90 \\
01 / 09 / 91 \\
12 / 04 / 92\end{array}$ & $\begin{array}{l}0001 \\
0001 \\
0001 \\
0001 \\
0001\end{array}$ & $M G / L$ & $\begin{array}{l}< \\
< \\
< \\
< \\
<\end{array}$ & $\begin{array}{l}0.01 \\
0.1 \\
0.01 \\
0.01 \\
0.01\end{array}$ & $\begin{array}{l}0.01 \\
0.1 \\
0.01 \\
0.01 \\
0.01\end{array}$ & $\begin{array}{l}- \\
- \\
- \\
-\end{array}$ \\
\hline ZIHC & $\begin{array}{l}02 / 02 / 89 \\
09 / 19 / 89 \\
04 / 06 / 90 \\
01 / 09 / 91\end{array}$ & $\begin{array}{l}0001 \\
0001 \\
0001 \\
0001\end{array}$ & MG/L & $\begin{array}{l}< \\
< \\
<\end{array}$ & $\begin{array}{l}0.01 \\
0.005 \\
0.006 \\
0.005\end{array}$ & $\begin{array}{l}0.01 \\
0.005 \\
0.005 \\
0.005\end{array}$ & $\begin{array}{l}- \\
- \\
-\end{array}$ \\
\hline
\end{tabular}

PARAMETER VALUE IHDICATOR (PVI): < - LESS TKAN DETECTION LIMIT

SAMPLE ID COOES

0001 - FILTERED SAMPLE (.45 HICRONS)

OTHER PARAMETER VALUE FLAGS:

H - HOLD TIME EXPIRED, VALUE SUSPECT

$J$ - ESTIMATED VALUE 
GROUNDHATER QUALITY DATA BY LOCATION

SITE: AMBOI AMBROSIA LAKE

LOCATION: 0706

MORTH COORDIHATE: 53612.0 FT

EAST COORDIHATE: $\quad 59942.0$ FT

06/10/80 TO 12/04/92

REPORT DATE: $08 / 04 / 94$

FORMATION OF COMPLETIOH: ALLUVIUM (AL)

HYDRAULIC FLON RELATIONSHIP: ON-SITE (O)

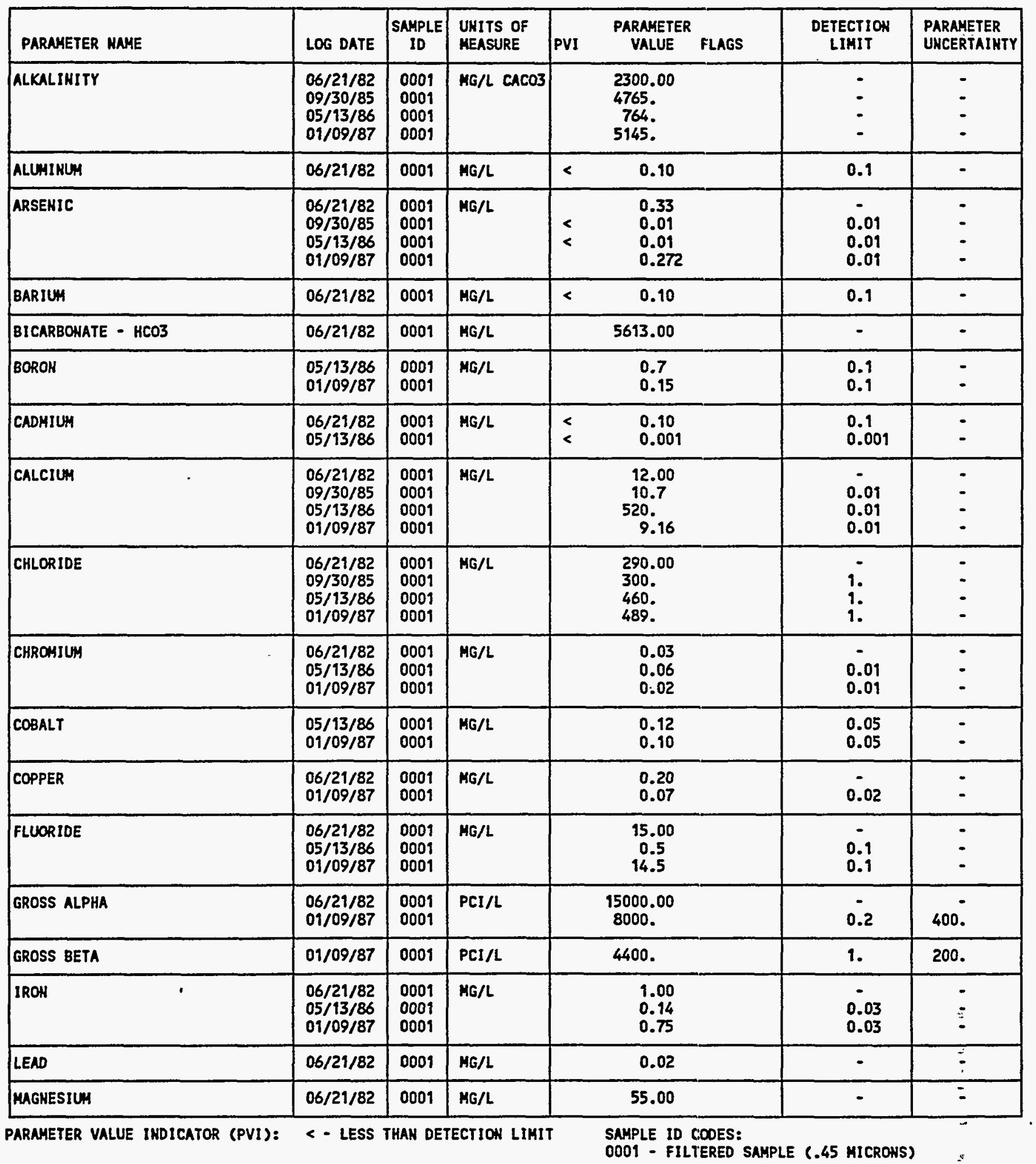


GROUKDWATER OUALITY DATA BY LOCATION

SITE: AMBOI AMBROSIA LAKE

LOCATION: 0706

NORTH COORDIHATE: 53612.0 FT

EAST COORDINATE: $\quad 59942.0$ FT

06/10/80 TO 12/04/92

REPORT DATE: $08 / 04 / 94$

FORMATION OF COMPLETION: ALLUVIUN (AL)

HYDRAULIC FLON RELATIONSHIP: ON-SITE (O)

\begin{tabular}{|c|c|c|c|c|c|c|c|}
\hline PARAMETER NAME & LOG DATE & $\underset{\text { SD }}{\text { SAMPLE }}$ & $\begin{array}{l}\text { UHITS OF } \\
\text { MEASURE }\end{array}$ & $\begin{array}{l}\text { PARAMETER } \\
\text { VALUEE }\end{array}$ & FLAGS & $\begin{array}{l}\text { DETECTION } \\
\text { LIMIT }\end{array}$ & $\begin{array}{l}\text { PARAMETER } \\
\text { UHCERTAIHTY }\end{array}$ \\
\hline MAGNESIUM & $\begin{array}{l}09 / 30 / 85 \\
05 / 13 / 86 \\
01 / 09 / 87\end{array}$ & $\begin{array}{l}0001 \\
0001 \\
0001\end{array}$ & MG/L & $\begin{array}{c}43.2 \\
273 . \\
42.1\end{array}$ & & $\begin{array}{l}0.001 \\
0.001 \\
0.001\end{array}$ & - \\
\hline MAMGAHESE & $\begin{array}{l}05 / 13 / 86 \\
01 / 09 / 87\end{array}$ & $\begin{array}{l}0001 \\
0001\end{array}$ & MG/L & $\begin{array}{l}0.22 \\
0.09\end{array}$ & & $\begin{array}{l}0.01 \\
0.01\end{array}$ & - \\
\hline HERCURY & $06 / 21 / 82$ & 0001 & MG/L & 0.002 & & 0.002 & - \\
\hline MOLYBDENUM & $\begin{array}{l}06 / 21 / 82 \\
09 / 30 / 85 \\
05 / 13 / 86 \\
01 / 09 / 87\end{array}$ & $\begin{array}{l}0001 \\
0001 \\
0001 \\
0001\end{array}$ & MG/L & $\begin{array}{c}225.00 \\
223 . \\
9.37 \\
220 .\end{array}$ & & $\begin{array}{l}0.01 \\
0.01 \\
0.01\end{array}$ & : \\
\hline HET GROSS ALPHA * & $01 / 09 / 87$ & 0001 & $\mathrm{PCI} / \mathrm{L}$ & 2415.96 & & - & - \\
\hline MITRATE & $\begin{array}{l}06 / 21 / 82 \\
05 / 13 / 86 \\
01 / 09 / 87\end{array}$ & $\begin{array}{l}0001 \\
0001 \\
0001\end{array}$ & HG/L & $\begin{array}{l}1.00 \\
25 . \\
25 .\end{array}$ & & $\begin{array}{l}1 . \\
1 .\end{array}$ & - \\
\hline PH & $\begin{array}{l}06 / 21 / 82 \\
09 / 30 / 85 \\
05 / 13 / 86 \\
01 / 09 / 87\end{array}$ & $\begin{array}{l}0001 \\
0001 \\
0001 \\
0001\end{array}$ & SU & $\begin{array}{l}8.40 \\
9.12 \\
7.25 \\
9.21\end{array}$ & & $\begin{array}{l}- \\
- \\
-\end{array}$ & : \\
\hline POTASSIUM & $\begin{array}{l}06 / 21 / 82 \\
09 / 30 / 85 \\
05 / 13 / 86 \\
01 / 09 / 87\end{array}$ & $\begin{array}{l}0001 \\
0001 \\
0001 \\
0001\end{array}$ & MG/L & $\begin{array}{c}9.00 \\
13.3 \\
15.8 \\
0.91\end{array}$ & & $\begin{array}{l}- \\
0.01 \\
0.01 \\
0.01\end{array}$ & : \\
\hline RADIUM-226 & $\begin{array}{l}06 / 21 / 82 \\
01 / 09 / 87\end{array}$ & $\begin{array}{l}0001 \\
0001\end{array}$ & $\mathrm{PCI} / \mathrm{L}$ & $\begin{array}{l}4.00 \\
130 .\end{array}$ & & 1. & 10. \\
\hline RADIUM-226 + RADIUH-228 & $01 / 09 / 87$ & 0001 & $\mathrm{PCI} / \mathrm{L}$ & 131.80 & & - & - \\
\hline RADIUH-228 & $01 / 09 / 87$ & 0001 & PCI/L & 1.8 & & 1. & 6.1 \\
\hline SELENIUM & $\begin{array}{l}06 / 21 / 82 \\
09 / 30 / 85 \\
05 / 13 / 86 \\
01 / 09 / 87\end{array}$ & $\begin{array}{l}0001 \\
0001 \\
0001 \\
0001\end{array}$ & $M G / L$ & $\begin{array}{l}0.037 \\
0.088 \\
0.01 \\
0.012\end{array}$ & & $\begin{array}{l}- \\
0.005 \\
0.005 \\
0.005\end{array}$ & $\begin{array}{l}- \\
-\end{array}$ \\
\hline SILICA - SIOZ & $06 / 21 / 82$ & 0001 & MG/L & 6.50 & & - & - \\
\hline SILVER & $\begin{array}{l}06 / 21 / 82 \\
01 / 09 / 87\end{array}$ & $\begin{array}{l}0001 \\
0001\end{array}$ & HG/L & $\begin{array}{l}0.15 \\
0.02\end{array}$ & & 0.01 & - \\
\hline scoIun & $\begin{array}{l}06 / 21 / 82 \\
09 / 30 / 85 \\
05 / 13 / 86 \\
01 / 09 / 87\end{array}$ & $\begin{array}{l}0001 \\
0001 \\
0001 \\
0001\end{array}$ & HG/L & $\begin{array}{l}7100.00 \\
7020 . \\
1140 . \\
7610 .\end{array}$ & & $\begin{array}{l}- \\
0.002 \\
0.002 \\
0.002\end{array}$ & $\begin{array}{l}- \\
- \\
-\end{array}$ \\
\hline SPECIFIC CONDUCTANCE & $\begin{array}{l}06 / 21 / 82 \\
09 / 30 / 85 \\
05 / 13 / 86 \\
01 / 09 / 87\end{array}$ & $\begin{array}{l}0001 \\
0001 \\
0001 \\
0001\end{array}$ & UHHO/CH & $\begin{array}{c}22200.00 \\
17000 . \\
4500 . \\
14500 .\end{array}$ & & : & - \\
\hline
\end{tabular}

- NET GROSS ALPHA (GROSS ALPHA - URANIUN) HITH 1 HG URANIUM = 686 PCI

PARAMETER VALUE INDICATOR (PVI): < - LeSS than DETECTION LIHIT 
GROUNDWATER QUALITY DATA BY LOCATION

SITE: AMBOI AMBROSIA LAKE

LOCATION: 0706

MORTH COORDIHATE: 53612.0 FT

EAST COORDIKATE: 59942.0 FT

06/10/80 TO 12/04/92

REPORT DATE: 08/04/94

FORHATION OF COMPLETIOH: ALLUVIUM (AL)

HYDRAULIC FLON RELATIONSHIP: ON-SITE (O)

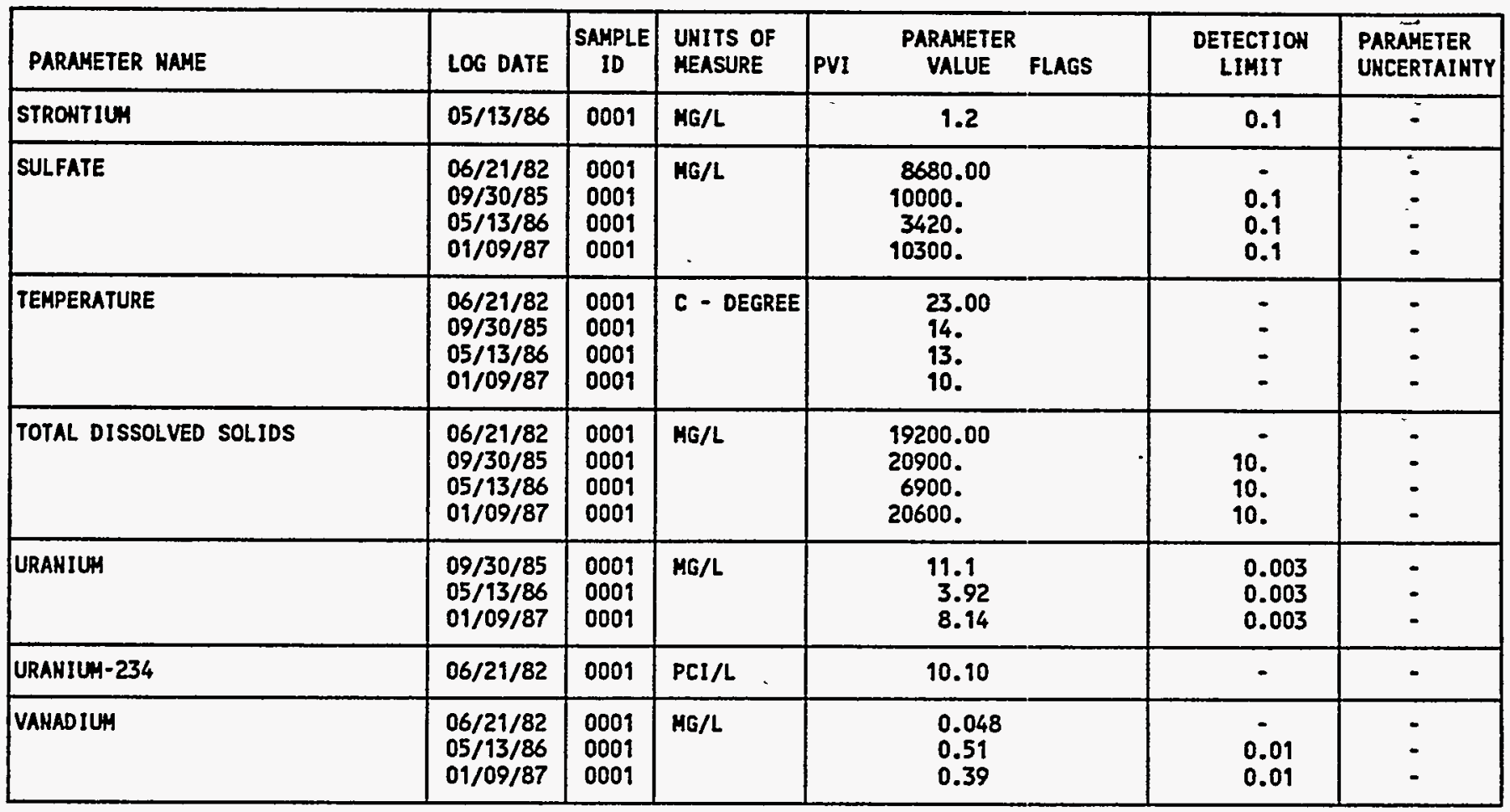

PARAMETER VALUE IHDICATOR (PVI):

< - LESS THAN DETECTION LIMIT

SAMPLE 1D COOES:

0001 - FILTERED SAMPLE (.45 MICRONS) 
GROUMDWATER OUALITY DATA BY LOCATION

SITE: AMBOI AMBROSIA LAKE

LOCATION: 0707

NORTH COORDIHATE: $\quad 53788.0$ FT

EAST COORDIHATE: $\quad 60309.0$ FT

$06 / 10 / 80$ TO $12 / 04 / 92$

REPDRT DATE: 08/04/94

FORHATION OF COMPLETION: URANIUM MILL TAILINGS (TA)

HYDRAULIC FLOW RELATIONSHIP: OH-SITE (O)

\begin{tabular}{|c|c|c|c|c|c|c|c|}
\hline PARAMETER NAME & LOG DATE & $\underset{\text { SD }}{\text { SAMPLE }}$ & $\begin{array}{l}\text { UNITS OF } \\
\text { MEASURE }\end{array}$ & PVI & $\begin{array}{l}\text { PARAMETER } \\
\text { VALUE FLAGS }\end{array}$ & $\begin{array}{l}\text { DETECTION } \\
\text { LIMIT }\end{array}$ & $\begin{array}{l}\text { PARAMETER } \\
\text { UHCERTAIHTY }\end{array}$ \\
\hline ALKALIHITY & $0.6 / 21 / 82$ & 0001 & HG/L CACO3 & & 770.00 & - & - \\
\hline ALUHINUM & $06 / 21 / 82$ & 0001 & MG/L & & 0.12 & - & - \\
\hline ARSENIC & $06 / 21 / 82$ & 0001 & HG/L & & 0.01 & - & - \\
\hline BARIUH & $06 / 21 / 82$ & 0001 & MG/L & $<$ & 0.10 & 0.1 & - \\
\hline BICARBONATE - HCO3 & $06 / 21 / 82$ & 0001 & MG/L & & 1879.00 & - & - \\
\hline CADHIUA & $06 / 21 / 82$ & 0001 & MG/L & $<$ & 0.01 & 0.01 & - \\
\hline CalcIU:M & $06 / 21 / 82$ & 0001 & MG/L & & 440.00 & - & - \\
\hline CHLORIDE & $06 / 21 / 82$ & 0001 & MG/L & & 213.00 & - & - \\
\hline CHROMIUM & $06 / 21 / 82$ & 0001 & MG/L & & 0.01 & - & - \\
\hline COPPER & $06 / 21 / 82$ & 0001 & MG/L & & 0.063 & - & - \\
\hline FLUORIDE & $06 / 21 / 82$ & 0001 & MG/L & & 5.00 & - & - \\
\hline IRON & $06 / 21 / 82$ & 0001 & $M G / L$ & & 1.46 & - & - \\
\hline LEAD & $06 / 21 / 82$ & 0001 & $M G / L$ & & 0.01 & - & - \\
\hline MAGHESIUM & $06 / 21 / 82$ & 0001 & MG/L & & 600.00 & - & - \\
\hline MERCURY & $06 / 21 / 82$ & 0001 & HG/L & $<$ & 0.002 & 0.002 & - \\
\hline MOLYBDENUH & $06 / 21 / 82$ & 0001 & $M G / L$ & & 67.00 & - & - \\
\hline NITRATE & $06 / 21 / 82$ & 0001 & MG/L & $<$ & 1.00 & 1. & - \\
\hline PH & $06 / 21 / 82$ & 0001 & SU & & 6.80 & - & - \\
\hline POTASSIU: & $06 / 21 / 82$ & 0001 & HG/L & & 12.00 & - & - \\
\hline RADILA-226 & $06 / 21 / 82$ & 0001 & $\mathrm{PCI} / \mathrm{L}$ & & 27.00 & - & - \\
\hline SELENIUH & $06 / 21 / 82$ & 0001 & $M G / L$ & & 0.018 & - & - \\
\hline SILICA - SIO2 & $06 / 21 / 82$ & 0001 & HG/L & & 9.50 & - & - \\
\hline SILVER & $06 / 21 / 82$ & 0001 & $M G / L$ & & 0.05 & - & - \\
\hline SODIUM & $06 / 21 / 82$ & 0001 & $M G / L$ & & 4000.00 & - & - \\
\hline SPECIFIC CONDUCTAKCE & $06 / 21 / 82$ & 0001 & UHKO/CH & & 13200.00 & - & - \\
\hline SULFATE & $06 / 21 / 82$ & 0001 & MG/L & & 9110.00 & - & - \\
\hline TEMPERATURE & $06 / 21 / 82$ & 0009 & C - DEGREE & & 15.00 & - & - \\
\hline TOTAL DISSOLVED SOLIDS & $06 / 21 / 82$ & 0001 & $\mathrm{MG} / \mathrm{L}$ & & 15400.00 & - & - \\
\hline URANIUH-234 & $06 / 21 / 82$ & 0001 & $\mathrm{PCI} / \mathrm{L}$ & & 21.50 & - & - \\
\hline VANADIUM & $06 / 21 / 82$ & 0009 & $M G / L$ & $<$ & 0.01 & 0.01 & - \\
\hline
\end{tabular}

PARAMETER VALUE INDICATOR (PVI):

< - LESS THAN DETECTION LIMIT

SAMPLE ID COOES

0001 - FILTERED SAMPLE (.45 MICROHS) 
GROUNDHATER QUALITY DATA BY LOCATION

SITE: AMBOI AMBROSIA LAKE

LOCATION: 0707

HORTH COORDIHATE: 53788.0 FT

EAST COORDIHATE: $\quad 60309.0$ FT

06/10/80 TO 12/04/92

REPORT DATE: 08/04/94

FORMATIOH OF COMPLETION: URANIUM MILL TAILIHGS (TA)

HYDRAULIC FLON RELATIONSHIP: ON-SITE (O)

\begin{tabular}{|c|c|c|c|c|c|c|c|c|}
\hline PARAMETER MAHE & LOG DATE & $\begin{array}{c}\text { SAMPLE } \\
\text { ID }\end{array}$ & $\begin{array}{l}\text { UNITS OF } \\
\text { MEASURE }\end{array}$ & PVI & $\begin{array}{l}\text { PARAMETER } \\
\text { VALUE }\end{array}$ & FLAGS & $\begin{array}{l}\text { DETECTION } \\
\text { LIMIT }\end{array}$ & $\begin{array}{l}\text { PARÄ̈ETER } \\
\text { UNCERTAINTY }\end{array}$ \\
\hline
\end{tabular}

PARAMETER VALUE INDICATOR (PVI): < - LESS THAN DETECTION LIMIT SAMPLE ID COOES: 
GROUNDWATER QUALITY DATA BY LOCATIOH

SITE: AMBO1 AMBROSIA LAKE

LOCATION: 0708

HORTH COORDINATE: 53295.0 FT

EAST COORDINATE: $\quad 59872.0$ FT

06/10/80 TO $12 / 04 / 92$

REPORT DATE: $08 / 04 / 94$

FORMATIOH OF COMPLETION: TRES HERMANOS - CI SANDSTOHE (TO) HYDRAULIC FLON RELATIONSHIP: DOWN GRADIENT (D)

\begin{tabular}{|c|c|c|c|c|c|c|}
\hline PARAMETER NAME & LOG DATE & $\begin{array}{c}\text { SAMPLE } \\
\text { ID }\end{array}$ & $\begin{array}{l}\text { UNITS OF } \\
\text { MEASURE }\end{array}$ & $\begin{array}{l}\text { PARAMETER } \\
\text { PVI VALUE }\end{array}$ & $\begin{array}{l}\text { DETECTIOH } \\
\text { LIMIT }\end{array}$ & $\begin{array}{l}\text { PARAMETER } \\
\text { UHCERTAIHTY }\end{array}$ \\
\hline ALKALINITY & $06 / 21 / 82$ & 0.001 & MG/L CACO3 & 320.00 & - & - \\
\hline ALUMINUM & $06 / 21 / 82$ & 0001 & $M G / L$ & 0.10 & 0.1 & - \\
\hline ARSENIC & $06 / 21 / 82$ & 0001 & HG/L & 0.002 & - & - \\
\hline BARIUM & $06 / 21 / 82$ & 0001 & MG/L & 0.10 & 0.1 & - \\
\hline BICARBONATE - HCO3 & $06 / 21 / 82$ & 0001 & $M G / L$ & 781.00 & - & - \\
\hline CADHIU: & $06 / 21 / 82$ & 0001 & HG/L & 0.01 & 0.01 & $\cdot$ \\
\hline CALCIUM & $06 / 21 / 82$ & 0001 & $M G / L$ & 470.00 & $\cdot \quad-$ & - \\
\hline CHLORIDE & $06 / 21 / 82$ & 0001 & HG/L & 260.00 & - & - \\
\hline CHROMIUH & $06 / 21 / 82$ & 0001 & HG/L & 0.01 & 0.01 & - \\
\hline COPPER & $06 / 21 / 82$ & 0001 & MG/L & 0.025 & 0.025 & - \\
\hline FLUORIDE & $06 / 21 / 82$ & 0001 & $M G / L$ & 2.00 & - & - \\
\hline GROSS ALPHA & $06 / 21 / 82$ & 0001 & PCI/L & 31000.00 & - & - \\
\hline IRON & $06 / 21 / 82$ & 0001 & MG/L & 0.26 & - & - \\
\hline LEAD & $06 / 21 / 82$ & 0001 & $M G / L$ & 0.01 & 0.01 & - \\
\hline MAGNESIUH & $06 / 21 / 82$ & 0001 & $M G / L$ & 333.00 & - & - \\
\hline HERCURY & $06 / 21 / 82$ & 0001 & MG/L & 0.002 & 0.002 & - \\
\hline MOLYBDENUM & $06 / 21 / 82$ & 0001 & MG/L & 10.30 & - & - \\
\hline NITRATE & $06 / 21 / 82$ & 0001 & MG/L & 24.00 & - & - \\
\hline PH & $06 / 21 / 82$ & 0001 & SU & 7.80 & - & - \\
\hline POTASSIUA & $06 / 21 / 82$ & 0001 & MG/L & 10.00 & - & - \\
\hline RADIUA-226 & $06 / 21 / 82$ & 0001 & $\mathrm{PCI} / \mathrm{L}$ & 5.00 & - & - \\
\hline SELENIUY & $06 / 21 / 82$ & 0001 & MG/L & 0.225 & - & - \\
\hline SILICA - S102 & $08 / 21 / 82$ & 0001 & MG/L & 6.80 & - & - \\
\hline SILVER & $06 / 21 / 82$ & 0001 & $M G / L$ & 0.019 & - & $\because$ \\
\hline SOOIUN & $06 / 21 / 82$ & 0001 & MG/L & 1590.00 & - & - \\
\hline SPECIFIC CONDUCTANCE & $06 / 21 / 82$ & 0001 & UAHO/CM & 8280.00 & - & - \\
\hline SULFATE & $06 / 21 / 82$ & 0001 & $M G / L$ & 4010.00 & - & - \\
\hline TEMPERATURE & $06 / 21 / 82$ & 0001 & C - DEGREE & 15.00 & - & - \\
\hline TOTAL DISSOLVED SOLIDS & $06 / 21 / 82$ & 0001 & MG/L & 7190.00 & - & - \\
\hline URAKIUM-234 & $06 / 29 / 82$ & 0001 & $\mathrm{PCl} / \mathrm{L}$ & 11.50 & - & - \\
\hline
\end{tabular}

PARAMETER VALUE INDICATOR (PVI): < - LESS THAN DETECTIOH LIMIT

SAMPLE ID COOES:

0001 - FILTERED SAMPLE (.45 MICROHS) 
GROUNDWATER QUALITY DATA BY LOCATION

SITE: AMBO1 AMBROSIA LAKE

LOCATION: 0708

MORTH COORDIHATE: 53295.0 FT

EAST COORDINATE: 59872.0 FT

$06 / 10 / 80$ TO $12 / 04 / 92$

REPORT DATE: 08/04/94

FORHATIOH OF COHPLETIOH: TRES HERHANOS - CI SAMDSTONE (TO)

HYORAULIC FLON RELATIONSHIP: DOWN GRADIENT (D)

\begin{tabular}{|c|c|c|c|c|c|c|c|}
\hline PARAMETER NAHE & LOG DATE & $\begin{array}{c}\text { SAMPLE } \\
\text { ID }\end{array}$ & $\begin{array}{l}\text { UNITS OF } \\
\text { MEASURE }\end{array}$ & PVI & $\begin{array}{l}\text { PARAMETER } \\
\text { VALUE FLAGS }\end{array}$ & $\begin{array}{l}\text { DETECTION } \\
\text { LIMIT }\end{array}$ & $\begin{array}{l}\text { PARAMETER } \\
\text { UNCERTAIHTY }\end{array}$ \\
\hline VAKADIUA & $06 / 21 / 82$ & 0001 & $M G / L$ & $<$ & 0.01 & 0.01 & - \\
\hline
\end{tabular}

PARAMETER VALUE INDICATOR (PVI): < - LESS THAN DETECTION LIMIT

SAMPLE 10 COOES:

0001 - FILIERED SAMPLE (.45 MICROHS) 
GROUNDHATER QUALITY DATA BY LOCATION

SITE: AMBO1 AMBROSIA LAKE

LOCATION: 0709

MORTH COORDINATE: 55212.0 FT

EAST COORDIHATE: 60324.0 FT

$06 / 10 / 80$ TO $12 / 04 / 92$

REPORT DATE: 08/04/94

FORMATIOH OF COMPLETION: ALLUVIUM (AL)

HYDRAULIC FLON RELATIONSHIP: UPGRADIEHT (U)

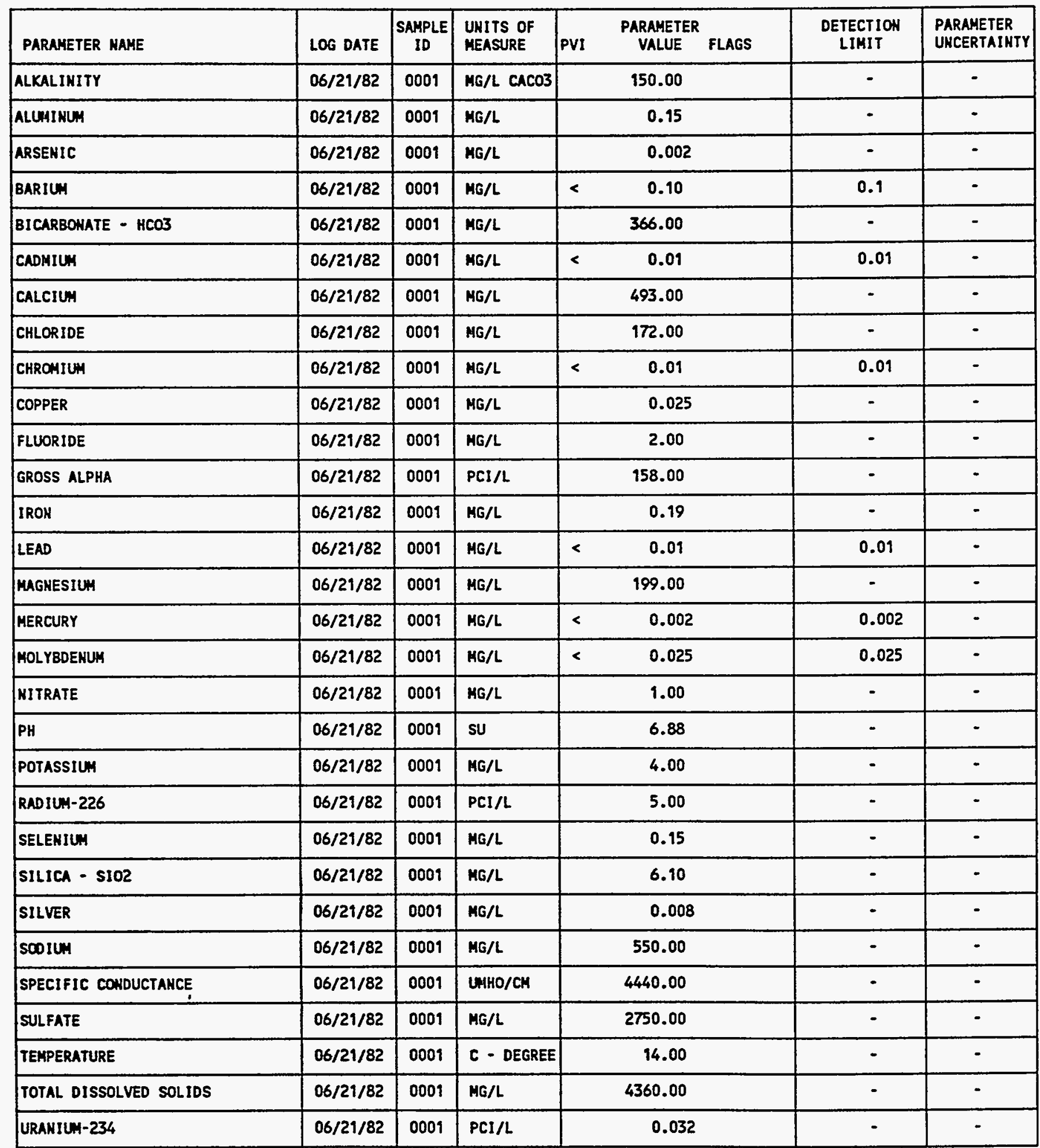

PARAMETER VALUE INDICATOR (PVI): < - LESS THAH DETECTION LIMIT

SAHPLE ID COOES:

0001 - FILTERED SAMPLE (.45 MICRONS) 
GROUNDHATER OUALITY DATA BY LOCATION

SITE: AMBOI AMBROSIA LAKE

LOCATION: O709

HORTH COORDINATE: 55212.0 FT

EAST COORDINATE: 60324.0 FT

06/10/80 TO 12/04/92

REPORT DATE: 08/04/94

FORHATION OF COMPLETIOH: ALLUVIUN (AL)

HYDRAULIC FLOW RELATIONSHIP: UPGRADIENT (U)

\begin{tabular}{|c|c|c|c|c|c|c|c|}
\hline PARAMETER HAME & LOG DATE & $\underset{\text { SNMPLE }}{\text { SN }}$ & $\begin{array}{l}\text { UNITS OF } \\
\text { MEASURE }\end{array}$ & PVI & $\begin{array}{l}\text { PARAMETER } \\
\text { VALUE FLAGS }\end{array}$ & $\begin{array}{l}\text { DETECTION } \\
\text { LIMIT }\end{array}$ & $\begin{array}{l}\text { PARAMETER } \\
\text { UNCERTAINTY }\end{array}$ \\
\hline VAHADIUA & $06 / 21 / 82$ & 0001 & MG/L & $<$ & 0.01 & 0.01 & - \\
\hline
\end{tabular}

PARAMETER VALUE IHDICATOR (PVI): < - LESS THAM DETECTION LIMIT

SAMPLE IO COOES:

0001 - FILTERED SAMPLE (.45 MICRONS) 
GROUKDHATER QUALITY DATA BY LOCATION

SITE: AMBO1 NMBROSIA LAKE

LOCATION: 0710

NORTH COORDINATE: 55599.0 FT

EAST COOROINATE: 61272.0 FT

O6/10/80 TO 12/04/92

REPORT DATE: 08/04/94

FORMATION OF COMPLETION: ALLUVIUNA (AL)

HYDRAULIC FLON RELATIONSHIP: UPGRADIENT (U)

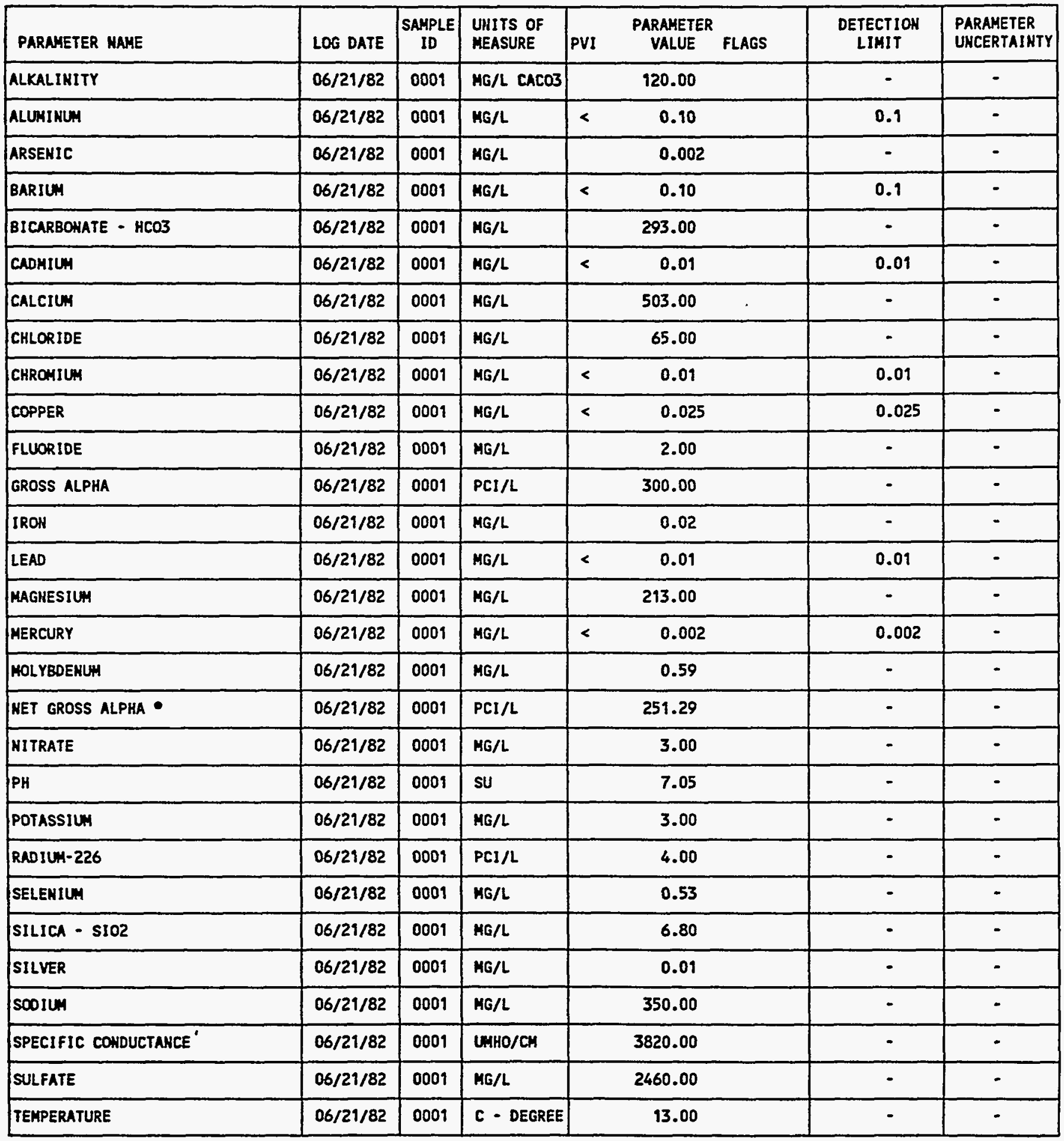

- MET GROSS ALPHA (GROSS ALPHA - URANIUM) HITH I MG URANIUH = 686 PCI

PARAMETER VALUE IMDICATOR (PVI): < - LESS THAN DETECTION LIMIT SAMPLE ID COOES:

OOO1 - FILTERED SAMPLE (.45 MICRONS) 
GROUHDWATER QUALITY DATA BY LOCATION

SITE: AMBOI AMBROSIA LAKE

LOCATIOH: 0710

HORTH COORDINATE: 55599.0 FT

EAST COORDIHATE: 69272.0 FT

06/10/80 TO 12/04/92

REPORT DATE: 08/04/94

FORMATION OF CONPLETION: ALLUVIUN (AL)

HYDRAULIC FLON RELATIOHSHIP: UPGRADIEHT (U)

\begin{tabular}{|c|c|c|c|c|c|c|c|}
\hline PARAMETER MAME & LOG DATE & $\underset{\text { SD }}{\text { SAMPLE }}$ & $\begin{array}{l}\text { UNITS OF } \\
\text { MEASURE }\end{array}$ & PVI & $\begin{array}{l}\text { PARAMETER } \\
\text { VALUE FLAGS }\end{array}$ & $\begin{array}{l}\text { DETECTIOH } \\
\text { LIMIT }\end{array}$ & $\begin{array}{l}\text { PARAMETER } \\
\text { UNCERTAIHTY }\end{array}$ \\
\hline TOTAL DISSOLVED SOLIDS & $06 / 21 / 82$ & 0001 & MG/L & & 3760.00 & - & - \\
\hline URAKIUM & $06 / 21 / 82$ & 0001 & MG/L & & 0.071 & - & - \\
\hline VARADIUA & $06 / 21 / 82$ & 0001 & MG/L & $<$ & 0.01 & 0.01 & - \\
\hline
\end{tabular}

PARAMETER VALUE IHDICATOR (PVI): < - LESS THAN DETECTION LIMIT

SAMPLE ID TCOOES:

0001 - FILTERED SAMPLE (.45 MICRONS) 
GROUNDHATER OUALIYY DATA BY LOCATION

SITE: AMBO1 AMBROSIA LAKE

LOCATIOH: 0711

NORTH COORDINATE: $\quad 44620.0$ FT

EAST COORDINATE: $\quad 58430.0$ FT

06/10/80 TO $12 / 04 / 92$

REPORT DATE: 08/04/94

FORMATION OF COMPLETIOH: TRES HERMANOS - B SANDSTONE (TB) HYDRAULIC FLOW RELATIONSHIP: UPGRADIENT (U)

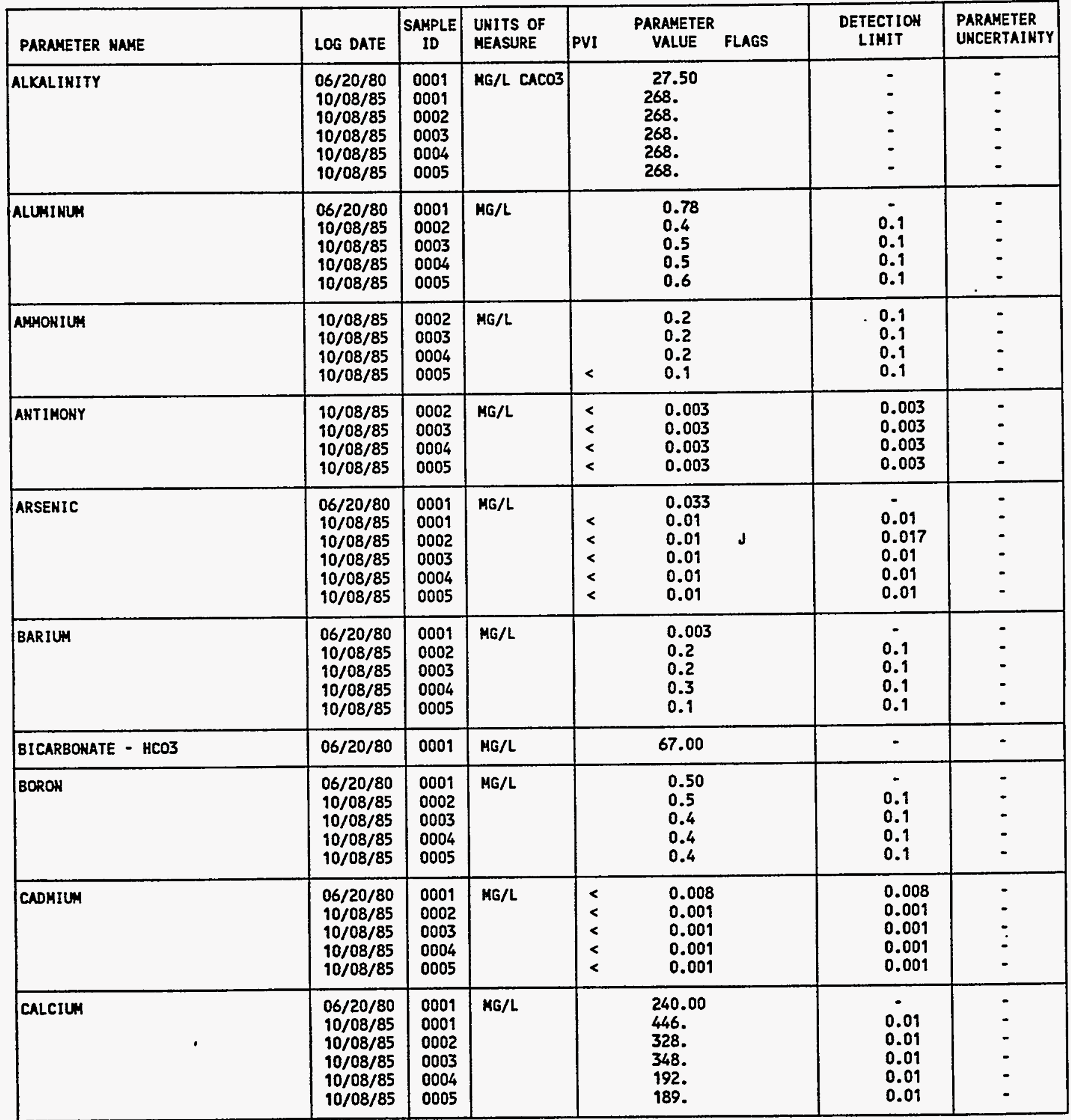

PARAMETER VALUE IMDICATOR (PVI): < - LESS THAN DETECTION LIMIT

SAMPLE ID COOES:

OTHER PARAMETER VALUE FLAGS:

0001 - FILTERED SAMPLE (.45 MICRONS)

J - ESTIMATED VALUE

0002 - FILTERED REPLICATE SAMPLE (.45 MICROHS)

0003 - FILTERED REPLICATE SAMPLE (.45 MICROHS)

0004 - FILTERED REPLICATE SAMPLE (.45 MICROHS) 
GROUNDHATER QUALITY DATA BY LOCATIOH

SITE: AMBOI AMBROSIA LAKE

LOCATION: 0711

MORTH COORDIHATE: 44620.0 FT

EAST COORDINATE: $\quad 58430.0$ FT

$06 / 10 / 80$ TO $12 / 04 / 92$

REPORT DATE: 08/04/94

FORMATION OF COMPLETIOH: TRES HERHANOS - 8 SAMDSTOHE (TB)

HYDRAULIC FLOH RELATIONSHIP: UPGRADIENT (U)

\begin{tabular}{|c|c|c|c|c|c|c|c|}
\hline PARAMETER NAME & LOG DATE & $\begin{array}{c}\text { SAMPLE } \\
\text { ID }\end{array}$ & $\begin{array}{l}\text { UHITS OF } \\
\text { MEASURE }\end{array}$ & PVI & $\begin{array}{l}\text { PARAMETEIR } \\
\text { VALUE FLAGS }\end{array}$ & $\begin{array}{l}\text { DETECTION } \\
\text { LIMIT }\end{array}$ & $\begin{array}{l}\text { PARAMETER } \\
\text { UHCERTAINTY }\end{array}$ \\
\hline CHLORIDE & $\begin{array}{l}06 / 20 / 80 \\
10 / 08 / 85 \\
10 / 08 / 85 \\
10 / 08 / 85 \\
10 / 08 / 85 \\
10 / 08 / 85\end{array}$ & $\begin{array}{l}0001 \\
0001 \\
0002 \\
0003 \\
0004 \\
0005\end{array}$ & MG/L & & $\begin{array}{l}109.00 \\
410 . \\
400 . \\
390 . \\
420 . \\
400 .\end{array}$ & $\begin{array}{l}1 . \\
1 . \\
1 . \\
1 .\end{array}$ & $\begin{array}{r}- \\
- \\
- \\
-\end{array}$ \\
\hline CHROHIUA & $\begin{array}{l}06 / 20 / 80 \\
10 / 08 / 85 \\
10 / 08 / 85 \\
10 / 08 / 85 \\
10 / 08 / 85\end{array}$ & $\begin{array}{l}0001 \\
0002 \\
0003 \\
0004 \\
0005\end{array}$ & MG/L & & $\begin{array}{l}0.16 \\
0.03 \\
0.05 \\
0.04 \\
0.04\end{array}$ & $\begin{array}{l}- \\
0.01 \\
0.01 \\
0.01 \\
0.01\end{array}$ & $\begin{array}{l}\ddot{-} \\
\dot{-} \\
\dot{-}\end{array}$ \\
\hline COBALT & $\begin{array}{l}06 / 20 / 80 \\
10 / 08 / 85 \\
10 / 08 / 85 \\
10 / 08 / 85 \\
10 / 08 / 85\end{array}$ & $\begin{array}{l}0001 \\
0002 \\
0003 \\
0004 \\
0005\end{array}$ & HG/L & $\begin{array}{l}< \\
< \\
< \\
< \\
<\end{array}$ & $\begin{array}{l}0.006 \\
0.05 \\
0.05 \\
0.05 \\
0.05\end{array}$ & $\begin{array}{l}0.006 \\
0.05 \\
0.05 \\
0.05 \\
0.05\end{array}$ & $\begin{array}{l}- \\
- \\
-\end{array}$ \\
\hline COPPER & $\begin{array}{l}06 / 20 / 80 \\
10 / 08 / 85 \\
10 / 08 / 85 \\
10 / 08 / 85 \\
10 / 08 / 85\end{array}$ & $\begin{array}{l}0001 \\
0002 \\
0003 \\
0004 \\
0005\end{array}$ & $M G / L$ & $\begin{array}{l}< \\
< \\
<\end{array}$ & $\begin{array}{l}0.001 \\
0.02 \\
0.02 \\
0.02 \\
0.02\end{array}$ & $\begin{array}{l}0.001 \\
0.02 \\
0.02 \\
0.02 \\
0.02\end{array}$ & $\begin{array}{l}- \\
- \\
-\end{array}$ \\
\hline CYAHIDE & $\begin{array}{l}10 / 08 / 85 \\
10 / 08 / 85 \\
10 / 08 / 85 \\
10 / 08 / 85\end{array}$ & $\begin{array}{l}0002 \\
0003 \\
0004 \\
0005\end{array}$ & $M G / L$ & $\begin{array}{l}< \\
< \\
<\end{array}$ & $\begin{array}{l}0.01 \\
0.01 \\
0.01 \\
0.01\end{array}$ & $\begin{array}{l}0.01 \\
0.01 \\
0.01 \\
0.01\end{array}$ & $\begin{array}{l}- \\
- \\
-\end{array}$ \\
\hline FLUORIDE & $\begin{array}{l}06 / 20 / 80 \\
10 / 08 / 85 \\
10 / 08 / 85 \\
10 / 08 / 85 \\
10 / 08 / 85\end{array}$ & $\begin{array}{l}0001 \\
0002 \\
0003 \\
0004 \\
0005\end{array}$ & $M G / L$ & & $\begin{array}{l}0.20 \\
0.8 \\
0.7 \\
0.7 \\
0.6\end{array}$ & $\begin{array}{l}- \\
0.1 \\
0.1 \\
0.1 \\
0.1\end{array}$ & $\begin{array}{l}- \\
- \\
- \\
-\end{array}$ \\
\hline IRON & $\begin{array}{l}06 / 20 / 80 \\
10 / 08 / 85 \\
10 / 08 / 85 \\
10 / 08 / 85 \\
10 / 08 / 85\end{array}$ & $\begin{array}{l}0001 \\
0002 \\
0003 \\
0004 \\
0005\end{array}$ & MG/L & $<$ & $\begin{array}{l}0.008 \\
0.37 \\
0.95 \\
0.04 \\
0.07\end{array}$ & $\begin{array}{l}0.008 \\
0.03 \\
0.03 \\
0.03 \\
0.03\end{array}$ & - \\
\hline LEAD & $\begin{array}{l}06 / 20 / 80 \\
10 / 08 / 85 \\
10 / 08 / 85 \\
10 / 08 / 85 \\
10 / 08 / 85\end{array}$ & $\begin{array}{l}0001 \\
0002 \\
0003 \\
0004 \\
0005\end{array}$ & $M G / L$ & $\begin{array}{l}< \\
< \\
< \\
<\end{array}$ & $\begin{array}{l}0.001 \\
0.01 \\
0.01 \\
0.01 \\
0.01\end{array}$ & $\begin{array}{l}0.001 \\
0.01 \\
0.01 \\
0.09 \\
0.01\end{array}$ & $\begin{array}{l}- \\
- \\
-\end{array}$ \\
\hline LEAD-210 & $\begin{array}{l}10 / 08 / 85 \\
10 / 08 / 85 \\
10 / 08 / 85 \\
10 / 08 / 85\end{array}$ & $\begin{array}{l}0002 \\
0003 \\
0004 \\
0005\end{array}$ & PCI/L & & $\begin{array}{l}1.4 \\
0.3 \\
0.2 \\
0.4\end{array}$ & $\begin{array}{l}1.5 \\
1.5 \\
1.5 \\
1.5\end{array}$ & $\begin{array}{l}1.1 \\
1.3 \\
1.2 \\
1.1\end{array}$ \\
\hline HAGMESILH & $\begin{array}{l}06 / 20 / 80 \\
10 / 08 / 85 \\
10 / 08 / 85\end{array}$ & $\begin{array}{l}0001 \\
0001 \\
0002\end{array}$ & $M G / L$ & & $\begin{array}{l}200.00 \\
246 . \\
217 .\end{array}$ & $\begin{array}{l}- \\
0.001 \\
0.001\end{array}$ & : \\
\hline PARAMETER VALUE IMDICATOR (PVI): & \multicolumn{4}{|c|}{ < - LESS THAN DETECTION LIMIT } & \multicolumn{3}{|l|}{$\begin{array}{l}\text { SAMPLE ID CODES: } \\
\text { O001 - FILTERED } \\
\text { O002 - FILTERED } \\
0003 \text { - FILTERED } \\
\text { D004 - FILTERED }\end{array}$} \\
\hline
\end{tabular}


GROUNDHATER QUALITY DATA BY LOCATION

SITE: AMBO1 AMBROSIA LAKE

LOCATION: 0711

NORTH COORDINATE: $\quad 44620.0$ FT

EAST COORDINATE: $\quad 58430.0$ FT

06/10/80 TO $12 / 04 / 92$

REPORT DATE: $08 / 04 / 94$

FORMATIOH OF COMPLETION: TRES HERMANOS - B SANDSTONE (TB)

HYDRAULIC FLON RELATIONSHIP: UPGRADIENT (U)

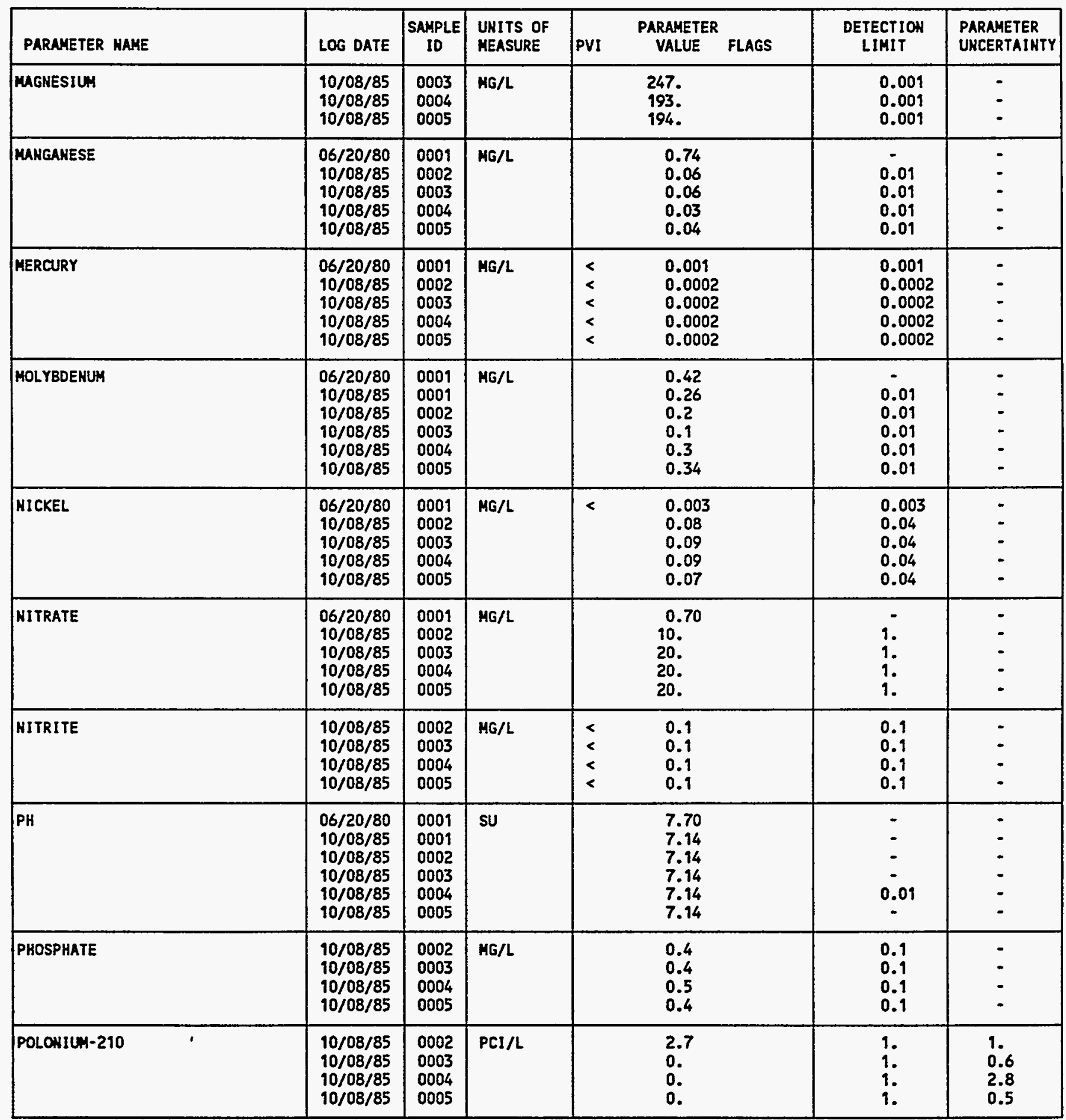

PARAMETER VALUE INDICATOR (PVI): < - LESS THAH DETECTION LIMIT

SAMPLE ID COOES

0001 - FILTERED SAMPLE (.45 MICROWS)

0002 - FILTERED REPLICATE SAMPLE (.45 MICRONS)

0003 - FILTERED REPLICATE SAMPLE (.45 MICROHS)

0004 - FILTERED REPLICATE SAMPLE (.45 MICRONS) 
GROUNDHATER OUALITY DATA BY LOCATIOH

SITE: AMBOI AMBROSIA LAKE

LOCATION: 0711

NORTH COORDIHATE: 44620.0 FT

EAST COORDINATE: $\quad \mathbf{5 8 4 3 0 . 0}$ FT

06/10/80 TO 12/04/92

REPORT DATE: 08/04/94

FORHATIOH OF COMPLETIOH: TRES HERMANOS - B SAHDSTOHE (TB)

HYDRAULIC FLON RELATIONSHIP: UPGRADIENT (U)

\begin{tabular}{|c|c|c|c|c|c|c|c|}
\hline PARÁMETER MAME & LOS DATE & $\begin{array}{c}\text { SAMPLE } \\
\text { ID }\end{array}$ & $\begin{array}{l}\text { UNITS OF } \\
\text { MEASURE }\end{array}$ & PVI & $\begin{array}{l}\text { PARAMETER: } \\
\text { VALUE FLAGS }\end{array}$ & $\begin{array}{l}\text { DETECTION } \\
\text { LIMIT }\end{array}$ & $\begin{array}{l}\text { PARAMETER } \\
\text { UHCERTAINTY }\end{array}$ \\
\hline POTASSIUN & $\begin{array}{l}06 / 20 / 80 \\
10 / 08 / 85 \\
10 / 08 / 85 \\
10 / 08 / 85 \\
10 / 08 / 85 \\
10 / 08 / 85\end{array}$ & $\begin{array}{l}0001 \\
0001 \\
0002 \\
0003 \\
0004 \\
0005\end{array}$ & $M G / L$ & & $\begin{array}{l}5.80 \\
5.96 \\
6.21 \\
5.95 \\
6.94 \\
6.58\end{array}$ & $\begin{array}{l}- \\
0.01 \\
0.01 \\
0.01 \\
0.01 \\
0.01\end{array}$ & $\begin{array}{l}- \\
- \\
- \\
-\end{array}$ \\
\hline RADIUH-226 & $\begin{array}{l}06 / 20 / 80 \\
10 / 08 / 85 \\
10 / 08 / 85 \\
10 / 08 / 85 \\
10 / 08 / 85\end{array}$ & $\begin{array}{l}0001 \\
0002 \\
0003 \\
0004 \\
0005\end{array}$ & PCI/L & & $\begin{array}{c}18.00 \\
1 . \\
0.4 \\
17 . \\
1 .\end{array}$ & $\begin{array}{l}. \\
1 . \\
1 . \\
1 .\end{array}$ & $\begin{array}{l}0 \\
0.4 \\
0.3 \\
1 . \\
0.4\end{array}$ \\
\hline RADIUM-226 + RADIUW-228 & $\begin{array}{l}10 / 08 / 85 \\
10 / 08 / 85 \\
10 / 08 / 85 \\
10 / 08 / 85\end{array}$ & $\begin{array}{l}0002 \\
0003 \\
0004 \\
0005\end{array}$ & $\mathrm{PCI} / \mathrm{L}$ & & $\begin{array}{r}1.00 \\
0.40 \\
17.00 \\
1.00\end{array}$ & - & $\dot{-}$ \\
\hline RADIUH-228 & $\begin{array}{l}10 / 08 / 85 \\
10 / 08 / 85 \\
10 / 08 / 85 \\
10 / 08 / 85\end{array}$ & $\begin{array}{l}0002 \\
0003 \\
0004 \\
0005\end{array}$ & PCI/L & & $\begin{array}{l}0 . \\
0 . \\
0 . \\
0 .\end{array}$ & $\begin{array}{l}1 . \\
1 . \\
1 .\end{array}$ & $\begin{array}{l}1.2 \\
1.3 \\
0.8 \\
0.9\end{array}$ \\
\hline SELENIUM & $\begin{array}{l}06 / 20 / 80 \\
10 / 08 / 85 \\
10 / 08 / 85 \\
10 / 08 / 85 \\
10 / 08 / 85 \\
10 / 08 / 85\end{array}$ & $\begin{array}{l}0001 \\
0001 \\
0002 \\
0003 \\
0004 \\
0005\end{array}$ & $M G / L$ & $\begin{array}{l}< \\
< \\
< \\
<\end{array}$ & $\begin{array}{l}0.12 \\
0.005 \\
0.005 \\
0.005 \\
0.005 \\
0.005\end{array}$ & $\begin{array}{l}- \\
0.005 \\
0.005 \\
0.005 \\
0.005 \\
0.005\end{array}$ & $\begin{array}{l}- \\
- \\
- \\
-\end{array}$ \\
\hline SILICA - SIO2 & $\begin{array}{l}10 / 08 / 85 \\
10 / 08 / 85 \\
10 / 08 / 85 \\
10 / 08 / 85\end{array}$ & $\begin{array}{l}0002 \\
0003 \\
0004 \\
0005\end{array}$ & $M G / L$ & & $\begin{array}{l}8 . \\
8 . \\
8 . \\
8 .\end{array}$ & $\begin{array}{l}2 . \\
2 . \\
2 . \\
2 .\end{array}$ & 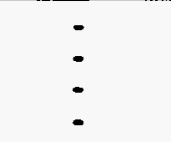 \\
\hline SILVER & $\begin{array}{l}06 / 20 / 80 \\
10 / 08 / 85 \\
10 / 08 / 85 \\
10 / 08 / 85 \\
10 / 08 / 85\end{array}$ & $\begin{array}{l}0001 \\
0002 \\
0003 \\
0004 \\
0005\end{array}$ & $M G / L$ & $\begin{array}{l}< \\
< \\
< \\
< \\
<\end{array}$ & $\begin{array}{l}0.002 \\
0.01 \\
0.01 \\
0.01 \\
0.01\end{array}$ & $\begin{array}{l}0.002 \\
0.01 \\
0.01 \\
0.01 \\
0.01\end{array}$ & $\begin{array}{l}- \\
- \\
- \\
-\end{array}$ \\
\hline soorum & $\begin{array}{l}06 / 20 / 80 \\
10 / 08 / 85 \\
10 / 08 / 85 \\
10 / 08 / 85 \\
10 / 08 / 85 \\
10 / 08 / 85\end{array}$ & $\begin{array}{l}0001 \\
0001 \\
0002 \\
0003 \\
0004 \\
0005\end{array}$ & $M G / L$ & & $\begin{array}{l}200.00 \\
286 . \\
216 . \\
467 . \\
780 . \\
762 .\end{array}$ & $\begin{array}{l}- \\
0.002 \\
0.002 \\
0.002 \\
0.002 \\
0.002\end{array}$ & $\begin{array}{l}- \\
- \\
- \\
-\end{array}$ \\
\hline SPECIFIC CONDUCTANCE & $\begin{array}{l}06 / 20 / 80 \\
10 / 08 / 85 \\
10 / 08 / 85 \\
10 / 08 / 85 \\
10 / 08 / 85 \\
10 / 08 / 85\end{array}$ & $\begin{array}{l}0001 \\
0001 \\
0002 \\
0003 \\
0004 \\
0005\end{array}$ & UнНО/CM & & $\begin{array}{l}2800.00 \\
3200 \\
3200 \\
3200 \\
3200 \\
3200\end{array}$ & $\begin{array}{l}- \\
- \\
- \\
-\end{array}$ & $\begin{array}{l}- \\
- \\
- \\
-\end{array}$ \\
\hline STROHTIUM & $10 / 08 / 85$ & 0002 & $M G / L$ & & 4.3 & 0.1 & $\because$ \\
\hline
\end{tabular}

PARAMETER VALUE InDICATOR (PVI): < - LESS THAK DETECTION LIMIT

SAMPLE ID COOES:

0001 - FILTERED SAMPLE (.45 MICROHS)

DO02 - FILTERED REPLICATE SAMPLE (.45 MICRONS)

0003 - FILTERED REPLICATE SAMPLE (.45 MICROWS)

0004 - FILTERED REPLICATE SAMPLE (.45 MICROHS) 
GROUNDHATER QUALITY DATA BY LOCATION

SITE: AHBOI AHBROSIA LAKE

LOCATIOH: 0711

NORTH COORDIHATE: 44620.0 FT

EAST COORDINATE: $\quad 58430.0$ FT

06/10/80 To $12 / 04 / 92$

REPORT DATE: $08 / 04 / 94$

FORMATION OF CONPLETION: TRES HERHANOS - B SANOSTONE (TB)

HYORAULIC FLON RELATIONSHIP: UPGRADIENT (U)

\begin{tabular}{|c|c|c|c|c|c|c|c|}
\hline PARAMETER MAME & LOG DATE & $\begin{array}{c}\text { SAMPLE } \\
\text { ID }\end{array}$ & $\begin{array}{l}\text { UNITS OF } \\
\text { HEASURE }\end{array}$ & PVI & $\begin{array}{l}\text { PARAMETER } \\
\text { VALUE FLAGS }\end{array}$ & $\begin{array}{l}\text { DETECTION } \\
\text { LIMIT }\end{array}$ & $\begin{array}{l}\text { PARAMETER } \\
\text { UNCERTAINTY }\end{array}$ \\
\hline STRONTIUA & $\begin{array}{l}10 / 08 / 85 \\
10 / 08 / 85 \\
10 / 08 / 85\end{array}$ & $\begin{array}{l}0003 \\
0004 \\
0005\end{array}$ & $M G / L$ & & $\begin{array}{l}4.1 \\
2 . \\
2 .\end{array}$ & $\begin{array}{l}0.1 \\
0.1 \\
0.1\end{array}$ & $\begin{array}{l}- \\
-\end{array}$ \\
\hline SULFATE & $\begin{array}{l}06 / 20 / 80 \\
10 / 08 / 85 \\
10 / 08 / 85 \\
10 / 08 / 85 \\
10 / 08 / 85 \\
10 / 08 / 85\end{array}$ & $\begin{array}{l}0001 \\
0001 \\
0002 \\
0003 \\
0004 \\
0005\end{array}$ & MG/L & & $\begin{array}{l}1600.00 \\
2070 . \\
1350 . \\
2040 . \\
2060 . \\
2060 .\end{array}$ & $\begin{array}{l}. \\
0.1 \\
0.1 \\
0.1 \\
0.1 \\
0.1\end{array}$ & $\begin{array}{l}- \\
- \\
- \\
.\end{array}$ \\
\hline SULFIDE & $\begin{array}{l}10 / 08 / 85 \\
10 / 08 / 85 \\
10 / 08 / 85 \\
10 / 08 / 85\end{array}$ & $\begin{array}{l}0002 \\
0003 \\
0004 \\
0005\end{array}$ & $\mathrm{MG} / \mathrm{L}$ & $\begin{array}{l}< \\
< \\
< \\
<\end{array}$ & $\begin{array}{l}0.1 \\
0.1 \\
0.1 \\
0.1\end{array}$ & $\begin{array}{l}0.1 \\
0.1 \\
0.1 \\
0.1\end{array}$ & $\begin{array}{l}- \\
- \\
-\end{array}$ \\
\hline TEMPERATURE & $\begin{array}{l}10 / 08 / 85 \\
10 / 08 / 85 \\
10 / 08 / 85 \\
10 / 08 / 85 \\
10 / 08 / 85\end{array}$ & $\begin{array}{l}0001 \\
0002 \\
0003 \\
0004 \\
0005\end{array}$ & C - DEGREE & & $\begin{array}{l}13.5 \\
13.5 \\
13.5 \\
13.5 \\
13.5\end{array}$ & $\begin{array}{l}- \\
- \\
- \\
-\end{array}$ & $\begin{array}{l}- \\
- \\
-\end{array}$ \\
\hline THORIUM-230 & $\begin{array}{l}10 / 08 / 85 \\
10 / 08 / 85 \\
10 / 08 / 85 \\
10 / 08 / 85\end{array}$ & $\begin{array}{l}0002 \\
0003 \\
0004 \\
0005\end{array}$ & PCI/L & & $\begin{array}{l}0.3 \\
0.1 \\
0.3 \\
0.2\end{array}$ & $\begin{array}{l}1 . \\
1 . \\
1 . \\
1 .\end{array}$ & $\begin{array}{l}0.4 \\
0.3 \\
0.4 \\
0.3\end{array}$ \\
\hline TIN & $\begin{array}{l}10 / 08 / 85 \\
10 / 08 / 85 \\
10 / 08 / 85 \\
10 / 08 / 85\end{array}$ & $\begin{array}{l}0002 \\
0003 \\
0004 \\
0005\end{array}$ & MG/L & $\begin{array}{l}< \\
< \\
<\end{array}$ & $\begin{array}{l}0.005 \\
0.005 \\
0.005 \\
0.005\end{array}$ & $\begin{array}{l}0.005 \\
0.005 \\
0.005 \\
0.005\end{array}$ & $\begin{array}{l}- \\
- \\
-\end{array}$ \\
\hline TOTAL DISSOLVED SOLIDS & $\begin{array}{l}06 / 20 / 80 \\
10 / 08 / 85 \\
10 / 08 / 85 \\
10 / 08 / 85 \\
10 / 08 / 85 \\
10 / 08 / 85\end{array}$ & $\begin{array}{l}0001 \\
0001 \\
0002 \\
0003 \\
0004 \\
0005\end{array}$ & MG/L & & $\begin{array}{l}2540.00 \\
3860 . \\
3910 . \\
3940 . \\
3960 . \\
3980 .\end{array}$ & $\begin{array}{l}10 . \\
10 . \\
10 . \\
10 . \\
10 .\end{array}$ & $\begin{array}{l}- \\
- \\
- \\
-\end{array}$ \\
\hline TOTAL ORGAMIC CARBON & $\begin{array}{l}10 / 08 / 85 \\
10 / 08 / 85 \\
10 / 08 / 85\end{array}$ & $\begin{array}{l}0002 \\
0004 \\
0005\end{array}$ & $M G / L$ & & $\begin{array}{l}82 . \\
90 \\
56 .\end{array}$ & $\begin{array}{l}1 . \\
1 . \\
1 .\end{array}$ & - \\
\hline TOTAL SUSPENDED SOLIDS & $06 / 20 / 80$ & 0001 & MG/L & & 46.00 & - & - \\
\hline URANIUY & $\begin{array}{l}06 / 20 / 80 \\
10 / 08 / 85 \\
10 / 08 / 85 \\
10 / 08 / 85 \\
10 / 08 / 85 \\
10 / 08 / 85\end{array}$ & $\begin{array}{l}0001 \\
0001 \\
0002 \\
0003 \\
0004 \\
0005\end{array}$ & HG/L & & $\begin{array}{l}0.10 \\
0.0069 \\
0.0138 \\
0.0127 \\
0.0028 \\
0.0006\end{array}$ & $\begin{array}{l}0.003 \\
0.003 \\
0.003 \\
0.003 \\
0.003\end{array}$ & $\begin{array}{l}\dot{-} \\
\dot{-} \\
\dot{-} \\
.\end{array}$ \\
\hline VAKADIUH & $\begin{array}{l}06 / 20 / 80 \\
10 / 08 / 85 \\
10 / 08 / 85 \\
10 / 08 / 85\end{array}$ & $\begin{array}{l}0001 \\
0002 \\
0003 \\
0004\end{array}$ & $M G / L$ & $\begin{array}{l}< \\
< \\
<\end{array}$ & $\begin{array}{l}0.003 \\
0.01 \\
0.01 \\
0.01\end{array}$ & $\begin{array}{l}0.003 \\
0.01 \\
0.01 \\
0.01\end{array}$ & $\begin{array}{l}- \\
- \\
-\end{array}$ \\
\hline
\end{tabular}

PARAMETER VALUE INDICATOR (PVI): < - LESS THAN DETECTION LIMIT

SAMPLE IO COOES:

0009 - FILTERED SAMPLE (.45 MICROHS)

OTHER PARAMETER VALUE FLAGS:

0002 - FILTERED REPLICATE SANPLE (.45 HICROHS)

0003 - FILTERED REPLICATE SAHPLE (.45 MICRONS)

0004 - FILTERED REPLICATE SAMPLE (.45 MICRONS) 
GROUMDWATER OUALITY DATA BY LOCATION

SITE: AMBO1 AMBROSIA LAKE

LOCATION: 0711

MORTH COORDIHATE: 44620.0 FT

EAST COORDINATE:

06/10/80 TO $12 / 04 / 92$

REPORT DATE: 08/04/94

FORMATION OF COMPLETIOH: TRES HERMAHOS - B SAHDSTONE (TB) HYDRAULIC FLOW RELATIONSHIP: UPGRADIENT (U)

\begin{tabular}{|c|c|c|c|c|c|c|c|}
\hline PARAMETER MAME & LOG DATE & $\begin{array}{c}\text { SAMPLE } \\
10\end{array}$ & $\begin{array}{l}\text { UNITS OF } \\
\text { MEASURE }\end{array}$ & PVI & $\begin{array}{l}\text { PARAMETEF! } \\
\text { VALUE FLAGS }\end{array}$ & $\begin{array}{l}\text { DETECTION } \\
\text { LIHIT }\end{array}$ & $\begin{array}{l}\text { PARÂMETER } \\
\text { UHCERTAIHTY }\end{array}$ \\
\hline VAHADIUY & $10 / 08 / 85$ & 0005 & MG/L & & 0.1 & 0.01 & - \\
\hline ZIMC & $\begin{array}{l}06 / 20 / 80 \\
10 / 08 / 85 \\
10 / 08 / 85 \\
10 / 08 / 85 \\
10 / 08 / 85\end{array}$ & $\begin{array}{l}0001 \\
0002 \\
0003 \\
0004 \\
0005\end{array}$ & $M G / L$ & & $\begin{array}{l}0.15 \\
0.013 \\
0.021 \\
0.011 \\
0.01\end{array}$ & $\begin{array}{l}- \\
0.005 \\
0.005 \\
0.005 \\
0.005\end{array}$ & - \\
\hline
\end{tabular}

PARAMETER VALUE IMDICATOR (PVI): < - LESS THAM DETECTIOH LIMIT

SAMPLE ID COOES:

D001 - FILTERED SAMPLE (.45 MICRONS)

0002 - FILTERED REPLICATE SAMPLE (.45 MICRONS)

0003 - FILTERED REPLICATE SAMPLE (.45 MICRONS)

0004 - FILTERED REPLICATE SAMPLE (.45 MICROHS) 
GROUNDWATER QUALITY DATA BY LOCATION

SITE: AMBO1 AMBROSIA LAKE

LOCATION: 0712

NORTH COORDIHATE: $\quad 58780.0$ FT

EAST COORDIHATE: $\quad 53420.0$ FT

06/10/80 TO $12 / 04 / 92$

REPORT DATE: 08/04/94

FORMATION OF COMPLETION: ALLUVIUN (AL)

IYORAULIC FLON RELATIOWSHIP: UNKNOWN (H)

\begin{tabular}{|c|c|c|c|c|c|c|c|}
\hline PARAHETER MAKE & LOG DATE & $\underset{\text { ID }}{\text { SAHPLE }}$ & $\begin{array}{l}\text { UNITS OF } \\
\text { MEASURE }\end{array}$ & PVI & $\begin{array}{l}\text { PARAMETER } \\
\text { VALUE FLAGS }\end{array}$ & $\begin{array}{l}\text { DETECTIOH } \\
\text { LIMIT }\end{array}$ & $\begin{array}{l}\text { PARAMETER } \\
\text { UNCERTAIHTY }\end{array}$ \\
\hline ALKALINITY & $06 / 25 / 80$ & 0001 & HG/L CACO3 & & 103.00 & - & - \\
\hline ALLMINUM & $06 / 25 / 80$ & 0009 & HG/L & & 1.80 & - & - \\
\hline ARSENIC & $06 / 25 / 80$ & 0001 & MG/L & & 0.091 & - & - \\
\hline BARIUN & $06 / 25 / 80$ & 0001 & HE/L & $<$ & 0.001 & 0.001 & - \\
\hline BICARBOHATE - HCO3 & $06 / 25 / 80$ & 0001 & $M G / L$ & & 252.00 & - & - \\
\hline BORON & $06 / 25 / 80$ & 0001 & MG/L & & 0.77 & - & - \\
\hline CAOMIUA & $06 / 25 / 80$ & 0001 & MG/L & $<$ & 0.008 & 0.008 & - \\
\hline CALCIUM & $06 / 25 / 80$ & 0001 & MG/L & & 490.00 & - & - \\
\hline CHLORIDE & $06 / 25 / 80$ & 0001 & $M G / L$ & & 1360.00 & - & - \\
\hline CHROMIUA & $06 / 25 / 80$ & 0001 & $M G / L$ & $<$ & 0.001 & 0.001 & - \\
\hline COBALT & $06 / 25 / 80$ & 0001 & HG/L & $<$ & 0.006 & 0.006 & - \\
\hline COPPER & $06 / 25 / 80$ & 0001 & $M G / L$ & $<$ & 0.001 & 0.001 & - \\
\hline FLUORIDE & $06 / 25 / 80$ & 0001 & MG/L & & 0.04 & - & - \\
\hline IRON & $06 / 25 / 80$ & 0001 & $M G / L$ & $<$ & 0.008 & 0.008 & - \\
\hline LEAD & $06 / 25 / 80$ & 0001 & HG/L & & 0.009 & - & - \\
\hline MAGNESIUM & $06 / 25 / 80$ & 0001 & $M G / L$ & & 460.00 & - & - \\
\hline MAKGAKESE & $06 / 25 / 80$ & 0001 & MG/L & & 0.54 & - & - \\
\hline MERCURY & $06 / 25 / 80$ & 0001 & MG/L & $<$ & 0.001 & 0.001 & - \\
\hline MOLYBDENUM & $06 / 25 / 80$ & 0001 & $M G / L$ & $<$ & 0.002 & 0.002 & - \\
\hline HICKEL & $06 / 25 / 80$ & 0001 & MG/L & $<$ & 0.003 & 0.003 & - \\
\hline NITRATE & $06 / 25 / 80$ & 0001 & MG/L & & 28.00 & - & - \\
\hline РН & $06 / 25 / 80$ & 0001 & SU & & 7.60 & - & - \\
\hline POTASSIUH & $06 / 25 / 80$ & 0001 & MG/L & & 13.00 & - & - \\
\hline RADIUH-226 & $06 / 25 / 80$ & 0001 & $\mathrm{PCl} / \mathrm{L}$ & & 4.32 & - & - \\
\hline SELENIU:H & $06 / 25 / 80$ & 0001 & MG/L & & 0.15 & - & - \\
\hline SILVER & $06 / 25 / 80$ & 0001 & MG/L & $<$ & 0.002 & 0.002 & - \\
\hline soorius & $06 / 25 / 80$ & 0001 & $M G / L$ & & 670.00 & - & - \\
\hline SPECIFIC CONDUCTANCE & $06 / 25 / 80$ & 0001 & UAHO/CM & & 7340.00 & - & - \\
\hline SULFATE & $06 / 25 / 80$ & 0001 & $M G / L$ & & 2610.00 & - & - \\
\hline TOTAL DISSOLVED SOLIDS & $06 / 25 / 80$ & 0001 & $M G / L$ & & 6380.00 & - & - \\
\hline
\end{tabular}

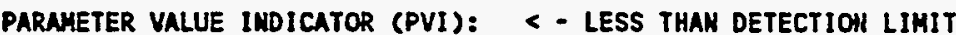

SAMPLE ID COOES:

0001 - FILTERED SAMPLE (.45 MICRONS) 
GROUHDWATER OUALITY DATA BY LOCATIOH

SITE: AMBOI AMBROSIA LAKE

LOCATIOH: 0712

NORTH COORDINATE: 58780.0 FT

EAST COORDIHATE: $\quad 53420.0$ FT

06/10/80 TO $12 / 04 / 92$

REPORT DATE: $08 / 04 / 94$

FORMATION OF COAPLETION: ALLUVIUH (AL)

HYDRAULIC FLOH RELATIONSHIP: UHKHONN (N)

\begin{tabular}{|c|c|c|c|c|c|c|c|}
\hline PARAMETER MAME & LOG DATE & $\begin{array}{c}\text { SAMPLE } \\
\text { ID }\end{array}$ & $\begin{array}{l}\text { UNITS OF } \\
\text { MEASURE }\end{array}$ & PVI & $\begin{array}{l}\text { PARAMETER } \\
\text { VALUE FLAGS }\end{array}$ & $\begin{array}{l}\text { DETECTION } \\
\text { LIMIT }\end{array}$ & $\begin{array}{l}\text { PARANETER } \\
\text { UNCERTAINTY }\end{array}$ \\
\hline TOTAL SUSPENDED SOLIDS & $06 / 25 / 80$ & 0001 & MG/L & & 372.00 & - & - \\
\hline URAKIUM & $06 / 25 / 80$ & 0001 & MG/L & & 0.044 & - & - \\
\hline VANADIUM & $06 / 25 / 80$ & 0001 & $M G / L$ & $<$ & 0.003 & 0.003 & - \\
\hline ZINC & $06 / 25 / 80$ & 0001 & $M G / L$ & & 0.14 & - & - \\
\hline
\end{tabular}

PARAMETER VALUE IMDICATOR (PVI):

< - LESS THAN DETECTION LIMIT

SAMPLE ID COOES:

OOOI - FILTERED SAMPLE (.45 HICRONS) 
GROUNDHATER QUALITY DATA BY LOCATION

SITE: AMBO1 AMBROSIA LAKE

LOCATIOH: 0713

NORTH COORDIHATE: 59395.0 FT

EAST COORDINATE: $\quad 50400.0$ FT

06/10/80 TO 12/04/92

REPORT DATE: 08/04/94

FORMATION OF COMPLETION: ALLUVIUM (AL)

HYDRAULIC FLON RELATIONSHIP: UNKNONN (N)

\begin{tabular}{|c|c|c|c|c|c|c|}
\hline PARAMETER MAME & LOG DATE & $\underset{\text { SAMPLE }}{\text { ID }}$ & $\begin{array}{l}\text { UNITS OF } \\
\text { MEASURE }\end{array}$ & \begin{tabular}{|c} 
PARAMETER \\
PVI \\
VALUE
\end{tabular} FLAGS & $\begin{array}{l}\text { DETECTIOH } \\
\text { LIMIT }\end{array}$ & $\begin{array}{l}\text { PARAMETER } \\
\text { UNCERTAINTY }\end{array}$ \\
\hline ALKALINITY & $06 / 25 / 80$ & 0001 & $\mathrm{HG} / \mathrm{L} \operatorname{CACO} 3$ & 41.40 & - & - \\
\hline ALUHIKUA & $06 / 25 / 80$ & 0001 & MG/L & 0.80 & - & - \\
\hline ARSENIC & $06 / 25 / 80$ & 0001 & $M G / L$ & 0.041 & - & - \\
\hline BARIUH & $06 / 25 / 80$ & 0001 & HG/L & 0.005 & - & - \\
\hline BICARBOHATE - HCO3 & $06 / 25 / 80$ & 0001 & MG/L & 101.00 & - & - \\
\hline BORON & $06 / 25 / 80$ & 0001 & $M G / L$ & 0.48 & - & - \\
\hline CADMIUY & $06 / 25 / 80$ & 0001 & $M G / L$ & 0.008 & 0.008 & - \\
\hline CALCIUM & $06 / 25 / 80$ & 0001 & MG/L & 470.00 & - & - \\
\hline CHLORIDE & $06 / 25 / 80$ & 0001 & MG/L & 70.00 & - & - \\
\hline CHROHIUM & $06 / 25 / 80$ & 0001 & $M G / L$ & 0.004 & - & - \\
\hline COBALT & $06 / 25 / 80$ & 0001 & MG/L & 0.006 & 0.006 & - \\
\hline COPPER & $06 / 25 / 80$ & 0001 & HG/L & 0.03 & - & - \\
\hline FLLORIDE & $06 / 25 / 80$ & 0001 & MG/L & 0.30 & - & - \\
\hline IRON & $06 / 25 / 80$ & 0001 & $M G / L$ & 0.008 & 0.008 & - \\
\hline LEAD & $06 / 25 / 80$ & 0001 & MG/L & 0.004 & - & - \\
\hline MAGNESIUH & $06 / 25 / 80$ & 0001 & MG/L & 190.00 & - & - \\
\hline MAKGANESE & $06 / 25 / 80$ & 0001 & MG/L & 0.49 & - & - \\
\hline MERCURY & $06 / 25 / 80$ & 0001 & MG/L & 0.001 & 0.001 & - \\
\hline MOLYBDENUA4 & $06 / 25 / 80$ & 0001 & MG/L & 0.002 & 0.002 & - \\
\hline NICKEL & $06 / 25 / 80$ & 0001 & MG/L & 0.003 & 0.003 & - \\
\hline HITRATE & $06 / 25 / 80$ & 0001 & MG/L & 0.90 & - & - \\
\hline $\mathrm{PH}$ & $06 / 25 / 80$ & 0001 & SU & 7.80 & - & - \\
\hline POTASSIUA & $06 / 25 / 80$ & 0001 & MG/L & 7.40 & - & - \\
\hline RNDIUM-226 & $06 / 25 / 80$ & 0001 & $\mathrm{PCI} / \mathrm{L}$ & 9.58 & - & - \\
\hline SELEKIUH & $0.6 / 25 / 80$ & 0001 & MG/L & 0.087 & - & - \\
\hline SILVER & $06 / 25 / 80$ & 0001 & $H G / L$ & 0.035 & - & - \\
\hline SOOIUH & $06 / 25 / 80$ & 0001 & MG/L & 190.00 & - & - \\
\hline SPECIFIC CONDUCTANCE & $0.6 / 25 / 80$ & 0001 & UMHO/CM & 3520.00 & - & - \\
\hline SULFATE & $06 / 25 / 80$ & 0001 & MG/L & 2290.00 & - & - \\
\hline TOTAL DISSOLVED SOLIDS & $06 / 25 / 80$ & 0001 & HG/L & 3560.00 & - & - \\
\hline
\end{tabular}

PARANETER VALUE INDICATOR (PVI): < - LESS THAH DETECTION LIMIT

SAMPLE ID COOES:

0001 - FILTERED SAMPLE (.45 MICRONS) 
GROUNDWATER QUALITY DATA BY LOCATIOW

SITE: AMBO1 AMBROSIA LAKE

LOCATIOH: 0713

HORTH COOROIHATE: 59395.0 FT

EAST COORDIHATE: $\quad 50400.0$ FT

$06 / 10 / 80$ TO $12 / 04 / 92$

REPORT DATE: $08 / 04 / 94$

FORMATIOH OF COMPLETION: ALLUVIUH (AL)

HYDRAULIC FLON RELATIONSHIP: UHKWOUN (H)

\begin{tabular}{|c|c|c|c|c|c|c|c|}
\hline PARAMETER MAME & LOG DATE & $\underset{\text { SAMPLE }}{\text { ID }}$ & $\begin{array}{l}\text { UHITS OF } \\
\text { MEASURE }\end{array}$ & PVI & $\begin{array}{l}\text { PARAMETER } \\
\text { VALUE FLAGS }\end{array}$ & $\begin{array}{l}\text { DETECTION } \\
\text { LIMIT }\end{array}$ & $\begin{array}{l}\text { PARANETER } \\
\text { UHCERTAINTY }\end{array}$ \\
\hline TOTAL SUSPENDED SOLIDS & $06 / 25 / 80$ & 0001 & MG/L & & 136.00 & - & - \\
\hline URAHIUH & $06 / 25 / 80$ & 0001 & $M G / L$ & & 0.024 & - & - \\
\hline VAMADIUA & $06 / 25 / 80$ & 0001 & MG/L & $<$ & 0.003 & 0.003 & - \\
\hline ZINC & $06 / 25 / 80$ & 0001 & MG/L & & 0.049 & - & - \\
\hline
\end{tabular}

PARAMETER VALUE INDICATOR (PVI): < - LESS THAN DETECTION LIMIT

SAMPLE ID COOES:

0001 - FILIERED SAHPLE (.45 MICRONS) 
GROUNDWATER OUALITY DATA BY LOCATION

SITE: AMBOI AMBROSIA LAKE

LOCATION: 0714

HORTH COORDINATE: 50050.0 FT

EAST COORDINATE: 53110.0 FT

06/10/80 TO $12 / 04 / 92$

REPORT DATE: 08/04/94

FORMATION OF COAPLETION: ALLUVIUA (AL)

HYDRAULIC FLON RELATIONSHIP: UHKNOWN (N)

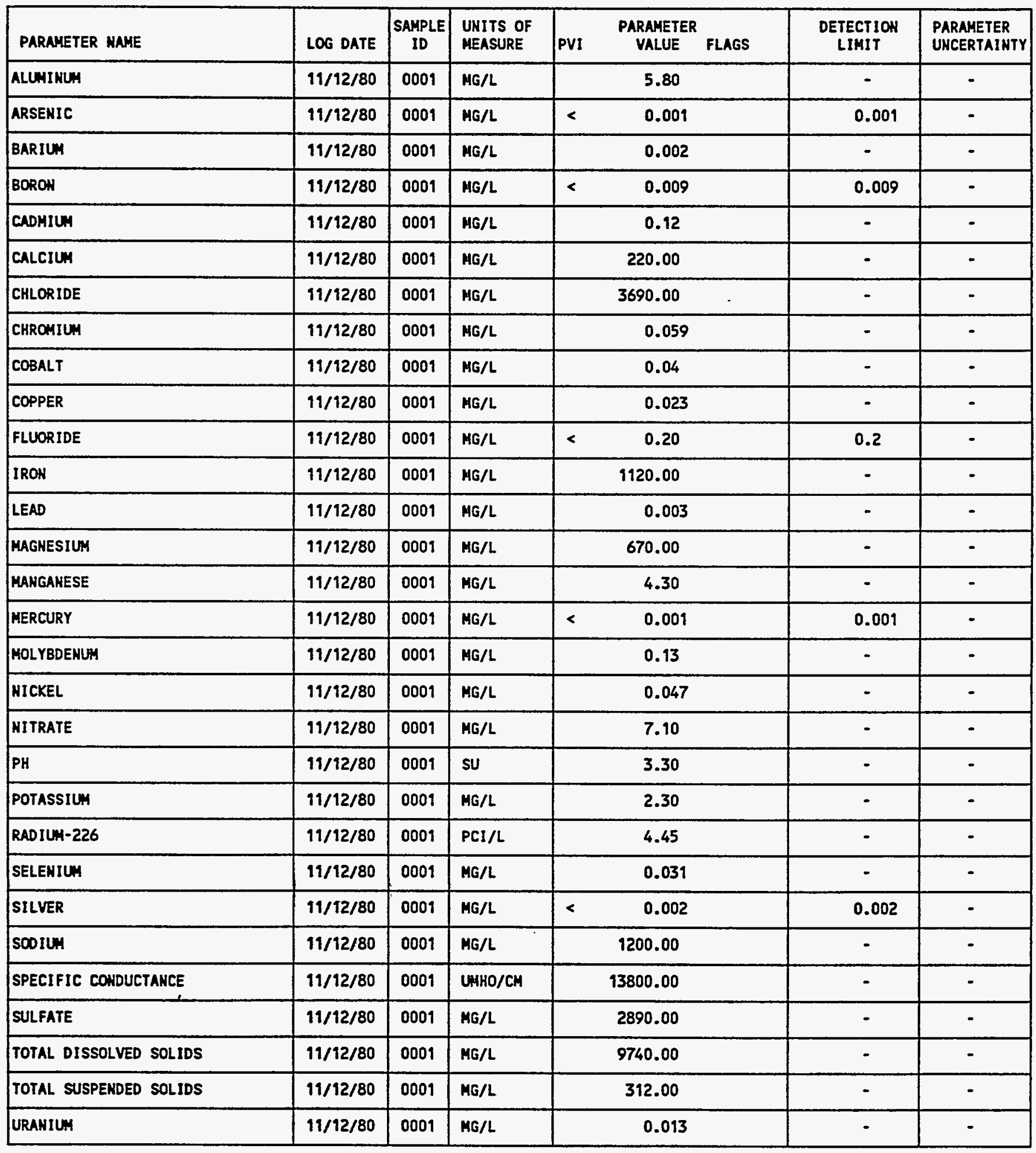

PARAMETER VALUE IHDICATOR (PVI): < - LESS THAN DETECTIOH LIMIT

SAMPLE ID COOES

0001 - FILTERED SAMPLE (.45 MICRONS) 
GROUNDHATER QUALITY DATA BY LOCATION

SITE: AMBO1 AMBROSIA LAKE

LOCATIOH: 0714

NORTH COORDINATE: 50050.0 FT

EAST COORDIKATE: $\quad 53110.0$ FT

06/10/80 TO 12/04/92

REPORT OATE: 08/04/94

FORMATION OF COAPLETION: ALLUVIUM (AL) HYDRAULIC FLOW RELATIONSHIP: UNKNOWN (N)

\begin{tabular}{|l|c|c|l|lc|c|c|}
\hline PARAMETER HAME & LOG DATE & $\begin{array}{l}\text { SAMPLE } \\
\text { I0 }\end{array}$ & $\begin{array}{l}\text { UHITS OF } \\
\text { MEASURE }\end{array}$ & PVI & $\begin{array}{c}\text { PARAMETER } \\
\text { VALUE }\end{array}$ & $\begin{array}{c}\text { DETAGS } \\
\text { LIMIT }\end{array}$ & $\begin{array}{l}\text { PARAMETER } \\
\text { UNCERTAINTY }\end{array}$ \\
\hline VAMADIUM & $11 / 12 / 80$ & 0001 & MG/L & $<$ & 0.003 & 0.003 & - \\
\hline ZINC & $11 / 12 / 80$ & 0001 & MG/L & & 0.43 & - & - \\
\hline
\end{tabular}

PARAHETER VALUE IMDICATOR (PVI):

< - LESS THAN DETECTION LIMIT

SAMPLE ID CODES:

0001 - FILTERED SNMPLE (.45 MICRONS) 
GROUNDHATER QUALITY DATA BY LOCATION

SITE: AMBOI AMBROSIA LAKE

LOCATION: 0715

NORTH COORDIHATE: 50680.0 FT

EAST COORDINATE: $\quad 52310.0$ FT

06/10/80 TO 12/04/92

REPORT DATE: 08/04/94

FORMATION OF COMPLETION: ALLUVIUM (AL)

HYDRAULIC FLOW RELATIONSHIP: UNKNOWN (N)

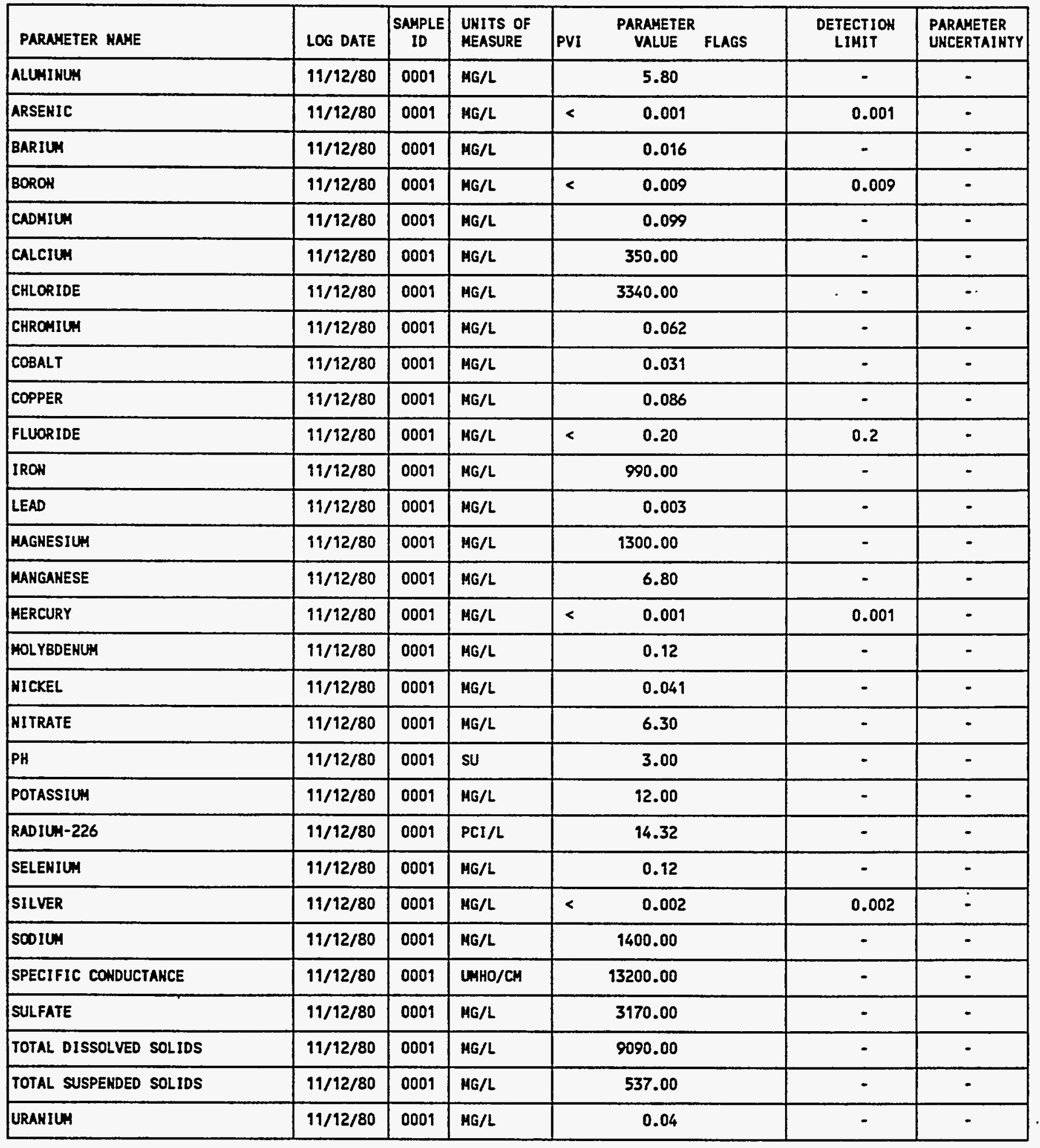

PARAMETER VALUE INDICATOR (PVI):

< - LESS THAN DETECTIOA LIMIT 
GROUNDHATER OUALITY DATA BY LOCATION

SITE: AMBO1 AMBROSIA LAKE

LOCATION: 0715

NORTH COORDINATE: 50680.0 FT

EAST COORDINATE: $\quad 52310.0$ FT

06/10/80 TO $12 / 04 / 92$

REPORT DATE: 08/04/94

FORMATIOH OF COMPLETIOH: ALLUVIUH (AL)

HYDRAULIC FLON RELATIONSHIP: UNKNOWH (H)

\begin{tabular}{|c|c|c|c|c|c|c|c|}
\hline PARAMETER NAME & LOG DATE & $\begin{array}{c}\text { SAMPLE } \\
10\end{array}$ & $\begin{array}{l}\text { UNITS OF } \\
\text { MEASURE }\end{array}$ & PVI & $\begin{array}{l}\text { PARAMETER } \\
\text { VALUE FLAGS }\end{array}$ & $\begin{array}{l}\text { DETECTIOH } \\
\text { LIMIT }\end{array}$ & $\begin{array}{l}\text { PARAMETER } \\
\text { UHCERTAIHTY }\end{array}$ \\
\hline VANADIUA & $11 / 12 / 80$ & 0001 & $M G / L$ & $<$ & 0.003 & 0.003 & - \\
\hline ZIнC & $11 / 12 / 80$ & 0001 & $M G / L$ & & 0.52 & - & - \\
\hline
\end{tabular}

PARAMETER VALUE IHDICATOR (PVI):

< - LESS THAN DETECTION LIMIT

SAMPLE ID COOES:

0001 - FILTERED SAMPLE (.45 MICRONS) 
GROUNDWATER QUALITY DATA BY LOCATIOH

SITE: AMBOI AMBROSIA LAKE

LOCATION: 0716

NORTH COORDINATE: $\quad 48800.0$ FT

EAST COORDINATE: $\quad 53510.0$ FT

06/10/80 TO 12/04/92

REPORT DATE: 08/04/94

FORMATION OF COMPLETION: ALLUVIUN (AL)

HYDRAULIC FLON RELATIONSHIP: UHKHOWN (N)

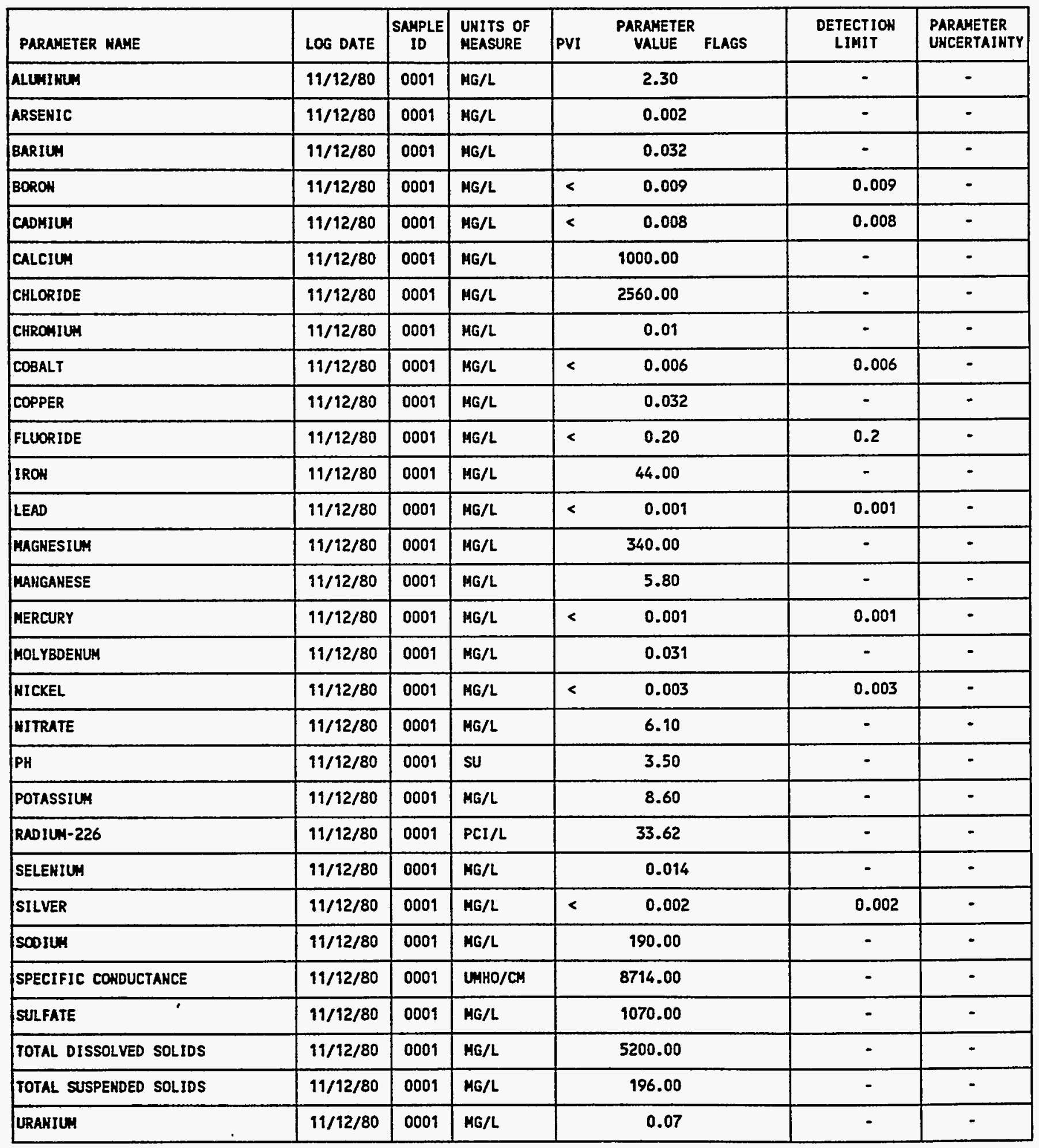

PARAMETER VALUE IMDICATOR (PVI): < - LESS THAN DETECTION LIMIT

SAMPLE ID COOES:

0001 - FILTERED SAMPLE (.45 MICRONS) 
GROUHDWATER QUALITY DATA BY LOCATION

SITE: AMBOI AMBROSIA LAKE

LOCATION: 0716

NORTH COORDINATE: 48800.0 FT

EAST COORDINATE: $\quad 53510.0$ FT

06/10/80 TO $12 / 04 / 92$

REPORT DATE: 08/04/94

FORMATION OF COMPLETION: ALLUVIUM (AL)

HYORAULIC FLOW RELATIOHSHIP: UHKWOWH (N)

\begin{tabular}{|c|c|c|c|c|c|c|c|}
\hline PARAMETER NAME & LOG DATE & $\begin{array}{c}\text { SAMPLE } \\
10\end{array}$ & $\begin{array}{l}\text { UNITS OF } \\
\text { MEASURE }\end{array}$ & PVI & $\begin{array}{l}\text { PARAMETER! } \\
\text { VALUE FLAGS }\end{array}$ & $\begin{array}{l}\text { DETECTION } \\
\text { LIHIT }\end{array}$ & $\begin{array}{l}\text { PARAMETER } \\
\text { UMCERTAIHTY }\end{array}$ \\
\hline VAHROIUH & $11 / 12 / 80$ & 0001 & MG/L & & 0.11 & - & - \\
\hline ZIHC & $11 / 12 / 80$ & 0001 & $M G / L$ & & 0.18 & - & - \\
\hline
\end{tabular}

PARAMETER VALUE IMDICATOR (PVI): < - LESS THAH DETECTION LIMIT

SAMPLE ID COOES:

0001 - FILTERED SAHPLE (.45 MICRONS) 
GROUNDWATER QUALITY DATA BY LOCATION

SITE: AMBOI AMBROSIA LAKE

LOCATIOH: 0717

NORTH COORDINATE: 48090.0 FT

EAST COORDINATE: $\quad 53510.0$ FT

06/10/80 TO $12 / 04 / 92$

REPORT DATE: 08/04/94

FORMATION OF COMPLETION: MANCOS SHALE (KM)

HYDRAULIC FLOU RELATIONSHIP: UNKNOWN (H)

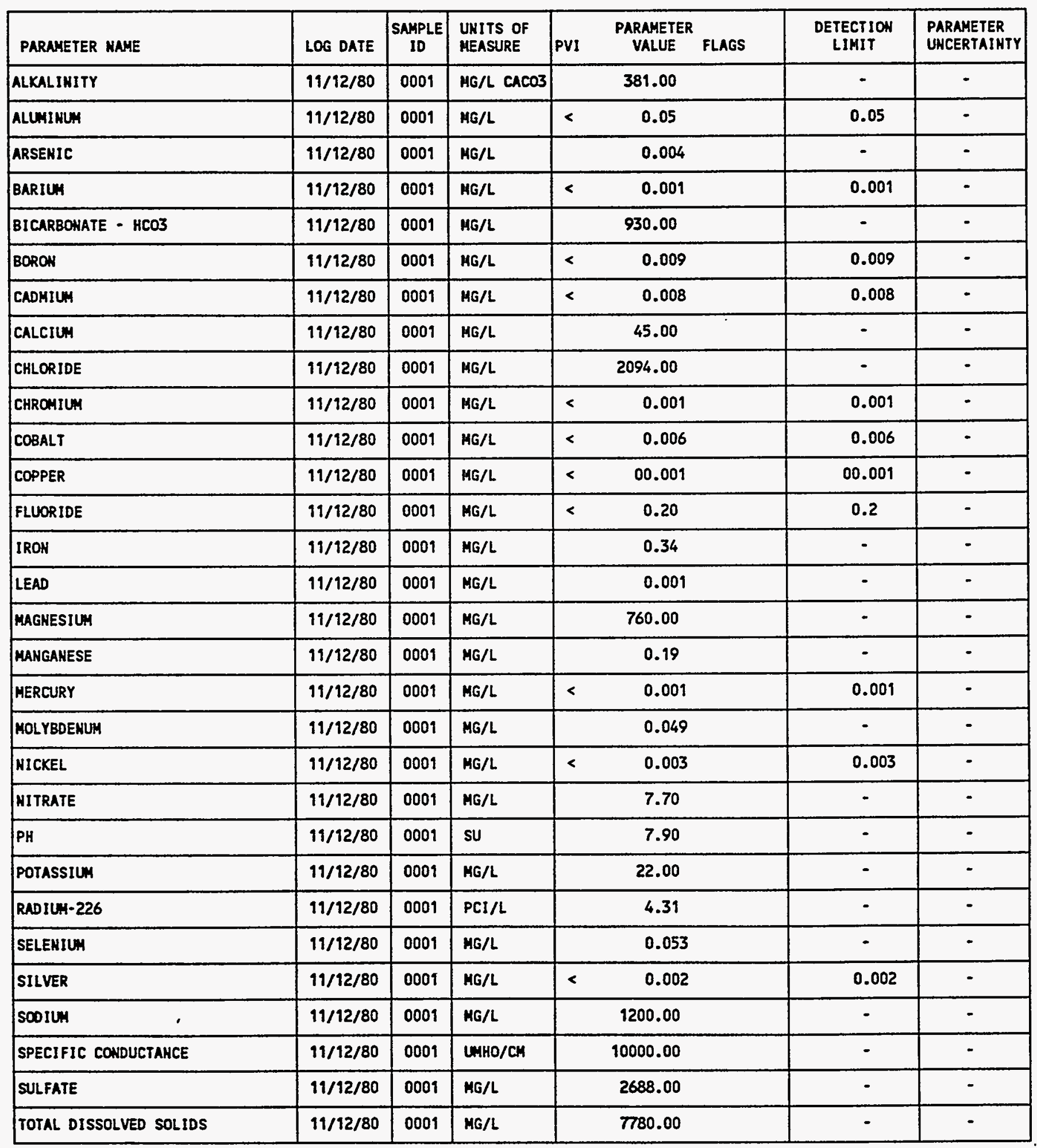

PARAMETER VALUE INDICATOR (PVI):

< - LESS THAN DETECTION LIMIT

SAMPLE ID CODES:

0001 - FILTERED SAMPLE (.45 MICRONS) 
GROUNDHATER QUALITY DATA BY LOCATION

SITE: AMBOI AMBROSIA LAKE

LOCATIOH: 0717

HORTH COORDINATE: 48090.0 FT

EAST COORDIHATE: $\quad 53510.0$ FT

06/10/80 TO 12/04/92

REPORT DATE: 08/04/94

FORMATION OF COMPLETION: MAHCOS SHALE (KH)

HYDRAULIC FLOW RELATIONSHIP: UHKHOWH (N)

\begin{tabular}{|c|c|c|c|c|c|c|c|}
\hline PARAMETER MAME & LOG DATE & $\underset{\text { SAMPLE }}{\text { SA }}$ & $\begin{array}{l}\text { UHITS OF } \\
\text { MEASURE }\end{array}$ & PVI & $\begin{array}{l}\text { PARAMETER } \\
\text { VALUE FLAGS }\end{array}$ & $\begin{array}{l}\text { DETECTION } \\
\text { LIMIT }\end{array}$ & $\begin{array}{l}\text { PARAMETER } \\
\text { UNCERTAINTY }\end{array}$ \\
\hline TOTAL SUSPENDED SOLIDS & $11 / 12 / 80$ & 0001 & MG/L & & 81.00 & - & - \\
\hline URAKIUA & $11 / 12 / 80$ & 0001 & MG/L & & 0.06 & - & - \\
\hline VAKADIUH & $11 / 12 / 80$ & 0001 & MG/L & $<$ & 0.003 & 0.003 & - \\
\hline ZINC & $11 / 12 / 80$ & 0001 & MG/L & & 0.23 & - & - \\
\hline
\end{tabular}

PARAMETER VALUE IMDICATOR (PVI): < - LESS THAN DETECTION LIMIT

SAMPLE ID COOES:

0001 - FILTERED SAMPLE (.45 MICRONS) 
GROUNDHATER QUALITY DATA BY LOCATIOH

SITE: AMBOI AMBROSIA LAKE

LOCATION: 0718

MORTH COORDINATE: $\quad 51470.0$ FT

EAST COORDINATE: $\quad 56950.0$ FT

06/10/80 TO $12 / 04 / 92$

REPORT DATE: 08/04/94

FORMATION OF COHPLETIOH: ALLUVIUM (AL)

HYDRAULIC FLOW RELATIOHSHIP: UNKNONN (N)

\begin{tabular}{|c|c|c|c|c|c|c|c|}
\hline PARAMETER MAME & LOG DATE & $\begin{array}{c}\text { SAMPLE } \\
\text { ID }\end{array}$ & $\begin{array}{l}\text { UNITS OF } \\
\text { MEASURE }\end{array}$ & PVI & $\begin{array}{l}\text { PARAMETER } \\
\text { VALUE FLAGS }\end{array}$ & $\begin{array}{l}\text { DETECTION } \\
\text { LIMIT }\end{array}$ & $\begin{array}{l}\text { PARAMETER } \\
\text { UNCERTAINTY }\end{array}$ \\
\hline ALKALINITY & $\begin{array}{l}06 / 25 / 80 \\
10 / 16 / 85 \\
05 / 17 / 86\end{array}$ & $\begin{array}{l}0001 \\
0001 \\
0001\end{array}$ & $\mathrm{HG} / \mathrm{L} \quad \mathrm{CACO} 3$ & & $\begin{array}{l}149.00 \\
476 . \\
817 .\end{array}$ & - & : \\
\hline ALUHINUM & $06 / 25 / 80$ & 0001 & MG/L & & 0.89 & - & - \\
\hline ARSENIC & $\begin{array}{l}06 / 25 / 80 \\
10 / 16 / 85 \\
05 / 17 / 86\end{array}$ & $\begin{array}{l}0001 \\
0001 \\
0001\end{array}$ & $M G / L$ & $<$ & $\begin{array}{l}0.18 \\
0.01 \\
0.01\end{array}$ & $\begin{array}{l}- \\
0.01 \\
0.01\end{array}$ & - \\
\hline BARIUN & $06 / 25 / 80$ & 0001 & MG/L & $<$ & 0.001 & 0.001 & - \\
\hline BICARBONATE - HCO3 & $06 / 25 / 80$ & 0001 & MG/L & & 363.00 & - & - \\
\hline BORON & $\begin{array}{l}06 / 25 / 80 \\
05 / 17 / 86\end{array}$ & $\begin{array}{l}0001 \\
0001\end{array}$ & MG/L & & $\begin{array}{l}0.91 \\
0.7\end{array}$ & 0.1 & - \\
\hline CADMIUH & $\begin{array}{l}06 / 25 / 80 \\
05 / 17 / 86\end{array}$ & $\begin{array}{l}0001 \\
0001\end{array}$ & MG/L & $<$ & $\begin{array}{l}0.008 \\
0.001\end{array}$ & $\begin{array}{l}0.008 \\
0.001\end{array}$ & - \\
\hline CALCIUH & $\begin{array}{l}06 / 25 / 80 \\
10 / 16 / 85 \\
05 / 17 / 86\end{array}$ & $\begin{array}{l}0001 \\
0001 \\
0001\end{array}$ & $M G / L$ & & $\begin{array}{l}460.00 \\
446 . \\
363 .\end{array}$ & $\begin{array}{l}- \\
0.01 \\
0.01\end{array}$ & $\div$ \\
\hline CHLORIDE & $\begin{array}{l}06 / 25 / 80 \\
10 / 16 / 85 \\
05 / 17 / 86\end{array}$ & $\begin{array}{l}0001 \\
0001 \\
0001\end{array}$ & MG/L & & $\begin{array}{l}184.00 \\
190 . \\
180 .\end{array}$ & 1. & - \\
\hline CHROAIUM & $\begin{array}{l}06 / 25 / 80 \\
05 / 17 / 86\end{array}$ & $\begin{array}{l}0001 \\
0001\end{array}$ & MG/L & $<$ & $\begin{array}{l}0.001 \\
0.04\end{array}$ & $\begin{array}{l}0.001 \\
0.01\end{array}$ & - \\
\hline COBALT & $\begin{array}{l}06 / 25 / 80 \\
05 / 17 / 86\end{array}$ & $\begin{array}{l}0001 \\
0001\end{array}$ & $M G / L$ & $<$ & $\begin{array}{l}0.006 \\
0.09\end{array}$ & $\begin{array}{l}0.006 \\
0.05\end{array}$ & - \\
\hline COPPER & $06 / 25 / 80$ & 0001 & $M G / L$ & & 0.015 & - & - \\
\hline FLUORIDE & $\begin{array}{l}06 / 25 / 80 \\
05 / 17 / 86\end{array}$ & $\begin{array}{l}0001 \\
0001\end{array}$ & $M G / L$ & & $\begin{array}{l}0.25 \\
0.4\end{array}$ & 0.1 & - \\
\hline IRON & $\begin{array}{l}06 / 25 / 80 \\
05 / 17 / 86\end{array}$ & $\begin{array}{l}0001 \\
0001\end{array}$ & MG/L & & $\begin{array}{l}0.022 \\
0.61\end{array}$ & 0.03 & - \\
\hline LEAD & $06 / 25 / 80$ & 0001 & HG/L & & 0.01 & - & - \\
\hline HAGNESIUA & $\begin{array}{l}06 / 25 / 80 \\
10 / 16 / 85 \\
05 / 17 / 86\end{array}$ & $\begin{array}{l}0001 \\
0001 \\
0001\end{array}$ & MG/L & & $\begin{array}{l}240.00 \\
395 . \\
246 .\end{array}$ & $\begin{array}{l}0.009 \\
0.001\end{array}$ & : \\
\hline MANGAKESE & $\begin{array}{l}06 / 25 / 80 \\
05 / 17 / 86\end{array}$ & $\begin{array}{l}0001 \\
0001\end{array}$ & MG/L & & $\begin{array}{l}0.012 \\
0.12\end{array}$ & 0.01 & - \\
\hline MERCURY & $06 / 25 / 80$ & 0001 & $M G / L$ & $<$ & 0.001 & 0.001 & - \\
\hline MOLYEDENUM & $\begin{array}{l}06 / 25 / 80 \\
10 / 16 / 85 \\
05 / 17 / 86\end{array}$ & $\begin{array}{l}0001 \\
0001 \\
0001\end{array}$ & MG/L & & $\begin{array}{l}0.046 \\
0.17 \\
0.22\end{array}$ & $\begin{array}{c}- \\
0.01 \\
0.01\end{array}$ & - \\
\hline NICKEL & $06 / 25 / 80$ & 0001 & MG/L & & 0.023 & - & - \\
\hline
\end{tabular}

PARAMETER VALUE INDICATOR (PVI): < - LESS THAN DETECTION LIMIT 
GROUHDHATER OUALITY DATA BY LOCATION

SITE: AMBOI AMBROSIA LAKE

LOCATION: 0718

NORTH COORDINATE: 51470.0 FT

EAST COORDIHATE: $\quad 56950.0$ FT

06/10/80 TO $12 / 04 / 92$

REPORT DATE: 08/04/94

FORMATIOH OF COHPLETION: ALLUVIUH (AL)

HYDRAULIC FLON RELATIONSHIP: UHKHOWN (N)

\begin{tabular}{|c|c|c|c|c|c|c|c|}
\hline $\begin{array}{c}\zeta \\
\text { PARAMETER NAME }\end{array}$ & LOG DATE & $\underset{10}{\text { SAHPLE }}$ & $\begin{array}{l}\text { UNITS OF } \\
\text { MEASURE }\end{array}$ & PVI & $\begin{array}{l}\text { PARAMETER } \\
\text { VALUE FLAGS }\end{array}$ & $\begin{array}{l}\text { DETECTION } \\
\text { LIMIT }\end{array}$ & $\begin{array}{l}\text { PARAMETER } \\
\text { UNCERTAINTY }\end{array}$ \\
\hline NITRATE & $\begin{array}{l}06 / 25 / 80 \\
05 / 17 / 86\end{array}$ & $\begin{array}{l}0001 \\
0001\end{array}$ & $M G / L$ & & $\begin{array}{l}10.00 \\
45 .\end{array}$ & 1. & - \\
\hline PH & $\begin{array}{l}06 / 25 / 80 \\
10 / 16 / 85 \\
05 / 17 / 86\end{array}$ & $\begin{array}{l}0001 \\
0001 \\
0001\end{array}$ & SU & & $\begin{array}{l}7.70 \\
7.15 \\
7.8\end{array}$ & - & - \\
\hline POTASSIUM & $\begin{array}{l}06 / 25 / 80 \\
10 / 16 / 85 \\
05 / 17 / 86\end{array}$ & $\begin{array}{l}0001 \\
0001 \\
0001\end{array}$ & HG/L & & $\begin{array}{l}8.40 \\
7.61 \\
14 .\end{array}$ & $\begin{array}{l}- \\
0.01 \\
0.01\end{array}$ & - \\
\hline RADIUH-226 & $06 / 25 / 80$ & 0001 & $\mathrm{PCI} / \mathrm{L}$ & & 2.65 & - & - \\
\hline SELEHIUH & $\begin{array}{l}06 / 25 / 80 \\
10 / 16 / 85 \\
05 / 17 / 86\end{array}$ & $\begin{array}{l}0001 \\
0001 \\
0001\end{array}$ & $M G / L^{-}$ & $<$ & $\begin{array}{l}0.95 \\
0.005 \\
0.008\end{array}$ & $\begin{array}{l}0.005 \\
0.005\end{array}$ & : \\
\hline SILVER & $06 / 25 / 80$ & 0001 & $H G / L$ & & 0.018 & - & - \\
\hline SooIun & $\begin{array}{l}06 / 25 / 80 \\
10 / 16 / 85 \\
05 / 17 / 86\end{array}$ & $\begin{array}{l}0001 \\
0001 \\
0001\end{array}$ & $M G / L$ & & $\begin{array}{l}1200.00 \\
1650 . \\
1830 .\end{array}$ & $\begin{array}{l}- \\
0.002 \\
0.002\end{array}$ & - \\
\hline SPECIFIC COHDUCTANCE & $\begin{array}{l}06 / 25 / 80 \\
10 / 16 / 85 \\
05 / 17 / 86\end{array}$ & $\begin{array}{l}0001 \\
0001 \\
0001\end{array}$ & UHHO/CH & & $\begin{array}{l}7140.00 \\
7000 . \\
5000 .\end{array}$ & - & - \\
\hline STROATIUA & $05 / 17 / 86$ & 0001 & $M G / L$ & & 0.7 & 0.1 & - \\
\hline SULFATE & $\begin{array}{l}06 / 25 / 80 \\
10 / 16 / 85 \\
05 / 17 / 86\end{array}$ & $\begin{array}{l}0001 \\
0001 \\
0001\end{array}$ & MG/L & & $\begin{array}{l}4290.00 \\
4940 . \\
4620 .\end{array}$ & $\begin{array}{l}- \\
0.1 \\
0.1\end{array}$ & : \\
\hline TEMPERATURE & $\begin{array}{l}10 / 16 / 85 \\
05 / 17 / 86\end{array}$ & $\begin{array}{l}0001 \\
0001\end{array}$ & C - DEGREE & - & $\begin{array}{l}12 . \\
13 .\end{array}$ & - & - \\
\hline TOTAL OISSOLVEO SOLIDS & $\begin{array}{l}06 / 25 / 80 \\
10 / 16 / 85 \\
05 / 17 / 86\end{array}$ & $\begin{array}{l}0001 \\
0001 \\
0001\end{array}$ & MG/L & & $\begin{array}{l}6920.00 \\
8080 . \\
7440 .\end{array}$ & $\begin{array}{l}10 \\
10 .\end{array}$ & - \\
\hline TOTAL SUSPEHDED SOLIDS & $06 / 25 / 80$ & 0001 & $M G / L$ & & 81.00 & - & - \\
\hline URAKIUA & $\begin{array}{l}06 / 25 / 80 \\
10 / 16 / 85 \\
05 / 17 / 86\end{array}$ & $\begin{array}{l}0001 \\
0009 \\
0001\end{array}$ & MG/L & & $\begin{array}{l}0.42 \\
0.712 \\
1.26\end{array}$ & $\begin{array}{l}0.003 \\
0.003\end{array}$ & 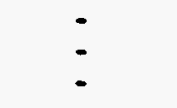 \\
\hline VAKADIUM & $\begin{array}{l}06 / 25 / 80 \\
05 / 17 / 86\end{array}$ & $\begin{array}{l}0001 \\
0001\end{array}$ & $\mathrm{HG} / \mathrm{L}$ & $<$ & $\begin{array}{l}0.003 \\
0.23\end{array}$ & $\begin{array}{l}0.003 \\
0.01\end{array}$ & - \\
\hline ZINC & $06 / 25 / 80$ & 0001 & MG/L & & 0.069 & - & - \\
\hline
\end{tabular}

PARAMETER VALUE IHDICATOR (PVI): < - LESS THAN DETECTION LIHIT

SAMPLE ID COOES:

0001 - FILTERED SAMPLE (.45 MICROHS) 
GROUNDHATER QUALITY DATA BY LOCATION

SITE: AMBOI AMBROSIA LAKE

LOCATION: 0719

HORTH COORDINATE: $\quad 47800.0$ FT

EAST COORDINATE: $\quad 54605.0$ FT

06/10/80 TO $12 / 04 / 92$

REPORT DATE: 08/04/94

FORMATION OF COMPLETION: ALLUVIUM (AL)

HYDRAULIC FLON RELATIONSHIP: UNKNOWH (N)

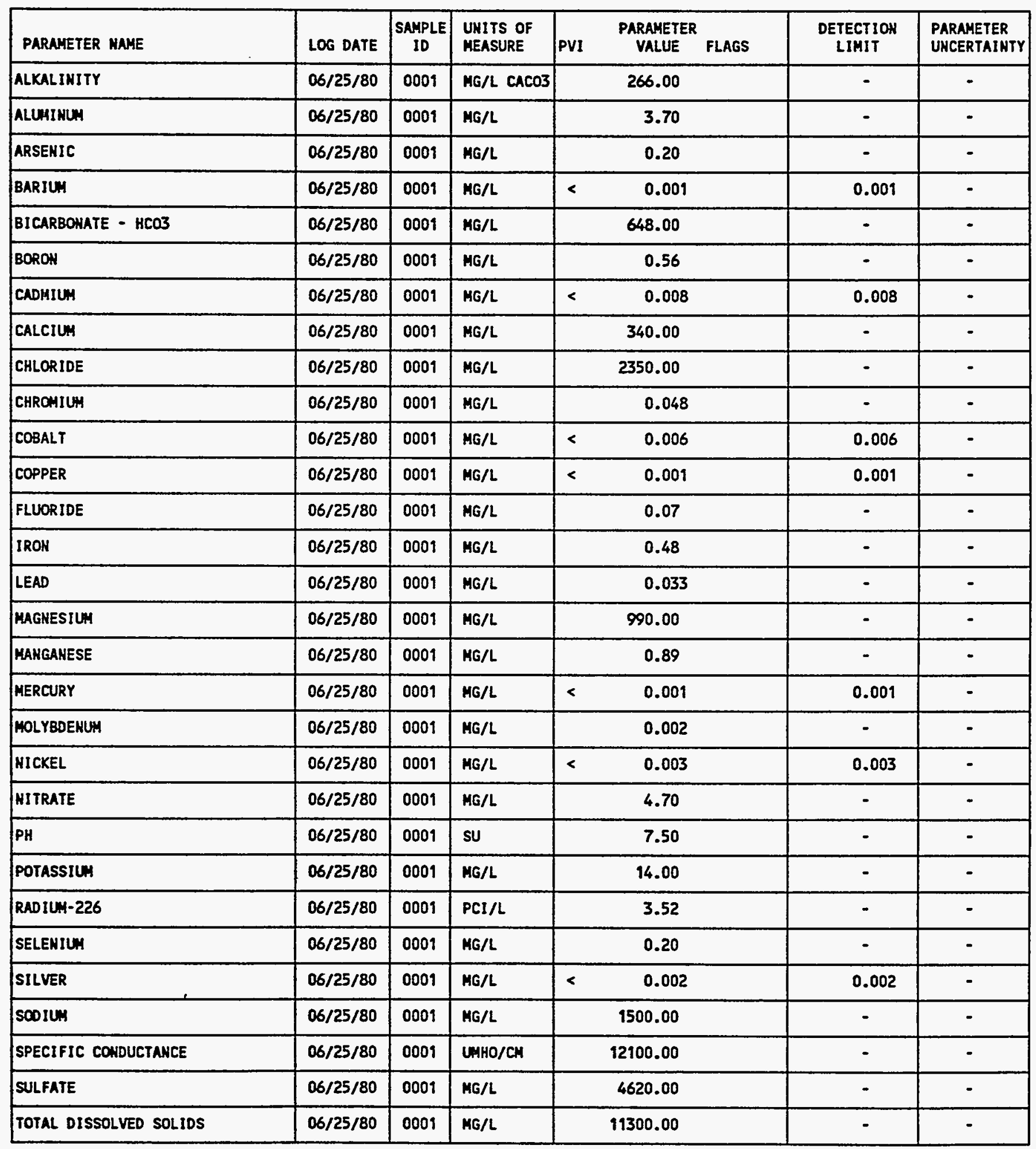

PARAMETER VALUE INDICATOR (PVI):

< - LESS THAN DETECTION LIMIT

SAMPLE ID COOES:

0001 - FILTERED SAMPLE (.45 MICRONS) 
GROUNDWATER QUALITY DATA BY LOCATIOH

SITE: AMBO1 AMBROSIA LAKE

LOCATIOH: 0719

NORTH COORDINATE: $\quad 47800.0$ FT

EAST COORDIHATE: 54605.0 FT

06/10/80 TO 12/04/92

REPORT DATE: 08/04/94

FORMATION OF COMPLETION: ALLUVIUM (AL)

HYDRAULIC FLOW RELATIONSHIP: UHKNOWH (H)

\begin{tabular}{|c|c|c|c|c|c|c|c|}
\hline PARAMETER NAME & LOG DATE & $\underset{\text { ID }}{\text { SAMPLE }}$ & $\begin{array}{l}\text { UHITS OF } \\
\text { MEASURE }\end{array}$ & PVI & $\begin{array}{l}\text { PARAMETER } \\
\text { VALUE FLAGS }\end{array}$ & $\begin{array}{l}\text { DETECTION } \\
\text { LIMIT }\end{array}$ & $\begin{array}{l}\text { PARAMETER } \\
\text { UHCERTAINTY }\end{array}$ \\
\hline TOTAL SUSPEHDED SOLIDS & $06 / 25 / 80$ & 0001 & MG/L & & 478.00 & - & - \\
\hline URAKIUA & $06 / 25 / 80$ & 0001 & MG/L & & 0.025 & - & - \\
\hline VANADIUM & $0.6 / 25 / 80$ & 0001 & MG/L & $<$ & 0.003 & 0.003 & - \\
\hline ZINC & $06 / 25 / 80$ & 0001 & MG/L & & 0.27 & - & - \\
\hline
\end{tabular}

PARAMETER VALUE IMDICATOR (PVI):

< - LESS THAN DETECTION LIMIT

SNMPLE ID COOES:

0001 - FII.TERED SAMPLE (.45 MICRONS) 
GROUNDWATER OUALITY DATA BY LOCATION

SITE: AMBOI AMBROSIA LAKE

LOCATION: OT2O

MORTH COORDINATE: $\quad 49600.0$ FT

EAST COORDIHATE: $\quad 54110.0$ FT

06/10/80 TO $12 / 04 / 92$

REPORT DATE: 08/04/94

FORMATION OF COHPLETION: ALLUVIUN (AL)

HYDRAULIC FLOW RELATIOHSHIP: UNKNOWH (N)

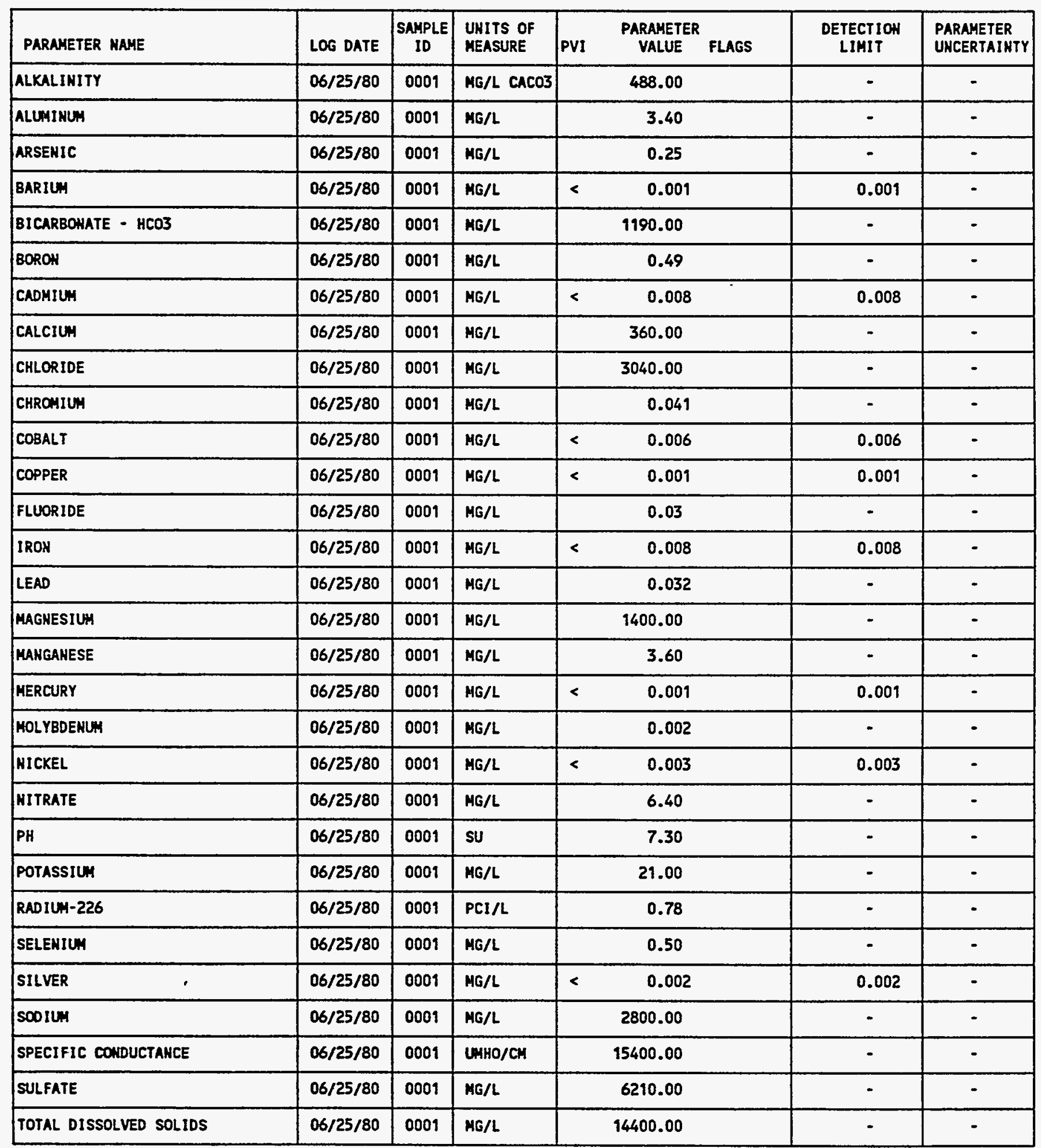

PARANETER VALUE IHDICATOR (PVI):

< - LESS THAN DETECTION LIMIT

SAMPLE ID COOES:

0001 - FILTERED SAMPLE (.45 MICRONS) 
GROUNDWATER QUALITY DATA BY LOCATION

SITE: AMBOI AMBROSIA LAKE

LOCATION: 0720

MORTH COORDIHATE: 49600.0 FT

EAST COORDINATE: $\quad 54110.0$ FT

06/10/80 TO $12 / 04 / 92$

REPORT DATE: 08/04/94

FORMATIOH OF COMPLETIOH: ALLUVIUM (AL)

HYDRAULIC FLOW RELATIONSHIP: UNKNOWN (H)

\begin{tabular}{|c|c|c|c|c|c|c|c|}
\hline PARAMETER HAME & LOG DATE & $\begin{array}{c}\text { SAMPLE } \\
\text { ID }\end{array}$ & $\begin{array}{l}\text { UHITS OF } \\
\text { MEASURE }\end{array}$ & PVI & $\begin{array}{l}\text { PARAHETER } \\
\text { VALUE FLAGS }\end{array}$ & $\begin{array}{l}\text { DETECTIOH } \\
\text { LIMIT }\end{array}$ & $\begin{array}{l}\text { PARAMETER } \\
\text { UNCERTAINTY }\end{array}$ \\
\hline TOTAL SUSPENDED SOLIDS & $06 / 25 / 80$ & 0001 & $M G / L$ & & 165.00 & - & $\cdots$ \\
\hline URAKIUNA & $06 / 25 / 80$ & 0001 & MG/L & & 0.18 & - & - \\
\hline VAKADIUA & $06 / 25 / 80$ & 0001 & MG/L & $<$ & 0.003 & 0.003 & $s-$ \\
\hline ZIHC & $06 / 25 / 80$ & 0001 & MG/L & & 0.26 & - & - \\
\hline
\end{tabular}

PARAMETER VALUE IMDICATOR (PVI): < - LESS THAK DETECTION LIHIT SAMPLE ID COOES:

0001 - FII.TERED SAMPLE (.45 MICROHS) 
GROUNDHATER OUALITY DATA BY LOCATION

SITE: AMBO1 AMBROSIA LAKE

LOCATION: 0721

MORTH COORDINATE: 51090.0 FT

EAST COORDINATE: $\quad 48190.0$ FT

06/10/80 TO 12/04/92

REPORT DATE: 08/04/94

FORMATION OF COHPLETION: TRES HERMANOS-A SANDSTONE (TR)

HYDRAULIC FLON RELATIOHSHIP: UNKHOWN (N)

\begin{tabular}{|c|c|c|c|c|c|c|}
\hline PARAMETER MAME & LOG DATE & $\begin{array}{c}\text { SAMPLE } \\
\text { ID }\end{array}$ & $\begin{array}{l}\text { UNITS OF } \\
\text { MEASURE }\end{array}$ & $\begin{array}{lc} & \text { PARAMETER } \\
\text { PVI } & \text { VALUE }\end{array}$ & $\begin{array}{l}\text { DETECTIOH } \\
\text { LIMIT }\end{array}$ & $\begin{array}{l}\text { PARAMETER } \\
\text { UNCERTAIHTY }\end{array}$ \\
\hline ALKALINITY & $10 / 02 / 80$ & 0001 & MG/L CACO3 & 26.60 & - & $\cdot$ \\
\hline ALCAIIIUK & $10 / 02 / 80$ & 0009 & $M G / L$ & 0.093 & - & - \\
\hline ARSENIC & $10 / 02 / 80$ & 0001 & MG/L & 0.001 & - & - \\
\hline BARIUH & $10 / 02 / 80$ & 0001 & MG/L & 0.053 & - & - \\
\hline BICARBONATE - HCO3 & $10 / 02 / 80$ & 0001 & MG/L & 65.00 & - & - \\
\hline BORON & $10 / 02 / 80$ & 0001 & MG/L & 0.33 & - & - \\
\hline CADHIUM & $10 / 02 / 80$ & 0001 & HG/L & 0.008 & 0.008 & - \\
\hline CALCILA & $10 / 02 / 80$ & 0001 & HG/L & 480.00 & - & - \\
\hline CHLORIDE & $10 / 02 / 80$ & 0001 & MG/L & 1264.00 & - & - \\
\hline CHROMIUM & $10 / 02 / 80$ & 0001 & MG/L & 0.039 & - & - \\
\hline COBALT & $10 / 02 / 80$ & 0001 & MG/L & 0.006 & 0.006 & - \\
\hline COPPER & $10 / 02 / 80$ & 0001 & HG/L & 0.002 & - & 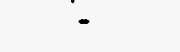 \\
\hline FLUORIDE & $10 / 02 / 80$ & 0001 & KG/L & 0.07 & - & - \\
\hline IRON & $10 / 02 / 80$ & 0001 & $M G / L$ & 0.01 & $\cdot$ & - \\
\hline LEAD & $10 / 02 / 80$ & 0001 & MG/L & 0.001 & 0.001 & - \\
\hline MAGNESIUM & $10 / 02 / 80$ & 0001 & $M G / L$ & 840.00 & - & - \\
\hline MANGAMESE & $10 / 02 / 80$ & 0001 & $M G / L$ & 3.80 & - & - \\
\hline HERCURY & $10 / 02 / 80$ & 0001 & MG/L & 0.001 & 0.001 & - \\
\hline HOL YBDEMAR & $10 / 02 / 80$ & 0001 & $M G / L$ & 0.002 & - & - \\
\hline HICKEL & $10 / 02 / 80$ & 0001 & $M G / L$ & 0.001 & 0.001 & - \\
\hline NITRATE & $10 / 02 / 80$ & 0001 & MG/L & 7.40 & - & - \\
\hline $\mathrm{PH}$ & $10 / 02 / 80$ & 0001 & SU & 6.60 & - & - \\
\hline POTASSIUH & $10 / 02 / 80$ & 0001 & $M G / L$ & 17.00 & - & - \\
\hline RADIUA-226 & $10 / 02 / 80$ & 0001 & PCI/L & 75.66 & - & - \\
\hline SELENIUM & $10 / 02 / 80$ & 0009 & MG/L & 0.22 & - & - \\
\hline SILVER & $10 / 02 / 80$ & 0001 & MG/L & 0.002 & 0.002 & - \\
\hline scolun & $10 / 02 / 80$ & 0001 & MG/L & 430.00 & - & - \\
\hline SPECIFIC CONDUCTAHCE & $10 / 02 / 80$ & 0001 & UHHO/CH & 8000.00 & - & - \\
\hline SULFATE & $10 / 02 / 80$ & 0001 & MG/L & 3200.00 & - & - \\
\hline TOTAL DISSOLVED SOLIDS & $10 / 02 / 80$ & 0001 & $M G / L$ & 6560.00 & - & - \\
\hline
\end{tabular}

PARAMETER VALUE INDICATOR (PVI):

$<$ - LESS THAH DETECTION LIMIT

SAMPLE ID COOES:

0001 - FILTERED SAMPLE (.45 MICROHS) 
GROUNDWATER QUALITY DATA BY LOCATIOH

SITE: AMBO1 AMBROSIA LAKE

LOCATIOH: 0721

NORTH COORDINATE: 51090.0 FT

EAST COORDINATE: $\quad 48190.0$ FT

06/10/80 TO $12 / 04 / 92$

REPORT OATE: 08/04/94

FORMATION OF COMPLETION: TRES HERMANOS-A SAMDSTONE (TR)

HYDRAULIC FLON RELATIONSHIP: UHKHONH (N)

\begin{tabular}{|c|c|c|c|c|c|c|c|}
\hline PARAMETER KAME & LOS DATE & $\underset{\text { ID }}{\text { SAMPLE }}$ & $\begin{array}{l}\text { UHITS OF } \\
\text { MEASURE }\end{array}$ & PVI & $\begin{array}{l}\text { PARAMETER: } \\
\text { VALUE FLAGS }\end{array}$ & $\begin{array}{l}\text { DETECTION } \\
\text { LIMIT }\end{array}$ & $\begin{array}{l}\text { PARĀHETER } \\
\text { UHCERTAINTY }\end{array}$ \\
\hline TOTAL SUSPEHDED SOLIDS & $10 / 02 / 80$ & 0001 & MG/L & & 46.00 & - & - \\
\hline URAKIUY & $10 / 02 / 80$ & 0001 & MG/L & & 0.02 & - & - \\
\hline VAKADIUM & $10 / 02 / 80$ & 0001 & MG/L & & 0.09 & - & - \\
\hline 2INC & $10 / 02 / 80$ & 0001 & MG/L & & 0.17 & - & - \\
\hline
\end{tabular}

PARAMETER VALUE IHDICATOR (PVI): < - LeSS THAN DETECTION LIMIT

SAMPLE IO COOES

0001 - FILTERED SAMPLE (.45 MICRONS) 
GROUNDHATER QUALITY DATA BY LOCATION

SITE: AMBOI AMBROSIA LAKE

LOCATION: 0722

NORTH COORDINATE: $\quad 47820.0$ FT

EAST COORDIHATE: $\quad 59070.0$ FT

06/10/80 TO $12 / 04 / 92$

REPORT DATE: 08/04/94

FORHATION OF COMPLETION: TRES HERMANOS-A SANDSTONE (TR)

HYDRAULIC FLON RELATIONSHIP: UPGRADIENT (U)

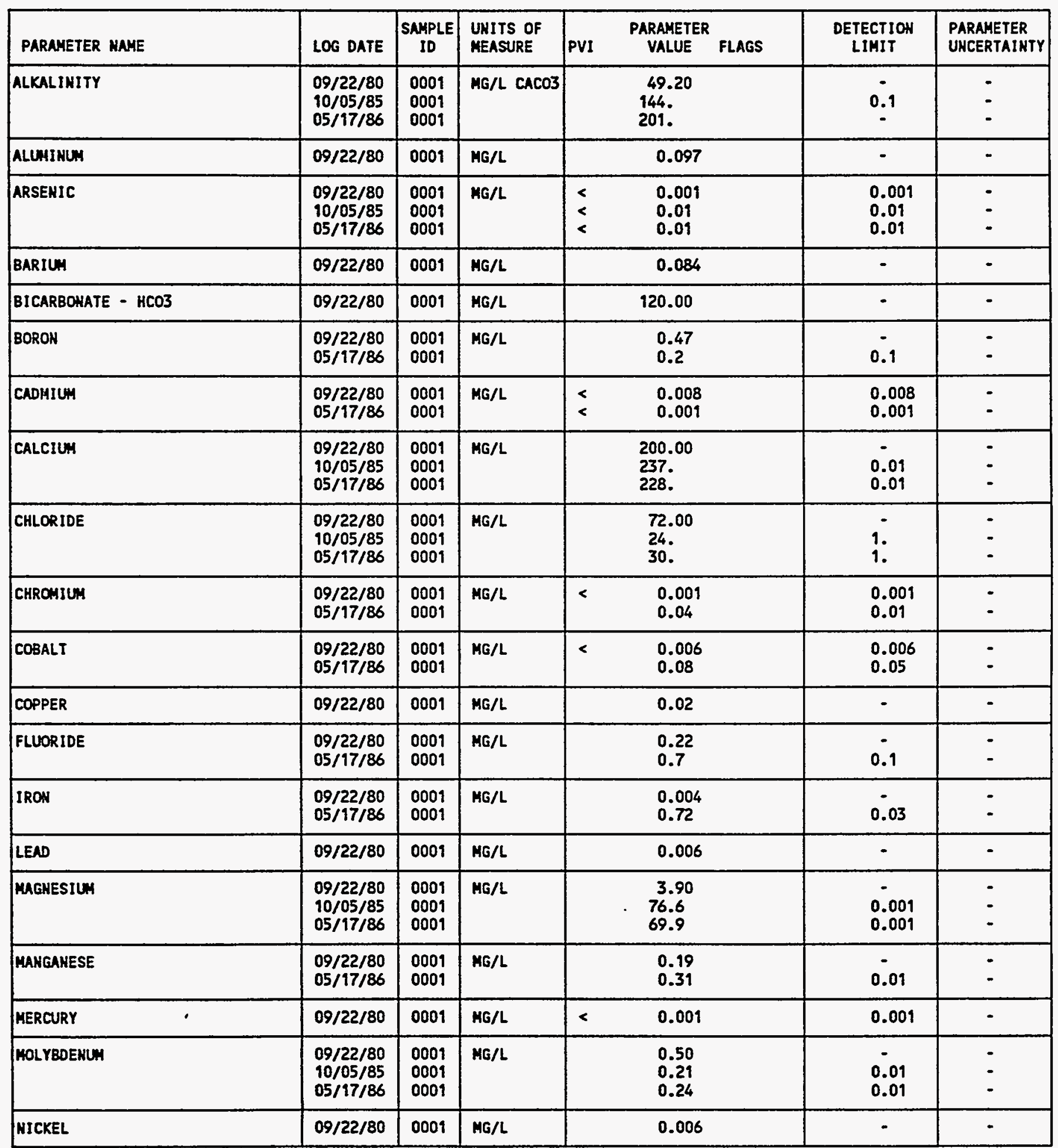

PARAMeter VAlUe IhDicator (PVI): < - LeSS than DETECTION LIMIT

SAMPLE ID COOES:

0001 - FILTERED SAMPLE (.45 MICRONS) 
GROUHDHATER OUALITY DATA BY LOCATIOH

SITE: AMBO1 AMBROSIA LAKE

LOCATION: 0722

HORTH COORDIHATE: $\quad 47820.0$ FT

EAST COORDIHATE: $\quad 59070.0$ FT

06/10/80 TO 12/04/92

REPORT DATE: 08/04/94

FORMATION OF COHPLETION: TRES HERMANOS-A SAHDSTOHE (TR)

HYDRAULIC FLON RELATIOHSHIP: UPGRADIENT (U)

\begin{tabular}{|c|c|c|c|c|c|c|c|}
\hline$\left.\right|_{\text {PARAYETER MAME }}$ & LOG DATE & $\underset{\text { ID }}{\text { SAMPLE }}$ & $\begin{array}{l}\text { UNITS OF } \\
\text { MEASURE }\end{array}$ & PVI & $\begin{array}{l}\text { PARAMETER } \\
\text { VALUE FLAGS }\end{array}$ & $\begin{array}{l}\text { DETECTION } \\
\text { LIMIT }\end{array}$ & $\begin{array}{l}\text { PARAMETER } \\
\text { UNCERTAINTY }\end{array}$ \\
\hline NITRATE & $\begin{array}{l}09 / 22 / 80 \\
05 / 17 / 86\end{array}$ & $\begin{array}{l}0001 \\
0001\end{array}$ & MG/L & & $\begin{array}{l}3.90 \\
73 .\end{array}$ & 1. & - \\
\hline PH & $\begin{array}{l}09 / 22 / 80 \\
10 / 05 / 85 \\
05 / 17 / 86\end{array}$ & $\begin{array}{l}0001 \\
0001 \\
0001\end{array}$ & su & & $\begin{array}{l}7.50 \\
7.87 \\
8.94\end{array}$ & - & $\begin{array}{r}\dot{-} \\
-\end{array}$ \\
\hline POTASSIUH & $\begin{array}{l}09 / 22 / 80 \\
10 / 05 / 85 \\
05 / 17 / 86\end{array}$ & $\begin{array}{l}0001 \\
0001 \\
0001\end{array}$ & MG/L & & $\begin{array}{l}10.00 \\
10 . \\
8.19\end{array}$ & $\begin{array}{l}- \\
0.01 \\
0.01\end{array}$ & : \\
\hline RADIUN-226 & $09 / 22 / 80$ & 0001 & PCI/L & & 7.61 & - & - \\
\hline SELEHIUM & $\begin{array}{l}09 / 22 / 80 \\
10 / 05 / 85 \\
05 / 17 / 86\end{array}$ & $\begin{array}{l}0001 \\
0001 \\
0001\end{array}$ & MG/L & $<$ & $\begin{array}{l}0.18 \\
0.005 \\
0.005\end{array}$ & $\begin{array}{l}0.005 \\
0.005\end{array}$ & 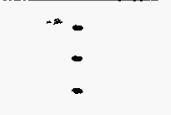 \\
\hline SILVER & $09 / 22 / 80$ & 0001 & MG/L & $<$ & 0.002 & 0.002 & - \\
\hline sooIun & $\begin{array}{l}09 / 22 / 80 \\
10 / 05 / 85 \\
05 / 17 / 86\end{array}$ & $\begin{array}{l}0001 \\
0001 \\
0001\end{array}$ & MG/L. & & $\begin{array}{l}240.00 \\
397 . \\
564 .\end{array}$ & $\begin{array}{l}- \\
0.002 \\
0.002\end{array}$ & $:$ \\
\hline SPECIFIC CONDUCTANCE & $\begin{array}{l}09 / 22 / 80 \\
10 / 05 / 85 \\
05 / 17 / 86\end{array}$ & $\begin{array}{l}0001 \\
0001 \\
0001\end{array}$ & UMHO/CM & & $\begin{array}{l}1840.00 \\
2300 . \\
2000\end{array}$ & - & $\begin{array}{l}- \\
=\end{array}$ \\
\hline STRONTIUH & $05 / 17 / 86$ & 0001 & $M G / L$ & & 3.3 & 0.1 & - \\
\hline SULFATE & $\begin{array}{l}09 / 22 / 80 \\
10 / 05 / 85 \\
05 / 17 / 86\end{array}$ & $\begin{array}{l}0001 \\
0001 \\
0001\end{array}$ & $\begin{array}{c}M G / L \\
.\end{array}$ & & $\begin{array}{l}750.00 \\
1550 . \\
1740 .\end{array}$ & $\begin{array}{l}0.1 \\
0.1\end{array}$ & - \\
\hline TEMPERATURE & $\begin{array}{l}10 / 05 / 85 \\
05 / 17 / 86\end{array}$ & $\begin{array}{l}0001 \\
0001\end{array}$ & C - DEGREE & & $\begin{array}{l}14 . \\
13 .\end{array}$ & $\dot{-}$ & - \\
\hline TOTAL DISSOLVED SOLIDS & $\begin{array}{l}09 / 22 / 80 \\
10 / 05 / 85 \\
05 / 17 / 86\end{array}$ & $\begin{array}{l}0001 \\
0001 \\
0001\end{array}$ & $\mathrm{MG} / \mathrm{L}$ & & $\begin{array}{l}1413.00 \\
2490 . \\
2590 .\end{array}$ & $\begin{array}{l}10 \\
10 .\end{array}$ & : \\
\hline TOTAL SUSPENDED SOLIDS & $09 / 22 / 80$ & 0001 & HG/L & & 117.00 & - & - \\
\hline URANIUN & $\begin{array}{l}09 / 22 / 80 \\
10 / 05 / 85 \\
05 / 17 / 86\end{array}$ & $\begin{array}{l}0001 \\
0001 \\
0001\end{array}$ & MG/L & & $\begin{array}{l}0.52 \\
0.0082 \\
0.0018 \quad \mathrm{~J}\end{array}$ & $\begin{array}{l}- \\
0.003 \\
0.003\end{array}$ & 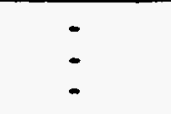 \\
\hline VAHADIUA & $\begin{array}{l}09 / 22 / 80 \\
05 / 17 / 86\end{array}$ & $\begin{array}{l}0001 \\
0001\end{array}$ & MG/L & & $\begin{array}{l}0.016 \\
0.36\end{array}$ & 0.01 & $\because$ \\
\hline ZINC & $09 / 22 / 80$ & 0001 & MG/L & & 0.99 & - & - \\
\hline
\end{tabular}

PARAMETER VALUE IMDICATOR (PVI): < - LESS THAN DETECTION LIMIT

SAMPLE ID COOES:

OOO1 - FILIERED SAMPLE (.45 MICROHS)

OTHER PARAMETER VALUE FLAGS:

J - ESTIMATED VALUE 
GRONHDWATER OUALITY DATA BY LOCATION

SITE: AMBO1 AMBROSIA LAKE

LOCATION: 0723

NORTH COORDIHATE: 48190.0 FT

EAST COORDINATE: $\quad 56540.0$ FT

D6/10/80 TO 12/04/92

REPORT DATE: 08/04/94

FORMATION OF COMPLETION: DAKOTA SANDSTOHE (KD)

HYDRAULIC FLON RELATIONSHIP: UNKNOWN (N)

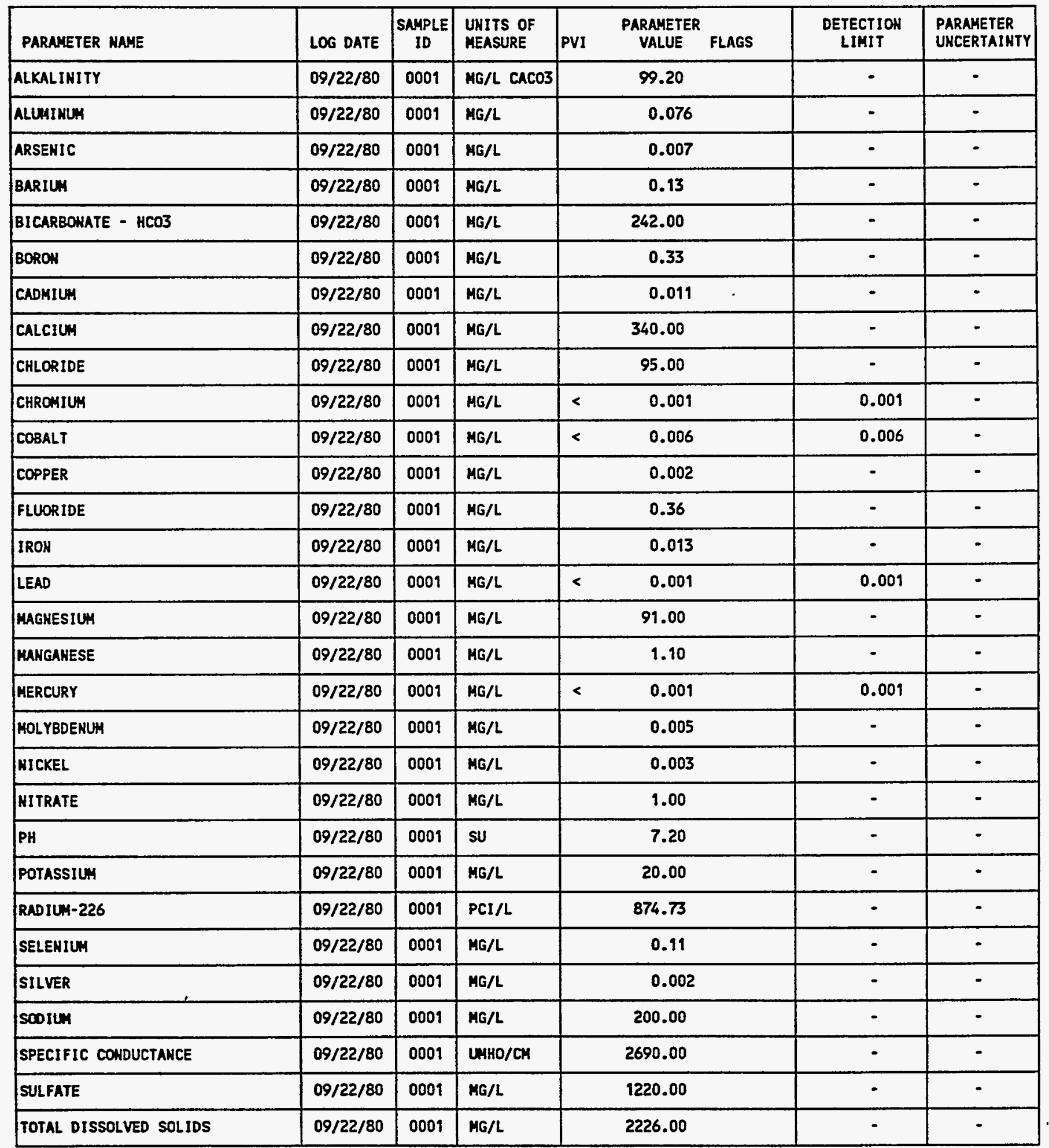

PARAMETER VALUE INDICATOR (PVI): < - LESS THAH DETECTION LIMIT

SAMPLE ID COOES:

0001 - FILTERED SAMPLE (.45 MICROHS) 
GROUNOHATER QUALITY DATA BY LOCATION

SITE: AMBOI AMBROSIA LAKE

LOCATIOH: 0723

MORTH COORDINATE: $\quad 48190.0$ FT

EAST COORDINATE: $\quad 56540.0$ FT

06/10/80 TO $12 / 04 / 92$

REPORT DATE: $08 / 04 / 94$

FORMATION OF COHPLETIOH: DAKOTA SANDSTOHE (KO)

HYDRAULIC FLON RELATIONSHIP: UHKMOWH (H)

\begin{tabular}{|c|c|c|c|c|c|c|c|}
\hline PARAMETER NAME & LOG DATE & $\begin{array}{c}\text { SAMPLE } \\
\text { ID }\end{array}$ & $\begin{array}{l}\text { UNITS OF } \\
\text { MEASURE }\end{array}$ & PVI & $\begin{array}{l}\text { PARAMETER } \\
\text { VALUE FLAGS }\end{array}$ & $\begin{array}{l}\text { DETECTION } \\
\text { LIMIT }\end{array}$ & $\begin{array}{l}\text { PARAMETER } \\
\text { UNCERTAINTY }\end{array}$ \\
\hline TOTAL SUSPEKDED SOLIDS & $09 / 22 / 80$ & 0001 & MG/L & & 2180.00 & - & - \\
\hline URAHILIM & $09 / 22 / 80$ & 0001 & MG/L & & 0.31 & - & - \\
\hline VAKADIUA & $09 / 22 / 80$ & 0001 & MG/L & & 0.015 & - & - \\
\hline ZIHC & $09 / 22 / 80$ & 0001 & MG/L & & 0.86 & - & - \\
\hline
\end{tabular}

PARAMETER VALUE IMDICATOR (PVI):

< - Less than Detection limit

SAMPLE ID COOES:

0001 - FII.TERED SAMPLE (.45 MICROHS) 
GROUNDWATER QUALITY DATA BY LOCATIOH

SITE: AMBOI AMBROSIA LAKE

LOCATIOH: 0724

NORTH COOROINATE: $\quad 49715.0$ FT

EAST COORDIHATE: $\quad 56105.0$ FT

06/10/80 TO 12/04/92

REPORT DATE: 08/04/94

FORMATION OF COMPLETION: DAKOTA SAKDSTONE (KD)

HYDRAULIC FLON RELATIONSHIP: UNKHOWN (N)

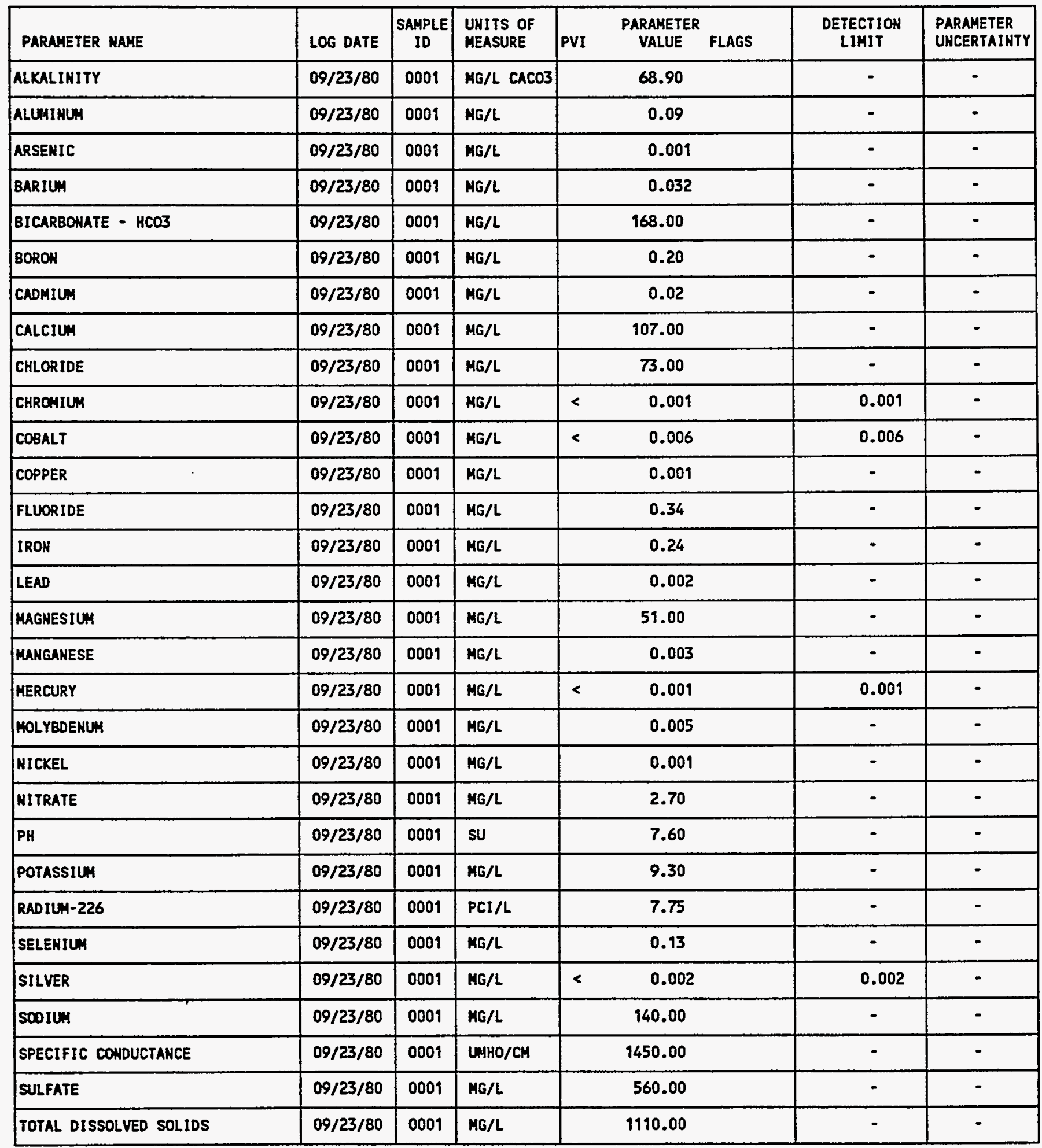

PARAMETER VALUE IMDICATOR (PVI): < - LESS THAN DETECTION LIMIT

SAMPLE ID COOES:

0001 - FILTERED SAMPLE (.45 MICRONS) 
GROUNDWATER QUALITY DATA BY LOCATIOH

SITE: AMBOI AMBROSIA LAKE

LOCATION: 0724

MORTH COORDIHATE: $\quad 49715.0$ FT

EAST COORDIHATE: $\quad 56105.0$ FT

06/10/80 TO $12 / 04 / 92$

REPORT DATE: 08/04/94

FORMATIOH OF COMPLETIOH: DAKOTA SAMDSTONE (KD)

HYDRAULIC FLON RELATIONSHIP: UNKNOWH (N)

\begin{tabular}{|c|c|c|c|c|c|c|c|}
\hline PARAMETER HAME & LOG DATE & $\underset{10}{\text { SAMPLE }}$ & $\begin{array}{l}\text { UNITS OF } \\
\text { MEASURE }\end{array}$ & PVI & $\begin{array}{l}\text { PARAMETER } \\
\text { VALUE FLAGS }\end{array}$ & $\begin{array}{l}\text { DETECTION } \\
\text { LIMIT }\end{array}$ & $\begin{array}{l}\text { PARAHETER } \\
\text { UNCERTAIHTY }\end{array}$ \\
\hline TOTAL SUSPENDED SOLIOS & $09 / 23 / 80$ & 0001 & $M G / L$ & & 56.00 & - & - \\
\hline URAKIUH & $09 / 23 / 80$ & 0001 & MG/L & & 0.08 & - & - \\
\hline VAKADIUM & $09 / 23 / 80$ & 0009 & MG/L & & 0.007 & - & - \\
\hline 2INC & $09 / 23 / 80$ & 0001 & MG/L & & 0.091 & - & - \\
\hline
\end{tabular}

PARAMETER VALUE INDICATOR (PVI): < - LESS THAN DETECTION LIMIT SAMPLE IO COOES:

OOO1 - FILTERED SAMPLE (.45 MICRONS) 
GROUNDHATER QUALITY DATA BY LOCATION

SITE: AMBO1 AMBROSIA LAKE

LOCATION: OT25

MORTH COORDINATE: $\quad 51880.0$ FT

EAST COORDINATE: $\quad 54950.0$ FT

06/10/80 TO 12/04/92

REPORT DATE: 08/04/94

FORMATION OF COMPLETION: DAKOTA SAMDSTONE (KD)

HYDRAULIC FLOW RELATIONSHIP: UNKNOWN (H)

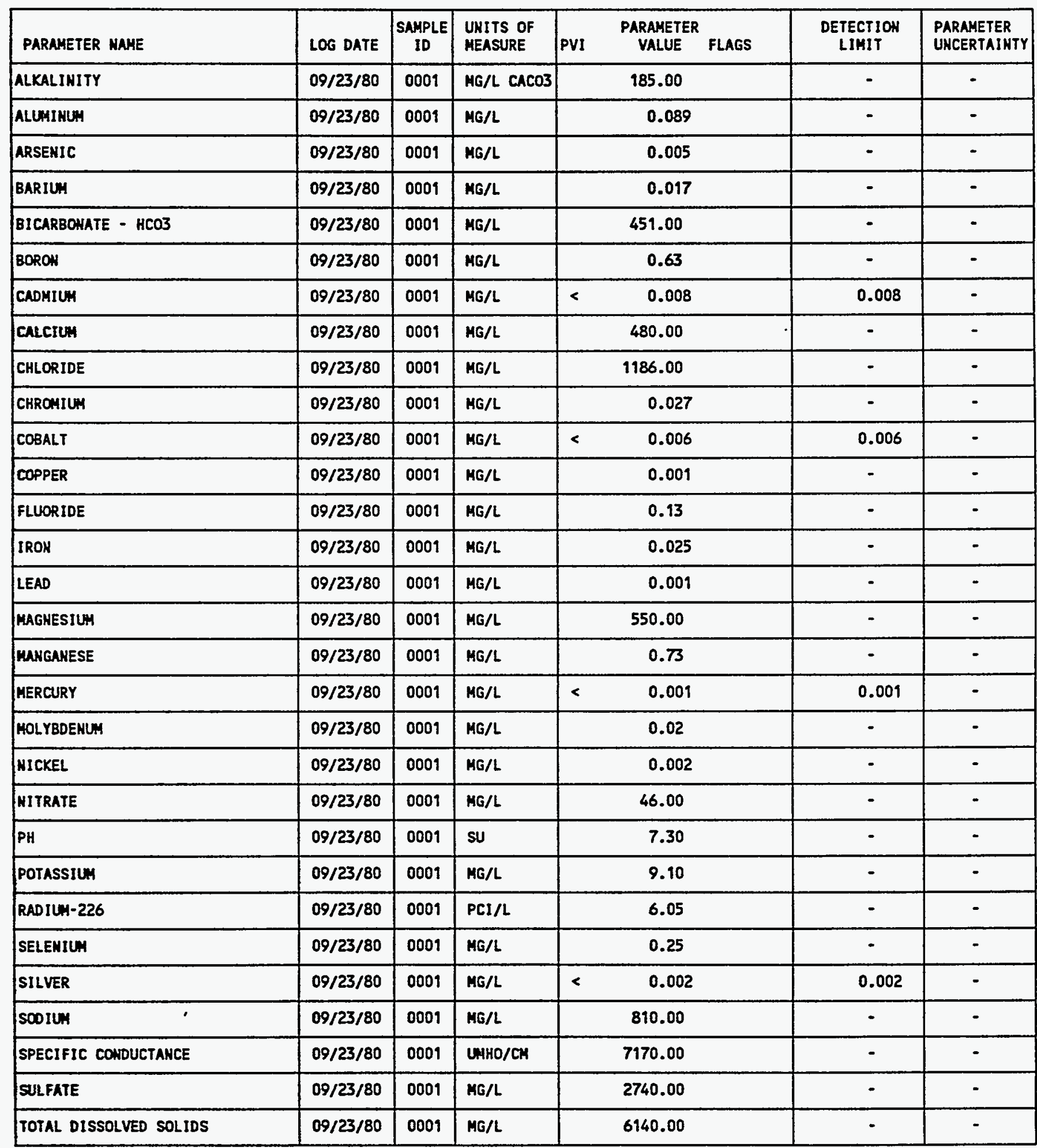

PARAMETER VALUE IMDICATOR (PVI): < - LESS THAM DETECTIOH LIMIT

SAMPLE ID COOES:

0001 - FILTERED SAMPLE (.45 MICROHS) 
GROUMDHATER QUALITY DATA BY LOCATIOH

SITE: AMBOI AMBROSIA LAKE

LOCATIOH: 0725

MORTH COORDIHATE: $\quad 51880.0$ FT

EAST COORDIHATE: $\quad 54950.0$ FT

06/10/80 TO $12 / 04 / 92$

REPORT DATE: 08/04/94

FORMATION OF COMPLETION: DAKOTA SAMDSTONE (KD)

HYDRAULIC FLON RELATIONSHIP: UMKNOWN (H)

\begin{tabular}{|c|c|c|c|c|c|c|c|}
\hline PARAMETER NAME & LOS DATE & $\underset{\text { SN }}{\text { SNPLE }}$ & $\begin{array}{l}\text { UNITS OF } \\
\text { MEASURE }\end{array}$ & PVI & $\begin{array}{l}\text { PARAMETER } \\
\text { VALUE FLAGS }\end{array}$ & $\begin{array}{l}\text { DETECTION } \\
\text { LIMIT }\end{array}$ & $\begin{array}{l}\text { PARAMETER } \\
\text { UNCERTAINTY }\end{array}$ \\
\hline TOTAL SUSPEKDED SOLIDS & $09 / 23 / 80$ & 0001 & MG/L & & 33.00 & - & - \\
\hline URAHIUA & $09 / 23 / 80$ & 0001 & $\mathrm{HG} / \mathrm{L}$ & & 0.11 & - & - \\
\hline VAKADIUA & $09 / 23 / 80$ & 0001 & MG/L & & 0.015 & - & $=$ \\
\hline ZINC & $09 / 23 / 80$ & 0009 & MG/L & & 0.16 & - & - \\
\hline
\end{tabular}

PARAMETER VALUE IHDICATOR (PVI): < - LESS THAN DETECTIOH LIMIT

SAMPLE ID COOES:

0001 - FILTERED SAMPLE (.45 MICRONS) 
GROUNDWATER OUALITY DATA BY LOCATION

SITE: AMBO1 AMBROSIA LAKE

LOCATION: OTZ6

NORTH COORDINATE: $\quad 53980.0$ FT

EAST COORDIHATE: $\quad \mathbf{4 8 5 6 0 . 0}$ FT

06/10/80 TO 12/04/92

REPORT DATE: 08/04/94

FORMATION OF COHPLETION: TRES HERMANOS-A SANDSTOHE (TR) HYDRAULIC FLOW RELATIOHSHIP: UNKNOWH (K)

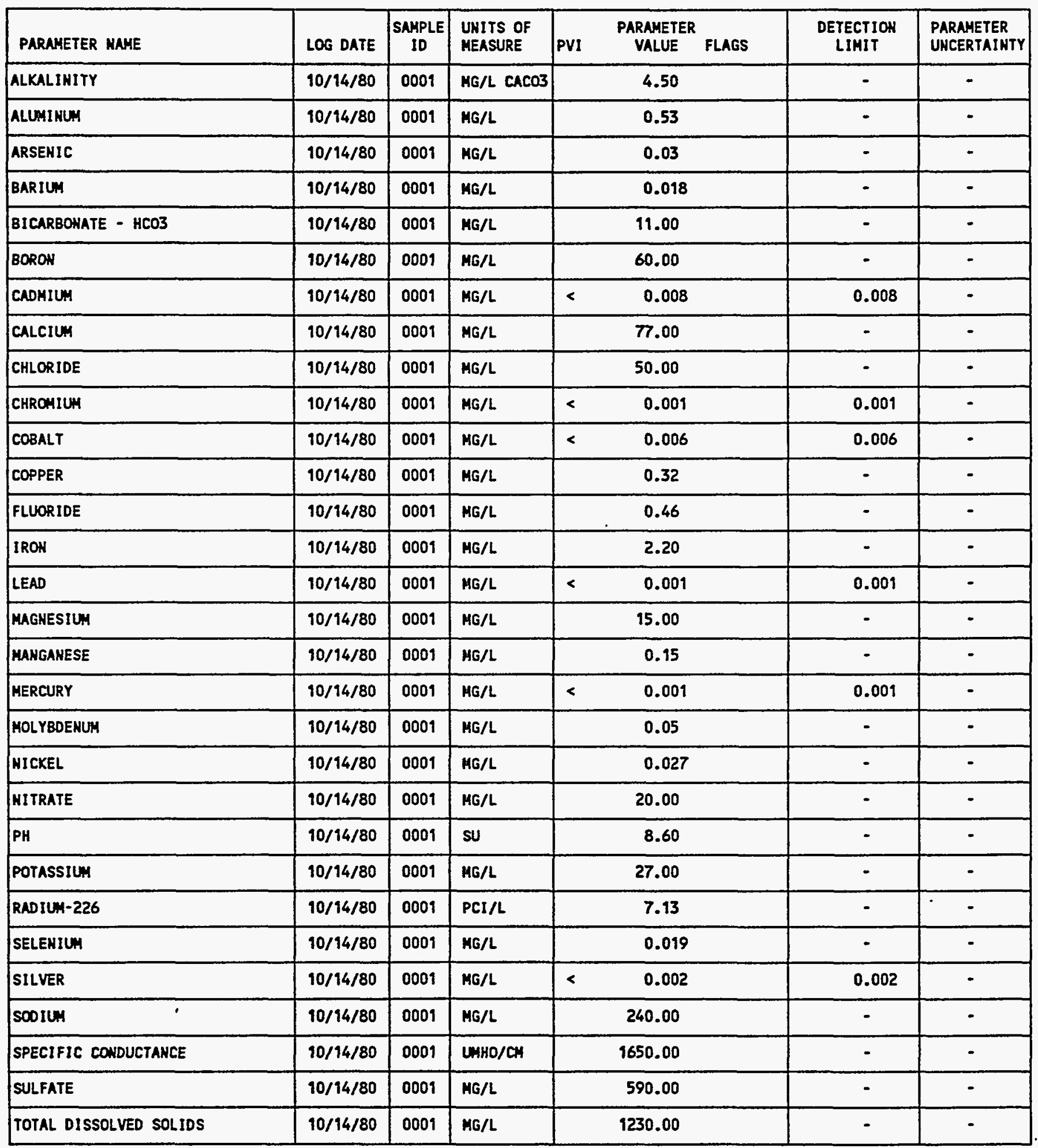

PARAMETER VALUE INDICATOR (PVI): < - LESS THAN DETECTIOH LIMIT 
GROUNDWATER QUALITY DATA BY LOCATION

SITE: AMBO1 AMBROSIA LAKE

LOCATION: 0726

NORTH COORDINATE: 53980.0 FT

EAST COORDINATE: $\quad 48560.0$ FT

06/10/80 TO $12 / 04 / 92$

REPORT DATE: 08/04/94

FORMATION OF COMPLETION: TRES HERMANOS-A SANDSTONE (TR)

HYDRAULIC FLON RELATIONSHIP: UNKHONN (K)

\begin{tabular}{|c|c|c|c|c|c|c|c|}
\hline PARAMETER NAME & LOG DATE & $\begin{array}{c}\text { SAMPLE } \\
\text { ID }\end{array}$ & $\begin{array}{l}\text { UNITS OF } \\
\text { MEASURE }\end{array}$ & PVI & $\begin{array}{l}\text { PARAMETER: } \\
\text { VALUE FLAGS }\end{array}$ & $\begin{array}{l}\text { DETECTION } \\
\text { LIMIT }\end{array}$ & $\begin{array}{l}\text { PARAMETER } \\
\text { UHCERTAINTY }\end{array}$ \\
\hline TOTAL SUSPEHDED SOLIDS & $10 / 14 / 80$ & 0001 & MG/L & & 248.00 & - & - \\
\hline URAKIUNA & $10 / 14 / 80$ & 0001 & MG/L & & 0.05 & - & - \\
\hline VANADIUH & $10 / 14 / 80$ & 0001 & $M G / L$ & & 0.051 & - & - \\
\hline ZIHC & $10 / 14 / 80$ & 0001 & MG/L & & 1.30 & - & - \\
\hline
\end{tabular}

PARAMETER VALUE IMDICATOR (PVI): \& - LESS THAH DETECTION LIMIT

SAMPLE ID COOES:

OOOI - FILTERED SAMPLE (.45 MICRONS) 
GROUNDWATER OUALITY DATA BY LOCATION

SITE: AMBO1 AMBROSIA LAKE

LOCATIOH: 0727

NORTH COORDIMATE: $\quad 68980.0$ FT

EAST COORDINATE: $\quad \mathbf{5 5 9 6 0 . 0}$ FT

$06 / 10 / 80$ TO $12 / 04 / 92$

REPORT DATE: $08 / 04 / 94$

FORHATION OF COMPLETION: MESTHATER CAMYON MEMBER (JH)

HYDRAULIC FLOW RELATIONSHIP: UNKHOWN (H)

\begin{tabular}{|c|c|c|c|c|c|c|c|}
\hline PARNMETER MNME & LOG DATE & $\begin{array}{c}\text { SAMPLE } \\
\text { ID }\end{array}$ & $\begin{array}{l}\text { UNITS OF } \\
\text { MEASURE }\end{array}$ & PVI & $\begin{array}{l}\text { PARAMETER } \\
\text { VALUE FLAGS }\end{array}$ & $\begin{array}{l}\text { DETECTIOW } \\
\text { LIMIT }\end{array}$ & $\begin{array}{l}\text { PARAMETER } \\
\text { UNCERTAINTY }\end{array}$ \\
\hline ALKALIKITY & $11 / 03 / 80$ & 0001 & MG/L CACO3 & & 44.30 & - & - \\
\hline ARSENIC & $11 / 03 / 80$ & 0001 & HG/L & & 0.058 & - & - \\
\hline BICARBOMATE - HCO3 & $11 / 03 / 80$ & 0001 & MG/L & & 108.00 & - & - \\
\hline BORON & $11 / 03 / 80$ & 0001 & HG/L & & 0.14 & - & - \\
\hline CADMIUM & $19 / 03 / 80$ & 0001 & $M G / L$ & $<$ & 0.008 & 0.008 & - \\
\hline CALCIUM & $11 / 03 / 80$ & 0001 & $M G / L$ & & 43.00 & - & - \\
\hline COBALT & $11 / 03 / 80$ & 0001 & MG/L & $<$ & 0.006 & 0.006 & - \\
\hline COPPER & $11 / 03 / 80$ & 0001 & MG/L & & 0.018 & - & - \\
\hline FLUORIDE & $11 / 03 / 80$ & 0001 & HG/L & $<$ & 0.20 & 0.2 & - \\
\hline IRON & $11 / 03 / 80$ & 0001 & $M G / L$ & & 0.10 & - & - \\
\hline LEAD & $11 / 03 / 80$ & 0001 & HG/L & $<$ & 0.001 & 0.001 & - \\
\hline MAGNESIUM & $11 / 03 / 80$ & 0001 & MG/L & & 0.11 & - & - \\
\hline MAMGAHESE & $19 / 03 / 80$ & 0009 & HG/L & & 0.027 & - & - \\
\hline POTASSIUN & $11 / 03 / 80$ & 0001 & $M G / L$ & & 29.00 & - & - \\
\hline RADIUN-226 & $11 / 03 / 80$ & 0001 & PCI/L & & 1.25 & - & - \\
\hline SELEMIUY & $11 / 03 / 80$ & 0001 & MG/L & $<$ & 00.019 & 00.019 & - \\
\hline SILVER & $11 / 03 / 80$ & 0001 & $M G / L$ & $<$ & 0.002 & 0.002 & - \\
\hline SOOILA & $91 / 03 / 80$ & 0001 & MG/L & & 320.00 & - & - \\
\hline SPECIFIC CONDUCTANCE & $11 / 03 / 80$ & 0001 & UHHO/CM & & 1980.00 & - & - \\
\hline SULFATE & $11 / 03 / 80$ & 0001 & MG/L & & 500.00 & - & - \\
\hline TOTAL DISSOLVED SOLIDS & $11 / 03 / 80$ & 0001 & $M G / L$ & & 1190.00 & - & - \\
\hline
\end{tabular}

PARAMETER VALUE INDICATOR (PVI): < - LESS THAN DETECTION LIMIT

SAMPLE IO COOES:

0001 - FILTERED SAMPLE (.45 MICRONS) 
GROUNDWATER OUALITY DATA BY LOCATIOH

SITE: AMBO1 AMBROSIA LAKE

LOCATIOH: 0727

HORTH COOROINATE: 68980.0 FT

EAST COORDIHATE: $\quad 55960.0$ FT

06/10/80 TO $12 / 04 / 92$

REPORT OATE: $08 / 04 / 94$

FORMATION OF COMPLETION: WESTHATER CANYON MEMBER (JM)

HYDRAULIC FLOW RELATIONSHIP: UNKHOWN (N)

\begin{tabular}{|c|c|c|c|c|c|c|c|}
\hline PARAMETER HAME & LOG DATE & $\begin{array}{c}\text { SAMPLE } \\
\text { ID }\end{array}$ & $\begin{array}{l}\text { UNITS OF } \\
\text { MEASURE }\end{array}$ & PVI & $\begin{array}{l}\text { PARAMETER } \\
\text { VALUE FLAGS }\end{array}$ & $\begin{array}{l}\text { DETECTION } \\
\text { LIMIT }\end{array}$ & $\begin{array}{l}\text { PARAMETER } \\
\text { UHCERTAINTY }\end{array}$ \\
\hline TOTAL SUSPEHDED SOLIDS & $11 / 03 / 80$ & 0001 & MG/L & & 6040.00 & - & - \\
\hline URAKIUA & $11 / 03 / 80$ & 0009 & MG/L & & 0.23 & - & - \\
\hline VANADIUH & $11 / 03 / 80$ & 0001 & $M G / L$ & $<$ & 0.003 & 0.003 & - \\
\hline ZIMC & $11 / 03 / 80$ & 0001 & MG/L & & 0.022 & - & - \\
\hline
\end{tabular}

PARAMETER VALUE IMDICATOR (PVI): < - LESS THAN DETECTION LIMIT

SAMPLE ID COOES:

0001 - FILTERED SAMPLE (.45 MICRONS) 
GROUNDHATER OUALITY DATA BY LOCATION

SITE: AMBOI AMBROSIA LAKE

LOCATION: 0728

MORTH COORDIHATE: 52550.0 FT

EAST COORDIHATE: $\quad 44940.0$ FT

06/10/80 TO $12 / 04 / 92$

REPORT DATE: 08/04/94

FORMATION OF COMPLETION: DAKOTA SANDSTONE (KD)

HYDRAULIC FLOW RELATIONSHIP: UNKHOWN (H)

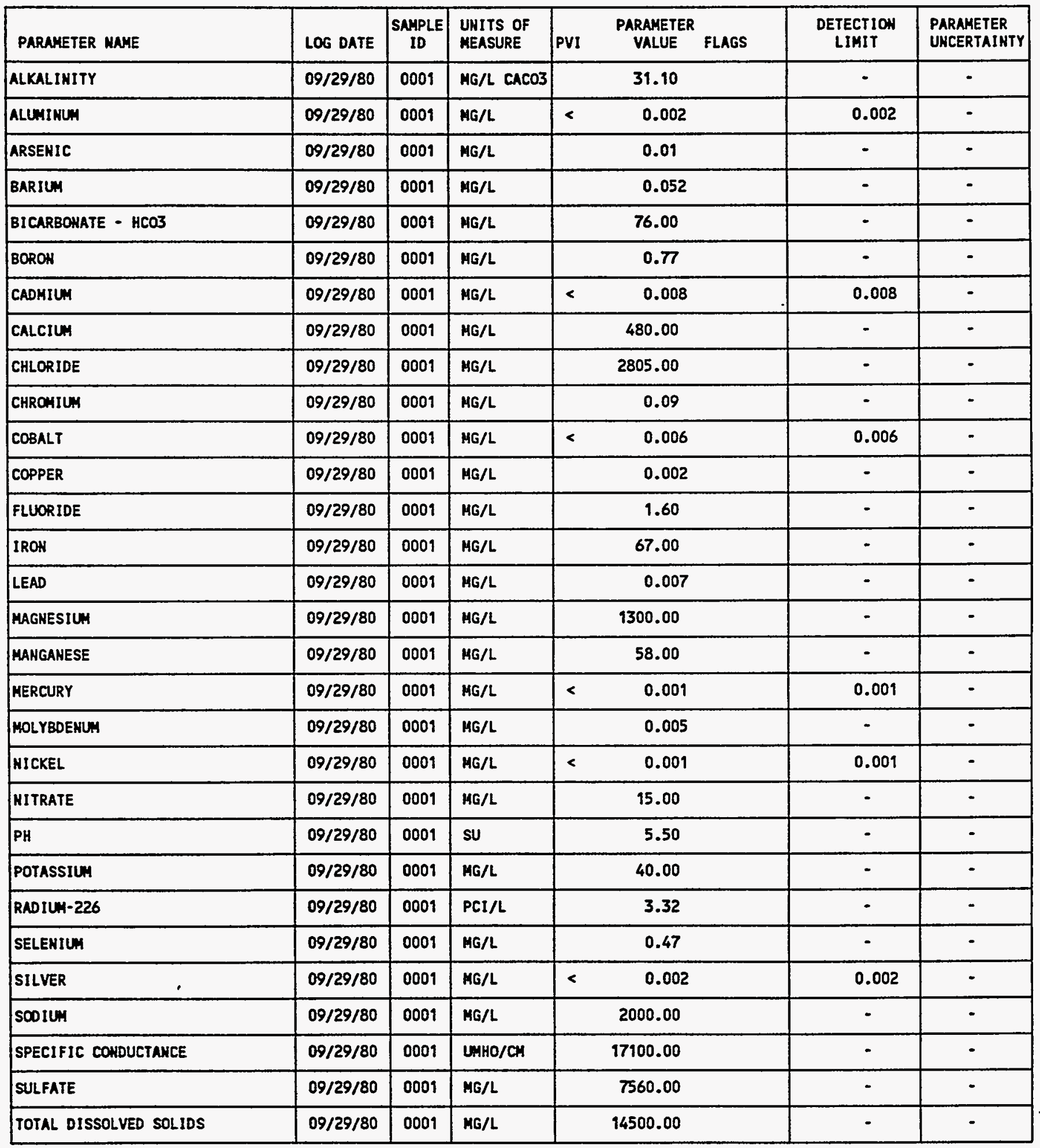

PARAMETER VALUE IMDICATOR (PVI):

< - LESS THAN DETECTION LIMIT

SAMPLE ID COOES:

0001 - FILTERED SAMPLE (.45 MICROHS) 
GROUNDWATER QUALITY DATA BY LOCATIOW

SITE: AMBO1 AMBROSIA LAKE

LOCATIOH: 0728

HORTH COORDINATE: 52550.0 FT

EAST COORDIHATE: $\quad 44940.0 \mathrm{FT}$

06/10/80 TO 12/04/92

REPORT OATE: 08/04/94

FORMATION OF COMPLETIOH: DAKOTA SAKDSTOHE (KD)

HYDRAULIC FLOW RELATIONSHIP: UHKNOWH (H)

\begin{tabular}{|c|c|c|c|c|c|c|c|}
\hline PARAMETER MAME & LOG DATE & SAMPLE & $\begin{array}{l}\text { UNITS OF } \\
\text { MEASURE }\end{array}$ & PVI & $\begin{array}{l}\text { PARAMETER } \\
\text { VALUE FLAGS }\end{array}$ & $\begin{array}{l}\text { DETECTION } \\
\text { LIMIT }\end{array}$ & $\begin{array}{l}\text { PARAMETER } \\
\text { UNCERTAINTY }\end{array}$ \\
\hline TOTAL SUSPENDED SOLIDS & $09 / 29 / 80$ & 0001 & HG/L & & 151.00 & - & - \\
\hline URAKIUN & $09 / 29 / 80$ & 0001 & MG/L & & 0.07 & - & - \\
\hline VANADIUY & $09 / 29 / 80$ & 0001 & MG/L & & 0.017 & - & - \\
\hline ZINC & $09 / 29 / 80$ & 0001 & MG/L & & 0.11 & - & - \\
\hline
\end{tabular}

PARAMETER VALUE IHDICATOR (PVI): < - LESS THAK DETECTION LIMIT

SAMPLE ID COOES:

0001 - FILTERED SAMPLE (.45 MICROHS) 
GROUNDWATER QUALITY DATA BY LOCATION

SITE: AMBOI AMBROSIA LAKE

LOCATION: 0729

NORTH COORDINATE: $\quad 53350.0$ FT

EAST COORDIHATE: $\quad 53510.0$ FT

06/10/80 TO 12/04/92

REPORT DATE: 08/04/94

FORMATION OF COMPLETION: ALLUVIUM (AL)

HYORAULIC FLOW RELATIONSHIP: UNKNOWN (N)

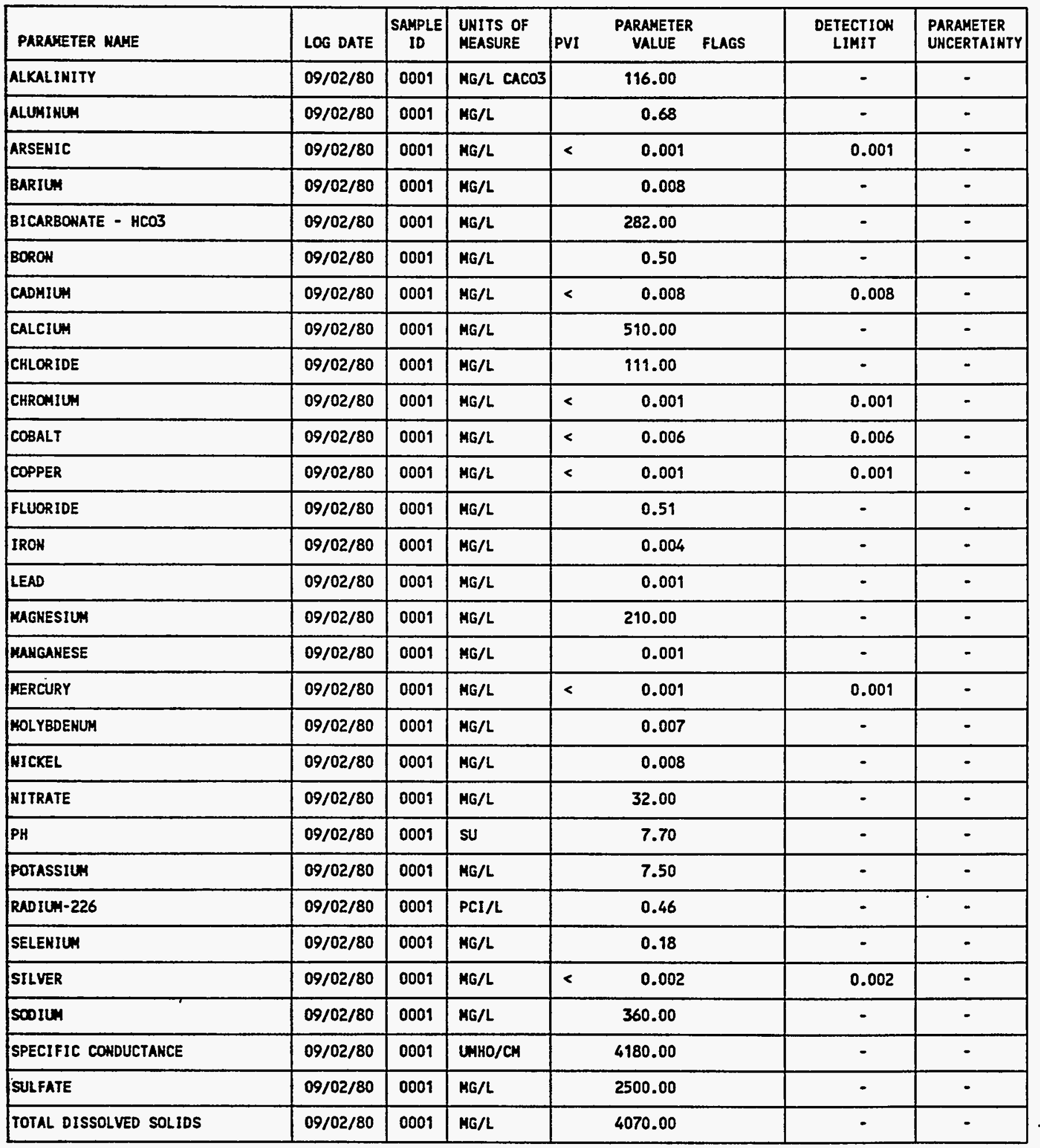

PARAMETER VALUE IHDICATOR (PVI):

< - LESS THAN DETECTION LIMIT

SAMPLE ID COOES:

0001 - FILTERED SAMPLE (.45 MICRONS) 
GROUNDHATER OUALITY DATA BY LOCATION

SITE: AMBOI AMBROSIA LAKE

LOCATION: 0729

NORTH COORDINATE: 53350.0 FT

EAST COORDINATE: $\quad 53510.0$ FT

06/10/80 TO 12/04/92

REPORT DATE: 08/04/94

FORMATIOH OF COAPLETIOH: ALLUVIUM (AL)

HYDRAULIC FLON RELATIOHSHIP: UHKHONH (H)

\begin{tabular}{|c|c|c|c|c|c|c|c|}
\hline PARAMETER MAME & LOG DATE & $\underset{\text { ID }}{\text { SAMPLE }}$ & $\begin{array}{l}\text { UNITS OF } \\
\text { MEASURE }\end{array}$ & PVI & $\begin{array}{l}\text { PARAMETER! } \\
\text { VALUE FLAGS }\end{array}$ & $\begin{array}{l}\text { DETECTIOH } \\
\text { LIMIT }\end{array}$ & $\begin{array}{l}\text { PARAMETER } \\
\text { UHCERTAINTY }\end{array}$ \\
\hline TOTAL SUSPENDED SOLIDS & $09 / 02 / 80$ & 0001 & MG/L & & 15.00 & - & - \\
\hline URAKIUH & $09 / 02 / 80$ & 0001 & MG/L & & 0.075 & - & - \\
\hline VAHADILA & $09 / 02 / 80$ & 0001 & $M G / L$ & & 0.018 & - & - \\
\hline ZIMC & $09 / 02 / 80$ & 0001 & MG/L & & 0.014 & - & - \\
\hline
\end{tabular}

PARAMETER VALUE INDICATOR (PVI): < - LESS THAN DETECTION LIMIT

SAMPLE ID COOES:

0001 - FILTERED SAHPLE (.45 MICRONS) 
GROUNDWATER QUALITY DATA BY LOCATION

SITE: MMBOI AMBROSIA LAKE

LOCATIOH: 0737

NORTH COOROINATE: 54291.3 FT

EAST COORDINATE: 60397.3 FT

06/10/80 TO 12/04/92

REPORT OATE: $08 / 04 / 94$

FORMATION OF COMPLETION: URAHIUM MILL TAILIMGS (TA)

HYDRAULIC FLOW RELATIONSHIP: ON-SITE (O)

\begin{tabular}{|c|c|c|c|c|c|c|c|}
\hline PARANETER NAME & LOG DATE & $\underset{10}{\text { SNMPLE }}$ & $\begin{array}{l}\text { UNITS OF } \\
\text { MEASURE }\end{array}$ & PVI & $\begin{array}{l}\text { PARAMETER } \\
\text { VALUE FLAGS }\end{array}$ & $\begin{array}{l}\text { DETECTIOH } \\
\text { LIMIT }\end{array}$ & $\begin{array}{l}\text { PARAMETER } \\
\text { UMCERTAINTY }\end{array}$ \\
\hline ALKALIHITY & $05 / 13 / 86$ & 0001 & MG/L CACO3 & & 6175 & - & - \\
\hline CADHIU: & $05 / 13 / 86$ & 0001 & $M G / L$ & $<$ & 0.001 & 0.001 & - \\
\hline CHLORIDE & $05 / 13 / 86$ & 0001 & MG/L & & 200. & 1. & - \\
\hline CHROMIUH & $05 / 13 / 86$ & 0001 & MG/L & & 0.03 & 0.01 & - \\
\hline COBALT & $05 / 13 / 86$ & 0001 & HG/L & & 0.1 & 0.05 & - \\
\hline FLUORIDE & $05 / 13 / 86$ & 0001 & MG/L & & 15. & 0.1 & - \\
\hline MANGAKESE & $05 / 13 / 86$ & 0001 & $M G / L$ & & 0.03 & 0.01 & - \\
\hline HOLYBDENUA & $05 / 13 / 86$ & 0001 & $M G / L$ & & 250. & 0.01 & - \\
\hline HITRATE & $05 / 13 / 86$ & 0001 & MG/L & & 10. & 1. & - \\
\hline PH & $05 / 13 / 86$ & 0001 & SU & & 9.74 & - & - \\
\hline POTASSIU: & $05 / 13 / 86$ & 0001 & MG/L & & 17.1 & 0.01 & - \\
\hline RADIUH-226 & $05 / 13 / 86$ & 0001 & PCI/L & & 71. & 1. & 11. \\
\hline SELENIUM & $05 / 13 / 86$ & 0001 & MG/L & $<$ & 0.005 & 0.005 & - \\
\hline THORIUH-230 & $05 / 13 / 86$ & 0001 & $\mathrm{PCI} / \mathrm{L}$ & & 58. & 1. & 11. \\
\hline URAHIUA & $05 / 13 / 86$ & 0001 & $M G / L$ & & 10.7 & 0.003 & - \\
\hline VANADIUH & $05 / 13 / 86$ & 0001 & $M G / L$ & & 0.57 & 0.01 & - \\
\hline
\end{tabular}

PARAMETER VALUE INDICATOR (PVI): < - LESS THAN DETECTION LIMIT

SAMPLE ID CODES:

0001 - FILTERED SAMPLE (.45 MICRONS) 
GROUHDWATER ' OUALITY DATA BY LOCATION

SITE: AMBO1 AMBROSIA LAKE

LOCATION: 0743

NORTH COORDINATE: 53782.5 FT

EAST COORDINATE: 60188.6 FT

06/10/80 TO 12/04/92

REPORT OATE: 08/04/94

FORMATION OF COMPLETION: URANIUM MILL TAILINGS (TA)

HYDRAULIC FLON RELATIOHSHIP: ON-SITE (O)

\begin{tabular}{|c|c|c|c|c|c|c|c|}
\hline $\begin{array}{l}\text { PARAMETER MAME } \\
\text { PAR }\end{array}$ & LOG DATE & $\begin{array}{c}\text { SAMPLE } \\
\text { ID }\end{array}$ & $\begin{array}{l}\text { UNITS OF } \\
\text { MEASURE }\end{array}$ & PVI & $\begin{array}{l}\text { PARAMETER } \\
\text { VALUE FLAGS }\end{array}$ & $\begin{array}{l}\text { DETECTION } \\
\text { LIMIT }\end{array}$ & $\begin{array}{l}\text { PARAHETER } \\
\text { UNCERTAINTY }\end{array}$ \\
\hline ALKALINITY & $05 / 13 / 86$ & 0001 & MG/L CACO3 & & 6371. & - & - \\
\hline ARSENIC & $05 / 13 / 86$ & 0001 & MG/L & $<$ & 0.01 & 0.01 & - \\
\hline CADMIUN & $05 / 13 / 86$ & 0001 & MG/L & $<$ & 0.001 & 0.001 & - \\
\hline CALCIUH & $05 / 13 / 86$ & 0001 & MG/L & & 16.2 & 0.01 & - \\
\hline CHLORIDE & $05 / 13 / 86$ & 0001 & MG/L & & 110. & 1. & - \\
\hline CHROHIUY & $05 / 13 / 86$ & 0001 & $M G / L$ & & 0.1 & 0.01 & - \\
\hline COBALT & $05 / 13 / 86$ & 0001 & HG/L & & 0.1 & 0.05 & - \\
\hline FLUORIDE & $05 / 13 / 86$ & 0001 & MG/L & & 19. & 0.1 & - \\
\hline IRON & $05 / 13 / 86$ & 0001 & MG/L & & 0.47 & 0.03 & - \\
\hline MAGHESIUM & $05 / 13 / 86$ & 0001 & MG/L & & 5.65 & 0.001 & - \\
\hline MAHGAMESE & $05 / 13 / 86$ & 0001 & $M G / L$ & & 0.02 & 0.01 & - \\
\hline MOLYBDEENUH & $05 / 13 / 86$ & 0001 & $M G / L$ & & 161. & 0.09 & - \\
\hline NITRATE & $05 / 13 / 86$ & 0001 & MG/L & & 6. & 1. & - \\
\hline $\mathrm{PH}$ & $05 / 13 / 86$ & 0001 & su & & 9.71 & - & - \\
\hline POTASSIUN & $05 / 13 / 86$ & 0001 & $M G / L$ & & 25. & 0.01 & - \\
\hline SELENIUH & $05 / 13 / 86$ & 0001 & MG/L & $<$ & 0.005 & 0.005 & - \\
\hline SCOIUH & $05 / 13 / 86$ & 0001 & $M G / L$ & & 5920. & 0.002 & - \\
\hline SPECIFIC CONDUCTAHCE & $05 / 13 / 86$ & 0001 & UHHO/CH & & 16500 & - & - \\
\hline STRONTIUM & $05 / 13 / 86$ & 0001 & MG/L & & 0.2 & 0.1 & - \\
\hline SULFATE & $05 / 13 / 86$ & 0001 & $\mathrm{MG} / \mathrm{L}$ & & 7890. & 0.1 & - \\
\hline TEMPERATURE & $05 / 13 / 86$ & 0001 & C - DEGREE & & 18. & - & - \\
\hline URANIUM & $05 / 13 / 86$ & 0001 & MG/L & & 2.17 & 0.003 & - \\
\hline VANADIUM & $05 / 13 / 86$ & 0001 & $M G / L$ & & 0.56 & 0.01 & - \\
\hline
\end{tabular}

PARAMETER VALUE INDICATOR (PVI):

< - LESS THAK DETECTION LIMIT

SAMPLE ID CODES:

D001 - FILTERED SAMPLE (.45 MICRONS) 
GROUHDWATER QUALITY DATA BY LOCATION

SITE: AMBO1 AMBROSIA LAKE

LOCATION: 0746

NORTH COORDINATE: 54193.0 FT

EAST COORDINATE: 60806.8 FT

06/10/80 TO $12 / 04 / 92$

REPORT DATE: $08 / 04 / 94$

FORMATION OF COHPLETION: URAMIUH MILL TAILIHGS (TA)

HYDRAULIC FLOW RELATIONSHIP: OW-SITE (O)

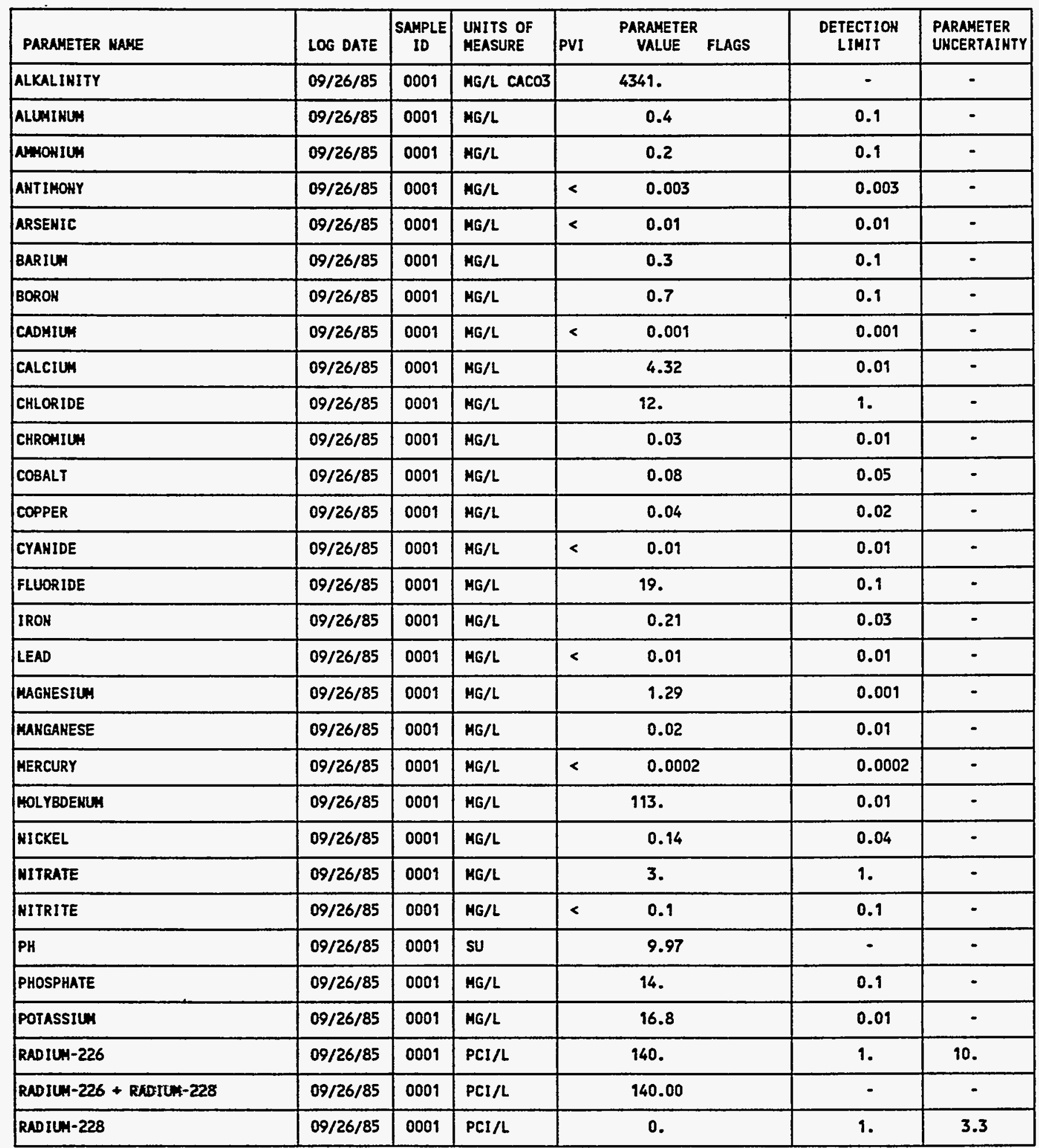

PARAMETER VALUE IHDICATOR (PVI): < - LESS THAH DETECTION LIMIT 
GROUHDWATER QUALITY DATA BY LOCATIOH

SITE: AMBO1 AMBROSIA LAKE

LOCATION: 0746

HORTH COORDINATE: 54993.0 FT

EAST COORDINATE: $\quad 60806.8$ FT

06/10/80 TO $12 / 04 / 92$

REPORT DATE: 08/04/94

FORMATION OF COHPLETION: URANIUA MILL TAILINGS (TA)

HYDRAULIC FLON RELATIONSHIP: ON-SITE (O)

\begin{tabular}{|c|c|c|c|c|c|c|c|}
\hline PARAMETER NAME & LOG DATE & $\underset{10}{\operatorname{SAMPLE}}$ & $\begin{array}{l}\text { UNITS OF } \\
\text { MEASURE }\end{array}$ & PVI & $\begin{array}{l}\text { PARAMETER } \\
\text { VALUE FLAGS }\end{array}$ & $\begin{array}{l}\text { DETECTION } \\
\text { LIMIT }\end{array}$ & $\begin{array}{l}\text { PARAMETER } \\
\text { UNCERTAINTY }\end{array}$ \\
\hline SELENIUM & $09 / 26 / 85$ & 0001 & HG/L & $<$ & 0.005 & 0.005 & - \\
\hline SILICA - SIOZ & $09 / 26 / 85$ & 0001 & MG/L & & 13. & 2. & - \\
\hline SILVER & $09 / 26 / 85$ & 0001 & $M G / L$ & $<$ & 0.01 & 0.01 & - \\
\hline SODIU, & $09 / 26 / 85$ & 0001 & MG/L & & 4810. & 0.002 & - \\
\hline SPECIFIC COHDUCTANCE & $09 / 26 / 85$ & 0001 & UNHO/CH & & 14000. & - & - \\
\hline STRONTIUH & $09 / 26 / 85$ & 0001 & MG/L & $<$ & 0.1 & 0.1 & - \\
\hline SULFATE & $09 / 26 / 85$ & 8001 & MG/L & & 5890. & 0.1 & - \\
\hline SULFIDE & $09 / 26 / 85$ & 0001 & MG/L & $<$ & 0.1 & 0.1 & - \\
\hline TEMPERATURE & $09 / 26 / 85$ & 0001 & C - DEGREE & & 18. & - & - \\
\hline THORIUM-230 & $09 / 26 / 85$ & 0001 & PCI/L & & 34. & 1. & 9.0 \\
\hline TIN & $09 / 26 / 85$ & 0001 & MG/L & $<$ & 0.005 & 0.005 & - \\
\hline TOTAL DISSOLVED SOLIDS & $09 / 26 / 85$ & 0001 & MG/L & & 13600. & 10. & - \\
\hline URAKIUH & $09 / 26 / 85$ & 0001 & $M G / L$ & & 1.32 & 0.003 & - \\
\hline VAMADIUH & $09 / 26 / 85$ & 0001 & MG/L & & 0.09 & 0.01 & - \\
\hline ZINC & $09 / 26 / 85$ & 0001 & MG/L & & 0.04 & 0.005 & - \\
\hline
\end{tabular}

PARAMETER VALUE IMDICATOR (PVI):

< - LESS THAN DETECTION LIMIT

SAMPLE ID CODES:

0001 - FILTERED SAMPLE (.45 MICRONS) 
GROUNDWATER QUALITY DATA BY LOCATIOH

SITE: AMBOI AMBROSIA LAKE

LOCATION: 0747

MORTH COORDINATE: 53990.5 FT

EAST COORDINATE: 60805.1 FT

$06 / 10 / 80$ TO $12 / 04 / 92$

REPORT DATE: 08/04/94

FORMATION OF COMPLETION: ALLUVIUM (AL)

HYORAULIC FLON RELATIONSHIP: ON-SITE (O)

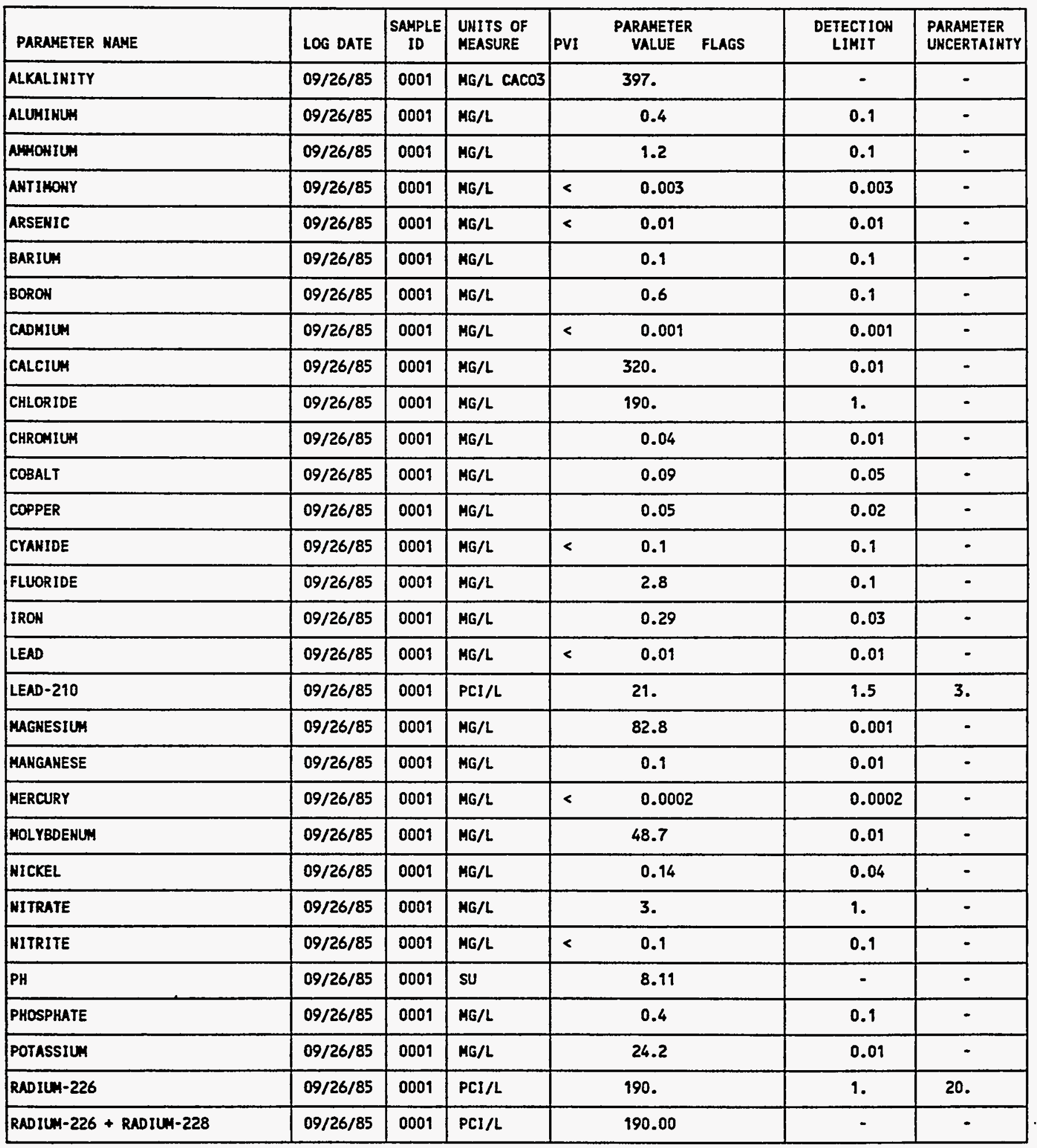

PARAMETER VALUE IMDICATOR (PVI):

< - LESS THAN DETECTION LIMIT

SAMPLE ID COOES:

0001 - FILTERED SAMPLE (.45 MICROHS) 
GROUKDHATER QUALITY DATA BY LOCATIOH

SITE: AMBOI AMBROSIA LAKE

LOCATION: 0747

HORTH COORDIHATE: 53990.5 FT

EAST COORDINATE: $\quad 60805.1$ FT

06/10/80 TO 12/04/92

REPORT DATE: 08/04/94

FORMATION OF COMPLETIOH: ALLUVIUH (AL)

HYORAULIC FLON RELATIONSHIP: ON-SITE (O)

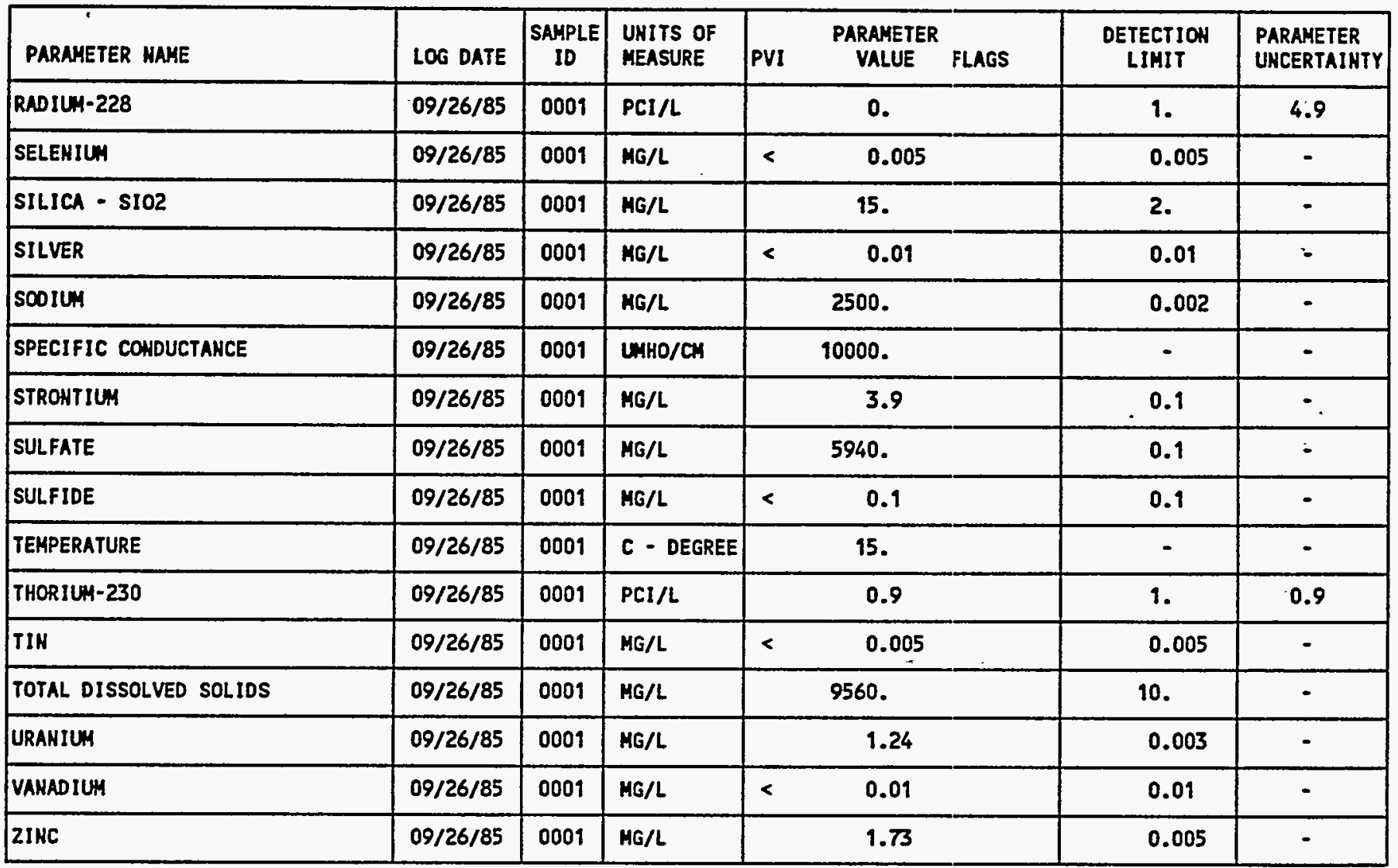

PARAMETER VALUE IMDICATOR (PVI): < - LESS THAN DETECTIOH LIMIT

SAMPLE ID COOES:

0001 - FILTIERED SAMPLE (.45 MICRONS) 
GROUNDWATER QUALITY DATA BY LOCATION

SITE: AMBOI AMBROSIA LAKE

LOCATION: 0748

NORTH COORDIHATE: 53985.9 FT

EAST COORDINATE: $\quad 60803.6$ FT

06/10/80 TO 12/04/92

REPORT DATE: $08 / 04 / 94$

FORMATION OF COMPLETION: ALLUVIUM (AL)

HYDRAULIC FLON RELATIONSHIP: OW-SITE (O)

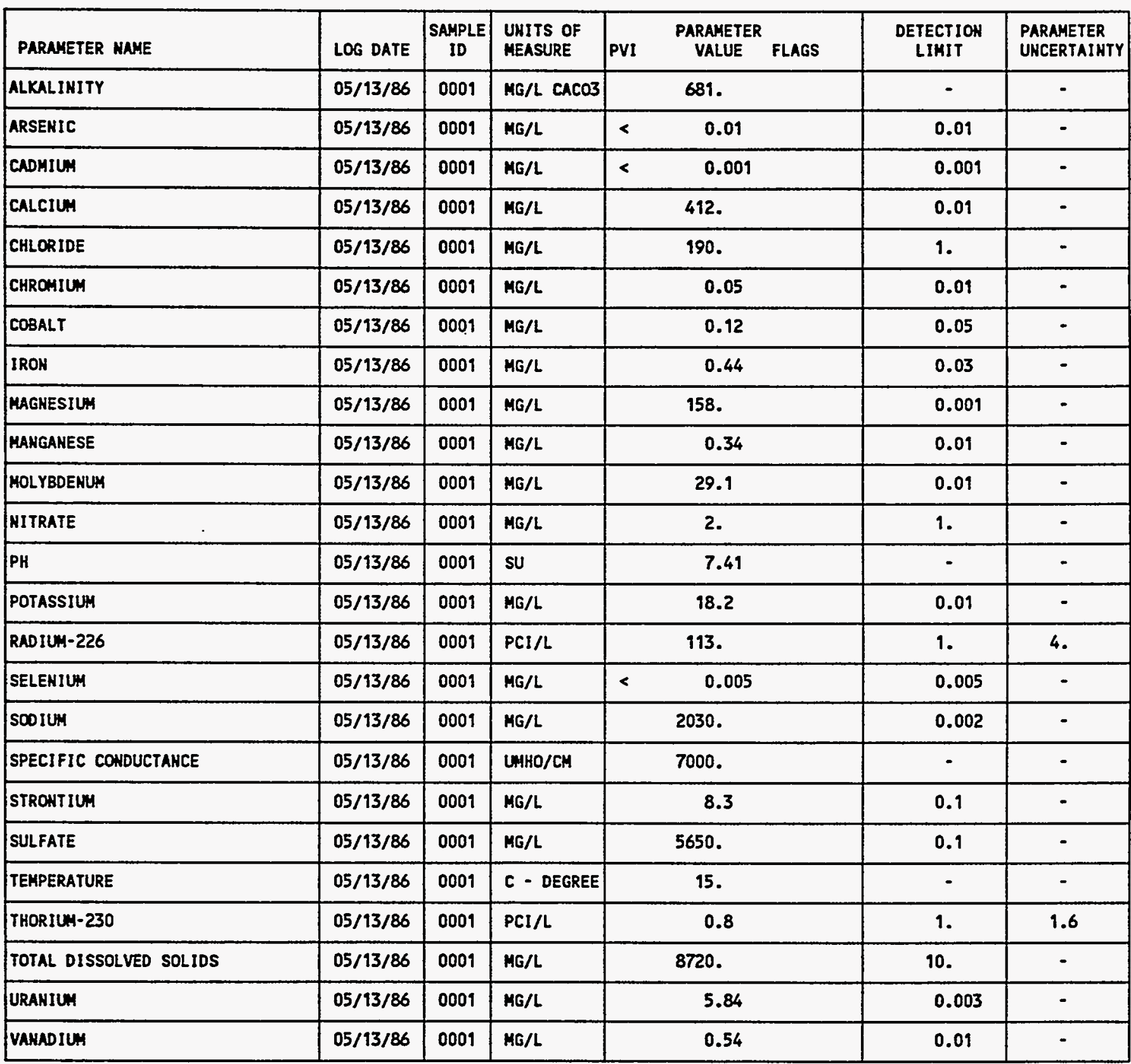

PARAMETER VALUE INDICATOR (PVI): < - LESS THAN DETECTION LIMIT

SAMPLE IO COOES:

0001 - FILTERED SAMPLE (.45 MICRONS) 
GROUNDHATER QUALITY DATA BY LOCATION

SITE: AMBO1 AMBROSIA LAKE

LOCATIOH: 0749

NORTH COORDIHATE: 53989.3 FT

EAST COORDIHATE: $\quad 60802.2$ FT

06/10/80 TO $12 / 04 / 92$

REPORT DATE: 08/04/94

FORMATIOH OF COMPLETIOH: ALLUVIUH (AL)

HYDRAULIC FLOW RELATIONSHIP: OH-SITE (O)

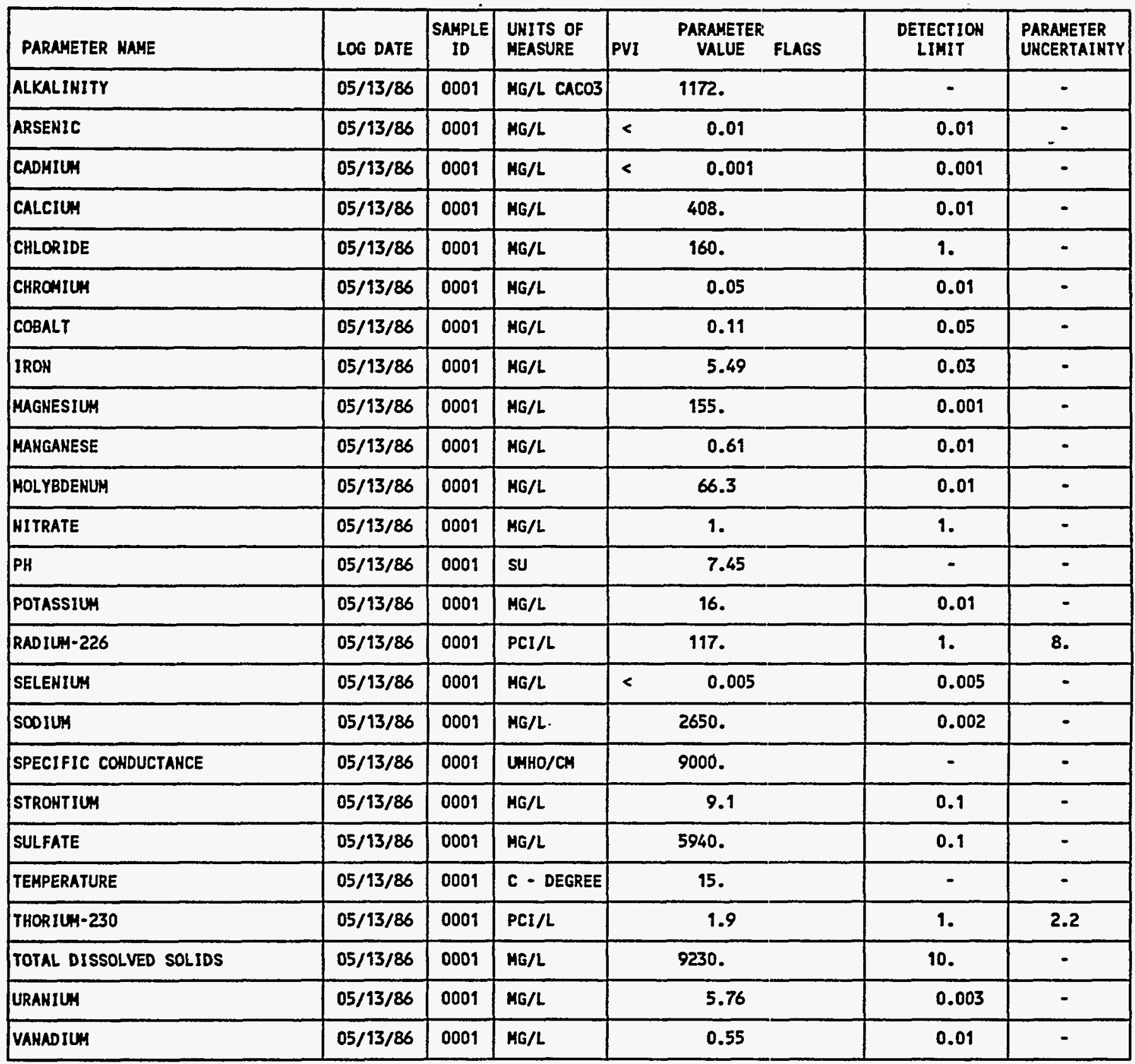

PARAMETER VALUE IRDICATOR (PVI): < - LESS THAM DETECTIOH LIMIT 
GROUNDWATER QUALITY DATA BY LOCATION

SITE: AMBOI AMBROSIA LAKE

LOCATIOH: 0730

HORTH COORDIHATE: $\quad 53977.5$ FT

EAST COORDINATE: 60809.1 FT

06/10/80 TO $12 / 04 / 92$

REPORT DATE: 08/04/94

FORMATION OF COMPLETION: URAMIUM MILL TAILINGS (TA)

HYDRAULIC FLON RELATIONSHIP: OH-SITE (O)

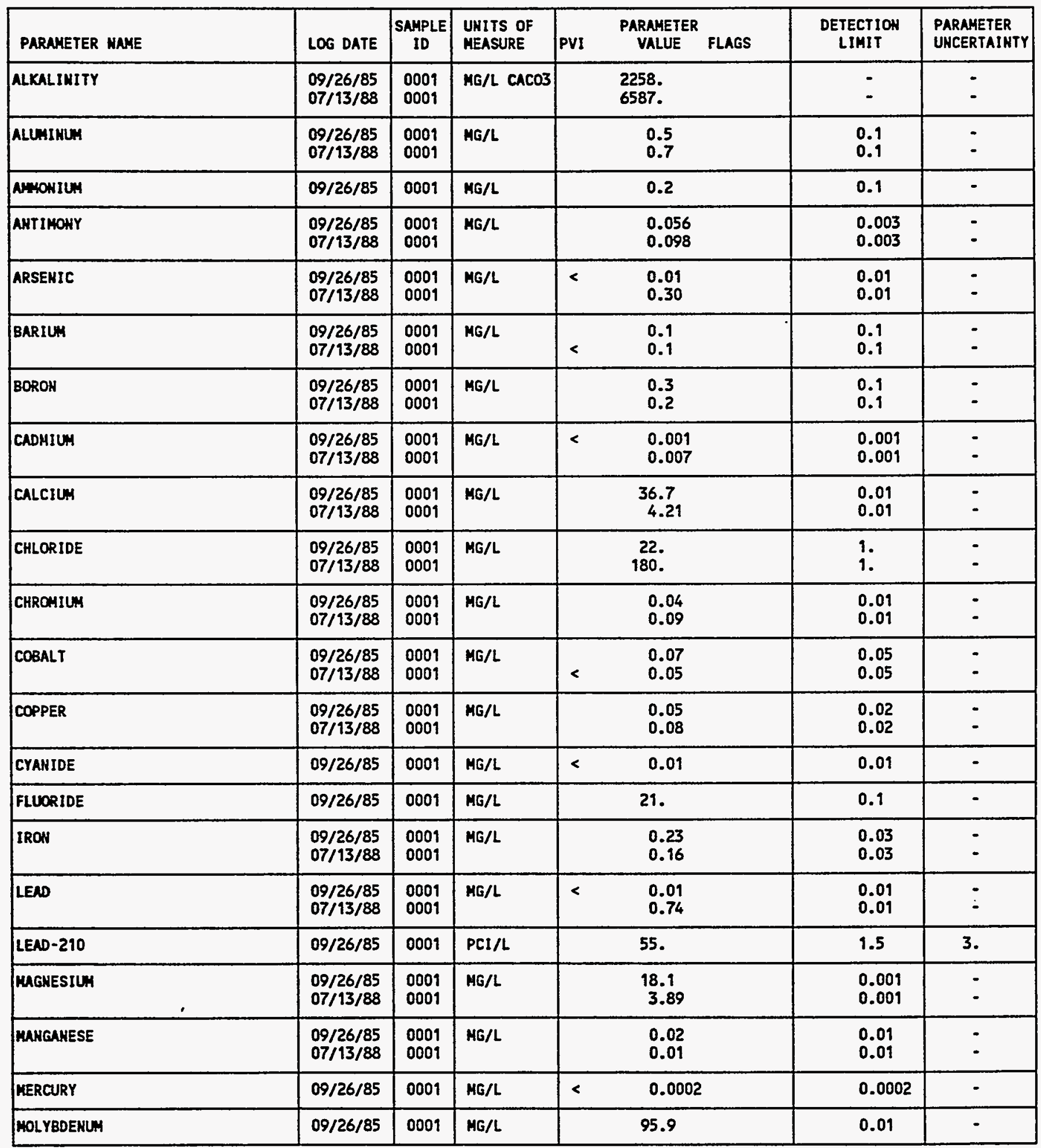

PARAMETER VALUe INDICATOR (PVI): < - LeSS than Detection LIMIT 
GROUNDHATER QUALITY DATA BY LOCATION

SITE: AMBOI AMBROSIA LAKE

LOCATION: 0750

MORTH COORDINATE: 53977.5 FT

EAST COORDINATE: 60801.1 FT

06/10/80 TO 12/04/92

REPORT DATE: 08/04/94

FORMATION OF COMPLETION: URANIUM MILL TAILINGS (TA)

HYDRAULIC FLOW RELATIONSHIP: OH-SITE (O)

\begin{tabular}{|c|c|c|c|c|c|c|c|}
\hline PARAMETER NAME & LOG DATE & $\underset{\text { ID }}{\text { SAMPLE }}$ & $\begin{array}{l}\text { UWITS OF } \\
\text { MEASURE }\end{array}$ & PVI & $\begin{array}{l}\text { PARAMETER: } \\
\text { VALUE FLAGS }\end{array}$ & $\begin{array}{l}\text { DETECTION } \\
\text { LIMIT }\end{array}$ & $\begin{array}{l}\text { PARAMETER } \\
\text { UHCERTAINTY }\end{array}$ \\
\hline MOLYYBDENUM & $07 / 13 / 88$ & 0001 & MG/L & & 120. & 0.01 & - \\
\hline HICKEL & $\begin{array}{l}09 / 26 / 85 \\
07 / 13 / 88\end{array}$ & $\begin{array}{l}0001 \\
0001\end{array}$ & MG/L & $<$ & $\begin{array}{l}0.13 \\
0.04\end{array}$ & $\begin{array}{l}0.04 \\
0.04\end{array}$ & $\dot{-}$ \\
\hline NITRATE & $09 / 26 / 85$ & 0001 & MG/L & & 1. & 1. & - \\
\hline HITRITE & $09 / 26 / 85$ & 0001 & MG/L & $<$ & 0.1 & 0.1 & - \\
\hline PH & $\begin{array}{l}09 / 26 / 85 \\
07 / 13 / 88\end{array}$ & $\begin{array}{l}0001 \\
0001\end{array}$ & SU & & $\begin{array}{r}9.61 \\
10.06\end{array}$ & - & 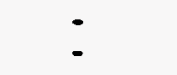 \\
\hline PHOSPHATE & $\begin{array}{l}09 / 26 / 85 \\
07 / 13 / 88\end{array}$ & $\begin{array}{l}0001 \\
0001\end{array}$ & MG/L & & $\begin{array}{r}4.8 \\
15.9\end{array}$ & $\begin{array}{l}0.1 \\
0.1\end{array}$ & $\dot{-}$ \\
\hline POTASSILM & $\begin{array}{l}09 / 26 / 85 \\
07 / 13 / 88\end{array}$ & $\begin{array}{l}0001 \\
0001\end{array}$ & MG/L & & $\begin{array}{l}22.3 \\
31 .\end{array}$ & $\begin{array}{l}0.01 \\
0.09\end{array}$ & - \\
\hline RADIUA-226 & $09 / 26 / 85$ & 0001 & $\mathrm{PCl} / \mathrm{L}$ & & 90. & 1. & 10. \\
\hline RADIUA-226 + RADIUA-228 & $09 / 26 / 85$ & 0001 & $\mathrm{PCI} / \mathrm{L}$ & & 90.10 & - & - \\
\hline RADIUHA-228 & $09 / 26 / 85$ & 0001 & $\mathrm{PCI} / \mathrm{L}$ & & 0.1 & 1. & 3.2 \\
\hline SELEHIUK & $\begin{array}{l}09 / 26 / 85 \\
07 / 13 / 88\end{array}$ & $\begin{array}{l}0001 \\
0001\end{array}$ & $M G / L$ & $<$ & $\begin{array}{l}0.005 \\
0.320\end{array}$ & $\begin{array}{l}0.005 \\
0.005\end{array}$ & - \\
\hline SILICA - S102 & $\begin{array}{l}09 / 26 / 85 \\
07 / 13 / 88\end{array}$ & $\begin{array}{l}0001 \\
0001\end{array}$ & HG/L & & $\begin{array}{l}9.0 \\
5.5\end{array}$ & $\begin{array}{l}2 . \\
2 .\end{array}$ & - \\
\hline SILVER & $\begin{array}{l}09 / 26 / 85 \\
07 / 13 / 88\end{array}$ & $\begin{array}{l}0001 \\
0001\end{array}$ & $M G / L$ & $<$ & $\begin{array}{l}0.01 \\
0.01\end{array}$ & $\begin{array}{l}0.01 \\
0.01\end{array}$ & - \\
\hline SOOIUH & $\begin{array}{l}09 / 26 / 85 \\
07 / 13 / 88\end{array}$ & $\begin{array}{l}0001 \\
0001\end{array}$ & $M G / L$ & & $\begin{array}{l}4190 . \\
5880 .\end{array}$ & $\begin{array}{l}0.002 \\
0.002\end{array}$ & - \\
\hline SPECIFIC COHDUCTAHCE & $\begin{array}{l}09 / 26 / 85 \\
07 / 13 / 88\end{array}$ & $\begin{array}{l}0001 \\
0001\end{array}$ & UMHO/CH & & $\begin{array}{l}10000 \\
16000\end{array}$ & - & - \\
\hline STRONTIU:A & $\begin{array}{l}09 / 26 / 85 \\
07 / 13 / 88\end{array}$ & $\begin{array}{l}0001 \\
0001\end{array}$ & MG/L & & $\begin{array}{l}0.5 \\
0.2\end{array}$ & $\begin{array}{l}0.1 \\
0.1\end{array}$ & : \\
\hline SULFATE & $\begin{array}{l}09 / 26 / 85 \\
07 / 13 / 88\end{array}$ & $\begin{array}{l}0001 \\
0001\end{array}$ & MG/L & & $\begin{array}{l}6550 . \\
6310 .\end{array}$ & $\begin{array}{l}0.1 \\
0.1\end{array}$ & - \\
\hline SULFIDE & $09 / 26 / 85$ & 0001 & $M G / L$ & $<$ & 0.1 & 0.1 & - \\
\hline TEMPERATURE & $\begin{array}{l}09 / 26 / 85 \\
07 / 13 / 88\end{array}$ & $\begin{array}{l}0001 \\
0001\end{array}$ & C - DEGREE & & $\begin{array}{l}17 . \\
18 .\end{array}$ & - & $\dot{-}$ \\
\hline TIN & $\begin{array}{l}09 / 26 / 85 \\
07 / 13 / 88\end{array}$ & $\begin{array}{l}0001 \\
0001\end{array}$ & MG/L & $<$ & $\begin{array}{l}0.005 \\
0.398\end{array}$ & $\begin{array}{l}0.005 \\
0.005\end{array}$ & - \\
\hline TOTAL DISSOLVED SOLIDS & $\begin{array}{l}09 / 26 / 85 \\
07 / 13 / 88\end{array}$ & $\begin{array}{l}0001 \\
0001\end{array}$ & MG/L & & $\begin{array}{l}13100 . \\
16700 .\end{array}$ & $\begin{array}{l}10 . \\
10 .\end{array}$ & - \\
\hline URAHIUH & $\begin{array}{l}09 / 26 / 85 \\
07 / 13 / 88\end{array}$ & $\begin{array}{l}0001 \\
0001\end{array}$ & MG/L & & $\begin{array}{l}6.27 \\
8.40\end{array}$ & $\begin{array}{l}0.003 \\
0.003\end{array}$ & $\because$ \\
\hline
\end{tabular}

PARAMETER VALUE IHDICATOR (PVI): < - LESS THAN DETECTION LIMIT

SAMPLE TO COOES:

0001 - FILTERED SAMPLE (.45 MICRONS) 
GROUNDHATER QUALITY DATA BY LOCATION

SITE: AMBOI AMBROSIA LAKE

LOCATION: OTSO

MORTH COORDINATE: $\quad 53977.5$ FT

EAST COORDINATE: 60801.1 FT

06/10/80 TO $12 / 04 / 92$

REPORT DATE: 08/04/94

FORMATION OF CONPLETION: URANIUH MILL TAILINGS (TA)

HYDRAULIC FLOU RELATIOHSHIP: OH-SITE (O)

\begin{tabular}{|c|c|c|c|c|c|c|c|}
\hline PARAMETER MAME & LOS DATE & $\mid \begin{array}{c}\text { SAMPLE } \\
\text { ID }\end{array}$ & $\begin{array}{l}\text { UNITS OF } \\
\text { MEASURE }\end{array}$ & PVI & $\begin{array}{l}\text { PARAMETER } \\
\text { VALUE FLAGS }\end{array}$ & $\begin{array}{l}\text { DETECTION } \\
\text { LIMIT }\end{array}$ & $\begin{array}{l}\text { PARAMETER } \\
\text { UHCERTAINTY }\end{array}$ \\
\hline VANADIUM & $\begin{array}{l}09 / 26 / 85 \\
07 / 13 / 88\end{array}$ & $\begin{array}{l}0009 \\
0001\end{array}$ & $M G / L$ & $<$ & $\begin{array}{l}0.01 \\
0.30\end{array}$ & $\begin{array}{l}0.01 \\
0.01\end{array}$ & - \\
\hline ZINC & $\begin{array}{l}09 / 26 / 85 \\
07 / 13 / 88\end{array}$ & $\begin{array}{l}0001 \\
0001\end{array}$ & $M G / L$ & & $\begin{array}{l}0.053 \\
0.030\end{array}$ & $\begin{array}{l}0.005 \\
0.005\end{array}$ & - \\
\hline
\end{tabular}

PARAMETER VALUE IHDICATOR (PVI):

< - LESS THAN DETECTION LIMIT

SAMPLE 10 COOES:

OOOI - FILTERED SAMPLE (.45 MICROHS) 
GROUNDHATER OUALITY DATA BY LOCATION

SITE: AMBOI AMBROSIA LAKE

LOCATION: 0751

MORTH COORDIHATE: 53972.8 FT

EAST COORDINATE: 60799.4 FT

06/10/80 TO $12 / 04 / 92$

REPORT DATE: 08/04/94

FORMATION OF COMPLETION: URAKIUM MILL TAILIHGS (TA)

HYORAULIC FLOH RELATIONSHIP: OH-SITE (O)

\begin{tabular}{|c|c|c|c|c|c|c|c|}
\hline PARAMETER NAME & LOG DATE & $\underset{\text { SD }}{\text { SAMPLE }}$ & $\begin{array}{l}\text { UNITS OF } \\
\text { MEASURE }\end{array}$ & PVI & $\begin{array}{l}\text { PARAMETER } \\
\text { VALUE FLAGS }\end{array}$ & $\begin{array}{l}\text { DETECTION } \\
\text { LIMIT }\end{array}$ & $\begin{array}{l}\text { PARAMETER } \\
\text { UHCERTAINTY }\end{array}$ \\
\hline ALKALIHITY & $\begin{array}{l}10 / 09 / 85 \\
10 / 09 / 85 \\
10 / 09 / 85 \\
10 / 09 / 85 \\
10 / 09 / 85 \\
07 / 13 / 88\end{array}$ & $\begin{array}{l}0001 \\
0002 \\
0003 \\
0004 \\
0005 \\
0001\end{array}$ & MG/L CACO3 & & $\begin{array}{l}4501 . \\
4501 . \\
4501 . \\
4501 . \\
4501 . \\
4512 .\end{array}$ & $\begin{array}{l}\text { - } \\
\dot{-} \\
-\end{array}$ & $:$ \\
\hline ALCMIHUM & $\begin{array}{l}10 / 09 / 85 \\
10 / 09 / 85 \\
10 / 09 / 85 \\
10 / 09 / 85 \\
10 / 09 / 85 \\
07 / 13 / 88\end{array}$ & $\begin{array}{l}0001 \\
0002 \\
0003 \\
0004 \\
0005 \\
0001\end{array}$ & $M G / L$ & & $\begin{array}{l}0.2 \\
0.4 \\
0.5 \\
0.4 \\
0.3 \\
0.8\end{array}$ & $\begin{array}{l}0.1 \\
0.1 \\
0.1 \\
0.1 \\
0.1 \\
0.1\end{array}$ & 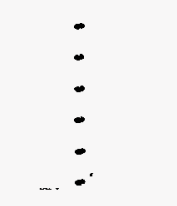 \\
\hline AMHONIUH & $\begin{array}{l}10 / 09 / 85 \\
10 / 09 / 85 \\
10 / 09 / 85 \\
10 / 09 / 85 \\
10 / 09 / 85\end{array}$ & $\begin{array}{l}0001 \\
0002 \\
0003 \\
0004 \\
0005\end{array}$ & $M G / L$ & & $\begin{array}{l}1.6 \\
1.6 \\
1.8 \\
1.6 \\
1.6\end{array}$ & $\begin{array}{l}0.1 \\
0.1 \\
0.1 \\
0.1 \\
0.1\end{array}$ & : \\
\hline ANTIMONY & $\begin{array}{l}10 / 09 / 85 \\
10 / 09 / 85 \\
10 / 09 / 85 \\
10 / 09 / 85 \\
10 / 09 / 85 \\
07 / 13 / 88\end{array}$ & $\begin{array}{l}0001 \\
0002 \\
0003 \\
0004 \\
0005 \\
0001\end{array}$ & MG/L & $\begin{array}{l}< \\
< \\
< \\
<\end{array}$ & $\begin{array}{l}0.003 \\
0.003 \\
0.003 \\
0.003 \\
0.003 \\
0.070\end{array}$ & $\begin{array}{l}0.003 \\
0.003 \\
0.003 \\
0.003 \\
0.003 \\
0.003\end{array}$ & $\begin{array}{l}- \\
- \\
- \\
-\end{array}$ \\
\hline ARSENIC & $\begin{array}{l}10 / 09 / 85 \\
10 / 09 / 85 \\
10 / 09 / 85 \\
10 / 09 / 85 \\
10 / 09 / 85 \\
07 / 13 / 88\end{array}$ & $\begin{array}{l}0001 \\
0002 \\
0003 \\
0004 \\
0005 \\
0001\end{array}$ & MG/L & & $\begin{array}{l}0.01 \\
0.01 \\
0.01 \\
0.01 \\
0.01 \\
0.10\end{array}$ & $\begin{array}{l}0.01 \\
0.01 \\
0.01 \\
0.01 \\
0.01 \\
0.01\end{array}$ & $\begin{array}{l}- \\
- \\
-\end{array}$ \\
\hline BARIUM & $\begin{array}{l}10 / 09 / 85 \\
10 / 09 / 85 \\
10 / 09 / 85 \\
10 / 09 / 85 \\
10 / 09 / 85 \\
07 / 13 / 88\end{array}$ & $\begin{array}{l}0001 \\
0002 \\
0003 \\
0004 \\
0005 \\
0001\end{array}$ & MG/L & $<$ & $\begin{array}{l}0.1 \\
0.1 \\
0.1 \\
0.1 \\
0.1 \\
0.1\end{array}$ & $\begin{array}{l}0.1 \\
0.1 \\
0.1 \\
0.1 \\
0.1 \\
0.1\end{array}$ & $\begin{array}{l}- \\
- \\
- \\
-\end{array}$ \\
\hline BORON & $\begin{array}{l}10 / 09 / 85 \\
10 / 09 / 85 \\
10 / 09 / 85 \\
10 / 09 / 85 \\
10 / 09 / 85 \\
07 / 13 / 88\end{array}$ & $\begin{array}{l}0001 \\
0002 \\
0003 \\
0004 \\
0005 \\
0001\end{array}$ & $M G / L$ & & $\begin{array}{l}0.4 \\
0.4 \\
0.3 \\
0.3 \\
0.4 \\
0.2\end{array}$ & $\begin{array}{l}0.1 \\
0.1 \\
0.1 \\
0.1 \\
0.1 \\
0.1\end{array}$ & $\begin{array}{l}- \\
- \\
- \\
-\end{array}$ \\
\hline CADHIUA & $\begin{array}{l}10 / 09 / 85 \\
10 / 09 / 85 \\
10 / 09 / 85 \\
10 / 09 / 85 \\
10 / 09 / 85 \\
07 / 13 / 88\end{array}$ & $\begin{array}{l}0001 \\
0002 \\
0003 \\
0004 \\
0005 \\
0001\end{array}$ & MG/L & $\begin{array}{l}< \\
< \\
< \\
<\end{array}$ & $\begin{array}{l}0.001 \\
0.001 \\
0.001 \\
0.001 \\
0.001 \\
0.006\end{array}$ & $\begin{array}{l}0.001 \\
0.001 \\
0.009 \\
0.009 \\
0.001 \\
0.001\end{array}$ & $\begin{array}{l}- \\
- \\
- \\
-\end{array}$ \\
\hline CALCILM & $10 / 09 / 85$ & 0001 & RG/L & & 1.94 & 0.01 & - \\
\hline
\end{tabular}

PARAMETER VALUE IHDICATOR (PVI): < - LESS THAH DETECTIOH LIMIT

SAMPLE ID COOES:

O001 - FILTERED SAMPLE (.45 MICROHS)

0002 - FILTERED REPLICATE SAMPLE (.45 MICRONS)

O003 - FILTERED REPLICATE SAMPLE (.45 MICRONS)

0004 - FILTERED REPLICATE SAMPLE (.45 MICRONS) 
GROUNDWATER QUALITY DATA BY LOCATION

SITE: AMBO1 AMBROSIA LAKE

LOCATIOH: 0751

MORTH COORDIHATE: 53972.8 FT

EAST COORDINATE: $\quad 60799.4$ FT

06/10/80 TO $12 / 04 / 92$

REPORT DATE: 08/04/94

FORMATION OF COMPLETION: URANIUN MILL TAILIHGS (TA)

HYDRAULIC FLON RELATIONSHIP: ON-SITE (O)

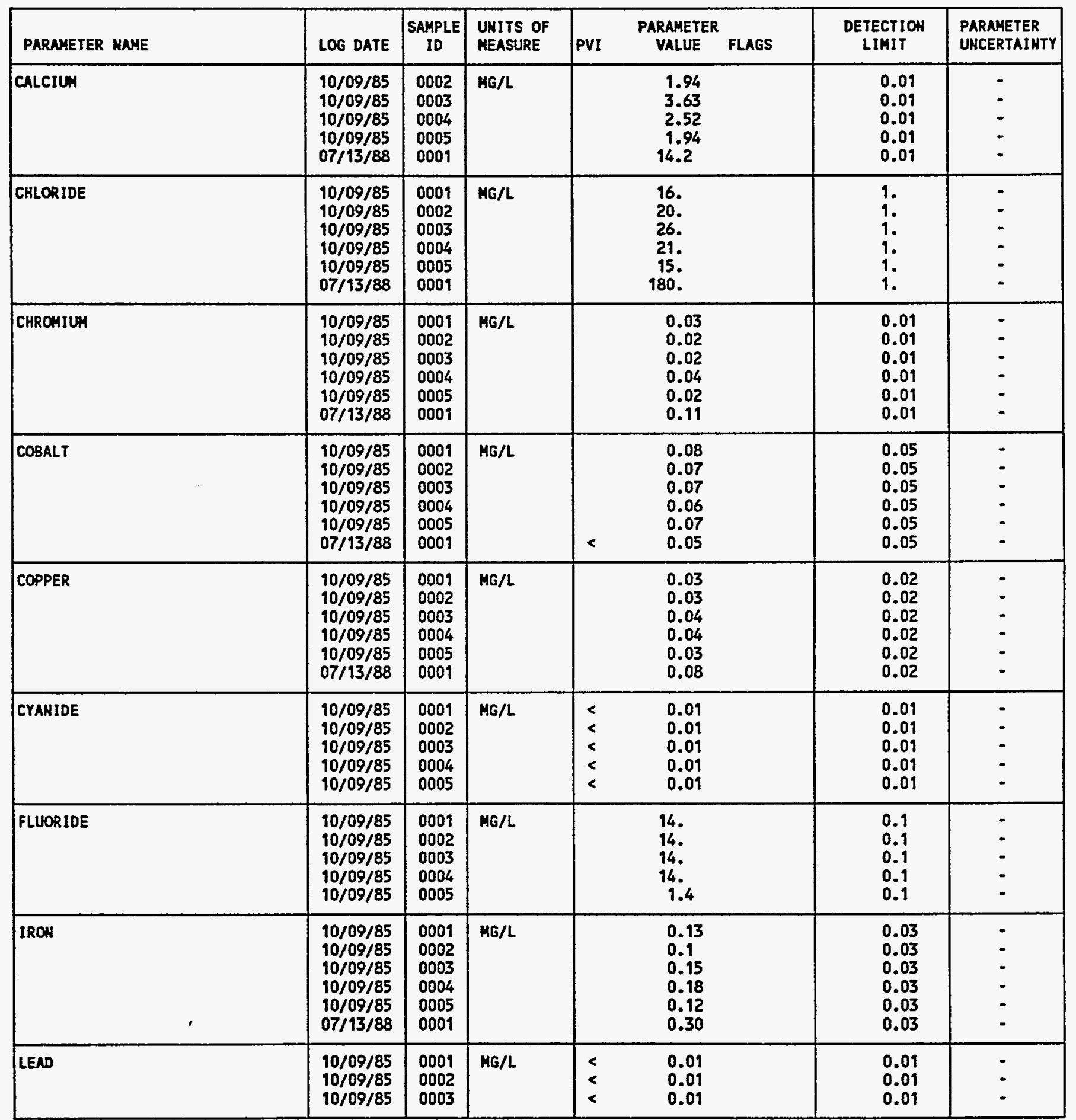

PARAMETER VALUE InDICATOR (PVI): < - LESS THAM DETECTION LIMIT

SAMPLE ID COOES:

0001 - FILTERED SAMPLE (.45 MICRONS)

0002 - FILTERED REPLICATE SAMPLE (.45 MICRONS)

0003 - FILTERED REPLICATE SAMPLE (.45 MICROWS)

0004 - FILTERED REPLICATE SAMPLE (.45 MICRONS) 
GROUNDWATER OUALITY DATA BY LOCATION

SITE: AMBOI AMBROSIA LAKE

LOCATION: 0751

HORTH COOROINATE: 53972.8 FT

EAST COORDIHATE: 60799.4 FT

06/10/80 TO 12/04/92

REPORT DATE: 08/04/94

FORHATION OF COHPLETIOH: URAHIUM MILL TAILIMGS (TA) HYDRAULIC FLOW RELATIOHSHIP: OH-SITE (O)

\begin{tabular}{|c|c|c|c|c|c|c|c|}
\hline PARAMETER MAME & LOG DATE & $\underset{10}{\text { SAMPLE }}$ & $\begin{array}{l}\text { UNITS OF } \\
\text { MEASURE }\end{array}$ & PVI & $\begin{array}{l}\text { Parameter } \\
\text { VALUE FLAGS }\end{array}$ & $\begin{array}{l}\text { DETECTIOH } \\
\text { LIMIT }\end{array}$ & $\begin{array}{l}\text { PARANETER } \\
\text { UNCERTAINTY }\end{array}$ \\
\hline LEAD & $\begin{array}{l}10 / 09 / 85 \\
10 / 09 / 85 \\
07 / 13 / 88\end{array}$ & $\begin{array}{l}0004 \\
0005 \\
0001\end{array}$ & $M G / L$ & $<$ & $\begin{array}{l}0.01 \\
0.01 \\
0.02\end{array}$ & $\begin{array}{l}0.01 \\
0.01 \\
0.01\end{array}$ & - \\
\hline LEAD-210 & $\begin{array}{l}10 / 09 / 85 \\
10 / 09 / 85 \\
10 / 09 / 85 \\
10 / 09 / 85\end{array}$ & $\begin{array}{l}0001 \\
0002 \\
0003 \\
0004\end{array}$ & $\mathrm{PCl} / \mathrm{L}$ & & $\begin{array}{l}52 . \\
48 . \\
90 . \\
52 .\end{array}$ & $\begin{array}{l}1.5 \\
1.5 \\
1.5 \\
1.5\end{array}$ & $\begin{array}{l}3 . \\
3 . \\
4 . \\
3 .\end{array}$ \\
\hline MAGHESIUH & $\begin{array}{l}10 / 09 / 85 \\
10 / 09 / 85 \\
10 / 09 / 85 \\
10 / 09 / 85 \\
10 / 09 / 85 \\
07 / 13 / 88\end{array}$ & $\begin{array}{l}0001 \\
0002 \\
0003 \\
0004 \\
0005 \\
0001\end{array}$ & $M G / L$ & & $\begin{array}{l}0.225 \\
0.196 \\
0.571 \\
0.282 \\
0.22 \\
31.5\end{array}$ & $\begin{array}{l}0.001 \\
0.001 \\
0.001 \\
0.001 \\
0.001 \\
0.001\end{array}$ & $\begin{array}{l}- \\
- \\
- \\
-\end{array}$ \\
\hline MAMGANESE & $\begin{array}{l}10 / 09 / 85 \\
10 / 09 / 85 \\
10 / 09 / 85 \\
10 / 09 / 85 \\
10 / 09 / 85 \\
07 / 13 / 88\end{array}$ & $\begin{array}{l}0001 \\
0002 \\
0003 \\
0004 \\
0005 \\
0001\end{array}$ & $M G / L$ & & $\begin{array}{l}0.03 \\
0.02 \\
0.02 \\
0.04 \\
0.04 \\
0.02\end{array}$ & $\begin{array}{l}0.01 \\
0.01 \\
0.01 \\
0.01 \\
0.01 \\
0.01\end{array}$ & $\begin{array}{l}: \\
: \\
- \\
-\end{array}$ \\
\hline MERCURY & $\begin{array}{l}10 / 09 / 85 \\
10 / 09 / 85 \\
10 / 09 / 85 \\
10 / 09 / 85 \\
10 / 09 / 85\end{array}$ & $\begin{array}{l}0001 \\
0002 \\
0003 \\
0004 \\
0005\end{array}$ & HG/L & $\begin{array}{l}< \\
< \\
< \\
< \\
<\end{array}$ & $\begin{array}{l}0.0002 \\
0.0002 \\
0.0002 \\
0.0002 \\
0.0002\end{array}$ & $\begin{array}{l}0.0002 \\
0.0002 \\
0.0002 \\
0.0002 \\
0.0002\end{array}$ & $\begin{array}{l}- \\
- \\
-\end{array}$ \\
\hline MOLYBDENUM & $\begin{array}{l}10 / 09 / 85 \\
10 / 09 / 85 \\
10 / 09 / 85 \\
10 / 09 / 85 \\
10 / 09 / 85 \\
07 / 13 / 88\end{array}$ & $\begin{array}{l}0001 \\
0002 \\
0003 \\
0004 \\
0005 \\
0001\end{array}$ & $M G / L$ & & $\begin{array}{c}99.0 \\
102 . \\
97.5 \\
102 . \\
104 . \\
118 .\end{array}$ & $\begin{array}{l}0.01 \\
0.01 \\
0.01 \\
0.01 \\
0.01 \\
0.01\end{array}$ & $\begin{array}{l}- \\
- \\
- \\
-\end{array}$ \\
\hline NICKEL & $\begin{array}{l}10 / 09 / 85 \\
10 / 09 / 85 \\
10 / 09 / 85 \\
10 / 09 / 85 \\
10 / 09 / 85 \\
07 / 13 / 88\end{array}$ & $\begin{array}{l}0001 \\
0002 \\
0003 \\
0004 \\
0005 \\
0001\end{array}$ & $M G / L$ & $<$ & $\begin{array}{l}0.13 \\
0.15 \\
0.18 \\
0.13 \\
0.13 \\
0.04\end{array}$ & $\begin{array}{l}0.04 \\
0.04 \\
0.04 \\
0.04 \\
0.04 \\
0.04\end{array}$ & $\begin{array}{l}- \\
- \\
- \\
-\end{array}$ \\
\hline NITRATE & $\begin{array}{l}10 / 09 / 85 \\
10 / 09 / 85 \\
10 / 09 / 85 \\
10 / 09 / 85 \\
10 / 09 / 85\end{array}$ & $\begin{array}{l}0001 \\
0002 \\
0003 \\
0004 \\
0005\end{array}$ & $M G / L$ & & $\begin{array}{l}3600 . \\
2600 \\
4900 \\
2500 \\
350\end{array}$ & $\begin{array}{l}1 . \\
1 . \\
1 . \\
1 .\end{array}$ & $\begin{array}{l}- \\
- \\
-\end{array}$ \\
\hline MITRITE & $\begin{array}{l}10 / 09 / 85 \\
10 / 09 / 85 \\
10 / 09 / 85 \\
10 / 09 / 85 \\
10 / 09 / 85\end{array}$ & $\begin{array}{l}0001 \\
0002 \\
0003 \\
0004 \\
0005\end{array}$ & $M G / L$ & $\begin{array}{l}< \\
< \\
< \\
< \\
<\end{array}$ & $\begin{array}{l}0.1 \\
0.1 \\
0.1 \\
0.1 \\
0.1\end{array}$ & $\begin{array}{l}0.1 \\
0.1 \\
0.1 \\
0.1 \\
0.1\end{array}$ & $\begin{array}{l}- \\
- \\
- \\
-\end{array}$ \\
\hline $\mathrm{PH}$ & $10 / 09 / 85$ & 0001 & su & & 10.13 & - & - \\
\hline
\end{tabular}

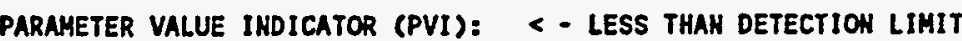

SAMPLE ID COOES:

0001 - FILTERED SAMPLE (.45 MICROHS)

0002 - FILTERED REPLICATE SAMPLE (.45 MICRONS)

0003 - FILTERED REPLICATE SAMPLE (.45 MICRONS)

0004 - FILTERED REPLICATE SAMPLE (.45 HICRONS) 
GROUNDWATER QUALITY DATA BY LOCATION

SITE: AMBO1 AMBROSIA LAKE

LOCATION: 0751

MORTH COORDINATE: $\quad 53972.8$ FT

EAST COORDINATE: 60799.4 FT

06/10/80 TO $12 / 04 / 92$

REPORT DATE: 08/04/94

FORMATION OF COAPLETION: URANIUH MILL TAILIMGS (TA) HYDRAULIC FLON RELATIONSHIP: ON-SITE (O)

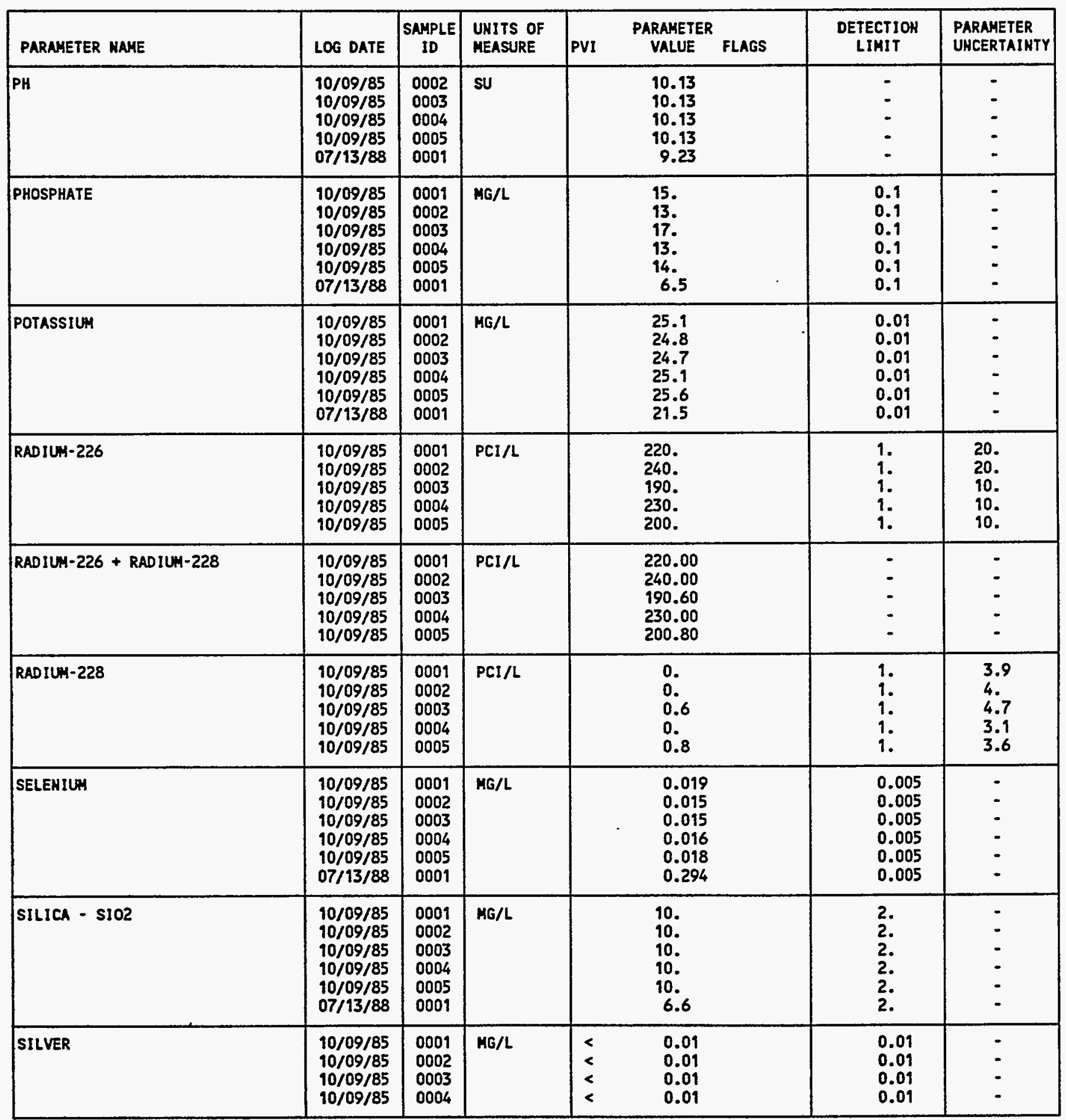

PARAMETER VALUE INDICATOR (PVI): < - LESS THAN DETECTIOH LIMIT

SAMPLE ID COOES:

0001 - FILTERED SAMPLE (.45 MICRONS)

0002 - FILTERED REPLICATE SAMPLE (.45 MICRONS)

D003 - FILTERED REPLICATE SAMPLE (.45 MICRONS)

0004 - FILTERED REPLICATE SAMPLE (.45 MICRONS) 
GROUNDWATER QUALITY DATA BY LOCATION

SITE: AMBO1 AMBROSIA LAKE

LOCATION: 0751

MORTH COORDINATE: 53972.8 FT

EAST COORDINATE: 60799.4 FT

06/10/80 TO $12 / 04 / 92$

REPORT DATE: $08 / 04 / 94$

FORMATION OF COMPLETIOH: URANIUM MILL TAILINGS (TA)

HYDRAULIC FLOW RELATIONSHIP: ON-SITE (O)

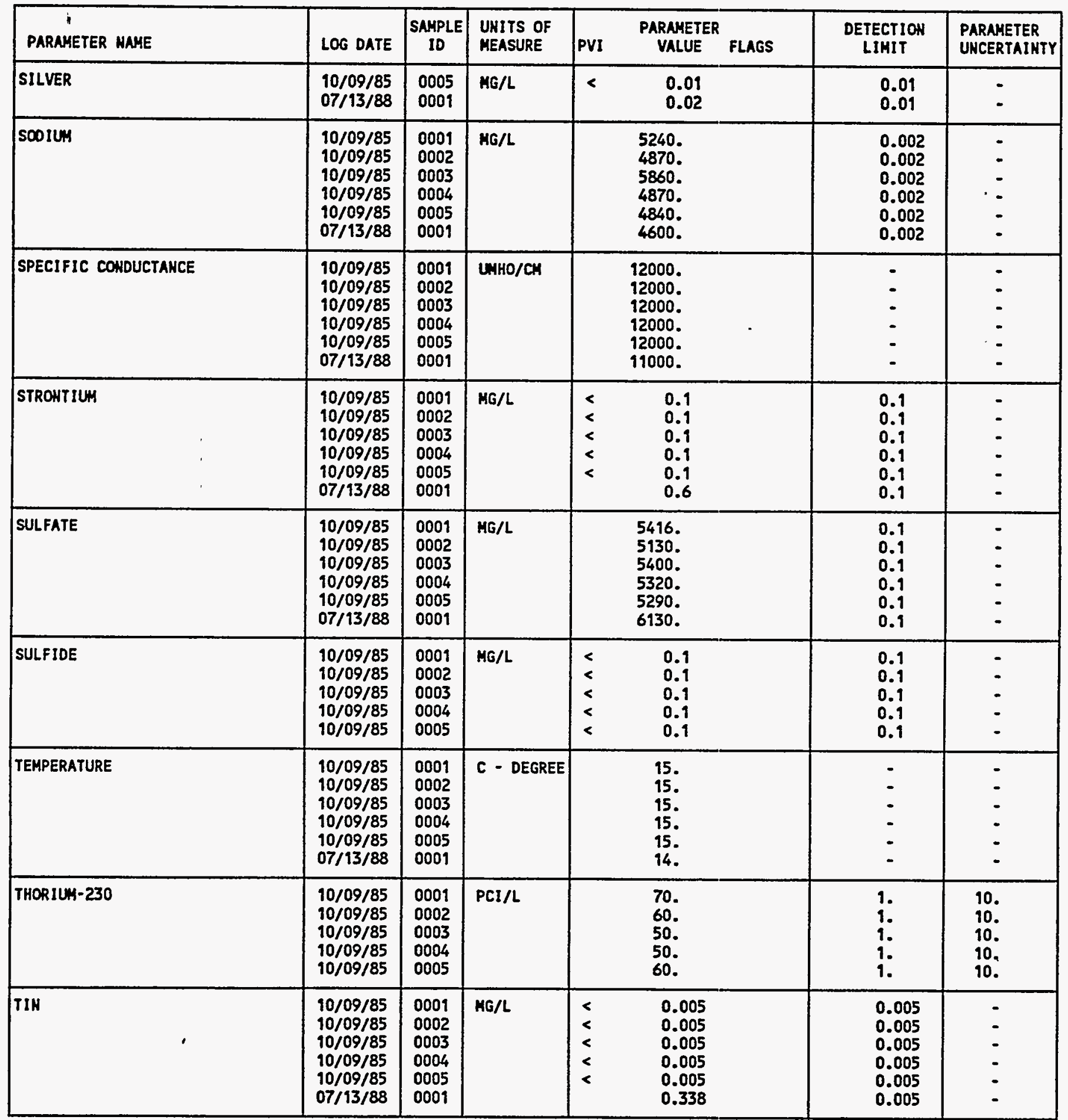

PARAMETER VALUE INDICATOR (PVI): < - LESS THAH DETECTION LIHIT

SAMPLE ID COOES:

0009 - FILVERED SAMPLE (.45 MICRONS) a

0002 - FILTERED REPLICATE SAMPLE (.45 MICROMS)

0003 - FILUERED REPLICATE SAMPLE (.45 MICRONS)

0004 - FILTERED REPLICATE SAMPLE (.45 MICRONS) 
GROUNDWATER QUALITY DATA BY LOCATION

SITE: AMBOI AMBROSIA LAKE

LOCATION: 0751

NORTH COORDINATE: $\quad 53972.8$ FT

EAST COORDINATE: $\quad 60799.4$ FT

06/10/80 TO $12 / 04 / 92$

REPORT DATE: 08/04/94

FORMATION OF COMPLETIOH: URANIUM MILL TAILINGS (TA)

HYORAULIC FLOU RELATIOHSHIP: OH-SITE (O)

\begin{tabular}{|c|c|c|c|c|c|c|c|}
\hline PARAMETER MAME & LOG DATE & $\underset{10}{\text { SAMPLE }}$ & $\begin{array}{l}\text { UNITS OF } \\
\text { MEASURE }\end{array}$ & PVI & $\begin{array}{l}\text { PARAMETER } \\
\text { VALUE FLAGS }\end{array}$ & $\begin{array}{l}\text { DETECTION } \\
\text { LIMIT }\end{array}$ & $\begin{array}{l}\text { PARAMETER } \\
\text { UHCERTAIHTY }\end{array}$ \\
\hline TOTAL OISSOLVED SOLIDS & $\begin{array}{l}10 / 09 / 85 \\
10 / 09 / 85 \\
10 / 09 / 85 \\
10 / 09 / 85 \\
10 / 09 / 85 \\
07 / 13 / 88\end{array}$ & $\begin{array}{l}0001 \\
0002 \\
0003 \\
0004 \\
0005 \\
0001\end{array}$ & $M G / L$ & & $\begin{array}{l}13300 . \\
12700 . \\
13100 . \\
13000 . \\
13000 \\
14300\end{array}$ & $\begin{array}{l}10 . \\
10 . \\
10 . \\
10 . \\
10 . \\
10 .\end{array}$ & : \\
\hline VAKADIUM & $\begin{array}{l}10 / 09 / 85 \\
10 / 09 / 85 \\
10 / 09 / 85 \\
10 / 09 / 85 \\
10 / 09 / 85 \\
07 / 13 / 88\end{array}$ & $\begin{array}{l}0001 \\
0002 \\
0003 \\
0004 \\
0005 \\
0001\end{array}$ & $M G / L$ & $\begin{array}{l}< \\
< \\
< \\
< \\
<\end{array}$ & $\begin{array}{l}0.01 \\
0.01 \\
0.01 \\
0.01 \\
0.01 \\
0.15\end{array}$ & $\begin{array}{l}0.01 \\
0.01 \\
0.01 \\
0.01 \\
0.01 \\
0.01\end{array}$ & : \\
\hline ZIKC & $\begin{array}{l}10 / 09 / 85 \\
10 / 09 / 85 \\
10 / 09 / 85 \\
10 / 09 / 85 \\
10 / 09 / 85 \\
07 / 13 / 88\end{array}$ & $\begin{array}{l}0001 \\
0002 \\
0003 \\
0004 \\
0005 \\
0001\end{array}$ & MG/L & & $\begin{array}{l}0.044 \\
0.017 \\
0.046 \\
0.031 \\
0.025 \\
0.018\end{array}$ & $\begin{array}{l}0.005 \\
0.005 \\
0.005 \\
0.005 \\
0.005 \\
0.005\end{array}$ & $\begin{array}{l}- \\
- \\
- \\
-\end{array}$ \\
\hline
\end{tabular}

PARAMETER VALUE INDICATOR (PVI): < - LESS THAN DETECTION LIMIT

SAMPLE ID COOES:

0009 - FILTERED SAMPLE (.45 MICRONS)

0002 - FILTERED REPLICATE SAMPLE (.45 MICROHS)

0003 - FILTERED REPLICATE SAMPLE (.45 MICRONS)

0004 - FILTERED REPLICATE SAMPLE (.45 MICRONS) 
GROUNDWATER OUALITY DATA BY LOCATIOH

SITE: AMBOI AMBROSIA LAKE

LOCATIOH: 0752

NORTH COORDINATE: 53802.6 FT

EAST COORDIHATE: 60808.1 FT

06/10/80 TO $12 / 04 / 92$

REPORT DATE: $08 / 04 / 94$

FORHATIOH OF COMPLETION: URAMIUH MILL TAILIHGS (TA)

HYDRAULIC FLON RELATIONSHIP: ON-SITE (O)

\begin{tabular}{|c|c|c|c|c|c|c|c|}
\hline PARAMETER MAME & LOG DATE & $\begin{array}{c}\text { SAMPLE } \\
\text { ID }\end{array}$ & $\begin{array}{l}\text { UNITS OF } \\
\text { MEASURE }\end{array}$ & PVI & $\begin{array}{l}\text { Parameter } \\
\text { VALUE FLAGS }\end{array}$ & $\begin{array}{l}\text { DETECTION } \\
\text { LIMIT }\end{array}$ & $\begin{array}{l}\text { PARAMETER } \\
\text { UNCERTAIKTY }\end{array}$ \\
\hline ALKALIKITY & $\begin{array}{l}09 / 26 / 85 \\
07 / 13 / 88\end{array}$ & $\begin{array}{l}0001 \\
0009\end{array}$ & MG/L CACO3 & & $\begin{array}{l}6828 . \\
1637\end{array}$ & - & $\dot{-}$ \\
\hline ALUMIHUM & $\begin{array}{l}09 / 26 / 85 \\
07 / 13 / 88\end{array}$ & $\begin{array}{l}0001 \\
0001\end{array}$ & $M G / L$ & & $\begin{array}{l}0.4 \\
0.2\end{array}$ & $\begin{array}{l}0.1 \\
0.1\end{array}$ & 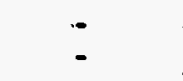 \\
\hline Amonius & $09 / 26 / 85$ & 0001 & $M G / L$ & $<$ & 0.1 & 0.1 & - \\
\hline AHTIMONY & $\begin{array}{l}09 / 26 / 85 \\
07 / 13 / 88\end{array}$ & $\begin{array}{l}0001 \\
0001\end{array}$ & MG/L & $<$ & $\begin{array}{l}0.003 \\
0.088\end{array}$ & $\begin{array}{l}0.003 \\
0.003\end{array}$ & - \\
\hline ARSEHIC & $\begin{array}{l}09 / 26 / 85 \\
07 / 13 / 88\end{array}$ & $\begin{array}{l}0001 \\
0001\end{array}$ & MG/L & $<$ & $\begin{array}{l}0.01 \\
0.11\end{array}$ & $\begin{array}{l}0.01 \\
0.01\end{array}$ & $\dot{-}$ \\
\hline BARIUM & $\begin{array}{l}09 / 26 / 85 \\
07 / 13 / 88\end{array}$ & $\begin{array}{l}0001 \\
0001\end{array}$ & $M G / L$ & $<$ & $\begin{array}{l}0.1 \\
0.1\end{array}$ & $\begin{array}{l}0.1 \\
0.1\end{array}$ & - \\
\hline BOROH & $\begin{array}{l}09 / 26 / 85 \\
07 / 13 / 88\end{array}$ & $\begin{array}{l}0001 \\
0001\end{array}$ & $M G / L$ & & $\begin{array}{l}0.4 \\
0.2\end{array}$ & $\begin{array}{l}0.1 \\
0.1\end{array}$ & - \\
\hline BROMIOE & $07 / 13 / 88$ & 0001 & HG/L & $<$ & 0.1 & 0.1 & $\cdots$ \\
\hline CADHIUH & $\begin{array}{l}09 / 26 / 85 \\
07 / 13 / 88\end{array}$ & $\begin{array}{l}0001 \\
0001\end{array}$ & MG/L & $<$ & $\begin{array}{l}0.001 \\
0.006\end{array}$ & $\begin{array}{l}0.001 \\
0.001\end{array}$ & - \\
\hline CALCIUA & $\begin{array}{l}09 / 26 / 85 \\
07 / 13 / 88\end{array}$ & $\begin{array}{l}0001 \\
0001\end{array}$ & $M G / L$ & & $\begin{array}{l}4.28 \\
4.05\end{array}$ & $\begin{array}{l}0.09 \\
0.01\end{array}$ & - \\
\hline CHLORIDE & $\begin{array}{l}09 / 26 / 85 \\
07 / 13 / 88\end{array}$ & $\begin{array}{l}0001 \\
0001\end{array}$ & HG/L & & $\begin{array}{l}6 . \\
280\end{array}$ & i. & - \\
\hline CHROMIUM & $\begin{array}{l}09 / 26 / 85 \\
07 / 13 / 88\end{array}$ & $\begin{array}{l}0001 \\
0001\end{array}$ & HG/L & & $\begin{array}{l}0.02 \\
0.04\end{array}$ & $\begin{array}{l}0.01 \\
0.01\end{array}$ & - \\
\hline COBALT & $\begin{array}{l}09 / 26 / 85 \\
07 / 13 / 88\end{array}$ & $\begin{array}{l}0009 \\
0001\end{array}$ & $M G / L$ & $<$ & $\begin{array}{l}0.07 \\
0.05\end{array}$ & $\begin{array}{l}0.05 \\
0.05\end{array}$ & - \\
\hline COPPER & $\begin{array}{l}09 / 26 / 85 \\
07 / 13 / 88\end{array}$ & $\begin{array}{l}0001 \\
0001\end{array}$ & MG/L & & $\begin{array}{l}0.04 \\
0.03\end{array}$ & $\begin{array}{l}0.02 \\
0.02\end{array}$ & : \\
\hline CYAHIDE & $09 / 26 / 85$ & 0001 & $M G / L$ & $<$ & 0.01 & 0.01 & - \\
\hline FLUORIOE & $\begin{array}{l}09 / 26 / 85 \\
07 / 13 / 88\end{array}$ & $\begin{array}{l}0001 \\
0001\end{array}$ & MG/L & & $\begin{array}{l}16 . \\
4.3\end{array}$ & $\begin{array}{l}0.1 \\
0.1\end{array}$ & - \\
\hline GROSS ALPHA & $07 / 13 / 88$ & 0001 & $\mathrm{PCI} / \mathrm{L}$ & & 3200. & 0.2 & 300. \\
\hline GROSS BETA & $07 / 13 / 88$ & 0001 & PCI/L & & 1100. & 1. & 100. \\
\hline IRON & $\begin{array}{l}09 / 26 / 85 \\
07 / 13 / 88\end{array}$ & $\begin{array}{l}0001 \\
0001\end{array}$ & MG/L & & $\begin{array}{l}0.18 \\
0.07\end{array}$ & $\begin{array}{l}0.03 \\
0.03\end{array}$ & - \\
\hline LEAD & $\begin{array}{l}09 / 26 / 85 \\
07 / 13 / 88\end{array}$ & $\begin{array}{l}0001 \\
0001\end{array}$ & MG/L & $<$ & $\begin{array}{l}0.01 \\
0.03\end{array}$ & $\begin{array}{l}0.01 \\
0.01\end{array}$ & $=$ \\
\hline LEAD-210 & $09 / 26 / 85$ & 0001 & PCI/L & & 66. & 1.5 & 3. \\
\hline MAGHESIUM & $09 / 26 / 85$ & 0001 & $M G / L$ & & 2.3 & 0.001 & - \\
\hline
\end{tabular}

PARAMETER VALUE IMDICATOR (PVI): < - LESS THAN DETECTION LIMIT

SAMPLE ID ODOES:

0001 - FILTERED SAMPLE (.45 MICRONS) 
GROUNDHATER QUALITY DATA BY LOCATION

SITE: AMBO1 AMBROSIA LAKE

LOCATION: 0752

MORTH COORDINATE: 53802.6 FT

EAST COORDINATE: $\quad 60808.1$ FT

06/10/80 TO $12 / 04 / 92$

REPORT DATE: 08/04/94

FORMATION OF COMPLETION: URANIUA MILL TAILIHGS (TA)

HYDRAULIC FLON RELATIONSHIP: ON-SITE (O)

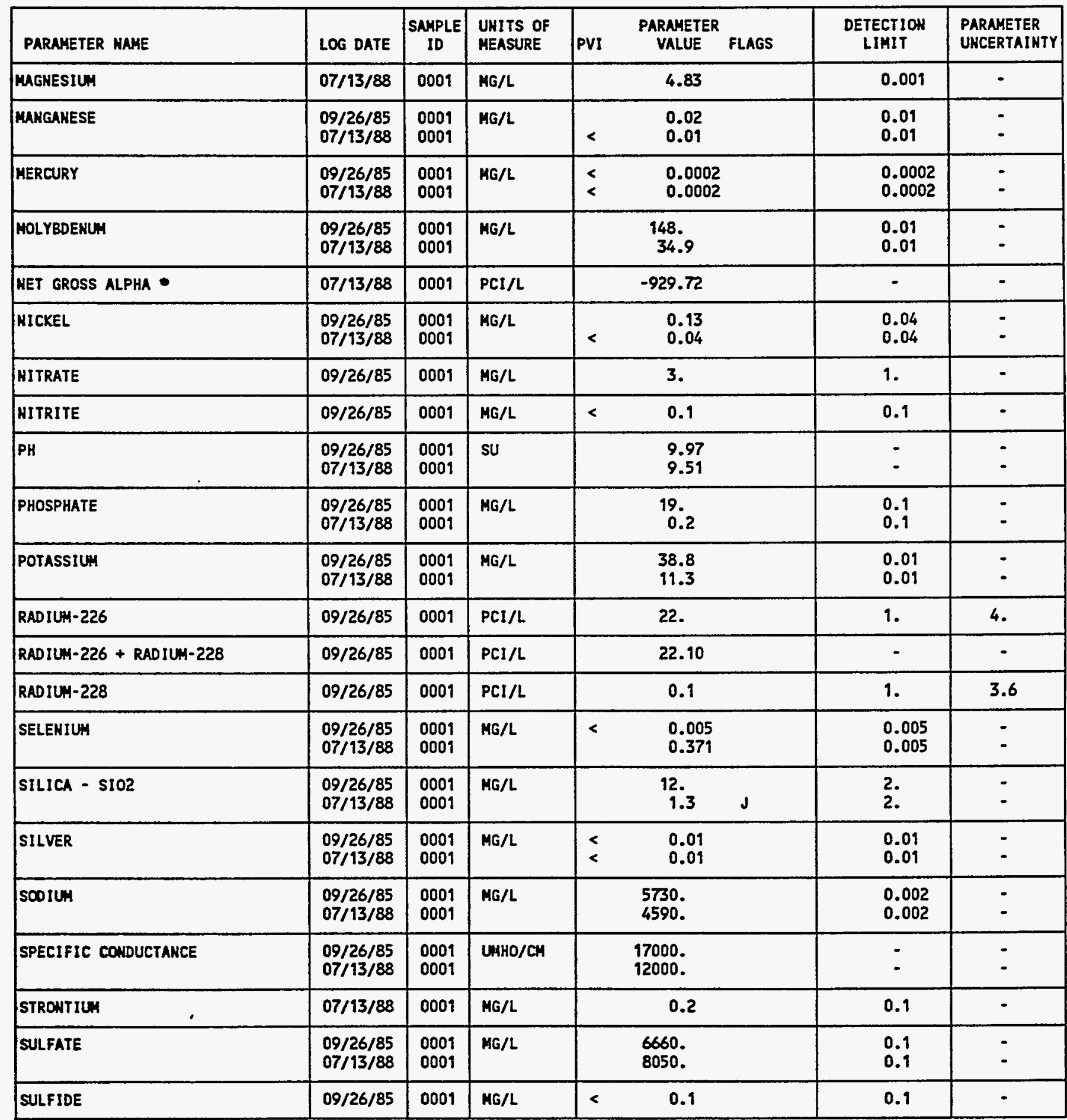

- NeT gROSS ALPHA (GROSS ALPHA - URANIUM) WITH 1 MG URAKIUM = 686 PCI

parameter Value indicator (PVI): < - less thah detection limit 
GROUHDHATER QUALITY DATA BY LOCATION

SITE: AMBO1 AMBROSIA LAKE

LOCATIOH: 0752

HORTH COORDIHATE: 53802.6 FT

EAST COORDIHATE: 60808.1 FT

06/10/80 TO $12 / 04 / 92$

REPORT DATE: 08/04/94

FORHATION OF COMPLETION: URAHIUM MILL TAILIHGS (TA)

HYDRAULIC FLON RELATIOHSHIP: ON-SITE (O)

\begin{tabular}{|c|c|c|c|c|c|c|c|}
\hline PARAMETER NAME & LOG DATE & $\underset{\text { SAMPLE }}{\text { ID }}$ & $\begin{array}{l}\text { UNITS OF } \\
\text { MEASURE }\end{array}$ & PVI & $\begin{array}{l}\text { PARAMETER } \\
\text { VALUE fLAGS }\end{array}$ & $\begin{array}{l}\text { DETECTION } \\
\text { LIMIT }\end{array}$ & $\begin{array}{l}\text { PARAMETER } \\
\text { UNCERTAJNTY }\end{array}$ \\
\hline TEMPERATURE & $\begin{array}{l}09 / 26 / 85 \\
07 / 13 / 88\end{array}$ & $\begin{array}{l}0001 \\
0001\end{array}$ & C - DEGREE & & $\begin{array}{l}16 . \\
16 .\end{array}$ & - & $\therefore$ \\
\hline THORIUH-230 & $09 / 26 / 85$ & 0001 & $\mathrm{PCI} / \mathrm{L}$ & & 420. & 1. & 30. \\
\hline TIH & $\begin{array}{l}09 / 26 / 85 \\
07 / 13 / 88\end{array}$ & $\begin{array}{l}0001 \\
0001\end{array}$ & MG/L & $<$ & $\begin{array}{l}0.005 \\
0.323\end{array}$ & $\begin{array}{l}0.005 \\
0.005\end{array}$ & $\dot{-}$ \\
\hline TOTAL DISSOLVED SOLIDS & $\begin{array}{l}09 / 26 / 85 \\
07 / 13 / 88\end{array}$ & $\begin{array}{l}0001 \\
0001\end{array}$ & $M G / L$ & & $\begin{array}{l}17200 \\
14000 .\end{array}$ & $\begin{array}{l}10 . \\
10 .\end{array}$ & - \\
\hline URAKIUM & $\begin{array}{l}09 / 26 / 85 \\
07 / 13 / 88\end{array}$ & $\begin{array}{l}0001 \\
0001\end{array}$ & MG/L & & $\begin{array}{l}8.65 \\
6.02\end{array}$ & $\begin{array}{l}0.003 \\
0.003\end{array}$ & $\dot{-}$ \\
\hline VAHADIUA & $\begin{array}{l}09 / 26 / 85 \\
07 / 13 / 88\end{array}$ & $\begin{array}{l}0001 \\
0001\end{array}$ & $M G / L$ & $<$ & $\begin{array}{l}0.01 \\
0.04\end{array}$ & $\begin{array}{l}0.01 \\
0.01\end{array}$ & $\dot{-}$ \\
\hline ZINC & $\begin{array}{l}09 / 26 / 85 \\
07 / 13 / 88\end{array}$ & $\begin{array}{l}0001 \\
0001\end{array}$ & $M G / L$ & & $\begin{array}{l}0.078 \\
0.037\end{array}$ & $\begin{array}{l}0.005 \\
0.005\end{array}$ & - \\
\hline
\end{tabular}

PARAMETER VALUE IHDICATOR (PVI): < - LESS THAN DETECTIOH LIMIT

SAMPLE ID COOES:

0001 - FILTERED SAMPLE (.45 MICRONS) 
GROUNDWATER QUALITY DATA BY LOCATION

SITE: AMBOI AMBROSIA LAKE

LOCATION: 0759

NORTH COORDINATE: 53623.2 FT

EAST COORDINATE: 60219.3 FT

06/10/80 TO $12 / 04 / 92$

REPORT DATE: 08/04/94

FORMATION OF COMPLETION: URAHIUY HILL TAILINGS (TA)

HYDRAULIC FLON RELATIONSHIP: ON-SITE (O)

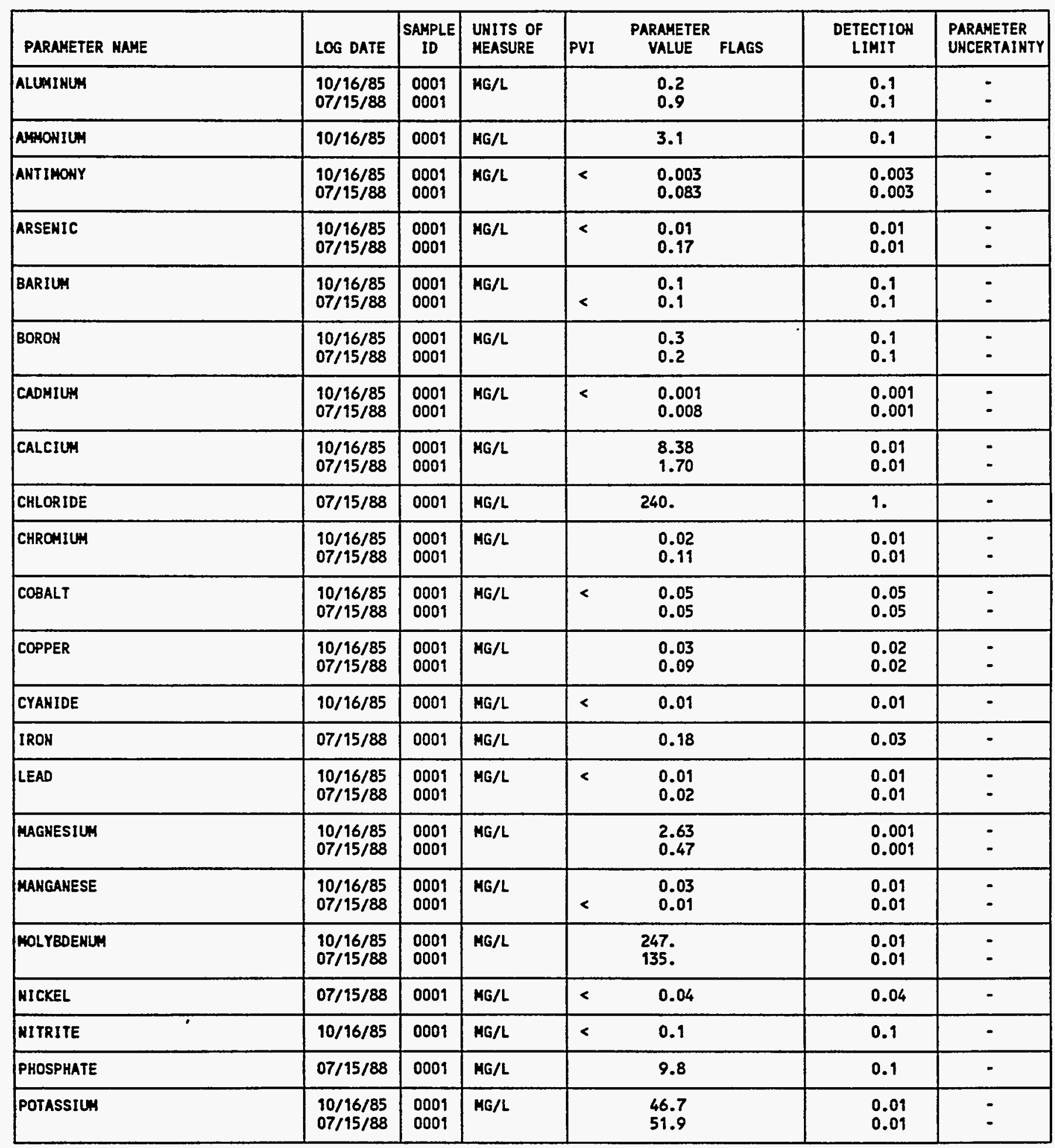

PARAMETER VALUE IMDICATOR (PVI): < - LESS THAN DETECTION LIMIT

SAMPLE ID COOES:

0001 - FILTERED SAMPLE (.45 MICRONS) 
GROUHDWATER QUALITY DATA BY LOCATIOH

SITE: AMBO1 AMBROSIA LAKE

LOCATION: 0759

HORTH COORDIHATE: 53623.2 FT

EAST COORDIHATE: 60219.3 FT

06/10/80 TO 12/04/92

REPORT DATE: 08/04/94

FORMATION OF COMPLETION: URANIUM MILL TAILIKGS (TA)

HYDRAULIC FLON RELATIONSHIP: OH-SITE (O)

\begin{tabular}{|c|c|c|c|c|c|c|c|}
\hline PARAMETER NAME & LOG DATE & $\underset{\text { SD }}{\text { SNPLE }}$ & $\begin{array}{l}\text { UNITS OF } \\
\text { MEASURE }\end{array}$ & PVI & $\begin{array}{l}\text { PARAMETER: } \\
\text { VALUE FLAGS }\end{array}$ & $\begin{array}{l}\text { DETECTION } \\
\text { LIMIT }\end{array}$ & $\begin{array}{l}\text { PARÄHETER } \\
\text { UNCERTAINTY }\end{array}$ \\
\hline SELENIUH & $\begin{array}{l}10 / 16 / 85 \\
07 / 15 / 88\end{array}$ & $\begin{array}{l}0001 \\
0001\end{array}$ & MG/L & & $\begin{array}{l}0.017 \\
0.403\end{array}$ & $\begin{array}{l}0.005 \\
0.005\end{array}$ & $\dot{-}$ \\
\hline SILICA - S102 & $07 / 15 / 88$ & 0001 & MG/L & & 10.7 & 2. & - \\
\hline SILVER & $\begin{array}{l}10 / 16 / 85 \\
07 / 15 / 88\end{array}$ & $\begin{array}{l}0001 \\
0001\end{array}$ & MG/L & $<$ & $\begin{array}{l}0.01 \\
0.02\end{array}$ & $\begin{array}{l}0.01 \\
0.01\end{array}$ & - \\
\hline SCOIUH & $\begin{array}{l}10 / 16 / 85 \\
07 / 15 / 88\end{array}$ & $\begin{array}{l}0001 \\
0001\end{array}$ & $M G / L$ & & $\begin{array}{l}9880 . \\
5780 .\end{array}$ & $\begin{array}{l}0.002 \\
0.002\end{array}$ & - \\
\hline STRONTIUM & $07 / 15 / 88$ & 0001 & MG/L & & 0.2 & 0.1 & - \\
\hline SULFATE & $\begin{array}{l}10 / 16 / 85 \\
07 / 15 / 88\end{array}$ & $\begin{array}{l}0001 \\
0001\end{array}$ & MG/L & & $\begin{array}{r}11000 \\
8200 .\end{array}$ & $\begin{array}{l}0.1 \\
0.1\end{array}$ & $\because$ \\
\hline SULFIDE & $10 / 16 / 85$ & 0001 & MG/L & $<$ & 0.1 & 0.1 & - \\
\hline TIN & $\begin{array}{l}10 / 16 / 85 \\
07 / 15 / 88\end{array}$ & $\begin{array}{l}0001 \\
0001\end{array}$ & MG/L & $<$ & $\begin{array}{l}0.005 \\
0.400\end{array}$ & $\begin{array}{l}0.005 \\
0.005\end{array}$ & $\dot{-}$ \\
\hline TOTAL DISSOLVED SOLIDS & $\begin{array}{l}10 / 16 / 85 \\
07 / 15 / 88\end{array}$ & $\begin{array}{l}0001 \\
0001\end{array}$ & MG/L & & $\begin{array}{l}25800 \\
18800\end{array}$ & $\begin{array}{l}10 . \\
10 .\end{array}$ & - \\
\hline URAHIUM & $07 / 15 / 88$ & 0001 & $M G / L$ & & 12.6 & 0.003 & - \\
\hline VAHADIUN & $\begin{array}{l}10 / 16 / 85 \\
07 / 15 / 88\end{array}$ & $\begin{array}{l}0001 \\
0001\end{array}$ & MG/L & $<$ & $\begin{array}{l}0.01 \\
0.17\end{array}$ & $\begin{array}{l}0.01 \\
0.01\end{array}$ & - \\
\hline ZINC & $\begin{array}{l}10 / 16 / 85 \\
07 / 15 / 88\end{array}$ & $\begin{array}{l}0001 \\
0001\end{array}$ & MG/L. & & $\begin{array}{l}0.025 \\
0.014\end{array}$ & $\begin{array}{l}0.005 \\
0.005\end{array}$ & - \\
\hline
\end{tabular}

PARAMETER VALUE INDICATOR (PVI): < - LESS THAN DETECTION LIMIT

SAMPLE ID COOES:

0001 - FIL'TERED SAMPLE (.45 MICROHS) 
GROUNDWATER QUALITY DATA BY LOCATION

SITE: AMBO1 AMBROSIA LAKE

LOCATION: 0773

NORTH COORDINATE: $55319.5 \mathrm{FT}$

EAST COORDINATE: 61688.9 FT

06/10/80 TO 12/04/92

REPORT DATE: 08/04/94

FORMATION OF COMPLETION: TRES HERMANOS - CI SAMDSTONE (TO)

HYDRAULIC FLON RELATIONSHIP: DOUN GRADIENT (D)

\begin{tabular}{|c|c|c|c|c|c|c|c|}
\hline PARAMETER NAME & LOG DATE & $\begin{array}{c}\text { SAMPLE } \\
\text { ID }\end{array}$ & $\begin{array}{l}\text { UNITS OF } \\
\text { MEASURE }\end{array}$ & PVI & $\begin{array}{l}\text { PARAMETER } \\
\text { VALUE FLAGS }\end{array}$ & $\begin{array}{l}\text { DETECTIOH } \\
\text { LIMIT }\end{array}$ & $\begin{array}{l}\text { PARAMETER } \\
\text { UHCERTAINTY }\end{array}$ \\
\hline ALKALINITY & $05 / 14 / 86$ & 0001 & MG/L CACO3 & & 2735 & - & $\cdot$ \\
\hline BORON & $05 / 14 / 86$ & 0001 & MG/L & & 0.1 & 0.1 & - \\
\hline CALCIUM & $05 / 14 / 86$ & 0001 & $M G / L$ & & 168. & 0.01 & - \\
\hline CHLORIDE & $05 / 14 / 86$ & 0001 & HG/L & & 10. & 1. & - \\
\hline CHROMIUN & $05 / 14 / 86$ & 0001 & $M G / L$ & & 0.05 & 0.01 & - \\
\hline COBALT & $05 / 14 / 86$ & 0001 & HG/L & & 0.07 & 0.05 & - \\
\hline MAGKESIUM & $05 / 14 / 86$ & 0001 & HG/L & & 0.022 & 0.001 & - \\
\hline MANGAKESE & $05 / 14 / 86$ & 0001 & $M G / L$ & & 0.03 & 0.01 & - \\
\hline MOLYBDENUM & $05 / 94 / 86$ & 0001 & $\mathrm{MG} / \mathrm{L}$ & & 0.32 & 0.01 & - \\
\hline MITRATE & $05 / 14 / 86$ & 0001 & $M G / L$ & & 2. & 1. & - \\
\hline PH & $05 / 14 / 86$ & 0001 & SU & & 12.41 & - & - \\
\hline POTASSIUH & $05 / 14 / 86$ & 0001 & HG/L & & 56.5 & 0.01 & - \\
\hline RADIUH-226 & $05 / 14 / 86$ & 0001 & PCI/L & & 22. & 1. & 2. \\
\hline TEMPERATURE & $05 / 14 / 86$ & 0001 & C - DEGREE & & 17. & - & - \\
\hline THORIUM-230 & $05 / 14 / 86$ & 0001 & PCI/L & & 0. & 1. & 0.1 \\
\hline TOTAL DISSOLVED SOLIDS & $05 / 14 / 86$ & 0001 & $M G / L$ & & 3040 & 10. & - \\
\hline URANIUN & $05 / 14 / 86$ & 0001 & MG/L & $<$ & $0.0003 \mathrm{~J}$ & 0.003 & - \\
\hline VANRDIUA & $05 / 14 / 86$ & 0001 & MG/L & & 0.21 & 0.01 & - \\
\hline
\end{tabular}

PARAMETER VALUE IHDICATOR (PVI): < - LESS THAN DETECTION LIMIT

SAMPLE ID COOES

0001 - FILTERED SAMPLE (.45 MICROHS)

OTHER PARAMETER VALUE fLAgS:

J - estimated VALUe 
GROUHDWATER QUALITY DATA BY LOCATION

SITE: AMBOI AMBROSIA LAKE

LOCATIOH: OTT7

MORTH COORDIHATE: 52881.7 FT

EAST COORDIHATE: $\quad 60119.8$ FT

06/10/80 TO $12 / 04 / 92$

REPORT OATE: $08 / 04 / 94$

FORMATION OF COMPLETION: TRES HERMAMOS - B SAMDSTOHE (TB)

HYDRAULIC FLON RELATIONSHIP: UPGRADIEHT (U)

\begin{tabular}{|c|c|c|c|c|c|c|c|c|}
\hline PARAMETER NAME & LOG DATE & $\begin{array}{c}\text { SAMPLE } \\
\text { ID }\end{array}$ & $\begin{array}{l}\text { UNITS OF } \\
\text { MEASURE }\end{array}$ & PVI & $\begin{array}{l}\text { PARAMETER } \\
\text { VALUE }\end{array}$ & FLAGS & $\begin{array}{l}\text { DETECTION } \\
\text { LIMIT }\end{array}$ & $\begin{array}{l}\text { PARAM̆ETER } \\
\text { UNCERTAINTY }\end{array}$ \\
\hline ALKALIHITY & $\begin{array}{l}04 / 06 / 88 \\
01 / 12 / 89 \\
09 / 19 / 89 \\
04 / 11 / 90 \\
01 / 22 / 91\end{array}$ & $\begin{array}{l}0001 \\
0001 \\
0001 \\
0001 \\
0001\end{array}$ & $M G / L C A C O 3$ & & $\begin{array}{l}337 . \\
256 . \\
105 . \\
69 . \\
47 .\end{array}$ & G & $\begin{array}{l}- \\
\dot{-} \\
\dot{-} \\
-\end{array}$ & $\begin{array}{l}- \\
- \\
-\end{array}$ \\
\hline ALUMINUM & $\begin{array}{l}04 / 06 / 88 \\
01 / 12 / 89 \\
09 / 19 / 89 \\
04 / 11 / 90 \\
01 / 22 / 91\end{array}$ & $\begin{array}{l}0001 \\
0001 \\
0001 \\
0001 \\
0001\end{array}$ & $M G / L$ & $\begin{array}{l}< \\
< \\
< \\
<\end{array}$ & $\begin{array}{l}0.28 \\
0.05 \\
0.1 \\
0.1 \\
0.05\end{array}$ & J & $\begin{array}{l}0.1 \\
0.1 \\
0.1 \\
0.1 \\
0.05\end{array}$ & $\begin{array}{l}- \\
- \\
- \\
-\end{array}$ \\
\hline AMHOHIUM & $\begin{array}{l}04 / 06 / 88 \\
01 / 12 / 89 \\
09 / 19 / 89 \\
04 / 11 / 90 \\
01 / 22 / 91\end{array}$ & $\begin{array}{l}0001 \\
0001 \\
0001 \\
0001 \\
0001\end{array}$ & $M G / L$ & & $\begin{array}{l}7.6 \\
5.09 \\
2.2 \\
1.6 \\
1.56\end{array}$ & $G$ & $\begin{array}{l}0.1 \\
0.1 \\
0.1 \\
0.1 \\
0.01\end{array}$ & : \\
\hline ANTIMONY & $\begin{array}{l}04 / 06 / 88 \\
01 / 12 / 89 \\
09 / 19 / 89 \\
04 / 11 / 90 \\
01 / 22 / 91\end{array}$ & $\begin{array}{l}0001 \\
0001 \\
0001 \\
0001 \\
0001\end{array}$ & $M G / L$ & & $\begin{array}{l}0.009 \\
0.007 \\
0.047 \\
0.099 \\
0.003\end{array}$ & G & $\begin{array}{l}0.003 \\
0.003 \\
0.003 \\
0.003 \\
0.003\end{array}$ & : \\
\hline ARSENIC & $\begin{array}{l}04 / 06 / 88 \\
01 / 12 / 89 \\
09 / 19 / 89 \\
04 / 11 / 90 \\
01 / 22 / 91\end{array}$ & $\begin{array}{l}0001 \\
0001 \\
0001 \\
0001 \\
0001\end{array}$ & $M G / L$ & $<$ & $\begin{array}{l}0.012 \\
0.005 \\
0.01 \\
0.01 \\
0.03\end{array}$ & $\begin{array}{l}\mathbf{J} \\
\mathbf{G I}\end{array}$ & $\begin{array}{l}0.01 \\
0.01 \\
0.01 \\
0.01 \\
0.03\end{array}$ & : \\
\hline BARIUM & $\begin{array}{l}04 / 06 / 88 \\
01 / 12 / 89 \\
09 / 19 / 89 \\
04 / 11 / 90 \\
01 / 22 / 91\end{array}$ & $\begin{array}{l}0001 \\
0001 \\
0001 \\
0001 \\
0001\end{array}$ & MG/L & $<$ & $\begin{array}{l}0.03 \\
0.02 \\
0.1 \\
0.1 \\
0.04\end{array}$ & $\begin{array}{l}\mathbf{J} \\
\mathbf{J} \\
\mathbf{G}\end{array}$ & $\begin{array}{l}0.1 \\
0.1 \\
0.1 \\
0.1 \\
0.01\end{array}$ & $\begin{array}{l}- \\
\dot{-} \\
- \\
-\end{array}$ \\
\hline BERYLLIUH & $\begin{array}{l}01 / 12 / 89 \\
09 / 19 / 89 \\
04 / 11 / 90 \\
01 / 22 / 91\end{array}$ & $\begin{array}{l}0001 \\
0001 \\
0001 \\
0001\end{array}$ & MG/L & $\begin{array}{l}< \\
< \\
< \\
<\end{array}$ & $\begin{array}{l}0.005 \\
0.01 \\
0.01 \\
0.005\end{array}$ & G & $\begin{array}{l}0.005 \\
0.01 \\
0.01 \\
0.005\end{array}$ & $\begin{array}{l}- \\
-\end{array}$ \\
\hline BORON & $\begin{array}{l}04 / 06 / 88 \\
01 / 12 / 89 \\
09 / 19 / 89 \\
04 / 11 / 90 \\
01 / 22 / 91\end{array}$ & $\begin{array}{l}0001 \\
0001 \\
0001 \\
0001 \\
0001\end{array}$ & MG/L & & $\begin{array}{l}0.11 \\
0.16 \\
0.3 \\
0.2 \\
0.29\end{array}$ & $\mathbf{G}$ & $\begin{array}{l}0.1 \\
0.1 \\
0.1 \\
0.1 \\
0.05\end{array}$ & $\begin{array}{l}- \\
- \\
-\end{array}$ \\
\hline BROMIDE & $\begin{array}{l}04 / 06 / 88 \\
01 / 12 / 89 \\
09 / 19 / 89 \\
04 / 11 / 90 \\
01 / 22 / 91\end{array}$ & $\begin{array}{l}0001 \\
0001 \\
0001 \\
0001 \\
0001\end{array}$ & MG/L & $\begin{array}{l}< \\
<\end{array}$ & $\begin{array}{l}0.1 \\
0.19 \\
0.1 \\
0.5 \\
0.4\end{array}$ & $\begin{array}{l}J \\
G\end{array}$ & $\begin{array}{l}0.1 \\
0.01 \\
0.1 \\
0.1 \\
0.1\end{array}$ & $\dot{-}$ \\
\hline CADHIUH & $\begin{array}{l}04 / 06 / 88 \\
01 / 12 / 89\end{array}$ & $\begin{array}{l}0001 \\
0001\end{array}$ & $M G / L$ & $<$ & $\begin{array}{l}0.006 \\
0.0001\end{array}$ & J & $\begin{array}{l}0.001 \\
0.001\end{array}$ & - \\
\hline
\end{tabular}

PARAMETER VALUE INDICATOR (PVI): < - LESS THAN DETECTION LIMIT

SAMPLE ID CISOES:

0001 - FILTIERED SAMPLE (.45 MICROWS)

OTHER PARAMETER VALUE FLAGS:

G - PH > 9, POSSIBLE GROUT CONTAMIHATIOH

1 - IMCREASED DETECTIOH LIMIT DUE TO REQUIRED DILUTION

J - estiMATED VALUE 
FORHATION OF COMPLETION: TRES HERMANOS - B SANDSTOHE (TB) HYDRAULIC FLON RELATIONSHIP: UPGRADIENT (U)

\begin{tabular}{|c|c|c|c|c|c|c|c|c|}
\hline PARAHETER MAME & LOG DATE & $\begin{array}{c}\text { SAMPLE } \\
\text { ID }\end{array}$ & $\begin{array}{l}\text { UNITS OF } \\
\text { MEASURE }\end{array}$ & PVI & $\begin{array}{l}\text { PARAMETER } \\
\text { VALUE }\end{array}$ & FLAGS & $\begin{array}{l}\text { DETECTIOH } \\
\text { LIMIT }\end{array}$ & $\begin{array}{l}\text { PARAMETER } \\
\text { UKCERTAINTY }\end{array}$ \\
\hline CADHIUY & $\begin{array}{l}09 / 19 / 89 \\
04 / 11 / 90 \\
01 / 22 / 91\end{array}$ & $\begin{array}{l}0009 \\
0001 \\
0001\end{array}$ & $M G / L$ & $<$ & $\begin{array}{l}0.002 \\
0.001 \\
0.001\end{array}$ & $\mathbf{G}$ & $\begin{array}{l}0.001 \\
0.001 \\
0.001\end{array}$ & : \\
\hline Calciun & $\begin{array}{l}04 / 06 / 88 \\
01 / 12 / 89 \\
09 / 19 / 89 \\
04 / 11 / 90 \\
01 / 22 / 91\end{array}$ & $\begin{array}{l}0001 \\
0001 \\
0001 \\
0001 \\
0001\end{array}$ & HG/L & & $\begin{array}{c}57.2 \\
42 . \\
133 . \\
83.7 \\
134 .\end{array}$ & G & $\begin{array}{l}0.01 \\
0.01 \\
0.01 \\
0.01 \\
0.5\end{array}$ & $\begin{array}{l}- \\
- \\
- \\
-\end{array}$ \\
\hline CHLORIDE & $\begin{array}{l}04 / 06 / 88 \\
01 / 12 / 89 \\
09 / 19 / 89 \\
04 / 11 / 90 \\
01 / 22 / 91\end{array}$ & $\begin{array}{l}0001 \\
0001 \\
0001 \\
0001 \\
0001\end{array}$ & $M G / L$ & & $\begin{array}{l}32 . \\
30 . \\
33 . \\
39 . \\
38 .\end{array}$ & G & $\begin{array}{l}1 . \\
1 . \\
1 . \\
1 . \\
0.5\end{array}$ & : \\
\hline COBALT & $\begin{array}{l}04 / 06 / 88 \\
01 / 12 / 89 \\
09 / 19 / 89 \\
04 / 11 / 90 \\
01 / 22 / 91\end{array}$ & $\begin{array}{l}0001 \\
0001 \\
0001 \\
0001 \\
0001\end{array}$ & MG/L & $\begin{array}{l}< \\
< \\
< \\
<\end{array}$ & $\begin{array}{l}0.01 \\
0.01 \\
0.05 \\
0.05 \\
0.03\end{array}$ & $\begin{array}{l}J \\
\text { J }\end{array}$ & $\begin{array}{l}0.05 \\
0.05 \\
0.05 \\
0.05 \\
0.03\end{array}$ & $\begin{array}{l}- \\
- \\
- \\
-\end{array}$ \\
\hline COPPER & $\begin{array}{l}04 / 06 / 88 \\
01 / 12 / 89 \\
09 / 19 / 89 \\
04 / 11 / 90 \\
01 / 22 / 91\end{array}$ & $\begin{array}{l}0001 \\
0001 \\
0001 \\
0001 \\
0001\end{array}$ & $M G / L$ & $\begin{array}{l}< \\
< \\
< \\
<\end{array}$ & $\begin{array}{l}0.02 \\
0.01 \\
0.02 \\
0.02 \\
0.01\end{array}$ & $\begin{array}{l}\text { J } \\
\text { G }\end{array}$ & $\begin{array}{l}0.02 \\
0.02 \\
0.02 \\
0.02 \\
0.01\end{array}$ & $\begin{array}{l}- \\
- \\
- \\
-\end{array}$ \\
\hline GROSS ALPHA & $\begin{array}{l}04 / 06 / 88 \\
01 / 12 / 89 \\
09 / 19 / 89 \\
04 / 11 / 90 \\
01 / 22 / 91\end{array}$ & $\begin{array}{l}0001 \\
0001 \\
0001 \\
0001 \\
0001\end{array}$ & $\mathrm{PCI} / \mathrm{L}$ & & $\begin{array}{l}6 . \\
18.8 \\
11 . \\
17 . \\
39.7\end{array}$ & $\begin{array}{l}\text { J } \\
\text { G }\end{array}$ & $\begin{array}{l}0.2 \\
1 . \\
1 . \\
1 . \\
1 .\end{array}$ & $\begin{array}{l}15 . \\
18.6 \\
17 . \\
13 . \\
36.5\end{array}$ \\
\hline GROSS BETA & $\begin{array}{l}04 / 06 / 88 \\
01 / 12 / 89 \\
09 / 19 / 89 \\
04 / 11 / 90 \\
01 / 22 / 91\end{array}$ & $\begin{array}{l}0001 \\
0001 \\
0001 \\
0001 \\
0001\end{array}$ & $\mathrm{PCI} / \mathrm{L}$ & & $\begin{array}{l}19 . \\
32.8 \\
21 . \\
28 . \\
36.8\end{array}$ & G & $\begin{array}{l}1 . \\
0.5 \\
0.5 \\
0.5 \\
0.5\end{array}$ & $\begin{array}{r}9.0 \\
24.2 \\
9.0 \\
11 . \\
17.9\end{array}$ \\
\hline
\end{tabular}

PARAMETER VALUE IMDICATOR (PVI): < - LESS THAN DETECTION LIMIT

OTHER PARAMETER VALUE FLAGS:

G - $\mathrm{PH}>$ 9, POSSIBLE GROUT CONTAMIHATION

$J$ - estimated VALUE 
GROUNDHATER QUALITY DATA BY LOCATION

SITE: AMBOI AMBROSIA LAKE

LOCATION: OTT7

HORTH COORDINATE: $\quad 52881.7$ FT

EAST COORDIHATE: 60119.8 FT

$06 / 10 / 80$ TO $12 / 04 / 92$

REPORT DATE: $08 / 04 / 94$

FORMATIOH OF COMPLETION: TRES HERMANOS - B SANDSTONE (TB)

HYDRAULIC FLON RELATIONSHIP: UPGRADIENT (U)

\begin{tabular}{|c|c|c|c|c|c|c|c|c|}
\hline PARAMETER MAME & LOG DATE & $\mid \begin{array}{c}\text { SAMPLE } \\
\text { ID }\end{array}$ & $\begin{array}{l}\text { UNITS OF } \\
\text { MEASURE }\end{array}$ & PVI & $\begin{array}{l}\text { PARAMETER } \\
\text { VALUE }\end{array}$ & FLAGS & $\begin{array}{l}\text { DETECTION } \\
\text { LIMIT }\end{array}$ & $\begin{array}{l}\text { PARAMETER } \\
\text { UNCERTAINTY }\end{array}$ \\
\hline IROH & $\begin{array}{l}04 / 06 / 88 \\
01 / 12 / 89 \\
09 / 19 / 89 \\
04 / 11 / 90 \\
01 / 22 / 91\end{array}$ & $\begin{array}{l}0001 \\
0001 \\
0001 \\
0001 \\
0001\end{array}$ & $M G / L$ & $\begin{array}{l}< \\
< \\
<\end{array}$ & $\begin{array}{l}0.03 \\
0.02 \\
0.05 \\
0.03 \\
0.03\end{array}$ & $\begin{array}{l}J \\
G\end{array}$ & $\begin{array}{l}0.03 \\
0.03 \\
0.03 \\
0.03 \\
0.03\end{array}$ & $\begin{array}{l}- \\
- \\
- \\
-\end{array}$ \\
\hline LEAD & $\begin{array}{l}04 / 06 / 88 \\
01 / 12 / 89 \\
09 / 19 / 89 \\
04 / 11 / 90 \\
01 / 22 / 91\end{array}$ & $\begin{array}{l}0001 \\
0001 \\
0001 \\
0001 \\
0001\end{array}$ & MG/L & $\begin{array}{l}< \\
< \\
<\end{array}$ & $\begin{array}{l}0.01 \\
0.001 \\
0.05 \\
0.01 \\
0.03\end{array}$ & $\begin{array}{l}\text { J } \\
\text { GI }\end{array}$ & $\begin{array}{l}0.01 \\
0.01 \\
0.01 \\
0.01 \\
0.03\end{array}$ & $\begin{array}{l}- \\
- \\
-\end{array}$ \\
\hline LEAD-210 & $\begin{array}{l}04 / 06 / 88 \\
01 / 12 / 89 \\
09 / 19 / 89\end{array}$ & $\begin{array}{l}0009 \\
0001 \\
0001\end{array}$ & $\mathrm{PCI} / \mathrm{L}$ & & $\begin{array}{l}0.4 \\
0.00 \\
0.1\end{array}$ & & $\begin{array}{l}1.5 \\
1.5 \\
1.5\end{array}$ & $\begin{array}{l}1.1 \\
5.35 \\
0.7\end{array}$ \\
\hline MAGNESIUH & $\begin{array}{l}04 / 06 / 88 \\
01 / 12 / 89 \\
09 / 19 / 89 \\
04 / 11 / 90 \\
01 / 22 / 91\end{array}$ & $\begin{array}{l}0001 \\
0001 \\
0001 \\
0001 \\
0001\end{array}$ & MG/L & $<$ & $\begin{array}{c}0.27 \\
1 . \\
20.5 \\
12.9 \\
22.5\end{array}$ & G & $\begin{array}{l}0.001 \\
1.001 \\
0.001 \\
0.001 \\
0.1\end{array}$ & $\begin{array}{l}- \\
- \\
- \\
-\end{array}$ \\
\hline MANGANESE & $\begin{array}{l}04 / 06 / 88 \\
01 / 12 / 89 \\
09 / 19 / 89 \\
04 / 11 / 90 \\
01 / 22 / 91\end{array}$ & $\begin{array}{l}0001 \\
0001 \\
0001 \\
0001 \\
0001\end{array}$ & MG/L & $\begin{array}{l}< \\
< \\
< \\
< \\
<\end{array}$ & $\begin{array}{l}0.01 \\
0.01 \\
0.01 \\
0.01 \\
0.01\end{array}$ & G & $\begin{array}{l}0.01 \\
0.01 \\
0.01 \\
0.01 \\
0.01\end{array}$ & $\begin{array}{l}- \\
- \\
-\end{array}$ \\
\hline HERCURY & $\begin{array}{l}04 / 06 / 88 \\
01 / 12 / 89 \\
09 / 19 / 89 \\
04 / 11 / 90 \\
01 / 22 / 91\end{array}$ & $\begin{array}{l}0001 \\
0001 \\
0001 \\
0001 \\
0001\end{array}$ & MG/L & $\begin{array}{l}< \\
< \\
< \\
< \\
<\end{array}$ & $\begin{array}{l}0.0002 \\
0.0001 \\
0.0002 \\
0.0002 \\
0.0002\end{array}$ & $\begin{array}{l}\text { J } \\
\mathbf{G}\end{array}$ & $\begin{array}{l}0.0002 \\
0.0002 \\
0.0002 \\
0.0002 \\
0.0002\end{array}$ & $\begin{array}{l}- \\
- \\
-\end{array}$ \\
\hline MOLYBDEHUM & $\begin{array}{l}04 / 06 / 88 \\
01 / 12 / 89 \\
09 / 19 / 89 \\
04 / 11 / 90 \\
01 / 22 / 91\end{array}$ & $\begin{array}{l}0001 \\
0001 \\
0001 \\
0001 \\
0001\end{array}$ & MG/L & & $\begin{array}{l}0.10 \\
0.028 \\
0.02 \\
0.06 \\
0.01\end{array}$ & G & $\begin{array}{l}0.01 \\
0.01 \\
0.01 \\
0.01 \\
0.01\end{array}$ & $\begin{array}{l}- \\
- \\
-\end{array}$ \\
\hline HET GROSS ALPHA * & $\begin{array}{l}04 / 06 / 88 \\
01 / 12 / 89 \\
09 / 19 / 89 \\
04 / 11 / 90 \\
01 / 22 / 91\end{array}$ & $\begin{array}{l}0001 \\
0001 \\
0001 \\
0001 \\
0001\end{array}$ & $\mathrm{PCI} / \mathrm{L}$ & & $\begin{array}{r}4.97 \\
12.63 \\
9.63 \\
16.31 \\
39.36\end{array}$ & & $\begin{array}{l}- \\
- \\
- \\
-\end{array}$ & : \\
\hline HICKEL & $\begin{array}{l}04 / 06 / 88 \\
01 / 12 / 89 \\
09 / 19 / 89 \\
04 / 11 / 90 \\
01 / 22 / 91\end{array}$ & $\begin{array}{l}0001 \\
0001 \\
0001 \\
0001 \\
0001\end{array}$ & MG/L & $\begin{array}{l}< \\
< \\
<\end{array}$ & $\begin{array}{l}0.02 \\
0.02 \\
0.04 \\
0.04 \\
0.04\end{array}$ & $\begin{array}{l}J \\
j \\
G\end{array}$ & $\begin{array}{l}0.04 \\
0.04 \\
0.04 \\
0.04 \\
0.04\end{array}$ & $\begin{array}{l}- \\
- \\
-\end{array}$ \\
\hline MITRATE & $04 / 06 / 88$ & 0009 & $M G / L$ & & 0.9 & J & 1. & - \\
\hline
\end{tabular}

- MET GROSS ALPHA (GROSS ALPHA - URANIUM) MITH 1 MG URANIUM = 686 PCI

PARAMETER VALUE IMDICATOR (PVI): < - LESS THAN DETECTION LIMIT

SAMPLE ID COOES:

0001 - FILTERED SAMPLE (.45 HICRONS)

OTHER PARAMETER VALUE FLAGS:

G - PH $>$ 9, POSSIBLE GROUT CONTAMINATION

1 - INCREASED DETECTION LIHIT DUE TO REQUIRED DILUTION

j- eSTIMATED VALUE 
GROUNDWATER QUALITY DATA BY LOCATION

SITE: AMBOI AMBROSIA LAKE

LOCATIOH: 0777

MORTH COORDIHATE: 52881.7 FT

EAST COOROINATE: $\quad 60119.8$ FT

$06 / 10 / 80$ TO $12 / 04 / 92$

REPORT DATE: $08 / 04 / 94$

FORMATION OF COMPLETION: TRES HERMLANOS - B SANDSTONE (TB)

HYDRAULIC FLON RELATIONSHIP: UPGRADIENT (U)

\begin{tabular}{|c|c|c|c|c|c|c|c|}
\hline PARAMETER MAME & LOG DATE & $\begin{array}{c}\text { SAMPLE } \\
\text { ID }\end{array}$ & $\begin{array}{l}\text { UNITS OF } \\
\text { MEASURE }\end{array}$ & PVI & $\begin{array}{l}\text { PARAMETER } \\
\text { VALUE FLAGS }\end{array}$ & $\begin{array}{l}\text { DETECTIOH } \\
\text { LIHIT }\end{array}$ & $\begin{array}{l}\text { PARAMETER } \\
\text { UNCERTAINTY }\end{array}$ \\
\hline NITRATE & $\begin{array}{l}01 / 12 / 89 \\
09 / 19 / 89 \\
04 / 11 / 90 \\
01 / 22 / 91\end{array}$ & $\begin{array}{l}0001 \\
0001 \\
0001 \\
0001\end{array}$ & HG/L & $<$ & $\begin{array}{l}1.94 \\
1 . \\
1.7 \\
2.2\end{array}$ & $\begin{array}{l}1 . \\
1 . \\
1 . \\
0.1\end{array}$ & :- \\
\hline NITRITE AND NITRATE & $\begin{array}{l}04 / 06 / 88 \\
04 / 11 / 90 \\
01 / 22 / 91\end{array}$ & $\begin{array}{l}0001 \\
0001 \\
0001\end{array}$ & MG/L & & $\begin{array}{l}0.9 \\
0.4 \\
0.58\end{array}$ & $\begin{array}{l}1 . \\
1.05 \\
0.05\end{array}$ & - \\
\hline PH & $\begin{array}{l}04 / 06 / 88 \\
01 / 12 / 89 \\
09 / 19 / 89 \\
04 / 11 / 90 \\
01 / 22 / 91\end{array}$ & $\begin{array}{l}0001 \\
0001 \\
0001 \\
0001 \\
0001\end{array}$ & su & & $\begin{array}{r}11.93 \\
11.65 \\
11.20 \\
9.92 \\
10.63\end{array}$ & $\begin{array}{l}- \\
- \\
- \\
-\end{array}$ & $\begin{array}{l}- \\
- \\
- \\
-\end{array}$ \\
\hline PKOSPHATE & $\begin{array}{l}04 / 06 / 88 \\
01 / 12 / 89 \\
09 / 19 / 89 \\
04 / 11 / 90 \\
01 / 22 / 91\end{array}$ & $\begin{array}{l}0001 \\
0001 \\
0001 \\
0001 \\
0001\end{array}$ & $M G / L$ & $\begin{array}{l}< \\
< \\
< \\
< \\
<\end{array}$ & $\begin{array}{l}0.1 \\
0.1 \\
0.1 \\
0.1 \\
0.05\end{array}$ & $\begin{array}{l}0.1 \\
0.1 \\
0.1 \\
0.1 \\
0.05\end{array}$ & $\begin{array}{l}- \\
- \\
- \\
-\end{array}$ \\
\hline POLONIUM-210 & $\begin{array}{l}04 / 06 / 88 \\
01 / 12 / 89 \\
09 / 19 / 89\end{array}$ & $\begin{array}{l}0001 \\
0001 \\
0001\end{array}$ & $\mathrm{PCI} / \mathrm{L}$ & & $\begin{array}{l}0.1 \\
0.466 \\
2.9\end{array}$ & $\begin{array}{l}1 . \\
1 .\end{array}$ & $\begin{array}{l}0.4 \\
1.26 \\
0.7\end{array}$ \\
\hline POTASSIUM & $\begin{array}{l}04 / 06 / 88 \\
01 / 12 / 89 \\
09 / 19 / 89 \\
04 / 11 / 90 \\
01 / 22 / 91\end{array}$ & $\begin{array}{l}0001 \\
0001 \\
0001 \\
0001 \\
0001\end{array}$ & MG/L & & $\begin{array}{r}18.6 \\
19.8 \\
13.7 \\
12.9 \\
9.0\end{array}$ & $\begin{array}{l}0.01 \\
0.01 \\
0.01 \\
0.01 \\
0.01\end{array}$ & $\begin{array}{l}- \\
- \\
-\end{array}$ \\
\hline RADIUM-226 & $\begin{array}{l}04 / 06 / 88 \\
01 / 12 / 89 \\
09 / 19 / 89 \\
04 / 11 / 90 \\
01 / 22 / 91\end{array}$ & $\begin{array}{l}0001 \\
0001 \\
0001 \\
0001 \\
0001\end{array}$ & $\mathrm{PCI} / \mathrm{L}$ & & $\begin{array}{l}0.9 \\
0.352 \\
0.2 \\
0.6 \\
1.2\end{array}$ & $\begin{array}{l}1 . \\
1 . \\
1 . \\
1 .\end{array}$ & $\begin{array}{l}0.3 \\
0.219 \\
0.2 \\
0.3 \\
0.5\end{array}$ \\
\hline RADIU:A-226 + RADIUM-228 & $\begin{array}{l}04 / 06 / 88 \\
01 / 12 / 89 \\
09 / 19 / 89 \\
04 / 11 / 90 \\
01 / 22 / 91\end{array}$ & $\begin{array}{l}0001 \\
0001 \\
0001 \\
0001 \\
0001\end{array}$ & $\mathrm{PCI} / \mathrm{L}$ & & $\begin{array}{l}1.20 \\
0.35 \\
0.40 \\
1.40 \\
3.20\end{array}$ & $\begin{array}{l}- \\
- \\
- \\
-\end{array}$ & $\begin{array}{l}- \\
- \\
-\end{array}$ \\
\hline RADIUN-228 & $\begin{array}{l}04 / 06 / 88 \\
01 / 12 / 89 \\
09 / 19 / 89 \\
04 / 11 / 90 \\
01 / 22 / 91\end{array}$ & $\begin{array}{l}0001 \\
0001 \\
0001 \\
0001 \\
0001\end{array}$ & $\mathrm{PCI} / \mathrm{L}$ & & $\begin{array}{l}0.3 \\
0.00 \\
0.2 \\
0.8 \\
2.0\end{array}$ & $\begin{array}{l}1 . \\
1 . \\
1 . \\
1 . \\
1 .\end{array}$ & $\begin{array}{l}0.7 \\
0.219 \\
0.9 \\
0.8 \\
1.9\end{array}$ \\
\hline SELENIUM & $\begin{array}{l}04 / 06 / 88 \\
01 / 12 / 89 \\
09 / 19 / 89 \\
04 / 11 / 90 \\
01 / 22 / 91\end{array}$ & $\begin{array}{l}0001 \\
0001 \\
0001 \\
0001 \\
0001\end{array}$ & $M G / L$ & $<$ & $\begin{array}{l}0.047 \\
0.005 \\
0.036 \\
0.005 \\
0.05\end{array}$ & $\begin{array}{l}0.005 \\
0.005 \\
0.005 \\
0.005 \\
0.05\end{array}$ & : \\
\hline SILICA - SIOZ & $04 / 06 / 88$ & 0001 & MG/L & & 39.9 & 2. & - \\
\hline
\end{tabular}

PARAMETER VALUE INDICATOR (PVI): < - LESS THAN DETECTION LIMIT

SAMPLE ID COOES:

0001 - FILTERED SAMPLE (.45 MICROHS)

OTHER PARAMETER VALUE FLAGS:

G - PH > 9, POSSIBLE GROUT CONTAMINATIOH

1 - INCREASED DETECTION LIHIT DUE TO REQUIRED DILUTION

J - ESTIMATED VALUE 
GROUHDHATER QUALITY DATA BY LOCATION

SITE: AMBOI AMBROSIA LAKE

LOCATION: 0777

NORTH COORDINATE: $\quad 52881.7$ FT

EAST COORDIHATE: $\quad 60119.8$ FT

06/10/80 TO $12 / 04 / 92$

REPORT DATE: 08/04/94

FORHATION OF COMPLETION: TRES HERHAHOS - B SAMDSTONE (TB) HYORAULIC FLOW RELATIOHSHIP: UPGRADIENT (U)

\begin{tabular}{|c|c|c|c|c|c|c|c|c|}
\hline PARAMETER MAME & LOG DATE & $\mid \begin{array}{c}\text { SAMPLE } \\
\text { ID }\end{array}$ & $\begin{array}{l}\text { UHITS OF } \\
\text { MEASURE }\end{array}$ & PVI & $\begin{array}{l}\text { PARAMETEIR } \\
\text { VALUE }\end{array}$ & FLAGS & $\begin{array}{l}\text { DETECTION } \\
\text { LIMIT }\end{array}$ & $\begin{array}{l}\text { PARAMETER } \\
\text { UNCERTAINTY }\end{array}$ \\
\hline SILICA - sIO2 & $\begin{array}{l}01 / 12 / 89 \\
09 / 19 / 89 \\
04 / 11 / 90 \\
01 / 22 / 91\end{array}$ & $\begin{array}{l}0001 \\
0001 \\
0001 \\
0001\end{array}$ & MG/L & & $\begin{array}{l}30.2 \\
24 . \\
22 . \\
24.2\end{array}$ & $\mathbf{G}$ & $\begin{array}{l}2 . \\
2 . \\
2 . \\
0.1\end{array}$ & $\begin{array}{l}- \\
-\end{array}$ \\
\hline SILVER & $\begin{array}{l}04 / 06 / 88 \\
01 / 12 / 89 \\
09 / 19 / 89 \\
04 / 11 / 90 \\
01 / 22 / 91\end{array}$ & $\begin{array}{l}0001 \\
0001 \\
0001 \\
0001 \\
0001\end{array}$ & MG/L & $\begin{array}{l}< \\
< \\
< \\
<\end{array}$ & $\begin{array}{l}0.01 \\
0.01 \\
0.01 \\
0.01 \\
0.01\end{array}$ & G & $\begin{array}{l}0.01 \\
0.01 \\
0.01 \\
0.01 \\
0.01\end{array}$ & $\begin{array}{l}- \\
- \\
-\end{array}$ \\
\hline scoitun & $\begin{array}{l}04 / 06 / 88 \\
01 / 12 / 89 \\
09 / 19 / 89 \\
04 / 11 / 90 \\
01 / 22 / 91\end{array}$ & $\begin{array}{l}0001 \\
0001 \\
0001 \\
0001 \\
0001\end{array}$ & MG/L & & $\begin{array}{c}599 . \\
840 . \\
816 . \\
1074 . \\
990 .\end{array}$ & GJ & $\begin{array}{l}0.002 \\
0.002 \\
0.002 \\
0.002 \\
5 .\end{array}$ & $\begin{array}{l}- \\
- \\
-\end{array}$ \\
\hline SPECIFIC CONDUCTANCE & $\begin{array}{l}04 / 06 / 88 \\
01 / 12 / 89 \\
09 / 19 / 89 \\
04 / 11 / 90 \\
01 / 22 / 91\end{array}$ & $\begin{array}{l}0001 \\
0001 \\
0001 \\
0001 \\
0001\end{array}$ & UМHO/CH & & $\begin{array}{l}2700 . \\
2525 . \\
1980 . \\
2100 . \\
2900 .\end{array}$ & G & $\begin{array}{l}- \\
- \\
-\end{array}$ & $\begin{array}{l}\dot{-} \\
\dot{-} \\
\dot{-}\end{array}$ \\
\hline STRONTIUM & $\begin{array}{l}04 / 06 / 88 \\
01 / 12 / 89 \\
09 / 19 / 89 \\
04 / 11 / 90 \\
01 / 22 / 91\end{array}$ & $\begin{array}{l}0001 \\
0001 \\
0001 \\
0001 \\
0001\end{array}$ & MG/L & & $\begin{array}{l}0.70 \\
1.51 \\
4.9 \\
3.67 \\
4.90\end{array}$ & G & $\begin{array}{l}0.1 \\
0.1 \\
0.1 \\
0.1 \\
0.01\end{array}$ & : \\
\hline SULFATE & $\begin{array}{l}04 / 06 / 88 \\
01 / 12 / 89 \\
09 / 19 / 89 \\
04 / 11 / 90 \\
01 / 22 / 91\end{array}$ & $\begin{array}{l}0001 \\
0001 \\
0001 \\
0001 \\
0001\end{array}$ & MG/L & & $\begin{array}{l}1210 . \\
1632 . \\
2030 . \\
2180 . \\
2250 .\end{array}$ & G & $\begin{array}{c}0.1 \\
0.1 \\
0.1 \\
0.1 \\
10 .\end{array}$ & $\begin{array}{l}- \\
- \\
-\end{array}$ \\
\hline SULFIDE & $\begin{array}{l}04 / 06 / 88 \\
01 / 12 / 89 \\
09 / 19 / 89 \\
04 / 11 / 90 \\
01 / 22 / 91\end{array}$ & $\begin{array}{l}0001 \\
0001 \\
0001 \\
0001 \\
0001\end{array}$ & HG/L & $\begin{array}{l}< \\
<\end{array}$ & $\begin{array}{l}0.1 \\
0.01 \\
0.1 \\
0.7 \\
8.4\end{array}$ & $\begin{array}{l}J \\
6\end{array}$ & $\begin{array}{l}0.1 \\
0.1 \\
0.1 \\
0.1 \\
0.1\end{array}$ & $\begin{array}{l}- \\
- \\
-\end{array}$ \\
\hline TEMPERATURE & $\begin{array}{l}04 / 06 / 88 \\
01 / 12 / 89 \\
09 / 19 / 89 \\
04 / 11 / 90 \\
01 / 22 / 91\end{array}$ & $\begin{array}{l}0001 \\
0001 \\
0001 \\
0001 \\
0001\end{array}$ & C - DEGREE & & $\begin{array}{l}12.5 \\
11.0 \\
14.0 \\
13 . \\
11 .\end{array}$ & G & $:$ & $\begin{array}{l}- \\
- \\
-\end{array}$ \\
\hline THALLIUA & $\begin{array}{l}01 / 12 / 89 \\
09 / 19 / 89 \\
04 / 11 / 90 \\
01 / 22 / 91\end{array}$ & $\begin{array}{l}0001 \\
0001 \\
0001 \\
0001\end{array}$ & $M G / L$ & $\begin{array}{l}< \\
< \\
<\end{array}$ & $\begin{array}{l}0.005 \\
0.01 \\
0.01 \\
0.03\end{array}$ & I & $\begin{array}{l}0.005 \\
0.01 \\
0.01 \\
0.03\end{array}$ & : \\
\hline THORIUA-230 & $\begin{array}{l}04 / 06 / 88 \\
01 / 12 / 89 \\
09 / 19 / 89\end{array}$ & $\begin{array}{l}0001 \\
0001 \\
0001\end{array}$ & $\mathrm{PCI} / \mathrm{L}$ & & $\begin{array}{l}0.0 \\
0.684 \\
0.0\end{array}$ & & $\begin{array}{l}1 . \\
1 .\end{array}$ & $\begin{array}{l}0.4 \\
0.166 \\
0.3\end{array}$ \\
\hline
\end{tabular}

OTHER PARAMETER VALUE FLAGS:

G - PH > 9, POSSIBLE GROUT CONTAMIHATIOH

I - INCREASED OETECTION LIMIT DUE TO REQUIRED DILUTION

d- estimated VAlue 
GROUNDHATER QUALITY DATA BY LOCATIOH

SITE: AMBOI AMBROSIA LAKE

LOCATIOH: OTT7

HORTH COORDIMATE: 52881.7 FT

EAST COORDINATE: $\quad 60119.8$ FT

06/10/80 TO 12/04/92

REPORT DATE: $08 / 04 / 94$

FORMATION OF COMPLETION: TRES HERMANOS - 8 SANDSTONE (TB)

HYDRAULIC FLOU RELATIONSHIP: UPGRADIENT (U)

\begin{tabular}{|c|c|c|c|c|c|c|c|c|}
\hline PARAMETER MAME & LOG DATE & $\begin{array}{c}\text { SAMPLE } \\
\text { ID }\end{array}$ & $\begin{array}{l}\text { UNITS OF } \\
\text { MEASURE }\end{array}$ & PVI & $\begin{array}{l}\text { PARAMETER } \\
\text { VALUE }\end{array}$ & FLAGS & $\begin{array}{l}\text { DETECTION } \\
\text { LIMIT }\end{array}$ & $\begin{array}{l}\text { PARAMETER } \\
\text { UNCERTAINTY }\end{array}$ \\
\hline TIK & $\begin{array}{l}04 / 06 / 88 \\
01 / 12 / 89 \\
09 / 19 / 89 \\
04 / 11 / 90 \\
01 / 22 / 91\end{array}$ & $\begin{array}{l}0001 \\
0001 \\
0001 \\
0001 \\
0001\end{array}$ & MG/L & $<$ & $\begin{array}{l}0.037 \\
0.002 \\
0.169 \\
0.099 \\
0.03\end{array}$ & $\begin{array}{l}\mathbf{J} \\
\text { GI }\end{array}$ & $\begin{array}{l}0.005 \\
0.005 \\
0.005 \\
0.005 \\
0.03\end{array}$ & $\begin{array}{l}- \\
- \\
- \\
-\end{array}$ \\
\hline TOTAL KJELDAHL NITROGEN & $01 / 22 / 91$ & 0001 & $M G / L$ & & 2. & G & 1. & - \\
\hline TOTAL ORGANIC CARBON & $\begin{array}{l}04 / 06 / 88 \\
01 / 12 / 89 \\
09 / 19 / 89 \\
04 / 11 / 90 \\
01 / 22 / 91\end{array}$ & $\begin{array}{l}0001 \\
0001 \\
0001 \\
0001 \\
0001\end{array}$ & MG/L & & $\begin{array}{c}26.0 \\
13 . \\
5.3 \\
14 . \\
10 .\end{array}$ & G & $\begin{array}{l}1 . \\
1 . \\
1 . \\
1 .\end{array}$ & $\begin{array}{l}- \\
- \\
-\end{array}$ \\
\hline URANIUM & $\begin{array}{l}04 / 06 / 88 \\
01 / 12 / 89 \\
09 / 19 / 89 \\
04 / 11 / 90 \\
01 / 22 / 91\end{array}$ & $\begin{array}{l}0001 \\
0001 \\
0001 \\
0001 \\
0001\end{array}$ & $M G / L$ & $\begin{array}{l}< \\
<\end{array}$ & $\begin{array}{l}0.0003 \\
0.009 \\
0.0020 \\
0.0010 \\
0.001\end{array}$ & $\begin{array}{l}\text { J } \\
\text { J } \\
\mathbf{J} \\
\mathbf{G}\end{array}$ & $\begin{array}{l}0.003 \\
0.003 \\
0.003 \\
0.003 \\
0.001\end{array}$ & $\begin{array}{l}- \\
- \\
- \\
-\end{array}$ \\
\hline VANADIUM & $\begin{array}{l}04 / 06 / 88 \\
01 / 12 / 89 \\
09 / 19 / 89 \\
04 / 11 / 90 \\
01 / 22 / 91\end{array}$ & $\begin{array}{l}0001 \\
0001 \\
0001 \\
0001 \\
0001\end{array}$ & $M G / L$ & $<$ & $\begin{array}{l}0.06 \\
0.01 \\
0.02 \\
0.01 \\
0.01\end{array}$ & G & $\begin{array}{l}0.01 \\
0.01 \\
0.01 \\
0.01 \\
0.01\end{array}$ & $\begin{array}{l}- \\
- \\
- \\
-\end{array}$ \\
\hline ZINC & $\begin{array}{l}04 / 06 / 88 \\
01 / 12 / 89 \\
09 / 19 / 89 \\
04 / 19 / 90 \\
01 / 22 / 91\end{array}$ & $\begin{array}{l}0001 \\
0001 \\
0001 \\
0001 \\
0001\end{array}$ & MG/L & $\begin{array}{l}< \\
< \\
<\end{array}$ & $\begin{array}{l}0.005 \\
0.01 \\
0.010 \\
0.007 \\
0.005\end{array}$ & $\mathbf{G}$ & $\begin{array}{l}0.005 \\
0.01 \\
0.005 \\
0.005 \\
0.005\end{array}$ & $\begin{array}{l}- \\
- \\
- \\
-\end{array}$ \\
\hline
\end{tabular}

PARAMETER VALUE INDICATOR (PVI):

< - LESS THAN DETECTION LIMIT

SAMPLE IO COOES:

0001 - FILTERED SAMPLE (.45 MICRONS)

OTHER PARAMETER VALUE FLAGS:

G - PH > 9, POSSIBLE GROUT CONTAMINATION

I - IHCREASED DETECTION LIMIT DUE TO REQUIRED DILUTION

$J$ - estimated VALUe 
GROUHDHATER QUALITY DATA BY LOCATIOH

SITE: AMBOI AMBROSIA LAKE

LOCATION: 0778

MORTH COORDIMATE: 52911.5 FT

EAST COORDINATE: 60205.4 FT

06/10/80 TO $12 / 04 / 92$

REPORT DATE: 08/04/94

FORMATION OF COMPLETION: TRES HERMAHOS - CI SANDSTONE (TO)

HYDRAULIC FLOW RELATIOHSHIP: DOWN GRADIENT (D)

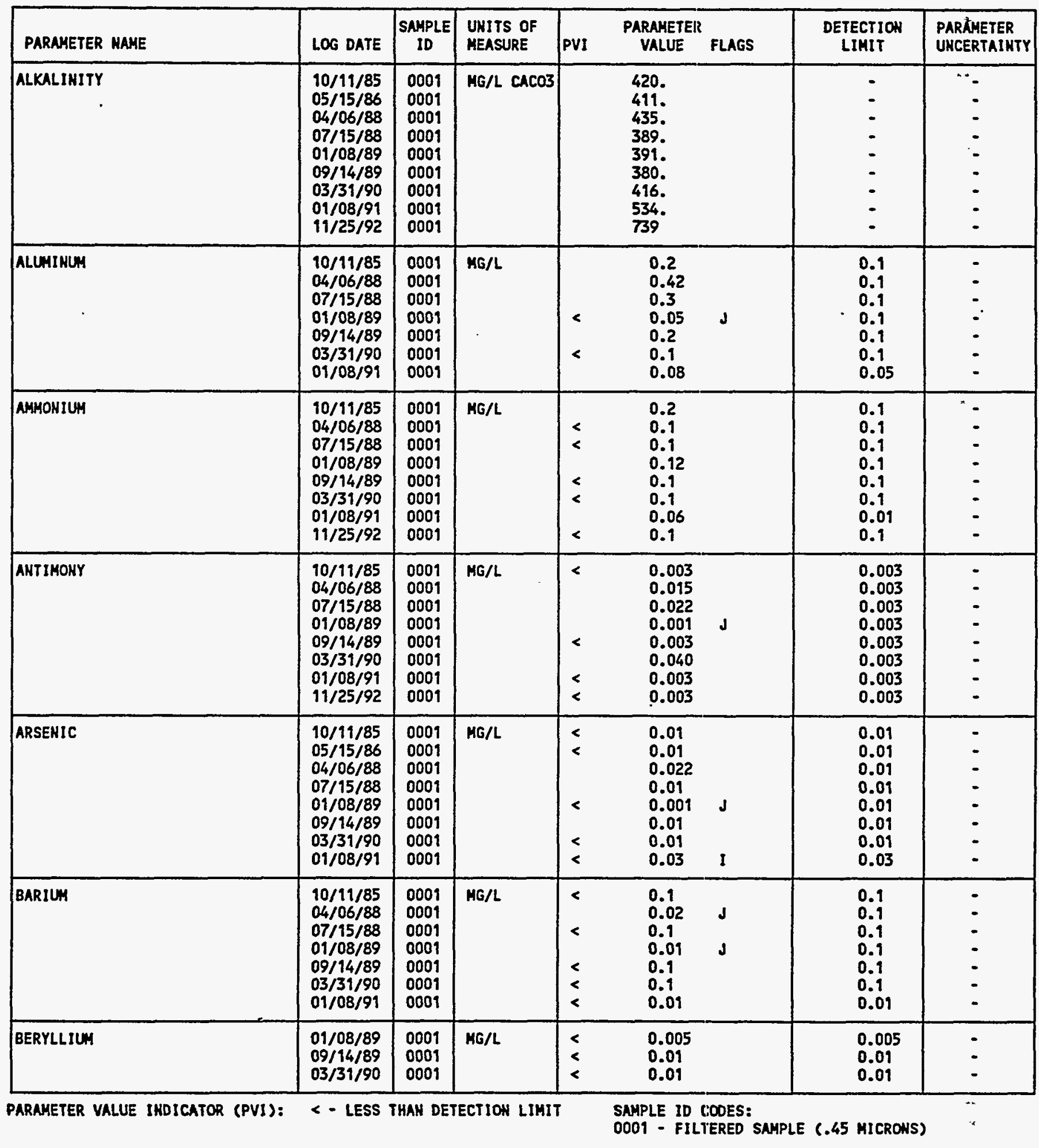

OTHER PARAMETER VALUE FLAGS:

I - INCREASED DETECTION LIMIT DUE TO REQUIRED DILUTIOH

J. ESTIMATED VALUE 
GROUNDWATER QUALITY DATA BY LOCATION

SITE: AMBOI AMBROSIA LAKE

LOCATION: 0778

NORTH COORDIHATE: 52911.5 FT

EAST COORDINATE: $\quad 60205.4$ FT

$06 / 10 / 80$ TO $12 / 04 / 92$

REPORT DATE: 08/04/94

FORMATION OF COHPLETIOH: TRES RERHANOS - CI SANDSTONE (TO) HYDRAULIC FLON RELATIONSHIP: DOWN GRADIENT (D)

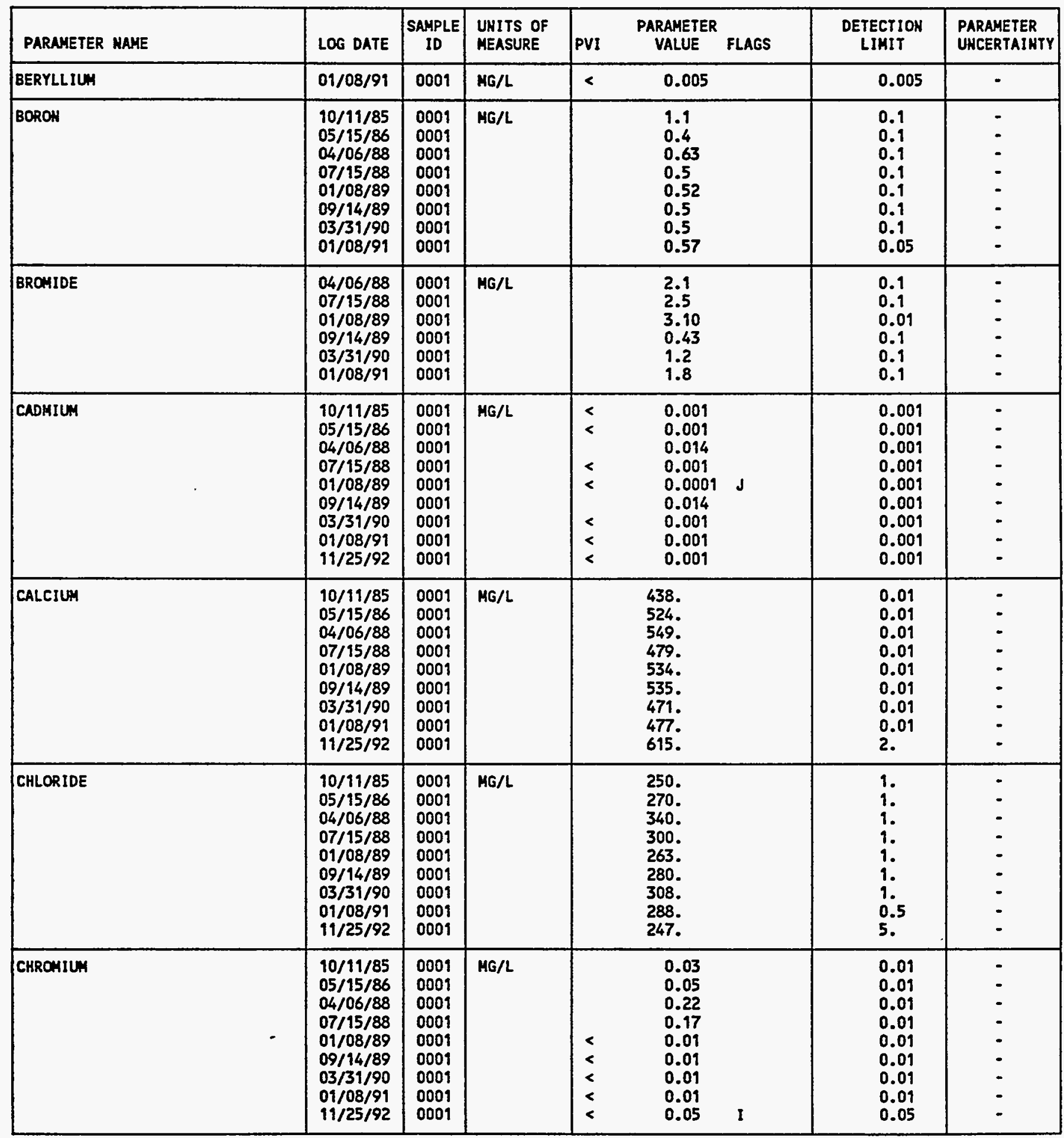

PARAMETER VALUE INDICATOR (PVI): < - LESS THAK DETECTION LIMIT

SAMPLE ID CODES:

0001 - FILTERED SAMPLE (.45 MICRONS)

OTHER PARAMETER VALUE FLAGS:

I - IMCREASED DETECTIOH LIMIT DUE TO REQUIRED DILUTIOH

d - estimated VALUE 
GROUHDWATER QUALITY DATA BY LOCATION

SITE: AMBO1 AMBROSIA LAKE

LOCATIOH: 0778

NORTH COORDINATE: 52911.5 FT

EAST COORDIHATE: 60205.4 FT

06/10/80 TO $12 / 04 / 92$

REPORT DATE: 08/04/94

FORMATIOM OF COAPLETIOH: TRES HERMAKOS - C1 SAKDSTONE (TO) HYDRAULIC FLON RELATIONSHIP: DOWH GRADIEHT (D)

\begin{tabular}{|c|c|c|c|c|c|c|c|}
\hline PARAMETER HAME & LOG DATE & $\begin{array}{c}\text { SAMPLE } \\
\text { IO }\end{array}$ & $\begin{array}{l}\text { UNITS OF } \\
\text { MEASURE }\end{array}$ & PVI & $\begin{array}{l}\text { PARAMETEF! } \\
\text { VALUE FLAGS }\end{array}$ & $\begin{array}{l}\text { DETECTIOH } \\
\text { LIMIT }\end{array}$ & $\begin{array}{l}\text { PARAMETER } \\
\text { UNCERTAINTY }\end{array}$ \\
\hline COBALT & $\begin{array}{l}10 / 11 / 85 \\
05 / 15 / 86 \\
04 / 06 / 88 \\
07 / 15 / 88 \\
01 / 08 / 89 \\
09 / 14 / 89 \\
03 / 31 / 90 \\
01 / 08 / 91 \\
11 / 25 / 92\end{array}$ & $\begin{array}{l}0001 \\
0001 \\
0001 \\
0001 \\
0001 \\
0001 \\
0001 \\
0001 \\
0001\end{array}$ & MG/L & $\begin{array}{l}< \\
< \\
< \\
< \\
< \\
< \\
<\end{array}$ & $\begin{array}{l}0.05 \\
0.08 \\
0.04 \\
0.05 \\
0.01 \\
0.05 \\
0.05 \\
0.03 \\
0.03\end{array}$ & $\begin{array}{l}0.05 \\
0.05 \\
0.05 \\
0.05 \\
0.05 \\
0.05 \\
0.05 \\
0.03 \\
0.03\end{array}$ & $\begin{array}{r}. \\
. \\
- \\
- \\
- \\
- \\
-\end{array}$ \\
\hline COPPER & $\begin{array}{l}10 / 11 / 85 \\
04 / 06 / 88 \\
07 / 15 / 88 \\
01 / 08 / 89 \\
09 / 14 / 89 \\
03 / 31 / 90 \\
01 / 08 / 91\end{array}$ & $\begin{array}{l}0001 \\
0001 \\
0001 \\
0001 \\
0001 \\
0001 \\
0001\end{array}$ & MG/L & $\begin{array}{l}< \\
< \\
< \\
<\end{array}$ & $\begin{array}{l}0.05 \\
0.04 \\
0.03 \\
0.01 \\
0.02 \\
0.02 \\
0.01\end{array}$ & $\begin{array}{l}0.02 \\
0.02 \\
0.02 \\
0.02 \\
0.02 \\
0.02 \\
0.01\end{array}$ & $\begin{array}{l}- \\
- \\
- \\
- \\
-\end{array}$ \\
\hline CYAHIDE & $\begin{array}{l}10 / 11 / 85 \\
01 / 08 / 89 \\
09 / 14 / 89 \\
03 / 31 / 90 \\
01 / 08 / 91\end{array}$ & $\begin{array}{l}0001 \\
0001 \\
0001 \\
0001 \\
0001\end{array}$ & MG/L & $<$ & $\begin{array}{l}0.01 \\
0.103 \\
0.01 \\
0.21 \\
0.27\end{array}$ & $\begin{array}{l}0.01 \\
0.01 \\
0.01 \\
0.01 \\
0.01\end{array}$ & $\begin{array}{l}- \\
- \\
- \\
-\end{array}$ \\
\hline FLUORIDE & $\begin{array}{l}10 / 11 / 85 \\
05 / 15 / 86 \\
04 / 06 / 88 \\
07 / 15 / 88 \\
01 / 08 / 89 \\
09 / 14 / 89 \\
03 / 31 / 90 \\
01 / 08 / 91 \\
11 / 25 / 92\end{array}$ & $\begin{array}{l}0001 \\
0001 \\
0001 \\
0001 \\
0001 \\
0001 \\
0001 \\
0001 \\
0001\end{array}$ & $M G / L$ & & $\begin{array}{l}0.5 \\
0.6 \\
0.43 \\
0.4 \\
0.5 \\
0.4 \\
0.4 \\
0.4 \\
0.4\end{array}$ & $\begin{array}{l}0.1 \\
0.1 \\
0.1 \\
0.1 \\
0.1 \\
0.1 \\
0.1 \\
0.1 \\
0.1\end{array}$ & $\begin{array}{l}- \\
- \\
- \\
- \\
- \\
- \\
-\end{array}$ \\
\hline GROSS ALPHA & $\begin{array}{l}04 / 06 / 88 \\
07 / 15 / 88 \\
01 / 08 / 89 \\
09 / 14 / 89 \\
03 / 31 / 90 \\
01 / 08 / 91 \\
11 / 25 / 92\end{array}$ & $\begin{array}{l}0001 \\
0001 \\
0001 \\
0001 \\
0001 \\
0001 \\
0001\end{array}$ & $\mathrm{PCI} / \mathrm{L}$ & & $\begin{array}{l}7800 . \\
9400 . \\
2880 . \\
4700 . \\
5900 . \\
4037 . \\
4650\end{array}$ & $\begin{array}{l}0.2 \\
0.2 \\
1 . \\
1 . \\
1 . \\
1.0 \\
1.0\end{array}$ & $\begin{array}{l}300 . \\
300 . \\
236 . \\
200 . \\
200 . \\
221 . \\
237 .\end{array}$ \\
\hline GROSS BETA & $\begin{array}{l}04 / 06 / 88 \\
07 / 15 / 88 \\
01 / 08 / 89 \\
09 / 14 / 89 \\
03 / 31 / 90 \\
01 / 08 / 91 \\
11 / 25 / 92\end{array}$ & $\begin{array}{l}0001 \\
0001 \\
0001 \\
0001 \\
0001 \\
0001 \\
0001\end{array}$ & PCI/L & & $\begin{array}{l}2700 . \\
2500 . \\
2950 . \\
2600 . \\
300 . \\
2005 . \\
2860 .\end{array}$ & $\begin{array}{l}1 . \\
1.5 \\
0.5 \\
0.5 \\
0.5 \\
0.5 \\
69.3\end{array}$ & $\begin{array}{l}100 . \\
100 . \\
132 . \\
100 \\
100 . \\
75.6 \\
110 .\end{array}$ \\
\hline IRON & $\begin{array}{l}10 / 11 / 85 \\
05 / 15 / 86 \\
04 / 06 / 88 \\
07 / 15 / 88 \\
01 / 08 / 89 \\
09 / 14 / 89\end{array}$ & $\begin{array}{l}0001 \\
0001 \\
0001 \\
0001 \\
0001 \\
0001\end{array}$ & MG/L & & $\begin{array}{l}0.09 \\
0.09 \\
0.16 \\
0.13 \\
0.04 \\
0.16\end{array}$ & $\begin{array}{l}0.03 \\
0.03 \\
0.03 \\
0.03 \\
0.03 \\
0.03\end{array}$ & $\begin{array}{l}- \\
- \\
- \\
-\end{array}$ \\
\hline
\end{tabular}

PARAMETER VALUE IMDICATOR (PVI): < - LESS THAN DETECTION LIMIT SAMPLE ID ICOOES:

0001 - FILTERED SAMPLE (.45 MICRONS)

OTHER PARAMETER VALUE FLAGS:

H - HOLD TIME EXPIRED, VALUE SUSPECT

J - estimated value 
GROUNDWATER QUALITY DATA BY LOCATION

SITE: AMBOI AMBROSIA LAKE

LOCATION: 0778

NORTH COORDIHATE: 52911.5 FT

EAST COORDIHATE: 60205.4 FT

$06 / 10 / 80$ TO $12 / 04 / 92$

REPORT DATE: $08 / 04 / 94$

FORMATION OF COHPLETION: TRES HERMANOS - CI SAHDSTONE (TO) HYORAULIC FLOW RELATIONSHIP: DOWN GRADIENT (D)

\begin{tabular}{|c|c|c|c|c|c|c|c|}
\hline PARANETER MAME & LOG DATE & $\begin{array}{c}\text { SAMPLE } \\
10\end{array}$ & $\begin{array}{l}\text { UNITS OF } \\
\text { MEASURE }\end{array}$ & PVI & $\begin{array}{l}\text { PARAHETER } \\
\text { VALUE FLAGS }\end{array}$ & $\begin{array}{l}\text { DETECTION } \\
\text { LIHIT }\end{array}$ & $\begin{array}{l}\text { PARAMETER } \\
\text { UNCERTAINTY }\end{array}$ \\
\hline IRON & $\begin{array}{l}03 / 31 / 90 \\
01 / 08 / 91 \\
11 / 25 / 92\end{array}$ & $\begin{array}{l}0001 \\
0001 \\
0001\end{array}$ & HG/L & & $\begin{array}{l}0.18 \\
0.08 \\
0.17\end{array}$ & $\begin{array}{l}0.03 \\
0.03 \\
0.03\end{array}$ & - \\
\hline LEAD & $\begin{array}{l}10 / 11 / 85 \\
04 / 06 / 88 \\
07 / 15 / 88 \\
01 / 08 / 89 \\
09 / 14 / 89 \\
03 / 31 / 90 \\
01 / 08 / 91\end{array}$ & $\begin{array}{l}0001 \\
0001 \\
0001 \\
0001 \\
0001 \\
0001 \\
0001\end{array}$ & MG/L & $<$ & $\begin{array}{l}0.01 \\
0.01 \\
0.01 \\
0.001 \\
0.02 \\
0.01 \\
0.03\end{array}$ & $\begin{array}{l}0.01 \\
0.01 \\
0.01 \\
0.01 \\
0.01 \\
0.01 \\
0.03\end{array}$ & $\begin{array}{l}- \\
- \\
- \\
- \\
-\end{array}$ \\
\hline LEAD-210 & $\begin{array}{l}10 / 11 / 85 \\
04 / 06 / 88 \\
07 / 15 / 88 \\
01 / 08 / 89 \\
09 / 14 / 89\end{array}$ & $\begin{array}{l}0001 \\
0001 \\
0001 \\
0001 \\
0001\end{array}$ & $\mathrm{PCl} / \mathrm{L}$ & & $\begin{array}{c}110 . \\
4.1 \\
1.2 \\
2.16 \\
0.9\end{array}$ & $\begin{array}{l}1.5 \\
1.5 \\
1.5 \\
1.5 \\
1.5\end{array}$ & $\begin{array}{l}4 . \\
1.1 \\
1.1 \\
1.61 \\
0.8\end{array}$ \\
\hline MAGNESIUM & $\begin{array}{l}10 / 11 / 85 \\
05 / 15 / 86 \\
04 / 06 / 88 \\
07 / 15 / 88 \\
01 / 08 / 89 \\
09 / 14 / 89 \\
03 / 31 / 90 \\
01 / 08 / 91 \\
11 / 25 / 92\end{array}$ & $\begin{array}{l}0001 \\
0001 \\
0001 \\
0001 \\
0001 \\
0001 \\
0001 \\
0001 \\
0001\end{array}$ & $M G / L$ & & $\begin{array}{l}171 . \\
202 . \\
191 . \\
192 . \\
205 . \\
195 . \\
176 . \\
228 . \\
205 .\end{array}$ & $\begin{array}{l}0.001 \\
0.001 \\
0.001 \\
0.001 \\
0.001 \\
0.001 \\
0.001 \\
0.5 \\
0.5\end{array}$ & $\begin{array}{l}- \\
- \\
- \\
- \\
- \\
- \\
-\end{array}$ \\
\hline MANGANESE & $\begin{array}{l}10 / 11 / 85 \\
05 / 15 / 86 \\
04 / 06 / 88 \\
07 / 15 / 88 \\
01 / 08 / 89 \\
09 / 14 / 89 \\
03 / 31 / 90 \\
01 / 08 / 91\end{array}$ & $\begin{array}{l}0001 \\
0001 \\
0001 \\
0001 \\
0001 \\
0001 \\
0001 \\
0001\end{array}$ & $M G / L$ & & $\begin{array}{l}0.06 \\
0.13 \\
0.06 \\
0.06 \\
0.01 \\
0.02 \\
0.02 \\
0.03\end{array}$ & $\begin{array}{l}0.01 \\
0.01 \\
0.01 \\
0.01 \\
0.01 \\
0.01 \\
0.01 \\
0.01\end{array}$ & $\begin{array}{l}- \\
- \\
- \\
- \\
- \\
-\end{array}$ \\
\hline HERCURY & $\begin{array}{l}10 / 11 / 85 \\
04 / 06 / 88 \\
07 / 15 / 88 \\
01 / 08 / 89 \\
09 / 14 / 89 \\
03 / 31 / 90 \\
01 / 08 / 91\end{array}$ & $\begin{array}{l}0001 \\
0001 \\
0001 \\
0001 \\
0001 \\
0001 \\
0001\end{array}$ & MG/L & $\begin{array}{l}< \\
< \\
< \\
< \\
<\end{array}$ & $\begin{array}{l}0.0002 \\
0.0005 \\
0.0003 \\
0.0001 \\
0.0002 \\
0.0002 \\
0.0002\end{array}$ & $\begin{array}{l}0.0002 \\
0.0002 \\
0.0002 \\
0.0002 \\
0.0002 \\
0.0002 \\
0.0002\end{array}$ & $\begin{array}{l}- \\
- \\
- \\
- \\
- \\
-\end{array}$ \\
\hline MOLYBJENCH & $\begin{array}{l}10 / 11 / 85 \\
05 / 15 / 86 \\
04 / 06 / 88 \\
07 / 15 / 88 \\
01 / 08 / 89 \\
09 / 14 / 89 \\
03 / 31 / 90 \\
01 / 08 / 91 \\
11 / 25 / 92\end{array}$ & $\begin{array}{l}0001 \\
0001 \\
0001 \\
0001 \\
0001 \\
0001 \\
0001 \\
0001 \\
0001\end{array}$ & $M G / L$ & $\begin{array}{l}< \\
< \\
<\end{array}$ & $\begin{array}{l}0.16 \\
0.13 \\
0.06 \\
0.06 \\
0.003 \\
0.02 \\
0.01 \\
0.01 \\
0.01\end{array}$ & $\begin{array}{l}0.01 \\
0.01 \\
0.01 \\
0.01 \\
0.01 \\
0.01 \\
0.01 \\
0.01 \\
0.01\end{array}$ & $\begin{array}{l}- \\
- \\
- \\
- \\
- \\
- \\
-\end{array}$ \\
\hline NET GROSS ALPHA - & $04 / 06 / 88$ & 0001 & $\mathrm{PCl} / \mathrm{L}$ & & 1399.62 & - & - \\
\hline
\end{tabular}

- MET GROSS ALPHA (GROSS ALPHA - URANIUM) MITH 1 HG URANIUM = 686 PCI

PARAMETER VALUE INDICATOR (PVI): < - LESS THAN DETECTION LIMIT

OTHER PARAMETER VALUE fLAGS:

I - INCREASED DETECTION LIHIT DUE TO REQUIRED DILUTION

J - ESTIMATED VALUE
SAMPLE ID COOES:

0001 - FILTERED SAMPLE (.45 MICRONS) 
GROUHDHATER QUALITY DATA BY LOCATIOH

SITE: AMB01 AMBROSIA LAKE

LOCATIOH: 0778

HORTH COORDIHATE: 52911.5 FT

EAST COORDIHATE: 60205.4 FT

06/10/80 TO $12 / 04 / 92$

REPORT DATE: 08/04/94

FORMATIOH OF COMPLETION: TRES HERMANOS - CI SAMDSTOME (TO)

HYDRAULIC FLON RELATIONSHIP: DOWN GRADIENT (D)

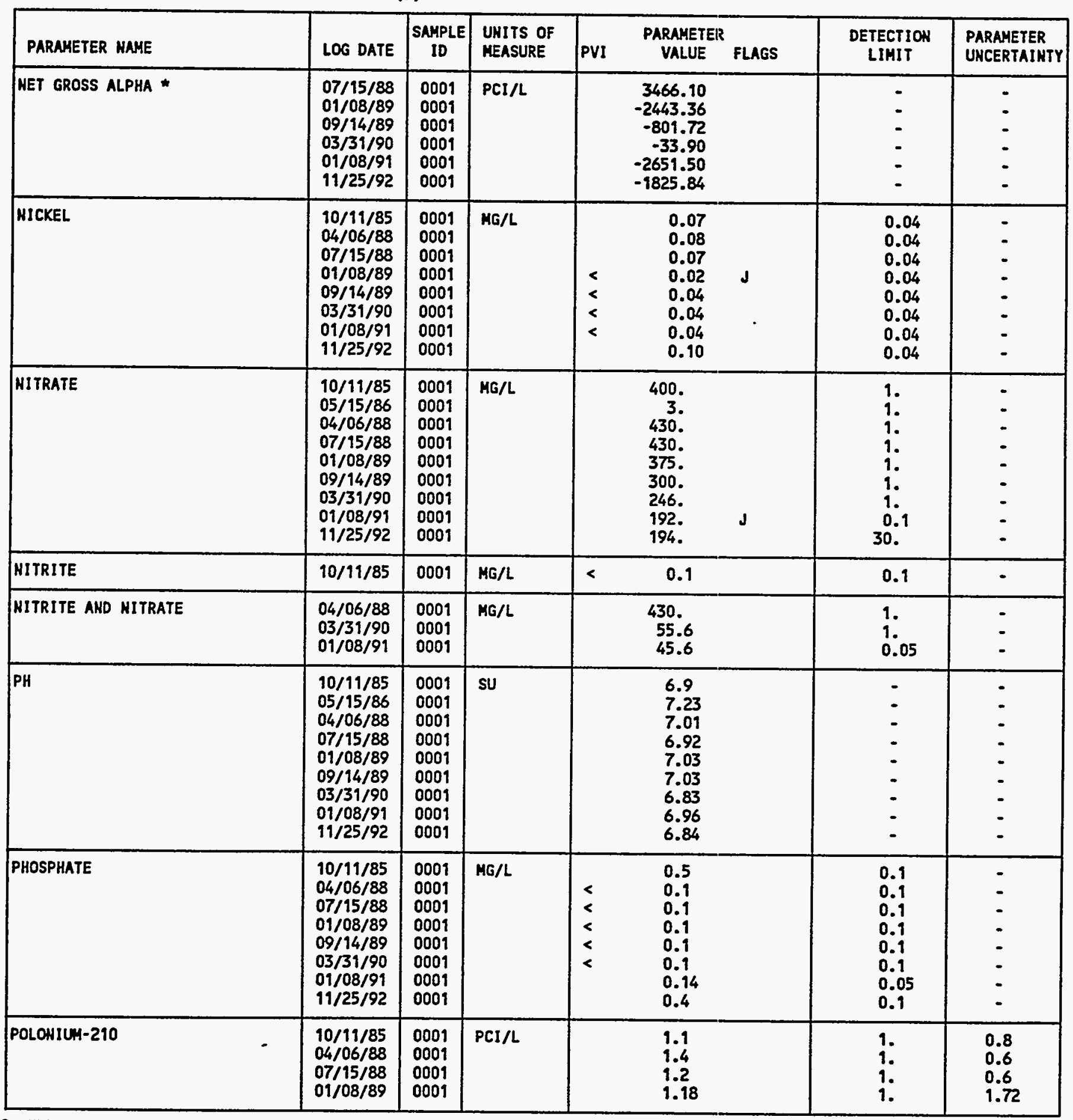

- het GROSS ALPHA (GROSS ALPHA - URAKIUH) WITH 1 MG URAKIUM = 686 PCI

PARAMETER VALUE IMDICATOR (PVI): < - LESS THAH DETECTIOH LIMIT SAMPLE ID LOOES:

0001 - FILTERED SAMPLE (.45 MICROHS)

OTHER PARAMETER VALUE fLAgS:

$J$ - estimated value 
GROUNDHATER QUALITY DATA BY LOCATION

SITE: AMBOI AMBROSIA LAKE

LOCATIOH: 0778

MORTH COORDIHATE: 52911.5 FT

EAST COORDIHATE: $\quad 60205.4 \mathrm{FT}$

$06 / 10 / 80$ TO $12 / 04 / 92$

REPORT DATE: 08/04/94

FORMATION OF COMPLETION: TRES HERHANOS - C1 SANDSTONE (TO) MYDRAULIC FLON RELATIONSHIP: DOWN GRADIENT (D)

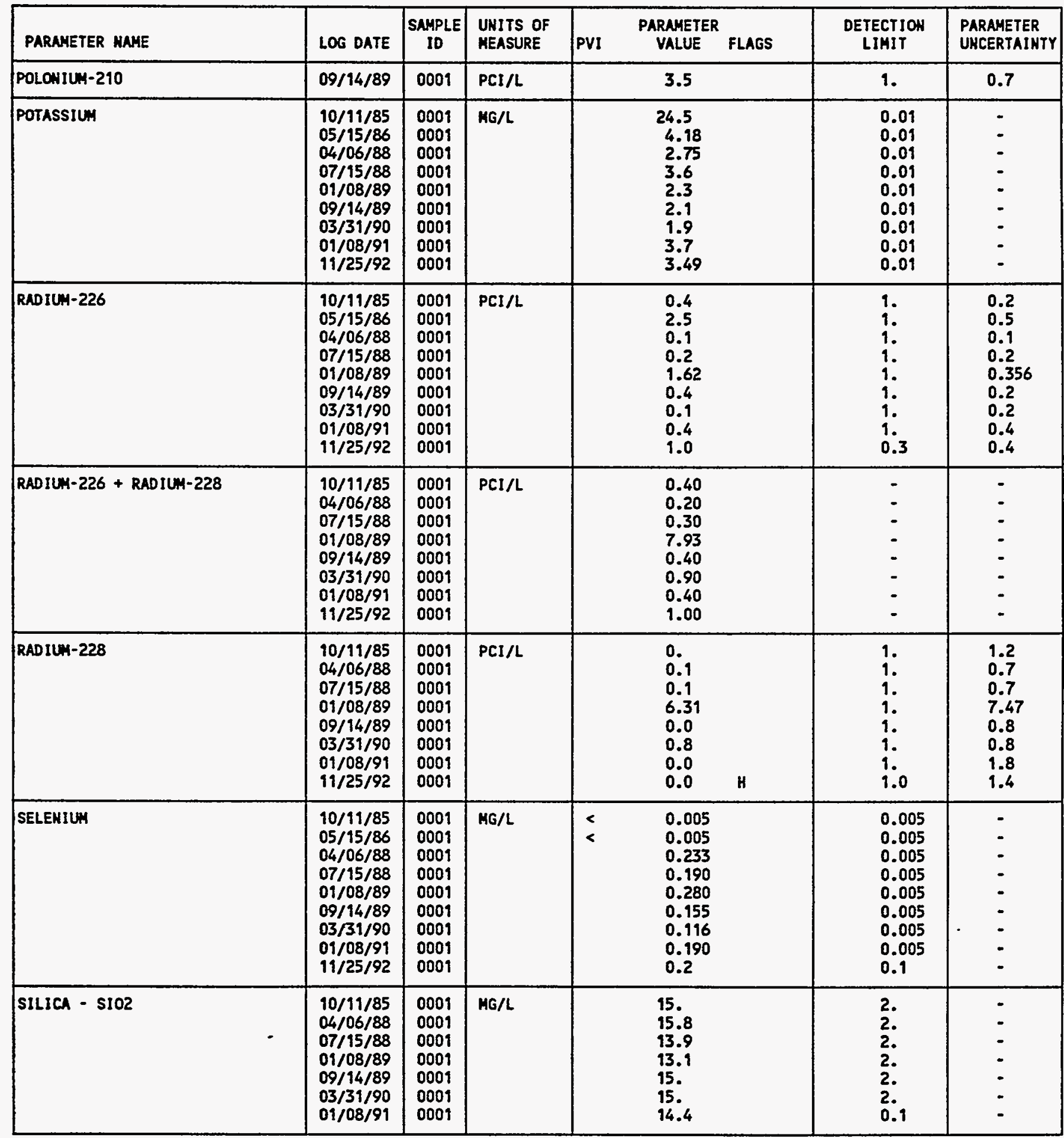

PARAMETER VALUE INDICATOR (PVI): < - LESS THAN DETECTION LIMIT

SAMPLE ID COOES

0001 - FILTERED SAMPLE (.45 MICROHS)

OTHER PARAMETER VALUE FLAGS:

H - HOLD TIME EXPIRED, VALLUE SUSPECT 
GROUKOWATER QUALITY DATA BY LOCATION

SITE: AMBOI AMBROSIA LAKE

LOCATION: 0778

HORTH COORDIHATE: 52911.5 FT

EAST COORDINATE: 60205.4 FT

06/10/80 TO $12 / 04 / 92$

REPORT DATE: 08/04/94

FORMATION OF COHPLETION: TRES HERMANOS - C1 SAHDSTOHE (TO) HYDRAULIC FLON RELATIONSHIP: DOWH GRADIENT (D)

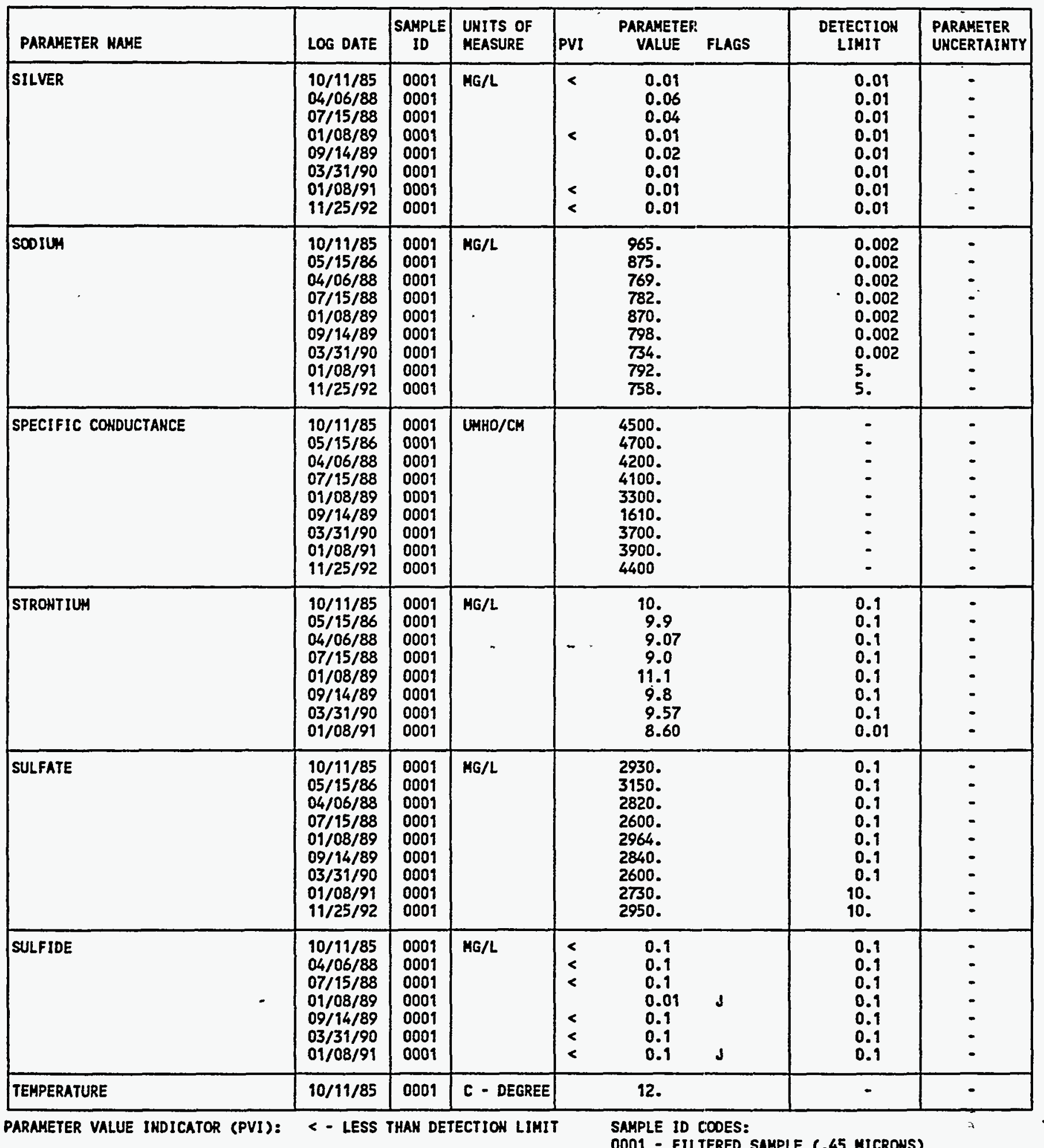

OTHER PARAMETER VALUE FLAGS:

J - ESTIMATED VALUE 
GROUMDHATER QUALITY DATA BY LOCATION

SITE: AMBO1 AMBROSIA LAKE

LOCATION: 0778

NORTH COORDINATE: 52911.5 FT

EAST COORDINATE: $\quad 60205.4$ FT

$06 / 10 / 80$ TO $12 / 04 / 92$

REPORT DATE: 08/04/94

FORMATION OF COMPLETION: TRES HERMANOS - C1 SANDSTONE (TO)

HYDRAULIC FLOW RELATIONSHIP: DOWN GRADIENT (D)

\begin{tabular}{|c|c|c|c|c|c|c|c|}
\hline PARAKETER NAME & LOG DATE & $\begin{array}{c}\text { SAMPLE } \\
10\end{array}$ & $\begin{array}{l}\text { UNITS OF } \\
\text { MEASURE }\end{array}$ & PVI & $\begin{array}{l}\text { PARAMETER } \\
\text { VALUE FLAGS }\end{array}$ & $\begin{array}{l}\text { DETECTIOH } \\
\text { LIMIT }\end{array}$ & $\begin{array}{l}\text { PARAMETER } \\
\text { UHCERTAINTY }\end{array}$ \\
\hline TEMPERATURE & $\begin{array}{l}05 / 15 / 86 \\
04 / 06 / 88 \\
07 / 15 / 88 \\
01 / 08 / 89 \\
09 / 14 / 89 \\
03 / 31 / 90 \\
01 / 08 / 91 \\
11 / 25 / 92\end{array}$ & $\begin{array}{l}0001 \\
0001 \\
0001 \\
0001 \\
0001 \\
0001 \\
0001 \\
0001\end{array}$ & C - DEGREE & & $\begin{array}{l}16 . \\
11.5 \\
12.0 \\
11.5 \\
12.0 \\
11.5 \\
12 . \\
11.0\end{array}$ & $\begin{array}{l}- \\
- \\
- \\
- \\
- \\
-\end{array}$ & $\begin{array}{l}- \\
- \\
- \\
- \\
- \\
-\end{array}$ \\
\hline THALLIUH & $\begin{array}{l}01 / 08 / 89 \\
09 / 14 / 89 \\
03 / 31 / 90 \\
01 / 08 / 91\end{array}$ & $\begin{array}{l}0001 \\
0001 \\
0001 \\
0001\end{array}$ & MG/L & $\begin{array}{l}< \\
< \\
<\end{array}$ & $\begin{array}{l}0.002 \\
0.01 \\
0.01 \\
0.03\end{array}$ & $\begin{array}{l}0.001 \\
0.01 \\
0.01 \\
0.03\end{array}$ & - \\
\hline THORIUM-230 & $\begin{array}{l}10 / 11 / 85 \\
05 / 15 / 86 \\
04 / 06 / 88 \\
07 / 15 / 88 \\
01 / 08 / 89 \\
09 / 14 / 89\end{array}$ & $\begin{array}{l}0001 \\
0001 \\
0001 \\
0001 \\
0001 \\
0001\end{array}$ & PCI/L & & $\begin{array}{l}5.5 \\
3 . \\
1.2 \\
0.0 \\
1.46 \\
0.0\end{array}$ & $\begin{array}{l}1 . \\
1 . \\
1 . \\
1 . \\
1 . \\
1 .\end{array}$ & $\begin{array}{l}1.4 \\
1 . \\
0.8 \\
0.3 \\
0.152 \\
0.2\end{array}$ \\
\hline TIN & $\begin{array}{l}10 / 11 / 85 \\
04 / 06 / 88 \\
07 / 15 / 88 \\
01 / 08 / 89 \\
09 / 14 / 89 \\
03 / 31 / 90 \\
01 / 08 / 91\end{array}$ & $\begin{array}{l}0001 \\
0001 \\
0001 \\
0001 \\
0001 \\
0001 \\
0001\end{array}$ & MG/L & $\begin{array}{l}< \\
< \\
<\end{array}$ & $\begin{array}{l}0.005 \\
0.030 \\
0.019 \\
0.005 \\
0.109 \\
0.029 \\
0.03\end{array}$ & $\begin{array}{l}0.005 \\
0.005 \\
0.005 \\
0.005 \\
0.005 \\
0.005 \\
0.03\end{array}$ & $\begin{array}{l}- \\
- \\
- \\
- \\
- \\
-\end{array}$ \\
\hline TOTAL DISSOLVED SOLIDS & $\begin{array}{l}10 / 11 / 85 \\
05 / 15 / 86 \\
04 / 06 / 88 \\
07 / 15 / 88 \\
01 / 08 / 89 \\
09 / 14 / 89 \\
03 / 31 / 90 \\
01 / 08 / 91 \\
11 / 25 / 92\end{array}$ & $\begin{array}{l}0001 \\
0001 \\
0001 \\
0001 \\
0001 \\
0001 \\
0001 \\
0001 \\
0001\end{array}$ & MG/L & & $\begin{array}{l}5610 . \\
5920 . \\
5720 . \\
5820 . \\
5506 . \\
5180 . \\
4990 . \\
5340 . \\
5550 .\end{array}$ & $\begin{array}{l}10 . \\
10 . \\
10 . \\
10 . \\
10 . \\
10 . \\
10 . \\
10 . \\
10 .\end{array}$ & $\begin{array}{l}- \\
- \\
- \\
- \\
- \\
- \\
- \\
-\end{array}$ \\
\hline TOTAL KJELDAHL NITROGEN & $\begin{array}{l}01 / 08 / 91 \\
11 / 25 / 92\end{array}$ & $\begin{array}{l}0001 \\
0001\end{array}$ & MG/L & & 1. & 1. & - \\
\hline TOTAL ORGANIC CARBON & $\begin{array}{l}10 / 11 / 85 \\
04 / 06 / 88 \\
07 / 15 / 88 \\
01 / 08 / 89 \\
09 / 14 / 89 \\
03 / 31 / 90 \\
01 / 08 / 91 \\
11 / 25 / 92\end{array}$ & $\begin{array}{l}0001 \\
0001 \\
0001 \\
0001 \\
0001 \\
0001 \\
0001 \\
0001\end{array}$ & HG/L & & $\begin{array}{l}81 . \\
136 . \\
113 . \\
18 . \\
97.9 \\
17.3 \\
18 . \\
16 .\end{array}$ & $\begin{array}{l}1 . \\
1 . \\
1 . \\
1 . \\
1 . \\
1 . \\
1 .\end{array}$ & $\begin{array}{l}- \\
- \\
- \\
- \\
- \\
-\end{array}$ \\
\hline URANIUM & $\begin{array}{l}10 / 11 / 85 \\
05 / 15 / 86 \\
04 / 06 / 88 \\
07 / 15 / 88 \\
01 / 08 / 89\end{array}$ & $\begin{array}{l}0001 \\
0001 \\
0001 \\
0001 \\
0001\end{array}$ & $M G / L$ & & $\begin{array}{l}11.8 \\
9.31 \\
9.33 \\
8.65 \\
7.760\end{array}$ & $\begin{array}{l}0.003 \\
0.003 \\
0.003 \\
0.003 \\
0.003\end{array}$ & $\begin{array}{l}- \\
- \\
- \\
-\end{array}$ \\
\hline
\end{tabular}

PARAMETER VALUE INDICATOR (PVI): < - LESS THAN DETECTIOH LIMIT

SAMPLE ID CODES:

0001 - FILTERED SAMPLE (.45 MICRONS)

OTHER PARAMETER VALUE FLAGS:

I - IHCREASED DETECTIOH LIMIT DUE TO REOUIRED DILUTION

- ESTIMATED VALUE 
GROUNDWATER OUALITY DATA BY LOCATION

SITE: AMBO1 AMBROSIA LAKE

LOCATIOH: 0778

HORTH COORDINATE: 52911.5 FT

EAST COORDIHATE: $\quad 60205.4$ FT

06/10/80 TO $12 / 04 / 92$

REPORT DATE: 08/04/94

FORMATION OF COMPLETION: TRES HERMANOS - C1 SAMDSTONE (TO) HYDRAULIC FLOW RELATIONSHIP: DOWH GRADIENT (D)

\begin{tabular}{|c|c|c|c|c|c|c|c|c|}
\hline PARAMETER MAME & LOG DATE & $\begin{array}{c}\text { SAMPLE } \\
\text { ID }\end{array}$ & $\begin{array}{l}\text { UNITS OF } \\
\text { MEASURE }\end{array}$ & PVI & $\begin{array}{l}\text { PARAMETER } \\
\text { VALUE }\end{array}$ & FLAGS & $\begin{array}{l}\text { DETECTIOW } \\
\text { LIMIT }\end{array}$ & $\begin{array}{l}\text { PARÁLMETER } \\
\text { UHCERTAINTY }\end{array}$ \\
\hline URANIUH & $\begin{array}{l}09 / 14 / 89 \\
03 / 31 / 90 \\
01 / 08 / 99 \\
11 / 25 / 92\end{array}$ & $\begin{array}{l}0001 \\
0001 \\
0001 \\
0001\end{array}$ & $M G / L$ & & $\begin{array}{l}8.02 \\
8.65 \\
9.75 \\
9.44\end{array}$ & H & $\begin{array}{l}0.003 \\
0.003 \\
0.001 \\
0.001\end{array}$ & $\begin{array}{l}- \\
- \\
-\end{array}$ \\
\hline VAKADILM & $\begin{array}{l}10 / 11 / 85 \\
05 / 15 / 86 \\
04 / 06 / 88 \\
07 / 15 / 88 \\
01 / 08 / 89 \\
09 / 14 / 89 \\
03 / 31 / 90 \\
01 / 08 / 91 \\
11 / 25 / 92\end{array}$ & $\begin{array}{l}0001 \\
0001 \\
0001 \\
0001 \\
0001 \\
0001 \\
0001 \\
0001 \\
0001\end{array}$ & MG/L & $\begin{array}{l}< \\
< \\
< \\
<\end{array}$ & $\begin{array}{l}0.01 \\
0.23 \\
0.09 \\
0.07 \\
0.01 \\
0.02 \\
0.02 \\
0.01 \\
0.2\end{array}$ & I & $\begin{array}{l}0.01 \\
0.01 \\
0.01 \\
0.01 \\
0.01 \\
0.01 \\
0.01 \\
0.01 \\
0.2\end{array}$ & $\begin{array}{l}- \\
\dot{-} \\
\dot{-} \\
\dot{-} \\
\dot{-} \\
-\end{array}$ \\
\hline ZINC & $\begin{array}{l}10 / 11 / 85 \\
04 / 06 / 88 \\
07 / 15 / 88 \\
01 / 08 / 89 \\
09 / 14 / 89 \\
03 / 31 / 90 \\
01 / 08 / 91\end{array}$ & $\begin{array}{l}0001 \\
0001 \\
0001 \\
0001 \\
0001 \\
0001 \\
0001\end{array}$ & $M G / L$ & $\begin{array}{l}< \\
< \\
<\end{array}$ & $\begin{array}{l}0.039 \\
0.035 \\
0.021 \\
0.01 \\
0.005 \\
0.009 \\
0.005\end{array}$ & & $\begin{array}{l}0.005 \\
0.005 \\
0.005 \\
0.01 \\
0.005 \\
0.005 \\
0.005\end{array}$ & $\begin{array}{l}\dot{-} \\
\dot{-} \\
\dot{-} \\
\dot{-} \\
-\end{array}$ \\
\hline
\end{tabular}

PARAMETER VALUE IHDICATOR (PVI): < - LESS THAN DETECTION LIMIT

OTHER PARAMETER VALUE FLAGS:

H - HOLD TIME EXPIRED, VALUE SUSPECT

1 - INCREASED DETECTION LIMIT DUE TO REQUIRED DILUTION 
GROUNDWATER OUALITY DATA BY LOCATIOH

SITE: AMBOI AMBROSIA LAKE

LOCATION: OT79

NORTH COORDINATE: 52914.0 FT

EAST COORDIHATE: 60284.7 FT

06/10/80 TO 12/04/92

REPORT DATE: $08 / 04 / 94$

FORMATION OF COMPLETION: TRES HERMANOS - C2 SANDSTONE (TT)

HYORAULIC FLON RELATIONSHIP: DOWN GRADIENT (D)

\begin{tabular}{|c|c|c|c|c|c|c|c|c|}
\hline PARAMETER NAME & LOS DATE & $\begin{array}{c}\text { SAMPLE } \\
\text { ID }\end{array}$ & $\begin{array}{l}\text { UNITS OF } \\
\text { MEASURE }\end{array}$ & PVI & $\begin{array}{l}\text { PARAMETER } \\
\text { VALUE }\end{array}$ & FLAGS & $\begin{array}{l}\text { DETECTION } \\
\text { LIMIT }\end{array}$ & $\begin{array}{l}\text { PARAMETER } \\
\text { UHCERTAIHTY }\end{array}$ \\
\hline ALKALINITY & $\begin{array}{l}10 / 10 / 85 \\
05 / 14 / 86 \\
04 / 06 / 88 \\
07 / 15 / 88 \\
01 / 11 / 89 \\
09 / 19 / 89\end{array}$ & $\begin{array}{l}0001 \\
0009 \\
0001 \\
0001 \\
0009 \\
0001\end{array}$ & $\mathrm{MG} / \mathrm{L} \mathrm{CACO3}$ & & $\begin{array}{l}672 . \\
747 . \\
808 . \\
895 . \\
850 . \\
788 .\end{array}$ & & $\begin{array}{l}- \\
: \\
-\end{array}$ & $\begin{array}{l}- \\
: \\
-\end{array}$ \\
\hline ALUAINUA & $\begin{array}{l}10 / 10 / 85 \\
04 / 06 / 88 \\
07 / 15 / 88 \\
01 / 11 / 89 \\
09 / 19 / 89\end{array}$ & $\begin{array}{l}0001 \\
0001 \\
0001 \\
0001 \\
0001\end{array}$ & $M G / L$ & $<$ & $\begin{array}{l}0.2 \\
0.32 \\
0.1 \\
0.05 \\
0.1\end{array}$ & $J$ & $\begin{array}{l}0.1 \\
0.1 \\
0.1 \\
0.1 \\
0.1\end{array}$ & : \\
\hline AMHONIU: & $\begin{array}{l}10 / 10 / 85 \\
04 / 06 / 88 \\
07 / 15 / 88 \\
01 / 19 / 89 \\
09 / 19 / 89\end{array}$ & $\begin{array}{l}0001 \\
0001 \\
0001 \\
0009 \\
0001\end{array}$ & $M G / L$ & & $\begin{array}{l}0.4 \\
0.4 \\
1.1 \\
1.11 \\
0.8\end{array}$ & & $\begin{array}{l}0.1 \\
0.1 \\
0.1 \\
0.1 \\
0.1\end{array}$ & $\begin{array}{l}- \\
- \\
- \\
-\end{array}$ \\
\hline ANTIHONY & $\begin{array}{l}10 / 10 / 85 \\
04 / 06 / 88 \\
07 / 15 / 88 \\
01 / 19 / 89 \\
09 / 19 / 89\end{array}$ & $\begin{array}{l}0001 \\
0001 \\
0001 \\
0001 \\
0001\end{array}$ & $M G / L$ & $<$ & $\begin{array}{l}0.003 \\
0.003 \\
0.025 \\
0.002 \\
0.007\end{array}$ & $\mathbf{J}$ & $\begin{array}{l}0.003 \\
0.003 \\
0.003 \\
0.003 \\
0.003\end{array}$ & $\begin{array}{l}- \\
- \\
-\end{array}$ \\
\hline ARSENIC & $\begin{array}{l}10 / 10 / 85 \\
05 / 14 / 86 \\
04 / 06 / 88 \\
07 / 15 / 88 \\
01 / 19 / 89 \\
09 / 19 / 89\end{array}$ & $\begin{array}{l}0001 \\
0001 \\
0001 \\
0001 \\
0001 \\
0009\end{array}$ & $M G / L$ & $<$ & $\begin{array}{l}0.01 \\
0.01 \\
0.025 \\
0.01 \\
0.001 \\
0.02\end{array}$ & J & $\begin{array}{l}0.01 \\
0.01 \\
0.01 \\
0.01 \\
0.01 \\
0.01\end{array}$ & $\begin{array}{l}- \\
- \\
- \\
-\end{array}$ \\
\hline BARIUM & $\begin{array}{l}10 / 10 / 85 \\
04 / 06 / 88 \\
07 / 15 / 88 \\
01 / 11 / 89 \\
09 / 19 / 89\end{array}$ & $\begin{array}{l}0001 \\
0001 \\
0001 \\
0001 \\
0001\end{array}$ & $M G / L$ & $<$ & $\begin{array}{l}0.3 \\
0.03 \\
0.1 \\
0.02 \\
0.1\end{array}$ & $\begin{array}{l}J \\
J\end{array}$ & $\begin{array}{l}0.1 \\
0.1 \\
0.1 \\
0.9 \\
0.1\end{array}$ & $\begin{array}{l}- \\
- \\
- \\
-\end{array}$ \\
\hline BERYLLIUH & $\begin{array}{l}01 / 99 / 89 \\
09 / 19 / 89\end{array}$ & $\begin{array}{l}0001 \\
0001\end{array}$ & $\mathrm{HG} / \mathrm{L}$ & $<$ & $\begin{array}{l}0.005 \\
0.01\end{array}$ & & $\begin{array}{l}0.005 \\
0.01\end{array}$ & - \\
\hline BORON & $\begin{array}{l}10 / 10 / 85 \\
05 / 14 / 86 \\
04 / 06 / 88 \\
07 / 15 / 88 \\
01 / 11 / 89 \\
09 / 19 / 89\end{array}$ & $\begin{array}{l}0001 \\
0001 \\
0001 \\
0001 \\
0001 \\
0001\end{array}$ & $M G / L$ & & $\begin{array}{l}0.7 \\
1.1 \\
1.06 \\
0.8 \\
0.80 \\
0.7\end{array}$ & & $\begin{array}{l}0.1 \\
0.1 \\
0.1 \\
0.1 \\
0.1 \\
0.1\end{array}$ & $\begin{array}{l}- \\
- \\
- \\
-\end{array}$ \\
\hline BROMIDE & $\begin{array}{l}04 / 06 / 88 \\
07 / 15 / 88 \\
01 / 19 / 89 \\
09 / 19 / 89\end{array}$ & $\begin{array}{l}0001 \\
0001 \\
0001 \\
0001\end{array}$ & MG/L & $\begin{array}{l}< \\
<\end{array}$ & $\begin{array}{c}0.1 \\
0.1 \\
41 . \\
0.1\end{array}$ & & $\begin{array}{l}0.1 \\
0.1 \\
0.01 \\
0.1\end{array}$ & : \\
\hline CADMIUN & $\begin{array}{l}10 / 10 / 85 \\
05 / 14 / 86 \\
04 / 06 / 88 \\
07 / 15 / 88\end{array}$ & $\begin{array}{l}0001 \\
0001 \\
0001 \\
0001\end{array}$ & $M G / L$ & $<$ & $\begin{array}{l}0.001 \\
0.001 \\
0.016 \\
0.001\end{array}$ & & $\begin{array}{l}0.001 \\
0.001 \\
0.001 \\
0.001\end{array}$ & - \\
\hline
\end{tabular}

PARAMETER VALUE INDICATOR (PVI): < - LESS THAN DETECTION LIMIT

SAMPLE ID COOES:

0001 - FILTERED SAMPLE (.45 MICRONS)

OTHER PARAMETER VALUE fLAGS:

J - ESTIMATED VALUE 
GROUNDWATER OUALITY DATA BY LOCATIOW

SITE: AMBO1 AMBROSIA LAKE

LOCATION: OTT9

MORTH COORDINATE: 52914.0 FT

EAST COORDIHATE: 60284.7 FT

06/10/80 TO 12/04/92

REPORT DATE: 08/04/94

FORHATIOH OF COMPLETIOH: TRES HERMANOS - C2 SANDSTOHE (TT) HYDRAULIC FLOA RELATIOHSHIP: DOWH GRADIENT (D)

\begin{tabular}{|c|c|c|c|c|c|c|c|c|}
\hline PARAMETER NAHE & LOG DATE & $\begin{array}{c}\text { SAMPLE } \\
10\end{array}$ & $\begin{array}{l}\text { UHITS OF } \\
\text { MEASURE }\end{array}$ & PVI & $\begin{array}{l}\text { PARAMETER } \\
\text { VALUE }\end{array}$ & FLAGS & $\begin{array}{l}\text { DETECTION } \\
\text { LIHIT }\end{array}$ & $\begin{array}{l}\text { PARAMETER } \\
\text { UHCERTAIHTY }\end{array}$ \\
\hline CADHIUH & $\begin{array}{l}01 / 11 / 89 \\
09 / 19 / 89\end{array}$ & $\begin{array}{l}0001 \\
0001\end{array}$ & MG/L & $<$ & $\begin{array}{l}0.0001 \\
0.015\end{array}$ & $\sqrt{ }$ & $\begin{array}{l}0.001 \\
0.001\end{array}$ & - \\
\hline CALCIUM & $\begin{array}{l}10 / 10 / 85 \\
05 / 14 / 86 \\
04 / 06 / 88 \\
07 / 15 / 88 \\
01 / 11 / 89 \\
09 / 19 / 89\end{array}$ & $\begin{array}{l}0001 \\
0001 \\
0001 \\
0001 \\
0001 \\
0001\end{array}$ & MG/L & & $\begin{array}{l}403 . \\
493 \\
525 \\
427 \\
483 \\
407\end{array}$ & & $\begin{array}{l}0.01 \\
0.01 \\
0.01 \\
0.01 \\
0.01 \\
0.01\end{array}$ & $\begin{array}{l}- \\
- \\
-\end{array}$ \\
\hline CHLORIDE & $\begin{array}{l}10 / 10 / 85 \\
05 / 14 / 86 \\
04 / 06 / 88 \\
07 / 15 / 88 \\
01 / 11 / 89 \\
09 / 19 / 89\end{array}$ & $\begin{array}{l}0001 \\
0001 \\
0001 \\
0001 \\
0001 \\
0001\end{array}$ & MG/L & & $\begin{array}{l}2 . \\
24 . \\
35 . \\
21 . \\
22 . \\
23 .\end{array}$ & & $\begin{array}{l}1 . \\
1 . \\
1 . \\
1 .\end{array}$ & $\begin{array}{l}- \\
- \\
- \\
-\end{array}$ \\
\hline CHROHIUH & $\begin{array}{l}10 / 10 / 85 \\
05 / 14 / 86 \\
04 / 06 / 88 \\
07 / 15 / 88 \\
01 / 11 / 89 \\
09 / 19 / 89\end{array}$ & $\begin{array}{l}0001 \\
0001 \\
0001 \\
0001 \\
0001 \\
0001\end{array}$ & MG/L & $<$ & $\begin{array}{l}0.04 \\
0.04 \\
0.22 \\
0.15 \\
0.01 \\
0.01\end{array}$ & & $\begin{array}{l}0.01 \\
0.01 \\
0.01 \\
0.01 \\
0.01 \\
0.01\end{array}$ & $\begin{array}{l}- \\
- \\
- \\
-\end{array}$ \\
\hline COBALT & $\begin{array}{l}10 / 10 / 85 \\
05 / 14 / 86 \\
04 / 06 / 88 \\
07 / 15 / 88 \\
01 / 11 / 89 \\
09 / 19 / 89\end{array}$ & $\begin{array}{l}0001 \\
0001 \\
0001 \\
0001 \\
0001 \\
0001\end{array}$ & HG/L & $<$ & $\begin{array}{l}0.07 \\
0.1 \\
0.06 \\
0.05 \\
0.01 \\
0.05\end{array}$ & $J$ & $\begin{array}{l}0.05 \\
0.05 \\
0.05 \\
0.05 \\
0.05 \\
0.05\end{array}$ & $\begin{array}{l}- \\
- \\
- \\
-\end{array}$ \\
\hline COPPER & $\begin{array}{l}-10 / 10 / 85 \\
04 / 06 / 88 \\
07 / 15 / 88 \\
01 / 11 / 89 \\
09 / 19 / 89\end{array}$ & $\begin{array}{l}0001 \\
0001 \\
0001 \\
0001 \\
0001\end{array}$ & $M G / L$ & $<$ & $\begin{array}{l}0.03 \\
0.03 \\
0.02 \\
0.01 \\
0.02\end{array}$ & J & $\begin{array}{l}0.02 \\
0.02 \\
0.02 \\
0.02 \\
0.02\end{array}$ & $\begin{array}{l}- \\
- \\
-\end{array}$ \\
\hline CYAHIDE & $\begin{array}{l}10 / 10 / 85 \\
01 / 11 / 89 \\
09 / 19 / 89\end{array}$ & $\begin{array}{l}0001 \\
0001 \\
0001\end{array}$ & MG/L & $\begin{array}{l}< \\
<\end{array}$ & $\begin{array}{l}0.01 \\
0.002 \\
0.01\end{array}$ & J & $\begin{array}{l}0.09 \\
0.01 \\
0.01\end{array}$ & : \\
\hline FLUORIDE & $\begin{array}{l}10 / 10 / 85 \\
05 / 14 / 86 \\
04 / 06 / 88 \\
07 / 15 / 88 \\
01 / 19 / 89 \\
09 / 99 / 89\end{array}$ & $\begin{array}{l}0001 \\
0001 \\
0001 \\
0001 \\
0001 \\
0001\end{array}$ & MG/L & & $\begin{array}{l}0.5 \\
0.4 \\
0.22 \\
0.4 \\
0.2 \\
0.2\end{array}$ & & $\begin{array}{l}0.1 \\
0.1 \\
0.1 \\
0.1 \\
0.1 \\
0.1\end{array}$ & $\begin{array}{l}- \\
\vdots \\
\vdots\end{array}$ \\
\hline GROSS ALPHA & $\begin{array}{l}04 / 06 / 88 \\
07 / 15 / 88 \\
01 / 11 / 89 \\
09 / 19 / 89\end{array}$ & $\begin{array}{l}0001 \\
0001 \\
0001 \\
0001\end{array}$ & PCI/L & & $\begin{array}{l}68 . \\
74 . \\
31.6 \\
15 .\end{array}$ & J & $\begin{array}{l}0.2 \\
0.2 \\
1 . \\
1 .\end{array}$ & $\begin{array}{l}46 . \\
50 . \\
44.9 \\
33 .\end{array}$ \\
\hline GROSS BETA & $\begin{array}{l}04 / 06 / 88 \\
07 / 15 / 88 \\
01 / 11 / 89 \\
09 / 19 / 89\end{array}$ & $\begin{array}{l}0001 \\
0001 \\
0001 \\
0001\end{array}$ & PCI/L & & $\begin{array}{l}77 . \\
57 . \\
64.9 \\
16 .\end{array}$ & & $\begin{array}{l}1 . \\
1 . \\
0.5 \\
0.5\end{array}$ & $\begin{array}{l}26 . \\
20 . \\
49.8 \\
18 .\end{array}$ \\
\hline
\end{tabular}

PARAMETER VALUE INDICATOR (PVI): < - LESS THAH DETECTION LIMIT

SAMPLE IO COOES:

0001 - FILTERED SAMPLE (.45 MICRONS)

OTHER PARAMETER VALUE FLAGS:

d. estimated VALUE 
GROUNDHATER QUALITY DATA BY LOCATION

SITE: AMBOT AMBROSIA LAKE

LOCATIOH: OT79

NORTH COORDINATE: 52914.0 FT

EAST COORDINATE: $\quad 60284.7$ FT

06/10/80 TO $12 / 04 / 92$

REPORT DATE: 08/04/94

FORMATION OF COHPLETION: TRES HERMANOS - C2 SANDSTONE (TT) HYDRAULIC FLON RELATIONSHIP: DOWH GRADIENT (D)

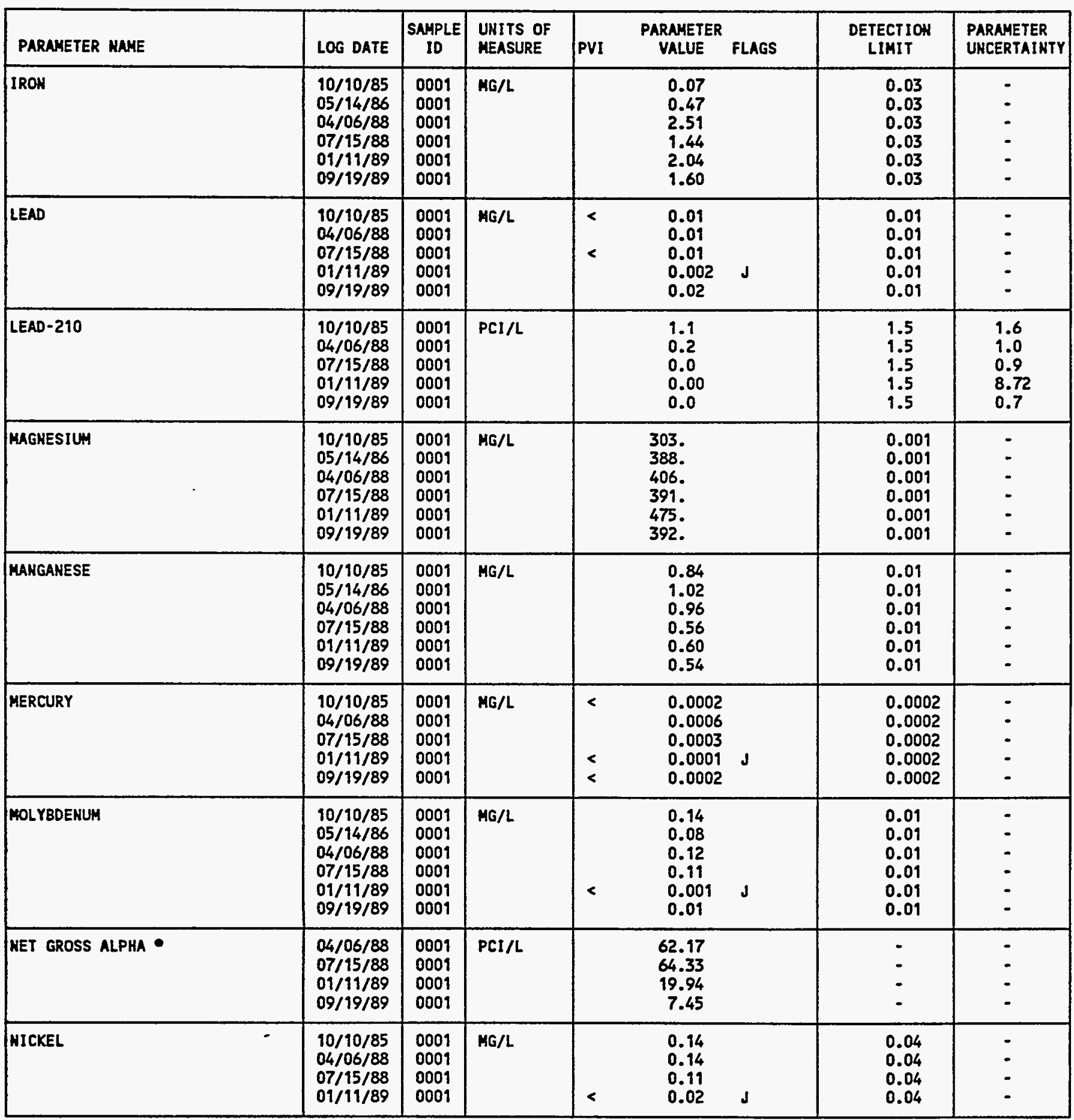

- MET gROSS ALPHA (GROSS ALPHA - URANIUM) UITH 1 HG URANIUM = 686 PCI

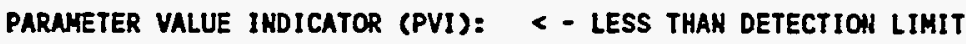


GROUHDUATER QUALITY DATA BY LOCATIOH

SITE: AMBOI AMBROSIA LAKE

LOCATION: 0779

MORTH COORDIHATE: 52914.0 FT

EAST COORDINATE: 60284.7 FT

06/10/80 TO $12 / 04 / 92$

REPORT DATE: $08 / 04 / 94$

FORMATIOH OF COMPLETIOH: TRES HERMANOS - C2 SANDSTOHE (TT) HYDRAULIC FLON RELATIONSHIP: DOWN GRADIEHT (D)

\begin{tabular}{|c|c|c|c|c|c|c|c|}
\hline $\begin{array}{c}\vdots \\
\text { PARAMETER MAME }\end{array}$ & LOG DATE & $\mid \begin{array}{c}\text { SNMPLE } \\
10\end{array}$ & $\begin{array}{l}\text { UNITS OF } \\
\text { MEASURE }\end{array}$ & PVI & $\begin{array}{l}\text { PARAMETER } \\
\text { VALUE FLAGS }\end{array}$ & $\begin{array}{l}\text { DETECTION } \\
\text { LIMIT }\end{array}$ & $\begin{array}{l}\text { PARAMETER } \\
\text { UNCERTAIHTY }\end{array}$ \\
\hline NICKEL & $09 / 19 / 89$ & 0001 & $M G / L$ & $<$ & 0.04 & 0.04 & - \\
\hline NITRATE & $\begin{array}{l}10 / 10 / 85 \\
05 / 14 / 86 \\
04 / 06 / 88 \\
07 / 15 / 88 \\
01 / 11 / 89 \\
09 / 19 / 89\end{array}$ & $\begin{array}{l}0001 \\
0001 \\
0001 \\
0001 \\
0001 \\
0001\end{array}$ & $M G / L$ & $<$ & $\begin{array}{l}2 . \\
1 . \\
0.2 \\
2.1 \\
2.29 \\
1 .\end{array}$ & $\begin{array}{l}1 . \\
1 . \\
1 . \\
1 .\end{array}$ & $\begin{array}{l}- \\
= \\
- \\
-\end{array}$ \\
\hline MITRITE & $10 / 10 / 85$ & 0001 & $M G / L$ & $<$ & 0.1 & 0.1 & - \\
\hline HITRITE AKD NITRATE & $04 / 06 / 88$ & 0001 & MG/L & & 0.2 & 1. & - \\
\hline PH & $\begin{array}{l}10 / 10 / 85 \\
05 / 14 / 86 \\
04 / 06 / 88 \\
07 / 15 / 88 \\
01 / 11 / 89 \\
09 / 19 / 89\end{array}$ & $\begin{array}{l}0001 \\
0001 \\
0001 \\
0001 \\
0001 \\
0001\end{array}$ & SU & & $\begin{array}{l}6.66 \\
6.62 \\
6.83 \\
6.71 \\
6.72 \\
6.68\end{array}$ & $\begin{array}{l}- \\
- \\
- \\
-\end{array}$ & $\begin{array}{l}- \\
- \\
- \\
-\end{array}$ \\
\hline PHOSPHATE & $\begin{array}{l}10 / 10 / 85 \\
04 / 06 / 88 \\
07 / 15 / 88 \\
01 / 11 / 89 \\
09 / 19 / 89\end{array}$ & $\begin{array}{l}0001 \\
0001 \\
0001 \\
0001 \\
0001\end{array}$ & $M G / L$ & $\begin{array}{l}< \\
< \\
< \\
<\end{array}$ & $\begin{array}{l}0.4 \\
0.1 \\
0.1 \\
0.1 \\
0.1\end{array}$ & $\begin{array}{l}0.1 \\
0.1 \\
0.1 \\
0.1 \\
0.1\end{array}$ & $\begin{array}{l}- \\
- \\
-\end{array}$ \\
\hline POLONIUMA-210 & $\begin{array}{l}10 / 10 / 85 \\
04 / 06 / 88 \\
07 / 15 / 88 \\
01 / 11 / 89 \\
09 / 19 / 89\end{array}$ & $\begin{array}{l}0001 \\
0001 \\
0001 \\
0001 \\
0001\end{array}$ & $\mathrm{PCI} / \mathrm{L}$ & & $\begin{array}{l}0 . \\
0.3 \\
0.0 \\
1.09 \\
1.3\end{array}$ & $\begin{array}{l}1 . \\
1 . \\
1 .\end{array}$ & $\begin{array}{l}0.6 \\
0.5 \\
0.4 \\
1.40 \\
0.5\end{array}$ \\
\hline POTASSIUM & $\begin{array}{l}10 / 10 / 85 \\
05 / 14 / 86 \\
04 / 06 / 88 \\
07 / 15 / 88 \\
01 / 11 / 89 \\
09 / 19 / 89\end{array}$ & $\begin{array}{l}0001 \\
0001 \\
0001 \\
0001 \\
0001 \\
0001\end{array}$ & $M G / L$ & & $\begin{array}{l}23.9 \\
25.7 \\
27.0 \\
21.1 \\
21.6 \\
20.6\end{array}$ & $\begin{array}{l}0.01 \\
0.01 \\
0.01 \\
0.01 \\
0.01 \\
0.01\end{array}$ & $\begin{array}{l}: \\
: \\
-\end{array}$ \\
\hline RADIUNA-226 & $\begin{array}{l}10 / 10 / 85 \\
04 / 06 / 88 \\
07 / 15 / 88 \\
01 / 11 / 89 \\
09 / 19 / 89\end{array}$ & $\begin{array}{l}0001 \\
0001 \\
0001 \\
0001 \\
0001\end{array}$ & $\mathrm{PCl} / \mathrm{L}$ & & $\begin{array}{l}4.4 \\
0.4 \\
0.7 \\
2.30 \\
0.0\end{array}$ & $\begin{array}{l}1 . \\
1 . \\
1 . \\
1 .\end{array}$ & $\begin{array}{l}0.7 \\
0.2 \\
0.3 \\
0.584 \\
0.1\end{array}$ \\
\hline RADIUH-226 + RADIUH-228 & $\begin{array}{l}10 / 10 / 85 \\
04 / 06 / 88 \\
07 / 15 / 88 \\
01 / 11 / 89 \\
09 / 19 / 89\end{array}$ & $\begin{array}{l}0001 \\
0001 \\
0001 \\
0001 \\
0001\end{array}$ & $\mathrm{PCI} / \mathrm{L}$ & & $\begin{array}{l}5.60 \\
3.00 \\
3.40 \\
2.30 \\
2.10\end{array}$ & $\begin{array}{l}- \\
- \\
- \\
-\end{array}$ & $\begin{array}{l}- \\
- \\
-\end{array}$ \\
\hline RADIUH-228 & $\begin{array}{l}10 / 10 / 85 \\
04 / 06 / 88 \\
07 / 15 / 88 \\
01 / 11 / 89 \\
09 / 19 / 89\end{array}$ & $\begin{array}{l}0001 \\
0001 \\
0001 \\
0001 \\
0001\end{array}$ & $\mathrm{PCI} / \mathrm{L}$ & & $\begin{array}{l}1.2 \\
2.6 \\
2.7 \\
0.00 \\
2.1\end{array}$ & $\begin{array}{l}1 . \\
1 . \\
1 . \\
1 .\end{array}$ & $\begin{array}{l}1.1 \\
0.8 \\
1.4 \\
5.64 \\
1.0\end{array}$ \\
\hline SELENIUH & $10 / 10 / 85$ & 0001 & MG/L & $<$ & 0.005 & 0.005 & $\because$ \\
\hline
\end{tabular}

PARAMETER VALUE IMDICATOR (PVI): < - LESS THAH DETECTIOH LIMIT

SAMPLE ID COOES:

0001 - FILTERED SAMPLE (.45 MICRONS)

OTHER PARAMETER VALUE FLAGS:

d - eSTIMATED VALUE 
GROUNDHATER QUALITY DATA BY LOCATION

SITE: AMBO1 AMBROSIA LAKE

LOCATION: 0779

NORTH COORDINATE: 52914.0 FT

EAST COORDINATE: 60284.7 FT

06/10/80 TO $12 / 04 / 92$

REPORT DATE: 08/04/94

FORMATION OF COMPLETIOH: TRES HERHANOS - C2 SAMDSTONE (TT)

HYDRAULIC FLON RELATIONSHIP: DOWN GRADIENT (D)

\begin{tabular}{|c|c|c|c|c|c|c|c|}
\hline PARAMETER MAHE & LOG DATE & $\begin{array}{c}\text { SAMPLE } \\
\text { ID }\end{array}$ & $\begin{array}{l}\text { UHITS OF } \\
\text { MEASURE }\end{array}$ & PVI & $\begin{array}{l}\text { PARAMETER } \\
\text { VALUE FLAGS }\end{array}$ & $\begin{array}{l}\text { DETECTIOH } \\
\text { LIMIT }\end{array}$ & $\begin{array}{l}\text { PARAMETER } \\
\text { UNCERTAIHTY }\end{array}$ \\
\hline SELENIUM & $\begin{array}{l}05 / 14 / 86 \\
04 / 06 / 88 \\
07 / 15 / 88 \\
01 / 11 / 89 \\
09 / 19 / 89\end{array}$ & $\begin{array}{l}0001 \\
0001 \\
0001 \\
0001 \\
0001\end{array}$ & MG/L & $\begin{array}{l}< \\
<\end{array}$ & $\begin{array}{l}0.005 \\
0.069 \\
0.092 \\
0.001 \\
0.047\end{array}$ & $\begin{array}{l}0.005 \\
0.005 \\
0.005 \\
0.005 \\
0.005\end{array}$ & $\begin{array}{l}- \\
- \\
- \\
-\end{array}$ \\
\hline SILVER & $\begin{array}{l}10 / 10 / 85 \\
04 / 06 / 88 \\
07 / 15 / 88 \\
01 / 11 / 89 \\
09 / 19 / 89\end{array}$ & $\begin{array}{l}0001 \\
0001 \\
0001 \\
0001 \\
0001\end{array}$ & $M G / L$ & $\begin{array}{l}< \\
<\end{array}$ & $\begin{array}{l}0.01 \\
0.05 \\
0.02 \\
0.01 \\
0.01\end{array}$ & $\begin{array}{l}0.01 \\
0.01 \\
0.01 \\
0.01 \\
0.01\end{array}$ & $\begin{array}{l}- \\
- \\
-\end{array}$ \\
\hline SPECIFIC CONDUCTANCE & $\begin{array}{l}10 / 10 / 85 \\
05 / 14 / 86 \\
04 / 06 / 88 \\
07 / 15 / 88 \\
01 / 11 / 89 \\
09 / 19 / 89\end{array}$ & $\begin{array}{l}0001 \\
0001 \\
0001 \\
0001 \\
0001 \\
0001\end{array}$ & LHHO/CH & & $\begin{array}{l}4000 . \\
4000 . \\
4150 . \\
4150 . \\
2750 . \\
710 .\end{array}$ & $\begin{array}{l}- \\
- \\
- \\
-\end{array}$ & $\begin{array}{l}- \\
- \\
-\end{array}$ \\
\hline STROHTIUM & $\begin{array}{l}10 / 10 / 85 \\
05 / 14 / 86 \\
04 / 06 / 88 \\
07 / 15 / 88 \\
01 / 11 / 89 \\
09 / 19 / 89\end{array}$ & $\begin{array}{l}0001 \\
0001 \\
0001 \\
0001 \\
0001 \\
0001\end{array}$ & $M G / L$ & & $\begin{array}{l}10 . \\
10.4 \\
9.88 \\
10.8 \\
12.0 \\
10.9\end{array}$ & $\begin{array}{l}0.1 \\
0.1 \\
0.1 \\
0.1 \\
0.1 \\
0.1\end{array}$ & $\begin{array}{l}- \\
- \\
- \\
-\end{array}$ \\
\hline SULFIOE & $\begin{array}{l}10 / 10 / 85 \\
04 / 06 / 88 \\
07 / 15 / 88 \\
01 / 11 / 89 \\
09 / 19 / 89\end{array}$ & $\begin{array}{l}0001 \\
0001 \\
0001 \\
0001 \\
0001\end{array}$ & $M G / L$ & $\begin{array}{l}< \\
< \\
<\end{array}$ & $\begin{array}{l}0.1 \\
0.1 \\
0.1 \\
0.53 \\
0.1\end{array}$ & $\begin{array}{l}0.1 \\
0.1 \\
0.1 \\
0.1 \\
0.1\end{array}$ & $\begin{array}{l}- \\
- \\
-\end{array}$ \\
\hline TEMPERATURE & $\begin{array}{l}10 / 10 / 85 \\
05 / 14 / 86 \\
04 / 06 / 88 \\
07 / 15 / 88 \\
01 / 11 / 89\end{array}$ & $\begin{array}{l}0001 \\
0001 \\
0001 \\
0001 \\
0001\end{array}$ & C - DEGREE & & $\begin{array}{l}12 . \\
14 . \\
13.0 \\
12.0 \\
11.0\end{array}$ & $\begin{array}{l}- \\
- \\
- \\
-\end{array}$ & $\begin{array}{l}- \\
- \\
-\end{array}$ \\
\hline
\end{tabular}

PARAMETER VALUE INDICATOR (PVI): < - LESS THAN DETECTION LIMIT SAMPLE ID COOES:

0001 - FILTERED SAMPLE (.45 MICROHS)

OTHER PARAMETER VALUE FLAGS:

$J$ - ESTIMATED VALUE 
GROUMDWATER QUALITY DATA BY LOCATION

SITE: AMBOI AMBROSIA LAKE

LOCATION: OT79

MORTH COORDIHATE: 52914.0 FT

EAST COORDINATE: $\quad 60284.7$ FT

06/10/80 TO $12 / 04 / 92$

REPORT DATE: 08/04/94

FORHATION OF CONPLETION: TRES HERMANOS - C2 SAHDSTONE (TT)

HYDRAULIC FLON RELATIONSHIP: DOWN GRADIENT (D)

\begin{tabular}{|c|c|c|c|c|c|c|c|}
\hline PARAMETER NAHE & LOG DATE & SAMPLE & $\begin{array}{l}\text { UHITS OF } \\
\text { MEASURE }\end{array}$ & PVI & $\begin{array}{l}\text { PARAMETEF? } \\
\text { VALUE FLAGS }\end{array}$ & $\begin{array}{l}\text { DETECTIOH } \\
\text { LIMIT }\end{array}$ & $\begin{array}{l}\text { PARAMETER } \\
\text { UNCERTAINTY }\end{array}$ \\
\hline TEMPERATURE & $09 / 19 / 89$ & 0001 & C - DEGREE & & 14.0 & - & - \\
\hline THALLIUA & $\begin{array}{l}01 / 11 / 89 \\
09 / 19 / 89\end{array}$ & $\begin{array}{l}0001 \\
0001\end{array}$ & $M G / L$ & $<$ & $\begin{array}{l}0.005 \\
0.01\end{array}$ & $\begin{array}{l}0.005 \\
0.01\end{array}$ & - \\
\hline THORIUH-230 & $\begin{array}{l}10 / 10 / 85 \\
04 / 06 / 88 \\
07 / 15 / 88 \\
01 / 11 / 89 \\
09 / 19 / 89\end{array}$ & $\begin{array}{l}0001 \\
0001 \\
0001 \\
0001 \\
0001\end{array}$ & $\mathrm{PCl} / \mathrm{L}$ & & $\begin{array}{l}0.2 \\
0.0 \\
0.7 \\
7.04 \\
0.1\end{array}$ & $\begin{array}{l}\text { i. } \\
\text { i. } \\
\text { i. }\end{array}$ & $\begin{array}{l}0.3 \\
0.4 \\
0.6 \\
0.710 \\
0.4\end{array}$ \\
\hline TIH & $\begin{array}{l}10 / 10 / 85 \\
04 / 06 / 88 \\
07 / 15 / 88 \\
01 / 11 / 89 \\
09 / 19 / 89\end{array}$ & $\begin{array}{l}0001 \\
0001 \\
0001 \\
0001 \\
0001\end{array}$ & MG/L & $<$ & $\begin{array}{l}0.005 \\
0.030 \\
0.040 \\
0.001 \\
0.142\end{array}$ & $\begin{array}{l}0.005 \\
0.005 \\
0.005 \\
0.005 \\
0.005\end{array}$ & $\begin{array}{l}- \\
- \\
- \\
-\end{array}$ \\
\hline TOTAL DISSOLVED SOLIDS & $\begin{array}{l}10 / 10 / 85 \\
05 / 14 / 86 \\
04 / 06 / 88 \\
07 / 15 / 88 \\
01 / 11 / 89 \\
09 / 19 / 89\end{array}$ & $\begin{array}{l}0001 \\
0001 \\
0001 \\
0001 \\
0001 \\
0001\end{array}$ & $M G / L$ & & $\begin{array}{l}5900 . \\
6490 . \\
6280 . \\
6730 . \\
6448 . \\
5950 .\end{array}$ & $\begin{array}{l}10 . \\
10 . \\
10 . \\
10 . \\
10 . \\
10 .\end{array}$ & $\begin{array}{l}- \\
- \\
:\end{array}$ \\
\hline TOTAL ORGANIC CARBOH & $\begin{array}{l}04 / 06 / 88 \\
07 / 15 / 88 \\
01 / 11 / 89 \\
09 / 19 / 89\end{array}$ & $\begin{array}{l}0001 \\
0001 \\
0001 \\
0001\end{array}$ & MG/L & . & $\begin{array}{r}258 . \\
263 . \\
65 . \\
189 .\end{array}$ & $\begin{array}{l}1 . \\
1 .\end{array}$ & : \\
\hline URANIUM & $\begin{array}{l}10 / 10 / 85 \\
05 / 14 / 86 \\
04 / 06 / 88 \\
07 / 15 / 88 \\
01 / 11 / 89 \\
09 / 19 / 89\end{array}$ & $\begin{array}{l}0001 \\
0001 \\
0001 \\
0001 \\
0001 \\
0001\end{array}$ & $M G / L$ & 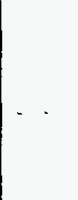 & $\begin{array}{l}0.0238 \\
0.0066 \\
0.0085 \\
0.0141 \\
0.017 \\
0.011\end{array}$ & $\begin{array}{l}0.003 \\
0.003 \\
0.003 \\
0.003 \\
0.003 \\
0.003\end{array}$ & $\begin{array}{l}- \\
- \\
-\end{array}$ \\
\hline VANADIUH & $\begin{array}{l}10 / 10 / 85 \\
05 / 14 / 86 \\
04 / 06 / 88 \\
07 / 15 / 88 \\
01 / 11 / 89 \\
09 / 19 / 89\end{array}$ & $\begin{array}{l}0001 \\
0001 \\
0001 \\
0001 \\
0001 \\
0001\end{array}$ & HG/L & $\begin{array}{l}< \\
<\end{array}$ & $\begin{array}{l}0.01 \\
0.34 \\
0.11 \\
0.07 \\
0.01 \\
0.01\end{array}$ & $\begin{array}{l}0.01 \\
0.01 \\
0.01 \\
0.01 \\
0.01 \\
0.01\end{array}$ & $\begin{array}{l}- \\
: \\
-\end{array}$ \\
\hline ZINC & $\begin{array}{l}10 / 10 / 85 \\
04 / 06 / 88 \\
07 / 15 / 88 \\
01 / 11 / 89 \\
09 / 19 / 89\end{array}$ & $\begin{array}{l}0001 \\
0001 \\
0001 \\
0001 \\
0001\end{array}$ & $M G / L$ & $<$ & $\begin{array}{l}0.074 \\
0.019 \\
0.016 \\
0.01 \\
0.007\end{array}$ & $\begin{array}{l}0.005 \\
0.005 \\
0.005 \\
0.01 \\
0.005\end{array}$ & $\begin{array}{l}- \\
- \\
- \\
- \\
-\end{array}$ \\
\hline
\end{tabular}

PARAMETER VALUE IMDICATOR (PVI): < - LESS THAN DETECTION LIMIT

SAMPLE ID COOES:

ODO1 - FILTERED SAMPLE (.45 MICRONS)

OTHER PARAMETER VALUE fLAGS:

$J$ - eSTIMATED VALUE 
GROUNDHATER QUALITY OATA BY LOCATION

SITE: AMBOI AMBROSIA LAKE

LOCATIOH: 0780

HORTH COORDIHATE: $\quad 53252.8$ FT

EAST COORDIHATE: $\quad 59057.6$ FT

$06 / 10 / 80$ TO $12 / 04 / 92$

REPORT DATE: 08/04/94

FORMATION OF COMPLETIOH: ALLUVIUM (AL)

HYDRAULIC FLON RELATIOHSHIP: DOWN GRADIENT (D)

\begin{tabular}{|c|c|c|c|c|c|c|c|}
\hline PARAHETER MAME & LOG DATE & $\underset{\text { SAMPLE }}{\text { ID }}$ & $\begin{array}{l}\text { UWITS OF } \\
\text { MEASURE }\end{array}$ & PVI & $\begin{array}{l}\text { PARAMETER } \\
\text { VALUE FLAGS }\end{array}$ & $\begin{array}{l}\text { DETECTION } \\
\text { LIMIT }\end{array}$ & $\begin{array}{l}\text { PARAMETER } \\
\text { UNCERTAINTY }\end{array}$ \\
\hline ALKALINITY & $\begin{array}{l}10 / 05 / 85 \\
05 / 13 / 86 \\
01 / 09 / 87\end{array}$ & $\begin{array}{l}0001 \\
0001 \\
0001\end{array}$ & MG/L CACO3 & & $\begin{array}{l}401 . \\
1917 . \\
609 .\end{array}$ & $\begin{array}{c}0.1 \\
- \\
-\end{array}$ & - \\
\hline ALUHINUM & $10 / 05 / 85$ & 0001 & ME/L & & 0.3 & 0.1 & - \\
\hline ANHONILN & $10 / 05 / 85$ & 0001 & MG/L & & 0.3 & 0.1 & - \\
\hline AKTIMONY & $10 / 05 / 85$ & 0001 & MG/L & $<$ & 0.003 & 0.003 & - \\
\hline ARSENIC & $\begin{array}{l}10 / 05 / 85 \\
05 / 13 / 86 \\
01 / 09 / 87\end{array}$ & $\begin{array}{l}0001 \\
0001 \\
0001\end{array}$ & MG/L & $<$ & $\begin{array}{l}0.01 \\
0.01 \\
0.005\end{array}$ & $\begin{array}{l}0.01 \\
0.01 \\
0.01\end{array}$ & : \\
\hline BARIUM & $10 / 05 / 85$ & 0001 & MG/L & $<$ & 0.1 & 0.1 & - \\
\hline BOROA & $\begin{array}{l}10 / 05 / 85 \\
05 / 13 / 86 \\
01 / 09 / 87\end{array}$ & $\begin{array}{l}0001 \\
0001 \\
0001\end{array}$ & $M G / L$ & & $\begin{array}{l}1.2 \\
1.5 \\
0.37\end{array}$ & $\begin{array}{l}0.1 \\
0.1 \\
0.1\end{array}$ & : \\
\hline CAOMIUH & $\begin{array}{l}10 / 05 / 85 \\
05 / 13 / 86\end{array}$ & $\begin{array}{l}0001 \\
0001\end{array}$ & MG/L & $<$ & $\begin{array}{l}0.001 \\
0.001\end{array}$ & $\begin{array}{l}0.001 \\
0.001\end{array}$ & - \\
\hline CAlciva & $\begin{array}{l}10 / 05 / 85 \\
05 / 13 / 86 \\
01 / 09 / 87\end{array}$ & $\begin{array}{l}0001 \\
0001 \\
0001\end{array}$ & $M G / L$ & & $\begin{array}{l}404 . \\
746 . \\
457 .\end{array}$ & $\begin{array}{l}0.01 \\
0.01 \\
0.01\end{array}$ & : \\
\hline CHLORIDE & $\begin{array}{l}10 / 05 / 85 \\
05 / 13 / 86 \\
01 / 09 / 87\end{array}$ & $\begin{array}{l}0001 \\
0001 \\
0001\end{array}$ & $M G / L$ & & $\begin{array}{l}210 . \\
280 \\
300\end{array}$ & $\begin{array}{l}1 . \\
1 . \\
1 .\end{array}$ & - \\
\hline CHROMIUM & $\begin{array}{l}10 / 05 / 85 \\
05 / 13 / 86 \\
01 / 09 / 87\end{array}$ & $\begin{array}{l}0001 \\
0001 \\
0001\end{array}$ & MG/L & & $\begin{array}{l}0.03 \\
0.03 \\
0.02\end{array}$ & $\begin{array}{l}0.01 \\
0.01 \\
0.01\end{array}$ & : \\
\hline COBALT & $\begin{array}{l}10 / 05 / 85 \\
05 / 13 / 86 \\
01 / 09 / 87\end{array}$ & $\begin{array}{l}0001 \\
0001 \\
0001\end{array}$ & MG/L & & $\begin{array}{l}0.07 \\
0.24 \\
0.09\end{array}$ & $\begin{array}{l}0.05 \\
0.05 \\
0.05\end{array}$ & - \\
\hline COPPER & $\begin{array}{l}10 / 05 / 85 \\
01 / 09 / 87\end{array}$ & $\begin{array}{l}0001 \\
0001\end{array}$ & $M G / L$ & & $\begin{array}{l}0.03 \\
0.03\end{array}$ & $\begin{array}{l}0.02 \\
0.02\end{array}$ & - \\
\hline CYAKIDE & $90 / 05 / 85$ & 0009 & $M G / L$ & $<$ & 0.001 & 0.01 & - \\
\hline FLUORIDE & $\begin{array}{l}10 / 05 / 85 \\
05 / 13 / 86 \\
01 / 09 / 87\end{array}$ & $\begin{array}{l}0001 \\
0001 \\
0001\end{array}$ & MS/L & & $\begin{array}{l}2.2 \\
1 . \\
0.69\end{array}$ & $\begin{array}{l}0.1 \\
0.1 \\
0.1\end{array}$ & - \\
\hline GROSS ALPHA & $01 / 09 / 87$ & 0001 & $\mathrm{PCI} / \mathrm{L}$ & & 2300. & 0.2 & 200. \\
\hline GROSS BETA & $01 / 09 / 87$ & 0001 & $\mathrm{PCI} / \mathrm{L}$ & & 980. & 1. & 50. \\
\hline IROH & $\begin{array}{l}10 / 05 / 85 \\
05 / 13 / 86 \\
01 / 09 / 87\end{array}$ & $\begin{array}{l}0001 \\
0001 \\
0001\end{array}$ & MG/L & & $\begin{array}{l}0.49 \\
4.13 \\
0.55\end{array}$ & $\begin{array}{l}0.03 \\
0.03 \\
0.03\end{array}$ & : \\
\hline LEAD & $10 / 05 / 85$ & 0001 & MG/L & $<$ & 0.01 & 0.01 & - \\
\hline
\end{tabular}

PARAMETER VALUE IHDICATOR (PVI): < - LESS THAN DETECTION LIMIT

SAMPLE ID CODES

0001 - FILTERED SAMPLE (.45 MICRONS)

OTHER PARAMETER YALUE FLAGS:

J - ESTIMATED VALUE 
GROUWDWATER QUALITY DATA BY LOCATION

SITE: AMBO1 AMBROSIA LAKE

LOCATIOH: 0780

NORTH COORDINATE: 53252.8 FT

EAST COORDIHATE: 59057.6 FT

06/10/80 TO 12/04/92

REPORT DATE: $08 / 04 / 94$

FORMATION OF COMPLETIOH: ALLUVIUH (AL)

HYDRAULIC FLON RELATIOHSHIP: DONK GRADIENT (D)

\begin{tabular}{|c|c|c|c|c|c|c|c|}
\hline PARAMETER NAME & LOG DATE & $\underset{10}{\text { SAMPLE }}$ & $\begin{array}{l}\text { UNITS OF } \\
\text { MEASURE }\end{array}$ & PVI & $\begin{array}{l}\text { PARAMETER: } \\
\text { VALUE FLAGS }\end{array}$ & $\begin{array}{l}\text { DETECTION } \\
\text { LIMIT }\end{array}$ & $\begin{array}{l}\text { PARAMETER } \\
\text { UHCERTAINTY }\end{array}$ \\
\hline MAGNESIUM & $\begin{array}{l}10 / 05 / 85 \\
05 / 13 / 86 \\
01 / 09 / 87\end{array}$ & $\begin{array}{l}0001 \\
0001 \\
0001\end{array}$ & MG/L & & $\begin{array}{l}222 . \\
442 . \\
290 .\end{array}$ & $\begin{array}{l}0.001 \\
0.001 \\
0.001\end{array}$ & $\dot{-}$ \\
\hline MANGAHESE & $\begin{array}{l}10 / 05 / 85 \\
05 / 13 / 86 \\
01 / 09 / 87\end{array}$ & $\begin{array}{l}0001 \\
0001 \\
0001\end{array}$ & MG/L & & $\begin{array}{l}0.56 \\
4.23 \\
0.33\end{array}$ & $\begin{array}{l}0.01 \\
0.01 \\
0.01\end{array}$ & : \\
\hline MERCURY & $10 / 05 / 85$ & 0001 & $M G / L$ & $<$ & 0.0002 & 0.0002 & - \\
\hline MOLYBDENUM & $\begin{array}{l}10 / 05 / 85 \\
05 / 13 / 86 \\
01 / 09 / 87\end{array}$ & $\begin{array}{l}0001 \\
0001 \\
0001\end{array}$ & HG/L & & $\begin{array}{l}3.17 \\
1.22 \\
3.6\end{array}$ & $\begin{array}{l}0.01 \\
0.01 \\
0.01\end{array}$ & $\because$ \\
\hline HET GROSS ALPHA • & $01 / 09 / 87$ & 0001 & $\mathrm{PCI} / \mathrm{L}$ & & -80.42 & - & - \\
\hline NICKEL & $10 / 05 / 85$ & 0001 & $M G / L$ & & 0.1 & 0.04 & - \\
\hline NITRATE & $\begin{array}{l}10 / 05 / 85 \\
05 / 13 / 86 \\
01 / 09 / 87\end{array}$ & $\begin{array}{l}0001 \\
0001 \\
0001\end{array}$ & $M G / L$ & & $\begin{array}{l}9.0 \\
75 . \\
140 .\end{array}$ & i. & - \\
\hline HITRITE & $10 / 05 / 85$ & 0001 & HG/L & $<$ & 0.1 & 0.1 & - \\
\hline PH & $\begin{array}{l}10 / 05 / 85 \\
05 / 13 / 86 \\
01 / 09 / 87\end{array}$ & $\begin{array}{l}0001 \\
0001 \\
0001\end{array}$ & su & & $\begin{array}{l}7.64 \\
7.28 \\
7.32\end{array}$ & $\dot{-}$ & - \\
\hline PHOSPHATE & $10 / 05 / 85$ & 0001 & $M G / L$ & & 0.3 & 0.1 & - \\
\hline POTASSIUA & $\begin{array}{l}10 / 05 / 85 \\
05 / 13 / 86 \\
01 / 09 / 87\end{array}$ & $\begin{array}{l}0001 \\
0001 \\
0001\end{array}$ & $M G / L$ & & $\begin{array}{l}9.54 \\
9.67 \\
5.85\end{array}$ & $\begin{array}{l}0.01 \\
0.01 \\
0.01\end{array}$ & - \\
\hline RADIUH-226 & $\begin{array}{l}05 / 13 / 86 \\
01 / 09 / 87\end{array}$ & $\begin{array}{l}0001 \\
0001\end{array}$ & $\mathrm{PCl} / \mathrm{L}$ & & $\begin{array}{l}2.5 \\
2.5\end{array}$ & 1. & $\begin{array}{l}0.7 \\
0.8\end{array}$ \\
\hline RADIUH-226 + RADIUM-228 & $01 / 09 / 87$ & 0001 & $\mathrm{PCI} / \mathrm{L}$ & & 3.10 & - & - \\
\hline RADIUN-228 & $01 / 09 / 87$ & 0001 & $\mathrm{PCI} / \mathrm{L}$ & & 0.6 & 1. & 1.4 \\
\hline SELENIUM & $\begin{array}{l}10 / 05 / 85 \\
05 / 13 / 86 \\
01 / 09 / 87\end{array}$ & $\begin{array}{l}0001 \\
0001 \\
0001\end{array}$ & MG/L & & $\begin{array}{l}0.127 \\
0.009 \\
0.537\end{array}$ & $\begin{array}{l}0.005 \\
0.005 \\
0.005\end{array}$ & - \\
\hline SILICA - SIOZ & $10 / 05 / 85$ & 0001 & MG/L & & 15. & 2. & $=$ \\
\hline SILVER & $\begin{array}{l}10 / 05 / 85 \\
01 / 09 / 87\end{array}$ & $\begin{array}{l}0001 \\
0001\end{array}$ & MG/L & $<$ & $\begin{array}{l}0.01 \\
0.01\end{array}$ & $\begin{array}{l}0.01 \\
0.01\end{array}$ & $\dot{-}$ \\
\hline Soolum & $\begin{array}{l}10 / 05 / 85 \\
05 / 13 / 86 \\
01 / 09 / 87\end{array}$ & $\begin{array}{l}0001 \\
0001 \\
0001\end{array}$ & $M G / L$ & & $\begin{array}{l}1140 \\
1430 \\
1540\end{array}$ & $\begin{array}{l}0.002 \\
0.002 \\
0.002\end{array}$ & : \\
\hline SPECIFIC CONDUCTANCE & $\begin{array}{l}10 / 05 / 85 \\
05 / 13 / 86\end{array}$ & $\begin{array}{l}0001 \\
0001\end{array}$ & UнHO/CH & & $\begin{array}{l}6000 \\
4400\end{array}$ & - & $\dot{-}$ \\
\hline
\end{tabular}

- NET GROSS ALPHA (GROSS ALPHA - URANIUM) WITH 1 MG URAHIUM = 686 PCI

PARAMETER VALUE IHDICATOR (PVI): < - LESS THAN DETECTIOH LIMIT 
GROUNDWATER OUALITY DATA BY LOCATION

SITE: ANBOI AMBROSIA LAKE

LOCATION: 0780

MORTH COORDINATE: 53252.8 FT

EAST COORDIHATE: $\quad 59057.6$ FT

06/10/80 TO $12 / 04 / 92$

REPORT DATE: 08/04/94

FORMATION OF COMPLETION: ALLUVIUN (AL)

HYDRAULIC FLON RELATIONSHIP: DOWH GRADIENT (D)

\begin{tabular}{|c|c|c|c|c|c|c|c|}
\hline PARAMETER MAME & LOG DATE & $\begin{array}{l}\text { SAMPLE } \\
\text { ID }\end{array}$ & $\begin{array}{l}\text { UNITS OF } \\
\text { MEASURE }\end{array}$ & PVI & $\begin{array}{l}\text { PARAMETER } \\
\text { VALUE FLAGS }\end{array}$ & $\begin{array}{l}\text { DETECTION } \\
\text { LIMIT }\end{array}$ & $\begin{array}{l}\text { PARAMETER } \\
\text { UNCERTAINTY }\end{array}$ \\
\hline SPECIFIC CONDUCTAHCE & $01 / 09 / 87$ & 0001 & LHнО/CH & & 5000. & - & - \\
\hline STRONTIUA & $\begin{array}{l}10 / 05 / 85 \\
05 / 13 / 86\end{array}$ & $\begin{array}{l}0001 \\
0001\end{array}$ & $M G / L$ & & $\begin{array}{l}7.8 \\
1.1\end{array}$ & $\begin{array}{l}0.1 \\
0.1\end{array}$ & - \\
\hline SULFATE & $\begin{array}{l}10 / 05 / 85 \\
05 / 13 / 86 \\
01 / 09 / 87\end{array}$ & $\begin{array}{l}0001 \\
0001 \\
0001\end{array}$ & $M G / L$ & & $\begin{array}{l}3610 . \\
4300 . \\
4440 .\end{array}$ & $\begin{array}{l}0.1 \\
0.1 \\
0.1\end{array}$ & : \\
\hline SULFIDE & $10 / 05 / 85$ & 0001 & HG/L & $<$ & 0.1 & 0.1 & - \\
\hline TEMPERATURE & $\begin{array}{l}10 / 05 / 85 \\
05 / 13 / 86 \\
01 / 09 / 87\end{array}$ & $\begin{array}{l}0001 \\
0001 \\
0001\end{array}$ & C - DEGREE & & $\begin{array}{c}14 . \\
12 . \\
9.0\end{array}$ & $:$ & : \\
\hline THORIUM-230 & $05 / 13 / 86$ & 0001 & $\mathrm{PCI} / \mathrm{L}$ & & 0.2 & 1. & 0.3 \\
\hline TIK & $10 / 05 / 85$ & $000 t$ & $M G / L$ & $<$ & 0.005 & 0.005 & - \\
\hline TOTAL DISSOLVED SOLIDS & $\begin{array}{l}10 / 05 / 85 \\
05 / 13 / 86 \\
01 / 09 / 87\end{array}$ & $\begin{array}{l}0001 \\
0001 \\
0001\end{array}$ & $M G / L$ & & $\begin{array}{l}6180 . \\
7250 \\
8300\end{array}$ & $\begin{array}{l}10 . \\
10 . \\
10 .\end{array}$ & : \\
\hline TOTAL ORGAHIC CARBOH & $10 / 05 / 85$ & 0001 & $M G / L$ & & 25. & 1. & - \\
\hline URANIUA & $\begin{array}{l}10 / 05 / 85 \\
05 / 13 / 86 \\
01 / 09 / 87\end{array}$ & $\begin{array}{l}0001 \\
0001 \\
0001\end{array}$ & MG/L & & $\begin{array}{l}2.8 \\
0.0933 \\
3.47\end{array}$ & $\begin{array}{l}0.003 \\
0.003 \\
0.003\end{array}$ & : \\
\hline VAHADIU:H & $\begin{array}{l}10 / 05 / 85 \\
05 / 13 / 86 \\
01 / 09 / 87\end{array}$ & $\begin{array}{l}0001 \\
0001 \\
0001\end{array}$ & $M G / L$ & & $\begin{array}{l}0.5 \\
0.5 \\
0.05\end{array}$ & $\begin{array}{l}0.01 \\
0.01 \\
0.01\end{array}$ & : \\
\hline ZIMC & $10 / 05 / 85$ & 0001 & MG/L & & 0.098 & 0.005 & - \\
\hline
\end{tabular}

PARAMETER VALUE IHDICATOR (PVI): < - LESS THAN DETECTIOH LIMIT

SAMPLE ID CODES:

0001 - FILTERED SAMPLE (.45 MICRONS) 
GROUHDHATER QUALITY DATA BY LOCATION

SITE: AMBO1 AMBROSIA LAKE

LOCATIOH: 0781

MORTH COORDIHATE: $\quad 53253.5$ FT

EAST COORDINATE: $\quad 59156.3$ FT

06/10/80 TO 12/04/92

REPORT OATE: 08/04/94

FORMATION OF COAPLETIOH: ALLUVIUH (AL)

HYORAULIC FLON RELATIOHSHIP: DOWH GRADIEHT (D)

\begin{tabular}{|c|c|c|c|c|c|c|c|}
\hline PARAMETER HAME & LOG DATE & $\begin{array}{c}\text { SAMPLE } \\
\text { ID }\end{array}$ & $\begin{array}{l}\text { UNITS OF } \\
\text { MEASURE }\end{array}$ & PVI & $\begin{array}{l}\text { PARAMETER } \\
\text { VALUE FLAGS }\end{array}$ & $\begin{array}{l}\text { DETECTION } \\
\text { LIMIT }\end{array}$ & $\begin{array}{l}\text { PARÁMETER } \\
\text { UNCERTAINTY }\end{array}$ \\
\hline ALKALINITY & $10 / 16 / 85$ & 0001 & MG/L CACO3 & & 241. & - & - \\
\hline ALUHIHUA & $10 / 16 / 85$ & 0001 & $M G / L$ & & 0.3 & 0.1 & - \\
\hline AMAONIUM & $10 / 16 / 85$ & 0001 & MG/L & & 0.5 & 0.1 & - \\
\hline AKTIMONY & $10 / 16 / 85$ & 0001 & MG/L & $<$ & 0.003 & 0.003 & - \\
\hline ARSEHIC & $10 / 16 / 85$ & 0001 & $M G / L$ & $<$ & 0.01 & 0.01 & - \\
\hline BARIUA & $10 / 16 / 85$ & 0001 & MG/L & & 0.1 & 0.1 & - \\
\hline BORON & $10 / 16 / 85$ & 0001 & MG/L & & 0.2 & 0.1 & - \\
\hline CADMIUH & $10 / 16 / 85$ & 0001 & $M G / L$ & $<$ & 0.001 & 0.001 & - \\
\hline CALCIUM & $10 / 16 / 85$ & 0001 & MG/L & & 56.1 & 0.01 & - \\
\hline CHLORIOE & $10 / 16 / 85$ & 0001 & $M G / L$ & & 20. & 1. & - \\
\hline CHROHIUH & $10 / 16 / 85$ & 0001 & $\mathrm{HG} / \mathrm{L}$ & & 0.17 & 0.01 & - \\
\hline COBALT & $10 / 16 / 85$ & 0001 & $M G / L$ & $<$ & 0.05 & 0.05 & - \\
\hline COPPER & $10 / 16 / 85$ & 0001 & MG/L & $<$ & 0.02 & 0.02 & - \\
\hline CYAHIDE & $10 / 16 / 85$ & 0001 & MG/L & $<$ & 0.01 & 0.01 & - \\
\hline FLUORIDE & $10 / 16 / 85$ & 0001 & $\mathrm{MG} / \mathrm{L}$ & & 1.1 & 0.1 & - \\
\hline IROH & $10 / 16 / 85$ & 0001 & MG/L & $<$ & 0.03 & 0.03 & - \\
\hline LEAD & $10 / 16 / 85$ & 0001 & HG/L & $<$ & 0.01 & 0.01 & - \\
\hline MAGHESIUM & $10 / 16 / 85$ & 0001 & $M G / L$ & & 1.84 & 0.001 & - \\
\hline MANGANESE & $10 / 16 / 85$ & 0001 & MG/L & $<$ & 0.01 & 0.01 & - \\
\hline MERCURY & $10 / 16 / 85$ & 0001 & $M G / L$ & $<$ & 0.0002 & 0.0002 & - \\
\hline MOLYBDENUH & $10 / 16 / 85$ & 0001 & MG/L & & 0.21 & 0.01 & - \\
\hline HICKEL & $10 / 16 / 85$ & 0001 & MG/L & $<$ & 0.04 & 0.04 & - \\
\hline NITRATE & $10 / 16 / 85$ & 0001 & $M G / L$ & & 14. & 1. & - \\
\hline NITRITE & $10 / 16 / 85$ & 0001 & MG/L & $<$ & 0.1 & 0.1 & - \\
\hline PH & $10 / 16 / 85$ & 0001 & su & & 11.18 & - & - \\
\hline PHOSPHATE & $10 / 16 / 85$ & 0001 & $H G / L$ & & 0.6 & 0.1 & - \\
\hline POTASSIUH & $10 / 16 / 85$ & 0001 & $M G / L$ & & 6.91 & 0.01 & - \\
\hline SELENIUM & $10 / 16 / 85$ & 0001 & $M G / L$ & $<$ & 0.005 & 0.005 & - \\
\hline SILICA - SIO2 & $10 / 16 / 85$ & 0001 & MG/L & & 5. & 2. & - \\
\hline SILVER & $10 / 96 / 85$ & 0001 & MG/L & $<$ & 0.01 & 0.01 & $i$ \\
\hline
\end{tabular}

PARAMETER VALUE IMDICATOR (PVI): < - LeSS THAN DETECTION LIMIT

SAMPLE 10 COOES:

0001 - FILTERED SAMPLE (.45 MICRONS) 
GROUNDWATER QUALITY DATA BY LOCATION

SITE: AMBO1 AMBROSIA LAKE

LOCATION: 0781

MORTH COORDINATE: 53253.5 FT

EAST COORDINATE: $\quad 59156.3$ FT

06/10/80 TO $12 / 04 / 92$

REPORT DATE: 08/04/94

FORMATION OF COMPLETION: ALLUVIUH (AL)

HYDRAULIC FLON RELATIOHSHIP: DOWN GRADIENT (D)

\begin{tabular}{|c|c|c|c|c|c|c|c|}
\hline PARAMETER NAHE & LOG DATE & $\underset{\text { ID }}{\text { SAMPLE }}$ & $\begin{array}{l}\text { UNITS OF } \\
\text { MEASURE }\end{array}$ & PVI & $\begin{array}{l}\text { PARAMETER } \\
\text { VALUE FLAGS }\end{array}$ & $\begin{array}{l}\text { DETECTION } \\
\text { LIMIT }\end{array}$ & $\begin{array}{l}\text { PARAMETER } \\
\text { UHCERTAINTY }\end{array}$ \\
\hline sootun & $10 / 16 / 85$ & 0001 & MG/L & & 220. & 0.002 & - \\
\hline SPECIFIC CONDUCTAKCE & $10 / 16 / 85$ & 0001 & UHHO/CM & & 1380. & - & - \\
\hline STRONTIUH & $10 / 96 / 85$ & 0001 & $\mathrm{MG} / \mathrm{L}$ & & 0.9 & 0.1 & - \\
\hline SULFATE & $10 / 16 / 85$ & 0001 & $\mathrm{MG} / \mathrm{L}$ & & 382. & 0.1 & - \\
\hline SULFIDE & $10 / 96 / 85$ & 0001 & MG/L & $<$ & 0.1 & 0.1 & - \\
\hline TEMPERATURE & $10 / 16 / 85$ & 0001 & C - DEGREE & & 10. & - & - \\
\hline THORILM-230 & $10 / 16 / 85$ & 0001 & $\mathrm{PCI} / \mathrm{L}$ & & 0.6 & 1. & 2. \\
\hline TIN & $10 / 16 / 85$ & 0001 & MG/L & $<$ & 0.005 & 0.005 & - \\
\hline TOTAL DISSOLVED SOLIDS & $10 / 16 / 85$ & 0001 & $M G / L$ & & 785 . & 10. & - \\
\hline TOTAL ORGANIC CARBOH & $10 / 16 / 85$ & 0001 & $M G / L$ & & 160. & 1. & - \\
\hline URAMIUN & $10 / 16 / 85$ & 0001 & $M G / L$ & & $0.0006 \mathrm{~J}$ & 0.003 & - \\
\hline VANADIUM & $10 / 16 / 85$ & 0001 & $\mathrm{HG} / \mathrm{L}$ & $<$ & 0.01 & 0.09 & - \\
\hline ZINC & $10 / 16 / 85$ & 0001 & $M G / L$ & $<$ & 0.005 & 0.005 & - \\
\hline
\end{tabular}

PARAMETER VALUE INDICATOR (PVI):

SAMPLE ID COOES

0001 - FILTERED SAMPLE (.45 MICRONS)

OTHER PARAMETER VALUE FLAGS:

$J$ - ESTIHATED VALUE 
GROUNDWATER QUALITY DATA BY LOCATIOH

SITE: AMBOI AMBROSIA LAKE

LOCATIOH: 0782

NORTH COORDIHATE: 55221.4 FT

EAST COORDINATE: 58792.8 FT

06/10/80 TO 12/04/92

REPORT DATE: 08/04/94

FORHATIOH OF COMPLETIOH: TRES HERMANOS - C2 SAHOSTONE (TT)

HYDRAULIC FLON RELATIONSHIP: CROSS GRADIEKT (C)

\begin{tabular}{|c|c|c|c|c|c|c|c|}
\hline PARAMETER NAME & LOG DATE & $\begin{array}{c}\text { SAMPLE } \\
\text { ID }\end{array}$ & $\begin{array}{l}\text { UNITS OF } \\
\text { MEASURE }\end{array}$ & PVI & $\begin{array}{l}\text { PARAMETER } \\
\text { VALUE FLAGS }\end{array}$ & $\begin{array}{l}\text { DETECTIOH } \\
\text { LIMIT }\end{array}$ & $\begin{array}{l}\text { PARAMETER } \\
\text { UNCERTAINTY }\end{array}$ \\
\hline ALKALINITY & $\begin{array}{l}10 / 09 / 85 \\
05 / 16 / 86\end{array}$ & $\begin{array}{l}0001 \\
0001\end{array}$ & MG/L Caco3 & & $\begin{array}{l}1072 . \\
449 .\end{array}$ & - & $\dot{-}$ \\
\hline ALUAINUY & $10 / 09 / 85$ & 0001 & $M G / L$ & & 0.4 & 0.1 & - \\
\hline AM4ONIU⿴囗十⺝ & $10 / 09 / 85$ & 0001 & $M G / L$ & & 0.8 & 0.1 & - \\
\hline AMTIMONY & $10 / 09 / 85$ & 0001 & ME/L & $<$ & 0.003 & 0.003 & - \\
\hline ARSENIC & $\begin{array}{l}10 / 09 / 85 \\
05 / 16 / 86\end{array}$ & $\begin{array}{l}0001 \\
0001\end{array}$ & MG/L & $<$ & $\begin{array}{l}0.01 \\
0.01\end{array}$ & $\begin{array}{l}0.01 \\
0.01\end{array}$ & $\dot{-}$ \\
\hline BARIUM & $10 / 09 / 85$ & 0001 & MG/L & & 0.3 & 0.1 & - \\
\hline BORON & $\begin{array}{l}10 / 09 / 85 \\
05 / 16 / 86\end{array}$ & $\begin{array}{l}0001 \\
0001\end{array}$ & HG/L & & $\begin{array}{l}0.2 \\
0.2\end{array}$ & $\begin{array}{l}0.1 \\
0.1\end{array}$ & - \\
\hline CADHILA & $\begin{array}{l}10 / 09 / 85 \\
05 / 16 / 86\end{array}$ & $\begin{array}{l}0001 \\
0001\end{array}$ & MG/L & $<$ & $\begin{array}{l}0.001 \\
0.001\end{array}$ & $\begin{array}{l}0.001 \\
0.001\end{array}$ & - \\
\hline CALCIUA & $\begin{array}{l}10 / 09 / 85 \\
05 / 16 / 86\end{array}$ & $\begin{array}{l}0001 \\
0001\end{array}$ & MG/L & & $\begin{array}{l}258 . \\
192 .\end{array}$ & $\begin{array}{l}0.01 \\
0.01\end{array}$ & - \\
\hline CHLORIDE & $\begin{array}{l}10 / 09 / 85 \\
05 / 16 / 86\end{array}$ & $\begin{array}{l}0001 \\
0001\end{array}$ & $M G / L$ & & $\begin{array}{l}20 . \\
12 .\end{array}$ & 1. & - \\
\hline CHROHIUH & $\begin{array}{l}10 / 09 / 85 \\
05 / 16 / 86\end{array}$ & $\begin{array}{l}0001 \\
0001\end{array}$ & HG/L & & $\begin{array}{l}0.04 \\
0.03\end{array}$ & $\begin{array}{l}0.09 \\
0.01\end{array}$ & - \\
\hline COBALT & $\begin{array}{l}10 / 09 / 85 \\
05 / 16 / 86\end{array}$ & $\begin{array}{l}0001 \\
0001\end{array}$ & $M G / L$ & $<$ & $\begin{array}{l}0.05 \\
0.05\end{array}$ & $\begin{array}{l}0.05 \\
0.05\end{array}$ & $\dot{-}$ \\
\hline COPPER & $10 / 09 / 85$ & 0001 & HG/L & . & 0.02 & 0.02 & - \\
\hline CYANIDE & $10 / 09 / 85$ & 0001 & MG/L & $<$ & 0.01 & 0.01 & - \\
\hline FLUORIDE & $\begin{array}{l}10 / 09 / 85 \\
05 / 16 / 86\end{array}$ & $\begin{array}{l}0001 \\
0001\end{array}$ & MG/L & & $\begin{array}{l}0.9 \\
0.6\end{array}$ & $\begin{array}{l}0.1 \\
0.1\end{array}$ & - \\
\hline IROH & $\begin{array}{l}10 / 09 / 85 \\
05 / 16 / 86\end{array}$ & $\begin{array}{l}0001 \\
0001\end{array}$ & HG/L & $<$ & $\begin{array}{l}0.03 \\
0.03\end{array}$ & $\begin{array}{l}0.03 \\
0.03\end{array}$ & - \\
\hline LEAD & $10 / 09 / 85$ & 0001 & $M G / L$ & $<$ & 0.01 & 0.01 & - \\
\hline LEAD-210 & $10 / 09 / 85$ & 0001 & $\mathrm{PCI} / \mathrm{L}$ & & 2.9 & 1.5 & 1.5 \\
\hline MAGNESIUM & $\begin{array}{l}10 / 09 / 85 \\
05 / 16 / 86\end{array}$ & $\begin{array}{l}0001 \\
0001\end{array}$ & $M G / L$ & & $\begin{array}{l}0.167 \\
0.161\end{array}$ & $\begin{array}{l}0.001 \\
0.001\end{array}$ & $\therefore$ \\
\hline MANGAKESE & $\begin{array}{l}10 / 09 / 85 \\
05 / 16 / 86\end{array}$ & $\begin{array}{l}0001 \\
0001\end{array}$ & $M G / L$ & & $\begin{array}{l}0.02 \\
0.01\end{array}$ & $\begin{array}{l}0.01 \\
0.01\end{array}$ & - \\
\hline MERCURY & $10 / 09 / 85$ & 0001 & MG/L & $<$ & 0.0002 & 0.0002 & - \\
\hline HOLYBDENUY & $\begin{array}{l}10 / 09 / 85 \\
05 / 16 / 86\end{array}$ & $\begin{array}{l}0001 \\
0001\end{array}$ & MG/L & & $\begin{array}{l}0.16 \\
0.17\end{array}$ & $\begin{array}{l}0.01 \\
0.01\end{array}$ & $=$ \\
\hline HICKEL & $10 / 09 / 85$ & 0001 & MG/L & & 0.06 & 0.04 & - \\
\hline
\end{tabular}

Parameter VALUE IhDICATOR (PVI): < - LeSS THAN DETECTION LIMIT

SAMPLE ID CODES:

0001 - FILTIERED SAHPLE (.45 MICRONS) 
GROUNDUATER QUALITY DATA BY LOCATION

SITE: AMBOI AMBROSIA LAKE

LOCATION: 0782

MORTH COORDINATE: 55221.4 FT

EAST COORDIHATE: $\quad 58792.8$ FT

06/10/80 TO $12 / 04 / 92$

REPORT DATE: 08/04/94

FORMATION OF CONPLETION: TRES HERHANOS - C2 SANDSTONE (TT) HYDRAULIC FLOW RELATIOHSHIP: CROSS GRADIENT (C)

\begin{tabular}{|c|c|c|c|c|c|c|c|}
\hline PARAMETER NAME & LOG DATE & $\begin{array}{c}\text { SAMPLE } \\
\text { ID }\end{array}$ & $\begin{array}{l}\text { UNITS OF } \\
\text { MEASURE }\end{array}$ & PVI & $\begin{array}{l}\text { PARAMETER } \\
\text { VALUE FLAGS }\end{array}$ & $\begin{array}{l}\text { DETECTION } \\
\text { LIMIT }\end{array}$ & $\begin{array}{l}\text { PARAMETER } \\
\text { UMCERTAINTY }\end{array}$ \\
\hline NITRATE & $\begin{array}{l}10 / 09 / 85 \\
05 / 16 / 86\end{array}$ & $\begin{array}{l}0001 \\
0001\end{array}$ & $M G / L$ & $<$ & $\begin{array}{l}4 . \\
1 .\end{array}$ & 1. & $\dot{-}$ \\
\hline MITRITE & $10 / 09 / 85$ & 0001 & MG/L & $<$ & 0.1 & 0.1 & - \\
\hline PH & $\begin{array}{l}10 / 09 / 85 \\
05 / 16 / 86\end{array}$ & $\begin{array}{l}0001 \\
0001\end{array}$ & SU & & $\begin{array}{l}12.2 \\
11.46\end{array}$ & - & - \\
\hline PHOSPHATE & $10 / 09 / 85$ & 0001 & MG/L & & 0.4 & 0.1 & - \\
\hline POLONIUN-210 & $10 / 09 / 85$ & 0001 & $\mathrm{PCI} / \mathrm{L}$ & & 0. & 1. & 0.6 \\
\hline POTASSIUH & $\begin{array}{l}10 / 09 / 85 \\
05 / 16 / 86\end{array}$ & $\begin{array}{l}0001 \\
0001\end{array}$ & MG/L & & $\begin{array}{l}24.9 \\
15.3\end{array}$ & $\begin{array}{l}0.01 \\
0.01\end{array}$ & $\dot{-}$ \\
\hline RADIU:A-226 & $\begin{array}{l}10 / 09 / 85 \\
05 / 96 / 86\end{array}$ & $\begin{array}{l}0001 \\
0001\end{array}$ & $\mathrm{PCI} / \mathrm{L}$ & & $\begin{array}{l}2.4 \\
1 .\end{array}$ & 1. & $\begin{array}{l}0.5 \\
0.4\end{array}$ \\
\hline RADIUH-226 + RADIUH-228 & $10 / 09 / 85$ & 0001 & $\mathrm{PCI} / \mathrm{L}$ & & 2.80 & - & - \\
\hline RADIUH-228 & $10 / 09 / 85$ & 0001 & $\mathrm{PCI} / \mathrm{L}$ & & 0.4 & 1. & 0.9 \\
\hline SELENIUM & $\begin{array}{l}10 / 09 / 85 \\
05 / 16 / 86\end{array}$ & $\begin{array}{l}0001 \\
0001\end{array}$ & MG/L & $<$ & $\begin{array}{l}0.005 \\
0.005\end{array}$ & $\begin{array}{l}0.005 \\
0.005\end{array}$ & - \\
\hline SILICA $=$ SIO2 & $10 / 09 / 85$ & 0001 & $\mathrm{MG} / \mathrm{L}$ & & 4. & 2. & - \\
\hline SILVER & $10 / 09 / 85$ & 0001 & $M G / L$ & $<$ & 0.01 & 0.01 & - \\
\hline SCOIUH & $\begin{array}{l}10 / 09 / 85 \\
05 / 16 / 86\end{array}$ & $\begin{array}{l}0001 \\
0001\end{array}$ & $M G / L$ & & $\begin{array}{l}264 . \\
292 .\end{array}$ & $\begin{array}{l}0.002 \\
0.002\end{array}$ & - \\
\hline SPECIFIC CONDUCTANCE & $\begin{array}{l}10 / 09 / 85 \\
05 / 16 / 86\end{array}$ & $\begin{array}{l}0001 \\
0001\end{array}$ & UNHO/CM & & $\begin{array}{l}4100 . \\
2450 .\end{array}$ & - & - \\
\hline STRONTIUN & $\begin{array}{l}10 / 09 / 85 \\
05 / 16 / 86\end{array}$ & $\begin{array}{l}0001 \\
0001\end{array}$ & MG/L & & $\begin{array}{l}2.6 \\
2.1\end{array}$ & $\begin{array}{l}0.1 \\
0.1\end{array}$ & - \\
\hline SULFATE & $\begin{array}{l}10 / 09 / 85 \\
05 / 16 / 86\end{array}$ & $\begin{array}{l}0001 \\
0001\end{array}$ & $M G / L$ & & $\begin{array}{l}516 . \\
633 .\end{array}$ & $\begin{array}{l}0.1 \\
0.1\end{array}$ & - \\
\hline SULFIDE & $10 / 09 / 85$ & 0001 & $M G / L$ & $<$ & 0.1 & 0.1 & - \\
\hline TEMPERATURE & $\begin{array}{l}10 / 09 / 85 \\
05 / 16 / 86\end{array}$ & $\begin{array}{l}0001 \\
0001\end{array}$ & C - DEGREE & & $\begin{array}{l}12.5 \\
16 .\end{array}$ & 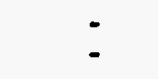 & - \\
\hline THORIUH-230 & $\begin{array}{l}10 / 09 / 85 \\
05 / 16 / 86\end{array}$ & $\begin{array}{l}0001 \\
0001\end{array}$ & $\mathrm{PCI} / \mathrm{L}$ & & $\begin{array}{l}0.5 \\
0.1\end{array}$ & 1. & $\begin{array}{l}0.5 \\
0.2\end{array}$ \\
\hline TIN & $10 / 09 / 85$ & 0001 & MG/L & $<$ & 0.005 & 0.005 & - \\
\hline TOTAL DISSOLVED SOLIDS & $\begin{array}{l}10 / 09 / 85 \\
05 / 16 / 86\end{array}$ & $\begin{array}{l}0001 \\
0001\end{array}$ & $M G / L$ & & $\begin{array}{l}1880 \\
1340 .\end{array}$ & $\begin{array}{l}10 . \\
10 .\end{array}$ & - \\
\hline TOTAL ORGANIC CARBON & $10 / 09 / 85$ & 0001 & $M G / L$ & & 18. & 1. & - \\
\hline URANIUM & $\begin{array}{l}10 / 09 / 85 \\
05 / 16 / 86\end{array}$ & $\begin{array}{l}0001 \\
0001\end{array}$ & MG/L & $<$ & $\begin{array}{l}0.0031 \\
0.0003 \mathrm{~J}\end{array}$ & $\begin{array}{l}0.003 \\
0.003\end{array}$ & - \\
\hline
\end{tabular}

PARAMETER VALUE IMDICATOR (PVI): < - LESS THAN DETECTION LIMIT 
GROUNDHATER QUALITY DATA BY LOCATION

SITE: AMBOI AMBROSIA LAKE

LOCATION: 0782

MORTH COORDINATE: 55221.4 FT

EAST COORDINATE: 58792.8 FT

06/10/80 TO $12 / 04 / 92$

REPORT DATE: 08/04/94

FORMATIOH OF COMPLETION: TRES HERMANOS - C2 SANDSTONE (TT)

HYDRAULIC FLOW RELATIONSHIP: CROSS GRADIENT (C)

\begin{tabular}{|c|c|c|c|c|c|c|c|}
\hline PARAMETER MAME & LOG DATE & $\underset{\text { SAMPLE }}{\text { SO }}$ & $\begin{array}{l}\text { UHITS OF } \\
\text { MEASURE }\end{array}$ & PVI & $\begin{array}{l}\text { PARAMETER } \\
\text { VALUE FLAGS }\end{array}$ & $\begin{array}{l}\text { DETECTION } \\
\text { LIHIT }\end{array}$ & $\begin{array}{l}\text { PARAMETER } \\
\text { UHCERTAINTY }\end{array}$ \\
\hline VANADIUY & $\begin{array}{l}10 / 09 / 85 \\
05 / 16 / 86\end{array}$ & $\begin{array}{l}0001 \\
0001\end{array}$ & MG/L & $<$ & $\begin{array}{l}0.01 \\
0.21\end{array}$ & $\begin{array}{l}0.01 \\
0.01\end{array}$ & $\because$ \\
\hline ZINC & $10 / 09 / 85$ & 0001 & $M G / L$ & $<$ & 0.005 & 0.005 & - \\
\hline
\end{tabular}

PARANETER VALUE IHDICATOR (PVI):

< - LESS THAH DETECTION LIHIT

SAMPLE ID COOES:

0001 - FILTERED SAMPLE (.45 MICRONS) 
GROUNDHATER OUALITY DATA BY LOCATION

SITE: AMBO1 AMBROSIA LAKE

LOCATION: 0784

NORTH COOROINATE: $54129.0 \mathrm{FT}$

EAST COORDINATE: $\quad 58763.8$ FT

06/10/80 TO $12 / 04 / 92$

REPORT DATE: 08/04/94

FORMATION OF COMPLETIOH: TRES HERMANOS - C2 SANDSTONE (TT)

HYDRAULIC FLON RELATIONSHIP: DOWN GRADIENT (D)

\begin{tabular}{|c|c|c|c|c|c|c|c|}
\hline PARAMETER NAME & LOG DATE & $\begin{array}{c}\text { SAMPLE } \\
\text { ID }\end{array}$ & $\begin{array}{l}\text { UHITS OF } \\
\text { MEASURE }\end{array}$ & PV & $\begin{array}{l}\text { PARAMETER } \\
\text { VALUE FLAGS }\end{array}$ & $\begin{array}{l}\text { DETECTIOH } \\
\text { LIMIT }\end{array}$ & $\begin{array}{l}\text { PARAMETER } \\
\text { UHCERTAINTY }\end{array}$ \\
\hline ALKALINITY & $05 / 15 / 86$ & 0001 & MG/L CACO3 & & 670. & - & - \\
\hline ARSENIC & $05 / 15 / 86$ & 0001 & MG/L & $<$ & 0.01 & 0.1 & - \\
\hline BOROH & $05 / 15 / 86$ & 0001 & MG/L & & 0.2 & 0.1 & - \\
\hline CADHIUH & $05 / 15 / 86$ & 0001 & HG/L & $<$ & 0.001 & 0.001 & - \\
\hline calcrum & $05 / 15 / 86$ & 0001 & MG/L & & 183. & 0.01 & - \\
\hline CHLORIDE & $05 / 15 / 86$ & 0001 & MG/L & & 95. & 1. & - \\
\hline CHROHIUM & $05 / 15 / 86$ & 0001 & $M G / L$ & & 0.11 & 0.01 & - \\
\hline COBALT & $05 / 15 / 86$ & 0001 & MG/L & $<$ & 0.05 & 0.05 & - \\
\hline FLUORIDE & $05 / 15 / 86$ & 0001 & ME/L & & 0.8 & 0.1 & - \\
\hline IRON & $05 / 15 / 86$ & 0001 & $M G / L$ & & 0.05 & 0.03 & - \\
\hline MAGNESIUM & $05 / 15 / 86$ & 0001 & MG/L & & 0.481 & 0.001 & - \\
\hline MAHGAMESE & $05 / 15 / 86$ & 0001 & MG/L & & 0.02 & 0.01 & - \\
\hline MOLYBDEENUM & $05 / 15 / 86$ & 0001 & MG/L & & 0.19 & 0.01 & - \\
\hline NITRATE & $05 / 15 / 86$ & 0001 & MG/L & & 5. & 1. & - \\
\hline $\mathrm{PH}$ & $05 / 15 / 86$ & 0001 & su & & 11.92 & - & - \\
\hline POTASSIUM & $05 / 15 / 86$ & 0001 & MG/L & & 23. & 0.01 & - \\
\hline RADIUH-226 & $05 / 15 / 86$ & 0001 & PCI/L & & 0.8 & 1. & 0.4 \\
\hline SELENIUN & $05 / 15 / 86$ & 0001 & MG/L & & 0.007 & 0.005 & - \\
\hline sootun & $05 / 15 / 86$ & 0001 & $M G / L$ & & 395. & 0.002 & - \\
\hline SPECIFIC CONDUCTANCE & $05 / 15 / 86$ & 0001 & UAHO/CN & & 3000. & - & - \\
\hline STRONTIUM & $05 / 15 / 86$ & 0001 & MG/L & & 2.8 & 0.1 & - \\
\hline SULFATE & $05 / 15 / 86$ & 0001 & MG/L & & 557. & 0.1 & - \\
\hline TEMPERATURE & $05 / 15 / 86$ & 0001 & $C$ - DEGREE & & 16. & - & - \\
\hline THORIUW-230 & $05 / 15 / 86$ & 0001 & PCI/L & & 1.6 & 1. & 0.7 \\
\hline TOTAL DISSOLVED SOLIDS & $05 / 15 / 86$ & 0001 & MG/L & & 1560. & 10. & - \\
\hline URANIUN & $05 / 15 / 86$ & 0001 & MG/L & $<$ & $0.0003 \mathrm{~J}$ & 0.003 & - \\
\hline VANADIUM & $05 / 95 / 86$ & 0009 & ME/L & & 0.38 & 0.01 & - \\
\hline
\end{tabular}

PARAMETER VALUE INDICATOR (PVI):

< - LESS THAN DETECTION LIMIT

SAMPLE ID COOES:

0001 - FILTERED SAMPLE (.45 MICRONS)

OTHER PARAMETER VALUE FLAGS:

$J$ - estimated VALUE 
GROUNDHATER OUALITY DATA BY LOCATIOH

SITE: AMBOI AMBROSIA LAKE

LOCATIOH: 0785

NORTH COORDINATE: 52770.8 FT

EAST COORDIHATE: 58816.5 FT

06/10/80 TO $12 / 04 / 92$

REPORT DATE: $08 / 04 / 94$

FORMATION OF COMPLETION: TRES HERHANOS - C2 SAMDSTONE (TT) HYDRAULIC FLON RELATIONSHIP: DOWH GRADIENT (D)

\begin{tabular}{|c|c|c|c|c|c|c|c|c|}
\hline PARAMETER MAME & LOG DATE & $\begin{array}{c}\text { SAMPLE } \\
\text { ID }\end{array}$ & $\begin{array}{l}\text { UNITS OF } \\
\text { MEASURE }\end{array}$ & PVI & $\begin{array}{l}\text { PARAMETER } \\
\text { VALUE }\end{array}$ & FLAGS & $\begin{array}{l}\text { DETECTION } \\
\text { LIHIT }\end{array}$ & $\begin{array}{l}\text { PARĂHETER } \\
\text { UNCERTAINTY }\end{array}$ \\
\hline ALKALINITY & $\begin{array}{l}10 / 15 / 85 \\
05 / 15 / 86 \\
01 / 09 / 87 \\
04 / 06 / 88 \\
07 / 15 / 88 \\
01 / 08 / 89 \\
09 / 19 / 89 \\
03 / 31 / 90 \\
01 / 15 / 91 \\
11 / 25 / 92\end{array}$ & $\begin{array}{l}0001 \\
0001 \\
0001 \\
0001 \\
0001 \\
0001 \\
0001 \\
0001 \\
0001 \\
0001\end{array}$ & $\mathrm{MG} / \mathrm{L} \mathrm{CACO} 3$ & & $\begin{array}{l}350 . \\
841 . \\
740 . \\
744 . \\
610 . \\
578 . \\
598 . \\
465 . \\
479 . \\
219\end{array}$ & & $\begin{array}{l}- \\
\vdots \\
i \\
\vdots \\
i \\
i \\
-\end{array}$ & $\begin{array}{l}- \\
= \\
= \\
- \\
- \\
- \\
-\end{array}$ \\
\hline ALUAIHUM & $\begin{array}{l}10 / 15 / 85 \\
04 / 06 / 88 \\
07 / 15 / 88 \\
01 / 08 / 89 \\
09 / 19 / 89 \\
03 / 31 / 90 \\
01 / 15 / 91\end{array}$ & $\begin{array}{l}0001 \\
0001 \\
0001 \\
0001 \\
0001 \\
0001 \\
0001\end{array}$ & $M G / L$ & $\begin{array}{l}< \\
< \\
< \\
<\end{array}$ & $\begin{array}{l}0.3 \\
0.44 \\
0.1 \\
0.05 \\
0.1 \\
0.1 \\
0.05\end{array}$ & $J$ & $\begin{array}{l}0.1 \\
0.1 \\
0.1 \\
0.1 \\
0.1 \\
0.1 \\
0.05\end{array}$ & $\begin{array}{l}- \\
- \\
- \\
-\end{array}$ \\
\hline AMHONIUM & $\begin{array}{l}10 / 15 / 85 \\
04 / 06 / 88 \\
07 / 15 / 88 \\
01 / 08 / 89 \\
09 / 19 / 89 \\
03 / 31 / 90 \\
01 / 15 / 91 \\
11 / 25 / 92\end{array}$ & $\begin{array}{l}0001 \\
0001 \\
0001 \\
0001 \\
0001 \\
0001 \\
0001 \\
0001\end{array}$ & $M G / L$ & $\begin{array}{l}< \\
< \\
< \\
<\end{array}$ & $\begin{array}{l}0.2 \\
0.1 \\
0.1 \\
0.18 \\
0.1 \\
0.1 \\
0.28 \\
0.3\end{array}$ & & $\begin{array}{l}0.1 \\
0.1 \\
0.1 \\
0.1 \\
0.1 \\
0.1 \\
0.01 \\
0.1\end{array}$ & $\begin{array}{l}- \\
- \\
- \\
-\end{array}$ \\
\hline AКT IMOHY & $\begin{array}{l}10 / 15 / 85 \\
04 / 06 / 88 \\
07 / 15 / 88 \\
01 / 08 / 89 \\
09 / 19 / 89 \\
03 / 31 / 90 \\
01 / 15 / 91 \\
11 / 25 / 92\end{array}$ & $\begin{array}{l}0001 \\
0001 \\
0001 \\
0001 \\
0001 \\
0001 \\
0001 \\
0001\end{array}$ & MG/L & $<$ & $\begin{array}{l}0.003 \\
0.032 \\
0.043 \\
0.003 \\
0.014 \\
0.075 \\
0.003 \\
0.003\end{array}$ & & $\begin{array}{l}0.003 \\
0.003 \\
0.003 \\
0.003 \\
0.003 \\
0.003 \\
0.003 \\
0.003\end{array}$ & $\begin{array}{l}- \\
: \\
- \\
-\end{array}$ \\
\hline ARSENIC & $\begin{array}{l}10 / 15 / 85 \\
05 / 15 / 86 \\
01 / 09 / 87 \\
04 / 06 / 88 \\
07 / 15 / 88 \\
01 / 08 / 89 \\
09 / 19 / 89 \\
03 / 31 / 90 \\
01 / 15 / 91\end{array}$ & $\begin{array}{l}0001 \\
0001 \\
0001 \\
0001 \\
0001 \\
0001 \\
0001 \\
0001 \\
0001\end{array}$ & $M G / L$ & $\begin{array}{l}< \\
< \\
< \\
<\end{array}$ & $\begin{array}{l}0.01 \\
0.01 \\
0.004 \\
0.026 \\
0.02 \\
0.001 \\
0.02 \\
0.12 \\
0.03\end{array}$ & $\begin{array}{l}2 \\
7 \\
J\end{array}$ & $\begin{array}{l}0.01 \\
0.01 \\
0.01 \\
0.01 \\
0.01 \\
0.01 \\
0.01 \\
0.01 \\
0.03\end{array}$ & $\begin{array}{l}- \\
- \\
- \\
- \\
- \\
- \\
-\end{array}$ \\
\hline BARILA & $\begin{array}{l}10 / 15 / 85 \\
04 / 06 / 88 \\
07 / 15 / 88 \\
01 / 08 / 89 \\
09 / 19 / 89 \\
03 / 31 / 90 \\
01 / 15 / 91\end{array}$ & $\begin{array}{l}0001 \\
0001 \\
0001 \\
0001 \\
0001 \\
0001 \\
0001\end{array}$ & $M G / L$ & $\begin{array}{l}< \\
< \\
< \\
<\end{array}$ & $\begin{array}{l}0.1 \\
0.03 \\
0.1 \\
0.02 \\
0.1 \\
0.1 \\
0.01\end{array}$ & $\begin{array}{l}\text { J } \\
\text { J }\end{array}$ & $\begin{array}{l}0.1 \\
0.1 \\
0.1 \\
0.1 \\
0.1 \\
0.1 \\
0.01\end{array}$ & $\begin{array}{l}- \\
- \\
- \\
- \\
-\end{array}$ \\
\hline BERYLLIUM & $01 / 08 / 89$ & 0001 & $M G / L$ & $<$ & 0.005 & & 0.005 & - \\
\hline
\end{tabular}

PARAMETER VALUE IMDICATOR (PVI): < - LESS THAK DETECTIOH LIHIT

SAMPLE ID COOES:

DO01 - FILTERED SAMPLE (.45 MICROHS)

OTHER PARAMETER VALUE FLAGS:

I - INCREASED DETECTIOH LIMIT DUE TO REQUIRED DILUTION

J - estimated VALUe 
GROUNDWATER OUALITY DATA BY LOCATION

SITE: AMBO1 AMBROSIA LAKE

LOCATION: 0785

MORTH COORDINATE: 52770.8 FT

EAST COORDINATE: $\quad 58816.5$ FT

06/10/80 TO $12 / 04 / 92$

REPORT DATE: 08/04/94

FORMTION OF COMPLETION: TRES HERHLANOS - C2 SANDSTONE (TT)

HYDRAULIC FLOW RELATIONSHIP: DOWH GRADIENT (D)

\begin{tabular}{|c|c|c|c|c|c|c|c|}
\hline PARAMETER NAME & LOG DATE & $\underset{\text { SOMPLE }}{\text { SA }}$ & $\begin{array}{l}\text { UHITS OF } \\
\text { MEASURE }\end{array}$ & PVI & $\begin{array}{l}\text { PARAMETER } \\
\text { VALUE FLAGS }\end{array}$ & $\begin{array}{l}\text { DETECTIOH } \\
\text { LIMIT }\end{array}$ & $\begin{array}{l}\text { PARAMETER } \\
\text { UNCERTAINTY }\end{array}$ \\
\hline BERYLLIUH & $\begin{array}{l}09 / 19 / 89 \\
03 / 31 / 90 \\
01 / 15 / 91\end{array}$ & $\begin{array}{l}0001 \\
0001 \\
0001\end{array}$ & MG/L & $\begin{array}{l}< \\
<\end{array}$ & $\begin{array}{l}0.01 \\
0.01 \\
0.005\end{array}$ & $\begin{array}{l}0.01 \\
0.01 \\
0.005\end{array}$ & - \\
\hline BORON & $\begin{array}{l}10 / 15 / 85 \\
05 / 15 / 86 \\
01 / 09 / 87 \\
04 / 06 / 88 \\
07 / 15 / 88 \\
01 / 08 / 89 \\
09 / 19 / 89 \\
03 / 31 / 90 \\
01 / 15 / 91\end{array}$ & $\begin{array}{l}0001 \\
0001 \\
0001 \\
0001 \\
0001 \\
0001 \\
0001 \\
0001 \\
0001\end{array}$ & $M G / L$ & & $\begin{array}{l}0.3 \\
0.3 \\
0.30 \\
0.63 \\
0.4 \\
0.42 \\
0.4 \\
0.3 \\
0.28\end{array}$ & $\begin{array}{l}0.1 \\
0.1 \\
0.1 \\
0.1 \\
0.1 \\
0.1 \\
0.1 \\
0.1 \\
0.05\end{array}$ & $\begin{array}{l}- \\
- \\
- \\
- \\
- \\
- \\
-\end{array}$ \\
\hline BROAIDE & $\begin{array}{l}04 / 06 / 88 \\
07 / 15 / 88 \\
01 / 08 / 89 \\
09 / 19 / 89 \\
03 / 31 / 90 \\
01 / 15 / 91\end{array}$ & $\begin{array}{l}0001 \\
0001 \\
0001 \\
0001 \\
0001 \\
0001\end{array}$ & MG/L & & $\begin{array}{l}0.3 \\
1.0 \\
3.25 \\
0.46 \\
1.1 \\
2.3\end{array}$ & $\begin{array}{l}0.1 \\
0.1 \\
0.01 \\
0.1 \\
0.1 \\
0.1\end{array}$ & $\begin{array}{l}- \\
- \\
- \\
-\end{array}$ \\
\hline CADHIUM & $\begin{array}{l}10 / 15 / 85 \\
05 / 15 / 86 \\
04 / 06 / 88 \\
07 / 15 / 88 \\
01 / 08 / 89 \\
09 / 19 / 89 \\
03 / 31 / 90 \\
01 / 15 / 91 \\
11 / 25 / 92\end{array}$ & $\begin{array}{l}0001 \\
0001 \\
0001 \\
0001 \\
0001 \\
0001 \\
0001 \\
0001 \\
0001\end{array}$ & $M G / L$ & $\begin{array}{l}< \\
<\end{array}$ & $\begin{array}{l}0.001 \\
0.001 \\
0.009 \\
0.002 \\
0.0011 \\
0.024 \\
0.001 \\
0.001 \\
0.0002\end{array}$ & $\begin{array}{l}0.001 \\
0.001 \\
0.001 \\
0.001 \\
0.001 \\
0.001 \\
0.001 \\
0.001 \\
0.0001\end{array}$ & $\begin{array}{l}- \\
- \\
- \\
- \\
- \\
-\end{array}$ \\
\hline CALCIUH & $\begin{array}{l}10 / 15 / 85 \\
05 / 15 / 86 \\
01 / 09 / 87 \\
04 / 06 / 88 \\
07 / 15 / 88 \\
01 / 08 / 89 \\
09 / 19 / 89 \\
03 / 31 / 90 \\
01 / 15 / 91 \\
11 / 25 / 92\end{array}$ & $\begin{array}{l}0001 \\
0001 \\
0001 \\
0001 \\
0001 \\
0001 \\
0001 \\
0001 \\
0001 \\
0001\end{array}$ & $M G / L$ & & $\begin{array}{l}198 . \\
531 . \\
497 . \\
561 . \\
391 . \\
423 . \\
375 . \\
370 . \\
255 . \\
311 .\end{array}$ & $\begin{array}{l}0.01 \\
0.01 \\
0.01 \\
0.01 \\
0.01 \\
0.01 \\
0.01 \\
0.01 \\
0.5 \\
0.5\end{array}$ & $\begin{array}{l}- \\
- \\
- \\
- \\
- \\
- \\
-\end{array}$ \\
\hline CHLORIDE & $\begin{array}{l}10 / 15 / 85 \\
05 / 15 / 86 \\
01 / 09 / 87 \\
04 / 06 / 88 \\
07 / 15 / 88 \\
01 / 08 / 89 \\
09 / 19 / 89 \\
03 / 31 / 90 \\
01 / 15 / 91 \\
11 / 25 / 92\end{array}$ & $\begin{array}{l}0001 \\
0001 \\
0001 \\
0001 \\
0001 \\
0001 \\
0001 \\
0001 \\
0001 \\
0001\end{array}$ & MG/L & & $\begin{array}{l}81 . \\
190 . \\
220 . \\
260 . \\
230 . \\
261 . \\
230 . \\
259 . \\
261 . \\
254 .\end{array}$ & $\begin{array}{l}1 . \\
i . \\
i . \\
i . \\
i . \\
i . \\
i . \\
i . \\
0.5 \\
5 .\end{array}$ & $\begin{array}{l}- \\
: \\
: \\
: \\
: \\
- \\
-\end{array}$ \\
\hline ChROAIUN & $\begin{array}{l}10 / 15 / 85 \\
05 / 15 / 86 \\
01 / 09 / 87 \\
04 / 06 / 88\end{array}$ & $\begin{array}{l}0001 \\
0001 \\
0001 \\
0001\end{array}$ & $M G / L$ & & $\begin{array}{l}0.02 \\
0.07 \\
0.02 \\
0.24\end{array}$ & $\begin{array}{l}0.01 \\
0.01 \\
0.01 \\
0.01\end{array}$ & $\begin{array}{l}- \\
- \\
-\end{array}$ \\
\hline
\end{tabular}

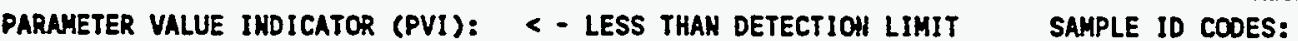

O001 - FILTERED SAMPLE (.45 MICRONS)

OTHER PARAMETER VALUE FLAGS:

H - HOLD TIME EXPIRED, VALUE SUSPECT 
GROUHDWATER QUALITY DATA BY LOCATION

SITE: AMBOI AMBROSIA LAKE

LOCATION: 0785

NORTH COORDINATE: $\quad 52770.8$ FT

EAST COORDINATE: $\quad 58816.5$ FT

06/10/80 TO 12/04/92

REPORT DATE: 08/04/94

FORMATION OF COMPLETION: TRES HERMANOS - C2 SAHDSTONE (TT)

HYDRAULIC FLON RELATIONSHIP: DOWN GRADIENT (D)

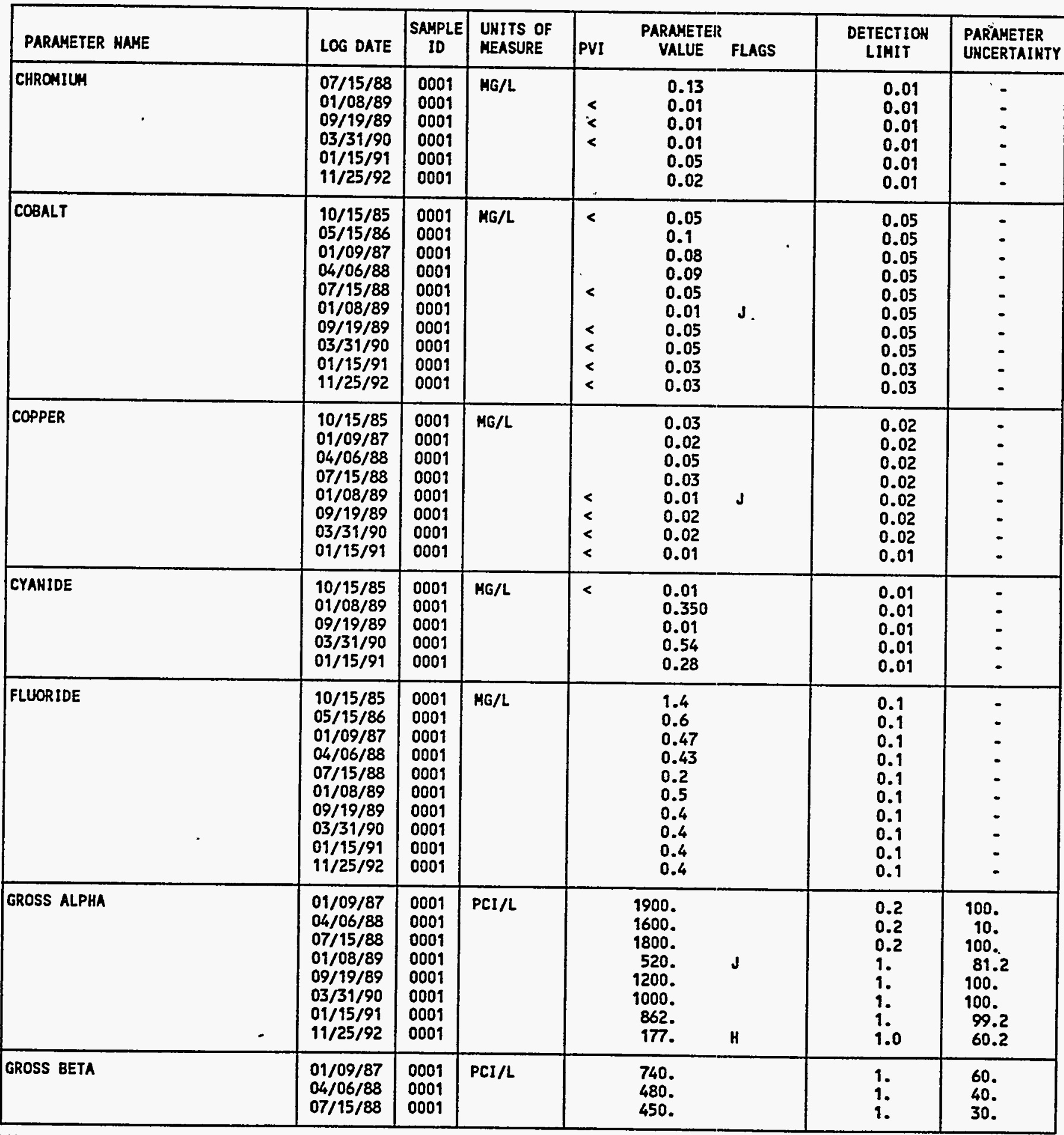

PARAMETER VALUE IHDICATOR (PVI): < - LESS THAN DETECTION LIMIT SAMPLE ID COOES:

0001 - FILTERED SAMPLE (.45 MICRONS) -

OTHER PARAMETER VALUE FLAGS:

H - HOLD TIME EXPIRED, VALUE SUSPECT

J - estimated VALUE 
GROUNDWATER QUALITY DATA BY LOCATION

SITE: AMBOI AMBROSIA LAKE

LOCATION: 0785

NORTH COORDINATE: 52770.8 FT

EAST COORDINATE: $\quad 58816.5$ FT

$06 / 10 / 80$ TO $12 / 04 / 92$

REPORT DATE: 08/04/94

FORHATION OF COMPLETION: TRES HERMAMOS - C2 SAMDSTONE (TT) HYORAULIC FLON RELATIONSHIP: DOWN GRADIENT (D)

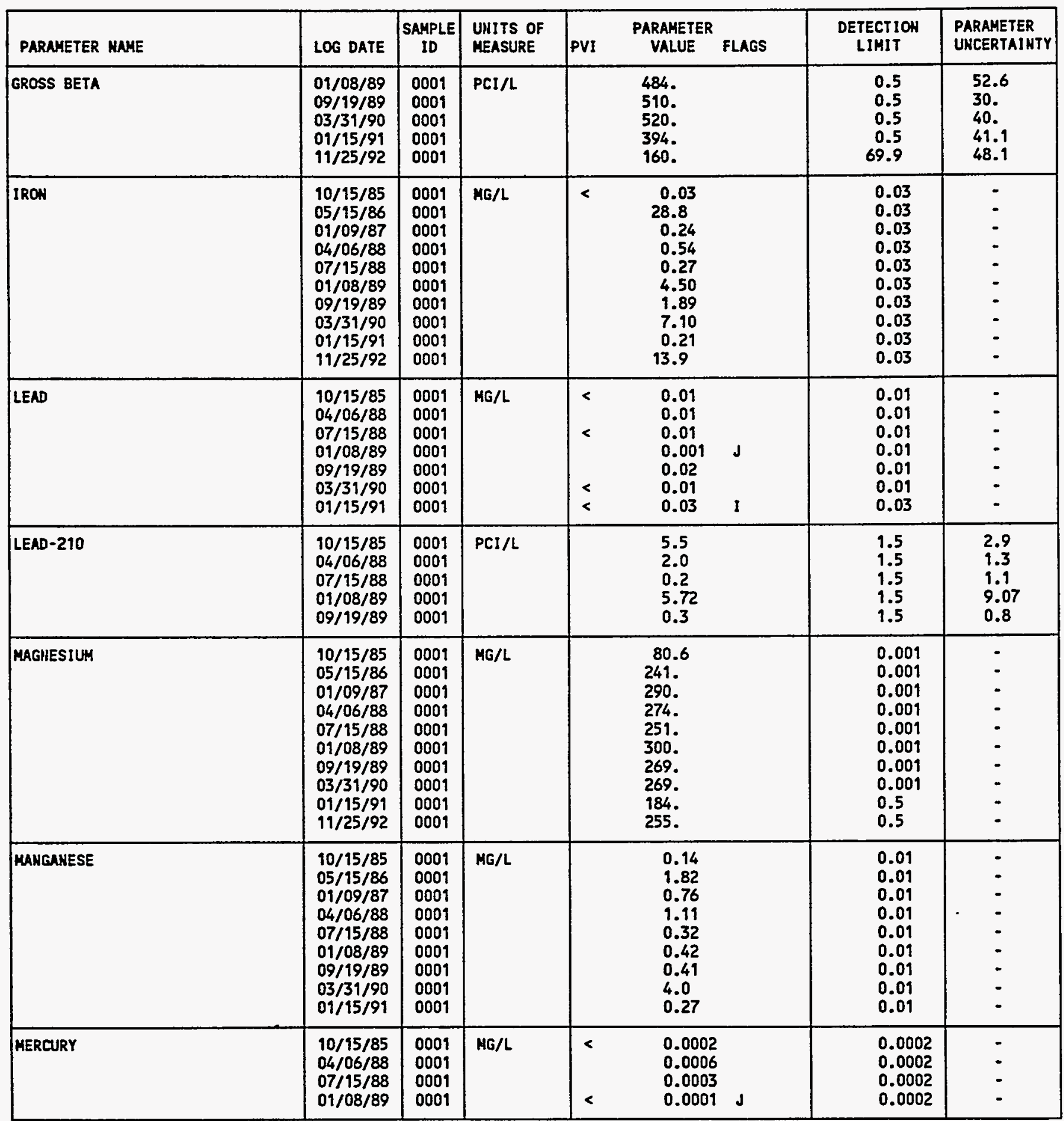

PARAIETER VALUE INDICATOR (PVI): < - LeSS than DETECTION LIMIT

SAMPLE ID COOES

0001 - FILTERED SAMPLE (.45 MICRONS)

OTHER PARAMETER VALUE FLAGS:

I - IMCREASED DETECTION LIMIT DUE TO REQUIRED DILUTION

J - estimated VALUE 
GROUNDWATER QUALITY DATA BY LOCATION

SITE: AMBOI AMBROSIA LAKE

LOCATION: 0785

NORTH COORDINATE: 52770.8 FT

EAST COORDINATE: $\quad 58816.5$ FT

06/10/80 TO $12 / 04 / 92$

REPORT DATE: 08/04/94

FORMATIOH OF COMPLETION: TRES HERMANOS - C2 SAMDSTONE (TT) HYDRAULIC FLOW RELATIOHSHIP: DOWH GRADIENT (D)

\begin{tabular}{|c|c|c|c|c|c|c|c|c|}
\hline PARAMETER MAME & LOG DATE & $\begin{array}{c}\text { SAMPLE } \\
\text { ID }\end{array}$ & $\begin{array}{l}\text { UHITS OF } \\
\text { MEASURE }\end{array}$ & PVI & $\begin{array}{l}\text { PARAMETERR } \\
\text { VALUE }\end{array}$ & FLAGS & $\begin{array}{l}\text { DETECTION } \\
\text { LIMIT }\end{array}$ & $\begin{array}{l}\text { PARAMETER } \\
\text { UNCERTAINTY }\end{array}$ \\
\hline MERCURY & $\begin{array}{l}09 / 19 / 89 \\
03 / 31 / 90 \\
01 / 15 / 91\end{array}$ & $\begin{array}{l}0001 \\
0001 \\
0001\end{array}$ & $M G / L$ & $\begin{array}{l}< \\
< \\
<\end{array}$ & $\begin{array}{l}0.000 ? \\
0.000 ? \\
0.0002\end{array}$ & & $\begin{array}{l}0.0002 \\
0.0002 \\
0.0002\end{array}$ & - \\
\hline HOLYBDENLM & $\begin{array}{l}10 / 15 / 85 \\
05 / 15 / 86 \\
01 / 09 / 87 \\
04 / 06 / 88 \\
07 / 15 / 88 \\
01 / 08 / 89 \\
09 / 19 / 89 \\
03 / 31 / 90 \\
01 / 15 / 91 \\
11 / 25 / 92\end{array}$ & $\begin{array}{l}0001 \\
0001 \\
0001 \\
0001 \\
0001 \\
0001 \\
0001 \\
0001 \\
0001 \\
0001\end{array}$ & $M G / L$ & & $\begin{array}{l}0.35 \\
0.2 \\
0.2 \\
0.14 \\
0.27 \\
0.019 \\
0.07 \\
0.04 \\
0.05 \\
0.04\end{array}$ & & $\begin{array}{l}0.01 \\
0.01 \\
0.01 \\
0.01 \\
0.01 \\
0.01 \\
0.01 \\
0.01 \\
0.01 \\
0.01\end{array}$ & $\begin{array}{l}- \\
: \\
- \\
: \\
- \\
-\end{array}$ \\
\hline HET GROSS ALPHA * & $\begin{array}{l}01 / 09 / 87 \\
04 / 06 / 88 \\
07 / 15 / 88 \\
01 / 08 / 88 \\
09 / 19 / 89 \\
03 / 31 / 90 \\
01 / 15 / 91 \\
11 / 25 / 92\end{array}$ & $\begin{array}{l}0001 \\
0001 \\
0001 \\
0001 \\
0001 \\
0001 \\
0001 \\
0001\end{array}$ & $\mathrm{PCI} / \mathrm{L}$ & & $\begin{array}{r}-363.80 \\
317.18 \\
654.38 \\
-721.66 \\
191.58 \\
324.29 \\
-359.08 \\
-109.06\end{array}$ & & $\begin{array}{l}- \\
- \\
i \\
\dot{-} \\
-\end{array}$ & $\begin{array}{l}- \\
- \\
- \\
- \\
- \\
-\end{array}$ \\
\hline HICKEL & $\begin{array}{l}10 / 15 / 85 \\
04 / 06 / 88 \\
07 / 15 / 88 \\
01 / 08 / 89 \\
09 / 19 / 89 \\
03 / 31 / 90 \\
01 / 15 / 91 \\
11 / 25 / 92\end{array}$ & $\begin{array}{l}0001 \\
0001 \\
0001 \\
0001 \\
0001 \\
0001 \\
0001 \\
0001\end{array}$ & MG/L & $\begin{array}{l}< \\
< \\
< \\
<\end{array}$ & $\begin{array}{l}0.04 \\
0.15 \\
0.07 \\
0.02 \\
0.04 \\
0.04 \\
0.04 \\
0.13\end{array}$ & $J$ & $\begin{array}{l}0.04 \\
0.04 \\
0.04 \\
0.04 \\
0.04 \\
0.04 \\
0.04 \\
0.04\end{array}$ & $\begin{array}{l}- \\
- \\
: \\
: \\
-\end{array}$ \\
\hline MITRATE & $\begin{array}{l}10 / 15 / 85 \\
05 / 15 / 86 \\
01 / 09 / 87 \\
04 / 06 / 88 \\
07 / 15 / 88 \\
01 / 08 / 89 \\
09 / 19 / 89 \\
03 / 31 / 90 \\
01 / 15 / 91 \\
11 / 25 / 92\end{array}$ & $\begin{array}{l}0001 \\
0001 \\
0001 \\
0001 \\
0001 \\
0001 \\
0001 \\
0001 \\
0001 \\
0001\end{array}$ & $\mathrm{MG} / \mathrm{L}$ & $\begin{array}{l}< \\
< \\
<\end{array}$ & $\begin{array}{c}20 . \\
1 . \\
5.3 \\
1.8 \\
4.6 \\
0.1 \\
1.4 \\
1.6 \\
3.1 \\
1.0\end{array}$ & $\begin{array}{l}3 \\
J\end{array}$ & $\begin{array}{l}1 . \\
1 . \\
1 . \\
1 . \\
1 . \\
1 . \\
1 . \\
1 . \\
0.1 \\
1.0\end{array}$ & $\begin{array}{l}- \\
- \\
- \\
- \\
- \\
- \\
-\end{array}$ \\
\hline HITRITE & $10 / 15 / 85$ & 0001 & MG/L & $<$ & 0.1 & & 0.1 & - \\
\hline MITRITE AND NITRATE & $\begin{array}{l}04 / 06 / 88 \\
03 / 31 / 90 \\
01 / 15 / 91\end{array}$ & $\begin{array}{l}0001 \\
0001 \\
0001\end{array}$ & MG/L & & $\begin{array}{l}1.8 \\
0.4 \\
0.38\end{array}$ & 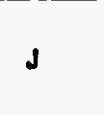 & $\begin{array}{l}1 . \\
1.05\end{array}$ & $\div$ \\
\hline PH & $\begin{array}{l}10 / 15 / 85 \\
05 / 15 / 86 \\
01 / 09 / 87 \\
04 / 06 / 88 \\
07 / 15 / 88\end{array}$ & $\begin{array}{l}0001 \\
0001 \\
0001 \\
0001 \\
0001\end{array}$ & su & & $\begin{array}{l}7.87 \\
7.35 \\
7.24 \\
7.18 \\
7.08\end{array}$ & & $\begin{array}{l}- \\
- \\
-\end{array}$ & $\begin{array}{l}- \\
- \\
-\end{array}$ \\
\hline
\end{tabular}

- het gross aLPHA (GROSS ALPHA - URAKIUH) WITH-1 MG URAHIUM = 686 PCI

PARAMETER VALUE INDICATOR (PVI): < - LESS THAN DETECTION LIMIT

OTHER PARAMETER VALUE FLAGS:

J - estIMATED VALUE
SAMPLE ID COOES:

0001 - FILTERED SAMPLE (.45 MICROHS) 


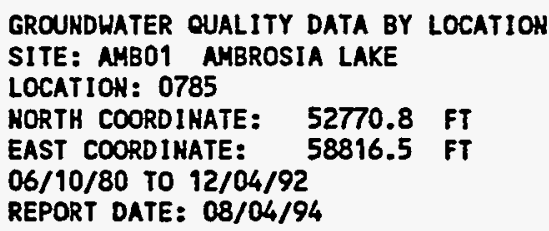

FORMATION OF CONPLETION: TRES HERHANOS - C2 SAKDSTONE (TT) HYDRAULIC FLON RELATIONSHIP: DOWN GRADIEHT (D)

\begin{tabular}{|c|c|c|c|c|c|c|c|c|}
\hline PARAMETER NAME & & LOG DATE & $\begin{array}{c}\text { SAMPLE } \\
10\end{array}$ & $\begin{array}{l}\text { UNITS OF } \\
\text { MEASURE }\end{array}$ & PVI & $\begin{array}{l}\text { PARAMETER } \\
\text { VALUE FLAGS }\end{array}$ & $\begin{array}{l}\text { DETECTION } \\
\text { LIMIT }\end{array}$ & $\begin{array}{l}\text { PARAMETER } \\
\text { UHCERTAINTY }\end{array}$ \\
\hline PH & & $\begin{array}{l}01 / 08 / 89 \\
09 / 19 / 89 \\
03 / 31 / 90 \\
01 / 15 / 91 \\
11 / 25 / 92\end{array}$ & $\begin{array}{l}0001 \\
0001 \\
0001 \\
0001 \\
0001\end{array}$ & su & & $\begin{array}{l}7.08 \\
6.94 \\
6.80 \\
7.06 \\
7.16\end{array}$ & $\begin{array}{l}- \\
- \\
- \\
-\end{array}$ & $\begin{array}{l}- \\
- \\
-\end{array}$ \\
\hline POTASSIUH & & $\begin{array}{l}10 / 15 / 85 \\
05 / 15 / 86 \\
01 / 09 / 87 \\
04 / 06 / 88 \\
07 / 15 / 88 \\
01 / 08 / 89 \\
09 / 19 / 89 \\
03 / 31 / 90 \\
01 / 15 / 91 \\
11 / 25 / 92\end{array}$ & $\begin{array}{l}0001 \\
0001 \\
0001 \\
0001 \\
0001 \\
0001 \\
0001 \\
0001 \\
0001 \\
0001\end{array}$ & $M G / L$ & & $\begin{array}{c}7.73 \\
11.7 \\
8.90 \\
8.25 \\
8.3 \\
8.2 \\
8.1 \\
8.6 \\
6.1 \\
7.57\end{array}$ & $\begin{array}{l}0.01 \\
0.01 \\
0.01 \\
0.01 \\
0.01 \\
0.01 \\
0.01 \\
0.01 \\
0.01 \\
0.01\end{array}$ & $\begin{array}{l}- \\
- \\
- \\
- \\
- \\
- \\
-\end{array}$ \\
\hline RADIUM-226 & & $\begin{array}{l}10 / 15 / 85 \\
01 / 09 / 87 \\
04 / 06 / 88 \\
07 / 15 / 88 \\
01 / 08 / 89 \\
09 / 19 / 89 \\
03 / 31 / 90 \\
11 / 25 / 92\end{array}$ & $\begin{array}{l}0001 \\
0001 \\
0001 \\
0001 \\
0001 \\
0001 \\
0001 \\
0001\end{array}$ & $\mathrm{PCl} / \mathrm{L}$ & & $\begin{array}{l}0.4 \\
2.0 \\
0.2 \\
0.6 \\
0.948 \\
0.1 \\
0.4 \\
0.8\end{array}$ & $\begin{array}{l}1 . \\
1 . \\
1 . \\
1 . \\
1 . \\
1 . \\
1.3\end{array}$ & $\begin{array}{l}1.9 \\
0.7 \\
0.2 \\
0.3 \\
0.365 \\
0.2 \\
0.3 \\
0.4\end{array}$ \\
\hline RADIUA-228 & - & $\begin{array}{l}10 / 15 / 85 \\
01 / 09 / 87 \\
04 / 06 / 88 \\
07 / 15 / 88 \\
01 / 08 / 89 \\
09 / 19 / 89 \\
03 / 31 / 90 \\
11 / 25 / 92\end{array}$ & $\begin{array}{l}0001 \\
0001 \\
0001 \\
0001 \\
0001 \\
0001 \\
0001 \\
0001\end{array}$ & $\mathrm{PCI} / \mathrm{L}$ & & $\begin{array}{l}0.4 \\
0.8 \\
0.8 \\
0.2 \\
9.56 \\
0.4 \\
0.5 \\
0.2\end{array}$ & $\begin{array}{l}1 . \\
1 . \\
1 . \\
1 . \\
1 . \\
1 . \\
1.0\end{array}$ & $\begin{array}{l}4.5 \\
1.1 \\
0.8 \\
0.8 \\
5.26 \\
0.9 \\
0.7 \\
1.5\end{array}$ \\
\hline
\end{tabular}

PARAMETER VALUE IMDICATOR (PVI): < - LESS THAN DETECTION LIHIT

SAMPLE ID COOES:

0001 - FILTERED SAMPLE (.45 MICRONS)

OTHER PARAMETER VALUE FLAGS:

H - HOLd TIME EXPIRED, VALUE SUSPECT 
GROUNDHATER QUALITY DATA BY LOCATION

SITE: AMBO1 AMBROSIA LAKE

LOCATION: 0785

MORTH COORDINATE: 52770.8 FT

EAST COORDINATE: $\quad 58816.5$ FT

06/10/80 TO $12 / 04 / 92$

REPORT DATE: 08/04/94

FORMATIOH OF COMPLETION: TRES HERMANOS - C2 SAKDSTONE (TT) HYDRAULIC FLOW RELATIOHSHIP: DOWH GRAOIENT (D)

\begin{tabular}{|c|c|c|c|c|c|c|c|c|c|}
\hline PARAMETER MAME & & LOG DATE & SAMPLE & $\begin{array}{l}\text { UNITS OF } \\
\text { MEASURE }\end{array}$ & PVI & $\begin{array}{l}\text { PARAMETEE: } \\
\text { VALUE }\end{array}$ & FLAGS & $\begin{array}{l}\text { DETECTION } \\
\text { LIMIT }\end{array}$ & $\begin{array}{l}\text { PARAMETER } \\
\text { UNCERTAINTY }\end{array}$ \\
\hline SELENIUH & & $\begin{array}{l}10 / 15 / 85 \\
05 / 15 / 86 \\
01 / 09 / 87 \\
04 / 06 / 88 \\
07 / 15 / 88 \\
01 / 08 / 89 \\
09 / 19 / 89 \\
03 / 31 / 90 \\
01 / 15 / 91 \\
11 / 25 / 92\end{array}$ & $\begin{array}{l}0001 \\
0001 \\
0001 \\
0001 \\
0001 \\
0001 \\
0001 \\
0001 \\
0001 \\
0001\end{array}$ & MG/L & $<$ & $\begin{array}{l}0.003 \\
0.005 \\
0.324 \\
0.123 \\
0.263 \\
0.180 \\
0.264 \\
0.162 \\
0.278 \\
0.06\end{array}$ & $\mathbf{J}$ & $\begin{array}{l}0.005 \\
0.005 \\
0.005 \\
0.005 \\
0.005 \\
0.005 \\
0.005 \\
0.005 \\
0.005 \\
0.03\end{array}$ & $\begin{array}{l}- \\
: \\
- \\
: \\
\vdots \\
-\end{array}$ \\
\hline SILICA - SIO2 & & $\begin{array}{l}10 / 15 / 85 \\
04 / 06 / 88 \\
07 / 15 / 88 \\
01 / 08 / 89 \\
09 / 19 / 89 \\
03 / 31 / 90 \\
01 / 15 / 91\end{array}$ & $\begin{array}{l}0001 \\
0001 \\
0001 \\
0001 \\
0001 \\
0001 \\
0001\end{array}$ & MG/L & & $\begin{array}{l}19 . \\
20.3 \\
14.5 \\
12.1 \\
11 . \\
9 . \\
7.6\end{array}$ & & $\begin{array}{l}2 . \\
2 . \\
2 . \\
2 . \\
2 . \\
2 . \\
0.1\end{array}$ & $\begin{array}{l}- \\
- \\
- \\
- \\
-\end{array}$ \\
\hline SILVER & & $\begin{array}{l}10 / 15 / 85 \\
01 / 09 / 87 \\
04 / 06 / 88 \\
07 / 15 / 88 \\
01 / 08 / 89 \\
09 / 19 / 89 \\
03 / 31 / 90 \\
01 / 15 / 91 \\
11 / 25 / 92\end{array}$ & $\begin{array}{l}0001 \\
0001 \\
0001 \\
0001 \\
0001 \\
0001 \\
0001 \\
0001 \\
0001\end{array}$ & MG/L & $\begin{array}{l}< \\
< \\
< \\
< \\
<\end{array}$ & $\begin{array}{l}0.01 \\
0.01 \\
0.08 \\
0.02 \\
0.01 \\
0.01 \\
0.01 \\
0.01 \\
0.02\end{array}$ & & $\begin{array}{l}0.01 \\
0.09 \\
0.01 \\
0.01 \\
0.09 \\
0.01 \\
0.01 \\
0.01 \\
0.01\end{array}$ & $\begin{array}{l}- \\
- \\
- \\
- \\
- \\
-\end{array}$ \\
\hline SOOIUH & & $\begin{array}{l}10 / 15 / 85 \\
05 / 15 / 86 \\
01 / 09 / 87 \\
04 / 06 / 88 \\
07 / 15 / 88 \\
01 / 08 / 89 \\
09 / 19 / 89 \\
03 / 31 / 90 \\
01 / 15 / 91 \\
11 / 25 / 92\end{array}$ & $\begin{array}{l}0001 \\
0001 \\
0001 \\
0001 \\
0001 \\
0001 \\
0001 \\
0001 \\
0001 \\
0001\end{array}$ & MG/L & & $\begin{array}{c}564 . \\
913 . \\
1240 . \\
855 . \\
1090 . \\
1250 . \\
1253 . \\
1270 . \\
1320 . \\
1270 .\end{array}$ & & $\begin{array}{l}0.002 \\
0.002 \\
0.002 \\
0.002 \\
0.002 \\
0.002 \\
0.002 \\
0.002 \\
5 . \\
20 .\end{array}$ & $\begin{array}{l}- \\
: \\
: \\
: \\
: \\
-\end{array}$ \\
\hline SPECIFIC CONDUCTANCE & & $\begin{array}{l}10 / 15 / 85 \\
05 / 15 / 86 \\
01 / 09 / 87 \\
04 / 06 / 88 \\
07 / 15 / 88 \\
01 / 08 / 89 \\
09 / 19 / 89 \\
03 / 31 / 90 \\
01 / 15 / 91 \\
11 / 25 / 92\end{array}$ & $\begin{array}{l}0001 \\
0001 \\
0001 \\
0001 \\
0001 \\
0001 \\
0001 \\
0001 \\
0001 \\
0001\end{array}$ & UHHO/CM & & $\begin{array}{l}2200 . \\
4500 . \\
4700 . \\
4800 \\
5000 \\
2900 . \\
2200 . \\
4750 . \\
5000 . \\
4720\end{array}$ & & $\begin{array}{l}- \\
: \\
- \\
- \\
- \\
-\end{array}$ & $\begin{array}{l}- \\
= \\
= \\
- \\
- \\
-\end{array}$ \\
\hline STRONTIUM & - & $\begin{array}{l}10 / 15 / 85 \\
05 / 15 / 86 \\
04 / 06 / 88 \\
07 / 15 / 88 \\
01 / 08 / 89\end{array}$ & $\begin{array}{l}0001 \\
0001 \\
0001 \\
0001 \\
0001\end{array}$ & MG/L & & $\begin{array}{l}2.2 \\
4.4 \\
8.26 \\
7.9 \\
9.3\end{array}$ & & $\begin{array}{l}0.1 \\
0.1 \\
0.1 \\
0.1 \\
0.1\end{array}$ & $\begin{array}{l}- \\
\dot{-} \\
-\end{array}$ \\
\hline
\end{tabular}

PARAMETER VALUE IMDICATOR (PVI): < - LESS THAN DETECTION LIMIT

SAMPLE ID COOES:

0001 - FILTERED SAMPLE (.45 MICRONS)

OTHER PARAMETER VALUE FLAGS:

H - HOLD TIME EXPIRED, VALUE SUSPECT

$J$ - estimated VAlUe 
GROUNDHATER QUALITY DATA BY LOCATION

SITE: AMBO1 AMBROSIA LAKE

LOCATION: 0785

NORTH COORDINATE: 52770.8 FT

EAST COORDIHATE: $\quad 58816.5$ FT

06/10/80 TO $12 / 04 / 92$

REPORT DATE: 08/04/94

FORHATION OF COMPLETION: TRES HERMANOS - C2 SANDSTOHE (TT) HYDRAULIC FLON RELATIONSHIP: DOWH GRADIENT (D)

\begin{tabular}{|c|c|c|c|c|c|c|c|}
\hline PARAMETER MAHE & LOG DATE & $\begin{array}{c}\text { SAMPLE } \\
\text { I0 }\end{array}$ & $\begin{array}{l}\text { UNITS OF } \\
\text { MEASURE }\end{array}$ & PVI & $\begin{array}{l}\text { PARAMETER } \\
\text { VALUE FLAGS }\end{array}$ & $\begin{array}{l}\text { DETECTIOH } \\
\text { LIMIT }\end{array}$ & $\begin{array}{l}\text { PARAMETER } \\
\text { UHCERTAIHTY }\end{array}$ \\
\hline STRONTIUH & $\begin{array}{l}09 / 9 / 89 \\
03 / 31 / 90 \\
01 / 15 / 91\end{array}$ & $\begin{array}{l}0009 \\
0001 \\
0001\end{array}$ & MG/L & & $\begin{array}{l}8.5 \\
7.63 \\
4.60\end{array}$ & $\begin{array}{l}0.1 \\
0.1 \\
0.01\end{array}$ & - \\
\hline SULFATE & $\begin{array}{l}10 / 15 / 85 \\
05 / 15 / 86 \\
01 / 09 / 87 \\
04 / 06 / 88 \\
07 / 15 / 88 \\
01 / 08 / 89 \\
09 / 19 / 89 \\
03 / 31 / 90 \\
01 / 15 / 91 \\
11 / 25 / 92\end{array}$ & $\begin{array}{l}0001 \\
0001 \\
0001 \\
0001 \\
0001 \\
0001 \\
0001 \\
0001 \\
0001 \\
0001\end{array}$ & MG/L & & $\begin{array}{l}1560 . \\
3370 . \\
3970 . \\
3510 . \\
3470 . \\
4182 . \\
4100 . \\
3880 . \\
4080 . \\
3700 .\end{array}$ & $\begin{array}{l}0.1 \\
0.1 \\
0.1 \\
0.1 \\
0.1 \\
0.9 \\
0.9 \\
0.1 \\
10 . \\
10 .\end{array}$ & $\begin{array}{l}- \\
: \\
: \\
: \\
: \\
-\end{array}$ \\
\hline SULFIDE & $\begin{array}{l}10 / 15 / 85 \\
04 / 06 / 88 \\
07 / 15 / 88 \\
01 / 08 / 89 \\
09 / 19 / 89 \\
03 / 31 / 90 \\
01 / 15 / 91\end{array}$ & $\begin{array}{l}0001 \\
0001 \\
0001 \\
0001 \\
0001 \\
0001 \\
0001\end{array}$ & MG/L & $\begin{array}{l}< \\
< \\
< \\
< \\
< \\
< \\
<\end{array}$ & $\begin{array}{l}0.1 \\
0.1 \\
0.1 \\
0.01 \\
0.1 \\
0.1 \\
0.1\end{array}$ & $\begin{array}{l}0.1 \\
0.1 \\
0.1 \\
0.1 \\
0.1 \\
0.1 \\
0.1\end{array}$ & $\begin{array}{l}- \\
- \\
- \\
- \\
-\end{array}$ \\
\hline TEMPERATURE & $\begin{array}{l}10 / 15 / 85 \\
05 / 15 / 86 \\
01 / 09 / 87 \\
04 / 06 / 88 \\
07 / 15 / 88 \\
01 / 08 / 89 \\
09 / 19 / 89 \\
03 / 31 / 90 \\
01 / 15 / 91 \\
11 / 25 / 92\end{array}$ & $\begin{array}{l}0001 \\
0001 \\
0001 \\
0001 \\
0001 \\
0001 \\
0001 \\
0001 \\
0001 \\
0001\end{array}$ & $C$ - DEGREE & & $\begin{array}{l}11 . \\
17 . \\
11 . \\
12.0 \\
13.0 \\
8.0 \\
14.0 \\
13 . \\
11.0 \\
10.0\end{array}$ & $\begin{array}{l}- \\
- \\
- \\
- \\
- \\
- \\
-\end{array}$ & $\begin{array}{l}- \\
: \\
- \\
: \\
- \\
- \\
-\end{array}$ \\
\hline THALLIUN & $\begin{array}{l}01 / 08 / 89 \\
09 / 19 / 89 \\
03 / 31 / 90 \\
01 / 15 / 91\end{array}$ & $\begin{array}{l}0001 \\
0001 \\
0001 \\
0001\end{array}$ & $M G / L$ & $<$ & $\begin{array}{l}0.003 \\
0.01 \\
0.01 \\
0.03\end{array}$ & $\begin{array}{l}0.001 \\
0.01 \\
0.01 \\
0.03\end{array}$ & $\begin{array}{l}- \\
.\end{array}$ \\
\hline THORIUM-230 & $\begin{array}{l}10 / 15 / 85 \\
04 / 06 / 88 \\
07 / 15 / 88 \\
01 / 08 / 89\end{array}$ & $\begin{array}{l}0001 \\
0001 \\
0001 \\
0001\end{array}$ & PCI/L & & $\begin{array}{r}1.5 \\
0.6 \\
0.2 \\
10.9\end{array}$ & $\begin{array}{l}1 . \\
1 . \\
1 .\end{array}$ & $\begin{array}{l}2.5 \\
0.6 \\
0.4 \\
0.750\end{array}$ \\
\hline TIII & $\begin{array}{l}10 / 15 / 85 \\
04 / 06 / 88 \\
07 / 15 / 88 \\
01 / 08 / 89 \\
09 / 19 / 89 \\
03 / 31 / 90 \\
01 / 15 / 91\end{array}$ & $\begin{array}{l}0001 \\
0001 \\
0001 \\
0001 \\
0001 \\
0001 \\
0001\end{array}$ & MG/L & $\begin{array}{l}< \\
< \\
<\end{array}$ & $\begin{array}{l}0.005 \\
0.030 \\
0.015 \\
0.005 \\
0.185 \\
0.080 \\
0.03\end{array}$ & $\begin{array}{l}0.005 \\
0.005 \\
0.005 \\
0.005 \\
0.005 \\
0.005 \\
0.03\end{array}$ & $\begin{array}{l}: \\
- \\
. \\
- \\
-\end{array}$ \\
\hline TOTAL DISSOLVED SOLIDS & $\begin{array}{l}10 / 15 / 85 \\
05 / 15 / 86 \\
01 / 09 / 87 \\
04 / 06 / 88\end{array}$ & $\begin{array}{l}0001 \\
0001 \\
0001 \\
0001\end{array}$ & MG/L & & $\begin{array}{l}2670 . \\
5690 . \\
6850 . \\
6950 .\end{array}$ & $\begin{array}{l}10 . \\
10 . \\
10 . \\
10 .\end{array}$ & $\begin{array}{l}- \\
- \\
-\end{array}$ \\
\hline
\end{tabular}

PARAMETER VALUE IMDICATOR (PVI): < - LESS THAN DETECTIOH LIMIT

OTHER PARAMETER VALUE FLAGS:

1 - INCREASED DETECTION LIMIT DUE TO REQUIRED DILUTION

$J$ - ESTIMATED VALUE 
GROUNDWATER QUALITY DATA BY LOCATION

SITE: AMBO1 AMBROSIA LAKE

LOCATION: 0785

MORTH COORDINATE: 52770.8 FT

EAST COORDINATE: $\quad 58816.5$ FT

06/10/80 TO 12/04/92

REPORT DATE: 08/04/94

FORMATIOH OF COMPLETION: TRES HERHANOS - C2 SAMDSTOHE (TT)

HYDRAULIC FLOH RELATIOHSHIP: DOWH GRADIENT (D)

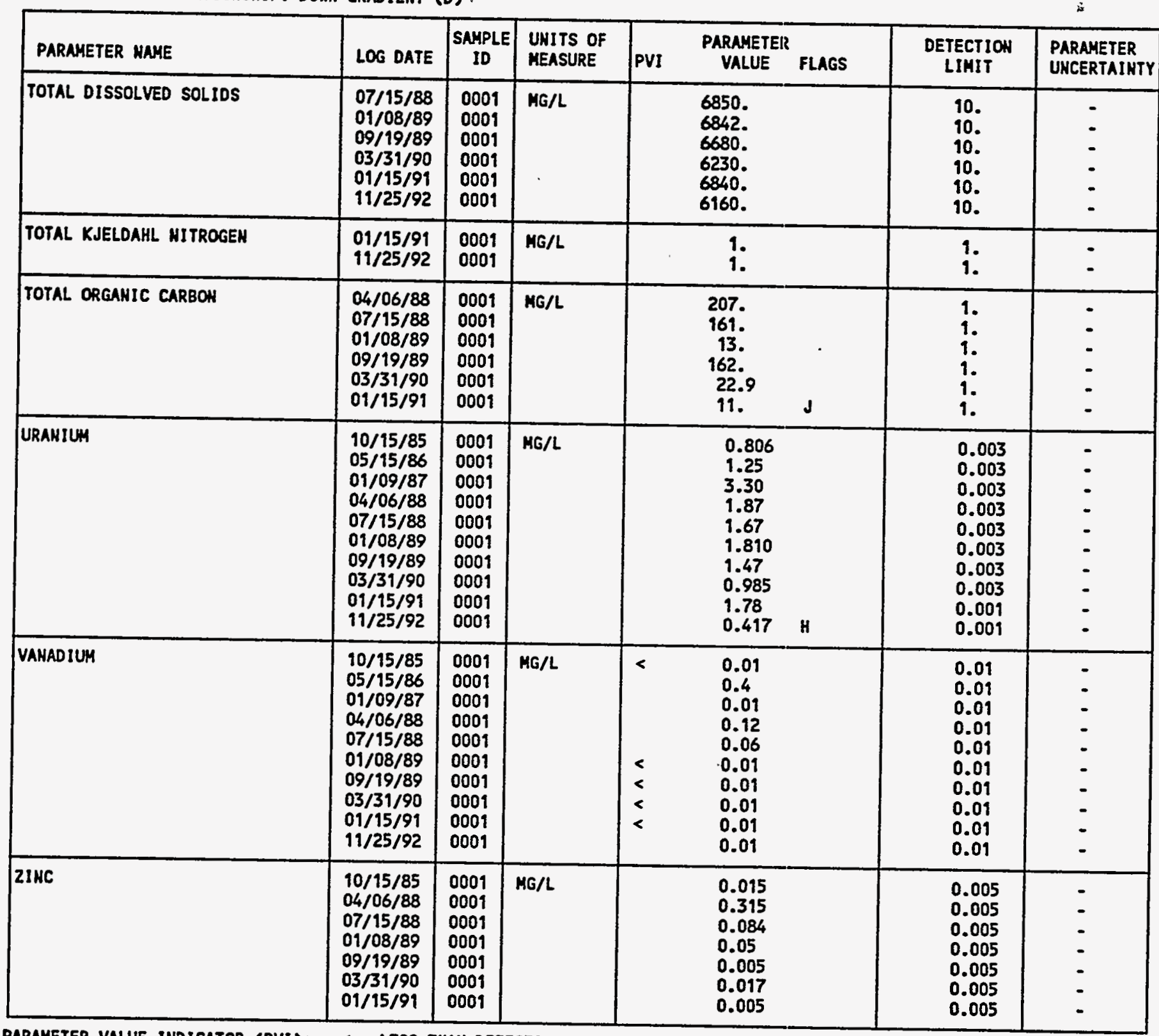

PARAMETER VALUE INDICATOR (PVI): < - LESS THAN DETECTION LIMIT SAMPLE ID CIODES:

0001 - FILTIERED SAMPLE (.45 MICRONS)

OTHER PARAMETER VALUE FLAGS:

H - HOLD TIME EXPIRED, VALUE SUSPECT

J - estimated VALUe 
GROUNDWATER QUALITY DATA BY LOCATION

SITE: AMBO1 AMBROSIA LAKE

LOCATION: 0786

HORTH COORDINATE: 52888.5 FT

EAST COORDINATE: $\quad 59760.9$ FT

$06 / 10 / 80$ TO $12 / 04 / 92$

REPORT DATE: $08 / 04 / 94$

FORMATION OF COMPLETION: TRES HERMANOS - CI SANDSTOHE (TO)

HYDRAULIC FLON RELATIONSHIP: DOWN GRADIENT (D)

\begin{tabular}{|c|c|c|c|c|c|c|c|c|}
\hline PARAMETER NAME & LOG DATE & $\begin{array}{c}\text { SAMPLE } \\
\text { ID }\end{array}$ & $\begin{array}{l}\text { UNITS OF } \\
\text { MEASURE }\end{array}$ & PVI & $\begin{array}{l}\text { PARAMETER } \\
\text { VALUE }\end{array}$ & FLAGS & $\begin{array}{l}\text { DETECTION } \\
\text { LIHIT }\end{array}$ & $\begin{array}{l}\text { PARAMETER } \\
\text { UNCERTAIHTY }\end{array}$ \\
\hline ALKALINITY & $\begin{array}{l}10 / 03 / 85 \\
10 / 03 / 85 \\
10 / 03 / 85 \\
10 / 03 / 85 \\
10 / 03 / 85 \\
05 / 14 / 86 \\
01 / 09 / 87 \\
07 / 15 / 88 \\
01 / 07 / 89 \\
09 / 19 / 89 \\
03 / 31 / 90 \\
01 / 16 / 91 \\
11 / 24 / 92\end{array}$ & $\begin{array}{l}0001 \\
0002 \\
0003 \\
0004 \\
0005 \\
0001 \\
0001 \\
0001 \\
0001 \\
0001 \\
0001 \\
0001 \\
0001\end{array}$ & MG/L CACO3 & & $\begin{array}{l}381 . \\
381 . \\
381 . \\
381 . \\
381 . \\
349 . \\
415 . \\
431 . \\
222 . \\
497 . \\
494 . \\
471 . \\
524\end{array}$ & & $\begin{array}{l}- \\
: \\
: \\
: \\
: \\
- \\
- \\
-\end{array}$ & $\begin{array}{l}- \\
: \\
- \\
: \\
- \\
- \\
- \\
- \\
-\end{array}$ \\
\hline ALUHINUM & $\begin{array}{l}10 / 03 / 85 \\
10 / 03 / 85 \\
10 / 03 / 85 \\
10 / 03 / 85 \\
10 / 03 / 85 \\
07 / 15 / 88 \\
01 / 07 / 89 \\
09 / 19 / 89 \\
03 / 31 / 90 \\
01 / 16 / 91\end{array}$ & $\begin{array}{l}0001 \\
0002 \\
0003 \\
0004 \\
0005 \\
0001 \\
0001 \\
0001 \\
0001 \\
0001\end{array}$ & $M G / L$ & $\begin{array}{l}< \\
< \\
<\end{array}$ & $\begin{array}{l}0.4 \\
0.3 \\
0.2 \\
0.2 \\
0.3 \\
0.2 \\
0.05 \\
0.1 \\
0.1 \\
0.05\end{array}$ & $J$ & $\begin{array}{l}0.1 \\
0.1 \\
0.1 \\
0.1 \\
0.1 \\
0.1 \\
0.1 \\
0.1 \\
0.1 \\
0.05\end{array}$ & $\begin{array}{l}: \\
: \\
: \\
: \\
- \\
- \\
-\end{array}$ \\
\hline AMKONIUH & $\begin{array}{l}10 / 03 / 85 \\
10 / 03 / 85 \\
10 / 03 / 85 \\
10 / 03 / 85 \\
10 / 03 / 85 \\
07 / 15 / 88 \\
01 / 07 / 89 \\
09 / 19 / 89 \\
03 / 31 / 90 \\
01 / 16 / 91 \\
11 / 24 / 92\end{array}$ & $\begin{array}{l}0001 \\
0002 \\
0003 \\
0004 \\
0005 \\
0001 \\
0001 \\
0001 \\
0001 \\
0001 \\
0001\end{array}$ & $\mathrm{MG} / \mathrm{L}$ & $\begin{array}{l}< \\
< \\
< \\
< \\
< \\
< \\
<\end{array}$ & $\begin{array}{l}0.1 \\
0.1 \\
0.2 \\
0.1 \\
0.1 \\
0.1 \\
0.10 \\
0.1 \\
0.1 \\
0.12 \\
0.2\end{array}$ & & $\begin{array}{l}0.1 \\
0.1 \\
0.1 \\
0.1 \\
0.1 \\
0.1 \\
0.1 \\
0.1 \\
0.1 \\
0.01 \\
0.1\end{array}$ & $\begin{array}{l}- \\
- \\
- \\
- \\
- \\
- \\
-\end{array}$ \\
\hline ARSENIC & $\begin{array}{l}10 / 03 / 85 \\
10 / 03 / 85 \\
10 / 03 / 85 \\
10 / 03 / 85 \\
10 / 03 / 85 \\
05 / 14 / 86 \\
01 / 09 / 87\end{array}$ & $\begin{array}{l}0001 \\
0002 \\
0003 \\
0004 \\
0005 \\
0001 \\
0001\end{array}$ & MG/L & $\begin{array}{l}< \\
< \\
< \\
< \\
< \\
<\end{array}$ & $\begin{array}{l}0.01 \\
0.01 \\
0.01 \\
0.01 \\
0.01 \\
0.01 \\
0.002\end{array}$ & J & $\begin{array}{l}0.01 \\
0.01 \\
0.01 \\
0.01 \\
0.01 \\
0.01 \\
0.01\end{array}$ & $\begin{array}{l}- \\
- \\
- \\
- \\
-\end{array}$ \\
\hline
\end{tabular}

PARAMETER VALUE IMDICATOR (PVI): < - LESS THAN DETECTION LIMIT

SAMPLE ID COOES:

OTHER PARAMETER VALUE FLAGS:

0001 - FILTERED SAMPLE (.45 MICRONS)

J- ESTIMATED VALUE

0002 - FILTERED REPLICATE SAMPLE (. 45 MICRONS)

0003 - FILTERED REPLICATE SAMPLE (.45 HICRONS)

0004 - FILTERED REPLICATE SAMPLE (.45 MICROHS) 
GROUHDHATER OUALITY DATA BY LOCATION

SITE: AMBOI AMBROSIA LAKE

LOCATION: 0786

NORTH COORDIHATE: $\quad 52888.5$ FT

EAST COORDINATE: $\quad 59760.9$ FT

06/10/80 TO $12 / 04 / 92$

REPORT DATE: $08 / 04 / 94$

FORMATION OF COMPLETIOH: TRES HERMANOS - CI SAMDSTONE (TO) HYDRAULIC FLOW RELATIOHSHIP: DOWN GRADIENT (D)

\begin{tabular}{|c|c|c|c|c|c|c|c|c|}
\hline $\begin{array}{l}1 \\
\text { PARAMETER HAME }\end{array}$ & LOG DATE & $\begin{array}{c}\text { SAMPLE } \\
\text { ID }\end{array}$ & $\begin{array}{l}\text { UNITS OF } \\
\text { MEASURE }\end{array}$ & PVI & $\begin{array}{l}\text { PARAMETEIl } \\
\text { VALUE }\end{array}$ & FLAGS & $\begin{array}{l}\text { DETECTION } \\
\text { LIMIT }\end{array}$ & $\begin{array}{l}\text { PARAMETER } \\
\text { UKCERTAIKTY }\end{array}$ \\
\hline ARSEHIC & $\begin{array}{l}07 / 15 / 88 \\
01 / 07 / 89 \\
09 / 19 / 89 \\
03 / 31 / 90 \\
01 / 16 / 91\end{array}$ & $\begin{array}{l}0001 \\
0001 \\
0001 \\
0001 \\
0001\end{array}$ & $M G / L$ & $\begin{array}{l}< \\
<\end{array}$ & $\begin{array}{l}0.01 \\
0.001 \\
0.02 \\
0.01 \\
0.03\end{array}$ & $\begin{array}{l}J \\
I\end{array}$ & $\begin{array}{l}0.01 \\
0.01 \\
0.01 \\
0.01 \\
0.03\end{array}$ & $\begin{array}{r}- \\
- \\
- \\
-\end{array}$ \\
\hline BARIU:M & $\begin{array}{l}10 / 03 / 85 \\
10 / 03 / 85 \\
10 / 03 / 85 \\
10 / 03 / 85 \\
10 / 03 / 85 \\
07 / 15 / 88 \\
01 / 07 / 89 \\
09 / 19 / 89 \\
03 / 31 / 90 \\
01 / 16 / 91\end{array}$ & $\begin{array}{l}0001 \\
0002 \\
0003 \\
0004 \\
0005 \\
0001 \\
0001 \\
0001 \\
0001 \\
0001\end{array}$ & $M G / L$ & $\begin{array}{l}< \\
< \\
< \\
<\end{array}$ & $\begin{array}{l}0.2 \\
0.2 \\
0.3 \\
0.2 \\
0.2 \\
0.1 \\
0.01 \\
0.9 \\
0.1 \\
0.01\end{array}$ & $J$ & $\begin{array}{l}0.1 \\
0.1 \\
0.1 \\
0.1 \\
0.1 \\
0.1 \\
0.1 \\
0.1 \\
0.1 \\
0.01\end{array}$ & $\begin{array}{l}- \\
- \\
- \\
- \\
- \\
- \\
-\end{array}$ \\
\hline BERYLLIUY & $\begin{array}{l}01 / 07 / 89 \\
09 / 19 / 89 \\
03 / 31 / 90 \\
01 / 16 / 91\end{array}$ & $\begin{array}{l}0001 \\
0001 \\
0001 \\
0001\end{array}$ & MG/L & $\begin{array}{l}< \\
< \\
< \\
<\end{array}$ & $\begin{array}{l}0.005 \\
0.01 \\
0.09 \\
0.005\end{array}$ & & $\begin{array}{l}0.005 \\
0.01 \\
0.01 \\
0.005\end{array}$ & - \\
\hline BORON & $\begin{array}{l}10 / 03 / 85 \\
10 / 03 / 85 \\
10 / 03 / 85 \\
10 / 03 / 85 \\
10 / 03 / 85 \\
05 / 14 / 86 \\
01 / 09 / 87 \\
07 / 15 / 88 \\
01 / 07 / 89 \\
09 / 19 / 89 \\
03 / 31 / 90 \\
01 / 16 / 91\end{array}$ & $\begin{array}{l}0001 \\
0002 \\
0003 \\
0004 \\
0005 \\
0001 \\
0001 \\
0001 \\
0001 \\
0001 \\
0001 \\
0001\end{array}$ & HG/L & & $\begin{array}{l}1.3 \\
1.3 \\
1.3 \\
1.4 \\
1.3 \\
1.4 \\
1.10 \\
1.1 \\
1.22 \\
1.1 \\
1.2 \\
0.88\end{array}$ & & $\begin{array}{l}0.1 \\
0.1 \\
0.1 \\
0.1 \\
0.1 \\
0.1 \\
0.1 \\
0.1 \\
0.1 \\
0.1 \\
0.1 \\
0.05\end{array}$ & $\begin{array}{l}- \\
- \\
- \\
- \\
- \\
- \\
- \\
-\end{array}$ \\
\hline BROAIDE & $\begin{array}{l}07 / 15 / 88 \\
01 / 07 / 89 \\
09 / 19 / 89 \\
03 / 31 / 90 \\
01 / 16 / 91\end{array}$ & $\begin{array}{l}0001 \\
0001 \\
0001 \\
0001 \\
0001\end{array}$ & $M G / L$ & & $\begin{array}{l}0.9 \\
1.43 \\
0.31 \\
1.0 \\
1.9\end{array}$ & & $\begin{array}{l}0.1 \\
0.01 \\
0.1 \\
0.1 \\
0.1\end{array}$ & $\begin{array}{l}- \\
- \\
- \\
-\end{array}$ \\
\hline CADMIUM & $\begin{array}{l}10 / 03 / 85 \\
10 / 03 / 85 \\
10 / 03 / 85 \\
10 / 03 / 85 \\
10 / 03 / 85 \\
05 / 14 / 86 \\
07 / 15 / 88 \\
01 / 07 / 89 \\
09 / 19 / 89 \\
03 / 31 / 90 \\
01 / 16 / 91 \\
11 / 24 / 92\end{array}$ & $\begin{array}{l}0001 \\
0002 \\
0003 \\
0004 \\
0005 \\
0001 \\
0001 \\
0001 \\
0001 \\
0001 \\
0001 \\
0001\end{array}$ & $M G / L$ & $\begin{array}{l}< \\
< \\
< \\
< \\
< \\
< \\
< \\
< \\
<\end{array}$ & $\begin{array}{l}0.001 \\
0.001 \\
0.003 \\
0.001 \\
0.001 \\
0.001 \\
0.001 \\
0.0001 \\
0.013 \\
0.001 \\
0.001 \\
0.0001\end{array}$ & H & $\begin{array}{l}0.001 \\
0.001 \\
0.001 \\
0.001 \\
0.001 \\
0.001 \\
0.001 \\
0.001 \\
0.001 \\
0.001 \\
0.001 \\
0.0001\end{array}$ & $\begin{array}{l}- \\
- \\
- \\
: \\
- \\
- \\
- \\
-\end{array}$ \\
\hline CALCIUA & $10 / 03 / 85$ & 0001 & $M G / L$ & & 404. & & 0.09 & - \\
\hline
\end{tabular}

PARAMETER VALUE IHDICATOR (PVI): < - LESS THAH DETECTION LIMIT

OTHER PARAMETER VALUE FLAGS:

H - HOLD TIME EXPIRED, VALUE SUSPECT

1 - INCREASED DETECTION LIMIT DUE TO REQUIRED DILUTIOH

J - estimated VAlue
SAMPLE ID COOES:

0001 - FILTERED SAMPLE (.45 MICRONS)

0002 - FILTERED REPLICATE SAMPLE (.45 MICRONS) 0003 - FILTERED REPLICATE SAMPLE (.45 MICROHS) 0004 - FILTERED REPLICATE SAMPLE (.45 MICRONS) 
GROUNDWATER QUALITY DATA BY LOCATIOH

SITE: AMBOI AMBROSIA LAKE

LOCATION: 0786

MORTH COORDINATE: $\quad 52888.5$ FT

EAST COORDINATE: 59760.9 FT

06/10/80 TO 12/04/92

REPORT DATE: 08/04/94

FORHATION OF COMPLETION: TRES HERMANOS - C1 SANDSTONE (TO) HYDRAULIC FLOW RELATIONSHIP: DOWH GRADIENT (D)

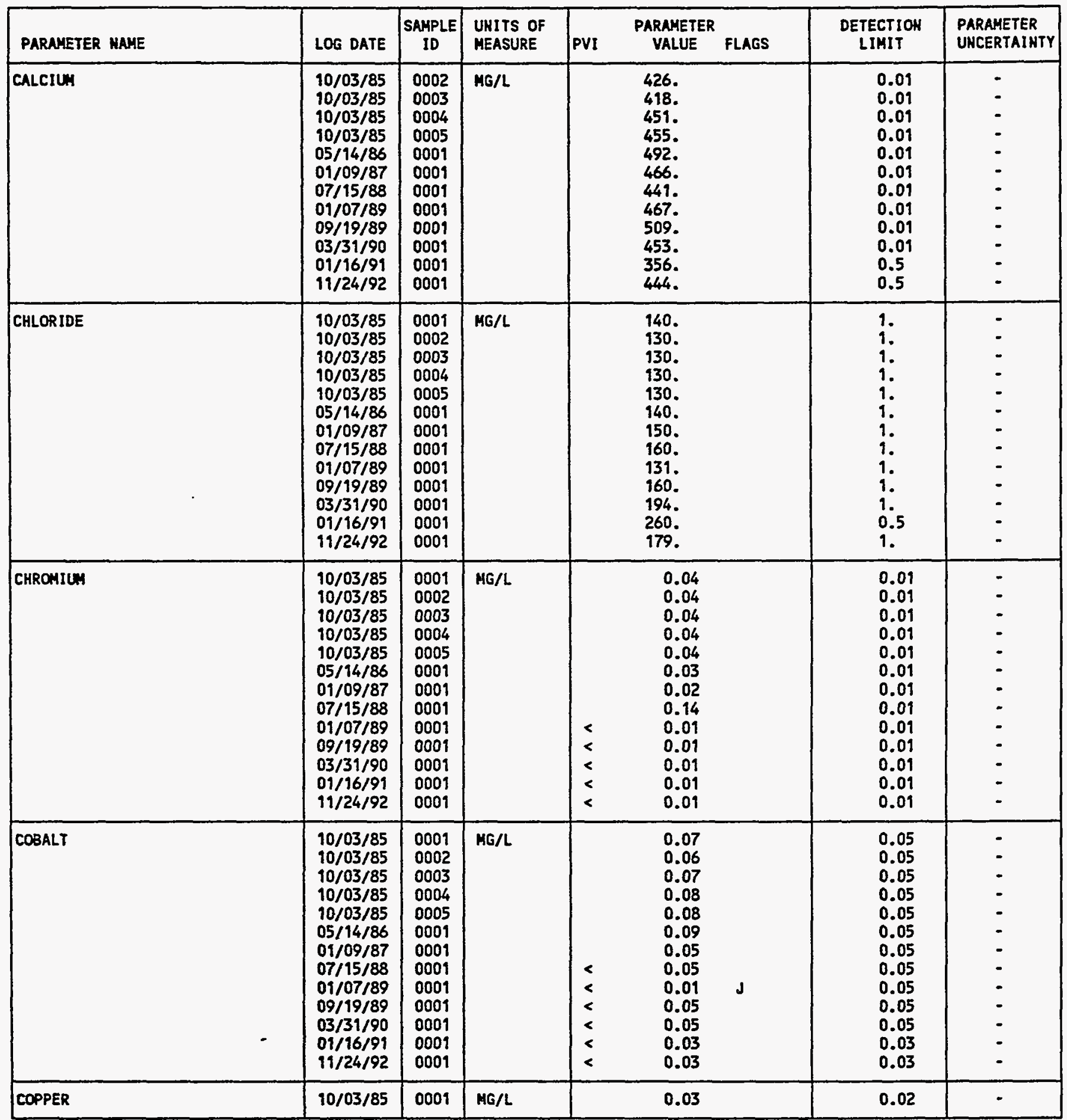

PARAMETER VALUE INDICATOR (PVI): < - LESS THAM DETECTION LIMIT

SAMPLE ID COOES:

OTHER PARAMETER VALUE FLAGS:

0001 - FILTERED SAMPLE (.45 MICROHS)

d - estimated VALUE

OOD2 - FILTERED REPLICATE SAMPLE (.45 MICRONS)

0003 - FILTERED REPLICATE SAMPLE (.45 MICRONS)

0004 - FILTERED REPLICATE SAMPLE (.45 MICROHS) 
GROUNDHATER QUALITY DATA BY LOCATION

SITE: AMBO1 AMBROSIA LAKE

LOCATION: 0786

MORTH COORDINATE: 52888.5 FT

EAST COORDINATE: $\quad 59760.9$ FT

06/10/80 TO $12 / 04 / 92$

REPORT DATE: $08 / 04 / 94$

FORMATION OF COMPLETION: TRES HERMANOS - CI SANDSTONE (TO) HYDRAULIC FLOW RELATIOHSHIP: DOWN GRADIENT (D)

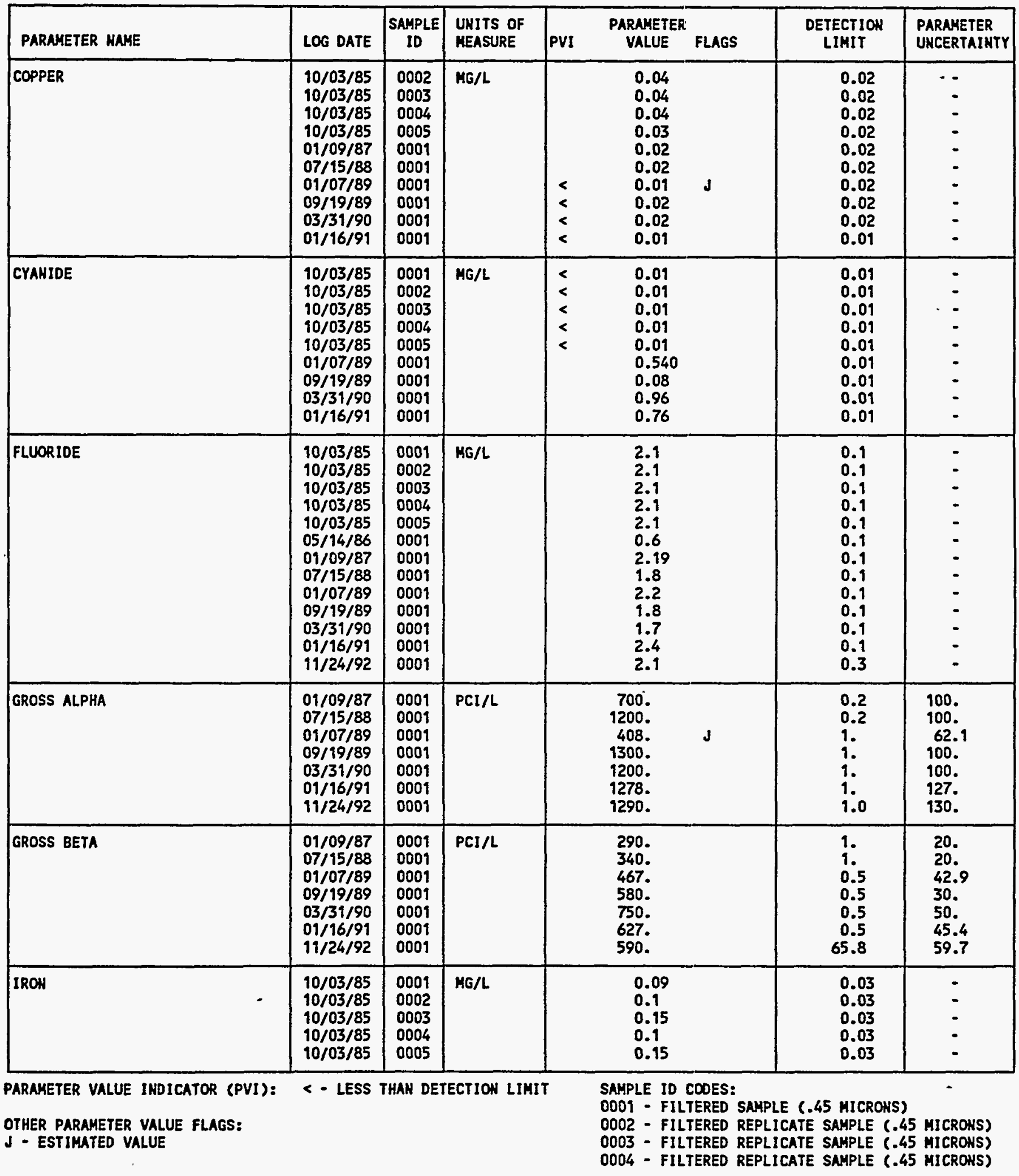


GROUNDWATER OUALITY DATA BY LOCATION

SITE: AMBOI AMBROSIA LAKE

LOCATION: 0786

NORTH COORDINATE: 52888.5 FT

EAST COORDINATE: 59760.9 FT

06/10/80 TO $12 / 04 / 92$

REPORT DATE: 08/04/94

FORMATION OF COMPLETION: TRES HERMANOS - C1 SAMDSTOHE (TO)

HYDRAULIC FLON RELATIONSHIP: DOWN GRADIENT (D)

\begin{tabular}{|c|c|c|c|c|c|c|c|}
\hline PARAMETER MAME & LOG DATE & $\begin{array}{c}\text { SAMPLE } \\
\text { ID }\end{array}$ & $\begin{array}{l}\text { UNITS OF } \\
\text { MEASURE }\end{array}$ & PVI & $\begin{array}{l}\text { PARAMETER } \\
\text { VALUE FLAGS }\end{array}$ & $\begin{array}{l}\text { DETECTION } \\
\text { LIMIT }\end{array}$ & $\begin{array}{l}\text { PARAKETER } \\
\text { UHCERTAINTY }\end{array}$ \\
\hline IRON & $\begin{array}{l}05 / 14 / 86 \\
01 / 09 / 87 \\
07 / 15 / 88 \\
01 / 07 / 89 \\
09 / 19 / 89 \\
03 / 31 / 90 \\
01 / 16 / 91 \\
11 / 24 / 92\end{array}$ & $\begin{array}{l}0001 \\
0001 \\
0001 \\
0001 \\
0001 \\
0001 \\
0001 \\
0001\end{array}$ & $M G / L$ & $<$ & $\begin{array}{l}0.18 \\
0.46 \\
0.30 \\
0.23 \\
0.38 \\
0.40 \\
0.03 \\
0.24\end{array}$ & $\begin{array}{l}0.03 \\
0.03 \\
0.03 \\
0.03 \\
0.03 \\
0.03 \\
0.03 \\
0.03\end{array}$ & $\begin{array}{l}- \\
- \\
- \\
- \\
-\end{array}$ \\
\hline LEAD & $\begin{array}{l}10 / 03 / 85 \\
10 / 03 / 85 \\
10 / 03 / 85 \\
10 / 03 / 85 \\
10 / 03 / 85 \\
07 / 15 / 88 \\
01 / 07 / 89 \\
09 / 19 / 89 \\
03 / 31 / 90 \\
01 / 16 / 91\end{array}$ & $\begin{array}{l}0001 \\
0002 \\
0003 \\
0004 \\
0005 \\
0001 \\
0001 \\
0001 \\
0001 \\
0001\end{array}$ & MG/L & $\begin{array}{l}< \\
< \\
< \\
< \\
< \\
< \\
< \\
<\end{array}$ & $\begin{array}{l}0.01 \\
0.01 \\
0.01 \\
0.01 \\
0.01 \\
0.01 \\
0.006 \\
0.02 \\
0.01 \\
0.03\end{array}$ & $\begin{array}{l}0.01 \\
0.01 \\
0.01 \\
0.01 \\
0.01 \\
0.01 \\
0.01 \\
0.01 \\
0.01 \\
0.03\end{array}$ & $\begin{array}{l}- \\
- \\
- \\
- \\
- \\
- \\
-\end{array}$ \\
\hline LEAD-210 & $\begin{array}{l}10 / 03 / 85 \\
10 / 03 / 85 \\
10 / 03 / 85 \\
10 / 03 / 85 \\
10 / 03 / 85 \\
07 / 15 / 88 \\
01 / 07 / 89 \\
09 / 19 / 89\end{array}$ & $\begin{array}{l}0001 \\
0002 \\
0003 \\
0004 \\
0005 \\
0001 \\
0001 \\
0001\end{array}$ & $\mathrm{PCI} / \mathrm{L}$ & & $\begin{array}{l}7.1 \\
8.3 \\
8.8 \\
8.2 \\
6.2 \\
0.3 \\
5.33 \\
0.4\end{array}$ & $\begin{array}{l}1.5 \\
1.5 \\
1.5 \\
1.5 \\
1.5 \\
1.5 \\
1.5 \\
1.5\end{array}$ & $\begin{array}{l}1.6 \\
1.7 \\
1.5 \\
1.5 \\
1.4 \\
1.0 \\
4.30 \\
0.7\end{array}$ \\
\hline MAGHESIUM & $\begin{array}{l}10 / 03 / 85 \\
10 / 03 / 85 \\
10 / 03 / 85 \\
10 / 03 / 85 \\
10 / 03 / 85 \\
05 / 14 / 86 \\
01 / 09 / 87 \\
07 / 15 / 88 \\
01 / 07 / 89 \\
09 / 19 / 89 \\
03 / 31 / 90 \\
01 / 16 / 91 \\
11 / 24 / 92\end{array}$ & $\begin{array}{l}0001 \\
0002 \\
0003 \\
0004 \\
0005 \\
0001 \\
0001 \\
0001 \\
0001 \\
0001 \\
0001 \\
0001 \\
0001\end{array}$ & MG/L & & $\begin{array}{l}279 . \\
278 . \\
280 . \\
283 . \\
286 . \\
274 . \\
291 . \\
270 . \\
320 . \\
296 . \\
284 . \\
214 . \\
278 .\end{array}$ & $\begin{array}{l}0.001 \\
0.001 \\
0.001 \\
0.001 \\
0.001 \\
0.001 \\
0.001 \\
0.001 \\
0.001 \\
0.001 \\
0.001 \\
0.5 \\
0.5\end{array}$ & $\begin{array}{l}- \\
: \\
: \\
: \\
- \\
- \\
- \\
- \\
-\end{array}$ \\
\hline MAKGANESE & $\begin{array}{l}10 / 03 / 85 \\
10 / 03 / 85 \\
10 / 03 / 85 \\
10 / 03 / 85 \\
10 / 03 / 85 \\
05 / 14 / 86 \\
01 / 09 / 87 \\
07 / 15 / 88 \\
01 / 07 / 89 \\
09 / 19 / 89 \\
03 / 31 / 90 \\
01 / 16 / 91\end{array}$ & $\begin{array}{l}0001 \\
0002 \\
0003 \\
0004 \\
0005 \\
0001 \\
0001 \\
0001 \\
0001 \\
0001 \\
0001 \\
0001\end{array}$ & $M G / L$ & & $\begin{array}{l}0.05 \\
0.06 \\
0.06 \\
0.06 \\
0.05 \\
0.07 \\
0.05 \\
0.06 \\
0.03 \\
0.04 \\
0.04 \\
0.02\end{array}$ & $\begin{array}{l}0.01 \\
0.01 \\
0.09 \\
0.01 \\
0.09 \\
0.01 \\
0.01 \\
0.01 \\
0.01 \\
0.09 \\
0.09 \\
0.01\end{array}$ & $\begin{array}{l}- \\
- \\
= \\
= \\
= \\
- \\
- \\
-\end{array}$ \\
\hline MERCURY & $10 / 03 / 85$ & 0001 & MG/L & $<$ & 0.0002 & 0.0002 & - \\
\hline $\begin{array}{l}\text { PARAMETER VALUE } \\
\text { OTHER PARAMETER } \\
\text { I - IMCREASED DE } \\
\text { J - ESTIMATED VAl }\end{array}$ & \multicolumn{4}{|c|}{ < - LESS THAN DETECTION LIMIT } & \multicolumn{3}{|l|}{$\begin{array}{l}\text { SAMPLE ID COOES: } \\
0001 \text { - FILTERED } \\
0002 \text { - FILTERED } \\
0003 \text { - FILTERED } \\
0004 \text { - FILTERED }\end{array}$} \\
\hline
\end{tabular}


GROUNDHATER QUALITY DATA BY LOCATION

SITE: AMBO1 AMBROSIA LAKE

LOCATIOH: 0786

NORTH COORDINATE: 52888.5 FT

EAST COORDINATE: $\quad 59760.9$ FT

06/10/80 TO $12 / 04 / 92$

REPORT DATE: $08 / 04 / 94$

FORMATION OF COMPLETIOH: TRES HERMANOS - C1 SAKDSTOHE (TO) HYDRAULIC FLON RELATIOHSHIP: DONH GRADIENT (D)

\begin{tabular}{|c|c|c|c|c|c|c|c|c|c|}
\hline PARAMETER MAME & & LOG DATE & $\begin{array}{c}\text { SAMPLE } \\
\text { ID }\end{array}$ & $\begin{array}{l}\text { UNITS OF } \\
\text { MEASURE }\end{array}$ & PVI & $\begin{array}{l}\text { PARAMETER } \\
\text { VALUE }\end{array}$ & FLAGS & $\begin{array}{l}\text { DETECTION } \\
\text { LIHIT }\end{array}$ & $\begin{array}{l}\text { PARAMETER } \\
\text { UHCERTAINTY }\end{array}$ \\
\hline MERCURY & & $\begin{array}{l}10 / 03 / 85 \\
10 / 03 / 85 \\
10 / 03 / 85 \\
10 / 03 / 85 \\
07 / 15 / 88 \\
01 / 07 / 89 \\
09 / 19 / 89 \\
03 / 31 / 90 \\
01 / 16 / 91\end{array}$ & $\begin{array}{l}0002 \\
0003 \\
0004 \\
0005 \\
0001 \\
0001 \\
0001 \\
0001 \\
0001\end{array}$ & HG/L & $\begin{array}{l}< \\
< \\
< \\
< \\
< \\
< \\
<\end{array}$ & $\begin{array}{l}0.0002 \\
0.0002 \\
0.0002 \\
0.0002 \\
0.0003 \\
0.0001 \\
0.0002 \\
0.0002 \\
0.0002\end{array}$ & J & $\begin{array}{l}0.0002 \\
0.0002 \\
0.0002 \\
0.0002 \\
0.0002 \\
0.0002 \\
0.0002 \\
0.0002 \\
0.0002\end{array}$ & $\begin{array}{l}= \\
- \\
= \\
= \\
= \\
=\end{array}$ \\
\hline MOLYBDENUM & & $\begin{array}{l}10 / 03 / 85 \\
10 / 03 / 85 \\
10 / 03 / 85 \\
10 / 03 / 85 \\
10 / 03 / 85 \\
05 / 14 / 86 \\
01 / 09 / 87 \\
07 / 15 / 88 \\
01 / 07 / 89 \\
09 / 19 / 89 \\
03 / 31 / 90 \\
01 / 16 / 91 \\
11 / 24 / 92\end{array}$ & $\begin{array}{l}0001 \\
0002 \\
0003 \\
0004 \\
0005 \\
0001 \\
0001 \\
0001 \\
0001 \\
0001 \\
0001 \\
0001 \\
0001\end{array}$ & MG/L & $<$ & $\begin{array}{l}0.2 \\
0.34 \\
0.22 \\
0.1 \\
0.2 \\
0.16 \\
0.1 \\
0.14 \\
0.070 \\
0.15 \\
0.17 \\
0.10 \\
0.18\end{array}$ & - & $\begin{array}{l}0.01 \\
0.01 \\
0.01 \\
0.01 \\
0.01 \\
0.01 \\
0.1 \\
0.01 \\
0.01 \\
0.01 \\
0.01 \\
0.01 \\
0.01\end{array}$ & $\begin{array}{l}- \\
- \\
- \\
- \\
- \\
- \\
- \\
- \\
-\end{array}$ \\
\hline NET GROSS ALPHA * & & $\begin{array}{l}01 / 09 / 87 \\
07 / 15 / 88 \\
01 / 07 / 89 \\
09 / 19 / 89 \\
03 / 31 / 90 \\
01 / 16 / 91 \\
11 / 24 / 92\end{array}$ & $\begin{array}{l}0001 \\
0001 \\
0001 \\
0001 \\
0001 \\
0001 \\
0001\end{array}$ & $\mathrm{PCI} / \mathrm{L}$ & & $\begin{array}{r}-109.48 \\
225.88 \\
-518.10 \\
456.22 \\
-89.68 \\
-697.68 \\
-431.86\end{array}$ & & $\begin{array}{l}- \\
- \\
- \\
- \\
-\end{array}$ & $\begin{array}{l}- \\
- \\
- \\
- \\
-\end{array}$ \\
\hline HICKEL & & $\begin{array}{l}10 / 03 / 85 \\
10 / 03 / 85 \\
10 / 03 / 85 \\
10 / 03 / 85 \\
10 / 03 / 85 \\
07 / 15 / 88 \\
01 / 07 / 89 \\
09 / 19 / 89 \\
03 / 31 / 90 \\
01 / 16 / 91 \\
11 / 24 / 92\end{array}$ & $\begin{array}{l}0001 \\
0002 \\
0003 \\
0004 \\
0005 \\
0001 \\
0001 \\
0001 \\
0001 \\
0001 \\
0001\end{array}$ & MG/L & $\begin{array}{l}< \\
< \\
< \\
< \\
<\end{array}$ & $\begin{array}{l}0.1 \\
0.1 \\
0.09 \\
0.09 \\
0.04 \\
0.08 \\
0.02 \\
0.04 \\
0.04 \\
0.04 \\
0.13\end{array}$ & J & $\begin{array}{l}0.04 \\
0.04 \\
0.04 \\
0.04 \\
0.04 \\
0.04 \\
0.04 \\
0.04 \\
0.04 \\
0.04 \\
0.04\end{array}$ & $\begin{array}{l}- \\
- \\
- \\
- \\
- \\
- \\
-\end{array}$ \\
\hline MITRATE & - & $\begin{array}{l}10 / 03 / 85 \\
10 / 03 / 85 \\
10 / 03 / 85 \\
10 / 03 / 85 \\
10 / 03 / 85 \\
05 / 14 / 86 \\
01 / 09 / 87 \\
07 / 15 / 88 \\
01 / 07 / 89 \\
09 / 19 / 89\end{array}$ & $\begin{array}{l}0001 \\
0002 \\
0003 \\
0004 \\
0005 \\
0001 \\
0001 \\
0001 \\
0001 \\
0001\end{array}$ & MG/L & $<$ & $\begin{array}{l}45 . \\
55 . \\
55 . \\
55 . \\
55 . \\
1 . \\
49 . \\
9.2 \\
24.3 \\
7.5\end{array}$ & & $\begin{array}{l}1 . \\
1 . \\
1 . \\
1 . \\
1 . \\
1 . \\
1 . \\
1 .\end{array}$ & $\begin{array}{l}- \\
: \\
- \\
- \\
- \\
- \\
-\end{array}$ \\
\hline
\end{tabular}

- het gross alPHa (GROSS ALPHA - URANIUH) WITH 1 HG URANIUH = 686 PCI

PARAMETER VALUE INDICATOR (PVI): < - LESS THAN DETECTION LIMIT

OTHER PARAMETER VALUE FLAGS:

$J$ - ESTIMATED VALUE
SAMPLE ID COOES:

0001 - FILTERED SAMPLE (.45 MICRONS)

0002 - FILTERED REPLICATE SAMPLE (.45 MICRONS)

0003 - FILTERED REPLICATE SAMPLE (.45 MICROHS)

0004 - FILTERED REPLICATE SAMPLE (.45 MICROHS) 
GROUNDWATER OUALITY DATA BY LOCATION

SITE: AMBOI AMBROSIA LAKE

LOCATION: 0786

NORTH COORDINATE: 52888.5 FT

EAST COORDINATE: $\quad 59760.9$ FT

06/10/80 TO $12 / 04 / 92$

REPORT DATE: $08 / 04 / 94$

FORMATION OF COMPLETION: TRES HERMANOS - CI SAHDSTOHE (TO)

HYDRAULIC FLOW RELATIONSHIP: DOWN GRADIEHT (D)

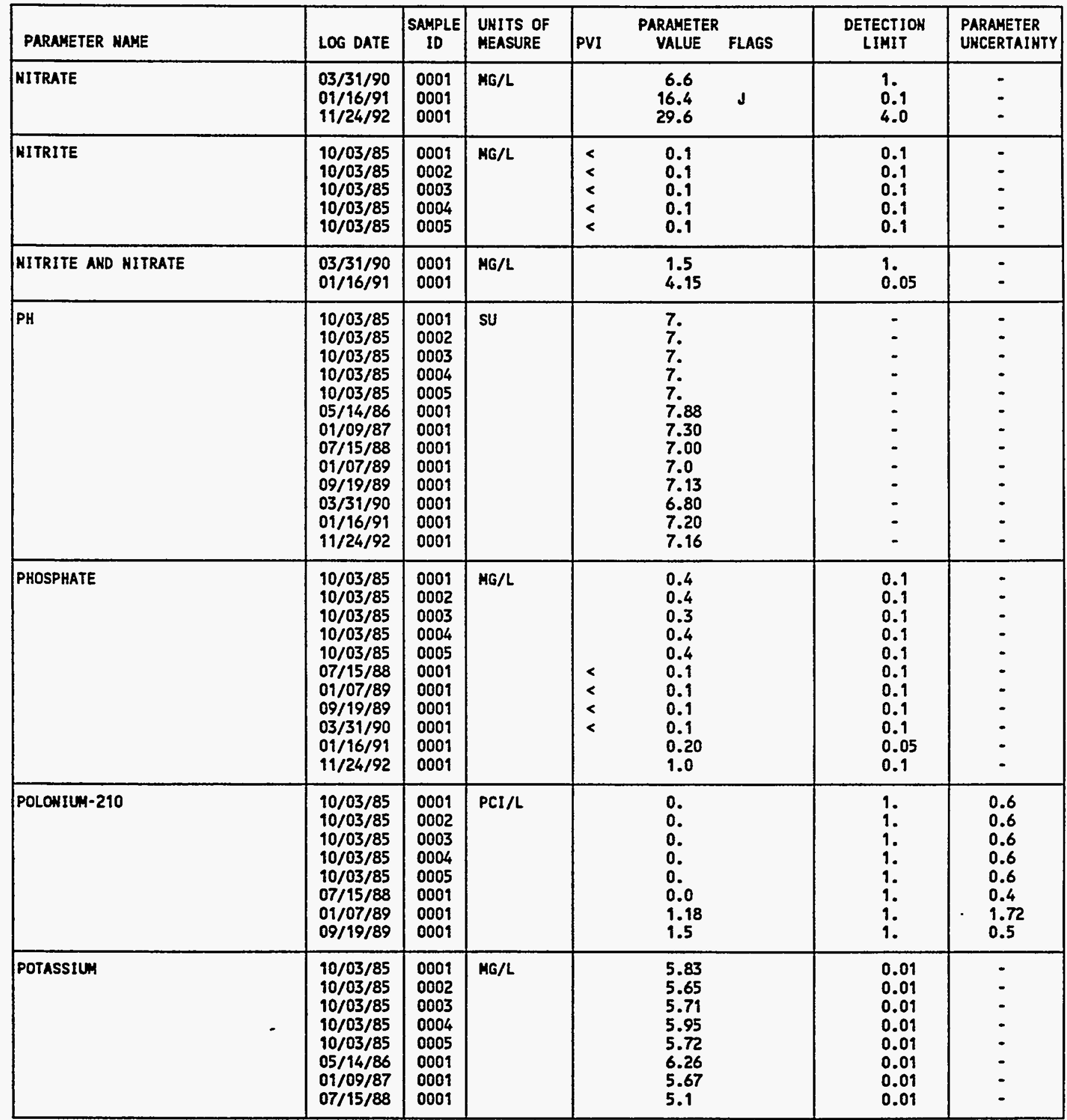

PARAMETER VALUE INDICATOR (PVI): < - LESS THAN DETECTION LIMIT

SAMPLE ID COOES:

0001 - FILTERED SAMPLE (.45 HICROHS)

OTHER PARAKETER YALUE FLAGS:

0002 - FILTERED REPLICATE SAMPLE (.45 MICROWS)

O003 - FILTERED REPLICATE SAMPLE (.45 MICROHS)

J - ESTIMATED VALUE

0004 - FILTERED REPLICATE SAMPLE (.45 MICROHS) 
GROUHDWATER QUALITY DATA BY LOCATION

SITE: AMBO1 AMBROSIA LAKE

LOCATIOH: 0786

NORTH COORDIHATE: 52888.5 FT

EAST COORDIHATE: 59760.9 FT

06/10/80 TO 12/04/92

REPORT DATE: 08/04/94

FORHATION OF COMPLETIOH: TRES HERMANOS - C1 SAHDSTONE (TO)

HYDRAULIC FLON RELATIOHSHIP: DOUH GRADIENT (D) :

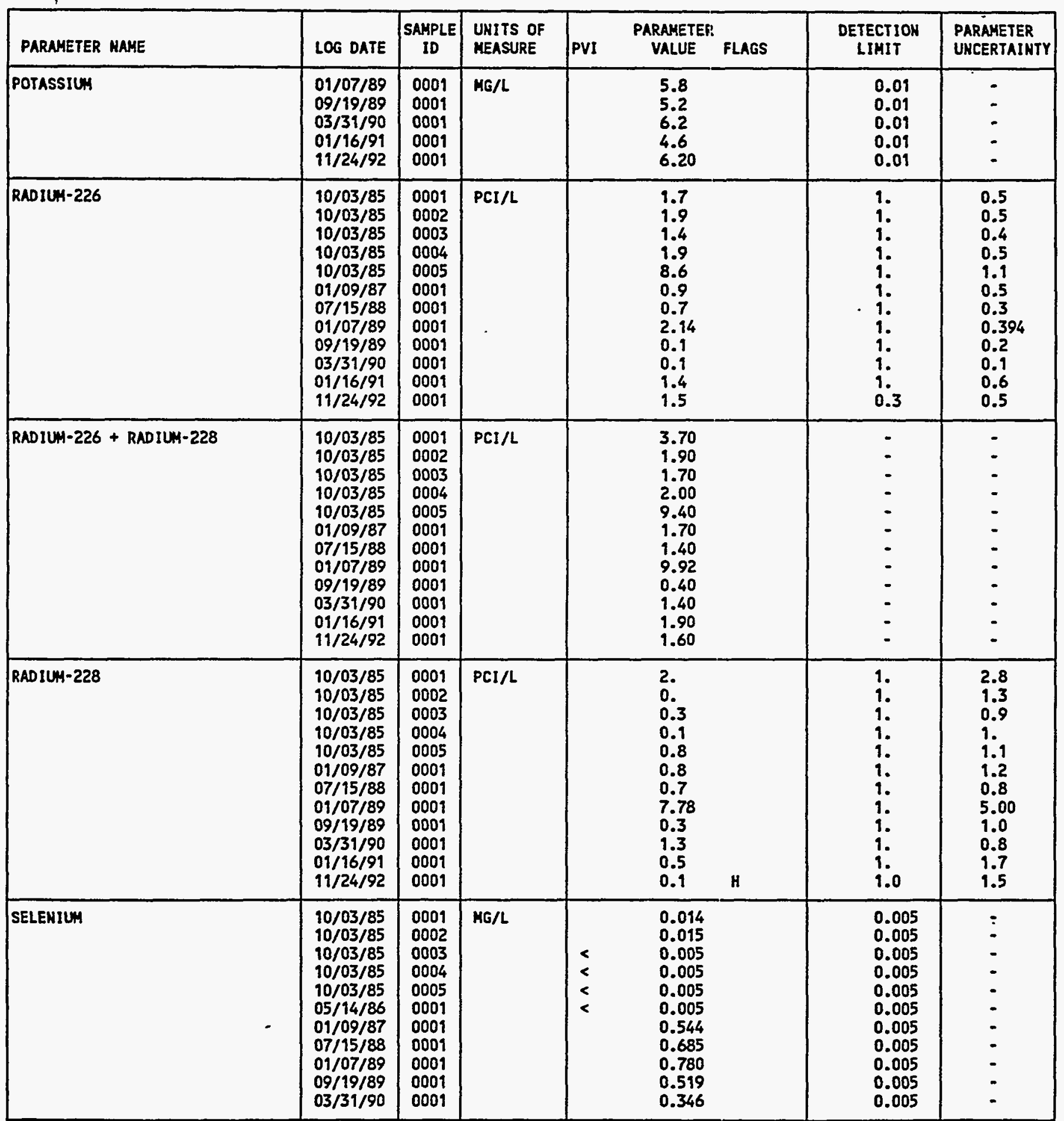

PARAMETER VALUE INDICATOR (PVI): < - LESS THAH DETECTION LIHIT SAMPLE ID CDOES:

OTHER PARAMETER VALUE FLAGS:

H - HOLD TIME EXPIRED, VALUE SUSPECT

0001 - FILTERED SAMPLE (.45 MICRONS)

DOO2 - FILTERED REPLICATE SAMPLE (.45 MICRONS)

0003 - FILTERED REPLICATE SAMPLE (.45 MICRONS)

0004 - FILTERED REPLICATE SAHPLE (.45 MICROHS) 
GROUNDWATER QUALITY DATA BY LOCATIOH

SITE: AMBO1 AMBROSIA LAKE

LOCATION: 0786

NORTH COORDINATE: 52888.5 FT

EAST COORDINATE: $\quad 59760.9$ FT

06/10/80 TO $12 / 04 / 92$

REPORT DATE: $08 / 04 / 94$

FORMATION OF COMPLETION: TRES HERMANOS - C1 SAMDSTOHE (TO) HYORAULIC FLON RELATIONSHIP: DOWN GRADIENT (D)

\begin{tabular}{|c|c|c|c|c|c|c|c|}
\hline PARAMETER MAME & LOG DATE & $\begin{array}{c}\text { SAMPLE } \\
\text { ID }\end{array}$ & $\begin{array}{l}\text { UNITS OF } \\
\text { MEASURE }\end{array}$ & PVI & $\begin{array}{l}\text { PARAMETER } \\
\text { VALUE FLAGS }\end{array}$ & $\begin{array}{l}\text { DETECTION } \\
\text { LIMIT }\end{array}$ & $\begin{array}{l}\text { PARAMETER } \\
\text { UHCERTAIHTY }\end{array}$ \\
\hline SELENILA & $\begin{array}{l}01 / 96 / 91 \\
11 / 24 / 92\end{array}$ & $\begin{array}{l}0001 \\
0001\end{array}$ & $H G / L$ & & $\begin{array}{l}0.420 \\
0.6\end{array}$ & $\begin{array}{l}0.005 \\
0.1\end{array}$ & - \\
\hline SILICA - SIO2 & $\begin{array}{l}10 / 03 / 85 \\
10 / 03 / 85 \\
10 / 03 / 85 \\
10 / 03 / 85 \\
10 / 03 / 85 \\
07 / 15 / 88 \\
01 / 07 / 89 \\
09 / 19 / 89 \\
03 / 31 / 90 \\
01 / 16 / 91\end{array}$ & $\begin{array}{l}0001 \\
0002 \\
0003 \\
0004 \\
0005 \\
0001 \\
0001 \\
0001 \\
0001 \\
0001\end{array}$ & MG/L & & $\begin{array}{l}11 . \\
10 . \\
11 . \\
13 . \\
12 . \\
12.3 \\
11.0 \\
7.5 \\
13 . \\
9.9\end{array}$ & $\begin{array}{l}2 . \\
2 . \\
2 . \\
2 . \\
2 . \\
2 . \\
2 . \\
2 . \\
2 . \\
0.1\end{array}$ & $\begin{array}{l}- \\
: \\
- \\
- \\
- \\
-\end{array}$ \\
\hline SILVER & $\begin{array}{l}10 / 03 / 85 \\
10 / 03 / 85 \\
10 / 03 / 85 \\
10 / 03 / 85 \\
10 / 03 / 85 \\
01 / 09 / 87 \\
07 / 15 / 88 \\
01 / 07 / 89 \\
09 / 19 / 89 \\
03 / 31 / 90 \\
01 / 16 / 91 \\
11 / 24 / 92\end{array}$ & $\begin{array}{l}0001 \\
0002 \\
0003 \\
0004 \\
0005 \\
0001 \\
0001 \\
0001 \\
0001 \\
0001 \\
0001 \\
0001\end{array}$ & $M G / L$ & $\begin{array}{l}< \\
< \\
< \\
< \\
< \\
< \\
< \\
< \\
< \\
<\end{array}$ & $\begin{array}{l}0.01 \\
0.01 \\
0.01 \\
0.01 \\
0.01 \\
0.01 \\
0.02 \\
0.01 \\
0.01 \\
0.01 \\
0.01 \\
0.01\end{array}$ & $\begin{array}{l}0.01 \\
0.01 \\
0.01 \\
0.01 \\
0.01 \\
0.01 \\
0.01 \\
0.01 \\
0.01 \\
0.01 \\
0.01 \\
0.01\end{array}$ & $\begin{array}{l}- \\
: \\
: \\
: \\
- \\
- \\
- \\
-\end{array}$ \\
\hline SOOILM & $\begin{array}{l}10 / 03 / 85 \\
10 / 03 / 85 \\
10 / 03 / 85 \\
10 / 03 / 85 \\
10 / 03 / 85 \\
05 / 14 / 86 \\
01 / 09 / 87 \\
07 / 15 / 88 \\
01 / 07 / 89 \\
09 / 19 / 89 \\
03 / 31 / 90 \\
01 / 16 / 91 \\
11 / 24 / 92\end{array}$ & $\begin{array}{l}0001 \\
0002 \\
0003 \\
0004 \\
0005 \\
0001 \\
0001 \\
0001 \\
0001 \\
0001 \\
0001 \\
0001 \\
0001\end{array}$ & $M G / L$ & & $\begin{array}{l}883 . \\
663 . \\
668 . \\
660 . \\
600 . \\
688 . \\
645 . \\
547 . \\
615 . \\
581 . \\
613 . \\
659 . \\
604 .\end{array}$ & $\begin{array}{l}0.002 \\
0.002 \\
0.002 \\
0.002 \\
0.002 \\
0.002 \\
0.002 \\
0.002 \\
0.002 \\
0.002 \\
0.002 \\
5 . \\
5 .\end{array}$ & $\begin{array}{l}- \\
: \\
: \\
- \\
- \\
- \\
- \\
-\end{array}$ \\
\hline SPECIFIC CONDUCTANCE & $\begin{array}{l}10 / 03 / 85 \\
10 / 03 / 85 \\
10 / 03 / 85 \\
10 / 03 / 85 \\
10 / 03 / 85 \\
05 / 14 / 86 \\
01 / 09 / 87 \\
07 / 15 / 88 \\
01 / 07 / 89 \\
09 / 19 / 89 \\
03 / 31 / 90 \\
01 / 16 / 91 \\
11 / 24 / 92\end{array}$ & $\begin{array}{l}0001 \\
0002 \\
0003 \\
0004 \\
0005 \\
0001 \\
0001 \\
0001 \\
0001 \\
0001 \\
0001 \\
0001 \\
0001\end{array}$ & ЧнНо/СК & & $\begin{array}{l}3600 . \\
3600 . \\
3600 . \\
3600 . \\
3600 . \\
2950 . \\
3400 . \\
3600 . \\
2550 . \\
1300 . \\
3620 . \\
3650 . \\
4080\end{array}$ & $\begin{array}{l}- \\
: \\
: \\
: \\
- \\
- \\
- \\
-\end{array}$ & $\begin{array}{l}- \\
i \\
- \\
\dot{-} \\
\dot{-} \\
- \\
- \\
-\end{array}$ \\
\hline STRONTIUA & $10 / 03 / 85$ & 0001 & HG/L & & 10. & 0.1 & - \\
\hline METER VALUE IHDICATOR (PVI): & \multicolumn{4}{|c|}{ < - LESS THAN DETECTION LIMIT } & $\begin{array}{l}\text { SAMPLE ID COOES: } \\
0001 \text { - FILTERED } \\
0002 \text { - FILTERED } \\
0003 \text { - FILTERED } \\
0004 \text { - FILTERED }\end{array}$ & $\begin{array}{l}\text { C.45 MICROH } \\
\text { TE SAMPLE } \\
\text { TE SAMPLE } \\
\text { TE SAMPLE }\end{array}$ & $\begin{array}{l}\text { HICRONS) } \\
\text { MICRONS) } \\
\text { HICRONS) }\end{array}$ \\
\hline
\end{tabular}


GROUMDHATER OUALITY DATA BY LOCATIOH

SITE: AMBO9 AMBROSIA LAKE

LOCATIOH: 0786

MORTH COORDINATE: 52888.5 FT

EAST COORDIHATE: 59760.9 FT

06/10/80 TO $12 / 04 / 92$

REPORT DATE: 08/04/94

FORMATIOH OF COHPLETION: TRES HERMANOS - CI SANDSTOHE (TO)

HYORAULIC FLOH RELATIONSHIP: DOWN GRADIEHT (D)

\begin{tabular}{|c|c|c|c|c|c|c|c|c|}
\hline PARAMETER NAHE & LOG DATE & $\mid \begin{array}{c}\text { SAMPLE } \\
\text { ID }\end{array}$ & $\begin{array}{l}\text { UNITS OF } \\
\text { MEASURE }\end{array}$ & PVI & $\begin{array}{l}\text { PARAMETER } \\
\text { VALUE }\end{array}$ & FLAGS & $\begin{array}{l}\text { DETECTION } \\
\text { LIMIT }\end{array}$ & $\begin{array}{l}\text { PARAMETER } \\
\text { UXCERTAINTY }\end{array}$ \\
\hline STRONTIUHS & $\begin{array}{l}10 / 03 / 85 \\
10 / 03 / 85 \\
10 / 03 / 85 \\
10 / 03 / 85 \\
05 / 14 / 86 \\
07 / 15 / 88 \\
01 / 07 / 89 \\
09 / 19 / 89 \\
03 / 31 / 90 \\
01 / 16 / 91\end{array}$ & $\begin{array}{l}0002 \\
0003 \\
0004 \\
0005 \\
0001 \\
0001 \\
0001 \\
0001 \\
0001 \\
0001\end{array}$ & MG/L & & $\begin{array}{c}10 . \\
11 . \\
11 . \\
11 . \\
9.0 \\
8.7 \\
11.0 \\
8.9 \\
9.93 \\
8.75\end{array}$ & & $\begin{array}{l}0.1 \\
0.1 \\
0.1 \\
0.1 \\
0.1 \\
0.1 \\
0.1 \\
0.1 \\
0.1 \\
0.01\end{array}$ & $\begin{array}{l}- \\
- \\
- \\
- \\
- \\
- \\
-\end{array}$ \\
\hline SULFATE & $\begin{array}{l}10 / 03 / 85 \\
10 / 03 / 85 \\
10 / 03 / 85 \\
10 / 03 / 85 \\
10 / 03 / 85 \\
05 / 14 / 86 \\
01 / 09 / 87 \\
07 / 15 / 88 \\
01 / 07 / 89 \\
09 / 19 / 89 \\
03 / 31 / 90 \\
01 / 16 / 91 \\
11 / 24 / 92\end{array}$ & $\begin{array}{l}0001 \\
0002 \\
0003 \\
0004 \\
0005 \\
0001 \\
0001 \\
0001 \\
0001 \\
0001 \\
0001 \\
0001 \\
0001\end{array}$ & $M G / L$ & & $\begin{array}{l}3010 . \\
3010 . \\
2960 . \\
3040^{\circ} \\
2930 . \\
3170 . \\
2900 . \\
2740 . \\
3235 . \\
2980 . \\
3130 . \\
3080 . \\
2790 .\end{array}$ & & $\begin{array}{l}0.1 \\
0.1 \\
0.1 \\
0.1 \\
0.1 \\
0.1 \\
0.1 \\
0.1 \\
0.1 \\
0.1 \\
0.1 \\
10 . \\
10 .\end{array}$ & $\begin{array}{l}- \\
: \\
- \\
- \\
- \\
- \\
- \\
-\end{array}$ \\
\hline SULF IDE & $\begin{array}{l}10 / 03 / 85 \\
10 / 03 / 85 \\
10 / 03 / 85 \\
10 / 03 / 85 \\
10 / 03 / 85 \\
07 / 15 / 88 \\
01 / 07 / 89 \\
09 / 19 / 89 \\
03 / 31 / 90 \\
01 / 16 / 91\end{array}$ & $\begin{array}{l}0009 \\
0002 \\
0003 \\
0004 \\
0005 \\
0001 \\
0001 \\
0001 \\
0001 \\
0001\end{array}$ & MG/L & $\begin{array}{l}< \\
< \\
< \\
< \\
< \\
< \\
< \\
< \\
<\end{array}$ & $\begin{array}{l}0.1 \\
0.1 \\
0.1 \\
0.1 \\
0.1 \\
0.1 \\
0.01 \\
0.1 \\
0.1 \\
0.1\end{array}$ & $\begin{array}{l}\text { J } \\
\text { J }\end{array}$ & $\begin{array}{l}0.1 \\
0.1 \\
0.1 \\
0.1 \\
0.1 \\
0.1 \\
0.1 \\
0.1 \\
0.1 \\
0.1\end{array}$ & $\begin{array}{l}- \\
: \\
: \\
: \\
- \\
-\end{array}$ \\
\hline TEMPERATURE & $\begin{array}{l}10 / 03 / 85 \\
10 / 03 / 85 \\
10 / 03 / 85 \\
10 / 03 / 85 \\
10 / 03 / 85 \\
05 / 14 / 86 \\
01 / 09 / 87 \\
07 / 15 / 88 \\
01 / 07 / 89 \\
09 / 19 / 89 \\
03 / 31 / 90 \\
01 / 16 / 91 \\
11 / 24 / 92\end{array}$ & $\begin{array}{l}0001 \\
0002 \\
0003 \\
0004 \\
0005 \\
0001 \\
0001 \\
0001 \\
0001 \\
0001 \\
0001 \\
0001 \\
0001\end{array}$ & C - DEGREE & & $\begin{array}{l}12 . \\
12 . \\
12 . \\
12 . \\
12 . \\
15 . \\
11 . \\
12.0 \\
12.0 \\
13.0 \\
12 . \\
11.5 \\
11.0\end{array}$ & & $\begin{array}{l}- \\
- \\
- \\
- \\
- \\
- \\
- \\
- \\
-\end{array}$ & $\begin{array}{l}- \\
- \\
- \\
- \\
- \\
- \\
- \\
-\end{array}$ \\
\hline THALLIUN & $\begin{array}{l}01 / 07 / 89 \\
09 / 19 / 89 \\
03 / 31 / 90 \\
01 / 16 / 91\end{array}$ & $\begin{array}{l}0001 \\
0001 \\
0001 \\
0001\end{array}$ & $\mathrm{HG} / \mathrm{L}$ & $\begin{array}{l}< \\
< \\
<\end{array}$ & $\begin{array}{l}0.001 \\
0.01 \\
0.01 \\
0.03\end{array}$ & I & $\begin{array}{l}0.001 \\
0.01 \\
0.01 \\
0.03\end{array}$ & $\begin{array}{l}- \\
\dot{-}\end{array}$ \\
\hline THORIUH-230 & $10 / 03 / 85$ & 0001 & $\mathrm{PCI} / \mathrm{L}$ & & 0.1 & & 1. & 0.3 \\
\hline
\end{tabular}

PARAMETER VALUE IMDICATOR (PVI): < - LESS THAN DETECTION LIMIT

SAMPLE ID CODOES:

OTHER PARAMETER VALUE FLAGS:

I - INCREASED DETECTION LIMIT DUE TO REQUIRED DILUTION

J - eSTIHATED VALUE

OOO1 - FILTERED SAMPLE (.45 MICRONS)

0002 - FILTERED REPLICATE SAMPLE (.45 MICRONS)

0003 - FILTIERED REPLICATE SAMPLE (.45 MICRONS)

0004 - FILTIERED REPLICATE SAMPLE (.45 MICROHS) 
GROUHDWATER QUALITY DATA BY LOCATION

SITE: AMBO1 AMBROSIA LAKE

LOCATION: 0786

HORTH COORDINATE: $\quad 52888.5$ FT

EAST COORDINATE: $\quad 59760.9$ FT

$06 / 10 / 80$ TO $12 / 04 / 92$

REPORT DATE: 08/04/94

FORHATIOH OF COMPLETION: TRES HERHANOS - CI SANDSTONE (TO) HYDRAULIC FLON RELATIONSHIP: DOWN GRADIENT (D)

\begin{tabular}{|c|c|c|c|c|c|c|c|}
\hline PARANETER MAME & LOG DATE & SAMPLE & $\begin{array}{l}\text { UKITS OF } \\
\text { MEASURE }\end{array}$ & PVI & $\begin{array}{l}\text { PARAMETER } \\
\text { VALUE FLAGS }\end{array}$ & $\begin{array}{l}\text { DETECTIOH } \\
\text { LIMIT }\end{array}$ & $\begin{array}{l}\text { PARAMETER } \\
\text { UHCERTAINTY }\end{array}$ \\
\hline THOR IUH-230 & $\begin{array}{l}10 / 03 / 85 \\
10 / 03 / 85 \\
10 / 03 / 85 \\
10 / 03 / 85 \\
07 / 15 / 88 \\
01 / 07 / 89 \\
09 / 19 / 89\end{array}$ & $\begin{array}{l}0002 \\
0003 \\
0004 \\
0005 \\
0001 \\
0001 \\
0001\end{array}$ & $\mathrm{PCI} / \mathrm{L}$ & & $\begin{array}{l}0.1 \\
0.4 \\
1.1 \\
2.5 \\
0.4 \\
0.790 \\
0.1\end{array}$ & $\begin{array}{l}1 . \\
1 . \\
1 . \\
1 . \\
1 . \\
1 .\end{array}$ & $\begin{array}{l}0.3 \\
0.4 \\
0.7 \\
1.4 \\
0.5 \\
0.290 \\
0.4\end{array}$ \\
\hline TIK & $\begin{array}{l}10 / 03 / 85 \\
10 / 03 / 85 \\
10 / 03 / 85 \\
10 / 03 / 85 \\
10 / 03 / 85 \\
07 / 15 / 88 \\
01 / 07 / 89 \\
09 / 19 / 89 \\
03 / 31 / 90 \\
01 / 16 / 91\end{array}$ & $\begin{array}{l}0001 \\
0002 \\
0003 \\
0004 \\
0005 \\
0001 \\
0001 \\
0001 \\
0001 \\
0001\end{array}$ & MG/L & $\begin{array}{l}< \\
< \\
< \\
< \\
< \\
<\end{array}$ & $\begin{array}{l}0.005 \\
0.005 \\
0.005 \\
0.005 \\
0.005 \\
0.013 \\
0.005 \\
0.096 \\
0.007 \\
0.03\end{array}$ & $\begin{array}{l}0.005 \\
0.005 \\
0.005 \\
0.005 \\
0.005 \\
0.005 \\
0.005 \\
0.005 \\
0.005 \\
0.03\end{array}$ & $\begin{array}{l}- \\
: \\
- \\
- \\
- \\
-\end{array}$ \\
\hline TOTAL KJELDAHL MITROGEH & $\begin{array}{l}01 / 16 / 91 \\
11 / 24 / 92\end{array}$ & $\begin{array}{l}0001 \\
0001\end{array}$ & MG/L & & 1. & $\begin{array}{l}1 . \\
1 .\end{array}$ & 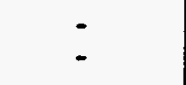 \\
\hline URANIUA & $\begin{array}{l}10 / 03 / 85 \\
10 / 03 / 85 \\
10 / 03 / 85 \\
10 / 03 / 85 \\
10 / 03 / 85 \\
05 / 14 / 86 \\
01 / 09 / 87 \\
07 / 15 / 88 \\
01 / 07 / 89 \\
09 / 99 / 89\end{array}$ & $\begin{array}{l}0001 \\
0002 \\
0003 \\
0004 \\
0005 \\
0001 \\
0001 \\
0001 \\
0001 \\
0001\end{array}$ & MG/L & & $\begin{array}{l}0.721 \\
0.775 \\
0.721 \\
0.695 \\
0.475 \\
0.699 \\
1.18 \\
1.42 \\
1.350 \\
1.23\end{array}$ & $\begin{array}{l}0.003 \\
0.003 \\
0.003 \\
0.003 \\
0.003 \\
0.003 \\
0.003 \\
0.003 \\
0.003 \\
0.003\end{array}$ & $\begin{array}{l}- \\
- \\
- \\
- \\
- \\
-\end{array}$ \\
\hline
\end{tabular}

PARAMETER VALUE IMDICATOR (PVI): < - LESS THAN DETECTION LIMIT

OTHER PARAMETER VALUE FLAGS:

1 - IHCREASED DETECTION LIMIT DUE TO REQUIRED DILUTION

J - estimated value
SAHPLE ID COOES:

0001 - FILTERED SAMPLE (.45 MICRONS)

0002 - FILTERED REPLICATE SAMPLE (.45 MICROHS)

0003 - FILTERED REPLICATE SAMPLE (.45 MICROHS)

0004 - FILTERED REPLICATE SAMPLE (.45 MICRONS) 
GROUNDHATER QUALITY DATA BY LOCATION

SITE: AMBOI AHBROSIA LAKE

LOCATION: 0786

HORTH COOROINATE: 52888.5 FT

EAST COORDINATE: 59760.9 FT

06/10/80 TO $12 / 04 / 92$

REPORT DATE: $08 / 04 / 94$

FORKATION OF COHPLETIOH: TRES HERMANOS - C1 SANDSTONE (TO) HYDRAULIC FLOW RELATIOHSHIP: DOWN GRADIENT (D)

\begin{tabular}{|c|c|c|c|c|c|c|c|c|}
\hline PARAMETER MAME & LOG DATE & $\begin{array}{c}\text { SNMPLE } \\
10\end{array}$ & $\begin{array}{l}\text { UNITS OF } \\
\text { MEASURE }\end{array}$ & PVI & $\begin{array}{l}\text { PARAMETER? } \\
\text { VALUE }\end{array}$ & FLAGS & $\begin{array}{l}\text { DETECTION } \\
\text { LIMIT }\end{array}$ & $\begin{array}{l}\text { PARAMETER } \\
\text { UNCERTAINTY }\end{array}$ \\
\hline URAKIUH & $\begin{array}{l}03 / 31 / 90 \\
01 / 16 / 91 \\
11 / 24 / 92\end{array}$ & $\begin{array}{l}0001 \\
0001 \\
0009\end{array}$ & MG/L & & $\begin{array}{l}1.88 \\
2.88 \\
2.51\end{array}$ & H & $\begin{array}{l}0.003 \\
0.001 \\
0.001\end{array}$ & - \\
\hline VANADIUA & $\begin{array}{l}10 / 03 / 85 \\
10 / 03 / 85 \\
10 / 03 / 85 \\
10 / 03 / 85 \\
10 / 03 / 85 \\
05 / 14 / 86 \\
01 / 09 / 87 \\
07 / 15 / 88 \\
01 / 07 / 89 \\
09 / 19 / 89 \\
03 / 31 / 90 \\
01 / 16 / 91 \\
11 / 24 / 92\end{array}$ & $\begin{array}{l}0001 \\
0002 \\
0003 \\
0004 \\
0005 \\
0001 \\
0001 \\
0001 \\
0001 \\
0001 \\
0001 \\
0001 \\
0001\end{array}$ & $M G / L$ & $\begin{array}{l}< \\
< \\
< \\
< \\
< \\
< \\
<\end{array}$ & $\begin{array}{l}0.01 \\
0.01 \\
0.01 \\
0.05 \\
0.1 \\
0.42 \\
0.01 \\
0.06 \\
0.01 \\
0.02 \\
0.01 \\
0.01 \\
0.05\end{array}$ & I & $\begin{array}{l}0.01 \\
0.01 \\
0.01 \\
0.01 \\
0.1 \\
0.01 \\
0.01 \\
0.01 \\
0.01 \\
0.01 \\
0.01 \\
0.01 \\
0.05\end{array}$ & $\begin{array}{l}- \\
- \\
- \\
- \\
- \\
- \\
- \\
-\end{array}$ \\
\hline ZIHC & $\begin{array}{l}10 / 03 / 85 \\
10 / 03 / 85 \\
10 / 03 / 85 \\
10 / 03 / 85 \\
10 / 03 / 85 \\
07 / 15 / 88 \\
01 / 07 / 89 \\
09 / 19 / 89 \\
03 / 31 / 90 \\
01 / 16 / 91\end{array}$ & $\begin{array}{l}0001 \\
0002 \\
0003 \\
0004 \\
0005 \\
0001 \\
0001 \\
0001 \\
0001 \\
0001\end{array}$ & $M G / L$ & $\begin{array}{l}< \\
<\end{array}$ & $\begin{array}{l}0.036 \\
0.05 \\
0.034 \\
0.038 \\
0.031 \\
0.166 \\
0.03 \\
0.005 \\
0.005 \\
0.005\end{array}$ & & $\begin{array}{l}0.005 \\
0.005 \\
0.005 \\
0.005 \\
0.005 \\
0.005 \\
0.005 \\
0.005 \\
0.005 \\
0.005\end{array}$ & $\begin{array}{l}- \\
- \\
- \\
- \\
- \\
-\end{array}$ \\
\hline
\end{tabular}

PARAMETER VALUE INDICATOR (PVI): < - LESS THAN DETECTION LIMIT

SAMPLE ID COOES:

OTHER PARAMETER VALUE FLAGS:

H - HOLD TIME EXPIRED, VALUE SUSPECT

I - IMCREASED DETECTION LIMIT DUE TO REQUIRED DILUTION

SAMPLE - FILTERED SAMPLE (.45 MICRONS)

0002 - FIL'TERED REPLICATE SAMPLE (.45 MICRONS)

0003 - FIL'TERED REPLICATE SAMPLE (.45 MICROWS)

0004 - FILPERED REPLICATE SAMPLE (.45 MICROHS) 
GROUNDHATER QUALITY DATA BY LOCATION

SITE: AMBOI AMBROSIA LAKE

LOCATION: 0787

NORTH COORDINATE: 52842.6 FT

EAST COORDINATE: 61202.9 FT

06/10/80 TO 12/04/92

REPORT DATE: 08/04/94

FORMATION OF COMPLETION: TRES HERHANOS - C2 SAMDSTOHE (TT)

HYDRAULIC FLOW RELATIONSHIP: DONN GRADIEHT (D)

\begin{tabular}{|c|c|c|c|c|c|c|c|c|}
\hline PARAMETER NAME & LOG DATE & $\begin{array}{c}\text { SAMPLE } \\
\text { ID }\end{array}$ & $\begin{array}{l}\text { UNITS OF } \\
\text { MEASURE }\end{array}$ & PVI & $\begin{array}{l}\text { PARAMETER } \\
\text { VALUE }\end{array}$ & FLAGS & $\begin{array}{l}\text { DETECTIOH } \\
\text { LIMIT }\end{array}$ & $\begin{array}{l}\text { PARAMETER } \\
\text { UHCERTAINTY }\end{array}$ \\
\hline ALKALIHITY & $\begin{array}{l}10 / 06 / 85 \\
05 / 15 / 86 \\
04 / 06 / 88 \\
07 / 15 / 88 \\
01 / 12 / 89 \\
09 / 19 / 89 \\
03 / 31 / 90 \\
01 / 16 / 91 \\
12 / 03 / 92\end{array}$ & $\begin{array}{l}0001 \\
0001 \\
0001 \\
0001 \\
0001 \\
0001 \\
0001 \\
0001 \\
0001\end{array}$ & HG/L CACO3 & & $\begin{array}{l}405 . \\
418 . \\
502 . \\
435 . \\
244 . \\
437 . \\
436 . \\
448 \\
466\end{array}$ & & $\begin{array}{l}- \\
- \\
- \\
- \\
- \\
-\end{array}$ & $\begin{array}{l}- \\
- \\
- \\
- \\
- \\
-\end{array}$ \\
\hline ALUHINUM & $\begin{array}{l}10 / 06 / 85 \\
04 / 06 / 88 \\
07 / 15 / 88 \\
01 / 12 / 89 \\
09 / 19 / 89 \\
03 / 31 / 90 \\
01 / 16 / 91\end{array}$ & $\begin{array}{l}0001 \\
0001 \\
0001 \\
0001 \\
0001 \\
0001 \\
0001\end{array}$ & MG/L & $\begin{array}{l}< \\
< \\
< \\
<\end{array}$ & $\begin{array}{l}0.6 \\
0.44 \\
0.1 \\
0.05 \\
0.1 \\
0.1 \\
0.05\end{array}$ & 小 & $\begin{array}{l}0.1 \\
0.1 \\
0.1 \\
0.1 \\
0.1 \\
0.1 \\
0.05\end{array}$ & $\begin{array}{l}- \\
- \\
- \\
- \\
-\end{array}$ \\
\hline ANT IMONY & $\begin{array}{l}10 / 06 / 85 \\
04 / 06 / 88 \\
07 / 15 / 88 \\
01 / 12 / 89 \\
09 / 19 / 89 \\
03 / 31 / 90 \\
01 / 16 / 91 \\
12 / 03 / 92\end{array}$ & $\begin{array}{l}0001 \\
0001 \\
0001 \\
0001 \\
0001 \\
0001 \\
0001 \\
0001\end{array}$ & MG/L & $<$ & $\begin{array}{l}0.003 \\
0.013 \\
0.025 \\
0.003 \\
0.003 \\
0.041 \\
0.003 \\
0.003\end{array}$ & & $\begin{array}{l}0.003 \\
0.003 \\
0.003 \\
0.003 \\
0.003 \\
0.003 \\
0.003 \\
0.003\end{array}$ & $\begin{array}{l}- \\
- \\
- \\
- \\
- \\
-\end{array}$ \\
\hline BARIUM & $\begin{array}{l}10 / 06 / 85 \\
04 / 06 / 88 \\
07 / 15 / 88 \\
01 / 12 / 89 \\
09 / 19 / 89 \\
03 / 31 / 90 \\
01 / 16 / 91\end{array}$ & $\begin{array}{l}0001 \\
0001 \\
0001 \\
0001 \\
0001 \\
0001 \\
0001\end{array}$ & $M G / L$ & $\begin{array}{l}< \\
< \\
< \\
<\end{array}$ & $\begin{array}{l}0.1 \\
0.03 \\
0.1 \\
0.02 \\
0.1 \\
0.1 \\
0.01\end{array}$ & $\begin{array}{l}\text { J } \\
\text { J }\end{array}$ & $\begin{array}{l}0.1 \\
0.1 \\
0.1 \\
0.1 \\
0.1 \\
0.1 \\
0.01\end{array}$ & $\begin{array}{l}- \\
- \\
- \\
- \\
-\end{array}$ \\
\hline BERYLLIU: & $\begin{array}{l}01 / 12 / 89 \\
09 / 19 / 89 \\
03 / 31 / 90\end{array}$ & $\begin{array}{l}0009 \\
0001 \\
0001\end{array}$ & $M G / L$ & $\begin{array}{l}< \\
<\end{array}$ & $\begin{array}{l}0.005 \\
0.09 \\
0.01\end{array}$ & & $\begin{array}{l}0.005 \\
0.01 \\
0.01\end{array}$ & $\begin{array}{l}- \\
-\end{array}$ \\
\hline
\end{tabular}

PARAMETER VALUE IMDICATOR (PVI): < - LESS THAN DETECTIOH LIMIT

SAMPLE ID COOES:

0009 - FILTERED SAMPLE (.45 MICRONS)

OTHER PARAMETER VALUE FLAGS:

1 - INCREASED DETECTION LIMIT DUE TO REOUIRED DILUTION

J - estimated VALUE 
GROUNDWATER OUALITY OATA BY LOCATION

SITE: AMBO1 AMBROSIA LAKE

LOCATION: 0787

HORTH COORDIHATE: 52842.6 FT

EAST COORDINATE: 61202.9 FT

06/10/80 TO $12 / 04 / 92$

REPORT DATE: 08/04/94

FORMATIOH OF COMPLETION: TRES HERHANOS - C2 SANDSTONE (TT)

HYDRAULIC FLOW RELATIONSHIP: DONN GRADIENT (D)

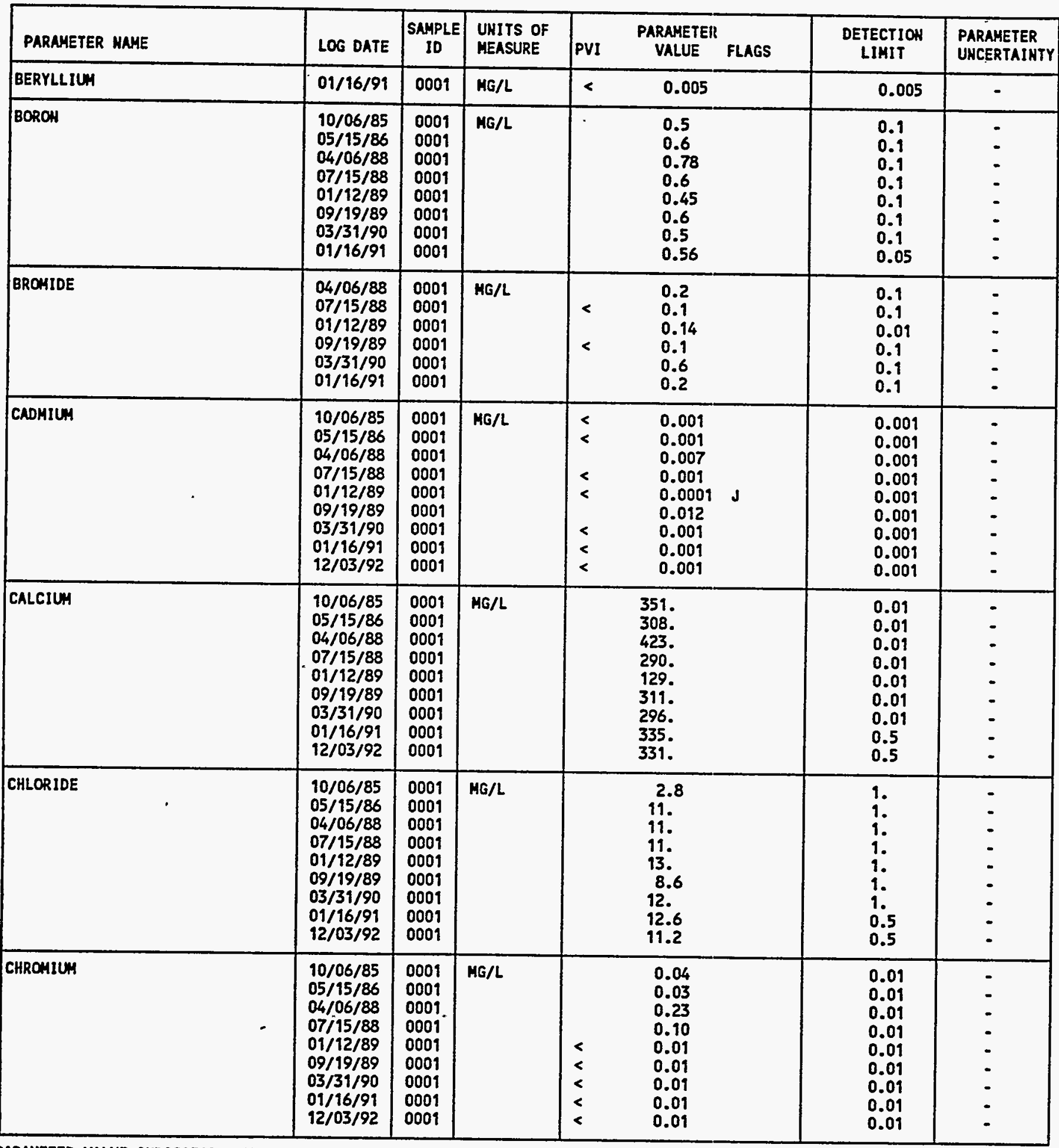

PARAMETER VALUe IHDicatoR (PVI): < - LeSS THAN DETECTION LIMIT

SAMPLE ID COOES:

DOO 1 - FILTERED SAMPLE (.45 MICROHS)

OTHER PARAMETER VALUE FLAGS:

J - estimated VALUE 
GROUNDWATER QUALITY DATA BY LOCATION

SITE: AMBOI AMBROSIA LAKE

LOCATION: 0787

HORTH COORDIHATE: 52842.6 FT

EAST COORDIHATE: 69202.9 FT

$06 / 10 / 80$ TO $12 / 04 / 92$

REPORT DATE: 08/04/94

FORMATION OF CONPLETION: TRES HERMANOS - C2 SANDSTONE (TT)

HYDRAULIC FLON RELATIONSHIP: DOWN GRADIENT (D)

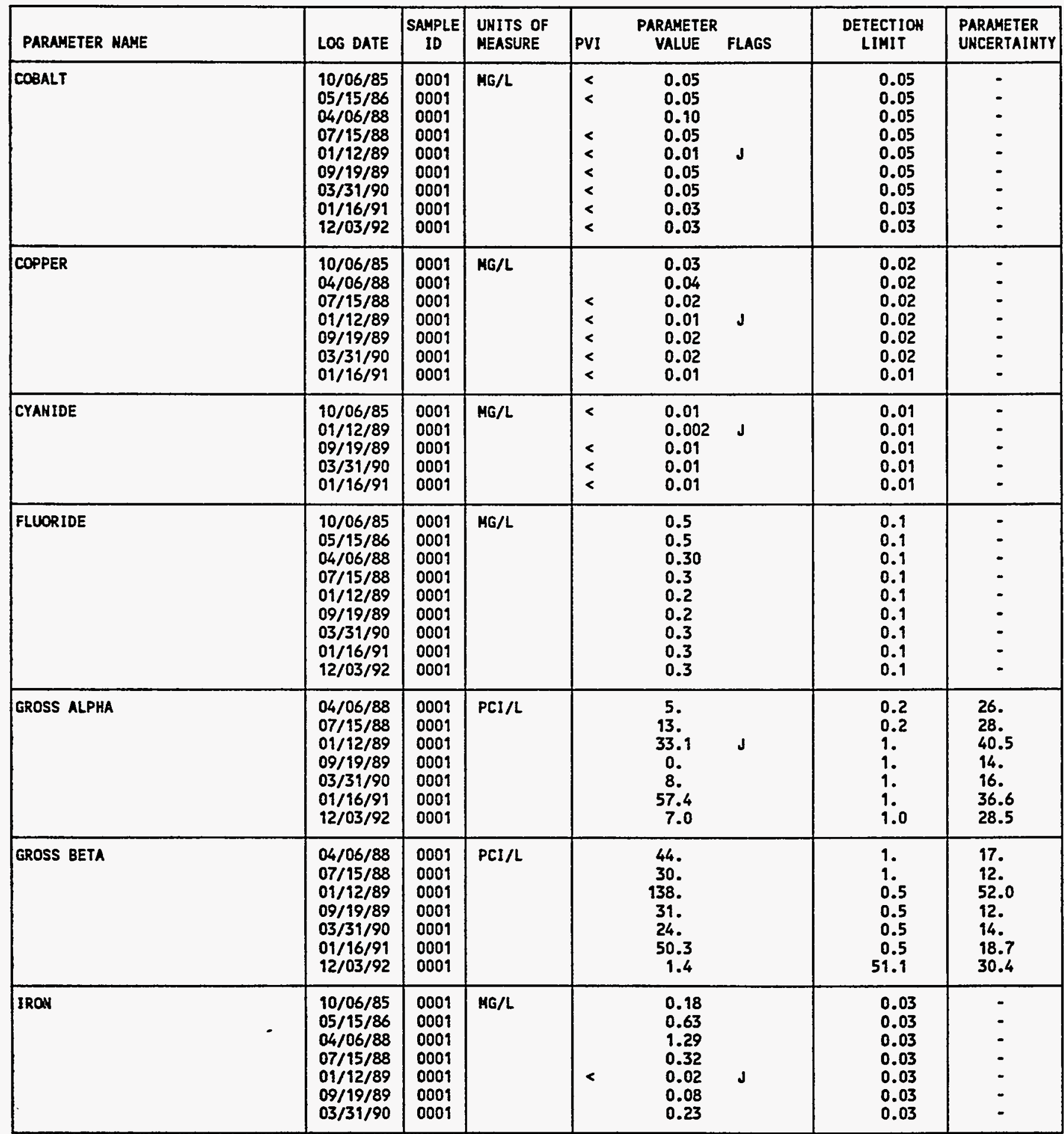

PARAMETER VALUE INDICATOR (PVI): < - LESS THAN DETECTION LIMIT

SAMPLE IO COOES:

0001 - FILTERED SAMPLE (.45 MICROHS)

OTHER PARAMETER VALUE FLAGS:

d- ESTIMATED VALUE 
GROUNDWATER QUALITY DATA BY LOCATION

SITE: AMBOI AMBROSIA LAKE

LOCATION: 0787

MORTH COORDIHATE: $\quad 52842.6$ FT

EAST COORDIHATE: 61202.9 FT

06/10/80 TO $12 / 04 / 92$

REPORT DATE: 08/04/94

FORHATIOH OF COMPLETIOH: TRES HERMANOS - C2 SAMDSTONE (TT) HYDRAULIC FLOU RELATIOHSHIP: DOWN GRADIENT (D)

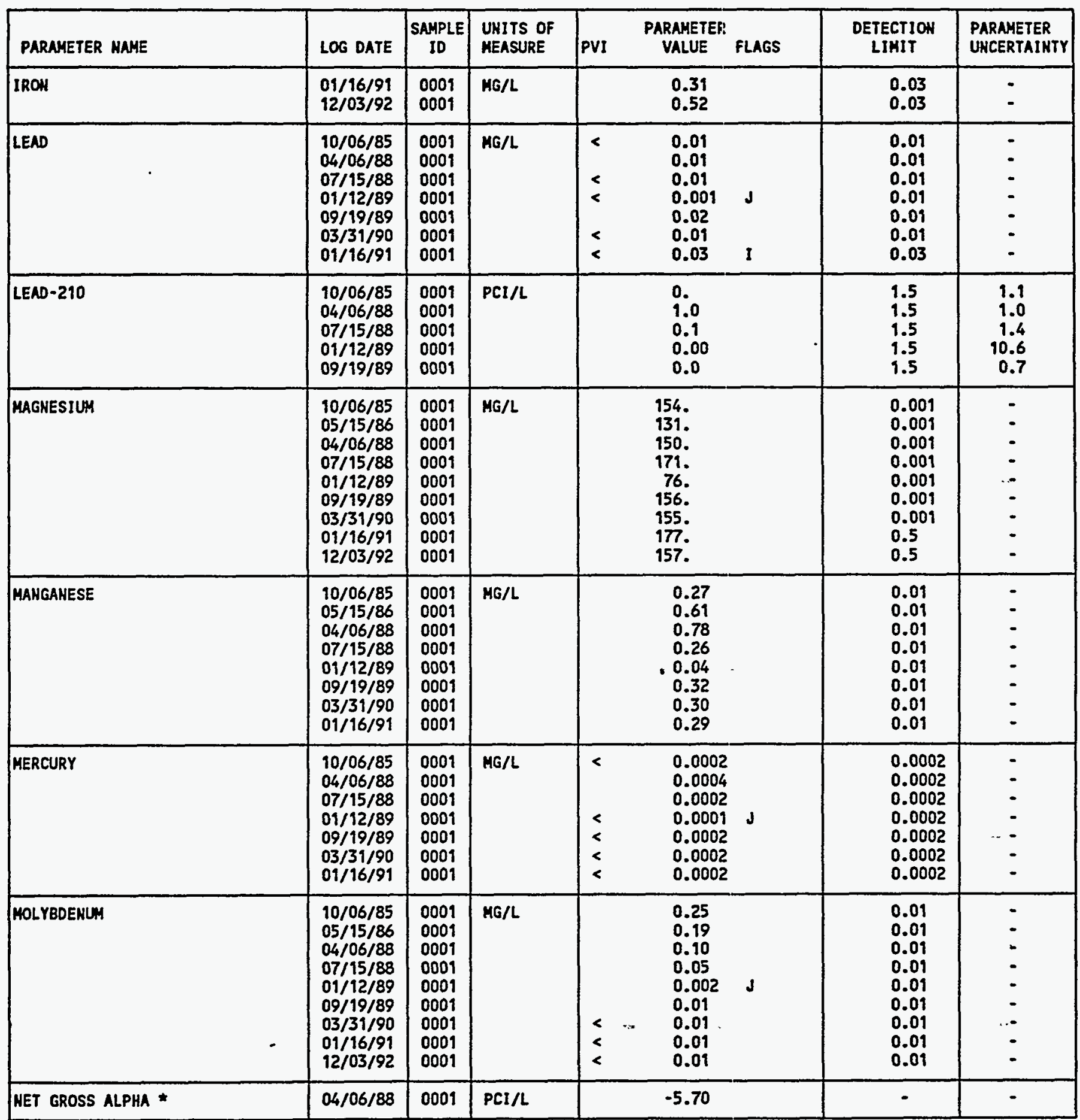

- MET gRoss alPHA (GROSS ALPHA - URAHIUH) WITH 1 Mg URANIUH = 686 PCI

PARAMETER VALUE IHDICATOR (PVI): < - LESS THAH DETECTION LIMIT
SAMPLE ID TOOES:

0009 - FILTERED SAMPLE (.45 MICROHS)

OTHER PARAMETER VALUE FLAGS:

1 - IHCREASED DETECTION LIMIT DUE TO REQUIRED DILUTIOH

J. Estimated VALUE 
GROUNDWATER QUALITY DATA BY LOCATION

SITE: AMBOI AMBROSIA LAKE

LOCATION: 0787

NORTH COORDINATE: 52842.6 FT

EAST COORDINATE: $\quad 61202.9$ FT

06/10/80 TO $12 / 04 / 92$

REPORT DATE: 08/04/94

FORMATIOH OF COMPLETIOH: TRES HERMANOS - C2 SAMDSTOHE (TT) HYDRAULIC FLON RELATIONSHIP: DOWN GRADIENT (D)

\begin{tabular}{|c|c|c|c|c|c|c|c|c|}
\hline PARAMETER NAME & LOG DATE & $\begin{array}{c}\text { SAMPLE } \\
\text { ID }\end{array}$ & $\begin{array}{l}\text { UNITS OF } \\
\text { MEASURE }\end{array}$ & PVI & $\begin{array}{l}\text { PARAMETER } \\
\text { VALUE }\end{array}$ & FLAGS & $\begin{array}{l}\text { DETECTION } \\
\text { LIMIT }\end{array}$ & $\begin{array}{l}\text { PARAMETER } \\
\text { UNCERTAIHTY }\end{array}$ \\
\hline HET GROSS ALPHA • & $\begin{array}{l}07 / 15 / 88 \\
01 / 12 / 89 \\
09 / 19 / 89 \\
03 / 31 / 90 \\
01 / 16 / 91 \\
12 / 03 / 92\end{array}$ & $\begin{array}{l}0001 \\
0001 \\
0001 \\
0001 \\
0001 \\
0009\end{array}$ & PCI/L & & $\begin{array}{r}8.20 \\
20.75 \\
-9.58 \\
4.84 \\
57.06 \\
4.26\end{array}$ & & $\begin{array}{l}- \\
- \\
-\end{array}$ & $\begin{array}{l}- \\
- \\
- \\
-\end{array}$ \\
\hline NICKEL & $\begin{array}{l}10 / 06 / 85 \\
04 / 06 / 88 \\
07 / 15 / 88 \\
01 / 12 / 89 \\
09 / 19 / 89 \\
03 / 31 / 90 \\
01 / 16 / 91 \\
12 / 03 / 92\end{array}$ & $\begin{array}{l}0001 \\
0001 \\
0001 \\
0001 \\
0001 \\
0001 \\
0001 \\
0001\end{array}$ & $M G / L$ & $\begin{array}{l}< \\
< \\
< \\
<\end{array}$ & $\begin{array}{l}0.08 \\
0.13 \\
0.05 \\
0.02 \\
0.04 \\
0.04 \\
0.04 \\
0.08\end{array}$ & J & $\begin{array}{l}0.04 \\
0.04 \\
0.04 \\
0.04 \\
0.04 \\
0.04 \\
0.04 \\
0.04\end{array}$ & $\begin{array}{l}- \\
: \\
- \\
: \\
-\end{array}$ \\
\hline NITRATE & $\begin{array}{l}10 / 06 / 85 \\
05 / 15 / 86 \\
04 / 06 / 88 \\
07 / 15 / 88 \\
01 / 12 / 89 \\
09 / 19 / 89 \\
03 / 31 / 90 \\
01 / 16 / 91 \\
12 / 03 / 92\end{array}$ & $\begin{array}{l}0001 \\
0001 \\
0001 \\
0001 \\
0001 \\
0001 \\
0001 \\
0001 \\
0001\end{array}$ & HG/L & $<$ & $\begin{array}{c}4 . \\
2 . \\
0.7 \\
3.0 \\
29.0 \\
1.0 \\
1.1 \\
1.8 \\
1.2\end{array}$ & $\begin{array}{l}7 \\
j\end{array}$ & $\begin{array}{l}1 . \\
9 . \\
1 . \\
1 . \\
1 . \\
1.0\end{array}$ & $\begin{array}{l}- \\
- \\
- \\
- \\
- \\
- \\
-\end{array}$ \\
\hline HITRITE & $10 / 06 / 85$ & 0001 & $M G / L$ & $<$ & 0.1 & & 0.1 & - \\
\hline HITRITE AND HITRATE & $\begin{array}{l}04 / 06 / 88 \\
03 / 31 / 90 \\
01 / 16 / 91\end{array}$ & $\begin{array}{l}0001 \\
0001 \\
0001\end{array}$ & $M G / L$ & & $\begin{array}{l}0.7 \\
0.2 \\
0.41\end{array}$ & $\begin{array}{l}J \\
J\end{array}$ & $\begin{array}{l}1 . \\
1.05 \\
0.05\end{array}$ & - \\
\hline $\mathrm{PH}$ & $\begin{array}{l}10 / 06 / 85 \\
05 / 15 / 86 \\
04 / 06 / 88 \\
07 / 15 / 88 \\
01 / 12 / 89 \\
09 / 19 / 89 \\
03 / 31 / 90 \\
01 / 16 / 91 \\
12 / 03 / 92\end{array}$ & $\begin{array}{l}0001 \\
0001 \\
0001 \\
0001 \\
0001 \\
0001 \\
0001 \\
0001 \\
0001\end{array}$ & SU & & $\begin{array}{l}7.08 \\
7 . \\
7.33 \\
7.55 \\
9.13 \\
7.80 \\
7.22 \\
7.62 \\
7.07\end{array}$ & & $\begin{array}{l}- \\
- \\
- \\
- \\
- \\
-\end{array}$ & $\begin{array}{l}- \\
- \\
- \\
- \\
- \\
- \\
-\end{array}$ \\
\hline PHOSPHATE & $\begin{array}{l}10 / 06 / 85 \\
04 / 06 / 88 \\
07 / 15 / 88 \\
01 / 12 / 89 \\
09 / 19 / 89 \\
03 / 31 / 90 \\
01 / 16 / 91 \\
12 / 03 / 92\end{array}$ & $\begin{array}{l}0001 \\
0001 \\
0001 \\
0001 \\
0001 \\
0001 \\
0001 \\
0001\end{array}$ & $M G / L$ & $\begin{array}{l}< \\
< \\
< \\
<\end{array}$ & $\begin{array}{l}0.5 \\
0.1 \\
0.1 \\
0.1 \\
0.1 \\
0.1 \\
0.13 \\
0.1\end{array}$ & H & $\begin{array}{l}0.1 \\
0.1 \\
0.1 \\
0.1 \\
0.1 \\
0.1 \\
0.05 \\
0.1\end{array}$ & $\begin{array}{l}- \\
: \\
- \\
: \\
-\end{array}$ \\
\hline POLONIUA-210 & $\begin{array}{l}10 / 06 / 85 \\
04 / 06 / 88 \\
07 / 15 / 88\end{array}$ & $\begin{array}{l}0001 \\
0001 \\
0001\end{array}$ & $\mathrm{PCI} / \mathrm{L}$ & & $\begin{array}{l}0.1 \\
0.1 \\
0.1\end{array}$ & & $\begin{array}{l}1 . \\
1 .\end{array}$ & $\begin{array}{l}0.6 \\
0.4 \\
0.5\end{array}$ \\
\hline
\end{tabular}

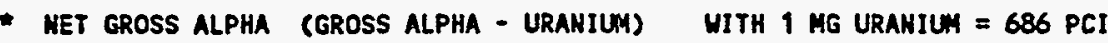

PARAMETER VALUE INDICATOR (PVI): < - LESS THAN DETECTION LIMIT

OTHER PARAMETER VALUE FLAGS:

H - HOLD TIME EXPIRED, VALUE SUSPECT

J - estimated value 
GROUNDWATER QUALITY DATA BY LOCATIOH

SITE: AMBOI AMBROSIA LAKE

LOCATIOH: 0787

HORTH COORDIHATE: 52842.6 FT

EAST COORDINATE: $\quad 61202.9$ FT

O6/10/80 TO 12/04/92

REPORT DATE: 08/04/94

FORMATION OF COMPLETION: TRES HERHANOS - C2 SAMDSTONE (TT) HYDRAULIC FLOW RELATIONSHIP: DOWH GRADIENT (D)

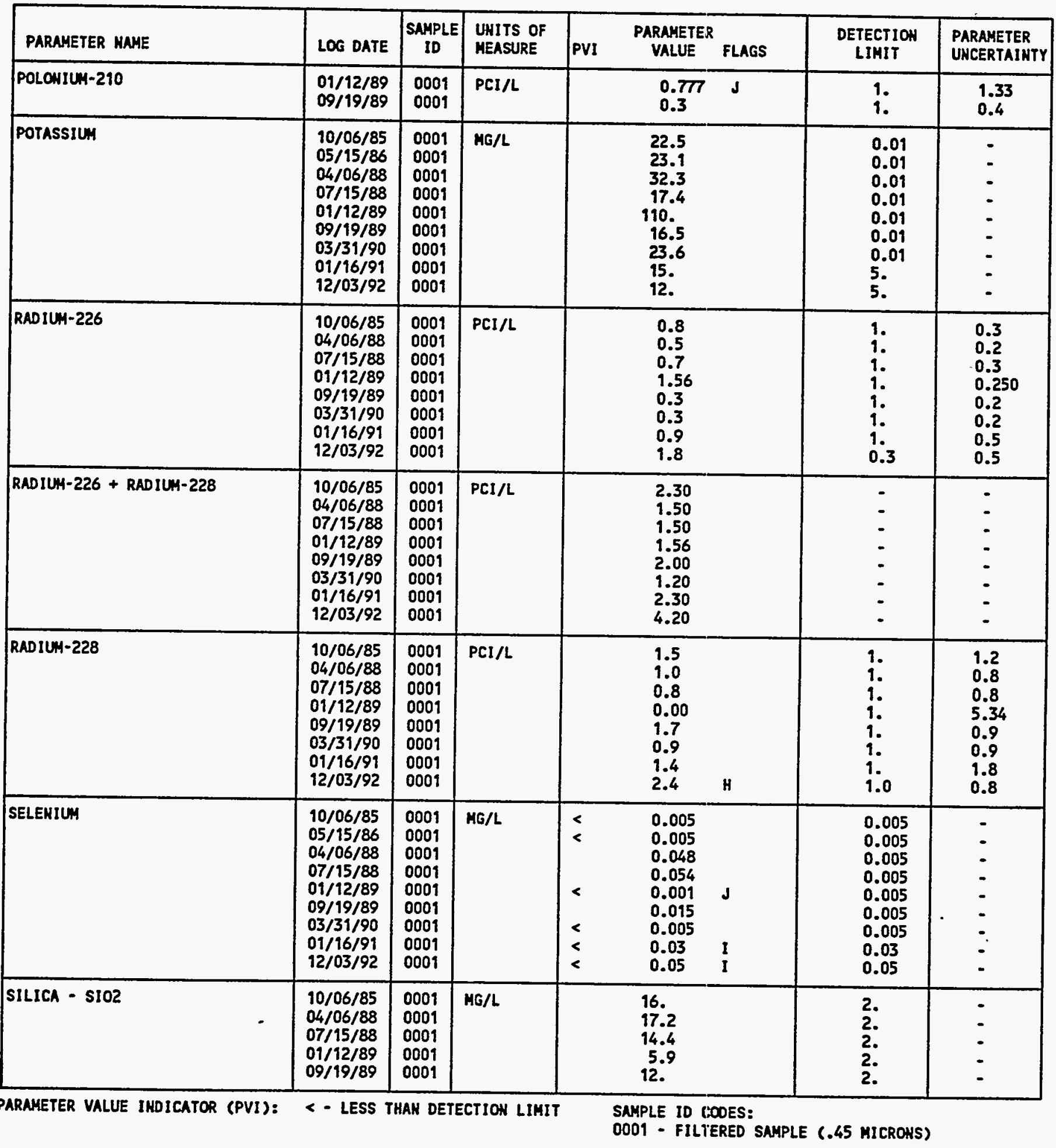

OTHER PARAMETER VALUE FLAGS:

H - HOLD TIME EXPIRED, VALUE SUSPECT

1 - IMCREASED DETECTION LIHIT DUE TO REQUIRED DILUTIOH

d- estimated value 
GROUNDWATER QUALITY DATA BY LOCATIOH

SITE: AMBO1 AMBROSIA LAKE

LOCATION: 0787

NORTH COORDINATE: 52842.6 FT

EAST COORDINATE: $\quad 61202.9$ FT

D6/10/80 TO $12 / 04 / 92$

REPORT OATE: $08 / 04 / 94$

FORMATION OF COMPLETION: TRES HERMANOS - C2 SANDSTOHE (TT)

HYDRAULIC FLON RELATIONSHIP: DOWN GRADIENT (D)

\begin{tabular}{|c|c|c|c|c|c|c|c|c|}
\hline PARAMETER MAME & & LOG DATE & $\begin{array}{c}\text { SAMPLE } \\
\text { I0 }\end{array}$ & $\begin{array}{l}\text { UNITS OF } \\
\text { MEASURE }\end{array}$ & PVI & $\begin{array}{l}\text { PARAMETER } \\
\text { VALUE FLAGS }\end{array}$ & $\begin{array}{l}\text { DETECTION } \\
\text { LIMIT }\end{array}$ & $\begin{array}{l}\text { PARAMETER } \\
\text { UNCERTAINTY }\end{array}$ \\
\hline SILICA - SIOZ & & $\begin{array}{l}03 / 31 / 90 \\
01 / 16 / 91\end{array}$ & $\begin{array}{l}0001 \\
0001\end{array}$ & HG/L & & 11.6 & $\begin{array}{l}2 . \\
0.1\end{array}$ & 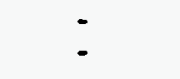 \\
\hline SILVER & & $\begin{array}{l}10 / 06 / 85 \\
04 / 06 / 88 \\
07 / 15 / 88 \\
01 / 12 / 89 \\
09 / 19 / 89 \\
03 / 31 / 90 \\
01 / 16 / 91 \\
12 / 03 / 92\end{array}$ & $\begin{array}{l}0001 \\
0001 \\
0001 \\
0001 \\
0001 \\
0001 \\
0001 \\
0001\end{array}$ & HE/L & $\begin{array}{l}< \\
< \\
< \\
< \\
< \\
<\end{array}$ & $\begin{array}{l}0.01 \\
0.09 \\
0.01 \\
0.01 \\
0.01 \\
0.01 \\
0.01 \\
0.01\end{array}$ & $\begin{array}{l}0.01 \\
0.01 \\
0.01 \\
0.01 \\
0.01 \\
0.01 \\
0.01 \\
0.01\end{array}$ & $\begin{array}{l}- \\
. \\
- \\
- \\
- \\
-\end{array}$ \\
\hline SOOIUM & & $\begin{array}{l}10 / 06 / 85 \\
05 / 15 / 86 \\
04 / 06 / 88 \\
07 / 15 / 88 \\
01 / 12 / 89 \\
09 / 19 / 89 \\
03 / 31 / 90 \\
01 / 16 / 91 \\
12 / 03 / 92\end{array}$ & $\begin{array}{l}0001 \\
0001 \\
0001 \\
0001 \\
0001 \\
0001 \\
0001 \\
0001 \\
0001\end{array}$ & MG/L & & $\begin{array}{l}463 . \\
755 . \\
497 . \\
599 \\
735 . \\
681 . \\
665 . \\
761 . \\
702 .\end{array}$ & $\begin{array}{l}0.002 \\
0.002 \\
0.002 \\
0.002 \\
0.002 \\
0.002 \\
0.002 \\
5 . \\
5 .\end{array}$ & $\begin{array}{l}- \\
: \\
: \\
- \\
-\end{array}$ \\
\hline SPECIFIC COHDUCTANCE & & $\begin{array}{l}10 / 06 / 85 \\
05 / 15 / 86 \\
04 / 06 / 88 \\
07 / 15 / 88 \\
01 / 12 / 89 \\
09 / 19 / 89 \\
03 / 31 / 90 \\
01 / 16 / 91 \\
12 / 03 / 92\end{array}$ & $\begin{array}{l}0001 \\
0001 \\
0001 \\
0001 \\
0001 \\
0001 \\
0001 \\
0001 \\
0001\end{array}$ & UNHO/CM & & $\begin{array}{l}3300 . \\
3200 . \\
3100 \\
3200 \\
2800 \\
150 \\
2400 \\
3200 \\
3570\end{array}$ & $\begin{array}{l}- \\
- \\
- \\
- \\
- \\
- \\
-\end{array}$ & $\begin{array}{l}- \\
- \\
- \\
- \\
- \\
-\end{array}$ \\
\hline STRONTIUN & & $\begin{array}{l}10 / 06 / 85 \\
05 / 15 / 86 \\
04 / 06 / 88 \\
07 / 15 / 88 \\
01 / 12 / 89 \\
09 / 19 / 89 \\
03 / 31 / 90 \\
01 / 16 / 91\end{array}$ & $\begin{array}{l}0001 \\
0001 \\
0001 \\
0001 \\
0001 \\
0001 \\
0001 \\
0001\end{array}$ & $M G / L$ & & $\begin{array}{l}8.6 \\
0.7 \\
5.25 \\
6.9 \\
9.20 \\
8.1 \\
7.62 \\
8.20\end{array}$ & $\begin{array}{l}0.1 \\
0.1 \\
0.1 \\
0.1 \\
0.1 \\
0.1 \\
0.1 \\
0.01\end{array}$ & $\begin{array}{l}- \\
\dot{-} \\
\dot{-} \\
- \\
-\end{array}$ \\
\hline SULFIDE & 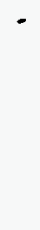 & $\begin{array}{l}10 / 06 / 85 \\
04 / 06 / 88 \\
07 / 15 / 88 \\
01 / 12 / 89 \\
09 / 19 / 89 \\
03 / 31 / 90 \\
01 / 16 / 91\end{array}$ & $\begin{array}{l}0001 \\
0001 \\
0001 \\
0001 \\
0001 \\
0001 \\
0001\end{array}$ & MG/L & $\begin{array}{l}< \\
< \\
< \\
< \\
<\end{array}$ & $\begin{array}{l}0.1 \\
0.1 \\
0.1 \\
0.13 \\
0.1 \\
0.1 \\
2.3\end{array}$ & $\begin{array}{l}0.1 \\
0.1 \\
0.1 \\
0.1 \\
0.1 \\
0.1 \\
0.1\end{array}$ & $\begin{array}{l}- \\
- \\
- \\
- \\
- \\
-\end{array}$ \\
\hline
\end{tabular}

PARAMETER VALUE INDICATOR (PVI):

$<$ - LESS THAN DETECTION LIMIT

SAMPLE ID COOES:

0001 - FILTERED SAMPLE (.45 MICRONS)

OTHER PARAMETER VALUE FLAGS:

$J$ - ESTIMATED VALUE 
GROUNDHATER QUALITY DATA BY LOCATION

SITE: AMBOI AMBROSIA LAKE

LOCATIOH: 0787

HORTH COORDIHATE: 52842.6 FT

EAST COORDINATE: 61202.9 FT

06/10/80 TO 12/04/92

REPORT DATE: 08/04/94

FORHATION OF COMPLETION: TRES HERMANOS - C2 SAHDSTOHE (TT) HYDRAULIC FLOW RELATIONSHIP: DOWH GRADIENT (D)

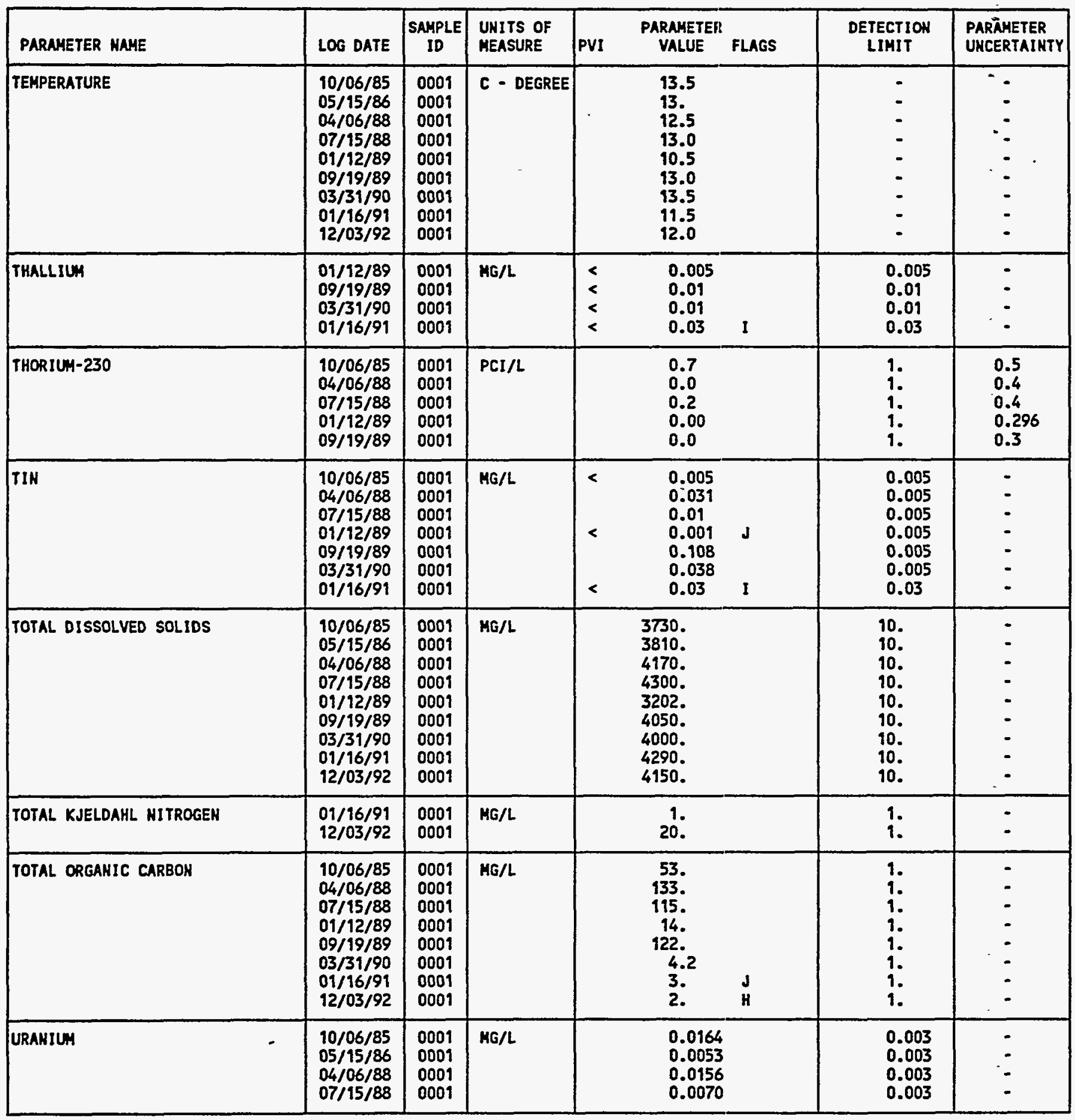

PARAMETER VALUE IMDICATOR (PVI): < - LESS THAN DETECTION LIMIT SAMPLE ID LCOES:

0001 - FILTERED SAMPLE (.45 MICROHS)

OTHER PARAMETER VALUE FLAGS:

H - HOLD TIME EXPIRED, VALUE SUSPECT

I - INCREASED DETECTIOH LIMIT DUE TO REQUIRED DILUTION

J estimated VAlue 
GROUNDHATER QUALITY DATA BY LOCATION

SITE: AMBO1 AMBROSIA LAKE

LOCATION: 0787

MORTH COOROINATE: 52842.6 FT

EAST COORDINATE: $\quad 61202.9$ FT

06/10/80 TO $12 / 04 / 92$

REPORT DATE: 08/04/94

FORMATION OF COMPLETION: TRES HERMANOS - C2 SAHDSTONE (TT)

HYDRAULIC FLON RELATIONSHIP: DOWH GRADIEKT (D)

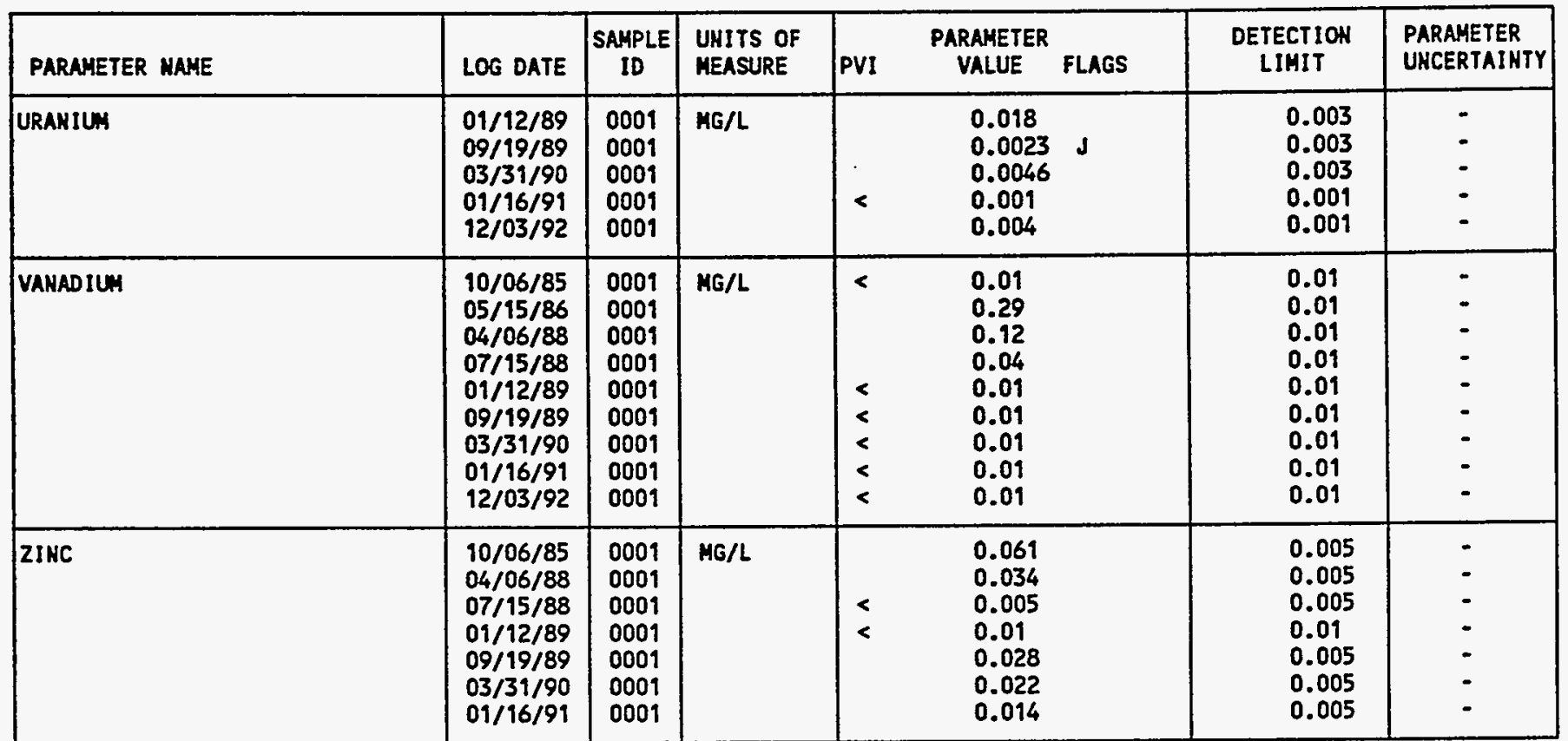

PARAMETER VALUE INDICATOR (PVI): < - LESS THAN DETECTIOH LIMIT

OTHER PARAMETER VALUE FLAGS:

$J$ - ESTIMATED VALUE 
GROUNDHATER QUALITY DATA BY LOCATION

SITE: AMBO1 AMBROSIA LAKE

LOCATION: 0791

NORTH COORDIHATE: 55739.9 FT

EAST COORDIHATE: 60567.0 FT

06/10/80 TO $12 / 04 / 92$

REPORT DATE: 08/04/94

FORMATION OF COMPLETION: TRES HERMAHOS - C1 SANDSTONE (TO) HYDRAULIC FLON RELATIOHSHIP: DONN GRADIENT (D)

\begin{tabular}{|c|c|c|c|c|c|c|c|}
\hline PARAMETER MAME & LOG DATE & $\underset{\text { ID }}{\text { SAMPLE }}$ & $\begin{array}{l}\text { UNITS OF } \\
\text { MEASURE }\end{array}$ & PV & $\begin{array}{l}\text { PARAMETER: } \\
\text { VALUE fLAGS }\end{array}$ & $\begin{array}{l}\text { DETECTIOH } \\
\text { LIMIT }\end{array}$ & $\begin{array}{l}\text { PARAMETER } \\
\text { UNCERTAINTY }\end{array}$ \\
\hline ALKALINITY & $\begin{array}{l}10 / 19 / 85 \\
05 / 21 / 86\end{array}$ & $\begin{array}{l}0001 \\
0001\end{array}$ & MG/L CACO3 & & $\begin{array}{l}451 . \\
2650 .\end{array}$ & - & - \\
\hline ALUHINUA & $10 / 19 / 85$ & 0001 & MG/L & & 0.6 & 0.1 & - \\
\hline AHWONIUH & $10 / 11 / 85$ & 0001 & $M G / L$ & & 1.6 & 0.1 & - \\
\hline ANTIMONY & $10 / 11 / 85$ & 0001 & HG/L & $<$ & 0.003 & 0.003 & - \\
\hline ARSENIC & $\begin{array}{l}10 / 11 / 85 \\
05 / 21 / 86\end{array}$ & $\begin{array}{l}0001 \\
0001\end{array}$ & MG/L & $<$ & $\begin{array}{l}0.02 \\
0.01\end{array}$ & $\begin{array}{l}0.01 \\
0.01\end{array}$ & - \\
\hline BARIUY & $10 / 11 / 85$ & 0001 & MG/L & & 0.1 & 0.1 & - \\
\hline BOROH & $\begin{array}{l}10 / 11 / 85 \\
05 / 21 / 86\end{array}$ & $\begin{array}{l}0001 \\
0001\end{array}$ & HG/L & & $\begin{array}{l}0.3 \\
0.3\end{array}$ & $\begin{array}{l}0.1 \\
0.1\end{array}$ & : \\
\hline CADMIUH & $\begin{array}{l}10 / 19 / 85 \\
05 / 21 / 86\end{array}$ & $\begin{array}{l}0001 \\
0001\end{array}$ & MG/L & $<$ & $\begin{array}{l}0.001 \\
0.001\end{array}$ & $\begin{array}{l}0.001 \\
0.001\end{array}$ & - \\
\hline CalCIUH & $\begin{array}{l}10 / 11 / 85 \\
05 / 21 / 86\end{array}$ & $\begin{array}{l}0001 \\
0001\end{array}$ & $M G / L$ & & $\begin{array}{l}34 . \\
181 .\end{array}$ & $\begin{array}{l}0.01 \\
0.01\end{array}$ & $\because$ \\
\hline CHLORIDE & $\begin{array}{l}10 / 11 / 85 \\
05 / 21 / 86\end{array}$ & $\begin{array}{l}0001 \\
0001\end{array}$ & MG/L & & $\begin{array}{l}31 . \\
38 .\end{array}$ & 9. & - \\
\hline CHRONIUN & $\begin{array}{l}10 / 11 / 85 \\
05 / 21 / 86\end{array}$ & $\begin{array}{l}0001 \\
0001\end{array}$ & MG/L & & $\begin{array}{l}0.21 \\
0.04\end{array}$ & $\begin{array}{l}0.01 \\
0.01\end{array}$ & - \\
\hline COBALT & $\begin{array}{l}10 / 11 / 85 \\
05 / 21 / 86\end{array}$ & $\begin{array}{l}0001 \\
0001\end{array}$ & $M G / L$ & $<$ & $\begin{array}{l}0.05 \\
0.06\end{array}$ & $\begin{array}{l}0.05 \\
0.05\end{array}$ & - \\
\hline COPPER & $10 / 11 / 85$ & 0001 & $M G / L$ & $<$ & 0.02 & 0.02 & - \\
\hline CYANIDE & $10 / 11 / 85$ & 0001 & MG/L & $<$ & 0.01 & 0.01 & - \\
\hline FLUORIDE & $\begin{array}{l}10 / 11 / 85 \\
05 / 21 / 86\end{array}$ & $\begin{array}{l}0001 \\
0001\end{array}$ & $M G / L$ & & $\begin{array}{l}1.1 \\
0.4\end{array}$ & $\begin{array}{l}0.1 \\
0.1\end{array}$ & - \\
\hline IRON & $\begin{array}{l}10 / 11 / 85 \\
05 / 21 / 86\end{array}$ & $\begin{array}{l}0001 \\
0001\end{array}$ & $\mathrm{HG} / \mathrm{L}$ & $<$ & $\begin{array}{l}0.03 \\
0.03\end{array}$ & $\begin{array}{l}0.03 \\
0.03\end{array}$ & - \\
\hline LEAD & $10 / 11 / 85$ & 0001 & $M G / L$ & $<$ & 0.01 & 0.01 & - \\
\hline LEAD-210 & $10 / 11 / 85$ & 0001 & $\mathrm{PCI} / \mathrm{L}$ & & 0.7 & 1.5 & 1.9 \\
\hline MAGHESIUM & $\begin{array}{l}10 / 11 / 85 \\
05 / 21 / 86\end{array}$ & $\begin{array}{l}0001 \\
0001\end{array}$ & $M G / L$ & & $\begin{array}{l}5.19 \\
0.022\end{array}$ & $\begin{array}{l}0.001 \\
0.001\end{array}$ & $\therefore$ \\
\hline MANGANESE & $\begin{array}{l}10 / 11 / 85 \\
05 / 21 / 86\end{array}$ & $\begin{array}{l}0001 \\
0001\end{array}$ & $M G / L$ & $<$ & $\begin{array}{l}0.02 \\
0.01\end{array}$ & $\begin{array}{l}0.01 \\
0.01\end{array}$ & : \\
\hline MERCURY & $10 / 11 / 85$ & 0001 & $M G / L$ & $<$ & 0.0002 & 0.0002 & - \\
\hline MOLYBDENUM & $\begin{array}{l}10 / 11 / 85 \\
05 / 21 / 86\end{array}$ & $\begin{array}{l}0001 \\
0001\end{array}$ & $M G / L$ & & $\begin{array}{l}0.1 \\
0.21\end{array}$ & $\begin{array}{l}0.01 \\
0.01\end{array}$ & - \\
\hline HICKEL & $10 / 11 / 85$ & 0001 & $M G / L$ & & 0.07 & 0.04 & $\because$ \\
\hline
\end{tabular}

PARAMETER VALUE IhDICATOR (PVI): < - LeSS THAH DETECTIOH LIMIT 
GROUNDWATER QUALITY DATA BY LOCATIOH

SITE: AMBOI AMBROSIA LAKE

LOCATION: 0791

NORTH COORDINATE: 55739.9 FT

EAST COORDIHATE: 60567.0 FT

O6/10/80 TO $12 / 04 / 92$

REPORT DATE: 08/04/94

FORMATION OF COHPLETION: TRES HERMANOS - CI SANDSTONE (TO)

HYDRAULIC FLOU RELATIOHSHIP: DOWH GRADIEKT (D)

\begin{tabular}{|c|c|c|c|c|c|c|c|}
\hline PARAMETER NAME & LOG DATE & $\underset{\text { ID }}{\text { SAMPLE }}$ & $\begin{array}{l}\text { UNITS OF } \\
\text { MEASURE }\end{array}$ & PVI & $\begin{array}{l}\text { PARAMETER } \\
\text { VALUE FLAGS }\end{array}$ & $\begin{array}{l}\text { DETECTION } \\
\text { LIMIT }\end{array}$ & $\begin{array}{l}\text { PARAMETER } \\
\text { UNCERTAINTY }\end{array}$ \\
\hline NITRATE & $\begin{array}{l}10 / 11 / 85 \\
05 / 21 / 86\end{array}$ & $\begin{array}{l}0001 \\
0001\end{array}$ & MG/L & & $\begin{array}{l}8 . \\
2 .\end{array}$ & $\begin{array}{l}1 . \\
1 .\end{array}$ & - \\
\hline MITRITE & $10 / 11 / 85$ & 0001 & MG/L & $<$ & 0.1 & 0.1 & - \\
\hline PH & $\begin{array}{l}10 / 11 / 85 \\
05 / 21 / 86\end{array}$ & $\begin{array}{l}0001 \\
0001\end{array}$ & su & & $\begin{array}{l}12.09 \\
12.46\end{array}$ & - & - \\
\hline PHOSPHATE & $10 / 11 / 85$ & 0001 & MG/L & & 0.4 & 0.1 & - \\
\hline POTASSIUH & $\begin{array}{l}10 / 11 / 85 \\
05 / 21 / 86\end{array}$ & $\begin{array}{l}0001 \\
0001\end{array}$ & MG/L & & $\begin{array}{l}22.3 \\
43.6\end{array}$ & $\begin{array}{l}0.01 \\
0.01\end{array}$ & - \\
\hline RADIUH-226 & $10 / 11 / 85$ & 0001 & $\mathrm{PCI} / \mathrm{L}$ & & 0.3 & 1. & 1.6 \\
\hline RADIUH-226 + RADIUH-228 & $10 / 11 / 85$ & 0001 & $\mathrm{PCI} / \mathrm{L}$ & & 1.50 & - & - \\
\hline RADIU:H-228 & $10 / 11 / 85$ & 0001 & PCI/L & & 1.2 & 1. & 3.8 \\
\hline SELEHIUH & $\begin{array}{l}10 / 11 / 85 \\
05 / 21 / 86\end{array}$ & $\begin{array}{l}0001 \\
0001\end{array}$ & MG/L & $<$ & $\begin{array}{l}0.016 \\
0.005\end{array}$ & $\begin{array}{l}0.005 \\
0.005\end{array}$ & - \\
\hline SILICA - SIOZ & $10 / 11 / 85$ & 0001 & $M G / L$ & & 6. & 2. & - \\
\hline SILVER & $10 / 11 / 85$ & 0001 & MG/L & $<$ & 0.01 & 0.01 & - \\
\hline SOOIUH & $\begin{array}{l}10 / 11 / 85 \\
05 / 21 / 86\end{array}$ & $\begin{array}{l}0001 \\
0001\end{array}$ & $M G / L$ & & $\begin{array}{l}690 . \\
1110 .\end{array}$ & $\begin{array}{l}0.002 \\
0.002\end{array}$ & - \\
\hline SPECIFIC COHDUCTAHCE & $\begin{array}{l}10 / 11 / 85 \\
05 / 21 / 86\end{array}$ & $\begin{array}{l}0001 \\
0001\end{array}$ & UHHO/CH & & $\begin{array}{l}3000 \\
8000\end{array}$ & - & 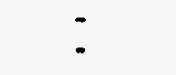 \\
\hline STROHT IUM & $\begin{array}{l}10 / 11 / 85 \\
05 / 21 / 86\end{array}$ & $\begin{array}{l}0001 \\
0001\end{array}$ & $\mathrm{HG} / \mathrm{L}$ & & $\begin{array}{l}1.5 \\
8.1\end{array}$ & $\begin{array}{l}0.1 \\
0.1\end{array}$ & - \\
\hline SULFATE & $\begin{array}{l}10 / 11 / 85 \\
05 / 21 / 86\end{array}$ & $\begin{array}{l}0001 \\
0001\end{array}$ & MG/L & & $\begin{array}{r}1200 . \\
240 .\end{array}$ & $\begin{array}{l}0.1 \\
0.1\end{array}$ & - \\
\hline SULFIDE & $10 / 11 / 85$ & 0001 & MG/L & $<$ & 0.1 & 0.1 & $\cdot$ \\
\hline TEMPERATURE & $\begin{array}{l}10 / 11 / 85 \\
05 / 21 / 86\end{array}$ & $\begin{array}{l}0001 \\
0001\end{array}$ & C - DEGREE & & $\begin{array}{l}11 . \\
15 .\end{array}$ & - & : \\
\hline THORIUN-230 & $10 / 11 / 85$ & 0001 & $\mathrm{PCI} / \mathrm{L}$ & & 1.1 & 1. & 2.5 \\
\hline TIN & $10 / 11 / 85$ & 0001 & $M G / L$ & $<$ & 0.005 & 0.005 & - \\
\hline TOTAL DISSOLVED SOLIOS & $\begin{array}{l}10 / 11 / 85 \\
05 / 21 / 86\end{array}$ & $\begin{array}{l}0001 \\
0001\end{array}$ & MG/L & & $\begin{array}{l}1950 . \\
3520 .\end{array}$ & $\begin{array}{l}10 . \\
10 .\end{array}$ & - \\
\hline URANIUA & $\begin{array}{l}10 / 11 / 85 \\
05 / 21 / 86\end{array}$ & $\begin{array}{l}0001 \\
0001\end{array}$ & $M G / L$ & $<$ & $\begin{array}{ll}0.0007 & \mathrm{~J} \\
0.0003 & \mathrm{~J}\end{array}$ & $\begin{array}{l}0.003 \\
0.003\end{array}$ & : \\
\hline VANADIUA & $\begin{array}{l}10 / 11 / 85 \\
05 / 21 / 86\end{array}$ & $\begin{array}{l}0001 \\
0001\end{array}$ & MG/L & $<$ & $\begin{array}{l}0.01 \\
0.3\end{array}$ & $\begin{array}{l}0.01 \\
0.01\end{array}$ & $\dot{-}$ \\
\hline ZINC & $10 / 11 / 85$ & 0001 & HG/L & $<$ & 0.005 & 0.005 & - \\
\hline
\end{tabular}

PARAMETER VALUE INDICATOR (PVI): < - LESS THAN DETECTION LIMIT

SAMPLE ID CODES:

0001 - FILTERED SAMPLE (.45 MICRONS)

OTHER PARAMETER VALUE FLAGS:

d- ESTIMATED VALUE 
GROUHDWATER QUALITY OATA BY LOCATION

SITE: AMBOI AMBROSIA LAKE

LOCATION: 0791

HORTH COORDIKATE: 55739.9 FT

EAST COORDINATE: 60567.0 FT

$06 / 10 / 80$ TO $12 / 04 / 92$

REPORT DATE: 08/04/94

FORMATION OF COMPLETION: TRES HERMANOS - C1 SAHDSTONE (TO) HYDRAULIC FLON RELATIOHSHIP: DOWN GRADIEHT (D)

\begin{tabular}{|l|l|l|l|l|l|l|l|}
\hline PARAMETER MAME & LOG DATE & $\begin{array}{c}\text { SAMPLE } \\
\text { ID }\end{array}$ & $\begin{array}{l}\text { UNITS OF } \\
\text { MEASURE }\end{array}$ & PVI & $\begin{array}{c}\text { PARAMETER } \\
\text { VALUE }\end{array}$ & $\begin{array}{c}\text { DETECTION } \\
\text { LIMIT }\end{array}$ & $\begin{array}{l}\text { PARAMETER } \\
\text { UHCERTAINTY }\end{array}$ \\
\hline
\end{tabular}

PARAMETER VALUE IndICATOR (PVI): < - LESS than DETECTION LIMIT SAMPLE ID COOES: 
GROUNDWATER QUALITY DATA BY LOCATION

SITE: AMBO1 AMBROSIA LAKE

LOCATIOH: 0792

MORTH COORDIHATE: 55724.9 FT -

EAST COORDIHATE: 60649.1 FT

06/10/80 To $12 / 04 / 92$

REPORT DATE: $08 / 04 / 94$

FORMATION OF COHPLETION: ALLUVIUN (AL)

HYDRAULIC FLON RELATIONSHIP: UPGRADIENT (U)

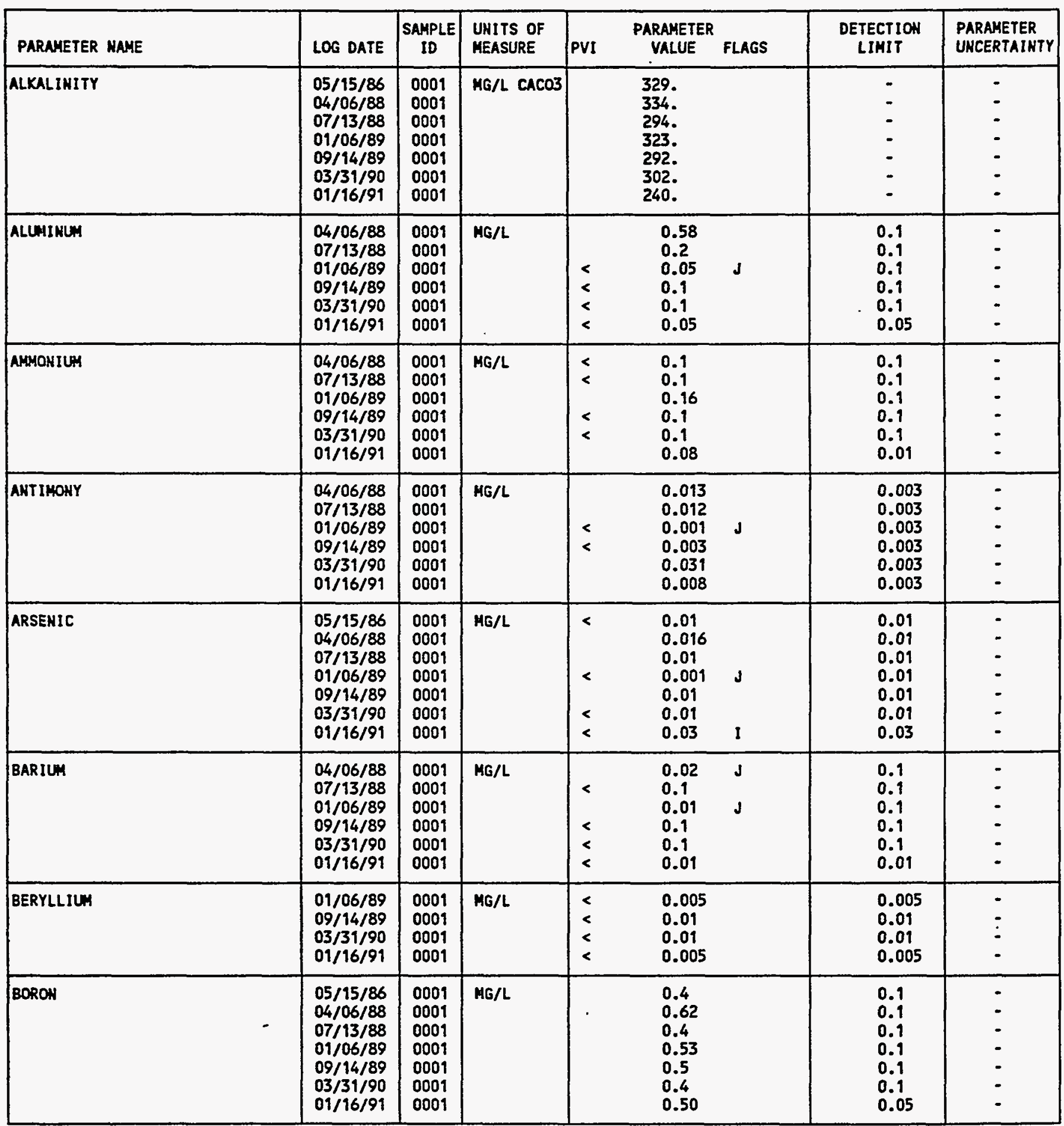

PARAMETER VALUE INDICATOR (PVI): < - LESS THAN DETECTION LIMIT

SAMPLE ID COOES:

OOO - FILTERED SAMPLE (.45 MICRONS)

OTHER PARAMETER VALUE FLAGS:

I - IHCREASED DETECTION LIMIT DUE TO REQUIRED DILUTION

- estimated value 
GROUNDHATER QUALITY DATA BY LOCATION

SITE: AMBOI AMBROSIA LAKE

LOCATIOH: 0792

NORTH COORDINATE: 55724.9 FT

EAST COORDINATE: 60649.1 FT

06/10/80 TO $12 / 04 / 92$

REPORT DATE: 08/04/94

FORHATION OF COMPLETIOH: ALLUVIUH (AL)

HYORAULIC FLON RELATIOHSHIP: UPGRADIENT (U)

\begin{tabular}{|c|c|c|c|c|c|c|c|c|}
\hline PARAMETER NAME & LOG DATE & $\begin{array}{c}\text { SAMPLE } \\
\text { ID }\end{array}$ & $\begin{array}{l}\text { UNITS OF } \\
\text { MEASURE }\end{array}$ & PVI & $\begin{array}{l}\text { PARAMETER } \\
\text { VALUE }\end{array}$ & FLAGS & $\begin{array}{l}\text { DETECTION } \\
\text { LIMIT }\end{array}$ & $\begin{array}{l}\text { PARAMETER } \\
\text { UHCERTAIHTY }\end{array}$ \\
\hline BROHIDE & $\begin{array}{l}04 / 06 / 88 \\
07 / 13 / 88 \\
01 / 06 / 89 \\
09 / 14 / 89 \\
03 / 31 / 90 \\
01 / 16 / 91\end{array}$ & $\begin{array}{l}0001 \\
0001 \\
0001 \\
0001 \\
0001 \\
0001\end{array}$ & MG/L & & $\begin{array}{l}0.3 \\
0.4 \\
0.70 \\
0.19 \\
0.3 \\
0.5\end{array}$ & & $\begin{array}{l}0.1 \\
0.1 \\
0.01 \\
0.1 \\
0.1 \\
0.1\end{array}$ & $\begin{array}{l}- \\
- \\
- \\
- \\
-\end{array}$ \\
\hline CADHIUM & $\begin{array}{l}05 / 15 / 86 \\
04 / 06 / 88 \\
07 / 13 / 88 \\
01 / 06 / 89 \\
09 / 14 / 89 \\
03 / 31 / 90 \\
01 / 16 / 91\end{array}$ & $\begin{array}{l}0001 \\
0001 \\
0001 \\
0001 \\
0001 \\
0001 \\
0001\end{array}$ & $M G / L$ & $\begin{array}{l}< \\
< \\
< \\
<\end{array}$ & $\begin{array}{l}0.001 \\
0.005 \\
0.001 \\
0.0001 \\
0.010 \\
0.001 \\
0.001\end{array}$ & $\boldsymbol{J}$ & $\begin{array}{l}0.001 \\
0.001 \\
0.001 \\
0.001 \\
0.001 \\
0.001 \\
0.001\end{array}$ & $\begin{array}{l}- \\
- \\
- \\
- \\
- \\
-\end{array}$ \\
\hline CALCIUM & $\begin{array}{l}05 / 15 / 86 \\
04 / 06 / 88 \\
07 / 13 / 88 \\
01 / 06 / 89 \\
09 / 14 / 89 \\
03 / 31 / 90 \\
01 / 16 / 91\end{array}$ & $\begin{array}{l}0001 \\
0001 \\
0001 \\
0001 \\
0001 \\
0001 \\
0001\end{array}$ & $M G / L$ & & $\begin{array}{l}502 . \\
712 . \\
524 . \\
500 . \\
481 . \\
417 . \\
484 .\end{array}$ & & $\begin{array}{l}0.01 \\
0.01 \\
0.01 \\
0.01 \\
0.01 \\
0.01 \\
0.5\end{array}$ & $\begin{array}{l}- \\
- \\
- \\
- \\
-\end{array}$ \\
\hline CHLORIDE & $\begin{array}{l}05 / 15 / 86 \\
04 / 06 / 88 \\
07 / 13 / 88 \\
01 / 06 / 89 \\
09 / 14 / 89 \\
03 / 31 / 90 \\
01 / 16 / 91\end{array}$ & $\begin{array}{l}0001 \\
0001 \\
0001 \\
0001 \\
0001 \\
0001 \\
0001\end{array}$ & $\mathrm{HG} / \mathrm{L}$ & & $\begin{array}{l}180 . \\
190 . \\
190 . \\
85 . \\
140 . \\
92 . \\
110 .\end{array}$ & & $\begin{array}{l}1 . \\
1 . \\
1 . \\
1 . \\
1 . \\
1 . \\
0.5\end{array}$ & $\begin{array}{l}- \\
- \\
- \\
- \\
-\end{array}$ \\
\hline CHROMIUM & $\begin{array}{l}05 / 15 / 86 \\
04 / 06 / 88 \\
07 / 13 / 88 \\
01 / 06 / 89 \\
09 / 14 / 89 \\
03 / 31 / 90 \\
01 / 16 / 91\end{array}$ & $\begin{array}{l}0001 \\
0001 \\
0001 \\
0001 \\
0001 \\
0001 \\
0001\end{array}$ & MG/L & $\begin{array}{l}< \\
< \\
<\end{array}$ & $\begin{array}{l}0.04 \\
0.28 \\
0.14 \\
0.01 \\
0.01 \\
0.01 \\
0.01\end{array}$ & & $\begin{array}{l}0.01 \\
0.01 \\
0.01 \\
0.01 \\
0.01 \\
0.01 \\
0.01\end{array}$ & $\begin{array}{l}- \\
- \\
- \\
- \\
-\end{array}$ \\
\hline COBALT & $\begin{array}{l}05 / 15 / 86 \\
04 / 06 / 88 \\
07 / 13 / 88 \\
01 / 06 / 89 \\
09 / 14 / 89 \\
03 / 31 / 90 \\
01 / 16 / 91\end{array}$ & $\begin{array}{l}0001 \\
0001 \\
0001 \\
0001 \\
0001 \\
0001 \\
0001\end{array}$ & $M G / L$ & $\begin{array}{l}< \\
< \\
< \\
< \\
<\end{array}$ & $\begin{array}{l}0.11 \\
0.12 \\
0.05 \\
0.01 \\
0.05 \\
0.05 \\
0.03\end{array}$ & $\mathbf{J}$ & $\begin{array}{l}0.05 \\
0.05 \\
0.05 \\
0.05 \\
0.05 \\
0.05 \\
0.03\end{array}$ & $\begin{array}{l}- \\
- \\
- \\
- \\
-\end{array}$ \\
\hline COPPER & $\begin{array}{l}04 / 06 / 88 \\
07 / 13 / 88 \\
01 / 06 / 89 \\
09 / 14 / 89 \\
03 / 31 / 90 \\
01 / 16 / 91\end{array}$ & $\begin{array}{l}0001 \\
0001 \\
0001 \\
0001 \\
0001 \\
0001\end{array}$ & $M G / L$ & $\begin{array}{l}< \\
< \\
< \\
<\end{array}$ & $\begin{array}{l}0.06 \\
0.03 \\
0.01 \\
0.02 \\
0.02 \\
0.01\end{array}$ & $\boldsymbol{J}$ & $\begin{array}{l}0.02 \\
0.02 \\
0.02 \\
0.02 \\
0.02 \\
0.01\end{array}$ & $\begin{array}{l}- \\
- \\
- \\
- \\
-\end{array}$ \\
\hline CYAHIDE & $\begin{array}{l}01 / 06 / 89 \\
09 / 14 / 89 \\
03 / 31 / 90\end{array}$ & $\begin{array}{l}0001 \\
0001 \\
0001\end{array}$ & MG/L & $\begin{array}{l}< \\
< \\
<\end{array}$ & $\begin{array}{l}0.002 \\
0.01 \\
0.01\end{array}$ & $\boldsymbol{J}$ & $\begin{array}{l}0.01 \\
0.01 \\
0.01\end{array}$ & 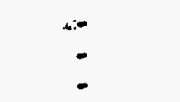 \\
\hline
\end{tabular}

PARAMETER VALUE INDICATOR (PVI): < - LESS THAN DETECTION LIMIT SAMLE ID (OODES:

0001 - FILVERED SAMPLE (.45 MICROHS)

OTHER PARAMETER VALUE FLAGS:

J ESTIMATED VALUE 
GROUHDWATER QUALITY DATA BY LOCATION

SITE: AMBOI AMBROSIA LAKE

LOCATION: 0792

NORTH COORDIHATE: 55724.9 FT

EAST COORDINATE: 60649.1 FT

$06 / 10 / 80$ TO $12 / 04 / 92$

REPORT DATE: 08/04/94

FORMATION OF COMPLETION: ALLUVIUM (AL)

HYDRAULIC FLOW RELATIOHSHIP: UPGRADIENT (U)

\begin{tabular}{|c|c|c|c|c|c|c|c|}
\hline PARAMETER NAME & LOG DATE & $\underset{\text { SAMPLE }}{\text { ID }}$ & $\begin{array}{l}\text { UNITS OF } \\
\text { MEASURE }\end{array}$ & PVI & $\begin{array}{l}\text { PARAMETER } \\
\text { VALUE FLAGS }\end{array}$ & $\begin{array}{l}\text { DETECTION } \\
\text { LIHIT }\end{array}$ & $\begin{array}{l}\text { PARAMETER } \\
\text { UHCERTAINTY }\end{array}$ \\
\hline CYANIDE & $01 / 16 / 91$ & 0001 & $M G / L$ & $<$ & 0.01 & 0.01 & - \\
\hline FLUORIDE & $\begin{array}{l}05 / 15 / 86 \\
04 / 06 / 88 \\
07 / 13 / 88 \\
01 / 06 / 89 \\
09 / 14 / 89 \\
03 / 31 / 90 \\
01 / 16 / 91\end{array}$ & $\begin{array}{l}0001 \\
0001 \\
0001 \\
0001 \\
0001 \\
0001 \\
0001\end{array}$ & $M G / L$ & & $\begin{array}{l}1.1 \\
1.05 \\
0.9 \\
1.0 \\
0.9 \\
0.9 \\
1.2\end{array}$ & $\begin{array}{l}0.1 \\
0.1 \\
0.1 \\
0.1 \\
0.1 \\
0.1 \\
0.1\end{array}$ & $\begin{array}{l}- \\
- \\
- \\
- \\
-\end{array}$ \\
\hline GROSS ALPHA & $\begin{array}{l}04 / 06 / 88 \\
07 / 13 / 88 \\
01 / 06 / 89 \\
09 / 14 / 89 \\
03 / 31 / 90\end{array}$ & $\begin{array}{l}0001 \\
0001 \\
0001 \\
0001 \\
0001\end{array}$ & $\mathrm{PCI} / \mathrm{L}$ & & $\begin{array}{c}2400 \\
1800 \\
1090 \\
560 \\
1400\end{array}$ & $\begin{array}{l}0.2 \\
0.2 \\
1 . \\
1 . \\
1 .\end{array}$ & $\begin{array}{c}10 . \\
100 . \\
91.1 \\
40 . \\
100\end{array}$ \\
\hline GROSS BETA & $\begin{array}{l}04 / 06 / 88 \\
07 / 13 / 88 \\
01 / 06 / 89 \\
09 / 14 / 89 \\
03 / 31 / 90\end{array}$ & $\begin{array}{l}0001 \\
0001 \\
0001 \\
0001 \\
0001\end{array}$ & $\mathrm{PCI} / \mathrm{L}$ & & $\begin{array}{l}790 . \\
670 \\
780 \\
280 \\
670\end{array}$ & $\begin{array}{l}1 . \\
1 . \\
0.5 \\
0.5 \\
0.5\end{array}$ & $\begin{array}{l}30 . \\
40 . \\
52.0 \\
20 . \\
30 .\end{array}$ \\
\hline IRON & $\begin{array}{l}05 / 15 / 86 \\
04 / 06 / 88 \\
07 / 13 / 88 \\
01 / 06 / 89 \\
09 / 14 / 89 \\
03 / 31 / 90 \\
01 / 16 / 91\end{array}$ & $\begin{array}{l}0001 \\
0001 \\
0001 \\
0001 \\
0001 \\
0001 \\
0001\end{array}$ & MG/L & $<$ & $\begin{array}{l}0.04 \\
0.18 \\
0.08 \\
0.02 \\
0.08 \\
0.25 \\
0.07\end{array}$ & $\begin{array}{l}0.03 \\
0.03 \\
0.03 \\
0.03 \\
0.03 \\
0.03 \\
0.03\end{array}$ & $\begin{array}{l}- \\
- \\
- \\
- \\
- \\
- \\
-\end{array}$ \\
\hline LEAD & $\begin{array}{l}04 / 06 / 88 \\
07 / 13 / 88 \\
01 / 06 / 89 \\
09 / 14 / 89 \\
03 / 31 / 90 \\
01 / 16 / 91\end{array}$ & $\begin{array}{l}0001 \\
0001 \\
0001 \\
0001 \\
0001 \\
0001\end{array}$ & $M G / L$ & $\begin{array}{l}< \\
< \\
< \\
<\end{array}$ & $\begin{array}{l}0.01 \\
0.01 \\
0.001 \\
0.02 \\
0.01 \\
0.03\end{array}$ & $\begin{array}{l}0.01 \\
0.01 \\
0.01 \\
0.01 \\
0.01 \\
0.03\end{array}$ & $\begin{array}{l}- \\
- \\
- \\
- \\
-\end{array}$ \\
\hline LEAD-210 & $\begin{array}{l}04 / 06 / 88 \\
07 / 13 / 88 \\
01 / 06 / 89 \\
09 / 14 / 89\end{array}$ & $\begin{array}{l}0001 \\
0001 \\
0001 \\
0001\end{array}$ & PCI/L. & & $\begin{array}{l}2.8 \\
0.8 \\
0.0818 \\
0.4\end{array}$ & $\begin{array}{l}1.5 \\
1.5 \\
1.5 \\
1.5\end{array}$ & $\begin{array}{l}1.0 \\
1.2 \\
5.22 \\
0.7\end{array}$ \\
\hline MAGNESIUM & $\begin{array}{l}05 / 15 / 86 \\
04 / 06 / 88 \\
07 / 13 / 88 \\
01 / 06 / 89 \\
09 / 14 / 89 \\
03 / 31 / 90 \\
01 / 16 / 91\end{array}$ & $\begin{array}{l}0001 \\
0001 \\
0001 \\
0001 \\
0001 \\
0001 \\
0001\end{array}$ & $M G / L$ & & $\begin{array}{l}82.5 \\
96.0 \\
103 . \\
102 . \\
105 . \\
101 . \\
143 .\end{array}$ & $\begin{array}{l}0.001 \\
0.001 \\
0.001 \\
0.001 \\
0.001 \\
0.001 \\
0.5\end{array}$ & $\begin{array}{l}- \\
- \\
- \\
- \\
- \\
-\end{array}$ \\
\hline MANGANESE & $\begin{array}{l}05 / 15 / 86 \\
04 / 06 / 88 \\
07 / 13 / 88 \\
01 / 06 / 89 \\
09 / 14 / 89 \\
03 / 31 / 90\end{array}$ & $\begin{array}{l}0001 \\
0001 \\
0001 \\
0001 \\
0001 \\
0001\end{array}$ & MG/L & $\begin{array}{l}< \\
<\end{array}$ & $\begin{array}{l}0.05 \\
0.04 \\
0.03 \\
0.01 \\
0.01 \\
0.01\end{array}$ & $\begin{array}{l}0.01 \\
0.01 \\
0.01 \\
0.01 \\
0.01 \\
0.01\end{array}$ & $\begin{array}{l}- \\
- \\
- \\
-\end{array}$ \\
\hline
\end{tabular}

PARAMETER VALUE INDICATOR (PVI): < - LESS THAN DETECTION LIMIT

OTHER PARAMETER VALUE FLAGS:

1 - INCREASED DETECTION LIMIT DUE TO REOUIRED DILUTION

d - estimated VALUE 
GROUNDHATER QUALITY DATA BY LOCATIOH

SITE: AMBO1 AMBROSIA LAKE

LOCATION: 0792

MORTH COORDINATE: 55724.9 FT

EAST COORDIHATE: 60649.1 FT

06/10/80 TO 12/04/92

REPORT DATE: 08/04/94

FORMATION OF COMPLETIOH: ALLUVIUH (AL)

HYDRAULIC FLON RELATIONSHIP: UPGRADIENT (U)

\begin{tabular}{|c|c|c|c|c|c|c|c|c|}
\hline PARAMETER NAME & LOG DATE & $\begin{array}{c}\text { SAMPLE } \\
\text { ID }\end{array}$ & $\begin{array}{l}\text { UNITS OF } \\
\text { MEASURE }\end{array}$ & PVI & $\begin{array}{l}\text { PARAMETER } \\
\text { VALUE }\end{array}$ & FLAGS & $\begin{array}{l}\text { DETECTION } \\
\text { LIMIT }\end{array}$ & $\begin{array}{l}\text { PARAMETER } \\
\text { UNCERTAINTY }\end{array}$ \\
\hline MANGAHESE & $01 / 16 / 91$ & 0001 & $\mathrm{MG} / \mathrm{L}$ & & 0.01 & & 0.01 & - \\
\hline MERCURY & $\begin{array}{l}04 / 06 / 88 \\
07 / 13 / 88 \\
01 / 06 / 89 \\
09 / 14 / 89 \\
03 / 31 / 90 \\
01 / 16 / 91\end{array}$ & $\begin{array}{l}0001 \\
0001 \\
0001 \\
0001 \\
0001 \\
0001\end{array}$ & MG/L & $\begin{array}{l}< \\
< \\
< \\
<\end{array}$ & $\begin{array}{l}0.0007 \\
0.000 ? \\
0.0001 \\
0.0002 \\
0.0002 \\
0.000 ?\end{array}$ & J & $\begin{array}{l}0.0002 \\
0.0002 \\
0.0002 \\
0.0002 \\
0.0002 \\
0.0002\end{array}$ & $\begin{array}{l}- \\
- \\
- \\
- \\
-\end{array}$ \\
\hline MOLYBDENUY & $\begin{array}{l}05 / 15 / 86 \\
04 / 06 / 88 \\
07 / 13 / 88 \\
01 / 06 / 89 \\
09 / 14 / 89 \\
03 / 31 / 90 \\
01 / 16 / 91\end{array}$ & $\begin{array}{l}0001 \\
0001 \\
0001 \\
0001 \\
0001 \\
0001 \\
0001\end{array}$ & MG/L & & $\begin{array}{l}1.87 \\
1.63 \\
1.26 \\
1.410 \\
1.19 \\
1.25 \\
1.17\end{array}$ & & $\begin{array}{l}0.01 \\
0.01 \\
0.01 \\
0.01 \\
0.01 \\
0.01 \\
0.01\end{array}$ & $\begin{array}{r}- \\
- \\
- \\
- \\
-\end{array}$ \\
\hline NET GROSS ALPHA * & $\begin{array}{l}04 / 06 / 88 \\
07 / 13 / 88 \\
01 / 06 / 89 \\
09 / 14 / 89 \\
03 / 31 / 90\end{array}$ & $\begin{array}{l}0001 \\
0001 \\
0001 \\
0001 \\
0001\end{array}$ & $\mathrm{PCI} / \mathrm{L}$ & & $\begin{array}{r}300.84 \\
-45.34 \\
-590.70 \\
-256.34 \\
199.50\end{array}$ & & $\begin{array}{l}- \\
- \\
- \\
-\end{array}$ & $\begin{array}{l}: \\
: \\
-\end{array}$ \\
\hline HICKEL & $\begin{array}{l}04 / 06 / 88 \\
07 / 13 / 88 \\
01 / 06 / 89 \\
09 / 14 / 89 \\
03 / 31 / 90 \\
01 / 16 / 91\end{array}$ & $\begin{array}{l}0001 \\
0001 \\
0001 \\
0001 \\
0001 \\
0001\end{array}$ & MG/L & $\begin{array}{l}< \\
< \\
<\end{array}$ & $\begin{array}{l}0.13 \\
0.04 \\
0.02 \\
0.04 \\
0.04 \\
0.04\end{array}$ & J & $\begin{array}{l}0.04 \\
0.04 \\
0.04 \\
0.04 \\
0.04 \\
0.04\end{array}$ & $\begin{array}{l}: \\
\dot{-} \\
-\end{array}$ \\
\hline MITRATE & $\begin{array}{l}05 / 15 / 86 \\
04 / 06 / 88 \\
07 / 13 / 88 \\
01 / 06 / 89 \\
09 / 14 / 89 \\
03 / 31 / 90 \\
01 / 16 / 91\end{array}$ & $\begin{array}{l}0001 \\
0001 \\
0001 \\
0001 \\
0001 \\
0001 \\
0001\end{array}$ & $M G / L$ & $\begin{array}{l}< \\
<\end{array}$ & $\begin{array}{l}1 . \\
1.6 \\
1.8 \\
0.2 \\
1.1 \\
0.9 \\
1.3\end{array}$ & $\begin{array}{l}j \\
j \\
J\end{array}$ & $\begin{array}{l}1 . \\
1 . \\
1 . \\
1 . \\
1 . \\
1.1\end{array}$ & $\begin{array}{l}- \\
- \\
- \\
- \\
-\end{array}$ \\
\hline MITRITE AND NITRATE & $\begin{array}{l}04 / 06 / 88 \\
03 / 31 / 90 \\
01 / 16 / 91\end{array}$ & $\begin{array}{l}0001 \\
0001 \\
0001\end{array}$ & MG/L & & $\begin{array}{l}1.6 \\
0.2 \\
0.31\end{array}$ & $\mathbf{J}$ & $\begin{array}{l}1 . \\
1.05 \\
0.05\end{array}$ & : \\
\hline $\mathrm{PH}$ & $\begin{array}{l}05 / 15 / 86 \\
04 / 06 / 88 \\
07 / 13 / 88 \\
01 / 06 / 89 \\
09 / 14 / 89 \\
03 / 31 / 90 \\
01 / 16 / 91\end{array}$ & $\begin{array}{l}0001 \\
0001 \\
0001 \\
0001 \\
0001 \\
0001 \\
0001\end{array}$ & SU & & $\begin{array}{l}7.35 \\
7.13 \\
7.08 \\
7.11 \\
7.05 \\
7.05 \\
7.54\end{array}$ & & $\begin{array}{l}- \\
- \\
- \\
- \\
- \\
-\end{array}$ & $\begin{array}{l}- \\
\vdots \\
\vdots \\
-\end{array}$ \\
\hline PHOSPHATE & $\begin{array}{l}04 / 06 / 88 \\
07 / 13 / 88 \\
01 / 06 / 89 \\
09 / 14 / 89 \\
03 / 31 / 90\end{array}$ & $\begin{array}{l}0001 \\
0001 \\
0001 \\
0001 \\
0001\end{array}$ & $M G / L$ & $\begin{array}{l}< \\
< \\
<\end{array}$ & $\begin{array}{l}0.1 \\
0.1 \\
0.1 \\
0.1 \\
0.1\end{array}$ & & $\begin{array}{l}0.1 \\
0.1 \\
0.1 \\
0.1 \\
0.1\end{array}$ & : \\
\hline
\end{tabular}

- het gross alPha (GROSS alPHa - URAhIUM) hith 1 mg URAMIUH = 686 PCI

PARAMETER VALUE IHDICATOR (PVI): < - LESS THAH DETECTION LIMIT 
GROUNDWATER QUALITY DATA BY LOCATIOH

SITE: AMBOI AMBROSIA LAKE

LOCATION: 0792

NORTH COORDINATE: 55724.9 FT

EAST COORDINATE: $\quad 60649.1$ FT

O6/10/80 TO $12 / 04 / 92$

REPORT DATE: 08/04/94

FORMATION OF COMPLETIOH: ALLUVIUN (AL)

HYDRAULIC FLOW RELATIONSHIP: UPGRADIENT (U)

\begin{tabular}{|c|c|c|c|c|c|c|c|}
\hline PARAMETER MAME & LOG DATE & $\begin{array}{c}\text { SAMPLE } \\
\text { ID }\end{array}$ & $\begin{array}{l}\text { UNITS OF } \\
\text { MEASURE }\end{array}$ & PVI & $\begin{array}{l}\text { PARAMETER } \\
\text { VALUE FLAGS }\end{array}$ & $\begin{array}{l}\text { DETECTIOH } \\
\text { LIMIT }\end{array}$ & $\begin{array}{l}\text { PARAMETER } \\
\text { UNCERTAIHTY }\end{array}$ \\
\hline PHOSPHATE & $01 / 16 / 91$ & 0001 & HG/L & & 0.05 & 0.05 & - \\
\hline POLONIUH-210 & $\begin{array}{l}04 / 06 / 88 \\
07 / 13 / 88 \\
01 / 06 / 89 \\
09 / 14 / 89\end{array}$ & $\begin{array}{l}0001 \\
0001 \\
0001 \\
0001\end{array}$ & $\mathrm{PCI} / \mathrm{L}$ & & $\begin{array}{l}2.1 \\
1.7 \\
0.196 \\
2.3\end{array}$ & $\begin{array}{l}1 . \\
1 . \\
1 . \\
1 .\end{array}$ & $\begin{array}{l}0.7 \\
0.7 \\
1.49 \\
0.6\end{array}$ \\
\hline POTASSIUH & $\begin{array}{l}05 / 15 / 86 \\
04 / 06 / 88 \\
07 / 13 / 88 \\
01 / 06 / 89 \\
09 / 14 / 89 \\
03 / 31 / 90 \\
01 / 16 / 91\end{array}$ & $\begin{array}{l}0001 \\
0001 \\
0001 \\
0001 \\
0001 \\
0001 \\
0001\end{array}$ & MG/L & & $\begin{array}{l}1.01 \\
0.62 \\
0.61 \\
0.8 \\
0.7 \\
0.8 \\
0.89\end{array}$ & $\begin{array}{l}0.01 \\
0.01 \\
0.01 \\
0.01 \\
0.01 \\
0.01 \\
0.01\end{array}$ & $\begin{array}{l}- \\
- \\
- \\
- \\
-\end{array}$ \\
\hline RADIUA-226 & $\begin{array}{l}05 / 15 / 86 \\
04 / 06 / 88 \\
07 / 13 / 88 \\
01 / 06 / 89 \\
09 / 14 / 89 \\
03 / 31 / 90\end{array}$ & $\begin{array}{l}0001 \\
0001 \\
0001 \\
0001 \\
0001 \\
0001\end{array}$ & $\mathrm{PCI} / \mathrm{L}$ & & $\begin{array}{l}0.8 \\
0.2 \\
0.4 \\
0.751 \\
0.1 \\
0.8\end{array}$ & $\begin{array}{l}1 . \\
1 . \\
1 . \\
1 .\end{array}$ & $\begin{array}{l}0.4 \\
0.2 \\
0.2 \\
0.309 \\
0.1 \\
0.3\end{array}$ \\
\hline RADIUN-226 + RADIUA-228 & $\begin{array}{l}04 / 06 / 88 \\
07 / 13 / 88 \\
01 / 06 / 89 \\
09 / 14 / 89 \\
03 / 31 / 90\end{array}$ & $\begin{array}{l}0001 \\
0001 \\
0001 \\
0001 \\
0001\end{array}$ & PCI/L & & $\begin{array}{l}0.30 \\
0.50 \\
6.85 \\
0.20 \\
2.50\end{array}$ & $\begin{array}{l}- \\
- \\
-\end{array}$ & $\begin{array}{l}- \\
- \\
-\end{array}$ \\
\hline RADIUH-228 & $\begin{array}{l}04 / 06 / 88 \\
07 / 13 / 88 \\
01 / 06 / 89 \\
09 / 14 / 89 \\
03 / 31 / 90\end{array}$ & $\begin{array}{l}0009 \\
0001 \\
0001 \\
0001 \\
0001\end{array}$ & $\mathrm{PCI} / \mathrm{L}$ & & $\begin{array}{l}0.1 \\
0.1 \\
6.10 \\
0.1 \\
1.7\end{array}$ & $\begin{array}{l}1 . \\
1 . \\
1 . \\
1 .\end{array}$ & $\begin{array}{l}0.8 \\
0.7 \\
4.20 \\
0.9 \\
0.9\end{array}$ \\
\hline SELEHIUN & $\begin{array}{l}05 / 15 / 86 \\
04 / 06 / 88 \\
07 / 13 / 88 \\
01 / 06 / 89 \\
09 / 14 / 89 \\
03 / 31 / 90 \\
01 / 16 / 91\end{array}$ & $\begin{array}{l}0001 \\
0001 \\
0001 \\
0001 \\
0001 \\
0001 \\
0001\end{array}$ & HG/L & $<$ & $\begin{array}{l}0.005 \\
0.889 \\
0.767 \\
1.800 \\
1.62 \\
2.22 \\
0.950\end{array}$ & $\begin{array}{l}0.005 \\
0.005 \\
0.005 \\
0.005 \\
0.005 \\
0.005 \\
0.005\end{array}$ & $\begin{array}{l}- \\
- \\
- \\
- \\
-\end{array}$ \\
\hline SILICA - SIOZ & $\begin{array}{l}04 / 06 / 88 \\
07 / 13 / 88 \\
01 / 06 / 89 \\
09 / 14 / 89 \\
03 / 31 / 90 \\
01 / 16 / 91\end{array}$ & $\begin{array}{l}0001 \\
0001 \\
0001 \\
0001 \\
0001 \\
0001\end{array}$ & HG/L & & $\begin{array}{l}19.7 \\
15.9 \\
15.5 \\
17 . \\
15 . \\
14.3\end{array}$ & $\begin{array}{l}2 . \\
2 . \\
2 . \\
2 . \\
2 . \\
0.1\end{array}$ & $\begin{array}{l}: \\
: \\
:\end{array}$ \\
\hline SILVER & $\begin{array}{l}04 / 06 / 88 \\
07 / 13 / 88 \\
01 / 06 / 89 \\
09 / 14 / 89 \\
03 / 31 / 90 \\
01 / 16 / 91\end{array}$ & $\begin{array}{l}0001 \\
0001 \\
0001 \\
0001 \\
0001 \\
0001\end{array}$ & HG/L & $\begin{array}{l}< \\
< \\
< \\
<\end{array}$ & $\begin{array}{l}0.11 \\
0.03 \\
0.01 \\
0.01 \\
0.01 \\
0.01\end{array}$ & $\begin{array}{l}0.01 \\
0.01 \\
0.01 \\
0.01 \\
0.01 \\
0.01\end{array}$ & $\begin{array}{l}- \\
- \\
- \\
-\end{array}$ \\
\hline soorus & $\begin{array}{l}05 / 15 / 86 \\
04 / 06 / 88\end{array}$ & $\begin{array}{l}0001 \\
0001\end{array}$ & $M G / L$ & & $\begin{array}{l}730 . \\
369 .\end{array}$ & $\begin{array}{l}0.002 \\
0.002\end{array}$ & - \\
\hline
\end{tabular}

PARAMETER VALUE IMDICATOR (PVI): < - LESS THAH DETECTION LIMIT SAMPLE ID COOES:

D001 - FILTERED SAMPLE (.45 MICROHS) 
GROUMDWATER QUALITY DATA BY LOCATION

SITE: AMBO1 AMBROSIA LAKE

LOCATIOH: 0792

MORTH COORDIHATE: 55724.9 FT

EAST COORDIHATE: 60649.1 FT

06/10/80 TO 12/04/92

REPORT DATE: 08/04/94

FORMATION OF COMPLETION: ALLUVIUH (AL)

HYDRAULIC FLOH RELATIONSHIP: UPGRADIEHT (U)

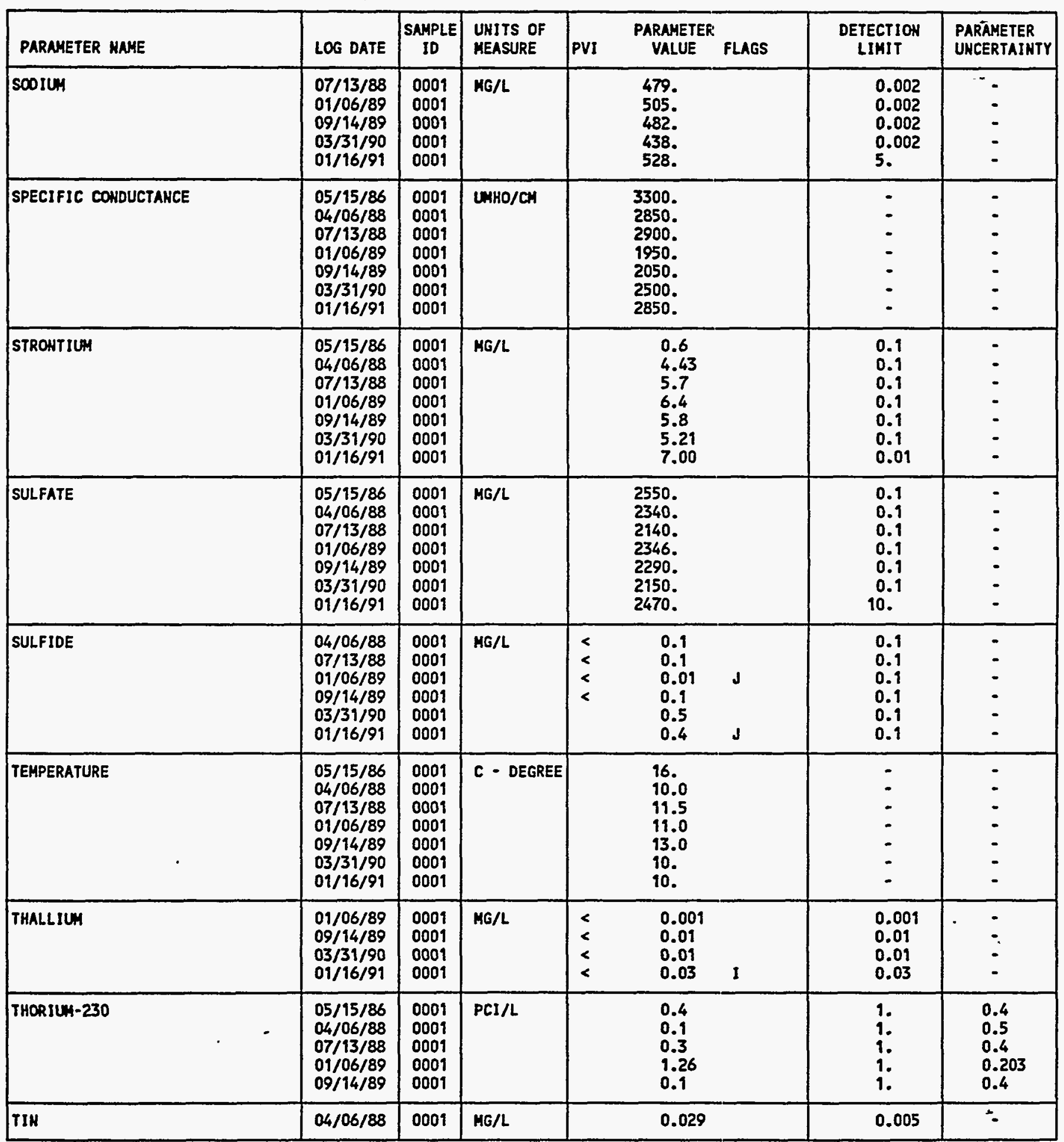

PARAMETER VALUE IMDICATOR (PVI): \& - LESS THAH DETECTION LIHIT 
GROUNDHATER QUALITY DATA BY LOCATION

SITE: AMBOI AMBROSIA LAKE

LOCATION: 0792

MORTH COORDINATE: 55724.9 FT

EAST COORDINATE: $\quad 60649.1$ FT

06/10/80 TO $12 / 04 / 92$

REPORT DATE: $08 / 04 / 94$

FORMATION OF COAPLETION: ALLUVIUN (AL)

HYDRAULIC FLON RELATIONSHIP: UPGRADIENT (U)

\begin{tabular}{|c|c|c|c|c|c|c|c|}
\hline PARAHETER NAME & LOG DATE & $\underset{10}{\text { SAMPLE }}$ & $\begin{array}{l}\text { UNITS OF } \\
\text { MEASURE }\end{array}$ & PVI & $\begin{array}{l}\text { PARAMETER } \\
\text { VALUE FLAGS }\end{array}$ & $\begin{array}{l}\text { DETECTION } \\
\text { LIMIT }\end{array}$ & $\begin{array}{l}\text { PARAMETER } \\
\text { UHCERTAIHTY }\end{array}$ \\
\hline TIN & $\begin{array}{l}07 / 13 / 88 \\
01 / 06 / 89 \\
09 / 14 / 89 \\
03 / 31 / 90 \\
01 / 16 / 91\end{array}$ & $\begin{array}{l}0001 \\
0001 \\
0001 \\
0001 \\
0001\end{array}$ & MG/L & $\begin{array}{l}< \\
<\end{array}$ & $\begin{array}{l}0.014 \\
0.005 \\
0.1 \\
0.014 \\
0.03\end{array}$ & $\begin{array}{l}0.005 \\
0.005 \\
0.9 \\
0.005 \\
0.03\end{array}$ & $\begin{array}{l}- \\
- \\
-\end{array}$ \\
\hline TOTAL DISSOLVED SOLIDS & $\begin{array}{l}05 / 15 / 86 \\
04 / 06 / 88 \\
07 / 13 / 88 \\
01 / 06 / 89 \\
09 / 14 / 89 \\
03 / 31 / 90 \\
01 / 16 / 91\end{array}$ & $\begin{array}{l}0001 \\
0001 \\
0001 \\
0001 \\
0001 \\
0001 \\
0001\end{array}$ & MG/L & & $\begin{array}{l}4110 . \\
4100 \\
4180 \\
3782 \\
3750 \\
3360 \\
4120\end{array}$ & $\begin{array}{l}10 . \\
10 . \\
10 . \\
10 . \\
10 . \\
10 . \\
10 .\end{array}$ & $\begin{array}{l}- \\
- \\
- \\
-\end{array}$ \\
\hline TOTAL KJELDAHL HITROGEN & $01 / 16 / 91$ & 0001 & MG/L & & 1. & 1. & - \\
\hline TOTAL ORGANIC CARBOH & $\begin{array}{l}04 / 06 / 88 \\
07 / 13 / 88 \\
01 / 06 / 89 \\
09 / 14 / 89 \\
03 / 31 / 90 \\
01 / 16 / 91\end{array}$ & $\begin{array}{l}0001 \\
0001 \\
0001 \\
0001 \\
0001 \\
0001\end{array}$ & MG/L & & $\begin{array}{c}95.7 \\
75.8 \\
7 . \\
79.6 \\
8.2 \\
6 .\end{array}$ & $\begin{array}{l}1 . \\
1 . \\
1 . \\
1 . \\
1 .\end{array}$ & $\begin{array}{l}- \\
- \\
- \\
-\end{array}$ \\
\hline URAKIUM & $\begin{array}{l}05 / 15 / 86 \\
04 / 06 / 88 \\
07 / 13 / 88 \\
01 / 06 / 89 \\
09 / 14 / 89 \\
03 / 31 / 90 \\
01 / 16 / 91\end{array}$ & $\begin{array}{l}0001 \\
0001 \\
0001 \\
0001 \\
0001 \\
0001 \\
0001\end{array}$ & $M G / L$ & & $\begin{array}{l}3.31 \\
3.06 \\
2.69 \\
2.450 \\
1.19 \\
1.75 \\
2.25\end{array}$ & $\begin{array}{l}0.003 \\
0.003 \\
0.003 \\
0.003 \\
0.003 \\
0.003 \\
0.001\end{array}$ & $\begin{array}{l}- \\
- \\
- \\
- \\
-\end{array}$ \\
\hline VAHADIUM & $\begin{array}{l}05 / 15 / 86 \\
04 / 06 / 88 \\
07 / 13 / 88 \\
01 / 06 / 89 \\
09 / 14 / 89 \\
03 / 31 / 90 \\
01 / 16 / 91\end{array}$ & $\begin{array}{l}0001 \\
0001 \\
0001 \\
0001 \\
0001 \\
0001 \\
0001\end{array}$ & MG/L & $\begin{array}{l}< \\
< \\
< \\
<\end{array}$ & $\begin{array}{l}0.26 \\
0.15 \\
0.06 \\
0.01 \\
0.01 \\
0.01 \\
0.01\end{array}$ & $\begin{array}{l}0.01 \\
0.01 \\
0.01 \\
0.01 \\
0.01 \\
0.01 \\
0.01\end{array}$ & $\begin{array}{l}: \\
: \\
: \\
-\end{array}$ \\
\hline ZIHC & $\begin{array}{l}04 / 06 / 88 \\
07 / 13 / 88 \\
01 / 06 / 89 \\
09 / 14 / 89 \\
03 / 31 / 90 \\
01 / 16 / 91\end{array}$ & $\begin{array}{l}0001 \\
0001 \\
0001 \\
0001 \\
0001 \\
0001\end{array}$ & $M G / L$ & $<$ & $\begin{array}{l}0.070 \\
0.016 \\
0.01 \\
0.005 \\
0.016 \\
0.024\end{array}$ & $\begin{array}{l}0.005 \\
0.005 \\
0.01 \\
0.005 \\
0.005 \\
0.005\end{array}$ & $\begin{array}{l}: \\
: \\
-\end{array}$ \\
\hline
\end{tabular}

PARAMETER VALUE IMDICATOR (PVI): < - LESS THAN DETECTION LIMIT

SAMPLE ID CODES:

0001 - FILTERED SAMPLE (.45 MICRONS)

OTHER PARAMETER VALUE FLAGS:

1 - INCREASED DETECTION LIMIT DUE TO REQUIRED DILUTIOH

ఓ - estimated VALUE 
GROUNDHATER QUALITY DATA BY LOCATIOH

SITE: AHBOI AMBROSIA LAKE

LOCATION: 0793

NORTH COOROINATE: 55493.0 FT

EAST COORDINATE: $60225.7 \mathrm{FT}$

06/10/80 TO $12 / 04 / 92$

REPORT DATE: 08/04/94

FORHATIOH OF COMPLETIOH: ALLUVIUH (AL)

HYDRAULIC FLON RELATIOHSHIP: UPGRADIENT (U)

\begin{tabular}{|c|c|c|c|c|c|c|c|c|}
\hline PARAMETER NAME & LOG DATE & $\begin{array}{c}\text { SAMPLE } \\
\text { ID }\end{array}$ & $\begin{array}{l}\text { UNITS OF } \\
\text { MEASURE }\end{array}$ & PVI & $\begin{array}{l}\text { Parametel? } \\
\text { VALUEE }\end{array}$ & FLAGS & $\begin{array}{l}\text { DETECTIOH } \\
\text { LIMIT }\end{array}$ & $\begin{array}{l}\text { PARAMETER } \\
\text { UNCERTAINTY }\end{array}$ \\
\hline ALKALINITY & $\begin{array}{l}05 / 15 / 86 \\
04 / 06 / 88 \\
07 / 13 / 88 \\
01 / 06 / 89 \\
09 / 19 / 89 \\
04 / 06 / 90 \\
01 / 16 / 91 \\
11 / 25 / 92\end{array}$ & $\begin{array}{l}0001 \\
0001 \\
0001 \\
0001 \\
0001 \\
0001 \\
0001 \\
0001\end{array}$ & $\mathrm{HG} / \mathrm{L} \mathrm{CACO}$ & & $\begin{array}{l}672 . \\
612 . \\
519 . \\
362 . \\
149 . \\
142 . \\
133 . \\
168\end{array}$ & & $\begin{array}{l}- \\
- \\
- \\
- \\
- \\
-\end{array}$ & $\begin{array}{l}\dot{-} \\
\dot{-} \\
\dot{-} \\
\dot{-} \\
-\end{array}$ \\
\hline ALUAINUM & $\begin{array}{l}04 / 06 / 88 \\
07 / 13 / 88 \\
01 / 06 / 89 \\
09 / 19 / 89 \\
04 / 06 / 90 \\
01 / 16 / 91\end{array}$ & $\begin{array}{l}0001 \\
0001 \\
0001 \\
0001 \\
0001 \\
0001\end{array}$ & MG/L & $\begin{array}{l}< \\
< \\
< \\
<\end{array}$ & $\begin{array}{l}0.56 \\
0.2 \\
0.05 \\
0.1 \\
0.1 \\
0.05\end{array}$ & J & $\begin{array}{l}0.1 \\
0.1 \\
0.1 \\
0.1 \\
0.1 \\
0.05\end{array}$ & $\begin{array}{l}- \\
- \\
- \\
-\end{array}$ \\
\hline AMHONIUH & $\begin{array}{l}04 / 06 / 88 \\
07 / 13 / 88 \\
01 / 06 / 89 \\
09 / 19 / 89 \\
04 / 06 / 90 \\
01 / 16 / 91 \\
11 / 25 / 92\end{array}$ & $\begin{array}{l}0001 \\
0001 \\
0001 \\
0001 \\
0001 \\
0001 \\
0001\end{array}$ & $M G / L$ & $\begin{array}{l}< \\
< \\
< \\
< \\
< \\
<\end{array}$ & $\begin{array}{l}0.1 \\
0.1 \\
0.16 \\
0.1 \\
0.1 \\
0.01 \\
0.1\end{array}$ & & $\begin{array}{l}0.1 \\
0.1 \\
0.1 \\
0.1 \\
0.1 \\
0.01 \\
0.1\end{array}$ & $\begin{array}{l}- \\
- \\
. \\
. \\
-\end{array}$ \\
\hline АКTIMOHY & $\begin{array}{l}04 / 06 / 88 \\
07 / 13 / 88 \\
01 / 06 / 89 \\
09 / 19 / 89 \\
04 / 06 / 90 \\
01 / 16 / 91 \\
11 / 25 / 92\end{array}$ & $\begin{array}{l}0001 \\
0001 \\
0001 \\
0001 \\
0001 \\
0001 \\
0001\end{array}$ & $M G / L$ & $\begin{array}{l}< \\
< \\
<\end{array}$ & $\begin{array}{l}0.010 \\
0.014 \\
0.001 \\
0.003 \\
0.045 \\
0.003 \\
0.003\end{array}$ & J & $\begin{array}{l}0.003 \\
0.003 \\
0.003 \\
0.003 \\
0.003 \\
0.003 \\
0.003\end{array}$ & $\begin{array}{l}- \\
- \\
- \\
- \\
-\end{array}$ \\
\hline ARSENIC & $\begin{array}{l}05 / 15 / 86 \\
04 / 06 / 88 \\
07 / 13 / 88 \\
01 / 06 / 89 \\
09 / 19 / 89 \\
04 / 06 / 90 \\
01 / 16 / 91\end{array}$ & $\begin{array}{l}0001 \\
0001 \\
0001 \\
0001 \\
0001 \\
0001 \\
0001\end{array}$ & $M G / L$ & $\begin{array}{l}< \\
< \\
< \\
<\end{array}$ & $\begin{array}{l}0.01 \\
0.016 \\
0.01 \\
0.001 \\
0.01 \\
0.01 \\
0.03\end{array}$ & J & $\begin{array}{l}0.01 \\
0.01 \\
0.01 \\
0.01 \\
0.01 \\
0.01 \\
0.03\end{array}$ & $\begin{array}{l}- \\
- \\
\dot{-} \\
\dot{-} \\
\dot{-}\end{array}$ \\
\hline BARIUM & $\begin{array}{l}04 / 06 / 88 \\
07 / 13 / 88 \\
01 / 06 / 89 \\
09 / 19 / 89 \\
04 / 06 / 90 \\
01 / 16 / 91\end{array}$ & $\begin{array}{l}0001 \\
0001 \\
0001 \\
0001 \\
0001 \\
0001\end{array}$ & $M G / L$ & $\begin{array}{l}< \\
< \\
< \\
<\end{array}$ & $\begin{array}{l}0.02 \\
0.1 \\
0.01 \\
0.1 \\
0.1 \\
0.01\end{array}$ & $\begin{array}{l}J \\
J\end{array}$ & $\begin{array}{l}0.1 \\
0.1 \\
0.1 \\
0.1 \\
0.1 \\
0.01\end{array}$ & $\begin{array}{l}- \\
- \\
- \\
-\end{array}$ \\
\hline BERYLLILH & $\begin{array}{l}01 / 06 / 89 \\
09 / 19 / 89 \\
04 / 06 / 90 \\
01 / 16 / 91\end{array}$ & $\begin{array}{l}0001 \\
0001 \\
0001 \\
0001\end{array}$ & MG/L & $\begin{array}{l}< \\
< \\
< \\
<\end{array}$ & $\begin{array}{l}0.005 \\
0.01 \\
0.01 \\
0.005\end{array}$ & & $\begin{array}{l}0.005 \\
0.01 \\
0.01 \\
0.005\end{array}$ & $\dot{-}$ \\
\hline BORON & $\begin{array}{l}05 / 15 / 86 \\
04 / 06 / 88 \\
07 / 13 / 88 \\
01 / 06 / 89\end{array}$ & $\begin{array}{l}0001 \\
0001 \\
0001 \\
0001\end{array}$ & MG/L & & $\begin{array}{l}0.6 \\
0.65 \\
0.5 \\
0.57\end{array}$ & & $\begin{array}{l}0.1 \\
0.1 \\
0.1 \\
0.1\end{array}$ & $\dot{-}$ \\
\hline
\end{tabular}

PARAMETER VALUE IMDICATOR (PVI): < - LESS THAN DETECTION LIMIT

SAMPLE IO COOES:

0001 - FILTERED SAMPLE (.45 MICRONS)

OTHER PARAMETER VALUE FLAGS:

1 - IMCREASED DETECTIOH LIMIT DUE TO REQUIRED DILUTIOH

J - estimated VALUE 
GROUNDWATER QUALITY DATA BY LOCATION

SITE: AMBOI AMBROSIA LAKE

LOCATION: 0793

NORTH COORDINATE: 55493.0 FT

EAST COORDINATE: 60225.7 FT

$06 / 10 / 80$ TO $12 / 04 / 92$

REPORT DATE: 08/04/94

FORHATION OF COMPLETION: ALLUVIUM (AL)

HYDRAULIC FLON RELATIOHSHIP: UPGRADIENT (U)

\begin{tabular}{|c|c|c|c|c|c|c|c|c|}
\hline PARAMETER MAME & LOG DATE & $\underset{\text { IO }}{\text { SAMPLE }}$ & $\begin{array}{l}\text { UHITS OF } \\
\text { HEASURE }\end{array}$ & PVI & $\begin{array}{l}\text { PARAMETER } \\
\text { VALUE }\end{array}$ & FLAGS & $\begin{array}{c}\text { OETECTION } \\
\text { LIMIT }\end{array}$ & $\begin{array}{l}\text { PARAMETER } \\
\text { UNCERTAIHTY }\end{array}$ \\
\hline BORON & $\begin{array}{l}09 / 19 / 89 \\
04 / 06 / 90 \\
01 / 16 / 91\end{array}$ & $\begin{array}{l}0001 \\
0001 \\
0001\end{array}$ & MG/L & & $\begin{array}{l}0.6 \\
0.6 \\
0.69\end{array}$ & & $\begin{array}{l}0.1 \\
0.1 \\
0.05\end{array}$ & - \\
\hline BROMIDE & $\begin{array}{l}04 / 06 / 88 \\
07 / 13 / 88 \\
01 / 06 / 89 \\
09 / 19 / 89 \\
04 / 06 / 90 \\
01 / 16 / 91\end{array}$ & $\begin{array}{l}0001 \\
0001 \\
0001 \\
0001 \\
0001 \\
0001\end{array}$ & $\mathrm{MG} / \mathrm{L}$ & & $\begin{array}{l}1.1 \\
1.6 \\
2.42 \\
0.19 \\
3.8 \\
9.1\end{array}$ & & $\begin{array}{l}0.1 \\
0.1 \\
0.01 \\
0.1 \\
0.1 \\
0.1\end{array}$ & $\begin{array}{l}- \\
- \\
- \\
-\end{array}$ \\
\hline CADMIUH & $\begin{array}{l}05 / 15 / 86 \\
04 / 06 / 88 \\
07 / 13 / 88 \\
01 / 06 / 89 \\
09 / 19 / 89 \\
04 / 06 / 90 \\
01 / 16 / 91 \\
11 / 25 / 92\end{array}$ & $\begin{array}{l}0001 \\
0001 \\
0001 \\
0001 \\
0001 \\
0001 \\
0001 \\
0001\end{array}$ & $M G / L$ & $\begin{array}{l}< \\
< \\
< \\
<\end{array}$ & $\begin{array}{l}0.009 \\
0.005 \\
0.001 \\
0.0002 \\
0.013 \\
0.009 \\
0.002 \\
0.05\end{array}$ & $\begin{array}{l}\mathbf{J} \\
\mathbf{I}\end{array}$ & $\begin{array}{l}0.001 \\
0.001 \\
0.001 \\
0.001 \\
0.001 \\
0.001 \\
0.001 \\
0.05\end{array}$ & $\begin{array}{l}- \\
- \\
- \\
- \\
-\end{array}$ \\
\hline CALCIUM & $\begin{array}{l}05 / 15 / 86 \\
04 / 06 / 88 \\
07 / 13 / 88 \\
01 / 06 / 89 \\
09 / 19 / 89 \\
04 / 06 / 90 \\
01 / 16 / 91 \\
11 / 25 / 92\end{array}$ & $\begin{array}{l}0001 \\
0001 \\
0001 \\
0001 \\
0001 \\
0001 \\
0001 \\
0001\end{array}$ & MG/L & & $\begin{array}{l}510 . \\
610 . \\
500 . \\
600 . \\
590 . \\
507 . \\
600 . \\
615 .\end{array}$ & & $\begin{array}{l}0.01 \\
0.01 \\
0.01 \\
0.01 \\
0.01 \\
0.01 \\
0.01 \\
2 .\end{array}$ & $\begin{array}{l}- \\
- \\
- \\
- \\
-\end{array}$ \\
\hline CHLORIDE & $\begin{array}{l}05 / 15 / 86 \\
04 / 06 / 88 \\
07 / 13 / 88 \\
01 / 06 / 89 \\
09 / 19 / 89 \\
04 / 06 / 90 \\
01 / 16 / 91 \\
11 / 25 / 92\end{array}$ & $\begin{array}{l}0001 \\
0001 \\
0001 \\
0001 \\
0001 \\
0001 \\
0001 \\
0001\end{array}$ & $M G / L$ & & $\begin{array}{l}230 . \\
170 . \\
200 . \\
208 . \\
430 . \\
518 . \\
643 . \\
77 .\end{array}$ & & $\begin{array}{l}1 . \\
1 . \\
1 . \\
1 . \\
1 . \\
1 . \\
0.5 \\
1 .\end{array}$ & $\begin{array}{l}- \\
- \\
- \\
- \\
-\end{array}$ \\
\hline CHROMIUM & $\begin{array}{l}05 / 15 / 86 \\
04 / 06 / 88 \\
07 / 13 / 88 \\
01 / 06 / 89 \\
09 / 19 / 89 \\
04 / 06 / 90 \\
01 / 16 / 91 \\
19 / 25 / 92\end{array}$ & $\begin{array}{l}0001 \\
0001 \\
0001 \\
0001 \\
0001 \\
0001 \\
0001 \\
0001\end{array}$ & $M G / L$ & $\begin{array}{l}< \\
< \\
< \\
<\end{array}$ & $\begin{array}{l}0.03 \\
0.28 \\
0.15 \\
0.01 \\
0.01 \\
0.01 \\
0.01 \\
0.01\end{array}$ & & $\begin{array}{l}0.01 \\
0.01 \\
0.01 \\
0.01 \\
0.01 \\
0.01 \\
0.01 \\
0.01\end{array}$ & $\begin{array}{l}- \\
- \\
- \\
= \\
-\end{array}$ \\
\hline COBALT & $\begin{array}{l}05 / 15 / 86 \\
04 / 06 / 88 \\
07 / 13 / 88 \\
01 / 06 / 89 \\
09 / 19 / 89 \\
04 / 06 / 90 \\
01 / 16 / 91 \\
11 / 25 / 92\end{array}$ & $\begin{array}{l}0001 \\
0001 \\
0001 \\
0001 \\
0001 \\
0001 \\
0001 \\
0001\end{array}$ & $M G / L$ & $\begin{array}{l}< \\
< \\
< \\
< \\
<\end{array}$ & $\begin{array}{l}0.08 \\
0.13 \\
0.05 \\
0.01 \\
0.05 \\
0.05 \\
0.03 \\
0.03\end{array}$ & J & $\begin{array}{l}0.05 \\
0.05 \\
0.05 \\
0.05 \\
0.05 \\
0.05 \\
0.03 \\
0.03\end{array}$ & $\begin{array}{l}\text { - } \\
\text { - } \\
\text { - } \\
- \\
-\end{array}$ \\
\hline COPPER & $04 / 06 / 88$ & 0001 & $M G / L$ & & 0.06 & & 0.02 & - \\
\hline
\end{tabular}

PARAMETER VALUE INDICATOR (PVI): < - LESS THAN DETECTION LIMIT

SAMPLE ID COOES:

0001 - FILTERED SAMPLE (.45 MICRONS)

OTHER PARAMETER VALUE FLAGS:

1 - IMCREASED DETECTION LIMIT DUE TO REQUIRED DILUTION

J. estimated value 
GROUNDWATER QUALITY DATA BY LOCATION

SITE: AMBOI AMBROSIA LAKE

LOCATION: 0793

NORTH COOROIHATE: $\quad 55493.0$ FT

EAST COORDIHATE: 60225.7 FT

O6/10/80 TO $12 / 04 / 92$

REPORT OATE: $08 / 04 / 94$

FORHATION OF COMPLETION: ALLUVIUM (AL)

HYDRAULIC FLON RELATIONSHIP: UPGRADIENT (U)

\begin{tabular}{|c|c|c|c|c|c|c|c|c|}
\hline PARAMETER MAME & LOG DATE & $\underset{\text { ID }}{\text { SAMPLE }}$ & $\begin{array}{l}\text { UNITS OF } \\
\text { MEASURE }\end{array}$ & PVI & $\begin{array}{l}\text { PARAMETER } \\
\text { VALUE }\end{array}$ & FLAGS & $\begin{array}{l}\text { DETECTION } \\
\text { LIMIT }\end{array}$ & $\begin{array}{l}\text { PARAMETER } \\
\text { UNCERTAIHTY }\end{array}$ \\
\hline COPPER & $\begin{array}{l}07 / 13 / 88 \\
01 / 06 / 89 \\
09 / 19 / 89 \\
04 / 06 / 90 \\
01 / 16 / 91\end{array}$ & $\begin{array}{l}0001 \\
0001 \\
0001 \\
0001 \\
0001\end{array}$ & MG/L & $\begin{array}{l}< \\
< \\
<\end{array}$ & $\begin{array}{l}0.03 \\
0.01 \\
0.02 \\
0.02 \\
0.01\end{array}$ & J & $\begin{array}{l}0.02 \\
0.02 \\
0.02 \\
0.02 \\
0.01\end{array}$ & $\begin{array}{l}- \\
- \\
-\end{array}$ \\
\hline CYANIDE & $\begin{array}{l}01 / 06 / 89 \\
09 / 19 / 89 \\
04 / 06 / 90 \\
01 / 16 / 91\end{array}$ & $\begin{array}{l}0001 \\
0001 \\
0001 \\
0001\end{array}$ & MG/L & $\begin{array}{l}< \\
< \\
<\end{array}$ & $\begin{array}{l}0.002 \\
0.01 \\
0.01 \\
0.01\end{array}$ & $J$ & $\begin{array}{l}0.01 \\
0.01 \\
0.01 \\
0.01\end{array}$ & - \\
\hline FLUORIDE & $\begin{array}{l}05 / 15 / 86 \\
04 / 06 / 88 \\
07 / 13 / 88 \\
01 / 06 / 89 \\
09 / 19 / 89 \\
04 / 06 / 90 \\
01 / 16 / 91 \\
11 / 25 / 92\end{array}$ & $\begin{array}{l}0001 \\
0001 \\
0001 \\
0001 \\
0001 \\
0001 \\
0001 \\
0001\end{array}$ & $M G / L$ & & $\begin{array}{l}2.2 \\
2.50 \\
2.3 \\
2.8 \\
2.4 \\
2.5 \\
2.9 \\
5.5\end{array}$ & & $\begin{array}{l}0.1 \\
0.1 \\
0.1 \\
0.1 \\
0.1 \\
0.1 \\
0.1 \\
0.2\end{array}$ & $\begin{array}{l}- \\
- \\
- \\
- \\
-\end{array}$ \\
\hline GROSS ALPHA & $\begin{array}{l}04 / 06 / 88 \\
07 / 13 / 88 \\
01 / 06 / 89 \\
09 / 19 / 89 \\
04 / 06 / 90 \\
01 / 16 / 91 \\
11 / 25 / 92\end{array}$ & $\begin{array}{l}0001 \\
0001 \\
0001 \\
0001 \\
0001 \\
0001 \\
0001\end{array}$ & PCI/L & & $\begin{array}{c}320 . \\
170 . \\
84.6 \\
69 . \\
66 . \\
115 . \\
3.2\end{array}$ & $\begin{array}{l}J \\
H\end{array}$ & $\begin{array}{l}0.2 \\
0.2 \\
1 . \\
1 . \\
1 . \\
1.0\end{array}$ & $\begin{array}{l}50 . \\
40 . \\
31.2 \\
34 . \\
30 . \\
54.3 \\
37.8\end{array}$ \\
\hline GROSS BETA & $\begin{array}{l}04 / 06 / 88 \\
07 / 13 / 88 \\
01 / 06 / 89 \\
09 / 19 / 89 \\
04 / 06 / 90 \\
01 / 16 / 91 \\
11 / 25 / 92\end{array}$ & $\begin{array}{l}0001 \\
0001 \\
0001 \\
0001 \\
0001 \\
0001 \\
0001\end{array}$ & $\mathrm{PCI} / \mathrm{L}$ & & $\begin{array}{l}120 . \\
62 . \\
63.8 \\
58 . \\
18 . \\
67.7 \\
0.0\end{array}$ & & $\begin{array}{l}1 . \\
1 . \\
0.5 \\
0.5 \\
0.5 \\
0.5 \\
98.9\end{array}$ & $\begin{array}{l}20 . \\
13 . \\
25.4 \\
21 . \\
21 . \\
24.0 \\
56.5\end{array}$ \\
\hline IRON & $\begin{array}{l}05 / 15 / 86 \\
04 / 06 / 88 \\
07 / 13 / 88 \\
01 / 06 / 89 \\
09 / 19 / 89 \\
04 / 06 / 90 \\
01 / 16 / 91 \\
11 / 25 / 92\end{array}$ & $\begin{array}{l}0001 \\
0001 \\
0001 \\
0001 \\
0001 \\
0001 \\
0001 \\
0001\end{array}$ & $\mathrm{HG} / \mathrm{L}$ & $\begin{array}{l}< \\
< \\
<\end{array}$ & $\begin{array}{l}0.04 \\
0.17 \\
0.08 \\
0.02 \\
0.09 \\
0.09 \\
0.03 \\
0.03\end{array}$ & 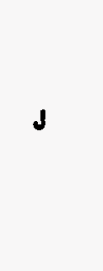 & $\begin{array}{l}0.03 \\
0.03 \\
0.03 \\
0.03 \\
0.03 \\
0.03 \\
0.03 \\
0.03\end{array}$ & $\begin{array}{l}: \\
\therefore \\
: \\
: \\
-\end{array}$ \\
\hline LEAD & $\begin{array}{l}04 / 06 / 88 \\
07 / 13 / 88 \\
01 / 06 / 89 \\
09 / 19 / 89 \\
04 / 06 / 90 \\
01 / 16 / 91\end{array}$ & $\begin{array}{l}0001 \\
0001 \\
0001 \\
0001 \\
0001 \\
0001\end{array}$ & MG/L & $\begin{array}{l}< \\
< \\
<\end{array}$ & $\begin{array}{l}0.01 \\
0.01 \\
0.001 \\
0.02 \\
0.01 \\
0.03\end{array}$ & $\begin{array}{l}\text { J } \\
\text { I }\end{array}$ & $\begin{array}{l}0.01 \\
0.01 \\
0.01 \\
0.01 \\
0.01 \\
0.03\end{array}$ & $\begin{array}{l}: \\
- \\
- \\
-\end{array}$ \\
\hline LEAD-210 & $\begin{array}{l}04 / 06 / 88 \\
07 / 13 / 88 \\
01 / 06 / 89\end{array}$ & $\begin{array}{l}0001 \\
0001 \\
0001\end{array}$ & $\mathrm{PCI} / \mathrm{L}$ & & $\begin{array}{l}1.8 \\
0.5 \\
5.09\end{array}$ & & $\begin{array}{l}1.5 \\
1.5 \\
1.5\end{array}$ & $\begin{array}{l}1.1 \\
1.1 \\
3.71\end{array}$ \\
\hline
\end{tabular}

PARAMETER VALUE INDICATOR (PVI): < - LESS THAN DETECTION LIMIT

SAMPLE ID COOES:

0001 - FILTERED SAMPLE (.45 MICRONS)

OTHER PARAMETER VALUE FLAGS:

H - HOLD TIME EXPIRED, VALUE SUSPECT

1 - IMCREASED DETECTION LIMIT DUE TO REQUIRED DILUTION

J - eStIMATED VALUE 
GROUNDWATER OUALITY DATA BY LOCATIOH

SITE: AMBO1 AMBROSIA LAKE

LOCATION: 0793

NORTH COORDINATE: 55493.0 FT

EAST COORDIHATE: 60225.7 FT

06/10/80 TO 12/04/92

REPORT DATE: 08/04/94

FORHATION OF COMPLETION: ALLUVIUM (AL)

HYDRAULIC FLON RELATIONSHIP: UPGRADIENT (U)

\begin{tabular}{|c|c|c|c|c|c|c|c|c|}
\hline PARAMIETER MAME & LOG DATE & $\underset{\text { SAMPLE }}{\text { SD }}$ & $\begin{array}{l}\text { UNITS OF } \\
\text { MEASURE }\end{array}$ & PVI & $\begin{array}{l}\text { PARAMETER } \\
\text { VALUE }\end{array}$ & FLAGS & $\begin{array}{l}\text { DETECTION } \\
\text { LIMIT }\end{array}$ & $\begin{array}{l}\text { PARAMETER } \\
\text { UHCERTAINTY }\end{array}$ \\
\hline LEAD-210 & $09 / 19 / 89$ & 0001 & PCI/L & & 0.0 & & 1.5 & 0.7 \\
\hline MAGESIUH & $\begin{array}{l}05 / 15 / 86 \\
04 / 06 / 88 \\
07 / 13 / 88 \\
01 / 06 / 89 \\
09 / 19 / 89 \\
04 / 06 / 90 \\
01 / 16 / 91 \\
11 / 25 / 92\end{array}$ & $\begin{array}{l}0001 \\
0001 \\
0001 \\
0001 \\
0001 \\
0001 \\
0001 \\
0001\end{array}$ & MG/L & & $\begin{array}{l}138 . \\
140 . \\
143 . \\
150 . \\
170 \\
158 . \\
194 . \\
178 .\end{array}$ & & $\begin{array}{l}0.001 \\
0.001 \\
0.001 \\
0.001 \\
0.001 \\
0.001 \\
0.5 \\
0.5\end{array}$ & $\begin{array}{l}- \\
\dot{-} \\
\dot{-} \\
\dot{-} \\
-\end{array}$ \\
\hline MAKGAKESE & $\begin{array}{l}05 / 15 / 86 \\
04 / 06 / 88 \\
07 / 13 / 88 \\
01 / 06 / 89 \\
09 / 19 / 89 \\
04 / 06 / 90 \\
01 / 16 / 99\end{array}$ & $\begin{array}{l}0001 \\
0001 \\
0001 \\
0001 \\
0001 \\
0001 \\
0001\end{array}$ & MG/L & $\begin{array}{l}< \\
< \\
<\end{array}$ & $\begin{array}{l}0.07 \\
0.04 \\
0.02 \\
0.01 \\
0.01 \\
0.01 \\
0.01\end{array}$ & - & $\begin{array}{l}0.01 \\
0.01 \\
0.01 \\
0.01 \\
0.01 \\
0.01 \\
0.01\end{array}$ & : \\
\hline MERCURY & $\begin{array}{l}04 / 06 / 88 \\
07 / 13 / 88 \\
01 / 06 / 89 \\
09 / 19 / 89 \\
04 / 06 / 90 \\
01 / 16 / 91\end{array}$ & $\begin{array}{l}0001 \\
0001 \\
0001 \\
0001 \\
0001 \\
0001\end{array}$ & $M G / L$ & $\begin{array}{l}< \\
< \\
< \\
< \\
<\end{array}$ & $\begin{array}{l}0.0003 \\
0.0002 \\
0.0001 \\
0.0002 \\
0.0002 \\
0.0002\end{array}$ & J & $\begin{array}{l}0.0002 \\
0.0002 \\
0.0002 \\
0.0002 \\
0.0002 \\
0.0002\end{array}$ & $\begin{array}{l}- \\
- \\
- \\
-\end{array}$ \\
\hline MOLYBDENUM & $\begin{array}{l}05 / 15 / 86 \\
04 / 06 / 88 \\
07 / 13 / 88 \\
01 / 06 / 89 \\
09 / 19 / 89 \\
04 / 06 / 90 \\
01 / 16 / 91 \\
11 / 25 / 92\end{array}$ & $\begin{array}{l}0001 \\
0001 \\
0001 \\
0001 \\
0001 \\
0001 \\
0001 \\
0001\end{array}$ & MG/L & & $\begin{array}{l}1.59 \\
2.01 \\
1.41 \\
1.450 \\
1.19 \\
0.25 \\
0.07 \\
0.06\end{array}$ & & $\begin{array}{l}0.01 \\
0.01 \\
0.01 \\
0.01 \\
0.01 \\
0.01 \\
0.01 \\
0.01\end{array}$ & $\begin{array}{l}- \\
- \\
- \\
- \\
-\end{array}$ \\
\hline HET GROSS ALPHA • & $\begin{array}{l}04 / 06 / 88 \\
07 / 13 / 88 \\
01 / 06 / 89 \\
09 / 19 / 89 \\
04 / 06 / 90 \\
01 / 16 / 91 \\
11 / 25 / 92\end{array}$ & $\begin{array}{l}0001 \\
0001 \\
0001 \\
0001 \\
0001 \\
0001 \\
0001\end{array}$ & $\mathrm{PCl} / \mathrm{L}$ & & $\begin{array}{r}50.40 \\
1.93 \\
-45.74 \\
7.95 \\
37.19 \\
97.85 \\
-18.07\end{array}$ & & $\begin{array}{l}- \\
- \\
- \\
-\end{array}$ & $\begin{array}{l}- \\
- \\
- \\
- \\
-\end{array}$ \\
\hline HICKEL & $\begin{array}{l}04 / 06 / 88 \\
07 / 13 / 88 \\
01 / 06 / 89 \\
09 / 19 / 89 \\
04 / 06 / 90 \\
01 / 16 / 91 \\
11 / 25 / 92\end{array}$ & $\begin{array}{l}0001 \\
0001 \\
0001 \\
0001 \\
0001 \\
0001 \\
0001\end{array}$ & MG/L & $\begin{array}{l}< \\
< \\
<\end{array}$ & $\begin{array}{l}0.14 \\
0.05 \\
0.02 \\
0.04 \\
0.04 \\
0.04 \\
0.08\end{array}$ & $J$ & $\begin{array}{l}0.04 \\
0.04 \\
0.04 \\
0.04 \\
0.04 \\
0.04 \\
0.04\end{array}$ & $\begin{array}{l}- \\
- \\
- \\
-\end{array}$ \\
\hline HITRATE & $\begin{array}{l}05 / 15 / 86 \\
04 / 06 / 88 \\
07 / 13 / 88 \\
01 / 06 / 89\end{array}$ & $\begin{array}{l}0001 \\
0001 \\
0001 \\
0001\end{array}$ & MG/L & & $\begin{array}{l}55 . \\
130 . \\
210 . \\
252 .\end{array}$ & & $\begin{array}{l}1 . \\
i .\end{array}$ & $\begin{array}{l}- \\
- \\
-\end{array}$ \\
\hline
\end{tabular}

- MET gROSS ALPHA (GROSS ALPHA - URANIUN) WITH 1 Mg URANIUM = 686 PCI

PARAHETER VALUE INDICATOR (PVI): < - LESS THAN DETECTIOH LIMIT SAMPLE ID CODES:

0001 - FILTERED SAMPLE (.45 MICROHS)

OtHeR PARAMETER VALUE flags:

J - ESTIMATED VALUE 
GROUNDWATER OUALITY DATA BY LOCATIOH

SITE: AMBO1 AMBROSIA LAKE

LOCATIOH: 0793

NORTH COORDINATE: 55493.0 FT

EAST COORDINATE: 60225.7 FT

06/10/80 To $12 / 04 / 92$

REPORT DATE: 08/04/94

FORMATION OF COMPLETION: ALLUVIUM (AL)

HYORAULIC FLOW RELATIONSHIP: UPGRADIENT (U)

\begin{tabular}{|c|c|c|c|c|c|c|c|}
\hline PARAMETER MAME & LOG DATE & SAMPLE & $\begin{array}{l}\text { UHITS OF } \\
\text { MEASURE }\end{array}$ & PVI & $\begin{array}{l}\text { Parameter } \\
\text { VALUE fLAGS }\end{array}$ & $\begin{array}{l}\text { DETECTION } \\
\text { LIMIT }\end{array}$ & $\begin{array}{l}\text { PARAMETER } \\
\text { UNCERTAINTY }\end{array}$ \\
\hline HITRATE & $\begin{array}{l}09 / 19 / 89 \\
04 / 06 / 90 \\
01 / 16 / 91 \\
11 / 25 / 92\end{array}$ & $\begin{array}{l}0001 \\
0001 \\
0001 \\
0001\end{array}$ & MG/L & & $\begin{array}{l}500 . \\
536 . \\
655 . \\
830 .\end{array}$ & $\begin{array}{c}1 . \\
1 . \\
0.1 \\
150 .\end{array}$ & : \\
\hline MITRITE AHD MITRATE & $\begin{array}{l}04 / 06 / 88 \\
04 / 06 / 90 \\
01 / 16 / 91\end{array}$ & $\begin{array}{l}0001 \\
0009 \\
0001\end{array}$ & $M G / L$ & & $\begin{array}{l}130 . \\
121 . \\
140 .\end{array}$ & $\begin{array}{l}1 . \\
1.05\end{array}$ & $\because$ \\
\hline PH & $\begin{array}{l}05 / 15 / 86 \\
04 / 06 / 88 \\
07 / 13 / 88 \\
01 / 06 / 89 \\
09 / 19 / 89 \\
04 / 06 / 90 \\
01 / 16 / 91 \\
11 / 25 / 92\end{array}$ & $\begin{array}{l}0001 \\
0001 \\
0001 \\
0001 \\
0001 \\
0001 \\
0001 \\
0001\end{array}$ & su & & $\begin{array}{l}7.37 \\
7.37 \\
7.28 \\
7.41 \\
7.22 \\
7.15 \\
7.76 \\
7.46\end{array}$ & 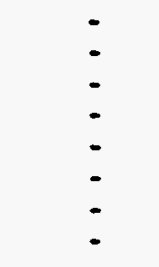 & $\begin{array}{l}- \\
- \\
- \\
- \\
-\end{array}$ \\
\hline PHOSPHATE & $\begin{array}{l}04 / 06 / 88 \\
07 / 13 / 88 \\
01 / 06 / 89 \\
09 / 19 / 89 \\
04 / 06 / 90 \\
01 / 16 / 91 \\
11 / 25 / 92\end{array}$ & $\begin{array}{l}0001 \\
0001 \\
0001 \\
0001 \\
0001 \\
0001 \\
0001\end{array}$ & HG/L & $\begin{array}{l}< \\
< \\
< \\
< \\
<\end{array}$ & $\begin{array}{l}0.1 \\
0.1 \\
0.1 \\
0.1 \\
0.1 \\
0.05 \\
0.2\end{array}$ & $\begin{array}{l}0.1 \\
0.1 \\
0.1 \\
0.1 \\
0.1 \\
0.05 \\
0.1\end{array}$ & $\begin{array}{l}- \\
- \\
- \\
- \\
-\end{array}$ \\
\hline POLONIUH-210 & $\begin{array}{l}04 / 06 / 88 \\
07 / 13 / 88 \\
01 / 06 / 89 \\
09 / 19 / 89\end{array}$ & $\begin{array}{l}0001 \\
0001 \\
0001 \\
0001\end{array}$ & $\mathrm{PCI} / \mathrm{L}$ & - & $\begin{array}{l}0.0 \\
0.0 \\
0.00 \\
0.0\end{array}$ & $\begin{array}{l}1 . \\
1 . \\
1 .\end{array}$ & $\begin{array}{l}0.4 \\
0.4 \\
1.38 \\
0.3\end{array}$ \\
\hline POTASSIUM & $\begin{array}{l}05 / 15 / 86 \\
04 / 06 / 88 \\
07 / 13 / 88 \\
01 / 06 / 89 \\
09 / 19 / 89 \\
04 / 06 / 90 \\
01 / 16 / 91 \\
11 / 25 / 92\end{array}$ & $\begin{array}{l}0001 \\
0001 \\
0001 \\
0001 \\
0001 \\
0001 \\
0001 \\
0001\end{array}$ & $M G / L=$ & & $\begin{array}{l}2.49 \\
1.68 \\
2.2 \\
1.9 \\
2.4 \\
2.8 \\
2.6 \\
4.16\end{array}$ & $\begin{array}{l}0.01 \\
0.01 \\
0.01 \\
0.01 \\
0.01 \\
0.01 \\
0.01 \\
0.01\end{array}$ & $\begin{array}{l}- \\
- \\
- \\
- \\
-\end{array}$ \\
\hline RADIUH-226 & $\begin{array}{l}05 / 15 / 86 \\
04 / 06 / 88 \\
07 / 13 / 88 \\
01 / 06 / 89 \\
09 / 19 / 89 \\
04 / 06 / 90 \\
01 / 16 / 91 \\
11 / 25 / 92\end{array}$ & $\begin{array}{l}0001 \\
0001 \\
0001 \\
0001 \\
0001 \\
0001 \\
0001 \\
0001\end{array}$ & $\mathrm{PCI} / \mathrm{L}$ & & $\begin{array}{l}0.7 \\
0.0 \\
0.4 \\
1.36 \\
0.2 \\
0.3 \\
1.6 \\
0.5\end{array}$ & $\begin{array}{l}1 . \\
1 . \\
1 . \\
1 . \\
1 . \\
1 . \\
0.3\end{array}$ & $\begin{array}{l}0.3 \\
0.1 \\
0.2 \\
0.450 \\
0.2 \\
0.2 \\
0.6 \\
0.3\end{array}$ \\
\hline RADIUH-226 + RADILH-228 & $\begin{array}{l}04 / 06 / 88 \\
07 / 13 / 88 \\
01 / 06 / 89 \\
09 / 19 / 89 \\
04 / 06 / 90 \\
01 / 16 / 91 \\
11 / 25 / 92\end{array}$ & $\begin{array}{l}0001 \\
0001 \\
0001 \\
0001 \\
0001 \\
0001 \\
0001\end{array}$ & PCI/L & & $\begin{array}{l}0.00 \\
0.60 \\
1.36 \\
0.70 \\
1.00 \\
2.30 \\
0.90\end{array}$ & $\begin{array}{l}- \\
- \\
- \\
:\end{array}$ & $\begin{array}{l}- \\
- \\
- \\
- \\
-\end{array}$ \\
\hline RADILH-228 & $04 / 06 / 88$ & 0001 & PCI/L & & 0.0 & 1. & 0.8 \\
\hline
\end{tabular}

PARAMETER VALUE IMDICATOR (PVI): < - LESS THAM DETECTION LIMIT SAMPLE ID COOES:

0001 - FILTERED SAMPLE (.45 MICRONS)

OTHER PARAMETER VALUE FLAGS:

$J$ - estimated VAlUe 
GROUNDHATER QUALITY DATA BY LOCATIOH

SITE: AMBO1 AMBROSIA LAKE

LOCATIOH: 0793

NORTH COORDINATE: $\quad 55493.0$ FT

EAST COORDINATE: 60225.7 FT

06/10/80 TO $12 / 04 / 92$

REPORT DATE: 08/04/94

FORHATION OF COAPLETION: ALLUVIUN (AL)

HYDRAULIC FLOH RELATIONSHIP: UPGRADIENT (U)

\begin{tabular}{|c|c|c|c|c|c|c|c|c|}
\hline PARAMETER MAME & & LOG DATE & $\begin{array}{c}\text { SAMPLE } \\
10\end{array}$ & $\begin{array}{l}\text { UNITS OF } \\
\text { MEASURE }\end{array}$ & PVI & $\begin{array}{l}\text { PARAMETER } \\
\text { VALUE FLAGS }\end{array}$ & $\begin{array}{l}\text { DETECTION } \\
\text { LIMIT }\end{array}$ & $\begin{array}{l}\text { PARAMETER } \\
\text { UNCERTAIHTY }\end{array}$ \\
\hline RADIUH-228 & & $\begin{array}{l}07 / 13 / 88 \\
01 / 06 / 89 \\
09 / 19 / 89 \\
04 / 06 / 90 \\
01 / 16 / 91 \\
11 / 25 / 92\end{array}$ & $\begin{array}{l}0001 \\
0001 \\
0001 \\
0001 \\
0001 \\
0001\end{array}$ & PCI/L & & $\begin{array}{l}0.2 \\
0.00 \\
0.5 \\
0.7 \\
0.7 \\
0.4\end{array}$ & $\begin{array}{l}1 . \\
1 . \\
1 . \\
1.0\end{array}$ & $\begin{array}{r}0.7 \\
18.9 \\
0.9 \\
0.9 \\
1.9 \\
1.3\end{array}$ \\
\hline SILVER & & $\begin{array}{l}04 / 06 / 88 \\
07 / 13 / 88 \\
01 / 06 / 89 \\
09 / 19 / 89 \\
04 / 06 / 90 \\
01 / 16 / 91 \\
11 / 25 / 92\end{array}$ & $\begin{array}{l}0001 \\
0001 \\
0001 \\
0001 \\
0001 \\
0001 \\
0001\end{array}$ & MG/L & $\begin{array}{l}< \\
< \\
< \\
< \\
<\end{array}$ & $\begin{array}{l}0.11 \\
0.03 \\
0.01 \\
0.01 \\
0.01 \\
0.01 \\
0.01\end{array}$ & $\begin{array}{l}0.01 \\
0.01 \\
0.01 \\
0.01 \\
0.01 \\
0.01 \\
0.01\end{array}$ & $\begin{array}{l}- \\
- \\
- \\
-\end{array}$ \\
\hline soolus & & $\begin{array}{l}05 / 15 / 86 \\
04 / 06 / 88 \\
07 / 13 / 88 \\
01 / 06 / 89 \\
09 / 19 / 89 \\
04 / 06 / 90 \\
01 / 16 / 91 \\
11 / 25 / 92\end{array}$ & $\begin{array}{l}0001 \\
0001 \\
0001 \\
0001 \\
0001 \\
0001 \\
0001 \\
0001\end{array}$ & $M G / L$ & & $\begin{array}{l}685 . \\
433 . \\
511 . \\
555 . \\
654 . \\
689 . \\
901 . \\
849 .\end{array}$ & $\begin{array}{l}0.002 \\
0.002 \\
0.002 \\
0.002 \\
0.002 \\
0.002 \\
5 . \\
5 .\end{array}$ & $\begin{array}{l}- \\
- \\
- \\
- \\
-\end{array}$ \\
\hline STRONTIUA & & $\begin{array}{l}05 / 15 / 86 \\
04 / 06 / 88 \\
07 / 13 / 88 \\
01 / 06 / 89 \\
09 / 19 / 89 \\
04 / 06 / 90 \\
01 / 16 / 91\end{array}$ & $\begin{array}{l}0001 \\
0001 \\
0001 \\
0001 \\
0001 \\
0001 \\
0001\end{array}$ & $M G / L$ & & $\begin{array}{c}1.1 \\
5.89 \\
8.1 \\
10.3 \\
5.8 \\
11.4 \\
12.5\end{array}$ & $\begin{array}{l}0.1 \\
0.1 \\
0.1 \\
0.1 \\
0.1 \\
0.1 \\
0.01\end{array}$ & $\begin{array}{l}- \\
- \\
- \\
- \\
-\end{array}$ \\
\hline
\end{tabular}

PARAMETER VALUE INDICATOR (PVI): < - LESS than DETECTIOH LIMIT

SAMPLE ID COOES:

0001 - FILTERED SAMPLE (.45 MICRONS)

OTHER PARAMETER VALUE fLAGS:

H - HOLO TIME EXPIRED, VALUE SUSPECT

d- estimated VALUE 
GROUNDWATER QUALITY DATA BY LOCATION

SITE: AMBO1 AMBROSIA LAKE

LOCATIOH: 0793

MORTH COORDIHATE: 55493.0 FT

EAST COORDINATE: 60225.7 FT

06/10/80 TO $12 / 04 / 92$

REPORT DATE: 08/04/94

FORHATION OF COMPLETION: ALLUVIUM (AL)

HYDRAULIC FLOW RELATIOHSHIP: UPGRADIENT (U)

\begin{tabular}{|c|c|c|c|c|c|c|c|c|}
\hline PARAMETER MAME & LOG DATE & $\mid \begin{array}{c}\text { SAMPLE } \\
\text { ID }\end{array}$ & $\begin{array}{l}\text { UNITS OF } \\
\text { MEASURE }\end{array}$ & PVI & $\begin{array}{l}\text { PARAMETER } \\
\text { VALUE }\end{array}$ & FLAGS & $\begin{array}{l}\text { DETECTION } \\
\text { LIMIT }\end{array}$ & $\begin{array}{l}\text { PARAMETER } \\
\text { UHCERTAINTY }\end{array}$ \\
\hline SULFATE & $\begin{array}{l}05 / 15 / 86 \\
04 / 06 / 88 \\
07 / 13 / 88 \\
01 / 06 / 89 \\
09 / 19 / 89 \\
04 / 06 / 90 \\
01 / 16 / 91 \\
11 / 25 / 92\end{array}$ & $\begin{array}{l}0001 \\
0001 \\
0001 \\
0001 \\
0001 \\
0001 \\
0001 \\
0001\end{array}$ & MG/L & ${ }^{\circ}$ & $\begin{array}{l}2530 . \\
2200 . \\
2020 . \\
2461 . \\
2480 . \\
2190 . \\
2520 . \\
2530 .\end{array}$ & & $\begin{array}{l}0.1 \\
0.1 \\
0.1 \\
0.1 \\
0.1 \\
0.1 \\
10.1 \\
10 .\end{array}$ & $\begin{array}{l}- \\
- \\
\because \\
-\end{array}$ \\
\hline SULFIDE & $\begin{array}{l}04 / 06 / 88 \\
07 / 13 / 88 \\
01 / 06 / 89 \\
09 / 19 / 89 \\
04 / 06 / 90 \\
01 / 16 / 91\end{array}$ & $\begin{array}{l}0001 \\
0001 \\
0001 \\
0001 \\
0001 \\
0001\end{array}$ & MG/L & $\begin{array}{l}< \\
< \\
< \\
< \\
<\end{array}$ & $\begin{array}{l}0.1 \\
0.1 \\
0.01 \\
0.1 \\
0.1 \\
0.6\end{array}$ & $\begin{array}{l}J \\
J\end{array}$ & $\begin{array}{l}0.1 \\
0.1 \\
0.1 \\
0.1 \\
0.1 \\
0.1\end{array}$ & : \\
\hline TEMPERATURE & $\begin{array}{l}05 / 15 / 86 \\
04 / 06 / 88 \\
07 / 13 / 88 \\
01 / 06 / 89 \\
09 / 19 / 89 \\
04 / 06 / 90 \\
01 / 16 / 91 \\
11 / 25 / 92\end{array}$ & $\begin{array}{l}0001 \\
0001 \\
0001 \\
0001 \\
0001 \\
0001 \\
0001 \\
0001\end{array}$ & C - DEGREE & & $\begin{array}{l}16 . \\
11.5 \\
12.0 \\
11.0 \\
12.5 \\
12.5 \\
11.0 \\
9.0\end{array}$ & & $\begin{array}{l}1 . \\
= \\
= \\
-\end{array}$ & $\begin{array}{l}- \\
- \\
- \\
- \\
-\end{array}$ \\
\hline THALLIUM & $\begin{array}{l}01 / 06 / 89 \\
09 / 19 / 89 \\
04 / 06 / 90 \\
01 / 16 / 91\end{array}$ & $\begin{array}{l}0001 \\
0001 \\
0001 \\
0001\end{array}$ & MG/L & $\begin{array}{l}< \\
<\end{array}$ & $\begin{array}{l}0.002 \\
0.01 \\
0.01 \\
0.03\end{array}$ & I & $\begin{array}{l}0.001 \\
0.01 \\
0.01 \\
0.03\end{array}$ & $\begin{array}{l}- \\
- \\
-\end{array}$ \\
\hline THORIUM-230 & $\begin{array}{l}05 / 15 / 86 \\
04 / 06 / 88 \\
07 / 13 / 88 \\
01 / 06 / 89 \\
09 / 19 / 89\end{array}$ & $\begin{array}{l}0001 \\
0001 \\
0001 \\
0001 \\
0001\end{array}$ & PCI/L & & $\begin{array}{l}0.3 \\
0.0 \\
0.2 \\
1.08 \\
0.1\end{array}$ & & $\begin{array}{l}1 . \\
1 . \\
1 . \\
1 . \\
1 .\end{array}$ & $\begin{array}{l}0.3 \\
0.5 \\
0.4 \\
0.507 \\
0.4\end{array}$ \\
\hline TIN & $\begin{array}{l}04 / 06 / 88 \\
07 / 13 / 88 \\
01 / 06 / 89 \\
09 / 19 / 89 \\
04 / 06 / 90 \\
01 / 16 / 91\end{array}$ & $\begin{array}{l}0001 \\
0001 \\
0001 \\
0001 \\
0001 \\
0001\end{array}$ & MG/L & $\begin{array}{l}< \\
< \\
<\end{array}$ & $\begin{array}{l}0.026 \\
0.013 \\
0.005 \\
0.1 \\
0.026 \\
0.03\end{array}$ & 1 & $\begin{array}{l}0.005 \\
0.005 \\
0.005 \\
0.1 \\
0.005 \\
0.03\end{array}$ & $\begin{array}{l}\dot{-} \\
\dot{-} \\
-\end{array}$ \\
\hline TOTAL DISSOLVED SOLIDS & $\begin{array}{l}05 / 15 / 86 \\
04 / 06 / 88 \\
07 / 13 / 88 \\
01 / 06 / 89 \\
09 / 19 / 89 \\
04 / 06 / 90 \\
01 / 16 / 91 \\
11 / 25 / 92\end{array}$ & $\begin{array}{l}0001 \\
0001 \\
0001 \\
0001 \\
0001 \\
0001 \\
0001 \\
0001\end{array}$ & MG/L & & $\begin{array}{l}4400 . \\
4160 . \\
4320 . \\
4296 . \\
3750 . \\
5050 . \\
5840 . \\
6220 .\end{array}$ & & $\begin{array}{l}10 . \\
10 . \\
10 . \\
10 . \\
10 . \\
10 . \\
10 . \\
10 .\end{array}$ & $\begin{array}{l}- \\
- \\
- \\
-\end{array}$ \\
\hline TOTAL KJELDAHL NITROGEN & $\begin{array}{l}01 / 16 / 91 \\
11 / 25 / 92\end{array}$ & $\begin{array}{l}0001 \\
0001\end{array}$ & MG/L & $<$ & 1. & & 1. & $\because$ \\
\hline TOTAL ORGAHIC CARBOH & $04 / 06 / 88$ & 0001 & $M G / L$ & & 110. & & 1. & - \\
\hline
\end{tabular}

PARAMETER VALUE IMDICATOR (PVI): \& L LESS THAN DETECTION LIMIT SAMPLE ID COOES:

0001 - FILTERED SAMPLE (.45 MICRONS)

OTHER PARAMETER VALUE FLAGS:

I - IHCREASED DETECTIOH LIMIT DUE TO REQUIRED DILUTION

J - estimated VALUE 
GROUNDWATER QUALITY OATA BY LOCATIOH

SITE: AMBOI AMBROSIA LAKE

LOCATION: 0793

MORTH COOROINATE: 55493.0 FT

EAST COORDINATE: $\quad 60225.7$ FT

06/10/80 TO $12 / 04 / 92$

REPORT DATE: 08/04/94

FORMATION OF COMPLETIOH: ALLUVIUH (AL)

IIYDRAULIC FLON RELATIONSHIP: UPGRADIENT (U)

\begin{tabular}{|c|c|c|c|c|c|c|c|c|}
\hline PARAMETER MAME & LOG DATE & $\underset{\text { ID }}{\text { SAMPLE }}$ & $\begin{array}{l}\text { UNITS OF } \\
\text { MEASURE }\end{array}$ & PVI & $\begin{array}{l}\text { PARAMETER } \\
\text { VALUE }\end{array}$ & FLAGS & $\begin{array}{l}\text { OETECTION } \\
\text { LIMIT }\end{array}$ & $\begin{array}{l}\text { PARAMETER } \\
\text { UNCERTAINTY }\end{array}$ \\
\hline TOTAL ORGAHIC CARBOA & $\begin{array}{l}07 / 13 / 88 \\
01 / 06 / 89 \\
09 / 19 / 89 \\
04 / 06 / 90 \\
01 / 16 / 91 \\
11 / 25 / 92\end{array}$ & $\begin{array}{l}0001 \\
0001 \\
0001 \\
0001 \\
0001 \\
0001\end{array}$ & MG/L & & $\begin{array}{l}97.8 \\
15 . \\
79.6 \\
25.9 \\
28 . \\
32 .\end{array}$ & $\mathbf{J}$ & $\begin{array}{l}1 . \\
1 . \\
1 . \\
1 . \\
1 .\end{array}$ & $\begin{array}{l}- \\
- \\
- \\
-\end{array}$ \\
\hline ZINC & $\begin{array}{l}04 / 06 / 88 \\
07 / 13 / 88 \\
01 / 06 / 89 \\
09 / 19 / 89 \\
04 / 06 / 90 \\
01 / 16 / 91\end{array}$ & $\begin{array}{l}0001 \\
0001 \\
0001 \\
0001 \\
0001 \\
0001\end{array}$ & MG/L & $<$ & $\begin{array}{l}0.068 \\
0.035 \\
0.01 \\
0.005 \\
0.008 \\
0.046\end{array}$ & & $\begin{array}{l}0.005 \\
0.005 \\
0.01 \\
0.005 \\
0.005 \\
0.005\end{array}$ & $\begin{array}{l}- \\
- \\
- \\
- \\
-\end{array}$ \\
\hline
\end{tabular}

PARAMETER VALUE INDICATOR (PVI): < - LESS THAN DETECTION LIMIT

SAMPLE ID COOES:

0001 - FILTERED SAMPLE (.45 MICROHS)

OTHER PARAMETER VALUE FLAGS:

H - HOLD TIME EXPIRED, VALUE SUSPECT

d - estimated VALUE

DATA FILE NAME: M: IDARTIAMBO1IGWQ10012.DAT 